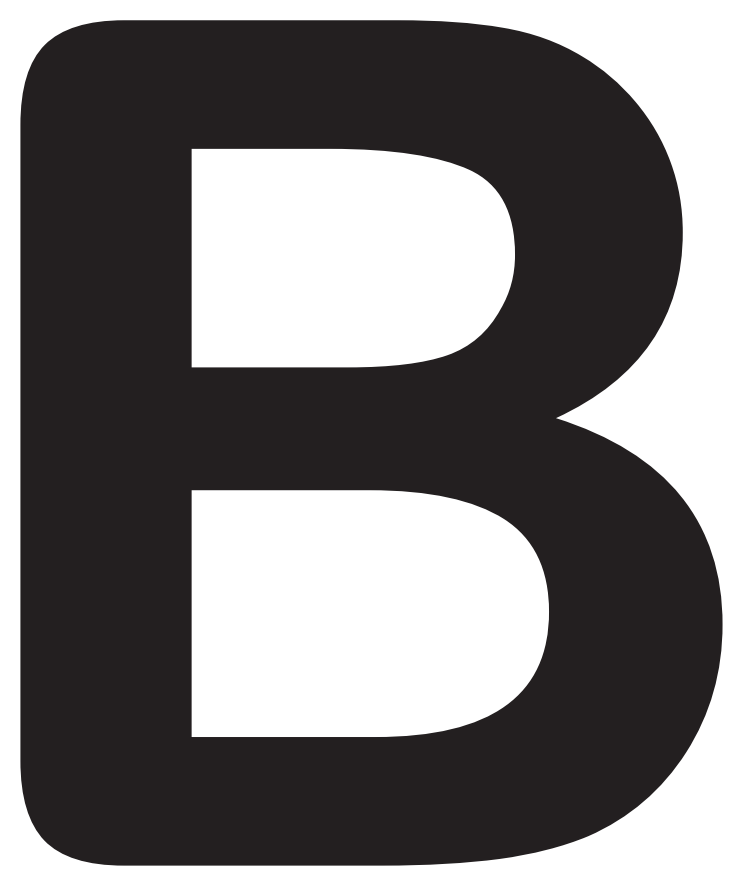

\title{
Scientific Sessions
}

(B)

Session numbers are prefixed by SS. Presentation numbers are prefixed by the letter $B$.

Sessions and abstracts are listed by days.

\footnotetext{
Thursday, March 7 .... 149 Friday, March 8 ........ 215 Saturday, March 9.... 265 Sunday, March 10 ..... 291 Monday, March 11..... 321
} 
Thursday, March 7 
$10: 30-12: 00$

Room B

\section{Cardiac}

SS 103

\section{CT and MRI in preoperative and postoperative evaluation}

Moderators:

G.A. Krombach; Giessen/DE

R. Sanz-Requena; Valencia/ES

\section{B-0001 10:30}

Multiparametric functional MRI for postoperative follow-up in patients with the ROSS-procedure: comparison of the aortic root replacement versus the subcoronary implantation technique C.O. Ritter', U. Baier ${ }^{1}$, S.P. Sommer', R. Leyh', H. Köstler', D. Hahn', M.J. Beer ${ }^{2}$; ${ }^{1}$ Würzburg/DE, ${ }^{2}$ Graz/AT (meinrad.beer@medunigraz.at)

Purpose: The ROSS procedure is an elegant therapeutic alternative for repair of severe aortic valve disease. Until now, only sparse information is available concerning the outcome of the different surgical approaches like the aortic root replacement (ARR) or the subcoronary implantation (SC) technique.

Methods and Materials: 62 consecutive MR examinations of 16 patients with operated severe aortic stenosis were acquired on a 1.5 T MR scanner 7-10 days (early), 3 (middle 1), 6 (middle 2) and 12 (late follow-up) months after ROSS operation. 8 patients ( $43 \pm 11 \mathrm{yrs}$ ) had the ARR-technique, 8 patients $(49 \pm 7 \mathrm{yrs})$ the SC. Morphometric and functional parameter were acquired by SSFP (cinematography, LGE) and GRE (flow analysis) sequences.

Results: Regression of LV mass was observed in all patients $(p<0.01)$ at early and middle follow-up without difference between both techniques $(p=0.141)$. Only slight changes of LV ejection fraction were observed without differences between both subgroups. Significant increases of the aortic orifice area were observed in the early and middle follow-up, more pronounced for patients with the SC technique $(4.6 \pm 1.4 \mathrm{~cm} 2 \mathrm{ARR}$ and $3.1 \pm 0.5 \mathrm{~cm} 2 \mathrm{SC}$ early follow-up versus $5.2 \pm 1.8 \mathrm{~cm} 2 \mathrm{ARR}$ and $4.0 \pm 1.1 \mathrm{~cm} 2$ middle follow-up). Mean aortic regurgitation was $11 \%$ with a slight increase during follow-up. Mild pulmonary regurgitation was observed in $88 \%$ of patients $(4.8 \pm 3.9 \mathrm{ml}$ for all) without changes during follow-up.

Conclusion: We observed significant morphometric improvements in the first year after the ROSS procedure irrespectively of the applied technique. Only mild aortic and pulmonary regurgitation developed. Significant increases of the aortic orifice area were observed.

\section{B-0002 10:39}

Flow pattern evaluation of apico-aortic conduit (CORREX) in high-risk patients with severe aortic stenosis: a cardiac magnetic resonance (MR) investigation

C. Mantini, A.R. Cotroneo, A. Tartaro, E. Di Gianfrancesco, C. Canosa, M. Foschi, D. Marinelli, G. Di Giammarco; Chieti/lT (c.mantini@rad.unich.it)

Purpose: Aortic valve bypass (AVB) relieves AS connecting left ventricular apex to descending thoracic aorta by a valved conduit (CORREX ${ }^{\mathrm{TM}}$ ). AVB is performed through a left thoracotomy, preferentially without cardiopulmonary bypass. Final distribution of flow through AVB and native aortic valve (NAV) is reported to be $60-70 \%$ to $30-40 \%$, although very little is known about true hemodynamics, including blood flow distribution through the ascending and descending aorta after the procedure. Methods and Materials: Ten high-risk AS patients underwent AVB utilising CORREX $^{\mathrm{TM}}$. In seven 30-day survivors, flow measurement was obtained using through-plane breath-hold phase-contrast MR sequences acquired in ascending aorta $(1 \mathrm{~cm}$ above the sinotubular junction), descending thoracic aorta $(3 \mathrm{~cm}$ proximally and distally to conduit anastomosis) and at ventricular portion of the conduit. MR was performed either before (mean $=2.5$ days; $\mathrm{SD}=2.4$ ) and after surgery (mean=11.7days; $S D=2.14$ ). Post-procedural left ventricular stroke volume (LVSV) was considered either discriminating the two left ventricular outflows and as a sum compared to preoperative LVSV through native aortic valve. Flow gradient (FG) across AVB was finally considered.

Results: Average age of patients was $77.4 \pm 7.7$ yo. Average aortic valve area was $0.62(S D=0.21) \mathrm{cm} 2$. LVSV through AVB was $68 \% \pm 10 \%$ of total. We registered a striking increase in postoperative LVSV even if it did not reach significance cause a too small group of patients. The backward flow at NAV preoperatively did not increase significantly after procedure (preop $=3.7 \mathrm{ml} ; \mathrm{SD}=2.4 \mathrm{vs}$ postop $=5.2 ; \mathrm{SD}=6.4$; $\mathrm{p}=\mathrm{ns})$. No $F G$ across AVB was recorded.
Conclusion: AVB relieves outflow obstruction in AS, resulting in balanced flow redistribution between NAV and AVB. It represents a valuable and safe alternative in high-risk patients with AS ineligible for standard AV replacement or TAVI.

\section{B-0003 10:48}

Pre- and postinterventional evaluation of cardiac function in patients suffering from mitral regurgitation using cardiac MRI

P. Krumm, C.S. Zuern, T.H. Wurster, C. Bretschneider, S. Mangold, B. Klumpp, A. May, C.D. Claussen, U. Kramer; Tübingen/DE (patrickkrumm@gmail.com)

Purpose: Consecutive mitral regurgitation after myocardial infarction and secondary ventricular dilatation results in a bad prognosis and high surgical risk due to cardiac comorbidity. Mitral clipping (MitraClip, Abbott Vascular) is a minimal-invasive percutaneous interventional palliative method to reduce mitral regurgitation. The purpose of this study was to evaluate pre- and postinterventional cardiac function and to quantify the degree of left atrial dilatation.

Methods and Materials: 21 patients were prospectively included in this study. Magnetic resonance imaging (MRI) was performed before and 6 weeks after intervention. Cardiac function was evaluated using steady-state free precession (SSFP) cine sequences by assessment of enddiastolic volume (EDV), ejection fraction (EF). Planimetry of the left atrium (LA) was performed in identical slices in four-chamber view. Paired statistical analysis was applied.

Results: 16 patients underwent postinterventional MRI. In paired analysis, mean difference in pre- and postinterventional MRI was for EDV -7.5 ml (95\% upper confidence interval [ $\mathrm{UCl}]-1.0 \mathrm{ml}, 95 \%$ lower $\mathrm{Cl}(\mathrm{LCl})-14.1 \mathrm{ml})$; for $\mathrm{EF}+3.9 \%$ (UCl $6.8 \%, \mathrm{LCl} 1.0 \%)$; for LA $-2.6 \mathrm{~cm}^{2}\left(\mathrm{UCl}-1.5 \mathrm{~cm}^{2}, \mathrm{LCl}-3.8 \mathrm{ml}\right)(\mathrm{p}<0.05)$.

Conclusion: The quantifiable left atrial dilatation decreased significantly as an indirect sign of reduced mitral regurgitation. EDV decreased in $81 \%$ of the patients. Postinterventional ejection fraction improved particularly in patients with initially low EF, in two patients EF did not improve. This can be considered as success of the palliative intervention in seriously ill patients. The clinical benefit of a successful MitraClip intervention can be monitored and validated using MRI in clinical follow-up examinations.

\section{B-0004 10:57}

Pre- and post-interventional analysis of myocardial strain in patients undergoing mitral valve clipping using cardiac MRI

P. Krumm, C.S. Zuern, T.H. Wurster, A. Seeger, S. Mangold, B. Klumpp, C.D. Claussen, A. May, U. Kramer; Tübingen/DE (patrickkrumm@gmail.com)

Purpose: Percutaneous interventional mitral clipping (MitraClip, Abbott Vascular) is performed to reduce mitral regurgitation. Effects of the intervention on myocardial strain were examined with a new feature tracking software.

Methods and Materials: 20 patients were prospectively included in this study. Magnetic resonance imaging (MRI) was performed before and 6 weeks after successful intervention. Myocardial strain analysis was performed using a dedicated feature tracking software (2D CPA MR, TomTec) based on steady-state free precession (SSFP) cine sequences in four-chamber view (4CV) and short axis (SA). Longitudinal myocardial strain was evaluated quantitatively using the timeto-peak (T2P) and peak value analysis. Paired statistical analysis was applied for pre- and post-interventional peak and T2P values for each myocardial segment and average values.

Results: 15 patients underwent post-interventional follow-up MRI. In paired strain analysis, arithmetic mean of differences for T2P was $63.3 \mathrm{~ms}( \pm 100,7 \mathrm{~ms})$, for strain peak values $3.2 \%( \pm 8.2 \%)$. Arithmetic mean of differences for peak values in apical segment 17 was $12.16 \%$, in segment 14 apical septal $9.2 \%$. Arithmetic mean of differences for T2P in apical segment 17 was $103.8 \mathrm{~ms}$, in segment 14 apical septal $188.9 \mathrm{~ms}$.

Conclusion: MitraClip intervention changes myocardial strain patterns: Iongitudinal strain T2P was reached $63.3 \mathrm{~ms}$ later in post-interventional analysis. However, peak values in longitudinal strain increased, particularly in apical segments. Increased strain values indicate a more effective myocardial contraction after clipping. A slower T2P for all myocardial segments might be due to the afterload is elevated relatively by reducing the regurgitation fraction with mitral clipping. 


\section{B-0005 11:06}

Pressure overloaded right ventricles: importance of trabeculae in evaluation of RV function by CMR

M.M.P. Driessen 1', V.J.M. Baggen', H.G. Freling², F.J. Meijboom', G.T. Sieswerda ${ }^{1}$, R.J. Snijder ${ }^{3}$, T. Leiner ${ }^{1}$, T.P. Willems ${ }^{2} ;{ }^{1}$ Utrecht/NL,

${ }^{2}$ Groningen/NL, ${ }^{3}$ Nieuwegein/NL (m.m.p.driessen-2@umcutrecht.nl)

Purpose: Cardiac magnetic resonance imaging (CMR) is the preferred method to evaluate right ventricular (RV) volumes and function. This study aimed to determine the impact and reproducibility of excluding trabeculae using semi-automatic software on right ventricular (RV) volumes and function assessed by CMR in patients with pressure overloaded RVs.

Methods and Materials: Four groups of 20 patients (pulmonary hypertension, arterial switch operation (ASO), tetralogy of Fallot (TOF), systemic RV) and 20 healthy subjects underwent short-axis multislice cine CMR. After manual contour tracing, trabeculae were excluded from the blood volume using semi-automatic pixel-intensity based software. Differences in volumes and function after excluding trabeculae were tested using paired samples $\mathrm{T}$-test. For intra- and interobserver agreement 25 datasets were re-analysed.

Results: Exclusion of trabeculae from the blood volume resulted in diminished end-diastolic and end-systolic volumes. This led to a significantly larger difference in $\mathrm{RV}$ volumes and absolute ejection fraction of patient groups, $4.7 \pm 1.6 \%$ in $\mathrm{PH}$ patients, $6.0 \pm 1.5$ in ASO patients, $7.2 \pm 1.7$ in TOF patients, and $10.1 \pm 2.3 \%$ in the systemic RV group, than in controls $(3.4 \pm 0.8 \%)$. For both methods there was a high inter- and intraobserver agreement in all measurements (correlation coefficients > 0.9; narrow limits of agreement).

Conclusion: Exclusion of trabeculae and papillary muscles from the blood volume results in a significant and clinically relevant change in RV volumes, RVEF and RV mass, for patients with pressure overloaded RVs. Using semi-automatic pixelintensity-based software, exclusion of trabeculae from the blood volume can be done reproducibly.

\section{B-0006 11:15}

Opacification ratios to detect changes in coronary flow by computed tomography angiography - comparison between pre- and poststented lesions

P.B. Rizzi', R.J. Cerci', P. Lemos², C.E. Rochitte², I. Gottlieb³, R.T. George' ${ }^{1}$ J. Texter', A.C. Lardo', J.A.C. Lima'; ${ }^{1}$ Baltimore, MD/US, ${ }^{2}$ São Paulo/BR,

3Rio de Janeiro/BR (paty.rizzi@gmail.com)

Purpose: To test whether percutaneous coronary revascularization (PCI) improves the opacification ratio between pre- and post-stenotic segments of coronary arteries using 64-MDCT coronary angiography (CTCA).

Methods and Materials: Twenty-one patients who underwent a CTCA prior to and a CTCA plus an invasive coronary angiography (ICA) after PCI were enrolled. Luminal CTCA opacification was measured in $\mathrm{HU}$ at no more than $3 \mathrm{~mm}$ proximal and distal to the stenosis or stents borders. Opacification ratio (ORCT) was defined as the ratio between distal over proximal measurements, and were also calculated for two equivalent segments in normal vessels. A mathematical model, accounting for technical variables influencing contrast enhancement, was applied to each scan and compared to true measurements. Pre- and post-treatment ORCT were compared using T-test.

Results: Final analysis included 28 stenotic and 16 normal coronary segments. There was significant improvement in mean stenotic ORCT values after $\mathrm{PCI}$ (ORCT (pre) $=0.82(95 \% \mathrm{Cl}: 0.75,0.89)$; ORCT $($ post $)=0.94(95 \% \mathrm{Cl}: 0.84,1.0)), p=0.014$. ORCT (pre) also differed from the ORCT in normal vessels (index ORCT (normal) $=0.94,95 \% \mathrm{Cl}=(0.90,0.99), \mathrm{p}=0.002)$ and from the calculated theoretical ORCT (index ORCT (model) $=0.95$ (95\% Cl: $0.92,0.07), p<0.001)$. No significant differences were detected in normal vessels from index to follow-up scans $(p=0.73)$, as well as from those in comparison with theoretical ratios $(p=0.95$ and $p=0.76$; index and follow-up, respectively).

Conclusion: Coronary ORCTs across lesions show significant improvement after $\mathrm{PCl}$. Theoretical models correcting variations in luminal enhancement due to technical factors can serve as references for the measurements across stenotic segments.

\section{B-0007 11:24}

\section{Additional value of cardiac computed tomography to assess} prosthetic valvular dysfunctions

A.A. Azarine, N. Hrynchyshyn, A. Samadi, L. Perdrix, G. Ashrafpoor, A. Redheuil, L. Macron, E. Mousseaux, B. Diebold; Paris/FR (arshid.azarine@egp.aphp.fr)

Purpose: To evaluate the potential contribution of cardiac multidetector computed tomography (MDCT) on prosthetic valve dysfunctions (PVD).
Methods and Materials: 38 patients with valvular prosthesis underwent contrastenhanced 64-row cardiac MDCT (Lightspeed VCT, GE Medical Systems, Milwaukee, USA) using a classical triphasic contrast injection protocol (iobitridol, Xenetix, Guerbet, France), within 2 days or less after a transthoracic \pm transoesophageal echocardiogram. A total of 50 prostheses were explored including 23 mechanical and 17 bioprosthetic valves. According to echocardiogram, 2 groups were defined: normal $(n=27)$ and PVD $(n=23)$ with 2 subgroups stenotic $(n=15)$ and regurgitant $(n=8)$. Multiplanar analysis of the MDCT dataset of these patients was performed during diastole and systole blinded to the echocardiographic findings. The results were compared to surgical data, when available $(n=16)$.

Results: MDCT demonstrated PVD in 29 cases: limitation in the opening/closure of the leaflets (12/2), annular tilting (8), calcification of leaflets (4), protrusive calcification of the ring (7), aspect of suture loosening (12), aspect of thrombosis (2) or pannus (4). MDCT found 22 PVD out of 23 in the PVD group and 6 PVD in the normal group: sub-annular calcifications or pannus (3), perivalvular abscesses (3), pseudoaneurysm (1). In 3 of the "normal group" who underwent further surgery, PVD was confirmed. MDCT was abnormal in 11/15 in the stenotic group and 8/8 in the regurgitant group.

Conclusion: MDCT is becoming a reliable and problem resolving complimentary technique in analyzing prosthetic valve dysfunction.

\section{B-0008 11:33}

Cardiac computed tomography angiography results in diagnostic and therapeutic change in prosthetic heart valve endocarditis J. Habets ${ }^{1}$, W. Tanis', L.A. van Herwerden', W.P.T.M. Mali',

R.B.A. van den Brink ${ }^{2}$, B.A.J.M. de Mol'2, S.A.J. Chamuleau', R.P.J. Budde'; ${ }^{1}$ Utrecht/NL, ${ }^{2}$ Amsterdam/NL (J.Habets@umcutrecht.nl)

Purpose: Echocardiography plays a key role in diagnosing prosthetic heart valve (PHV) endocarditis but can miss signs of PHV endocarditis which advocates for novel imaging techniques to improve diagnostic accuracy and patient outcome. The aim of this study was to determine the complementary diagnostic value of cardiac computed tomography angiography (CTA) to the clinical routine workup including transthoracic and transesophageal echocardiography (TTE/TEE) in patients with suspected PHV endocarditis and assess its impact on patient treatment.

Methods and Materials: A diagnostic prospective cross-sectional study which included patients with suspected PHV endocarditis. Besides the clinical routine workup (including TTE/TEE), CTA was performed to assess its complementary diagnostic and therapeutic value with an expert panel as reference standard. Diagnostic accuracy was determined for PHV endocarditis in general, vegetations and peri-annular complications (mycotic aneurysms/abscesses) with surgical findings or clinical follow-up as reference standard.

Results: Twenty-eight patients were included. Addition of CTA to the normal clinical routine workup (including TTE and TEE) resulted in a diagnostic change in six patients $(21 \%)$ and treatment change in seven patients $(25 \%)$. Diagnostic accuracy of routine clinical workup plus CTA was superior to clinical routine workup alone for the detection of PHV endocarditis in general, vegetations and peri-annular extension. Conclusion: This study demonstrates that CTA and clinical workup including TTE and TEE are complementary in patients with PHV endocarditis. CTA changes treatment strategy. Therefore, CTA imaging has to be performed after clinical routine workup in every patient with suspected PHV endocarditis.

\section{B-0009 $11: 42$}

Anatomical variance of coronary venous system in dual source computed tomography

M. Krupinski, M. Kuniewicz, J. Lelakowski, M. Irzyk, B. Laskowicz, M. Urbanczyk; Krakow/PL

Purpose: The aim of the study was to assess the anatomical variance of coronary venous system using dual source computed tomography (DSCT) in patients scheduled for cardiac resynchronisation therapy (CRT).

Methods and Materials: 32 patients ( 27 males, age $65 \pm 11$ years) with heart failure were included in the study. Anatomical variants were divided in three groups, dependent on the continuity of the cardiac venous system at the crux cordis. Cardiac veins were analysed in order to determine their number, distance from coronary sinus, diameter and angle of insertion.

Results: $16(50 \%)$ patients revealed separate insertion of the coronary sinus (CS) and small cardiac vein in right atrium, $13(40.5 \%)$ presented with continuity of anterior and posterior venous system at the crux cordis and in three patients $(9.5 \%)$ posterior interventricular vein (PIV) did not terminate to the CS. Posterior vein of the left ventricle (PVLV) was present in all (100\%) patients and left marginal vein (LMV) in $19(59.4 \%)$ cases. The mean distance from CS to PVLV and LMV was $30.8 \pm 15.1 \mathrm{~mm}$ and $73.2 \pm 18.8 \mathrm{~mm}$, respectively. The angle of insertion of PVLV to 
great cardiac vein (GCV) was $58^{\circ}$ and for the LMV revealed $61.5^{\circ}$. The diameter of insertion of PVLV and LMV to GCV was $2.7 \pm 1.3$ and $2.2 \pm 1.1$, respectively. In subsequently performed CRT procedure left ventricle (LV) lead was implanted in PVLV in $20(62.5 \%)$ cases and in LMV in 12 (37.5\%) patients.

Conclusion: Coronary venous system variance can be assessed in DSCT and its knowledge facilitates proper LV lead placement in CRT.

\section{B-0010 11:51}

Feasibility of MRI in patients with implanted subcutaneous loop

recorder type REVEAL XTß: data from the surprise study

L. Christensen, A. Christensen, H. Christensen; Copenhagen/DK

(louisamchristensen@gmail.com)

Purpose: Implanted devices such as pacemakers and loop-recorders have until recently been a contraindication to MRI. MRI compatible loop recorders have been marketed, and the use of these devices is increasing. We aimed to report our experience with performing MRI in the patient population having received a $\mathrm{MRI}$ compatible loop-recorder in relation to a study protocol investigating the true frequency of atrial fibrillation after stroke or TIA by implantation of a REVEAL XT® loop recorder (Medtronic Inc). subcutaneously on the thorax for up to 3 years of continuous monitoring.

Methods and Materials: We retrospectively revised all MRI reports in this patient group during the study period and registered problems with performing the investigation, including whether any reverse affects were reported.

Results: Out of the 84 patients who have been implanted, 12 patients have had $\mathrm{MRI}$, one patient on three occasions constituting a total of $15 \mathrm{MRIs} ; 13$ examinations were MRI of the brain, one of the knee and one of the heart. None of the patients experienced any heat, pain or movement of the device; the MRI examination of the heart was cancelled due to the formation of artefacts on the images of the heart; an effect not reported using CT of the thorax/heart.

Conclusion: MRI appears not to cause any safety problems in the patients having the REVEAL XT® implanted. Artefacts may occur in MRI investigation of the heart; however, CT can be used as an alternative in most cases.

Author Disclosures:

L. Christensen: Grant Recipient; Grant from Medtronic. Speaker; Speaker for

Medtronic. H. Christensen: Research/Grant Support; Grant from Medtronic.

Speaker; Speaker for Medtronic.

\section{$10: 30-12: 00$}

Room C

Neuro

\section{SS 111}

\section{From structure to function}

Moderators:

S.J. Bakke; Oslo/NO

B. Ertl-Wagner; Munich/DE

\section{B-0011 10:30}

Regional cortical thickness is associated with neurocognitive profile in progressive myoclonus epilepsy type 1 (EPM1, UnverrichtLundborg disease)

S. Suoranta ${ }^{1}$, E. Niskanen², M. Äikiä¹, P. Koskenkorva ${ }^{1}$, M. Könönen ${ }^{1}$, R. Kälviäinen', R. Vanninen'; ${ }^{1}$ Kuopio/FI, ${ }^{2}$ Vaasa/FI (sanna.suoranta@fimnet.fi)

Purpose: To investigate the relationship between cortical thickness and neurocognitive function in patients with progressive myoclonus epilepsy type 1, EPM1. Methods and Materials: Sixty-three genetically verified EPM1 patients underwent T1-weighted 3-dimensional imaging. The regional cortical thicknesses $(\mathrm{CTH})$ were analysed. The patients were clinically evaluated using the Unified Myoclonus Rating Scale (UMRS) test pattern. The Wechsler Adult Intelligence Scale (WAIS-R) was used to assess the patients' neurocognitive abilities and intelligence. Patients were divided into sub-groups by their myoclonus in action and global disability scores. Results: The EPM1 patients showed heterogeneous findings in clinical and neuropsychological evaluation; the mean myoclonus in action scores were $48.3 \pm$ 28.9, range 2-122 (maximum 160). Cognitive functioning of EPM1 patients varied from high average to defective, mean Full Scale IQ score was $80.2 \pm 14.4$, range 50-114. No signs of memory impairment were found. Utilising the CTH analysis, we observed that the EPM1 patients with a more severe form of the disorder had thinner cortex than the patients with no disability or milder form of the disease. The affected anatomical areas included those responsible for processing of verbal and complex visual data (Broca's and Wernicke's areas, fusiform gyrus, retrosplenial and perirhinal cortex) and higher-order cognitive functions (posterior cingulate and anterior prefrontal cortex).

Conclusion: The observed associations provide neuroanatomical-biological background for cognitive phenotypes in Unverricht-Lundborg disease. CTH analysis can supply new information on visually normal MR images. Further evaluation is needed for the application of CTH in individual patients.

Author Disclosures:

S. Suoranta: Research/Grant Support; EVO grants 5772751 and 5063523 from the Kuopio University Hospital, Strategic Funding from the University of Eastern Finland from the UEF-Brain consortium.

\section{B-0012 10:39}

Language impairment and reduced structural connectivity in

Rolandic epilepsy

R. Besseling, J. Jansen, W.H. Backes; Maastricht/NL

(r.m.h.besseling@gmail.com)

Purpose: Rolandic epilepsy (RE) is a childhood epilepsy with mild seizure semiology and epileptic discharges originating from the sensorimotor (Rolandic) area. A serious co-morbidity in RE is language impairment, for which we aim to find a correlate using structural connectivity.

Methods and Materials: Diffusion MRI was performed at 3.0 Tesla and $2 \times 2 \times 2 \mathrm{~mm}$ voxel size with $b=1200 \mathrm{~s} / \mathrm{mm}^{2}$ and 66 diffusion gradient directions in 23 children with RE with proven language deficits and 23 matched controls. Constrained spherical deconvolution provided voxel-wise tract orientations and whole-brain tractography (5M streamlines). For each of the 4 Rolandic areas (bilateral pre- and postcentral gyri), the streamlines to any of the 70 regions, obtained by automatic cortical parcellation (Freesurfer), were selected from the whole-brain tractogram. For reliable connections (top $20 \%$ of number of streamlines), connectivity was quantified by tract fractional anisotropy (FA), compared between groups, and correlated to language scores.

Results: In the left hemisphere, reduced tract FA was found for connections between the Rolandic areas and inferior frontal gyrus (Broca's area) and supramarginal gyrus (Wernicke's area), but not for contralateral homologue regions. For the aberrant connection between the left postcentral and inferior frontal gyrus, tract FA decreased with languages score in the RE group $(p=0.04)$.

Conclusion: In RE structural connectivity between Rolandic and language areas is disturbed and correlates with the language dysfunction. This provides a neuronal link between the location of the epileptic discharges and the language impairment. This research was supported by the Dutch Epilepsy Foundation.

\section{B-0013 10:48}

Relative contributory role of Interictal/Ictal SPECT, interictal PET, MR spectroscopy and T2 relaxometry in localisation of seizure focus in temporal lobe epilepsies: a metaanalysis and systematic review V. Venugopal ${ }^{1}$, S.K. Puri ${ }^{1}$, I. Afshan ${ }^{1}$, A.D. Prabhu ${ }^{2} ;{ }^{1}$ New Delhi/IN,${ }^{2}$ Chennai/IN

Purpose: To perform a meta-analysis to quantitatively assess the seizure localisation potential of SPECT, PET, MR spectroscopy (MRS) and T2 relaxometry (T2R) in patients with temporal lobe epilepsy (TLE).

Methods and Materials: A structured search strategy of PubMed, EMBASE, Cochrane Central Register and other databases was used to English language studies in which the diagnostic potential of FDG-PET, SPECT, MRS and T2R were assessed in patients with TLE. Studies were selected independently by three radiologists. Quality Assessment of Diagnostic Accuracy Studies tool was employed to assess the quality of the studies. Meta-Disc version 1.4 was used to describe and calculate sensitivity, specificity, summary receiver operating characteristic (SROC) curves and area under the curve (AUC) for these modalities. The meta-analysis was performed in accordance with the PRISMA guidelines.

Results: The analysis noted gross heterogeneity among the studies regarding study design, methodology and interpretation criteria. A total of 54 studies met the inclusion criteria. For ictal SPECT, interictal PET and interictal SPECT pooled sensitivity was $83(95 \% \mathrm{Cl} 80-85), 80$ (95\% Cl 78-82) and 58\%(95\% Cl 50-62), respectively. For MRS and T2R, pooled sensitivity was $76(95 \% \mathrm{Cl} 74-78)$ and $79 \%$ (95 Cl 76-81), respectively. Thus ictal SPECT had a slightly better sensivity, specificity and odds ratio. However, SROC analysis showed the diagnostic value of ictal SPECT in TLE has no significant difference compared with PET, MRS or T2R. Conclusion: Although interictal SPECT is found to have a slightly better diagnostic value in this study, no statistically significant difference is seen in the diagnostic potential compared with other modalities. 


\section{B-0014 10:57}

Subtle changes in normal appearing white matter precede development of white matter lesions

B.F.J. Verhaaren, M. de Groot, R. de Boer, S. Klein, A. Hofman,

A. van der Lugt, M.A. Ikram, W.J. Niessen, M.W. Vernooij; Rotterdam/NL (b.verhaaren@erasmusmc.nl)

Purpose: It is unknown whether white matter lesions (WMLs) develop instantaneously in previously normal brain areas or whether development of WMLs is a gradual process, in which tissue changes are already present before they become apparent on MRI as WMLs. We investigated whether WMLs in the general population are preceded by changes in the white matter.

Methods and Materials: In 689 participants from the general population (mean age 67 years), we performed two MRI scans (including DTI and FLAIR sequences) 3.5 years apart using the same 1.5-T scanner. Using automated tissue segmentation, we identified normal appearing white matter (NAWM) at baseline. We assessed which NAWM areas converted into WMLs during follow-up, and differentiated these areas into WML growth and de-novo WMLs. Fractional anisotropy (FA), mean diffusivity (MD) and FLAIR intensity of areas converting to WMLs and areas of persistent NAWM were compared using three approaches: a whole-brain analysis, a regionally matched approach and a voxel-wise approach.

Results: All three approaches showed that low FA, high MD and high FLAIR intensity at baseline were associated with WML development during follow-up. Compared to persistent NAWM areas, NAWM areas converting to WMLs had lower FA 0.337 vs. $0.387, p<0.001)$, higher $\mathrm{MD}\left(0.910^{*} 10-3 \mathrm{~mm} 2 / \mathrm{s}\right.$ vs. $0.729^{*} 10-3 \mathrm{~mm} 2 / \mathrm{s}, \mathrm{p}<$ $0.001)$ and higher normalised FLAIR-intensity $(1.233$ vs. $-0.340, p<0.001)$. This applied to both NAWM areas of growing and de-novo WMLs.

Conclusion: Subtle white matter changes are present before WMLs develop. This suggests that WMLs develop gradually and that WMLs are only the tip of the iceberg of white matter pathology.

\section{B-0015 11:06}

Retrograde degeneration of optic nerve and visual pathway in open angle glaucoma: a morphologic evaluation using $3 \mathrm{~T}$ MRI S. Sidek, F. A. Rahman, K. Rahmat, N. Mohd Ramli, N. Mohd Ramli; Kuala Lumpur/MY (bhkimy_7@hotmail.com)

Purpose: This study aimed at assessing glaucoma disease and the severity causing retrograde degeneration and atrophy of the optic nerve and optic radiation by using volumetric assessment and diffusion tensor imaging with 3 T MRI. To measure the optic nerve volume, fractional anisotropy (FA) and mean diffusivity (MD) for optic nerve and optic radiation in patients with bilateral mild and severe glaucoma and comparison made with normal subjects.

Methods and Materials: 90 subjects with ages ranging from 40 to 80 years ( 30 normal subjects, 30 mild glaucoma, 30 severe glaucoma) underwent 3 T MRI. Images were acquired with AX FSPGR 3D sequence (TE 1.868; TR $6.8241 .2 \mathrm{~mm}$ ) and DTI. The acquired images were post-processed using NIFTI (Neuroimaging Informatics Technology Initiative) format. Using NeuRoi software, each optic nerve volume was measured. Using Functool GE DTI software, the FA and MD values were obtained from each optic nerve and optic radiation. We evaluated 180 optic nerves and 180 optic radiations.

Results: Multiple comparisons show significant difference of the optic nerve volume and FA and MD of the optic nerve and optic radiation, between normal subject and mild glaucoma group ( $\left.\mathrm{p}^{2} 0.001\right)$, between normal subject and severe glaucoma group $\left(p^{2} 0.001\right)$ and between mild glaucoma group and severe glaucoma group ( $\left.p^{2} 0.001\right)$. Conclusion: Retrograde degeneration of optic nerve and optic radiation occurs in glaucoma and the volume loss, reduction of the FA values and increasing MD values correlate with disease severity. These findings highlight the importance of aggressive therapy for those patients at highest risk.

\section{B-0016 11:15}

High blood pressure and cerebral white matter lesion progression in the general population

B.F.J. Verhaaren, M.W. Vernooij, R. de Boer, A. Hofman, W.J. Niessen, A. van der Lugt, M.A. Ikram; Rotterdam/NL (b.verhaaren@erasmusmc.nl)

Purpose: High blood pressure is considered to be important in the development of cerebral white matter lesions (WMLs) in the elderly. Yet, current studies are still limited in demonstrating causality. We sought to overcome these limitations in a longitudinal MRI study.

Methods and Materials: In 678 participants from the population-based Rotterdam Scan Study, we investigated the relationship of systolic blood pressure (SBP), diastolic blood pressure (DBP) and pulse pressure (PP) with annual WML progression.
Mean SBP, DBP and PP were assessed over a 5-year period before longitudinal MRI scanning. WML progression was measured with a fully automated quantification method using two MRI scans 3.5 years apart. For the analyses we used linear regression models and evaluated adjustments for age, sex, cardiovascular factors and pre-existing WML volume. In addition, we evaluated WML progression related to control of hypertension.

Results: Both SBP and DBP were significantly associated with annual WML progression (0.08 (95\%-Cl: $0.03 ; 0.14) \mathrm{mL} /$ year per SD increase in SBP; and 0.08 $(0.03 ; 0.14) \mathrm{mL} /$ year per $\mathrm{SD}$ increase in $\mathrm{DBP})$. PP was only borderline significantly associated with WML progression. After adjustment for pre-existing WML volume, only SBP remained significantly associated: $0.08(0.03 ; 0.13), \mathrm{mL} / \mathrm{year}$ per SD. Uncontrolled hypertensives had $0.17(0.01 ; 0.32) \mathrm{mL} /$ year more progression than controlled hypertensives, and $0.14(0.03 ; 0.25) \mathrm{mL} /$ year more than normotensives. There was no significant difference between controlled hypertensives and normotensives.

Conclusion: Our results confirm that high blood pressure precedes WML progression and further support control of high blood pressure as a way to prevent WML development.

\section{B-0017 11:24}

MRI texture analysis in Unverricht-Lundborg disease reveals more complex and heterogeneous textural appearance in thalami, putamen and amygdala than in controls

K.K. Holli', S. Suoranta ${ }^{2}$, E. Niskanen ${ }^{3}$, P. Dastidar', H. Eskola', R. Vanninen²; 1Tampere/FI, ${ }^{1}$ Kuopio/FI, ${ }^{3}$ Vaasa/FI (sanna.suoranta@kuh.fi)

Purpose: Unverricht-Lundborg disease (EPM1) caused by mutations in cystatin $B$ gene is a rare autosomal recessive neurodegenerative disorder characterised by stimulus-sensitive myoclonus and tonic-clonic epileptic seizures. MRI of the brain is usually normal at the time of diagnosis. Texture analysis (TA) allows one to calculate mathematical patterns, i.e. texture features from MR images which can be used to detect, characterise and classify the possible underlying subtle changes in deep grey matter in EPM1 patients.

Methods and Materials: Ten EPM1 patients and ten age and sex matched control subjects underwent three-dimensional T1-weighted MRI (1.5 T) of the brain. TA was conducted with MaZda software. Spherical 3D volumes of interest (VOIs) were placed bilaterally in the thalamus, caudate nucleus, putamen, amygdala, and hippocampus.

Results: The deep grey matter structures were normal when assessed visually. TA revealed significantly differing $(p<0.05$, Mann-Whitney $U$ test) texture features in EPM1 patients. The features measuring complexities of image texture differed significantly bilaterally in thalami and in the right putaminal VOI indicating that patients have a more complex and heterogeneous textural appearance in these regions than controls. First-order histogram-based features measuring the intensity and contrast of given regions were also significantly different in thalamus, the right putaminal VOI and left amygdaloid VOI. No changes were found in hippocampi or caudate nuclei.

Conclusion: TA provides an additional and sensitive tool for extracting subtle structural information from MR images of EPM1 patients. Our MRI TA findings were parallel with previous studies with evoked potentials and PET, indicating thalamocortical abnormalities in EPM1.

\section{B-0018 11:33}

Structural MRI-assisted diagnosis of major depressive disorder using surface-based volumetric parameter classification approach L. Qiu, S. Lui, X. Huang, J. Zhang, X. Yang, Q. Yue, L. Zou, Q. Gong; Chengdu/CN (q/h20050616@gmail.com)

Purpose: To explore the diagnostic value of surface-based multiple morphometric parameters (including cortical thickness, pial area and volume) in first-episode, treatment-naive major depressive disorder (MDD) patients.

Methods and Materials: Thirst-one first-episode, treatment-naive MDD patients and 31 age, gender and years of education-matched controls were included in present study. Image processing was performed using the Freesurfer software. Three morphometric parameters including volume, thickness and surface area at each spatial location on the cortical surface was used to discriminate depressed patients from controls using a support vector machine (SVM) analytic approach, and a spatially distributed pattern of regions with maximal classification weights was identified.

Results: In the right hemisphere, all the three morphometric parameters showed above chance classification (accuracy for cortical thickness $=78 \%$, $p^{2} 0.001$; accuracy for volume $=72 \%$, $\mathrm{p}^{2} 0.001$; accuracy for pial area $=66 \%, \mathrm{p}^{2} 0.008$ ) for first-episodedepressed patients. While, in the left hemisphere, only cortical thickness (diag- 
nostic accuracy $=72 \%, \mathrm{p}^{2} 0.001$ ) and pial area (diagnostic accuracy $=69 \%, \mathrm{p}^{2} 0.003$ ) provided statistically significant classification for the first-episode, treatment-naive MDD patients.

Conclusion: Our results confirmed that volume-related parameters could differentiate the first-episode, treatment-naïve MDD patients from controls, and the right cortical thickness provided highest prediction accuracy, which might be useful for detecting early MDD. The spatial patterns detected using SVM may help further exploration of the specific genetic and neuropathological underpinnings of MDD as the three morphologic parameters are believed to be genetically and phenotypically independent.

\section{B-0019 11:42}

Resting-state networks, and peculiarities of the brain metabolism in patients with Parkinson's disease (PD) and different level of cognitive impairment $(\mathrm{Cl})$ : fMRI and ${ }^{1} \mathrm{H}$ MRS study

Z.Z. Rozhkova, O.M. Omelchenko; Kiev/UA (invivo@ukr.net)

Purpose: We use fMRI data for characteristics of the cortical connectivity in the absence of external input and MRS data for the characteristics of the local metabolic state of the brain.

Methods and Materials: Three groups of PD patients are studied by $\mathrm{AMRI}$ and ${ }^{1} \mathrm{H}$ MRS with 1.5 TSIGNA (GE). The $1^{\text {st }}$ group (DPDG): 13 PD patients with dementia (MMSE< 25). The $2^{\text {nd }}$ group (CIPDG): 15 PD patients with mild $\mathrm{Cl}\left(30>\mathrm{MMSE}^{325}\right)$. The $3^{\text {rd }}$ group (NPDG): 12 PD patients with normal cognitive function (MMSE> 30). $\mathrm{fMRI}$ data are obtained with block design parametric paradigm of EPI: TR/ $T E=3000 / 56 \mathrm{~ms}$, phases per location $=72$. Spectra are recorded in the white matter of the anterior (APCG), and in the posterior portions of cingulate gyrus (PPCG). Results: In the NPDG connections between the APCG and the PPCG, bilatera inferior parietal gyrus was found. In the NPDG activation of APCG decreased, but connectivity patterns persisted. In CIPDG activated clusters were found precuneally, and in PPCG no connection to the parietal lobe or APCG was seen. In DPDG no activation in PPCG but smaller clusters in the cuneal and precuneal region were found. In APCG the mean values of NAA/Cr in DPDG, CIPDG, and NPDG are: (1.68+-0.02), (2.04+-0.03), and (2.32+-0.05); Cho/Cr: (0.84+-0.02), (0.81+-0.05), and (0.53+-0.03). In PPCG the mean values of NAA/Cr in DPDG, CIPDG, and NPDG are: (1.14+-0.12), (1.81+-0.02), and (1.98+-0.04), and Cho/Cr: (0.96+-0.02), $(0.77+-0.03)$, and $(0.68+-0.03)$. We have found the progressive decreasing NAA $\mathrm{Cr}$ in the PPCG and increasing of Cho/Cr for the patient of NPDG, CIPDG, and DPDG that is associated with poorer cognitive function.

Conclusion: Bringing together $\mathrm{fMRI}$ in the resting state and ${ }^{1} \mathrm{H}$ MRS data we obtain very useful markers of neuronal dysfunction and neuronal loss in patients with PD and different levels of $\mathrm{Cl}$.

\section{B-0020 11:51}

Relationship among fractional anisotropy (FA) values, EEG activity and cognitive status in mild cognitive impairment $(\mathrm{MCl})$ and

Alzheimer's disease (AD) patients

F. Pitocco, G. Curcio, F. Scrascia, Y. Errante, A. Giona, C. Mallio,

F. Vernieri, B. Beomonte Zobel, C. Quattrocchi, L. Scarciolla; Rome/IT

(l.scarciolla@unicampus.it)

Purpose: Our aim was to investigate the relationship of white matter DTI-derived measures with quantitative electroencephalographic (EEG) data and cognitive status in subjects affected by $\mathrm{MCl}$ and $\mathrm{AD}$.

Methods and Materials: 55 subjects (28 AD, $14 \mathrm{MCl}$ and 13 healthy controls) underwent a MRI (1.5T, Avanto, Siemens, Erlangen, Germany) protocol including DTI. FA was assessed in frontal, temporal and occipital white matter (WM) areas bilaterally and in the corpus callosum (genu and splenium). Subjects also underwent a complete neuropsychological test evaluation and resting eyes-closed EEG for the quantitative study of its spectral content by means of fast Fourier transform (FFT). FFT was computed for different classical EEG bands (Delta, Theta, Alpha, Beta). Results: We found significant differences between patients and healthy controls: FA values on bilateral frontal lobe WM and genu of the corpus callosum $(p<0.001)$ Delta spectral power on central-temporal and frontal areas $(p<0.005)$; Theta spectral power on temporal areas $(p<0.03)$. These spectral power changes correlated negatively with global cognitive functioning as measured by MMSE. The increase of Delta band in AD patients showed tendency to correlate with FA measures at the bilateral frontal and temporal WM areas, while Theta band in $\mathrm{MCl}$ patients correlated with FA measures at the right frontal and left temporal WM areas and at the corpus callosum genu.

Conclusion: Our findings suggest that decrease of FA values of the white matter and the spectral power changes of EEG can together increase the diagnostic sensitivity in the detection of early degenerative dysfunction in $A D$.
$10: 30-12: 00$

Room D1

Chest

SS 104

\section{Lung cancer: from tissue characterisation to treatment}

Moderators:

E. Castañer; Sabadell/ES

N. Tacelli; Brussels/BE

\section{B-0021 10:30}

Prognostic value of CT perfusion parameters in patients with early stage resectable non-small cell lung cancer

L. Calandriello, A.R. Larici, A. del Ciello, G. Rindi, G. Petrone, L. Bonomo; Rome/IT (Icalan@hotmail.it)

Purpose: To correlate perfusion parameters and histopathological factors with prognosis at 2-year follow-up in patients with resectable non-small cell lung cancer (NSCLC) who underwent CT perfusion (CTP) examination before surgery and to correlate perfusion parameters with histopathological factors.

Methods and Materials: 20 patients with resectable NSCLC (stage IA-IIB) underwent CTP examination with 64 row CT and contrast medium injection before surgery; blood flow (BF), blood volume (BV), and peak enhancement intensity (PEI) were calculated. The histopathological evaluation assessed each tumour's pathological stage (IA-IIIA), tumour grade (G1-G3) and extent of necrosis $(<10 \%->$ $10 \%)$. All patients underwent a 24-month follow-up yielding two Results: patients with and without recurrent disease. The Chi-square, Mann-Whitney and Fisher exact test were used for statistics.

Results: 5 patients had no recurrence of disease and 15 patients showed recurrent disease at 24-month follow-up. Statistical analysis showed that CTP parameters $(\mathrm{BF}, \mathrm{BV}$ and $\mathrm{PEI})$ were significantly lower $\left(\mathrm{p}^{2} 0.05\right)$ in patients with relapse. Tumours with higher grade (G2-3), more advanced stage (IIB.IIIA) and more extensive necrosis $(>10 \%)$ were at higher risk of relapse $\left(\mathrm{p}^{2} 0.05\right)$. The analysis also showed significantly lower perfusion parameters (BF, BV and $\mathrm{PEI})$ in patients with more extensive necrosis $(>10 \%)\left(p^{2} 0.05\right)$.

Conclusion: Perfusion parameters (BF, BV and PEI) have been shown to be significantly lower in patients with progressive disease at two-year FUP due to a more extensive necrosis and so they could be regarded as predictive factors in patients with NSCLC eligible for surgery.

\section{B-0022 10:39}

Dynamic volume perfusion CT in patients with lung cancer: baseline perfusion characteristics of different histological subtypes

J. Shi ${ }^{1}$, C. Fink ${ }^{2}$, G. Schmid-Bindert ${ }^{3}$, L.R. Pilz ${ }^{3}$, P. Apfaltrer ${ }^{3}$, H. Haubenreisser ${ }^{3}$, U. Haberland ${ }^{4}$, S.O. Schönberg ${ }^{3}$, T. Henzler ${ }^{3} ;{ }^{1}$ Shanghai/CN, ${ }^{2}$ Celle/DE, ${ }^{3}$ Mannheim/DE, ${ }^{4}$ Forchheim/DE (thomas.henzler@medma.uni-heidelberg.de)

Purpose: Recent studies indicate that baseline tumour perfusion may help to identify patients responding to targeted therapy. This study prospectively evaluated dynamic volume perfusion CT (dVPCT) tumour baseline characteristics of untreated patients with lung cancer.

Methods and Materials: 173 consecutive patients (131 men, mean-age $60.5 \pm 9$ years with newly diagnosed lung cancer underwent dVPCT prior to tumour biopsy. Tumour blood volume (BV), blood flow (BF), mean transit time (MTT), permeability, size and volume were quantitatively measured using the deconvolution model and compared to histopathology. The Wilcoxon rank sum test and Pearson's correlation analysis was used for statistical analysis.

Results: Histopathology revealed adenocarcinoma (AC) in 88 patients, squamous cell carcinoma (SCC) in 54 patients, and small cell lung cancer (SCLC) in 31 patients. Tumour permeability was significantly different between AC, SCC and SCLC (all $p<0.05$ ). Tumour BF was significantly higher in patients with $A C$ than in patients with SCLC $(p=0.001)$. No significant difference was found between AC and SCC, as well as between SCLC and SCC (both p>0.05). Tumour BF and BV significantly correlated in all three tumour histologies (SCC: $r=0.704, p<0.001$; SCLC: $r=0.647, p<0.001$; $A C: r=0.614, p<0.001)$. Correlation of $B F$ and permeability was higher in patients with SCLC $(r=0.643, p<0.001)$ than in patients with $A C$ and SCC $(r=0.451, p<0.001 ; r=0.213, p<0.001$, respectively). No meaningful correlations were found between dVPCT parameters and tumour size and volume. Conclusion: dVPCT parameters significantly differ between AC, SCC and SCLC and are independent from tumour size and volume and thus provide additional functional information for staging and treatment planning. 
Author Disclosures:

U. Haberland: Employee; Siemens Healthcare.

\section{B-0023 10:48}

Correlation between grade and histological subtype of pulmonary adenocarcinoma and [18 F] fluorodeoxyglucose standardised uptake value after evaluation by PET/CT

L. Xu, A. Burke, S. Feigenberg; Baltimore, MD/US (Ixu1@umm.edu)

Purpose: PET-CT is routinely used for localisation and staging of lung tumours. This study addresses ${ }^{(18)} \mathrm{F}$ fluorodeoxyglucose standardised uptake values (SUVs) correlation with subtypes of pulmonary adenocarcinomas classified by the newest classification system.

Methods and Materials: We retrospectively reviewed SUV and histological parameters of 68 adenocarcinomas. Tumours were classified as well-differentiated (adenocarcinoma in situ (AIS) and minimally invasive), moderately differentiated (lepidic predominant adenocarcinoma (LPA) and other types including papillary and acinar), and poorly differentiated (mucinous, colloid, enteric, solid, and micropapillary). Mitotic figures and apoptotic cells were quantitated; degree, type of inflammation, and necrosis were semiquantitated; the presence of lymphovascular invasion was assessed.

Results: There were 68 tumours ( 38 men and 30 women). There were 8 welldifferentiated, 16 LPA, 21 predominately acinar and papillary, 4 mucinous, and 19 high-grade adenocarcinomas. Mean SUV was $3.1 \pm 2.0$ in well-differentiated tumours, $3.4 \pm 2.0$ in LPA, $3.8 \pm 2.6$ in acinar papillary, $6.8 \pm 2.6$ in invasive mucinous adenocarcinoma, and $7.9 \pm 3.4$ in poorly differentiated tumours. Poorly differentiated tumours had significantly higher SUVs than the others $(p<.0001)$. There was a positive correlation with apoptotic score $(p<.0001)$, mitotic count $(p=.0003)$, tumor necrosis $(p=.0006)$, angioinvasion and lymphocytic infiltrate $(p=0.01)$.

Conclusion: High-grade adenocarcinomas have a higher SUV, although there is extensive overlap in range. SUV do not distinguish between invasive and noninvasive tumors with lepidic spread. The increase in SUV in poorly differentiated tumors is related to high mitotic and apoptotic count, which also correlates with lymphovascular invasion and tumor necrosis.

\section{B-0024 10:57}

Which response criteria best help predict survival of patients with EGFR-mutant lung adenocarcinoma following EGFR tyrosine kinase inhibitor?

J. Lee, H. Lee, J.-H. Kim, M.-J. Ahn, H. Kang, K. Lee; Seoul/KR

(zhyun84.lee@samsung.com)

Purpose: To identify differences in radiologic assessment methods and determine optimal imaging criteria for response evaluation in lung adenocarcinoma patients with sensitizing EGFR mutations treated with EGFR tyrosine kinase inhibitor (TKIs). Methods and Materials: We retrospectively assessed patients with EGFR-mutant stage IV lung adenocarcinoma, who underwent TKIs every 3 weeks until disease progression. All 76 patients with at least one measurable lung lesions were quantitatively analysed in terms of tumour size, attenuation, volume, and texture parameters (uniformity and entropy) based on the whole tumour volume, on baseline contrast-enhanced CT scans and on follow-up CT scans. Patients are classified into responders and nonresponders by hierarchical clustering procedures according to the percent changes between baseline CT and follow-up CT 2 cycles later, in terms of each imaging parameter and every conceivable combination of imaging parameters. Time-to-progression (TTP) was stratified by each assessment model using the Kaplan-Meier method and a Cox regression model.

Results: Responders based on tumour volume had a longer TTP than nonresponders (mean PFS, 10.4 months versus 6.6 months, $P=.048$ ). Responders based on combination of tumour volume and texture parameters had a longer TTP than nonresponders (mean PFS, 10.7 months versus 6.6 months, $P=.009$ ). In case of other assessment models, the TTPs of responders were not different from the TTPs of nonresponders (all Ps >.05).

Conclusion: Combination of tumour volume and texture parameters reflecting heterogeneity of the tumour blood supply more earlier helped predict treatment response in EGFR-mutant lung adenocarcinoma patients treated with EGFR TKIs.
B-0025 11:06

Radiogenomic approach to identify anaplastic lymphoma kinasepositive lung adenocarcinoma

C. Jeong, H. Lee, J. Han, J. Jeong, K. Lee, K. Park, M.-J. Ahn, B.-T. Kim; Seoul/KR (chaejin.jeong@samsung.com)

Purpose: A subset (3-7\%) of non-small cell lung carcinomas (NSCLCs) harbors the EML4-ALK fusion gene. The importance of recognising this gene rearrangement was highlighted by clinical trials of crizotinib, the ALK inhibitor, showing encouraging clinical outcomes. The purpose of this study was to determine CT and metabolic characteristics in patients with ALK-positive NSCLCs and to devise an effective screening strategy.

Methods and Materials: We retrospectively reviewed radiologic and clinicopathologic data from patients with NSCLCs, in whom immunohistochemistry for ALK rearrangement was conducted. Patient characteristics and lesion size, marginal characteristics, solidity, density from CT, and SUVmax from PET-CT were assessed. Pathologic features such as differentiation, histologic subtype, EGFR and KRAS mutation status were also reviewed.

Results: Forty-one patients with ALK-rearranged NSCLCs tended to be younger $(p<.001)$ than ALK-negative patients $(n=180)$ and showed significant association with no exposure to tobacco $(p=.008)$. ALK-positive tumours were significantly less differentiated $(\mathrm{p}=.016)$. The SUVmax, lesion size, or tumour density did not significantly difference according to ALK status. Of ALK-positive tumours, only $29 \%$ showed spiculated margin, whereas $57 \%$ of ALK-negative tumours had spiculated margin ( $(\mathrm{p}=.002)$. Two histologic findings, namely the presence of signet-ring cells $(44 \%)$ and abundant extracellular mucus $(81 \%)$, helped explain the difference of tumour margin in ALK-positive tumours.

Conclusion: ALK-positive lung adenocarcinomas share certain clinical characteristics, and some radiologic findings may help identify cases for positive ALK testing. However, none of radiologic parameters were completely sensitive or specific to ALK rearrangement, and radiomorphology should not replace confirmatory molecular or immunohistochemical study.

\section{B-0026 11:15}

Mucinous vs nonmucinous adenocarcinoma in situ (AIS): imaging features, EGFR mutation and EGFR gene copy number status, and prognosis

D. Kim, H.-J. Lee, J. Wi, S. Lee, C. Park, J. Goo; Seoul/KR

(phemuto_45@naver.com)

Purpose: In IASCL/ATS/ERS classification, lung adenocarcinoma in situ (AIS) refers to a small localised adenocarcinoma $(</=3 \mathrm{~cm})$ that shows lepidic growth without stromal, vascular, or pleural invasion. As mucinous AIS are very rare, there have been few comparative studies between nonmucinous (non-AIS) and mucinous AIS (muc-AIS). Therefore, we compared imaging features, EGFR gene status, and prognosis between two types of AIS.

Methods and Materials: 13 patients with muc-AIS and 85 patients with nonAIS underwent surgical resection and comprised our study population. Nodule characteristics and mean HUs on CT, and SUVmax on PET of all nodules were compared. EGFR mutation and gene copy numbers were evaluated through direct DNA sequencing and FISH, respectively. Survival and disease recurrence were also assessed. Statistical comparisons were performed using Chi-square and t-tests. Results: Nodule characteristics were significantly different $(p<0.0001)$ : non-AIS were mainly non-solid $(60 \%)$; muc-AIS were mainly pure-solid $(54 \%)$. Mean HUs of muc-AIS $(-212 \pm 82)$ were higher than those of non-AIS $(-539 \pm 110)(p<0.0001)$. In all non-AIS and muc-AIS, the SUVmax was lower than 2.5. EGFR mutation was only found in non-AIS $(64 \%)(p=0.0003)$. EGFR gene copy numbers in non-AIS was various, whereas muc-AIS only showed disomy (100\%). Mean postoperative follow-up period was 3 years (range, 3-109 months) with no patient deaths. Among 22 patients who were followed over 5 years, no patients showed definite tumour recurrence.

Conclusion: All non-AIS appear as ground-glass nodules and muc-AIS can appear as solid nodules on CT. Both non-AIS and muc-AIS showed low glucose metabolism on PET, and EGFR mutation and amplification were exclusively found in non-AIS. Long disease-free survival was seen in both non-AIS and muc-AIS.

Author Disclosures:

J. Goo: Consultant; INFINITT Healthcare Co, Ltd. 


\section{B-0027 11:24}

Validation of a computed-tomography-based risk model for the prediction of cardiovascular disease in a lung cancer screening setting P.M. Jairam, P.A. de Jong, W.P.T.M. Mali, Y. van der Graaf; Utrecht/NL (pjairam@umcutrecht.nl)

Purpose: The routine-radiological-risk (Triple-R) score is a radiological risk model for the prediction of the absolute 5-year cardiovascular disease (CVD) risk. This score is based on additional imaging findings in routine non-cardiovascular chest computed tomography (CT) scans. The triple-R score demonstrated good prediction of individual risk in hospital populations. Good performance of the triple-R score in a screening setting emerges the possibility to enhance the benefits of chest CT-based screening of heavy smokers. Adding Triple-R to lung cancer screening results in multiple disease screening without the need for additional imaging. The aim of the present study was to validate the Triple-R score within a lung cancer screening setting.

Methods and Materials: A case-cohort study was used within a lung cancer screening trial. In a cohort of 3559 male participants, 263 fatal and non-fatal cardiovascular events (cases) occurred during mean follow-up of 27 months (max. 45 months). From the cohort, 341 subjects were randomly drawn (subcohort). According to Triple-R, CT's for the cases and subcohort were graded for left anterior descending coronary, mitral valve and descending aorta calcifications and heart size was measured. Discrimination and calibration was assessed over a mean follow-up period of 27 months.

Results: Mean age of cases and subcohort were, respectively, 62 (52-78) and $61(52-78)$ years. Discrimination was acceptable with a c-statistic of $0.68(95 \% \mathrm{Cl}$ 0.65-0.72). Triple-R demonstrated a good overall calibration.

Conclusion: CT-based cardiovascular Triple $\mathrm{R}$ score performed well in this lung cancer screening cohort, which is supporting future use in a clinical and lung cancer screening setting.

\section{B-0028 11:33}

\section{Diagnostic accuracy of virtual 18 F-FDG PET/CT bronchoscopy}

for the detection of lymph node metastases in non-small cell lung cancer patients

M.C. Herbrik' ${ }^{1}$ C. Buchbender ${ }^{2}$, J. Treffert ${ }^{3}$, B. Geiger ${ }^{4}$, V. Hartung-Knemeyer', H. Kühl', G. Antoch' ${ }^{2}$, M. Forsting ${ }^{1}$, T.A. Heusner ${ }^{2} ;{ }^{1}$ Essen/DE, ${ }^{2}$ Düsseldorf/DE, ${ }^{3}$ Knoxville, TN/US, ${ }^{4}$ Princeton, NJ/US (m.herbrik@gmx.de)

Purpose: Bronchoscopically guided lymph node biopsy is a common procedure in bronchial carcinoma patients. To plan those biopsies a virtual "fly-through" 3D FDG-PET/CT bronchoscopy has been shown to be technically feasible. The diagnostic accuracy of this procedure, using histopathology as reference standard, has not been evaluated yet. The aim of this study was to determine the diagnostic accuracy of a virtual 3D FDG-PET/CT bronchoscopy for the detection of thoracic lymph node metastases in non-small cell lung cancer (NSCLC) patients.

Methods and Materials: 30 consecutive NSCLC patients prospectively underwent whole body FDG-PET/CT. From these data virtual 3D FDG-PET/CT bronchoscopies were reconstructed. The sensitivity, specificity, the PPV, NPV and accuracy of FDGPET/CT bronchoscopy for the detection of lymph node metastases were evaluated on a compartment basis (according to Mountain and Dresler). Compartments with high rates of false positive results were identified. Histopathology (sampled by mediastinoscopy) served the standard of reference.

Results: The sensitivity, specificity, PPV, NPV and accuracy of virtual FDG-PET/ CT bronchoscopy for the detection of lymph node metastases were $74 \%, 82 \%$, $67 \%, 87 \%$ and $77 \%$, respectively. In the $4 \mathrm{~L}$ compartment there was a high rate (9/30) of false positive results.

Conclusion: Virtual "fly-through" 3D FDG-PET/CT bronchoscopy yields a relatively high diagnostic accuracy for the detection of regional lymph node metastases. It may be used to plan bronchoscopically guided lymph node biopsies. Results of this procedure in the localisation $4 \mathrm{~L}$ should be rated with caution due a high rate of false positive results.

\section{B-0029 11:42}

\section{Percutaneous transthoracic CT-guided biopsy using large core} needles: initial experience

U.C. Lalii, J.E. Wildberger, M. Bendek, M. Das; Maastricht/NL (u.lalji@mumc.nl)

Purpose: The use of large core needle biopsy in percutaneous transthoracic interventions may be advantageous in terms of histological evaluation (larger specimens) and mutational analysis (more cells for analysis). The aim of this study was to retrospectively evaluate the success rate and safety in percutaneous transthoracic CT guided needle biopsy using these devices.
Methods and Materials: 20 patients (8 male, 12 female, age 38- 76, mean 66 years) with suspected malignancy underwent percutaneous transthoracic CTguided needle biopsy under local anaesthesia using dedicated large core biopsy needles (10G Spirotome, Medinvents). Location and size of the tumour, number of pleural passes, number of biopsies, and complications (pneumothorax, bleeding) were recorded and compared to our standard core biopsy database (18G; $n=121$ ). Results: Location varied from pleural to hilar location. Mean tumour size was 2.8 $\mathrm{cm}$ (range $0.7-9.2 \mathrm{~cm}$ ). Only one pleural passage (mean pleura to lesion length $3.0 \mathrm{~cm}$ (range $9.2 \mathrm{~cm}-0 \mathrm{~cm}$ ) and one biopsy were necessary in all patients. Large core biopsy was successful in $100 \%$ ( $18 \mathrm{G}$ in $87 \%$ ). Pneumothorax was found in $50 \%(18 \mathrm{G}$ in $51 \%)$. No clinically relevant complications were recorded, especially no hemoptoe occurred. Minor intraparenchymal bleeding was present in 10 of 20 patients (18 $\mathrm{G}$ in 60 of 121 patients).

Conclusion: Large core biopsy with $10 \mathrm{G}$ is technically feasible and safe. Our preliminary experience did not show higher complication rates compared to standard $18 \mathrm{G}$ biopsy. Histological specimens obtained may increase confidence in pathological diagnosis and might be helpful especially for further immunohistochemical and mutational analysis.

\section{B-0030 11:51}

Primary lung cancer treated using radiofrequency ablation: two-year outcome data

J. Beeson, N. Wickings, V. Anikin, S. Kaul, P. Dalal; London/UK (p.dalal@rbht.nhs.uk)

Purpose: Percutaneous image-guided radiofrequency ablation (RFA) is being increasingly used as an alternative treatment option for patients with inoperable primary lung cancer (NSCLC), but there are little published outcome data. We report safety and efficacy of RFA in patients with NSCLC (stages 1-4) at 1 and 2 years. Methods and Materials: Under general anaesthesia 38 patients underwent RFA of 50 pulmonary tumours. Survival was evaluated prospectively at 3, 6, 9.12 and 24 months whilst complications were assessed 1 month post procedure.

Results: There were no cases of procedure-related mortality. Complications included pneumothorax requiring intercostal drain (ICD) insertion ( $n=20,53 \%)$, small pneumothorax not requiring $\operatorname{ICD}(n=3,8 \%)$ and pleural effusion $(n=2,5 \%)$. One-year overall survival and cancer specific $(C / S)$ for tumours classified as Stage $1(n=20)$ were 100 and $100 \%$, respectively. Stage $2(n=7) 85 \%$ and $100 \%$, respectively, and stage $4(n=11) 64$ and $73 \%$, respectively. Two-year survival rates (overall \& C/S) for Stage 1 were 85 and $95 \%$, in Stage 2 patients 43 and $86 \%$ and for those at Stage 4,45 and $64 \%$, respectively.

Conclusion: RFA is a safe, effective and well-tolerated treatment option in patients with inoperable lung cancer. Our study also shows that ablation therapy may be useful in higher stage tumours.

$10: 30-12: 00$

Room D2

Interventional Radiology

\section{SS 109}

\section{Chemoembolisation and radioembolisation of liver} tumours

Moderators:

A. Denys; Lausanne/CH

P.M. Paprottka; Munich/DE

\section{B-0031 10:30}

Repeated transarterial chemoembolisation in the treatment of liver metastases of colorectal cancer: local tumour control and survival data T. Gruber-Rouh, N.N.N. Naguib, M. Beeres, B. Bodelle, S. Zangos, N. Nour Eldin, R. Hammerstingl, T.J. Vogl; Frankfurt/DE

Purpose: To evaluate local tumour control and survival data after transarterial chemoembolisation with different drug combinations in the palliative treatment of liver metastases in patients with colorectal cancer.

Methods and Materials: A total of 564 patients (mean age,60.3 years) with unresectable liver metastases of $\mathrm{CRC}$ were repeatedly treated with chemoembolisation in 4-week intervals. In total, 3384 chemoembolisation procedures were performed (mean, 6 sessions per patient). Of 564 patients, $66.4 \%$ had multiple metastases, $10.6 \%$ had one metastasis, $11.2 \%$ had two metastases, and $11.8 \%$ had three or four metastases. The local chemotherapy protocol consisted of mitomycin $\mathrm{C}$ alone (43.1\%), mitomycin C with gemcitabine $(27.1 \%)$, mitomycin C with irinotecan (15.6\%), or mitomycin C with irinotecan and cisplatin (15.6\%). Embolisation was 
performed with lipiodol and starch microspheres for vessel occlusion. Tumour response was evaluated with $\mathrm{MRI}$. The change in tumour size was calculated and the response was evaluated according to the RECIST. Survival rates from first chemoembolisation session were calculated according to the Kaplan-Meier method. Results: Evaluation of local tumour control resulted in partial response $(n=94)$, stable disease $(n=272)$, and progressive disease $(n=198)$. The 1-year survival rate after chemoembolisation was $62 \%$, the 2 -year survival rate was $28 \%$, and the 3 -year survival rate was $7 \%$. Median survival was 14.3 months. There was no statistically significant difference between the three treatment protocols.

Conclusion: Chemoembolisation is a minimally invasive therapy option for palliative treatment of liver metastases in patients with CRC, with similar results among three chemoembolisation protocols.

\section{B-0032 10:39}

Holmium-166 radioembolisation: results of a phase 1 , dose escalation study in patients with unresectable, chemorefractory liver metastases - the HEPAR trial

M.L.J. Smits, J.F.W. Nijsen, M.A.A.J. van den Bosch, M.G.E.H. Lam, M.A.D. Vente, W.P.T.M. Mali, A.D. van het Schip, B.A. Zonnenberg, J.F. Prince; Utrecht/NL (j.prince@umcutrecht.nl)

Purpose: Holmium-166 (166Ho) poly (L-lactic acid) microspheres are unique because their distribution can be imaged in vivo with both SPECT and MRI. In this phase 1 clinical trial, we aimed to assess the safety and the maximum tolerated radiation dose (MTRD) of $166 \mathrm{Ho}-\mathrm{RE}$ in patients with liver metastases.

Methods and Materials: Patients with unresectable, chemorefractory liver metastases were enrolled for intra-arterial $166 \mathrm{Ho}-\mathrm{RE}$. Patients were treated in cohorts of three, with escalating aimed whole-liver absorbed doses of $20-80 \mathrm{~Gy}$. Cohorts were extended to maximally six patients if dose-limiting toxicity occurred. Toxicity was scored according to the Common Terminology Criteria for Adverse Events v.3 (CTCAE). Patients were assigned a dose in the order of study entry, with dose escalation until dose-limiting toxicity was encountered in at least two patients of a dose cohort. The primary endpoint was the MTRD. The study is registered with ClinicalTrials.gov, NCT01031784.

Results: Fifteen patients underwent $166 \mathrm{Ho}-\mathrm{RE}$ with mean estimated whole-liver absorbed doses of 18 Gy (20-Gy cohort), 35 Gy (40-Gy cohort), 58 Gy (60-Gy cohort), and 73 Gy (80-Gy cohort). The 20-Gy cohort was extended to six patients because of the occurrence of a pulmonary embolism. In the 80-Gy cohort, dose limiting toxicity was encountered in two patients. The MTRD was determined to be 60 Gy.

Conclusion: $166 \mathrm{Ho}-\mathrm{RE}$ is feasible and safe for the treatment of patients with unresectable and chemorefractory liver metastases and offers image-guided treatment. $166 \mathrm{Ho}-\mathrm{RE}$ should be performed with a aimed whole-liver absorbed dose of $60 \mathrm{~Gy}$.

\section{Author Disclosures:}

J.F.W. Nijsen: Patent Holder; Patents regarding holmium microspheres.

M.A.D. Vente: Employee; Sirtex Medical. A.D. van het Schip: Patent Holder;

Patents regarding holmium microspheres. B.A. Zonnenberg: Patent Holder;

Patents regarding holmium microspheres.

\section{B-0033 10:48}

Repetitive transarterial chemoembolisation as a palliative treatment option for liver metastases from cutaneous malignant melanoma: indications, outcomes and role in patient's management

T. Gruber-Rouh, P. Weisser, N.N.N. Naguib, K. Eichler, M. Harth, S. Zangos, N-E.A. Nour-Eldin, T.J. Vogl; Frankfurt/DE

Purpose: To evaluate local tumor control and survival data after TACE with three different chemotherapeutic protocols in the palliative treatment of patients with liver metastases from malignant melanoma.

Methods and Materials: The study protocol was approved by the ethical committee, and informed consent was obtained from all patients prior to treatment. A total of 67 patients (mean age, 55.2 years; range, 28-77 years) with unresectable liver metastases of malignant melanoma who did not respond to systemic therapy were repeatedly treated with TACE in 4-week intervals. In total, 456 chemoembolisation procedures were performed (mean, 7 sessions per patient; range, 3-12 sessions). The local chemotherapy protocol consisted of mitomycin alone $(14.9 \% ; n=10)$, mitomycin with gemcitabine $(43.3 \% ; n=29)$ or mitomycin, gemcitabine and cisplatin $(41.8 \%, \mathrm{n}=28)$. Embolisation was performed with lipiodol and degradable starch microspheres. Local tumor response was evaluated by MRI according to the modified RECIST criteria. Survival data were calculated according to the Kaplan-Meier method.

Results: The local tumor control was: partial response (PR) in 1.5\% $(n=1)$, stable disease (SD) in $50.7 \%(n=34)$ and progressive disease (PD) in $47.8 \%(n=32)$ of patients. The 1-year survival rate after chemoembolisation was $68 \%$, and the 2 -year survival rate was $36 \%$. The median and mean survival times from the start of TACE treatment were 18 and 31.8 months. There was no statistically significant difference between the three treatment protocols.

Conclusion: Chemoembolisation is a potentially palliative treatment option in achieving local control in selected patients with liver metastases from malignant melanoma.

\section{B-0034 10:57}

Experimental study on transarterial administration of survivin siRNA combined with transarterial chemoembolisation in rats with hepatocellular carcinoma (HCC)

T.J. Vogl ${ }^{1}$, J. Qian ${ }^{2}$, A. Tran'1, E. Oppermann ${ }^{1}$, U. Imlau ${ }^{1}$, Y. Hamidavi ${ }^{1}$,

H. Korkusuz ${ }^{1}$, W.O. Bechstein ${ }^{1} ;{ }^{1}$ Frankfurt a. Main/DE, ${ }^{2}$ Wuhan/CN

(t.vogl@em.uni-frankfurt.de)

Purpose: To evaluate the effects of transarterial administration of survivin siRNA combined with transarterial chemoembolisation (TACE) for treating hepatocellular carcinoma $(\mathrm{HCC})$ in rats.

Methods and Materials: Subcapsular implantation of a solid Morris hepatoma $3924 \mathrm{~A}$ in the liver was carried out in 20 male ACI rats (day 0). Tumour volume (V1) was measured by MRI (day 12). After laparotomy and retrograde placement of a catheter into the gastroduodenal artery (day 13), the following different agents were injected into the hepatic artery: TACE $(0.1 \mathrm{mg}$ of mitomycin $+0.1 \mathrm{ml}$ of lipiodol +5.0 mg of degradable starch microspheres) $+2.5 \mathrm{nmol}$ survivin siRNA $(n=10)$ (group $A$, $n=10$ ); TACE alone (group $B, n=10$ ). Tumour volume (V2) was assessed by MRI (day 25), tumour growth ratio (V2/V1) was calculated. Western blot analysis was performed to assess the protein expression level of survivin in each treatment. The progressional potential of the tumours was assessed for quantification of positive VEGF tumour cells via immunohistochemical analysis.

Results: Mean tumour growth ratio (V2/V1) was 1.1313+0.1381 in group A, and $3.1911+0.1393$ in group B. Compared with group B, group A showed significant inhibition of tumour growth $(P<0.01)$. Survivin expression level was lower in group $A$ than in group $B$. There was a significant difference between groups $A$ and $B$ in survivin expression by western blot analysis $(P<0.01)$. Higher expression of VEGF in HCC was observed in group $B$ vs. group $A$ by immunohistochemical examination $(P<0.01)$.

Conclusion: Combined TACE and transarterial administration of survivin siRNA is more effective than TACE alone for inhibiting the growth of HCC in rats.

\section{B-0035 11:06}

Role of transarterial chemoembolisation as bridging strategy in T2 HCC patients on the waiting list

E. Bozzi, I. Bargellini, F. Turini, A. Cicorelli, R. Cioni, C. Bartolozzi; Pisa/IT (elenabozzi@libero.it)

Purpose: To retrospectively evaluate long-term outcomes of T2 stage HCC patients who underwent orthotopic liver transplantation (OLT) after transarterial chemoembolisation (TACE), compared with patients transplanted without bridging therapy. Methods and Materials: The study included 168 cirrhotic patients (147 males, mean age 55.8 years) with T2 HCC, who underwent OLT from 1996 to 2010. In patients treated by TACE, latest CT examination prior to OLT was reviewed to assess tumour response according to $\mathrm{mRECIST}$. Patients were divided into three groups: A) no bridging therapy, B) complete response (CR) after TACE, C) partial response, stable or progressive disease after TACE. Overall (OS), recurrence-free (RFS) and disease-free (DFS) survivals were calculated by Kaplan-Meier analysis and compared by log-rank test.

Results: Fifty-six patients were in group A, 60 patients in group $B$ and 52 patients in group C; preprocedural data were comparable among groups. Five-year OS, RFS and DFS were $75.2 \%, 92.7 \%$ and $73.2 \%$, respectively. Survivals were significantly higher in group $\mathrm{B}$ compared with group $\mathrm{C}$ and comparable between group $\mathrm{A}$ and B. In tumours $>3 \mathrm{~cm}$, DFS censored for peri-operative deaths $(n=7)$ was higher in group $B$ compared with group $A(p=0.05)$.

Conclusion: In T2 transplanted patients, CR after pre-operative TACE represents a favourable prognostic indicator for survival and tumour recurrence. TACE may be beneficial for T2 HCC patients with overall tumour size $>3 \mathrm{~cm}$. 


\section{B-0036 11:15}

Semi-automatic volumetric tumour segmentation for hepatocellular carcinoma: comparison between C-arm cone-beam computed tomography and MRI

V. Tacher', M. Lin ${ }^{2}$, M. Chao', L. Gjesteby', N. Bhagat ${ }^{1} ;{ }^{1}$ Baltimore, MD/US, ${ }^{2}$ Briarcliff Manor, NY/US

Purpose: To evaluate the accuracy and reproducibility of a semi-automatic tumour segmentation software in measuring tumour volume of hepatocellular-carcinoma (HCC) before the first trans-arterial chemo-embolisation (TACE) on contrastenhancement magnetic-resonance-imaging (CE-MRI) and intra-procedural dualphase C-arm cone-beam computed-tomography (DP-CBCT) images.

Methods and Materials: $19 \mathrm{HCCs}$ were targeted in 19 patients (one per patient) who underwent baseline diagnostic CE-MRI and an intra-procedural DP-CBCT. The images were obtained from CE-MRI (arterial-phase of an intra-venous contrast medium injection) and DP-CBCT (delayed-phase of an intra-arterial contrast medium injection) before the actual embolisation. Three readers measured tumour volumes using a semi-automatic 3D-volumetric segmentation software which used a region-growing method employing non-Euclidean radial basis functions. Segmentation time and spatial position were recorded. The tumour volume measurements between images sets were compared using linear-regression and Student t-test, and evaluated with Intraclass-Correlation analysis (ICC). The inter-rater Dice Similarity Coefficient (DSC) accessed the segmentation spatial localisation.

Results: All 19 HCCs were analysed. On CE-MRI and DP-CBCT examinations, respectively, 1.there was no statistically significant difference in the mean segmented tumour volumes $\left(87 \pm 8 \mathrm{~cm}^{3}[2-873]\right.$ and $\left.92 \pm 10 \mathrm{~cm}^{3}[1-954], \mathrm{p}=0.30\right)$ and the mean time required for segmentation $(66 \pm 45$ seconds [21-173] and $85 \pm 34$ seconds [17-214],p=0.19), 2.the ICCs were 0.99 and 0.974 , showing a strong correlation among readers, and the inter-rater DSCs showed a good to excellent inter-user agreement on the spatial localisation of the tumour segmentation $(0.70 \pm 0.07$ and $0.74 \pm 0.05, p=0.07)$.

Conclusion: This study shows a strong correlation, accuracy and reproducibility of semi-automatic tumour segmentation software in measuring tumour volume on CE-MRI and DP-CBCT images. The use of the segmentation software on DP-CBCT and CE-MRI can be a valuable and highly accurate tool to measure the volume of hepatic tumours.

\section{B-0037 11:24}

Transcatheter arterial chemoembolisation of hepatocellular carcinoma in transplant patients: smaller bead diameter and hypervascularity lead to higher tumour necrosis

M. Hakky, S. Amirifeli, C. Wald, C. Molgaard, H. Ahari; Burlington, MA/US

Purpose: Transcatheter arterial chemoembolisation of hepatocellular carcinoma (HCC) with doxorubicin-loaded drug-eluting beads (DEB) is used as bridge therapy in patients awaiting transplant. Our purpose was to assess the influence of bead size and tumour vascularity on degree of tumour necrosis and successful bridging. Methods and Materials: Consecutive Barcelona Clinic Liver Cancer Stage A transplant listed patients with HCC underwent chemoembolisation. 13 patients treated with "large" DEB of 300-500 $\mu \mathrm{m}$ and 500-700 $\mu$ m diameter, 21 patients with "small" DEB of $100-300 \mu \mathrm{m}$ and $300-500 \mu \mathrm{m}$ diameter. Average age of small bead patients: $58.2 \pm 4.5$ years, male:female ratio $86 \%: 14 \%$ versus large bead patients: $57.9 \pm 12.3$ years, male:female ratio $85 \%: 15 \%$.Patients evaluated with imaging in 3-month intervals. Tumour vascularity assessed angiographically prior to first chemoembolisation. Explant pathology used to determine degree of necrosis. Results: $4 / 21(19 \%)$ small bead patients and $3 / 13(23 \%)$ large bead patients progressed beyond transplant criteria. 8 small bead patients and 9 large bead patients were transplanted $184 \pm 101$ and $169 \pm 96$ days after chemoembolisation, respectively. $4 / 8(50 \%)$ small bead patients and $3 / 9(33 \%)$ large bead patients had no arterial enhancement on imaging prior to transplant. The 4 small bead patients showed complete pathologic necrosis versus only 2 out of the 3 large bead patients. 2 small bead patients and 3 large bead patients had angiographically hypovascular lesions. Incomplete necrosis was seen in all $5 / 5$ (100\%) hypovascular lesions versus $6 / 12(50 \%)$ hypervascular lesions.

Conclusion: Chemoembolisation using smaller drug-eluting beads is associated with improved ability to bridge early stage HCC patients to transplant. Relative hypovascular HCC responds less well to chemoembolisation independent of bead size.
B-0038 11:33

Initial RECIST tumour reduction as a predictive parameter for transarterial chemoembolisation treatment success in patients with hepatocellular carcinoma

D.B. Hasdemir, B.C. Meyer, N. Schweitzer, A. Vogel, C. von Falck, H. Rosenthal, F. Wacker, T. Rodt; Hannover/DE (hasdemir.davut@mh-hannover.de)

Purpose: To test the hypothesis that initial tumour reduction based on RECIST criteria in patients with intermediate stage hepatocellular carcinoma has prognostic value for the maximum response to transarterial chemoembolisation (TACE) treatment. Methods and Materials: A data base review was performed for patients who underwent TACE procedures at our institution over three consecutive years. 63 intermediate stage hepatocellular carcinoma patients with RECIST follow-up were identified, who had no previous TACE. All patients were treated with a TACE procedure $(n=198)$ using doxorubicin and cisplatin as chemotherapeutic agents and lipiodol. Individual RECIST response throughout up to five TACE procedures was correlated to overall survival and maximum tumour response.

Results: Patients received an average of 3.1 TACE procedures (SD 2.8). KaplanMeier analysis yielded a mean overall survival of $843 \mathrm{~d}(95 \% \mathrm{Cl} 682-1004 \mathrm{~d}) .19$ patients (30.2\%) did not show any response, 44 patients $(69.8 \%)$ showed response. Maximum RECIST tumour response was assigned to individual treatment intervals (TACE 1: 19 patients (30.2\%); TACE 2: 6 patients $(9.5 \%)$; TACE 3: 8 patients (12.7\%); TACE 4: 5 patients (7.9\%); TACE 5: 0 patients $(0 \%) ; 6$ patients TACE $6-17(9.5 \%)$. A Pearson correlation was calculated between maximum tumour response and tumour response of the initial TACE procedures (TACE1: 0.606 ( $p$ $<0.01)$; TACE 2: $0.701(p<0.01)$; TACE 3: 0.498 ( $p<0.05)$.

Conclusion: Initial RECIST tumour reduction shows significant correlation to maximum tumour response. We conclude that it can be applied as a prognostic parameter for TACE treatment.

\section{B-0039 11:42}

Intraprocedural parenchymal blood volume measurement using repetitive $\mathrm{C}$-arm $\mathrm{CT}$ in patient with hepatocellular carcinoma and breast cancer during TACE: therapy response and evaluation T.J. Vogl, S. Blösser, P. Schäfer, E. Mbalisike, S. Zangos; Frankfurt a. Main/DE (t.vogl@em.uni-frankfurt.de)

Purpose: To evaluate potential correlations between initial intraprocedural tumour blood volume measurement and prognostic parameters of patients with primary and secondary liver malignancies focusing on tumour response.

Methods and Materials: 43 patients with liver malignancy from breast cancer $(n=24)$, and hepatocellular carcinoma (HCC)/cholangiocellular carcinoma (CCC) $(n=19)$ were included in this study. Each patient underwent an initial blood volume measurement using C-arm-CT and MRI followed by TACE+MRI in 4-week intervals. Images were evaluated by measuring and correlating tumour size in MRI and parenchymal blood volume (PBV) (RECIST).

Results: The preinterventional blood volume measurement of breast cancer patients demonstrated a mean blood volume of $94.05 \mathrm{ml} / 1000 \mathrm{ml}$ (SD 43.6), whereas 4-week follow-up PBV after TACE was $62.9 \mathrm{ml} / 1000 \mathrm{ml}(-31.15 \mathrm{ml} / 1000 \mathrm{ml})$. Mean tumour regression after 3 TACEs was $14.52 \%$. The HCC/CCC-groups showed a mean initial PBV of $57.3 \mathrm{ml} / 1000 \mathrm{ml}$ (SD 64.5). After TACE it was changed to $82.2 \mathrm{ml} / 1000 \mathrm{ml}(+24.9 \mathrm{ml} / 1000 \mathrm{ml}$ increase); after second TACE PBV decreased to $73.32 \mathrm{ml} / 1000 \mathrm{ml})(-8.9 \mathrm{ml} / 1000 \mathrm{ml})$. In the breast cancer group, initial tumour blood-volume $>100 \mathrm{ml} / 1000 \mathrm{ml}$ dropped by $-9.06 \%, 50-100 \mathrm{ml} / 1000 \mathrm{ml}$ dropped by $-22.24 \%$ and $<50 \mathrm{ml} / 1000 \mathrm{ml}$ dropped by $-0.23 \%$ in size (based on initial PBV). In the breast cancer group, postinterventional MRI follow-up showed stable disease (SD) in 21 patients, partial remission (PR) in 3 patients. In the HCC group, 9 patients had SD, 2 patients had progressive disease (PD). In the CCC group, 7 patients had SD and 1 had PD.

Conclusion: The second PBV measurement in the breast cancer group is a significant predictor for tumour regression, whereas the $\mathrm{HCC} / \mathrm{CCC}$ groups required 2 or more TACEs to show a decrease in blood volume and tumour size. 


\section{B-0040 11:51}

Study on the effect of chemoembolisation combined with microwave ablation for the treatment of hepatocellular carcinoma in rats

T.J. Vogl', J. Qian², U. Imlau', Y. Hamidavi', H. Korkusuz',

E. Oppermann ${ }^{1}$, W.O. Bechstein $1{ }^{1}{ }^{1}$ Frankfurt a. Main/DE, ${ }^{2}$ Wuhan/CN

(t.vogl@em.uni-frankfurt.de)

Purpose: To assess the effect of transcatheter arterial chemoembolisation (TACE) combined with microwave ablation for the treatment of hepatocellular carcinoma in an animal model.

Methods and Materials: All experiments were approved by the German government and the Institutional Animal Research Review Board. After subcapsular liver implantation of Morris Hepatoma $3924 \mathrm{~A}$ in $30 \mathrm{ACl}$ rats, the animals were randomly assigned to three interventional treatment groups as follows: (A) TACE $(0.1 \mathrm{mg}$ of mitomycin $+0.1 \mathrm{ml}$ of lipiodol $+5.0 \mathrm{mg}$ of degradable starch microspheres) + microwave ablation ( $2450 \mathrm{MHz} ; 35 \mathrm{~W}, 45 \mathrm{~s})(\mathrm{n}=10)$; (B) TACE alone (control group $1, n=10$ ); (C) microwave ablation alone (control group 2, $n=10$ ). Tumour volume before (V1) and after the treatment (V2) was assessed by MRI and the tumour growth ratio (V2/V1) was calculated. Immunohistochemical analysis was performed for quantification of positive MMP-9 and VEGF tumour cells in each treatment. Results: Mean tumour growth ratio was $1.3385+0.1949$ in group A, $3.1901+$ 0.1323 in group $B$, and $4.1779+0.1862$ in group $C$, respectively. Compared with the ratio in groups $B$ and $C$ (controls), the ratio in group $A$ showed significant reduction in tumour growth $(P<0.01)$. Lower expression of MMP-9 and VEGF in hepatocellular carcinoma was observed in group A vs. groups $B$ and $C$ (controls) $(P<0.05)$. Conclusion: Treatment with combined TACE/microwave ablation, compared with the treatment of TACE or microwave ablation alone, significantly inhibits tumour growth and intrahepatic metastases in an animal model of hepatocellular carcinoma.

10:30 - 12:00

Room E1

\section{Musculoskeletal}

\section{SS 110}

\section{Shoulder and hand}

Moderators:

C. Schüller-Weidekamm; Vienna/AT

M. Shahabpour; Brussels/BE

\section{B-0041 10:30}

Diagnosing adhesive capsulitis of shoulder on sonography alone: is it possible?

A. Tandon, S. Dewan, S. Bhatt, A.K. Jain; New Delhi/IN (sakshii.21@gmail.com)

Purpose: Adhesive capsulitis (AC) has been a diagnosis of exclusion on sonography due to lack of specific diagnostic criteria. Multiple static and dynamic parameters for sonographic diagnosis of $\mathrm{AC}$ are introduced.

Methods and Materials: Shoulder sonography was performed prospectively on 60 patients with painful shoulder and 30 asymptomatic controls by two radiologists. Static [coracohumeral ligament (CHL) thickness, increased soft tissue in rotator interval] and dynamic parameters [restriction of abduction and external rotation] for adhesive capsulitis were evaluated. Patients were divided into three groups: adhesive capsulitis (AC) $(n=30)$, painful shoulders (PS) $(n=30)$ and control group $(C L)(n=30)$ based on MRI diagnosis.

Results: Sonographic visualisation of $\mathrm{CHL}(96.7 \%)$ and its mean thickness (1.2 $\mathrm{mm}$ ) were highest in the $\mathrm{AC}$ group $(P<0.01)$. A cut-off value of $0.7 \mathrm{~mm}$ was found to be accurate (sensitivity $93.1 \%$ specificity $94.4 \%$ ) for diagnosing AC. Increased soft tissue in the rotator interval was seen in the AC group (sensitivity $86.2 \%$, specificity $92.8 \%$ ). On dynamic sonography, restriction of external rotation was specific (sensitivity of $96.7 \%$ and specificity $90 \%$ ), whereas restriction in abduction was non-specific (specificity 6.7\%) for AC. Inter-observer agreement was substantial for $\mathrm{CHL}$ visualisation (kappa 0.66 ). The overall sensitivity and specificity sonography for diagnosing adhesive capsulitis taking MRI as reference standard was $100 \%$ and $87 \%$, respectively.

Conclusion: Sonography, using the stated static and dynamic parameters was highly accurate in the diagnosis of adhesive capsulitis and in differentiating it from other causes of painful shoulder. It has the potential to be adopted as a first line modality.
B-0042 10:39

MR imaging of glenoid bone loss

K.R. Lee, J.F. Griffith, W.A. Ng, M. Tong, S. Nimish; Hong Kong/HK (leekalok2909@yahoo.com.hk)

Purpose: CT, using an en-face glenoid image, accurately quantifies glenoid bone loss in anterior shoulder dislocation. An anterior straight line and reduction in glenoid width are recognised CT signs of glenoid bone loss. It would be useful if MR could replace $\mathrm{CT}$ in this respect, especially as a high-resolution en-face image of the glenoid can be obtained during standard MR assessment. This study investigates the agreement between MR, CT and arthroscopy in measuring glenoid bone loss. Methods and Materials: Institutional approval for this study was granted. 176 patients (158 males, 18 females; mean $26.8 \pm 12.3$ years) with anterior shoulder dislocation underwent both shoulder MRI and CT examination. 65 of these patients also had arthroscopy performed. MR assessment of glenoid bone loss involved use of a best-fit circle.

Results: Excellent correlation existed between CT and MRI for length of anterior straight line $(r=0.97 p<0.0001)$, glenoid width $(r=0.95, p<0.0001)$ and severity of glenoid bone loss particularly using best-fit circle width $(r=0.82, p<0.0001)$ rather than best-fit circle area $(r=0.83, p<0.0001)$. There was a marginally better correlation between the CT and the arthroscopy $(r=0.91, p<0.0001)$ than MR and arthroscopy $(r=0.84, p<0.0001$ a) for glenoid bone loss.

Conclusion: MR assessment, particularly using glenoid width, is almost as accurate as CT assessment for glenoid bone loss. In general, MR can replace CT in this respect.

\section{B-0043 10:48}

Rotator cuff tendon tear size and retraction and quantification of muscle fatty infiltration using chemical shift-based water-fat MR imaging S. Lee, L. Nardo, R. Lucas, D. Karampinos, J. Carballido-Gamio, A. Lai, C.B. Ma, T. Link, R. Krug; San Francisco, CA/US (Sonia.Lee@ucsf.edu)

Purpose: This study aimed to define the relationship between rotator cuff tendon tear size and retraction and muscle fatty infiltration quantified using chemical shiftbased water-fat MR imaging.

Methods and Materials: The shoulders of 50 patients (26 men, 24 women; mean 52.44 years; range 17-65 years) with shoulder pain were imaged using $3 \mathrm{~T}$ MR scanner. Sequences included oblique-sagittal T1-, T2- and PD-weighted fast spin echo (FSE), coronal and axial T2 FSE and a gradient echo with 6 echo shifts to generate fat fraction maps using the IDEAL algorithm. Two radiologists evaluated supraspinatus, infraspinatus and subscapularis tendons according a 5-point scoring system: $0=$ normal; 1 =tendinosis; 2 partial thickness tear; $3=$ full-thickness tear and $4=$ full-thickness tear with retraction and graded muscles according to Goutallier classification. Tears sizes were measured. The retraction was classified into (i) retraction $<1 \mathrm{~cm}$; (ii) retraction $>1 \mathrm{~cm}$, but lateral to the glenoid; and (iii) retraction medial to the glenoid. Comparisons between IDEAL fat fraction values and tear variables were assessed with Spearman Rank Correlation (SRC)

Results: A significant correlation between the clinical grading and the fat fraction values was found for all the evaluated muscles: $p<0.0001$, SCR of 0.75 and 0.70 , 0.68 for supraspinatus, infraspinatus and subscapularis muscles, respectively. The fatty infiltration values correlated with both size of the tear $(p=0.031$ SCR of 0.48$)$, and with the degree of tendon retraction ( $p=0.015$; SCR of 0.54$)$.

Conclusion: Fat fraction using chemical shift-based water-fat MR imaging correlates with rotator cuff tear size and degree of retraction.

\section{B-0044 10:57}

Comparison of 3D turbo spin-echo space sequences with conventional 2D MRI sequences to assess the shoulder joint J.K. Kloth ${ }^{1}$, M. Winterstein ${ }^{1}$, M. Akbar ${ }^{1}$, E. Meyer ${ }^{2}$, D. Paul ${ }^{2}$, H.-U. Kauczor ${ }^{1}$, M.-A. Weber ${ }^{1} ;{ }^{1}$ Heidelberg/DE, ${ }^{2}$ Erlangen/DE (jost.kloth@med.uni-heidelberg.de)

Purpose: To determine the accuracy and reliability of three-dimensional (3D) intermediate- and T1-weighted turbo spin-echo (TSE) sampling perfection with application-optimised contrasts using different flip-angle evolution (SPACE) compared with conventional 2D sequences in assessment of the shoulder-joint. Methods and Materials: Ninety-nine subjects were examined on a 3-Tesla MRI system with both conventional 2D-TSE in T1-, T2- and fat-saturated proton-density (PD)-weighting (measurement time 26:12 min) and 3D-TSE SPACE in T1- and PD-weighting with $0.6 \mathrm{~mm}$ slide thickness (measurement time 15:52 min). All examinations were assessed independently by two reviewers for pathologies of the rotator cuff, biceps tendon, cartilage and bone-marrow, as well as the presence of effusion and bursitis. Concordance between 2D- and 3D-sequences and inter-observe agreement was evaluated using kappa statistics. 
Results: Concordance between 2D and 3D was almost perfect for tendinopathies of the rotator-cuff $(\mathrm{K}=0.88)$, omarthrosis $(\mathrm{K}=1)$ and adjacent bone marrow $(\mathrm{K}=0.873)$ and substantial for rotator-cuff injuries $(\mathrm{K}=0.621-0.726)$, tendinopathies of the biceps tendon ( $\mathrm{k}=0.787$ ), effusion ( $\mathrm{k}=0.779)$, bursitis $(\mathrm{k}=0.704)$ and well as cartilage tears $(\mathrm{k}=0.719)$. Inter-observer agreement was almost perfect for omarthrosis $(\mathrm{k}=1)$ and tendinopathies $(k=0.81)$, substantial for effusion $(k=0.701)$ and bone marrow $(K=0.778)$ but moderate and fair for the other mentioned pathologies. Kappacoefficients were generally higher for conventional 2D TSE sequences (0.333-1) when compared to 3D TSE SPACE sequences (-0.012-1).

Conclusion: Due to substantial and almost perfect concordance with conventional 2D TSE sequences for common pathologies, shorter examination-time up to $11 \mathrm{~min}$ and therefore increasing patient comfort, shoulder MRI examination-time can be reduced by nearly $40 \%$ using 3D-SPACE without loss of information.

Author Disclosures:

H. Kauczor: Advisory Board; Siemens. Investigator; Boehringer Ingelheim.

Speaker; Siemens, Boehringer Ingelheim, Bayer, Bracco.

\section{B-0045 11:06}

Evaluation of MR-arthrography in identifying bony Bankart lesions: usefulness of thin-slice T1 weighted GRE sequences

G. Foti, E. Oliboni, L. Romano; Negrar/IT (eugenio.oliboni.84@hotmail.it)

Purpose: To evaluate diagnostic accuracy of MR arthrography in identifying bony Bankart lesions using as reference standard high-resolution CT.

Methods and Materials: MR arthrogragraphy of 48 consecutive patients suffering from gleno-humeral instability and/or shoulder luxations were retrospectively reviewed by 2 readers in order to identify bony Bankart lesions. In addition to standard protocol, thin-slice $(0.8 \mathrm{~mm})$ fat-saturated T1 GRE sequence was acquired on axial plane. MRI imaging findings were compared with high-resolution CT images, considered as reference standard, considering a value of $p<0.05$ as statistically significant. Also, additional findings regarding tendons and ligament involvement were assessed.

Results: A bony Bankart lesion was identified in 14 of 48 patients using CT. At MRI, Glenoid fracture was correctly identified using standard protocol in 10/14 cases $(71.4 \%)$, whereas it was depicted by thin-slice GRE sequence in 13/14 cases (sensitivity $92.8 \%$ ); MRI pointed out no false-positive findings (specificity 100\%). Associated imaging findings included inferior gleno-humeral ligament tear $(n=6)$, recent Hill-Sachs humeral fracture $(n=3)$, and rotator cuff partial tears $(n=3)$. Thin slice T1-Weighed GRE sequence performed similarly to CT in identifying bony Bankart lesions ( $p>0.05$ ), while it allowed obtaining better results if compared with the standard sequences of our MR-arthrography protocol $(p=0.025)$.

Conclusion: Thin-slice fat-saturated T1 GRE sequence at MR arthrography, allowing multiplanar isotropic reconstructions, can be employed to depict bony Bankart lesions in patients suffering from gleno-humeral instability and shoulder luxations.

\section{B-0046 11:15}

Superior labral dimension of the glenoid labrum on direct MR arthrography (MRA): relationship with presence of SLAP

T. Im ${ }^{1}$, J.-A. Choi ${ }^{1}$, G. Lee ${ }^{1}$, J. Oh'1 , S. Hong ${ }^{2}$, H. Kang ${ }^{1} ;{ }^{1}$ Seongnam/KR, ${ }^{2}$ Seoul/KR (hifi310@gmail.com)

Purpose: To evaluate the relationship between superior labral dimension of glenoid labrum on direct MRA and SLAP lesion.

Methods and Materials: Direct MRA of shoulder was performed in 295 patients for chronic pain or instability, who underwent arthroscopy and whose records were reviewed. MR images were analysed by a radiologist; superior labral dimension was measured on coronal T1-weighted fat-suppressed (T1 FS) and non-fat-suppressed (T1WI) images, at the plane where long head of biceps tendon disappeared and labrum appeared the largest. Transverse and longitudinal dimensions were measured as base and height of the inverted triangular-shaped superior labrum and compared between patients who had SLAP on arthroscopy (SLAP group) vs. those who did not (non-SLAP group). Statistical analysis was done using Student's T-test. Results: In 295 patients, 132 patients (44.7\%) had SLAP. Mean values of base and height of labrum on T1WI were $8.91 \mathrm{~mm}$ (SD 0.17) and $5.28 \mathrm{~mm}$ (SD 0.11) in SLAP group, $8.96 \mathrm{~mm}$ (SD 0.17) and $5.04 \mathrm{~mm}$ (SD.088) in non-SLAP group, respectively. Mean values of base and height on T1 FS images were $8.68 \mathrm{~mm}$ (SD $0.16)$ and $5.21 \mathrm{~mm}$ (SD 0.10) in SLAP group, $8.76 \mathrm{~mm}$ (SD 0.16) and $4.95 \mathrm{~mm}$ (SD 0.08) in non-SLAP group, respectively. In the SLAP group, the mean labral height tended to be larger than that of non-SLAP group although the difference was not statistically different.

Conclusion: Labral dimensions on coronal MRA showed no statistical difference between SLAP and non-SLAP patients; there was a tendency for height to be larger in SLAP group than in non-SLAP group.

\section{B-0047 11:24}

Evaluation of concomitant osseous carpal injuries in patients with distal radial fractures in MDCT

A. von Schneider-Egestorf, C. von Falck, B. Meyer, F. Wacker, H. Rosenthal; Hannover/DE (amelievonschneider@hotmail.com)

Purpose: To investigate the prevalence and type of concomitant carpal bone fractures in patients with acute intra-articular distal radial fractures.

Methods and Materials: Evaluation of 1295 diagnostic MDCT scans of the wrist acquired between 01/2005 and 10/2011. The final study group included 603 patients with acute distal radial fractures (Frykman III-VIII). All images were analysed in consensus by a radiology resident and an experienced musculoskeletal radiologist. Results: $20.4 \%$ of all patients with distal intraarticular radial fractures had simultan carpal fractures. Among the total number of 144 fractures, the most common fracture type was the dorsal avulsion fracture of the triquetrum $(44.2 \%)$, followed by fractures of the lunate $(14.6 \%)$, trapezium fractures $(11.1 \%)$, scaphoideum fractures $(10.4 \%)$, hamate fractures $(7.7 \%)$ and pisiforme fractures $(4.9 \%)$. Significantly higher prevalence of fractures in the proximal carpal row. Trapezial ridge fractures were seen in 11 cases.

Conclusion: Intraarticular distal radial fractures are frequently associated with simultan carpal fractures. Based on our findings the diagnostic MDCT is highly recommended for patients with distal intraarticular radial fractures.

\section{B-0048 11:33}

Normal anatomy and variants of the trapeziometacarpal joint at MR imaging in asymptomatic volunteers

A. Hirschmann, R. Sutter, A. Schweizer, C.W.A. Pfirrmann; Zurich/CH (anna.hirschmann@balgrist.ch)

Purpose: To prospectively characterise the MR anatomy and variants of the trapeziometacarpal (TMC) joint in asymptomatic volunteers.

Methods and Materials: 34 asymptomatic volunteers (17 women, 17 men, mean age 33.9 \pm 9 .2years) underwent MR imaging of the thumb after approval by the local ethical committee. Two musculoskeletal radiologists independently classified visibility and signal intensity $(\mathrm{SI})$ characteristics of the anterior oblique (AOL/beak ligament), the posterior oblique (POL), the intermetacarpal (IML) and the dorsoradial ligaments (DRL) on a 3-point Likert scale. The thickness of all ligaments, cartilage integrity and presence of joint fluid were assessed. The alignment of the first metacarpal base with the trapezium was quantified on sagittal and coronal planes. Results: On intermediate-balanced fat-saturated images the POL $(65 \% / 74 \%$ reader $1 /$ reader 2$)$ and DRL (58\%/64\%) were commonly of intermediate SI, while the IML had a striated appearance in $91 \% / 76 \%$ of subjects. The AOL showed a variable SI (36\%/42\% low, $27 \% / 27 \%$ increased,36\%/30\% striated). The IML was the thickest ligament with a mean of $2.9 \mathrm{~mm} / 3.1 \mathrm{~mm}$ and the DRL the thinnest $(1.2 \mathrm{~mm} / 1.4 \mathrm{~mm})$. There was a mean dorsal shift $(1.8 \mathrm{~mm} / 2.0 \mathrm{~mm})$ and radial shift $(2.8 \mathrm{~mm} / 3.4 \mathrm{~mm})$ of the metacarpal base. The AOL was significantly thicker in men $(1.7 \mathrm{~mm})$ than in women (1.2 mm, $P=0.02)$. Radial shift was significantly larger in men $(3.4 \mathrm{~mm})$ than in women $(2.2 \mathrm{~mm}, \mathrm{P}=0.02)$. No shift in palmar or ulnar direction was seen. Conclusion: The TMC ligaments showed a considerable variability. The IML is typically striated and thicker compared to the other ligaments. Radial and dorsal shift of the TMC joint is a normal finding at MR imaging

\section{B-0049 11:42}

Safety of dorsal wrist arthroscopy portals: a magnetic resonance study R. Cazzato, R. Del Vescovo, F. Giurazza, R.F. Grasso, B. Beomonte Zobel; Rome/IT (r.delvescovo@unicampus.it)

Purpose: Aim of our study was to assess and compare the distance of commonly used dorsal wrist arthroscopy portals to radial and ulnar neurovascular structures. Methods and Materials: 40 patients were prospectively evaluated with T1-weighted spin-echo (SE) magnetic resonance (MR) sequences in the coronal and transverse planes of the radiocarpal joint. The distances between portals and nearest superficial nerve branch and vascular bundle were measured. Statistical analysis of the data was performed with SPSS.

Results: The median age of patients was 39 years. The median distance of the nearest nerve branch to portal 1-2 was $2 \mathrm{~mm}$; portal 3-4, $19.49 \mathrm{~mm}$; portal 4-5, $14.66 \mathrm{~mm}$; portal 6/R, $9.66 \mathrm{~mm}$; and portal $6 / \mathrm{U}, 4.33 \mathrm{~mm}$. The median distance of the nearest vascular bundle to portal 1-2 was $7.66 \mathrm{~mm}$; portal 3-4, $9.66 \mathrm{~mm}$; portal 4-5, $8.49 \mathrm{~mm}$; portal 6/R, $7.33 \mathrm{~mm}$; and portal 6/U, $6.33 \mathrm{~mm}$. In terms of distance to the nearest vascular bundle, the 3-4 portal was safer than the 1-2 portal $(P<$ $0.0001)$, 4-5 portal $(P=0.008), 6 / R(P<0.0001)$, and $6 / U$ portals $(P<0.0001)$. In terms of distance to nearest nerve branch, the $3-4$ portal was safer than the 1-2 
portal $(P<0.0001), 4-5$ portal $(P<0.0001), 6 / R(P<0.0001)$, and $6 / U$ portals $(P<$ 0.0001 ). No statistical significant differences were found between the two genders. Conclusion: In wrist arthroscopy, the 3-4 portal is safer than any other dorsal portal, followed by the $4-5$ portal. The 1-2 and $6 / U$ portals are the most dangerous in terms of proximity to neurovascular structures.

\section{B-0050 11:51}

Comparison of three-dimensional indirect isotropic MR arthrography and conventional MR arthrography for the diagnosis of rotator cuff tears J. Lee, Y. Yoon, S. Ji; Seoul/KR (zhyun84.lee@samsung.com)

Purpose: To compare the diagnostic accuracy of a three-dimensional (3D) indirect isotropic T1-weighted fast spin-echo (FSE) MR arthrography with that of a conventional two-dimensional (2D) sequences of indirect MR arthrography for the diagnosis of rotator cuff tears.

Methods and Materials: 149 patients who had undergone indirect shoulder MR arthrography followed by arthroscopic surgery were enrolled. Both conventional 2D and $3 \mathrm{D}$ isotropic T1-weighted FSE sequences were performed. Two radiologists evaluated the images for the presence of supraspinatus-infraspinatus and subscapularis tendon tears. Using the arthroscopic findings as the reference standard, the statistical differences between the sensitivities, specificities, and accuracies of both methods were analysed by McNemar test. Kappa values were also analysed to compare accuracy of grading supraspinatus-infraspinatus tendon tear between both methods.

Results: Arthroscopy confirmed 123 supraspinatus-infraspinatus tears and 75 subscapularis tears. The sensitivity and specificity of conventional sequences were $95 \%$ and $87 \%$, respectively, for supraspinatus-infraspinatus tears, $78 \%$ and $91 \%$ for subscapularis tears. The sensitivity and specificity of 3D sequence were $95 \%$ and $89 \%$, respectively, for supraspinatus-infraspinatus tears, $81 \%$ and $91 \%$ for subscapularis tears. There were no significant differences between two methods. The kappa values for grading supraspinatus-infraspinatus tear were 0.704 between conventional sequences and arthroscopic findings, 0.666 between 3D sequence and arthroscopic findings, and 0.829 between conventional and 3D sequences. The diagnostic accuracies for grading supraspinatus-infraspinatus tendon tear were not significantly different.

Conclusion: 3D indirect isotropic MR arthrography may show similar accuracy for the diagnosis of rotator cuff tears as conventional indirect MR arthrography with shorter imaging time.

\section{$10: 30-12: 00$} Room E2

\section{GI Tract}

\section{SS 101a}

\section{Acute bowel diseases: challenges and solutions}

\section{Moderators:}

J. Dormagen; Oslo/NO

S. Leschka; St. Gallen/CH

\section{B-0051 10:30}

The emperor's new clothes? An evaluation of the accuracy of transabdominal ultrasound in diagnosing acute appendicitis P. Yoong ${ }^{1}$, C.A. Johnson ${ }^{2}$, S. Fernando ${ }^{1}$, J.W. Graham ${ }^{1} ;{ }^{1} K^{\prime} i n g ' s ~ L y n n / U K$, ${ }^{2}$ London/UK (c.a.yoong@gmail.com)

Purpose: To determine the accuracy of transabdominal ultrasound in diagnosing acute appendicitis.

Methods and Materials: A retrospective review of ultrasound reports was performed between December 2007 and May 2012 (54 months). Scans were included in the study when the keyword "appendix" or "appendicitis" was present. Using established positive ultrasound findings for appendicitis, scans were divided into three groups: "positive", "indeterminate" and "negative". Correlation was made with histopathology reports and clinical notes. The sensitivity, specificity, positive and negative predictive values were calculated.

Results: There were a total of 317 ultrasound scans (314 patients). $89.6 \%$ ( $n=284)$ were performed by a consultant radiologist. The mean patient age was 26.2 (range $2-89)$ years and $82.6 \%(n=262)$ were female. $40 \%$ of scanned patients $(n=127)$ proceeded to have an appendicectomy during their admission. There was a sensitivity of $23 \%$ and specificity of $93 \%$ for the diagnosis of histologically confirmed acute appendicitis. $37 \%$ (40/110) of patients with negative scans showed acute appendicitis on histology.
Conclusion: Ultrasound was insensitive for diagnosing appendicitis, contrary to the findings in most of the available literature. A substantial proportion of patients with "normal" scans had acute appendicitis. Although ultrasound remains useful in identifying co-existing abdominal pathology, clinical evaluation and blood markers should play the primary role in diagnosis.

\section{B-0052 10:39}

Added value of ultrasound re-evaluation for patients with equivocal CT findings of acute appendicitis: a preliminary study

J. Sim, H. Kim, J. Yeon, B. Suh, K. Kim, Y. Ha, S. Paik;

Sungnam-si, Gyeonggi-do/KR (sunnyandluna@naver.com)

Purpose: A prospective study to estimate the additional diagnostic value of ultrasound (US) re-evaluation for patients with equivocal computed tomography (CT) findings of acute appendicitis.

Methods and Materials: Between April 2011 and October 2011, a total of 869 consecutive patients with suspected appendicitis who were referred for CT were included in the study. The likelihood of appendicitis was prospectively categorised into five categories. US re-evaluation was recommended for patients in the equivocal appendix and probably not appendicitis groups. The overall negative appendectomy rate of study period was compared with the rate during the same period of the previous year, and negative appendectomy rates of the US and nonUS evaluation groups were also compared.

Results: Among 869 patients, 71 (8.2\%) patients had equivocal finding of appendicitis and $63(7.9 \%)$ patients diagnosed as probably not appendicitis. The sensitivity and specificity of CT combined with US re-evaluation (100\% and $98.1 \%$, respectively) among patients with confirmed appendicitis exceeded those of CT only (93\%, 99\%; equivocal group considered as negative appendicitis and $100 \%$, $89.9 \%$; as positive, respectively), and this was statistically significant $(P<0.0001)$. After adding US re-evaluation, the overall negative appendectomy rate in our institution decreased from $3.4 \%$ to $2.3 \%$.

Conclusion: For patients with equivocal CT findings of acute appendicitis, US re-evaluation can improve diagnostic accuracy and decrease the rate of negative appendectomies.

\section{B-0053 10:48}

Reduced Z-axis coverage at multidetector-row CT to decrease radiation dose and maintain diagnostic accuracy in adults suspected of acute appendicitis

N. Brassart ${ }^{1}$, C. Winant ${ }^{2}$, D. Tack ${ }^{3}$, P. Gevenois ${ }^{1}$, V. de Maertelaer ${ }^{1}$, C. Keyzer ${ }^{1}$; 1'Brussels/BE, ${ }^{2}$ Mons/BE, ${ }^{3}$ Baudour/BE (nbrassar@gmail.com)

Purpose: To compare diagnostic performance of two reduced Z-axis coverages to that on the abdomen and pelvis for the diagnosis of acute appendicitis as well as alternative diseases at unenhanced CT.

Methods and Materials: This study included 152 patients suspected of appendicitis who were enrolled in two ethical-committee-approved previous trials. Based on scans covering the entire abdomen and pelvis (L-set), two additional sets of images were generated with two reduced $Z$-axis coverages: $1^{\circ}$ ) from the top of the iliac crests to the upper aspect of the pubis (S-set); and $2^{\circ}$ ) from the diaphragmatic roots to the upper aspect of the pubis (M-set). Two readers independently coded during separate sessions the appendix visualisation, measured its diameter, and proposed a diagnosis (appendicitis or alternative disease). Final diagnosis was based on surgical findings or clinical follow-up. Pearson chi-square, Fisher exact, Mc Nemar tests, and logistic regression were used.

Results: Forty-six patients had a definite diagnosis of appendicitis and 53 an alternative diseases. For the diagnosis of appendicitis, sensitivity, specificity, positive and negative predictive values, and accuracy were not significantly different between sets for both readers ( $p$ from 0.857 to 1.000 ). The probability of giving a correct alternative diagnosis was lower on S-set than on L-set (OR:0.573, $p=0.009$ ) for both readers, but there was no significant difference between $\mathrm{L}$ - and $\mathrm{M}$-set $(p=0.824)$, Z-axis coverage being reduced by $25 \%$ on M-set.

Conclusion: Restricted coverage could be recommended for the diagnosis of appendicitis as well as alternative diseases, whereas pelvic focused CT is insufficient for alternative diseases. 


\section{B-0055 10:57}

The effectiveness of multidetector computed tomography in patients that underwent immediate intestinal resection due to acute abdominal pain M. İnci, F. Özkan, S. Bozkurt, M. Yüksel, O. Peker; Kahramanmaraş/TR (drfatihinci@gmail.com)

Purpose: The aim of this study was to evaluate the role and additional diagnostic contribution of multidetector computed tomography (MDCT) findings in patients with acute abdominal pain caused by small and large bowel pathologies that required immediate intestinal resection.

Methods and Materials: A total of 42 patients who were admitted to our hospital's emergency room between January 2011 and July 2012 with a complaint of acute abdominal pain caused by non-traumatic small and large bowel pathologies and who underwent immediate intestinal resection were included in our study. The definitive diagnosis and MDCT findings of patients were retrospectively reviewed. Results: Of these patients, 23 (54.8\%) were males and $19(45.2 \%)$ were females. The mean age was $49 \pm 3.4$ years, ranging from 29 to 64 years. The sensitivity of MDCT in diagnosing intestinal pathologies leading to acute abdominal pain and which required immediate intestinal resection was $93.7,83.3$, and $85.7 \%$ in patient groups of intestinal perforation, acute mesenteric ischaemia, and intestinal obstruction, respectively. A total concordance between the MDCT findings and definitive diagnosis was found in 37 of 42 cases; partial discordance was seen in 3 of 42 cases $(7.1 \%)$ and total discordance was seen in 2 of 42 cases $(4.7 \%)$. The overall sensitivity of MDCT in the diagnosis of intestinal pathologies that required immediate intestinal resection was found to be $88.1 \%$.

Conclusion: MDCT is a rapid, reliable, highly accurate and cost-effective modality in the differential diagnosis of small and large bowel pathologies leading to acute abdominal pain and requiring immediate intestinal resection.

\section{B-0056 11:06}

In the evaluation of patients with obscure gastrointestinal bleed, does MDCT angiography have a role?

C.B. Kulkarni, S. Moorthy, S.K. Pullara, R.R. Kannan, P.V. Ramchandran,

S. Srinivasan; Kochi/IN (drchinmaykulkarni@gmail.com)

Purpose: To prospectively determine the sensitivity of MDCT angiography in detecting and diagnosing the cause of obscure gastrointestinal bleed (OGIB).

Methods and Materials: Our study included 50 patients (males 30, females 20) in the age range of 3-82 years (average age: 58.52 years) who were referred to our department as part of their workup for clinically evident gastrointestinal (Gl) bleed or as part of workup for anaemia. All patients underwent conventional upper endoscopy and colonoscopy. Following a noncontrast scan, all patients underwent triple-phase contrast CT scan using a 64-slice CT scan system. The diagnostic performance of MDCT was compared with the results of capsule endoscopy, $99 \mathrm{~m}$ technetium-labelled red blood cell scintigraphy (99mTc-RBC scintigraphy), digital subtraction angiography and surgery whenever available.

Results: The sensitivity, specificity, positive predictive value, and negative predictive values of MDCT for detection of bleed were $72.2,42.8,81.2$, and $44.4 \%$, respectively. Capsule endoscopy was done in 15 patients and was positive in 10 patients; it had a sensitivity of $71.4 \%$. Eleven patients had undergone $99 \mathrm{mTc}-\mathrm{RBC}$ scintigraphy prior to CT scan, and the result was positive in seven patients (sensitivity $70 \%$ ). Digital subtraction angiography was performed in only eight patients. All except one patient showed findings consistent with the lesions detected on MDCT. Conclusion: MDCT angiography is a sensitive tool that allows rapid detection and localisation of OGIB. It can be used as the first-line investigation in patients with negative endoscopy and colonoscopy studies. MDCT and capsule endoscopy have complementary roles in the evaluation of OGIB.

\section{B-0057 11:15}

Venous mesenteric ischaemia vs acute arterial mesenteric ischaemia with reperfusion: similarities and differences

D. Berritto ${ }^{1}$, F. lacobellis ${ }^{1}$, M.P. Belfiore ${ }^{1}$, M.A. Mazzei ${ }^{2}$, L. Saba ${ }^{3}$, R. Di Mizio ${ }^{4}$ S. Cappabianca ${ }^{1}$, A. Rotondo', R. Grassi $1{ }^{1}{ }^{1}$ Naples $/ I T,{ }^{2}$ Siena/lTT, ${ }^{3}$ Cagliari/lT, ${ }^{4}$ Penne/IT (daniela.berritto@libero.it)

Purpose: To explore similarities and differences between venous mesenteric ischaemia and the reperfusion after acute arterial occlusion in an experimental model studied by a 7T MRI system, establishing radiological findings that may help to distinguish between these 2 conditions and select the correct therapeutic approach. Methods and Materials: Thirty rats were divided in two groups that underwent different surgical models and a monitoring of ischaemic damage. Group.i. underwent superior mesenteric vein (SMV) ligation, whereas group II underwent superior mesenteric artery (SMA) ligation followed by reperfusion after 1 hour. At different time-points 3 rats for each group were imaged with $7 \mathrm{~T}$ MRI, euthanized and macroscopically/histologically analysed.

Results: In the first hour it was not possible to detect MRI findings that helped in distinguishing these two conditions: in both groups hyper-intense signal in T2-W sequences in the intestinal wall was detected, as well as the presence of free fluid. In the second hour, the MRI begun to show differences: in the group II, in the $100 \%$ of the rats, the hypotonic reflex ileus (HRI) was found, whereas this finding was not present after SMV occlusion. In the fourth hour the congested mesentery is detectable only in those rats with SMV occlusion while the paralytic ileus was visible in both groups from the sixth hour. Only in 1 case of VMI was observed wall pneumatosis after eighth hours.

Conclusion: Results of this study suggest that the analysis of mesentery and the presence/absence of the $\mathrm{HRI}$ analysed by MRI may help in distinguishing between these two conditions.

\section{B-0058 11:24}

Non-occlusive mesenteric ischaemia (NOMI) with and without reperfusion: $\mathrm{CT}$ features useful for diagnosis and their prognostic value M.A. Mazzei', G. Imbriaco', S. Guerrini', N. Cioffi Squitieri', E. Foderà', D. Berritto ${ }^{2}$, P. Mercuri', F.G. Mazzei', L. Volterrani' ${ }^{1}{ }^{1}$ Siena/IT, ${ }^{2}$ Naples $/ I T$ (mamazzei@gmail.com)

Purpose: NOMI is a dynamic condition. Its appearance at imaging may be different depending on the time in which $\mathrm{CT}$ examination is performed and on the presence of reperfusion event. The aim of our work has been to assess the efficacy of MDCT for the diagnosis of NOMI, with and without reperfusion event.

Methods and Materials: Clinical and imaging data from 20 patients (16 males, 4 females) with a dismissal summary of NOMI were retrospectively reviewed. All patients had at least 1 abdominal MDCT examination $(n=28)$ followed by surgery $(n=8)$, autopsy $(n=2)$, angiography $(n=1)$ or endoscopy $(n=9)$. The CT scans were evaluated for evidence of vessels, mesentery, bowel and cavity features. The mean diameter of the SMA of NOMI cases were compared with that of 30 controlled cases. Results: The average SMA diameter of NOMI cases were statistically smaller than that of controls (K-S test: $D=0.75$, Prob=3.7E-08). A correlation between the reperfusion event and mesenteric fat stranding $(p=0.026)$, bowel wall thickening $(p=3.2 \mathrm{E}-05)$ and high attenuation of the bowel wall on unenhanced CT images $(p=2.8 \mathrm{E}-04)$ was demonstrated by Fisher's exact tests. Normal mesenteric vessels plus wall thickening was significantly correlated with lower mortality $(p=0.034)$. Conclusion: The evaluation of mesentery, bowel and peritoneal CT features, in addition to the vessel findings will supply additional information to recognise NOMI and in discriminating the presence of a reperfusion event.

\section{B-0059 11:33}

Diagnosis of acute arterial mesenteric ischaemia with and without reperfusion using 7T-MRI in an animal model

D. Berritto ${ }^{1}$, F. lacobellis ${ }^{1}$, M.P. Belfiore ${ }^{1}$, M.A. Mazzei ${ }^{2}$, L. Saba ${ }^{3}$, M. Scaglione ${ }^{4}$ S. Cappabianca ${ }^{1}$, A. Rotondo', R. Grassi' $;{ }^{1}$ Naples $/ I T$, ${ }^{2}$ Siena/lT, ${ }^{3}$ Cagliari/lT, ${ }^{4}$ Castel Volturno/IT (daniela.berritto@libero.it)

Purpose: This study, based on an animal model, was designed to define the evolution of 2 different mechanisms of acute arterial mesenteric ischaemia (AAMI): the acute occlusion of superior mesenteric artery (SMA) and the acute occlusion followed by reperfusion, and to investigate the MRI findings in a timeline.

Methods and Materials: Eight rats underwent SMA ligation and then divided into two groups. In group.i. the ischaemia was maintained for 8 hours; in group II, 1 hour after SMA occlusion the ligation was removed, and the following reperfusion was monitored for 8 hours. MRI was performed using a 7-T MRI system.

Results: Free fluid, hypotonic reflex ileus, dilated loops gas-fluid filled and bowel wall hyperintensity are always present in all rats with mesenteric ischaemia independently from the mechanism. Exploring the 2 mechanisms (presencelabsence of reperfusion) we found that in those rats without reperfusion the signal intensity is normal at the first and second hour and becomes hyper-intense at the fourth and eighth hour; after reperfusion the hyper-intense signal is clearly visible form the first hour. Another MRI finding is the presence of bowel wall thickening that is visible from the second hour in case of reperfusion, but was never found in the timeline in those rats without reperfusion. These 2 findings suggest that the early hyper-intensity of the wall in T2-W sequence and bowel wall thickening may be considered signs of acute mesenteric ischaemia with reperfusion.

Conclusion: Results of this preliminary study in animal model suggest that MRI can adequately assess the different pathological changes that occur in the AAMI without or with reperfusion. 


\section{B-0060 11:42}

The potential benefit of abdominal $\mathrm{CT}$ to help differentiate between strains of clostridium difficile

S.N. Reddy, S. Taori, I.R. Poxton, F. Ewing, D. Brown, J.T. Murchison; Edinburgh/UK

Purpose: Severe Clostridium difficile infection (CDI) is increasingly being assessed by computed tomography (CT). Our aim was to assess imaging findings in patients affected by different $\mathrm{C}$. difficile strains.

Methods and Materials: All C. difficile positive faecal samples from 1 August 2010 to 31 July 2011 were cultured and PCR ribotyped. A retrospective review of prospectively collected clinical and radiological data was then performed on all positive, symptomatic, hospitalised adult in-patients.

Results: $453 \mathrm{CDI}$ patient episodes were identified in 350 symptomatic patients. Overall 72 CT-scans were performed, 55 scans demonstrated colonic involvement, with pancolitis in only $31 \%$. Colonic changes were present in $35 \%$ of episodes prior to the availability of a $C$. difficile positive result (median 2 days). Ribotype 001 (73 episodes) was the commonest identified $C$. difficile strain and a CT was performed in only $11 \%$, compared with ribotype 078 , the $6^{\text {th }}$ commonest strain (19 episodes) where CT was performed in $52 \%$. Patients with 078 had a greater colonic wall thicknesses (median $16 \mathrm{~mm}$ ) compared with the remaining cohort (median $11 \mathrm{~mm}$ ). Ascites was present in $64 \%$ of the scanned 078 patients compared with $31 \%$ in the remaining cohort.

Conclusion: $\mathrm{CDI}$ should be considered in the differential diagnosis of colitides, even prior to a $C$. difficile positive result. Increased colonic wall thickness and ascites were more common in the 078 episode group with a greater proportion of CT scans performed. 078 appears to stimulate a greater inflammatory response within the colon, which has not been previously described.

10:30 - 12:00

Room F1

\section{Oncologic Imaging}

\section{SS 116}

\section{Perfusion CT and MRI: ready for clinical practice}

Moderators:

S. Delorme; Heidelberg/DE

F.A. Gallagher; Cambridge/UK

\section{B-0061 10:30}

Functional MRI changes in follow-up after external beam radiation therapy of the prostate

A.M. Weidner; Mannheim/DE (anja.weidner@medma.uni-heidelberg.de)

Purpose: To evaluate changes visible in different magnetic resonance imaging (MRI) modalities in patients with prostate cancer after radiation therapy (RT). Methods and Materials: 43 examinations in 15 patients including T2-weighted imaging (T2), diffusion-weighted imaging (DWI), dynamic contrast-enhanced imaging (DCE) and spectroscopy (MRS) before and at several time points after RT were performed. Apparent diffusion coefficient (ADC) values, plasma flow (PF), mean transit time (MTT), maximum choline+creatine/citrate-ratio (CC/CR) and number of pathological spectra were evaluated.

Results: In initial MR we found a signal loss in T2, a decreased ADC, a decreased MTT and voxel with increased CC/CR in prostate cancer. Signal loss in T2-weighted imaging in benign tissue was partially reversible after radiation, cancer areas equalled benign tissue. In prostate cancer nodules we found a significant increase in ADC from a mean value of $835 \times 10-6 \mathrm{~mm} 2 / \mathrm{s}$ on initial MRI to $1527 \times 10-6 \mathrm{~mm} 2 / \mathrm{s}$ more than 2 years after RT. PF decreased from $38 \mathrm{ml} / 100 \mathrm{ml} / \mathrm{min}$ to $5 \mathrm{ml} / 100 \mathrm{ml} / \mathrm{min}$ and MTT increased significantly from $78 \mathrm{~s}$ to $254 \mathrm{~s}$ in prostate cancer nodules. No changes were found for PF and MTT in benign tissue. MRS showed inconsistent results with a tendency for decreased CC/CR from 2.5 to 1.0 and a decrease in number of pathological voxel from $100 \%$ to $20 \%$. PSA decreased to values less than $2 \mathrm{ng} / \mathrm{ml}$ in all patients.

Conclusion: We found characteristic changes in every MRI modality after RT. MRS showed inconsistent results, so it might be less useful for RT monitoring than DWI or DCE.
B-0062 10:39

Acute changes in rectal cancer perfusion CT parameters during short-course radiotherapy correlate with radiological response to therapy J.M. Franklin, E.J. Hill, T. Pwint, F.V. Gleeson, R.A. Sharma, E.M. Anderson; Oxford/UK (jamiemfranklin@hotmail.com)

Purpose: Acute perfusion changes measured with perfusion $\mathrm{CT}(\mathrm{pCT})$ have been shown to predict subsequent response to chemoradiotherapy. The purpose of this study was to assess $\mathrm{pCT}$ as a predictive test for subsequent radiological response to short course radiotherapy (SCRT) in patients with rectal cancer.

Methods and Materials: Seven patients were recruited to this pilot study. All patients had baseline pCT and repeat pCT on day 4-6 SCRT (25Gy/5[[Unable to Display Character: \&\#9839;]]), before delivery of the radiotherapy fraction. pCT was performed on a GE Lightspeed VCT 64-slice CT using a standardised protocol. Blood volume (BV), blood flow (BF) and mean transit time (MTT) were derived within tumour ROI. MR imaging with high-resolution T2W sequences was performed before and 8 weeks after treatment and used to assess treatment response.

Results: One patient was excluded from analysis due to an inadequate baseline pCT. Patients with complete MRI response to SCRT $(n=3)$ had an average percentage (\%) decrease in MTT $(-25.3 \%$; range $-4.5 \%$ to $-36.3 \%)$ and average $\%$ increase in BF (+65.3\%; range +26.4 to $+97.0 \%)$. Conversely, non-complete responders $(n=4)$ had an average \% increase in MTT $(12.4 \%$; range $-35.3 \%$ to $+66.7 \%)$ and average \% decrease in $\mathrm{BF}(-1.6 \%$; range -26.1 to $+39.5 \%)$. There was a trend toward a significant difference in $\mathrm{BF}$ changes from baseline between complete and non-complete responders ( $p=0.06$; Student's T-test).

Conclusion: An acute increase in tumour blood flow following SCRT in patients with rectal cancer is associated with a complete radiological response at 8 weeks.

\section{B-0063 10:48}

CT perfusion for evaluation of early treatment response of liver metastases after ${ }^{90} \mathrm{Y}$-radioembolisation

C.S. Reiner, F. Morsbach, B.-R. Sah, N. Schäfer, T. Pfammatter, H. Alkadhi; Zurich/CH (caecilia.reiner@usz.ch)

Purpose: To evaluate computed tomography (CT) perfusion for the assessment of early treatment response after ${ }^{90} \mathrm{Y}$-radioembolisation of patients with liver metastases.

Methods and Materials: 17 consecutive patients ( 10 males, 7 females; age range, 35-79y) with liver metastases underwent dynamic contrast-enhanced CT liver perfusion (128-slice dual source CT, four-dimensional spiral mode, scan range $14.8 \mathrm{~cm}$, 15 scans, cycle time 3 seconds) before and 4 weeks after ${ }^{90}$ Y-radioembolisation. The parameter arterial liver perfusion (ALP) was calculated from CT perfusion and tumour diameters were measured. Success of ${ }^{0} Y$-radioembolisation was evaluated on follow-up imaging (mean follow-up time 124 days) based on the Response Evaluation Criteria in Solid Tumours (Version 1.1). The paired t-test was used to compare the tumour parameters pre- and post-90Y-radioembolisation for different response groups.

Results: In the responder group $(\mathrm{n}=6)$ mean ALP pre- ${ }^{90} \mathrm{Y}$-radioembolisation was $34.02 \pm 12.48$ and significantly decreased to $20.40 \pm 11.00 \mathrm{ml} / 100 \mathrm{ml} / \mathrm{min}$ post${ }^{90} Y$-radioembolisation $(p=0.029)$. In the non-responder group $(n=11)$ mean ALP pre- ${ }^{9} \mathrm{Y}$-radioembolisation was not significantly different from mean ALP post${ }^{90}$-radioembolisation $(12.08 \pm 7.96$ vs. $15.80 \pm 9.37 \mathrm{ml} / 100 \mathrm{ml} / \mathrm{min}, \mathrm{p}=0.064)$. The mean tumour diameter showed no significant difference between pre- and post-90Yradioembolisation for both groups (responder: $p=0.242$, non-responder: $p=0.107$ ). Conclusion: CT perfusion shows early decrease of ALP in liver metastases responding to ${ }^{90} Y$-radioembolisation, while tumour diameter remains unchanged early after treatment. Hence, CT perfusion may be a valuable technique for early response assessment after ${ }^{90} \mathrm{Y}$-radioembolisation.

\section{B-0064 10:57}

First-pass perfusion CT on 256-detector row CT in the quantitative assessment of perfusion map for solid malignant neoplastic liver lesions: preliminary results

O. Minutolo, D. Ippolito, A.C. Cadonici, P.A. Bonaffini, C.R.G.L. Talei Franzesi, S. Sironi; Monza/lT (orazio.minutolo@yahoo.com)

Purpose: To determine the value of CT perfusion technique as diagnostic tool in the assessment of lesions vascularization parameters in patients with neoplastic liver disease.

Methods and Materials: A total of 50 lesions histologically proven hepatocellular carcinoma and 30 liver metastases were evaluated with 256-slice scanner CT perfusion technique (Brilliance iCT Philips). Perfusion CT images were acquired at a single liver level with following protocol ( 16 slice/scan; total of 40 scans; bolus 
injection of $50 \mathrm{ml}$ of iodinated contrast with flow rate of $5 \mathrm{ml} / \mathrm{sec}$ ). Philips's workstation was used to generate colour permeability maps showing perfusion of enhancing tumours. The parameters were calculated pixel-by-pixel using maximum slope method, and quantitative maps of arterial and portal perfusion were created. The following parameters were calculated: hepatic perfusion (HP), peak enhancement intensity (PEI), time to peak (TTP) and blood volume (BV). Correlation between CT perfusion parameters and clinical pathological classification was performed. Results: The quantitative analysis of $50 \mathrm{HCC}$ lesions showed the following values: $\mathrm{HP}(\mathrm{ml} / \mathrm{s} / 100 \mathrm{~g}): 47 \pm 13.8 ; \mathrm{PEI}(\mathrm{HU}): 39.6 \pm 30.1 ; \mathrm{TTP}(\mathrm{sec}): 18.7 \pm 4.1$ and BV $(\mathrm{ml} / 100$ $\mathrm{mg}): 22.5 \pm 4.6$. The corresponding values calculated in liver metastases were: HP $(\mathrm{ml} / \mathrm{s} / 100 \mathrm{~g}): 16.1 \pm 14.3$, PEI $(\mathrm{HU}): 24.23 \pm 26.25$, TTP $(\mathrm{sec}): 15.58 \pm 4.90$, BV $(\mathrm{ml} / 100$ $\mathrm{mg})$ : $3.47 \pm 3.85$. A significant $(\mathrm{p}<0.001)$ increase of HP, PEI, TTP and BV was observed in $\mathrm{HCC}$ lesions in comparison with metastases, due to neoangiogenesis associated with HCC growth, and in relation to necrotic phenomenon that typically occurs in metastatic lesion.

Conclusion: CT perfusion technique represents a complementary diagnostic tool that offers quantitative information about arterial and portal perfusion of liver tumours, and a feasible technique that provides an in vivo information about the microvascular structures related to biological changes in liver tumours.

\section{B-0065 11:06}

\section{CT perfusion imaging in stage II-III non-small cell lung cancer} patients undergoing chemo-radiotherapy-evaluation of metabolic tumour activity

M. Das, B. Reymen, P. Lambin, H. Sharifi, J.E. Wildberger, D. De Ruysscher, W. van Elmpt; Maastricht/NL (m.das@mumc.nl)

Purpose: Perfusion CT techniques have been developed to quantify vasculature inside tumours, whereas FDG-PET/CT shows the metabolic activity. We investigated whether the metabolic active regions of primary non-small cell lung cancer (NSCLC) tumour show different vasculature properties compared with metabolically active regions.

Methods and Materials: Twelve patients with stage II-III NSCLC from two clinical trials (10 from NCT01024829 and 2 from NCT01210378) prior to (chemo-) radiotherapy treatment were evaluated. On the same day, patients underwent FDG-PET/ CT (Siemens Biograph 40 PET/CT) and perfusion CT imaging (Siemens Definition Flash CT). Tumour volumes were delineated on the FDG-PET/CT scans by a radiation oncologist. FDG-PET/CT and perfusion $\mathrm{CT}$ imaging were registered and metabolic uptake regions on the FDG-PET/CT were defined in 2 regions inside the primary tumour: low (0-50\% of the maximum SUV) and high metabolic uptake ( $>50 \%$ of the maximum SUV). Blood flow (BF), blood volume (BV) and permeability (PMB) were calculated for the entire tumour (Siemens Volume Perfusion CT software tool)

Results: Differences (mean $\pm 1 S D$ ) were observed between low and high metabolically active regions: average $B F(\mathrm{ml} / 100 \mathrm{ml} / \mathrm{min})$ was $28 \pm 23 \mathrm{vs} .41 \pm 29(p=0.012)$, and average $B V(\mathrm{ml} / 100 \mathrm{ml})$ was $4.7 \pm 3.2$ vs. $7.1 \pm 4.4(p=0.007), P M B \_A(m l / 100$ $\mathrm{ml} / \mathrm{min}$ ) was $13 \pm 19$ vs. $20 \pm 27(\mathrm{p}=0.002)$.

Conclusion: CT perfusion imaging is capable of differentiating between tumour regions that are metabolically active showing increased blood flow, blood volume and permeability and those with less metabolic activity. These findings yield the potential to use perfusion imaging in staging and follow-up of NSCLC for antiangiogenic therapy.

Author Disclosures:

M. Das: Consultant; Medrad. Grant Recipient; Siemens, Bayer. Speaker;

Siemens, Bayer, Medrad. J.E. Wildberger: Consultant; Medrad, Bayer,

Siemens. Grant Recipient; Bayer, Siemens. Speaker; Medrad, Bayer, Siemens.

\section{B-0066 11:15}

Imaging of antivascular effects of multikinase inhibitors (MKI) in patients with metastatic renal cell cancer (mRCC) using dynamic contrast- enhanced CT (DCE-CT, perfusion CT): a pilot study

A. Sterzik, M. Staehler, J. Casuscelli, M. Karpitschka, F. Schwarz, M.F. Reiser,

A. Graser; Munich/DE (alexander.sterzik@med.uni-muenchen.de)

Purpose: To assess the early antivascular effects of MKIs in patients with mRCC with DCE-CT.

Methods and Materials: 20 patients with mRCC underwent perfusion CT imaging of representative metastatic lesions using 4D-spiral mode acquisition: $10 \mathrm{~cm}$ z-axis coverage, acquisition time $41 \mathrm{sec}, 100 \mathrm{kVp}$ (abdomen), $80 \mathrm{kVp}$ (chest), 100 $\mathrm{mAs}$, on a dual-source CT scanner. Two subsequent DCE-scans were performed before and 7 days after start of treatment with Sunitib (Sutent ${ }^{\oplus ;} ;=15$ ) or Pazopanib (Votrient $\left.{ }^{\circledR} ; n=5\right)$. Therapy-induced changes of tumour perfusion were quantitatively assessed using a volumetric approach. Blood flow (BF), blood volume (BV) and permeability-surface area product (PS) were calculated applying maximum slope and modified Patlak approaches. Quantitative analysis of perfusion parameters and qualitative assessment for the presence of motion artefacts before and after automated motion correction was performed by two independent readers.

Results: 28 intraabdominal and 22 intrathoracic lesions were assessed. Motion artefacts were significantly reduced after automated motion correction $(p<0.001)$. Between baseline and follow-up scan, perfusion CT showed a significant reduction of $B F(159 \pm 74 \mathrm{vs} .78 \pm 52 \mathrm{ml} / 100 \mathrm{mg} / \mathrm{min}, \mathrm{p}<0.001)$, $B V(19 \pm 10.2$ to $10.8 \pm 7.4 \mathrm{ml} / 100$ $\mathrm{ml}, \mathrm{p}<0.001)$, but not of PS $(20.1 \pm 11.5$ to $15.6 \pm 11.6 \mathrm{ml} / \mathrm{min} / \mathrm{ml} \mathrm{p}=0.17)$. A high correlation was found between the 2 observers regarding quantitative perfusion parameters $(r=0.92 ; p<0.001)$.

Conclusion: Perfusion parameters measured in $\mathrm{mRCC}$ patients before and early after the start of antiangiogenic therapy are reproducible between different readers. Automated software-based motion correction significantly improves image quality in acquisitions with long breathhold times.

Author Disclosures:

A. Sterzik: Research/Grant Support; Bayer AG Healthcare. M. Staehler:

Speaker; Bayer AG, GlaxoSmithKline. A. Graser: Advisory Board; Siemens AG, Pfizer Pharma, Bayer AG. Speaker; Siemens AG, Pfizer Pharma, Bracco, Bayer AG, Guerbet.

\section{B-0067 11:24}

Early vs late recurrent glioblastomas: added value of T1-weighted perfusion MR imaging compared with T2-weighted perfusion MR imaging S.J. Kang, H.S. Kim, S.J. Kim, C.G. Choi; Seoul/KR (marie0975@hanmail.net)

Purpose: To determine whether dynamic contrast-enhanced (DCE) perfusion MR images have added value in distinguishing early ( $<12$ weeks after chemoradiation) or late (> 12 weeks after chemoradiation) recurrent glioblastomas (RGMs) from treatment-related changes (TRCs), compared with dynamic susceptibility contrast (DSC) perfusion MR images.

Methods and Materials: This study was approved by the IRB. Conventional, DSC, and DCE MR images in 99 patients with RGMs and 82 patients with TRCs were retrospectively reviewed. Two experienced neuroradiologists independently reviewed conventional plus DSC MR images (image set 1) and, 10 weeks later, reviewed conventional plus DCE MR images (image set 2) to differentiate RGM from TRC based on the scoring system of tumour perfusion volume fraction (TPVF). The TPVF was segmented using optimum $50 \%$ cutoff value derived from bimodal histogram. The alternative free-response receiver operating characteristic curve $(\mathrm{Az})$ analysis and weighted kappa statistics were obtained to evaluate reader performance and agreement.

Results: The optimum 50\% cutoff values for differentiating early and late RGMs from TRC were 1.69 and 1.53 for normalised cerebral blood volume and 0.062 and 0.057 for normalised wash-in, respectively. In late recurrence, the two observers demonstrated significant improvement in Az, sensitivities, and specificities from image set 1 to set 2 . In early recurrence, $A z$, sensitivities, and specificities increased with the addition of DCE images but did not reach statistical significance. Interobserver agreement increased from image set 1 to set 2 (0.65 vs 0.76).

Conclusion: DCE perfusion MR imaging significantly improve the diagnosis of late RGM compared with DSC perfusion MR imaging

\section{B-0068 11:33}

Monitoring the antivascular effects of a novel multimodal therapy with dynamic contrast-enhanced MRI (DCE-MRI) in an experimental human squamous cell carcinoma model

A. Sterzik, P. Paprottka, P. Zengel, S. Roßpunkt,

E. Baloch, M. Moser, M.F. Reiser, K. Nikolaou, C. Cyran;

Munich/DE (alexander.sterzik@med.uni-muenchen.de)

Purpose: To investigate with immunohistochemical validation whether DCE-MRI is useful for monitoring the antiangiogenetic effects of a novel experimental multimodal therapy on pharyngeal squamous cell carcinoma xenografts in rats.

Methods and Materials: Athymic rats $(n=14)$ with subcutanously implanted human hypopharynx-carcinoma-cells ( $\mathrm{FaDu}$ ) were assessed with 3-T dynamic gadobutrolenhanced MRI at baseline on d0 and after a 7-day course of a novel therapy regimen, consisting of combined weight-adapted daily administration of the COX-IIinhibitor Celecoxib ${ }^{\circledR}(25 \mathrm{mg} / \mathrm{kg})$, the matrix metalloproteinase-inhibitor Galardin ${ }^{\circledR}(50$ $\mathrm{mg} / \mathrm{kg})$ and the uPA-inhibitor Mesupron ${ }^{\circledR}(0.03 \mathrm{mg} / \mathrm{kg})$. Quantitative measurements of tumour blood flow (tBF), tumour blood volume (tBV) and permeability-surface area product (PS) were calculated, using a two-compartment model. DCE-MRI values were correlated with immunohistochemical results.

Results: tBF as well as tBV in triple-therapy animals dropped significantly from day 0 to day 7 (tBF: $37.8 \pm 15$ to $21.3 \pm 7.1 \mathrm{ml} / 100 \mathrm{ml} / \mathrm{min}$; $p<0.01$; tBV: $17.1 \pm 4.4$ to 
$7.7 \pm 3.3 \mathrm{ml} / \mathrm{mg} ; \mathrm{p}<0.01)$. No significant effects on PS were noticed in either therapy or control group ( $p>0.05$ ). Immunohistochemical analysis showed a markedly reduced tumour vascularity in the therapy group (CD31+ cell count: $534 \pm 88$ vs. $1228 \pm 309 ; p<0.05)$ with fewer proliferating cells (\%Ki- $67+$ cells: $17.9 \pm 3.9 \%$ vs. $21.9 \pm 3.5 \% ; p<0.05)$, resulting in a delayed tumour growth $(p<0.05)$. Modest but significant correlations could be found between $\mathrm{tBF} / \mathrm{tBV}$ and immunohistochemical tumour vascularity (tBF: $r=0.66 ; p<0.01 ; \mathrm{tBV}: r=0.54 ; p<0.05$ ) as well as tBV and immunohistochemical tumour proliferation $(r=0.56 ; p<0.05)$.

Conclusion: DCE-MRI seems a suitable tool for non invasive monitoring the antivascular effects of this new therapy regimen in this experimental setting.

Author Disclosures:

A. Sterzik: Research/Grant Support; Bayer Schering Healthcare. C. Cyran: Research/Grant Support; Bayer Schering Healthcare.

\section{B-0069 11:42}

Fractional 3D quantification of early contrast-enhancing tumour fractions on dynamic MRI acquisitions in soft tissue sarcoma: prospective comparison with partial tissue-stained pathological tumour viability estimates

A.K. Singh, W. Cai, A. Imanzadeh, S. Saini, G. Harris; Boston, MA/US (ahsingh@partners.org)

Purpose: To assess the feasibility and accuracy of dynamic MRI-based viableappearing tumour fraction quantification by advanced post processing methods in treated and excised soft-tissue sarcomas by comparing with pathologically derived-viable tumour fraction estimates obtained by partial tissue staining method. Methods and Materials: In this on-going prospective study, post-gadolinium, dynamic T1-weighted sequence from MRI data sets of 16 treated for pathologically proven soft-tissue sarcomas was performed $2-5$ days before surgical excision. Dynamic post contrast sequences where recurring series of 15-20 second acquisitions that were acquired repetitively for 7 minutes after start of injection were subjected to automated post processing for quantification of viable tumour. Post excision pathology percentage estimate for viable tumour tissue was taken as standard of reference for comparing MRI-derived viable tumour tissue estimates using correlation and Bland Altman (BA) analysis.

Results: In all the 16 cases, the viable-appearing tumour volume percentage derived from MRI dynamic contrast acquisitions was within 1.96 standard deviation (SD) [-82.0 to 115.7] of the BA plot indicating similarity to pathological tumour viability estimates and good correlation $(r=0.774 ; p<0.0001 ; 95 \%$ confidence interval $=0.7421$ to 0.9772 ). There were 4 outliers outside $95 \%$ confidence limits of agreement in the BA plot.

Conclusion: 3D-Quantification of MR early contrast-enhancing and wash out fractions in soft-tissue sarcoma showed similarity to percentage of tumour viability estimate reported on pathology report post-tumour excision in this on-going prospective study and thus may provide a more objective measurement for assessing tissue viability pending validation on a larger cohort.

Author Disclosures:

G. Harris: Advisory Board; Fovia Inc.

\section{B-0070 11:51}

A perfusion CT-derived patient-specific arterial input function for pharmacokinetic modelling of dynamic contrast-enhanced MRI

J.M. Franklin, M. Enescu, E.J. Hill, R.A. Sharma, J.A. Schnabel, F.V. Gleeson, E.M. Anderson; Oxford/UK (jamiemfranklin@hotmail.com)

Purpose: Pharmacokinetic modelling of dynamic contrast-enhanced MRI (dceMRI) data requires estimation of the arterial input function (AIF). Due to the methodological challenges of deriving an individual patient AIF directly from dceMRI, a population-averaged AIF is typically used, e.g. AlFOrton. This study aimed to assess the variability and benefit of an AIF derived directly from contemporaneous perfusion $\mathrm{CT}(\mathrm{pCT})$ examinations.

Methods and Materials: Eight patients with rectal cancer had dceMRI and pCT examinations twice before and once following pelvic radiotherapy (25Gy/5[[Unable to Display Character: \&\#9839;] in one week). AIFs were derived from the pCT data (AIFpCT) by modelling the time-attenuation curves of external iliac arteries. The peak enhancement (PE) and area under the initial uptake curve in the first $30 \mathrm{~s}$ (AIUC30) was computed for each AIF. ANOVA and t-test analyses were used to assess variation of AIFpCT and compare AIFpCT and AIFOrton for each patient. Results: There was no significant intra-patient variability of AIUC30 $(p=0.75)$ or PE $(p=0.63)$ between AIFpCT. A significant difference was observed between the average AIFpCT over the three time points and AIFOrton PE ( $p=0.023)$, but no significant difference between the average AIFpCT and AIFOrton AIUC30 $(p=0.17)$.
Maximum difference of AIUC30 and PE between the AIFOrton and AIFpCT was typically greater than maximum variability observed between AIFpCT (mean maximum difference AIUC30 0.96mM[[Unable to Display Character: \&\#61599;]]min vs $0.76 \mathrm{mM}[[$ Unable to Display Character: \&\#61599;]]min, $p=0.23$; mean maximum difference $P E 2.66 \mathrm{mM}$ vs $2.15 \mathrm{mM}, \mathrm{p}=0.39$ ).

Conclusion: Peak enhancement of AIFOrton differed significantly from AIFpCT, while there was no significant intra-patient variability of AIFpCT. Incorporating a patient-specific AIFpCT into dceMRI analyses may thus improve pharmacokinetic modelling.

10:30 - 12:00 Room F2

Breast

SS 102

\section{Breast MRI: improving accuracy and tissue characterisation}

Moderators:

R. Schulz-Wendtland; Erlangen/DE

M. Telesca; Rome/lT

\section{B-0071 10:30}

BI-RADS ${ }^{\circledR}$-adapted combined contrast-enhanced magnetic resonance and diffusion-weighted imaging reading for multiparametric imaging of breast lesions at $3 \mathrm{~T}$ improves diagnostic accuracy

K. Pinker-Domenig, H. Bickel, T.H. Helbich, S. Gruber, S. Trattnig, W. Bogner; Vienna/AT (katja.pinker-domenig@meduniwien.ac.at)

Purpose: To develop and assess a combined reading for contrast-enhanced magnetic resonance (CE-MRI) and diffusion-weighted imaging (DWI) adapted to the Breast Imaging Reporting and Data System (BI-RADS) for multiparametric MRI of the breast at $3 \mathrm{~T}$.

Methods and Materials: 247 patients with histopathologically verified breast lesions were included in this IRB-approved prospective study. All patients underwent CE-MR and DWI at 3 T. MRI was classified according to BI-RADS and assessed for apparent diffusion coefficient (ADC) values. A reading method that adapted ADC thresholds to the assigned BI-RADS classification was developed. Sensitivity, specificity, diagnostic accuracy and the area-under-curve were calculated. BIRADS-adapted reading was compared to previously published reading methods in the same population. Inter- and intra-reader variability was assessed.

Results: Sensitivity of BI-RADS-adapted reading was not different from the high sensitivity of CE-MRI ( $p=0.4)$. BI-RADS-adapted reading maximized specificity $(89.4 \%)$, which was significantly higher compared to CE-MRI $(p<0.001)$. Previously published reading methods did not perform as well as the BI-RADS-adapted reading except for a logistic regression model. BI-RADS-adapted reading was more sensitive in NMLE and was more robust to inter- and intra-reader variability. Conclusion: Multiparametric $3 \mathrm{~T} \mathrm{MRI}$ of the breast using a BI-RADS-adapted reading is fast, simple to use and significantly improves the diagnostic accuracy of MRI of the breast.

\section{B-0072 10:39}

The additional value of expert reading of 3 Tesla breast MRI in patients with microcalcifications on mammography L.G. Merckel', H.M. Verkooijen', N.H.G.M. Peters' ${ }^{1}$, R.M. Mann², W.B. Veldhuis', K.M. Duvivier', T. van Dalen ${ }^{1}$, P.H.M. Peeters', M.A.A.J. van den Bosch';

${ }^{1}$ Utrecht/NL, ${ }^{2}$ Nijmegen/NL (L.G.Merckel-2@umcutrecht.nl)

Purpose: Ductal carcinoma in situ (DCIS) is mainly diagnosed through microcalcifications detected on mammography. We investigated the added diagnostic value of $3 \mathrm{~T}$ breast MRI in patients presenting with microcalcifications on mammography. Methods and Materials: Between January 2006 and May 2009, 141 patients with mammographically detected microcalcifications underwent contrast-enhanced $3 \mathrm{~T}$ breast MRI before undergoing breast biopsy in the context of a multicentre randomised trial (MONET trial). All histologically confirmed index lesions were reviewed by an expert breast radiologist according to the BI-RADS ${ }^{\circledR}$ lexicon. Added diagnostic value of breast MRI was evaluated using receiver-operating characteristic (ROC) analysis. Furthermore, it was investigated which BI-RADS descriptors were most strongly associated with the diagnosis of pure DCIS in patients with microcalcifications.

Results: Overall 52/141 (37\%) lesions turned out to be malignant; 30 (21\%) patients had pure DCIS and $22(23 \%)$ had mixed or pure invasive breast cancer. Expert 
$\mathrm{MRI}$ reading in addition to baseline characteristics and conventional imaging led to a strong improvement in AUC from $0.62(95 \% \mathrm{Cl} 0.52-0.71)$ to $0.82(95 \% \mathrm{Cl}$ $0.74-0.90)$. Non-mass classification was positively associated $(p<0.05)$ with the presence of pure DCIS when compared with non-malignant lesions. Segmental non-mass-like enhancement had the highest predictive value $(82 \%, 95 \% \mathrm{Cl} 48-97)$ for the presence of DCIS

Conclusion: 3 T breast MRI of mammographically detected microcalcifications is of added diagnostic value for the diagnosis of DCIS when performed by expert radiologists and if classification is based upon the ACR BI-RADS ${ }^{\circledR}$ lexicon for breast MRI.

\section{B-0073 10:48}

Which threshold level is appropriate for analysis of the computeraided detection system of breast MRI? Effect of pathologic tumour characteristics and biological markers

S. Song ${ }^{1}$, B. Seo ${ }^{1}$, K.-W. Hwang ${ }^{2}$, K. $\mathrm{Cho}^{3}$, O. Woo ${ }^{3} ;{ }^{1}$ Ansan/KR, ${ }^{2}$ Incheon/KR, ${ }^{3}$ Seoul/KR (akaeuny@hanmail.net)

Purpose: The purpose was to investigate which threshold is appropriate for CAD analysis for breast MRI and whether pathologic characteristics and biological markers influence the CAD analysis.

Methods and Materials: We evaluated preoperative MRI with CAD of 132 breast cancers (82 invasive cancers and 50 DCIS) who underwent surgical treatment. Two breast radiologists analysed CAD findings and measured the tumour extent at three types of thresholds, 30,50 , and $100 \%$. To assess which threshold would be appropriate, we compared the measured tumour extents at each threshold. We assessed whether pathologic or biologic findings could influence the results of CAD analysis. Spearman rank correlation and Pearson's Chi-square test were used. Results: For invasive cancers, mean tumour size was $22.28 \mathrm{~mm}$ on pathologic examination and $22.57 \mathrm{~mm}$ on MRI. The size of invasive cancers on the CAD analysis was $21.80 \mathrm{~mm}$ at $30 \%$ threshold, 18.68 at $50 \%$ and 9.67 at $100 \%$. For DCIS cases, mean tumour size was $24.96 \mathrm{~mm}$ on pathologic examination and 25.80 $\mathrm{mm}$ on MRI. The size of DCIS was $27.06 \mathrm{~mm}$ at $30 \%$ threshold, 21.54 at $50 \%$ and 8.28 at $100 \%$. These differences were statistically significant $(P<0.05)$. For both of invasive cancers and DCIS cases, $30 \%$ threshold was the best to demonstrate whole tumour extent. For invasive cancers, CAD analysis was influenced by tumour grade. For DCIS, CAD analysis was influenced by biologic marker.

Conclusion: In CAD analysis, $30 \%$ threshold is the most appropriate for both invasive cancers and DCIS. Tumour grade and biological markers can influence tumour detection on the CAD system.

\section{B-0074 10:57}

The additional value of diffusion-weighted imaging (DWI) in MRmammography: should we still look at curves?

P.A.T. Baltzer ${ }^{1}$, M. Dietzel ${ }^{2}$, J.L. Halder ${ }^{3}$, W.A. Kaiser ${ }^{3} ;{ }^{1}$ Vienna/AT, ${ }^{2}$ Erlangen/DE, ${ }^{3}$ Jena/DE

Purpose: For differential diagnosis of breast lesions in dynamic contrast-enhanced MRI, morphology on enhanced early phase (EP) images and enhancement curve types (EP and delayed phase, DP) are assessed. Accordingly, the major part of the dynamic images is used for assessment of DP only. Diffusion-Weighted Imaging (DWI) is an alternative and fast quantitative technique. We investigated whether integration of Apparent Diffusion Coefficient (ADC) values may replace acquisition of DP images.

Methods and Materials: Consecutive breast MRI examinations (1.5 T, dynamic enhanced T1w images, temporal resolution 1 min., DWI: 2 b-values: 0\&1000s/ $\mathrm{mm} 2, \mathrm{ADC}$ calculated automatically) of consecutive patients classified as BI-RADS 3-5 in mammography/ultrasound were investigated in this IRB-approved study. EP enhancement (\%) and DP Washout (\%) were analysed. Classification accuracy of single parameters and possible combinations was investigated using ROC and binary logistic regression analysis. Reference standard was histopathology or imaging follow-up $>24$ months.

Results: Included were 127 patients showing 144 lesions (102 malignant, 42 benign). Highest Area under the ROC curve (AUC) was reached by single-parameter Washout (0.849) and ADC (0.884). Combination of initial enhancement and ADC reached highest AUC of 0.898 without significant differences to ADC values only (P>0.05).

Conclusion: Analysis of DP data in a clinical setting does not add quantitative value for lesion differentiation if compared with DWI measurements. A protocol including only initial enhancement and DWI measurements would take about $5 \mathrm{~min}$. examination time and would also allow morphologic lesion assessment.

\section{B-0075 11:06}

Apparent diffusion coefficient (ADC) measurements of breast lesions: most suspect area versus complete lesion assessment

P.A.T. Baltzer ${ }^{1}$, M. Dietzel ${ }^{2}$, J.L. Halder ${ }^{3}$, W.A. Kaiser ${ }^{3} ;{ }^{1}$ Vienna/AT, ${ }^{2}$ Erlangen/DE, ${ }^{3}$ Jena/DE

Purpose: Diffusion-Weighted Imaging (DWI) is an increasingly used quantitative imaging technique in breast MRI. For the analysis of dynamic-contrast-enhancement a careful examination of lesions is standard of care, as the most suspicious enhancing part of the lesion ("hotspot") provides the best diagnostic information. Accordingly, we investigated whether such "hotspots-analysis" might be beneficial for the assessment of DWI in breast lesions.

Methods and Materials: Consecutive DWI examinations of the breast (1.5 T, dedicated breast coil, TR $3500 \mathrm{~ms}$, TEeff $80 \mathrm{~ms}$, b-values: 0, 1000s $/ \mathrm{mm} 2$ ) of patients referred due to unclear/suspicious findings were investigated in this IRB-approved study. ADC values were calculated by scanner software. Regions of Interest were drawn around enhancing lesions (ROII) and the most suspicious lesion subpart (lowest ADC value, ROIs) on ADC maps by two blinded observers in consensus. Reference standard was histopathology or imaging follow-up > 24 months. Diagnostic accuracy was compared by ROC analysis.

Results: Included were 144 lesions (102 malignant, 42 benign) in 127 patients. Area under the ROC curve was significantly $(\mathrm{P}=0.006)$ higher for ROls $(0.884)$ compared with ROII (0.834).

Conclusion: Our systematic comparison revealed a higher diagnostic accuracy of $A D C$ values obtained from lesion subparts showing the highest diffusion restriction. These results encourage careful ADC sampling of breast lesions instead of complete lesion ADC averaging.

\section{B-0076 11:15}

Combined contrast-enhanced MRI (CE-MRI) and 3D multivoxel proton magnetic resonance spectroscopy (3D-1H-MRSI) at 3 Tesla enables an improved characterisation of breast tumours

K. Pinker-Domenig, S. Gruber, W. Bogner, B. Brück, H. Bickel, P.A.T. Baltzer, T. Helbich; Vienna/AT (katja.pinker@meduniwien.ac.at)

Purpose: To develop and assess a combined reading for CE-MRI and 3D-1H-MRS at 3 Tesla in patients with breast tumours.

Methods and Materials: In this IRB-approved prospective study 113 patients with an imaging abnormality were examined at $3 \mathrm{~T}$ with $3 \mathrm{D}-1 \mathrm{H}-\mathrm{MRSI}(10 \times 10 \times 10 \mathrm{~mm}$; TA $11 \mathrm{~min}$ ) followed by a T2-weighted and a high resolution T1-weighted sequence with a single dose of contrast agent (Dotarem, Guerbet). Lesion morphology and EH-kinetics were assessed and classified according to BIRADS. 3D-1H-MRSI findings were defined as positive if the SNR of the choline resonance peak was 32.55. For the combined reading a lesions was classified as malignant if at least two of three values, i.e. lesion morphology, enhancement kinetics, and SNR were positive. Sensitivity, specificity and diagnostic accuracy of CE-MRI, 3D-1H-MRSI and the combined reading were calculated. Histopathology was used as the standard of reference.

Results: There were 39 benign and 74 malignant lesions. Median SNR of the benign and malignant lesions was 0.95 and 9.98. CE-MRI alone had a sensitivity of $97 \%$, a specificity of $64 \%$ and diagnostic accuracy of $86 \%$. Using an SNR threshold of $2.55 \mathrm{MRSI}$ had a sensitivity of $93 \%$, a specificity of $92 \%$ and diagnostic accuracy of $92 \%$. Using the combined imaging protocol we kept an excellent sensitivity of $97 \%$, increased specificity from $64 \%$ to $74 \%$ as compared with CE-MRI resulting in a diagnostic accuracy of $89 \%$.

Conclusion: Combined CE-MRI and 3D-1H-MRSI at 3 T allows an increase in specificity while maintaining the excellent sensitivity of MRI of the breast.

\section{B-0077 11:24}

Application of breast MRI vs. classical prognostic factors to predict survival in patients with primary breast cancer

M. Dietzel', P.A.T. Baltzer ${ }^{2}$, R. Zoubi ${ }^{3}$, H. Habrecht ${ }^{4}$, C. Jerowski ${ }^{4}$

I.B. Runnebaum ${ }^{4}$, W.A. Kaiser ${ }^{4} ;{ }^{1}$ Erlangen/DE, Jena/DE, ${ }^{2} J e n a / D E$, Vienna/AT, ${ }^{3}$ Bielefeld/DE, Jena/DE, ${ }^{4}$ Jena/DE (dietzelmatthias2@hotmail.com)

Purpose: To identify the potential of breast MRI vs. classical prognostic factors to predict disease-related death in breast cancer.

Methods and Materials: Patients receiving pre-therapeutic MRI staging of primary breast cancer during 4 consecutive years at our primary care multidisciplinary breast cancer centre were included. In each patient classical prognostic factors were identified (TNM-staging, tumour typing, tumour grading, progesterone/estrogen receptors [\%], HER2NEU score, associated DCIS component) and follow-up was performed. The endpoint was defined as disease-related death. For quantitative 
analysis of breast MRI a dedicated CAD software was used (computer-aided analysis). It enables semi-automatic analysis of enhancement characteristics of the whole tumour (3D analysis of early vs. delayed phase) and the "hot-spot" (i.e. voxel with highest washin/washout ratio). Potential of breast MRI characteristics vs. classical prognostic factors to predict disease-related death was investigated separately and in combination using Cox regression. To identify significant and independent predictors for disease-related death, backward feature selection was applied ( $p^{\text {entry }} /$ removal: $<0.05 />0.01$ )

Results: Two-hundred and twenty-three patients were included (disease-related death: $n=24 / 10.8 \%$; censored: $n=187 / 83.9 \%$; loss to follow-up: $n=12 / 5.4 \%$ ). Mean follow-up was 4.7 years. If tested separately, $C R$ analysis identified significant potential both for breast MRI and classical prognostic factors to predict diseaserelated death $(p<0.001)$. If breast MRI characteristics and classical prognostic factors were used in combination, predictive performance could be further increased significantly $(p<0.05)$ : after feature selection five classical prognostic factors (TNM stage, tumour typing, HER2NEU score) and six breast MRI characteristics (hot-spot: time to peak enhancement, washout ratio; 3D analysis: tumour volume; tumour voxels showing washout plus weak/intermediate washin and tumour voxels showing plateau and weak washin) remained in this final model as significant and independent coefficients $(p<0.05)$

Conclusion: Breast MRI has a significant potential to predict disease-related death in breast-cancer patients. It not only can be used as a stand-alone tool for this task but also adds significant predictive value to classical prognostic factors to stratify this endpoint.

\section{B-0079 11:33}

Diagnosis of breast lesions using proton MR-spectroscopy at 1.5 and 3 Tesla: a systematic review and meta-analysis

P.A.T. Baltzer ${ }^{1}$, M. Dietzel ${ }^{2}$, W.A. Kaiser ${ }^{3} ;{ }^{1}$ Vienna/AT, ${ }^{2}$ Erlangen/DE, ${ }^{3}$ Jena/DE

Purpose: To perform a systematic review and meta-analysis to estimate the accuracy of breast proton magnetic resonance spectroscopy (MRS) in the diagnosis of breast lesions and to identify variables that influence accuracy of MRS.

Methods and Materials: A comprehensive search of the Pubmed database was performed on articles listed until 01/06/2012. The medical subjects heading (MeSH) and text words for the terms "breast", "spectroscopy" and "magnetic resonance" were used. Investigations including $>10$ subjects at $1.5 \mathrm{~T}$ or $3 \mathrm{~T}$ applying $1 \mathrm{D}$ single voxel MRS or spatially resolved MRS for differentiation between benign and malignant breast lesions were eligible. A reference standard had to be established either by histopathology or imaging follow-up 312 months.

Results: Nineteen studies were used for general data pooling, including 1183 patients and 1198 lesions (773 malignant, 452 benign). Pooled sensitivity and specificity were $73 \%(95 \% \mathrm{Cl} 70-76 \%)$ and $88 \%$ (95\% Cl $85-91 \%)$, respectively. Pooled diagnostic odds ratio (DOR) was $34.30(95 \% \mathrm{Cl} 16.71-70.43)$. For breast cancer versus benign lesions, the SROC's AUC of MRS was 0.88 and the point $Q^{*}$ was 0.81 . There was evidence of between studies heterogeneity regarding sensitivity and DOR $(P<0.001)$. No significant influences of higher field strength, postcontrast acquisition or qualitative against quantitative MRS measurements were identified. Egger's test confirmed significant publication bias in studies including small numbers of patients $(P<0.001)$.

Conclusion: Breast MRS shows variable sensitivity and high specificity in the diagnosis of breast lesions independent from the technical MRS approach. Due to significant publication bias, pooled diagnostic measures might be overestimated.

\section{B-0080 11:42}

Volumetric assessment of MRI enhancement kinetics of invasive breast cancers with a post-processing software with correlation of hormonal receptor subtypes

L.C.H. Leong ${ }^{1}$, E. Gombos ${ }^{2} ;{ }^{1}$ Singapore/SG, ${ }^{2}$ Boston, MA/US

Purpose: The aim of the study was to use an MRI computer-aided detection postprocessing software to assess the enhancement patterns by percentage tumour volume in invasive breast cancers of different hormonal receptor subtypes.

Methods and Materials: Pathology database search was performed on women with newly diagnosed breast cancers confirmed by core biopsies and had breast MRI assessment from March 2005 to March 2009. Only malignancies presenting as breast masses were included for the analysis. In-situ cancers, rare types of breast cancers such as metaplastic carcinoma or phyllodes tumour and nonepithelial malignancies were excluded. Enhancement kinetics data were analysed prospectively for the worst enhancement pattern and enhancement patterns by percentage tumour volume. The findings were then compared against the ER, PR, Her2 and triple negative hormonal receptor subtypes.
Results: 194 breast cancer masses from 191 women were evaluated. Analysis for the most suspicious enhancement kinetics pattern showed no statistical correlation with any of the receptor subtypes. There was higher tumour component by percentage volume showing early rapid enhancement in estrogen receptor (ER) negative $(64.1 \%$ vs $53.6 \%, p=0.013)$, progesterone receptor $(P R)$ negative $(65.4 \%$ vs $52.5 \%, p=0.001)$ and triple negative tumours $(65.3 \%$ vs $54.6 \%, p=0.028)$ compared to ER positive, PR positive and non-triple negative tumours, respectively. Conclusion: Volumetric analysis of MRI enhancement kinetics was more useful in showing the vascular properties of breast tumours. The analysis revealed that ER negative, PR negative and triple-negative malignant masses had a greater volume containing rapid type initial enhancement.

$10: 30-12: 00$ Room G/H

\section{Genitourinary}

SS 107

\section{Prostate imaging}

S. Morozov; Moscow/RU

J. Richenberg; Brighton/UK

\section{B-0081 10:30}

Reliability of the PI-RADS scoring system for functional prostate MRI M. Quentin, L. Schimmoeller, C. Arsov, R. Rabenalt, R. Lanzman, G. Antoch, P. Albers, D. Blondin; Düsseldorf/DE (michael.quentin@med.uni-duesseldorf.de)

Purpose: Recently the European Society of Urogenital Radiology (ESUR) published guidelines to standardise prostate MRI. These guidelines contain a simple 5-point scale scoring system called (PI-RADS, prostate imaging, reporting, and data system) for estimating the probability of a lesion to be malignant. This recommendation has not been validated so far which is the aim of the present study. Methods and Materials: 167 lesions which have been histologically confirmed with MRI-guided prostate biopsy were retrospectively scored by three blinded radiologists using the PI-RADS scoring system. 56 lesions revealed prostate cancer, while 108 lesions were benign. Inter-observer reliability was evaluated using Kappa statistics (K).

Results: With respect to T2-weighted images, diffusion weighted imaging (DWI) and dynamic contrast-enhanced imaging (DCE-MRI) $\mathrm{k}$ were $0.55,0.64$, and 0.65, respectively. $\mathrm{K}$ values in tumour were higher in T2-weighted images, and in DWI than those in non-tumour lesions. A total score of ten or more points in summation of the individual scores of each sequence as the cut off for malignancy was leading to $86 \%$ sensitivity, $68 \%$ specificity, $58 \%$ positive predictive value, and $90 \%$ negative predictive value.

Conclusion: The use of the PI-RADS scoring system for functional prostate MRI is feasible and comes along with a good inter-observer reliability.

\section{B-0082 10:39}

Preoperative multiparametric magnetic resonance imaging (mp-MRI) reduces positive surgical margins after robotic-assisted laparoscopic prostatectomy (RALP): experience in 136 patients

S. Alessi, G. Petralia, G. Musi, A. Alconchel, G. Bardo, S. Raimondi, G. Renne, O. De Cobelli, M. Bellomi; Milan/IT (Sarah.Alessi@ieo.it)

Purpose: To investigate whether preoperative multiparametric magnetic resonance imaging (mp-MRI) reduces positive surgical margins after robotic-assisted laparoscopic prostatectomy (RALP)

Methods and Materials: The study was approved by the local ethics committee and all patients gave their informed consent. Between March 2011 and May 2012, 136 consecutive patients (median age: $61.1 \mathrm{yr}$, range: $47-73$ ) with biopsy-proven prostate adenocarcinoma were prospectively enrolled in the study, undergoing $\mathrm{mp}-\mathrm{MRI}$ before RALP. Mp-MRI imaging was performed at $1.5 \mathrm{~T}$, including anatomic T2-weighted images, diffusion weighted (DW) and dynamic contrast-enhanced (DCE) sequences. All patients were retrospectively matched with a group of 136 controls who underwent RALP without preoperative mp-MRI. Paired t-tests and the non-parametric Wilcoxon signed rank test were used to compare age, PSA and Gleason Score between cases and controls. Percentages of positive margins in each group were compared with the McNemar's test. $P$ values $<0.05$ were considered statistically significant for all tests. The post-operative histopathological examination was used as the standard of reference for the presence of positive surgical margins.

Results: In the 136 enrolled patients, 12 (8.82\%) had positive surgical margins. Among the 136 controls, 25 (18.38\%) had positive margins. We found evidence of 
a statistically significant reduction $(p=0.03)$ of positive surgical margins in patients who performed preoperative $\mathrm{mp}-\mathrm{MRI}$.

Conclusion: In this study, preoperative $\mathrm{mp}-\mathrm{MRI}$ reduced the percentage of positive surgical margins after RALP. Further studies are desirable to confirm this encouraging observation.

\section{B-0083 10:48}

The cost-effectiveness of MRI and MR-guided biopsy versus TRUS-

guided biopsy in the diagnosis of prostate cancer

S. Crienen, M. de Rooij, J.O. Barentsz, J.A. Witjes, J.P.C. Grutters,

M.M. Rovers; Nijmegen/NL (m.derooij@rad.umcn.nl)

Purpose: The current diagnostic pathway for prostate cancer (elevated serum PSA, followed by transrectal ultrasound-guided biopsy (TRUS-GB)) tends to miss significant tumours and leads to overdiagnosis and understaging. Multiparametric magnetic resonance imaging (mp-MRI) followed by magnetic resonance-guided biopsy (MR-GB) can potentially improve diagnosis. Cost-effectiveness of these two diagnostic strategies has never been studied before. The aim of this study was to develop a decision analytic model, using a healthcare perspective, to assess cost-effectiveness of mp-MRI possibly followed by MR-GB versus TRUS-GB in prostate cancer diagnosis.

Methods and Materials: We developed a decision analytic tree with a Markov model to compare the MRI-strategy with TRUS-GB. Input data were derived from systematic literature searches (TRUS-GB accuracy, transition probabilities, utilities different treatment options, survival data), meta-analysis (MRI accuracy), and expert opinion (decision tree and cost information). We performed a sensitivity analysis to assess the uncertainty surrounding the effects and overall, diagnostic, and treatment costs. We plotted a cost-effectiveness acceptability curve to present the probability a strategy is cost-effective over different willingness to pay values. Results: Total expected mean cost per patient over 10 years of MRI strategy ( $€$ 3106) were lower than those for the TRUS-GB (€ 3233). QALYs for the MRI strategy (6.18) were higher than for the TRUS-GB strategy (6.05). Probabilistic sensitivity analysis showed that in $69 \%$ of simulations, MRI was more effective than TRUS-GB. Conclusion: The use of mp-MRI and MR-GB appears to be an efficient strategy in prostate cancer detection when compared to TRUS-GB, with even less costs.

\section{B-0084 10:57}

Quantitative shear wave elastography: detection and characterisation of prostate cancer on 105 patients

J.-M. Correas, A. Khairoune, A.-M. Tissier, V. Vassiliu, A. Méjean, O. Hélénon; Paris/FR (jean-michel.correas@nck.aphp.fr)

Purpose: To evaluate the performance of TransRectal UltraSound (TRUS) quantitative Shear Wave Elastography (SWE) for prostate lesion detection and characterisation.

Methods and Materials: 105 patients presenting increased PSA values $(9.1 \mathrm{ng} /$ $\mathrm{mL} \pm 7.2$ ) were prospectively enrolled. The prostate was scanned using TRUS with SWE (Supersonic Imagine ${ }^{\mathrm{TM}}$, France) for stiff area detection. Elasticity measurements were taken at the level of the 12 systematic biopsy sites and for each nodule detected by either B-mode or SWE. Ratios between nodules and adjacent tissue were calculated. SWE measurements were correlated to pathology (systematic sextant $(n=12)$ and targeted ( $n=2-7)$ biopsies).

Results: 48 patients exhibited significant prostate cancer (core size $^{3} 3 \mathrm{~mm}$, Gleason score ${ }^{36}$ ). 1238 sextant biopsies (164 positive cores) and targeted biopsies on 137 nodules (43 adenocarcinomas, 94 adenomatous hyperplasia/focal prostatitis) were performed. Prostate cancer nodules exhibited higher stiffness $(64 \pm 40 \mathrm{kPa})$ than adjacent gland $(20 \pm 14 \mathrm{kPa})$. Stiffness ratio was significantly higher for cancer $(3.5 \pm 2.0)$ compared with benign nodules $(1.3 \pm 0.9 ; p<0.002)$. The cut-off values for highest NPV were for stiffness values $35 \mathrm{kPa}$ and for elasticity ratio combined with absolute values, 1.7 and $47 \mathrm{kPa}$. SWE performance (Sensitivity (Se), Specificity (Spe), Positive Predictive Value (PPV), Negative Predictive Value (NPV) and Accuracy (Acc)) was for SWE values Se=96\%; Spe=79\%; PPV=45\%; NPV=97\%; AcC $=82 \%$; for combined SWE ratio and values: $\mathrm{Se}=90 \%$; Spe $=94 \%$; $P P V=77 \%$; $\mathrm{NPV}=98 \%$; Acc $=93 \%$. AUC ROC curve was 0.92 for SWE values and 0.92 for combined SWE ratio and SWE values.

Conclusion: SWE is an additional diagnostic imaging method that can be added to routine prostate TRUS for cancer detection and targeted biopsies.

Author Disclosures:

J.-M. Correas: Advisory Board; Toshiba Medical Systems, Philips Healthcare. Investigator; Bracco BR127 protocole. Speaker; Toshiba Medical Systems,

Philips Healthcare, Supersonic Imagine, Guerbet.
B-0085 11:06

3 T multi-parametric ultrahigh b values (b 2000) MR imaging for detection and localisation of peripheral and transition zone prostate cancer

G. Manenti, M. Nezzo, S. Altobelli, M. Antonicoli, S. Capuani, G. Simonetti; Rome/IT (guggi@tiscali.it)

Purpose: To retrospectively determine the accuracy of T2 weighted+DWI (10 b-value apparent-diffusion-coefficient (ADC) mapping and b 2000 images), T2W+DWI+DCE-MRI and T2W + DWI + MRSI +DCE-MRI protocol before sextant and TRUS-MRI image fusion-guided biopsies for detection and localisation of cancer nodules $>0.5 \mathrm{~cm}^{3}$ in peripheral and transition zone.

Methods and Materials: 40 patients with biopsy-proven prostate cancer (median age 72.2; age range, 47-84 years) with a median Gleason score of 4 were evaluated with a 3 T MRI using both phased array and endo-rectal coil. Localisation accuracy and $\mathrm{ROI}$-based receiver operating characteristic $(\mathrm{ROC})$ curves were calculated. Results: MRI examination showed 58 nodules in 40 patients (26 in the PZ and 32 in the TZ). Twenty-nine nodules were malignant (29/58.50\%). AUC value of the $\mathrm{b} 2000 \mathrm{ratio} / \mathrm{b} 1000$ ratio was not significantly higher than that of $A D C(0.82$ vs $0.71, p=0.052)$. Using T2W+DWI+MRSI+DCE-MRI the accuracy in tumour detection was improved compared with T2WI+DWI (AUC: 0.94 versus 0.81 for T2WI+DWI, $p=0.022$.

Conclusion: The DWI appears to be the best method to detect nodules of prostate cancer compared with 3D spectroscopic imaging and T2WI. The use of 3D spectroscopic imaging and DWI slightly improves the localisation of the nodules. MRI reanalysis identifies in ADC values the most accurate method to identify prostate cancer.

\section{B-0086 11:15}

Analysis of the dependence on b-values of DWI signal model outcomes in peripheral healthy and cancerous prostate tissues S. Lucarini, L.N. Mazzoni, S. Chiti, S. Busoni, C. Gori, I. Menchi; Florence/IT (slucarini@iol.it)

Purpose: To establish the influence of the b-values adopted during the diffusionweighted (DW) acquisition on apparent diffusion coefficient (ADC), perfusion fraction (PF), slow and fast diffusion coefficient (Dslow, Dfast).

Methods and Materials: 63 consecutive patients with prostate cancer who underwent MR examination (1.5 T Siemens AERA) were retrospectively included in the study. After T2W-TSE, the MR protocol included: DW-EPI ( $b=0,50,100,150$, $200,250,400,650,800,1000,1400,1800,2300 \mathrm{~s} / \mathrm{mm}^{2}$ ) and 3D-spectroscopic imaging sequence (3D-MRSI, TE/TR=120/930 ms). Two observers manually defined the ROIs on DW-images. Peripheral prostate tissues were considered healthy (HP) and cancerous ( $\mathrm{PCa}$ ) on the basis of ultrasound-guided biopsy, T2W images findings (Choline+Creatine)/Citrate in 3D-MRSI (healthy if $<0.6$, Ca if $>1.2$ ). Mono-exponential and bi-exponential models were fitted to DW-signals and all the parameters were estimated in $\mathrm{HP}$ and $\mathrm{PCa}$ tissues, using two different ranges of b-values (group A: 0-2300 s/mm², group B: 0-800 s/mm²). ANOVA with Bonferroni contrast was performed to establish differences among groups (threshold $p=0.05$ ). Results: 6 patients were excluded (non-adequate signal quality in 3D-MRSI). 118 ROls were defined: 45-HP, 73-PCa tissues. ANOVA showed significant differences of all the parameters between group $\mathrm{A}$ and $\mathrm{B}$, both in $\mathrm{HP}$ and $\mathrm{PCa}$ tissues. Mean \pm SD of $A D C$, Dslow, Dfast $\left(10^{-3} \mathrm{Dmm}^{2} / \mathrm{s}\right)$ and PF (\%) in group A and $B$ were (HP-PCa, group $A ; B$ ): $1.63 \pm 0.22-1.25 \pm 0.32 ; 1.78 \pm 0.21-1.44 \pm 0.32$ (ADC), $0.27 \pm 0.20-0.33 \pm 0.25 ; 1.06 \pm 0.59-0.81 \pm 0.60$ (Dslow), $2.70 \pm 0.64-3.13 \pm 1.10$; 9.82 $\pm 9.80-9.27 \pm 7.53$ (Dfast), $76 \pm 11-59 \pm 17 ; 34 \pm 31-30 \pm 26$ (PF).

Conclusion: All the parameters considered in this study showed a significant dependence on the chosen b-values. This observation can be important when comparing DWI quantitative outcomes acquired in different centres where different sequences are adopted.

\section{B-0087 11:24}

Comparison of diffusional kurtosis imaging and mono-exponential DWI model in distinguishing healthy from cancerous peripheral prostate tissues

S. Lucarini, L.N. Mazzoni, S. Chiti, S. Busoni, C. Gori, I. Menchi; Florence/IT (slucarini@iol.it)

Purpose: To compare sensitivity and specificity of diffusional kurtosis (DK) imaging and mono-exponential model in distinguishing healthy from cancerous prostate tissues.

Methods and Materials: Our retrospective study included 61 patients with biopsyproven prostate cancer who underwent prostate MR examination (1.5 T Siemens, 
AERA). The MR protocol included: T2W-TSE, DW-EPI ( $b=0,50,100,150,200$, 250, 400, 650, 800, 1000, 1400, 1800, $\left.2300 \mathrm{~s} / \mathrm{mm}^{2}\right)$, 3D-spectroscopic imaging sequence (3D-SI, TE/TR=120/930 ms). ROls were defined on DW-images by two observers in consensus. Peripheral cancerous (PCa) and healthy peripheral (HP) tissues were discriminated by means of ultrasound-guided biopsy, T2W images findings and (Choline+Creatine)/Citrate in 3D-SI (healthy if $<0.6$, Ca it $>1.2$ ). Monoexponential and DK models were fitted to DW-signals. Apparent diffusion coefficient $(A D C)$, corrected diffusion coefficient $(D)$ and DK-metric $(K)$ were estimated in HP and PCa tissues. A ROC analysis was performed to evaluate sensitivity and specificity of each parameter in distinguishing HP from PCa tissues.

Results: 6 patients were excluded (non-adequate signal quality in 3D-SI). $111 \mathrm{RO}$ were defined, 41 in HP, 70 in PCa tissues. Mean \pm SD in HP-PCa tissues were: $1.65 \pm 0.21-1.24 \pm 0.3010^{-3} \mathrm{Dmm}^{2} / \mathrm{s}$ (ADC), $2.00 \pm 0.23-1.62 \pm 0.3810^{-3} \mathrm{Dmm}^{2} / \mathrm{s}$ (D), $0.70 \pm 0.08-0.93 \pm 0.16(\mathrm{~K})$. The areas under the curve (AUCs) with $95 \%$ confidence interval of ADC, D, K (ROC analysis) were 0.84 (0.77-0.92), 0.82 (0.74-0.90), 0.87 (0.80-0.93), respectively.

Conclusion: $\mathrm{K}$ showed the best performance in distinguishing HP from PCa tissues with the highest AUC. Moreover, being $\mathrm{K}$ a measure of the non-Gaussian diffusion behaviour and since it is higher in PCa than in HP tissues, this study demonstrates an increment of the diffusion complexity in PCa vs HP tissues.

\section{B-0088 11:33}

MRI+MRSI reliably detects and excludes high-grade prostate cancer in patients with elevated PSA

G.M. Villeirs, J. Schatteman, P.J. De Visschere, G.O. De Meerleer, N. Lumen, W. Oosterlinck; Gent/BE (Geert.Villeirs@UGent.be)

Purpose: To prospectively assess the value of T2-weighted MR imaging with spectroscopic imaging (MRI+MRSI) in the detection and exclusion of high-grade prostate carcinoma (HGPC), defined as Gleason $4+3$ or higher, in patients with elevated PSA.

Methods and Materials: Histopathological evidence of prostate cancer (PC) or extended follow-up data (mean 4.5 years, range $0.25-9.2$ years) were available in 889 men (mean PSA $11.7 \mathrm{ng} / \mathrm{mL}$, range $2.5-200 \mathrm{ng} / \mathrm{mL}$ ) who underwent MRI+MRSI. Non-evidence of PC was defined as absence of histopathologically proven PC after at least 2 years of follow-up.

Results: 526 patients were diagnosed with PC within 100 days after MRI+MRSI, of whom 148 had HGPC (28.1\%). The sensitivity and negative predictive value (NPV) for detecting or excluding HGPC was $90.5 \%$ (134/148) and 96.7\% (410/424), respectively. Eight additional HGPCs ( 3 cT1c, 3 pT2 and 2 pT3) were detected within the first two years of follow-up (mean 0.8 years, range $0.3-1.9$ years). The NPV remained $96.0 \%(407 / 424)$ at 1 year and $94.8 \%(402 / 424)$ at 2 years. Fourteen more patients were diagnosed with HGPC between 2.1 and 7.6 years of follow-up (mean 3.5 years). Ten of them underwent a repeat MRI+MRS that was positive in all 10 cases. The NPV at 4 years was $92.5 \%$ (392/424) but probably biased by newly occurring HGPCs.

Conclusion: MRI+MRS is an excellent modality to both detect and exclude highgrade prostate carcinoma in patients with elevated PSA.

\section{B-0089 11:42}

Correlation between 1H-MR spectroscopy and haematochemical evaluation (PSA) in patients undergoing radiation therapy for prostate cancer

F. Barchetti, V. Panebianco, A. Pace, V. Forte, V. Forte, V. Tombolini, C. Catalano; Rome/IT (flavio.barchetti@live.it)

Purpose: Evaluation and correspondence between the achievement of the metabolic Nadir detected with $1 \mathrm{H}$-Spectroscopy (MRSI) and the haematochemical one (PSA) in order to demonstrate glandular atrophy in patients with prostate cancer who underwent radiotherapy.

Methods and Materials: Between January 2011 and January 2012, 33 patients with unresectable prostate cancer treated with radiotherapy underwent multiparametric MRI before radiation treatment and at 3, 6, 12/18 and 24 months after the treatment. The protocol included high-resolution T2-weighted morphological sequences, Diffusion sequences, 3D CSI spectroscopic sequences and GRE T1-weighted perfusional sequences. The data obtained from MRSI were analysed by dedicated software evaluating both (choline+creatine)/citrate ratio and Choline/Citrate ratio. All patients underwent PSA evaluation during the follow-up.

Results: We found metabolic glandular atrophy in 9 out of 33 patients (ratio equal to 0 ) already at 3 months after treatment, no response during all follow-up in 9 patients, partial response going to metabolic atrophy in 8 patients (ratio tends to 0 only at the end of the follow-up) and partial response going to relapse in 7 patients (ratio reducing to non pathological values at 3 and 6 months and tends to pre-treatment values at 12 and even more at 24 months).

Conclusion: Through the analysis of the data obtained from MRSI, it was possible to determine the response to radiation therapy earlier and with greater accuracy than the haematochemical evaluation of the PSA.

\section{B-0090 11:51}

The role of $3 \mathrm{~T}$ diffusion imaging in prostate cancer recurrence after radical prostatectomy

F. Barchetti, V. Panebianco, V. Forte, A. Pace, V. Tombolini, C. Catalano; Rome/IT (flavio.barchetti@live.it)

Purpose: To validate the role of $3 \mathrm{~T}$ Diffusion-Weighted Imaging (DWI) in the detection of local prostate cancer recurrence after radical prostatectomy (RP). Methods and Materials: High-resolution T2-weighted imaging, DWI and dynamiccontrast enhanced MRI (DCE-MRI) were performed with a $3 \mathrm{~T}$ magnet in 262 patients after RP. Twenty out of 262 patients evaluated were excluded. The population was divided on the basis of PSA restitution after radiation therapy in a group of 126 patients (group A) and of transrectal ultrasound biopsy in a group of 116 patients (group B).

Results: In Group A in identifying local recurrence combined T2-weighted and DCE-MRI (T2+DCE) showed sensitivity $98 \%$, specificity $94 \%$ and accuracy $93 \%$; combined T2 weighted and DW-imaging with b-value $3000 \mathrm{~s} / \mathrm{mm} 2$ (T2+DW3) displayed sensitivity $97 \%$, specificity $95 \%$ and accuracy $92 \%$, while with b-value 1000 s/mm2 (T2+DW1) sensitivity $93 \%$, specificity $89 \%$ and accuracy $88 \%$. In group B in detecting local cancer recurrence, T2+DCE showed sensitivity $100 \%$, specificity $97 \%$ and accuracy $91 \%$; T2+DW3 displayed sensitivity $98 \%$, specificity $96 \%$ and accuracy $89 \%$; T2+DW 1 have sensitivity $94 \%$, specificity $92 \%$ and accuracy $86 \%$. Conclusion: DWI can be proposed either as a further imaging study or as a reliable alternative to DCE-MRI in detecting local prostate cancer recurrence after RP.

10:30 - 12:00

Room I/K

\section{Abdominal Viscera}

\section{SS 101b}

\section{Liver fibrosis and cirrhosis: elastography and biomarkers}

Moderators:

R. Faschingbauer; Innsbruck/AT

T.J. Kroencke; Berlin/DE

\section{B-0091 10:30}

Estimation of reference values for liver elasticity in biopsy-proven normal liver using Supersonic Shear Wave imaging: measurement reliability and effect of steatosis

C. Suh, S. Kim, K. Kim; Seoul/KR

Purpose: To determine the reference values of liver elasticity in the biopsy-proven normal liver with Supersonic shear wave imaging (SSI) and to evaluate the measurement reliability and effect of steatosis.

Methods and Materials: We retrospectively identified 238 patients who underwent SSI (Supersonic Imagine, Aix Provenace, France) and subsequent ultrasoundguided liver biopsy on the same day. After the exclusion of patients with any abnormal hepatic pathology $(n=42)$ except for simple hepatic steatosis, 196 subjects (123 nonsteatotic, 73 steatotic) were included in the study. Three consecutive liver elasticity measurements were obtained in each patient. Median value expressed as $\mathrm{kPa}$ was used as a representative measurement of liver elasticity. The reference range of elasticity was determined according to Clinical and Laboratory Standards Institute guideline C28-A3 for determining reference intervals for quantitative clinical laboratory tests. The mean values of the liver elasticity were compared between nonsteatotic and steatotic livers using the student t-test. Measurement reliability was evaluated by using ICC.

Results: The mean elasticity values in subjects with the biopsy-proven normal (nonsteatotic and steatotic) liver were $4.37 \mathrm{kPa}$ with the estimated reference range of elasticity of $2.58-6.16 \mathrm{kPa}$. There was no significant difference in the mean elasticity between the nonsteatotic $(4.39 \mathrm{kPa})$ and steatotic liver $(4.33 \mathrm{kPa})(\mathrm{P}=.69)$. The overall ICC value of the elasticity measurements (nonsteatotic and steatotic) was 0.92 . Measurement reliability of the steatotic liver $(I C C=0.89)$ was not significantly different from those for the nonsteatotic liver (ICC $=0.94)(P=.34)$.

Conclusion: Hepatic elasticity values measured with SSI in histologically proven normal liver ranged from 2.58 to $6.16 \mathrm{kPa}$ with a high measurement reliability. 
Simple steatosis in the liver did not significantly affect either elasticity values or measurement reliability.

\section{B-0092 10:39}

Accuracy of a new real-time shear wave elastography technique in the assessment of significant liver fibrosis: preliminary results G. Ferraioli, C. Tinelli, B. Dal Bello, R. Lissandrin, M. Zicchetti, C. Filice; Pavia/IT (giovanna.ferraioli@unipv.it)

Purpose: To evaluate the performance of a new real-time shear wave elastography (RTSWE) technique in the assessment of significant liver fibrosis.

Methods and Materials: Consecutive patients with chronic viral hepatitis scheduled for liver biopsy (LB) (group 1), and healthy volunteers (group 2) were studied. In group 1 RTSWE using the iU22 ultrasound system (Philips Medical Systems, Bothell, WA, USA) with a convex broadband probe and ElastPQ technique, transient elastography (TE) using FibroScan ${ }^{\mathrm{TM}}$ (Echosens, Paris, France) and ultrasoundassisted LB were consecutively performed. In group 2, RTSWE and TE were carried out. Fibrosis was staged according to the METAVIR scoring system. For statistical analysis, group 1 was divided into group 1 A (F0-F1 stages), and group 1B (F2-F4 stages). In group 1, AUCs were calculated for F0-F1 versus F2-F4, for both RTSWE and TE.

Results: 77 subjects (61 males and 16 females) and 40 subjects (19 males and 21 females) were studied in groups 1 and 2, respectively. Median values of measurements were significantly lower in group 2 with respect to groups $1 \mathrm{~A}$ and 1B. When comparing measurements in group $1 \mathrm{~A}$ with measurements in group 1B, AUCs were $0.86(95 \% \mathrm{Cl}, 0.75-0.93)$ for RTSWE and $0.77(0.66-0.87)$ for TE $(p=0.13)$. The optimal cut-off values for the diagnosis of significant fibrosis $(F>2)$ were $5.6 \mathrm{kPa}$ for RTSWE and $7.3 \mathrm{kPa}$ for TE. Sensitivity and specificity were $78.6 \%$ (59.0-91.7) and 89.7\% (75.8-97.1) for RTSWE, and 69.0\% (49.2-84.7) and 83.3\% (68.6-93.0) for TE, respectively.

Conclusion: These results show that RTSWE is accurate in identifying patients with significant fibrosis. With both TE and RTSWE, healthy volunteers show significant lower values with respect to patients with no significant fibrosis.

\section{B-0093 10:48}

MR elastography for predicting progression of cirrhosis

U. Motosugi, T. Takamura, T. Ichikawa, K. Sano, H. Morisaka, S. Ichikawa,

T. Araki; Yamanshi/JP (utaroh-motosugi@nifty.com)

Purpose: To assess the efficacy of MR elastography in predicting progression of cirrhosis, in patients with Child-Pugh class A disease.

Methods and Materials: A total of 131 patients with type $C$ hepatitis and ChildPugh class A disease were included. All patients underwent MR elastography and liver function tests and were followed up every 3 months, for re-evaluation of their Child-Pugh class. The endpoint was set to the time when the Child-Pugh class A of the patients progressed to class B. Univariate and multivariate Cox proportion hazards models were used to determine the independent risk factor for cirrhosis progression.

Results: The median follow-up period was 18 months (range 3[[Unable to Display Character: \&\#8210;]]27 months). Results of multivariate Cox proportional hazards analysis showed that liver stiffness measured by MR elastography was an independent risk factor for predicting cirrhosis progression as well as prothrombin activity. The progression rate was significantly higher in patients with liver stiffness of $>$ $5.4 \mathrm{kPa}$ than in those with liver stiffness of $25.4 \mathrm{kPa}$ (log rank test, $\mathrm{p}<0.001$ ). The rate of cirrhosis progression to Child-Pugh class B per year-person was $44.8 \%$ in patients with liver stiffness of $>5.4 \mathrm{kPa}$, whereas it was $5.5 \%$ in patients with liver stiffness of $25.4 \mathrm{kPa}$.

Conclusion: Liver stiffness measured by MR elastography was an effective predictor of progression of cirrhosis from Child-Pugh class A to B disease in patients with type $\mathrm{C}$ hepatitis.

\section{B-0094 10:57}

Evaluation of shear wave elastography for liver fibrosis quantification

A. Guibal, T. Lefort, C. Cohen-Bacrie, G. Renosi, J. Scoazec, J. Dumortier,

P. Valette; Lyon/FR

Purpose: Shear wave elastography (SWE) is a new method for quantitative tissue elasticity evaluation. The purpose of this study was to evaluate SWE used for liver fibrosis quantification.

Methods and Materials: One hundred and seventy patients (aged 21-82, mean 56 years) who have had liver biopsy as part of their follow-up for chronic liver disease or transplantation were included in the study. Supersonic shear imaging (SSI) was performed before biopsy. The average of 3 consecutive measurements in the area provided for biopsy was compared with staging of fibrosis derived from METAVIR score.

Results: $148 / 170$ patients (87\%) had successful liver evaluation by SWE. 57 (39\%) were liver transplanted, $30(20 \%)$ had NASH disease, $17(11 \%)$ alcoholic liver disease, 16 (11\%) chronic hepatitis C, 6 (4\%) chronic hepatitis B, $22(15 \%)$ other diseases. Areas under the ROC curves (confidence interval 95\%) were $0.904(0.845-0,946)$ for patients ${ }^{3} \mathrm{~F} 2 ; 0.958(0.912-0,984)$ for patients ${ }^{3} \mathrm{~F} 3$ and $0.988(0.955-0.999)$ for cirrhotic patients $(p<0.0001)$. The optimal cutoff values were greater than $8.8 \mathrm{kPa}$ for diagnosis of significant fibrosis $\left({ }^{3} \mathrm{~F} 2\right)$, and greater than $18.1 \mathrm{kPa}$ for cirrhosis.

Conclusion: Shear wave elastography with SSI is a promising method which allows a non-invasive measurement of liver elasticity to determinate fibrosis stages. With SSI, follow-up would be easier and liver biopsy could be avoided.

Author Disclosures:

C. Cohen-Bacrie: Employee; Supersonic Imagine.

\section{B-0095 11:06}

Evaluation of shear wave elastography to monitor development of fibrosis after liver transplantation

O. Kolokythas ${ }^{1}$, R. Bhattacharya ${ }^{1}$, I.W. Liou ${ }^{1}$, A. Kang ${ }^{1}$, P. Bhargava ${ }^{1}$,

L.M. Mitsumori' ${ }^{1}$, C. Cuevas ${ }^{1}$, M.F. Bruce ${ }^{2} ;{ }^{1}$ Seattle, WA/US,

${ }^{2}$ Aix-en-Provence/FR (matt.f.bruce@gmail.com)

Purpose: Initial investigation of transient elastography (TE) for following liver transplantation has been encouraging. Recent studies have demonstrated advantages of shearwave elastography (SWE) in the ease of use, reproducibility and accuracy over TE in estimating liver fibrosis. The purpose of this study was to investigate SWE in the assessment of liver fibrosis as alternative non-invasive method to liver biopsy following liver transplantation.

Methods and Materials: SWE was used to assess liver stiffness in 40 (age 2463) post liver transplant patients receiving biopsy. SWE was performed just before biopsy over the right hepatic lobe at the site of biopsy. The stage of fibrosis assessed with a four-point scoring system and inflammation as a marker for rejection were compared against the mean of three SWE acquisitions. Spearman correlation was used for statistical analysis.

Results: All 40 patients had successful SWE acquisitions. The Spearman correlation coefficient showed a correlation of 0.73 with fibrosis stage ( $p$ value $<0.01$ ). No correlation $(0.32$ with $p$ value $<0.5)$ was observed with inflammation.

Conclusion: SWE appears to be a promising alternative to liver biopsy in evaluating the recurrence of fibrosis after liver transplantation.

Author Disclosures:

O. Kolokythas: Advisory Board; Philips Healthcare. Consultant; Philips Healthcare. Equipment Support Recipient; Philips Healthcare, Supersonics Imagine. Grant Recipient; Bracco Diagnostics. M.F. Bruce: Employee; Supersonic Imagine.

\section{B-0096 11:15}

The efficacy of Gadoxetate Disodium-enhanced magnetic resonance (MR) imaging in staging liver fibrosis

D.S. Feier ${ }^{1}$, C. Balassy², N. Bastati-Huber ${ }^{2}$, J. Stift², R. Badea ${ }^{1}$,

A. Ba-Ssalamah ${ }^{2} ;{ }^{1}$ Cluj-Napoca/RO, ${ }^{2}$ Vienna/AT

Purpose: Liver-specific, contrast-enhanced magnetic resonance imaging (CE-MRI) may provide anatomic and functional information in the setting of diffuse chronic liver diseases (CLD). We aimed to evaluate the efficacy of dynamic gadoxetate disodium-enhanced (Gd-EOB-DTPA) MRI in the staging of liver fibrosis (LF) in patients with diffuse CLD and to investigate the factors that may influence its efficacy. Methods and Materials: For this purpose, we analysed retrospectively 102 patients (mean age, 54.86 years; $58.7 \%$ males) with histologically proven LF according to METAVIR scoring system (F0-23 patients; F1-11 patients; F2-14 patients; F3-13 patients; and F4-41 patients). Patients underwent dynamic contrast-enhanced 3 Tesla MRI with GD-EOB-DTPA and images were analysed before contrast injection and in the hepatobiliary-phase (20 minutes after contrast administration). Signal intensity of the liver was defined using region-of-interest measurements and relative enhancement (RE) was calculated.

Results: RE correlated strongly with fibrosis $(r=-0.65, p<0.0001)$, moderately with necro-inflammatory activity $(r=-0.41, p=0.002)$ and iron load $(r=-0.21, p=0.05)$, but not with steatosis $(r=-0.12, p=0.32)$. In multivariate analysis, only fibrosis independently influenced the values of RE $(p<0.0001)$. RE performed well in the staging of LF, with an area under the ROC curve (AUROC) of 0.81 for $F 1$ (Se:70\%, Sp:85\%, PPV:57.4\%, NPV:92.3\%), 0.82 for F2 (Se:74.6\%, Sp:75\%, PPV:95.6\%, NPV:91.2\%), 0.85 for F3 (Se:73.5\%, Sp:87\%, PPV:97.2\%, NPV:93\%) and 0.83 
for F4 (Se:83.3\%, Sp:79.7\%, PPV:96.8\%, NPV:83\%). Discordance between RE and histology was observed in $28(27.5 \%)$ patients.

Conclusion: Using RE measurements, contrast-enhanced MRI can accurately stage LF in CLD patients. Histologically proven steatosis, inflammation and iron load have no influence on the enhancement.

\section{B-0097 11:24}

Differential portal venous flow response to terlipressin in normal and cirrhotic rats: non-invasive assessment using phase-contrast MRI

M. Chouhan, A. Bainbridge, N. Davies, R. Mookerjee, R. Jalan,

S. Walker-Samuel, M. Lythgoe, S. Punwani, S.A. Taylor; London/UK (manil.chouhan@gmail.com)

Purpose: Portal venous flow (PVF) measurement using phase-contrast MRI (PCMRI) is technically feasible at 9.4T. The purpose of this study was to assess the sensitivity of PC-MRI to expected changes in portal flow after terlipressin administration and study the functional response in normal and cirrhotic rats.

Methods and Materials: Eight Sprague-Dawley rats were randomised to bile duct ligation (BDL) procedure $(n=4)$ or sham laparotomy $(n=4)$. After 4 weeks, PVF was measured using respiratory-gated $2 \mathrm{D} \mathrm{PC}$-MRI $\left(2 \mathrm{~mm}\right.$ slice thickness, $10^{\circ}$ flip angle and $22 \mathrm{~cm} / \mathrm{s}$ velocity encoding) with a 9.4T Agilent system. Terlipressin (known to reduce PVF) was administered intravenously at a dose of $10 \mathrm{mcg} / 100 \mathrm{~g}$ and PCMRI measurements repeated sequentially for 30-40 minutes post-administration. Bulk PVF was normalised to explanted liver weight, obtained after termination of the experiment. Data were analysed using paired and unpaired Student t-tests. Results: Baseline liver weight normalised mean PVF in sham $(143.53 \pm 14.42 \mathrm{ml} /$ $\mathrm{min} / 100 \mathrm{~g})$ vs BDL $(79.51 \pm 44.74 \mathrm{ml} / \mathrm{min} / 100 \mathrm{~g})$ rats was not statistically significant $(\mathrm{p}=0.059)$. The reduction in PVF post-terlipressin was significant in sham (mean reduction of $63.48 \pm 14.28 \mathrm{ml} / \mathrm{min} / 100 \mathrm{~g} ; \mathrm{p}<0.05$ ), but not in $\mathrm{BDL}$ (mean reduction of $55.44 \pm 35.71 \mathrm{ml} / \mathrm{min} / 100 \mathrm{~g} ; \mathrm{p}=0.053$ ) rats. Significant differences in post-terlipressin nadir PVF in sham $(80.05 \pm 20.18 \mathrm{ml} / \mathrm{min} / 100 \mathrm{~g})$ vs BDL $(24.07 \pm 14.66 \mathrm{ml} / \mathrm{min} / 100$ g) rats were, however, demonstrated $(p<0.05)$.

Conclusion: Expected reductions in PVF were detected non-invasively using PCMRI in both normal and cirrhotic rats. Data are suggestive of a lower baseline PVF in cirrhotics, which go on to demonstrate a different, more labile haemodynamic response to terlipressin compared with normal rats.

\section{B-0098 11:33}

Evaluation of T1rho as a potential MR biomarker for liver cirrhosis: comparison of healthy control subjects and patients with liver cirrhosis

I. Rauscher ${ }^{1}$, C. Ganter ${ }^{1}$, P. Martirosian ${ }^{2}$, E.J. Rummeny ${ }^{1}$, K. Holzapfel'; ${ }^{1}$ Munich/DE, ${ }^{2}$ Tübingen/DE

Purpose: The purpose of this study was to compare mean liver T1rho values in patients with liver cirrhosis and healthy control subjects in order to evaluate T1rho as a potential MR biomarker for liver cirrhosis.

Methods and Materials: Ten healthy control subjects ( 6 female, 4 male) and 17 patients with clinically diagnosed and/or biopsy-proven liver cirrhosis (4 female, 13 male) were examined at 1.5 T (Magnetom Avanto, Siemens). T1rho-weighted images were acquired using a 2D Turbo FLASH sequence (TR/TE $3000 / 1.31 \mathrm{~ms}$, FA $8^{\circ}$, FoV $309 \times 380 \mathrm{~mm}$, resolution $2 \times 2 \times 6 \mathrm{~mm}$, acquisition time $15 \mathrm{~s}$ ) with Spin-Lock preparation. T1rho maps were calculated from five breath-hold measurements, performed with different Spin-Lock times (4, 8, 16, 32, $48 \mathrm{~ms})$. Mean liver T1rho values of healthy control subjects and patients with liver cirrhosis were calculated by two radiologists in consensus and were compared using two-tailed student t-test. In addition, a receiver operating characteristic (ROC) curve analysis was performed to evaluate the utility of mean liver T1rho for the prediction of liver cirrhosis.

Results: Mean liver T1rho values in patients with liver cirrhosis $(57.7 \mathrm{~ms} \pm 8.1$ $\mathrm{ms}$ ) were significantly higher than those of healthy control subjects $(48.6 \mathrm{~ms} \pm 3.5$ $\mathrm{ms} ; \mathrm{p}=0.006$ ). According to the ROC analysis at a threshold value of $51.4 \mathrm{~ms}$ the sensitivity and specificity of mean liver T1 rho in predicting liver cirrhosis were 86.7 and $88.9 \%$, respectively. The area under the ROC curve was 0.87 .

Conclusion: Mean liver T1rho values in patients with liver cirrhosis were significantly higher than in healthy subjects suggesting a potential role of liver T1rho as a MR biomarker for liver cirrhosis.

\section{B-0099 11:42}

Biomarkers of nonalcoholic fatty liver disease diagnosed by 1H SRM 3 T J. Martin-Rodriguez, J. Arrebola, J. Gonzalez-Calvin; Granada/ES (joseluismartin.rx@hotmail.com)

Purpose: Epidemiological studies showed that non-alcoholic fatty liver disease (NAFLD) is a possible precursor of cirrhosis and it has been associated with metabolic syndrome, diabetes and cardiovascular disease. There is a need to find a biomarker which proves to be reliable, non-invasive and easy to perform in clinical practice.

Methods and Materials: Our aim was to know whether serum alanine aminotransferase (ALT) is a reliable biomarker of liver fat content in NAFLD, and to determine if the current threshold of normality for ALT is appropriate to assess the presence of liver fat. This is a cross-sectional, randomised, prospective, population-based study. We studied 120 healthy subjects with alcohol consumption less than $50 \mathrm{~g} /$ week. Quantification of liver fat content was by spectroscopy $1 \mathrm{H} \mathrm{MR} 3$ T. $5 \%$ of liver fat content as the upper limit of normal for the diagnosis of hepatic steatosis. Results: There was an excellent positive correlation between liver fat content and serum levels of ALT $(r=0.73, p<0.0001)$. Results of the backward step-wise regression analysis showed that serum ALT was the most important predictor of hepatic steatosis (B coefficient=3.25). All subjects with ALT values $>37 \mathrm{U} / \mathrm{L}$ had hepatic steatosis (PPV:100\%) and none of the subjects with ALT $<20 \mathrm{U} / \mathrm{L}$ had steatosis (NPV: $100 \%$ ). ROC curves were created; the best cut-off value for the diagnosis of NAFLD was ALT: 23 U/L (sensitivity: $94.67 \%$, specificity $73.91 \%$, PPV: $85.84 \%$, NPV: $89.47 \%$ ).

Conclusion: Serum ALT levels could be a reliable biomarker of NAFLD if the upper limit of normal for ALT is set at $23 \mathrm{U} / \mathrm{L}$.

\section{B-0100 11:51}

Absolute quantification of phosphorus compounds in the liver on a clinical $3 \mathrm{~T}$ scanner

A. Laufs ${ }^{1}$, R. Livingstone ${ }^{2}$, S. Kahl', B. Nowotny', B. Klueppelholz',

G. Giani', J. Bunke ${ }^{3}$, J.-H. Hwang', M. Roden'; ; Düsseldorf/DE, ${ }^{2}$ Vellore/IN,

${ }^{3}$ Hamburg/DE (alessandra.laufs@uni-duesseldorf.de)

Purpose: Hepatic energy metabolism plays an important role in insulin resistance and liver diseases. The aim was to establish a robust and fast method to detect and to quantify liver ATP content in molar concentrations for use in a clinical setting, i.e. large cohort studies.

Methods and Materials: Healthy volunteers $(n=86 ; 58 \pm 12$ years; $B M I: 25.6 \pm 3.1$ $\mathrm{kg} / \mathrm{m}^{2}$ ) consented to the approved protocol. All experiments were performed on a 3-Tesla MRI scanner (Philips Achieva 3.0 T X-series, The Netherlands). ${ }^{31} \mathrm{P}$ spectra were acquired using a $14-\mathrm{cm}$ diameter ${ }^{31} \mathrm{P}$ surface coil, with the built-in ${ }^{1} \mathrm{H}$ body coil used for NOE enhancement and decoupling (TR: 4 sec; acquisition time: 13 minutes). Localisation in the liver was achieved using ISIS. Separately, intra- and interday variability were measured on 6 volunteers. The absolute quantification was established using matching phantoms and an external reference (MPA).

Results: All peaks were well resolved with the mean SNR ( $\gamma$-ATP) of 16. The concentrations were found to be $1.97 \pm 0.61 \mathrm{mmol} / /$ (phosphomonoester), $7.80 \pm$ $2.11 \mathrm{mmol} / \mathrm{l}$ (phosphodiester), $1.97 \pm 0.52 \mathrm{mmol} / \mathrm{l}$ (inorganic phosphate) and 2.70 $\pm 0.59 \mathrm{mmol} / \mathrm{l}(\mathrm{\gamma}$-ATP). The intra- and interday reproducibility was $4.5 \%$, respectively, $1 \%$ (PME) $9 \%$, respectively, 10\% (PDE), 11\%, respectively, $2 \%$ (Pi) and $9 \%$, respectively, $3 \%$ (Y-ATP).

Conclusion: The absolute quantification for large cohort studies has been successfully established on a clinical scanner. The obtained concentrations from this study agree with the concentrations from previous studies. Thus, the current method can reliably detect concentration changes above $10 \%$ in phosphorus compounds in human liver.

$10: 30-12: 00$ Room L/M

\section{Physics in Radiology}

\section{SS 113}

\section{Multi modality imaging and MR safety}

Moderators:

O. Ciraj-Bjelac; Belgrade/RS

O. Speck; Magdeburg/DE

\section{B-0101 10:30}

MR tumour perfusion measurements: dual echo versus multi-echo approach

V. Hietschold, M. Riebisch, A. Abramyuk, M. Laniado, N. Abolmaali;

Dresden/DE (Volker.Hietschold@Uniklinikum-Dresden.de)

Purpose: Gadolinium chelates (CM) lead to concentration-dependent shortening of $\mathrm{T} 1$ as well as $\mathrm{T}^{*}$ relaxation times. T1 shortening is more influenced by the interstitial CM, whereas T2* contains more information about the capillaries. By sequence weighting, the one or other aspect can be pronounced, by means of 
double echo sequences both effects can be separated one from the other. We present an extension of this concept to multi-echo measurements.

Methods and Materials: Brain perfusion measurements were performed in 10 patients as part of an ongoing study investigating the reproducibility of perfusion parameters on a 3 Tesla MR scanner (Siemens Magnetom Verio). Three slices were recorded with a temporal resolution of $2 \mathrm{~s}$ by means of a four echo FLASH sequence $\left(\mathrm{TR}=52 \mathrm{~ms}, \mathrm{TE}=1.2 / 5 / 10 / 15 \mathrm{~ms}\right.$, alpha $\left.=301 / 470^{\circ}\right)$. Evaluations of contrast to noise ratios (CNR) during the bolus passage of Omniscan (GE Healthcare) through different tissues were done with an inhouse software written in IDL (Exelis VIS). Results: The CNR is improved by a factor of about 1.8 calculating delta-R2 ${ }^{*}$ from four echoes compared to calculations based on two echos. Signal intensity estimations for $\mathrm{TE}=0$ do not significantly benefit by the increased number of echoes. Conclusion: Multi-echo measurements allow for a larger dynamic range of T2 shortening compared to single or double echo sequences, at the same time giving more signal. By extending the concept to keyhole and/or EPI sequences, covering of entire tumours could be achieved.

\section{B-0102 10:39}

PET/MR imaging of the pelvis in the presence of endoprostheses: reducing image artefacts and increasing accuracy through inpainting C.N. Ladefoged, F. Andersen, S. Keller, J. Löfgren, A.E. Hansen, S. Holm, L. Hojgaard, T. Beyer; Copenhagen/DK (thomas.beyer@cmi-experts.com)

Purpose: In combined PET/MR, attenuation correction (AC) is performed indirectly. Implant-induced susceptibility artefacts challenge MR-based AC. We evaluate the accuracy of MR-AC in PET/MR patients with metallic endoprostheses.

Methods and Materials: We select patients with uni- or bi-lateral endoprostheses from 100 consecutive referrals for whole body (WB) PET/MR imaging (mMR, Siemens Healthcare). Simultaneous WB-PET/MR scanning was performed at 120 min p.i. of $300 \mathrm{MBq}$ [18 F]-FDG: 4 min/bed including Dixon Water Fat segmentation (DWFS)). MR-AC was performed using a) original MR-images (DWFS), b) as a) with implant-induced air-pockets filled with soft-tissue, c) as b) superimposed with co-registered endoprostheses from CT and d) as a) with implant-induced airpockets filled with metal. PET reconstruction after MR-AC (a-d) used AW-OSEM (3iterations, 21subsets, 2 mm Gaussian) on 344-matrices. Mean/max standardised uptake value (SUVbw) was calculated from interest volumes with $40 \%$ isocontour levels over the hips, pelvic muscle and bladder.

Results: In 100 patients we found 1 and 2 uni- and bi-lateral endoprosthesis, respectively. Associated MR artefacts were much larger than the implants: $20.9 \%$ versus $2.5 \%$ of the attenuating voxels in (a) of the central hip region.

MR-AC using (b) and (c) recovered FDG-distribution pattern compared to uncorrected PET (noAC) images and (a), while (d) resulted in severe overestimation (> $470 \%$ SUVmax). The relative changes in SUVmax/mean in the reference regions (bladder, pelvic muscle) from (b-d) compared to (a) were insignificantly small. Conclusion: Endoprostheses cause PET/MR artefacts and bias PET quantification. Artefacts and bias can be corrected by automated inpainting with a single softtissue composition prior to MR-AC, thus restoring quantitative activity distribution.

\section{Author Disclosures:}

T. Beyer: Founder; cmi-experts $\mathrm{GmbH}$.

\section{B-0103 10:48}

Combined PET/MR imaging: the effect of ignoring bone during MR-

based attenuation correction in oncology imaging

J. Saa, J. Löfgren, R. Sersar, M. Aznar, C. Ladefoged, F. Andersen, R. Larsen,

T. Beyer; Copenhagen/DK (thomas.beyer@cmi-experts.com)

Purpose: In combined PET/MR, PET attenuation correction (AC) is based on tissue segmentation following in-/opposed phase MR imaging (in_opp). Bone tissue is not represented in routine MR-based AC (MR-ACin_opp). We evaluate PET quantification accuracy following MR-AC without and with accounting for bone tissue using separate CT.

Methods and Materials: Nine whole-body patients underwent an [18 F]-FDGPET/CT exam (mCT, Siemens) followed by a PET/MR scan (mMR, Siemens) at 119 min p.i. of $300 \mathrm{MBq}$ [18 F]-FDG. PET (/MR) images were reconstructed using standard MR-AC (ACin_opp) and four modified MR-AC maps. These were created by co-registering (b-spline) the CT images to (ACin_opp) and adding CT bone mask values representing cortical bone: $1200 \mathrm{HU}$ (ACcort), spongiosa bone: $350 \mathrm{HU}$ (ACspong), average CT value (ACmean) and original CT values (ACorgCT). PET images were reconstructed after MR-AC using AW-OSEM (3iterations, 21subsets, $2 \mathrm{~mm}$ Gaussian) on 344-matrices. Mean/max standardised uptake values (SUVbw) were calculated in anatomical reference regions and PET-positive lesions.

Results: Mean CT attenuation was $(734 \pm 158) \mathrm{HU}(\min / \max 475 / 900 \mathrm{HU})$. Visual assessment of the different AC-PET images indicated most prominent differences to standard MR-AC (ACin_opp) in the skeletal system. Maximum changes in SUVmax were observed for (ACcort) in the upper spine: from $0.7 \pm 0.2$ to $3.0 \pm 0.4$ and the femoral head: from $0.2 \pm 0.1$ to $1.4 \pm 0.4$. Changes in MR-AC had no prominent impact on SUV changes: neither in reference soft tissues nor in three PET-positive soft tissue lesions in the pelvis and neck.

Conclusion: Standard MR-AC without accounting for bone attenuation appears acceptable for whole body PET/MR imaging when bony lesions are not the primary diagnostic targets.

Author Disclosures:

T. Beyer: Employee; cmi-experts $\mathrm{GmbH}$, cmi-experts $\mathrm{GmbH}$.

\section{B-0104 10:57}

Assessment of the performance of TOF-PET and PET

reconstructions on edge definition of cold regions in the presence of solitary hot spots and low count-rate studies

Y. Bouchareb 1 , M. Masoomi ${ }^{2}$, M. Newell ${ }^{1} ;{ }^{1}$ London/UK, ${ }^{2}$ Portsmouth/UK

(byassine06@yahoo.co.uk)

Purpose: To investigate an additional requirement in low count rate studies which is to define the edges of cold regions in the presence of solitary hot spots.

Methods and Materials: Phantom PET/CT scans were performed on the Philips Gemini TOF scanner. In low count rate studies corresponding to typical clinical scans of obese patients and gating or dynamic studies, it is crucial to consider accurate TOF reconstruction kernels, number of iterations and number of subsets. The NEMA image quality phantom was used; spheres 10, 17 and $28 \mathrm{~mm}$ were filed with F-18 and spheres 13, 22 and $37 \mathrm{~mm}$ were filled with water. Spheres to background $(2.1 \mathrm{kBq} / \mathrm{mL}$ ) ratio was $4: 1$ (total activity was $22.6 \mathrm{MBq})$. Three TOFListMode-EM reconstructions using 3 iterations (20 and 33 subsets) and kernel width of $14.1 \mathrm{~cm}$ and $18.7 \mathrm{~cm}$ were performed. Images were also reconstructed using non-TOF (LOR-RAMLA and 3D-RAMLA) methods for comparison. Activity line profiles $(A L P)$, contrast recovery coefficients $(C R C)$ and Noise were used to analyse the reconstructed images.

Results: ALP extracted through diameters of cold spheres indicated an improvement of 5,11 and $15 \%$ in TOF-ListMode-EM reconstructed sphere diameters over nonTOF-LOR-RAMLA ( 3 ietrations/33 subsets), nonTOF-LOR-RAMLA (3 iterations/20 subsets) and nonTOF-3D-RAMLA reconstructions, respectively. The TOF-ListMode-EM reconstruction using $18.7 \mathrm{~cm}$ kernel and 3 iterations with 33 subsets achieved the highest CRC for spheres of diameters $<=28 \mathrm{~mm}$ and better background noise reduction.

Conclusion: TOF-ListMode-EM reconstruction with stated kernel width, number of iterations and subsets improves edge definition of cold regions and image contrast in low count studies (e.g. gated imaging).

\section{B-0105 11:06}

The incidence of biological effects from 3.0 Tesla (T) MRI compared

to $1.5 \mathrm{~T}$ : an observational study in 911 consecutive outpatients

F. Alghamdi, P. Bertrand, L. Barantin, M.A. Lauvin, X. Cazals, F. Domengie,

R. Bibi, D. Herbreteau, J.-P. Cottier; Tours/FR (faisal.gh.30@gmail.com)

Purpose: To compare the acute biological effects of a 3.0 Tesla $(\mathrm{T})$ magnetic resonance imaging (MRI) to $1.5 \mathrm{~T} \mathrm{MRI}$

Methods and Materials: After MRI examination, 911 patients (449 with 3.0 T MRI and 462 with $1.5 \mathrm{~T} \mathrm{MRI}$ ) were presented with a verbal rating scale questionnaire consisting of 11 symptoms related to MRI examination. Chi-square tests were used to assess the relationship between the strength of the magnetic field (MF) and the incidence of the symptoms. A $P$ value of $<.05$ was considered significant. Results: There was no statistically significant relationship between the strength of the MF and the incidence of the symptoms related to static MF exposure, such as vertigo $(P=.13)$, nausea $(P=.35)$, headache $(P=.21)$, and a metallic taste $(P=.64)$. A warm feeling induced by radiofrequency (RF) was significantly higher in the 3.0 T MRI $(P<.0001)$ with a significant correlation between the mean specific energy absorption rate (SAR) and a warm feeling in the $3.0 \mathrm{~T} \mathrm{MRI}(P<.0001)$. Numbness/ tingling related to the gradient MF was significantly higher in the 3.0 T MRI $(P=.027)$. Conclusion: The thermal effect induced by the RF and the numbness/tingling induced by the gradient field were significantly higher in the 3.0 T MRI than in the 1.5 T MRI. There was no statistically significant difference between the symptoms related to static MF exposure from the 3.0 T MRI and 1.5 T MRI. 


\section{B-0106 11:15}

Static magnetic fields in 1.5 and 3 T MR scanners do not influence perception of pain and touch compared with placebo exposition

A. Pomschar, K. Kamm, R. Ruscheweyh, R. Laubender, M.F. Reiser, A. Straube,

B. Ertl-Wagner; Munich/DE (Andreas.Pomschar@med.uni-muenchen.de)

Purpose: Potential biological effects of static magnetic fields have been in the focus of public debate with the European Physical Agents (Electromagnetic Fields) Directive 2004/40/EC having the potential to seriously limit MR imaging in Europe. Data on potential effects are still very scarce. We aimed to investigate the influence of homogeneous static magnetic fields in 1.5 and 3 Tesla MR scanners on pain and touch perception compared with placebo exposition.

Methods and Materials: 18 healthy volunteers (age: $23 \pm 1.8$ years, 9 females) underwent three experimental sessions on separate days in randomised order, using field strengths of OT (placebo scanner), 1.5 $\mathrm{T}$ and $3 \mathrm{~T}$. Participants were blinded to field strength. No imaging sequences were acquired. In each session, a Quantitative Sensory and Pain Testing programme was performed before and after a 10-min exposure to the static magnetic field. It included thresholds for mechanical detection, mechanical pain, pressure pain, heat pain and phasic heat pain intensity ratings. A linear mixed-effects model was used for statistical analysis. Results: For 1.5 and $3 \mathrm{~T}$ compared with the placebo control, statistical analysis revealed no significant effect of the magnetic field for all tested qualities, thresholds for mechanical detection ( $p=0.82 / 0.09)$, mechanical pain $(p=0.83 / 0.53)$, pressure pain $(p=0.97 / 0.28)$, heat pain $(p=0.53 / 0.38)$ and phasic heat pain intensity ratings $(\mathrm{p}=0.56 / 0.75)$.

Conclusion: Short exposure to static magnetic fields in clinical 1.5 T and $3 \mathrm{~T} \mathrm{MRI}$ scanners had no significant effect on pain and touch perception compared with a placebo exposure.

\section{B-0107 11:24}

Safety and efficiency of low-field magnetic resonance imaging in patients with cardiac rhythm management devices

C. Schukro, M. Lee; Vienna/AT

Purpose: Low-field magnetic resonance imaging (MRI), i.e. MRI with a magnetic field strength $<0.5$ Tesla, has been reported to be safe in patients with pacemakers; however, there are no data about the safety of low-field MRI in patients with implantable cardioverter defibrillators (ICD) and/or cardiac resynchronization therapy (CRT). We aimed to investigate the safety and efficiency of routine low-field MRI in patients with different devices for cardiac rhythm management (i.e. pacemakers and ICD, including devices with CRT).

Methods and Materials: MRI scans of 191 regions of interest were evaluated with a field strength of 0.2 Tesla in 167 patients $(62.3 \%$ male; age at MRI scan $75.9 \pm 9.2$ years; time since device implantation $4.1 \pm 3.2$ years) with cardiac rhythm management devices (152 pacemakers, 8 ICD, 4 ICD with CRT, and 3 CRT pacemakers). This analysis included 27 pacemaker-dependent patients $(16.2 \%), 20$ patients with 1.5-Tesla-MR conditional pacemakers (12\%) and 10 patients with abandoned leads $(6 \%)$

Results: Except for one examination, which was interrupted because of recurrent severe nausea, all MRI scans could be analysed efficiently. No induction of arrhythmia or inhibition of pacemaker function occurred. Compared to the device interrogation before MRI, there were no significant changes in battery voltage $(2.85 \pm 0.13 \mathrm{~V}$, before and after MRI), pacing capture threshold, sensing of intrinsic ECG, lead impedance, and ICD-shock impedance after completed examination. Conclusion: Low-field MRI examinations ( 0.2 Tesla) were safe and efficient in patients with devices for cardiac rhythm management, even in case of pacemakerdependency or the presence of abandoned leads.

\section{B-0108 11:33}

Possible hazardous effect of "MR safe" metallic implants for workers

N. Oberhofer ${ }^{1}$, P. Ferrari2; ${ }^{1}$ Bolzano/IT, ${ }^{2}$ Mattarello/IT (nadia.oberhofer@asbz.it)

Purpose: to investigate if test conditions for medical implants certified as "MR safe" for patient examination also consider the extremely high gradient field exposure of devices implanted in MR-workers during their activities near the magnet bore and possible hazards.

Methods and Materials: Starting from a case report of a nurse with a nitinol (nickel-titanium alloy) stapes piston implant certified as suitable for MR exam until $3 \mathrm{~T}$, reporting a strong, several days' persisting pain after she had approached a 1.5 T gantry, some databases dedicated to the MR compatibility of devices have been consulted. Reference literature for MR testing of different implants has been analysed with respect to test conditions.
Results: In the evaluated literature the implant test conditions were generally focused on patient scanning, usually considering patient table moving into the gantry at low speed a few $\mathrm{cm} / \mathrm{s}$, perpendicularly to the field lines of the magnetic fringe field. However, implanted in a worker, during movement at normal walking speed near the magnet bore any implant is exposed to extremely high magnetic field gradients, which for metallic non ferromagnetic implants results in an opposing force due to Lenz' law.

Conclusion: In general MR-testing procedures of medical implants certified as "MR safe" for patient examination in mid-field scanners $(1.5 \mathrm{~T}-3.0 \mathrm{~T})$ do not consider the possible hazardous effect of Lenz' law on metallic devices implanted in workers during activities near the magnet bore.

We suggest including into the implant and device compatibility test the particular worker's scenario too.

\section{B-0109 11:42}

Swedish national study on modality choice and justification of CT, MRI and US examinations in children

B. Isberg', H. Jorulf2, U. Svahn', S. Richter', W. Leitz'; ' 'Stockholm/SE, ${ }^{2}$ Uppsala/SE (bengt.isberg@ptj.se)

Purpose: The aim of this retrospective study was to evaluate the level of justification regarding choice of method for computed tomography (CT), magnetic resonance imaging (MRI) and ultrasound (US) as well as the quality of requests for examinations of children in Sweden.

Methods and Materials: Request forms from all Swedish hospitals were collected for CT, MRI and US examinations ( $N=3149$ ) of children, performed during 2 weeks in 2011. 18 physicians choose examination based on request information and judged quality. All requests were evaluated by at least 2 observers. Outcomes were assessed for health care regions and levels, examined body parts, age classes and gender.

Results: $96 \%$ of requests were considered adequate. In $51 \%$ of CT, $68 \%$ of MRI and in $88 \%$ of US examinations the observers agreed on the same examination as requested and performed by the hospital. In $14 \%$ of CT, $5 \%$ of MRI and in $2 \%$ of US examinations the observers agreed on examinations, not being the same as requested and performed. In $30 \%$ of CT, $24 \%$ of MRI and in $9 \%$ of US the observers disagreed, at least one observer choose another examination than requested and performed.

Conclusion: The level of agreement on examination can be considered as an estimation of the level of consensus. The low level of agreement for CT mandates increased efforts in making guidelines to improve routines and to reduce radiation doses to patients. This study was initiated and funded by the Swedish Radiation Protection Authority, Department of Radiation Protection/ Medical Exposures.

\section{B-0110 11:51}

In vitro comparison of ultrasound-based elastography techniques S. Franchi-Abella 1 , J.-M. Correas ${ }^{2}$, C. Elie ${ }^{2} ;{ }^{1}$ Le Kremlin-Bicêtre/FR, ${ }^{2}$ Paris/FR (stephanie.franchi@bct.aphp.fr)

Purpose: Ultrasound elastography techniques are based on two different physical principal: strain and Shear Wave Velocity (SWV). The purpose of this study was to assess the performance of these two techniques in vitro on a calibrated elasticity phantom using five ultrasound diagnostic imaging system to improve clinical comprehension and use of elastography.

Methods and Materials: An operator performed all acquisitions on a tissuemimicking phantom developed for elastography calibration (CIRS 049 A) containing spherical inclusions embedded in an homogeneous background (BG) of known elasticity ( $30 \pm 5 \mathrm{kPa}$ ). Two inclusions were harder than the $B G$ and two were softer $(44 \pm 6 \mathrm{kPa}, 75 \pm 10 \mathrm{kPa}, 12 \pm 3 \mathrm{kPa}$ and $18 \pm 4 \mathrm{kPa})$. Protocol was performed using high-frequency linear transducer on four different inclusions. Qualitative assessment was done on elastograms. Semiquantitative measurements were based on the ratio of strain or elasticities between the BG and each inclusion and were compared with theoretical ratio. Quantitative evaluation was performed with SWV techniques. Results: All techniques are adequate when assessing whether or not an inclusion is harder or softer than the BG. Semi-quantitative ratio of stiffness between two structures is not reproducible and strongly depends on the manufacturer. It is more accurate for SWV techniques. Direct quantitative evaluation of stiffness using SWV exhibits biases for soft targets but can still be used to precisely measure stiffness if bias is taken into account.

Conclusion: Limitations and advantages of the different elastography techniques must be known while performing an elastography acquisition in clinical practice. 


\section{Vascular}

\section{SS 115}

\section{Carotid plaque evaluation}

\section{Moderators:}

M.A. Aschauer; Graz/AT

D. Filippiadis; Athens/GR

\section{B-0111 10:30}

Arterial stiffness is associated with carotid intraplaque haemorrhage in the general population: the Rotterdam study

M. Selwaness, Q. van den Bouwhuijsen, F.U.S. Mattace-Raso, G. Verwoert,

A. Hofman, O.H. Franco, J.J. Wentzel, J. Witteman, A. van der Lugt;

Rotterdam/NL (m.selwaness@erasmusmc.nl)

Purpose: Aging and vascular risk factors are important determinants of carotid intraplaque haemorrhage (IPH), but whether arterial stiffness is linked to IPH is unknown. In a population-based study, we investigated the association between arterial stiffness, as assessed by pulse wave velocity (PWV), and carotid atherosclerotic plaque composition.

Methods and Materials: Overall, 1,059 participants from the general population with wall thickening $(>2.5 \mathrm{~mm})$ on ultrasound underwent PWV measurement and 1.5 T magnetic resonance imaging (MRI) for carotid plaque characterisation. Multisequence scanning was used to assess the presence of IPH, lipid core and calcification in both carotid arteries. Generalized estimation equation analyses, adjusted for age, gender, mean arterial pressure (MAP), heart rate (HR), carotid wall thickening and traditional cardiovascular risk factors were used to assess the association between PWV and plaque characteristics.

Results: The mean age of the subjects was $74.5 \pm 8.0$ ( $53.3 \%$ men). Atherosclerotic plaques were present in 1,978 carotid arteries, of which $552(28 \%)$ had IPH. PWV adjusted for age, gender, MAP and HR was associated with the presence of IPH (OR per SD, 1.22; 95\% Cl, 1.06-1.40) and calcification (OR per SD 1.15; 95\% Cl, 1.00-1.31), but not with the presence of a lipid core. However, only IPH remained significant after additional adjustment for traditional cardiovascular risk factors (OR per SD, $1.19 ; 95 \% \mathrm{Cl}, 1.03-1.39$ )

Conclusion: PWV, appears to be an independent determinant of IPH. The current findings suggest that arterial stiffness contributes to the development of different atherosclerotic plaque characteristics.

\section{B-0112 10:39}

Evaluation of carotid atherosclerotic plaque with lab-based x-ray phase-contrast imaging

H. Hetterich'1 , S. Fill', M. Willner'², F. Bamberg', J. Herzen²,

A. Hipp ${ }^{2}$, M.F. Reiser ${ }^{1}$, F. Pfeiffer ${ }^{2}$, T. Saam ${ }^{1} ;{ }^{1}$ Munich/DE,

${ }^{2}$ Garching/DE (holger.hetterich@med.uni-muenchen.de)

Purpose: Grating-based phase-contrast imaging (gb-PCl) is a novel imaging technique that uses the phase-shift information of $x$-rays passing through matter. This technique provides an excellent soft tissue contrast and might therefore become a valuable tool for vessel wall imaging. The aim of this experimental ex-vivo study was to evaluate the potential of gb-PCl for atherosclerotic plaque imaging in human carotid arteries.

Methods and Materials: Seven postmortem human carotid artery specimens were imaged at a laboratory set-up using a conventional broadband $\mathrm{x}$-ray tube and a grating interferometer followed by histology processing. $\mathrm{Gb}-\mathrm{PCl}$ and histology data were matched using anatomical landmarks. Signal characteristics of necrotic core (NC), fibrous cap (FC), intraplaque haemorrhage (IPH) and calcifications (Ca) were established by reviewer 1 . Sensitivity, specificity and accuracy of gb-PCl for plaque detection and the potential for accurate plaque quantification were assessed by reviewers 2 and 3 .

Results: We found characteristic signal criteria in all analysed plaque components. Applying these criteria gb-PCI had a good sensitivity for the detection of FC/NC, $\mathrm{IPH}$ and $\mathrm{Ca}$ (all $>80 \%$ ) and excellent specificity and accuracy (all $>90 \%$ ) with good inter-reader agreement ([[Unable to Display Character: \&\#1082;]] $0.72, p<$ 0.0001). There were excellent correlations for quantitative measurements of FC, $\mathrm{NC}$ and $\mathrm{Ca}$ between gb-PCl and histopathology $\left(\mathrm{R}^{3} 0.92\right)$. Inter-reader reproducibility was excellent with $\mathrm{ICC}^{3} 0.98$ for all measurements.

Conclusion: $\mathrm{Gb}-\mathrm{PCl}$ can identify and quantify atherosclerotic plaque components in a lab-based set-up with excellent correlation to histopathology.
B-0113 10:48

Meta-analysis and systematic review of the predictive value of carotid plaque haemorrhage by magnetic resonance imaging on cerebrovascular events

T. Saam 1 , H. Hetterich'1, V. Hoffmann², C. Yuan ${ }^{3}$, M. Treitl', M. Dichgans' ${ }^{1}$,

H. Poppert ${ }^{1}$, M.F. Reiser ${ }^{1}$, F. Bamberg ${ }^{1} ;{ }^{1}$ Munich/DE, ${ }^{2}$ Neuherberg/DE,

${ }^{3}$ Seattle, WA/US (Tobias.Saam@med.uni-muenchen.de)

Purpose: Several small studies suggested that MR-based identification of carotid plaque haemorrhage $(\mathrm{CPH})$ is associated with the occurrence of cerebrovascular events. Thus, we conducted a systematic review and meta-analysis to determine precise estimates of the predictive value of $\mathrm{CPH}$ for cerebrovascular events.

Methods and Materials: We searched PubMed, EMBASE and the Cochrane Library through February 2012 for studies that followed $>35$ individuals after baseline MRI. Independent observers abstracted information on population characteristics, MR techniques, outcome events and study quality. Risk estimates of the presence of $\mathrm{CPH}$ (T1w hyperintensities) for cerebrovascular events (TIA, stroke, amaurosis fugax) were derived in random effect regression analysis and causes of heterogeneity were determined in meta-regression analysis. Also, publication bias was assessed using funnel plots.

Results: Among 218 published articles, we identified 8 eligible studies including 689 participants $(73.1 \pm 2.5$ years, $77.9 \%$ male) who underwent carotid MRI (all 1.5 $\mathrm{T})$. Prevalence of $\mathrm{CPH}$ was high (49.0\%). Over a median follow-up of 19.6 months, 108 cerebrovascular events occurred (15.7\% event rate). Presence of $\mathrm{CPH}$ was associated with a $\sim 6$-fold higher risk for events (HR: 5.69; 95\%-Cl: 2.98-10.87) with a trend for heterogeneity $(p=0.07)$ but no evidence of publication bias $(p>0.05)$. Event rate in subjects with detectable $\mathrm{CPH}$ was $30.6 \%$.

Conclusion: Presence of $\mathrm{CPH}$ on MRI strongly predicts cerebrovascular events despite heterogeneity in populations, endpoints, and categorisation of MRI findings. Homogenization of future studies is warranted to allow for sufficient assessment of level of evidence for intervention trials.

\section{B-0114 10:57}

Genetic loci for coronary calcification and serum lipids relate to aortic and carotid calcification

D. Bos, M.A. Ikram, A. Isaacs, B.F.J. Verhaaren, A. Hofman, C.M. van Duijn, J.C.M. Witteman, A. van der Lugt, M.W. Vernooij; Rotterdam/NL (d.bos@erasmusmc.nl)

Purpose: Atherosclerosis in different vessel beds shares lifestyle and environmental risk factors. We investigated whether this also holds for genetic risk factors. Hence, we used genetic loci for coronary artery calcification (CAC) and serum lipid levels - one of the strongest risk factors for atherosclerosis - to assess their relationship with atherosclerosis in different vessel beds.

Methods and Materials: In 1,987 persons from the population-based Rotterdam study we used three SNPs (single nucleotide polymorphisms) for CAC and 132 SNPs for total cholesterol, low-density lipoprotein cholesterol, high-density lipoprotein cholesterol or triglycerides. To quantify atherosclerotic calcification, as marker of atherosclerosis, all participants underwent non-enhanced CT of the aortic arch and carotid arteries. We investigated associations between genetic risk scores of the joint effect of the SNPs and calcification.

Results: The joint effect of CAC-SNPs was associated with larger calcification volumes in all vessel beds [difference in calcification volume per SD increase in genetic risk score $0.15(95 \% \mathrm{Cl}: 0.11 ; 0.20)$ in aorta, $0.14(95 \% \mathrm{Cl}: 0.10 ; 0.18)$ in extracranial carotids, $0.11(95 \% \mathrm{Cl}: 0.07 ; 0.16)$ in intracranial carotids]. The joint effect of total cholesterol-SNPs, LDL-SNPs and of all lipid SNPs together was associated with larger calcification volumes in both the aortic arch and the carotid arteries, but attenuated after adjusting for the lipid fraction and lipid lowering medication. Conclusion: The genetic basis for carotid artery calcification overlaps with the most important loci of coronary artery calcification. Furthermore, serum lipids share a genetic predisposition with both calcification in the aortic arch and the carotid arteries, providing novel insights into the aetiology of atherosclerosis.

\section{B-0115 11:06}

Is carotid intima-media (IMT) and extra media thickness (EMT) a surrogate marker of early atherosclerosis in patients with inflammatory bowel disease?

A. Oikonomou, E. Astrinakis, P. Zezos, N. Courcoutsakis, A. Mpampali,

I. Karatzoglou, G. Kouklakis, P. Prassopoulos; Alexandroupolis/GR (aoikonom@med.duth.gr)

Purpose: IMT, EMT and echogenicity of the intima-media complex (EC) reflect early changes in the arterial wall and may appear years before atherosclerotic 
plaque has formed correlating with cardiovascular risk factors. The present study aims at investigating whether there are differences in the carotid wall thickness and echogenicity between patients with IBD and well-matched healthy controls by high-resolution ultrasonography of the carotid arteries.

Methods and Materials: 31 IBD patients (19 male, mean age: $36.9 \mathrm{yrs,} \mathrm{crohn/}$ Ulcerative colitis: 19/12, 17 smokers) and 29 healthy controls ( 15 male, mean age: $36.1 \mathrm{yrs}, 16$ smokers) underwent high-resolution ultrasonography of the carotid arteries. The following variables were recorded in bilateral common carotid arteries: 1) IMT at 5, 10 and $15 \mathrm{~mm}$ from the carotid bulb, 2) EMT (the distance between the common carotid wall and the internal jugular vein) and 3) EC.

Results: Common carotid artery IMT $(0.000<p<0.01$, T-test) and EMT ( $p<$ 0.001 , T-test) were significantly higher in IBD patients compared with controls. No difference in EC was found between IBD patients and controls. No difference in IMT, EMT and EC was found between Crohn's and ulcerative colitis patients. Smokers IBD patients had significantly higher IMT and EMT values than smokers controls $(0.002<\mathrm{p}<0.022$, T-test $)$

Conclusion: IBD appears to be a risk factor for development of early atherosclerosis as showed by greater values of carotid IMT and EMT in IBD patients compared with healthy controls irrespective of smoking history and type of IBD disease.

\section{B-0116 11:15}

Assessment of microvasculature in atherosclerotic plaque with dynamic contrast-enhanced-MRI: model comparison, reproducibility and validation with histology

M.E. Kooi ${ }^{1}$, M.E. Gaens ${ }^{1}$, R.H.M. van Hoof ${ }^{1}$, J.C. Sluimer ${ }^{1}$, S. Heeneman',

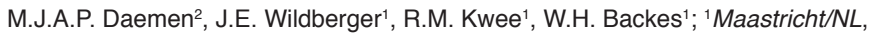
${ }^{2}$ Amsterdam/NL (eline.kooi@mumc.nl)

Purpose: Dynamic contrast-enhanced (DCE)-MRI can be used to identify atherosclerotic plaques with increased microvasculature, which is thought to be an important feature of plaque vulnerability. The purpose is to compare four pharmacokinetic models (Tofts, Extended Tofts, Patlak, and Extended Graphical model) for their ability to describe DCE-MRI of plaques, to determine the scan-rescan reproducibility, and to validate the results with histology.

Methods and Materials: Forty-five consecutive patients with $30-99 \%$ carotid stenosis underwent DCE-MRI. Plaque enhancement was measured dynamically using a gadolinium-based contrast agent. Pharmacokinetic parameters ( $\mathrm{K}^{\text {trans }}$, ve, vp) were determined by fitting the models to concentration-time curves. The relative fit errors and parameter uncertainties were determined to find the most suitable model. Sixteen patients were scanned twice to determine the reproducibility. Carotid endarterectomy specimens of sixteen patients were collected for histologic validation.

Results: Relative fit uncertainties for $\mathrm{K}^{\text {trans }}$ and vp were $10 \pm 1 \%$ (mean $\pm \mathrm{SE}$ ) and $20 \pm 2 \%$, respectively, for the Patlak model, which was significantly lower than for the other models. The reproducibility (coefficient of variation) for the Patlak model was $16 \%$ for $K^{\text {trans }}$ and $26 \%$ for vp. Significant correlations were found between $K^{\text {trans }}$ and plaque microvasculature as quantified by histology (Pearson's $\rho=0.72, p=0.005$ ). Conclusion: The Patlak model is most reliable to describe carotid plaque enhancement. Correlation with histology validated $\mathrm{K}^{\text {trans }}$ as an indicator of plaque microvasculature and the reproducibility of $\mathrm{K}^{\text {trans }}$ was good.

\section{Author Disclosures:}

M.E. Kooi: Research/Grant Support; Grant from CTMM (Parisk), financed by public and private funding (e.g. Philips Healthcare contributed to private funding).

\section{B-0117 11.24}

\section{Effect of expansive arterial remodelling on clinical presentation and} plaque composition: an in vivo MRI study of the carotid arteries A. Helck $^{1}$, M. Buchholz ${ }^{1}$, A. Rominger ${ }^{1}$, C. Yuan², M.F. Reiser ${ }^{1}$, K. Nikolaou', M. Dichgans ${ }^{1}$, A. Karpinska ${ }^{1}$, T. Saam ${ }^{1} ;{ }^{1}$ Munich/DE, ${ }^{2}$ Seattle, WA/US (andreas.helck@med.uni-muenchen.de)

Purpose: Expansive remodelling is considered to be a feature of vulnerable atherosclerotic plaques in coronary arteries. Most knowledge of carotid lesions is based on endarterectomy specimens which do not provide insight into remodelling patterns. Via MRI, we sought to evaluate the association between ischaemic stroke and expansive remodelling.

Methods and Materials: 54 patients with carotid plaques $\left({ }^{3} 2 \mathrm{~mm}\right.$ thickness by ultrasound) and unilateral ischaemic stroke in the territory of the ICA were included. All patients received black-blood 3.0 Tesla- MRI with fat-saturated pre-/post-contrast T1w-, PDw-, T2w- and TOF-images using surface coils and parallel imaging techniques. Quantitative measurements of lumen, wall, outer wall, haemorrhage, calcification and necrotic core were determined by consensus-reading of 2 expe- rienced reviewers for both sides and for common and internal carotid artery (CCA/ ICA), respectively. The modified remodelling index (RI) was calculated by dividing the outer vessel area at the site of the carotid plaque by the lumen area measured on TOF images in a normal reference segment distally (ICA) or proximal (CCA). Results: The CCA on the symptomatic side compared to the asymptomatic side had significantly larger maximum necrotic core and haemorrhage areas $(P<0.01)$. No other significant differences were found. The ICA on the symptomatic side compared to the asymptomatic had significantly larger wall, maximum necrotic core and haemorrhage areas $(P<0.01)$. No other significant differences were found. More specifically, the RI did not differ significantly either in the CCA (1.61 vs. 1.61, $\mathrm{P}=0.8$ ) or in the ICA (2.57 vs. 2.34, $\mathrm{P}=0.23$ ).

Conclusion: In this series of subjects no association was found between expansive remodelling and ischaemic stroke.

\section{B-0118 11:33}

Determination of the vascular input function using magnitude or phase-based MRI: influence on dynamic contrast-enhanced MRI model parameters in carotid plaques

R.H.M. van Hoof ${ }^{1}$, M.T.B. Truijman' ${ }^{1}$, E. Hermeling ${ }^{1}$, R.J. van Oostenbrugge ${ }^{1}$, R.J. van der Geest ${ }^{2}$, M.J.A.P. Daemen ${ }^{3}$, J.E. Wildberger ${ }^{1}$,

W.H. Backes ${ }^{1}$, M.E. Kooi ${ }^{1} ;{ }^{1}$ Maastricht/NL, ${ }^{2}$ Leiden/NL, ${ }^{3}$ Amsterdam/NL (r.vanhoof@maastrichtuniversity.nl)

Purpose: A reliable vascular input function (VIF) is important for quantitative analysis of atherosclerotic carotid plaque microvasculature using dynamic contrastenhanced (DCE) MRI. In tumour imaging and brain perfusion studies, it has been demonstrated that a phase-based VIF (ph-VIF) is less sensitive to flow artefacts compared to magnitude-based VIF (m-VIF). The purpose is (1) to compare m-VIF and ph-VIF and (2) to investigate the influence of different VIFs on DCE MRI model parameters in carotid plaques.

Methods and Materials: 21 patients with $30-99 \%$ carotid stenosis underwent 3 T DCE MRI. Data from four patients, scanned with a high temporal resolution were used to construct group-averaged $\mathrm{m}$-VIF and ph-VIF of the jugular vein. The other 17 patients were used for calculating $K^{\text {rans }}$ (measure of plaque microvasculature) using $\mathrm{m}$-VIF and ph-VIF. The effect of neglecting flow on the m-VIF was estimated using the Bloch equations.

Results: Peak concentrations of $m$-VIFs were on average 4-fold lower than for ph-VIFs $(p<0.001)$. Despite these differences, strong and significant correlation between $K^{\text {rans }}$ determined using group averaged $\mathrm{m}$-VIF and ph-VIF was found (Pearson's correlation 0.91, $\mathrm{p}<0.001$ ). Taking into account a theoretical flow of 8 $\mathrm{cm} / \mathrm{sec}$, the discrepancy in peak concentration of $\mathrm{m}$-VIF and ph-VIF disappeared. Conclusion: Our data suggest a strong influence of flow when calculating $\mathrm{m}$-VIF of the jugular vein. Despite this, strong correlation between $K^{\text {rans }}$ parameters determined using m-VIF and ph-VIF was found. It is expected that a phase-based VIF results in a quantitative more realistic value of $K^{\text {rans }}$

\section{B-0119 11:42}

Low does CT angiography using iterative reconstruction for the assessment of carotid artery stenosis

L. Crush, M. O'Keeffe, B. Normoyle, F. O'Neill, E.M. Canniff, P.D. Mc Laughlin, S. O'Neill, O. O'Connor, M.M. Maher; Cork/IE (Icrush03@gmail.com)

Purpose: To assess the feasibility of low-dose CT angiography (CTA), with a substantial dose reduction of $50 \%$, using iterative reconstruction in the assessment of patients suspected of carotid artery stenosis (CAS).

Methods and Materials: Following approval by Institutional Research Ethics Committee, twenty patients referred for the assessment of CAS were recruited. CTA of the carotid arteries was performed on a 64-slice CT scanner (General Electric, LightSpeed XTE). To facilitate a robust gold standard, each patient received two CT scans, a conventional dose (CD) scan (50-70\% standard protocol dose) and a low-dose (LD) protocol (30-50\% standard protocol dose). CD and LD studies had a combined $100 \mathrm{ml}$ of iodinated contrast followed by $20 \mathrm{ml}$ saline. The dose length product (DLP) for CD and LD was noted and the effective dose (ED) calculated. Intravascular attenuation, contrast to noise ratio (CNR), signal to noise (SNR) ratio and $\%$ stenosis of the internal carotid artery (ICA) were estimated by two radiologists. Results: The mean DLP for CD and LD were $687.96 \mathrm{mGycm}$ vs $341.32 \mathrm{mGycm}$ $(p<0.001)$. LD resulted in a $49.6 \%$ reduction in effective dose $(p<0.001)$. While there was a statistically significant decrease in CNR and SNR in the LD protocol, overall image quality remained satisfactory. There was agreement between $C D$ and LD for all occluded ICAs $(n=7)$ and for estimation of $\%$ stenosis (less than $3 \%$ difference) in 7 of 8 cases (87\%).

Conclusion: Low-dose CTA with ASIR reconstruction can achieve a radiation dose reduction approaching $50 \%$ while maintaining image quality and diagnostic accuracy. 


\section{B-0120 11:51}

Determining the vulnerable plaque: correlation between 18 F-FDG PET and dynamic contrast-enhanced MRI in atherosclerotic plaques of symptomatic patients

M.T.B. Truijman, R.M. Kwee, R.H.M. van Hoof, R.J. van Oostenbrugge, W.H. Mess, J.E. Wildberger, W.H. Backes, J.A. Bucerius, M.E. Kooi; Maastricht/NL (martine.truijman@mumc.nl)

Purpose: Identifying vulnerable atherosclerotic plaques in symptomatic patients with moderate $(30-69 \%)$ carotid artery stenosis can contribute to clinical decision making. Hallmarks of plaque vulnerability are inflammation and increased neovascularisation. Inflammation can be assessed with ${ }^{18} \mathrm{~F}$-FDG PET, while neovascularisation can be quantified with dynamic contrast-enhanced (DCE) MRI. We aimed to investigate the correlation between inflammation as assessed by ${ }^{18} \mathrm{~F}$-FDG PET and neovascularisation as assessed by DCE-MRI.

Methods and Materials: Fifty-eight patients with transient ischaemic attack (TIA) or minor stroke in the carotid territory and ipsilateral carotid plaque causing a moderate stenosis were included. All patients underwent $1.5 \mathrm{~T}$ multi-sequence MR imaging. Quantification of neovascularisation was done using a custom-made Matlab program which calculates $K^{\text {rans }}$. A 3D PET-CT scan was performed on all patients one hour after injection of $2.75 \mathrm{MBq} / \mathrm{kg}$ body weight ${ }^{18} \mathrm{~F}-\mathrm{FDG}$. Dedicated fusion software was used to calculate mean blood-normalised ${ }^{18} \mathrm{~F}$-FDG standard uptake values (SUV) of the plaque.

Results: Of the 58 patients, 9 were excluded due to poor image quality of the DCE-MRI. In total, we analysed 49 patients. The mean $K^{\text {rans }}$ and mean normalised SUV were $0.110( \pm 0.027)$ and $1.446( \pm 0.255)$, respectively. We found a weak but significant positive correlation between the mean normalised SUV and the mean $K^{\text {rans }}$ (Spearman's $r=0.302, p=0.035$ ).

Conclusion: There is a weak but significant positive correlation between $K^{\text {rans }}$, which is a marker for neovascularisation and SUV, which is a marker for inflammation. Future studies are warranted to investigate whether DCE-MRI and/or 18 F-FDG PET can be used to predict cerebrovascular events.

10:30 - 12:00 Room P

\section{Emergency Radiology}

\section{SS 117}

\section{An update on emergency thoraco-abdominal imaging}

Moderators:

B. Feragalli; Chieti/IT

A. Huete; Santiago/CL

\section{B-0121 10:30}

Emergency CT: what is missed at first reading?

A. Platon, G. Varnay, C.D. Becker, P.-A. Poletti; Geneva/CH

(alexandra.platon@hcuge.ch)

Purpose: To evaluate the accuracy of the preliminary $\mathrm{CT}$ interpretation made by the radiology residents in the emergency department.

Methods and Materials: During four periods of 20 consecutive days, attending radiologists prospectively recorded any disparity between their own $\mathrm{CT}$ interpretation (reference standard) and the preliminary interpretation delivered by the emergency radiology residents. Disparities were recorded during both regular hours and on-call time. A misinterpretation was considered major when potentially life-threatening within hours after admission. The total number of $\mathrm{CT}$ performed during the survey was recorded and further classified into neuroradiological and body examinations. A rate of disparities was calculated for all $\mathrm{CT}$ examinations and also for body and neuroradiological cases.

Results: A total of 3044 emergency CT examinations were performed during the survey: $1568(51 \%)$ were body CT and $1476(49 \%)$ neuroradiological cases. Disparities were reported in $145(4.8 \%)$ of all CT examinations: $0.8 \%(25 / 3044)$ were major misinterpretations (17 body, 8 neuro). There were 100/1568 (6.3\%) disparities reported in body CT versus $45 / 1476$ (3\%) in neuroradiological examinations $(p=0.001)$. No significant difference was found when comparing disparities recorded during regular working hours $(47 / 1083(4.3 \%))$ versus on-call period (98/1961 (5\%)), $\mathrm{p}=0.47$.

Conclusion: Our results show that disparities occur most frequently in emergency body CT examinations, but major disparities represent a small proportion of all misinterpretations. The detailed analysis of our data allows comparison and benchmarking over time and offers a basis of reference for optimising the training of radiology residents in emergency centres.

\section{B-0122 10:39}

Traumatic aortic injury: what is changing?

J.M. Artigas ${ }^{1}$, M. Martí De Gracia ${ }^{2}$, J.A. Soto ${ }^{3}$, A. Garcia-Valiente ${ }^{1}$, M. Marini ${ }^{4}$,

P. Parrilla'; ' Zaragoza/ES, ${ }^{2}$ Madrid/ES, ${ }^{3}$ Boston, MA/US, ${ }^{4}$ A Coruña/ES (jmartigas@salud.aragon.es)

Purpose: To emphasize the role of MDCT in traumatic aortic injury (TAI), including MDCT-based grading systems and current clinical work-up.

Methods and Materials: Review of our last 21 cases of TAl studied with MDCT in light of recent literature.

Results: TAl usually appeared as a result of motor vehicle accidents and predominantly affected aortic isthmus in young males (average: 41 years), most often in the context of severe multiple trauma (ISS average: 43 ). Our cases were classified retrospectively into four groups according to MDCT findings, mainly aortic contour preservation. Grade I: intimal tear, thrombus, or intramural haematoma $<1 \mathrm{~cm}$, without aortic external contour abnormality (2 cases/9, $5 \%$ ); grade II: the same finding, but $>1 \mathrm{~cm}$ (large intimal flap) (2/9, $5 \%$ ); grade III: presence of pseudoaneurysm $(9 / 43 \%)$; grade IV: aortic wall rupture with active contrast extravasation, regardless of the presence or not of a pseudoaneurysm ( $8 / 38 \%)$. The presence of hypotension and mediastinal haematoma thicker than $15 \mathrm{~mm}$ was also considered. Mortality in groups I and II was largely related to other severe associated injuries. Survival for groups III and IV was 50\% and 33\%, respectively. Survivors were younger people (mean: 25 years) without haemodynamic instability on arrival at ED and no other life-threatening associated injuries.

Conclusion: TAl is a dynamic process. Whole body MDCT evaluation eliminates the need for conventional angiography and while prospective validation becomes available, the proposed classification system allows to decide between immediate surgical/endovascular repair, a more conservative approach with initial medical treatment and delayed repair, or nonsurgical management with follow-up imaging.

\section{B-0123 10:48}

Comparison between dedicated coronary $\mathrm{CT}$ angiogram and triple rule out using $\mathbf{3 2 0}$ multislice $\mathrm{CT}$ in cases of acute chest pain

Y. Ragab, H. Hamza, H. Awadalla; Cairo/EG (yragab61@hotmail.com)

Purpose: To compare between dedicated and triple rule-out CT angiography (CTA) regarding the quality of coronary artery opacification using 230 multidetector $\mathrm{CT}$. Methods and Materials: 18 dedicated coronary CT angiographic studies and 18 triple rule-out CT angiographic studies were included. Two radiologists, blinded to type of CTA, scored coronary artery image quality. Up to 14 coronary artery segments were scored for motion artefacts, grading from 1 (no motion artefact) to 4 (severe motion artefact). The quality of opacification was also scored. The average of all segments and dedicated larger and smaller coronary artery segments was compared.

Results: The average motion-artefact scores per vessel segment for dedicated and triple rule-out studies were 1.54 and 1.61 , respectively $(P=.5)$. For larger segments, the average motion-artefact score was 1.32 for dedicated CTA compared to 1.46 for triple rule-out CTA $(P=.2)$. The average coronary artery opacification for dedicated studies was 1.04 for all segments and 1.02 for larger segments, compared to triple rule-out studies, which had scores of $1.07(P=.4)$ for all segments and $1.05(P=.4)$ for larger segments. No statistically significant differences in favour of dedicated CTA were identified.

Conclusion: The quality of images obtained by triple rule-out CTA is comparable to that of dedicated coronary CTA, showing no statistically significant difference in motion artefacts or opacification. So it may be an alternative and useful diagnostic study in a selected group of patients with acute chest pain.

\section{B-0124 10:57}

Can the presence of active or recent bleeding in CT angio of acute lower intestinal bleeding be predicted?

M. Marti de Gracia ${ }^{1}$, J.M. Artigas Martín', J.A. Soto ${ }^{3}$, A. Borobia', A. Vicente', G. Garzón'; ' ${ }^{1}$ Madrid/ES, ${ }^{2}$ Zaragoza/ES, ${ }^{3}$ Boston, MA/US (mmarti.hulp@salud.madrid.org)

Purpose: To identify factors that predict the presence of recent or active bleeding (ROAB) at CTA performed for acute lower intestinal bleeding (ALIB).

Methods and Materials: Prospective study (24 months) that included patients with ALIB and CTA as the first test. A three-phase MTDC protocol was used. Two radiologists determined if signs of ROAB were present. Variables studied were: transfusion 
requirements, the use of anticoagulant/anti-platelet therapy or coagulopathy, cause and location of the bleed. Data were analysed, and ROC curves were derived. Results: 130 patients $(64 \mathrm{~W}, 66 \mathrm{M})$ with median age of 76 years were included. CTA findings were: active bleeding $(n=53)$, recent bleeding $(n=17)$, potential cause of bled $(n=45)$, negative $(n=15)$. Patients received anticoagulants $(n=38)$, anti-platelet therapy $(n=23)$, coagulopathy $(n=14)$, normal coagulation $(n=55)$, transfusion $(n=76)$. Cause of bleeding was: colitis $(n=33)$, neoplasms $(n=19)$, diverticula $(n=21)$, vascular lesion $(n=28)$, others $(n=14)$, systemic disease $(n=10)$, no cause $(n=5)$. Lesion Location was: small bowel $(n=16)$, right-transverse colon $(n=25)$, descending-recto-sigmoid colon $(n=59)$, multi-focal $(n=14)$, stomach $(n=1)$, no clear location $(n=15)$. There was a statistically significant (SS) association ( $p$ $<.0 .001$ ) between the need for transfusions and the likelihood of finding ROAB. The association between anticoagulant/anti-platelet therapy, coagulopathy, gender or age and positive CTA findings was not SS. Lesions in the right colon, small bowe and vascular lesions had a higher likelihood of a positive CTA finding.

Conclusion: The need for transfusion therapy is a predictor of a positive finding of ROAB in CTA of ALIB.

\section{B-0125 11:06}

Accuracy of the AAST organ injury scale as a CT imaging checklist

for traumatic liver and spleen injuries

G. Homann ${ }^{1}$, C.M. Toschke ${ }^{2}$, P. Gaßmann ${ }^{3}$, U. Hanning ${ }^{1}$, W. Heindel ${ }^{1}$, V. Vieth ${ }^{1}$;

${ }^{1}$ Münster/DE, ${ }^{2}$ Ahlen/DE, ${ }^{3}$ Mainz/DE (georgandreas.homann@ukmuenster.de)

Purpose: Detection of abdominal injury is a very important component in trauma management, so an accelerated assessment of liver and spleen injuries including their degree of severity is necessary. There is a good case to believe that in stress situations this subjective performance results in diagnostic mistakes. Structured objective scores as the Organ Injury Scale (OIS) drawn up by the AAST are a valuable guidance for objective trauma assessment. Aim of our study was to evaluate retrospectively whether a structured approach using the OIS differs to real-time-diagnosis.

Methods and Materials: The OIS was applied by an experienced radiologist on CT data of 53 patients which underwent CT laparotomy after abdominal trauma. These results were correlated with the original text of the initial CT findings. Finally, we compared both with the intraoperative findings gathered from the surgery report. Results: Regarding the original CT report we found a mean divergence of $0.68 \pm$ 0.8 to the OIS found in the surgery report for liver injuries $(0.69 \pm 1.17$ for spleen injuries). In comparison with the structured approach, where we detected a divergence of $0.8 \pm 0.68(0.47 \pm 0.77$ for spleen injuries $)$. There was no significant difference, but we detected a lower rate of over-diagnoses in both approaches.

Conclusion: A structured approach to abdominal trauma using an imaging checklist does not lead to a significantly higher detection rate, but reduces the rate of overdiagnoses and may be more precise in grading the severity grade plus streamlines the emergeny-CT-procedure.

\section{B-0126 11:15}

\section{Comparison of efficacy and safety between distal embolisation} and augmented embolisation techniques for treating blunt splenic injuries with active haemorrhage

Y.-C. Wong, L.-J. Wang, C.-H. Wu, H.-W. Chen; Taoyuan/TW

Purpose: To compare the efficacy and complication between distal embolisation and augmented embolisation (distal and proximal embolisations) techniques for treating blunt splenic injuries (BSI).

Methods and Materials: Within a two-year period, 26 consecutive patients of BS in whom haemodynamics was stable but CT showed contrast extravasation were included in this prospectively study. We randomised them into distal embolisation group and augmented embolisation group. They were followed up twice by CT at 1 week and 3 months after embolisation. We compared their demographics, injury grades, clinical parameters, hemograms, successful rates and splenic infarct volumes between the two groups. Embolisation failure was defined as persistent hypotension that required surgery or detection of splenic contrast extravasation on follow-up CT. Complication was defined as post-embolisation splenic infarct or splenic abscess.

Results: All underwent first follow-up CT, 24 underwent second follow-up CT. Of 26 patients, 17 received distal embolisation, 9 received augmented embolisation. Their basic demographics, CT injury grades, clinical parameters and hemograms did not differ. Their mean systolic blood pressure was significantly elevated after embolisation. Two $(2 / 17,12 \%)$ of the distal embolisation group failed and required a second augmented embolisation. None of the augmented group failed but the difference did not reach statistical significance. The mean splenic infarct volume of distal embolisation group and augmented embolisation group was $10.9 \%$ and $6.6 \%$ respectively $(p=0.481)$. None developed splenic abscess.

Conclusion: Augmented embolisation is effective and safe for treating BSI. A larger series of study are needed to prove that it is more successful than distal embolisation.

Author Disclosures:

Y. Wong: Research/Grant Support; Chang Gung Medical Research Project.

L. Wang: Research/Grant Support; Chang Gung Medical Research Project.

C. Wu: Research/Grant Support; Chang Gung Medical Research Project.

H. Chen: Research/Grant Support; Chang Gung Medical Research Project.

\section{B-0127 11:24}

Diagnostic performance of MDCT in the evaluation of bowel

obstruction: can the radiologist's experience make a difference?

A. Ferri, R. Basilico, N. Civitareale, A. Lella, V. Calamita, A. Cotroneo; Chieti/IT

Purpose: To evaluate the diagnostic performance of MDCT in the detection of site and cause of bowel obstruction and in the diagnosis of bowel wall ischaemia or infarction, by assessing the added value of the radiologist's experience.

Methods and Materials: We retrospectively analysed the MDCT reports of 127 patients admitted to our hospital for the clinical suspect of bowel obstruction and we included 98 patients with MDCT, surgical and histological diagnosis of bowel obstruction. We calculated the diagnostic accuracy of the MDCT reports in the evaluation of the cause and the site of obstruction and the sensitivity, specificity and diagnostic accuracy values in the detection of bowel wall ischaemia or infarction. An experienced abdominal radiologist reviewed the MDCT examinations and compared her results with the original MDCT reports.

Results: Bowel obstruction causes were adhesions (36.3\%), neoplasms (22.7\%), hernias $(13.6 \%)$, volvulus $(12.5 \%)$ and other causes $(14.7 \%)$. The diagnostic accuracy in determining the cause of bowel obstruction was $88.7 \%$ for the MDCT reports and $91 \%$ for the experienced reader; the diagnostic accuracy for the site of obstruction was high for both MDCT reports (91.2\%) and the experienced reader $(93.5 \%)$ with values of $71 \%$ and $80 \%$. The sensitivity, specificity and diagnostic accuracy of MDCT reports in identifying bowel wall ischaemia or infarction were, respectively, $73,96 \% 90 \%$ : the sensitivity value significantly increased to $82 \%$ for the abdominal radiologist.

Conclusion: MDCT is very accurate in diagnosing the cause and the site of bowel obstruction. The radiologist's experience in abdominal disease represents an added value in the diagnosis of bowel wall ischaemia or infarction.

\section{B-0128 11:33}

Diagnosis of bowel obstruction performed by MSCT in emergency department compared with the surgical findings

E. Capalbo, F. Sajadidhekrodi, M. Cosentino, M. Peli, M. Lovisatti, A. Kluzer, S. Fornari, M. Cariati; Milan/IT (emanuelacapalbo@tiscalinet.it)

Purpose: To evaluate the diagnostic performance of MSCT performed in emergency department, and to characterise side and type of bowel obstruction compared with surgical findings.

Methods and Materials: In first semester of 2012 we performed 686 abdominal CT in patients came to our emergency department with clinical suspect of acute abdomen. The exams were performed with contrast medium, using a GE 16 slices. We have selected 91 positive exams for bowel obstruction performed in 57 men and 34 women, mean age 62.4 years (range 19-87). We calculated sensitivity, specificity, positive (PPV) and negative (NPV) predictive values and diagnostic accuracy. Diagnostic concordance of nature and site has been evaluated using K Cohen's coefficient.

Results: The causes of bowel obstruction are: $27 \%$ inflammation, $24 \%$ at full speed, $14 \%$ tumours, $11 \%$ volvulus, $8 \%$, hernias $7 \%$ invaginations, $3 \%$ gallstone ileus and $1 \%$ fitobezoar. The diagnosis of nature is indeterminate in $5 \%$ of patients. The localisation has been in $41 \%$ to small intestine, $34 \%$ to sigma/rectum and $21 \%$ to colon in particular $8 \%$ to right and $13 \%$ to left. The site is not properly defined in $4 \%$ of cases. The sensitivity of MSCT is $92.5 \%$, specificity is $84.6 \%$, PPV $94.8 \%$, NPV $88.5 \%$, and diagnostic accuracy $90.5 \%$. The K Cohen's coefficient is 0.83 for nature and 0.91 for site of bowel obstruction.

Conclusion: MSCT identifies the intestinal obstruction, both cause and localisation, with a good concordance compared with surgical definitive diagnosis. It is today the gold-standard diagnostic in emergency department to define the patient's therapy planning.

Author Disclosures:

E. Capalbo: Author; Emanuela Capalbo. 


\section{B-0129 11:42}

Accuracy of ultrasonographic diagnosis of acute appendicitis in pregnant women

N. Kokhanovsky, A.-R. Zeina, N. Reindorp, A. Levit-Kantor, Y. Glick, A. Nachtigal; Hadera/IL (nataliakoh@hotmail.com)

Purpose: To assess the reliability and value of the sonographic examinations in the diagnosis of acute appendicitis in pregnant women.

Methods and Materials: We obtained sonographic examinations performed on 68 pregnant women with suspected acute appendicitis retrospectively for 16 months (Sep. 2008 - Feb. 2010). The average age of study subjects was 27.3 years, with an average gestational age of 26 weeks. Sonography was the first imaging modality employed. Surgery or clinical follow-up was the gold standard for the evaluation of sonographic performance. All examinations were performed using grey-scale graded compression in the left lateral decubitus position. The sonographic criteria for acute appendicitis were detection of a noncompressible, blind-ended, tubular, structure measuring greater than $6 \mathrm{~mm}$ in maximal diameter.

Results: 4 patients had positive sonographic findings of acute appendicitis $(5.9 \%)$. Sonographic findings were correlated with surgical findings and clinical follow-up. The diagnosis was confirmed in all 4 patients (100\%). In the 64 patients with negative sonographic findings of acute appendicitis, the result for 1 patient (1.6\%) proved false-negative, whereas the remaining $63(98.4 \%)$ improved on clinical follow-up. Accordingly, sensitivity was $80.0 \%$, specificity was $100 \%$ and overall accuracy was $98.5 \%$. Moreover, in 4 out of the 64 patients with negative sonographic findings for acute appendicitis, a different pathologic finding such as acute pyelonephritis was identified.

Conclusion: Our experience suggests that ultrasonographic examination with the optimal study protocol is a highly accurate method for the diagnosis of acute appendicitis in pregnant women and should be performed as the first imaging test.

\section{B-0130 11:51}

Quality assessment of out sourced after-hours computed tomography teleradiology reports in a central London University Hospital J. Hohmann 1 , P. de Villiers' ${ }^{1}$, C. Urigo'², D. Sarpi', C. Newerla ${ }^{3}$, J. Brookes';

${ }^{1}$ London/UK, ${ }^{2}$ Lugano/CH, ${ }^{3}$ Basle/CH (jhohmann@uhbs.ch)

Purpose: The study assessed the quality of out sourced after-hours computed tomography teleradiology service reports.

Methods and Materials: We evaluated 1028 patients over a time period of five months (437 females, 591 males, mean age: 51 years) who were referred either by the A\&E or by the other in-house departments from $7 \mathrm{pm}$ to 8 am for different reasons. Reporting was done by a teleradiology service provider located in the UK and Australia. Reports were assessed during the routinely performed morning meeting by a panel of in-house radiologists. Assessment was done by a five-point agreement scale $(5=$ "No disagreement", 1 = ".unequivocal potential for serious morbidity or threat to life"). In 811 (79\%) patients no disagreement was found, 164 $(16 \%)$ were rated as category $4,40(4 \%)$ as category 3 (".likelihood of harm is low"). In $13(1.3 \%)$ patients a decision of category 2 was made (".strong likelihood of moderate morbidity but not threat to life"). No category 1 decision was made. A follow-up of the category 2 patients was done.

Results: In $8(0.8 \%)$ patients the in-house reports were correct, in $2(0.2 \%)$ patients the teleradiology service provider was right and in $3(0.3 \%)$ patients the final diagnoses remained unclear.

Conclusion: There was a small rate $(0.8 \%)$ of proven serious misinterpretations by the teleradiology service provider, but these were less than in comparable studies with preliminary in-house staff reports (1.6-24.6\%).
$10: 30-12: 00$

Room Q

\section{Radiographers}

SS 114

Importance of education in practice

Moderators:

R. Ribeiro; Lisbon/PT

T. Roding; Haarlem/NL

\section{B-0131 10:30}

Risk management in radiology: applying failure mode effects and criticality analysis in computer tomography

M. Antoniutti, C. Maso, N. Vecchiato, S. Doratiotto; Treviso/IT

Purpose: This study intended to verify the usefulness of the application of failure mode and effects analysis criticality (FMECA) in the computed tomography (CT) process.

Methods and Materials: A multidisciplinary safety team conducted an FMECA to identify and reduce the criticality, the near misses and errors in the CT process. The six sequential steps in the FMECA process are applied: define the topic, assemble a safety team, develop a process map, conduct a risk analysis for each subprocess, develop and implement an action plan to redesign the process, monitor, sustain, share, and reevaluate the improvement. Each failure mode identified was scored according to its potentiality, the severity and the impact with the risk priority number (RPN). The RPN is given by the product of the numerical ratings of the severity, the probability of occurrence, and the probability of detection.

Results: Failure modes with higher RPN were: homonyms within the scheduler CT (RPN: 360 ), sending images in the PACS under the name of a homonymous (RPN: 360), sending images in PACS for reporting but not for displaying (RPN: 280), incomplete filling of forms (RPN:192), sending incomplete images of the PACS (RPN:160), it is not always possible to standardise protocols body CT (RPN:160) and reflux of contrast medium in saline (RPN:126). These failure modes were prioritized for corrective action in the CT process.

Conclusion: FMECA is an effective and reliable method to pro-active examine complex processes in the radiology department, and $\mathrm{CT}$ is used as an particular example for which FMECA process.

\section{B-0132 10:39}

How important is the experience in breast imaging for radiographers performing breast-MRI?

P. Clauser, V. Londero, C. Molinari, R. Girometti, S. Da Dalt, C. Zuiani,

M. Bazzocchi; Udine/IT (clauser.p@hotmail.it)

Purpose: To evaluate whether radiographers' experience in breast imaging helps in reducing significant artefacts when acquiring dynamic breast-MRI.

Methods and Materials: One experienced radiologist prospectively evaluated a total of 270 breast-MRI examinations performed by three different radiographers (R1, R2 and R3) over the period September 2011-June 2012 (90 examinations each). R1 had a 5 -year experience in breast-MRI and 15 years of experience in breast imaging; R2 and R3 had no specific training in breast imaging, and 5- (R2) and 2-year (R3) experience in breast MRI. For each examination the radiologist, who was blinded to radiographer's experience, was asked to assess the presence or absence of significant artefacts, defined as motion artefacts affecting image quality and/or diagnostic evaluation. The prevalence of significant artefacts in R1, R2 and R3 examinations was tested with the Chi-square test.

Results: Significant artefacts were found in 45/270 examinations $(16.6 \% ; \mathrm{Cl} 195 \%=12.7-21.6 \%)$. In particular, significant artefacts affected $6 / 90$ examinations $(6.6 \% ; \mathrm{Cl} 195 \%=3.1-13.8 \%)$ performed by $\mathrm{R} 1,22 / 90$ examinations (24.4\%;Cl95\%=16.7-34.2\%) performed by R2 and $17 / 90$ (18.8\%, Cl95\%=12.1$28.2 \%)$ performed by $R 3$, with a significant difference between $R 1$ and $R 2(p<0.05)$. Conclusion: Artefacts potentially affecting image quality and diagnostic evaluation are less frequent in breast-MRI examinations performed by those radiographers who combine experience in breast imaging with specific experience in breast-MRI. A possible explanation is a better understanding of disease specific problems and of the importance of empathise with the patient. 


\section{B-0133 10:48}

Radiography authorship: a European and international review

B. Snaith; Wakefield/UK (bev.snaith@midyorks.nhs.uk)

Purpose: To establish the current authorship within international radiography journals and identify publication themes, patterns of authorship and geographical spread.

Methods and Materials: A bibliometric review was performed of articles published over 8 years (2004-11) in 4 international English-language radiography journals: the Journal of Medical Imaging and Radiation Science, Radiography, South African Radiographer and The Radiographer. Each journal encompasses both diagnostic and therapy disciplines. Analysis of article type and subject was compared at a journal, author and country level.

Results: 835 articles met the inclusion criteria, with 1999 contributing authors and 43 countries represented. European authors contributed $43.8 \%(n=366 / 835)$ of all articles; however, variation was noted in author and country productivity. Of the European originating articles $50.8 \%(n=186 / 366)$ were presenting the findings of research, with the remainder comprising Journal (29.5\%); Clinical (13.9\%) or Opinion (13.9\%) articles.

Conclusion: Europe is well established in radiography publication; however variation exists and strategies to explore further expansion will be discussed.

Author Disclosures:

B. Snaith: Other; Editorial board member and reviewer.

\section{B-0134 10:57}

The influence of clinical placement setting on academic achievement within an undergraduate diagnostic radiography programme

E. Lewis, M. Hardy; Bradford/UK (e.lewis2@bradford.ac.uk)

Purpose: Practice-based learning within the clinical setting forms a substantial part of diagnostic radiography degree courses within the UK. Previous studies have attempted to evaluate the quality of the clinical learning environment by examining student perceptions without considering the impact upon academic achievement. This CoRIPS funded study attempts to objectively measure the impact of substantive clinical placement on the academic progression and achievement of undergraduate radiography students whilst controlling for demographic variables.

Methods and Materials: This study examined data from 6 consecutive cohorts of students (178 students in total) at a single UK Higher Education Institution (HEI) where students are allocated to one of 6 Hospital Trusts for the majority of their clinical placement. Data were compiled retrospectively from student academic records and random effects analytical modelling was undertaken to control for demographic variables, including age, ethnicity, gender and baseline academic qualification. Degree classification and aggregated mean mark for each year of the programme were used as a measure of academic achievement and progression. Results: Initial analysis suggests that the mean academic progression from year 1 to year 3 varies between clinical placements. Further analysis of the data is ongoing but will consider each of the variables independently and in combination to provide an insight into the different influences on academic performance.

Conclusion: Academic achievement is a universal measure of success. However, the impact of clinical placement on learning success has not been previously considered in radiography undergraduate education.

\section{B-0135 11:06}

Use of radiation in operating theatres in Finland

P. Heikkilä̈, A. Henner' ${ }^{1}$ P. Tenkanen-Rautakoski², M. Pirinen², S.-M. Ahonen';

1'Oulu/FI, ${ }^{2} \mathrm{Helsinki/FI} \mathrm{(anja.henner@oamk.fi)}$

Purpose: Nurses and medical doctors use mobile C-arms in operating theatres. Aim of this study was to find out how much radiation and which procedures are used and who is responsible for radiation safety.

Methods and Materials: The data were collected in spring 2012 by an electronic questionnaire made for this survey. 31 operating theatre units answered the enquiry. Seven sections were included in questionnaire: radiation user's organisation, use of radiation, $\mathrm{C}$-arm techniques, radiation dose monitoring of the staff, use of radiation shields, radiation protection during pregnancy (patient or the staff member), safe use of radiation.

Results: There was lack in training. Responsibilities in radiation user's organisation were divided; radiation safety officer was mostly physicist or radiologist. Physicist was responsible for quality assurance. Practical nurse or nurse was mostly in charge of the use of $\mathrm{C}$-arm. Radiation was used with orthopaedic and traumatological procedures by surgeon, nurse or practical nurse. $84 \%$ of the respondents had personal dose monitoring and the dosimeter was placed on thyroid shield, apron or left arm. Group dosimeters were used either on the shields or they were hanged on
C-arm. Staff without lead shields exit the room. Lead shields for all patients were used in $58 \%$ of cases. Pregnant staff member was transferred to other duties $(61 \%)$. Conclusion: There is a lack in radiation protection education. The right way to use of radiation dosimeter is not clearly understood in all operating theatre units. The use of radiation in operating theatres in Finland needs improvements.

\section{B-0136 11:15}

Information-seeking behaviour of radiographers

L. Sancho' ${ }^{1}$ A.F.C.L. Abrantes' ${ }^{1}$, L.P.V. Ribeiro' ${ }^{1}$ R.P.P. Almeida' ${ }^{1}$, S.I. Rodrigues ${ }^{1}$, C.A. Silva ${ }^{2} ;{ }^{1}$ Faro/PT, ${ }^{2}$ Evora/PT (luis.sancho@sapo.pt)

Purpose: Understand how the radiographers act when faced with information needs resulting from the professional practice. Identify the preferences and informational habits of these individuals, as well as ascertain their capabilities in the use of electronic information resources.

Methods and Materials: A 33-question self-applied questionnaire was used as the instrument to explore radiographers' information needs and behaviour. A total of 142 valid questionnaires were collected in health institutions across the country. The data was than interpreted and statistically analysed using the Statistical Package for the Social Sciences V.20 software.

Results: $86 \%$ of radiographers admit to having experienced, throughout practice on medical environment, informational needs that led them to consult one or more sources. The search for information is mostly done through the use of nonspecialized sources (e.g. Google) (57\%). Although $73 \%$ of respondents claim to conduct research activities in electronic databases, $22 \%$ mentioned the need to learn more about research strategies. The use of specialized health libraries is indicated by a minority of radiographers $(10 \%)$. However, books are still present in $90 \%$ of these professionals' private collections.

Conclusion: The majority of radiographers use informational sources to suppress doubts related to the practice in radiology. Nevertheless, their knowledge on this matter is still relatively elementary, and a better learning of the searching resources is needed so radiographers can justify their professional actions with solid and valid scientific basis (evidence-based radiology).

\section{B-0137 11:24}

Dose reduction in computer tomography, the effects of technologist training on the number of extra anatomic images

T.W.F. Pappot, M.E.J. Pijl; Arnhem/NL (Tpappot@rijnstate.nl)

Purpose: To determine the effects of technologist training on the number of images beyond the intended anatomic area of interest.

Methods and Materials: In two 4-week periods, one before and one after a 10-minute technologist training, all body CTs were evaluated. Techs were made aware of dose issues arising from images beyond boundaries as prescribed in protocols. Dose length product (DLP), the total number of reconstructed images and the number of images cranial and caudal of the prescribed protocol were noted. Scans, short of prescribed anatomic coverage, were also counted. Pathological findings in extra anatomic images were noted. Dose per image was calculated: DLP / total number of images. Effective DLP (within anatomic boundaries) and extra DLP (beyond anatomic boundaries) were calculated.

Results: In total 571 examinations were evaluated, 287 prior to the tech training and 284 after. There were no significant statistical differences between both periods in patient characteristics or scan type. Before and after training the median number of effective images was equal $(133 ; p=.51)$ and the median effective DLP was similar ( 232 vs $237 \mathrm{mGy} . \mathrm{cm} ; \mathrm{p}=.59$ ). The median number of extra images per scan decreased $53 \%$ from 15 prior to training to 6 after $(p<.001)$. Accordingly, the extra DLP was reduced $50 \%(p<.001)$. The number of too short scans increased from $14(4.9 \%)$ to $25(8.7 \%)(p=.06)$. No additional pathological findings were detected in extra anatomic images.

Conclusion: Brief technologist training results in a significant $(p<.001)$ decrease in the number of extra anatomic images in body CT.

\section{B-0138 11:33}

Evidence-based practice in radiology

M. Dias' ${ }^{1}$, A.F.C.L. Abrantes', C.A. Silva², R.P.P. Almeida', L.P.V. Ribeiro',

J. Pinheiro', K.B. Azevedo'; ; ${ }^{1}$ Faro/PT, ${ }^{2}$ Evora/PT (rui_ppa@hotmail.com)

Purpose: This study aimed to evaluate the radiographers' knowledge on evidencebased radiology, how they conduct their research on this thematic and how they apply it.

Methods and Materials: A self-applied questionnaire made by Ahonen \& Liikanen (2010) to assess evidence-based radiology was applied to radiographers working in public hospitals and healthcare centres. Data analysis was made by descrip- 
tive statistics, T-student tests for comparing groups, and Mann-Whitney tests for verifying hypothesis and item relations.

Results: Most radiographers completely agree (37.5\%) or partially agree (40\%) that evidence-based radiology makes part of their job requirements, but only $32.5 \%$ consider it absolutely useful for developing their skills. Most research projects and papers $(72.5 \%)$ are done by radiographers during their degree to develop their knowledge and quality of services rendered. Age and academic degree do not have statistical significance $(p<0.05)$ for evidence-based radiology; however, there is a slight increase for radiographers with higher academic degrees and for those who have greater access to research papers and literature.

Conclusion: Evidence-based practice is not applied by most radiographers as a work method; however, the underlying knowledge on this thematic suggests it would be well received and developed to enhance skill, knowledge and the department general quality.

\section{B-0139 11:42}

Radiographers quality assurance work: resistance and cooperation

R. Gullien, J.G. Andersen, A.E. Haakull; Oslo/NO (uxraul@ous-hf.no)

Purpose: Explaining the radiographer-chosen improvement initiatives in quality assurance work (QAW), adjustment and progression of these initiatives and changing possible resistance to cooperation.

Methods and Materials: Different implementation strategies and improvemen initiatives were used in the QAW to prevent a feeling of insecurity, threatening, uneasiness, less valuable, to avoid conflicts and to enable education, training, self-assessment, improve oneself. Manager implements measurements, strategies and improvement initiatives. The employees came up with suggestions of actions, brainstorm. Voting was performed and top three were selected. Combination of suggested and specific initiatives over time: continuous individual feedback over time (baseline, after implementation, follow-up), individual assessment measured by experience in mammography, theoretical and practical training, lectures, examples, individual positioning training, reading guidelines and articles, individual feedback on positioning technique, tailored training for new employees, different checklist for employees (new/"old", inexperienced/experienced), checklist for updating knowledge, self-study, workshops, training in self-evaluation, new internal guidelines, frequent feedback if necessary, practical training with supervisor, temporarily get another kind of work, meetings and discussions, inform about QAW when job interview. Results: Positive changes in cognition, self-knowledge, personal skills, confidence in the situation, comfortable, demystified, attitude change; related to knowledge and awareness. Teamwork manager and employee with focus on improvement work and differentiate between work and employee.

Conclusion: Using various strategies are beneficial, different methods to meet different needs. Top-down, bottom-up, threat-relaxation, tentative-participation, resistance-cooperation. Dialogue and cooperation between staff and management; between the radiographers are essential for success.

\section{B-0140 11:51}

Radiographers' knowledge about adverse reactions to iodinated contrast media

A. Santos, L. Capitolina, I. Silva, A. Saraiva; Coimbra/PT

(adelinosantos@estescoimbra.pt)

Purpose: EudraVigilance-Pharmacovigilance is a data processing network and management system for reporting and evaluating suspected adverse reactions of medicinal products in the European Economic Area. The main purpose of this paper is to assess the level of knowledge of radiographers relatively adverse reactions of iodinated contrast media (ICM).

Methods and Materials: An original electronic survey was used, which is composed of four dimensions of study to gauge radiographers' knowledge regarding the chemistry of ICM (I), potential adverse reactions (II), possible procedures to be taken in emergency situations (III) and actions to be taken under the National Pharmacovigilance System (IV), in a first phase. And throughout various European countries (Northern, Central and Mediterranean), in a second stage.

Results: Only $11.5 \%$ of respondents were able to correctly answer the four questions on chemistry of ICM. However, a significant relationship between those who know the classification of ADR and who has knowledge of chemistry ICM still exists. Concerning the procedures to be taken in case of ADR, we found that only $13.3 \%$ of subjects were able to correctly identify usual emergency procedures in this type of situation and, regrettably, only $3 \%$ of the sample proved able to correctly identify all adverse reactions suggested by the questionnaire.

Conclusion: The only method that enables epidemiological continuing surveillance safety of all drugs is the spontaneous reporting of adverse events. This pioneer study can be a starting point for future investigations into this matter to understand the reason for this shouting lack of knowledge in relation to ADR.

$10: 30-12: 00$

Room Z

\section{Computer Applications}

\section{SS 105}

IT infrastructure, learning support and teleradiology

Moderators:

B. Gibaud; Rennes/FR

A. Simisker; Tartu/EE

\section{B-0141 10:30}

\section{Development of the ViSion ontology}

D.J. Vining ${ }^{1}$, U. Salem 1 , C. Popovici' ${ }^{2}$, L. Jiang ${ }^{3}$, C. Duran ${ }^{1}$, A. Pitici², I. Aghenitei², M. Jurca ${ }^{2}$, R. Rosu ${ }^{2} ;{ }^{1}$ Houston, TX/US, ${ }^{2}$ Chapel Hill, NC/US, ${ }^{3}$ Beijing/CN (dvining@mdanderson.org)

Purpose: Radiology reporting is often practiced in English but the translation of reports to native languages could be beneficial to patients. Furthermore, diagnoses must be coded with standardised ontologies (i.e., controlled vocabularies with defined relationships) to enable electronic medical records. We present a structured reporting solution with an integrated ontology tool that is capable of automatically translating and coding reports.

Methods and Materials: We developed a reporting system, called ViSion, which allows a radiologist to capture key images, tag images with anatomy and pathology terms and assemble a multimedia structured report. The terminology employed has been developed with an integrated ontology tool. The anatomy terms are organised in a hierarchy, and each anatomy is associated with a set of pathologies. The pairing of anatomy and pathology terms comprises a diagnosis. Each diagnosis can be further described with secondary characteristics. The ViSion ontology was created in English but translated to foreign languages using Google Translate followed by a radiologist's review. Hence, the ability to translate a ViSion structured report to multiple languages becomes a trivial task. Furthermore, all of the diagnoses have been crossed-referenced to other standardised medical ontologies (e.g., ICD-10-CM).

Results: The ViSion ontology currently consists of 906 anatomy terms, 1472 pathology terms, 1380 secondary characteristic terms and 5653 anatomy-pathology term combinations constituting unique diagnoses. Each term has been translated to foreign languages and cross-referenced to other ontologies.

Conclusion: ViSion is a multimedia structured reporting system that operates in multiple languages and provides a method to automatically code radiological diagnoses.

Author Disclosures:

D.J. Vining: Founder; VisionSR, Inc. C. Popovici: Employee; Eloquentix, Inc. A. Pitici: Employee; Eloquentix, Inc. I. Aghenitei: Employee; Eloquentix, Inc. M. Jurca: Employee; Eloquentix, Inc. R. Rosu: CEO; Eloquentix, Inc.

\section{B-0142 10:39}

IT infrastructure to support secondary use of routinely acquired clinical imaging data for research

K. Leung, F. Van der Lijn, H.A. Vrooman, W.J. Niessen, M.C.J.M. Sturkenboom; Rotterdam/NL (k.y.e.leung@erasmusmc.nl)

Purpose: To exploit the ever increasing quantity of routinely acquired imaging data generated in clinical practice for research, we propose an infrastructure for the automated anonymization, extraction and processing of image data stored in clinical data repositories.

Methods and Materials: As proof of principle, we used this infrastructure to implement a pipeline for measuring brain volumes in clinical MRI. This fully automated system consists of four steps: subject selection using PACS query, anonymization of privacy sensitive information and removal of facial features, quality assurance on DICOM header and image information, and biomarker extraction. 1114 examinations were selected based on MRI protocol: dementia (246), multiple sclerosis (446) and a general healthy elderly population study (422). We evaluated the effectiveness of the infrastructure in accessing and successfully extracting biomarkers from routinely acquired clinical imaging data. To prove the validity, we compared brain volumes from dementia and multiple sclerosis patients with the healthy population. Results: Success rates of $82.5 \%, 82.3 \%$ and $100 \%$ are achieved for the three protocol groups, respectively, indicating large amounts of routinely acquired clinical imaging data can be used for research purposes. In line with the literature, 
brain volumes (expressed as percentages of intracranial volume) were found to be significantly smaller in patients scanned under dementia $(79.7 \%)$ and multiple sclerosis $(81.4 \%)$ protocols, when compared with healthy controls $(82.8 \%)$.

Conclusion: This demonstrates that quantitative image biomarkers can be reliably extracted from routinely acquired clinical imaging data. This enables secondary use of clinical images for quantitative biomarker discovery at a hitherto unprecedented scale.

\section{B-0143 10:48}

Interactive methods improve radiology long-term learning

J.C. Pueyo, J. Etxano, P. Slon, R. Zalazar, J. Ros, L. García del Barrio,

G. Bastarrika, A. Villanueva; Pamplona/ES (jetxano@unav.es)

Purpose: Personal remote response systems (clickers) have shown to be an interesting tool to encourage participation. The aim of this study is to evaluate whether the use of clickers improves learning and retention of radiology within a group of medical students.

Methods and Materials: 175 medical students attended 17 radiology lessons. Each lesson was about 145 radiological images. At the beginning of each lesson, students have a PDF with all the images corresponding to that day. At the end of each lesson, there were five interactive questions of those images using clickers. Three months later, there was an examination with multiple-choice questions. Half of the questions were images that had already been interactively worked with clickers. The other half were images of the PDFs not worked with clickers. Incorrect answer value was -0.25 . To evaluate the difference in the degree of learning achieved between both methods, we analysed the index of correct and false answers of each group.

Results: The averages of the correct answers were significantly higher in the group of interactive teaching than in the passive education group (63 vs $53 \%, p$ $<0.005$ ). The percentage of incorrect answers of the interactive teaching group was significantly lower than in the other $(23.4 v$ v. $27.4 p<0.05)$. The percentage of unanswered questions was significantly lower in the interactive group (13\% vs $19.5 \% \mathrm{p}<0.005)$.

Conclusion: Interactive learning with remote response devices (clickers) is an effective method in teaching radiology, because it significantly improves learning and retention of knowledge.

\section{B-0144 10:57}

The radiology events register (RaER): incident reporting in radiology

C. Mandel ${ }^{1}$, T. Schultz ${ }^{2}$, N. Hannaford ${ }^{2}$, J. Grimm ${ }^{3} ;{ }^{1}$ Melbourne/AU,

${ }^{2}$ Adelaide/AU, ${ }^{3}$ Sydney/AU (mandel@ausdoctors.net)

Purpose: Incident reporting is a vital tool in high-risk industries. Adoption in healthcare is patchy and lags behind other industries. This paper provides an overview of the establishment of a radiology incident reporting system in Australia and New Zealand and the issues associated with encouraging healthcare professionals to report incidents.

Methods and Materials: In 2006 the Royal Australian and New Zealand College of Radiologists established an incident-reporting database, the radiology events register (RaER), the first dedicated radiology incident reporting system. The source of incident reports and incident trends over time have been analysed.

Results: Over 4000 incidents have been recorded, including data transferred from other organisations' incident databases. A variety of strategies have been implemented to increase reporting and include: convening a biennial conference; promotion in lectures; publication of case reports; the use of incentives; and the mandating of reporting as part of training. Given the large numbers of radiology tests and procedures performed each year, and the estimated rate of adverse events in healthcare services, the majority of incidents are not being reported in RaER. The low numbers of reports per month are consistent with significant barriers to reporting that need to be overcome.

Conclusion: Incident reporting is an essential safety tool in healthcare. Radiology can benefit from dedicated incident databases, but convincing radiologists and other healthcare providers to report incidents is a significant hurdle. Leadership, feedback, promotion, incorporation of findings into daily practice, incentives and education are essential to encourage reporting.
B-0145 11:06

The liver imaging atlas: an interactive multimodality web-based reference and educational resource of liver imaging

O. Kolokythas, S. Zaidi, S. Osman, A.P. Tornow, T.T. Pham,

S. Bastawrous, G.S. Phillips, P. Bhargava, D.L. Coy; Seattle, WA/US (orpheus@u.washington.edu)

Purpose: To create an interactive reference of liver imaging that includes a comprehensive spectrum of liver pathology in CT, MR and ultrasound imaging modalities. We have developed and demonstrate an interactive imaging tool that is freely available via the world wide web with the aim to address these diagnostic and educational challenges.

Methods and Materials: A web-based editing and entering tool was developed to allow systematic uploading and annotation of CT, MR and ultrasound cases with hepatic pathology. A user interface was developed to allow for various search modes and presentation schemes of multimodality imaging pathology.

Results: The interactive website www.liveratlas.org allows the authors to upload and categorise liver imaging cases by their modality-specific imaging features and diagnostic categories by means of an innovative tagging system. This system enables users to search, filter, and display cases by selecting imaging features, disease categories, diagnosis, or by entering search terms as free text. A quiz mode feature allows radiology educators and trainees to use the atlas as a teaching or self-assessment tool. Cases can be shared via email or saved as a bookmark, allowing users and educators to prepare presentations or training sessions.

Conclusion: The website www.liveratlas.org is an interactive comprehensive reference tool to rapidly and effectively access comprehensive CT, MR and ultrasound imaging of liver pathology. It allows radiologists in training and in practice to find examples of common and rare pathologies, explore and focus their differential diagnoses in specific clinical scenarios, test themselves and select cases to save and share.

Author Disclosures:

O. Kolokythas: Advisory Board; Philips Healthcare. Consultant; Philips Healthcare. Equipment Support Recipient; Philips Healthcare, Supersonic Imagine. Grant Recipient; Bracco Diagnostics.

\section{B-0146 11:15}

Evaluation of teleradiology and telemedicine regarding financial and personnel benefit for regions with low population density

M.C. Spoerl, N. Hosten; Greifswald/DE (mark_spoerl@hotmail.com)

Purpose: The benefit of telemedicine and especially teleradiology are numerous. We conducted an analysis concerning financial benefits and staffing policy. Methods and Materials: Regional telemedicine projects from a pool of 52 telemedicine systems at 14 facilities were analysed, focused on interdisciplinary tumourboards in Greifswald and Eberswalde, where specialists such as radiologists, radiotherapists and pathologists are required to attend. Statistic data including cases discussed, external cases discussed, speaking time of external participants, demonstration of imaging and total duration was collected. The study also evaluates acquisition and maintenance costs compared with reduction of travel and personnel expenses.

Results: Acquisition costs for a telemedicine setup vary between 7,800 Euro for a desktop setup and between 25,000 and 40,000 Euro for a conference room setup (Cisco Systems, San Francisco). In addition maintenance costs of about 9\% of the acquisition costs occur. Representing telemedical solutions for low-density areas, the Eberswalde tumourboard processes an average of 35 cases per week with up to 10 external cases. A reduction in travel distance of 24,300 kilometres and a reduction of travel time of 362 hours annually can be achieved. Converted into travel expenses and saved medical labour time it results in 25,000 Euros annually. Conclusion: Telemedicine and especially teleradiology are sufficient tools to guarantee the best medical care to areas with low population density and a deficit of medical experts like Mecklenburg-West Pomerania and the Barnim Plateau. They provide a economic method to reduce costs and optimise staffing difficulties. This Project was financed by INTERREG IV A.

\section{B-0147 11:24}

Fast search for radiology cases in hospital systems via contentbased image retrieval

G. Langs ${ }^{1}$, R. Donner ${ }^{1}$, M. Holzer', D. Markonis², H. Mueller², E. Birngruber'; ${ }^{1}$ Vienna/AT, ${ }^{2}$ Sierre/CH (georg.langs@meduniwien.ac.at)

Purpose: Efficient and quick access to comparable cases and corresponding radiology reports is crucial during radiological assessment and increases quality. Currently, in most cases search queries within a PACS are limited to meta data. 
This limits the effectiveness of searches, since they do not use visual information in the images, such as patterns that correspond to specific diseases.

Methods and Materials: We present a computational framework for content-based image and report retrieval of radiological data. The system searches for cases that exhibit similar pathology patterns and displays them together with the radiology reports. It fully automatically identifies the anatomical location of an image and retrieves cases with similar pathology. We performed evaluation with approximately 61.000 image series sampled from a hospital PACS (3 TB of imaging data).

Results: The system identified the position of a query case with a median error of $2.4 \mathrm{~cm}$ within a search time below a second. On a pilot set, lung pathology retrieval achieved $67 \%$ correct diseases in the top ranked 3 cases.

Conclusion: A fast and efficient way to retrieve relevant data from hospital image data bases improves reporting quality and speed in radiology. Retrieval plays an increasingly important role in the clinical radiology environment. The computational system performs retrieval based on image content and is able to provide results that match in both anatomy and pathology. It supports radiologists to find relevant cases based on regions of interest in a matter of seconds, even if no specific keywords are given.

\section{B-0148 11:33}

Internet-based surveys among referring in-hospital clinicians and radiologists: a useful tool to evaluate report quality and interdisciplinary co-operation

C. Neumann, O. Khalifa, D. Tscholakoff, G. Mostbeck; Vienna/AT

(christian.neumann.nch@wienkav.at)

Purpose: To evaluate expectations of and satisfaction with radiology reports of clinicians and radiologists according to COVER/ROVER (JML Bosmans et al, Radiology 2011) and quality of interdisciplinary co-operation (IC) with departments of radiology in 2 major tertiary community hospitals.

Methods and Materials: Adopted COVER/ROVER and IC surveys (surveymonkey. com, Portland, OR/US) were e-mailed to 533 referring physicians and 26 radiologists of hospital A ( 1081 beds) and to 300 physicians and 12 radiologists of hospital B (1120 beds). Regarding the IC questionnaire, 37 questions were formulated covering aspects of scheduling, interdisciplinary communication, access to reports and images, security aspects, radiation protection and contrast media knowledge. Results: COVER response rates were $17.3 \%$ and $20.3 \%$ in hospitals A and B, respectively. ROVER response rates were $53.8 \% / 91.7 \%$. IC-survey response rate by clinicians was $13.5 \% / 16.0 \%$. Results of COVER were similar in hospitals $A$ and $\mathrm{B}$ and compared well with Bosmans et al regarding structure of reports, urge for immediate reporting of acute findings, need for a conclusion and recommendations for further management. ROVER surveys stress the importance of learning how to write reports. The IC results were different between hospitals $A$ and $B$ regarding scheduling and interdisciplinary communication. There is a need for clinician training in IT applications for access to images and reports.

Conclusion: Our ROVER/COVER results emphasize the key role of in-hospital radiology as a gatekeeper in the diagnostic work-up and interdisciplinary communication. The IC survey helps to identify friction points between radiology, referring physicians and IT staff.

\section{B-0149 11:42}

\section{Cross regional diagnostic radiology network for preoperative} assessment of colorectal cancer: preliminary experiences

L.C.O. Blomqvist ${ }^{1}$, C. Kling-Hassler', A. Sjövall', T. Almqvist ${ }^{1}$, P. Asp ${ }^{1}$,

H. Walfridsson ${ }^{2}$, R. Sundqvist ${ }^{1}$, U. Hertin ${ }^{1} ;{ }^{1}$ Stockholm/SE, ${ }^{2}$ Sollentuna/SE (lennart.k.blomqvist@ki.se)

Purpose: To assess the effect on quality of radiological reports when performed as part of a regional expert colorectal cancer diagnostic network.

Methods and Materials: Radiologists from ten different institutions in the Stockholm-Gotland region, with two million inhabitants, are recruited. After a work-shop and separate teaching sessions, the radiologists form a virtual network responsible for reporting preoperative cross-sectional imaging in patients with newly diagnosed colorectal cancer, including access to double-reading and on-line expert consultation. The network uses a common web-based RIS/PACS system with streaming technology enabling real-time communication with direct feedback. The project is divided into two phases where radiologists in the baseline phase are reporting to the individual institutions and a network phase where the reports are performed scheduled collectively in the network. In the network phase, the radiologist also have the opportunity to discuss general issues weekly together.

Results: After integration of the individual RIS/PACS system the radiologist are, at the time of writing, collecting data for the baseline phase.
Conclusion: Diagnostic radiology expert networks is attractive to improve communication, in particular during double-reading. It can also improve the educational process, fast-track reporting, expert consultation as well as providing a more patient -oriented regional equality, potentially resulting in better treatment of diseases that requires multidisciplinary and multi-institutional communication.

Author Disclosures:

P. Asp: Employee; RxEye AB. H. Walfridsson: Owner; ICOX. U. Hertin:

Employee; RxEye AB. Owner; RxEye AB.

\section{4:00 - 15:30} Room C

\section{Head and Neck}

\section{SS 208}

\section{Head and neck cancer: functional imaging and hybrid modalities}

Moderators:

N. Abolmaali; Dresden/DE

S. Steens; Nijmegen/NL

\section{B-0150 14:00}

CT perfusion of head and neck lymph nodes: differentiation between untreated and treated lymphoma

A.M. Tawfik' ${ }^{1}$, N.M. Batouty', A.A. Razek ${ }^{1}$, S. Eteiba', M.A. Shaheed', T.J. Vogl ${ }^{2}$. ${ }^{1}$ Mansoura/EG, ${ }^{2}$ Frankfurt a. Main/DE (ahm_m_tawfik@hotmail.com)

Purpose: To evaluate whether CT perfusion (CTp) of cervical nodal lymphoma could be used to differentiate between viable lymphoma tissue in primary untreated lymphoma and non-viable scar tissue after completion of therapy.

Methods and Materials: 64-multislice CTp of the head and neck was performed for ten patients with cervical nodal lymphoma before receiving any treatment and nine patients after finishing chemotherapy treatment for lymphoma, who were in complete remission and met the inclusion criterion of CT and clinical evidence of previously enlarged cervical lymph nodes. All patients had pathologic proof of lymphoma. Quantitative values of blood flow (BF), blood volume (BV), mean transit time (MTT) and permeability surface area product (PS) for cervical lymphoma nodes ( 1 to 5 enlarged nodes for each patient) were calculated using deconvolution-based CTp software. Values were statistically compared between untreated (23 nodes) and treated lymphoma nodes (17 nodes).

Results: The mean BF, BV and PS were significantly higher and MTT significantly lower for untreated than treated lymphoma nodes, all $p$ values $<0.0001$. The mean BF values were 128.5 and $40 \mathrm{ml} / 100 \mathrm{~g} / \mathrm{min}$, mean BV 7.8 and $1.6 \mathrm{ml} / 100 \mathrm{~g}$, mean PS 22.6 and $1.6 \mathrm{ml} / 100 \mathrm{~g} / \mathrm{min}$ and the mean MTT vales were 2.6 and 4.1 seconds for untreated and treated lymphoma nodes, respectively.

Conclusion: Untreated lymphoma nodes are characterised by significantly higher BF, BV and PS and lower MTT than treated lymphoma nodes. CTp may be used for assessment of the activity of lymphoma and identification of residual activity after treatment.

\section{B-0151 14:09}

\section{Prediction of treatment response in head and neck carcinomas} using IVIM-DWI: evaluation of lymph node metastasis

T. Hauser ${ }^{1}$, M. Essig', F.B. Laun', M. Münter ${ }^{3}$, A. Jensen', K.H. Fritsche', B. Stieltjes ${ }^{1} ;{ }^{1}$ Heidelberg/DE, ${ }^{2}$ Erlangen/DE, ${ }^{3}$ Stuttgart/DE

Purpose: To obtain diffusion and microperfusion measures in lymph node metastases of head and neck squamous cell carcinomas (HNSCC) using intravoxel incoherent motion (IVIM) imaging. These parameters were used to characterise lymph nodes in the staging phase and longitudinal follow-up was performed to evaluate the potential predictive value of these parameters considering therapy response. Methods and Materials: Thirteen patients with lymph node metastases of histological confirmed locally advanced HNSCC were examined using diffusion weighted imaging (DWI) before a combined radiotherapy. DWI imaging was performed at 3 T using eight different b-values ranging from 0 to $800 \mathrm{~s} / \mathrm{mm} 2$. A follow-up period of at least 10.5 months (range: $10.5-25.5$ months) was available in all patients. One patient with a macroscopically necrotic lymph node was excluded from analyses. An $\mathrm{ROI}$ analysis was performed in all patients. Using the IVIM approach, the perfusion fraction $f$ and the diffusion coefficient $D$ were extracted.

Results: A locoregional failure (LRF) was present in 3 of 12 patients within 10.5-month follow-up. The initial $f$-value was significantly higher $(\mathrm{p}<0.01)$ in patients with LRF $(14.5 \pm 0.6 \%$ vs. $7.5 \pm 2.7 \%)$. The initial diffusion coefficient $D$ did 
not differ significantly $(p=0.37)$ between the two groups $\left(0.97 \pm 0.15 \times 10^{-3} \mathrm{~mm}^{2} / \mathrm{s}\right.$ vs. $\left.0.88 \pm 0.14 \times 10^{-3} \mathrm{~mm}^{2} / \mathrm{s}\right)$.

Conclusion: Our results indicate that a high initial perfusion fraction $f$ in lymph nodes may predict poor treatment response in patients with HNSCC due to locoregional failure. The baseline diffusion coefficient $D$ showed no significant differences between groups.

\section{B-0152 14:18}

Combined perfusion-diffusion-weighted MRI for staging of recurrent papillary thyroid cancer: a feasibility study

K.N.A. De Paepe, R. Hermans, F. De Keyzer, V. Vander Poorten,

S. Nuyts, E. Hauben, B. Decallonne, M. Bex, V. Vandecaveye; Leuven/BE (katja.depaepe@uzleuven.be)

Purpose: To evaluate diffusion-weighted (DWI) and dynamic contrast-enhanced (DCE) magnetic resonance imaging (MRI) for diagnosis of recurrent papillary thyroid cancer (PTC) in comparison to ultrasound and peroperative and histopathological findings.

Methods and Materials: Twenty-eight patients with suspicion for recurrent PTC underwent 3 T MRI, including DWI ( $\left.b=0-50-100-500-750-1000 \mathrm{sec} / \mathrm{mm}^{2}\right)$ and DCE-MRI (25 runs; 6 seconds each; pre-, during and post-contrast). Interpretation criteria were: b1000-DWI for detection of soft tissue lesions and lymph nodes (LN); characterisation with apparent diffusion coefficient (ADC) calculated from all (ADCavg), low (ADClow) and high b-values (ADChigh); characterisation by DCE-MRI with arterial slope (AS), area under the curve (AUC) and c-peak. The $A D C$ and DCE-parameters of tumoural and benign lesions were compared with a Mann-Witney $U$ test. Receiver-under-the-operator-curve analysis determined a threshold from which the accuracy of functional MR-imaging was compared to ultrasound in correlation to histopathology.

Results: Only ADChigh and AS showed statistical difference between recurrent tumour and benign lesions (ADChigh: $p=0.002$; AS: $p<0.0001)$. Per-neck region, b1000-DWI combined with AS yielded highest sensitivity of $93 \%$ with $98 \%$ specificity. Ultrasound showed a sensitivity of $72 \%$ and specificity of $98 \%$. Per patient, functional MRI corrected ultrasound in only 4 patients for diagnosing tumour recurrence. However, functional MRI findings correctly changed treatment planning in 13 of 28 patients [excluding tumour recurrence $(n=2)$, mediastinal metastatic LN $(n=5)$, laryngeal metastasis $(n=2)$, ipsilateral nodal skip metastasis to level II $(n=2)$, contralateral nodal metastases $(n=2)]$.

Conclusion: Combining b1000-DWI with DCE-MRI may show additional value to ultrasound for diagnosing and planning treatment in recurrent PTC.

\section{B-0153 14:27}

Diagnostic evaluation of patients with squamous cell cancer of the head and neck after free flap reconstructions - the usefulness of functional imaging techniques

M. Członkowski, A. Trojanowska, P. Trojanowski, J. Klatka, A. Drop; Lublin/PL (m.czlonkowski@interia.pl)

Purpose: To evaluate patients operated on head and neck squamous cell cancer and reconstructed using free tissue transfer flaps

Methods and Materials: We evaluated 34 patients with T4 tumours of oral cavity, oropharynx, hypopharynx and larynx. All patients underwent surgical resection of the tumour and reconstruction with free tissue transfer flap: musculocutaneous, fasciocutaneous, osseous and osseocutaneous. Patients underwent post-operative radiotherapy. In the whole group we performed CT and/or MR study 3, 6 and 12 months after the completion of therapy. In patients suspected for recurrence based on CT/MR findings, MR diffusion-weighted imaging and CT perfusion were additionally performed. In case of positive findings these patients were qualified for surgery. Results: In 11 out of 35 patients imaging study was suggestive for recurrence (4 nodal and 7 local). In this group additional functional imaging was performed. MR DWI and CT perfusion findings were indicative of nodal recurrence in two cases and patients were operated on. Recurrence was not proven on histology in either case. In 7 patients with suspected local recurrence additional functional imaging was performed in 5 cases and the recurrence has been proven histologically in 3 cases. Conclusion: We have to be aware that functional imaging may be of little value in patients reconstructed with free flaps. Although diagnostic imaging plays a prime role in post-treatment follow-up, positive findings must be evaluated with caution. Careful reading together with ENT surgeon is vital.

\section{B-0154 14:36}

The role of MRI and FDG PET/CT imaging in the detection of unknown primary tumours with cervical metastases

A. Lévai; Budapest/HU

Purpose: Our aim was to assess the usefulness of PET/CT and MRI in the detection of unknown primary tumour in patients with malignant cervical lymphadenopathy. Methods and Materials: 421 patients were referred for FDG PET/CT with suspected or known malignancy in the head and neck region from 2006 to 2012. 43 patients had undergone correlative MRI within 2 months of FDG PET/CT. Histopathology findings were used to evaluate the performance of FDG PET and MRI. We used standardised score system to classify radiological findings in PET /CT and MRI. Results: In identification of the primary tumour site MRI succeeded in $52 \%$, and PET /CT in 53\%. MRI had 4 false-negative cases, while PET/CT had only 1 . Of the 43 patients, 3 had primary carcinoma other than the head and neck. The primary tumour stage was determined low except for 2 cases, where PET/CT understaged the disease. In 6 cases, PET/CT and MRI revealed different nodal stage. Details of nodal characteristics were detectable more with MRI.

Conclusion: PET/CT and MRI both appeared similarly accurate in the detection of an occult primary tumour in the head and neck, as well as in estimation of the nodal stage. PET/CT was beneficial in detecting distant disease and second primary tumour. MRI was more predictive in detection of primary cancers. The criterias of our standardised characterisation score system that we used in PET /CT and MRI can be a path for radiologists and nuclear medicine physicians to analyse images of cervical metastasis of an unknown primary tumour.

\section{B-0155 14:45}

Diffusion-weighted MR imaging in characterisation of head and neck lesions

F.U. Öztürk, F.Y. Dönmez, F. Kural, S. Çetiner, M. Ağıldere; Ankara/TR (drkural@hotmail.com)

Purpose: The purpose of our study is to evaluate the usefulness of diffusionweighted imaging (DWI) in the tissue characterisation of head and neck lesions, to investigate the difference in apparent diffusion coefficient (ADC) values for distinguishing malignant and benign head and neck lesions, lymphoma and carcinoma, malignant lymph nodes and benign lymph nodes, and to determine cut-off values for these distinctions.

Methods and Materials: This study included 95 head and neck lesions in 88 patients. Routine MR imaging and DWI were performed on a 1.5 T MR unit using single-shot echo-planar imaging with b factors of 0,400 and $800 \mathrm{sec} / \mathrm{mm} 2$. ADC values were calculated and the groups were compared using Kruskal-Wallis test. Results: There was a statistically significant difference between malignant and benign lesions, lymphoma and carcinoma, and malignant lymph nodes and benign lymph nodes. When an ADC value of $1.13 \times 10-3 \mathrm{~mm} 2 / \mathrm{s}$ or lower was used for predicting malignancy, the sensitivity, specificity and accuracy were $85.7 \%, 71.7 \%$ and $78.9 \%$, respectively. If an ADC value of $0.85 \times 10-3 \mathrm{~mm} 2 / \mathrm{s}$ was used as a threshold value for differentiating lymphoma from carcinoma the best results were obtained with an accuracy of $83.7 \%$, sensitivity of $92.9 \%$ and specificity of $78.3 \%$. When an $A D C$ value of $0.95 \times 10-3 \mathrm{~mm} 2 / \mathrm{s}$ was used as a threshold value for differentiating malignant lymph nodes from benign lymph nodes the highest accuracy of $82.8 \%$, with $90.5 \%$ sensitivity and $71.4 \%$ specificity was obtained.

Conclusion: DWI can reliably be used to characterise head and neck lesions based on their ADC values.

\section{B-0156 14:54}

Comparison of contrast-enhanced PET/CT and PET/MRI with only T2-weighted images in patients with head and neck cancer - how much MR information is needed in PET/MRI?

F.P. Kuhn, M. Hüllner, S.S. Kollias, G.K. Von Schulthess, P. Veit-Haibach; Zurich/CH (felix.kuhn@usz.ch)

Purpose: In general, in MRI contrast media is given for lesion detection and characterisation. In multi-modality imaging, the information about malignancy may derive from the PET-component. We evaluated if a single T2-weighted (T2w) MR pulse sequence in PET/MRI might be equivalent to contrast-enhanced (ce) PET/ CT for lesion characterisation in patients with head and neck cancer.

Methods and Materials: Eighty consecutive patients referred for primary staging or re-staging of head and neck cancer underwent sequential whole body 18 F-FDG PET with CT-based attenuation correction, ceCT and 2-point Dixon-based T2w axial MRI (IDEAL pulse sequence, GE Healthcare) of the head and neck in a tri-modality PET/CT-MR system. PET-positive lesions were assessed by CT and MRI for their anatomical localisation, conspicuity and additional information for characterisation. 
Results: In 38 patients with at least one PET-positive lesion, 60 lesions were evaluated. No significant difference was found between T2w and CT regarding lesion localisation and size. Furthermore, no significant difference was found between T2w and CT regarding conspicuity of tumours (Wilcoxon signed rank test, $p=0.16$ ) and PET-positive lymph nodes $(\mathrm{p}=0.16)$. In $\mathrm{CT}$, fewer artefacts $(7 \%)$ reduced the diagnostic confidence compared to T2w $(23.2 \%)$. Overall lesion characterisation with T2w was considered superior to CT in $32 \%$ of lesions, equal to CT in $56 \%$ and inferior to CT in $12 \%$.

Conclusion: PET/MRI with only axial T2w images and without the application of contrast media has the potential to replace cePET/CT as the primary diagnostic imaging tool for the evaluation of head and neck cancers.

Author Disclosures:

G.K. Von Schulthess: Advisory Board; GE Healthcare. Consultant; Icon Medical Imaging plc. Research/Grant Support; GE Healthcare. Speaker; Occasional remunerated speaker for GE Healthcare and others.

\section{B-0157 15:03}

\section{Comparison of contrast-enhanced PET/MRI and contrast-enhanced} PET/CT in patients with head and neck cancers

F.P. Kuhn, M. Hüllner, S.S. Kollias, G.K. Von Schulthess, P. Veit-Haibach; Zurich/CH (felix.kuhn@usz.ch)

Purpose: PET/MR has the potential to become a powerful tool in clinical oncological imaging. The purpose of this prospective study was to evaluate the diagnostic performance of contrast-enhanced (ce) MRI in patients with head and neck cancers in comparison with ceCT to characterise PET-positive lesions.

Methods and Materials: Eighty consecutive patients referred for primary staging or re-staging of head and neck cancers underwent sequential whole body 18 F-FDG PET with CT-based attenuation correction (AC), contrast-enhanced CT and conventional diagnostic MRI (2-point Dixon-based T2- and native/contrast-enhanced T1-weighted sequences) of the head and neck in a tri-modality PET/CT-MR system. PET-positive lesions were assessed by CT and MRI for their anatomical localisation, conspicuity and additional information for characterisation.

Results: In 38 patients with at least one PET-positive lesion, 60 lesions were evaluated. No significant difference was found between MRI and CT regarding lesion localisation. MRI performed significantly better than CT regarding conspicuity of tumors (Wilcoxon signed rank test, $\mathrm{p}<0.01$ ) and similarly regarding conspicuity of PET-positive lymph nodes $(p=0.08)$. In $C T$, fewer artefacts $(7 \%)$ reduced the diagnostic confidence compared to MRI (16\%). Overall lesion characterisation with MRI was considered superior to CT in $33 \%$ of lesions, equal to CT in $58 \%$ and inferior to CT in $9 \%$.

Conclusion: CeMRI outperformed ceCT in terms of conspicuity and characterisation of PET-positive head and neck tumor lesions and performed similarly in cervical lymph node assessment. Hence, cePET/MRI has the potential to replace cePET/CT as the primary diagnostic imaging tool for the evaluation of head and neck cancers.

\section{Author Disclosures:}

G.K. Von Schulthess: Advisory Board; GE Healthcare. Consultant; Icon Medical Imaging plc. Research/Grant Support; GE Healthcare. Speaker; Occasional remunerated speaker for GE Healthcare and others.

\section{B-0158 15:12}

Initial clinical results of simultaneous PET/MRI in comparison with

$\mathrm{PET} / \mathrm{CT}$ in patients with head and neck cancer

P. Stumpp, K. Kubiessa, S. Purz, M. Gawlitza,

A. Kühn, K.G. Steinhoff, A. Boehm, R. Kluge, T. Kahn;

Leipzig/DE (patrick.stumpp@medizin.uni-leipzig.de)

Purpose: Describing diagnostic capability of simultaneous PET/MRI in comparison with PET/CT and their single components CT, MRI and PET in an initial prospective study based on 17 patients with head-and-neck cancer.

Methods and Materials: 17 patients with head-and-neck cancer received an ${ }^{18} \mathrm{~F}-\mathrm{FDG}-\mathrm{PET} / \mathrm{CT}$ for staging or follow-up and an additional simultaneous PET/ MRI scan with a whole body imaging part and a dedicated examination of the neck. Sole MRI, CT and PET components of the multimodal acquisitions plus the PET/MRI and PET/CT examinations were evaluated independently, blinded and in a randomised order by two readers. Results were compared with the reference standard, and sensitivity, specificity, positive (PPV) and negative predictive value (NPV) were calculated.

Results: 23 malignant tumours and 55 benign changes were found with the reference standard. For PET/CT the two reader groups showed a sensitivity of $78.3 \%$ and $87 \%$, a specificity of $85.5 \%$ and $89.1 \%$, a PPV of $71.4 \%$ and $75 \%$ and a NPV of $90.7 \%$ and $94 \%$. For PET/MRI sensitivity was $78.3 \%$ and $82.6 \%$, specificity
$81.8 \%$ and $94.5 \%$, PPV $65.5 \%$ and $85.7 \%$ and NPV $91.2 \%$ and $93.8 \%$. Evaluation of the single PET part from PET/CT revealed highest sensitivity of $95.7 \%$, whereas evaluation of the sole MRI component from PET/MRI showed best specificity of $96.4 \%$. There was a high interrater agreement in all modalities (Cohen's kappa coefficient: $0.61-0.82)$.

Conclusion: PET/MRI of patients with head and neck cancer yielded good diagnostic capability, similar to PET/CT. Further studies on larger cohorts to prove these first results seem justified.

\section{B-0159 15:21 \\ Diagnostic accuracy of ${ }^{18} \mathrm{~F}-\mathrm{FDG}-\mathrm{PET} / \mathrm{CT}$ and fused ${ }^{18} \mathrm{~F}-\mathrm{FDG}-\mathrm{PET}-\mathrm{MR}$ images for $\mathbf{T}$ and $\mathbf{N}$ staging of primary oral malignancies \\ P. Heusch ${ }^{1}$, C. Buchbender ${ }^{1}$, C. Sproll1 ${ }^{1}$, J. Terjung ${ }^{2}$, A. Scherer ${ }^{1}$, \\ G. Antoch ${ }^{1}$, J. Handschel ${ }^{1}$, T.A. Heusner ${ }^{1} ;{ }^{1}$ Düsseldorf/DE, \\ ${ }^{2}$ Essen/DE (philipp.heusch@med.uni-duesseldorf.de)}

Purpose: To compare ${ }^{18} \mathrm{~F}-\mathrm{FDG}-\mathrm{PET}-\mathrm{MRI}$ (PET and MRI fused on a software basis) ${ }^{18} \mathrm{~F}-\mathrm{FDG}-\mathrm{PET} / \mathrm{CT}$ regarding their diagnostic performance in primary tumour $(\mathrm{T})$ and nodal $(\mathrm{N})$ staging in patients with oral malignancies.

Methods and Materials: 20 patients [ 8 females, 12 males, age $65 \pm 10$ years] before oral tumour resection and bilateral neck dissection prospectively underwent ${ }^{18} \mathrm{~F}$ FDG- PET/CT and 3 Tesla MRI. PET datasets were fused with contrast-enhanced T1-weighted MR images using a special software (Medifusion ${ }^{\mathrm{TM}}$ ). The sensitivity, specificity, positive predictive value (PPV), negative predictive value (NPV) and accuracy for the correct $T$ stage and $\mathrm{N}$ stage were calculated for ${ }^{18} \mathrm{~F}-\mathrm{FDG}-\mathrm{PET} / \mathrm{CT}$, ${ }^{18} \mathrm{~F}$-FDG-PET-MRI and ${ }^{18} \mathrm{~F}$-FDG-PET-MRI side-by-side with DWI. Histopathology served as the reference standard.

Results: The sensitivity, specificity, PPV, NPV and accuracy for determining the correct T stage was 46\%, 40\%, 63\%, 25\% and $44 \%$ for ${ }^{18} \mathrm{~F}-\mathrm{FDG}$ PET/CT, 50\%, $50 \%$, $75 \%, 25 \%$ and $50 \%$ for ${ }^{18} \mathrm{~F}-$ FDG-PET-MRI and $36 \%, 40 \%, 57 \%, 22 \%$ and $38 \%$ for ${ }^{18} \mathrm{~F}-\mathrm{FDG}-\mathrm{PET}-\mathrm{MRI}$ plus DWI, respectively. The respective values for determining the correct $\mathrm{N}$ stage were $36 \%, 43 \%, 50 \%, 30 \%$ and $39 \%$ for ${ }^{18} \mathrm{~F}-\mathrm{FDG}-\mathrm{PET} / \mathrm{CT}$, $46 \%, 43 \%, 56 \%, 33 \%$ and $44 \%$ for ${ }^{18}$ F-FDG-PET-MRI and $46 \%, 57 \%, 63 \%, 40 \%$ and $50 \%$ for ${ }^{18} \mathrm{~F}$-FDG-PET-MRI plus DWI. There were no significant differences between the results of ${ }^{18} \mathrm{~F}$-FDG-PET/CT, ${ }^{18} \mathrm{~F}$-FDG-PET-MRI and ${ }^{18} \mathrm{~F}$-FDG-PET-MRI plus DWI ( $p>$.05).

Conclusion: Software-based ${ }^{18} \mathrm{~F}$-FDG-PET-MRI and ${ }^{18} \mathrm{~F}$-FDG-PET-MRI plus DWI compared to ${ }^{18} \mathrm{~F}$-FDG-PET/CT images may not increase the diagnostic accuracy regarding the correct $\mathrm{T}$ and $\mathrm{N}$ stage in patients with oral malignancies.

\begin{tabular}{ll}
\hline 14:00 - 15:30 Room D1 &
\end{tabular}

Chest

SS 204

\section{Airways and infiltrative lung diseases}

Moderators:

G.R. Ferretti; Grenoble/FR

C. Mueller-Mang; Vienna/AT

\section{B-0160 14:00}

How does iterative image reconstruction effect emphysema

quantification with chest CT?

H. Jafarov ${ }^{1}$, C. Fink ${ }^{2}$, H. Haubenreisser ${ }^{1}$, M. Meyer ${ }^{1}$,

P. Apfaltrer ${ }^{1}$, S.O. Schönberg ${ }^{1}$, T. Henzler ${ }^{1} ;{ }^{1}$ Mannheim/DE,

${ }^{2}$ Celle/DE (hashim.jafarov@umm.de)

Purpose: Emphysema quantification with chest CT is crucial for surgical and interventional therapy but highly influenced by image noise and reconstruction kernels. Thus, we prospectively evaluated the effect of iterative reconstruction (IR) on emphysema quantification with chest CT.

Methods and Materials: Chest CT raw data of 39 patients referred for emphysema quantification were reconstructed with standard filtered back projection (FBP) and an IR technique (IRIS, Siemens) using corresponding soft, medium sharp and sharp reconstruction kernels (B30, B50, B70 and I30, 150, I70). Each data set was analysed using dedicated software for emphysema quantification (Pulmo-3D, Siemens). The following parameters were automatically analysed and compared between the different reconstructions: volume of the segmented lung (LV), mean lung density (MLD), full-width at half-maximum of the frequency distribution of lung-densities (FWHM), ratio of pixels with low attenuation (-950 HU=Emphysema index, El). Results: LV and MLD showed no significant differences between FBP and IR as well as between the different reconstruction kernels (all $p>0.05$ ). In contrast, 
FWHM and El both showed statistically significantly lower values using IR instead of standard FBP (FWHM: B30//30=111.3 vs. 92.1; B50/50=167.6 vs. 115 ; B70/ $I 70=197.8$ vs. $137.5 ; \mathrm{El}: \mathrm{B} 30 / 130=4.8$ vs. $2.8 ; B 50 / 50=11.3$ vs. $5.8 ; B 70 / 170=20$ vs. 6.6). There was a significant lower variation between the different kernels using IR when compared to FBP. Image noise was reduced by $27 \%$ when compared to FBP. Conclusion: Variation of quantitative emphysema chest CT parameters between different reconstruction kernels is significantly reduced with IR when compared to FBP and may increase the robustness for therapy planning

\section{B-0161 14:09}

Assessing pulmonary perfusion in emphysema: automated quantification of perfused blood volume in dual-energy CTPA F.G. Meinel, A. Graef, S.F. Thieme, F. Bamberg, C. Neurohr, M.F. Reiser, T.R.C. Johnson; Munich/DE (felix.meinel@med.uni-muenchen.de)

Purpose: To determine whether automated quantification of lung perfused blood volume (PBV) in dual-energy computed tomography pulmonary angiography (DECTPA) can be used to assess the severity and regional distribution of pulmonary hypoperfusion in emphysema.

Methods and Materials: We retrospectively analysed 40 consecutive patients (mean age $67 \pm 13$ years) with pulmonary emphysema, no cardiopulmonary comorbidities and a DE-CTPA negative for pulmonary embolism. Automated quantification of global and regional pulmonary PBV was performed using the syngo dual-energy application (Siemens Healthcare). We further quantified the global and regional percentage of voxels with a CT density <-900 HU. Emphysema severity was rated visually and pulmonary function tests were obtained by chart review. Results: Global pulmonary PBV showed a moderate but highly significant negative correlation with residual volume (RV) in \% of predicted $R V(r=-0.62, p=0.002$, $\mathrm{n}=23$ ) and a positive correlation with forced expiratory volume in 1 second (FEV1) in $\%$ of predicted FEV1 $(r=0.67, p<0.001, n=23)$. Global PBV values strongly correlated with diffusing lung capacity for carbon monoxide ( $D L C O, r=0.80, p<0.001$, $n=15)$. Pulmonary PBV values decreased with visual emphysema severity $(r=-0.46$, $\mathrm{p}=0.003, \mathrm{n}=40$ ). Moderate negative correlations were found between global PBV values and parenchymal hypodensity in a per-patient $(r=-0.63, p<0.001, n=40)$ and per-region analyses $(r=-0.62, p<0.001, n=40)$.

Conclusion: DE-CTPA allows simultaneous assessment of lung morphology, parenchymal density and pulmonary PBV. In patients with pulmonary emphysema, automated quantification of pulmonary PBV in DE-CTPA can be used for a quick, reader-independent estimation of global and regional pulmonary perfusion, which correlates with pulmonary function tests.

Author Disclosures:

T.R.C. Johnson: Equipment Support Recipient; Siemens Healthcare. Research/ Grant Support; Bayer Healthcare.

\section{B-0162 14:18}

Densitometry on MDCT in cystic fibrosis: radiological evidence for emphysema

M.O. Wielpütz ${ }^{1}$, O. Weinheimer ${ }^{2}$, M. Eichinger ${ }^{1}$, M. Wiebel', J. Biederer ${ }^{1}$, H.-U. Kauczor' ${ }^{1}$, C.-P. Heussel', M.A. Mall', M. Puderbach ${ }^{1} ;{ }^{1}{ }^{1}$ Heidelberg/DE, 2Mainz/DE (mark.wielpuetz@web.de)

Purpose: The present study was conducted to employ computational densitometry based on multi-detector computed tomography (MDCT) of the chest to characterise and quantify emphysema in cystic fibrosis (CF), identical to its routine clinical application in chronic-obstructive pulmonary disease (COPD). Results were validated against pulmonary function testing (PFT, i.e. forced expiratory volume in $1 \mathrm{~s}$ percent predicted [FEV1\%], residual volume [RV] and total lung capacity [TLC]). Patients without lung disease (NORMAL) served as controls.

Methods and Materials: MDCT from $\mathrm{n}=41 \mathrm{CF}$ (median FEV1\%=46, median age 20a) and $n=20$ NORMAL (FEV1\%=102, 30a) were subjected to densitometry. Lung volume (LV) and emphysema volume (EV) were segmented (threshold -950 Hounsfield units), and the emphysema index was computed (EI). All results were correlated with paralleled PFT (median gap 0d, range 0-73d).

Results: Mean LV was $4681 \mathrm{ml}$ in CF and $3967 \mathrm{ml}$ in NORMAL (n.s). Significant EV was found in CF (mean $457 \mathrm{ml}$ ) compared to NORMAL $(78 \mathrm{ml})(p<0.05)$. Median El was elevated to $7 \%$ in $\mathrm{CF}$ patients, but $1 \%$ in NORMAL. El correlated well with FEV $1 \%$ in CF ( $r s=-0.55)$ and NORMAL ( $r s=0.67)$, but with RV ( $r s=0.69)$, and RV/ TLC ( $r s=0.47)$ in CF only $(p<0.05)$. Importantly, El increased markedly with age in $\mathrm{CF}$ ( $\mathrm{rs}=0.67, \mathrm{p}<0.001)$, starting at $13 a$.

Conclusion: Our results indicate the development of progressive emphysema in chronic CF, which should be considered for new therapeutic approaches. Densitometry may introduce new quantitative and prognostic parameters into severity assessment of CF lung disease.

\section{B-0163 14:27}

The effect of inspiration on airway dimensions measured in CT images from the Danish Lung Cancer Screening Trial

J. Petersen', M.M.W. Wille', L.H. Thomsen², A. Feragen', A. Dirksen²,

M. de Bruijne ${ }^{3} ;{ }^{1}$ Copenhagen/DK, ${ }^{2}$ Hellerup/DK, ${ }^{3}$ Rotterdam/NL (phup@diku.dk)

Purpose: Airway dimensions measured from CT are increasingly being used to investigate diseases such as chronic obstructive pulmonary disease (COPD). In this study, we investigate the effect of differences in inspiration level on such measurements in voluntary inspiration breathhold scans.

Methods and Materials: We selected from the Danish Lung Cancer Screening Trial 978 subjects without COPD who were scanned annually for 5 years with lowdose multi-slice CT. Using in-house developed software, the lungs and airways were automatically segmented and corresponding airway branches were found in all scans of the same subject using image registration. Mixed effect models were used to predict the relative change in lumen diameter (LD) and wall thickness (WT) in airways of generation 0 (trachea) to 6 based on relative changes in the segmented total lung volume (TLV).

Results: On average, 1.0, 2.0, 3.9, 7.6, 15.0, 25.0 and 27.3 airways per subject were included from generations $0,1,2,3,4,5$ and 6 , respectively. Relative changes in LD were positively related to changes in TLV and coefficients increased with generation: $0.20(+-0.02), 0.19(+-0.02), 0.21(+-0.01), 0.25(+-0.01), 0.29(+-0.01)$ $0.34(+-0.01), 0.37(+-0.01)$. Relative changes in WT were inversely related to changes in TLV and generation: $-0.01(+-0.02), 0.01(+-0.01),-0.02(+-0.01),-0.03$ (+- 0.01), -0.05 (+-0.01), -0.09 (+- 0.00), -0.08 (+-0.00).

Conclusion: Subjects who inspire deeper prior to scanning tend to have larger LD and smaller WT. This effect is more pronounced in higher generation airways. Thus, adjustment for inspiration level is needed to accurately assess airway dimensions.

Author Disclosures:

M. de Bruijne: Grant Recipient; AstraZeneca.

\section{B-0164 14:36}

Chronic bronchitis in large airway: airway wall measurements on thin-slice low-dose CT

X. Xie, A.E. Dijkstra, J.M. Vonk, M. Oudkerk, H.J.M. Groen, R. Vliegenthart; Groningen/NL (x.xueqian@rad.umcg.nl)

Purpose: Chronic bronchitis (CB) is usually caused by smoking and characterised by chronic inflammation and remodelling of the airway wall, commonly in large airways. The study purpose is to determine differences in airway wall thickness (AWT) and wall area percentage (WA\%) between subjects with and without CB symptoms. Methods and Materials: 50 heavy smokers with CB symptoms (cough, mucus, dyspnoea and wheezing) and 50 heavy smokers without CB symptoms were randomly selected from 1,413 participants in a lung cancer screening trial. Airway walls were measured on images in thin-slice low-dose CT with a dedicated software tool, for airways with a luminal diameter $35 \mathrm{~mm}$ in 5 selected bronchi (RB1, RB4, RB10, LB1+2 and LB10). Differences in measurements between the groups were assessed by t-test. The association between CB symptoms and AWT and WA\% was analysed using multiple linear regression adjusted for age, body mass index, smoking habit, amount of emphysema, and lung function.

Results: Mean AWT measured at 5 bronchi was $1.55 \pm 0.44 \mathrm{~mm}$ and $1.42 \pm 0.40 \mathrm{~mm}$ in subjects with and without $C B$ symptoms, respectively $(P<0.001)$. WA\% was $47 \pm 12 \%$ and $43 \pm 11 \%$, respectively $(P<0.001)$. With adjustment for confounders, a significant positive association between both airway wall measurements (AWT and $\mathrm{WA} \%$ ) and $\mathrm{CB}$ symptoms was found for airways with a luminal diameter from 5 to $10 \mathrm{~mm}(P<0.01)$. In airways with a luminal diameter ${ }^{310} \mathrm{~mm}$, no significant association was found $(P>0.05)$.

Conclusion: Patients with chronic bronchitis symptoms have thicker airway walls of airways between 5 and $10 \mathrm{~mm}$ diameter, not in larger diameter

\section{B-0165 14:45}

Value of inspiratory and expiratory lung volume und lung density for detection of bronchiolitis obliterans syndrome (BOS): a feasibility study S. Dettmer ${ }^{1}$, O. Otten ${ }^{1}$, C. de Wall' ${ }^{1}$, J.-M. Kuhnigk ${ }^{2}$, F. Wacker ${ }^{1}$, H.-O. Shin ${ }^{1}$; ${ }^{1}$ Hannover/DE, ${ }^{2}$ Bremen/DE (sabine-dettmer@t-online.de)

Purpose: To evaluate whether quantitative assessment of lung density and volume in computed tomography (CT) show differences in patients with and without BOS after lung transplantation.

Methods and Materials: 210 CT examinations were carried out in lung transplant patients in full inspiration/expiration using a 64 row MDCT $(120 \mathrm{kVp}$, rotation time $0.8 \mathrm{~s}$, pitch 0.984 , collimation $1.25 \mathrm{~mm}$, reconstruction increment $1 \mathrm{~mm}$, standard reconstruction kernel). 26/184 examinations were performed in patients with/with- 
out BOS. Lung volume (vol), mean lung density (MLD), $10^{\text {th }}, 20^{\text {th }}, 30^{\text {th }}, 40^{\text {th }}, 50^{\text {th }}$ percentile (P10-P50), peak, full width half maximum (FWHM), percentage of high (>-300 HU, HAV) and of low attenuation values (<-950 HU, LAV) were assessed with dedicated software (MeVis Pulmo). Measurements of patients with/without BOS and patients without BOS with normal ( $F E V 1>90 \%$ of baseline, BOS0)/slightly reduced values for FEV1 (FEV1 81-90\% of baseline, BOSO-p) were compared with independent t-test.

Results: Vol, MLD, P10, P20, P40, P50, peak, and FWHM ( $\mathrm{p}^{20.005)}$ in expiration scan as well as difference in volume between inspiration and expiration $(p<$ 0.001 ) were significantly different in patients with/without BOS. In inspiration, P10 $(p=0.017)$, FWHM $(p=0.036)$ and LAV $(p=0.026)$ showed significant differences at higher levels. Comparing patients with BOSO and BOSO-p, P10 might be used for distinction $(\mathrm{p}=0.029)$.

Conclusion: Expiratory lung volume and density showed significant differences in patients with/without BOS. P10 value might be used as an imaging biomarker for early detection of BOS.

\section{Author Disclosures:}

S. Dettmer: Grant Recipient; Integrated Research and Treatment Center Transplantation at Medical School Hannover. C. de Wall: Grant Recipient; Integrated Research and Treatment Center Transplantation at Medical School Hannover. H. Shin: Grant Recipient; Integrated Research and Treatment Center Transplantation at Medical School Hannover.

\section{B-0166 14:54}

Value of density mappings in computed tomography for detection of bronchiolitis obliterans syndrome (BOS)

S. Dettmer, T. Kaireit, O. Solyanik, C. de Wall, F. Wacker, H.-O. Shin; Hannover/DE (sabine-dettmer@t-online.de)

Purpose: The aim of this study was to prospectively evaluate the diagnostic value of density mappings for detection of BOS following lung transplantation.

Methods and Materials: $132 \mathrm{CT}$ examinations were carried out in lung transplant patients in full inspiration / expiration using a 64-row MDCT (120 kVp, rotation time $0.8 \mathrm{~s}$, pitch 0.984 , collimation $1.25 \mathrm{~mm}$, reconstruction increment $1 \mathrm{~mm}$, standard reconstruction kernel). 27 / 105 examinations were performed in patients with / without BOS (BOS 0: 80, BOS Op: 25, BOS 1: 11, BOS 2: 5, BOS 3: 11). The assessment of AT was performed using both, a threshold-based method in expiration (HU range: $-910 \mathrm{HU}$ to $-850 \mathrm{HU}$ ) and density mappings of $\mathrm{HU}$ values in inspiration and expiration after registration applying a threshold of $-910 \mathrm{HU}$ to $-750 \mathrm{HU}$ and $\mathrm{HU}$ differences ${ }^{2} 70 \mathrm{HU}$ between expiration and inspiration. With both methods, measurements of patients with / without BOS were compared with independent t-test. Results: AT quantified with density mappings was significantly different in patients with / without BOS. Mean AT was $28 \%$ in patients with BOS and $18 \%$ in patients without BOS. Comparing patients with and without BOS AT might be used for distinction $(p=0.002)$. AT quantified with the standard threshold-based method also showed differences between both groups, but with a lower significance $(p=0.019)$. Conclusion: Measurement of AT with density mappings of HU-values showed a significantly better distinction between patients with / without BOS than the standard threshold-based method.

\section{Author Disclosures:}

S. Dettmer: Grant Recipient; Integrated Research and Treatment Center Transplantation at Medical School Hannover. C. de Wall: Grant Recipient; Integrated Research and Treatment Center Transplantation at Medical School Hannover. H. Shin: Grant Recipient; Integrated Research and Treatment Center Transplantation at Medical School Hannover.

\section{B-0167 15:03}

Feasibility of krypton ventilation for $\mathrm{CT}$ imaging of lung ventilation: preliminary animal data

A.H. Mahnken ${ }^{1}$, G. Jost ${ }^{2}$, H. Pietsch'; ${ }^{1}$ Marburg/DE,

2Berlin/DE (mahnken@med.uni-marburg.de)

Purpose: To assess the feasibility and safety of krypton ventilation imaging by intra-individual comparison to xenon ventilation CT.

Methods and Materials: Seven male New Zealand white rabbits (4.4-6.0 kg) were included in this study. After intubation and mechanical ventilation unenhanced CT scans were obtained in end-inspiratory breath-hold. Thereafter, xenon $(30 \%)$ and krypton $(70 \%)$ ventilation CT was performed in random order. After 2-minute washin of gas "A" CT imaging was performed (128x0.6 mm; $80 \mathrm{kV} ; 140 \mathrm{mAseff})$. After a 30-minute wash-out period and another 2-minute wash-in of gas "B" another CT scan was performed using the same scan protocol. Heart rate and oxygen saturation were measured. Unenhanced and krypton or xenon data were registered and sub- tracted using a non-rigid image registration tool (HepaCare, Siemens, Forchheim, G). Subtraction data were analysed using syngo PulmoCT-Software (Siemens). Results: One animal had to be excluded from data analysis due to problems during intubation. Oxygen saturation in the krypton group was $99.3 \%$ vs. $99.1 \%$ after xenon inhalation $(p=0.4993)$. Corresponding values for heart rate were $128 \pm 36$ and $132 \pm 38$, respectively $(p=0.6036)$. Xenon resulted in an average $34.6 \pm 6.4 \mathrm{HU}$ increase of enhancement, whereas krypton achieved a mean increase of $21.9 \pm 1.9$ $\mathrm{HU}$ in enhancement $(\mathrm{p}=0.0055)$.

Conclusion: The use of krypton for lung ventilation imaging appears to be feasible and safe. Despite the use of a markedly higher concentration of krypton, enhancement is significantly lower when compared with xenon CT ventilation imaging, but the use of krypton could avoid unwanted side-effects of xenon.

Author Disclosures:

A.H. Mahnken: Research/Grant Support; Siemens. Speaker; Bayer. G. Jost: Employee; Bayer Healthcare. H. Pietsch: Employee; Bayer Healthcare.

\section{B-0168 $15: 12$}

Quantification of idiopathic pulmonary fibrosis by semiquantitative HRCT score: correlation between radiologists and pulmonary function tests

L. Navarro Vilar, M.L. Domingo Montañana, R. Peris Sanchez,

E. Fernandez Fabrellas, C. Ramírez Fuentes, J. Vilar Samper; Valencia/ES (linavi85@gmail.com)

Purpose: Measuring the extent of idiopathic pulmonary fibrosis by a visual highresolution computed tomography (HRCT) score method and its assessment. Correlation between score evaluation of radiologists and its correlation with the pulmonary function tests.

Methods and Materials: Thirty-five patients with IPF were analysed retrospectively using HRCT and pulmonary function tests at diagnosis onset. In the HRCT images, the lungs were divided into six zones (upper, middle and lower zones of the right and left lung). The images were scored by consensus of the two radiologists on a scale of $0-6$ based on four patterns of pulmonary fibrosis: ground glass, reticular abnormalities, distortion and honeycombing. The score was applied as follows: 0 (absent), $1(210 \%), 2(11-20 \%), 3(21-30 \%), 4(31-40 \%), 5(41-50 \%)$ or $6(>51 \%)$. These scores were also summed into a pattern CTtotalscore. Pulmonary function tests were performed within 1 month and included \%FEV1, \%FVC, FEV1/FVC, total lung capacity (\%TLC), diffusing lung capacity for carbon monoxide (\%DLCO) and DLCO adjusted for alveolar volume (KCO). Statistical analysis was made using Pearson's correlation

Results: We found a significant correlation between radiologists for the total score $(r=0.9)$, ground glass $(r=0.92)$, reticular abnormalities $(r=0.48)$ and distortion $(r=0.64)$ $(p<0.01)$. There was a significant correlation between the extent of honeycombing pattern and DLCO $(r=-0.48, p=0.004)$. The total score is correlated with DLCO $\%$ $(r=-0.41, p=0.01)$ and TLC $(r=-0.36, p=0.03)$.

Conclusion: The visual score HRCT, with high correlation between radiologists, allows a reliable quantification of the extent and severity of the IFP. The honeycombing score and the total HRCT score show the highest correlation with the pulmonary function tests. The DLCO reflects better the severity of the disease.

\section{B-0169 15:21}

High-resolution computed tomography (HRCT) of the lungs in brain dead pigs: a feasibility study

G. Bozovic ${ }^{1}$, S. Steen', T. Sjöberg', C. Schaefer-Prokop², J. Verschakelen ${ }^{3}$, Q. Liao', R. Siemund', I. Björkman-Burtscher ${ }^{1} ;{ }^{1}$ Lund/SE, ${ }^{2}$ Amersfoort/NL, ${ }^{3}$ Leuven/BE (Gracijela.Bozovic@med.lu.se)

Purpose: Brain death has adverse effects on lung perfusion and ventilation with possible damage of lung parenchyma pre-transplantation. We wanted to assess brain dead subjects treated with a new drug regime with HRCT as a pre-transplant work-up.

Methods and Materials: Eleven pigs were decapitated (DC) assuring brain death, attached to ventilator and treated with a new drug regime optimising circulation thereby preventing lung edema. Thirteen non-decapitated pigs (N-DC) attached to ventilator, supported with conventional treatment served as controls. All were monitored 24h and thereafter examined with chest HRCT. Images were analysed by two radiologists using a pre-defined questionnaire assessing parenchymal and airway changes. In consensus, an overall conclusion inferred presence of edema, infection/atelectasis or airway pathology. Severity was estimated with a subjective scale.

Results: After $24 \mathrm{~h}$ there were no significant differences between the groups regarding mean arterial pressure (MAP), arterial oxygen/fraction of inspired oxygen ( $\mathrm{PaO} / \mathrm{FiO} 2)$, amount of infused fluid, urine production or clinical signs of edema. 
Both groups showed parenchymal changes in a comparable extent (consolidation in 6/11 (DC) and 10/13 (N-DC) and GGO in 6/11 (DC) and 9/13 (N-DC), respectively). Overall conclusion appraised the presence of oedema in 2/11 (DC) but none of the $\mathrm{N}$-DC pigs, signs of old/ recent infection in 6/11 (DC) and 7/13 (N-DC) and possible hypersensitive pneumonitis in 1/13 (N-DC).

Conclusion: After 24h, there were no significant differences in clinical and imaging findings between the groups supporting the successful hemodynamic optimising with the new drug regime. HRCT allows evaluation of pre-transplant lungs.

14:00 - 15:30

Room D2

Interventional Radiology

\section{SS 209}

\section{Ablation and biopsy of the prostate and the kidney}

Moderators:

J.J. Fütterer; Nijmegen/NL

J. Kettenbach; Berne/CH

\section{B-0170 14:00}

1.5 $\mathrm{T}$ magnetic resonance-guided transgluteal biopsies of the prostate in patients with clinically suspected prostate cancer: technique and feasibility

B. Bodelle, N.N.N. Naguib, K. Eichler, T.J. Vogl, S. Zangos;

Frankfurt/DE (bbodelle@googlemail.com)

Purpose: Feasibility and safety of magnetic resonance-guided prostate biopsies (MRGBx) with a transgluteal approach in patients with cancer suspicious prostatic regions (CSRs).

Methods and Materials: 25 men who were clinically suspected for prostate cancer with elevated prostate-specific antigen (PSA) levels and at least one prior negative transrectal ultrasound-guided prostatic biopsy (TRUSBx) underwent diagnostic MRI of the prostate. CSRs were identified and MRGBx with a transgluteal approach in a large closed-bore 1.5-T MR system were manually performed in coaxial technique. Success rate, biopsy findings, side effects, procedure time, repositioning of the needle guide and the length of the biopsy channel were documented. Follow-up was performed after 24 months.

Results: 40 CSRs were detected $(n=25)$. All MRGBx procedures were technically successful. Biopsies per CSR: $3.3 \pm 1.5$ (range, 1-7). Histopathological analysis: adenocarcinoma in $35 \%$ (14 of 40 ), acute or chronic prostatitis in $30 \%$ (12 of 40 ), adenofibromyomatous changes in $22.5 \%$ (9 of 40 ) and no identifiable pathology in $17.5 \%$ (7 of 40 ) of CSRs. Pathological overlap for chronic prostatitis and adenofibromyomatous changes in one patient with biopsies in 2 CSRs. No missed prostate cancer after MRGBx in follow-up. Mean procedure time was $31 \pm 7$ minutes (range, 21-46 minutes). Side effects were hematuria $(n=7)$, hematospermia $(n=3)$, combined hematuria/-spermia $(n=2)$, infection $(n=1)$.

Conclusion: MRGBx with a transgluteal approach is feasible, safe and a promising technique for histological clarification of prostate cancer in patients with elevated PSA after negative TRUSBx. MRGBx offers a reasonable alternative to repeated TRUSBx for clarification of prostate cancer.

\section{B-0171 14:09}

Magnetic resonance-guided biopsy (MRGB) in combination with multiparametric MRI (mpMRI) after negative transrectal guided ultrasound (TRUS) biopsy of the prostate

S. Polanec, T. Helbich, K. Pinker-Domenig, S. Leitner, P. Brader; Vienna/AT

Purpose: The performance of mpMRI after previous negative TRUS biopsy resulted in a better diagnostic accuracy and has increased the opportunities for image-guided techniques like MRGB. We hypothesise that MRGB obviates falsenegative biopsies. The purpose of our study was to demonstrate the feasibility of combined mpMRI and MRGB of the prostate and to assess diagnostic accuracy. Methods and Materials: Forty-one patients (mean age of 65 years, range 44-75 years; mean PSA level: $8.17 \mathrm{ng} / \mathrm{ml}$, range from $0.7-30.3 \mathrm{ng} / \mathrm{ml}$ ) were enrolled in this IRB-approved study and subjected to combined mpMRI and MRGB. All patients had at least one prior negative TRUS biopsy. MRGB was performed in a closed 1.5T MR unit and the needle was inserted rectally. All patients had a follow-up between 2 and 61 months.

Results: Prostate cancer (PCa) was detected in 11 patients $(26.9 \%)$ with a median Gleason score of 7 (range from 6-9). The PCa occurred in nine cases in the peripheral zone and one in the transition zone and central zone. In 30 patients no
PCa was detected. In the follow-up no new PCa was diagnosed and the surveillance rate of patients with a detected PCa was $100 \%$.

Conclusion: Combined mpMRI and MRGB is feasible, improves diagnostic accuracy and obviates false-negative biopsies for early diagnosis of PCa.

\section{B-0172 14:18}

MRI-guided biopsy of the prostate: evaluation of patient acceptance and adverse effects

T. Franiel, N. Egbers, C. Schwenke, A. Maxeiner, C. Stephan, B. Hamm; Berlin/DE (tobias.franiel@charite.de)

Purpose: To evaluate the acceptance, adverse effects, and complications of prostate biopsy guided by magnetic resonance imaging (MRI-GB) compared with biopsy guided by transrectal ultrasound (TRUS-GB).

Methods and Materials: Fifty-four men with persistent suspicion of prostate cancer and at least one negative TRUS-GB underwent MRI of the prostate in the setting of a prospective clinical study. Subsequently, targeted biopsy of suspicious areas was performed using MRI guidance (range of biopsy specimens, 1-9). One week after outpatient MRI-GB, patients were administered a questionnaire by telephone. The questionnaire items included pain intensity compared with prior TRUS-GB performed in accordance with current guidelines, haematuria, hemospermia, rectal bleeding, fever, chills, and other complications. Finally, patients were asked for an overall assessment with regard to the preferred biopsy procedure.

Results: MRI-GB was preferred by $65 \%$ (35/54) of the patients, and $82 \%(44 / 54)$ stated that they would undergo MRI-GB again. Pain intensity and duration of bleeding were significantly lower for MRI-GB compared with TRUS-GB $(p<0.01)$. Haematuria was the most common side effect of both biopsy methods (MRI, $59 \%$; TRUS, $80 \%$ ). Complications of MRI-GB were arterial bleeding and infection with fever in one case each. After TRUS-GB, epididymitis developed in one case and infection with fever in two cases.

Conclusion: Patients prefer MRI-GB of the prostate to TRUS-GB due to lower pain intensity and fewer side effects, indicating that MRI-guided biopsy is a suitable option for patients with persistent suspicion of prostate cancer after prior negative TRUS-GB.

Author Disclosures:

B. Hamm: Advisory Board; Siemens, BAYER.

\section{B-0173 14:27}

MRI+US fusion-guided prostate biopsy and ablation

H. Amalou, S. Xu, P. Pinto, B. Wood; Bethesda, MD/US (amalouh@cc.nih.gov)

Purpose: To demonstrate status of clinical trial using fusion guidance for IR procedures and show the feasibility of MRI + ultrasound fusion guidance for prostate biopsy and laser ablation.

Methods and Materials: Over 600 patients underwent fusion-guided biopsy of the prostate based upon pre-procedural MRI imaging and PSA suspicion. Fusionguided laser ablation was studied in custom phantoms with CT, MRI, and ultrasound confirmation of laser ablation zones.

Results: Fusion guidance significantly increased the cancer detection rate versus random blind sextant biopsy alone. Gleason scores correlated with imaging findings. Biopsy locations could be mapped to specific locations on the MRI for later use in patients undergoing watchful waiting. Laser ablation can be performed accurately with ultrasound guidance and with MRI + ultrasound guidance without requiring the physical presence of the MRI gantry.

Conclusion: Fusion guidance for focal prostate biopsy adds value for certain prostate cancer patients and prostate cancer thermal ablation with laser can be guided by fusion imaging without an MRI gantry.

Author Disclosures:

H. Amalou: Patent Holder; NIH may own IP in the field. Research/Grant Support; Cooperative research and development agreement NIH and Philips Healthcare. S. Xu: Patent Holder; NIH may hold IP in the field. Research/ Grant Support; NIH and Philips Healthcare have a cooperative research and development agreement. P. Pinto: Patent Holder; NIH may have IP in the field. Research/Grant Support; NIH and Philips have a cooperative research and development agreement. B. Wood: Patent Holder; NIH may have intellectual property in the field. Research/Grant Support; $\mathrm{NIH}$ and Philips Healthcare have a cooperative research and development agreement. 


\section{B-0174 14:36}

Non-invasive focal therapy of organ confined prostate cancer: phase I study using magnetic resonance guided focused ultrasound technology and excision pathology for efficacy assessment A. Napoli, V. Panebianco, M. Anzidei, F. Boni, V. Noce, L. Bertaccini, G. Cartocci, F. Ciolina, C. Catalano; Rome/lT (alessandro.napoli@uniroma1.it)

Purpose: To assess safety and initial effectiveness of non-invasive high-intensity 3 T MR-guided focused Ultrasound (MRgFUS) treatment of localised prostate cancer in a phase I, treat and resection designed exploratory study.

Methods and Materials: On the basis of a power analysis, 11 patients with biopsyproven focal T2 prostate cancer (low-to-intermediate risk: Gleason max $3+4$ ), confirmed on a previous multiparametric MR exam, including dynamic contrast enhanced (DCE) imaging, underwent MRgFUS. All patients were scheduled to laparoscopic prostatectomy; MRgFUS treatment was carried out on MR identifiable lesions ( $\max 2$ ) using a patient-specific energy and real-time MR thermometry monitor for correct location. Non-perfused volume (VPV) in the post-ablative MRI was than compared with excision pathology for necrosis assessment.

Results: No significant complications were observed in all subjects during or immediately after the procedure. Procedure was validated by pathologist that demonstrated extensive coagulative necrosis at the site of sonication surrounded by normal prostatic tissue with inflammatory changes; these features positively compared with immediate post-ablative MRI scan and NPV. At histology $10 \mathrm{pa}-$ tients were free of residual viable tumour within the treated area; in the remaining patient, $10 \%$ of residual tumour was observed within the NPV. There was a variable amount of isolated cancer tissue within non-treated parenchyma that was identifiable neither at MRI nor at biopsy.

Conclusion: Conclusion Results of our Phase I study suggest MRgFUS ablation as a safe and effective modality to determine $>90 \%$ necrosis of identifiable prostate cancer; other prospective studies are needed to extend success rate in larger cohort.

\section{B-0175 14:45}

Technical challenges, pitfalls and obstacles on performing prostatic artery embolisation for benign prostatic hyperplasia

H.A.M.R. Rio Tinto, T. Bilhim, L. Fernandes, J. Pereira, J.M. Pisco; Lisbon/PT

Purpose: To evaluate the pre-embolisation workup and the safety of prostatic arterial embolistion (PAE) for prostatic benign hyperplasia $(\mathrm{BPH})$ and its implications during and after the procedure.

Methods and Materials: Our patient evaluation includes clinical history and examination, severity of low urinary tract symptoms with IPSS, quality of life (QoL), erectile/sexual function, uroflowmetry, digital examination, transrectal ultrasound and PSA. An Angio-CT (CTA) is performed in patients within the inclusion criteria to assess vascular anatomy. We selectively catheterise the prostatic artery and embolise. The end points are near stasis in prostatic vessels and obliteration of prostatic artery supply observed in the control angiogram. Follow-up of clinical parameters is performed at one, three and six months, one and two years.

Results: In more 300 cases performed by now we achieved technical success in the large majority of patients with a clinical failure of about $20-30 \%$. In more than $95 \%$ of cases we find a very good correlation between the anatomical data obtained with CTA and digital subtraction angiography (DSA). In more than $50 \%$ of cases we found important anastomosis with direct implications during the embolisation procedure. Conclusion: PAE is a challenging and safe procedure to treat benign prostatic hyperplasia. Clinical evaluation and CTA are essential as pre-embolisation workup.

\section{B-0176 14:54}

Mid-term results of percutaneous image-guided radiofrequency ablation of renal tumours

P. Balageas, F. Cornelis, Y. Le Bras, J.-M. Ferrière, A. Ravaud, N. Grenier; Bordeaux/FR (balageaspierre@gmail.com)

Purpose: To evaluate the mid-term survival and outcomes after radiofrequency ablation (RFA) of renal tumours.

Methods and Materials: Institutional review board approval was obtained for this single institution retrospective study; informed patient consent was not required according to local laws. Between 2002 and 2009, 93 patients (112 tumours), with a median age of 73.5 years (20-87), consecutively treated with percutaneous radiofrequency ablation under US or CT-guided were selected from our institutional database. After a subset analysis, 62 patients (71 tumours) meet the inclusion criteria for survival and oncological efficacy including 25 patients $(40.3 \%)$ with solitary kidney and 7 cystic cancers. Maximal tumour diameters were between 8 to $46 \mathrm{~mm}$ (median: $23 \mathrm{~mm}$ ).
Results: RFA was technically possible for all patients. Mean follow-up was 38.8 months (range : 18-78 months). Primary and secondary technique effectiveness were 95.2 and $98.4 \%$ per patient, respectively, and one-session complete ablation was obtained for all tumours smaller than $2 \mathrm{~cm}$. The rates of local tumour progression and metastasis evolution were, respectively, 3.2 and $9.7 \%$ per patient. There was no difference in glomerular filtration rate from baseline before procedure at 2 and 6 months. The major complication rate was $5.9 \%$ per session.

Conclusion: Based on these mid-term results, percutaneous renal RFA seems to be safe and effective with interesting nephron-sparing results.

\section{B-0177 15:03}

Retrospective study of renal tumors treated with radio frequency ablation at Uppsala University Hospital since 2007: which factors affect ablation results?

V. Acosta Ruiz, A. Magnusson, P. Dahlman, E. Brekkan, M. Lönnemark; Uppsala/SE (vanessa.acosta_ruiz.6628@student.uu.se)

Purpose: To identify the factors leading to complete tumor ablation. Our hypothesis was that electrode placement and tumor size could be factors that affected ablation success. Radio frequency ablation is a relatively new method used for treatment of renal masses. Several studies have identified factors that affect ablation results; larger $(>3 \mathrm{~cm})$ and centrally located tumors are more often prone to recurrence. Methods and Materials: 52 percutaneous CT guided RFA treated renal tumors were retrospectively studied. Patients were followed up with contrast-enhanced CT scans at 3, 6, 12 months and yearly after treatment. Collection of data included patient demographics, tumor data (detection method, renal anatomical position, histopathological diagnosis, size), RFA treatment data (electrode placement) and follow-up results (tumor relapse). Data was statistically analyzed using the Mann Whitney and Fischer's exact tests.

Results: After a first ablation session $75 \%$ tumors were completely ablated. Reablation of incompletely ablated tumors increased total success rate to $85 \%$. Incompletely ablated tumors had a greater median tumor diameter, a shorter mean distance to the collecting system and a greater percentage of clear cell carcinomas in comparison to completely ablated tumors. Electrode placement was more often categorised as optimal for the complete (92\%) than the incomplete ablated tumor group $(60 \%)$.

Conclusion: Small tumor size $(<3 \mathrm{~cm})$, optimal electrode placement and a long distance to the collecting system $(>10 \mathrm{~mm})$ seem to be factors that increase the frequency of complete ablation. These variables may be useful to assess in the future when considering potential patients for RFA.

\section{B-0178 15:12}

Irreversible electroporation (IRE) of the pig kidney with involvement of the renal pelvis - technical aspects, clinical outcome and 3d lesion analysis

C.M. Sommer, M.F. Wachter, S. Fritz, D. Vollherbst, U. Stampfl, N. Bellemann, T. Gockner, T. Mokry; Heidelberg/DE (christof.sommer@med.uni-heidelberg.de)

Purpose: To evaluate irreversible electroporation (IRE) of the pig kidney with involvement of the renal pelvis.

Methods and Materials: IRE was performed with a commercial system. In 10 kidneys of 5 pigs, 1 or 2 IRE (s) of the central renal tissue with involvement of the renal pelvis was/were performed. Different applicator configurations were used: 2 applicators $(n=11 ; I), 3$ applicators $(n=2 ; I I)$ or 6 applicators $(n=3 ; I I I)$. One hour after IRE, pigs underwent contrast-enhanced CT with subsequent sacrifice. Technical aspects (applicator spacing, tip exposure), clinical outcome (complications, blood samples) and 3d lesion analysis (short axis, volume, circularity) were performed. Results: Applicator spacing and tip exposure were $14.3 \pm 2.8 \mathrm{~mm}$ and $20 \mathrm{~mm}$ in I, $12.3 \pm 1.9 \mathrm{~mm}$ and $20 \mathrm{~mm}$ in II, and $16.4 \pm 3.5 \mathrm{~mm}$ and $30 \mathrm{~mm}$ in III, respectively. In III, 2 small perirenal haematomas were identified. Damage to pelvic structures was not observed on CT and gross pathology. Before and within 1 hour after IRE, creatinine, haemoglobin, white blood cell count, creatine kinase MB, pulse rate, mean arterial blood pressure and ECG were not significantly different. Lesion short axis and volume were $20.6 \pm 3.6 \mathrm{~mm}$ and $5.4 \pm 1.8 \mathrm{~cm}^{3}$ in I, respectively, $31.9 \pm 8.2 \mathrm{~mm}$ and $12.2 \pm 5.5 \mathrm{~cm}^{3}$ in II, and $39.3 \pm 2.4 \mathrm{~mm}$ and $68.8 \pm 10.0 \mathrm{~cm}^{3}$ in III with significant differences $(p<0.01$ and $p<0.01)$. Circularity was $0.8 \pm 0.2(0.6-0.9)$ in $I, 0.7 \pm 0.1$ $(0.6-0.9)$ in II, and $0.7 \pm 0.1(0.6-0.8)$ in III without significant differences.

Conclusion: IRE of the pig kidney with involvement of the renal pelvis is feasible and safe. There is no negative impact on the clinical outcome. Lesion size is significantly different depending on applicator configuration. 
Author Disclosures:

C.M. Sommer: Research/Grant Support; This study was technically and financially supported by AngioDynamics ${ }^{\circledR}$ Inc., Queensbury, USA. S. Fritz: Research/Grant Support; This study was technically and financially supported by AngioDynamics ${ }^{\circledR}$ Inc., Queensbury, USA.

\section{B-0179 15:21}

Retrograde ureteral stent exchange: comparison between the direct grasping and the simple snare technique

R. Cazzato, R. Grasso, G. Luppi, E. Faiella, R. Del Vescovo, F. Giurazza,

B. Beomonte Zobel; Rome/IT (r.cazzato@unicampus.it)

Purpose: To compare the modified-snare (MS) technique and the direct-grasping (DG) technique during retrograde exchange of double-J ureteral stent under fluoroscopic guidance.

Methods and Materials: 44 patients (24 men, 20 women; mean age, 68.9 years; range, $40-85$ years) were included. Ureteral obstructions were due to compression by pelvic malignancies. All stents had been previously placed via an anterograde approach through percutaneous nephrostomy sites. Stents were retrieved by either the MS or the DG technique. Time needed to grasp each single stent was recorded. Fisher's test was performed to compare procedural time with both techniques. A $p$ value $<0.05$ was considered significant.

Results: A total of 68 ureteral stents were successfully exchanged in 44 patients. All stents were successfully externalised either with the MS technique (42 stents in 28 patients) or with the DG technique (26 stents in 16 patients). The mean time needed to retrieve a ureteral double-J stent was $4.4 \mathrm{~min}$ (range, 3-7 $\mathrm{min}$ ) for the DG technique and $7.8 \mathrm{~min}$ (range, $4-12 \mathrm{~min}$ ) for the MS technique $(p=0.029)$. Conclusion: Compared to the MS technique, the DG technique is an easier, quicker and less expensive technique for retrograde exchange of ureteral stents.

\section{4:00 - 15:30} Room E1

\section{Musculoskeletal}

\section{SS 210}

\section{Lower limb: tricks for improved imaging}

Moderators:

A. Klauser; Innsbruck/AT

L.M. Sconfienza; Milan/IT

\section{B-0180 14:00}

MR imaging of articular cartilage at the ankle: utility of axial traction

J.S. Bauer, P. Jungmann, C. Schäffeler, M. Sauerschnig, A. Mann, T. Baum,

E.J. Rummeny, K. Wörtler; Munich/DE

Purpose: To determine the impact of axial traction during MR imaging of the ankle cartilage at $3.0 \mathrm{~T}$ in volunteers with respect to motion artefacts, image quality and cartilage surface delineation.

Methods and Materials: Optimised coronal and sagittal T1-weighted (w) turbo spin echo (TSE) sequences with a DRIVE pulse (in-plane spatial resolution 0.21 $\mathrm{mm}$ ), a sagittal fat-saturated intermediate-weighted (IMfs) TSE sequence and a $3 \mathrm{D}$ gradient echo sequence were acquired at 3.0 $\mathrm{T}$ in 8 volunteers with and without axial traction of $6 \mathrm{~kg}$. Joint space width was measured at four locations in the tibiotalar joint. Motion artefacts, general image quality, delineation of the cartilage surface, cartilage matrix and the confidence of diagnosing cartilage pathology were evaluated independently by two radiologists. Differences were assessed using a Wilcoxon test.

Results: With axial traction, joint space width increased significantly $(1.7 \pm 0.5 \mathrm{~mm}$ vs. $0.3 \pm 0.2 \mathrm{~mm}$ ). Consequently, delineation of the cartilage surface was rated superior in $7 / 8$ volunteers $(p<0.05)$. No differences were found in motion artefacts, general image quality, and artefacts within the cartilage matrix ( $p>0.1)$. Comparing the different sequences, Cartilage surface and matrix was best visualised by the T1-w TSE sequences with a DRIVE pulse (surface average of all regions and readers in sag T1-DRIVE vs. IMfs on a four-point scale $1.3 \pm 0.6$ vs. $3.0 \pm 1.0, p<0.05$ ). Conclusion: Axial traction of $6 \mathrm{~kg}$ increases the joint space width the tibiotalar joint, and thus better visualises the articular cartilage surface. Highest spatial resolution and visualisation of both cartilage surface and matrix were achieved by a T1-w TSE sequence with a DRIVE pulse.
B-0181 14:09

Do sagittal and coronal alignment of the hindfoot change in upright weight-bearing position? Comparison of upright weight-bearing and supine non-weight-bearing computed tomography

A. Hirschmann, C.W.A. Pfirrmann, N. Espinosa, G. Klammer, F.M. Buck; Zurich/CH (anna.hirschmann@balgrist.ch)

Purpose: To prospectively quantify changes in the coronal and sagittal alignment of the hindfoot between upright weight-bearing computed tomography (WBCT) and supine non-weight-bearing CT (NWBCT).

Methods and Materials: Institutional Review Board approval and informed consent of all patients were obtained. WBCT and NWBCT scans of the foot were obtained of 22 patients ( 8 women, 14 men; median age 46 years, range 19-75 years) using a conventional 64-row CT unit (Philips Brilliance 64, Best, Netherlands) for NWBCT and a novel cone-beam extremity CT for WBCT (Verity, Planmed, Helsinki, Finland). Two musculoskeletal radiologists independently performed the following measurements: hindfoot alignment angle (HFA); fibulocalcaneal (FCD) and medial talocalcaneal (TCD) distance in the coronal plane; talocalcaneal overlap (TCO), naviculocalcaneal distance (NCD) in the sagittal plane. Significant changes between WBCT and NWBCT were sought using Wilcoxon signed-rank test. A p value of < 0.05 was considered statistically significant.

Results: Significant differences were found for all measurements between WBCT and NWBCT. Measurement results were as follows (WBCT/NWBCT): HFA (median $20.2^{\circ} / 19.5^{\circ}$ valgus, range $4.9-84.0^{\circ} / 1.3^{\circ}-53.0^{\circ}$, mean difference $6.3^{\circ}, p=0.036$ ), FCD (median $0 \mathrm{~cm} / 0.4 \mathrm{~cm}$, range $-1.2-1.1 \mathrm{~cm} /-0.8-1.5 \mathrm{~cm}$, mean difference 0.4 $\mathrm{cm}, \mathrm{p}=0.001$ ), TCD (median $0.2 \mathrm{~cm} / 0.3 \mathrm{~cm}$, range $0.1-0.6 \mathrm{~cm} / 0.1-0.7 \mathrm{~cm}$, mean difference $0.1 \mathrm{~cm}, \mathrm{p}=0.004$ ), TCO (median $0.1 \mathrm{~cm} / 0.5 \mathrm{~cm}$, range $-0.3-1.1 \mathrm{~cm} /-0.3$ $2.1 \mathrm{~cm}$, mean difference $0.4 \mathrm{~cm}, \mathrm{p}=0.001$ ), NCD (median $1.6 \mathrm{~cm} / 2.9 \mathrm{~cm}$, range $0.8-2.9 \mathrm{~cm} / 0.7-2.2 \mathrm{~cm}$, mean difference $0.3 \mathrm{~cm}, \mathrm{p}=0.007$ ).

Conclusion: Sagittal and coronal alignments of the hindfoot significantly change in upright weight-bearing position. Differences can be visualised and measured using WBCT.

\section{B-0182 14:18}

MRI in plantar plate disease evaluation: diagnostic value of 'stress test' C. Ottonello, I. D'Ambrosio, P. Giuliani, P. Ronconi; Rome/IT (ottonello@seram.org)

Purpose: To evaluate the role of MRI, even in joint hyperextension, "stress test" (ST), in the study of plantar plate (PP) disease.

Methods and Materials: 20 patients (10 symptomatic, $58.7 \pm 9.4$ years, 10 asymptomatic, $59.5 \pm 10.0$ years) performed MRI (Artoscan, Esaote, Genoa, Italy), in standard position and with ST. Adjacent flexor tendon was also evaluated. All symptomatic patients were subjected to surgery.

Results: Asymptomatic patients: 1/10 was positive for PP disfunction (dorsal subluxation demonstrated only on ST), without plantar plate tear. Symptomatic patients: 6/10 demonstrated plantar plate tear/disfunction on standard MRI and on ST, 2/4 negative for PP tear on standard examination showed the lesion only on ST, 2/4 negative for PP tear both on standard examination and ST showed dorsal subluxation on ST due to microinstability for plantar plate failure, without tear. Surgical revision confirmed the plantar plate tear in MRI positive cases (on MRI standard and/or on ST); in the 2 MRI negative cases surgery confirmed PP laxity without tear. No significant flexor tendons tear in 20/20.

Conclusion: Standard RM reported in asymptomatic patients specificity $100 \%$ and high NPV (90\%), incremented by the ST (NPV and specificity $100 \%)$. In symptomatic patients PP evaluation on standard MRI reported sensitivity $50 \%$, while ST increased sensitivity to $100 \%$; in the evaluation of dorsal subluxation of metatarsophalangeal joint, the sensitivity was $60 \%$ (standard RM) but reached $100 \%$ with ST. Therefore, ST would seem to significantly increase MRI diagnostic accuracy in the evaluation of PPD. 


\section{B-0183 14:27}

Stable or unstable tear of the medial meniscus of the knee: can weight-bearing MRI solve the problem?

A. La Marra, S. Mariani, E. Costantini, A. Conchiglia, A. Barile, C. Masciocchi; L'Aquila/IT (alicelm@hotmail.it)

Purpose: To assess the role of 1.5 T, dedicated low-field standard and upright-MRI in the evaluation of stable or unstable tears of medial meniscus in comparison with arthroscopy.

Methods and Materials: We retrospectively reviewed and analysed $20 \mathrm{MRI}$ exams (group A) of normal knee and $77 \mathrm{MRI}$ exams (group B) of knee with clinical evidence of tears of the medial meniscus. In the same session, after conventional $1.5 \mathrm{~T}$ and "dedicated" $0.27 \mathrm{~T}$ supine MRI exam, the patients underwent upright weight-bearing examination with the same dedicated MRI unit. In all cases sagittal and coronal SE T1-W were used. All patients underwent arthroscopy within 21 days from MRI exam. Results: In group A, no statistically significant anatomical modifications of shape, intensity and position of the medial meniscus between standard $1.5 \mathrm{~T}$, dedicated supine and upright MRI were observed. In group B, the images acquired in the supine position (dedicated and $1.5 \mathrm{~T} \mathrm{MRI}$ ) documented in 31 cases a traumatic tear (group B1) and in 46 cases a degenerative tear (group B2). In group B1, weightbearing MRI showed presence of unstable tear only in 19 out 31 cases. In group B2, weight-bearing MRI showed a degenerative unstable meniscal tear only in 29 out of 46 cases. Arthroscopy confirmed weight-bearing MRI diagnosis in all cases. Conclusion: Our preliminary results show that upright weight-bearing MRI has wide potentials for the study of dynamic modifications in case of meniscal stable or unstable tear.

\section{B-0184 14:36}

Periosteal high volume image-guided injection of recalcitrant medial collateral ligament injuries: a retrospective case series analysis O. Drumm, O. Chan, P. Malliaras, D. Morrissey, N. Maffulli; London/UK (odrumm@yahoo.co.uk)

Purpose: We evaluate a novel injection method, periosteal high volume imageguided injection, in the management of recalcitrant medial collateral ligament (MCL) injury of the knee.

Methods and Materials: The injection, comprising $10 \mathrm{ml}$ of local anaesthetic with $25-50 \mathrm{mg}$ of hydrocortisone, is directed at the periosteal attachment of the MCL. 28 patients in total received the intervention between June 2009 and November 2011 at a London hospital. Each was asked to complete a study-specific questionnaire including a visual analogue scale (VAS) and the International Knee Documentation Committee (IKDC) subjective knee form (to describe symptoms pre-injection and at follow-up).

Results: $68 \%$ of patients responded $(n=19)$. Three patients were omitted according to the exclusion criteria. $31.3 \%$ of patients $(n=5)$ were professional athletes. Mean duration of symptoms was $5.4(\mathrm{SD}=6.6)$ months. Patients reported a mean improvement on the VAS of $75.4 \%$ (SD=23.7). There was a significant improvement in IKDC scores (mean difference of $42 \%, S D=14.2)$ pre- and post-injection ( $p=0.01$ ). $62.5 \%$ of patients $(n=10)$ had returned to their previous level of sport at follow-up including all the professional athletes. Knee symptoms returned to normal in $50 \%$ $(n=8)$, and improved substantially in a further $37.5 \%(n=6)$. The remaining $12.5 \%$ $(n=2)$ experienced an initial improvement but symptoms recurred.

Conclusion: Periosteal high volume image-guided injection is a useful treatment for recalcitrant MCL injury, a common sports injury that can result in lengthy periods of missed training and competition. Results are encouraging, particularly amongst the professional athletes studied.

\section{B-0185 14:45}

Ischiofemoral impingement, do you want to believe?

R. Prada, R. Oca, A. Rocha, L. Fernández, M. Costas, G. Tardáguila; Vigo/ES (roqueoca@hotmail.com)

Purpose: To find out if there are anatomic and imaging MR criteria to diagnose ischiofemoral impingement.

Methods and Materials: 290 MRls of the hip were retrospectively reviewed from August 2011 to September 2012. A total of 9 ischiofemoral impingement were diagnosed and 20 normal patients were included as control. Ischiofemoral space, quadratus femoris space and femoral inclination angle were measured independently by two blinded musculoskeletal radiologists. The degree of oedema and fatty infiltration in the quadratus femoris muscle were also assessed visually. Differences in ischiofemoral space, quadratus femoris space and femoral inclination angle were studied between pathological and control cases. The interobserver reliability was obtained for quantitative variables.
Results: All affected subjects were women between 14 and 52 years old (mean age: 40). Ischiofemoral space was significantly narrower in patients with ischiofemoral impingement when compared with control subjects $(13.1 \pm 4.06 \mathrm{~mm}$ vs $24.9 \pm$ $4.55 \mathrm{~mm}$, respectively; $p<0.01$ ). Quadratus femoris space was also significantly narrower in affected subjects $(9.4 \pm 2.97 \mathrm{~mm}$ vs $16.4 \pm 1.08 \mathrm{~mm} ; \mathrm{p}<0.01)$. Inclination angle was not statistically different $(p>0.05)$. The degree of oedema and fatty infiltration was inversely proportional to values of ischiofemoral and quadratus femoris space. The interobserver agreement ( $\mathrm{k}$ ) was 0.8 for ischiofemoral space, 0.63 for quadratus femoris space and 0.53 for inclination angle.

Conclusion: Ischiofemoral impingement can be accurately diagnosed following anatomic and imaging MR criteria.

\section{B-0186 14:54}

US-guided viscosupplementation of the hip: therapeutic efficacy in patients affected by femoro-acetabular impingment

C. Martini', F. Lacelli', E. Fabbro', G. Ferrero', G. Serafini2; ${ }^{1}$ Genoa/lT, 2Pietra Ligure/IT (chiarapio@libero.it)

Purpose: US-guided viscosupplementation is a safe and effective therapy for symptomatic osteoarthritis $(\mathrm{OA})$ of the hip and it allows to replace NSAIDs therapy and to delay prosthetic intervention. Femoro-acetabular impingement (FAI) is a cause of $\mathrm{OA}$. The purpose of this work is to evaluate the efficacy of viscosupplementation as therapy of OA in patients affected by femoro-acetabular impingement.

Methods and Materials: 352 patients selected by orthopaedists for hip pain and plain films signs of OA (degrees II-IV of K-L) underwent viscosupplementation ( $4 \mathrm{ml}$ of intermediate weight hyaluronic acid for three times every 30days). Each patient was evaluated before the treatment and 1, 3, 6 and 12 months after the treatment by means of VAS and Oxford Score. Hence, these patients were divided into responsive (increase Oxford score $=/>10$ and/or reduction VASO/>3) and non-responsive (increase Oxford score $<10$ and/or reduction VASO $<3$ ). Then we retrospectively evaluated the plain films of the hip these patients had done before the therapy, in order to find the signs of FAI.

Results: $281 / 352(79.8 \%)$ patients were included into the "responsive" group; $71 / 352(20.2 \%)$ in the "non-responsive" one. In the "responsive" group we found signs of FAl on the plain films of $37 / 281$ patients $(11.4 \%)$; in the "non-responsive" in $43 / 71(60.5 \%)$.

Conclusion: Despite viscosupplementation is an effective therapy of $O A$, there is an high prevalence of non-responsive among patients affected by FAI.

\section{B-0187 15:03}

A new way to measure lower limb length and alignment using 3D models based on biplanar linear radiography: a comparison with measurements on supine $\mathrm{CT}$ scans and upright full-length radiographs R. Guggenberger, C.W. Pfirrmann, P. Koch, F. Buck; Zurich/CH (romanguggenberger@yahoo.de)

Purpose: To compare lower limb length and alignment angle on supine computed tomography (CT), upright full-length radiography (UFR) and 3D models based on upright biplanar radiography (BLR).

Methods and Materials: IRB-approved study involving 51 consecutive patients (22 male, 29 female; mean age 68.8years; range 43-92years) scheduled for total knee replacement. Lower limb length and alignment angles were measured on CT, UFR and $3 \mathrm{D}$ models based on BLR using standard and extended length measurements by two independent readers. Measurements of different modalities were compared by paired t-tests, Pearson correlation coefficients and Bland-Altman analyses. Results: Mean limb lengths were $783 \mathrm{~mm}(\mathrm{SD} \pm 56.1 \mathrm{~mm}$, range 639-927 mm), $785 \mathrm{~mm}(\mathrm{SD} \pm 53.0 \mathrm{~mm}, 655-924 \mathrm{~mm}), 780 \mathrm{~mm}(\mathrm{SD} \pm 55.4 \mathrm{~mm}, 633-921 \mathrm{~mm})$ and $783 \mathrm{~mm}(\mathrm{SD} \pm 55.9 \mathrm{~mm}, 636-924 \mathrm{~mm}$ ) for CT, UFR and 3D models using standard and extended measurements, respectively. Mean alignment angles were $2.3^{\circ}$ $\left(\mathrm{SD} \pm 5.5^{\circ}\right.$, range $\left.-12^{\circ}-20^{\circ}\right), 2.5^{\circ}\left(\mathrm{SD} \pm 6.7^{\circ},-17^{\circ}-18^{\circ}\right), 3.4^{\circ}\left(\mathrm{SD} \pm 6.6^{\circ},-14^{\circ}-18^{\circ}\right)$, respectively. Length and angle measurements differed significantly when comparing standard 3D models to CT and UFR, respectively $(p<0.001)$. Pearson correlation coefficients were $0.992,0.993,0.995$ and 0.998 for limb length and $0.968,0.983$ and 0.984 for angle measurements in CT, UFR and $3 D$ models, respectively. Bland-Altman analyses for CT and UFR compared to 3D models showed a small positive mean difference in length using standard measurements $(2.7 \mathrm{~mm}, \mathrm{SD} \pm 5.5$; $5.4 \mathrm{~mm}, \mathrm{SD} \pm 13.1)$ and a small negative mean difference in angle measurements $\left(-1.1^{\circ}, \mathrm{SD} \pm 2.5 ;-0.9^{\circ}, \mathrm{SD} \pm 3.1\right)$. No bias for length measurements was seen using extended measurements.

Conclusion: Upright 3D models allow for lower limb length and alignment angle measurements comparable supine CT scans and UFR. 


\section{B-0188 $\quad 15: 12$}

Dynamic contrast-enhanced MRI can assess vascularity within pseudarthrotic clefts and predicts good clinical outcome

M.-A. Weber ${ }^{1}$, K. Bloess ${ }^{1}$, I. Burkholder ${ }^{2}$, D. Bender',

G. Schmidmaier ${ }^{1}$, H.-U. Kauczor ${ }^{1}$, O. Schoierer ${ }^{1} ;{ }^{1}$ Heidelberg/DE,

${ }^{2}$ Zweibrücken/DE (MarcAndre.Weber@med.uni-heidelberg.de)

Purpose: To prospectively evaluate whether dynamic contrast-enhanced (DCE) MRI can assess vascularity within pseudarthrotic clefts and predicts clinical outcome better than the clinical non-union scoring system (NUSS).

Methods and Materials: Sixty-four patients (mean age, 49.3 years) with nonunion of an extremity fracture in computed-tomography received 3-Tesla MRI including DCE (coronal T1-weighted fat-saturated VIBE, TR/TE=3.76/1.35 ms, 17 measurements, $2 \mathrm{~mm}$ slice thickness) after $0.1 \mathrm{mmol} / \mathrm{kg}$ body-weight of gadoterat. We assessed vascularity within the pseudarthrotic cleft using a region-of-interest analysis. Signal intensity curves were subdivided into those with more intense contrast-agent uptake in the pseudarthrotic cleft than in normal adjacent muscle tissue (vascularised non-union) and those with similar or less uptake. The pharmacokinetic parameters of the Tofts model (Ktrans, Kep, iAUC, Ve) were correlated to the clinical outcome at one-year follow-up $(n=37)$.

Results: Despite inserted osteosynthesis material, DCE parameters could be evaluated in $\mathrm{n}=60$ at first visit. Sensitivity/specificity of vascularised non-unions as indicator of good clinical outcome was $77.3 \% / 46.7 \%$ compared to $95.5 \% / 26.7 \%$ using NUSS. Logistic regression revealed non-significant impact of NUSS on clinical outcome $(p=0.27$, odds ratio $=0.97)$. At first examination, median iAUC (initial area under the enhancement curve) was 24.13 in patients with good outcome compared with 10.96 in non-responders $(p=0.032)$, while Ktrans, Kep, and Ve were not significantly different. Using a receiver operating characteristic analysis, sensitivity/specificity of iAUC at the optimal cut-off value of 17.5 to predict outcome was $68.2 \% / 76.9 \%$. All pharmacokinetic parameters did not change significantly at the one-year control $(n=18)$.

Conclusion: DCE MRI can assess vascularity in pseudarthrotic clefts. A vascularised non-union indicates good clinical outcome.

\section{B-0189 15:21}

Osteoidosteoma - post-procedural MR-imaging characteristics

following percutaneous MR-guided laser ablation in an open 1.0 Tesla MRI

S. Fuchs, B. Gebauer, L. Stelter, M. Maurer, D. Renz, P. Schwabe, B. Hamm,

F. Streitparth; Berlin/DE (simon.fuchs@charite.de)

Purpose: Imaging-guided thermal ablation has become the therapy of choice among the different treatment strategies of symptomatic Osteoid Osteoma lesions. In this descriptive, prospective study, our objective was to evaluate typical post-procedural MR-imaging characteristics following MR-guided laser ablation of Osteoid Osteoma.

Methods and Materials: 35 patients with Osteoid Osteoma treated with MR-guided laser ablation underwent follow-up MR-Imaging immediately after the procedure as well as after $3,6,12,24,36$ and 48 months. Imaging protocol included fatsaturated T2W SPIR TSE, non-contrast and contrast-enhanced T1w TSE images and subtraction images. MR images were jointly reviewed by two radiologists, regarding the appearance of treated areas, nidal enhancement and presence of side effects such as bone oedema and soft tissue enhancement. Imaging was correlated to clinical status.

Results: Mean follow-up time was 13.9 months. 28 patients showed a postprocedural target-sign appearance with subsequent "positive inward fusion" of the different zonal compartments. Post-procedural MR-imaging can support evaluation of therapeutic success. Characteristic MR findings correlated well with the clinical status.

Conclusion: Interpretation of post-procedural follow-up MR-imaging after laser ablation of Osteoid Osteoma may contribute to understanding of histopathological post-therapeutic changes and cornerstones of therapeutic success.
14:00 - 15:30

Room E2

\section{GI Tract}

\section{SS 201a}

\section{Bowel imaging: protocol optimisation and intervention}

Moderators:

V. Cantisani; Rome/IT

P. Popovic; Ljubljana/SI

\section{B-0190 14:00}

Abdominal and pelvic $\mathrm{CT}$ : is positive enteric contrast still necessary? Preliminary results of a clinical investigation

S. Kammerer, A. Knauer, B. Buerke, C. Schuelke, W.L. Heindel, J. Wessling; Münster/DE (sara.kammerer@ukmuenster.de)

Purpose: Evaluating diagnostic accuracy in abdominal CT depending of enteric preparation with positive, neutral and without contrast.

Methods and Materials: Abdominal MSCT (1.5 mm slice thickness, 85 seconds scan delay) of the first 1000 patients were included. Patients were classified into three groups: A. positive enteric contrast with barium sulphate $(n=351)$, B. neutral enteric contrast with mineral water $(n=373)$ and $C$. without any enteric contrast $(n=276)$. Independent evaluation by two radiologists included segmental delineation of the intestine and the influence of enteric preparation on diagnosis and reliability using three-point confidence scales. Furthermore, confidence for delineation of dedicated enteric pathologies (stratification, etc). was noted. For statistical analysis datasets were assigned to five groups: oncology, inflammation, vascular, trauma and Gl-pathology using appropriate methods.

Results: Overall, there was no difference for segmental delineation from duodenum to rectum between patients with positive, neutral and without enteric contrast $(p>$ 0.05 ). The influence of enteric contrast on diagnosis was rated low in all groups (no influence; $976 / 1000, p>0.05$ ). Inferior performance was observed in a subgroup of 32 patients with mesenteric abscesses and fistulas prepared with neutral or without enteric contrast. In 177/1000 examinations specific pathologic enteric findings were observed with no statistical difference between the three groups.

Conclusion: The preliminary results suggest that positive enteric contrast may be replaced by neutral water in abdominal and pelvic $\mathrm{CT}$, whereas no enteric contrast preparation seems to be inferior. However, positive enteric contrast should be further applied in patients with suspected extramural inflammatory disease.

\section{B-0191 14:09}

Value of oral effervescent powder administration for multidetector

CT evaluation of the oesophagus

K.I. Ringe, S. Meyer, F. Wacker, H.-J. Raatschen

Hannover/DE (ringe.kristina@mh-hannover.de)

Purpose: To assess the value of oral effervescent powder administration for CT evaluation of the oesophagus, quantitatively and qualitatively.

Methods and Materials: 42 patients $(27 \mathrm{~m} / 15 \mathrm{f}$, mean age $57 \mathrm{y})$ referred for thoracoabdominal staging CT were included in this prospective IRB-approved study. Contrast-enhanced imaging was performed on a 64-slice CT-scanner after oral administration of $3 \mathrm{~g}$ effervescent powder immediately before image acquisition. Oesophageal distension was assessed at three levels (proximal/middle/distal) by volumetry of the inner (ID) and outer diameter (OD). Two blinded readers separately evaluated oesophageal distension in the corresponding segments on a three-point scale. At an interval of two weeks, both readers in consensus decided on the number of diagnostic oesophageal segments in each patient in terms of the possibility to decide upon a potentially underlying pathology. Findings were compared with results from a matched control group. Quantitative and qualitative results were compared (T-Test, Mann-Whitney U Test). Inter-observer variability was calculated (weighted-Cohen- $k$ ).

Results: ID and OD were significantly larger in all oesophageal segments after effervescent powder administration as compared to the control group $(p<0.05$; mean ID: 1.13 vs. 0.14 , mean OD: 2.17 vs. 1.26 ). Further, oesophageal distension was rated significantly better, with a good inter-observer variability (mean weighted $\mathrm{K}=0.75)$. At consensus evaluation, 93 oesophageal segments $(74 \%)$ were rated as diagnostic in the effervescent group, versus 49 segments (38\%) in the control group $(p<0.0001)$

Conclusion: Correctly timed oral administration of effervescent powder results in good distension of the oesophagus, allowing readily assessment of the wall at contrast-enhanced CT as compared to studies without effervescent powder. 


\section{B-0192 14:18}

Prospective study of using balloon duodenography catheters in CT enteroclysis for small bowel diseases

K.C.H. Lau, L.M.F. Tee; Hong Kong/HK

Purpose: To determine the usefulness of using balloon duodenography catheters (BDC) for CT enteroclysis (CTE), with a comparison of small bowel distension in using BDC and nasogastric catheters (NC). One of the limitations of CTE is the suboptimal distension of the bowels, especially the distal ileum. Using BDC, there is less reflux of contrast into the stomach and should have more contrast in the distal small bowel.

Methods and Materials: The study protocol was approved by the HA KWC research and ethics committee and all patients gave written informed consent for the examination and this study. From May 2011 to July 2012, CTE was performed in 60 consecutive patients with random allocation of 27 patients using BDC and 33 patients using NC. All catheter insertions and CTE examinations were performed according to department protocol. Small bowel distension was differentiated into four regions (proximal jejunum, distal jejunum, proximal ileum and distal ileum) and given a score from 1 to $3(1-<20 \mathrm{~mm}, 2-20-30 \mathrm{~mm}, 3->30 \mathrm{~mm})$.

Results: Distension of proximal and distal ileum was better with BDC than NC ( $p$ $<0.05$ : statistically significant difference). No significant difference was present for others sites $(p>0.05)$.

Conclusion: Our study shows statistically significant better distension of the proximal and distal ileum, with the use of BDC when compared with NC. This is of particular use, as the distal ileum is a common site of small bowel diseases. With better bowel distension, this would suggest that using BDC would help to further improve the accuracy of CTE.

\section{B-0193 14:27}

Role of preoperative imaging with multidetector computed tomography (MDCT) in the management of patients with gastroesophageal reflux disease (GERD) symptoms, candidate to sleeve surgical revision

M. Rengo, D. Caruso, F. Vecchietti, M.M. Maceroni, G. Silecchia, A. Laghi; Latina/IT (marco.rengo@gmail.com)

Purpose: To evaluate if multidetector computed tomography (MDCT) can be helpful and useful in the decision-making process in sleeve patients with gastroesophageal reflux disease (GERD) symptoms and to demonstrate the reproducibility and accuracy of the technique.

Methods and Materials: Twenty-three patients submitted to LSG, complaining upper Gl symptoms and/or weight regain and scheduled for a sleeve surgical revision were investigated. All patients underwent MDCT scan, upper GI barium swallow study and endoscopy. MDCT was compared with barium and endoscopy features as concern: oesophageal dilatation, neo-fundus development and volume, hiatal hernia, sleeve size in toto and atrum dilatation. All patients underwent laparoscopic sleeve revision. Surgical findings were considered "as gold standard".

Results: A total of 21 patients with hiatal hernia, neo-fundus or sleeve dilatation underwent surgical correction. All findings identified at MDCT were confirmed by intraoperative findings. The presence of hiatal hernia was significantly underestimated by both barium and endoscopy with a sensitivity of $57.1 \%$ and $50 \%$, respectively $(\mathrm{P}=0.04, \mathrm{P}=0.02)$.

Conclusion: MDCT is an accurate method for the detection of hiatal hernias and quantification of gastric volumes and can be considered as non invasive method to guide surgery.

\section{B-0194 14:36}

Accuracy of MDCT in preoperative definition of maximum tumour diameter in patients with gastric cancer

M.A. Mazzei, S. Guerrini, N. Salvini, P. Mercuri, A. Parrinello, N. Cioffi Squitieri, D. Marrelli, F.G. Mazzei, L. Volterrani; Siena/IT (mamazzei@gmail.com)

Purpose: The maximum tumour diameter (Dmax) is a prognostic factor in patients with gastric cancer, considering its dependence on the depth of invasion. The aim of our work has been to evaluate the accuracy of MDCT in the preoperative definition of Dmax in patients with gastric cancer, assuming surgical specimen measurements as gold standard, in order to obtain a pre- surgery prognostic evaluation.

Methods and Materials: Pre-surgery CT examinations of 104 patients (mean age 68.81, range 25-90) with diagnosis of gastric cancer were evaluated retrospectively and in a blind fashion by a radiologist with expertise in the oncologic field. The Dmax measured was obtained through 2D multiplanar curved reconstruction (ADW 4.6 GE Healthcare). The results were compared with macroscopic data after surgery.
Results: The mean value of Dmax obtained by surgical specimen was $57.45 \mathrm{~mm}$ (range 10-250) versus $70.73 \mathrm{~mm}$ (range 16-195.3) of Dmax measured through MDCT. If the Dmax values were stratified into three groups (group 1 smaller than $45 \mathrm{~mm}$, group 2 between 45 and $80 \mathrm{~mm}$, group 3 bigger than $80 \mathrm{~mm}$ ), a correlation with MDCT results of $62.22,65.90$ and $100 \%$, respectively, was found.

Conclusion: MDCT is an accurate technique to obtain an appropriate preoperative definition of Dmax, within the limits of tumour bigger than $45 \mathrm{~mm}$. The revaluation of each case with Dmax smaller than $45 \mathrm{~mm}$ will supply additional information about the discrepancy (retraction of the stomach following immersion into formalin, diffusion in the submucosal layer).

\section{B-0195 14:45}

Percutaneous endoscopic gastrostomy (PEG) and jejunostomy (PEJ) placement guided by CT fluoroscopy with or without simultaneous endoscopy in otherwise untreatable patients C.G. Trumm ${ }^{1}$, R.-T. Hoffmann², R.A. Lang ${ }^{3}$, H. Winter', R. Weidenhagen', K.-W. Jauch ${ }^{1}$, M.F. Reiser ${ }^{1}$, F.W. Spelsberg' ${ }^{1}{ }^{1}$ Munich/DE, ${ }^{2}$ Dresden/DE, ${ }^{3}$ Ottobeuren/DE (christoph.trumm@med.uni-muenchen.de)

Purpose: Percutaneous endoscopic gastrostomy (PEG) or jejunostomy (PEJ) is substantial for patients with swallowing disorders to maintain enteral nutrition or to palliatively decompress intractable small bowel obstruction. Endoscopic placement can be impossible due to previous (gastric) operation, obesity, hepato-splenomegaly, peritoneal carcinosis, inadequate transillumination or obstructed passage. Computed tomography (CT) fluoroscopic guidance with or without endoscopy can enable the placement of a PEG/PEJ if endoscopically guided placement fails. Methods and Materials: 101 consecutive patients were referred to our department for feeding support $(n=87)$ or decompression $(n=14)$. Reasons were: ENT tumour $(n=51)$, oesophageal cancer $(n=19)$, mediastinal mass $(n=2)$, and neurological disorders $(n=15)$. Decompression tubes were placed because of cancer $(n=13)$ or Crohn's disease $(n=1)$. The following approaches were chosen: I. CT fluoroscopy and simultaneous gastroscopy $(n=61)$, II. CT fluoroscopy and inflation of the stomach via nasogastric tube $(n=29)$, and III. direct puncture under CT fluoroscopic guidance $(n=11)$.

Results: CT fluoroscopy-guided PEG/PEJ was feasible in 89 of 101 patients. No procedure-related mortality was observed. One tube was misplaced into the colon in a patient with a history of gastrectomy. No complication was seen after removal. Minor complications were dislodgement $(n=17)$, peristomal leakage $(n=7)$, wound infection $(n=1)$, superficial skin infection $(n=6)$ and tube obstruction $(n=2)$.

Conclusion: CT fluoroscopy-guided PEG/PEJ is feasible and safe, and provides adequate feeding support or decompression. It offers the benefits of minimally invasive therapy even in patients with contraindications to established endoscopic methods, combining the advantages of both techniques. Long-term complications - mainly tube-related problems - are easily treated.

\section{B-0196 14:54}

CT assessment of post-resection arterial stumps for right-sided colorectal cancer: a potential marker of quality of resection?

T.L. Kaye, D.G. Jayne, N.P. West, D.J.M. Tolan; Leeds/UK

(thomas.kaye@leedsth.nhs.uk)

Purpose: There is evidence that the quality and extent of colonic resection affects tumour recurrence rates and patient survival. This is currently evaluated by pathological analysis of the resected specimen. Analysis of post-resection arterial stumps via routine follow-up CT could act an alternative in vivo marker.

Methods and Materials: Staging and follow-up CT scans from 156 patients with right-sided colorectal tumours were analysed. Pre-operative arterial anatomy and post-operative arterial stumps were demonstrated using multi-planar reformats and volume rendering techniques. Methods of stump identification, stump length and stump orientation were recorded.

Results: Ileocolic $(98.7 \%)$, middle colic $(94.2 \%)$ and right colic artery $(23.7 \%)$ identification was comparable with catheter angiogram studies. Mean time interval between staging and analysed follow-up CT was 25 months. Post-resection ileocolic stumps were consistently identified ( $88.4 \%$ of cases). In the vast majority of cases this was via a thrombosed vessel $(97 \%)$. There was a wide variation in ileocolic stump length (2.5 - $74.3 \mathrm{~mm}$, mean $27.6 \mathrm{~mm}$, standard deviation $13.5 \mathrm{~mm}$ ). Conclusion: To the best of our knowledge this is the largest study to date demonstrating arterial stumps post colonic cancer resection. It is the first to consistently identify stumps years after colonic resection using routine surveillance (portal venous) CT. CT-based stump length analysis may therefore have potential as an in vivo marker of extent and quality of surgical resection. 


\section{B-0197 15:03}

Evaluation of Crohn's disease recurrence in patients with ileocolic anastomosis: value of computed tomography enterography with water enema

F. Paparo, M. Revelli, C. Puppo, I. Garello, L. Bacigalupo,

L. Rollandi, R. Piccazzo, A. Garlaschi, G.A. Rollandi; Genoa/lT

(francesco.paparo@galliera.it)

Purpose: To investigate the value of computed tomography enterography with water enema (CTE-WE) in the assessment of the status of the anastomotic site in patients with Crohn's disease $(C D)$ who had previously undergone ileocolic resection. Methods and Materials: 51 CTE-WE examinations of CD patients (mean age: $52.8 \pm 13.3$ years; 26 [51\%] men and 25 women [49\%]) with ileocolic anastomosis (latero-lateral $=10[19.61 \%]$, termino-lateral $=27[52.94 \%]$, termino-terminal $=14$ [27.45\%]) were retrieved from our institutional radiology database, and reviewed in consensus by two gastrointestinal radiologists. The presence of $C D$ anastomotic recurrence and its phenotype (inflammatory, stricturing, penetrating), and the presence of inflammatory lesions involving other bowel segments beside the anastomotic site were recorded. Endoscopy and medical records were used as reference standards.

Results: CT signs of CD recurrence were detected in 45 patients, including: 32 (71.12\%) isolated anastomotic recurrences, $11(24.44 \%)$ anastomotic recurrences + synchronous inflammatory lesions (SILs), 2 (4.44\%) inflammatory lesions not involving the anastomotic site. Phenotypes of the $43 \mathrm{CD}$ anastomotic recurrences were: $22(51.1 \%)$ inflammatory (non-stricturing non-penetrating), 16 (37.2\%) stricturing, $4(9.3 \%)$ penetrating. No relation was found between the phenotype of $C D$ recurrence and the type of the anastomosis (LL, TL, TT) $(P=0.1252$; Chi-square test). Conclusion: CTe-WE allowed a complete characterisation of the anastomotic site and the detection of SILs, which had a relatively high prevalence in our study cohort $(24.44 \%)$

\section{B-0198 15:12}

Crohn's disease activity: correlation of inflammatory mediators with overall small-bowel motility

S. Bickelhaupt ${ }^{1}$, S. Pazahr ${ }^{1}$, J.M. Froehlich ${ }^{1}$, R. Cattin², H. Bouquet ${ }^{3}$, G. Rogler ${ }^{1}$, P. Frei', A. Boss', M. Patak'; ${ }^{1}$ Zurich $/ \mathrm{CH},{ }^{2} \mathrm{Biel} / \mathrm{CH},{ }^{3} \mathrm{Berne} / \mathrm{CH}$

Purpose: Active Crohn's disease (CD) increases the level of inflammatory markers of which C-reactive protein (CRP) and calprotectin are commonly used to monitor disease activity. The aim was to evaluate the correlation between CRP and calprotectin levels and overall small bowel motility in patients with Crohn's disease assessed with MRI.

Methods and Materials: 13 patients with Crohn's disease (4f/9m, mean 42y) were included in this IRB-approved prospective study. MRI (1.5-T, Philips Achieva) was performed after a 1 -h preparation of $1000 \mathrm{ml}$ Mannitol-Solution (3\%). Cine T2w-2D-SSFP motility acquisitions (TR 2.47/TE 1.23/250 ms slice repetition time) were performed in free breathing over 69-84sec. Randomly chosen small-bowel segments were analysed in two abdominal quadrants using dedicated MR-motility assessment software (Motasso). Contraction frequency, amplitude, luminal diameter and amplitude diameter ratio (occlusion ratio, ADR) were evaluated as well as CRP ( $\mathrm{ng} / \mathrm{ul}$ ) and Calprotectin (ug/g) levels. Pearson's correlation was calculated. Results: Calprotectin was determined in mean 12 days $(\mathrm{SEM} \pm 10.09)$ before, CRP 15 days $(S D \pm 28.80)$ before MRI. A significant inverse linear correlation was found between the contraction frequency and both the level of CRP $(r=-0.701, p=0.008)$ and calprotectin $(r=-0.805, p=0.001)$. Expansion of the mean small bowel diameter significantly correlated with calprotectin levels $(r=0.857, p=<0.001)$ but not with CRP ( $r=0.447, p=0.126)$. The absolute amplitude of the contractions did not correlate neither with the level of CRP $(r=-0.527, p=0.064)$ nor with calprotectin $(r=-0.612, p=0.026)$. The ratio describing relative luminal occlusion during contraction (ADR) significantly correlated with calprotectin $(r=0.736, p=0.004)$ and with CRP $(r=0.577, p=0.039)$.

Conclusion: Alterations of overall small bowel motility during active phases of $C D$ significantly correlate with the level of calprotectin and CRP.

Author Disclosures:

H. Bouquet: Employee; Fromer Employee of SOHARD AG.
B-0199 15:21

Pilot study to assess the diagnostic performance of MRI in the identification of adhesions between the abdominal wall and small bowel loops, using a time-efficient protocol

A. Gupta, A. Hansmann, P.F.C. Lung, R. Tandon, R. llangovan, M. Marshall; Middlesex/UK (anikahansmann@nhs.net)

Purpose: Abdominal adhesions following surgery are the commonest cause of small bowel obstruction and may complicate subsequent surgery. Accurate identification of adhesions has been reported using MR fluoroscopy sequences. We report our results utilising a time-efficient protocol.

Methods and Materials: 10 patients scheduled for open colorectal surgery and who had undergone previous abdominal surgery were recruited over 4 months (mean age 44, range 29-59). The abdomen was divided into 9 segments prior to MR and surgery enabling accurate correlation. Using a 1.5 T scanner (SiemensAvanto), T2 sagittal, axial, sagittal true-FISP 30 s cine sequences were obtained (slice thickness $1 \mathrm{~cm}$ ), in inspiration, expiration and abdominal strain. Scanning time was 8 min. Adhesions were identified by finding angulation, fixation or altered peristalsis. 4 readers analysed the images independently, providing a consensus opinion on the number and location of adhesions. Laparotomy was the gold standard.

Results: 90 abdominal segments were evaluated with adhesions identified in $44 \%$ at laparotomy and $34 \%$ on consensus MR. 31 segments demonstrated adhesions on MR; $74 \%$ correlated at surgery, whilst the remainder were found in adjacent segments (26\%). Using a $2 \times 2$ table, sensitivity was $60 \%$, specificity $86 \%$, with positive and negative predictive values of $77 \%$ and $73 \%$, respectively. Overall accuracy was $74 \%$.

Conclusion: Our results suggest utilisation of a time-efficient MR protocol in identifying adhesions between the bowel and anterior abdominal wall is feasible in a busy unit, and may represent a non-invasive application in the research of adhesion-preventative techniques. Reader performance rose during our study and further improvements may be realised in a larger trial.

14:00 - 15:30 Room F1

Oncologic Imaging

\section{SS 216}

\section{New biomarkers for tumour quantification}

Moderators:

C.J. Herold; Vienna/AT

C. Keyzer; Brussels/BE

\section{B-0200 14:00}

3D CT-histogram analysis enables distinguishing affected and FDGnegative lymph nodes in patients with lung cancer

P. Flechsig ${ }^{1}$, C. Kratochwil' ${ }^{1}$, J. Moltz², C.-P. Heussel', R. Talanow ${ }^{1}$, H.-U. Kauczor', U. Haberkorn', F.L. Giesel ${ }^{1} ;{ }^{1}$ Heidelberg/DE, ${ }^{2}$ Bremen/DE

Purpose: Patients with lung cancer often suffer additional chronic lung diseases, and often these patients present with FDG avid mediastinal lymph nodes due to inflammatory reasons rather than malignancy. FDG-PET is the leading imaging modality for $\mathrm{N}$-staging in lung cancer patients; however, in patients with doubtful FDG-PET status, 3D CT histogram analysis might give an additional value to diagnostic imaging. In this investigation $3 \mathrm{D}$ CT histogram analysis was used to identify metastatic lymph node involvement in lung cancer patients in correlation to FDG-PET/CT.

Methods and Materials: 38 lymph nodes from 32 patients aged 43-76 years diagnosed with lung cancer were investigated. FDG-PET/CT was performed prior to surgery/biopsy according to clinical schedule. Lymph node assessments were acquired using FDG-uptake and 3D CT histogram analysis on the basis of non-enhanced CT scans. For computing the histograms, the lymph nodes were segmented by a semi-automatic algorithm; the segmentation result was corrected manually if required in certain cases. Both imaging findings were correlated with the results from the histological gold standard study.

Results: 21 positive and 17 negative histologically proven lymph nodes were successfully analysed by $3 \mathrm{D}$ CT histogram. The histological positive lymph nodes presented a median CT HU value of 34.5 while histological negative lymph nodes presented a median CT HU value of 7.6. Findings were independent of the FDGuptake value.

Conclusion: $3 \mathrm{D}$ histogram analysis seems to be a very promising and valuable imaging surrogate for $\mathrm{N}$-staging stratification in patients with lung cancer with doubtful glucose uptake in FDG-PET. 


\section{B-0201 14:09}

Tumour vascularization imaging without contrast agents: the potential of IVIM-MRI

M. lima' ${ }^{1}$, O. Reynaud², T. Tsurugizawa ${ }^{2}$, L. Ciobanu², J.-R. Li², F. Geffroy², B. Djemai ${ }^{2}$, D. Le Bihan'; ${ }^{1}$ Kyoto/JP, ${ }^{2}$ Gif-sur-Yvette/FR (mamiiima1@gmail.com)

Purpose: To investigate the potential of IVIM MRI to evaluate perfusion and diffusion in a 9L glioma rat brain tumour model using a 17.2T MRI scanner.

Methods and Materials: 14 fischer rats were studied. IVIM MRI images were acquired with $72 \mathrm{~b}$ values ( $25 \mathrm{~b}$ values; 2 to $160 \mathrm{~s} / \mathrm{mm}^{2}, 35 \mathrm{~b}$ values; 172.5 to 935 $\mathrm{s} / \mathrm{mm}^{2}$, and $12 \mathrm{~b}$ values; 1150 to $3025 \mathrm{~s} / \mathrm{mm}^{2}$ ). The signal attenuation curve was first fitted using a biexponential diffusion model to estimate slow and fast components fraction (fslow, Dfast and Dslow) of the pure diffusion part of the signal (for $b>500 \mathrm{~s} / \mathrm{mm}^{2}$ ). Then, the diffusion component was removed from the signal and the remaining signal was fitted using the IVIM model for $b<200 \mathrm{~s} / \mathrm{mm}^{2}$ to get estimates of perfusion fraction, $\mathrm{f}$, and pseudo-diffusion, $\mathrm{D}^{*}$. Fitting was performed on a ROI basis and on a pixel-by-pixel basis to generate diffusion and perfusion maps. All rats were killed and histology (CD31) was obtained for comparison with IVIM parameters.

Results: IVIM maps clearly highlighted areas with high and low fraction perfusion within tumours which were generally heterogeneous, as confirmed by histology. Interestingly, we found a negative correlation between the IVIM perfusion fraction and fslow, suggesting that cell density in high perfused areas is less than in other tumour parts and normal brain tissue.

Conclusion: This study confirms that IVIM MRI can delineate high perfusion areas in tumours and provide information on tissue structure from diffusion parameters.

\section{B-0202 14:18}

Intravoxel incoherent motion (IVIM) diffusion-weighted MRI for monitoring the therapeutic efficacy of a vascular disrupting agent (CKD-516) in rabbit VX2 liver tumours

I. Joo, J. Lee, J. Han, B. Choi; Seoul/KR

Purpose: To evaluate the diagnostic value of intravoxel incoherent motion (IVIM) diffusion-weighted imaging (DWI) in the quantitative assessment of therapeutic efficacy of a vascular disrupting agent (VDA) (CKD-516) in rabbit VX2 liver tumours. Methods and Materials: In 29 VX2 liver tumour-bearing rabbits (23 in the treated group and 6 in the control group), IVIM-DWIs were performed at a 3 T scanner using $12 \mathrm{~b}$ values before and 4 hours, 24 hours, 3 days, and 7 days after CKD-516 administration. Apparent diffusion coefficient (ADC) and true diffusion coefficient (D), pseudo-diffusion coefficient $\left(D^{*}\right)$, and perfusion fraction (f) of tumours were compared between the control and treated groups and among time points. Correlation between change in tumour size and IVIM-DWI parameters was analysed to determine which IVIM-DWI parameters can be predictors for tumour response. Results: The treated group showed a significantly larger increase in ADC at 24 hours, a decrease of $D^{*}$ at 4 hours, and a decrease of $f$ at 4 hours and 24 hours, than did the control group $(P<0.05)$. In the treated group, compared to baseline values, $D^{*}$ and $f$ significantly decreased at 4 hours and then recovered at 24 hours and $D$ significantly increased at 24 hours $(P<0.005)$. In addition, the greater decrease in $f$ at 4 hours correlated with the smaller increase in tumour size during the 7 days $(\mathrm{rho}=0.53, \mathrm{P}=0.04)$.

Conclusion: The therapeutic effect induced by VDA could be effectively evaluated using IVIM-DWI, and $f$ could be an early predictive indicator for tumour response.

Author Disclosures:

I. Joo: Research/Grant Support; The current study was supported by Grants by Chong Keun Dang Corp., Seoul, Korea. J. Lee: Research/Grant Support; The current study was supported by Grants by Chong Keun Dang Corp., Seoul, Korea.

\section{B-0203 14:27}

Texture analysis on contrast-enhanced computed tomography combined with FDG-PET in predicting the response to chemotherapy of advanced non-small cell lung cancer

M. Ravanelli, F. Ferraroni, D. Farina, M. Morassi, P. Tessitore, P. Rossini,

R. Maroldi; Brescia/IT (marcoravanelli@hotmail.it)

Purpose: To assess whether tumour heterogeneity, quantified by texture analysis on contrast-enhanced CT (CECT), combined with FDG uptake at PET-CT can predict the response to chemotherapy in advanced non-small cell lung cancer (NSCLC). Methods and Materials: Pre-treatment CECT studies and FDG-PET studies of twenty-six patients with advanced NSCLC who had undergone first-line chemotherapy were retrospectively reviewed and analysed. Tumour heterogeneity was measured by texture analysis on CECT images; mean SUV from each patient was measured on FDG-PET. Treatment response was evaluated after three cycles of platinum-based chemotherapy, according to RECIST. The predictive value of texture and PET parameters have been investigated by multivariate analysis.

Results: Fourteen out of 25 patients were non-responders (4 progressors, 10 stable disease). Combining heterogeneity and SUV in a unique parameter (heterogeneity*SUV), the latter was significantly higher in responders than in non-responders (medians 192.7 vs 92.8 , respectively; $0<0.0001$ ) and showed a high predictive value: odds ratio (probability of response) of third vs first tertile was 49 (p0.003). Responses in first tertile were $1 / 8$, in the second $3 / 9$, in the third $7 / 8$ ( $\mathrm{p} 0.007, \mathrm{p}$ for trend 0.003 ). Tumour heterogeneity alone showed a significantly weaker predictive value, while SUV alone was not predictive of treatment response. Conclusion: The combination of texture features of tumour heterogeneity on CECT and the metabolic information provided by FDG-PET has the potential to be an independent predictor of response to first-line chemotherapy in advanced NSCLC. In particular, tumour SUV combined with heterogeneity provides an additional value compared with tumor heterogeneity alone.

\section{B-0204 14:36}

Texture analysis of advanced non-small cell lung cancer on contrastenhanced computed tomography: prediction of the response to the first-line chemotherapy

M. Ravanelli, M. Morassi, D. Farina, E. Roca, R. Maroldi; Brescia/IT

(marcoravanelli@hotmail.it)

Purpose: To assess whether tumour heterogeneity, quantified by texture analysis on contrast-enhanced CT (CECT), can predict the response to chemotherapy in advanced non-small cell lung cancer (NSCLC).

Methods and Materials: Fifty-three pre-treatment CECT studies of patients with advanced NSCLC who had undergone first-line chemotherapy were retrospectively reviewed and analysed. Treatment response was evaluated after three cycles of platinum-based chemotherapy, according to RECIST. Tumour uniformity on CT images has been assessed by a three-step method: image thresholding; multiscale filtering by Laplacian-of-Gaussian filter; quantification of texture features on filtered images using first-order statistics. The predictive and prognostic values of resulting texture parameters have been investigated by multivariate analysis.

Results: Thirty-one out of 53 patients were non-responders ( 7 progressors, 24 with stable disease). Average overall survival was 13 months (4-35), minimum follow-up was 12 months. In the adenocarcinoma group ( $n=31)$, the tumour uniformity multiplied by the grey-level of unfiltered images (GL*uni index) has been shown to be the unique independent variable predictive of treatment response. Dividing the GL*uni index (8.5-46.6) into tertiles, lesions in the second and the third tertiles had a 8-fold higher probability of treatment response compared to those in the first (p0.02). TNM stage, sex, age and performance status did not show predictive value. No association between texture features and response to treatment was observed in the non-adenocarcinoma group. GL*uni index was not prognostic. Conclusion: Texture features of tumour heterogeneity on CECT images in advanced lung adenocarcinoma has the potential to be a predictive indicator of response to platinum-based chemotherapy.

\section{B-0205 14:45}

Textural analysis of lymphoma on unenhanced computed tomography: initial evidence for a relationship with tumour glucose metabolism, stage, end of treatment status and survival S. Babikir, B. Ganeshan, A.M. Groves, I. Kayani; London/UK (b.ganeshan@ucl.ac.uk)

Purpose: To provide initial evidence in lymphoma for a relationship between tumour heterogeneity, as assessed by CT texture analysis (CTTA), and tumour glucose metabolism, stage, end of treatment status (EOTS) and survival.

Methods and Materials: This retrospective, pilot study comprised 50 consenting lymphoma patients with consecutive routine 18 F-fluorodeoxyglucose (FDG) PET-CT scans. Texture analysis using TexRAD, (a commercially available texture analysis software), of non-contrast-enhanced CT images with lesions involved: 1) image filtration extracting fine, medium and coarse texture-scales, and 2) texture quantification using proportion positive pixels (PPP), standard-deviation (SD), and kurtosis (K). Corresponding tumour FDG uptake (standardised uptake value (SUVavg and SUVmax)), clinical-stage and EOTS from PET-CT and clinical reports were collected. Survival was assessed by optimised Kaplan-Meier analysis. Results: 7, 19, 9 and 15 patients had stages I, II, III and IV, respectively. Fine to coarse textures correlated with SUVavg (baseline-scan: SD at coarse-scale, $r s=0.362 p=0.011$; interim-scan: PPP at medium-scale, $r s=-0.766 p=0.0001)$ and clinical-stage (baseline-scan: $K$ at fine-scale, $r s=0.424 p=0.002$ ). Baseline coarse textures predicted EOTS (PPP at coarse-scale, $p=0.006 ; \mathrm{K}$ at coarse scale, $p=$ 
0.032). Median (range) overall and disease-free survival was 34 (8-52) and 31 (356) months, respectively. Medium texture-scale predicted overall (baseline-scan: $\mathrm{K}, \mathrm{p}=0.0002$, interim-scan: $\mathrm{K}, \mathrm{p}=0.0008$ ) and disease-free survival (baseline-scan: $\mathrm{K}, \mathrm{p}=0.0031$, interim-scan: $\mathrm{K}, \mathrm{p}=0.046)$. SUVmax predicted disease-free survival (baseline-scan: $p=0.024)$. Clinical-stage did not predict EOTS and survival. Conclusion: Evidence provided suggests that CTTA offers an objective measurement of lymphoma severity on CT. The potential risk stratification and prognostic assessment implications may allow optimal selection of PET-CT candidates in lymphoma.

Author Disclosures:

B. Ganeshan: Shareholder; Shareholder and director of TexRAD Ltd, an imaging software company that is developing and commercialising the texture analysis software considered in this study.

\section{B-0206 14:54}

Improvement in both sensitivity and specificity of readers with next generation of mammography CAD

V. Nikitin, I. Lossev, A. Filatov, N. Bagotskaya; Longmont, CO/US

(vadim.nikitin@parascript.com)

Purpose: The purpose of the study was to compare radiologist performance for screening mammography with and without Parascript ${ }^{\circledR}$ AccuDetect ${ }^{\circledR}$ computeraided detection (CAD) software.

Methods and Materials: Each of 240 mammography cases (including 120 cancer cases and 120 normal cases) was reviewed by 12 radiologists without and with CAD (AccuDetect, version 6.1). The mammography cases were obtained with GE Senographe Essential and Philips MicroDose Mammography L30 full-field digital mammography systems. Each radiologist initially interpreted a case without CAD noted the findings and assigned BI-RADS category 0,1 or 2 to the case. For each radiologist, the unassisted interpretation was "locked" before the radiologist turned on the CAD marks. After turning on the CAD marks, the radiologist could add, remove or adjust the findings noted on the unassisted interpretation as well as change the BI-RADS score for the case. Sensitivity and specificity calculations for unassisted and CAD-assisted interpretations were based on the BI-RADS category assignment dichotomization: (BI-RADS $=0$ ) versus (BI-RADS $=1$ or 2 ). Results: Average increase in sensitivity due to assistance of CAD is $1.5 \%$, which translates into $18.8 \%$ of those initially missed cancers that were recognised with CAD assistance. Average increase in specificity due to assistance of CAD is $4.9 \%$. Both increases in sensitivity and specificity are statistically significant

Conclusion: The study demonstrates that both sensitivity and specificity of radiologists increased with assistance of AccuDetect CAD system.

Author Disclosures:

V. Nikitin: Employee; Parascript. I. Lossev: Employee; Parascript. A. Filatov: Employee; Parascript. N. Bagotskaya: Employee; Parascript.

\section{B-0207 15:03}

\section{Assessing the contribution of hypoxia to R2* differences between} cancerous and normal prostate tissue

A. Johnson, A. Latifoltojar, V. Hamy, H. Fitzke, K. Shmueli, S. Punwani; London/UK (aj372@cam.ac.uk)

Purpose: R2* BOLD MRI is proposed as a potential tool for mapping tumour hypoxia in prostate cancer. However, $\mathrm{R} 2^{*}$ has a complex relationship with tissue oxygenation that remains unexplored in prostate cancer. $R 2^{*}$ is the sum of $\mathrm{R} 2$ (primarily reflecting tissue structure) and R2' (highly sensitive to static magnetic field inhomogeneities, e.g. from deoxyhaemoglobin). Whilst R2* changes are observed for prostate cancer, they may reflect tissue structure or oxygenation differences. This study aimed to clarify the nature of $\mathrm{R} 2^{*}$ change by quantifying and comparing R2* and its components (R2 and R2') for cancerous and normal prostate tissue. Methods and Materials: Twenty patients underwent $1.5 \mathrm{~T}$ multi-parametric $(\mathrm{mp})$ prostate MRI (DWI, DCE and T2-weighted), supplemented by dual echo-time T2* and T2 quantitation and followed by biopsy. Tumour and healthy regions of interest (identified on mp-MRI and confirmed by biopsy) were contoured in consensus by two experienced observers on matched T2- and T2*-weighted images. Mean $\mathrm{R} 2{ }^{*}, \mathrm{R} 2$ and $\mathrm{R} 2$ ' were calculated and compared between cancerous and normal tissue using the student's t-test.

Results: R2* and R2 were significantly greater for cancer than normal tissue (R2*: $18 \pm 5 s-1$ [mean $\pm S D$ ] vs. $13 \pm 7 s-1$ respectively, $p=0.0130 ; R 2: 11 \pm 2 s-1$ vs. $6 \pm 2 \mathrm{~s}-1$ respectively, $\mathrm{p}<0.0001)$. No significant difference was found in R2' $(7 \pm$ $4 \mathrm{~s}-1$ vs. $7 \pm 7 \mathrm{~s}-1$ respectively, $\mathrm{p}=0.5597$ ).

Conclusion: Prostate tumour R2* values predominantly reflect differences in R2 (tissue structure) and not R2' (tissue hypoxia).

\section{B-0208 15:12}

Scatter amplitude is a good landmark for tumour localisation and treatment assessment in time-domain diffuse optical tomography during neo-adjuvant chemotherapy in breast cancer

M. Van de Giessen, B.E. Schaafsma, J.R. Kroep, M.N.J.M. Wasser, C.J.H. Van de Velde, B.P.F. Lelieveldt, J. Dijkstra, A.L. Vahrmeijer; Leiden/NL (m.vandegiessen@lumc.nl)

Purpose: Neo-adjuvant chemotherapy response may be monitored in a noninvasive way using multi-spectral diffuse optical tomography (DOT). A clinically feasible measurement method in these $3 \mathrm{D}$ volumes is proposed and compared with previously published whole breast measurements.

Methods and Materials: Twelve breast cancer patients were included, which received neo-adjuvant chemotherapy. Four DOT scans were obtained: before therapy, after 3 and 9 weeks and pre-operative (18 weeks). Deoxyhaemoglobin $(\mathrm{Hb})$, oxyhaemoglobin $(\mathrm{HbO} 2)$ and percentages water and lipids were measured in volumes as measured in DCE-MRI scans before therapy, centred on areas of high scattering amplitude and approximate location in pre-treatment DCE-MRI and/ or x-ray mammography. Patients were divided in responders and poor responders based on post-operative pathological assessment.

Results: Responders (R) and poor responders (PR) showed statistically significant $(\mathrm{P}<0.05)$ different development of $\mathrm{Hb}$ values at 3,9 and 18 weeks within measurement volumes relative to the first DOT scan: 0.66/1.08 (R/PR, 3 weeks), 0.56/1.07 (9 weeks), 0.55/1.48 (18 weeks). For $\mathrm{HbO} 2$ results were similar, but only significant for 9 and 18 weeks: $0.71 / 1.03$ ( 3 weeks), 0.53/0.89 ( 9 weeks), 0.56/1.06 (18 weeks). Percentages water and lipids were not significantly different. Whole breast measurements did not show statistically significant differences for $\mathrm{Hb}$ and $\mathrm{HbO} 2$ between responders and poor responders.

Conclusion: Using the scatter amplitude to determine the tumour location in DOT volumes enables the measurement of $\mathrm{Hb}$ and $\mathrm{HbO} 2$ such that these parameters differ significantly between responders and poor responders. This potentially allows effectiveness assessment of neo-adjuvant treatment in breast cancer patients already at 3 weeks.

\section{B-0209 15:21}

Software validation of metastatic sarcoma lesion assessment using CT volumetric density tumour trajectory

L.R. Folio ${ }^{1}$, V.M. Derderian ${ }^{1}$, E.C. Jones ${ }^{1}$, M.S. Merchant ${ }^{1}$, A.M. Venkatesan ${ }^{1}$ E. Lotan'; ${ }^{1}$ Bethesda, MD/US, ${ }^{2}$ Tel Hashomer/IL (vana.derderian@nih.gov)

Purpose: Recent studies suggest that tumour assessment methods accounting for both tumour density and volume (SACT, MASS, Choi) add value beyond assessing axial dimensions (RECIST) or selective regions of interest (ROI). We have developed a comprehensive tumour response method termed Total Volume of Viable Tumour (TVVT) that assesses rate of change in Hounsfield unit (HU) density over treatment course (tumour trajectory), thereby quantifying viable tumour burden as a Volume of Interest (VOI). Steeper trajectories may translate to earlier determination of response versus progression.

Methods and Materials: We evaluated the efficacy of experimental volume/HU (TVVT) software and criteria within our Picture Archiving and Communication System (Carestream Health, Rochester, NY) to circumscribe, segment and characterise volumetric density distribution throughout all parts of measurable lesions on CT. Comparison of response and tumour trajectory assessment between RECIST criteria and TVVT was performed in a test set of 10 patients with various sarcomas under radiologist supervision. Histograms depicting range and absolute $\mathrm{HU}$ for whole tumor volumes were analysed on a subset of patients.

Results: Preliminary investigation shows feasibility of semi-automatic segmentation to outline and quantify density distribution in whole tumour volumes. Initial assessment of our set of metastatic lesions suggests that hypervascular sarcomas are a worthwhile study cohort. Histogram shifts appear to correlate with qualitative visual estimates of tumor necrosis whereas RECIST diameters demonstrate little or no differences.

Conclusion: Comprehensive tumour analysis using volumetric density algorithms such as TVVT should more accurately reflect tumour burden and trajectory over selective linear or ROI measurements, leading to improved image-based tumour staging and response assessment. Semi-automated quantification of whole tumour burden should decrease inter-observer measurement variability, enabling more convenient, reliable imaging results for multiple contiguous vascular metastases. 


\section{Breast}

\section{SS 202}

\section{Improvements in preoperative staging of breast \\ cancer}

Moderators:

R.A. Kubik-Huch; Baden/CH

A. Zytoon; Shebin El-Kom/EG

\section{B-0210 14:00}

Usefulness of ultrasonography (US) and ultrasonography-guided fine-needle aspiration biopsy (FNAB) for axillary staging in breast cancer: is the breast imaging reporting and data system (BI-RADS) categorisation applicable?

H. Ahn, S. Kim, B. Yun, M. Kim, M. Jang, S. Park, S.-W. Kim, E. Kang; Gyeonggi-do/KR (ach0224@gmail.com)

Purpose: To evaluate accuracy of US and US-guided-FNAB for diagnosing axillary metastasis in breast carcinoma patients and to determine applicability of the BI-RADS categorisation.

Methods and Materials: We retrospectively reviewed data on 858 patients who underwent preoperative or prechemotherapy axillary US for axillary staging from January 2009 to December 2011. US-guided FNAB was performed if lymph nodes showed any suspicious findings according to the BI-RADS categorisation: even cortical thickening with preserved hilum, category $4 \mathrm{a}(\mathrm{C} 4 \mathrm{a})$; even cortical thickening with compressed hilum or uneven cortical thickening with preserved hilum, C4b; uneven cortical thickening with compressed hilum, C4c; hypoechoic mass with loss of hilum, C5. US and FNAB findings were compared using sentinel lymph node biopsy (SLNB) and axillary lymph node dissection (ALND) data.

Results: Of 858 patients, $212(24.7 \%)$ showed suspicious nodes on US and underwent US-guided FNAB. Of these 212 patients, 91 (42.9\%) showed positive, $112(52.8 \%)$ showed negative, and 9 showed insufficient findings on FNAB. Of 858 patients, 767 patients either with normal axillary US $(n=646)$ or without positive findings on FNAB $(n=121)$ underwent SLNB. Of these patients, $169(22 \%)$ had metastatic lymph node. Of 91 patients with positive FNAB findings, 57 underwent neoadjuvant chemotherapy and 34 underwent ALND. The sensitivity, specificity, PPV and NPV of US were $47.6 \%, 86 \%, 60.4 \%$ and $79.7 \%$, respectively. The PPV of US was $26.3 \%$ in C4a, $67.4 \%$ in C4b, $46.4 \%$ in C4c, $93.3 \%$ in C5. The sensitivity, specificity, PPV and NPV of US-guided-FNAB were $71.7 \%, 100 \%, 100 \%$ and $70.2 \%$, respectively.

Conclusion: Axillary US showed high specificity and NPV. A categorisation similar to BI-RADS could be applicable for assessing axillary metastasis in breast cancer patients.

\section{B-0211 14:09}

Improving diagnostic yield in axillary lymphadenopathy sampling: core biopsy and vacuum-assisted core biopsy

R. Salvador, X. Salvador, I. Miranda, O. Dominguez, L. Romero; Barcelona/ES (rafasalvador@telefonica.net)

Purpose: To analyse and compare the tolerance of the method and the results obtained by sampling axillary lymph nodes with two different devices: large-needle (CB) and vacuum-assisted core biopsy (VACB), directed to patients where previous fine needle aspiration (FNAB) has failed to obtain material, or those with different cancers to determine the origin of the metastasic node.

Methods and Materials: 22 consecutive patients, suspicious or proved to have breast cancer were included, all of them with enlarged lymph nodes and signed the informed consent. The two groups were alternatively sampled by large needle (14G) core biopsy and vacuum-assisted needle (14G) biopsy to obtain 2 cylinders in each procedure. Eleven patients in each group. The size of the sample (weight), as well as the time of the procedure from the injection of anaesthetic to the final collection of the 2 samples was recorded. Discomfort, as well as the acceptance of the method was also recorded and scored in a 4-grade scale.

Results: The size of the samples obtained by vacuum-assisted method was bigger than that by core-needle (between twice and three times bigger). But the time for both procedures was similar (always 4-5 minutes). No differences were found in pain or any discomfort and tolerance of the method.

Conclusion: Vacuum-assisted axillary lymph node biopsy is a procedure to obtain samples at least as safely as with core-needle biopsy and deserves bigger speci- men for diagnosis, especially when receptors have to be studied in defining the origin of axillary lymph node metastases.

Author Disclosures:

R. Salvador: Speaker; Member of the medical Advisoy Board of Philips Healthcare.

\section{B-0212 14:18}

Gadofosveset-enhanced axillary MRI for nodal staging in breast cancer R. Schipper, M.B.I. Lobbes, L.M. van Roozendaal, C.J.G. Castro, B. de Vries, E.M. Heuts, K.B.M.I. Keymeulen, M.L. Smidt, R.G.H. Beets-Tan; Maastricht/NL (marc.lobbes@mumc.nl)

Purpose: Aim of this study was to determine the diagnostic performance of gadofosveset-enhanced MRI for axillary lymph node staging in breast cancer patients. Methods and Materials: Ten women diagnosed with invasive breast cancer underwent both non-enhanced and gadofosveset-enhanced 3DT1-weighted MRI. A radiologist, blinded for clinical data, evaluated all lymph nodes with regard to size, morphologic features and gadofosveset uptake. Lymph nodes depicted on MRI were matched with nodes removed during surgery. Nodal status was investigated by a blinded pathologist. Sensitivity, specificity, PPV, and NPV values were calculated. Results: A total of 152 lymph nodes were extracted during sentinel lymph node biopsy or axillary lymph node dissection of which 116 were matched with lymph nodes depicted on MRI. Histopathological examination resulted in 21 macro-metastases and 8 micro-metastases. With contrast-enhanced MRI, 20 lymph nodes were rated as true positive, 83 as true-negative, 4 as false positive, and 9 as false negative. This resulted in an overall node-by-node sensitivity, specificity, PPV and NPV of, respectively, 67\% (95\% Cl 49-80), 95\% (95\% Cl 89-98), 83\% (95\% Cl 64-93) and $89 \%(95 \% \mathrm{Cl} 81-94)$. If ignoring micro-metastases, MRI showed a sensitivity of $87 \%(95 \% \mathrm{Cl} 68-96)$ and a specificity of $96 \%(95 \% \mathrm{Cl} 89-98)$. Calculated PPV and NPV were $83 \%(95 \% \mathrm{Cl} 64-93)$ and $97 \%$ (95\% Cl 90-99), respectively.

Conclusion: This feasibility study concerning gadofosveset-enhanced MRI of axillary lymph nodes showed promising initial results. These results warrant larger studies to confirm these promising results.

\section{B-0213 14:27}

Assessment of 18 Fluorodesoxyglucose positron emission tomography (18 FDG PET-CT) vs contrast-enhanced magnetic resonance imaging at 3 Tesla ( 3 T CE-MRI) for lymph node staging in breast cancer patients

H. Magometschnigg, H. Bickel, G.J. Wengert, G. Karanikas, P. Brader, K. Pinker-Domenig; Vienna/AT

Purpose: To assess sensitivity and specificity of prone breast ${ }^{18} \mathrm{FDG}-\mathrm{PET}-\mathrm{CT}$ for detection of lymph node metastases and to compare it with 3 T CE-MRI of the breast. Methods and Materials: 74 patients with breast cancer were included in this IRB-approved prospective study. All patients underwent 3 T CE-MRI and ${ }^{18} \mathrm{FDG}$ PET-CT of the breast in the prone position. Patients were injected with ca. 300 $\mathrm{MBq}{ }^{18} \mathrm{~F}$-FDG. CT data were only used for attenuation correction. In MRI lymph nodes were considered positive for malignancy if there was enlargement $>1 \mathrm{~cm}$, thickened cortex, loss of fatty hilum, matted nodes or an irregular node contour. In PET-CT a lymph node was classified as positive when ${ }^{18} \mathrm{FDG}$-uptake was greater than blood-pool activity. The evaluation of nodes was performed on a patient-bypatient basis. All nodes were histopathologically verified.

Results: ${ }^{18}$ FDG-PET-CT achieved a sensitivity of $79 \%$ and a specificity of $98 \%$. PPV was 0.95 ( $\mathrm{Cl}$ 0.76-0.99), and NPV was 0.91 ( $\mathrm{Cl} 0.8-0.96$ ). Diagnostic accuracy was 0.92 . CE-MRI had a sensitivity of $74 \%$ and a specificity of $84 \%$. PPV was 0.68 (Cl 0.48-0.83) and NPV was 0.88 ( $\mathrm{Cl}$ 0.76-0.94). Diagnostic accuracy was 0.81 . 24 nodes were positive and 50 nodes were negative for malignancy. The mean SUVmax of lymph node metastases was 5.52 (range 1.14 - 12.75).

Conclusion: ${ }^{18}$ FDG-PET-CT improves identification of lymph node metastases as compared with 3 T CE-MRI. In patients with lymph nodes classified as malignant by ${ }^{18}$ FDG-PET-CT of the breast a sentinel lymph node biopsy can be omitted and surgeons can proceed immediately to ALND. 


\section{B-0214 14:36}

Imaging of axillary lymph nodes in breast cancer patients: How do enhancement kinetics of contrast-enhanced lymph nodes apparent on dynamic MR-mammography correlate with standardised uptake value of 18 F-FDG PET/CT?

J. Krammer, C.G. Kaiser, A. Schnitzer, S.O. Schönberg, K. Wasser; Mannheim/DE (Julia.Krammer@umm.de)

Purpose: Focusing on contrast-enhanced lymph nodes (LN) apparent on dynamic MR-mammography (dMRM) we intended to answer the following questions: 1) Is there a correlation between the maximum standardised uptake value (SUVmax) of 18 F-FDG PET/CT and maximum signal-intensity increase as well as type of signal-intensity curve (1-3) on dMRM? 2) Do contrast-enhanced LN negative on 18 F-FDG PET/CT have a significantly lesser signal-intensity increase and/or lower curve grading on dMRM compared to 18 F-FDG PET/CT positive LN?

Methods and Materials: 25 breast cancer patients who underwent both 18 F-FDG PET/CT and dMRM were analysed. The mean study interval between the examinations was 7 days. The signal-intensity increase and curve type of axillary $\mathrm{LN}^{3}$ $0.5 \mathrm{~cm}$ in short-axis and with predominant contrast enhancement on dMRM were analysed and correlated with the SUVmax on 18 F-FDG PET/CT.

Results: 30 LN in 21 patients were 18 F-FDG PET/CT positive. There was no correlation between the maximum signal-intensity increase and SUVmax of these $\mathrm{LN}(\mathrm{r}=0.04, \mathrm{p}=0.05) .14 \mathrm{LN}$ in 9 patients did not show an appropriate correlate on 18 F-FDG PET/CT (not visible or SUVmax²blood pool). These LN did not reveal a significantly lower curve grading or lesser signal-intensity increase compared to LN positive on 18 F-FDG PET/CT.

Conclusion: There is no association between the uptake of 18 F-FDG and the vascularisation of axillary $L N$ on contrast-enhanced dMRM as signal-intensity increase and curve type do not correlate with SUVmax. Unspecific contrast-enhanced LN on dMRM negative on 18 F-FDG PET/CT can frequently show enhancement kinetics equivalent to those rated positive on 18 F-FDG PET/CT.

\section{B-0215 14:45}

Ultrasound elastography in the diagnostic assessment of axillary lymph nodes in women presenting to a breast imaging centre

L. Sim, L. Leong; Singapore/SG (gdrssj@sgh.com.sg)

Purpose: To evaluate the performance of elastography in distinguishing benign and metastatic axillary lymph nodes.

Methods and Materials: 67 women with 72 sonographically visible axillary lymph nodes undergoing biopsy at our breast imaging centre were evaluated independently with conventional ultrasound, elastography and combined ultrasound and elastography (CEUS). The elastogram was classified as benign or malignant, based on the strain pattern, the length and area ratios of the lesion seen on elastography versus ultrasound. Validation of radiological diagnosis was by histopathology. The sensitivity, specificity, PPV, NPV and accuracy of each test were compared individually and with CEUS. To obtain a parameter for diagnostic performance, ROC curves were plotted.

Results: Of the 72 axillary lymph nodes biopsied, 33 had metastases and 39 were benign. The sensitivity, specificity and accuracy of conventional ultrasound were $93.9 \%, 30.8 \%$ and $59.7 \%$, respectively. The sensitivity, specificity and accuracy of elastography were $93.9 \%, 97.4 \%$ and $95.8 \%$, respectively, and that of combined ultrasound and elastography were $97 \%, 92.3 \%$ and $94.4 \%$, respectively. The sensitivities of all 3 tests were similar but the specificity and accuracy obtained by elastography and CEUS were significantly better than conventional ultrasound $(P$ $<0.0005$ ). Elastography correctly diagnosed $96 \%$ of histologically benign lymph nodes which were deemed malignant on ultrasound.

Conclusion: The use of elastography alone or combined with ultrasound has a higher specificity and accuracy than conventional ultrasound in evaluating axillary lymph nodes. Given the high specificity of elastography, biopsy could have been avoided in $96 \%$ of cases classified as malignant on ultrasound but benign on elastography.

\section{B-0216 14:54}

The Z011 trial: is this the end of axillary ultrasound in the preoperative assessment of breast cancer patients?

T.P. Farrell, M. Stenson, P.A. Carroll, M. Griffin, E.M. Connolly, S.A. O'Keeffe; Dublin/IE (farreltp@tcd.ie)

Purpose: To evaluate correlation between the number of abnormal nodes on axillary ultrasound (AxUS) and final nodal burden on histology and calculate the sensitivity, specificity and utility of AxUS-FNAC in preoperative axillary staging in breast cancer.
Methods and Materials: 567 breast cancer patients between 2009 and 2011 underwent preoperative AxUS. Suspicious nodes as per protocol were biopsied, negative axillae proceeded to sentinel lymph node biopsy (SLNB). Patients were stratified based on number of abnormal nodes identified on AxUS (1, 2, > 2 nodes) and the number on final histology was noted. Sensitivity, specificity, positive predictive and negative predictive values for AxUS-FNAC/core biopsy were calculated. Results: 266 (46.9\%) patients had positive axillary nodes on final histology with 169 detected by AxUS-FNAC (sensitivity $86.2 \%$, specificity $100 \%$, PPV $100 \%$, NPV $68.6 \%$ ). Patients with nodal metastases identified by AxUS-FNAC had a mean nodal burden of 7.1 nodes on histology (SEM=0.6, 95\% $\mathrm{Cl}=5.9-8.3 ; 1$ node identified on AxUS $=4.8$ nodes on final histology, 2 nodes on AxUS=9.9 nodes, $>2$ nodes $=9.6$ nodes) with correlation between AxUS and histology node numbers ( $r=0.39,95 \%$ $\mathrm{Cl}=0.24-0.51$, $\mathrm{p}$-value $<0.0001$ ). AxUS-FNAC detected $63.5 \%$ of node positive patients. Mean nodal burden of patients diagnosed on SLNB with negative AxUS or FNAC was 2.4 nodes.

Conclusion: The Z011 trial questioned the role of AxUS in preoperative axillary staging of breast cancer in cases with 22 positive SLN. In this study, a single FNAC positive node correlated with 4.8 nodes on final histology suggesting that AxUS continues to be essential in guiding appropriate management of the axilla in breast cancer.

\section{B-0217 15:03}

Surgical impact of preoperative breast MRI in women under $\mathbf{4 0}$

A. Porto ${ }^{1}$, A. Petrillo', R. Fusco', S. Filice'1, M. D’Aiuto', R.M. Trimboli'2,

F. Sardanelli2; ${ }^{1}$ Naples/IT, ${ }^{2}$ San Donato Milanese/IT (annamaria.porto@libero.it)

Purpose: Evaluate surgical impact of preoperative MRI in young patients. Methods and Materials: We reviewed a single-institution database of 283 consecutive patients under 40 treated for breast cancer. Excluding 37 patients (13\%) who received neoadjuvant chemotherapy, 124/246 (50\%) underwent conventional imaging (Cl), mammography/ultrasonography (Cl-group), whereas 122/246 (50\%) underwent $\mathrm{Cl}$ and dynamic breast MRI (Cl\&MRI-group). Pathology of surgical specimens served as reference standard. $X^{2}$ and McNemar tests were used. Results: Mastectomy rate was 110/246 (45\%) overall, 46/124 (37\%) for Cl group and $64 / 122(52 \%)$ for $\mathrm{Cl}$ and MRI group ( $\mathrm{p}=0.015)$. Of $122 \mathrm{Cl}$ and MRI patients, 46 would have undergone mastectomy due to $\mathrm{Cl}$, while MRI determined 18 additional mastectomies, increasing mastectomy rate from $38 \%$ to $52 \%(p<0.021)$. In the $\mathrm{Cl}$ group, 9 patients had multifocal, multicentric, synchronous or bilateral cancers, 9 $(100 \%)$ at mammography and $8(89 \%)$ at ultrasound; 2 mastectomies were due to false positives at both conventional tests $(2 / 124,1.6 \%)$. In the $\mathrm{Cl}$ and MRI group, 30 patients had multifocal, multicentric, or synchronous bilateral cancers, $5(17 \%)$ at mammography, $15(50 \%)$ at ultrasound, and $29(97 \%)$ at MRI (3 only MRI-detected synchronous bilateral cancers) $(p<0.002) ; 2$ mastectomies were due to MRI false positives (rate $2 / 122,1.6 \%$, not significantly different from $\mathrm{Cl}$ group, $\mathrm{p}=0.987$ ). Conclusion: MRI was more sensitive than $\mathrm{Cl}$ for multifocal, multicentric, or synchronous bilateral cancers. The additional mastectomy rate due to MRI was $14 \%$. Adding MRI, the percentage of young patients conservatively treated remained near to $50 \%$. By using solely $\mathrm{Cl}$ or using $\mathrm{Cl}$ and $\mathrm{MRI}$, the inappropriate mastectomy rate was equal or lower than $2 \%$.

\section{B-0218 15:12}

Imaging features in mammography and breast ultrasound are related to HER-2 receptor over-expression of primary invasive breast cancer A. Adams ${ }^{1}$, K.G.A. Gilhuijs ${ }^{1}$, K.E. Pengel ${ }^{2}$, C.E. Loo ${ }^{2}$, W.P.T.M. Mali', S.G. Elias'; 1Utrecht/NL, ${ }^{2}$ Amsterdam/NL (a.adams@umcutrecht.nl)

Purpose: To investigate what mammography and ultrasound imaging features are related to over-expression of the HER-2 receptor in invasive breast cancer. These features could give insight in molecular basis of imaging phenotype, facilitate prebiopsy patient recruitment for early-phase HER-2 targeting molecular imaging trials, and ultimately aid in predicting therapy response and prognosis.

Methods and Materials: Mammography and breast ultrasound imaging features of 598 early invasive breast cancers were scored according to the $4^{\text {th }}$ edition of the BI-RADS lexicon, HER-2 status was determined on surgical specimens. Relevant imaging features to predict HER-2 over-expression were first identified by conducting a systematic review and meta analysis, and then applied in multivariable logistic regression models.

Results: HER-2 over-expression was found in 80 cancers (13\%). On multivariable analysis, the presence of microcalcifications (OR $3.5,95 \% \mathrm{Cl} 2.1-5.8)$ on mammography, and posterior mass attenuation (OR $0.5,95 \% \mathrm{Cl} 0.3-0.9)$ on ultrasound were strong, independent predictors. Less strong predictors were breast density category $3 / 4$ (OR $0.9,95 \% \mathrm{Cl} 0.5-1.6$ ) and mass presence (OR $0.8,95 \% \mathrm{Cl} 0.4$ 1.4) on mammography, and circumscribed mass margin (OR $0.8,95 \% \mathrm{Cl} 0.3-2.1)$ 
and irregular mass shape (OR 1.3, 95\% $\mathrm{Cl} 0.8-2.1)$ on ultrasound. The area under the ROC curve was $0.69(95 \% \mathrm{Cl} 0.63-0.75, \mathrm{p}<0.001)$, and 0.67 after adjustment for over-optimism. Post-test probabilities of HER-2 over-expression were $23-37 \%$ for cancers with calcifications, without posterior mass attenuation, and 5-10\% for cancers without calcifications, with posterior mass attenuation.

Conclusion: Microcalcifications on mammography, and posterior acoustic attenuation of masses on ultrasound were predictors of HER-2/neu over-expression, indicating that imaging characteristics reflect molecular expression patterns in breast cancer.

\section{B-0219 15:21}

Is availability of breast MRI-guided intervention associated with improved results of breast MRI screening of the contralateral breast in women with newly diagnosed breast carcinoma?

V. Freitas ${ }^{1}$, S. Kulkarni², S. Ghai², R. Fleming ${ }^{2}$, A. Scaranelo², P. Crystal ${ }^{2}$;

${ }^{1}$ Rio De Janeiro/BR, ${ }^{2}$ Toronto, ON/CA (pavel.crystal@utoronto.ca)

Purpose: The aim of this study was to evaluate the association between availability of MRI-guided breast intervention and yield of contralateral screening breast MRI in women with newly diagnosed breast carcinoma.

Methods and Materials: Results of 1621 consecutive preoperative breast MRI studies were compared between periods without (2004-2006) and with (2007-2010) MRI-guided intervention capabilities.

Results: MRI detected otherwise occult contralateral cancers in $38(2.3 \%)$ of 1621 patients. Cancer detection rate in 2007-2010 was $2.8 \%(30 / 1073)$ which is significantly higher $(p<.04)$ than $1.5 \%(8 / 548)$ cancer detection rate in 2004-2006. MRI performed at the time when MRI-guided intervention was available detected DCIS in $23 \%(7 / 30)$ of all cancers versus $13 \%(1 / 8)$ of DCIS detected at the time period when MRI-guided biopsy was not available. Mean size of 23 invasive cancers diagnosed in 2007-2010 was $15 \mathrm{~mm}$ (range 4-71 mm) versus mean size of $21 \mathrm{~mm}$ (range 7-39 mm) for 7 invasive cancers diagnosed in 2004-2006. Small cancers (DCIS and invasive cancers $<10 \mathrm{~mm}$ ) comprised 53\% (16/30) of cancers diagnosed in 2007-2010 versus $25 \%$ (2/8) of cancers diagnosed in 2004-2006. Rate of benign image-guided biopsies for MRI-detected lesions in 2007-2010 was $3.7 \%(40 / 1073)$ similarly to the $3.6 \%(20 / 548)$ of benign biopsies in 2004-2006. Conclusion: Availability of MRI-guided intervention is associated with higher cancer detection yield and higher number of otherwise occult DCIS and small invasive cancers detected by screening MRI of the contralateral breast in women with newly diagnosed breast carcinoma.

Neuro

\section{SS 211}

\section{Infection and inflammation}

Moderators:

E. Marco de Lucas; Sancibrian/ES

G. Schroth; Berne/CH

\section{B-0220 14:00}

Evaluation of plaque detection and optimum time of enhancement in acute attack multiple sclerosis after contrast injection

H. Hashemi, H. Ghanaati, S. Behzadi, M. Harirchian, M. Yaghoobi, M. Shakiba,

A. Jalali, K. Firouznia; Tehran/IR (k_firouznia@yahoo.com)

Purpose: This study aimed at evaluating the characteristics of multiple sclerosis (MS) plaques (number, size and signal intensity) and also to detect the optimum time of enhancement after injection of Gadobutrol.

Methods and Materials: Sixty-two relapsing-remitting type MS patients, (56 females, 6 males) with the mean age of $31 \pm 7$ years were enrolled into this study. The patients underwent $\mathrm{T} 1$ weighted magnetic resonance imaging (MRI) scan without contrast. Subsequently, Gadobutrol was injected $(0.1 \mathrm{mmol} / \mathrm{kg})$ and MRI scanning was repeated after 30 seconds, 5, 10, 15 and 30 minutes of Gadobutrol injection. Results: Enhancing plaques were seen in 42 patients. The mean number of enhancing plaques was $4 \pm 8$ plaques after 30 seconds of contrast injection. This figure increased to $7 \pm 13$ plaques after 15 minutes and $6 \pm 10$ plaques after 30 minutes. The signal intensity and size of plaques increased progressively, and the maximum signal intensity and plaque size were seen after 30 minutes $(p<0.001)$. Conclusion: The maximum number of enhancing plaques in MS patients was detected 15 minutes after contrast administration and the size and signal intensity of the lesions also increased remarkably at this time.
B-0221 14:09

Respiratory syncytial virus-related encephalitis: brain MR study with diffusion imaging

A. Pak, S. Suh, G. Son, Y. Lee, H. Seo, K. Kim, B. Eun, N. Lee, H. Seol; Seoul/KR (suppy@korea.ac.kr)

Purpose: Respiratory syncytial virus (RSV) is a common pathogen of acute respiratory infection causing significant morbidity and mortality in childhood. Among the major complications, encephalitis has been sporadically reported. We describe the incidence, clinical and MRI imaging findings of RSV-related encephalitis in this report.

Methods and Materials: We retrospectively reviewed the medical records and imaging findings of patients with RSV-positive enzyme assay, admitted at the paediatric department in three urban tertiary-care hospitals of our medical centre for 7 years. There were 3856 cases with a diagnosis of RSV-bronchiolitis. Among them, $28(28 / 3856,0.73 \%)$ had taken brain MRI due to neurologic symptoms. 8 of $28(28.6 \%)$ showed positive imaging findings. 5 of 8 positive MR imaging cases revealed non-RSV related pathology such as $\mathrm{SDH}$, diffuse brain atrophy due to status epilepticus, periventricular leukomalacia, pre-existed ventriculomegaly, hypoxic brain injury.

Results: There were three RSV-related encephalitis. The incidence of this entity is $0.08 \%(3 / 3856)$ for RSV positive enzyme assay, $10.7 \%(3 / 28)$ for brain MRI examination, and $37.5 \%(3 / 8)$ for abnormal MRI findings. The imaging findings of RSV-related encephalitis are as following: rhombenmesencephalitis pattern, rhombenmesencephalitis with acute disseminated encephalitis pattern, and limbic encephalitis pattern.

Conclusion: Encephalitis rarely occurs with RSV bronchiolitis. However, when brain MR is needed in suspicion of neurologic involvement, RSV encephalitis is not uncommon among abnormal MR cases and mimics other viral and limbic encephalitis on imaging study. Physicians should be aware of this entity for proper diagnosis and neurologic care of RSV-positive patients.

\section{B-0222 14:18}

Cortical-juxtacortical lesions in clinically isolated syndromes: distribution and diagnostic value

J.P. Salazar, C. Auger, D. Pareto, R. Mitjana, M. Tintore, J. Corral; Barcelona/ES

Purpose: To evaluate presence and spatial distribution of cortical-juxtacortical lesions in patients with a clinical isolated syndrome (CIS) by using MRI-based lesion probability maps and to determine its impact to demonstrate lesion dissemination in space (DIS) according to the 2010 McDonald criteria for multiple sclerosis (MS). Methods and Materials: $65 \mathrm{CIS}$ patients aged between 20 and 50 years old (mean 35.9 years) who underwent brain and spinal cord 3 T MRI within the first five months after symptoms onset. The following sequences were obtained: 1) PD and T2-weighted; 2) T2-fast FLAIR; 3) Un-enhanced and contrast-enhanced T1-weigthed; and 4) 3D double inversion-recovery. Cortical-juxtacortical lesions in each patient were identified, manually outlined and segmented into a binarized mask. A cortical-juxtacortical MRI-based lesion probability map was obtained. The 2010 McDonald criteria for DIS were assessed in each patient.

Results: Cortical-juxtacortical lesions were identified in 24 patients $(60 \%)$. Frontal lobe was the most affected area with an incidence of $37 \%$ of the lesions. Temporal and parietal lobes had also a significant incidence (20\% and $23 \%)$. According to the MS 2010 McDonald diagnostic criteria, identification of cortico-juxtacortical lesion contributed to demonstrate DIS in $20 \%$.

Conclusion: Cortical-juxtacortical lesions are a common MRI finding in patients with CIS, predominantly involving the frontal, parietal and temporal lobes. Its demonstration has a high relevance for demonstrating DIS according to the MS 2010 McDonald diagnostic criteria.

\section{B-0223 14:27}

Regional MRI perfusion measures predict motor/executive function in patients with clinically isolated syndrome

E. Papadaki, P. Simos, T. Panou, V. Mastorodemos, E. Amanakis, T. Maris,

A. Karantanas, A. Plaitakis; Iraklion/GR (fpapada@otenet.gr)

Purpose: The aim of this study is to explore whether CNS hemodynamic disturbances, detected in patients with clinically isolated syndrome (CIS), correlate with executive function.

Methods and Materials: Thirty CIS patients and forty-three healthy subjects matched for age, gender, education level, FSIQ, and place of residence were administered computerized tests of visuomotor learning and set-shifting ability. Regional cerebral blood volume (CBV), cerebral blood flow (CBF), and mean transit time (MTT) values were estimated using the dynamic susceptibility contrast- 
enhanced T2*-weighted MRI (DSC-MRI) technique in 20 regions of interest in normal appearing white matter (NAWM) and normal deep grey matter (NDGM) structures bilaterally, that serve as integral components of brain circuits responsible for visuomotor learning and executive functions.

Results: CIS patients showed significantly elevated reaction time (RT) on both tasks, while their CBV values were globally increased. Significant, positive correlation coefficients were found between error rates on the inhibition condition of the visuomotor learning task and CBV values in both thalami and occipital, and periventricular NAWM, bilaterally. On the set shifting condition of the respective task significant positive associations were found between CBV values in the semioval centre and periventricular NAWM bilaterally and error rates, between CBF values in both thalami and error rates, and between CBF values in the left parietal NAWM and right caudate and RT.

Conclusion: Impaired executive function in CIS patients, including inhibition and set shifting abilities, correlated positively with elevated regional CBV values thought to reflect active widespread brain inflammatory processes.

\section{B-0224 14:36}

The "central vein sign": is there a place for susceptibility-weighted imaging in possible multiple sclerosis?

T. Kau, M. Taschwer, M. Schönfelder, J.R. Weber, K.A. Hausegger; Klagenfurt/AT (t.kau@gmx.at)

Purpose: Susceptibility-weighted imaging (SWI) may have the potential to depict the perivenous extent of white matter lesions (WMLs) in multiple sclerosis (MS). We aimed to assess the discriminatory value of the "central vein sign" (CVS). Methods and Materials: In a lesion-based 3 T MRI study, 28 WMLs in 14 patients were prospectively included applying the following criteria: 1) at least one circumscribed lesion $>5 \mathrm{~mm}, 2$ ) not more than eight non-confluent lesions $>3 \mathrm{~mm}$. FLAIR images served as gold standard for the identification of WMLs which were classified as periventricular, subcortical, or infratentorial. Only defined WMLs with a maximum diameter of $>5 \mathrm{~mm}$ were correlated with their SWI equivalent for CVS evaluation. Results: Five patients fulfilled the revised McDonald criteria for MS and nine patients were given an alternative diagnosis with non-MS-WMLs most probably representing focal gliosis. A total of 28 lesions $>5 \mathrm{~mm}$ (19 MS-WMLs and 9 nonMS-WMLs) were detected. Consensus reading found a central vein in 16 out of 19 MS-WMLs (84\%) and in one out of nine non-WMLs (11\%), respectively. The CVS proved to be a highly significant discriminator $(p<0.001)$ between MS-WMLs and non-MS-WMLs with a sensitivity, specificity, positive and negative predictive values, and accuracy of $84 \%, 89 \%, 94 \%, 73 \%$, and $86 \%$, respectively. The frequency of CVS positive lesions was highest in the periventricular zone (7/10), even more markedly in MS-WMLs alone (6/7). Inter-rater agreement was good (kappa $=0.77$ ). Conclusion: Even though the CVS is not exclusively found in MS-WMLs, SWI may be a useful adjunct in patients with possible MS.

\section{B-0225 14:45}

Single- and multi-voxel proton spectroscopy in patients with pyogenic brain abscess

P.-H. Lai, C.-W. Ko, S.-H. Hsu, J.-H. Fu, P.-C. Wang, H.-B. Pan; Kaohsiung/TW (pinghonglai@gmail.com)

Purpose: The purpose of our study was to evaluate the equivalence of multi-voxel spectroscopy (MVS) and single-voxel spectroscopy (SVS) in the detection of the metabolites of pyogenic abscesses.

Methods and Materials: The magnetic resonance spectroscopy (MRS) criteria for diagnosing pyogenic abscesses are presence of cytosolic amino acids (AAs) with or without lactate (Lac), succinate (Suc), acetate (Ac), and lipid (Lip). Forty-two patients with pyogenic abscesses were studied using SVS and MVS methods. The abscess cavity was evaluated by SVS and MVS. Two neuroradiologists reviewed the MRS data. A $\kappa$ value was calculated to express inter-reader agreement of the abscesses metabolites. After consensus judgment of two readers, diagnostic accuracy was calculated on MVS with SVS as reference. A $\mathrm{k}$ value was calculated to express inter-technique agreement of the abscesses metabolites.

Results: Resonance of AAs with or without other metabolites was observed in $76 \%$ abscesses on SVS and MVS. The consistency of spectral interpretation of the two readers was very good ( $\mathrm{k}$ ranged from 0.95 to 1 ). After consensus judgment of two readers, sensitivities and negative predictive values of MVS ranged from $91 \%$ (Ac) to $100 \%$ (AAs, Suc, Lac, Lip) with SVS as reference. Specificity and positive predictive values of MVS were $100 \%$ for detecting all five metabolites. The inter-technique $\mathrm{k}$ values of the two readers in consensus were very good $(\mathrm{K}$ ranged from 0.90 to 1 ).
Conclusion: SVS and MVS provide similar metabolites among pyogenic brain abscesses subjects, and they possess comparable ability for diagnosing pyogenic brain abscesses.

\section{B-0227 14:54}

Directional diffusivity changes describing micro-structural damage in normal appearing and lesioned cervical cord white matter in multiple sclerosis

N. Berkovitz, P. Gottlieb, S. Tal; Zrifin/lL (nadavber@gmail.com)

Purpose: To evaluate the DTI parameters of relapsing-remitting multiple sclerosis (RRMS) patients and the normal appearing cervical spinal cord (NASC) with focus on Eigenvalue data. Longitudinal diffusivity $(\lambda 1)$ has been associated with axonal loss and Wallerian degeneration while transverse diffusivity $(\lambda 2, \lambda 3)$ with demyelination.

Methods and Materials: DTI of the cervical spine was performed. DTI parameters were measured bilaterally at the level of $\mathrm{C} 2-3$ in anterior, lateral and posterior spinal cord regions of interest (ROIs). Values were compared between white matter in NASC and white matter (NWM) in non-MS patients. When existing, a single cervical MS lesion was measured. Values were correlated with clinical parameters of EDSS and disease duration.

Results: There were 10 RRMS and 7 control patients. Eight lesions were measured. Anterior ROls showed a significantly lower FA considered to contain grey matter. NASC had significant higher mean $\lambda 1, \lambda 2$ and ADC values than NWM. Lesions had significantly higher $\lambda 2, \lambda 3$ and lower FA values than NASC. Lesions were higher in $\lambda 1, \lambda 2$ and $A D C$ with a lower FA than NWN, without anterior ROIs. FA changes between NASC and lesions correlated with $\lambda 2$ and $\lambda 3$. FA in NASC and NWM show correlation with $\lambda 1, \lambda 2$ and $\lambda 3$. Clinical parameters did not correlate. Conclusion: NASC shows an increased longitudinal and transverse diffusivity compared to NWM. In lesions, the transverse diffusivity is increased, reducing FA. NASC shows both axonal and myelin-related damage while for a focal lesion it is the myelin damage that has increased significantly.

\section{B-0228 15:03}

Cognitive deficits in clinically isolated syndrome correlate with perfusion changes indicative of inflammation

E. Papadaki, P. Simos, T. Panou, V. Mastorodemos, E. Amanakis, T. Maris,

A. Karantanas, A. Plaitakis; Iraklion/GR (fpapada@otenet.gr)

Purpose: Cognitive deficits occur in patients with clinically isolated syndrome (CIS), which represents the earliest clinical stage of multiple sclerosis (MS), but their pathophysiology remains unclear. The aim of this study was to investigate the relation between hemodynamic measurements and memory function in patients with $\mathrm{CIS}$ and relapsing remitting-multiple sclerosis (RR-MS).

Methods and Materials: Thirty patients with CIS, thirty RR-MS patients and forty-three healthy subjects, matched for age, gender, education level, and FSIQ, were administered tests of short-term and working memory for digits, passages and geometric patterns. Using the dynamic susceptibility contrast-MRI (DSC-MRI) technique, cerebral blood volume (CBV), cerebral blood flow (CBF), and mean transit time (MTT) values were estimated in twenty cerebral regions of interest, placed in normal appearing white matter (NAWM) and normal deep gray matter (NDGM) structures, bilaterally.

Results: Both CIS and RR-MS groups showed significantly impaired scores on verbal working memory and secondary verbal memory. In CIS patients, globally elevated CBV and MTT values were detected, while their memory performance correlated inversely with CBV values in the left frontal NAWM, right thalamus, right caudate and corpus callosum, brain structures known to be involved in cognitive function.In contrast, among RR-MS patients, correlations of CBV values with memory indices were positive in the right frontal and periventricular NAWM and in the caudate.

Conclusion: As the hemodynamic changes, detected in CIS, are indicative of inflammation, the observed cognitive disturbances may relate to widespread brain inflammatory processes that prevail in early MS.

\section{B-0229 15:12}

$3 \mathrm{~T}$ MRS alterations in brain metabolism in HAART-naive HIV infection

A. Trofimova, T. Trofimova, G. Kataeva, S. Medvedev, N. Belyakov,

V. Rassokhin, A. Korotkov, E. Malakhova; St. Petersburg/RU

(suhackaya@yandex.ru)

Purpose: To study regional changes in brain metabolites during early HIV infection compared with later stage HIV infection and HIV-seronegative controls. 
Methods and Materials: 2D ${ }^{1} \mathrm{H}$ MR spectroscopy (TE 144) with a preselected volume at the supraventricular level was performed in 15 patients with early HIV infection (plasma level of CD4-lymphocytes more than 350 cells per mcl., HAART naive); 19 patients with later stage of HIV (without HAART/neurologic impairment) and 20 HIV-seronegative controls. Protocol included ax T2, ax FLAIR, 3DT1 pre-, postcontrast. Metabolites ratios were analysed in white and grey matter in 3 anatomic regions of both hemispheres - anterior and posterior frontal, parietal. Statistics were calculated using STATISTICA software package, factorial ANOVA with $\mathrm{p}<0.01$.

Results: Naa/Cr was decreased in white matter in both early HIV $(2,100 \pm 0.25)$, later HIV $(2,107 \pm 0.26)$ when compared with HIV-negative controls $(2,174 \pm 0.27)$. In the cortex $\mathrm{Naa} / \mathrm{Cr}$ was decreased in anterior frontal region in early HIV $(1,577 \pm 0.017)$, later HIV $(1,604 \pm 0.015)$ compare with HIV-negative controls $(1,652 \pm 0.017)$. Naa/ Cho was decreased in white matter in early HIV $(2,068 \pm 0.44)$, later HIV $(2,153 \pm 0.45)$ when comparedwith controls $(2,335 \pm 0.44)$ and was decreased in grey matter in early HIV $(1,878 \pm 0.44)$, later HIV $(1,929 \pm 0.39)$ compared with HIV-negative controls $(1,985 \pm 0.38)$. There was no difference between $\mathrm{HIV}$ groups in $\mathrm{Naa} / \mathrm{Cr}$, $\mathrm{Naa} / \mathrm{Cho}$. Cho/Cr was significantly different in 3 groups, most increased in early $\operatorname{HIV}(1,058 \pm 0.20)$ compared with later HIV $(1,001 \pm 0.15)$ and controls $(0.950 \pm 0.14)$ without regional predominance.

Conclusion: The results suggest that HIV infection produces brain metabolites alterations early in the course of disease, with predominance of inflammatory changes in early HIV infection.

\section{4:00 - 15:30} Room I/K

\section{Abdominal Viscera}

\section{SS 201b}

\section{Hepatocellular carcinoma: diagnosis and management}

Moderators:

G. Brancatelli; Palermo/IT

M. Bruegel; Munich/DE

\section{B-0230 14:00}

Intravoxel incoherent motion diffusion-weighted MR imaging of hepatocellular carcinoma: correlation with enhancement degree and histological grade

S. Woo, J. Lee, J.-H. Yoon, I. Joo, J. Han, B. Choi; Seoul/KR

(j_crew7@hotmail.com)

Purpose: To compare intravoxel incoherent motion (IVIM)-derived parameters with the apparent diffusion coefficient (ADC) in predicting the histological grade of hepatocellular carcinomas (HCC), and to evaluate the relationship between IVIMderived parameters and arterial enhancement (AE) degree.

Methods and Materials: This retrospective study was IRB approved and informed consent was waived. Forty patients with 42 surgically confirmed HCCs underwent diffusion-weighted imaging (DWI) with eight b-values $\left(0-800 \mathrm{sec} / \mathrm{mm}^{2}\right)$. ADCtotal, $\mathrm{D}$ (diffusion coefficient), $\mathrm{D}^{*}$ (pseudodiffusion coefficient), and $f$ (perfusion fraction) were calculated. Two radiologists determined the enhancement degree in consensus and the percentage of AE of HCCs. The relationship between the parameters and histological grade and AE was assessed using Spearman's or Pearson's correlation test. Receiver operating characteristics (ROC) analysis of discrimination between fair and poor grade HCCs was performed for D and ADCtotal.

Results: $\mathrm{D}$ and $\mathrm{ADC}$ total were both significantly correlated with histological grade: $r=-0.604, p<0.001$ and $r=-0.448, p=0.002$, respectively. $\mathrm{D}$ and ADCtotal were both significantly lower in poor $\left(D=0.99 \pm 0.13 \times 10^{-3} \mathrm{~mm}^{2} / \mathrm{s}, A D C=1.13 \pm 0.14\right.$ $\left.\times 10^{-3} \mathrm{~mm}^{2} / \mathrm{s}\right)$ than in fair grade HCCs $\left(D=1.18 \pm 0.16 \times 10^{-3} \mathrm{~mm}^{2} / \mathrm{s}, A D C=1.25\right.$ $\left.\pm 0.17 \times 10^{-3} \mathrm{~mm}^{2} / \mathrm{s}\right)(p<0.001, p=0.029$, respectively $)$. However, ROC analysis demonstrated a greater $A z$ value of $D(0.836)$ for differentiating poor grade HCCs from fair grade HCCs than ADCtotal $(0.733)(p=0.037)$. The percentage of $\mathrm{AE}$ and $f$ were correlated $(r=0.621, p<0.001)$.

Conclusion: IVIM-derived diffusion coefficient of HCCs showed significantly better diagnostic performance in predicting histological grade than ADCtotal, and significant correlation was observed between $f$ and the percentage of $\mathrm{AE}$.

\section{B-0231 14:09}

Accuracy of contrast-enhanced imaging in the pretransplantation staging of hepatocellular carcinoma (HCC) and pathologic predictive factors of HCC recurrence after liver transplantation (LT)

G. Besutti, A. Pecchi, M. De Santis, G. Tarantino, F. Di Benedetto, P. Torricelli; Modena/IT (giulia.besutti@libero.it)

Purpose: To assess the accuracy of imaging in HCC pretransplantation staging, with pathologic evaluation of the explanted liver as the reference standard and to identify pathologic predictive factors for HCC recurrence after LT.

Methods and Materials: 175 patients who underwent LT for HCC between October 2004 and November 2011 and survived more than 2 months were included. 119 of them had previously undergone locoregional therapies or resection. Imaging findings were retrospectively evaluated on pretransplantation contrast-enhanced CT/ RM performed within 75 days before LT (mean 42 days). Histopathological findings were obtained by a review of the pathologic reports. Incidence of recurrence was evaluated. Pathologic predictive factors for recurrence and association between pretransplantation imaging and explanted liver pathology were estimated by using linear regression analysis and $\mathrm{X} 2$ test.

Results: A significant association was found between imaging and pathology in the assessment of the presence of viable tumour $\left(\mathrm{p}^{2} 0.001\right.$, also in patients who undergone previous local therapies), number of nodules ( $\left.\mathrm{p}^{2} 0.001\right)$, diameter of the largest nodule $\left(p^{2} 0.001\right)$, overall size $\left(p^{2} 0.001\right)$ and lobar distribution $(p=0.03)$. Recurrence was found in 24 (13.7\%) patients. A significant association was found between imaging detection of viable tumour and HCC recurrence $(p=0.002)$. Pathological findings associated with recurrence were the presence of viable tumour $(p=0.003)$, HCC type rather than combined HCC-cholangiocarcinoma $\left(p^{2} 0.001\right)$, capsule contact $\left(\mathrm{p}^{2} 0.001\right)$ and vascular invasion $\left(\mathrm{p}^{2} 0.001\right)$.

Conclusion: Contrast-enhanced imaging can be considered accurate in the pretransplantation staging of HCC. Presence of viable tumour, HCC type, capsule contact and vascular invasion were predictive factors for HCC recurrence after LT.

\section{B-0232 14:18}

Detection of HCC and liver metastases with BR14: final results of a multicentre phase IIA study

J. Hohmann ${ }^{1}$, A. Müller ${ }^{2}$, J. Skrok ${ }^{3}$, K.-J. Wolf ${ }^{2}$, A. Martegani ${ }^{4}$, C.F. Dietrich ${ }^{5}$ T. Albrecht ${ }^{2} ;{ }^{1}$ Basle/CH, ${ }^{2}$ Berlin/DE, ${ }^{3}$ Baltimore, MD/US, ${ }^{4} \mathrm{Como} / \mathrm{IT}$, ${ }^{5}$ Bad Mergentheim/DE (jhohmann@uhbs.ch)

Purpose: The study was primarily designed to find the optimal dose range of BR14 to detect malignant focal liver lesions. Secondary objectives were the evaluation of the safety profile and comparison with contrast-enhanced MRI (CE MRI).

Methods and Materials: 25 patients (9f, $16 \mathrm{~m}$, mean age: $66 \mathrm{y}$ ) with known HCC or liver metastases were examined in three centres during a time period of three months. Each patient underwent a baseline and at least three contrast-enhanced US (CEUS) with ascending dose levels $(0.25 \mathrm{ml}, 1.0 \mathrm{ml}, 4.0 \mathrm{ml})$ of BR14. CE MRI was done 4 weeks prior or post-study examination. Lesions were recorded in on a liver map, with respect to localisation, size and suggested lesion type. Examination quality was documented and safety parameters were assessed.

Results: The number of lesions detected with BR14 CEUS increased with dose, while the number of missed lesions and the lesion size decreased. Despite the increasing contrast enhancement no other image quality parameter showed a substantial difference. No significant changes were found for the analysed safety parameters and no serious adverse events were reported.

Conclusion: We finally conclude that the recommended dose level of BR14 is between $1.0 \mathrm{ml}$ and $4.0 \mathrm{ml}$ for which the lesion detection was comparable to the CE MRI. In addition, we found a higher number of especially small lesions with higher doses of BR14 which might be due to a higher sensitivity of CEUS for the detection of liver metastases. However, this is an interesting and debatable finding.

Author Disclosures:

J. Hohmann: Grant Recipient. T. Albrecht: Grant Recipient.

\section{B-0233 14:27}

Integrating contrast-enhanced sonography (CEUS) in the follow-up algorithm of patients with percutaneously ablated hepatocellular carcinoma (HCC)

O. Catalano, P. Vallone, V. Granata, F. Izzo, V. Albino, A. Nunziata, A. Petrillo; Naples/IT (orlandcat@tin.it)

Purpose: $\mathrm{HCC}$ patients treated with percutaneous ablation require close follow-up for detecting tumour recurrence. We illustrate our single-centre experience on using CEUS as a follow-up tool alternated with CT. 
Methods and Materials: During 2005-2011 588 patients with 1-3 HCCs were treated with radiofrequency ablation (alone or combined with ethanol injection). Patients had complete ablated tumours at $1 \mathrm{mo}$. CT was followed-up serially, using alternated CEUS (one sulphur hexafluoride-based microbubbles injection per lobe) and CT every 3 months. The following patterns of recurrence were considered: A, enhancing tissue within the lesion; B, enhancing tissue adherent to the lesion; C, enhancing tissue within the same liver segment of the treated nodule; D, enhancing tissue within a different segment (progression). Patients with positive CEUS underwent confirmatory CT (standard reference).

Results: Median follow-up was 29 months. There were 221 recurrences. Three pattern A recurrences ( 2 detected by CEUS and 1 by CT), 86 pattern B recurrences ( 44 detected by CEUS and 42 by CT), 70 pattern $C$ recurrences ( 32 detected by CEUS and 38 by CT), and 62 pattern $D$ recurrences ( 23 detected by CEUS and 39 by CT). CT detected additional nodules in 16/101 patients with positive CEUS. Conclusion: CEUS follow-up of HCC patients after ablation is feasible. Since $72 \%$ recurrences develop in the same segment of the necrotic nodule, CEUS proves to be effective despite the less comprehensive liver survey compared to CT. Including CEUS in patient follow-up may allow decreasing the number of CT examinations.

\section{B-0234 14:36}

Hepatocellular carcinoma $(\mathrm{HCC})$ treated with transarterial chemoembolisation (TACE): volume CT perfusion (VCTP) imaging in the detection of early changes - preliminary results

J. Steiner, T. Wimmer, D. Bohlsen, F. Quehenberger, H. Schöllnast; Graz/AT (juergensteiner1@gmx.at)

Purpose: To describe the early changes after transarterial chemoembolisation (TACE) of hepatocellular carcinomas (HCC) using volume CT perfusion (VCTP) imaging.

Methods and Materials: This prospective study was approved by the Institutional Review Board and informed consent was obtained from all patients. VCTP imaging was performed in 11 patients who underwent TACE of HCC. VCTP imaging of the HCC was performed on the day before TACE and 5 - 7 days after TACE to assess early changes. All VCTP studies were performed on a 320 channel CT scanner (Aquilion One, Toshiba Medical Systems) and perfusion of the HCC was calculated using commercial body perfusion software (Toshiba Medical Systems). Tube voltage was $100 \mathrm{kV}$, tube current was $100 \mathrm{~mA}$, and rotation time was 0.5 sec. Hepatic arterial flow (HAF), portal venous flow (PVF), and hepatic arterial flow fraction (HAFF) of the HCC were calculated before and after TACE. Perfusion values before and after TACE were compared using the t-test.

Results: Mean HAF $( \pm$ SD) decreased from $121 \pm 76$ to $95 \pm 21 \mathrm{~mL} / \mathrm{min} / 100 \mathrm{~mL}$ $(22 \%, p=0.210)$, mean PVF $( \pm S D)$ increased from $174 \pm 79$ to $259 \pm 190 \mathrm{~mL}$ $\mathrm{min} / 100 \mathrm{~mL}(50 \%, \mathrm{p}=0.064)$, and HAFF decreased from $46 \pm 13$ to $37 \pm 12 \%$ $(20 \%, p<0.002)$

Conclusion: VCTP imaging is a non-invasive technique for evaluation of the early changes of HCC after TACE and may be used as imaging tool to assess early response. Especially the HAFF may be an accurate parameter for assessment of early response.

\section{B-0235 14:45}

Role of dynamic contrast-enhanced perfusion MR imaging in monitoring of patients with $\mathrm{HCC}$ lesions treated by radiofrequency ablation or transarterial chemoembolisation

C. Trattenero, D. Ippolito, M. Colombo, P.A. Bonaffini, R. Corso, S. Sironi; Milan/IT (chiaratratte@gmail.com)

Purpose: To prospectively investigate the role of dynamic contrast-enhanced (DCE) perfusion MR imaging in detection of blood flow changes related to therapeutic effects of transarterial chemoembolisation (TACE) and radiofrequency ablation (RFA) in patients with HCC lesions.

Methods and Materials: Fifty-four patients with known cirrhotic liver disease and biopsy-proven HCC lesions that underwent TACE or RFA treatment were prospectively enrolled in our study. Upper abdominal MRI examination (1.5 T Achieva, Philips) with multiplanar T2 and T1 sequences and dynamic contrast-enhanced THRIVE was performed about 1 month after the treatment. Philips's workstation was used to generate colour permeability maps showing perfusion images of hepatic parenchyma and of treated lesions that were compared with $\mathrm{T} 2, \mathrm{~T} 1$ weighted images and THRIVE sequences. After placing region of interest (ROI) in normal hepatic parenchyma, correctly treated lesions and along the contours of $\mathrm{HCC}$ recurrence lesions, following parameters were calculated and statistically analysed: relative arterial, venous and late enhancement (RAE, RVE, RLE), maximum enhancement (ME), maximum relative enhancement (MRE) and time to peak (TTP)
Results: Twenty-one out of 54 patients had a residual disease and values of perfusion parameters measured within tumour tissue were: REA 44.66, RVE 60.50, RLE 52.72, ME 553.21, MRE 65.95 and TTP 140.61. The same parameters calculated in those correctly treated were: REA -1.24, RVE 5.93, RLE 16.9, ME 203.24, MRE 25.78, TTP 165.87. A significant difference $(p<0.001)$ was found for all the evaluated parameters between residual viable tumour tissue $(p<0.001)$ compared to successfully treated lesions, due to the presence of residual arterial vascular structure in viable portion of treated HCC.

Conclusion: MRI perfusion images is a useful non-invasive tool in quantitative and qualitatively assessment of patients with treatment response of $\mathrm{HCC}$ lesions.

\section{B-0236 14:54}

\section{Diagnostic efficacy of combined dynamic perfusion MRI with ADC} mapping in the assessment of therapeutic effects of HCC-treated lesions: preliminary results

D. Ippolito, C. Trattenero, P. Bonaffini, C. Capraro, R. Corso, S. Sironi; Monza/IT (davide.atena@tiscalinet.it)

Purpose: To correlate kinetic parameters derived from dynamic contrast-enhanced MR perfusion imaging with apparent diffusion coefficient (ADC) for the assessment of multiparametric approach to interventional treatment of $\mathrm{HCC}$ lesions.

Methods and Materials: Fifty-four patients with known cirrhotic liver disease and biopsy-proven diagnosis of $\mathrm{HCC}$ lesion, that underwent TACE or RFA treatment, were prospectively enrolled. MR study was performed 4 weeks after treatment and consists of multiplanar standard protocol with $\mathrm{T} 2$ and $\mathrm{T} 1$ sequences, dynamic contrast-enhanced (THRIVE), using a 1.5 T MRI system (Achieva, Philips), including also diffusion weighted imaging (DWI). Philips's workstation was used to generate colour permeability maps showing perfusion of enhancing tumours and quantitative ADC maps. After placing regions of interests (ROIs) on the site of maps which best corresponded to the enhanced regions of lesion, the following parameters were calculated: relative enhancement, maximum enhancement, maximum relative enhancement, time to peak and ADC values. Correlation between MR imaging, parametric values and clinical pathological classification was statistically analysed. Results: Sixteen out of 54 patients had residual disease and values of parameters measured within residual tumour tissue were: REA 44.66, VE 60.50, RLE 52.72, ME $553.21(\%)$, MRE 65.95 (\%), TTP (s) 140.61 , and $982.21 \pm 103.93 \times 10^{-3} \mathrm{~mm}^{2 /}$ $\mathrm{sec}$. Corresponding values obtained in the remaining 26 cases, correctly treated, were: REA -1.24, RVE 5.93, RLE 16.9, E 203.24, RE 25.78, TTP 165.87 and $1682.72 \pm 149.74 \times 10^{-3} \mathrm{~mm}^{2} / \mathrm{sec}$. A significant difference $(\mathrm{p}<0.001)$ was found for all obtained parameters between residual viable tumour tissue $(p<0.001)$ compared to successfully treated lesion, due to the presence of residual arterial vascular structure in viable portion of treated HCC.

Conclusion: This feasibility study shows that quantitative multiparametric MR images analysis could provide an in vivo marker of biological characteristics of $\mathrm{HCC}$ useful in assessing the therapeutic treatment response.

\section{B-0237 15:03}

Recurrence patterns of hepatocellular carcinoma $(\mathrm{HCC})$ after liver transplantation (LT) and variations in dynamic imaging and histopathological characteristics between the primary and the intrahepatic recurrent $\mathrm{HCC}$

G. Besutti, A. Pecchi, M. De Santis, G. Tarantino, F. Di Benedetto, P. Torricelli; Modena/IT (giulia.besutti@libero.it)

Purpose: To describe the patterns of recurrence of HCC after LT and to evaluate the variation in imaging and histopathological characteristics between the primary $\mathrm{HCC}$ and the intrahepatic recurrence.

Methods and Materials: 24 cases of recurrent HCC on a total of 175 patients who underwent LT for HCC between October 2004 and November 2011 were reviewed. Imaging characteristics of the primary $\mathrm{HCC}$ were retrospectively evaluated on dynamic contrast-enhanced imaging (CT/MRI) performed within 75 days before LT (mean 42 days). Patterns and characteristics of recurrence were retrospectively evaluated on follow-up dynamic contrast-enhanced imaging (CT/MRI). Histopathological characteristics of the primary $\mathrm{HCC}$ and recurrence were obtained by a review of the pathologic reports. The association between the variations in histopathological and imaging characteristics of primary and recurrent HCC was estimated using linear regression analysis.

Results: The initial recurrence was extrahepatic in 14 (58.3\%, lung, bone, lymph nodes, brain, spleen, adrenal), intrahepatic in $7(29.2 \%)$ and both intrahepatic and extrahepatic (peritoneum, lung, bone, lymph nodes) in $3(12.5 \%)$ patients. Of 14 patients with initial or late intrahepatic recurrence, $2(14.3 \%)$ showed a variation in imaging characteristics between the primary HCC (hypervascular) and the recurrence (hypovascular), 1 (7.1\%) showed a variation in histopathological char- 
acteristics (from moderate to poor differentiation), but no association was found between imaging and histopathological variations.

Conclusion: The most common pattern of recurrence was extrahepatic location, followed by intrahepatic recurrence and both intrahepatic and extrahepatic diffusion. Variations in dynamic-imaging characteristics of intrahepatic recurrence were not associated with variations in histopathological characteristics.

\section{B-0238 15:12}

Accuracy of mRECIST versus RECIST 1.1 in predicting outcome in hepatocellular carcinoma treated with Sorafenib

G. Gallusi, M. Di Martino, C. Lombardo, M. Del Monte, R. Di Miscio, A.F. Attili,

C. Catalano; Rome/IT (dimase81_6@hotmail.com)

Purpose: Both in SHARP and Asia-Pacific trials, a poor radiological response rate (RRR) ( $p=0.17$ and 0.05 , respectively), assessed with RECIST, contrasted with a significant elongation of overall survival (OS) ( $p=0.014$ and 0.001 , respectively) in patients treated with Sorafenib. So far, reliable criteria to determine the response of hepatocellular carcinoma (HCC) to Sorafenib are lacking. The aim of this study was to compare RECIST1.1 and mRECIST ability in the estimation of the response to therapy in patients with advanced HCC treated with Sorafenib.

Methods and Materials: From August 2008 to July 2012, 58 cirrhotic patients with advanced $\mathrm{HCC}$ received Sorafenib at starting dose of $400 \mathrm{mg}$ bid and were followed until death occurred. Using RECIST1.1 and mRECIST, 27 patients who had undergone a 4-phase CT scan/dynamic MR before and after (30-100 days) the start of treatment were retrospectively analysed. RRR was evaluated according to RECIST1.1 and mRECIST, to determine the ability of each method in predicting the response of HCC to Sorafenib, taking OS as end-point.

Results: The objective response $[\mathrm{OR}=$ complete response $(\mathrm{CR})+$ partial response (PR)], stable disease (SD) and progressive disease (PD) rates according to RECIST1.1 and mRECIST were 14\%, 25\%, 59\% and 25\%,18\%, 55\%, respectively. In CR+PR versus SD+PD patients, median OS was 24.3 months (both with RECIST1.1 and $\mathrm{mRECIST}$ ) versus 10.9 (with RECIST1.1) and 10.1 month (with mRECIST). OR was significantly associated with OS only according to mRECIST ( $p=0.007)$. Conclusion: RRR according to mRECIST, but not to RECIST1.1, sensibly correlates to outcome in cirrhotic patients with HCC treated with Sorafenib.

\section{B-0239 15:21}

Could antiplatelet therapy prevent hepatocellular carcinoma? 7 Tesla liver magnetic resonance imaging study in a mouse model of hbvrelated chronic hepatitis

P. Marra, A. Esposito, G. Sitia, A. Palmisano, T. Canu, F. De Cobelli,

L.G. Guidotti, A. Del Maschio; Milan/IT (marrapaolo87@libero.it)

Purpose: A dysfunctional T-cell response in chronic hepatitis B (CHB) causes cellular injury and viral persistence with increased risk of hepatocellular carcinoma (HCC). Accumulation of virus-specific cytotoxic lymphocytes in the liver is promoted by platelets. To assess the effectiveness of antiplatelet therapy in hepato-carcinogenesis prevention we used preclinical liver magnetic resonance imaging (MRI) in a mouse model of $\mathrm{CHB}$.

Methods and Materials: 220 transgenic mice with $\mathrm{CHB}$ were randomised to receive placebo (Vehicle $=110$ mice) or the experimental therapy with aspirin and clopidogrel $(\mathrm{Asp} / \mathrm{Clo}=110$ mice $) .220$ healthy transgenic mice formed two control groups (Sham = 110 irradiated mice; $\mathrm{Ctrl}=110$ unmanipulated mice). 20 mice per group underwent in vivo liver $\mathrm{MRI}$ at 9 and 15 months after $\mathrm{CHB}$ induction with a 7 Tesla scanner. Fat-sat T2w turbo-RARE and T1w RARE sequences were acquired after intravenous administration of Gd-EOB-DTPA (Primovist $(B)$ ). The remaining mice were used for biochemical, histological and survival analysis.

Results: In-vivo MRI demonstrated a significant reduction in incidence of HCC in the Asp/Clo versus placebo group both at 9 months ( $5 \%$ vs $20 \%, p<0.001)$ and 15 months $(30 \%$ vs $65 \%$, p < 0.001$)$. At the same time points $\mathrm{HCC} /$ total-liver volume ratio was, respectively, 3.8 and 5.4 fold smaller in Asp/Clo mice $(p<0.001)$. Accordingly histological analyses revealed that Asp/Clo therapy reduced immunopathology, hepatocellular regeneration and fibrosis. The experimental therapy also improved survival (mice died at day 520: $75 \%$ Vehicle vs $20 \%$ Asp/Clo, $p<0.0005$ ).

Conclusion: A prophylactic therapy with aspirin and clopidogrel in transgenic mice with $\mathrm{CHB}$ inhibits hepato-carcinogenesis and improves survival.
14:00 - 15:30

Room L/M

Physics in Radiology

\section{SS 213}

\section{Innovations in CT technology and data processing}

Moderators:

C. Leidecker; Forchheim/DE

R. Padovani; Udine/IT

\section{B-0240 14:00}

Performance evaluation of a new CT detector with minimal electronic noise for low dose abdominal perfusion imaging

E. Klotz, U. Haberland, B. Schmidt; Forchheim/DE

Purpose: $80 \mathrm{kV}$ are not routinely used for abdominal CT perfusion imaging with conventional detectors because non-linear effects occur when the electronic noise of the detector becomes larger than the photon noise. We evaluated a new detector design (Stellar, Siemens Healthcare) with minimal electronic noise for this application.

Methods and Materials: Inserts with iodine concentrations of 0.5 and $1.0 \mathrm{mg} /$ $\mathrm{ml}$ were placed in a $30 \mathrm{~cm}$ water-phantom and examined with $100 \mathrm{kV}$ and $80 \mathrm{kV}$ on two otherwise identical scanners equipped with a conventional and a Stellar detector (SOMATOM Definition Flash). Scans were performed using standard and periodic spiral dynamic mode (10 repetitions) varying image mAs from 15 to 250 (CTDI 0.3 to $5 \mathrm{mGy}$ ). We measured iodine contrast and noise. Dose normalised contrast-to-noise ratio $\mathrm{CNRn}=$ contrast/(noise*sqrt (dose)) was used as an objective measure for comparison.

Results: CNRn at $80 \mathrm{kV}$ was at least $8 \%$ lower for the conventional detector at all dose settings. Electronic noise became dominant at $60 \mathrm{mAs}$ and $\mathrm{CNRn}$ decreased by another $35 \%$ at $15 \mathrm{mAs}$ with increasingly prominent artefacts. For the Stellar detector CNRn did not decrease by more than $5 \%$ down to $15 \mathrm{mAs}$ and there were no relevant image artefacts. Using $80 \mathrm{kV}$ the same CNR of the clinical default setting (100 kV, $120 \mathrm{mAs})$ was obtained at $65 \%$ of the dose.

Conclusion: The Stellar detector allows the routine use of $80 \mathrm{kV}$ for abdominal perfusion imaging. For identical CNR this reduces the dose by $35 \%$ compared to $100 \mathrm{kV}$.

Author Disclosures:

E. Klotz: Employee; Siemens Healthcare, Germany. U. Haberland: Employee; Siemens Healthcare, Germany. B. Schmidt: Employee; Siemens Healthcare, Germany.

\section{B-0241 14:09}

Performance of a next-generation solid-state detector with fully integrated readout electronics for MDCT: quantitative analysis of image quality and radiation exposure reduction potential in comparison with its predecessor

G. Pahn, L.-S. Veloza, H.-P. Schlemmer, H.-U. Kauczor, W. Stiller; Heidelberg/DE (gregor.pahn@med.uni-heidelberg.de)

Purpose: Quantitative assessment of image quality and radiation exposure reduction potential of a next-generation solid-state MDCT detector with fully integrated readout electronics in comparison with its predecessor.

Methods and Materials: Using standard CT image quality phantoms and an automated quantitative image quality analysis software developed in-house, image quality and radiation exposure (RE) of an MDCT system (Somatom Definition Flash, Siemens) either equipped with UFC ${ }^{\mathrm{TM}}$ or new "Stellar" detectors has been assessed for different combinations of acquisition and reconstruction parameters. Subsequently, measurements and analysis were repeated for "Stellar" detectors without altering parameters apart from noise-weighted reduction of tube currenttime product resulting in image noise levels equal to those achieved when using their predecessor.

Results: Image noise of both detectors was comparable for small absorption crosssections (Øwater $=24 \mathrm{~cm}$ ) while being $18-35 \%$ lower for large ones $(\varnothing$ water $=40 \mathrm{~cm})$ when using "Stellar" detectors. CT number stability and uniformity declined with increasing subject cross-section, particularly at $80 \mathrm{kVp}$, though not as much for "Stellar" detectors. When noise of "Stellar" acquisitions was increased to UFC ${ }^{\mathrm{TM}}$

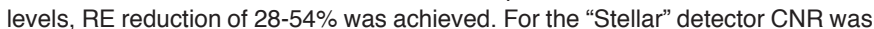
slightly higher (same RE) or slightly lower (reduced RE), while spatial resolution was always equal for both detectors (up to $20 \mathrm{lp} / \mathrm{cm}$ at MTF10\%).

Conclusion: Overall image quality improves when a next-generation MDCT detector is employed instead of using its predecessor. For acquisition settings intrinsically 
associated with high image noise the new detector exhibits much lower noise levels, offering great potential for radiation exposure reduction without adversely affecting overall image quality.

Author Disclosures:

H.-U. Kauczor: Advisory Board; Siemens Medical Solutions. Investigator; Boehringer Ingelheim. Speaker; Siemens Medical Solutions, Boehringer Ingelheim, Bayer, Bracco. W. Stiller: Equipment Support Recipient; Siemens Medical Solutions.

\section{B-0242 14:18}

Integrated circuit detector technology in abdominal computed

tomography: value in obese patients

F. Morsbach ${ }^{1}$, S. Bickelhaupt ${ }^{1}$, S. Raetzer ${ }^{1}$, B. Schmidt ${ }^{2}$, H. Alkadhi'; ${ }^{1}$ Zurich/CH, ${ }^{2}$ Forchheim/DE

Purpose: To assess the effect of an integrated circuit (IC) detector for abdominal CT imaging in obese patients on image quality.

Methods and Materials: An abdominal phantom with various extension rings was used simulating abdominal diameters of up to $600 \mathrm{~mm}$. The phantom was scanned on a CT equipped with either conventional discreet circuit detector (DC) or with an IC detector (at $120 \mathrm{kVp}$ and $150 \mathrm{mAs} / 75 \mathrm{mAs}$ ). Datasets were reconstructed with filtered back projection (FBP) and iterative reconstruction (IR). Images were evaluated by two blinded readers based on image quality and image noise. Image noise was additionally assessed in 20 patients undergoing abdominal CT with the IC detector who underwent previous CT at similar protocol setting with a DC detector. Results: Readers found improvements in image quality and subjective image noise in IC detector-acquired images compared with the DC detector in the largest phantom, regardless of reconstruction algorithm. Objective image noise was also significantly $(P<0.001)$ reduced for the largest phantom. A noise reduction of up to $44.5 \%$ was achieved for the IC detector when scanned with $75 \mathrm{mAs}$ and reconstructed with IR as compared with $\mathrm{DC}$ detector-acquired images. In patients, noise was significantly $(P<0.05)$ reduced by on average $6.4 \%$ with the IC detector. A significant correlation was found between noise and abdominal diameter in DC detector images $(r=0.604, P=0.005)$, whereas no such correlation was found for the IC detector $(\mathrm{P}=0.060)$.

Conclusion: Use of an IC detector appears particularly beneficial for abdominal CT imaging in large body diameters by reducing image noise.

\section{B-0243 14:27}

Comparison of filtered back projection (FBP) with hybrid and iterative reconstruction algorithms (HIR and IR) in low dose protocols including high and low kV settings in CT angiography by testing the detectability of simulated endoleak and thrombus in a phantom study

Z. Deák, J. Grimm, F. Mück, M. Treitl, M.F. Reiser, S. Wirth; Munich/DE (zsuzsanna.deak@med.uni-muenchen.de)

Purpose: To evaluate the dose reduction potential of HIR and IR in comparison with FBP.

Methods and Materials: An anthropomorphic cardiac phantom (QRM Corp., Germany) was scanned using a 64-row MDCT scanner (CT 750HD; GE-Healthcare, USA). The phantom's calcification insert was replaced with a model simulating a stented aortic aneurysm with endoleak and intraluminal thrombus. Images were obtained at tube voltages of $120 \mathrm{kV}, 100 \mathrm{kV}$ and $80 \mathrm{kV}$ using automated tube current modulation with incrementally increasing noise indices $(\mathrm{Nl}=16,25,34,43,52,61$, 70 ) adjusted to a primary reconstruction slice thickness of $2.5 \mathrm{~mm}$. After acquisition, images of $0.625 \mathrm{~mm}$ slice thickness were secondarily re-reconstructed with FBP, HIR (ASIR $®$ with $50 \%$ blending, GE-Healthcare) and IR (Veo®, GE-Healthcare). Twenty-one radiologists blinded to image reconstruction methods independently analysed the detectability of simulated vascular pathologies (endoleaks $<1 \mathrm{~cm}^{2}$ and intraluminal thrombus $<3 \mathrm{~mm}$ ). Contrast-to-noise ratio was measured for the stented aorta. A two-by-two table was used to calculate sensitivity, specificity value. Results: Sensitivity and specificity for vascular pathologies were $>90 \%$ with FBP at $3.45 \mathrm{mGy}, 1.23 \mathrm{mGy}$ and $1.46 \mathrm{mGy}$, with HIR at $1.83 \mathrm{mGy}, 0.84 \mathrm{mGy}$ and 1.02 $\mathrm{mGy}$, with IR at $0.41 \mathrm{mGy}, 0.46 \mathrm{mGy}$ and $0.55 \mathrm{mGy}$, at $120 \mathrm{kV}, 100 \mathrm{kV}$ and $80 \mathrm{kV}$. CNR of aorta showed corresponding results.

Conclusion: Simulated vascular pathologies were generally detectable at higher noise level than suggested by manufacturer. In comparison to FBP, the iterative and hybrid reconstruction algorithm allowed for a dose reduction by $88 \%$ and $47 \%$ at $120 \mathrm{kV}, 63 \%$ and $32 \%$ at $100 \mathrm{kV}$ and $70 \%$ and $30 \%$ at $80 \mathrm{kV}$, respectively, in our phantom study.
Author Disclosures:

S. Wirth: Speaker; GE Healthcare.

\section{B-0244 14:36}

Influence of sinogram affirmed iterative reconstruction of CT data on image noise characteristics and low-contrast detectability in comparison with filtered back projection: an objective approach C. von Falck ${ }^{1}$, V. Bratanova ${ }^{1}$, T. Rodt ${ }^{1}$, B. Meyer ${ }^{1}$, S. Waldeck ${ }^{2}$, F. Wacker ${ }^{1}$, H.-o. Shin'; ${ }^{1}$ Hannover/DE, ${ }^{2}$ Koblenz/DE (c.v.falck@gmx.de)

Purpose: To systematically evaluate the influence of sinogram-affirmed iterative reconstruction (SAFIRE) of MDCT data on image noise and low-contrast detectability using an objective approach.

Methods and Materials: A low-contrast phantom for MDCT was scanned on a 128-slice scanner at different dose levels $(25.50,100,150,200 \mathrm{mAs})$. The datasets were reconstructed using the standard kernel B30f (FBP) and an iterative algorithm (SAFIRE). A software phantom was used to insert virtual lesions with a contrast of $-20 \mathrm{HU}$. Low-contrast detectability was determined using the multiscale structural similarity index (MS-SSIM*).

Results: The use of SAFIRE led to an improvement in lesion detectability for all dose levels and lesions sizes. The relative improvement of low-contrast performance as compared with the FBP was inversely related to the dose level, declining from $208 \%( \pm 37 \%), 259 \%( \pm 30 \%)$ and $309 \%( \pm 35 \%)$ at $25 \mathrm{mAs}$ to $106 \%( \pm 6 \%)$, $119 \%( \pm 9 \%)$ and $123 \%( \pm 8 \%)$ at $200 \mathrm{mAs}$ for SAFIRE filter strengths of 1.3 and 5 , respectively $(p<0.05)$. There was no statistically significant influence of lesion size. The datasets reconstructed using SAFIRE reached at least the low-contrast performance of the datasets reconstructed with FBP at a relative dose of $50 \%$. The use of SAFIRE led to a shift of noise granularity towards a coarser image noise while higher dose levels showed a shift towards finer noise.

Conclusion: The use of SAFIRE improves low-contrast detectability when compared with FBP allowing for a dose reduction of approximately $50 \%$ without compromising low-contrast detectability or spatial resolution. The unfamiliar imaging appearance of iteratively reconstructed datasets may in part be explained by a different, coarser noise characteristic.

Author Disclosures:

C. von Falck: Research/Grant Support; Visage Imaging, Siemens Healthcare. T. Rodt: Research/Grant Support; Visage Imaging. B. Meyer: Research/ Grant Support; Visage Imaging. F. Wacker: Research/Grant Support; Siemens Healthcare, Visage Imaging.

\section{B-0245 14:45}

CTDI measurements using radiochromic films

N. Tomic, N. Sharoubim, F. DeBlois, J. Seuntjens; Montreal, QC/CA (nada.tomic@mail.mcgill.ca)

Purpose: We demonstrate a new method for computed tomography dose index (CTDI) measurements acquired on computed tomography (CT) scanners using XR-QA model radiochromic film dosimetry system. The method was tested comparing CTDI values measured and CT scanner listed for six different CT scanners at various locations.

Methods and Materials: Dose profiles were measured with XR-QA radiochromic film strips, sandwiched between acrylic rods cut in half and placed within CTDI body phantom. Films strips were scanned before and after irradiation with the Epson Perfection V500 photo scanner in reflective mode. The reference dosimetry system was calibrated in terms of air kerma in air. The measured reflectance change is converted into air kerma using a calibration curve. Air kerma was converted into absorbed dose using the appropriate ratio of mass-energy absorption coefficients water-to-air for a given beam quality, defined by half-value layer following the AAPM TG-61 protocol.

Results: Dose values for each of the five CTDI phantom film positions were obtained by averaging dose profiles from each film piece over the length of 10 $\mathrm{cm}$, and were used to calculate weighted CTDIvol. The average difference for six scanners between calculated and listed CTDIvol was $3.9 \%$ with a maximum observed difference of $6.9 \%$.

Conclusion: In contrast to ionisation chamber measurement, the proposed method requires only a single exposure, does not need stem effect correction, and provides an acceptable accuracy in comparison to CT scanner listed CTDI values. Measured dose profiles allow for measurements of DLP and peak doses at the same time. 


\section{B-0246 14:54}

Physical characterisation of iterative reconstruction algorithms in computed tomography and assessment of image quality for the optimisation of a dual-energy abdominal protocol

L. Berta, L. Mascaro, M. Cataldo, R. Maroldi; Brescia/IT (berta.luca@gmail.com)

Purpose: To assess the quality of $\mathrm{CT}$ images reconstructed with sinogram affirmed iterative reconstructions (SAFIRE) and comparing their performances with Iterative reconstructions in image space (IRIS) and standard filtered back projection (FBP) algorithms on a Siemens Somatom Definition Flash. Results were used to optimise a dual energy (DE) abdominal protocol.

Methods and Materials: FBP, IRIS and the 5 strength levels in SAFIRE (S1-S5) reconstructions were characterised in contrast, noise and spatial resolution for medium-smooth filters using a tungsten wire in air, water and Catphan phantoms. Standard deviation (SD), contrast-to-noise ratios (CNR), noise power spectra (NPS), modulation transfer function (MTF), and the non-prewhitening matched filter signal-to-noise ratio (SNRNPW), a quasi-ideal observer metric in the frequency domain assumed to be the most reliable image quality reference, were calculated for all kernels and used for dose saving evaluations.

Results: The highest CNR in DE protocol resulted with a composition factor of 0.6 . Compared to FBP, the average SD reductions were $51 \pm 2 \%$ with SAFIRE S5 and $30 \pm 2 \%$ with IRIS kernels. A shift towards low frequencies in the NPS was found for all iterative filters, giving to images a different texture. Differences between MTF values were within $5 \%$ in all FBP-Iterative coupled filter comparisons. The optimal kernels, from SNRNPW analysis of abdominal protocol, resulted I31-S5 for SAFIRE and I-44 for IRIS with a potential dose reduction of $42 \%$ and $30 \%$, respectively. Conclusion: Iterative reconstructions and DE acquisition strongly improve image quality reducing the noise and improving the contrast without affecting the spatial resolution.

\section{B-0248 15:03}

The impact of tube voltage, scan direction and beam collimation on the performance of automatic tube current modulation systems in paediatric and adult CT

N. Buls ${ }^{1}$, J. Schoenaers ${ }^{2}$, G. Van Gompel' ${ }^{1}$ K. Nieboer ${ }^{1}$, J. de Mey ${ }^{1}{ }^{1}$ Jette/BE, ${ }^{2}$ Hasselt/BE (Nico.Buls@uzbrussel.be)

Purpose: To report on the impact of CT scan parameter settings on the performance of an Automatic Tube Current Modulation system for paediatric and adult CT. Methods and Materials: A previously designed phantom was used which has an ellipsoidal cone shape with varying diameters that represents infant to adult sizes over five age categories (0-5y, 5-10y, 5-15y, 15-20y). Phantom material consists of two-component polyurethane prepolymer that shows an excellent attenuation agreement with ICRU-44 soft tissue for typical CT photon spectra. The impact of following scan parameters was assessed on a 750HD scanner (GE Healthcare): beam width, scan direction and tube voltage. ATCM performance was evaluated by assessing the noise variance in a central region of interest $(2 \mathrm{~cm})$ over the different age categories.

Results: For all tube potentials, the noise variance (mean VAR $=1.38$ ) was significantly lower $(p<0.05)$ when the phantom was scanned in the craniocaudal direction compared with the caudocranial direction (mean VAR $=1.66$ ). In the craniocaudal direction, a $80 \mathrm{kVp}$ scan resulted in the lowest noise variance (VAR $=0.49$ ) for the smallest age category $(0-5 y)$, whereas a $120 \mathrm{kVp}$ scan showed the highest variance $(\mathrm{VAR}=0.64)$. This in contrary to the largest age group $(15-20 \mathrm{y})$ where the $120 \mathrm{kVp}$ scan resulted in the lowest noise variance (VAR $=0.58$ ), compared with $80 \mathrm{kVp}(\mathrm{VAR}=0.82)$. The beam collimation width selection $(20$ and $40 \mathrm{~mm})$ had no impact on noise variance

Conclusion: The phantom can be used for assessment and optimisation of ATCM systems for patients of various sizes

\section{B-0249 15:12}

3D spatial resolution evaluation of clinical CT using a spherical phantom

T. Mäkelä, V. Sairanen, J.I. Peltonen, M. Kortesniemi; Helsinki/FI

(viljami.sairanen@helsinki.fi)

Purpose: Computed tomography (CT) scanners used in clinical imaging provides three-dimensional data and versatile visualisations for diagnostic use. However, the standard spatial resolution evaluation using the modulation transfer function (MTF) is often measured parallel to Cartesian axes and thus does not represent all possible directions. The aim of the study was to investigate the feasibility of using a spherical contrast phantom and directional MTF in the resolution assessments. Methods and Materials: The contrast phantom consisted of a Teflon (PTFE) sphere with a diameter of $20 \pm 0.05 \mathrm{~mm}$ placed within a polystyrene shell. The phantom was scanned with a multi-slice CT (MSCT) and a cone-beam CT (CBCT). A Matlab program was written for analysing image data and producing directional, radially averaged (rMTF) and frequency-wise averaged (fMTF) curves. 10\% fMTF value in XY-plane for both devices was compared with corresponding values measured with a tungsten wire reference method.

Results: $10 \%$ fMTF value in XY-plane was $0.71 \pm 0.01 \mathrm{lp} / \mathrm{mm}$ for the MSCT whereas for the CBCT it was $1.82 \pm 0.12 \mathrm{lp} / \mathrm{mm}$. Reference value for the MSCT was $0.68 \mathrm{Ip} /$ $\mathrm{mm}$ and for the CBCT $1.71 \mathrm{lp} / \mathrm{mm}$. 10\% fMTF value over the full solid angle for the MSCT was $0.74 \pm 0.03 \mathrm{lp} / \mathrm{mm}$ and for the СВCT $2.00 \pm 0.23 \mathrm{lp} / \mathrm{mm}$.

Conclusion: Directional MTF evaluation provides similar results as the standard method. However, it can be expanded to describe scanner's spatial resolution over the full solid angle without increasing the scanning time. Combined with fMTF's versatility and method's easy implementation in a clinical environment, the 3D MTF serves modern CT imaging well.

\begin{tabular}{ll}
\hline $14: 00-15: 30$ & Room N/O
\end{tabular}

Vascular

SS 215

\section{Major vessel imaging}

Moderators:

W.R. Jaschke; Innsbruck/AT

O. Pellerin; Paris/FR

\section{B-0250 14:00}

Low dose runoff CTA: what protocol is preferred without special reconstruction algorithms?

E. Kondratyev, G. Karmazanovsky; Moscow/RU (evgenykondratiev@gmail.com)

Purpose: To compare two low-dose scanning protocols, and to estimate effects on the signal, noise, effective radiation dose (ED), quality of acquired data.

Methods and Materials: 90 patients were divided into three groups. The scanning protocol was $120 \mathrm{kVp} 200 \mathrm{mAs}$ in first group, $80 \mathrm{kVp} 200 \mathrm{mAs}$ in second group, and $120 \mathrm{kV} 50 \mathrm{mAs}$ in third group, special reconstruction algorithms have not been used. For quantitative and qualitative evaluation we used visual scores (1$3)$, mean arterial attenuation, noise, contrast-to-noise ratio (CNR) in three arterial segments and ED.

Results: The BMI ranged from 21 to $38 \mathrm{~kg} / \mathrm{m} 2$ and were not significantly different between groups $(p=0.07,0.614,0.297$, respectively). The mean intraarterial attenuation in 1, 2, 3 groups was $315 \pm 6 \mathrm{HU}, 448 \pm 76 \mathrm{HU}, 334 \pm 33 \mathrm{HU}$, respectively, but significantly higher in second group $(p=0.0003)$. Noise in second group $(64 \pm 20$ $\mathrm{HU}$ ) was significantly higher than that in third group $(45 \pm 14 \mathrm{HU}, \mathrm{p}=0.0002)$, and first group $(29 \pm 8.5 \mathrm{HU}, \mathrm{p}=0.0002)$. The CNR in second $(6.64 \pm 2.3)$ and third $(6.36 \pm 2.1)$ groups was significantly lower than that in first group (11.7, $\mathrm{p}=0.0001)$. Image quality was comparable between three techniques, except aorto-iliac segment in second group. Effective dose was $27.1 \pm 5.9 \mathrm{mZv}, 8.1 \pm 1.8 \mathrm{mZv}$ and $6.4 \pm 1.02 \mathrm{mZv}$, respectively, differences were significant $(\mathrm{p}=0.0001)$.

Conclusion: Average radiation dose reduction up to $77 \%$ was achieved using " 80 $\mathrm{kV}$ " and " $50 \mathrm{mAs}$ " protocol providing sufficient image quality. Application of the 80 $\mathrm{kV}$ protocol in people with $\mathrm{BMI}>30$ is limited, especially in evaluation of aorto-iliac segment due to higher noise and attenuation levels. While at $50 \mathrm{mAs}$ protocol such limitation is not observed. This allows to recommend it as the preferred protocol for low-dose runoff CTA in case of special reconstruction algorithms.

\section{B-0251 14:09}

Value of contrast-enhanced MRA of the peripheral arteries at $3 \mathrm{~T}$ : results of a large European multicentre trial comparing meglumine gadoterate to gadobutrol-MRA with DSA

C. Loewe ${ }^{1}$, J. Arnaiz², D. Krause ${ }^{3}$, L. Marti Bonmati ${ }^{4}$, M. Aschauer ${ }^{5}$, A. Tartaro ${ }^{6}$, M. Lombardi 7 , M. Burrel ${ }^{8}$, R. Izzillo ${ }^{9} ;{ }^{1}$ Vienna/AT, ${ }^{2}$ Santander/ES, ${ }^{3}$ Dijon/FR, ${ }^{4}$ Valencia/ES, ${ }^{5} \mathrm{Graz} / \mathrm{AT},{ }^{6} \mathrm{Chieti} / \mathrm{IT},{ }^{7} \mathrm{Pisa} / \mathrm{IT}$, ${ }^{8}$ Barcelona/ES, ${ }^{9} \mathrm{Saint}$-Denis/FR (christian.loewe@meduniwien.ac.at)

Purpose: This subanalysis of a multicentre trial aimed to assess the value of contrast-enhanced MRA at $3 \mathrm{~T}$ for diagnosis and treatment planning in peripheral arterial occlusive disease (PAOD).

Methods and Materials: This multicentre trial including 189 patients was primarily aimed to compare the degrees of agreement in stenosis detection between contrast-enhanced MRA and DSA using two different contrast agents (Dotarem ${ }^{\circledR}$ or Gadovist $($ ). In this subanalysis, parameters related to stenosis detection and grading, specificity, sensitivity, positive/negative predictive values calculation (ac- 
curacy parameters), factors important for treatment planning and patient outcome were investigated. These factors included diagnostic confidence, stenosis length and vessel diameter. Additionally, signal/contrast to noise ratios (SNR/CNR) were calculated. Image data from 156 patients eligible for evaluation (per protocol population) were assessed by two independent readers in a centralized reading. Results: The patient management (angioplasty $64 \%$ vs $51 \%$, surgery $23 \%$ vs $26 \%$ ), diagnostic confidence ( $86.3 \%$ vs $86.2 \%)$, stenosis length and vessel diameters were similar in both groups. The global mean stenosis length was $54.26 \mathrm{~mm}$, and $5.32 \mathrm{~mm}$ for normal diameter, $1.49 \mathrm{~mm}$ for narrowest diameter. Both contrast agents were well tolerated.

Conclusion: This subanalysis demonstrates the value of peripheral MRA at $3 \mathrm{~T}$ for diagnosis and treatment planning in patients suffering from PAOD without any difference between Dotarem ${ }^{\circledR}-$ MRA and Gadovist ${ }^{\circledR}-$ MRA

\section{Author Disclosures:}

C. Loewe: Investigator; Multicenter trials sposored by Bayer, Guerbet. Speaker; Siemens, Bracco, GE Healthcare, Bayer, Guerbet, Covidien. J. Arnaiz: Investigator; Multicenter trial sponsored by Guerbet. D. Krause: Investigator; Multicenter trial sponsored by Guerbet. L. Marti Bonmati: Investigator; Multicenter trial sponsored by Guerbet. M. Aschauer: Investigator; Multicenter trial sponsored by Guerbet. A. Tartaro: Investigator; Multicenter trial sponsored by Guerbet. M. Lombardi: Investigator; Multicenter trial sponsored by Guerbet. M. Burrel: Investigator; Multicenter trial sponsored by Guerbet. R. Izzillo: Investigator; Multicenter trial sponsored by Guerbet.

\section{B-0252 14:18}

Nonenhanced quiescent-interval single-shot (QISS) MRA in assessment of peripheral artery disease: comparison with digital subtraction angiography (DSA)

R.S. Lanzman ${ }^{1}$, P. Heusch ${ }^{1}$, J. Aissa ${ }^{1}$, P. Schmitt ${ }^{2}$, J. Klasen ${ }^{1}$,

D. Blondin ${ }^{1}, \mathrm{G}$. Antoch ${ }^{1}$, P. Kröpil1; ${ }^{1}$ Düsseldorf/DE,

Erlangen/DE (Rotem.Lanzman@med.uni-duesseldorf.de)

Purpose: To evaluate a nonenhanced ECG-gated quiescent-interval single-shot MR angiography (QISS-MRA) of the lower extremity in patients with peripheral artery disease using digital subtraction angiography (DSA) as the reference standard.

Methods and Materials: 19 patients (mean age $68.2 \pm 12.1$ years) with peripheral artery disease were included in this study. Nonenhanced QISS-MRA of the distal aorta and run-off vessels was acquired at $1.5 \mathrm{~T}$ with $3 \mathrm{~mm}$ slice thickness with 0.6 mm overlap and an inplane resolution of $1.0 \times 1.0 \mathrm{~mm}$, resulting in a total scan time of approx. 9 min. ECG-gating was applied for synchronization of the quiescent interval with the period of maximum systolic inflow. The degree of stenosis was assessed by two readers using a 4-point scale (grade 1, normal appearing vessel; grade 2 , vessel narrowing < 50\%; grade 3 , stenosis 50\%-99\%; grade 4 , vessel occlusion) for 15 predefined anatomical segments. DSA served as the reference standard. Results: QISS-MRA was performed successfully in all patients. 226 arterial segments that were evaluated by DSA were considered for further analysis. With QISS-MRA the degree of stenosis was assessed correctly in 212 of 226 (93.8\%), overestimated in 13 of $226(5.8 \%)$ segments and underestimated in 2 of 226 $(0.9 \%)$ segments. As compared to DSA, QISS-MRA had a high sensitivity ( $98.6 \%)$, specificity $(97.5 \%)$ as well as positive and negative predictive values $(94.4 \%$ and $99.4 \%$, respectively) for detection of significant stenosis ( $350 \%)$.

Conclusion: ECG-gated QISS-MRA is a fast and reliable imaging technique for nonenhanced assessment of stenosis of the lower extremities.

Author Disclosures:

P. Schmitt: Employee; Siemens AG.

\section{B-0253 14:27}

Magnetic resonance thrombus characterisation can identify patients with rapid abdominal aortic aneurysm expansion

V.L. Nguyen ${ }^{1}$, T. Leiner ${ }^{2}$, M.C.J. Wishaupt ${ }^{1}$, F.A.M.V. Hellenthal ${ }^{1}$,

R.J. van der Geest ${ }^{3}$, E.M. Kooi ${ }^{1}$, G.W.H. Schurink ${ }^{1} ;{ }^{1}$ Maastricht/NL, ${ }^{2}$ Utrecht/NL,

${ }^{3}$ Leiden/NL (v.nguyen@mumc.nl)

Purpose: High signal intensity (SI) on T1-weighted (T1w) magnetic resonance imaging (MRI) of intraluminal thrombus in abdominal aortic aneurysm (AAA) indicates unorganised haemorrhagic thrombus. The purpose of the present study was to investigate whether AAA with high thrombus signal intensity on $\mathrm{T} 1 \mathrm{w} \mathrm{MRI}$ is associated with higher aneurysm expansion rate.

Methods and Materials: Twenty-one patients (15 males; age, mean age $( \pm S D)$ : $71 \pm 8$ years) with known AAA (Mean Dmax $( \pm S D): 50 \pm 6 \mathrm{~mm}$ ) underwent MRI examination at $1.5 \mathrm{~T}$ twice within a period of $190 \pm 27$ days. Two blinded observers independently analysed thrombus SI relative to muscle on MR images and patients were categorised as having either high thrombus SI (group I) or low thrombus SI (group II). Aneurysm expansion rates were expressed as the change in aneurysm surface area $\left(\mathrm{mm}^{2}\right)$ per day between baseline and follow-up.

Results: Interobserver agreement was high for the assessment of thrombus with high SI Kappa $=0.93)$. Patients in group.i. $(n=8)$ had higher aneurysm expansion rate compared with patients in group II $(n=13)\left(0.84 \pm 0.5 \mathrm{vs} .0 .38 \pm 0.5 \mathrm{~mm}^{2} / \mathrm{day}\right.$, respectively; $P=0.03$ ). Baseline maximal diameter was similar for both groups $(P=0.2)$

Conclusion: AAA with high thrombus SI on T1w TFE MRI, which indicates unorganised haemorrhagic thrombus, is associated with higher AAA expansion rates. We conclude that thrombus morphology can play an important role in the evolution of $A A A$ and that MR thrombus imaging may be a valuable tool for risk stratification of AAA. This study was founded by the Dutch Heart Foundation (2008B042).

\section{B-0254 14:36}

CT angiography of abdominal aorta with low kV CT protocol and low contrast medium volume: a feasibility study

C.R.G.L. Talei Franzesi, D. Ippolito, P. Bonaffini, A.C. Cadonici, A. Masetto, S. Sironi; Monza/IT (ctfdoc@hotmail.com)

Purpose: To reduce the radiation dose exposure and contrast medium volume using low-kV CT angiography protocol ( $100 \mathrm{kV} ; 40 \mathrm{ml})$ in the evaluation of abdominal aorta, maintaining high diagnostic performance.

Methods and Materials: A total of 60 patients with abdominal vascular disease were prospectively enrolled. All patients underwent 256MDCT scan examination of abdominal aorta (Brilliance iCT, Philips). 30 patients were scanned using lowdose radiation protocol (100 kV; automated tube current modulation) and ultralow-contrast volume $(40 \mathrm{ml} ; 4 \mathrm{ml} / \mathrm{s} ; 350 \mathrm{mgl} / \mathrm{ml})$ and a control group of 30 patients underwent standard CT-angiography protocol $(120 \mathrm{kV}$; automated tube current modulation) and standard contrast volume $(120 \mathrm{~mL})$. Density measurements were performed on abdominal aorta, renal arteries and common iliac arteries. The radiation dose exposure (dose length product, DLP), the intravessels density and the signal-to-noise ratio (SNR) were calculated, compared and statistically analysed. Results: All exams were considered diagnostic, allowing the correct visualisation of main aortic branch and vascular wall. Higher density measurements were obtained in low-kV protocol: mean attenuation value of abdominal aorta $343 \mathrm{HU}$, renal arteries $331 \mathrm{HU}$ and common iliac arteries; in comparison to control group: mean value of abdominal aorta $319 \mathrm{HU}$, renal arteries $294 \mathrm{HU}$ and common iliac arteries $323 \mathrm{HU}$. A reduction of $65 \%(p<0.05)$ of radiation dose exposure in low-kV protocol (335 DLP; 5.8 CTDIvol) was obtained among control group (973 DLP; 19.4 CTDIvol). Conclusion: Low kV protocol and ultra-low contrast medium volume $(40 \mathrm{~mL})$ reduce the radiation exposure (over 65\%) and the renal damage, allowing a relevant reduction of contrast material volume, enabling to evaluate more easily young patients or patients with renal failure.

\section{B-0255 14:45}

An international multi-centre comparison of the non-contrast MR angiography technique time-spatial labelling inversion pulse (timeSLIP) against contrast-enhanced CT angiography for assessing renal artery stenosis: the renal artery contrast-free trial (REACT) T.S.E. Albert ${ }^{1}$, I. Parienty ${ }^{2}$, V. Catala ${ }^{3}$, H. Xue ${ }^{4}$, V. Katabathina ${ }^{5}$, J.E. Lopera ${ }^{5}$, Z.Y. Jin ${ }^{4}$, N. Yellin 1 , M. Akahane $6 ;{ }^{1}$ Monterey, CA/US, ${ }^{2}$ Antony/FR, ${ }^{3}$ Barcelona/ES, ${ }^{4}$ Beijing/CN, ${ }^{5}$ San Antonio, TX/US, ${ }^{6}$ Tokyo/JP (timothy.albert@gmail.com)

Purpose: The non-contrast-enhanced MR angiography (NC-MRA) technique time-SLIP may provide a safe alternative for evaluating renal artery stenosis (RAS). This international multi-centre trial tested the hypothesis that NC-MRA is accurate and robust for assessing RAS in comparison to contrast-enhanced CT angiography (CTA)

Methods and Materials: Four centres (US, Europe, Asia) enrolled 64 patients (average \pm SD: $58 \pm 13$ years, $34(53 \%)$ males). Each patient received an abdominal CTA and abdominal NC-MRA using time-SLIP with balanced SSFP. All images were visually assessed for quality (arterial signal intensity) and absence or presence of RAS ( $</=50 \%$ or $>50 \%$ stenosis). In addition, arteries with any visible disease had stenotic severity quantified. Two blinded readers evaluated each study. No arteries were excluded from the analysis.

Results: NC-MRA image quality was excellent in $46 / 64$ patients $(72 \%)$ and good in $15 / 64$ patients (23\%). Using CTA as 'gold-standard' 21 of 128 arteries $(16.4 \%)$ had RAS. NC-MRA correctly classified 15/21 with RAS and correctly classified 97/107 as not having disease $(</=50 \%)$ : sensitivity $71 \%$, specificity $91 \%$, and accuracy $88 \%\left(X^{2}=0.56, p=0.45\right.$, no difference). Of the 16 misclassified arteries only 3 had clinically relevant misclassification (CTA $>/=70 \%$ and NC-MRA $</=50 \%$ or NC-MRA 
$>/=70 \%$ and $\mathrm{CTA}</=50 \%$ ). On average measured stenotic severity ( $\mathrm{n}=27$ arteries) was similar between NC-MRA $(63 \pm 17 \%)$ and CTA $(60 \pm 17 \%)(p=0.41)$.

Conclusion: To our knowledge this is the first international multi-centre trial to validate a NC-MRA technique. Compared with CTA the NC-MRA technique timeSLIP is robust and accurate for assessing renal artery stenosis.

Author Disclosures:

T.S.E. Albert: Consultant; Toshiba Medical Systems Corporation. Research/ Grant Support; Study funded by Toshiba Medical Systems Corporation. I. Parienty: Research/Grant Support; Toshiba Medical Systems Corporation. V. Catala: Research/Grant Support; Toshiba Medical Systems Corporation. H. Xue: Research/Grant Support; Toshiba Medical System Corporation. V. Katabathina: Research/Grant Support; Toshiba Medical Systems Corporation. J.E. Lopera: Research/Grant Support; Toshiba Medical Systems Corporation. Z.Y. Jin: Research/Grant Support; Toshiba Medical System Corporation. M. Akahane: Research/Grant Support; Toshiba Medical Systems Corporation.

\section{B-0256 14:54}

Zonal variations in the size of the atherosclerotic aortic arch during cardiac cycle and their implications on endovascular stenting S. Puppala, A. Shah, C. Dadnam; Leeds/UK (asimshah@doctors.org.uk)

Purpose: We aim to assess the expansile differences between the Ishimaru zones of the aortic arch throughout the cardiac cycle. This study further focuses on the implications of this variation in diameters of the aorta for sizing endovascular procedures.

Methods and Materials: Cardiac gated CT examinations of atherosclerotic ascending, arch and descending aorta, performed for a number of reasons, were evaluated. Presence of calcified and non calcified plaques in the arch was used as markers for atherosclerosis. Patients with acute aortic syndromes, connective tissue syndromes and aortitis were excluded. The best systolic and diastolic images were interrogated and true transverse slices were obtained using a 3D workstation. Differences between the systolic and diastolic images for the short axis, long axis and an average of the two were calculated.

Results: A total of 135 zones were evaluated in 27 patients. The average variation between diastole and systole diameters was 1 . Zone $0-0.17 \mathrm{~mm}$; 2 . Zone $1-0.0$ $\mathrm{mm}$; 3. Zone $2-0.38 \mathrm{~mm}$; 5 . Zone $4-0.58 \mathrm{~mm}$.

Conclusion: While the average variations were well under $1 \mathrm{~mm}$ for all zones the maximum was noted in zone $4(0.58)$ and the least in zone $1(0.0)$. The study shows limited variations in atherosclerotic aortas supporting the theory of reduced expansibility in them secondary to the disease process. This in turn suggest that we need not undertake gated examinations of the aorta prior to endovascular procedures thus avoiding the hazards of radiation.

\section{B-0257 15:03}

CT angiography for the evaluation of the thoracic aorta: dynamic changes in the cardiac cycle with implications for thoracic endograft treatment

J. Vallejos, C. Capunay, P. Carrascosa, J.C. Parodi, M.I. Sagarduy, P. Cortines; Vicente López/AR (javiervallejos@diagnosticomaipu.com.ar)

Purpose: The aim of this study was to demonstrate that there are significant differences in diameter and area in the descending aorta in the different phases of the cardiac cycle.

Methods and Materials: Twenty-five patients who had an indication of CTA were evaluated with a multidetector 256-slice CT. Two phases were scanned in systole and diastole so as to perform measurements in both. Measurements were done a $\mathrm{t} 1 \mathrm{~cm}, 4 \mathrm{~cm}$ and $8 \mathrm{~cm}$ from the left subclavian artery. Differences between systole and diastole were calculated at different levels using Student's T test.

Results: Comparison between systolic and diastolic phases showed a statistically significant difference in diameters and area, especially at $4 \mathrm{~cm}$ from the left subclavian artery: at $1 \mathrm{~cm}$ from the subclavian artery: DT mean difference of $0.7 \mathrm{~mm}$ $( \pm 1.2)$, two-sided $p=0.0024$. DAP: mean difference of $1.4 \mathrm{~mm}( \pm 1.2)$; two-sided $p<0.0001$. Area: mean difference $57.06 \mathrm{~cm} 2( \pm 39.04)$; two-sided $p<0.0001$. At $4 \mathrm{~cm}$ of the subclavian artery: DT mean difference of $1.83 \mathrm{~mm}( \pm 1.12)$, two-sided $\mathrm{p}=<0.0001$. DAP: mean difference of $1.68 \mathrm{~mm}( \pm 0.98)$; two-sided $p<0.0001$. Area: mean difference $74.30 \mathrm{~cm} 2( \pm 59.71)$; two-sided $p<0.0001$.

Conclusion: Measurements of the descending aorta at $4 \mathrm{~cm}$ from the left subclavian artery showed statistically significant differences between systole and diastole. The largest diameters of the aorta are the ones of the systolic phase. The systolic phase is the appropriate phase to determine the aortic diameters.
B-0258 15:12

Peripheral artery occlusion disease (PAOD): comparison of CE-MRA versus DSA in grading of stenosis and planning therapy

T.J. Vogl, C. Wurz, S. Zangos, A. Thalhammer, T. Schmitz-Rixen;

Frankfurt a. Main/DE (t.vogl@em.uni-frankfurt.de)

Purpose: To compare contrast-enhanced magnetic resonance angiography (MRA) with conventional digital subtraction angiography (DSA) for detecting stenoses and planning of therapy in patients with peripheral artery occlusion disease (PAOD). Methods and Materials: In this retrospective study, 71 patients ( 20 women/51 men; mean: 68 years) with established PAOD underwent both imaging modalities in a maximum interval of 40 days. DSA was the standard of reference. The pelvic and leg arteries were divided into 31 anatomic segments, which were graded on a scale from 1 to 4 ( $1=$ no stenosis, $2=$ stenoses $<70 \%, 3=$ stenoses ${ }^{3} 70 \%, 4=$ occlusion). The pelvic and leg vessel systems were categorised with the TASC II-score into five grades (none, TASC-A, TASC-B, TASC-C and TASC-D) for detecting whether the therapeutic consequences would be the same for both imaging modalities. Results: 1,937 vessel segments were evaluated. MRA and DSA agreed in the grading of 1,802 segments $(93.03 \%)$, and differed in 69 cases in one category, in 28 cases in two, in 38 cases in three categories. In discriminating between hemodynamically relevant ( $370 \%$ /occlusion) and non-haemodynamically relevant findings ( $<70 \% /$ nonstenosis) MRA achieved a sensitivity of $90.59 \%$ and a specificity of $96.61 \%$. Evaluation of TASC II-classification for the aorto-iliac region showed that in 52 of 56 patients TASC II-class was the same for DSA and MRA $(92.86 \%$; $\mathrm{K}=0.88$ ), for the femoral-popliteal region the result was nearly the same, the evaluation showed agreement in 52 of 56 patients $(92.86 \%$; $\mathrm{k}=0.90)$.

Conclusion: There was almost perfect agreement between MRA and DSA in the TASC classification. Thus, the therapeutic consequences are predominantly the same, irrespective of the modality used.

\section{B-0259 15:21}

lodine load reduction in CT aorta angiography with gemstone spectral imaging: comparison with standard CT aorta angiography

X. Luo, J. Wu, J. Sun, M. Chen; Yangzhou/CN (luoxianfu@gmail.com)

Purpose: To compare quantitative and subjective image quality between computed tomographic aorta angiography (CTAA) with a gemstone spectral imaging (GSI) technique with reduced iodine load and standard CTAA.

Methods and Materials: 86 patients underwent CT scanning on HDCT (Discovery CT750 HD, GE Healthcare). All cases were randomised into two groups: standard CTAA (100-120 kVp) with $500 \mathrm{mg} / \mathrm{kg}$ per body weight of iodine $(\mathrm{n}=46)$ and GSI scanning CTAA (image reconstruction at $50 \mathrm{keV}$ ) with the same injection volume as in the standard protocol but composed of contrast medium and saline in a 1:1.5 fashion $(n=40)$. Signal intensity and noise of aorta were measured; signal-to-noise ratio (SNR) and contrast-to-noise ratio (CNR) were calculated. A five-point scale was used to subjectively evaluate vascular enhancement and image noise.

Results: Compared with standard CTAA, GSI CTAA demonstrated higher signal intensity in all aorta arteries (all $P<0.05$ ), inferior noise only in segmental arteries $(P<0.05)$, higher SNR and CNR (both $P<0.05$ ), and compatible effective dose $(P>0.05)$. The five-point score was higher in the standard CTAA protocol $(P<$ 0.05). The inter-reader agreement regarding the dichotomized diagnostic versus nondiagnostic scale was similar $(P>0.05)$ between two groups.

Conclusion: GSI CTAA with image reconstruction at 50 kev allows a significant reduction of $60 \%$ iodine load while improving intravascular signal intensity, maintaining SNR and with comparable radiation dose. 
14:00 - 15:30

Room P

\section{Cardiac}

\section{SS 203}

\section{Planning cardiac interventions}

Moderators:

I. Arkhipova; Moscow/RU

P. Mildenberger; Mainz/DE

\section{B-0260 14:00}

Anatomical angulation between the long axis of the left ventricle and left ventricular outflow tract as possible predictor of postprocedural complications in patients undergoing transapical aortic valve implantation B. Foldyna, M. Haensig, C. Lücke, D. Holzhey, S. Nitzsche, M. Borger, F.W. Mohr, M. Gutberlet, L. Lehmkuhl; Leipzig/DE (b.foldyna@gmail.com)

Purpose: To examine whether the angle between the left ventricular long axis and left ventricular outflow tract (aLV-LVOT) allows prediction of the occurrence of paravalvular leakage $(\mathrm{PL})$, its impact on procedural time and elevated postoperative CK-MB levels in patients undergoing transapical valve implantation (TA-AVI). Methods and Materials: 75 consecutive high-risk patients with severe aortic stenosis scheduled for TA-AVI were included. All patients underwent preoperative cardiac computed tomography (CT) and received Edwards-Sapien ${ }^{T M}$ prosthesis. The aLV-LVOT was measured in systole and diastole using multiplanar reconstruction of the preoperative MSCT data. The aLV-LVOT was correlated to occurrence of the $\mathrm{PL}$, to the procedure duration and postoperative CK-MB levels. All measurements were performed by two experienced radiologists to calculate interobserver variability. Results: Cardiac CT was available in 75 patients (age $81.9 \pm 5.8$ years, $71 \%$ female). The mean aLV-LVOT was $61.8 \pm 9.8^{\circ}$ and $61.3 \pm 10.1^{\circ}$ in systole and diastole, respectively, without a significant change during the cardiac cycle. Paravalvular leakage was found in a total of 35 patients $\left(I^{\circ}=29 / 38.7 \%,\left\|I^{\circ}=6 / 8 \%,\right\| I^{\circ}=0\right)$. Patients with relevant $\left({ }^{3} I^{\circ}\right)$ paravalvular leakage showed significantly steeper mean aLV-LVOT than patients without or $I^{\circ}$ paravalvular leakage (Mean difference: $12.2^{\circ} \pm 4.1^{\circ} ; p<$ $0.001)$. The aLV-LVOT was not associated with the prolonged procedural time nor the postoperative CK-MB levels $(p=n . s)$.

Conclusion: In TA-AVI the aLV-LVOT angle had a significant effect on the occurrence of paravalvular leakage. A steeper angle was associated with a significant higher grade of PL; thus, it might have a significant impact on the future design of valve stents and new delivery devices.

\section{B-0261 14:09}

Temporal dynamics of the aortic root and the aortic valve: implications for transcatheter aortic valve implantation

T. Jurencak, B. Kietselaer, C. Mihl, V. Van Ommen, L. van Garsse, J. Wildberger, M. Das; Maastricht/NL (t.jurencak@gmail.com)

Purpose: To determine temporal changes of dimensions of the aortic root and the aortic valve during the cardiac cycle and to investigate its effect on Transcatheter Aortic Valve Implantation (TAVI) therapy planning.

Methods and Materials: MDCT examination with retrospective ECG-gating was performed in 52 consecutive patients referred for pre-TAVI assessment. Images were reconstructed at eleven time points throughout the cardiac cycle. Ten anatomical structures within the aortic root and the aortic valve were measured at each time point. Temporal changes for each structure were evaluated by paired-samples T-test and annular changes in dimensions were investigated in respect to prosthesis sizing. Results: Four out of ten anatomical structures show significant changes in dimension $(p<0.05)$ during the cardiac cycle. We observed significant dimensional changes in the shortest diameter of the aortic annulus $(\Delta=3.7 \pm 1.3 \mathrm{~mm})$; the sinotubular junction $(\Delta=2.9 \pm 1.1 \mathrm{~mm})$; the aortic root at the left coronary ostium level $(\Delta=3.5 \pm 0.9 \mathrm{~mm})$ and the aortic sinus diameter $(\Delta=2.8 \pm 0.9 \mathrm{~mm})$, respectively. The largest dimensions were measured at $20 \%$ of the RR interval. Prosthesis sizing based on systolic or diastolic measurements would lead to selection of a different valve size in more than $20 \%$ of subjects.

Conclusion: Temporal changes in annular dimensions can have important impact on prosthesis sizing. Imaging in systole ( $20 \%$ RR interval) provides maximal aortic root dimension measurements and should therefore be used for prosthesis sizing.

\section{B-0262 14:18}

Aortic annulus area assessment by multidetector computed tomography for predicting paravalvular regurgitation in patients undergoing balloon-expandable transcatheter aortic valve implantation - a comparison with transthoracic and transoesophageal echocardiography G. Pontone, D. Andreini, E. Bertella, S. Mushtaq, S. Cortinovis, A. Annoni, A. Formenti, G. Ballerini, M. Pepi; Milan/IT (gianluca.pontone@ccfm.it)

Purpose: Transcatheter aortic valve implantation (TAVI) is a valid alternative to surgery in high risk patients with severe aortic stenosis (AS). Aortic annulus (AoA) sizing is crucial for TAVI success. The aim of the study is to compare AoA dimensions measured by multidetector computed tomography (MDCT) versus those obtained with transthoracic (TTE) and transoesophageal echocardiography (TEE) for predicting paravalvular aortic regurgitation (PVR) after TAVI.

Methods and Materials: AoA maximum diameter, minimum diameter and area were assessed with MDCT and compared to TTE and TEE diameter and area for predicting PVR after TAVI in 151 patients (45 men, age 81.2 \pm 6.4 years).

Results: AoA maximum, minimum diameter and area detected by MDCT were $25.04 \pm 2.39 \mathrm{~mm}, 21.27 \pm 2.10 \mathrm{~mm}$, and $420.87 \pm 76.10 \mathrm{~mm}^{2}$, respectively. AoA diameter and area measured by TTE and TEE were $21.14 \pm 1.94 \mathrm{~mm}, 353.82 \pm 64.57$ $\mathrm{mm}^{2}, 22.04 \pm 1.94 \mathrm{~mm}$ and $384.33 \pm 67.30 \mathrm{~mm}^{2}$, respectively. A good correlation was found between AoA diameters and area evaluated by MDCT versus TTE and TEE $(0.61,0.65$ and 0.69 , and $0.61,0.65$ and 0.70 , respectively) with a mean difference of $3.90 \pm 1.98 \mathrm{~mm}, 0.13 \pm 1.67 \mathrm{~mm}$ and $67.05 \pm 55.87 \mathrm{~mm}^{2}$, and $3.0 \pm 2.0$ $\mathrm{mm}, 0.77 \pm 1.70 \mathrm{~mm}$ and $36.54 \pm 56.43 \mathrm{~mm}^{2}$, respectively. Grade 32 PVR occurred in 46 patients and was related to male gender, higher BMI, pre-procedural aortic regurgitation and lower mismatch between the nominal area of the implanted prosthesis and AoA-AMDCT.

Conclusion: Mismatch between prosthesis area and AoA area detected by MDCT is a better predictor of PVR as compared to echocardiography mismatch. Specific MDCT-based sizing recommendations should be developed.

\section{B-0263 14:27}

Transcatheter aortic valve replacement: performance of an aortic valvular complex model for semi-automated quantification of aortic annulus dimensions at cardiac CT

P. Blanke ${ }^{1}$, E.-M. Spira ${ }^{1}$, D. Bernhardt'², R. lonasec ${ }^{3}$, U. Ebersberger ${ }^{4}$, T. Flohr ${ }^{2}$, M. Langer ${ }^{1}$, U.J. Schoepf 5 ; ${ }^{1}$ Freiburg/DE, ${ }^{2}$ Forchheim/DE, ${ }^{3}$ Princeton, NJ/US, ${ }^{4}$ Munich/DE, ${ }^{5}$ Charleston, SC/US (philipp.blanke@uniklinik-freiburg.de)

Purpose: To evaluate the accuracy and time-effectiveness of semi-automated model-based annulus computation compared with manual planimetry for assessment of aortic annulus dimensions prior to transcatheter aortic valve replacement (TAVR). Methods and Materials: Retrospectively ECG-gated dual-source cardiac CT data of 50 consecutive TAVR candidates $(82.5 \pm 5.8$ years) were included. Data were reconstructed at $300 \mathrm{~ms}$ past the R-peak and were analysed using an automatic $3 \mathrm{D}$ aortic valve model, fitted to CT-data by discriminative learning methods and incremental search. It contains the aortic root's surface, commissures and hinges and allows for automated morphologic identification of the annulus plane, defined by the most basal hinge points of the aortic cusp. Manual planimetric measurement using multiplanar reformations was used as the reference standard. Data were analysed using linear regression and Bland-Altman plots. Hypothetical prosthesis sizing ( $23 \mathrm{~mm}$ prosthesis for $<22 \mathrm{~mm}$ aortic annulus; $26 \mathrm{~mm}: 22-25 \mathrm{~mm} ; 29 \mathrm{~mm}$ : $>25 \mathrm{~mm}$ ) was compared using k-statistics.

Results: Aortic valve hinge-points were correctly identified in $47 / 50$ patients (94\%). Mean effective annulus diameter was $24.5 \pm 2.3 \mathrm{~mm}$ by model-based analysis and $24.6 \pm 2.2 \mathrm{~mm}$ by manual assessment $(p=n . s)$. Excellent correlation was found between both methods $(r=0.98, p<0.01)$. Bland-Altman analysis revealed no systematic bias. Agreement for prosthesis sizing was found in $44 / 50$ patients $(k=0.80)$. Mean analysis time was significantly $(p<0.001)$ reduced for model-based measurements ( $28 \pm 11 \mathrm{sec}$ vs. $92 \pm 13 \mathrm{sec}$ ).

Conclusion: Semi-automated morphologic aortic annulus quantification derived from an aortic valve model enables fast and accurate procedural planning in excellent agreement with manual planimetry and has the potential to improve cardiac imaging workflow in the evaluation of patients prior to TAVR.

Author Disclosures:

D. Bernhardt: Employee; Siemens. R. Ionasec: Employee; Siemens. T. Flohr: Employee; Siemens. U.J. Schoepf: Consultant; GE; Bracco, Bayer Healthcare, Medrad. 


\section{B-0264 14:36}

Dimensions of the aortic root and the aortic valve for transcatheter aortic valve implantation: non-ECG-triggered multi-detector CT high-pitch scans compared with retrospectively ECG-gated low-pitch cardiac scans

T. Jurencak, B. Kietselaer, C. Mihl, P. Nelemans, V. Van Ommen, L. van Garsse, J. Wildberger, M. Das; Maastricht/NL (t.jurencak@gmail.com)

Purpose: To compare low-pitch retrospectively ECG-gated (gated) and high-pitch non-ECG-triggered (non-gated) multidetector CT (MDCT) protocols in terms of measurements of the aortic root and the aortic valve and consequently prosthesis sizing in Transcatheter Aortic Valve Implantation (TAVI).

Methods and Materials: 46 consecutive TAVI candidates underwent MDCT examination with retrospectively gated helical scanning of the aortic valve region followed by a non-gated whole aorta CT angiography. Eleven anatomical structures within the aortic root and the aortic valve which are deemed to be crucial for TAVI planning were measured throughout the cardiac cycle and compared with measurements from non-ECG-gated scans. Differences in annular dimensions measured in both types of scans were tested in terms of relevance for prosthesis sizing.

Results: Substantial differences in measurements were found for the aortic root at the right coronary ostium level $(p<0.05)$, the sinotubular junction and the aortic sinuses diameter $(p<0.001)$. The maximal annular dimensions occur in early systole. Measurements of diameters in high-pitch scans agree only to diastolic images. However, measurements of annular perimeter in non-ECG-triggered highpitch scans agree to systolic images. The highest agreement in prosthesis sizing according to measures from both CT protocols in systole and diastole was noted if annular perimeter was followed.

Conclusion: Even though the results of annular perimeter are comparable at first glance, only a dedicated gated CTA protocol offers the full potential to perform all measurements at the optimal time point and should be recommended for prosthesis sizing in TAVI.

\section{B-0265 14:45}

Is aortic valve calcium distribution a predictor for outcome after transcutaneous aortic valve implantation?

P. Apfaltrer ${ }^{1}$, G. Schymik ${ }^{2}$, T. Germann ${ }^{1}$, H. Schroefel ${ }^{2}$, M. Meyer ${ }^{1}$,

C. Fink', S.O. Schönberg', T. Henzler ${ }^{1} ;{ }^{1}$ Mannheim/DE,

${ }^{2}$ Karlsruhe/DE (paul.apfaltrer@umm.de)

Purpose: To examine possible relationships between severity of aortic valve calcification, the degree of aortic-valve-regurgitation (AVR) and occurrence of MACE events after transcatheter-aortic-valve-implantation (TAVI).

Methods and Materials: 58 patients ( 25 men, $83.3 \pm 6.1$ years) with symptomatic aortic stenosis were included in this study. 15 patients received a CoreValve ${ }^{\circledR}$ aortic valve prosthesis and in 43 patients an Edwards SAPIEN ${ }^{\mathrm{TM}}$ prosthesis was implanted. Prior to TAVI, all patients underwent a thoracic-pelvine ECG-gated-CTangiography for therapy planning. The aortic-valve calcification score (AS) and aortic-valve calcium volume (AV) was measured and compared to post-procedural AVR measured by echocardiography and to the 30 -day incidence of major-adversecardiac-events (MACE).

Results: The overall incidence of MACE after TAVI was 29.3\%. There was no significant correlation between the amount of AS and AV and MACE after TAVI (both $p>0.05$ ). Post-interventional AVR did not correlate with pre-procedural measured AS and AV (both $p>0.05$ ). Patients with post-procedural AVR showed no significant differences in $A S$ and $A V$ than patients without evidence of $A V R$ after TAVI ( $698.5 \pm 113.6$ vs. $658.8 \pm 114.1$ and $1.20 \pm 0.78$ vs. $1.30 \pm 0.95$; both $p>0.05$ ). Conclusion: The degree of aortic-valve calcification is neither associated with MACE nor with post-procedural AVR after TAVI. Further risk scores are required to predict patient outcomes after TAVI.

Author Disclosures:

G. Schymik: Consultant; Edwards Lifescience. Speaker; dwards Lifescience, Philips, Medtronic.

\section{B-0266 14:54}

Influence of observer experience and training on proficiency in cardiac CT interpretation

J.M. Kerl' ${ }^{1}$, U.J. Schoepf ${ }^{2}$, R.W. Bauer ${ }^{1}$, P. Costello², T.J. Vogl'1, C. Herzog';

${ }^{1}$ Frankfurt a. Main/DE, ${ }^{2}$ Charleston, SC/US (matthias.kerl@kgu.de)

Purpose: To assess the influence of experience and training on the proficiency in coronary CT angiography (CCTA) interpretation of practitioners with different levels of experience.
Methods and Materials: This study was IRB approved and HIPAA compliant. Three radiologists with 8 years, 5 years, and 12 months of experience in reading CCTA, a first year radiology resident, and an interventional cardiologist without previous experience independently analysed two case series of 50 catheter-correlated CCTA studies for coronary artery stenosis $(0 \%, 249 \%, 50 \%-74 \%, 75 \%-99 \%$, or $100 \%)$. Results of the first case series were unblinded and presented along with catheter angiography results to each reader before proceeding to the second series. Diagnostic accuracy on a per-segment basis was compared for all readers and both case series, respectively.

Results: Correlation coefficients between CCTA and catheter angiography initially ranged between good $(r=0.87)$ and poor $(r=0.26)$, depending on reader experience, and significantly $(p<0.05)$ improved in the second case series (range: $r=0.91$ to $\mathrm{r}=0.51$ ). Diagnostic accuracy was significantly ( $\mathrm{p} 12$ months of experience (range: 97.8-96.5\%) as compared to the inexperienced readers (range: $90.7-93.1 \%)$ ) and only slightly $(p=0.26)$ increased in the second case series. However, PPV significantly $(p<0.05)$ increased. For both inexperienced readers diagnostic accuracy (range: $95.6-95.9 \%)$ significantly $(p<0.05)$ increased and equalized $(p=0.04)$ in the second case series, but sensitivity and PPV still remained rather low (range: $62.7-66.1 \% / 78.0-84.1 \%)$.

Conclusion: The level of experience is the strongest determinant of proficiency in CCTA interpretation. Limited one-time training improves proficiency, but not to clinically satisfactory levels.

Author Disclosures:

J.M. Kerl: Speaker; Siemens AG. U.J. Schoepf: Other; Research Consultant, Bayer AG Research Grant, Bayer AG Research Consultant, Bracco Group Research Grant, Bracco Group Research Consultant, General Electric Company Research Grant, General Electric Com. R.W. Bauer: Speaker; Siemens AG.

\section{B-0267 15:03}

Utility of cardiac magnetic resonance in the identification of patients eligible for early implantation of cardioverter defibrillator after acute myocardial infarction

E. Belloni', E. Emanuele ${ }^{2}$, G. Marchesi ${ }^{3}$, D. Aschieri' ${ }^{3}$ S. Ferraro ${ }^{3}$, E. Michieletti ${ }^{3}$, G.Q. Villani ${ }^{3}$, P. Scagnelli'; ${ }^{1}$ Castel San Giovanni/lTT, ${ }^{2}$ Robbio/IT, ${ }^{3}$ Piacenza/IT (belloni.elena@alice.it)

Purpose: To evaluate the utility of cardiac magnetic resonance (CMR) in early identification of irreversible left ventricle (LV) damage after acute myocardial infarction (AMI) and in prediction of absent future improvement of LV ejection fraction (LVEF), to identify who should benefit from early implantable cardioverter defibrillator (ICD) implantation.

Methods and Materials: 29 patients underwent CMR within $5 \pm 3$ days after first revascularized $\mathrm{AMI}$ and at 3 months. The number of $\mathrm{LV}$ wall segments with transmural necrosis (TN) and microvascular obstruction (MO) at LE imaging was evaluated. MO was defined as hypointensity within a hyperintense LE area. Patients were subdivided in 2 groups according to the LVEF at Cine imaging: gr.1=LVEF $35 \%$ $(n=10) ;$ gr.2=LVEF $>35 \%(n=19)$.

Results: In the acute phase, the number of segments with TN and MO was higher in gr. 1 than in gr.2 (TN:gr. $1=6.1 \pm 0.5$, gr.2=2.5 \pm 2.8 , unpaired $t$-test, $P<0.001 ; \mathrm{MO}$ : gr.1=3.1 \pm 2.3 , gr.2=1.0 \pm 1.9 , unpaired t-test, $P=0.02$ ). In the whole group of 29 patients, the number of segments with $\mathrm{TN}$ and $\mathrm{MO}$ correlated inversely with LVEF (TN: Spearman's rho $=-0.79, P<0.001$ ) (MO: Spearman's rho $=-0.59, P<0.001$ ). At 3 months, LVEF decreased in gr.1 and increased in gr.2 (gr.1: from $33.1 \pm 2.2 \%$ to $29.6 \pm 3.2 \%$, paired t-test, $P=0.017$ ) (gr.2: from $50.0 \pm 9.4 \%$ to $54.4 \pm 8.4 \%$ paired t-test, $P=0.006$ ).

Conclusion: At CMR, the presence of TN (with or without MO) in at least $6 \mathrm{LV}$ wall segments in patients with LVEF'35 immediately after AMI seemed to be correlated with irreversibile myocardial damage and absent LVEF improvement at 3 months. CMR could be a useful tool in the stratification of patients with revascularized AMI, to identify who should benefit from early ICD implantation.

\section{B-0268 15:12}

Optimisation of contrast injection protocol for CT angiography in transcatheter aortic valve implantation (TAVI): preliminary results D. Di Marco', M. Giglio², R. Fachechi', S. Sironi', P. Spagnolo ${ }^{2} ;{ }^{1}$ Monza/IT, ${ }^{2}$ Milan/IT (daniela.dimarco@gmail.com)

Purpose: To evaluate the feasibility of a BMI-adjusted injection protocol on vessel attenuation and image quality for CT angiography of patients referred for TAVI. Methods and Materials: 64-MDCT angiography with retrospective gating was performed in 45 patients with a BMI-adapted contrast medium (CM) bolus: BMI $<22,40 \mathrm{~mL} \mathrm{CM}$ at $3.5 \mathrm{~mL} / \mathrm{s}$; 22.1-26.5, $55 \mathrm{~mL}$ at $4.5 \mathrm{~mL} / \mathrm{s} ;>26.6,70 \mathrm{~mL}$ at 5.5 $\mathrm{mL} / \mathrm{s} .45$ control patients matched for BMl who had previously undergone routine 
MDCT-examination with a fixed CM protocol of $100 \mathrm{ml}$ at $5 \mathrm{ml} / \mathrm{s}$ served as reference group. Two radiologists independently evaluated the image sets in terms of diagnostic confidence (based on a 3-point scale) and arterial contrast opacification in Hounsfield units (HUs). Attenuation values in the ascending aorta and in the aorto iliac vessels were measured and correlated with $\mathrm{BMI}$; the results in the two protocol groups were compared.

Results: there was no significant difference between image quality grade and mean attenuation values of the two groups $(346 \pm 82 \mathrm{HU}$ vs $357 \pm 63 \mathrm{HU}$; p not significant). Without BMI adaptation, vessels attenuation correlated strongly with BMI $(r=-0.61 ; p<0.001)$. No correlation between BMI and vessel attenuation ( $r$ $=-0.22$; $p$ not significant) was found with the BMl-adapted protocol.

Conclusion: the BMI-adjusted injection protocol allows a significant reduction of the amount of $\mathrm{CM}$ injected while maintaining image quality. It should be recommended in high-risk TAVI patients in order to avoid unnecessarily high iodine administration and prevent radiocontrast-induced nephropathy.

\section{B-0269 15:21}

Assessment of aortic valve area in patients with severe aortic stenosis using cardiac dual-source computed tomography: comparison with transthoracic echocardiography and cardiac magnetic resonance

H. An, S. Ko, H. Hwang, M. Song, J. Choi, J. Shin; Seoul/KR

(erantis@hanmail.net)

Purpose: To compare aortic valve area (AVA) in severe aortic stenosis (AS), using dual-source CT (DSCT) in comparison with transthoracic echocardiography (TTE) and cardiac MR (CMR) and to determine whether degree of valvular calcification is related to planimetric AVA obtained with DSCT.

Methods and Materials: 114 patients (66 male, mean age 60 years) with severe AS who underwent TTE, DSCT and CMR were included in the study. The AVA estimated by planimetry (DSCT, CMR) and continuity equation (TTE). Mean AVAs measured by each method were compared with paired t-test, Bland-Altman analysis and correlation coefficient. Valvular calcium grade and calcium volume score on DSCT were correlated with planimetric AVA by DSCT and valvular morphologic characteristics.

Results: The mean AVA derived by DSCT, TTE and CMR were $0.94 \pm 0.16$, $0.80 \pm 0.17$, and $0.80 \pm 0.15 \mathrm{~cm}^{2}$, respectively. DSCT-derived AVA correlated fairly with TTE-derived $(r=0.46)$ and moderately with CMR-derived $(r=0.65)$. There was good $(r=0.74, p<0.001)$ agreement between valvular calcium grade and volume score. DSCT-derived AVA correlated fairly ( $r=0.39$ and 0.30 , respectively, $p<0.01$ ) with valvular calcium grade and volume score. DSCT-derived AVA with severe degree of valvular calcification $\left(n=38,0.86 \pm 0.16 \mathrm{~cm}^{2}\right)$ was significantly smaller than those of no $\left(\mathrm{n}=6,1.06 \pm 0.17 \mathrm{~cm}^{2}\right)$, mild $\left(\mathrm{n}=31,1.0 \pm 0.15 \mathrm{~cm}^{2}\right)$ and moderate $\left(n=39,0.94 \pm 0.13 \mathrm{~cm}^{2}\right)$ degree of calcification. There was no difference in valvular calcium score between bicuspid $(n=59)$ and tricuspid $(n=55)$ valves $(2489 \pm 2242$ versus $1928 \pm 1459, p=0.116)$. TTE-derived AVA was smaller in $\operatorname{BAV}\left(0.76 \pm 0.18 \mathrm{~cm}^{2}\right)$ than TAV $\left(0.83 \pm 0.14 \mathrm{~cm}^{2}\right)$ but DSCT- $\left(0.92 \pm 0.16 \mathrm{~cm}^{2}\right.$ and $\left.0.96 \pm 0.15 \mathrm{~cm}^{2}\right)$ and CMR $\left(0.78 \pm 0.16 \mathrm{~cm}^{2}\right.$ and $\left.0.81 \pm 0.12 \mathrm{~cm}^{2}\right)$-derived AVAs were not different.

Conclusion: DSCT significantly overestimates the AVA compared to TTE and $\mathrm{CMR}$ in calcified severe AS. Both degree and volume score of valvular calcification associate fairly with AVA on DSCT.

\section{$14: 00-15: 30$} Room Q

\section{Radiographers}

\section{SS 214}

\section{Managing quality and dose in CT}

Moderators:

E. Agadakos; Athens/GR

A. Yule; Cardiff/UK

\section{B-0270 14:00}

Personalised low dose chest CT applying adaptive iterative dose reduction algorithm (AIDR 3D) integrated automatic exposure control techniques with 320-slice MDCT: initial clinical trial

X. Chen; Guangzhou/CN (hoshi_chen@tom.com)

Purpose: To investigate the feasibility of individualised low dose chest CT with AIDR3D integrated automatic exposure control (AEC) techniques.

Methods and Materials: 48 subjects (age: 13-84 yrs; BMI: 15.62-30.84) who underwent chest CT previously with conventional protocol (group A:AEC with target noise SD12.5 and FBP reconstruction) and referred to follow-up chest CT within two months were enrolled. AEC (SD25) integrated with AIDR3D was used in follow-up chest CT protocol (group B), the raw data of which were reconstructed again with FBP (group $\mathrm{C}$ ). The max/min $\mathrm{mAs}$ of each subject and their correlation with BMI were investigated to validate whether system could personalise tailor tube current. The chest images were divided into upper, middle and lower parts which were evaluated separately by two radiologists with 3-point scale (3-good, 2-acceptable, 1-poor). Image noise was measured in these parts with 8-mm diameter ROI placed on specific anatomic structures. Effective dose (ED) was compared. Results: In group B, max/min mAs correlated positively with BMI ( $p=0.001)$; image quality scores of upper, middle and lower part of chest (lung kernel: 2.90, 2.90, 2.90, $\mathrm{p}=1.000$; mediastinum kernel: $2.63,2.60,2.69, \mathrm{p}=0.710$ ) showed no significant difference, the mean noise of which was 32.01, 32.35, 34.09 for lung/16.58, 14.37, 16.66 for mediastinum. More cases in group $\mathrm{B}$ than in group $\mathrm{C}$ achieved diagnostic (scored $\left.{ }^{3} 2\right)$ image quality (100\%, 96\% vs 100\%, 40.82\% for lung and mediastinum, respectively). CTDI, DLP, ED of group $B$ was much lower than group $A$ (1.371 vs $10.435 \mathrm{mGy}, 51.09$ vs $394.555 \mathrm{mGy}^{*} \mathrm{~cm}, 0.72$ vs $\left.5.52 \mathrm{mSv}\right), 87 \%$ radiation dose was saved in the follow-up chest CT.

Conclusion: Personalised low dose chest CT is feasible by applying AIDR 3D integrated $A E C$ techniques, which could reduce $87 \%$ radiation dose compared with conventional exam and achieve uniform image quality.

\section{B-0271 14:09}

Feasibility of low dose protocol at 640 -slice dynamic volume CT for diagnosing central airways narrowing

Z.-X. Ding, Z. Wang; Hangzhou/CN (wang12345zhen@sina.com)

Purpose: To compare the radiation dose and image quality of the central airways using 640-slice dynamic volume CT at two different protocols.

Methods and Materials: Sixteen patients with small BMI $(\mathrm{BMI}<25)$ underwent 640 -slice dynamic volume CT examinations. Eight patients employed $100 \mathrm{kv}$ protocol, acquisition time, 5.1 seconds; and remaining eight objects employed $80 \mathrm{kv}$ protocol, acquisition time, 5.2 seconds. Image acquisition time was selected according to Texp time (from maximal end-inspiration to the end of forceful exhalation). MPR and $3 \mathrm{D}$ reconstruction images were created, and reviewed by two experienced radiologists with blinded, who measured the tracheal lumen to determine the presence of central airways narrowing. The 3D reconstruction of confidence was graded on a 4-point scale from 0 (no confidence) to 3 (highest of confidence. The MPR noise was assessed by measuring the SD of the pre-sternal soft tissue. The DLP was used to compare radiation doses.

Results: The MPR image noise was ranked higher for CT images of $80 \mathrm{KV}$ protocol than $100 \mathrm{KV}$ protocol. There was no significant difference in confidence between $80 \mathrm{KV}$ and $100 \mathrm{KV}$ protocol $(P>0.05)$. Excessive central airway collapse (reduction $>30 \%$ ) was seen in 3 patients with tracheomalacia and remaining 13 subjects had centre airway stenosis. DLP of $80 \mathrm{KV}$ protocol was $244.9 \mathrm{mGy} . \mathrm{cm}$ and $100 \mathrm{KV}$ protocol was 539.8 mGy.cm.

Conclusion: The 640-slice dynamic volume CT is a promising method for diagnosing central airway narrowing. The $80 \mathrm{KV}$ protocol performs as well as the $100 \mathrm{KV}$ protocol for the 4D dynamic imaging in small BMI objects.

\section{B-0272 14:18}

Conducting a national diagnostic reference level survey for computed tomography examinations: the Portuguese experience J. Santos ${ }^{1}$, S. Foley' ${ }^{2}$, G. Paulo', M.F. McEntee ${ }^{3}$, L. Rainford ${ }^{2} ;{ }^{1}$ Coimbra/PT, ${ }^{2}$ Dublin/IE, ${ }^{3}$ Sydney/AU (joanasantos@estescoimbra.pt)

Purpose: Research has shown that CT technology, if not correctly applied, has radiation protection implications. This study aimed to identify the frequency of CT examinations, current protocols and establish national diagnostic reference levels (DRLs) for commonly performed paediatric examinations.

Methods and Materials: A national contact list was formulated and a website created. A questionnaire survey of practice across all CT sites was completed, preceded by a pilot study and invitations to the clinical sites inviting participation. Demographic data were collated, collected over a seven-month period and protocols were reviewed (including methods of paediatric age categorisation). The $75^{\text {th }}$ percentiles for CT volume index (CTDIvol) and dose-length product (DLP) values of commonly performed examinations were identified.

Results: A 21\% response rate resulted from the national survey, from which $55 \%$ were public institutions. Head (22\%), thorax (10\%) and abdominal (12\%) CT examinations were identified as the most commonly performed. National DRLs are recommended in CTDIvol (mGy) and DLP (mGy.cm) for the head $75 \mathrm{mGy} / 1010$ mGy.cm, thorax 13.6 mGy/470 mGy.cm and abdomen $18.3 \mathrm{mGy} / 800 \mathrm{mGy} . \mathrm{cm}$, respectively. Specific paediatric protocols and age categorisation methods varied 
considerably, therefore paediatric CT DRLs were not defined based on this survey and optimisation research is continuing for paediatric examinations.

Conclusion: Portuguese DRLs for adult CT examinations have been identified and recommended based on the national survey. The majority are higher than European recommendations, suggesting substantial potential for optimisation. A lack of standardisation of practice for paediatric CT examinations was identified justifying further research.

\section{B-0273 14:27}

Comparison of image quality between left and right arm injection of contrast agent in computed tomography of carotid arteries

D. Hribar, N. Djuric, S. Sterzaj, T. Holc, M. Kovacic, A. Janezic; Ljubljana/SI

(dejan.hribar@kclj.si)

Purpose: Artefacts in carotid computed tomography (CTA) can degrade image quality and may be related to the arm side where contrast agent is injected. The objective of this study is to evaluate the differences in image quality with the use of left versus right-arm injection side.

Methods and Materials: 50 patients were included in the study (27 males, 23 females, age $67.6 \pm 12.65$ years). 40-MSCT was used (spiral technique, $120 \mathrm{kV}$, $150 \mathrm{mAs}, \mathrm{AEC}$, slice thickness $0.75 \mathrm{~mm}$, reconstruction increment $0.4 \mathrm{~mm}$ ). All patients received $80 \mathrm{~mL}$ of $\mathrm{CM}$ followed by a $40-\mathrm{mL}$ saline chaser bolus, both administered IV at $4 \mathrm{~mL} / \mathrm{sec}$. Contrast agent was injected either into the right arm $(n=25)$ or into the left arm $(n=25)$. We determined the relationship between the injection side and the intensity of perivenous streak artefacts and venous reflux. Results: Streak artefacts in the aortic arch and at the origin of the great vessels were demonstrated in $80 \%$ of patients with left arm injection (55\% without influence on the diagnosis, $35 \%$ with possible influence). There was significantly less artefacts in patients with right-arm injection (44\% all without influence on the diagnosis). The amount of refluxed contrast agent was slightly greater with left-arm injection (40\%) versus right-arm injection (32\%).

Conclusion: Our results are similar to other researches. Perivenous streak artefacts and venous reflux could not be prevented with right- or left-arm injections although right-arm injection of contrast agent may provide better image quality in CT angiography of carotid arteries.

\section{B-0274 14:36}

Dose assessment in paediatric head computed tomography examinations

C.L.D.C. Fernandes, S.I. Rodrigues, A.F.C.L. Abrantes, L.P.V. Ribeiro,

R.P.P. Almeida, C.A. Silva, K.B. Azevedo; Faro/PT (cindy_lineuza@hotmail.com)

Purpose: To determine if the doses used in paediatric head CT scanning are appropriate and according to the reference dose levels by International Commission on Radiological Protection (ICRP) and Département federal de l'intérieur (DFI). Methods and Materials: A retrospective study was conducted in two radiology departments from public hospitals using a sample of 535 patients under 15 years submitted to head CT examinations between 2008 and 2011, performed with different equipments: Somatom Emotion $2(n=100)$, Somatom Emotion $6(n=250)$ and Somatom Emotion $16(n=185)$. The influence of patient age and cephalic perimeter were considered. Multiple data analysis was used in order to assess radiation dose levels and the concordance of these with diagnostic reference levels.

Results: The results were obtained for four different paediatric groups, divided according to patient age: less than 1 year; 1 to 5 years; 6 to 10 years and 11 to 15 years. The mean values obtained of dose length-product from some groups are lower than the diagnostic reference levels (720 mGy.cm from 6 to 10 years and 807 mGy.cm from 11 to 15 years). However, until 5 years the values are higher than the recommendations (569 mGy.cm for less than 1 year and 590 mGy.cm from 1 to 5 years).

Conclusion: This work identifies possible gaps and all possible methods should be applied to reduce the radiation dose provided without deteriorating image quality. A set of recommendations are needed to optimise the radiation dose since the children have a superior radiosensitivity.

\section{B-0275 14:45}

Dose values in eye lens in paediatric brain computed tomography: influence of different protocols

C. $\underline{\text { Carriço }}^{1}$, M. Inácio', A. Kristiansen², M. Larsen², J. Santos ${ }^{1}$, S. Holm²,

G. Paulo ${ }^{1} ;{ }^{1}$ Coimbra/PT, ${ }^{2}$ Odense/DK (catarina.carrico@hotmail.com)

Purpose: The number of head computed tomography (CT) examinations in paediatrics had an exponential increase. Taking into account the highest radiosensitive of children tissues and organs, radiology departments should permanently optimise CT protocols in order to minimize exposure, especially to more radiosensitive organs as eye lens. The main goals of this study were to evaluate the influence of different paediatric head CT protocols, used in Portugal and Denmark, regarding CT dose values and eye lens exposure.

Methods and Materials: ACT Siemens Somatom Definition AS 64 slices was used and after a quality control, CTDI (CT dose index) and DLP (dose length product) values were determined. Different protocols were applied in a paediatric phantom and some exposure parameters were changed. The dose values in the eye lens were measured using an Unfors EDD-30 dosimeter. Radiologists from Portugal and Denmark blindly assessed the image quality obtained with different protocols. Results: The best compromise between image quality and CTDIvol (75.71 mGy) and DLP (513 mGy.cm) values was observed in the supra tentorial image of the Portuguese protocol, with a change of slice thickness to $5 \mathrm{~mm}$. According to the results obtained, the eye lens dose values decrease $(20.98 \%)$ when the gantry orientation is supra-orbital-meatal.

Conclusion: The best image is not necessarily the one that has higher dose. This study enabled a cooperation and exchange of experiences between Portuguese and Danish students, where they have developed good practices in paediatric head CT.

\section{B-0276 14:54}

Influence of different arm positioning of MSCT of chest by apply automatic tube current modulation technique

Z.-X. Ding, Z. Wang; Hangzhou/CN (wang12345zhen@sina.com)

Purpose: The purpose of this study was to examine the influence of different arm positioning on automatic tube current modulation of multi-slice CT on chest image quality and radiation dosage.

Methods and Materials: Sixty patients underwent scanning by using Care Dose 4D thorax CT protocol in which both arms were raised above the shoulder region using standard-position (Group 1) and postural manoeuver (Group 2), respectively. Objective and subjective image quality was assessed. Individual recorded the CTDIV values and the tube current-time product per exposed section. Effective radiation dose was calculated.

Results: Compared with the image quality in the standard-position group, the image quality in the postural manoeuver group was decreased on subjective image quality but within acceptable diagnostic limits. The average effective dose in the postural manoeuver group was $3.37 \mathrm{mSv}$; the dose in the standard-position was $17 \%$ lower than this.

Conclusion: Both arms were raised above the shoulder region scanning using Care Dose 4D thorax CT protocol can reduce the overall dosage to patients and the image quality without adverse influences, and may be more feasible in clinical practice.

\section{B-0277 15:03}

The effectiveness of lead apron for radiation protection in $\mathrm{CT}$

N. Weber, P. Monnin, C. Elandoy, S. Ding; Lausanne/CH

(nicolas.weber@hesav.ch)

Purpose: To determine the effectiveness of lead shielding for radiation protection in CT.

Methods and Materials: Three 32-cm diameter CTDI (PMMA) phantoms, of 15 $\mathrm{cm}$ long, placed side by side, were scanned over a length of $28 \mathrm{~cm}$ with a GE 64 CT scanner. Absorbed dose was estimated with thermoluminescent dosimeters positioned in the middle, and at two levels in periphery of the phantom ( $1 \mathrm{~cm}$ inside): top and bottom. Additionally, the dose was measured on the surface of the phantom ("skin dose"). TLD were placed over a length of $28.5 \mathrm{~cm}$, with $14.5 \mathrm{~cm}$ in the scatter field. Three acquisitions were performed: 1) without lead apron, 2) with a lead apron at $0 \mathrm{~cm}$ from the edge of the primary field and 3) with a lead apron at $2.5 \mathrm{~cm}$. Results: In scattered radiation, no significant difference of dose was found between without shielding and with the apron at $0 \mathrm{~cm}$, in the middle of the phantom. However, in the presence of the apron the dose is lowered by as much as $12 \%$ at the bottom of the phantom, whereas the skin dose is increased by $10 \%$ (tunnel effect). This indicates that the scanner table is the main source of external scattered radiation. When the apron is placed at $2.5 \mathrm{~cm}$, the results are intermediate to the one obtained with the shielding at $0 \mathrm{~cm}$ and without shielding.

Conclusion: The effectiveness of lead shielding is very low and limited to regions near the scanner table. 


\author{
B-0278 15:12 \\ Optimisation of local diagnostic reference levels in head computed \\ tomography \\ M. Monteiro, A. Carvalho, J. Matias, J. Santos; Coimbra/PT \\ (mmonteiro@estescoimbra.pt)
}

Purpose: The increasing use of computed tomography (CT) scans resulted in the exposure of patients to high level exposure to ionising radiation compared with other modalities. Considering the frequency of head CT examinations, the establishment of diagnostic reference levels (DRL) and optimisation of dose levels without loss of diagnostic image quality is crucial.

Methods and Materials: The local DRLs of head CT in a public hospital of the center of Portugal were established by the analyses of patients CT dose index (CTDI) and dose length product (DLP) values. To the optimisation process a anthropomorphic standard patient phantom (Radon Woman) was used and image quality was subsequently evaluated by radiographers with more than 15 years of CT experience. Results: We obtained a reduction in the value of CTDI up to $38 \%$ without significant loss of image quality for diagnostic purposes in comparison with hospital protocol used in this institution.

Conclusion: Through the optimisation of the head protocol the CT dose values in patient can be reduced maintaining the image quality for diagnostic.

\section{B-0279 15:21}

Comparison of radiation dose and image quality between sequential and spiral brain $\mathrm{CT}$

I. Pace, F. Zarb; Msida/MT (francis.zarb@um.edu.mt)

Purpose: To evaluate and compare sequential CT to spiral CT examinations of the brain in terms of radiation dose and image quality.

Methods and Materials: The study was carried out prospectively on 40 patients referred for brain CT. Radiation dose and image quality data were gathered from an equal number of CT examinations performed with either sequential $(n=20)$ or spiral $(n=20)$ techniques. Radiation dose data in terms of CTDlvol and DLP were recorded by the scanning radiographers at the end of each examination. ACT brain image evaluation set was compiled which was evaluated by four radiologists. The radiologists evaluated the quality of the images by visually grading the reproduction of anatomical structures as outlined in EUR 16262: European guidelines on quality criteria for CT.

Results: Mean CTDIvol and DLP readings were significantly higher $(p<0.05)$ for sequential scans over spiral scans (CTDIvol: $22.06 \mathrm{mGy}$ vs. $14.94 \mathrm{mGy}$ and DLP: $304.60 \mathrm{mGycm}$ vs. $229.10 \mathrm{mGycm})$. The mean image quality grading scores for all criteria for the sequential technique were significantly $(p<0.05)$ higher than those obtained by the spiral technique.

Conclusion: Spiral acquired CT brain images have lower radiation doses. The quality of the images produced using this technique, however, produces images of a lower quality to those obtained using the sequential technique. Further research is recommended investigating whether the level of image quality produced by the spiral technique is sufficient for diagnosis in order for patients to benefit from associated radiation dose reductions.

\section{$14: 00-15: 30$}

Room Z

\section{Computer Applications}

\section{SS 205}

\section{Quantitative image analysis and optimisation}

\section{Moderators:}

L. Faggioni; Pisa/IT

M. Fatehi; Tehran/IR

\section{B-0280 14:00}

Consistent automatic adipose tissue quantification in MR abdomen images

D. Smeets, W. Van Hecke, D. Loeckx; Leuven/BE (dirk.smeets@icometrix.com)

Purpose: The amount and distribution of body fat are well-known health risk factors. For use as clinical biomarker, an accurate and reproducible quantification, differentiating between visceral (VAT) and subcutaneous adipose tissue (SAT), is indispensible. This is performed by the proposed method.

Methods and Materials: Four datasets of 2 subjects, each containing T1 water and fat images are acquired using Dixon-based fat suppression. First, a coarse body mask is obtained by thresholding and morphological operations. Next, the body mask is classified into 4 classes (fat, water, air, and outliers) by a multivariate Gaussian intensity model, regularized by a Markov random field. To increase robustness to image variability, the statistical model parameters are automatically updated with the EM algorithm. The air voxels are rejected from the mask and the adipose tissue segmentation is refined using a new 3-class Gaussian intensity model. To separate SAT from VAT, an intra-abdominal mask is created by calculating the convex hull around the water tissue (water fraction of at least $50 \%$ ).

Results: The adipose tissue quantification is, even under heavy motion, visually satisfactory. It is, moreover, highly consistent over the two datasets of each subject, as demonstrated by the high ICC ( $\mathrm{r}=0.997)$ and the small difference in fat, SAT, and VAT percentage of total body volume of $0.26 \%, 0.14 \%$, and $0.12 \%$, respectively. Conclusion: An automatic adipose tissue quantification method based on spatially regularized multivariate Gaussian mixture models is demonstrated to provide consistent measurements for SAT and VAT.

\section{B-0281 14:09}

Computational texture analysis in interstitial lung disease: comparison of descriptors and classification accuracy

J. Ofner ${ }^{1}$, C. Mueller-Mang ${ }^{1}$, A. Burner', D. Markonis²,

A. Depeursinge ${ }^{2}, \mathrm{H}$. Mueller ${ }^{2}$, G. Langs ${ }^{1} ;{ }^{1}$ Vienna/AT, ${ }^{2}$ Sierre/CH (joachim.ofner@meduniwien.ac.at)

Purpose: The purpose of this work is the comparison of local texture features for fully automated quantification of Interstitial Lung Disease (ILD) related pathological tissue in High Resolution Computed Tomography (HRCT) images. Different features are compared, and a method for the identification of an optimal feature set for discrimination of lung texture classes is proposed.

Methods and Materials: The approach was evaluated on 97 HRCT images of pathological lung tissue. Slice thickness was $1 \mathrm{~mm}$ and inter-slice distance 10 $\mathrm{mm}$. Regions of normal and four pathologic patterns (emphysema, ground glass, fibrosis, micronodules) were annotated by two radiologists. We extracted sets of texture features (Local Binary Patterns (LBP), Haralick features, wavelets, textons) and evaluated the classification performance on voxel level and region level with a tenfold cross validation. A Random Forest classifier was used as classifier, and corresponding feature importances served as feature selection criterion.

Results: On voxel level Haralick features performed best with $69.59 \%$ classification accuracy (chance level: $20 \%$ ). On region level LBPs reached the highest classification performance of $77.45 \%$. The most discriminative features of the texture classes are intensity histogram values in the range between -1024 and $-800 \mathrm{HU}$. Conclusion: Experiments reveal that LBPs on region level outperform classical and computationally more complex co-occurrence matrices. Furthermore, intensity is an essential feature for description of ILD-related textures. In general region-level features outperform voxel-level features.

\section{B-0282 14:18}

Quantification of correlations between somatostatin receptors expression in biopsies from neuroendocrine tumours and PET/CT uptake values in the context of personalised medicine

M. Athelogou', D. Kaemmerer', A. Lupp ${ }^{3}$, V. Prasad ${ }^{4}$, R. Schoenmeyer ${ }^{1}$, G. Binnig', R. Baum ${ }^{2} ;{ }^{1}$ Munich/DE, ${ }^{2}$ Bad Berka/DE, ${ }^{3}$ Jena/DE, ${ }^{4}$ Berlin/DE (mathelogou@definiens.com)

Purpose: Somatostatin receptors (SSTR) are expressed in nearly all neuroendocrine tumours (NET), especially in gastroenteropancreatic NET (GEP-NET). Diagnosis, prognosis and treatment of NET patients are primarily based on SSTR expression and PET/CT. Therefore objective quantifications of scoring methods and correlations between PET/CT uptake values and SSTR expression in biopsies are needed. A method for such quantifications is introduced.

Methods and Materials: Automated image analysis is used for the detailed quantification of SSTR expression in each virtual slide from NET biopsies of 30 patients by using Definiens software. Tumour regions are separated from nontumour regions and staining intensity is calculated. Cells, nuclei and membranes are segmented and classified. The results are correlated with SSTR expression scoring calculated by visual inspection. All the previous data are correlated with corresponding PET/CT uptake values.

Results: Image analysis results of 237 virtual SSTR stained tissue slides show positive correlations with visual calculated SSTR expression scoring ( $p$-values $<$ 0.005). Correlations between the PET/CT uptake values and SSTR image analysis results show also $p$-values $<0.05$.

Conclusion: Automated image analysis is used to quantify correlations between patient data of in vivo and in vitro modalities like PET/CT and SSTR expression. These results are very promising and quantitatively support our hypothesis of a "strong correlation" between SSTR stained biopsy tissue and PET/CT uptake values. This quantification is necessary for diagnosis and prognosis to establish patient individual treatment for NET and especially for GEP-NET. 


\section{B-0283 14:27}

3D analysis CT software in volumetric and densitometric analysis of urinary stones

M. Moschetta, A. Scardapane, M. Telegrafo, G. Angelelli, A.A. Stabile lanora; Bari/IT (marco.moschetta@gmail.com)

Purpose: To assess the reliability of 3D analysis CT software in determining urinary stones volume and density.

Methods and Materials: CT images of 140 patients for a total of 219 urinary stones (116 calyceal, 22 pelvic, 81 ureteral) were evaluated. All exams were performed using a 320 MDCT with a low dose protocol and without injection of contrast material. CT scans were independently evaluated by two expert radiologists. For each stone, volumetric [SV (stone volume) $=\mathrm{I}$ (length) $\mathrm{x} \mathrm{w}$ (width) $\mathrm{xt}$ (thickness) $\mathrm{x} \pi \times 0.52]$ and densitometric [selecting a ROI on axial plane] measurements were calculated. The obtained data were then compared with the measurements performed by the 3D analysis CT software (Vitrea fX 2.1). Differences between the two systems were evaluated by the analysis of variance (Anova) test. The interobserver agreement was calculated using the Cohen's kappa ( $\mathrm{k}$ ) test.

Results: The volumetric and densitometric measurements of urinary stones obtained without 3D analysis software showed a lower interobserver agreement $(\mathrm{k}=$ $0.54)$ than that resulting from the use of the software $(k=0.98)$. Statistical significant differences were found between volumetric and densitometric measurements as performed with and without the 3D software for each urinary stone $(p<0.05)$.

Conclusion: Volumetric and densitometric evaluation of the urinary stones provided by $3 \mathrm{D}$ analysis software is reliable and reproducible and could significantly influence the therapeutic choices.

\section{B-0284 14:36}

The 3DSlicer open-source platform for segmentation, registration, quantitative imaging and 3D visualisation of biomedical image data S. Pujol' 1 , S. Pieper ${ }^{2}$, R. Kikinis ${ }^{1} ;{ }^{1}$ Boston, MA/US, ${ }^{2}$ Cambridge, MA/US (spujol@bwh.harvard.edu)

Purpose: 3DSlicer is an open-source platform for medical image computing and image-guided intervention used in biomedical and clinical research worldwide. The software aims at delivering cutting-edge medical image analysis technology to the clinical and scientific community. This abstract introduces translational clinical researchers to the capabilities of 3DSlicer.

Methods and Materials: 3DSlicer is a multidisciplinary platform that integrates standard radiological viewing capabilities and state-of-the-art tools for segmentation, registration, quantitative analysis and $3 \mathrm{D}$ visualisation of multi-modal imaging data. 3DSlicer is developed through a multi-institution effort of several large-scale consortia, including NA-MIC,NAC and NCIGT. The platform is built upon a set of open-source software packages allowing developers to create tools within a robust architectural framework. 3DSlicer integrates 97 modules, and is freely available for Windows, Linux and MacOS under a BSD-style open-source license. Hands-on workshops based on the comprehensive training compendium of the software are delivered on a regular basis at national and international venues. 3DSlicer's extensible architecture allows plugin development, such as SlicerRT for radiotherapy research and SlicerIGT for image-guided therapy.

Results: 3DSlicer is being used in numerous academic institutions and research laboratories worldwide. Clinical applications include neurosurgery, prostate cancer, atrial fibrillation, Huntington's disease and traumatic brain injury. More than 2,000 clinicians and scientists have attended 3DSlicer training workshops, and the latest version of the software 3DSlicer version 4 has been downloaded over 37,000 times since its release in late 2011

Conclusion: 3DSlicer is a multi-institution effort of several large-scale consortia to share and disseminate the latest advances in medical image computing to clinical researchers.

\section{B-0285 14:45}

Can the CT acquisition protocol be used in combination with the study description to optimise patient dose tracking for CT examinations? T. De Bondt' 1 , Q. Collier', R. Salgado ${ }^{1}$, M. Geldof', F. Deferme ${ }^{1}$, J. Valette ${ }^{2}$, A. Jomier ${ }^{2}$, W. Van Hecke 1 , P.M. Parizel' ${ }^{1} ;{ }^{1}$ Antwerp/BE, ${ }^{2}$ Mutzig/FR (timo.debondt@ua.ac.be)

Purpose: To improve the dose alert triggering mechanism for CT scans, in order to decrease the incidence of irrelevant alerts and increase the reporting of clinically relevant alerts.

Methods and Materials: We prospectively evaluated dose information for CT examinations on two scanners using dedicated software (DoseWatch ${ }^{\circledR}$ ), over a 7 -month period (10273 studies). We selected the most common studies and de- termined the number of alerts triggered, based on the currently used, exclusively study description-based threshold method. The default setting of this threshold is twice the median for each separate descriptor. Then, logical combinations of study description and protocol were made and linked by a RSNA Radlex elements, for a total of $62 \%$ of all examinations. The mapped data sets were then used to determine the number of alerts triggered when using a threshold method based on a combination of study description and acquisition protocol. The impact of both strategies on relevant dose information was assessed.

Results: Considering only the relevantly mapped scans, we found a $51 \%$ decrease, from 959 to 466 alerts, using the Radlex-based alert triggering. Of these, $23(5 \%)$ were not previously reported by the old method. The new alerts could all be attributed to patient or examination-related factors. Of the 470 alerts (49\%) that were no longer reported, none were found to have any clinical relevance.

Conclusion: The combination of study description and acquisition protocol in the threshold method for CT dose measurements helps to decrease the number of alerts, while maintaining patient safety.

Author Disclosures:

J. Valette: Employee; SerphyDose. A. Jomier: Employee; SerphyDose.

\section{B-0286 14:54}

A patient dose managing solution identifies erroneous parameter settings in CT acquisition protocols, and contributes towards lowering radiation exposure

T. De Bondt' ${ }^{1}$, Q. Collier', R. Salgado', M. Geldof', F. Deferme', J. Valette ${ }^{2}$, A. Jomier ${ }^{2}$, W. Van Hecke' ${ }^{1}$, P.M. Parizel' ${ }^{1} ;{ }^{1}$ Antwerp/BE, ${ }^{2}$ Mutzig/FR (timo.debondt@ua.ac.be)

Purpose: To optimise CT protocols and parameter settings, by comparing the radiation dose between 2 different CT scanners of the same type.

Methods and Materials: Using dedicated software (DoseWatch $\circledast$ ), dose information from CT examinations performed on two 64-slice LightSpeed VCT scanners over an 7-month period was prospectively evaluated. We compared the administered radiation doses for 61 equivalent acquisition protocols on the 2 machines, and identified acquisition protocols in which there was a significant dose variation. For these protocols, we performed a rigorous imaging parameter check, including comparison of the number of examinations, acquisition types, number of irradiation events, $\mathrm{mA}, \mathrm{KVp}$, scanning length, exposure time and pitch factor. These data were then linked to the protocol setup.

Results: We found significant parameter variations in 5 out of $61 \mathrm{CT}$ acquisition protocols. In 4 cases, the variations could not be attributed to a difference in the acquisition protocol set-up, and a thorough inspection did not lead to any improvement. For 1 acquisition protocol (helical skull), we found that the noise index was erroneously configured on one of the CT machines. After correction of this parameter, the median dose length product (DLP) on this machine was reduced from 771 mGy.cm to 446 mGy.cm (42\% decrease), based on measurements during 2 months prior to correction (148 cases) and 5 months after correction (364 cases). Conclusion: The use of a patient dose managing system for CT can identify suboptimal parameter settings, and helps to significantly reduce the radiation dose without loss of relevant diagnostic information.

Author Disclosures:

J. Valette: Employee; SerphyDose. A. Jomier: Employee; SerphyDose.

\section{B-0287 15:03}

Evaluation of the quality of abdominal computed tomography images reconstructed with iterative reconstruction technique L. Henriksson, A. Persson, M. Sandborg; Linköping/SE (lilian.henriksson@lio.se)

Purpose: Image quality evaluation of abdominal computed tomography (CT) examinations reconstructed with filtered back projection (FBP) and Sinogram affirmed iterative reconstruction (SAFIRE).

Methods and Materials: The study included 110 examinations from our RIS/PACS system, out of which 55 had been reconstructed with FBP and 55 with SAFIRE. Automated tube current modulation had been activated with $30 \%$ lower quality reference $\mathrm{mAs}$ ( $\mathrm{qRef} \mathrm{mAs}$ ) for SAFIRE. Comparisons were made regarding radiation dose (volume CT dose index, CTDIvol), image noise and subjective image quality. Criteria based on the European guidelines on quality criteria for computed tomography were used for the subjective assessment. A pilot study including 16 examinations was performed before the main study as preparation for the subjective assessment. CTDIvol and image noise were compared using a two-tailed Student's $t$-test $(p<0.001$ was considered significant). Visual grading characteristics (VGC) were used to evaluate the subjective image assessment. Interobserver agreement was assessed using Cohen's linearly weighted kappa $(\kappa)$. 
Results: CTDIvol and image noise was significantly lower for the SAFIRE examinations, compared to the FBP examinations $(p<0.001)$. VGC showed significantly better results for the SAFIRE examinations regarding the visual reproduction of the kidney contours, the intrahepatic vessel contours and for the overall diagnostic acceptability $(95 \% \mathrm{Cl}$ of $0.6052-0.7408,0.6272-0.7546$ and $0.5237-0.6687$, respectively). A varying interobserver agreement was found ( $\mathrm{k} 0.094$ to 0.763 ).

Conclusion: The scan and examination parameters used for the SAFIRE examinations in this study made it possible to lower the qRef mAs by $30 \%$ without image quality loss.

\section{B-0288 15:12}

Improved image quality of virtual non-contrast dual-energy CT images using a new iodine subtraction algorithm

A. Helck, N. Hummel, F. Meinel, S.F. Thieme, M.F. Reiser, A. Graser, T.R.C. Johnson; Munich/DE (andreas.helck@med.uni-muenchen.de)

Purpose: To evaluate a new algorithm for calculation of virtual non-contrast images (VNC) from dual-energy CT scans and determine whether VNC can replace non-contrast scans.

Methods and Materials: 43 patients received a contrast-enhanced dual-energy (DE) CT of the abdomen in portal venous phase. VNC were calculated using the standard VNC (sVNC) and a new VNC algorithm (nVNC). Overall image quality, identifiability of vessels and homogeneity of liver parenchyma were graded by two experienced radiologists in consensus using a 5-point rating scale with a true non-contrast CT (tNC) scan as standard of reference ( $1=$ =no difference; $2=$ minimal,

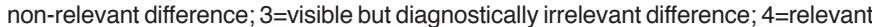
difference; $5=0$ obvious difference). Image noise was measured in liver parenchyma, retroperitoneal fat and aortic lumen. Wilcoxon signed-rank non-parametric test was used to assess statistical significance.

Results: The $\mathrm{nVNC}$ was superior compared to the standard VNC regarding overall image quality and identifiability of vessels $(2.4 \mathrm{vs} .3 .2 / \mathrm{p}<0.001$ and $1.4 \mathrm{vs} .1 .9 / \mathrm{p}$ $<0.001$ ), whereas no significant difference was observed concerning the homogeneity of the liver parenchyma (nVNC: $2.5 / \mathrm{sVNC} 2.4$ ). There was no significant difference regarding image noise with an average value of $7.5 \mathrm{HU}(\mathrm{nVNC})$ and 7.4 $\mathrm{HU}$ (sVNC), respectively, compared to $9.8 \mathrm{HU}$ (tNC).

Conclusion: The new algorithm provides a better image quality and an improved assessment of vessels and small structures. The nVNC images represent adequate substitutes for TNC images, rendering an omission of non-contrast acquisitions feasible, corresponding to a substantial dose reduction.

\section{B-0289 15:21}

Image quality of monoenergetic low-keV datasets for lower extremity dual-energy CT angiography

S. Sudarski, D. Schneider, P. Apfaltrer, S.O. Schönberg, T. Henzler;

Mannheim/DE (sonja_sudarski@gmx.de)

Purpose: To compare image quality of conventional 120-keV polyenergetic images (PEIs) and monoenergetic images (MEls) for dual-energy CT angiography (DE-CTA) datasets of the lower extremity.

Methods and Materials: We retrospectively evaluated DE-CTA datasets obtained from 18 patients ( 13 men, $67 \pm 10$ years). MEls were calculated in $10-\mathrm{keV}$ intervals from 120 to $40 \mathrm{keV}$ using the 80 - and $140-\mathrm{keV}$ image data. Attenuation and image noise were measured in the external iliac, femoral, popliteal and one lower leg artery. Contrast-to-noise ratio (CNR) and signal-to-noise ratio (SNR) were calculated for each vessel. Differences in these parameters were compared between the different monoenergetic datasets. The two best MEls were compared to 120-keV-PEls. Results: SNR and CNR of 70- and 60-keV MEls were highest when compared to other low-keV MEIs. In 70-keV MEls, CNR in external iliac, femoral and popliteal arteries was higher compared to PEls $(+29 \%,+19 \%$ and $+12 \%$; $p<0.05)$, while CNR did not change in lower leg arteries ( $p>0.05)$. SNR in external iliac and lower leg arteries was equal in 70-keV MEls and PEls, while in femoral and popliteal arteries it was lower $(-9 \%,-10 \% ; p<0.05) .60-k e V$ MEls showed a higher CNR in lower leg arteries compared to PEls $(+17 \%$; $p<0.05)$, while SNR did not differ $(p>0.05)$. In the other three arteries, 60-keV MEls showed significantly not only higher CNR but also lower SNR.

Conclusion: In lower extremity DE-CTA studies, 60- and 70-keV MEls show improved CNR at equal SNR levels compared to conventional PEIs. Thus, reduction of radiation dose seems to be achievable without compromising image quality. 
Friday, March 8 


\section{Chest}

\section{SS 504}

\section{Lung nodules}

Moderators:

S. Diederich; Düsseldorf/DE

N. Karabulut; Denizli/TR

\section{B-0290 10:30}

$70 \mathrm{kV}$ computed tomography of the thorax: valence for computerassisted nodule evaluation and radiation dose - first clinical results B. Schulz ${ }^{1}$, B. Bodelle ${ }^{1}$, T. Lehnert ${ }^{1}$, F. Al-Butmeh ${ }^{1}$, M. Sedlmair ${ }^{2}$, R. Banckwitz ${ }^{2}$, D. Klement ${ }^{1}$, T.J. Vogl ${ }^{1}$, M.J. Kerl' ${ }^{1}$; ${ }^{1}$ Frankfurt a. Main/DE, ${ }^{2}$ Erlangen/DE (mail@borisschulz.com)

Purpose: To evaluate radiation dose and efficiency of computer-assisted detection (CAD) technique of pulmonary nodules in low dose chest CT performed at $70 \mathrm{kVp}$. Methods and Materials: CAD was performed upon chest CT with $70 \mathrm{kVp}$ and $100 \mathrm{kVp}$ (gold standard) at a tube current of $87 \mathrm{mAs}$ (collimation: $64 \times 0.6 \mathrm{~mm}$ ). Detection rate for pulmonary nodules and size measurements of both techniques were compared to each other. Radiation dosage in terms of effective dose $(E)$ was measured using an Alderson-Rando Phantom.

Results: 74 patients with 301 nodules were included in the study. CAD detection rate was similar for $70 \mathrm{kVp}(94.5 \%)$ and $100 \mathrm{kVp}(92.4 \%)$. Mean transversal nodule diameter was $5.5 \mathrm{~mm}(70 \mathrm{kVp})$, respectively, $5.7 \mathrm{~mm}(100 \mathrm{kVp})$ with an average volume of $0.12 \mathrm{ml}$ (both techniques). Derived from the phantom measurements patient examinations resulted in an $\mathrm{E}$ of $0.51 \mathrm{mSv}(70 \mathrm{kVp})$, resp., $2.02 \mathrm{mSv}(100 \mathrm{kVp})$. Conclusion: $70 \mathrm{kVp}$ low dose chest CT is suitable for CAD-based lung nodule analysis at a quarter of the radiation burden of the standard technique. Based on the accurate CAD measurements $70 \mathrm{kVp} C T$ can be used for detection of pulmonal lesions and follow-up examinations.

\section{Author Disclosures:}

M. Sedlmair: Employee; Siemens Healthcare. R. Banckwitz: Employee; Siemens Healthcare. M.J. Kerl: Consultant; Siemens Healthcare.

\section{B-0291 10:39}

Improved accuracy of lung cancer diagnosis for fast-growing lung nodules in CT lung cancer screening by optimisation of volumedoubling time cut-off

M.A. Heuvelmans ${ }^{1}$, M. Oudkerk¹, G.H. De Bock ${ }^{1}$, X. Xie' ${ }^{1}$, P.M.A. Van Ooijen ${ }^{1}$, M.J.W. Greuter ${ }^{1}$, P.A. De Jong ${ }^{2}$, H.J.M. Groen ${ }^{1}$, R. Vliegenthart ${ }^{1} ;{ }^{1}$ Groningen/NL, ${ }^{2}$ Utrecht/NL (m.a.heuvelmans@student.rug.nl)

Purpose: To investigate whether optimisation of volume-doubling time (VDT) cutoff for fast-growing nodules in a lung cancer screening trial can improve accuracy of lung cancer diagnosis.

Methods and Materials: All subjects underwent at least two low-dose computed tomography (CT) scans during the baseline-round screening and regular secondround screening in year 2 of the NELSON lung cancer screening study and were referred to a pulmonologist because of a solid nodule with volume $350 \mathrm{~mm}^{3}$ and VDT < 400 days. Histology was the reference for diagnosis, or, to confirm benignity, stability of the nodule volume on subsequent CT scans for at least two years after referral to a pulmonologist. Nodule volume and VDT were semi-automatically calculated by software. Reduction of false-positive rate was evaluated at maintained sensitivity for lung cancer diagnosis with the VDT cut-off of 400 days as reference. Results: 68 fast-growing nodules were included (61 individuals), of which 27 nodules $(40 \%)$ were malignant. Mean follow-up of non-resected benign nodules was 4.4 (range 2.7-5.7) years. The optimal VDT cut-off for the 3-month follow-up $\mathrm{CT}$ in the baseline round was 232 days. Using this cut-off, false-positive referrals reduced by $33 \%$ (20 versus 30 false-positive referrals). For the regular secondround screening, VDTs varied more among malignant nodules, precluding lowering of the VDT cut-off of 400 days.

Conclusion: Lowering the VDT cut-off of the 3-month follow-up CT in baseline lung cancer screening from 400 to 232 days may reduce false-positive referrals, without apparent loss in sensitivity.

\section{B-0292 10:48}

AIDR improves characterisation of sub-centimetre pulmonary GGO on low dose HRCT

S. Hashemi Amroabadi', H. Mehrez², R.S. Cobbold',

N.S. Paul'; ; Toronto, ON/CA, ${ }^{2}$ Markham, ON/CA

(sayedmasoud.hashemiamroabadi@mail.utoronto.ca)

Purpose: Persistent sub-centimetre GGO are pre-malignant. HRCT improves detection of small nodules and low dose $\mathrm{CT}$ (LDCT) reduces patient exposure but both decrease CNR essential for characterisation of small GGO. We investigate the use of adaptive iterative dose reduction (AIDR) in low dose HRCT (LD-HRCT) for diagnosis of small GGO.

Methods and Materials: LDCT ( $120 \mathrm{kV}, 25 \mathrm{mAs}, 64 \times 0.5 \mathrm{~mm}$ ) was used in 3 stages: 1) computer-simulated lung nodules 5,8 and $10 \mathrm{~mm}$ spheres each of CT\# $100,-630$ and $-800 \mathrm{HU}$; 2) identical synthetic nodules, placed in an anthropomorphic chest phantom and 3) 22 in vivo pulmonary nodules (13 GGO, 9 solid); $5.1 \mathrm{~mm}$ diameter (4.0-6.9), mean CT\# -327 HU (8 to -738). Raw data reconstructions are as follows (slice thickness/overlap mm): 0.5/0.5, 1/1, 2/1, 2/2, 3/1, 4/2, 4/4, 5/2.5 and $5 / 5$ with FBP. In vivo images were also reconstructed with AIDR. CT\# and CNR were determined for each protocol.

Results: CT\# accuracy decreases with increased slice thickness for all nodules: $5 / 5$ reconstructions decrease $\mathrm{CT} \#>-400 \mathrm{HU}$ for $5 \mathrm{~mm}$ solid $(+100 \mathrm{HU})$ nodules and $\sim-130 \mathrm{HU}$ for $5 \mathrm{~mm}$ GGO (-630 HU and $-800 \mathrm{HU}$ ), Spearman's coefficient $=-1.0, p<0.005$. HRCT $(<3.0 / 1.5 \mathrm{~mm})$ has accurate nodule CT\# (error $<10 \%)$. AIDR increases CNR for GGO nodules by $63 \%$, for solid nodules by $45 \%$ and improves accurate CT\# with LD-HRCT compared to FBP (paired-test, $p<0.0001$ ). Conclusion: CNR for sub-centimetre GGO is improved with $3 / 1.5 \mathrm{~mm}$ reconstructions for FBP and AIDR. AIDR enhances CNR by $45-6 \%$ and improves accuracy in $\mathrm{GGO}$ diagnosis with LD-HRCT.

Author Disclosures:

H. Mehrez: Employee; Toshiba Medical Systems.

\section{B-0293 10:57}

Comparison of capabilities for differentiating malignant SPNs from benign SPNs among dynamic first-pass perfusion area-detector CT, dynamic first-pass MRI and FDG-PET/CT

Y. Ohno ${ }^{1}$, S. Seki', M. Nishio', H. Koyama', T. Yoshikawa', S. Matsumoto', Y. Fujisawa ${ }^{2} ;{ }^{1}$ Kobe/JP, ${ }^{2}$ Ohtawara/JP (yosirad@kobe-u.ac.jp)

Purpose: To compare differentiation capability of malignant nodules from benign nodules among dynamic first-pass perfusion area-detector $C T$ (perfusion ADCT) analysed by newly developed and previously developed models, dynamic first-pass MRI and FDG-PET/CT.

Methods and Materials: 45 consecutive patients ( 26 males, 19 females) with 85 nodules underwent dynamic perfusion ADCT, dynamic MRI at $1.5 \mathrm{~T}$ scanner, PET/ $\mathrm{CT}$, and microbacterial and/or pathological examinations. 85 nodules were classified into two groups based on the final diagnoses: malignant $(n=62)$ and benign nodules $(n=23)$. For quantitative assessment of perfusion $C T$, nodule perfusions by single-input maximum-slope model (NPSMS) and dual-input maximum slope model (NPDMS) were calculated. For semi-quantitative assessment of dynamic $\mathrm{MRI}$, maximum enhancement ratio (MER) and maximum slope of enhancement ratio (MSER) were calculated according to the past literatures. On PET/CT, maximum value of SUV (SUVmax) was determined. To compare diagnostic capability among all indexes, ROC analyses were performed, and feasible threshold values were determined. Finally, sensitivity, specificity and accuracy were compared with each other using McNemar's test.

Results: Area under the curves of NPDMS $(A z=0.85)$ and MSER $(A z=0.83)$ were significantly larger than that of $S U V \max (A z=0.72, p<0.05)$. When feasible threshold values adopted, accuracy of NPDMS (87.1 [78/85]\%) was significantly higher than that of NPSMS $(80.0[68 / 85] \%)$ and SUVmax $(75.3[64 / 85] \%)$.

Conclusion: First-pass ADCT analysed by dual-input maximum slope model was significantly more accurate method for differentiation of malignant nodules from benign nodules than that by single-input maximum slope model and PET/CT, and at least as valuable as dynamic first-pass MRI.

Author Disclosures:

Y. Ohno: Research/Grant Support; Toshiba Medical Systems, Bayer Pharma, Philips Healthcare. T. Yoshikawa: Research/Grant Support; Toshiba Medical Systems. S. Matsumoto: Research/Grant Support; Toshiba Medical Systems. Y. Fujisawa: Employee; Toshiba Medical Systems. 


\section{B-0294 11:06}

Can dual-energy index help to differentiate malignant and benign lesions of lung?

A. Kannivelu, S. Srinivasan, H.S. Teh, H.T. Koay, C.H.A. Tan, T.W. Choy,

K.K. Yeow, S.K.J. Lim; Singapore/SG (sivasu2@gmail.com)

Purpose: To determine the feasibility of dual-energy index in the differentiation of malignant and benign lesions using their attenuation values obtained in $100 \mathrm{kVp}$ and $140 \mathrm{kVp}$ images.

Methods and Materials: Forty-three consecutive patients who had lung lesions detected on dual-energy CT thorax imaging (SOMATOM Definition FLASH, Siemens AG, Erlangen, Germany) and histopathological finding were analysed. Images were acquired at $100 \mathrm{kVp}$ and $140 \mathrm{kVp}$. CT Hounsfield units (HU) at $100 \mathrm{kVp}, 140$ $k V p$ were measured, and dual-energy index (DEI) was calculated. Results were analysed using ANOVA and independent sample t-test.

Results: Thirty-one patients had malignant lesions (adenocarcinoma (ADC), $n=19$; squamous cell carcinoma ( $\mathrm{SCC}$ ), $n=7$; others $(\mathrm{OTH}), \mathrm{n}=5$ ), and 12 patients had benign lesions. Mean DEI values of malignant and benign groups were 0.00981 $( \pm 0.00442)$ and $0.00472( \pm 0.00255)$, respectively $(P<0.05)$. In malignancy group, mean DEl values of individual subgroups were $0.01097 \pm 0.00486$ (ADC), 0.007046 $\pm 0.00152(\mathrm{SCC})$ and $0.00981 \pm 0.00256(\mathrm{OTH})$, respectively. Difference between the ADC and the SCC subgroups was found to be statistically significant $(P<0.05)$. Conclusion: Dual-energy index obtained using dual-energy CT imaging may provide an objective quantitative parameter in characterisation of lung lesion, allowing distinction between a malignant tumour from benign lesion, as well as differentiation of different histological subtype of malignant lung lesions.

\section{B-0295 11:15}

Features of resolving and non-resolving indeterminate pulmonary nodules on follow-up CT: the NELSON study

Y. Zhao, M. Dorrius, M. Heuvelmans, P. van Ooijen, M. Oudkerk, R. Vliegenthart; Groningen/NL (m.a.heuvelmans@student.rug.nl)

Purpose: This study aimed at analysing computed tomography (CT) features associated with resolution of indeterminate pulmonary nodules in low-dose CT lung cancer screening.

Methods and Materials: The Dutch Belgium Trial for Lung Cancer Screening Study (NELSON) was institutional review board approved. For this retrospective evaluation of indeterminate pulmonary nodules found at baseline, patient consent was waived. Indeterminate nodules were re-examined by CT at 3 months. Regular repeat screening CT rounds were at years 2,4 and 6 . This study included only solid, intraparenchymal nodules. A nodule was defined as resolving if it had completely disappeared on a subsequent CT. Nodule resolution was regarded as spontaneous, not the effect of treatment. CT features of resolving nodules and non-resolving (stable and malignant) nodules were compared.

Results: At baseline, 1059 solid, intraparenchymal nodules (805 participants) were identified as indeterminate. During subsequent screening rounds, 9.3\% (99/1059) of the nodules disappeared, $75.8 \%$ of these before the 3-month CT and $93.0 \%$ before the year 2 screening. Non-peripheral nodules and nodules with non-smooth margin were more likely to resolve than peripheral and smooth nodules (19.7 vs $7.6 \%, p<0.001$, and 13.6 vs $7.5 \%, p<0.005$, respectively).

Conclusion: Resolving pulmonary nodules share CT features with malignant nodules. A 3-month repeat CT for indeterminate pulmonary nodules can identify most resolving nodules.

\section{B-0296 11:24}

Stratification of pure ground-glass opacity (GGO) nodular lung adenocarcinoma using quantitative analysis of $\mathrm{CT}$ imaging metrics to select patients for limited-resection more precisely

J. Son, H. Lee, J. Kim, J. Han, K. Lee, O. Kwon, B. Kim, H. Kim, Y. Shim; Seoul/KR (jiye.son@samsung.com)

Purpose: To determine whether quantitative analysis of CT imaging metrics can help differentiate among adenocarcinoma in situ (AIS), minimally invasive adenocarcinoma (MIA), invasive adenocarcinoma showing pure ground-glass nodule (GGN) on CT scan

Methods and Materials: We reviewed CT and PET images of tumours and pathologic specimens from 180 consecutive patients who underwent complete resection for lung adenocarcinomas showing GGN with little solid component on CT. Tumour size in lung setting/mediastinal setting, tumour disappearance ratio (TDR), volume, mass, skewness/kurtosis on the histogram, and texture parameters (uniformity and entropy) were assessed from CT, and SUVmax was measured on PET. On pathology, the histopathological classification was determined as AIS,
MIA, invasive adenocarcinoma, and the extents of invasive carcinoma component were quantified. Association between imaging parameters and pathologic classification was examined.

Results: 177 patients $(98 \%)$ were pathologic stage $1 \mathrm{~A}$, and 2 were $1 \mathrm{~B}$ and the other one was $2 \mathrm{~A}$. Of all 190 tumours, 38 were AIS $(20 \%), 56$ were MIA $(29 \%)$, and 96 were invasive adenocarcinoma ( $51 \%)$. Based on univariate analysis, tumour size in lung setting, uniformity, and entropy were able to significantly separate tumours according to pathologic classification, whereas TDR, volume, mass, or SUVmax were not. On multivariate analysis, uniformity and entropy were remained as independent factors to stratify the pathologic subgroups of patients (odds ratio $[\mathrm{OR}]=0.001, P=.007$, and $\mathrm{OR}=2.4, P=.01$, respectively).

Conclusion: Texture parameters on $\mathrm{CT}$ imaging metrics, reflecting tumour heterogeneity, appear to help stratify pure GGN lung adenocarcinoma.

\section{B-0297 11:33}

Non-solid, part-solid or solid? Classification of pulmonary nodules in thoracic $\mathrm{CT}$ by radiologists and a computer-aided diagnosis system C. Jacobs' ${ }^{1}$, E.M. van Rikxoort'2, J.-M. Kuhnigk'1, E.T. Scholten ${ }^{3}$,

P.A. de Jong ${ }^{4}$, C. Schaefer-Prokop 5 , M. Prokop², B. van Ginneken²; ${ }^{1}$ Bremen/DE, ${ }^{2}$ Nijmegen/NL, ${ }^{3} \mathrm{Haarlem} / \mathrm{NL},{ }^{4} \mathrm{Utrecht} / \mathrm{NL},{ }^{5} \mathrm{Amersfoort} / \mathrm{NL}$ (colin.jacobs@mevis.fraunhofer.de)

Purpose: Classifying pulmonary nodules into solid, part-solid and non-solid is crucial for patient management. A computer algorithm is compared to a radiologist on a large data set obtained from a multi-center lung cancer screening trial. Methods and Materials: Low-dose chest CT scans (16x0.75 mm, 120-140 kVp, 30 $\mathrm{mAs}$ ) with part-solid, non-solid, and solid nodules with a diameter between 7 and $30 \mathrm{~mm}$ were randomly selected from two sites participating in the Dutch-Belgian NELSON lung cancer screening trial. The set contained 137 scans, including 50 part-solid, 50 non-solid and 52 solid nodules. The nodule-type recorded in the screening database was used as a reference standard. An automated classification system for characterisation of nodules was designed using morphometric features. The accuracy of the computer algorithm was evaluated in three ways: classifying nodules (1) as solid or subsolid, (2) as solid, part-solid or non-solid, and, (3) for the subsolid lesions only, as part-solid or non-solid. An experienced thoracic radiologist independently performed the same classification.

Results: The accuracy of the automated system to differentiate between solid and subsolid nodules was 0.88 , compared to 0.95 for the radiologist. The computer classified the nodules as solid, part-solid or non-solid with an accuracy of 0.72 versus 0.80 for the radiologist. The software reached an accuracy of 0.71 in differentiating part-solid from non-solid nodules, where the radiologist had an accuracy of 0.77 . Conclusion: A novel automated characterisation tool for pulmonary nodules shows promising performance and could aid radiologists in selecting the appropriate workup for pulmonary nodules.

Author Disclosures:

C. Jacobs: Research/Grant Support; MeVis Medical Solutions AG.

\section{B-0298 11:42}

Solid pulmonary nodules detected by low-dose CT: effect of semiautomatic software on volumetry in vitro and in vivo

Y. Zhao, M. Dorrius, P. van Ooijen, M. Oudkerk, R. Vliegenthart; Groningen/NL (r.vliegenthart@umcg.nl)

Purpose: This study aimed at comparing accuracy of semi-automatic software systems for volumetry and volume doubling time (VDT) calculation of solid pulmonary nodules, detected by low-dose computed tomography (CT).

Methods and Materials: Scans were obtained from an anthropomorphic lung phantom with 60 artificial solid nodules (volume:65-905 $\mathrm{mm}^{3}$ ) and from 25 participants of the NELSON lung cancer screening trial. Semi-automated software (P1) was used for volumetry of the artificial nodules to evaluate accuracy of measured volumes. Subsequently, NELSON participants with at least one pulmonary nodule on the baseline and consecutive screening $\mathrm{CT}$ were randomly selected. The volume of each pulmonary nodule was determined for both time points using $\mathrm{P} 1$ and two additional software packages (P2 and P3). Manual modification was performed if software assessment was visually inaccurate. Pulmonary nodules were classified according to the NELSON categorisation protocol based on size and VDT.

Results: P1 accurately assessed the volume of the artificial nodules, with mean volume underestimation of $4 \pm 6 \%$. The participants had 147 pulmonary nodules (P1-derived volume:12-437 $\mathrm{mm}^{3}$ ). Significant volume differences were found between software packages $(p<0.001)$. Compared with $\mathrm{P} 1, \mathrm{P} 2$ and $\mathrm{P} 3$ overestimated volume by a mean of $38 \pm 24 \%$ and $50 \pm 22 \%$, respectively. At baseline, there was consensus on nodule size categorisation in $80 \%$ for P1-P2 and $74 \%$ for P1-P3. At 
follow-up, consensus on VDT categorisation was present in 27\% for P1-P2 and $26 \%$ for P1-P3.

Conclusion: Accurate volumetry of pulmonary nodules can be performed with semi-automated software. Different software packages yield significantly different volumetry results, most pronounced in case of VDT determination.

\section{B-0299 11:51}

Feasibility of training radiographers to detect nodules in $\mathrm{CT}$ lung cancer screening

A. Nair ${ }^{1}$, N. Gartland', B. Barton', E. Ifeakor ${ }^{1}$, J. Hodgkinson ${ }^{1}$, R. Vliegenthart ${ }^{2}$,

P.A. de Jong ${ }^{3}$, M. Prokop ${ }^{4}$, A. Devaraj'; ${ }^{1}$ London/UK, ${ }^{2}$ Groningen/NL,

${ }^{3}$ Utrecht/NL, ${ }^{4}$ Nijmegen/NL (arjun7764@gmail.com)

Purpose: To evaluate the feasibility of training radiographers for the task of detecting lung nodules on $\mathrm{CT}$, and assess the effect of continuous feedback.

Methods and Materials: Four experienced CT radiographers were tutored on the appearances of lung nodules and their mimics. $100 \mathrm{CTs}$ obtained from the NELSON trial were then arbitrarily divided into subsets of 10 . Radiographers were asked to identify all nodules. After each subset, a radiologist reviewed the radiographers' readings and provided feedback. The sensitivity and specificity of radiographers' readings were calculated for each subset. The reference standard included all positive nodules identified by the NELSON trial readers, as well as extra nodules identified by the radiographers that were classified as genuine nodules by two UK Lung Screening trial (UKLS) radiologists in consensus.

Results: Of 417 opacities (14-79 per subset), 282 (67.6\%) nodules formed the reference standard. The median (and individual) radiographers' sensitivity and specificity over the 100 scans were $62.1 \%(39.7 \%, 48.6 \%, 75.5 \%$ and $78.0 \%)$ and $67.7 \%(63.9 \%, 65.3 \%, 70.1 \%$ and $74.3 \%)$, respectively. Sensitivity and specificity varied between $22.7-86.4 \%$ and $50.0-92.9 \%$, respectively, in the first subset, to $48.0-88.0 \%$ and $35.0-80.0 \%$, respectively, in the final subset, showing no consistent trend between subsets $(\mathrm{p}=0.0005-1.0000)$.

Conclusion: Initial radiographer performance in lung nodule detection is highly variable. Some readers are capable of achieving accuracy rates similar to those reported for radiologists. However, after initial training no learning effect was demonstrated, indicating that initial radiographer performance may be guided by pre-existing ability and experience.

\section{$10: 30-12: 00$}

Room D2

\section{Interventional Radiology}

\section{SS 509}

\section{Neurovascular and spine interventions}

Moderators:

E.R. Gizewski; Innsbruck/AT

L. Pierot; Reims/FR

\section{B-0300 10:30}

Carotid artery stenting: results and analysis of a single-centre experience after 12 years

F. Fanelli, A. Cannavale, E. Boatta, M. Corona, C. Cirelli, F.M. Salvatori; Rome/IT (alessandro.cannavale@hotmail.com)

Purpose: To report 12-year experience in carotid artery stenting (CAS) with cerebral protection devices evaluating technical aspects, results and outcomes.

Methods and Materials: 636 patients who underwent CAS with a minimum follow-up of 6 months were retrospectively analysed. CAS Indications were in accordance with the NASCET criteria. A bilateral CAS was performed in two sessions in 47 patients. A cerebral protection device (CPD) was used in $94.7 \%$ of the cases whereas no CPDs were employed in 36 cases $(5.3 \%)$ for anatomical complexity. Pre and post CAS medication was administered according to standard protocols. Results: Technical success was achieved in all cases. After a mean follow-up of $76 \pm 32.4$ months, 478 patients $(70 \%)$ are alive, with a 30 -day mortality of $1.9 \%$. In-stent restenosis occurred in $9 / 683$ cases $(1.3 \%)$ and solved with angioplasty in $2 / 9$ cases $(22 \%)$ or re-stenting in $7 / 9(78 \%)$. Primary patency rate was $98.7 \%$; secondary patency rate $100 \%$. Neurological events occurred in $3.2 \%$ of cases. Results were analysed correlating neurological complications with the operator's experience, plaque morphology and patient's age. Neurological disorders were 1.9 $\%$ in asymptomatic patients and $4.5 \%$ in symptomatic ones $(\mathrm{p}=0.001)$ and they proved to be higher in older patients (>80y) $6.4 \%$ vs $2.7 \%$ of patients $<80$ years $(p<0.05)$. The overall neurological adverse events were $6.1 \%$ in the presence of complex plaque morphology and $1.0 \%$ in case of stable plaques $(p<0.001)$.
Conclusion: Our study confirmed that CAS can be considered a valid method for the treatment of the carotid pathology; nevertheless patient's accurate evaluation is mandatory.

\section{B-0301 10:39}

Additional value of mechanical thrombectomy devices in thromboembolic complications during endovascular occlusion of intracranial aneurysms

M.H.J. Voormolen, T. van der Zijden, T. Menovsky, P.N.M. Parizel; Antwerp/BE (maurits.voormolen@uza.be)

Purpose: Evaluation of mechanical thrombectomy devices during endovascular occlusion of intracranial aneurysms.

Methods and Materials: Thrombo-embolic events are one of the most frequent complications of endovascular occlusion of intracranial aneurysms, For small, not flow obstructing thrombus we use Aggrastat (Tirofiban) intravenous. Until recently, intra-arterial thrombolysis was one of the few interventional methods to dissolve the thrombo-occlusion of intracranial arteries, but with a high risk of haemorrhage. Mechanical thrombectomy devices (MTD) for stroke treatment became available in our hospital from 2010. In our hospital, 495 endovascular occlusions of 445 intracranial aneurysms have been performed in 377 patients from January 2006 until August 2012. In our series we retrospectively evaluated the results of intra-arterial thrombectomy versus thrombolysis in per procedural thrombo-occlusive events. Results: Overall, 54 patients (11\%) had thrombo-embolic events, of whom 27 (5.5\%) had permanent neurological events or death. In 17 cases (3.4\%) a thromboocclusion of a major intracranial artery occurred. In 10 cases intra-arterial thrombolysis was used, resulting in 5 deaths (50\%), 3 permanent neurological deficits (30\%) and 2 patients without sequel. Since October 2010, 7 mechanical thrombectomies have been done (4 Trevo stents, 3 Solitaire stents), resulting in one permanent neurological deficit (14\%), 1 TIA and 5 patients without clinical sequel. We had no technical MTD problem or problems with the occluded aneurysms.

Conclusion: Thrombo-embolic occlusion of intracranial arteries during endovascular aneurysm occlusion can be a potential lethal complication. Intra-arterial thrombolysis has a very high complication rate. Mechanical thrombectomy devices remove the thrombus safe and with good clinical outcome.

\section{B-0302 10:48}

Stent-assisted endovascular occlusion of intracranial aneurysms M.H.J. Voormolen, T. Van der Zijden, T. Menovsky, P. Parizel; Antwerp/BE (maurits.voormolen@uza.be)

Purpose: Evaluation of additional stent support during endovascular occlusion of intracranial aneurysms: indications, procedural complications and follow-up. Methods and Materials: From January 2006 until August 2012, 71 intracranial aneurysms in 58 patients ( 42 females, 16 males; mean/median age 54 years (range 20-79)) were treated in our hospital by endovascular occlusion with stent support: SAH (3), dissecting aneurysm (3), neurological deficit (16), incidental finding (19), additional to bled aneurysm (9) and additional coiling (21). Aneurysms ranged in maximal size between 2 and $30 \mathrm{~mm}$ (mean/median $9 \mathrm{~mm}$ ). Aneurysms were located in the cavernous sinus $(n=14)$, supraclinoid internal carotid artery $(n=33)$, anterior communicating artery $(n=3)$, middle cerebral artery $(n=2)$, pericallosal artery $(n=2)$ and posterior circulation $(n=17)$. Patients were preloaded with aspirin and clopidogrel (multiplate test control). Clinical and angiographic follow-up was done at 3- to 12-month intervals after treatment.

Results: Single stents were placed in 49 cases, 2 stents in 12 and flow-diverter stents in 10. In 14 cases no additional coil aneurysm occlusion was performed. Seventeen procedural complications occurred (24\%): 3 thrombo-embolic, 5 in-stent thrombosis, 3 haemorrhages, 3 side-branch occlusions and 3 technical device problems. Most had no clinical sequel, but 2 patients died $(3 \%)$ and 5 patients had permanent neurological deficit $(7 \%)$. Five aneurysms $(7 \%)$ needed second additional stenting during follow-up. Mean follow-up was 9 months (range 0-39 months). Conclusion: Endovascular intracranial aneurysm occlusion with stent assistance is a delicate procedure with a higher complication rate compared to regular coil occlusion. However, this technique is useful in complex otherwise difficult or untreatable intracranial aneurysms. 


\section{B-0303 10:57}

Long-term results of microsurgical and endovascular therapy of intracranial aneurysms in patients following subarachnoid haemorrhage

K. Bojanowski', J. Baron ${ }^{2}$, B. Kostkiewicz', M. Zawadzki', J. Walecki';

${ }^{1}$ Warsaw/PL, ${ }^{2}$ Katowice/PL (jerzy.walecki@cskmswia.pl)

Purpose: The aim of this paper was to evaluate long-term clinical outcomes and radiologic findings in patients following microsurgical treatment and endovascular embolisation of intracranial aneurysms.

Methods and Materials: A total of 66 subjects aged $20-75$ (42 women and 24 men) were evaluated. Patients were divided into two groups according to the type of procedure they underwent: 36 patients had open surgery and 30 patients had undergone endovascular embolisation. The assessment was performed from 6 months to 4 years after treatment. All patients underwent a follow-up cerebral arteriography and clinical evaluation.

Results: A total occlusion or a neck remnant was discovered in a majority of patients following microsurgical procedures and endovascular treatment $(94 \%$ and $3 \%$ in contrast to $87 \%$ and $7 \%$, respectively). No recurrent aneurysms were revealed. The Mann-Whitney $\mathrm{U}$ test did not reveal any statistical significance in long-term treatment results regarding self-reliance ( $p$-value $=0.2$ ). The amount of blood extravasated following the rupture of an aneurysm was a significant independent factor influencing the clinical outcome and determined the long-term results in both groups ( $\mathrm{p}$-value $<$ 0.05). A correlation between a patient's age and the clinical outcome following a microsurgical procedure was proved. The outcome was better in younger patients ( $p$-value< 0.05 ). This was not observed in patients after endovascular treatment. Conclusion: Microsurgical and endovascular therapy are comparable methods for treating cerebral aneurysms; however, embolisation is correlated with a higher rate of complications in elderly patients. The final clinical outcome depends on the amount of extravasated blood following a subarachnoid haemorrhage.

\section{B-0304 11:06}

Embolisation of cerebral aneurysms with hydrogel-coated coils: systematic review and meta-analysis

A. Pałys, Z. Serafin, W. Lasek; Bydgoszcz/PL (serafin@cm.umk.pl)

Purpose: Hydrogel-coated coils for endovascular treatment of cerebral aneurysms were developed to reduce the risk of aneurysm recurrence and the rate of retreatment. The aim of this review was to verify the efficacy and safety of hydrogel-coated coils.

Methods and Materials: A literature search was performed by two independent researches for papers published up to August 2012 on follow-up results of intracranial aneurysm embolisation with hydrogel-coated coils. Analysis included aneurysm presentation at follow-up compared to initial treatment result, the rate of rebleeding and retreatment, and specific complications. Pooled rates of major recurrences and total recurrences were calculated.

Results: Of 41 articles on the use of hydrogel-coated coils for embolisation of cerebral aneurysms, 13 (1575 aneurysms) were included. Significant heterogeneity was found regarding patients populations, methods of aneurysm coiling, follow-up schedules, and recurrence definitions. A cumulative follow-up rate was $76 \%(95 \% \mathrm{Cl}, 74-78 \%$, range $51-100 \%)$. Pooled major recurrence rate was $13 \%$ $(95 \% \mathrm{Cl}, 11-15 \%$, range $2-27 \%)$ and pooled total recurrence rate was $17 \%(95 \%$ $\mathrm{Cl}, 15-19 \%$, range $3-29 \%$ ). Control groups consisting of aneurysms treated with bare platinum coils were analysed in only in four articles (436 aneurysms). Pooled major recurrence rate for bare platinum coils was $33 \%(95 \% \mathrm{Cl}, 28-38 \%)$ and was significantly higher than this for hydrogel-coated coils.

Conclusion: Embolisation of cerebral aneurysms with hydrogel-coated coils results in lower rate of major recurrence, than with bare platinum coils. More high-quality standardised prospective studies are needed to define specific indications for the use of hydrogel-coated coils.

\section{B-0305 11:15}

Effect of antiplatelet therapy on radiographic outcome of positivity on diffusion-weighted imaging in elective endovascular coiling of unruptured cerebral aneurysm

T. Matsushige, K. Shinagawa, Y. Akiyama, T. Okazaki, S. Sakamoto, Y. Kiura, K. Sugiyama, K. Kurisu; Hiroshima/JP

Purpose: Our aim was to evaluate the radiographic outcome of high signal lesions on diffusion-weighted imaging (DWI) in the elective endovascular coiling of intracranial unruptured aneurysm with analysing the correlation in periprocedural antiplatelet therapy.

Methods and Materials: We retrospectively reviewed 33 patients initially undergoing coiling of unruptured aneurysm between 2007 and 2012. The mode of antiplatelet therapy was categorised into two groups: single in 13 patients and multiple in 20. All patients underwent 3.0 Tesla MRI, scheduled before, 24-48 hours after and within 3 months after the procedure. A total of 91 high signal lesions on DWI were serially numbered and the signal change on conventional MRI in the follow-up was evaluated. The quantitative parameters on DWI (location, volume and value of apparent diffusion coefficient: ADC) for each lesion were measured. We used Fisher exact test and the Mann-Whitney $U$ test to compare differences between two groups; statistical significance was assigned when $\mathrm{p}<0.05$.

Results: There was statistically significant difference in the presence of DWI lesions (multiple: $65 \%$ versus single: $100 \%, p=0.03$ ). In each lesion, the volume was significantly small $\left(0.074 \pm 0.019 \mathrm{~cm}^{3}\right.$ versus $\left.0.149 \pm 0.113 \mathrm{~cm}^{3}, \mathrm{p}=0.009\right)$ and the permanent signal change was less observed in multiple antiplatelets use $(5.2 \%$ versus $24.5 \%, p=0.02)$. There was negative correlation between the volume and the value of $A D C(r=-0.48, p<.0001)$.

Conclusion: Periprocedural use of multiple antiplatelet agents is expected to reduce the volume of thromboembolism and permanent signal change in the follow-up.

\section{B-0306 11:24}

MR-guided lumbosacral nerve root injection therapy using an open

1.0 Tesla MRI system: a clinical investigation

F. Streitparth ${ }^{1}$, M. De Bucourt ${ }^{1}$, T. Hartwig' ${ }^{1}$, T. Walter ${ }^{1}$, M. Maurer ${ }^{1}$,

D. Renz', B. Gebauer', B. Hamm ${ }^{1}$, U. Teichgräber ${ }^{2} ;{ }^{1}$ Berlin/DE,

2Jena/DE (florian.streitparth@charite.de)

Purpose: To evaluate the accuracy, safety and efficacy of MR-guided periradicular nerve root injection therapy using an open 1.0 Tesla MRI system with fast dynamic imaging.

Methods and Materials: Between April 2008 and November 2011 a total of 249 MR-guided periradicular nerve root injections were performed in 141 patients suffering from lumbosacral radicular pain. All interventions were performed in an open 1.0 Tesla MRI. An interactive PDW TSE sequence was used for real-time guidance. An in-room monitor, wireless MR-mouse for operator-controlled multiplanar navigation, a flexible surface coil and MR-compatible 20-G needle were used. Informed consent was obtained from all patients. Clinical outcome was evaluated by clinical follow-up and questionnaire prior to injection therapy (baseline) and to 6 months after using a numeric visual analog scale (VAS).

Results: All procedures were technically successful. No major complications occurred. At 6 months, of 103 patients (57 males, 46 females; mean age, 49.5 ; range, $20-80$ ) enrolled in the outcome analysis, $14.6 \%$ reported complete remission of radicular pain, $53.4 \%$ significant relief of pain, $22.3 \%$ mild relief and $9.7 \%$ no relief of pain. We found a significant decrease of the VAS-score from pre-intervention to 6 months follow-up $(p<.0001)$. No significant difference in outcome was seen between patients with degenerative foraminal stenoses and patients with herniated disks. Conclusion: MR fluoroscopy-guided periradicular injection therapy of the lumbosacral spine under open 1.0 Tesla MRI-guidance is accurate, safe and efficient in the symptomatic treatment of radicular pain.

\section{B-0307 11:33}

Vertebral split fractures: the role of percutaneous vertebroplasty

L. Huwart' 1 , O. Hauger ${ }^{2}$, P.-Y. Marcy', P. Foti' ${ }^{1}$, M.-E. Amoretti',

P. Brunner ${ }^{3}$, N. Amoretti'; ${ }^{1}$ Nice/FR, ${ }^{2}$ Bordeaux/FR, ${ }^{3}$ Monaco/MC

(huwart.laurent@wanadoo.fr)

Purpose: The treatment of vertebral split fractures remains controversial, consisting of either corset or internal fixation. The aim of this study was to evaluate CT- and fluoroscopy-guided percutaneous vertebroplasty in the management of vertebral split fractures.

Methods and Materials: Institutional Review Board approval and informed consent were obtained for this study. Sixty-two consecutive adult patients who had posttraumatic vertebral split fractures (A2 according to the $A O$ classification) without neurological symptoms were prospectively treated by percutaneous vertebroplasty. All these procedures were performed by an interventional radiologist under computed tomography (CT) and fluoroscopy guidance using only local anaesthesia. Postoperative outcome was assessed using the visual analogue scale (VAS) and Oswestry disability index (ODI) scores.

Results: Vertebroplasty was performed on thoracic and lumbar vertebrae. The filling of the vertebral body was satisfactory in $100 \%$ of the patients, with seven discal cement leakages (14\%). The mean VAS measurements \pm standard deviation (SD) significantly decreased from $7.9 \pm 1.5$ preoperatively to $3.3 \pm 2.1$ at 1 day, 2.2 \pm 2.0 at 1 month, and $1.8 \pm 1.4$ at 6 months $(P<0.001)$. The mean ODI scores \pm SD had also a significant improvement: $62.3 \pm 17.2$ preoperatively and $15.1 \pm 6.0$ at the 6-month follow-up $(P<0.001)$. Radiological union was observed within the 6 months following vertebroplasty in all the cases.

Conclusion: The results of our study showed that type A2 vertebral fractures could be successfully treated by CT- and fluoroscopy-guided percutaneous vertebroplasty with an excellent functional recovery. 


\section{B-0308 11:42}

Percutaneous vertebroplasty in vertebra plana: does the presence of intravertebral cleft impact the efficiency of the procedure?

L. Huwart ${ }^{1}$, P.-Y. Marcy ${ }^{1}$, P. Foti' ${ }^{1}$, M.-E. Amoretti ${ }^{1}$, O. Hauger ${ }^{2}$,

P. Brunner ${ }^{3}$, N. Amoretti ${ }^{1} ;{ }^{1}$ Nice/FR, ${ }^{2}$ Bordeaux/FR, ${ }^{3}$ Monaco/MC

(huwart.laurent@wanadoo.fr)

Purpose: The purpose of this study was to assess if the presence of intravertebral cleft impact the efficiency of percutaneous vertebroplasty in 100 adult patients with vertebra plana.

Methods and Materials: 100 adult patients (28 men and 72 women; mean age: 83 years) with vertebra plana were prospectively treated by CT- and fluoroscopyguided percutaneous vertebroplasty under only local anaesthesia. A 13-gauge 10-cm Trocar t'am (Thiebaud, France) was placed into the vertebral body with unilateral approach. Then polymethylmethacrylate was injected intra-vertebrally: if present, the intravertebral cleft should be filled. Follow-up was done using visual analogue scale (VAS) for the severity of pain at day 1, 1 month, 6 months and 1 year. Results: Vertebra plana ranged from D6 to $L 4$ with $82 \%$ at the thoracolumbar junction. In the group with cleft ( $n=58 / 100$ patients), VAS scores \pm standard deviations (SDs) were: preoperatively, $8.1 \pm 1.5$; at day $1,1.3 \pm 1.4$; at 1 month, 1.2 \pm 1.2 ; at 6 months, $1.3 \pm 1.4$; and at 1 year, $1.6 \pm 1.5$. In the group without cleft ( $n$ $=42$ ), VAS scores \pm SDs were: $7.5 \pm 1.2,2.4 \pm 2.1,2.0 \pm 1.8,1.9 \pm 1.6$ and $2.0 \pm$ 1.7. The decrease between pre- and post-operative VAS values was significantly higher in the group with cleft than without it ( $p<0.05$ in all cases).

Conclusion: Percutaneous vertebroplasty could be safe and effective in patients with vertebra plana, with better results when intravertebral cleft was present.

\section{B-0309 11:51}

Percutaneous ablation of spinal osteoid osteoma: report of $\mathbf{3 7}$ patients G. Tsoumakidou, J. Garnon, I. Enescu, X. Buy, A. Gangi; Strasbourg/FR (gtsoumakidou@yahoo.com)

Purpose: To evaluate the efficacy and safety of percutaneous image-guided laser ablation of spinal osteoid osteoma.

Methods and Materials: From 1994 till December 2011, 37 patients (mean age: 23 years, min: 5, max: 45) with spinal osteoid osteoma (mean nidus size: $8 \mathrm{~mm}$ ) underwent percutaneous laser ablation. Procedures were performed under spinal or general anaesthesia under combined CT and fluoroscopic guidance. Mean distance from the closest nerve root was $7.5 \mathrm{~mm}$ ( $\min : 1 \mathrm{~mm}$, max: $40 \mathrm{~mm}$ ). In 22 patients, the nidus was close to a nerve root or the spinal cord (distance less than $7 \mathrm{~mm}$ ) and temperature monitoring and active cooling were necessary. An average energy of $1260 \mathrm{~J}$ was delivered using an infrared diode laser generator, with a 2-Watt continuous power.

Results: Percutaneous laser ablation was technically feasible in all cases. Primary clinical success (complete pain relief) was noted in all cases. Two patients presented a recurrence and were re-treated percutaneously successfully. No neurological complications were observed. Mean follow-up was 69 months (max: 210 months, min: 2 months).

Conclusion: Percutaneous laser ablation of spinal osteoid osteoma can be achieved safely. Use of thermal insulation and temperature monitoring techniques is mandatory to protect the adjacent nervous structures.

\section{0:30 - 12:00} Room E1

\section{Musculoskeletal}

\section{SS 510}

\section{Tendon, muscle and tissue composition}

\section{Moderators:}

J. Healy; London/UK

A. Mileto; Durham, NC/US

\section{B-0310 10:30}

Efficacy of intra-tendinous injection of platelet-rich plasma to treat tendinosis: comprehensive assessment on a murine model

B. Dallaudiere' ${ }^{1}$, M. Lempicki', L. Pesquer ${ }^{2}$, P. Meyer ${ }^{2}$, E. Schouman-Claeys ${ }^{1}$, J.-M. Serfaty ${ }^{1} ;{ }^{1}$ Paris/FR, ${ }^{2}$ Bordeaux/FR (bendallau64@hotmail.fr)

Purpose: Tendinopathy shows early disorganized collagen fibres with neo-angiogenesis on histology. Peri-tendinous injection of corticosteroid is the commonly accepted strategy despite the absence of inflammation in tendinosis. The aim of our study was to assess the potential of intratendinous injection of platelet-rich plasma (PRP) to treat tendinopathy in a murine model of patellar and Achilles tendinopathy, and to evaluate its local toxicity.

Methods and Materials: Thirty rats (120 patellar and Achilles tendons) were used for the study. We induced tendinosis $(T+)$ in 80 tendons by injecting under ultrasonography (US) guidance Collagenase $1{ }^{\circledR}$ (day $0=\mathrm{D} 0$, patellar $=40$ and Achilles $=40$ ). Clinical examination and tendon US were performed at D3, immediately followed by either PRP (PRPT+, $n=40$ ) or physiological serum (PST+, $\mathrm{n}=40$, control) US-guided intratendinous injection. Follow-up at D6, D13, D18 and D25 using clinical, US and histology, and comparison between the 2 groups were performed. To study PRP toxicity, we compared the 40 remaining normal tendons (T-) after injecting PRP in 40 (PRPT-).

Results: All PRPT+ showed a better joint mobilization compared to PST+ at D6 $(p=0.005), D 13(p=0.02), D 18(p=0.003)$ and D25 $(p=0.01)$. Similar results were found regarding US and histology, with thinner diameters (D6, $p=0.003$; D25, $p^{2} 0.004$ ) and less disorganised collagen fibres and neovessels ( $D 6, p=0.003$; $\mathrm{D} 25, \mathrm{p}=0.0003$ ) in PRPT + compared to PST+. Comparison between PRPT- and $T$ - showed no PRP toxicity on tendon $(p=0.18)$.

Conclusion: Our study suggests that mono-injection of PRP in tendinosis rapidly accelerates tendon's healing, with no local toxicity.

\section{B-0311 10:39}

High volume image-guided injections in patellar tendinopathy S.L. Morton, O. Chan, D. Morrissey, J. King, T. Crisp, N. Maffulli; London/UK

Purpose: To quantify the effect of high volume image-guided injection (HVIGI) to manage recalcitrant patellar tendinopathy (PT).

Methods and Materials: 20 patients with proximal PT after failed conservative management were recruited (mean symptom duration 19.8 months). 12 males $(n=11)$ were followed retrospectively over 9 months; 8 males $(n=8)$ were followed prospectively over 12 weeks. The diagnosis of PT was confirmed with ultrasound and power Doppler (average thickness of prospective subjects $10.3 \mathrm{~mm}$, average neovascularisation 3.8 on modified Ohberg scale). All subjects were injected under real-time ultrasound guidance with $10 \mathrm{ml}$ of $0.5 \%$ Marcaine mixed with 25 $\mathrm{mg}$ hydrocortisone followed by a mean of $30 \mathrm{ml}$ normal saline at the interface between the PT and Hoffa's body, adjacent to the area of neovascularisation. The same supervised rehabilitation programme was followed by all patients. The VISA-P questionnaire was administered for pre-injection severity and at follow-up. Results: There was instant resolution of neovascularisation in all subjects. The VISA-P score statistically significantly improved from a mean of 45.5 to 64.0 for ( $p$ $<0.01$ ), which is likely to be clinically significant. The retrospective data increased by 19.9 points compared to 16.4 in the prospective subjects followed over a shorter time period. Over $60 \%$ of the prospective subjects agreed/strongly agreed that their symptoms/strength had improved, with over $33 \%$ returning to their required level of sport within 12 weeks.

Conclusion: High volume image-guided injections should be considered in the management of recalcitrant chronic PT. Randomised controlled trials are needed to consolidate these findings.

\section{B-0312 10:48}

Biomechanical properties of the calcaneal tendon in vivo assessed by transient shear wave elastography

S. Aubry ${ }^{1}$, J.-R. Risson ${ }^{1}$, A. Kastler ${ }^{2}$, B. Barbier-Brion ${ }^{1}$, M. Runge ${ }^{1}$, B. Kastler ${ }^{1}$; ${ }^{1}$ Besancon/FR, ${ }^{2}$ Clermont-Ferrand/FR (radio.aubry@free.fr)

Purpose: To assess elastic and anisotropic properties of normal calcaneal tendon in vivo by transient shear wave elastography (SWE).

Methods and Materials: This study was approved by Institutional Review Board, and written informed consent was obtained from all volunteers. Eighty healthy subjects over 18 were prospectively included. Data on patients' height, weight, sporting activities and take-off foot were assessed. The thickness, width and cross-sectional area of the calcaneal tendons were measured on axial grayscale ultrasound. The shear wave propagation velocity (Vmean) was measured by three radiologists on axial and sagittal SWE images at four different degrees of ankle flexion, enabling to calculate elasticity modulus (Emean), and relative anisotropy coefficient (A) values.

Results: In complete plantar flexion, Vmean was $6.8 \pm 1.4 \mathrm{~m} \cdot \mathrm{s}^{-1}$ and $5.1 \pm 0.8 \mathrm{~m} \cdot \mathrm{s}^{-1}$, respectively, on the sagittal and axial SWE image, resulting in an elastographic anisotropy $A$ of $0.38 \pm 0.31$. Emean was equal to $147.6 \pm 62.6 \mathrm{kPa}$ and $78.7 \pm 26.9 \mathrm{kPa}$ on the sagittal and axial SWE image, respectively. Men and young people had significantly harder tendons. The best interobserver correlation coefficient of Emean and Vmean was 0.43 and 0.46 , respectively, in the sagittal SWE for position 1. Vmean and Emean significantly increase when the tendon is stretched by ankle 
dorsiflexion. The maximal values in sagittal SWE were Vmean $=16.1 \pm 0.7 \mathrm{~m} \cdot \mathrm{s}^{-1}$, Emean $=779.5 \pm 57.1 \mathrm{kPa}$ and $\mathrm{A}=1.87 \pm 0.72$

Conclusion: SWE allows the elastic properties of the calcaneal tendon to be evaluated quantitatively in vivo, but interobserver reproducibility is questionable. It confirms the tendinous elastographic anisotropy and stiffness augmentation of stretched tendon, although age and sex may influence these properties.

\section{B-0313 10:57}

Muscle elastography in patients affected by multiple sclerosis

G. Illomei, G. Spinicci, M. Arru, M. Marrosu; Cagliari/IT

(giovanni.illomei@radiologiadelcorso.it)

Purpose: The purpose of the study was to investigate the use of real time elastography in evaluating the muscle stiffness comparing to Ashworth scale. The aim of the study is to create an elastography score for MS patients.

Methods and Materials: We investigated 101 MS patients, 61 women and 40 men. Disability score assessed by Expanded Disability Status Score of Kurtzke scale ranged from 0 and 8.5 with a mean value of 3.5. In all patients a neurological examination was performed and referred to Ashworth scale score by a neurologist. All patients underwent real time elastography on Esaote my Lab Twice with transducer with a high frequency probe 4-13 $\mathrm{MHz}$. The muscles examined were quadriceps of both legs. The study was a double-blinded one, and it was approved by local Ethic Committee.

Results: There was full concordance between the Ashworth scale evaluation and elastography score. However, patients classified as score 0 in the Ashworth scale can be spitted in $0 \mathrm{a}$ (total normality of muscle fibers elasticity) and $0 \mathrm{~b}$ (initial compromising of muscle fibers elasticity). The main result of the study was the creation of an elastography score of muscle stiffness in MS patients that can be compared with Ashworth scale.

Conclusion: Ashworth scale is at present the only method to evaluate muscle stiffness in MS patients. Our study demonstrated for the first time that it is possible to have an imaging method to assess this clinical examination giving new possibilities to follow the evolving of the disease in these patients.

\section{B-0314 11:06}

Dixon-based MRI for assessment of muscle-fat content in-vitro and in patients with achillodynia in comparison to healthy volunteers M.A.F. Fischer, C.W.A. Pfirrmann, N. Espinosa, D.A. Raptis, F.M. Buck; Zurich/CH (michaelalexander.fischer@usz.ch)

Purpose: To prospectively evaluate dual gradient-echo MRI with dixon-based generation of fat-/water-images (MRIDIXON) for quantification of the muscle-fat content (MFC) in-vitro and in patients with achillodynia compared to asymptomatic volunteers.

Methods and Materials: MRIDIXON was used to measure the MFC of 15 phantoms containing titrated mixtures of organic muscle/fat from $0-100 \%$ as well as of the gastrocnemius (DixonGASTRO) and tibialis anterior (DixonTIBIALIS) muscles in 30 patients ( 13 women; mean age, $57 \pm 15$ years) with achillodynia and in 20 matched asymptomatic volunteers ( 10 women; mean age, $30 \pm 14$ years) at $1.5 \mathrm{~T}$. Accuracy of MRIDIXON in quantification of MFC was assessed in-vitro as well as in-vivo using single-voxel MR-spectroscopy (MRS) as the standard of reference. DixonGASTRO and DixonTIBIALIS were related to visual grading of the MFC (grades 0-4) in both muscles (VisualGASTRO and VisualTIBIALIS).

Results: Correlation of MFC derived from MRIDIXON, phantoms and MRS was excellent with correlation coefficient $\left(R^{2}\right)$ ranging from 0.93 to 0.99 and mean measurement bias ranging from -0.1 to $+0.8 \%$. Mean DixonGASTRO/DixonTIBIALIS was $7.0 \pm 4.7 / 4.9 \pm 2.3$ in patients and $3.6 \pm 0.7 / 3.6 \pm 0.5$ in volunteers. VisualGASTRO/ VisualTIBIALIS ranged from 0 to $2 / 0$ to 3 and 0 to $1 / 0$ to 1 , respectively. No significant difference was seen for DixonTIBIALIS between patients and volunteers $(p=.07)$, whereas DixonGASTRO was significantly higher in patients $(p<.01)$. Both, VisualGASTRO and VisualTIBIALIS did not differ in patients and volunteers (all, $p>.05$ ). Conclusion: MRIDIXON allows for accurate quantification of MFC and demonstrates a significantly higher fat content in the gastrocnemius muscle in patients suffering of achillodynia than in asymptomatic volunteers, outer performing visual assessment of MFC.
B-0315 11:15

Postmortal 31P magnetic resonance spectroscopy of the skeletal muscle: a-ATP/Pi ratio as a forensic tool?

J. Yamamura ${ }^{1}$, T.M. Schidt ${ }^{1}$, R. Fischer ${ }^{1}$, Z.J. Wang ${ }^{2}$, M. Lorenzen ${ }^{1}$;

${ }^{1}$ Hamburg/DE, ${ }^{2}$ Dallas, TX/US

Purpose: Phosphor magnetic resonance spectroscopy ( ${ }^{31} \mathrm{P}$ MRS) is an established method for metabolic examinations of resting and exercising skeletal muscle. The aim of this study was to investigate the temporal pattern of phosphor metabolites in the Adductor magnus muscle post mortem and to check the value of MRS as a forensic tool especially for the determination of the time of death.

Methods and Materials: 21 corpses, died of natural cause, were examined (13 m, 8; age: $70.5 \pm 8.7 y$, weight $74 \pm 18 \mathrm{~kg}$ ). A control group of 3 male subjects (mean: $38.7 \pm 24.5 \mathrm{y}$, range: $24-67 \mathrm{y}$, mean body weight: $81 \pm 17 \mathrm{~kg}$ ) was examined at a single time point. ${ }^{31} \mathrm{P}$ MRS was performed on a $1.5 \mathrm{~T}$ MRI (TR $700 \mathrm{~ms}$, TE $0.35 \mathrm{~ms}$, averages 256 , flip angle $90^{\circ}$ ). A standard ${ }^{31} \mathrm{P}$ surface coil in the patient table, placed under the thigh, was employed. To measure the concentration of the phosphor metabolites scans were repeated in intervals of one hour over a period from 2 to $24 \mathrm{~h}$ post mortem (p.m). The core temperature was rectally measured throughout the MRI examination.

Results: The mean core temperature decreased from $36.0^{\circ} \mathrm{C}$ to $25.7^{\circ} \mathrm{C}$. In vivo and ex vivo spectra showed characteristically different metabolite concentrations. a-ATP/Pi ratio decreased exponentially from 0.445 to $0.032\left(r^{2}=0.997, p<0.001\right)$. Conclusion: There is a characteristic postmortal time pattern of the phosphor metabolites. Especially the a-ATP/Pi ratio could be useful as a forensic tool because of its significant exponential postmortal time course.

\section{B-0316 11:24}

Visceral fat by DXA: current methods and potential clinical implications A. Bazzocchi, D. Diano, F. Ponti, A. Andreone, C. Sassi, U. Albisinni, G. Battista; Bologna/IT (dani84.dd@libero.it)

Purpose: Our aim was to investigate the potential impact of visceral fat assessment by DXA in the clinical management of patients submitted to different therapeutic programs for obesity.

Methods and Materials: We prospectively recruited 169 patients affected by obesity and submitted to medical and/or surgical treatment. Body mass index (BMI) and body composition were analyzed with anthropometric and whole-body DXA evaluations simultaneously performed at baseline and after one year. A new software available to estimate android visceral fat was applied to last-generation DXA equipment.

Results: Ninety-one patients ruled out the study. Among patients ending the follow-up period (28-males, 50-females, 53.4 \pm 13 .0-year-old, 40.5 $\pm 9.1-\mathrm{Kg} / \mathrm{m} 2$ ) BMI decreased in 69/78-88.5\% controls, while regained in 9/78-11.5\%. An opposite trend between $\mathrm{BMI}$ and total fat mass/non-bone lean mass (TFM/TLM), android ratio (AFM/ALM), and android visceral/subcutaneous fat (V/S) was found in 7/78-9.0\%, $11 / 78-14.1 \%$ and $31 / 78-39.7 \%$, respectively. BMI was significantly correlated with TFM/TLM and AFM/ALM ( $r=0.717$ and 0.713 respectively), while it was far from a statistically significant connection with V/S $(r=0.011, p=0.896)$. Although slightly better correlations were demonstrated between TFM/TLM (and AFM/ALM) and $\mathrm{V} / \mathrm{S}$, these were extremely low $(r=0.146, p=0.061)$. The corresponding values for $\triangle \mathrm{BMI}$ and $\triangle \mathrm{DXA}$ measures showed similar results.

Conclusion: The evolution of the most important metabolic markers of body composition is not predictable by the only BMI. Moreover, a wide range of variation is observed in relationships between DXA measures and BMI. Today, DXA has reached a new important frontier in the analysis of body composition, bridging the historical gap of visceral fat assessment.

\section{B-0317 11:33}

Post-exercise intramyocellular acetylcarnitine levels in endurance trained and sedentary subjects measured with ${ }^{1} \mathrm{H}-\mathrm{MRS}$

L. Lindeboom, B.M.W. Brouwers, T. van de Weijer, C.I.H.C. Nabuurs, M.E. Kooi, M.K.C. Hesselink, P. Schrauwen, J.E. Wildberger, V.B. Schrauwen-Hinderling; Maastricht/NL (eline.kooi@mumc.nl)

Purpose: Long echo time acquisition strategies facilitate the determination of acetylcarnitine concentration in skeletal muscle in vivo with ${ }^{1} \mathrm{H}$-magnetic resonance spectroscopy (MRS). Rapid post-exercise normalisation of acetylcarnitine in the exercised muscle may be indicative of good tuning of substrate supply and use, as we expect to find in endurance trained subjects. We therefore investigated the effect of acute exercise on acetylcarnitine concentrations in trained and sedentary subjects. Methods and Materials: Four endurance trained and four sedentary subjects cycled for 30 minutes at $50 \%$ of their predetermined Wmax. Acetylcarnitine con- 
centrations were determined dynamically before and after exercise using a whole body MRI-system (Achieva, 3 T, Philips Healthcare). Point-RESolved spectroscopy (PRESS) was used to acquire spectra from a voxel in the M. vastus lateralis. The total creatine $(\mathrm{t}-\mathrm{Cr})$ peak was used as internal reference. Post-exercise acquisition of spectra started approximately 15 minutes after cycling.

Results: Exercise-induced elevations of acetylcarnitine levels were similar in both groups ( $\Delta$ post-pre $3.1 \pm 1.9 \mathrm{mmol} / \mathrm{kg}$ wet weight $(\mathrm{ww})$ ). However, post-exercise acetylcarnitine kinetics were significantly different $(p<0.05)$. In sedentary subjects, the concentration continued to increase during recovery towards a plateau at 40 minutes $(8.5 \pm 0.3 \mathrm{mmol} / \mathrm{kgww})$. In contrast, the acetylcarnitine concentration started to decrease from $5.8 \pm 0.5 \mathrm{mmol} / \mathrm{kgww}$ at 20 minutes after exercise in the trained subjects towards $4.4 \pm 1.0 \mathrm{mmol} / \mathrm{kgww}$ at 40 minutes.

Conclusion: We demonstrate the dynamic character of acetylcarnitine in a situation of changing substrate demand/supply. The current protocol can differentiate between two metabolically different groups, which opens a window towards investigation of acetylcarnitine in relation to metabolic flexibility and insulin resistance.

\section{B-0318 11:42}

In vivo differentiation of muscle precursor cells using MR relaxometry

N.C. Chuck, F. Azzabi Zouraq, D. Eberli, A. Boss; Zurich/CH

(natalie.chuck@usz.ch)

Purpose: To assess the differentiation process of muscle precursor cells (MPCs) applying magnetic-resonance-imaging (MRI) relaxometry and diffusion measurements in a mouse model in correlation with histology, immunohistochemistry and organ bath.

Methods and Materials: Human MPCs were isolated from biopsies of the M.rectus abdominis. The MPCs were mixed with a collagen carrier and injected subcutaneously in nude mice. Animals with collagen-only-injections served as controls. MRI was conducted on a 4.7T small-animal-scanner (Bruker BioSpec) at 5 time points between day 3 and 21 post-injection. Relaxometry measurements comprised T1 and T2 measurements using multi-echo-spin-echo (TE 11-99 ms) and saturationrecovery-sequences (recovery time $118-4000 \mathrm{~ms}$ ) and $\mathrm{T}^{*}$ quantification with a multi-echo-gradient-echo-sequence (TE1 $=4.5 \mathrm{~ms}, \Delta \mathrm{TE}=7 \mathrm{~ms}, 12$ echoes). Diffusion imaging was performed with a spin-echo-sequence including diffusion gradients with $b=0.400$ and $800 \mathrm{~s} / \mathrm{mm}^{2}$. Relaxation times and $A D C$ values were measured in the skeletal muscles. Evaluations were performed with custom-made Matlab scripts. Animals were harvested after 28 days and the engineered muscle tissue was assessed by histology, organ-bath and immunofluorescence.

Results: Relaxation and diffusion properties of the engineered tissue could reliably be measured at all time points. Relaxometry measurements revealed a decrease of T1, T2 and T2* relaxation-time during differentiation (initial measurement: T1 $2242.6 \mathrm{~ms} \pm 116, \mathrm{~T} 2: 224.3 \mathrm{~ms} \pm 66, \mathrm{~T} 2^{*}: 33.5 \mathrm{~ms} \pm 4$; final measurement: $\mathrm{T} 1: 1386.64$ $\mathrm{ms} \pm 88, \mathrm{~T} 2: 31.9 \mathrm{~ms} \pm 4, \mathrm{~T} 2^{\star}: 10.8 \mathrm{~ms} \pm 0.8$ ), thereby approaching the physiological relaxation properties of muscular tissue. ADC showed a decrease from $2.24 \mathrm{~mm}^{2 /}$ $\mathrm{s} \pm 0.26$ to $1.51 \mathrm{~mm}^{2} / \mathrm{s} \pm 0.05$. Cell differentiation and myofibre formation was confirmed by histology, immunohistochemistry and contractility confirmed by organ bath. Conclusion: We demonstrated that MRI relaxometry measurements are able to describe the differentiation of muscle precursor cells. This method might offer the possibility to non-invasively assess the effectiveness of cellular therapies for muscular disorders in near future.

\section{B-0319 11:51}

MRI findings of the bone marrow related to unloading with longitudinal follow-up

L. Nardo', D. Sandman', W. Virayavanich', S. Lee', A. Lai', L. Zhang',

R. Souza', M. Guindani ${ }^{2}$, T. Link ${ }^{1} ;{ }^{1}$ San Francisco, CA/US, ${ }^{2}$ Houston, TX/US

(lorenzo.nardo@ucsf.edu)

Purpose: To longitudinally evaluate bone marrow changes on MRI related to unloading in patients with 3-to-6 weeks of lower extremity unloading.

Methods and Materials: 112 MRI knee examinations in 30 patients (14 men, 16 women, aged 20-53 years) were performed following 3-6 weeks of ipsilateral lower extremity unloading related to knee or ankle injury. All patients had MRI studies before the unloading period and consecutive MRI follow-up studies 1.5-2 months, 2-6 months, $6-12$ months and $>12$ months after unloading. Bone marrow signal abnormalities were analysed by 3 radiologists on T2 FSE sequences and scored according to (i) severity of signal abnormalities, (ii) morphology and (iii) increased vascularity in the knee joint. In addition to Mcnemar's test, Spearman rank correlation test (SRC) and Kendall's tau (KT) comparing individual scores were calculated. Results: All 30 patients presented abnormal bone marrow findings after unloading which were characterised by increasing severity and vascularity with a peak at 2-6 months $(p<0.001)$. These findings decreased within one year $(p<0.001)$ and disappeared after more than 12 months. The unloading-findings were more severe at the patella and femur than at the tibia $(p<0.001)$. High scores of severity were associated with confluent and patchy patterns $(\mathrm{SCR}=0.923, \mathrm{p}<0.01$ and $\mathrm{KT}=0.877 \mathrm{p}<0.01$ ).

Conclusion: Bone marrow changes at the knee related to disuse are most prominent 2-6 months following unloading when both patchy and confluent hyperintense patterns are present. Manifestations of unloading are most prevalent within the patella and the femur and associated with increased vascularity.

10:30 - 12:00 Room F1

\section{Oncologic Imaging}

SS 516

\section{Cutting edge imaging in oncology: when and how?}

\section{Moderators:}

K. Coenegrachts; Bruges/BE

E.J. Rummeny; Munich/DE

\section{B-0320 10:30}

Virtual 3D 18 F-FDG PET/CT panendoscopy for assessing the upper airways of head and neck cancer patients: initial results

P. Heusch ${ }^{1}$, C. Buchbender ${ }^{1}$, B. Geiger ${ }^{2}$, J. Treffert ${ }^{3}$, T. Lauenstein ${ }^{4}$, M. Forsting ${ }^{4}$, G. Antoch ${ }^{1}$, T.A. Heusner ${ }^{1} ;{ }^{1}$ Düsseldorf/DE, ${ }^{2}$ Princeton, NJ/US, ${ }^{3}$ Knoxville, TN/US, ${ }^{4}$ Essen/DE (philipp.heusch@med.uni-duesseldorf.de)

Purpose: To evaluate whether a virtual 3D 18 F-FDG PET/CT panendoscopy is technically feasible and can be used for a non-invasive visualisation of the upper airways and pharyngeal and laryngeal malignancies.

Methods and Materials: From whole body 18 F-FDG PET/CT datasets including a dedicated head and neck investigation protocol of 40 consecutive patients (mean age: $61 \pm 9$ years) with histopathologically proven pharyngeal and laryngeal malignancies virtual 3D 18 F-FDG PET/CT panendoscopies were reconstructed using an investigational software application. The feasibility to completely assess specific spaces of the upper airways by a virtual "fly-through" and to detect primary tumours was tested; the time for an automated segmentation of the virtual $18 \mathrm{~F}$ FDG PET/CT panendoscopies was measured.

Results: The nasopharynx, oropharynx, laryngopharynx, epiglottis, subglottis and the tracheobronchial tree were accessible in all 40 , the laryngopharynx, aryepiglottical folds, piriform sinus, glottis, and oral cavity in $37,37,37,33$ and 16 patients, respectively. In all 12 patients with restricted fiberoptical evaluation due to a primarily intubation the subglottical space was entirely accessible via virtual panendoscopy. The primary tumour was depicted in $36 / 40$ patients $(90 \%)$. The mean processing time for virtual $18 \mathrm{~F}-\mathrm{FDG} P \mathrm{PET} / \mathrm{CT}$ panendoscopies was $145 \pm 98 \mathrm{~s}$.

Conclusion: Virtual 18 F-FDG PET/CT panendoscopy of the upper airways is technically feasible and can detect pharyngeal and laryngeal malignancies. The tool allows for a complete evaluation of the subglottical space when optical panendoscopy is incomplete due to intubation. It may be used for planning endoscopy-guided biopsies and surgery in the future.

\section{B-0321 10:39}

18 F-FLT- PET in the diagnosis of gastric cancer

T. Staniuk ${ }^{1}$, M. Studniarek ${ }^{2}$, W. Zegarski', E. Srutek ${ }^{1}$, B. Malkowski';

'Bydgoszcz/PL, ${ }^{2}$ Bojano/PL (mstud@gumed.edu.pl)

Purpose: The aim of the study was to evaluate the usefulness of 18 F-FLT PET/ $\mathrm{CT}$ in the detection and differentiation of gastric cancer.

Methods and Materials: 94 consecutive patients with newly diagnosed advanced gastric cancer were examined with FLT PET. For quantitative and comparative analysis, the maximal standardised uptake value (SUVmax) was calculated for the tumours and not invaded gastric wall. On the basis of pathological assessment of removed specimen the tumours were divided into 4 groups: adenocarcinoma tubulare (G2 and G3), mucinous adenocarcinoma, undifferentiated carcinoma and adenocarcinoma tubulare $\mathrm{p}$. mucocellulare. This is not purely WHO classification, it is applied in our oncology center.

Results: For each of the four types of gastric cancer ( $\mathrm{N}$ cases) the minimal, maximal and mean value of SUVmax (SD) was found: adenocarcinoma tubulare (57), SUVmax 1.5-23.1 (7.46 \pm 4.57$)$, mucinous adenocarcinoma (17), SUVmax 2.3-10.3 $(5.5 \pm 2.4)^{*}$, undifferentiated carcinoma (6), SUVmax 3.1-13.6 (7.28 \pm 3.25$)$, adenocarcinoma tubular p. mucocellulare (14), SUVmax 2-25.3 (7.7 \pm 6.99$)$ and normal gastric wall (22), SUVmax 1.01-2.55 (1.84 \pm 0.35$)$. It significantly differs from AdenoCa tubular and normal gastric wall and from all types of tumour. All gastric 
cancer types showed higher accumulation of FLT than non infiltrated gastric wall as measured by SUVmax. Only tumours with relatively low cellular density (mucinous type) accumulated FLT significantly less than other ones.

Conclusion: This cancer type has different clinical cours and a different way of dissemination, so we conclude that FLT PET can be useful in predicting prognosis, planning treatment and monitoring response in patients with gastric cancer.

\section{B-0322 10:48}

First clinical experience in restaging of patients with recurrent prostate cancer by 11C-choline-PET/MR: comprehensive diagnostic MR protocol and comparison with 11C-choline-PET/CT

M. Eiber, M. Souvatzoglou, E.J. Rummeny, M. Schwaiger, A.J. Beer; Munich/DE (matthias.eiber@tum.de)

Purpose: Whole body integrated PET/MR might provide an alternative imaging tool to $11 \mathrm{C}$-choline-PET/CT for restaging prostate cancer.

Methods and Materials: 34 patients underwent a single injection/dual imaging protocol on a PET/CT scanner (5 min pi) and a subsequent PET/MR scan (Siemens Biograph mMR; Ø47 min pi). PET/MR were performed in 3-4 bed positions including simultaneous MR acquisition of a coronal Dixon-T1w-VIBE for attenuation correction, a coronal T1w-SE and a axial fat-sat T2w-haste-sequence. During an additional PET scan over the pelvis axial/coronal T2w, axial DWI (b values: 0,400 , $800 \mathrm{~s} / \mathrm{mm} 2$ ) and axial T1w-TWIST-DCE-sequences were acquired. Finally, the whole body was covered with axial contrast-enhanced fat-saturated T1w-GRE sequences. PET/MR and PET/CT were read independently and all suspicious lesions (local recurrence, lymph node and bone metastases) were rated on a three-point-scale (1: definitely a metastasis, 2: probably a metastasis, 3: indeterminate).

Results: Despite a considerable longer imaging time for 11C-choline-PET/MR (Ø42 $\mathrm{min}$ ) compared to PET/CT (Ø21 $\mathrm{min}$ ) the protocol was well tolerated by all patients. Detection rate for local recurrence was substantially higher and more conclusive for PET/MR which detected 18 regions in 12 patients (rating 1.215) compared to $\mathrm{PET} / \mathrm{CT}$ with 12 regions in 8 patients (rating 1.325). For lymph node metastases a nearly similar performance was found with 52 and 55 regions, respectively (rating 1.180 and 1.637). PET/MR detected bone metastases in 17 regions in 5 patients (rating 1.183), whereas $\mathrm{PET} / \mathrm{CT}$ found 14 regions in 4 patients (rating 1.045). PET/ MR was able to demonstrate choline-negative non-sclerotic bone metastases in two patients which were not identified by PET/CT.

Conclusion: These data indicate that 11C-choline-PET/MR is diagnostic feasible for restaging of prostate cancer with potential higher detection rates for local recurrence and bone metastases.

\section{Author Disclosures}

M. Eiber: Speaker; Siemens AG. E.J. Rummeny: Grant Recipient; Philips

M. Schwaiger: Research/Grant Support; DFG, EU. Speaker; Siemens AG.

A.J. Beer: Grant Recipient; DFG. Speaker; Siemens AG.

\section{B-0323 10:57}

Apparent diffusion coefficient for evaluating early tumour response to neoadjuvant chemo-radiotherapy in locally advanced cervical cancer: correlation with histopathology. Preliminary results

M. Miccò, A.L. Valentini, B. Gui, A.M. De Gaetano, M. lacobucci, L. Bonomo; Rome/IT (marta.iacobucci@gmail.com)

Purpose: To evaluate diagnostic accuracy of diffusion-weighted magnetic resonance imaging (DWI) in predicting response to neo-adjuvant chemo-radiotherapy (nCRT) in patients with locally advanced cervical carcinoma using apparent diffusion coefficient (ADC). Cervical lesions ADC were correlated with post-surgical histopathology.

Methods and Materials: 24 women (FIGO>IB Bulky) underwent MRI and DWI prior to, after 2 weeks and at the end of $\mathrm{nCRT}$, using $1.5 \mathrm{~T}$ scanner. Cervical lesion volume and ADC were measured at each assessment. Radical hysterectomy was performed 4 weeks after MRI. Treatment response was determined based on histopathology and was classified as complete response (CR), residual (RD) or stable disease (SD). Mean ADCs (mADC), ADC increase and volume reduction (VR) rates were compared using histopathology as reference standard Results: According to histology, 13/24 (54\%) had CR, 11/24 (46\%) had RD < 1 $\mathrm{cm}$. 2 patients presented SD. Before therapy, in the study population mADC was $0.96 \pm 0.06 \times 10^{-3} \mathrm{~mm}^{2} / \mathrm{s}$ but it was lower in $\operatorname{SD}\left(0.76 \pm 0.1 \times 10^{-3} \mathrm{~mm}^{2} / \mathrm{s}\right)$. After 2 weeks of nCRT, mADC correlated with tumour response: a) in $\mathrm{CR}$ with $23 \%$ percent change $\left(1.20 \pm 0.02 \times 10^{-3} \mathrm{~mm}^{2} / \mathrm{s}\right.$ vs $\left.0.98 \pm 0.06 \times 10^{-3} \mathrm{~mm}^{2} / \mathrm{s} ; \mathrm{P}<0.001\right)$; b) in RD with $10 \%$ percent change $\left(1.05 \pm 0.02 \times 10^{-3} \mathrm{~mm}^{2} / \mathrm{s}\right.$ vs $0.93 \pm 0.06 \times 10^{-3} \mathrm{~mm}^{2} / \mathrm{s} ; \mathrm{P}<$ 0.001 ). Tumour volume decreased in $\mathrm{CR}$ and $\mathrm{RD}$ with reduction rate of $48 \%$ and $46 \%$, respectively. At the end of CRT, no significant differences on ADC between
CR and RD (mADC $1.20 \pm 0.07 \times 10^{-3} \mathrm{~mm}^{2} / \mathrm{s}$ vs $1.17 \pm 0.06 \times 10^{-3} \mathrm{~mm}^{2} / \mathrm{s} ; \mathrm{P}>1$ ) were observed. All CR had local inflammation at histology.

Conclusion: ADC is early indicator of tumour response in patients with advanced cervical cancer.

\section{B-0324 11:06}

The value of DWI in differentiating ocular tumours from retinal detachment K. Erb, G. Willerding, M. Taupitz, B. Hamm, P. Asbach;

Berlin/DE (katharina.erb@charite.de)

Purpose: Most patients with an ocular tumour present with retinal detachment. It can be difficult to distinguish ocular tumours from retinal detachment with noncontrast and contrast-enhanced T1- and T2-weighted sequences due to retinal effusion. In this study, we hypothesize that DWI can help to differentiate ocular melanoma from retinal detachment.

Methods and Materials: Thirty patients with ocular melanoma were prospectively investigated with ocular MRI including DWI in this IRB approved study. On the DWI of different $b$ values $\left(b=0,1000 \mathrm{~mm}^{2} / \mathrm{sec}\right)$ a ROI was placed into the ocular tumour and another ROI into the retinal detachment. ADC values of the ROIs were calculated.

Results: Twenty-three patients (61\%) had retinal detachment. The mean ADC in ocular melanoma $\left(922 \times 10^{-6} \mathrm{~mm}^{2} / \mathrm{sec}\right)$ differs significantly from the mean ADC in retinal detachment $\left(2035 \times 10^{-6} \mathrm{~mm}^{2} / \mathrm{sec}\right)$

Conclusion: DWI helps significantly to delineate ocular melanoma from retina detachment. Therefore, we recommend including DWI in ocular MRI if an ocular tumour is suspected.

\section{B-0325 11:15}

Dosimetric impact of MR vs CT delineation of boost volume in breast cancer

M. Betti, L. Orlandini, C. Biagini, M. de Liguoro, F. Mungai, L. Natale, L. Cionini; Sesto Fiorentino/IT (margherita.betti@lacittadellasalute.it)

Purpose: Postoperative radiotherapy after conservative surgery in breast cancer usually includes focal over irradiation (boost) to the surgical bed (SB). Localisation of SB with $\mathrm{CT}$ results is difficult in several cases due to insufficient intrinsic contrast between soft tissues provided by this imaging modality. To prevent marginal misses large boost volume are delineated, resulting in an increase of dose to normal tissue. MR imaging provides superior soft tissue contrast and the potential to better differentiate between normal tissue and SB. The purpose of this study is to evaluate how MR imaging contouring dosimetrically affect the radiotherapy treatment, compared to CT.

Methods and Materials: 12 boost volumes were drawn on CT and on MR images, elastically co-registered with MiradaRtx System. RT treatment plan was optimised for standard and hypofractionated schedules, using CT volumes as baseline. The target volume dose distribution on CT and MR were compared and dose volume histograms (DVHs) cutoff point V98\% (volume covered by $98 \%$ of prescription dose) was used at this purpose.

Results: The volumes of the SB varied considerably from CT to MR (concordance index between 0.36 and 0.68). The dosimetrical difference between MR and CT boost dose in terms of $\mathrm{V} 98 \%$ ranged between $10 \%$ and $30 \%$ for both standard and hypo-fractionated plans. The analysis revealed smaller differences in V98\% when boost volumes were delineated on MR image.

Conclusion: MR imaging to delineate SB volume may add to the accuracy in delivering boost dose.

\section{B-0326 $11 \cdot 24$}

$\mathrm{PET} / \mathrm{MR}$ vs $\mathrm{PET} / \mathrm{CT}$ in lung cancer $\mathrm{N}$ staging: initial experience with a three-segment model attenuation correction

A. Kohan, C. Rubbert, J.L. Vercher Conejero, S. Partovi, R. Jones, K. Herrmann, P. Faulhaber; Cleveland, OH/US (aak107@case.edu)

Purpose: Current attenuation correction (AC) methods for PET/MRI include a T1-weighted imaging and segmentation model accounting for air, soft tissue and lung (3-segment model) and a Dixon 5 segment model of air, lung, bone, soft tissue and fat. Staging of lung cancer with PET/MRI may be challenging since both primary tumour and adenopathies occur at tissue interfaces between soft tissue and lung. Particularly, $\mathrm{N}$-staging may be affected by errors in segmentation. The purpose of this study was to assess the accuracy of PET/MRI in determining nodal stage in lung cancer patients.

Methods and Materials: Eleven patients with suspected or known lung cancer were enrolled in our IRB-approved protocol to undergo both PET/MR and PET/CT for (re)staging of lung cancer at the same time point. Data of both examinations 
were randomised and analysed by four blinded readers independently ( 2 radiologists; 2 nuclear physicians; 4, 15, 6 and 12 yrs of experience). JACC guidelines were applied to determine $\mathrm{N}$ stage. Kappa statistics were performed to compare observer performance for PET/CT and PET/MRI, respectively.

Results: For the $\mathrm{N}$ staging in $\mathrm{PET} / \mathrm{CT}$, inter-observer agreement was almost perfect with a kappa of 0.86 . Inter-observer agreement with PET/MRI was still substantial yielding a kappa of 0.70 .

Conclusion: Despite our small study population, PET/MR with a three-segment model AC showed promising results with a substantial inter-reader agreement for $\mathrm{N}$ staging only slightly inferior to PET/CT. Interpretation of PET/MRI might still be subject to a learning curve and optimisation of AC methods.

Author Disclosures:

A. Kohan: Research/Grant Support; Philips Healthcare. C. Rubbert: Research/ Grant Support; Philips Healthcare. J.L. Vercher Conejero: Research/ Grant Support; Philips Healthcare. S. Partovi: Research/Grant Support; Philips Healthcare. R. Jones: Research/Grant Support; Philips Healthcare. K. Herrmann: Research/Grant Support; Philips Healthcare. P. Faulhaber: Research/Grant Support; Philips Healthcare.

\section{B-0327 11:33}

Incidental findings in the general adult population using whole body MRI as a screening method

S. Ulus, E. Karaarslan; Istanbul/TR (silaulus@hotmail.com)

Purpose: Whole body MRI (WB-MRI) is a safe and reliable imaging method and is recommended as an advanced check-up procedure. The aim of this study is to describe the technique and to evaluate incidental findings in asymptomatic adult population.

Methods and Materials: Between March 2008 and December 2011, 118 consecutive subjects ( 71 males, 47 females, mean age 47.4 ) undergoing thorough check-up were prospectively included in the study. MRI was performed with Siemens MagnetomAvanto (SQ engine, $45 \mathrm{mT} / \mathrm{sec}$ ) with $205 \mathrm{~cm}$ moving table, parallel imaging and automatic image composing software. Statistical analysis was performed with NCSS. For comparison of quality data, Chi-square and odds ratio were used. Results: In 80 subjects (67\%), 114 benign (63.5\%) and 6 malign (3.5\%) lesions were detected. Six subjects underwent operation $(5 \%)$ for adrenal carcinoma, renal carcinoma, pancreatic mucinous cystadenoma, intradural schwannoma (2), and enchondroma of the femur. In one subject, tuberculosis pneumonia was detected and medical treatment was given. In 3 subjects focal nodular hyperplasia of the liver, and in one an unclassified solid mass in spleen were encountered and they are in follow-up. The most common incidental lesions were liver cyst (12), liver haemangioma (9), renal cyst (13), and thyroid nodule (6). No significant difference was observed for the incidence of benign and malign lesions between subjects aged ${ }^{3} 40$ years and $<40$ years $(p=0.209$ and $p=0.898$, respectively).

Conclusion: $7 \%$ of the population screened by WB-MRI underwent further surgical and medical treatment. WB-MRI is a useful and non-invasive tool for the detection of incidental lesions in asymptomatic subjects.

\section{B-0328 11:42}

Bone metastasis at a glance: a new advanced visualisation algorithm for fast and easy detection in thoracoabdominal CT

D.F. Toth, M. Toepker, M.E. Mayerhoefer, J. Furtner, U. Asenbaum, G. Karanikas, M. Weber, C. Czerny, H. Ringl; Vienna/AT (daniel.toth@meduniwien.ac.at)

Purpose: To retrospectively assess the detection rate of bone metastases in thoracoabdominal CT achieved by the combined reading of advanced visualisation of cancellous bone (AVCB) and multiplanar reformats (MPRs) compared with reading MPRs only.

Methods and Materials: The study was approved by the local Institutional Review Board. 156 consecutive patients with confirmed cancer that received a whole body PET/CT examination for clinical purposes were included $(n=156 ; 93$ male and 63 female patients; mean age, $59.8 \mathrm{y} \pm 14.9$; range, $11-85 \mathrm{y}$ ). The CT scan of these examinations was subjected to the AVCB algorithm that segmented the bones, removed the cortical layer, and allowed a free view on the cancellous bone. The PET images served as part of the reference standard only. 15 patients were used as a training set. Four radiologists independently evaluated the remaining patients by reporting half of the patients using AVCB and MPRs together, and the other half using MPRs only. Radiologists were blinded to patient names and patient order was randomised. Results were compared to a standard of reference created by one senior radiologists and one nuclear medicine specialist. Logistic regression was used for statistical analysis.

Results: 349 lesions were found in 103 patients, with 203 classified as malignant. The detection rate for all bone lesions was 35\% (247/698) for MPRs and, at 74\%
(521/698), significantly higher using AVCB and MPRs $(P<.001)$. The average reading time decreased from 85 to 43 seconds $(P<.001)$.

Conclusion: Advanced visualisation of cancellous bone significantly increases the ability to detect bone metastases and reduces the time for diagnosis.

Author Disclosures:

D.F. Toth: Patent Holder. H. Ringl: Patent Holder.

\section{B-0329 11:51}

Comparison of the utility of whole body MRI with and without contrast-enhanced quick $3 \mathrm{D}$ and double $\mathrm{RF}$ fat suppression techniques, conventional whole body MRI, PET/CT and conventional examination for assessment of recurrence in NSCLC patients Y. Ohno ${ }^{1}$, S. Seki', M. Nishio ${ }^{1}$, H. Koyama ${ }^{1}$, T. Yoshikawa ${ }^{1}$, S. Matsumoto', S. Satou' ${ }^{2}$ K. Sugimura $1{ }^{1}{ }^{1}$ Kobe/JP, ${ }^{2}$ Ohtawara/JP (yosirad@kobe-u.ac.jp)

Purpose: To compare diagnostic capabilities for the assessment of recurrence in non-small cell lung cancer (NSCLC) patients by contrast-enhanced whole body MRI (CE-WB-MRI) with and without CE-Quick 3D and double RF fat suppression technique (DFS), FDG-PET/CT and conventional radiological examinations. Methods and Materials: A total of 134 pathologically proven and completely resected NSCLC patients (78 males, 56 females; mean age: 72 years) underwent FDG-PET/CT, CE-WB-MRI with and without Quick $3 \mathrm{D}$ and DFS at 3 T as well as conventional radiological examinations. The probability of recurrence was assessed with a 5-point scoring system on a per-patient basis, and final diagnosis was made by consensus between two readers. The capability for overall recurrence assessment by all the methods was compared by means of ROC analysis and their sensitivity, specificity and accuracy by means of McNemar's test.

Results: Although areas under the curve did not show any significant differences, specificity (100\%) and accuracy (95.5\%) of CE-WB-MRI with CE-Quick 3D and DFS were significantly higher than those of FDG-PET/CT (specificity: $93.6 \%, p=0.02$; accuracy: $89.6 \%, p=0.01$ ) and conventional radiological examinations (specificity: $92.7 \%, p=0.01$; accuracy: $91.0 \%, p=0.03)$. In addition, specificity of CE-WB-MRI without CE-Quick 3D and DFS (100\%) was significantly higher than that of FDGPET/CT ( $p=0.02)$ and conventional radiological examinations $(p=0.01)$. Conclusion: Specificity and accuracy of CE-WB-MRI with CE-Quick 3D and DFS for assessment of recurrence in NSCLC patients are at least as high as, or higher than those of others.

Author Disclosures:

Y. Ohno: Research/Grant Support; Toshiba Medical Systems. M. Nishio: Research/Grant Support; Toshiba Medical Systems. T. Yoshikawa: Research/ Grant Support; Toshiba Medical Systems. S. Matsumoto: Research/Grant Support; Toshiba Medical Systems. S. Satou: Employee; Toshiba Medical Systems.

$10: 30-12: 00$ Room F2

Breast

SS 502

\section{Elastography and other advances in breast ultrasound}

Moderators:

D. Djilas-Ivanovic; Sremska Kamenica/RS

P. Skaane; Oslo/NO

\section{B-0330 10:30}

Quantitative shear wave elastography: evaluating an additional use with conventional ultrasound in diagnosis of breast lesions

J. Moon, K.-S. Jung, S. Koh, J.-Y. Jung; Anyang-si/KR (geenie-m@hanmail.net)

Purpose: We assessed whether the additional use of quantitative shear wave elastography (QSWE) would improve diagnostic accuracy of conventional ultrasound (US).

Methods and Materials: Two experienced radiologists performed conventional US and QSWE of 115 patients with 133 lesions. BI-RADS categories were assessed and quantitative lesion elasticity was measured in terms of Young's modulus $(\mathrm{kPa})$ for each lesion. Pathologic results were reference standard. We compared diagnostic accuracy of conventional US and US with QSWE, including sensitivity, specificity and area under the curve (AUC) using a receiver operating characteristic (ROC) curve analysis. In addition, we prospectively investigated two values, 
mean elasticity and maximum elasticity of each lesion, which are the most reliable indicators in QSWE.

Results: For differentiation of malignant from benign lesions, conventional US was associated with sensitivity of $100 \%$, specificity of $43.6 \%$ and the AUC was 0.718 . The AUC was 0.8909 for mean elasticity values and 0.8880 for maximum elasticity values. The cutoff value was $60.7 \mathrm{kPa}$ for mean elasticity values, with $84.4 \%$ sensitivity and $90.1 \%$ specificity. The cutoff value for maximum elasticity was 81.3 $\mathrm{kPa}$, with $88.1 \%$ sensitivity and $88.12 \%$ specificity. Additional use of QSWE with conventional US was associated with a sensitivity of $97.0 \%$, specificity of $92.0 \%$, and accuracy of $75.2 \%$.

Conclusion: Additional use of QSWE increases the diagnostic accuracy of conventional US. Both mean elasticity and maximum elasticity show no difference as a decisive value but optimal cutoff points of these two values are different.

\section{B-0331 10:39}

Quantitative ShearWave ultrasound elastography: initial experience in palpable breast masses

S. Hari, S. Naga, A. Dhar, M. Jana; New Delhi/IN

Purpose: To determine the appearance of breast masses at shear wave elastography (SWE) and to assess the correlation between quantitative values of lesion stiffness and pathologic results.

Methods and Materials: Using the Aixplorer® ultrasound (US) system (SuperSonic Imagine, Aix en Provence, France), 144 breast masses were identified in 131 consecutive patients. Two orthogonal elastography images were obtained of each mass. Quantitative lesion elasticity was measured in terms of the Young modulus (in kilopascals). Pathologic results were available in all cases. B-mode US images were classified according to ACR-BI-RADS. BI-RADS categories 1-3 were taken as benign while BI-RADS categories 4 and 5 were classified as malignant. Results: All breast lesions were detected at SWE. The mean elasticity value for benign lesions ( $n=91$ ) was $29.89 \mathrm{kPa}$ (SD27.24) while that for malignant lesions $(n=53)$ was 188.97 (SD 63.22). The mean size of the lesions on US was $23.63 \mathrm{~mm}$ with (SD15.39). A cut-off mean elasticity point of $92.25 \mathrm{kPa}$ was set as threshold for benign/malignant differentiation using receiver operating curve. The sensitivity, specificity, PPV, NPV and accuracy of conventional ultrasound versus SWE were $96.2 \%$ vs $96.2 \%, 80.2 \%$ vs $95.6 \%, 73.9 \%$ vs $92.7 \%, 97.3 \%$ vs $97.7 \%$ and $86.1 \%$ vs $95.8 \%$, respectively. Complicated cysts were differentiated from solid lesions because they had elasticity values of $0 \mathrm{kPa}$ (no signal was retrieved from liquid areas). Conclusion: The elasticity values of benign and malignant lesions were significantly different allowing for accurate characterisation. The diagnostic performance of SWE is comparable with B-mode US, with SWE having a superior specificity.

\section{B-0332 10:48}

Can the ring sign improve the performance of shearwave elastography in benign/malignant differentiation of screen detected solid breast masses?

M. Szewcyk-Bieda, A. Evans, S. Vinnicombe, P. Whelehan, K. Thomson; Dundee/UK (a.z.evans@dundee.ac.uk)

Purpose: Shear wave elastography (SWE) can differentiate benign from malignant solid breast masses. Small, low-grade cancers can have benign (low) mean stiffness values but often show a ring of slightly stiffer tissue around the lesion. We aimed to establish whether taking the presence of a ring sign to indicate malignancy improves the discriminatory performance of SWE in screen detected solid breast masses. Methods and Materials: The ring sign was defined as the presence of stiffer borders on at least 2 aspects of the lesion. The presence or the absence of the ring sign was independently assessed by two breast radiologists, blinded to pathology outcomes, in a consecutive series of 145 screen-detected solid masses. A third radiologist arbitrated in discordant cases. The discriminatory performance of SWE using a threshold mean stiffness value of 50 kilopascals $(\mathrm{kPa})$ was compared with taking either a threshold mean stiffness of $50 \mathrm{kPa}$ or a ring sign as positive. Results: Of 145 masses, 42 were benign and 103 were malignant. In 122 (84\%), the ring sign findings of two radiologists were concordant. For mean stiffness threshold of $50 \mathrm{kPa}$, sensitivity was $83 \%$ and specificity was $55 \%$. When either mean stiffness over $50 \mathrm{kPa}$ or a ring sign was counted as positive, sensitivity was $91 \%$ and specificity was $45 \%$. The increase in sensitivity was statistically significant (Fisher's exact test, $p=0.048)$. The drop in specificity was not $(p=0.25)$.

Conclusion: The ring sign can improve the sensitivity of shear wave elastography in differentiating benign from malignant screen-detected solid breast masses.

\section{B-0334 10:57}

Influence of menopause in women on breast elasticity measured by elasticity parameters with sonoelastography

X. Wang, Y. Wang, P. Xu; Shanghai/CN (wangxiaohong426@yahoo.com.cn)

Purpose: To assess the value of elasticity parameters on breast sonoelastography by influence of menopause factor

Methods and Materials: 286 female patients (premenopausal vs postmenopausal: 192 vs 108) with 300 breast lesions were conducted routine ultrasound and elastographic ultrasound preoperatively. The elastic parameters of strain ratio (SR, SR1 was calculated using the same-level and normal-appearing breast region as reference; SR2 was calculated using the subcutaneous fat as reference) were then obtained, with the pathologic diagnosis served as golden standard. The sensitivity, specificity, and area under the curve were analysed by receiver operating characteristic $(\mathrm{ROC})$ curve with or without the influence of menopause factor.

Results: Histopathology revealed 180 malignant and 120 benign lesions. Without the factor of menopause, SR1 and SR2 exhibited moderate diagnostic performance (area under the curve, 0.803 vs 0.782 ). When the modified SR 1 cutoff points of 2.35 and 2.52 were used in the groups of premenopausal and postmenopausal, respectively, the sensitivity, specificity and accuracy were remarkably improved (79.2\%, 92\%, $85.7 \%$ vs $64.2 \%, 81.4 \%, 77.3 \%, p<0.05)$. No statistical differences were found in the diagnostic value of SR2 with or without the influence of menopause factor (sensitivity, specificity and accuracy: $59.2 \%, 77.8 \%, 70.3 \%$ vs $70 \%, 73.9 \%, 72.7 \%)$.

Conclusion: Menopause has an important influence on breast elasticity measured by elasticity parameters, especially in SR1. Taking this factor into consideration may help to improve the diagnostic performance of breast sonoelastography in a more comprehensive way.

\section{B-0335 11:06}

Stiffness measured by Shear Wave elastography: a biomarker of the early chemotherapy response in a human breast cancer model? F. Chamming's, M. Le Frère-Belda, v. Fitoussi, L. Pidial, H. Latorre-Ossa, J.-L. Gennisson, O. Clément, C.-A. Cuenod, L.S. Fournier; Paris/FR (foucauld.chammings@egp.aphp.fr)

Purpose: To assess if stiffness as measured by shear-wave elastography (SWE), could be an early biomarker of response to chemotherapy in a breast cancer model. Methods and Materials: A human invasive ductal carcinoma was implanted subcutaneously in 10 athymic female mice. After 8 weeks of growth, 2 tumours were removed for baseline pathological analysis and 8 were treated by oral chemotherapy during 5 weeks. Ultrasound was performed every week before and during treatment, using a superficial 8-MHz probe. Maximum diameter and mean elasticity value of the tumor were measured. Kinetics of changes in diameter and elasticity before and under treatment were calculated as the slope of diameter or elasticity over time. At the end of the treatment, tumours were removed for pathological analysis. Results: Before treatment, we observed a progressive stiffness increase along tumour growth (slope: $8.8 \mathrm{kPa} /$ week). Under chemotherapy, we observed an early stabilisation of tumour stiffness (slope: $-0.8 \mathrm{kPa}$ per week). Furthermore, a significant decrease of elasticity values was seen after four weeks of treatment $(p=0.03)$. We also observed an early progressive decrease of diameter showing that there was a good response to treatment. After five weeks of treatment, we observed modifications of the pathological features of the tumor, i.e. mainly a decrease of cellular density.

Conclusion: In our model, we observed an early stabilisation of tumour elasticity with an inversion of elasticity slope under treatment showing that SWE could be an interesting tool to assess early tumor response to chemotherapy.

Author Disclosures:

F. Chamming's: Grant Recipient; Société Française de Radiologie. Other; Supersonic imagine. J. Gennisson: Consultant; Supersonic Imagine.

\section{B-0336 11:15}

SONAZOID ${ }^{\circledR}$ (perflubutane) microbubble-enhanced ultrasound for differential diagnosis of breast lesions: pooled analysis of phase 2 and 3 clinical studies

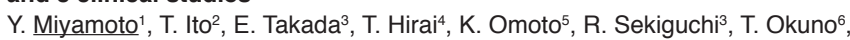
S. Kanazawa 1 , F. Moriyasu $1 ;{ }^{1}$ Tokyo/JP, ${ }^{2}$ Osaka/JP, ${ }^{3 \top}$ ochigi/JP, ${ }^{4}$ Nara/JP,

${ }^{5}$ Saitama/JP, ${ }^{6}$ Kobe/JP (miyamoto@jikei.ac.jp)

Purpose: Two clinical studies (Phase 2 and Phase 3) were performed to assess the efficacy and safety of contrast-enhanced ultrasound withSONAZOID ${ }^{\circledR}$ (Perflubutane) microbubbles for differential diagnosis of breast lesions compared with unenhanced ultrasound and contrast-enhanced MRI. 
Methods and Materials: A total of 151 patients were enrolled at 5 centers in Japan. All patients provided written informed consent before entering the studies. Unenhanced ultrasound, contrast-enhanced ultrasound, and contrast-enhanced MR images were independently assessed by three blinded reviewers, who viewed the different images in a randomised sequence and were also blinded to subject characteristics. The observation period for safety assessment was 3 days after the contrast agent administration.

Results: Among 140 patients included in the diagnostic analysis, the accuracy of contrast-enhanced ultrasound (86.7\%) was significantly higher than that of unenhanced ultrasound $(66.9 \%)$ and contrast-enhanced MRI $(69.5 \%)$ (both $p<0.001)$. Sensitivity and specificity of contrast-enhanced ultrasound $(92.9 \%, 84.0 \%)$ were also superior to unenhanced ultrasound $(86.5 \%, 58.5 \%)$ and contrast-enhanced MRI (86.5\%, 62.2\%) (sensitivity: $p=0.177$ and $p=0.160)$ (specificity: both $p<$ 0.001 ). The incidence of adverse events was $9.9 \%$, with adverse drug reactions in $3.3 \%$ (all mild).

Conclusion: Compared with unenhanced ultrasound and contrast-enhanced MRI, contrast-enhanced ultrasound with SONAZOID ${ }^{\circledR}$ (Perflubutane) microbubbles achieved significantly better diagnostic accuracy and specificity for breast lesions without causing serious adverse reactions.

\section{B-0337 11:24}

US-based automated breast volume scanner (ABVS): improvement of breast lesion detection in $\mathbf{5 1}$ patients - initial study

X. Wang, Y. Wang, P. Xu; Shanghai/CN (wangxiaohong426@yahoo.com.cn)

Purpose: To investigate the clinical application of ultrasound (US)-based automated breast volume scanner (ABVS) in detection of breast lesions.

Methods and Materials: Conventional handheld ultrasound, US-based ABVS and mammography were performed on 41 patients with 65 breast lesions by three different physicians. Two other physicians blinded to the results of conventional ultrasound, mammography and histopathology obtained the number of lesions detected by the three devices, respectively. All the lesions were then classified according to ACR BI-RADS assessment categories. Sensitivity, specificity, and accuracy were then calculated, with histopathology serves as the reference standard. Results: US-based ABVS showed the best detection ability among the three modalities. The number of breast lesions depicted by conventional handheld ultrasound, US-based ABVS and mammography were 65,67 , and 48 , respectively. ABVS helped to confirm all the subjects detected by the other two devices only, and two more lesions were found as well. More importantly, 3 (18.8\%) mammographically occult breast cancers were detected by ABVS. The retraction phenomenon in the coronal view was highly suggestive of malignant tumour in diagnosis. The sensitivity, specificity, and accuracy of conventional handheld ultrasound were $75.0 \%(12 / 16)$, $87.8 \%$ (43/49), and $84.6 \%$ (55/65), respectively. However, ABVS displayed high specificity and accuracy (93.9\% [46/49], 89.2\% [58/65], respectively).

Conclusion: US-based automated breast volume scanner greatly improves the detectable rate of breast lesions. With standardised workflow and good reproducibility, ABVS is expected to be a potential frontier in breast screening in the future.

\section{B-0338 11:33}

Useful of real-time virtual sonography (RVS) on the evaluation of MRI-detected lesions of the breast in second-look sonography

S. Nakano, K. Fujii, M. Yoshida, J. Kousaka, Y. Mori, T. Ando, R. Tetsuka,

T. Fukutomi, T. Ishiguchi; Nagakute/JP (snakano1@aichi-med-u.ac.jp)

Purpose: The aim of this study was to verify the utility of second-look sonography using real-time virtual sonography (RVS), a coordinated sonography with an MRI system that uses an image fusion technique on the sonographic evaluation of MRI-detected lesions of the breast.

Methods and Materials: Of the 196 consecutive patients who were examined with breast MRI in our hospital, those patients who underwent second-look sonography to identify MRI-detected lesions were enrolled in this study. MRI was performed using a 1.5-T imager with the patient in a supine position. To assess the ef[[Unable to Display Character: \&\#64257;]]cacy bene[[Unable to Display Character: \&\#64257;]]ts of RVS, the correlations between lesion detection rates on second-look sonography using conventional B-mode or RVS were analysed.

Results: Of the 196 patients, 55 (28\%) demonstrated 67 lesions initially detected by MRI, followed by second-look sonography. The detection rates of 24 malignant and 43 benign lesions were $33 \%(8 / 24)$ and $23 \%(10 / 43)$ by conventional B-mode; RVS increased these rates signi[[Unable to Display Character: \&\#64257;]]cantly, to $92 \%(22 / 24)$ and $88 \%(38 / 43)$, respectively $(p<0.001)$. Interestingly, $61 \%$ of MRI-detected lesions were situated around mammary fascia, and $90 \%$ of these were detected with second-look sonography using RVS. Of the seven lesions with no sonographic correlates, [[Unable to Display Character: \&\#64257;]]ve could be biopsied by marking MRI information onto the body surface using RVS.

Conclusion: Our results suggest that the additional use of RVS on second-look sonography signi[[Unable to Display Character: \&\#64257;]]cantly increases the sonographic detection rate of MRI-detected lesions without operator dependence.

\section{B-0339 11:42}

Targeting accuracy of real-time virtual sonography (RVS) in sonographic identification of enhancing lesions in breast MRI

S. Nakano, K. Fujii, M. Yoshida, J. Kousaka, Y. Mori, T. Ando, R. Tetsuka,

T. Fukutomi, T. Ishiguchi; Nagakute/JP (snakano1@aichi-med-u.ac.jp)

Purpose: The purpose of this study was to evaluate the targeting accuracy of RVS to sonographically identify enhancing lesions by breast MRI.

Methods and Materials: Between December 2008 and May 2009, RVS was performed in 51 consecutive patients with 63 enhancing lesions. MRI was performed with the patients in the supine position using a 1.5-T imager with a body surface coil to achieve the same position as with sonography. To assess the accuracy of the RVS, the following three issues were analyzed: (i) The sonographic detection rate of enhancing lesions, (ii) the comparison of the tumor size measured by sonography and the MRI-MPR and (iii) the positioning errors as the distance from the actual sonographic position to the expected MRI position in 3-D.

Results: Among the 63 enhancing lesions, $42(67 \%)$ lesions were identi[[Unable to Display Character: \&\#64257;]]ed by conventional B-mode, whereas the remaining $21(33 \%)$ initial conventional B-mode occult lesions were identi[[Unable to Display Character: \&\#64257;]]ed by RVS alone. The sonographic size of the lesions detected by RVS alone was signi[[Unable to Display Character: \&\#64257;]] cantly smaller than that of lesions detected by conventional B-mode $(p<0.001)$. The mean tumor size provided by RVS was $12.3 \mathrm{~mm}$ for real-time sonography and $14.1 \mathrm{~mm}$ for MRI-MPR $(r=0.848, p<0.001)$. The mean positioning errors for the transverse and sagittal planes and the depth from the skin were 7.7, 6.9 and 2.8 $\mathrm{mm}$, respectively. The overall mean 3D positioning error was $12.0 \mathrm{~mm}$.

Conclusion: Our results suggest that RVS has good targeting accuracy to directly compare a sonographic image with MRI results without operator dependence.

$10: 30-12: 00$

Room G/H

\section{Genitourinary}

\section{SS 507}

\section{New frontiers in GU imaging}

Moderators:

G. Hagen; Oslo/NO

C.M.A. Hoeks; Nijmegen/NL

\section{B-0340 10:30}

Functional evaluation of transplanted kidneys using arterial spin labelling (ASL) MRI at $1.5 \mathrm{~T}$ and $3 \mathrm{~T}$ : results in 105 patients

R.S. Lanzman, D. Blondin, P. Heusch, P. Bilk, P. Kröpil, F. Miese, G. Antoch, H.-J. Wittsack; Düsseldorf/DE (rotemshlomo@yahoo.de)

Purpose: The purpose of our study was to investigate arterial spin labelling (ASL) MRI for functional assessment of transplanted kidneys at 1.5 T and $3 \mathrm{~T}$.

Methods and Materials: 105 renal allograft recipients (mean age 50.8 \pm 14.7 years) were included in this study. ASL MRI was performed at $1.5 \mathrm{~T}(\mathrm{n}=71)$ and $3 \mathrm{~T}(\mathrm{n}=34)$ using a single-slice paracoronal FAIR TrueFisp sequence ( 30 averages, inversion time $1200 \mathrm{~ms}$, slice thickness $8 \mathrm{~mm}$, matrix $128 \times 128$ ). For quantification of ASL perfusion, $\mathrm{T} 1$ relaxation times were determined at $1.5 \mathrm{~T}$ and $3 \mathrm{~T}$ using the variable flip angle approach. ROIs were drawn by a single reviewer on ASL parameter maps for quantification of cortical perfusion. ASL perfusion was correlated with allograft function as determined by the estimated glomerular filtration rate (eGFR) and compared between patients with good or moderate allograft function (group a; eGFR $>30 \mathrm{ml} / \mathrm{min} / 1.73 \mathrm{~m}^{2}$ ) and patients with heavily impaired allograft function (group b; eGFR $230 \mathrm{ml} / \mathrm{min} / 1.73 \mathrm{~m}^{2}$ ).

Results: ASL perfusion and eGFR were comparable at $1.5 \mathrm{~T}(36.1 \pm 22.6 \mathrm{ml} /$ $\mathrm{min} / 1.73 \mathrm{~m}^{2}$ and $\left.237 \pm 101 \mathrm{ml} / 100 \mathrm{~g} / \mathrm{min}\right)$ and $3 \mathrm{~T}\left(42.3 \pm 22.2 \mathrm{ml} / \mathrm{min} / 1.73 \mathrm{~m}^{2}\right.$ and $248 \pm 66 \mathrm{ml} / 100 \mathrm{~g} / \mathrm{min})$. ASL perfusion was significantly higher in group a $(283.9 \pm 59.4 \mathrm{ml} / 100 \mathrm{~g} / \mathrm{min})$ as compared to group b $(195.6 \pm 62.0 \mathrm{ml} / 100 \mathrm{~g} / \mathrm{min})$ $(p<0.0001)$. ASL perfusion values exhibited a significant correlation with eGFR $(r=0.59, p<0.0001)$. 
Conclusion: Cortical ASL perfusion values correlate significantly with allograft function, highlighting the potential of ASL MRI for functional evaluation of renal allografts at $1.5 \mathrm{~T}$ and $3 \mathrm{~T}$.

\section{B-0341 10:39}

Assessment of novel chemokine-directed therapy of renal allograft rejection in a murine animal model with multiparametric functional MRI M. Notohamiprodjo, A. Kalnins, M. Kolb, A. Wagner, M.F. Reiser, K. Nikolaou, J. Andrassy; Munich/DE (mike.notohamiprodjo@med.uni-muenchen.de)

Purpose: Renal allograft rejection is associated with chemokine ligand 2 (CCL2)dependent glomerular and interstitial macrophage recruitment. CCL2 blockade with the Oligonucleotide/Spiegelmer mNOX-E36-3'PEG inhibits leucocyte recruitment. The purpose of this study was to evaluate MR renography and diffusion-weighted imaging (DWI) for assessment of this novel chemokine-directed therapy in an experimental murine animal model.

Methods and Materials: An orthotopic renal transplant mouse-model with acute allograft rejection (balb/c into C57/BL6) was used. 5 animals were treated with low-dose cyclosporin A in combination with mNOX. 7 animals did not receive treatment. 3 syngenic controls (C57/BL6 into C57/BL6) were performed. Imaging was conducted at $3 \mathrm{~T}$ with a dedicated mouse coil. Diffusion-weighted imaging was performed with b values ranging 0 to $800 \mathrm{~s} / \mathrm{mm} 2$. MR renography was performed with a TWIST-sequence with a temporal resolution of 1.5 seconds following a bolus of $10 \mu \mathrm{l}$ gadobutrol in $100 \mu \mathrm{l} \mathrm{NaCl}$. Significance between subgroups was assessed with Wilcoxon's rank-sum test.

Results: Syngenic allograft showed substantially higher plasma flow $(53.2 \pm 17.2$ $\mathrm{ml} / 100 \mathrm{ml} / \mathrm{min})$ and significantly lower $(p<0.05)$ ADC $(1.20 \pm 0.02 \mathrm{~mm} 2 / \mathrm{s})$ than native kidneys $(44.1 \pm 7.1 \mathrm{ml} / 100 \mathrm{ml} / \mathrm{min}$ and $1.29 \pm 0.09 \mathrm{~mm} / \mathrm{s})$. Allograft-ADC of treated animals $(0.98 \pm 0.14 \mathrm{~mm} 2 / \mathrm{s})$ was substantially higher than of untreated animals $(0.76 \pm 0.17 \mathrm{~mm} 2 / \mathrm{s})$. Cortical plasma flow $(24.5 \pm 5.5 \mathrm{ml} / 100 \mathrm{ml} / \mathrm{min})$ was significantly lower $(p<0.05)$ for untreated animals compared to treated animals $(37.4 \pm 7.6 \mathrm{ml} / 100 \mathrm{ml} / \mathrm{min})$. Volume of distribution and mean transit time did not show significant differences between the subgroups.

Conclusion: Chemokine-directed treatment ameliorates acute renal allograft rejection. Functional MRI is able to non-invasively assess these therapy effects in this experimental model and holds high potential to become an alternative to invasive organ biopsy.

\section{B-0342 10:48}

\section{Assesment cisplatin-induced interstitial nephropathy using} diffusion-weighted MRI

R. Del Vescovo, F. Giurazza, R.L. Cazzato, C.L. Piccolo, R.F. Grasso, B. Beomonte Zobel; Rome/IT (r.delvescovo@unicampus.it)

Purpose: To assess the potential of noninvasive diffusion-weighted magnetic resonance imaging (DW-MRI) to depict interstitial kidney changes in patients (pts) under cisplatin-based chemotherapy.

Methods and Materials: We analysed ADC maps of 34 oncologic patient $(5$ woman and 29 men; mean age 56 years; $50 \%$ affected by advanced lung cancer) treated with cisplatin-based chemotherapy comparing with age- and sex-matched control group's ADC maps. The creatinine clearance $(\mathrm{CrCl})$ used to determinate renal function was based on the formula of Crockfort (normal value $>60 \mathrm{~mL} / \mathrm{min}$ ). The ADC values were calculated using a ROI positionated manually on the cortex of each pole in axial and coronal images. For statistical analysis t-test was used. A p value of less than 0.05 was considered significant.

Results: There was no anatomical difference visible with conventional MR imaging of the cortex and medulla relationship. In pts treated with cisplatin there was a reduction of $A D C$ values in the cortex, but no significant changes in medulla. The $A D C$ values of healthy volunteer were significantly higher comparing with patients treated with cisplatin $(p<0.04)$. There was no significant difference between the $A D C$ values of left and right kidney in each patient except in one who present a unilateral ureteral obstruction. No significant correlation was found between the creatinine clearance and ADC values obtained in both groups.

Conclusion: DW-MRI may allow a noninvasive detection of changes in kidneys in pts with interstitial nephropathy during and after chemotherapy treatment.

\section{B-0343 10:57}

Value of diffusion-weighted-MR-imaging for the detection of nephritis H.J. Michaely', M. Reichert', B. Henninger ${ }^{2}$, S. Haneder ${ }^{1}$, S.O. Schönberg'; 1Mannheim/DE, ${ }^{2}$ Innsbruck/AT (henrik.michaely@umm.de)

Purpose: To evaluate diffusion-weighted-MR-imaging (DWI-MRI) for the detection and assessment of infectious renal disease.
Methods and Materials: In a retrospective study, 21 patients with non-tumorous increased signal intensity of the kidneys on DWI sequences $(b=800 \mathrm{~s} / \mathrm{mm} 2)$ and corresponding ADC-decrease was identified. As a control group served 60 patients without clinical signs of renal infection. All patients underwent routine MRI-protocol of the abdomen including EPI-sequence for the DWI (b.0/400/800), T2w HASTE, T1w VIBE post-contrast (Dotarem). Confirmation of renal infection was established on the basis of clinical criteria. T1w- and T2w-images were assessed and compared to DWI for the presence of altered signal and the degree of the visibility of pathology was graded on an three-point scale.

Results: In all 21 patients with positive DWI-findings, a renal infection could be confirmed while none of the patients in the control group showed a suspicious signal pattern on DWI. T2w-imaging and contrast-enhanced T1w-imaging displayed obvious pathologic signal in $3 / 21(14 \%)$ and $11 / 19(58 \%)$ patients, slightly pathologic signal in $17 / 21(81 \%)$ and $7 / 19(37 \%)$, respectively. No pathologic change was seen in $1 / 21(4 \%)$ and $1 / 19(5 \%)$. The median visibility score of 2 for the DWI-imaging and the $\mathrm{T} 1 \mathrm{w}$-images was significantly higher than the median visibility score of 1 for the T2w-imaging ( $p=0.0001$ (DWI vs. T2w) and $p=0.078$ (T1w vs. T2w). No significant differences were found between the visibility scores of T1w imaging and DWI. Conclusion: DWI of the kidneys seems to be highly sensitive for the detection of infections within the kidney.

Author Disclosures:

H.J. Michaely: Advisory Board; Siemens Body MR.

\section{B-0344 11:06}

DCE-MRI assessment of kidney function and renal masses: single slice versus whole organ/tumour

M. Notohamiprodjo, A.D. Helck, S. Winter, M. Staehler,

M. Braunagel, M. Ingrisch, M.F. Reiser, K. Nikolaou;

Munich/DE (mike.notohamiprodjo@med.uni-muenchen.de)

Purpose: To investigate feasibility dynamic-contrast-enhanced (DCE)-MRI assessment of kidney function and renal masses on a single slice compared with investigation of the whole organ or tumour.

Methods and Materials: Fourteen patients with renal tumours (11 renal cell carcinomas, 2 angiomyolipoma, 1 oncocytoma) before and after partial nephrectomy underwent DCE-MRI at 3 T (Magnetom VERIO, Siemens Healthcare Sector) with a time-resolved angiography with stochastic trajectories (TWIST)-sequence (voxelsize 2.6x2.6x2.6 mm 3 , temporal resolution $2.5 \mathrm{~mm}$ ) with half-dose Gadobutrol (Bayer Healthcare Pharmaceuticals). 28 kidney datasets and 14 tumour datasets were assessed using in-house built IDL-software PMI 0.4. Using 2-compartment-models with semi-automated segmentation, renal perfusion and filtration as well as tumour perfusion and permeability were calculated for a single central Region-of-interest on a single slice or the whole organ or tumour. Statistical analysis was performed with paired t-tests and Pearson's correlation coefficient.

Results: There were no significant differences between single slice / whole kidney (plasma-flow $275.8 \pm 105.6$ vs. $234 \pm 96.7 \mathrm{ml} / 100 \mathrm{ml} / \mathrm{min}$, plasma-volume $28.2 \pm 8.9$ vs. $25.0 \pm 9.0 \mathrm{ml} / 100 \mathrm{ml}$, glomerular-filtration-rate $16.9 \pm 9.2$ vs. $15.9 \pm 8.0 \mathrm{ml} / 100 \mathrm{ml} /$ $\mathrm{min}$ ) as well as between single slice/ whole tumour (plasma-flow $122.5 \pm 85.6 \mathrm{vs}$. $120.5 \pm 95.2 \mathrm{ml} / 100 \mathrm{ml} / \mathrm{min}$, plasma-volume $18.9 \mathrm{vs} .13 .0 \mathrm{ml} / 100 \mathrm{ml}$ and permeabilitysurface-product $5.4 \pm 7.9$ vs. $5.4 \pm 8.3 \mathrm{ml} / 100 \mathrm{ml} / \mathrm{min}$ ). Correlations between single slice and whole organ/tumour was were excellent and significant for all parameters $(r=0.95$ to $0.99, p<0.01)$.

Conclusion: Single slice assessment of kidney function and renal masses with DCE-MRI does not show significant differences to the more time-consuming analysis of the whole organ/tumour. Incorporation of single slice analysis of DCE-MRI data in future studies appears feasible and justifiable.

\section{B-0345 11:15}

In vivo sodium (23Na) imaging of the human kidneys at $7.0 \mathrm{~T}$ : preliminary results

S. Haneder ${ }^{1}$, V. Juras², H.J. Michaely' ${ }^{1}$ S.O. Schönberg ${ }^{1}$, S. Trattnig², Š. Zbýňn'; ${ }^{1}$ Mannheim/DE, ${ }^{2}$ Vienna/AT

Purpose: To evaluate the feasibility of in vivo high-resolution (HR) ${ }^{23} \mathrm{Na}$ imaging of the renal cortico-medullary ${ }^{23} \mathrm{Na}$ gradient and to measure ${ }^{23} \mathrm{Na}-\mathrm{T}^{*}$ relaxation times in human kidneys at an ultra-high field strength of 7.0T.

Methods and Materials: In this prospective, IRB-approved study, 8 healthy volunteers (4 females, 4 males; mean age $29.0 \pm 3.5$ years) were scanned on a 7.0T whole body MR system (Siemens, Erlangen, Germany). For HR ${ }^{23} \mathrm{Na}-\mathrm{MRI}$ a $3 \mathrm{D}$ gradient-echo-sequence with variable echo time scheme (vTE-GRE) (spatial resolution- $4 \times 4 \times 5 \mathrm{~mm}^{3} ; 24$ slices; TE=4.19 ms; mean TR=49 ms; mean averages=34; mean measurement time-41:57 $\mathrm{min}$ ) was used. The $\mathrm{T} 2^{*}$ times were calculated using a multi-echo 3D vTE-GRE sequence (resolution- $4 \times 4 \times 15 \mathrm{~mm}^{3} ; 12$ slices; 10 echoes; 
$\mathrm{TE}=2.64 \mathrm{~ms}$ to $60.42 \mathrm{~ms}$; TR=75 $\mathrm{ms} ; 24$ averages; measurement time=46:50 $\mathrm{min}$ ). All HR images were corrected for inhomogeneous sensitivity of coil. The ${ }^{23} \mathrm{Na}-\mathrm{SNR}$ values are given on a pixel-by-pixel basis for $20 \mathrm{~mm}$ from the renal cortex in the direction of the medullary pyramid. The $\mathrm{T}^{*}$ maps were calculated by fitting the ${ }^{23} \mathrm{Na}$ signal decay mono-exponentially on a pixel-by-pixel basis using least squares fitting routine written in IDL.

Results: The mean cortico-medullary ${ }^{23} \mathrm{Na}-\mathrm{SNR}$ for all 16 healthy kidneys increased from the renal cortex $(34.1 \pm 10.2)$ towards the renal medullary pyramid $(88.8 \pm$ 20.9). The cortico-medullary increase ranged inter-individually from 50.8 to $65.7 \%$. The mean ${ }^{23} \mathrm{Na}-\mathrm{T}^{*}$ of all volunteers was $19.9 \pm 1.8 \mathrm{~ms}$.

Conclusion: The feasibility of ultra-high field, $\mathrm{HR}{ }^{23} \mathrm{Na}-\mathrm{MRI}$ of healthy human kidneys was demonstrated. Moreover, high ${ }^{23} \mathrm{Na}-\mathrm{SNR}$ provided by $7.0 \mathrm{~T}$ allowed the in vivo measurements of ${ }^{23} \mathrm{Na}-\mathrm{T}^{*}$ times in human kidneys for the first time.

\section{B-0346 11:24}

Arterial spin labelling for the prostate: initial experience at 3 T MRI

S. Takahashi ${ }^{1}$, N. Aoyama' ${ }^{1}$, T. Kimura ${ }^{2}$, K. Kitajima ${ }^{1}$, Y. Ueno ${ }^{1}$, S. Sato ${ }^{3}$,

K. Sugimura $1 ;{ }^{1}$ Kobe/JP, ${ }^{2}$ Otawara/JP, ${ }^{3}$ Osaka/JP (staka@med.kobe-u.ac.jp)

Purpose: To assess the value of ASL-perfusion MRI for the prostate using dynamic contrast-enhanced (DCE) study findings as the reference standard.

Methods and Materials: 22 male patients (mean age $69.2 \pm 7.6$ years) with suspected prostate cancer were prospectively included in this study. Single-slice 2D-pulsed ASL images were acquired during our routine prostate screening protocol on a 3 T MRI unit, including the DCE study. First, ROI was drawn for the obturator muscle on a control image of ASL sequence (Scont.ref). Then, ROIs were drawn for the femoral artery, the obturator muscle, the normal $\mathrm{PZ}$ and the regions where DCE showed early enhancement on the tagged ASL image. These values were divided with Scont.ref, yielding an ASL ratio (ASLR). Analysis of the variance was performed to assess differences in ASLR for each tissue or region. A radiologist scored the degree of enhancement on DCE and compare to the contrast ratio of ASLR and the visual assessment of ASL signals.

Results: A mean ASLR of the femoral artery was $15.8 \pm 6.9 \%$, where those of the normal $\mathrm{PZ}$ and the obturator muscle were $0.6 \pm 0.5 \%$, and $0.6 \pm 0.5 \%$, respectively. On the other hand, the enhanced regions on DCE showed a mean ASLR of $0.98 \pm 1.0 \%$. Visual assessment of the ASL image detected 7 out of 12 well-enhanced regions. Contrast ratio of ASLR demonstrated significant positive correlation to the degree of enhancement on DCE (Spearman; $r=0.54, p=.007$ ).

Conclusion: ASL may allow detecting the region of the higher tissue blood flow in the prostate without contrast materials.

Author Disclosures:

T. Kimura: Employee; Toshiba Medical systems. S. Sato: Employee; Toshiba Medical systems.

\section{B-0347 11:33}

A new look at the female pelvis: ultra-high-field (7T) MR imaging

L. Umutlu, O. Kraff, A. Fischer, S. Kinner, S. Maderwald, M. Forsting, M.E. Ladd, T.C. Lauenstein; Essen/DE (Lale.Umutlu@uk-essen.de)

Purpose: Aim of this study was to investigate the feasibility of contrast-enhanced 7 Tesla MRI of the female pelvis.

Methods and Materials: 10 healthy female volunteers were examined on a $7 \mathrm{~T}$ whole body MR system (Magnetom 7T, Siemens) utilising an 8-channel transmit/ receive radiofrequency body coil. The examination protocol included 1) T1w fs $2 \mathrm{D}$ FLASH, 2) dynamic T1w fs 3D FLASH, 3) T2w TSE sequences. For qualitative image analysis of T1w MRI, the delineation of pelvic anatomy, pelvis vasculature, tissue contrast and overall image quality was assessed and for T2w MRI the zonal anatomy of the uterus and the conspicuity of the ovaries were evaluated (fivepoint-scale analysis: $5=$ excellent to $1=$ non-diagnostic). Image impairment due to various artefacts was assessed. Contrast ratios between junctional zone and myometrium were obtained via ROI analysis for T2w MRI.

Results: Of all three sequences, 2D FLASH MRI offered best overall image quality (mean contrast-enhanced 4.75) and highest tissue contrast (mean contrastenhanced 4.92). For the T1w sequences, $2 \mathrm{D}$ FLASH imaging was rated with higher scores for all assessed structures than 3D FLASH MRI. T2w TSE imaging provided a moderate to high conspicuity of the zonal anatomy of the uterus with mean scores ranging from 3.5 for endometrium to 4.67 for myometrium. Overall image impairment was rated strongest for T2w MRI (2.95) and least for 2D FLASH MRI (mean 4.24). Conclusion: Our results indicate the successful transformation of the associated high SNR into high spatiotemporal resolution T1w pelvis MRI at $7 \mathrm{~T}$ and valuable non-contrast diagnostics of pelvis vasculature.
B-0348 11:42

Bridging septa in T2-weighted MRI of healthy volunteers: is there an association with the peri- and pararenal fat thickness?

B. Mensel, R. Bülow, F. Weber, J.-P. Kühn, N. Hosten, K. Hegenscheid; Greifswald/DE (birger.mensel@uni-greifswald.de)

Purpose: To analyse the distribution of perirenal bridging septa (BS) and their correlation with para- and perirenal fat thickness (PPFT).

Methods and Materials: 200 healthy volunteers (76 men and 124 women) with a mean age of $50.7 \pm 13$.2 years underwent T2-weighted imaging of the abdomen. Two observers rated the BS according to a 4-point scale $(0=$ no BS visible to $3=$ maximum extent of BS). In addition, the PPFT was measured on T1-weighted images. For both variables a possible correlation was evaluated and analysis of the intra- and interobserver variability was performed.

Results: In 94 subjects (47.0\%) BS were visible. Male subjects showed BS statistical significantly more often compared to female subjects $(P<0.001)$. The distribution according to the stage of BS was: stage 1: $40(20.0 \%)$, stage 2: 23 (11.5\%) and stage 3: $31(15.5 \%)$ subjects. The PPFT was greater in men $(14.19$ $\mathrm{mm}$, IQR: 9.39-20.61 mm) compared to women (7.32 mm, IQR: 6.04-11.30 mm, P $<0.001)$. Corresponding correlation coefficients between the stage of BS and PPFT were $r=0.780(P<0.001)$ and $r=0.431(P<0.001)$. Greater PPFT was related to a higher stage of $B S$ in both sexes $(P<0.001)$ with significant differences between all adjacent stages for male subjects $(P<0.05)$. Female subjects showed significant differences only between stage 0 and $1(P<0.05)$. Intra- and interobserver variability for the stage of $B S$ and PPFT was very good.

Conclusion: A higher PPFT is associated with higher BS stage. MRI allows a reliable classification of PH and PPFT measurement.

\section{B-0349 11:51}

Feasibility of 3 T MR diffusion tensor imaging (DTI) for reconstructing anatomic connectivity of sacral plexus

V. lotti ${ }^{1}$, A. Pecchi' ${ }^{1}$ L. Nocetti ${ }^{1}$, F. Fiocchi' ${ }^{1}$, C. Alboni ${ }^{2}$, P. Torricelli' ${ }^{1}{ }^{1}$ Modena/IT, 2Sassuolo/IT (valeiotti@yahoo.it)

Purpose: To investigate the feasibility of reconstructing anatomic connectivity of sacral plexus using 3 T-MR diffusion tensor imaging (MR-DTI) with three-dimensional (3D) tractography approach.

Methods and Materials: 3 T-MR-DTI was performed on 10 women volunteers. The sequence was acquired on oblique axial plane parallel to the sacrum, from the level of the $L 4$ nerve to the coccyges. Main diffusion directions reflecting the fibre orientation were determined using a Sense-SSEPI with diffusion-sensitised gradients (b600 mm2s-1) along 16 directions. A deterministic bre-tracking algorithm was used to show fibre architecture, compared with anatomic atlas. Three ROIs were placed at different levels of the spinal cord nerves: one in the middle of each tract-of-interest, accompanied by "AND" selection ROIs at both ends of the nerve tract ROI. Diffusion-weighted Ssh-EPI images are processed on a dedicated workstation for data pre-processing with MedINRIA deterministic software.

Results: Tractography of the sacral plexus was feasible for all 10 volunteers, giving 3D insight into the general anatomy and organisation of the nerves $L 4$ to S4. Main artefacts in fibre reconstruction, caused by bladder over-distension, by presence of air in rectum and by constant small movements due to breathing and/or spontaneous contractions of pelvic organs, never invalidate the feasibility of tractography. Fibres were reconstructed from medulla to the pelvic course of main branches of sacral-plexus nerves. Emerging branches of pudendal nerve were found in 6 volunteers.

Conclusion: 3 T-MR-DTI allows a precise anatomic reconstruction of lumbar-sacral plexus. These findings are encouraging for using DTI as a mean to investigate sacral-plexus, especially in female pelvis. 
Neuro

\section{SS 511}

\section{Stroke-related arterial disease}

Moderators:

E. Avdagic; Sarajevo/BA

P. Barsi; Budapest/HU

\section{B-0350 10:30}

Atherosclerotic calcification is related to cognitive decline: the Rotterdam study

D. Bos, M.W. Vernooij, A. Hofman, G.P. Krestin, J.C.M. Witteman,

A. van der Lugt, M.A. Ikram; Rotterdam/NL (d.bos@erasmusmc.nl)

Purpose: Increasing evidence suggests that vascular disease, and as such atherosclerosis, may play an important role in the pathogenesis of cognitive impairment and dementia. In this study, we investigate the relationship between arterial calcification, as marker of atherosclerosis, in four vessel beds and cognitive decline over a 6.4-year interval.

Methods and Materials: From the population-based Rotterdam study, 2,413 participants (mean age 69.5 years) underwent computed tomography of the coronaries, aortic arch, extracranial and intracranial carotid arteries to quantify atherosclerotic calcification. Cognitive function was assessed at baseline and at follow-up (mean follow-up time: 6.4 years) using MMSE and a neuropsychological test battery that assessed the following domains: global cognition, memory, executive function, information processing speed and motor speed. Per participant, we calculated the difference between both time points in standardised scores for each cognitive domain. Relationships between atherosclerotic calcification and cognition were assessed using linear regression models and adjusted for relevant confounders (age, sex, education, APOE-e4-status and cardiovascular risk factors).

Results: Calcification in all vessel beds, except in the extracranial carotid arteries, was associated with a decreased MMSE score. Larger calcification volumes were also associated with a decrease in global cognitive function and executive function. Most prominent were the associations of intracranial carotid calcification with global cognition and executive function [multivariable-adjusted cognitive decline per SD increase in calcification volume $-0.03(95 \% \mathrm{Cl}:-0.06 ;-0.01)$ and $-0.03(95 \% \mathrm{Cl}$ : $-0.06 ;-0.01)$, respectively].

Conclusion: Atherosclerotic calcification, in particular intracranial carotid artery calcification, is an important risk factor for cognitive decline and thus potentially also for dementia.

\section{B-0351 10:39}

Does clinical severity in acute stroke reliably predict large vessel occlusion? Results from a prospective cohort study of CT angiography (CTA) in hyper-acute stroke

C. Hansen, C. Ovesen, H. Christensen, I. Havsteen, J. Nielsen, A. Christensen; Copenhagen/DK (hchr0039@gmail.com)

Purpose: The availability of endovascular services is increasing, but still not available in the majority of TPA centres. The aim of this study was to describe the relations between NIHSS and large vessel occlusions in patients with hyper-acute ischaemic stroke.

Methods and Materials: A prospective single hospital registry based on consecutive patients admitted for TPA work up with routine CTA was started on July 1, 2009; this analysis is based on patients admitted before December 2011. Bispebjerg University Hospital has a TPA-service with a catchment area of app. 1.7 mio. inhabitants on even dates. CT scans are performed using 64-section MDCT (Brilliance-64, Philips Healthcare) with CTA from the aortic arch to the vertex. All images were systematically reviewed by a blinded neuroradiologist; sensitivity, specificity, positive predictive value and negative predictive value were calculated at all levels of NIHSS.

Results: Five-hundred and seventy-three patients with acute ischaemic stroke were entered into the registry. At a NIHSS score equal to or larger than 10 , the sensitivity was equal to $43.8 \%$, the specificity was $89.6 \%$ the positive predictive value was $63.2 \%$ and the negative predictive value was $89.6 \%$ in predicting an acute occlusion. Conclusion: If the cut off is set at NIHSS 10, there is a $10 \%$ risk of missing an occlusion and a $37 \%$ risk of transporting a patient with a TPA drip for no reason. The relation is between occurrence of large vessel occlusions and NIHSS is a continuum.

\section{B-0352 10:48}

Value of monoenergetic low KV dual-energy CT datasets for improved image quality of cervical and cerebral CT-angiography

D. Schneider, P. Apfaltrer, H. Haubenreisser, M. Meyer,

S. Sudarski, C. Fink, S.O. Schönberg, T. Henzler;

Mannheim/DE (david.schneider@stud.uni-heidelberg.de)

Purpose: High vessel attenuation as well as a high contrast-to-noise (CNR) ratio is a prerequisite for highly diagnostic cervical and cerebral CT-angiographic examinations. The purpose of this study was to evaluate the value of calculated low-kV-monoenergetic dual-energy CT (DECT) angiographic datasets.

Methods and Materials: 41 patients $(21$ men, mean age $58 \pm 14)$ that underwent DECT-angiography of the cervical $(n=7)$ or cerebral vessels $(n=34)$ were retrospectively included in this study. 80 and 140-kV DECT data were used to calculate low-kV monoenergetic image datasets from 120 to $40 \mathrm{keV}$ (10-keV intervals). Vessel and soft tissue attenuation and image noise were measured in various regions of interest and the CNR was subsequently calculated. Differences in image attenuation and CNR were compared between the different monoenergetic datasets. Vessel attenuation and CNR of the best monoenergetic datasets were then compared to conventional $120-\mathrm{kV}$ polyenergetic datasets.

Results: In comparison to conventional polyenergetic 120-kV datasets, above all, $60-\mathrm{keV}$ monoenergetic cervical datasets yielded to a significant increase in vessel attenuation and CNR $(+40 \%,+18 \%$; all $p<0.01)$. For cerebral vessel assessment, in particular $70-\mathrm{keV}$ monoenergetic datasets improved vessel attenuation and CNR $(+3 \%,+10 \%$; all $p<0.01)$ when compared to conventional $120-k V$ datasets. Conclusion: Calculated $60-\mathrm{keV}$ monoenergetic image data significantly improves vessel attenuation as well as the CNR of cervical DECT-angiographic studies, whereas $70-\mathrm{kV}$ monoenergetic datasets are favourable to improve vessel attenuation and the CNR of cerebral DECT-angiographic studies. Thus, lowering the amount of iodinated contrast-material might become feasible using monoenergetic-low-kV images, which is particularly important for combined perfusion and angiography protocols.

\section{B-0353 10:57}

Black blood MRI in patients with and without suspected primary angiitis of the central nervous system

T. Saam, T. Pfefferkorn, M. Habs, C. Opherk, A. Straube,

M. Dichgans, M.F. Reiser, K. Nikolaou, J. Linn;

Munich/DE (Tobias.Saam@med.uni-muenchen.de)

Purpose: Single case reports suggest that black blood MRI (T1-weighted fat and blood suppressed sequences with and without contrast injection; BB-MRI) may visualise intracranial vessel wall contrast enhancement in primary angiitis of the central nervous system (PACNS). In this single-centre observational pilot study we prospectively investigated the value of BB-MRI in the diagnosis of large artery PACNS.

Methods and Materials: In this prospective, mono-centric study 12 patients with suspected large artery PACNS received a standardised diagnostic programme including BB-MRI. 10 patients without any evidence of PACNS and without intracranial stenosis served as control group. Vessel wall contrast enhancement was graded (grade 0-2) by two experienced readers blinded to clinical data and correlated to the final diagnosis.

Results: Four of 12 included patients received a final diagnosis of PACNS. All of them showed moderate (grade 1) to strong (grade 2) vessel wall contrast enhancement at the sites of stenosis. A moderate (grade 1) vessel wall CE grade was also observed in 6 of the remaining 8 patients in whom alternative diagnoses were made: arteriosclerotic disease $(n=4)$, intracranial dissection $(n=1)$, and Moyamoya disease $(n=1)$. None of the patients in the control group had vessel wall contrast enhancement.

Conclusion: Our pilot study demonstrates that vessel wall contrast enhancement is a frequent finding in PACNS and its mimics, but it is absent in patients without evidence of intracranial vascular disease. Larger trials will be necessary to evaluate the utility of BB-MRI in the diagnostic workup of PACNS.

\section{B-0354 11:06}

$3 \mathrm{~T}$ MRI reveals extra- and intracranial involvement in giant cell arteritis (GCA)

S. Siemonsen, C. Brekenfeld, T. Klink, J. Fiehler, T. Bley;

Hamburg/DE (s.siemonsen@uke.uni-hamburg.de)

Purpose: Vessel wall enhancement (VWE) of the superficial extracranial arteries can be frequently detected in GCA by high-resolution MRI. The frequency and amount of intracranial, intradural involvement is still unclear. We hypothesized 
that with an optimised MR-protocol, mural inflammatory changes of superficial extracranial and intradural arteries can be detected in patients with GCA.

Methods and Materials: 10 patients with suspected GCA underwent 3.0 Tesla MRI, including fat saturated (fs) T1w scans pre- and post-contrast, optimised for assessment of intradural VWE, and high-resolution fsT1w scans to evaluate superficial extracranial vessels. Temporal artery biopsies were available in 4 cases. VWE of intradural and extracranial vessels was evaluated by two observers

Results: In 5 patients, GCA was diagnosed clinically, in 4 biopsy proven. A clear VWE of superficial extracranial vessels was detected in 8 cases. Clear VWE of the intradural internal carotid artery (ICA) was detected in 6 cases, 5 with corresponding vessel wall thickening. Slight enhancement of the right medial cerebral artery was seen in 1 case. In one patient with GCA, no VWE of intradural or extracranial vessels was detected. One patient who did not receive a temporal artery biopsy presented with VWE of intradural and extracranial arteries, but the final clinical diagnosis was connective tissue disease.

Conclusion: VWE of extracranial vessels is a frequent finding in GCA. Additionally, VWE of intradural arteries, mainly the ICA, can be found. Therefore, high-resolution MRI seems to be a feasible technique to visualise vasculitic involvement of the extra- and intracranial arteries in GCA.

\section{B-0355 11:15}

BOLD fMRI of cerebrovascular reserve in patients with severe stenosis of the middle cerebral artery: preliminary results

A. Krainik, A. Attye, M. Villien, K. Boubagra, K. Garambois, J. Bouvier,

J. Warnking, F. Tahon, O. Detante; Grenoble/FR (akrainik@chu-grenoble.fr)

Purpose: To measure cerebrovascular reserve using $\mathrm{fMRI}$ (CVR fMRI) in patients with severe stenosis of the middle cerebral artery (MCA) to identify those at risk of haemodynamical stroke.

Methods and Materials: Among 16 patients with severe intracranial arterial stenosis, 7 patients ( 4 females; $61.9 \pm 18.5$ years) had a stenosis of the right $(n=4)$ or left $(n=3)$ MCA only. CVR fMRI was performed using BOLD contrast with a block-design hypercapnic challenge (CO2 7\%) at $3 \mathrm{~T}$. Averaged end-tidal CO2 pressure (EtCO2) was used as regressor for statistical analyses. We measured \%BOLD/mm $\mathrm{mg}$ EtCO2 on segmented grey matter of MCA territories. We calculated a laterality index with LIMCA $=($ Left_CVR-Right_CVR)/(Left_CVR+Right_CVR). LI were compared to those obtained in 100 volunteers that provided a $99 \%$ confidence interval for I LIMCA $<0.08$. Basal perfusion was measured using dynamic susceptibility contrast. Results: Basal perfusion measurements were symmetrical in all patients. No adverse reaction to hypercapnia was detected. CVR values ranged from 0.13 to 0.37 with $\mathrm{m} \pm \mathrm{sd}=0.25 \pm 0.07$. Among these 7 patients, three patients had a normal ILIMCAI $<0.08$ ranging from 0.02 to $0.05(0.04 \pm 0.01)$, and four patients had an abnormal ILIMCAI> 0.08 ranging from 0.13 to $0.17(0.16 \pm 0.02)$. All abnormal LI values identified the MCA territory ipsilateral to the stenosis.

Conclusion: These preliminary results show that among patients with severe stenosis of MCA, several patients have abnormal ILIMCAI in the territory ipsilateral to the stenotic artery, suggesting significant CVR impairment that might be at risk of haemodynamic stroke. CVR fMRI should be considered to better define treatment strategy, including intravascular stenting.

\section{Author Disclosures:}

J. Bouvier: Research/Grant Support; Philips.

\section{B-0356 11:24}

Intracranial artery calcification on CT predicts poor outcome after stroke: results from a prospective cohort study

C. Ovesen, H. Christensen, A.-M. Abild, C. Hansen, I. Havsteen, J. Nielsen,

A. Christensen; Copenhagen/DK (anderschristensenemail@hotmail.com)

Purpose: Intracranial atherosclerosis has been reported a significant risk factor of recurrent stroke. We investigated if intracranial calcifications (ICC) predicted recurrent vascular events and death in long-term follow-up.

Methods and Materials: Cerebral CT (CT-C) and CT-angiography (CTA) from consecutive patients admitted to a single centre with acute ischaemic stroke within 4.5 hours in the period from April 2009 to December 2011 with follow-up until August 2012 were included in the analysis. ICC was recorded using a four-point grading scale of calcifications in the intracranial segment of the internal carotid artery. Recurrence was defined as stroke, transitory ischaemic attack (TIA), or myocardial infarction (MI) documented by a discharge card; poor outcome was recurrence or death.

Results: First degree calcifications was found in 426/661 patients; 2 . degree in $123 / 661 ; 67$ had 3 . degree, and 45 had 4 . degree. Mean follow-up was 701 days (range 238-1192 days), 13 patients were lost to follow-up, all visitors to Denmark. Fifty-three patients died and 42 suffered recurrent ischaemic stroke, of whom 18 intracerebral haematoma, $8 \mathrm{TCl}$ and $12 \mathrm{MI}$. Kaplan-Meier curves for recurrent stoke and death, recurrent stroke alone and death alone stratified for degree of calcification showed a significant difference between the 4 groups with increasing number of events with increasing degree of calcification. In univariate analysis, the risk of the combined endpoint of death or recurrence was increased in patients with any degree of calcifications, $\mathrm{HR} 1.76(95 \% \mathrm{Cl}: 1.15-2.86, \mathrm{p}=0.006)$.

Conclusion: ICC predicts death and vascular recurrences after ischaemic stroke.

\section{B-0357 11:33}

Intracranial artery stenosis on CT angiography (CTA) predicts poor outcome after stroke: results from a prospective cohort study

C. Ovesen, H. Christensen, A.-M. Abild, C. Hansen, I. Havsteen, J. Nielsen, A. Christensen; Copenhagen/DK (hchr0039@gmail.com)

Purpose: Intracranial stenosis has been reported a risk factor of recurrent stroke. We investigated if intracranial stenosis predicted vascular events (stroke, myocardial infarction, TIA) and death in a Danish population of patients with ischaemic stroke. Methods and Materials: Cerebral CT (CT-C) and CT-angiography (CTA) (from consecutive patients admitted with acute ischaemic stroke to our institution in the period from April 2009 to December 2011 and followed up till August 2012) were included into the analysis. Intracranial stenosis was graded after standardised WASID criteria. Recurrence (stroke, myocardial infarction, TIA) was identified based on discharge cards, and recurrence or death constituted a poor outcome. Results: A total of 661 patients were included into the analysis, of whom 86 had a total of 91 stenoses: in 51 patients: $30-50 \%$ stenosis, in $24: 50-70 \%$, and in 16 : > $70 \%$. Mean follow-up was 701 days (range 238-1192 days), 13 patients were lost to follow-up, all visitors to Denmark. Fifty-three patients died within the follow-up period; 42 suffered recurrent ischaemic stroke, 18 intracerebral haematoma, $8 \mathrm{TCl}$ and $12 \mathrm{MI}$. Kaplan-Meier curves for time to recurrence or death stratified for degree of stenosis showed significant difference between groups (log rank test, $\mathrm{P}=0.009$ ). In univariate analysis, any degree of stenosis increased the risk of poor outcome hazard ratio $(\mathrm{HR}) 1.8(95 \% \mathrm{Cl}: 1.15-2.81, \mathrm{P}=0.01)$. Patients with $>70 \%$ stenosis showed the highest risk of poor outcome, HR 3.3 (95\% Cl: 1.45-7.7, p=0.004). Conclusion: Intracranial stenosis increases the risk of poor outcome. Stenosis > $50 \%$ was observed in $6 \%$ of patients.

\section{B-0358 11:42}

Contribution of the temporal maximum intensity projection (tMIP) datasets for measurement of the middle cerebral artery (MCA) occlusion and predictive value for results of systemic thrombolytic therapy

J. Baxa, R. Tupý, V. Rohan, B. Kreuzberg, J. Ferda; Pilsen/CZ (baxaj@fnplzen.cz)

Purpose: The goal of retrospective study was to compare possibilities to measure the extent of MCA occlusion (thrombus length) in patients with acute stroke and predictive value for recanalization and clinical outcome after systemic therapy.

Methods and Materials: We analysed 63 patients with MCA occlusions (25 males, average age 72.3 years, range $23-91$ years) detected with multimodal CT examination (non-contrast $\mathrm{CT}$, perfusion CT and conventional CTA). Conventional CTA (slice $0.6 \mathrm{~mm}$ ) and temporal maximum intensity projection (tMIP) datasets (slice $1.5 \mathrm{~mm}$ ) reconstructed over the full time span from volume CT perfusion were used for thrombus length measurement. Results of used techniques were compared and correlated with results of systemic thrombolytic therapy ( 24 hours recanalization and 3-month clinical outcome).

Results: There was no statistically significant difference in measurement results using conventional CTA and tMIP, but in 23 subjects (36\%) measurement of thrombus was not possible using conventional CTA due to unclear peripheral border of thrombus. We proved statistically significant correlation of whole extent of thrombus and recanalization success $(p=0.0241)$. The involvement of $M 2$ section was main negative factor for recanalization $(p=0.0068)$. The strong significant correlation of thrombus length and 3-month clinical outcome was proved $(p=0.0079)$ with cut-off value of $11 \mathrm{~mm}$.

Conclusion: Our results confirm the benefits of detailed evaluation of cerebral arteries using tMIP datasets, which enable to measure thrombus length in all subjects in comparison to conventional CTA. The thrombus length was established as negative factor for recanalization and clinical outcome after systemic thrombolytic therapy. 


\section{B-0359 11:51}

MRI findings in small vessel disease and their relationship to cognitive impairment

P. Benjamin, A.J. Lawrence, B. Patel, A.W. Chung, T.R. Barrick, A.D. Mackinnnon, R. Morris, H.S. Markus; London/UK (philipbenjamin@doctors.net.uk)

Purpose: Cerebral small vessel disease (SVD) is a common cause of cognitive impairment. White matter hyperintensities (WMH) and lacunar infarcts are the commonest magnetic resonance imaging (MRI) parameters observed in clinical practice. The relationship between these findings on MRI and cognitive impairment remains unclear. The aim of our study was to determine the relationship between lacune count, total lacune volume and location of lacunes with cognition.

Methods and Materials: Patients with lacunar stroke and WMHs were recruited to the prospective SCANS study. Baseline data $(n=121)$ was used for this analysis. Multimodal MRI and neuropsychological testing were performed. Lacunes were identified and counted by a neuroradiologist on a T1 image, then mapped using an automated region growing technique. Lacunar volume was calculated from the resulting masks, and lacunar location automatically determined by projection onto standard atlases. T2 WMH volume and brain volume were also measured. Results: In multiple regression controlling for WMH volume and brain volume, both the lacune number and the total volume were related to a significant decrease in executive function (number: Beta $=-0.21, p<0.006$; volume: Beta $=-0.16, p<0.04$ ) and processing speed (number: Beta $=-0.26, p<0.001$; volume: Beta $=-0.24, p<$ $0.004)$. Lacunes in the thalamus were associated with impaired processing speed performance $(p<0.002)$.

Conclusion: One can infer a number of things relating to cognitive impairment when looking at an MRI scan of a patient with cerebral small vessel disease. 1) The number of lacunes are a better predictor of cognitive status than diffuse WMH and 2) thalamic lacunes, in particular, are associated with impaired processing speed.

\section{$10: 30-12: 00$}

Room N/O

\section{Vascular}

\section{SS 515}

\section{Novel tools for blood flow evaluation}

Moderators:

J. Barkhausen; Lübeck/DE

F. Garaci; Rome/IT

\section{B-0361 10:30}

Assessment of blood flow haemodynamics of liver cirrhosis patients after treatment with a TIPS stent-graft using a flowsensitive 4D MRI imaging at 3 Tesla

Z. Stankovic ${ }^{1}$, M.F. Russe ${ }^{2}$, B. Jung ${ }^{2}$, W. Euringer ${ }^{2}$, Z. Csatari²,

L. Stehlin ${ }^{2}$, M. Langer ${ }^{2}$, M. Markl1; ${ }^{1}$ Chicago, IL/US,

${ }^{2}$ Freiburg/DE (zoran.stankovic@uniklinik-freiburg.de)

Purpose: To perform qualitative and quantitative analysis of altered haemodynamics of liver cirrhosis patients after TIPS-stent-graft implantation and volunteers using time-resolved flow-sensitive 4D MRI at $3 \mathrm{~T}$.

Methods and Materials: Our study group consisted of 5 liver cirrhosis patients including an examination before and after stent-graft implantation as well as 10 volunteers. 3D liver flow characteristics in the arteries and portal vein were evaluated using flow-sensitive $4 \mathrm{D}$ MRI at $3 \mathrm{~T}$ MRI with a spatial resolution of $1.6 \times 2.1 \times 2.4$ $\mathrm{mm}^{3}$ and temporal resolution of $62.4 \mathrm{~ms}$. Hepatic flow visualisation was performed using 3D streamlines and time-resolved particle traces. Quantitative evaluation included retrospective extraction of regional maximum and mean velocities, flow volume, vessel area as well as pulsatility and resistance indices.

Results: 3D streamlines and particle traces visualisation in the arterial and portal venous system could successfully be performed for almost all patients and volunteers. Quantitative results reveal maximum and mean velocities tend to be lower in MRI compared to Doppler Ultrasound, area showed significant lower values for US $(p<0.01)$. Comparing the changes after TIPS-stent-graft implantation the data results demonstrated an increase of velocities and flow volume in the venous system as well as hepatic artery.

Conclusion: Flow-sensitive 4D MRI enabled a qualitative and quantitative evaluation of arterial and portal venous haemodynamics of liver cirrhosis patients before and after TIPS-stent-graft implantation including a volunteer group. Flow-sensitive 4D MRI may be a standardised method with complete volumetric and functional coverage of the blood flow haemodynamics providing additional information in patients after TIPS-stent-graft implantation.
B-0362 10:39

Evaluation of atherosclerosis and endothelial functions in nonalcoholic hepatosteatosis patients: comparison with ultrasound and biochemical markers

M.G. Yilmabasar, A. Hayirlioglu, U. Ozdamarlar, I. Kuru; Istanbul/TR (yilmabasar@gmail.com)

Purpose: In this study, we aimed to evaluate the endothelial functions in patients with nonalcoholic fatty liver disease (NAFLD).

Methods and Materials: In this observational case-control study, a total of $51 \mathrm{pa}-$ tients with NAFLD and age and sex-matched 21 healthy controls were enrolled. In both patients and controls, levels of asymmetric dimethylarginine (ADMA), systemic endothelial function (brachial artery flow-mediated dilation) (FMD) and carotid artery intima-media thickness (C-IMT, mm) were measured. Endothelial functions of the brachial and carotid arteries were evaluated by vascular ultrasound.

Results: Carotid intima media thickness was significantly higher in patients with NAFLD than controls $(0.67 \pm 0.09$ versus $0.52 \pm 0.11 \mathrm{~mm}, \mathrm{P}<0.001)$. The average C-IMT measurements were found in groups of control, simple steatosis and NAFLD with (borderline and definitive) NASH as $0.52 \pm 0.11,0.63 \pm 0.07$ and $0.68 \pm 0.1 \mathrm{~mm}$, respectively. The differences between groups were significant $(P$ $<0.001)$. Measurement of brachial artery FMD was significantly lower in patients with NAFLD compared to controls $(7.3 \pm 4.8$ versus $16.5 \pm 7.1 \%, P<0.001)$. FMD measurements in groups of control, the simple steatosis and NAFLD with NASH as $16.5 \pm 7.1,9.64 \pm 6.63$ and $7.03 \pm 4.57 \%$, respectively, and the differences were statistically significant $(P<0.001)$. The increase in $C-I M T$ and decrease in FMD were independent of metabolic syndrome and it was also more evident in patients with simple steatosis and NASH compared to controls.

Conclusion: Our data suggested that NAFLD is associated with endothelial dysfunction and increased early atherosclerosis compared to healthy subjects.

\section{B-0363 10:48}

CT angiography in renal donors using automated $k V p$ selection in combination with sinogram-affirmed iterative reconstruction: evaluation of radiation dose and image quality M.P.F. Botelho, F.D. Gonzalez-Guindalini, P. Entezari, H. Chalian, V. Yaghmai; Chicago, IL/US (v-yaghmai@northwestern.edu)

Purpose: This study aimed to assess image quality and radiation dose of CT angiography (CTA) in kidney donors obtained with automated kVp selection and sinogram-affirmed iterative reconstruction (SAFIRE), using scans with fixed $k V p$ and filtered back projection reconstruction algorithm as the comparison.

Methods and Materials: Mean attenuation, contrast-to-noise ratio (CNR) and signal-to-noise ratio (SNR) at abdominal aorta, right renal artery (RRA) and left renal artery (LRA) were measured in 24 kidney donors who had undergone CTA with tube current modulation, $120 \mathrm{kVp}$, filtered back projection reconstruction algorithm (Group A) and were compared with 24 BMI-matched kidney donors who had undergone CTA with automated kVp selection and SAFIRE (Group B). Mean CTDIvol was calculated for the unenhanced and arterial phases. Student's t-test was used for analysis.

Results: $B M I$ was comparable between the groups $(P=0.38)$. Lower $k V p$ was automatically selected in $19 / 24$ of Group B patients $(79.1 \%)$. Mean attenuation level was significantly different between Groups A and B in abdominal aorta $(300+41.49$ vs $389.22+93.89, P=0.0004)$, RRA (292.56 \pm 52.15 vs $361.61 \pm 90.1, P=0.0043)$ and LRA (292.28 \pm 49.45 vs $359.73 \pm 78.29, P=0.0017)$. CNR and SNR at RRA and LRA were significantly higher in Group B compared with Group A ( $P=0.0043$ and $P=0.0061$, respectively). Mean CTDIvol was $15.08 \pm 3.6 \mathrm{mGy}$ and $10.27 \pm 3.7 \mathrm{mGy}$ for Groups A and B, respectively ( $P=0.0005)$.

Conclusion: Both SNR and CNR were improved by automated $k V p$ selection with SAFIRE in kidney donors undergoing abdominal CTA while receiving significantly lower radiation dose.

Author Disclosures:

M.P.F. Botelho: Research/Grant Support; Educational Grant Support by Siemens Healthcare. F.D. Gonzalez-Guindalini: Research/Grant Support; Educational Grant Support by Siemens Healthcare. H. Chalian: Research/Grant Support; Educational Grant Support by Siemens Healthcare. 


\section{B-0364 10:57}

Comparison of high pitch (3) and standard pitch (0.6) CT angiography using 128-slice dual-source $\mathrm{CT}$ in patients with peripheral arterial disease

K. Choo, J. Park, J. Kim, J. Roh; Busan/KR (speedsmile@hanmail.net)

Purpose: We investigated the effect of high-pitch (3) CT angiography with good image quality on qualitative and quantitative parameters and reduced the radiation dose in patients with peripheral arterial disease.

Methods and Materials: Eighty patients were divided into two groups; the protocol was high pitch in 40 patients group and standard pitch in the other 40 patients group. For quantitative analysis we calculated the mean arterial attenuation, the mean difference between maximum and minimum attenuation values, contrast-tonoise ratio (CNR) and figure of merit (FOM). In addition, visual scores were used for qualitative evaluation.

Results: There were no significant intergroup differences in mean arterial attenuation (high pitch vs. standard pitch: $428.6 \pm 85.6$ vs. $434.3 \pm 86.9 \mathrm{HU}$ ) and in the mean difference between maximum and minimum attenuation (high pitch vs. standard pitch: $240.1 \pm 90.0$ vs. $220.1 \pm 98.4 \mathrm{HU}$ ). Both the mean CNR and the mean FOM were not significantly different (high pitch vs. standard pitch: $27.0 \pm 10.8$ vs. 28.0 $\pm 8.6,2.2 \pm 1.96$ vs. $1.5 \pm 0.8$, respectively), and there was no significant intergroup difference in visual scores. The mean dose-length product was significantly lower at high pitch than at standard pitch $(408.4 \pm 43.4$ vs. $619.8 \pm 188.4 .7 \mathrm{mGy} \cdot \mathrm{cm})$. Conclusion: The high-pitch protocol CT angiography with 128-slice dual-source CT allows for reduction of the radiation dose by approximately $34 \%$ versus standard pitch protocol CT angiography with 128-slice dual-source CT without deterioration of vascular enhancement and image quality.

\section{B-0365 11:06}

Differential diagnosis of cervical artery dissection and intra-arterial thrombosis using MRI and MRA

M. Dreval, M. Krotenkova, L. Kalashnikova, L. Dobryinina, R. Konovalov; Moscow/RU

Purpose: This study aimed at exploring MRI and 3D-TOF MRA in differential diagnosis of intra-arterial thrombosis and cervical artery dissection (internal carotid artery and vertebral artery) in different periods of disease using the contrast index. Methods and Materials: A total of 40 patients with extracranial artery dissection manifested by ischaemic stroke or isolated headache $(20$ men, 20 woman, mean age $37.3 \pm 6.1$ years) and 8 patients ( 5 men, 3 woman, mean age $52.4 \pm 8.8$ ) with ischaemic stroke due to intra-arterial thrombosis were studied. Scanning protocol included 3D-TOF MRA sequence for extracranial internal and vertebral arteries and T1 fat suppression ( $f / s$ ) sequence for neck in axial plane. MRT/MRA were carried in dynamics and obtained data were analysed in 6 time intervals: 1-3 days, 4-7 days, 8-14 days, 15-21 days, 22-60 days and 61-90 days. Intramural haematoma (IMH), intra artery thrombus (IAT) and $\mathrm{m}$. sternocleidomastoid signal intensity was measured and contrast index calculated.

Results: After statistical analysis of the data with the use of non-parametric statistical methods (Mann-Whitney $U$ test) it was revealed that value of the contrast index of IMH was higher than that of IAT within 2-5 time interval (4 to 60 days) ( $p$ $<0.05)$. The contrast index did not differ significantly within first 3 days and during 3 months. The subjective visual analysis showed the same results.

Conclusion: MRI sequences as T1 f/s and 3D-TOF MRA could be useful for differential diagnosis of IMH and IAT within the interval of 4 days to 2 months of the disease.

\section{B-0366 11:15}

Impact of organ-specific dose reduction on image quality of head and neck CT-angiography

R.S. Lanzman, L. Schimmöller, P. Heusch, S. Dietrich,

F. Miese, J. Aissa, T.A. Heusner, G. Antoch, P. Kröpil;

Düsseldorf/DE (Patric.Kroepil@med.uni-duesseldorf.de)

Purpose: Organ-specific dose reduction (OSDR) algorithms can reduce radiation on radiosensitive organs up to $59 \%$. This study evaluates the influence of a new OSDR algorithm on image quality (IQ) of head and neck CT-angiography (CTA) in clinical routine.

Methods and Materials: 62 consecutive patients $(68.1 \pm 13.2$ years) were randomised into 2 groups and imaged on a 128-row CT-scanner. Group A $(n=31)$ underwent conventional CTA and group $B(n=31)$ CTA with a novel OSDR algorithm (XCare ${ }^{\mathrm{TM}}$, Siemens), which lowers the radiation exposure of radiosensitive organs (e.g. eye lens, thyroid gland) by reducing the tube current for anterior projections. Subjective and objective IQ was statistically compared. Subjective IQ was rated on a 5-point-scale. Signal-to-noise ratio (SNR) and contrast-to-noise ratio (CNR) was calculated with ROI measurements.

Results: SNR of the common carotid artery and middle cerebral artery was $53.6 \pm 22.7$ and $43.3 \pm 15.3$ (group A) versus $54.1 \pm 20.5$ and $46.2 \pm 14.6$ (group B). CNR was $40.0 \pm 19.3$ and $29.7 \pm 12.0$ (group A) compared to $40.7 \pm 16.8$ and $32.9 \pm 10.9$ (group B). Subjective IQ was excellent in both groups (mean score $4.4 \pm 0.7$ versus $4.4 \pm 0.6$ ). Differences between the two groups were not significant.

Conclusion: The OSDR algorithm XCare ${ }^{\mathrm{TM}}$ does not compromise IQ of head and neck CTA. Its application can be recommended for CTA in clinical routine to protect the thyroid gland and ocular lenses from unnecessary high radiation.

\section{B-0367 11:24}

Precision of pulmonary vein diameter measurements assessed by CE-MRA and steady-state-free precession imaging

F. Henes, P. Bannas, M. Regier, J. Buhk, K. Müllerleile, G. Adam, M. Groth; Hamburg/DE (f.henes@uke.uni-hamburg.de)

Purpose: To evaluate inter- and intraobserver reliability of pulmonary vein (PV) diameter measurements by contrast-enhanced magnetic resonance angiography (CE-MRA) and ECG-gated 2D unenhanced steady-state-free precession sequences (SSFP).

Methods and Materials: CE-MRA and SSFP of $60 \mathrm{PV}$ in 17 patients were evaluated. PV diameters were measured in transverse and coronal orientation at predefined levels by two independent readers. Intraclass correlation coefficient (ICC) and Bland-Altman analysis (BA) were used to assess inter- and intraobserver reliability and variances. F-test was performed for comparison of intra- and interobserver variances. Pearson's correlation coefficient and BA were used to compare CEMRA and SSFP. A t-test was used to determine possible significant differences between the measurements.

Results: There was moderate correlation $(R=0.68$, transverse; $R=0.66$, coronal) without significant difference in diameter measurements between CE-MRA and SSFP ( $p=0.15$, transverse; $p=0.25$, coronal). Intraobserver limits of agreement (LAG) ranged between $\pm 0.50 \mathrm{~cm}$ (transverse) and $\pm 0.86 \mathrm{~cm}$ (coronal) for CE-MRA versus $\pm 0.40 \mathrm{~cm}$ (transverse) and $\pm 0.67 \mathrm{~cm}$ (coronal) for SSFP. Interobserver agreement showed LAG ranging between $\pm 0.59 \mathrm{~cm}$ (transverse) and $\pm 0.83 \mathrm{~cm}$ (coronal) for CE-MRA versus $\pm 0.34 \mathrm{~cm}$ (transverse) and $\pm 0.75 \mathrm{~cm}$ (coronal) for SSFP. Only transverse SSFP measurements revealed ICC $>0.75$. Intra- and interobserver variances did not reveal significant differences between CE-MRA and SSFP in any orientation (all p-values $>0.05$ ).

Conclusion: SSFP and CE-MRA enable comparable precision of PV diameter measurements. However both methods revealed a wide range of intra- and interobserver agreement, which has to be thoroughly considered in the clinical use.

\section{B-0368 11:33}

The application of CT angiography with whole brain perfusion imaging in the evaluation of patients with transient ischaemic attack H. Shi, F. Yang, X. Ma, W. Guo, S. Dong, M. Qiao; Beijing/CN (shihuiping@yahoo.com)

Purpose: To investigate the 320-row low-dose volume CT perfusion imaging combined with CT angiography in the evaluation of cerebral microcirculation changes of transient ischaemic attack (TIA).

Methods and Materials: Sixteen cases of TIA patients (male 11, female 5) underwent 320 row of dynamic volume CT perfusion imaging combined with CT angiography during $40 \mathrm{ml} 370 \mathrm{mgl} / \mathrm{ml}$ contrast agent injection and acquired the blood flow perfusion parameters such as regional cerebral flow ( $\mathrm{rCBF}$ ), regional cerebral volume ( $\mathrm{rCBV}$ ), mean transit time (MTT), time to peak (TTP) and delay time (DLY) and dynamic 4D-CTA images. The measured region including the ischaemia side local lesions and the corresponding healthy side brain tissue perfusion parameters. The dynamic 4D-CTA images were reconstructed to show vascular stenosis site and vascular collateral circulations.

Results: The stenosis of one side middle cerebral artery in 13 cases, one side posterior cerebral artery in 3 cases. The CBF, CBV, MTT, TTP and DLY in stenosis and healthy side had statistically significant difference $(p<0.05)$ and the parameters were $33.9 \pm 6.4$ and $35.7 \pm 6.9 \mathrm{ml} /(100 \mathrm{~g} \cdot \mathrm{min}), 2.03 \pm 0.15$ and $2.11 \pm 0.18$ $\mathrm{ml} / 100 \mathrm{~g}, 3.88 \pm 0.66$ and $3.69 \pm 0.72 \mathrm{~s}, 14.43 \pm 4.20$ and $13.68 \pm 4.08 \mathrm{~s}, 2.09 \pm 0.85 \mathrm{~s}$ and $1.51 \pm 0.58 \mathrm{~s}$.

Conclusion: The one-stop CTP/CTA imaging technique significantly improves the accuracy and efficiency in the evaluation of cerebral arteries stenosis, infarct localisation and abnormal blood perfusion and is a perfect imaging tool for the TIA diagnosis. 


\section{B-0369 11:42}

Multicentre, intra-individual comparison of single dose gadobenate dimeglumine and double dose gadopentetate dimeglumine for MR angiography of the peripheral arteries

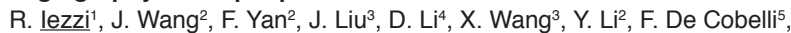

G. Pirovano ${ }^{6} ;{ }^{1}$ Rome/lTT, ${ }^{2}$ Shanghai/CN, ${ }^{3}$ Peking/CN, ${ }^{4}$ Hefei/CN, ${ }^{5} \mathrm{Milan} / \mathrm{IT}$,

${ }_{6}^{6}$ Princeton, NJ/US (miles.kirchin@bracco.com)

Purpose: To prospectively compare single dose gadobenate dimeglumine with double dose gadopentetate dimeglumine for CE-MRA in patients with peripheral arterial occlusive disease (PAOD).

Methods and Materials: Institutional Review Board approval and informed patient consent was obtained. Sixty-eight patients $(53 \mathrm{M} / 15 \mathrm{~F} ; 62.4 \pm 15.68$ years) with suspected moderate-to-severe PAOD were enrolled for randomised 3-station CEMRA with $0.1 \mathrm{mmol} / \mathrm{kg}$ gadobenate dimeglumine and $0.2 \mathrm{mmol} / \mathrm{kg}$ gadopentetate dimeglumine. Three blinded readers assessed images for vessel anatomical delineation, disease detection/exclusion, and global preference. Diagnostic performance (sensitivity, specificity, accuracy, PPV and NPV) for detection of $351 \%$ stenosis was determined for 53 patients who underwent DSA. The non-inferiority of single dose gadobenate dimeglumine was assessed using the Wilcoxon signed rank, McNemar, and Wald tests. Quantitative enhancement was compared.

Results: No differences $\left(\mathrm{p}^{3} 0.25\right)$ were noted for any qualitative parameter at any station. Equivalence was reported in at least $62 / 64$ patients (93.8\% 3-reader agreement) for diagnostic preference. Superiority for gadobenate dimeglumine was reported by all readers for all diagnostic performance indicators (sensitivity: $80.4-88.0 \%$ vs. $75.2-85.8 \%$; specificity: $89.8-96.0 \%$ vs. $88.7-94.8 \%$; accuracy: $87.4-91.7 \%$ vs. $84.9-90.6 \%$; PPV: $84.0-92.8 \%$ vs. $82.3-90.8 \%$; NPV: $88.5-92.4 \%$ vs. $85.7-91.1 \%)$ with significance $\left(\mathrm{p}^{2} 0.05\right)$ shown by reader 1 for sensitivity, accuracy and NPV. No differences $(p>0.05)$ were noted by any reader for quantitative enhancement in the pelvis although significantly $(p<0.05)$ greater enhancement with gadobenate dimeglumine was noted by 2 readers in the thigh.

Conclusion: The image quality and diagnostic performance achieved on peripheral CE-MRA with $0.1 \mathrm{mmol} / \mathrm{kg}$ gadobenate dimeglumine is at least equivalent to that achieved with $0.2 \mathrm{mmol} / \mathrm{kg}$ gadopentetate dimeglumine.

10:30 - 12:00 Room P

\section{Cardiac}

\section{SS 503}

\section{Advances in coronary CT angiography}

Moderators:

S. Bohata; Brno/CZ

L. Natale; Sesto Fiorentino/IT

\section{B-0370 10:30}

Evaluation of a novel algorithm for coronary motion compensation in prospective and retrospective coronary CT angiography (CCTA)

J.-L. Sablayrolles ${ }^{1}$, J. Feignoux', P. Ayestaran², A. Sagniez'; ' ${ }^{2}$ Saint Denis/FR,

'Buc/FR (jl.sablayrolles@me.com)

Purpose: Assessment of coronaries on patients with high heart rate (HR) using present generation $\mathrm{CT}$ is challenging due to an increase of vessel motion. The purpose of this study was to evaluate the performance of a novel coronary motion compensation algorithm on patients with a HR $>60 \mathrm{bpm}$.

Methods and Materials: Prospective or Retrospective CCTA scans on 15 patients with minimum HR of 60 (mean: 72) where acquired using a Discovery CT750 HD (GE Healthcare, Waukesha, WI) and reconstructed using conventional CCTA reconstruction algorithm (STD) and novel coronary motion compensation algorithm, SnapShot Freeze (SSF). Two senior cardiovascular radiologists evaluated diagnostic image quality of 13 coronary segments in a blinded manner using a 4-grade scale (1 non-diagnostic, 4 excellent). 186 coronary segments were assessed.

Results: Overall number of diagnostic segments was higher using SSF compared with STD $(95 \%$ vs. $85 \%)$. Overall average score of SSF reconstructed segments was significantly higher compared with STD reconstruction (2.7 vs. 2.4, T-Test $p=0.0002)$. Specifically, average score for right coronary segments ( $2.8 \mathrm{vs} .2 .3, \mathrm{~T}-$ Test $\mathrm{p}=0.0001$ ), left coronary segments ( $2.9 \mathrm{vs.} .2 .7$, T-Test $\mathrm{p}=0.023$ ) and circumflex coronary segments ( $2.8 \mathrm{vs.} 2.6$, T-Test $p=0.04$ ) were significantly higher with SSF compared with STD, whereas average score of acute marginal coronary ( $2.7 \mathrm{vs}$. 2.5 , T-Test $p=0.17$ ) and ramus intermedius coronary ( 2.6 vs. 2.2 , T-Test $p=0.09$ ) showed no significant difference between SSF and STD. On 9 patients ramus intermedius coronary was missing.
Conclusion: SnapShot Freeze increases the diagnostic confidence of CCTA scans for patients with high heart rates.

Author Disclosures:

J.-L. Sablayrolles: Speaker; General Electric Healthcare. P. Ayestaran: Employee; General Electric Healthcare. A. Sagniez: Employee; General Electric Healthcare.

\section{B-0371 10:39}

Estimating effective dose for cardiac CT with patient-specific dose maps in comparison with DLP conversion ("k factor") method

J.H. Yanof', C. Thompson' 1 , J. Wiegert'2, K. Yaddanapudi', S. Halliburton'; ${ }^{1}$ Cleveland, OH/US, ${ }^{2}$ Hamburg/DE

Purpose: The widely adopted DLP conversion ("k") factors, developed by Jessen et. al., used to estimate effective dose are based on a standard reference phantom. The aim of this study was to compare effective doses (ED) estimated from patientspecific $\mathrm{CT}$ dose maps with those estimated using $\mathrm{k}$ factors.

Methods and Materials: Nine cardiac CT data sets (mean patient age $56.6 \mathrm{yrs}$; $5 \mathrm{~F} / 4 \mathrm{M}$; BMI $22-42 \mathrm{~kg} / \mathrm{m}^{2}$ ) were obtained with a 256-slice scanner (iCT, Philips Healthcare). These contrast-enhanced coronary scans were performed with prospectively ECG triggered axial techniques. A full field of view $(500 \mathrm{~mm})$ image reconstruction was used with scan lengths ranging from 120 to $600 \mathrm{~mm}$. Each image data set was 'voxelized' to create a patient-specific "virtual phantom" with attenuation characteristics assigned based on CT numbers per ICRU 44. A Monte Carlo dosimetry simulation - using original scan parameters and modelled scanner geometry - was used to compute absorbed dose values for each voxel of the virtual phantoms to create individual dose maps. Dose maps were segmented by two consulting observers to determine patient-specific organ doses. Average EDs computed as 1) weighted sum of organ doses (ICRP 60) and 2) k x DLP were compared with linear regression and percent differences.

Results: ED values differed by an absolute mean percentage of $33.5 \%$. The regression line was EDdosemap $=1.41 \mathrm{EDK}-0.98\left(\mathrm{R}^{2}=0.78\right)$.

Conclusion: A standard effective dose estimate based on a $\mathrm{k}$-factor can be significantly different from an estimate based on a patient-specific dose map.

Author Disclosures:

J.H. Yanof: Employee; Philips Healthcare. C. Thompson: Research/Grant Support; Support from Philips. J. Wiegert: Employee; Philips Research. S. Halliburton: Consultant; Philips Healthcare.

\section{B-0372 10:48}

Impact of a new detector technology (Stellar, Siemens Healthcare) on image noise in coronary CTA

S.D.C. Deseive ${ }^{1}$, A.-L. Menges ${ }^{1}$, S. Martinoff' ${ }^{1}$, M. Hadamitzky ${ }^{1}$, T. Flohr ${ }^{2}$, B. Schmidt ${ }^{2}$, C. Thierfelder ${ }^{2}$, T. Allmendinger ${ }^{2}$, J. Hausleiter ${ }^{1} ;{ }^{1}$ Munich/DE, ${ }^{2}$ Erlangen/DE (simon.deseive@gmx.de)

Purpose: To evaluate the potential of a novel detector technology (Stellar, Siemens Healtcare) in combination with iterative image reconstruction (Safire, Siemens Healthcare) for the reduction of image noise in coronary CTA.

Methods and Materials: Twenty (12 male) consecutive non-obese patients (group A) were scanned using a $2^{\text {nd }}$ generation Dual Source CT system (Definition Flash, Siemens Healthcare) and matched to a control group (group B) of similar age, BMI and sex scanned prior to installation of the new Detector. Tube voltage and current were set to $100 \mathrm{kV}$ and $370 \mathrm{mAs}$ in both groups. Image noise, signal intensity, signal-to-noise ratio (SNR) and contrast-to-noise ratio (CNR) were obtained as quantitative image quality parameters for all datasets.

Results: Mean age and BMI were 58.2 and 24.6 in group A and 57.9 and 24.5 in group B. Image noise was $30 \%$ lower in group A compared with group B (18.6 vs. $26.3 \mathrm{HU}$ ), while signal intensity was comparable (484.5 vs. $479.7 \mathrm{HU})$ resulting in significantly higher SNR (27.4 vs. 19.4$)$ and CNR (21.6 vs. 15.7$)$.

Conclusion: In combination with iterative image reconstruction, image noise can effectively be reduced with the novel detector technology.

Author Disclosures:

T. Flohr: Employee; Siemens Healthcare. B. Schmidt: Employee; Siemens Healthcare. C. Thierfelder: Employee; Siemens Healthcare. T. Allmendinger: Employee; Siemens Healthcar. J. Hausleiter: Research/Grant Support; Siemens Healthcare. Speaker; Siemens Healthcare. 


\section{B-0373 10:57}

Radiation dose reduction in computed tomography myocardial perfusion imaging using iterative reconstruction and patient-tailored imaging

M. Williams ${ }^{1}$, S. Golay ${ }^{1}$, N.W. Weir', S. Mirsadraee', E.J.R. Van Beek', J. Reid², G. McKillop ${ }^{1}$, N. Uren ${ }^{1}$, D.E. Newby ${ }^{1} ;{ }^{1}$ Edinburgh/UK, ${ }^{2}$ Melrose/UK

Purpose: We assessed the application of iterative reconstruction and patienttailored imaging on radiation dose in computed tomography (CT) myocardial perfusion imaging (CTP).

Methods and Materials: Participants underwent rest CT coronary angiography and adenosine stress CTP using a 320-multidetector scanner (Aquilion ONE, Toshiba). Radiation dose reduction techniques included iterative reconstruction (AIDR3D), tube current adjustment-based scout attenuation and half-segment reconstruction. Dose length product (DLP) and image noise were recorded. Subjective image quality was assessed ( 1 , excellent; 4 , uninterpretable) and summed for 17 myocardial segments.

Results: For 28 patients before and 18 after introduction of the protocol there was no difference in gender $\left(68 \%\right.$ male vs $72 \%$, p0.754), body mass index $\left(28 \mathrm{~kg} / \mathrm{m}^{2}\right.$ $(95 \% \mathrm{Cl} 27.30)$ vs 28 (25.30), p0.512), heart rate at rest (56 beats/minute (53.58) vs 56 (52.60), p0.970) or stress (73 (69.77) vs 72 (66.78), p0.837), or z-axis collimation at rest $(133 \mathrm{~mm}(127,138)$ vs $129(124,135)$, p0.956) or stress $(115(108,123)$ vs $120(112,130), p 0.961)$. There was a significant reduction in tube current $(470$ $\mathrm{mA}(453,486)$ vs $371(290,452), \mathrm{p} 0.021)$, tube voltage $(96 \%$ at $120 \mathrm{kV}$ vs $33 \%, \mathrm{p}$ $<0.001)$ and DLP at rest $(225$ mGy.cm $(213,238)$ vs $144(102,186)$, p0.001) and stress $(584(474,693)$ vs $242(171,312), p<0.001)$. There was no difference in image noise at rest (30 Hu (26.34) vs 33 (31.36), p0.167) and a small increase at stress (26 (22.31) vs 39 (33.45), p0.001). There was no difference in subjective image quality (median 17 [IQR 17.21] vs 17 [17.19], p0.523).

Conclusion: Iterative reconstruction and patient-tailored imaging led to a $60 \%$ reduction in CTP radiation dose, while maintaining image quality.

\section{B-0374 11:06}

Radiation dose reduction in cardiac CTA using a novel iterative reconstruction algorithm in 320-row detector CT

T. Durmus ${ }^{1}$, R. Luhur 1 , J. Blobel' ${ }^{2}$ J. Mews ${ }^{2}$, A. Lembcke ${ }^{1} ;{ }^{1}$ Berlin/DE, 2Neuss/DE (tahir.durmus@charite.de)

Purpose: Radiation exposure reduction in cardiac CTA using a novel iterative reconstruction algorithm (AIDR3D) with automatic exposure control (AEC).

Methods and Materials: 60 patients were examined on a 320-row detector CT (AquilionONE, Toshiba Medical Systems) using 100 kV, 8-12 cm z-axis, $350 \mathrm{~ms}$ acquisition time, $0.5 \mathrm{~mm}$ slice thickness and $320 \mathrm{~mm}$ FoV. Contrast medium injection was performed using a standardised protocol with $0.66 \mathrm{mg}$ lodine per $\mathrm{kg}$ body weight. Group 1 (G1) consisted of 30 patients (BMl=25.7 $\pm 3.6 \mathrm{~kg} / \mathrm{m} 2, \mathrm{HF}=54.9 \pm 7.4$ bpm) examined using BMl adapted tube current (140-580 mA) and a FBP reconstruction algorithm in combination with advanced noise reduction filters. In group 2 (G2) 30 patients (BMl=25.7 $\pm 3.7 \mathrm{~kg} / \mathrm{m} 2, \mathrm{HF}=58.3 \pm 7.8 \mathrm{bpm}$ ) were examined using the novel reconstruction algorithm AIDR3D. For AEC a target noise of $33 \mathrm{HU}$ was predetermined. Analysis was performed using circular regions of interests placed into the descending aorta (DA), left ventricular lumen (LV) and myocardial wall (MW). Signal- and contrast-to-noise-ratio (SNR, CNR) were calculated. Doselength-products (DLP) were recorded and effective doses were estimated. Results in $\mathrm{G} 1$ and $\mathrm{G} 2$ were compared using an unpaired t-test.

Results: Both SNR and CNR differed significantly in both groups: SNR in G1 and G2 were $19.5 \pm 6.6$ and $23.0 \pm 5.3$, respectively, in $D A(p=0.025)$ and $12.7 \pm 4.4$ and $20.4 \pm 4.5$, respectively, in LV $(p<0.001)$. CNR in G1 and G2 were $9.3 \pm 3.7$ and $19.3 \pm 5.0$, respectively $(p<0.001)$. Effective dose was in $\mathrm{G} 2(0.88 \pm 0.34 \mathrm{mSv}) 24 \%$ less than in $\mathrm{G} 1(1.15 \pm 0.46 \mathrm{mSv} ; \mathrm{p}<0.011)$.

Conclusion: AIDR3D with AEC allows for significant radiation dose reduction compared with advanced FBP reconstruction with $\mathrm{BMI}$-adapted exposure. Image quality parameters in the AIDR3D group were superior to the reference group indicating further dose reduction potential.

Author Disclosures:

J. Blobel: Employee; Toshiba Medical Systems. J. Mews: Employee; Toshiba Medical Systems.
B-0375 11:15

Computed tomography coronary angiography with a consistent dose below $2 \mathrm{mSv}$ using double high-pitch spiral acquisition in patients with atrial fibrillation

Q. Wang, Y. Chen, B. He; Beijing/CN (doctorwq@126.com)

Purpose: To evaluate the feasibility and imaging quality of double prospectively ECG-triggered high-pitch spiral acquisition mode for coronary computed tomography angiography (CTCA) in patients with atrial fibrillation (AF).

Methods and Materials: 47 patients ( 11 women, 36 men; mean age $64.5 \pm 12.1$ years) were enrolled for CTCA examinations using a dual-source CT with $2 \times 128$ $\times 0.6 \mathrm{~mm}$ collimation, $0.28 \mathrm{~s}$ rotation time and a pitch of 3.4. Double high-pitch mode was prospectively triggered first at $60 \%$ and later at $30 \%$ of the R-R interval within two cardiac cycles. Image quality was evaluated using a four-point scale (1 $=$ excellent, $4=$ non-assessable).

Results: From 672 coronary artery segments, $77.5 \%$ (521/672) was rated as score of $1,20.8 \%(140 / 672)$ as score of $2,1.2 \%(8 / 672)$ as score of 3 and $0.4 \%(3 / 672)$ was rated as 'non-assessable'. The average image quality score was $1.25 \pm 0.38$ on a per segment basis. Mean dose-length product (DLP) for CTCA was $92.6 \pm 28.2$ $\mathrm{mGy} \cdot \mathrm{cm}$, the effective dose was $1.30 \pm 0.39 \mathrm{mSv}(0.64-1.97 \mathrm{mSv})$.

Conclusion: In patients with AF, double prospectively ECG-triggered high-pitch spiral acquisition could be a feasible and valuable scan mode for CTCA with a consistent dose below $2 \mathrm{mSv}$ as well as diagnostic imaging quality.

\section{B-0376 11.24}

Coronary artery calcium scoring from dual-source chest CT: potentials for ungated, high-pitch scan technique in routine clinical practice

F. Molinari, A. Hutt, M. Rémy-Jardin, J.-B. Faivre, A. Simeone, E. Algeri, J. Rémy; Lille/FR (martine.remy@chru-lille.fr)

Purpose: To assess the concordance between ungated, high-pitch (thoracic) and ECG-gated (cardiac) examinations in the assessment of coronary artery calcium (CAC) score.

Methods and Materials: 185 smokers (mean heart rate: $71.9 \mathrm{bpm}$ ) underwent dual-source CT examinations with acquisition of two sets of images during the same session: (a) an ungated, high-pitch scan over the entire thorax (pitch: 3.0, temporal resolution: $75 \mathrm{~ms}$ ) (group 1); (b) a prospectively ECG-gated scan over the cardiac cavities (group 2). Two operators independently analysed the CAC load on both examinations and categorised the population according to the Agatston score. Results: The interobserver reproducibility was good for both techniques (group 1: kappa $=0.90$, group 2: $k a p p a=0.89$ ). The mean absolute values of the Agatston score did not significantly differ between groups $(p=0.18)$. There was good reproducibility between the two techniques in classifying the population according to the commonly used cutoffs (kappa: 0.90). The ungated technique adequately categorised 172 out of the 185 patients (93); in the 13 discordant cases $(13 / 185 ; 7)$, the ungated studies yielded lower scores than gated studies, with no more than one score category misplaced by the ungated studies. BMI and heart rates did not significantly differ between concordant and discordant scores $(p=0.81, p=0.21$, respectively). Differences in CAC scores between techniques were correlated to differences in the CAC scores at the level of the right coronary artery $(r=0.98 ; p=.0001)$.

Conclusion: Despite the mean heart rate of the studied population, a good concordance in assessing the CAC scores was found between ungated, high-pitch and ECG-gated examinations.

\section{B-0377 11:33}

Diagnostic accuracy of free-breathing coronary CTA using dualsource $\mathrm{CT}$ high-pitch acquisition: comparison of single and double scans with conventional angiography

M.C. So ${ }^{1}$, W.L. Chin ${ }^{2}$, C.M. Wong ${ }^{1}$, C.W. Tai ${ }^{1}$, M.W. Leung ${ }^{1} ;{ }^{1}$ Hong Kong/HK, ${ }^{2}$ Singapore/SG (ninamc_so@yahoo.com.hk)

Purpose: To retrospectively investigate whether two subsequent prospectively ECG-gated high-pitch CT acquisitions can increase the diagnostic accuracy of coronary CT angiography (CCTA) for free-breathing patients compared with a single acquisition and conventional angiography.

Methods and Materials: Twenty-one patients ( 8 male, 13 female, mean age 77.2 years) with heart rates $<60 \mathrm{bpm}$ unable to hold their breath underwent two immediately subsequent free-breathing CCTA scans in dual-source CT prospectively ECG-triggered high-pitch mode, followed by conventional angiography within 1 month. CCTA data and conventional angiograms were evaluated by segment by two different observers. CCTA image quality was rated on a four-point scale $(1=$ excellent to $4=$ unevaluable) with $350 \%$ diameter stenosis considered significant. 
Discrepancies between CT and conventional angiogram results were resolved by consensus together with a third observer. Sensitivity, specificity and accuracy of single and double high-pitch scans were calculated on a per segment basis. Estimated effective radiation doses were calculated using dose length product $\times 0.014$. Results: CCTA images were diagnostic for $86 \%$ (243/283) and 97\% (276/283) of segments for single and double scans, respectively, and sensitivity, specificity and accuracy were $100 \%, 88 \%, 90 \%$ and $98 \%, 95 \%, 95 \%$, respectively. The mean estimated effective radiation dose was $1.3 \mathrm{mSv}$ and $2.5 \mathrm{mSv}$, respectively.

Conclusion: In patients with heart rates ${ }^{2} 60 \mathrm{bpm}$, free-breathing CCTA using dual source CT double scans in high-pitch acquisition mode was associated with high diagnostic accuracy for the assessment of coronary artery stenosis at a very low effective radiation dose.

\section{Author Disclosures:}

W.L. Chin: Employee; Siemens Medical Solutions.

\section{B-0378 11:42}

One beat coronary CT angiography using 640 slices multidetector

CT scanner in patients with atrial fibrillation

A. Gennarelli, A. Di Sibio, M. Perri, F. Di Stasio, V. Felli, M. Di Luzio,

A.V. Giordano, E. Di Cesare, C. Masciocchi; L'Aquila/IT

Purpose: To evaluate image quality and ionising radiation doses of CT coronary angiography using 640-slices multidetector CT in patients with atrial fibrillation (AF). Methods and Materials: 28 patients that have persistent AF with median heart rate of 75 bpm underwent coronary CT angiography using 640-slices multidetector CT with adaptive iterative dose reduction system. All CT examinations were performed using a one-beat prospective gating; in patients, that presented heart rate $>65 \mathrm{bpm}$, was used a broad phase window width (30-85\% of R-R). The effective dose was assessed for every patient. Coronary arteries were subdivided into 15 segments using the American Heart Association's (AHA) model. Image quality was evaluated by two expert radiologists on a workstation (Vitrea, Toshiba) using a 4-score grading system: score 1 (absence of motion artefacts), score 2 (presence of mild artefacts), score 3 (presence of moderate artefacts) and score 4 (presence of severe artefacts). Median score of both radiologist and inter-rater reliability ( $\mathrm{k}$ coefficient) were assessed.

Results: Image quality was judged excellent (score 1) in $65.4 \%$ of coronary artery segments (275/420), good (score 2) in 22.7\% (95/420), fair (score 3 ) in $7.1 \%$ (30/420) and low (grade 4$)$ in $4.8 \%$ (20/420). Mean effective dose was $3.35 \mathrm{mSv}$ (range between 1.3 and $7.9 \mathrm{mSv})$. Inter-rater reliability was excellent $(\mathrm{k}=0.85)$.

Conclusion: High image quality and low ionising radiations effective doses demonstrate that the newest generation of CT scanner overcome the limitation at coronary $\mathrm{CT}$ angiography that atrial fibrillation poses so far.

\section{B-0379 11:51}

Influence of AIDR on accuracy of plaque characterisation and lumen assessment during CT coronary angiography

A. Ursani, H. Mehrez, H. Kashani, N. Paul; Toronto, ON/CA (ali.ursani@uhn.ca)

Purpose: To determine the impact of Adaptive Iterative Dose Reduction (AIDR) on accurate lumen assessment in a custom-built arterial plaque phantom.

Methods and Materials: Custom-built $10 \mathrm{~cm}$ coronary artery phantom with $6 \times 3.5$ $\mathrm{mm}$ (G1) and $6 \times 5 \mathrm{~mm}$ (G2) vessels, lumen stenosis 0, 30, 50, 70, 90 and $100 \%$ due to $400 \mathrm{HU}$ and $800 \mathrm{HU}$ plaque; and $3 \times 3.5 \mathrm{~mm}$ (G3) and $3 \times 5 \mathrm{~mm}$ (G4) vessels with lumen stenosis $30,50,70 \%$ due to $-60 \mathrm{HU}$ and $60 \mathrm{HU}$ plaque. The lumen measured $+400 \mathrm{HU}$. Volume CT (vCT) was performed; spatial resolution $350 \mu \mathrm{m}$ with low- and high-resolution kernels (FC04, FC30, FC90), $120 \mathrm{kVp}$ and $150 \mathrm{~mA}$. 0.5/0.35 mm axial images were reconstructed \pm AIDR. In-line histography mapped average plaque CT\# $(\mathrm{HU})$ and average lumen $\mathrm{CT \#}(\mathrm{HU})$ and vessel profile. IN was measured and image contrast $(C)$ calculated.

Results: Lumen stenosis contrast data for soft filter kernel FC04 and calcified plaque $800 \mathrm{HU}$ : Without AIDR: G1:30\%=894/705; 50\%=910/602; $70 \%=784 / 550 ; 90 \%=600 / 400 ;$ G2: $30 \%=919 / 550 ; 50 \%=915 / 500$; $70 \%=864 / 400 ; 90 \%=413 / 360$. With AIDR:

G1: $30 \%=870 / 602 ; 50 \%=890 / 530 ; 70 \%=770 / 490 ; 90 \%=580 / 345 . \mathrm{G} 2: 30 \%=900 / 620$ $50 \%=898 / 544 ; 70 \%=856 / 562 ; 90 \%=400 / 330$ AIDR improved mean contrast $\sim 10 \%$ for calcified plaque and $15 \%$ for non-calcified plaque for all reconstructions with $30-70 \%$ luminal stenosis (paired t-test, $\mathrm{p}^{2} 0.001$ ). AIDR improved accurate representation of $90 \%$ lumen stenosis in $3.5 \mathrm{~mm}$ vessels.

Conclusion: AIDR improves plaque image contrast by $10-15 \%$ and visualisation of high-grade stenosis in $3.5 \mathrm{~mm}$ coronaries. This promises superior performance for accurate coronary plaque characterisation and lumen assessment.
10:30 - 12:00

Room Q

\section{Radiographers}

\section{SS 514}

\section{The radiographer's role as health care team member}

Moderators:

V. Syrgiamiotis; Athens/GR

C. Vandulek; Kaposvár/HU

\section{B-0380 10:30}

How do radiographers interact with children in the radiographic examination?

B. Björkman ${ }^{1}$, K. Enskär', M. Golsäter ${ }^{1}$, R.J. Simeonsson'²; Jönköping/SE,

${ }^{2}$ Chapel Hill, NC/US (bjbe@hhj.hj.se)

Purpose: The verbal interaction between the child and the radiographer in the radiographic examination is an unexplored, but important area of clinical practice. The aim of this study was to investigate the nature of that interaction between child and radiographer and the extent to which it varied as a function of the child's age. Methods and Materials: The participants in the study were 20 female radiographers and 32 children (3-15 years) examined for acute injuries. The verbal interactions of child and radiographer during the examination were videotaped and analysed using the Roter Interaction Analysis System (RIAS). Descriptive and non-parametric statistics were used to analyse the interaction data.

Results: The results revealed that $80 \%$ of the verbal interaction was carried out by the radiographer either to the child or to an escorting parent, while $17 \%$ was carried out by the child and $3 \%$ by the parent. Of the radiographers' communication directed to children, $78 \%$ was categorised as task-focused exchange and $22 \%$ as socio-emotional exchange. Of children's utterances, $34 \%$ was categorised as a task-focused exchange and $66 \%$ involved socio-emotional exchange. The distribution of task-focused and socio-emotional utterances varied with regard to children's age. More frequent utterances and more frequent socio-emotional exchange were found for interactions involving younger children.

Conclusion: These findings reinforce the need for radiographers to be flexible and sensitive in their interactions with children and to recognise the child's individual and developmental capabilities when engaging them in radiographic procedures.

\section{B-0381 10:39}

Requesting x-rays at the right time to utilise the capacity of the radiology department: an everyday challenge

M. Mowinckel-Nilsen; Os/NO (milh@helse-bergen.no)

Purpose: Historic data shows that delayed ordering of x-rays makes it difficult to optimally utilise the capacity of the Radiology department in a small orthopaedic hospital. What could be done to improve this?

Methods and Materials: A method called patient-focused redesign (developed by Leicester Royal Infirmary, adapted to Norwegian conditions by Ringerike Hospital $\mathrm{HF}$ ) was used as a tool to find a better workflow in ordering $\mathrm{x}$-rays. A multidisciplinary team was assembled, and a project outline was made with a process demarcation. The team prepared a communication plan, mapped the current workflow, had a creative phase redesigning a new workflow, and measured the situation before the new workflow was tested in the hospital. An analysis of the old and new workflow was made. Evaluation was done after the new workflow had been in place for 1.5 years; new measurements were done to see the effects of the changes in the workflow. Results: Numbers of steps in the workflow were halved, saving time for several professions. X-ray examinations not ordered on time were reduced by $76.5 \%$. Examinations ordered a week or more before the images were taken, which is within the desired timeframe, were increased by $64.1 \%$.

Conclusion: Patient-focused redesign is a good method for finding better and more efficient workflows; it can save time, increase the exploitation ratio of the equipment, and ultimately increase the quality of the radiographic service. 


\section{B-0382 10:48}

Risk management in computed tomography using an incident reporting system

M. Antoniutti, S. Doratiotto; Treviso/IT (sdoratiotto@alice.it)

Purpose: Improving the management of patient safety by adopting best management practices for the governance of the system and the corporate network.

Methods and Materials: Analysis of the critical situation in the diagnostic computed tomography (CT) performed in 2009-2011 with incident reporting system (IR) using the instrument of audit and definition of improvement actions.

Results: Improving actions have been taken together with a method of monitoring the most critical issues with greater severity or frequency of occurrence in the CT process. Critical areas: high number of booking errors from the radiological studies of central booking, inappropriate requests by general practitioners (GPs). Improvement action (IA): organization of the audit with GPs, the final shared document has been spread through the Internet site. High number of patients not adequately prepared for the examination (non-hydrated, non-fasting, incomplete clinical information, and incomplete filling of the informed consent form examination). IA: a letter from the chief of radiology was sent to all departments to alert that requests for CT must be sent in. Radiology correctly completed and audit with GPs. Errors found in PACS. IA: correction of PACS errors and permanent organization of audit. Conclusion: This project helped the development of effective interventions closely related to the understanding of the organization's critical work in the CT process with the need to streamline the planning CT scan of the list to avoid an incongruous workload and to give each patient the right welcome. Internal audits and permanent working groups have been proposed to actuate effective remedial actions.

\section{B-0383 10:57}

Is a radiographer-led immediate reporting service for emergency

department referrals a cost-effective initiative?

M. Hardy ${ }^{1}$, B. Snaith ${ }^{2}{ }^{1}$ Bradford/UK, ${ }^{2}$ Wakefield/UK

(M.L.Hardy1@bradford.ac.uk)

Purpose: Demand for both Emergency Department (ED) and radiology services continues to increase across the UK while simultaneously, healthcare organisations are being asked to evaluate the quality of care provided and constrain service costs. National guidance on radiograph reporting times recommends ED radiographs are reported on day of patient attendance but in practice, delays in reporting persist. This study considers whether a radiographer-led immediate reporting service for ED referrals could provide a cost-effective service improvement solution.

Methods and Materials: A pragmatic multi-centre randomised controlled trial was undertaken. 1502 patients were recruited and randomly assigned to an immediate or delayed reporting arm and treated according to group assignment. Patient health gain was measured in terms of change in utilities derived from EQ-5D responses at baseline and 8-week follow-up. Resources used and the costs of an immediate reporting service were analysed at the patient level and compared to standard reporting practices.

Results: 1688 radiographic examinations were performed (1502 patients). 79 discordant radiographic interpretations were identified ( $n=79 / 1688 ; 4.7 \%)$. Interpretive errors were significantly reduced within immediate reporting arm. No significant difference was noted in the relative improvement in patient perceived health status between the 2 arms of the study. The average cost saving per patient in the immediate reporting arm £23.40.

Conclusion: Radiographer-led immediate reporting of ED radiographs is a cost-effective service development and its universal introduction could make a significant contribution to the current drive to increase service productivity within current budget constraints.

\section{B-0384 11:06}

An investigation into the ability of Irish radiographers: in the identification of wrist and scaphoid fractures

A.P. Tyrrell', L. Rainford ${ }^{2}$; ${ }^{1}$ Kilkenny/IE, ${ }^{2}$ Dublin/IE (louise.rainford@ucd.ie)

Purpose: To investigate the ability of Irish radiographers in identifying and locating wrist/scaphoid fractures, to add to the evidence base for role expansion.

Methods and Materials: In total, 25 radiographers across two university teaching and two large regional hospitals in Ireland participated. The computer application Ziltron demonstrated 71 plain film wrist radiographs in a Free-Response Operating Characteristic (FROC) study. The data set contained 35 abnormal images (fracture (s) present) and 36 normal (non-fracture). "Truth" was determined by the Radiology report. Fracture were categorised: obvious to subtle. Statistical analysis was performed by JAFROC application.
Results: Analysis of the results for the area under the curve (AUC) demonstrated statistically significant differences in the accuracy of fracture identification between groups ( $p$ value $<0.032$ ), when categorised with respect to post-graduation clinical experience. Radiographers with greater and less than 3 years clinical experience scored mean Az scores of 0.737 and 0.716 , respectively. A trend in increased performance and increased clinical experience was also demonstrated with a mean increase of $9.2 \%$ and $8.8 \%$ in terms of sensitivity and specificity, respectively. Conclusion: Radiographers with increased clinical experience performed the most accurately with respect to AUC, sensitivity and specificity findings. Hospital category did not influence the radiographer's performance in this investigation: university teaching centre versus large regional centre. The acceptable standard following post-graduate image interpretation training is $80-95 \%$ in terms of accuracy of skeletal fracture identification. Graduate training programmes/CPD modules in clinical decision making are recommended as is a survey on a national scale to incorporate a larger sample size.

\section{Author Disclosures:}

A.P. Tyrrell: Grant Recipient; Alan Tyrrell. Research/Grant Support; Irish Institute of Radiography and Radiation Therapy Student Research Grant Winner 2012.

\section{B-0385 11:15}

Workloads of reporting radiographers and sonographers: costeffectiveness compared with consultant radiologist W.P. Hedges ${ }^{1}$, S.H. Khan'; ; ${ }^{1}$ Lancaster/UK, ${ }^{2}$ Blackburn/UK (will.hedges@doctors.org.uk)

Purpose: To accurately measure the workload of radiographers and sonographers for the first time in the UK and compare them with that of consultant radiologists in a DGH.

Methods and Materials: Reporting data for sonographers, radiologists and reporting radiographers at a large DGH was taken from electronic records. These were converted into an RVU score using an adapted version of the Pitman-Jones RVU system. Reporting workload was calculated by dividing the total RVU scores by the number of PA spent reporting images by members of each group.

Results: There were 9 sonographers working $80.5 \mathrm{PA} /$ week, 2 reporting radiographers worked $10 \mathrm{PA} /$ week, and 14 consultant radiologists working 158.21 PA/ week during the period April 2010 - March 2011. Sonographer workload amounted to 1,955.9 RVU per PA per year (RVU/PA/year). Radiographer workload was to 4,277 RVU/PA/year. Crude consultant radiologist workload was 4,106 RVU/PA/ year, but they spend $42.49 \%$ of their time on non-reporting activities, giving a net score of 7,140 RVU/PA/year.

Conclusion: The reporting radiographers and sonographers make a valuable and significant contribution dealing with the increasing radiology workload. However, radiologists are more productive per PA. Differences in pay grades makes costeffectiveness between the groups more equal.

\section{B-0386 11:24}

Radiographer's expectations for role development: a national survey in view of radiography students in final year of graduation

C.M. Mendes, A.F.C.L. Abrantes, R.P.P. Almeida, L.P.V. Ribeiro, S.I. Rodrigues; Faro/PT (celinemendes@msn.com)

Purpose: To investigate the role development expectations of graduate radiographers with a view to predicting the potential impact of a misalignment of these expectations on quality of the service delivery and staff retention.

Methods and Materials: A self-applied questionnaire development by Williamson and Mundy (2009) in UK was validated for the Portuguese language by the backtranslation method with permission of the authors and assigned to radiography students in final year of graduation in the Portuguese health schools. A total of 84 valid questionnaires, in the Likert scale format, utilised 20 attitude questions in relation to main themes of research (expectation, valence and knowledge) and were interpreted and statistically analyzed through descriptive statistics and Spearman's rho correlation.

Results: The radiography students stated an expectation for role development opportunities with $66.7 \%$ indicating that these expectations would be realized within $2(50.0 \%)$ and $5(29.8 \%)$ years of graduation. A significant and strong positive correlation $(r=0.791, p=0.000)$ between job satisfaction and expectation for role development in 5 years after graduation was seen. Farther, there were no significant differences between the health schools for the expectation, valence and knowledge. Conclusion: There is an expectation and value assigned for role development opportunities. Expectation is seen to be intrinsically linked with job satisfaction suggesting that misalignment of this would have a potentially negative impact on motivation and retention of the future radiography workforce. However, there is 
a lack of social recognition of the radiographer, suggesting the need to promote measures to increase the profession recognition.

\section{B-0387 11:33}

Job satisfaction among radiographers in Switzerland: a national survey N. Richli Meystre, P. Lehmann, N. Mamboury; Lausanne/CH (nicole.richli@hesav.ch)

Purpose: The technological improvements in radiology over the past decade have resulted in an important rise in demands for radiological examinations and treatments. An increasing number of well qualified radiographers are needed and healthcare authorities are worried about the growing shortage of radiographers. Job (in)satisfaction has been highlighted as a contributing factor to turnover, respectively, to long-time retention. The aim of this survey was to appreciate workplace conditions and job satisfaction among radiographers.

Methods and Materials: All radiographers working in Switzerland were invited to participate with an online questionnaire. Different factors contributing to job satisfaction were scored from -2 (very unsatisfied) up to +2 (very satisfied).

Results: 753 radiographers completed the questionnaire. The response rate is estimated at $26 \% .62 \%$ are working in diagnostic, $23 \%$ in nuclear medicine and $15 \%$ in radiooncology. $37 \%$ are working for more than 10 years in the same place. The overall job satisfaction level was scored at 0.66. The highest levels were reached for the diversity of job activities $(+1.19)$, the quality of radiology facilities $(+1.16)$, teamworking $(+1.03)$ and autonomy $(+1.03)$. The lowest levels were reached for collaboration with radiologists $(+0.23)$, salary $(+0.17)$ and career opportunities $(0)$. Conclusion: Radiographers in Switzerland are rather satisfied about their job and the workplace conditions. In order to avoid shortage, efforts should be taken to increase the number of radiographer students and in supporting returnees. Jobenrichment measures are seen as a necessity for retention in a profession with low career opportunities.

\section{B-0388 11:42}

Progression of quality improvement works in radiography by radiographers

R. Gullien, J.G. Andersen, A.E. Haakull; Oslo/NO (uxraul@ous-hf.no)

Purpose: To visualise the ongoing progression over years in quality improvement work in radiography by radiographers and share our experiences.

Methods and Materials: 1) As a basis through the entire improvement work, the National Quality Assurance Manual (QAM) that specifies how to measure the quality assurance work was used. 2) Practiced the method in the department as basic measurement individual and overall measurements (static/snapshot). 3) Individual and overall measurements recorded several times over a period to see changes (dynamics/moving range) (local). 4) Implemented targeted improvements. 5) Measured inter-variability among the quality control (QC) radiographers. 6) Training the $Q C$ radiographers to avoid differences between them. 7) National meeting with representatives from all counties (national). 8) Comparison across the counties and input to the audit of the QAM. 9) Cooperation with hospital outside Norway, benchmarking the system, comparison across countries (international). Results: Radiographers were trained by self-assessment: their knowledge was updated, their skills were developed, their attitude to regular audit was changed and improved, their own performances against international and national standard and objectives were reviewed. Targeted initiatives against unacceptable variances in performance are necessary. Time set aside to allow radiographic quality assurance work, analysing, evaluating and act accordingly. QC radiographers have the responsibility of ensuring the essential quality improvement and assurance work. Conclusion: Progression is from snapshot/static to dynamics/moving range, from general to focused measures, from local to national, from national to international. Quality improvements became more and more complex over time. Advancements and developments are important in addition to the continuous implementation of basic training and instructions.

\section{B-0389 11:51}

Radiographer contribution to the interpretation of trauma radiographs: a survey of UK practice

B. Snaith ${ }^{1}$, M. Hardy ${ }^{2}$, E. Lewis ${ }^{1} ;{ }^{1}$ Wakefield/UK, ${ }^{2}$ Bradford/UK

(bev.snaith@midyorks.nhs.uk)

Purpose: Radiographer abnormality detection schemes (RADS) were introduced in the UK in the mid-1980s with the development of the "red dot scheme". This presentation reports the findings of a new survey and establishes the current position of RADS (including "red dot", commenting and reporting) across the UK and provides insight into the influence of technology on the professional development and practice in this field.

Methods and Materials: In August 2011, a postal questionnaire was distributed to 521 hospital sites across the UK operating both a radiography and trauma service. Information was sought relating to: the type of trauma and radiography service operated; details of the RADS operated, including education and audit to support radiographer participation; the mandatory/voluntary status of the RADS; and the imaging technology operating within the organization.

Results: A total of $328(n=328 / 521 ; 63.0 \%)$ responses were received within the defined timeframe. Analysis of the data is ongoing but will include a descriptive summary of current practice across the UK with regard to "red dot", commenting and reporting by radiographers and assessment of the impact on the implementation of digital imaging technology across the UK on service levels compared to an earlier survey undertaken in 2007.

Conclusion: Radiographer contribution to the interpretation of trauma images is an expectation of UK radiographer practice. Whether the profession has moved forward in its readiness to progress beyond "red dot" and embrace commenting is yet to be seen and this presentation will provide an insight into practice boundaries and variations that exist.

14:00 - 15:30

Room A

\section{Genitourinary}

\section{SS 607}

\section{Female pelvis imaging}

\section{Moderators:}

L.S. Fournier: Paris/FR

A.G. Rockall; London/UK

\section{B-0390 14:00}

Diagnostic and prognostic value of ${ }^{18} \mathrm{~F}-\mathrm{FDG}$ PET/MR over MR and

$\mathrm{PET} / \mathrm{CT}$ in recurrent gynaecologic malignancies prior to pelvic

exenteration

I.A. Burger ${ }^{1}$, H.A. Vargas ${ }^{2}$, O.F. Donati², V. Andikyan², H. Schoder ${ }^{2}$, D. Chi ${ }^{2}$,

E. Sala ${ }^{2}$, H. Hricak'; ' ${ }^{2}$ urich/CH, ${ }^{2}$ New York, NY/US (irene.burger@usz.ch)

Purpose: To assess the incremental value of fused MRI $\left[{ }^{18} \mathrm{~F}\right]-\mathrm{FDG}-\mathrm{PET}$ compared to MRI or PET/CT for local disease extent prior to pelvic exenteration for recurrent gynaecological malignancies, and to determine if FDG uptake metrics [SUVmax, total lesion glycolysis (TLG) and metabolic tumour volume (MTV)] can be used as predictive biomarkers for overall survival.

Methods and Materials: 31 patients undergoing FDG-PET/CT and pelvic MRI alone within 90 days prior to pelvic exenteration were retrospectively selected. The presence of bladder, rectum and pelvic sidewall invasion was determined on PET/CT, 3 months later on MRI and subsequently on fused MRI/PET images. For each patient SUVmax, TLG and MTV were measured. Areas under the curve (AUCs) for detecting pelvic organ or side wall invasion were calculated. Kaplan Meier graphs were used to determine associations between SUVmax, TLG, MTV and overall survival.

Results: The AUCs for bladder, rectum and pelvic side wall invasion were 0.90 , 0.92 and 0.77 for PET/CT, 0.86, 0.97 and 0.89 for MRI and 0.94, 0.99 and 0.99 , respectively, for fused MRI/PET. There was a significant association between TLG/MTV and overall survival $(p=0.05 / p=0.001)$, but not for SUVmax $(p=0.317)$. Conclusion: In patients undergoing pelvic exenteration for recurrent gynaecological malignancies, fused MRI/PET was more accurate than PET/CT or MRI alone for assessment of local disease extent. TLG and MTV were predictive biomarkers for overall survival, but SUVmax was not associated with overall survival.

\section{B-0391 14:09}

Diagnostic value of quantitative measurement of microvascular changes achieved using perfusion MR imaging in the assessment of tumour grading in endometrial carcinoma

O. Minutolo, D. Ippolito, A.C. Cadonici, P.A. Bonaffini, C. Capraro, S. Sironi; Monza/IT (orazio.minutolo@yahoo.com)

Purpose: Diagnostic value of dynamic contrast-enhanced perfusion MRI in detection and characterisation of endometrial cancer, correlated with tumour grading, being the histopathological analysis as the standard of reference.

Methods and Materials: A total of 80 patients with histologically proven endometrial carcinoma underwent a dedicated pelvic MRI examination (1.5 T system, Achieva, Philips), using high-resolution pelvic phased array multicoil. Each patient scan 
consists of multiplanar T2 and T1 sequences and dynamic contrast-enhanced THRIVE. Philips's workstation was used to generate colour permeability maps showing perfusion of enhancing tumours. After the placing of regions of interests (ROIs) on the site of the maps which best corresponded to the enhanced regions of lesion, the following parameters were calculated: relative enhancement, maximum enhancement, maximum relative enhancement and time to peak.

Results: Pathological results of all patients were obtained postoperatively and compared with DSCE-MRI perfusion parameters. At histological examination 21 patients had G1 tumour, 44 had G2, 14 had G3 and 1 had squamous cell carcinoma. Tumour locations visualised on T2w and DSCE-MRI were consistent with histopathological findings and the quantitative analysis showed the following

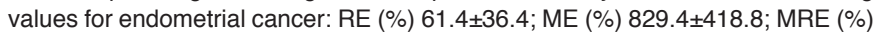
$69.4 \pm 42.2$ and TTP $(\mathrm{sec}) 179.5 \pm 27.4$. The corresponding values calculated in normal endometrium were: RE (\%) 157.1 \pm 61.3 ; ME (\%) 1634.5 \pm 625.7 ; MRE (\%) 128.8 \pm 51.6 and TTP $(\mathrm{sec}) 193.6 \pm 23.8$. A significant $(p<0.001)$ decrease of each evaluated parameters was obtained for tumour lesions in comparison with normal myometrium, furthermore a significant higher values $(p<0.001)$ for RE, ME, MRE were obtained for G1 tumours (less aggressive) than those with G2 or G3 grading. Conclusion: Conventional MRI combined with perfusion DSCE represents a feasible technique that provides non-invasive, quantitative parameters of vascularization, useful in the assessment of diagnosis of patient with endometrial cancer, offering quantitative and qualitative information about biological characteristics of endometrial cancer, related to tumour aggressiveness.

\section{B-0392 14:18}

Influence of pathophysiological parameters with kinematic MRI in evaluation of female pelvic floor

M. Palumbo, S. Carbone, L.S. Maltese, D. D’Elia, A. Luceri, V. Ricci,

L. Volterrani; Siena/IT (palumbomichele81@hotmail.it)

Purpose: To evaluate the correlation between pelvic floor morphometry obtained by Cinematic MR imaging (CineMRI) and physiological and pathophysiological parameters.

Methods and Materials: Two groups of patients were recruited in a period of 6 months: 18 patients without symptoms (group $A$, age $41.83 \pm 15.05$ years), who performed MRI of the pelvis for other indications, 13 patients (group B, age 60.84 \pm 11.30 years) with clear symptoms attributable to pelvic floor dysfunction. All patients were given a questionnaire designed to obtain physiological data (age, gender, $\mathrm{BMI}$ ) and pathological (diabetes, comorbidities, previous pelvic surgery). The MRI provided a morphological study with T2-weighted sequences in the axial sagittal and coronal plane and an Cinematic FIESTA (Fast Imaging Employing SteadyState Acquisition) in the sagittal plane at rest and during the phase of maximum straining, to measure the line pubo-coccygeal ( $P C L)$, the line $\mathrm{H}$ (pelvis diastasis) and the M (lower pelvis).

Results: BMI correlated significantly with $\mathrm{H}(\mathrm{p}=0.037)$ and $\mathrm{PCL}(\mathrm{p}=0.026)$, while age correlated with $\mathrm{H}(\mathrm{p}=0.009)$ and $\mathrm{M}(\mathrm{p}=0.004)$. The parity but not the number of children causes a significant increase in pelvic lowering $(p=0.038)$ as well as previous pelvic surgery $(p=0.038)$

Conclusion: The pathophysiological changes are predictive of morphometric parameters obtained with MRI. Because of its fast acquisition a cinematic sequence could be advisable in all study protocols of female pelvis.

\section{B-0393 14:27}

Origin of the uterine artery in females using three-dimensional reconstructed contrast-enhanced MR angiography before uterine artery embolisation: where to look for it

N.N.N. Naguib, N.-E.A. Nour-Eldin, T. Lehnert, B. Schulz, S. Zangos, T.J. Vogl; Frankfurt a. Main/DE (nagy3n@yahoo.com)

Purpose: To describe the patterns of origin of the uterine artery from the internal iliac artery (IIA) before uterine artery embolisation (UAE) using three-dimensional (3D) reconstructed contrast-enhanced MR angiography (CE-MRA).

Methods and Materials: The study was retrospectively performed on 84 females (mean age 45.5 year). Pre-embolisation CE-MRA was performed before UAE. 3D images were reconstructed using Syngo-VesselView application. For each uterine artery the point of origin was identified and the angle which the artery makes with its main stem of origin was calculated.

Results: The uterine artery was detected in 165 (98.2\%) of the 168 (84 patients) arteries. The artery originated directly from the IIA anterior division in 141 arteries of the detected arteries (85.5\%), from the IIA bifurcation point in 7 arteries (4.2\%), from the IIA main stem in 3 arteries $(1.8 \%)$ and 1 uterine artery $(0.6 \%)$ originated from the IIA posterior division. The uterine artery originated indirectly from the anterior division with a common segment in 13 arteries (7.9\%). The common origin was with the obturator in 12 cases and with the internal pudendal in one case. The angle of origin of the uterine artery on the right side showed a mean of $53.2^{\circ}$ (SD: 25.23, range: $0^{\circ}-98^{\circ}$ ). On the left side the angle showed a mean value of $54.8^{\circ}$ (SD: 17.72 , range: $19^{\circ}-91^{\circ}$ ).

Conclusion: The uterine artery arises typically directly from the IIA anterior division. Atypical origins like origin from the IIA main stem or from the IIA posterior division should be searched for when the artery could not be found in its typical location.

\section{B-0394 14:36}

Fibre tracking evaluation of sacral nervous pathways 3D architecture in women affected by endometriosis

M. Sergi, V. Vinci, S. Bernardo, P. Sollazzo, M. Saldari, L. Manganaro; Rome/IT (mariaeleonora.sergi@gmail.com)

Purpose: To evaluate feasibility of tractography to study sacral nerves in women affected by pelvic endometriosis.

Methods and Materials: We enrolled 2 groups of patient: 25 women with US diagnosis of endometriosis and a control-group of 10 women. With $3 \mathrm{~T} \mathrm{MRI}$, we reconstructed nervous pathway postprocessing DTI images. We analysed 2 main parameters: 3D architecture of nervous pathways and fractional anisotropy values (FA). We analysed fibres length and branches morphology, thereafter we assigned to each a range of values obtaining 3 main classes of pathways: $1^{\text {st }}$ class (normality, $3-4), 2^{\text {nd }}$ class (mild alteration, 5-6) and $3^{\text {rd }}$ class (severe alteration, 7-8).

Results: Analysing 3D nervous pathways, we obtained a homogeneous distribution of the control group (10pt) in the first class (normal pathways). All cases of endometriosis of the posterior compartments (18/25 patients) presented an abnormal pathway ( $3^{\text {rd }}$ class), while remaining cases with anterior-medial localisation were equally distributed in $1^{\text {st }}$ and $2^{\text {nd }}$ class. We obtained low FA values in both groups; however, in case-control group range values were included between 0.47 and 0.34 while in patient groups FA values were lower between 0.36 and 0.24 .

Conclusion: Fibre tracking allowed a complete evaluation of the female pelvis defining the global situation of the patient and leastly formulating a potential explanation to symptoms. Fibrotic reaction, adhesions and nerves growth stimulation factors may explain both of the altered branch morphology and the lower FA values. We suggest tractography as a complementary tool for the female pelvis study, representing the only non-invasive way to study nervous pathways.

\section{B-0395 14:45}

How to improve magnetic resonance accuracy in detection of deep infiltrating colorectal endometriosis: correlation with laparoscopy and histopathology

M. Miccò, A.L. Valentini, B. Gui, V. Ninivaggi, M. Marino, L. Bonomo; Rome/IT (ninivaggi.valeria@hotmail.it)

Purpose: To verify whether MRI accuracy in diagnosing deep infiltrating colorectal endometriosis (DICE) can be improved using an association of two selected findings. Laparoscopy or histopathology was taken as the gold standard.

Methods and Materials: Imaging database of our Institute was retrospectively reviewed. T2-weighted MR images were evaluated by two experienced radiologists using two criteria. First, not thickened or thickened intestinal wall surrounded by fat-plane interface represents a normal result; nodules/hypointense plaque-like lesions in the adjacent fat-plane joint to thickened intestinal wall are considered an abnormal result. Second, not thickened or thickened intestinal wall surrounded or not by fat-plane interface represents a normal result; nodules/hypointense plaque-like lesions in the adjacent fat-plane joint to thickened intestinal wall showing "radiating retracting shape" are considered an abnormal result. A third radiologist looked in medical history for laparoscopy and histopathology. MRI results were compared with laparoscopy or histopathology findings by $2 \times 2$ tables and were statistically analysed ( $\mathrm{K}$ statistics).

Results: 33/50 patients underwent laparoscopy. Intestinal resection was performed in 11/33 patients; in 22/33 superficial foci, adhesions or nodules were removed. MRI sensitivity, specificity, negative predictive value and positive predictive value were $100 \%, 27.2 \%, 100 \%$ and $40.7 \%$, respectively (agreement $=51.5 \%$; $\mathrm{k}$ value $=0.20$; $p<0.055)$ when using the first, and $100 \%, 95.4 \%, 100 \%$ and $91.6 \%$, respectively (agreement $=96.9 \% ; \mathrm{K}$ value $=0.93 ; \mathrm{p}<0.0000$ ) when using the second criterion. Conclusion: Nodules/hypointense plaque-like lesions joint to thickened intestinal wall showing "radiating retracting shape" improve MRI accuracy in DICE diagnosis. 


\section{B-0396 14:54}

Preliminary functional study with MR defecography in patients suffering from deep endometriosis

P. Sollazzo, S. Bernardo, M. Sergi, V. Vinci, M. Saldari, L. Manganaro; Rome/IT (valeriavinci87@yahoo.it)

Purpose: We evaluated feasibility of $3 \mathrm{~T}$ system in the detection of endometriosis implants in the Douglas Pouch, in order to offer a precise preoperative diagnosis of deep infiltrative endometriosis involving this particular region.

Methods and Materials: From October 2010 and April 2011, we enrolled 19 women coming with either ultrasound or anamnestic suspect of endometriosis. Pelvic MRI examination was performed on $3 \mathrm{~T}$ system. We theoretically divided female pelvis in three compartments and concentrated on the posterior one, evaluating, in particular, the uterosacral ligaments and the specific signs of posterior cul-de-sac obliteration. Results: MRI diagnosed posterior cul-de-sac obliteration in 15/19 patients. We detected in 5/15 cases nodular endometriosis implants in PCS; signs of fibrotic plaque on the uterine serosal surface were identified in $4 / 15$ cases; $3 / 15$ cases showed a tethered appearance of the rectum, $6 / 15$ cases presented an elevation of the posterior vaginal fornix. In $3 / 15$ cases strands between uterus and intestine was clearly depictable, at last, involvement of utero-sacral ligaments (USLs) and signs of utero-sacral ligaments involvement were identified in 6/15 cases. Moreover, glandular-like collection localised behind uterus was identified in 7/15 cases. MRI findings were compared with laparoscopy, thus obtaining a sensitivity value of $93 \%$, and specificity value of $75 \%$

Conclusion: Precise preoperative mapping of posterior cul-de-sac region is essential for a preoperative planning. In our work 3 T MRI revealed excellent in the evaluation of posterior cul-de-sac obliteration associated with an optimal evaluation of the utero-sacral ligaments, due to the higher contrast spatial resolution.

\section{B-0397 15:03}

Diffusion-weighted magnetic resonance imaging of the female pelvis: do apparent diffusion coefficient values of the fat tissue change over the menstrual phases?

F. Fornasa, A. Gasparini, F. Pantalone, M. Bellotti, A. Dibenedetto, C. Cicciò; San Bonifacio/IT (francescafornasa@libero.it)

Purpose: To establish whether the apparent diffusion coefficient (ADC) measured with DWI in the pelvic fat tissue of healthy reproductive-age women significantly varies from the proliferative to the periovulatory phase of the menstrual cycle. Methods and Materials: 27 women (18-40 years), not assuming oral contraceptives nor referring any vaginal discharge or abnormalities of their menstrual cycles, underwent pelvic DWI (1.5 T, 8-channel coil, sagittal scans, b values: 0 and 800 $\mathrm{mm} / \mathrm{sec} 2$ ) both at the $5^{\text {th }}$ day after the beginning of the menstruation and repeatedly every day in the periovulatory phase; among these latter examinations, the one corresponding to the $14^{\text {th }}$ day before the subsequent cycle was retrospectively selected. An ADC value resulting from the average of three measurements obtained at slightly different position was calculated for each examination within a circularshaped, 4-mm diameter region of interest totally included in the adipose tissue of the ischiorectal fossa. The statistical significance of the differences between the $A D C$ values measured in the two menstrual phases was determined using the Student's t-test per paired data.

Results: The ADC values measured in the pelvic adipose tissue in the proliferative phase of the menstrual cycle (mean: $0.765 \mathrm{~mm} 2 / \mathrm{sec}$, range: $0.26-1.26$ ) were higher than those obtained in the periovulatory phase (mean: $0.558 \mathrm{~mm} 2 / \mathrm{sec}$, range: $0.24-0.89)$. The difference was highly significant at statistical analysis $(p<0.001)$. Conclusion: The ADC values measured with DWI in the pelvic adipose tissue of healthy reproductive-age women are significantly higher during the proliferative than in the periovulatory phase of the menstrual cycle.

\section{B-0398 15:12}

Effect of different birthing positions on pelvic bony dimensions in healthy women and pregnant patients in MR pelvimetry

S. Zangos, K. Eichler, A. Schollenberger, A. Reitter, F. Louwen, P. Siebenhandl, T. Gruber-Rouh, T.J. Vogl; Frankfurt a. Main/DE (zangos@em.uni-frankfurt.de)

Purpose: The aim of this study was to evaluate whether pelvic dimensions changes with pregnancy or different birthing positions.

Methods and Materials: MR pelvimetry was performed in 40 nonpregnant female volunteers and in 40 women with a foetus in a breech presentation in a 1.5-T MR imaging system (Magnetom Espree; Siemens, Erlangen). Images were taken in the supine and hand-to-knee position. The obstetric conjugate, sagittal inlet, outlet and midpelvic diameter, and interspinous, intertuberous, and transverse diameters were measured on the MR console and compared among positions. The obstetric conjugate and the sagittal inlet and outlet were both assessed in the midsagittal plane. Results: MR pelvimetry in the different positions proved feasible in all subjects, yielding diagnostic quality images in every volunteer and pregnant woman, although the hand-to-knee positions were found difficult to maintain. In the hand-to-knee position, pelvic inlet reduced from $13.3 \mathrm{~cm}$ to $13.0 \mathrm{~cm}$ and obstetric conjugate from $12.6 \mathrm{~cm}$ to $12.3 \mathrm{~cm}$. In contrast, sagittal midpelvic diameter enlarged from $13.3 \mathrm{~cm}$ to $13.6 \mathrm{~cm}$, pelvic outlet anteroposterior diameter from 11.5 to $12.0 \mathrm{~cm}$ and sagittal pelvic outlet diameter from $8.7 \mathrm{~cm}$ to $9.1 \mathrm{~cm}$.

Conclusion: Our results showed that a hand-to-knee birthing position expands sagittal midpelvic diameter, pelvic outlet anteroposterior diameter and sagittal pelvic outlet diameter, suggesting facilitation of labour and delivery.

\section{B-0399 15:21}

Retrospective analysis of elements that can predict short- and longterm outcome in patients affected by uterine leiomyomas and treated with MRgFUS

F. Ciolina, A. Napoli, F. Zaccagna, F. Boni, L. Bertaccini, V. Noce,

B. Cavallo Marincola, C. Catalano; Rome/IT (federica.ciolina@gmail.com)

Purpose: To retrospectively evaluate predictive factors to obtain an optimal treatment of uterine fibroids treated using Magnetic Resonance guided Focused Ultrasound (MRgFUS).

Methods and Materials: 68 fibroids $(52 \pm 18 \mathrm{~mm})$ in 55 symptomatic women (age

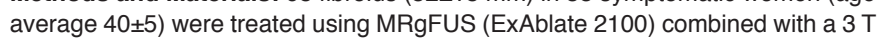
MR unit (GE). T2 SI and T1 SI of fibroids were measured on pre-treatment examination before and after administration of gadolinium (Gd-BOPTA, Bracco); also the distance between centre of myoma and skin, skin thickness, maximum diameter and volume of fibroids were recorded. The day of treatment the non-perfused volume (NPV) was recorded at the end of the treatment. It was considered treatment time in terms of energy $(\mathrm{J})$ used and duration of single sonication. All parameters were related to final NPV and volume shrinkage at 12 months to established short- and long-term results of treatment.

Results: Difference between T1 SI of the fibroids and healthy myometrium significantly correlates with volume shrinkage $(45 \%)$ and NPV $(60 \%, r=0.85)$. T2 SI on pre-treatment studies, distance between skin surface and centre of myoma $(53 \pm 19$ $\mathrm{mm})$ and skin thickness $(13 \pm 7 \mathrm{~mm})$ show a mild correlation with volume reduction at 12 months ( $36+18 \mathrm{~mm}$, respectively $r=-72.5 p=0.047$ and $r=69.7 p=0.03)$. Moreover, there was positive correlation between measured parameters, sonication duration and energy necessary to obtain $a>50 \%$ NPV $(70 \pm 15 \%, r=62.8, p=0.021)$. Conclusion: SI ratio on T1 $\mathrm{w}$ of uterine fibroids and myometrium on screening MRI should be considered as predictor of positive outcome for MRgFUS treatment.

\section{Neuro}

\section{SS 611}

\section{Alzheimer's and Parkinson's}

Moderators:

B. Góraj; Nijmegen/NL

T. Meindl; Landshut/DE

\section{B-0400 14:00}

$\mathrm{MCl}$ to $\mathrm{AD}$ conversion and the role of myoinositol (MI) and

$\mathrm{N}$-acetylaspertate (NAA) as biomarkers for this process

J. Walecki, M. Barcikowska, J.B. Ćwikła, T. Gabrylewicz, T. Bulski; Warsaw/PL (jerzy.walecki@cskmswia.pl)

Purpose: The objective of this study was to evaluate metabolic changes in patients suffering from $\mathrm{MCl}$ using MR proton spectroscopy. These metabolic changes seem to be biomarkers for clinical conversion from $\mathrm{MCl}$ to $\mathrm{AD}$. Among the many modalities which diagnose preclinical and early AD (e.g. PET amyloid imaging) $1 \mathrm{H}$ MRS still remains vital.

Methods and Materials: MRS was performed on a group of $51 \mathrm{MCl}$ diagnosed patients. All of them had clinical and biochemical tests as well as standard neuropsychological examinations. An $\mathrm{MCl}$ diagnosis was established following the criteria proposed by the Mayo Clinical Group. 1H MRS was performed with a single-voxel method using the PRESS sequence. Results were presented in ratios and quantitatively (LC model).

Results: All patients had at least two annual clinical check-ups. In the beginning, 14 subjects had amnestic $\mathrm{MCl}$ and the others had multidomain MCl. During clinical 
follow-up checks (median 3 years) 15 subjects had stable disease (SD), 20 had progression of disease $(\mathrm{PD})$ and 16 developed $\mathrm{AD}$. Baseline metabolic ratios $(1 \mathrm{H}$ MRS) between the 3 groups of patients indicated significant differences in the left frontal lobe in $\mathrm{ml} / \mathrm{H} 20$ ratio, between patients with SD $(0.27)$ and DP $(0.22)(P=$ $0.03)$. In groups of patients with $D P$ and $A D$ there was a significant difference in $\mathrm{NAA} / \mathrm{Cr}(1.77$ vs. 1.43$),(P=0.02)$.

Conclusion: $\mathrm{MI}$ and NAA seem to be very sensitive biomarkers for conversion from $\mathrm{MCl}$ to $\mathrm{AD}$. The significance of our metabolic ratio results needs further study.

\section{B-0401 14:09}

Evaluation of posterior cingulate region with FDG-PET and MR advanced techniques (MRS, PWI and DTI) in patients with $\mathrm{MCl}$ correlation of the methods

A. Zimny, J. Bladowska, A. Macioszek, P. Szewczyk, E. Trypka, R. Wojtynska, J. Leszek, M.J. Sasiadek; Wroclaw/PL (abernac@wp.pl)

Purpose: Posterior cingulate region (PCR) is an area of the earliest pathological changes in mild cognitive impairment (MCl). $\mathrm{PET}$ is the only imaging method included in diagnostic criteria of dementia. The aim of the study was to compare FDG-PET and MR measurements.

Methods and Materials: Fifty five patients diagnosed with $\mathrm{MCl}$ ( $66.5 \mathrm{yrs}$ ) according to clinical criteria and PET scan and 20 age-matched controls (69 yrs) underwent MR examination including MRS, PWI and DTI. Values of metabolite ratios (NAA/ $\mathrm{Cr}, \mathrm{Cho} / \mathrm{Cr}, \mathrm{ml} / \mathrm{Cr}$ ), cerebral blood volume (rCBV) and fractional anisotropy (FA) were compared to the rates of glucose metabolism within PCR.

Results: Compared to controls, $\mathrm{MCl}$ patients showed significant $(\mathrm{p}<0.05)$ glucose hypometabolism and decreased rCBV and FA values. FDG-PET results correlated significantly only with rCBV values. Compared to FDG-PET, PWI showed similar and DTI higher accuracy in distinguishing MCl from controls. According to FDG-PET findings 2 groups of $\mathrm{MCl}$ patients were established: with normal (30 subjects) and lower (25 subjects) glucose metabolism within PCR. MCl subjects with normal PET scan showed normal MRS and PWI results but significantly decreased FA values while $\mathrm{MCl}$ subjects with glucose hypometabolism revealed normal MRS findings but lower rCBV and FA values.

Conclusion: Advanced MR techniques such as PWI and DTI may be regarded as competitive techniques to FDG-PET, with PWI showing similar and DTI higher accuracy in distinguishing $\mathrm{MCl}$ from the control group. DTI was the only method to show changes in $\mathrm{MCl}$ patients with normal FDG-PET scan and normal PWI and MRS findings.

\section{B-0402 14:18}

The relation of uric acid with brain atrophy and cognition: the Rotterdam scan study

B.F.J. Verhaaren, M.W. Vernooij, A. Dehghan, H. Vrooman,

R. de Boer, W.J. Niessen, A. van der Lugt, M.A. Ikram; Rotterdam/NL (b.verhaaren@erasmusmc.nl)

Purpose: Uric acid has been associated with focal vascular brain disease such as lacunar infarcts and white matter lesions. However, it is unknown whether uric acid also relates to global brain changes such as brain atrophy. We therefore studied the relation of uric acid with brain atrophy and whether this is coupled with worse cognitive function.

Methods and Materials: In 814 persons of the population-based Rotterdam study (mean age 62.0 years), we studied the relation of uric acid levels with brain tissue atrophy and cognition, using linear regression models, adjusted for age, sex and putative confounders. Brain atrophy was assessed using automated processing of MRI. Cognition was assessed using a validated neuropsychological test-battery and we computed compound scores of cognitive domains.

Results: Higher uric acid levels were associated with white matter atrophy (difference in Z-score of white matter volume per standard deviation increase in uric acid: -0.07 (95\% confidence interval: $-0.12 ;-0.01)$ ). This was particularly marked when comparing hyperuricaemic to normo-uricaemic persons (Z-score difference: -0.27 $(-0.43 ;-0.11))$. Worse cognition was primarily found in persons with hyperuricaemia $(-0.28(-0.48 ;-0.08))$

Conclusion: Hyperuricaemia is related to white matter atrophy and worse cognition.

\section{B-0403 14:27}

MR spectroscopy in mild and moderate cognitive impairment as illness outcome predictor: preliminary experience

J. Forner Giner', A. Alberich-Bayarri', G. Garcia Marti', A. Pomar-Nadal', J. Rayón-Aledo ${ }^{2}$, L. Martí-Bonmatíi'; ${ }^{1}$ Valencia/ES, ${ }^{2}$ Madrid/ES (aalberich.val@quiron.es)

Purpose: To evaluate Alzheimer's disease premature diagnosis based on spectroscopy MRI

Methods and Materials: We carried out a prospective study on 178 patients (116 women and 62 men) whose ages ranged 27 to 68 years (mean age $65 \pm 12$ ). Every patient presented mild or moderate cognitive impairment according to Reisberg score (3-4 grade). Standard brain MR and spectroscopy brain MR were performed using a 3 T intensity MRI device (Archieva TX, Philips Healthcare, Best, The Netherlands). Spectroscopy MR was focused in posterior parietal and frontal lobes gray substance using a unique volume $(20 \times 20 \times 15 \mathrm{~mm})$ and two different echotimes, TE (32 and 136). Morphology and volume of temporal lobes were evaluated through FLAIR coronal images which were used to quantify temporal horn width and so classify patients in four groups. JMRUI (Java Magnetic Resonance User Interface) was used to calculate quantitative parameters in MR spectroscopy. MR was used to measure: calculation of NAA ( $\mathrm{N}$-acetyl aspartate), $\mathrm{Cr}$ (creatinine), Cho (choline) and $\mathrm{ml}$ (myo-inositol) metabolites ratios. We used analysis of variance to compare metabolite ratios between the different patient groups previously defined. Results: We found significant differences $\left(p^{2} 0.05\right)$ between groups in NAA/Cho concentration $(p=0.022)$, both in long and in short eco times and frontal and parietal anatomical regions. No other significant differences between groups were found. Conclusion: MR spectroscopy could increase efficiency in Alzheimer's disease premature diagnosis as well as turning out to be a useful additional tool for the clinician next to clinical history.

\section{B-0404 14:36}

Differentiating multiple system atrophy and Parkinson's disease on

3 T MRI: a novel use of decision tree

S. Rajandran Nair, L.K. Tan, N. Ramli, S.Y. Lim, K. Rahmat, H. Mohd Nor; Kuala Lumpur/MY

Purpose: Differentiation of multiple system atrophy (MSA) and Parkinson's disease (PD) remains a clinical challenge. The purpose of this study was to develop a decision tree using parameters obtained from standard MRI and diffusion tensor imaging (DTI) to distinguish these clinical entities.

Methods and Materials: We performed standard MRI brain and DTI at 3.0 T on 26 PD and 13 MSA patients. Linear, volumetry and DTI (fractional anisotropy, FA and mean diffusivity, MD) of putamen, substantia nigra, pons, middle cerebellar peduncles (MCP) and cerebellum were measured. A three-node decision tree was developed, with the aim of $100 \%$ specificity at node $1,100 \%$ sensitivity at node 2 and highest combined sensitivity and specificity at node 3 .

Results: Mean MCP width, anteroposterior diameter of pons and mean FA MCP with cut-off values of $14.6 \mathrm{~mm}, 21.8 \mathrm{~mm}$ and 0.55 respectively, were selected for the decision tree from nine parameters (mean width, FA and MD of MCP; anteroposterior diameter of pons; cerebellar FA and volume; pons and mean putamen volume; mean FA substantia nigra compacta-rostral) that showed statistically significant ( $P$ $<0.05$ ) differences between MSA and PD. The decision tree accurately classified 12 out of 13 MSA patients with an overall $92 \%$ sensitivity, $96 \%$ specificity, $92 \%$ PPV and $96 \%$ NPV.

Conclusion: The decision tree could be used as a diagnostic algorithm in the differentiation of MSA and PD.

\section{B-0405 14:45}

Subthalamic nuclei 3 Tesla T2-relaxometry correlated to UPDRS scores in pre-deep brain stimulation (DBS) patients with Parkinson disease with consideration to the laterality of the disease

T.M.L. Lönnfors-Weitzel, T. Weitzel, J. Slotboom, C. Kiefer, A. Kaelin-Lang, C. Pollo, R. Wiest; Berne/CH (tarja.loennfors@insel.ch)

Purpose: Degeneration within the subthalamic nucleus (STN) in Parkinson's disease (PD) is a potential marker for targeting DBS. We aimed to investigate whether pre- and postoperative Unified Parkinson's Disease Rating Scale (UPDRS) scores are related to T2 relaxation (T2r) of the STN and whether there is a correlation of laterality between clinical assessment and $\mathrm{T} 2 \mathrm{r}$.

Methods and Materials: 12 patients and 14 controls were examined at 3 Tesla by a multicontrast spinecho sequence. STN and control volumes relaxation times were analysed UPDRS scores were determined during "on" and "off" pre- and postoperatively. 
Results: The preoperative UPDRS motor scale during the off periods correlated significantly T2r in the STN (Pearson corr.; $r=0.72, \mathrm{Cl} 99 \%$ ). Outcome, estimated as a difference of preoperative UPDRS motor scale and postoperative UPDRS motor scale correlated with T2r in the STN (Pearson corr. $r=0.82, \mathrm{Cl} 99 \%$ ). UPDRS improvement correlated with the laterality between the right to left STN (Pearson corr.; $r=0.73, \mathrm{Cl} 99 \%$ ).

Conclusion: T2r values correlated with pre-DBS UPDRS motor scales in the STN. The laterality of right vs. left STN T2r predicts the outcome for UPDRS II. T2r reflects the lateralisation of PD that may be further incorporated into planning of unilateral DBS.

\section{B-0406 14:54}

\section{Increased regional grey matter volume in Parkinson's disease} patients with excessive daytime sleepiness: an MRI study

M. Chondrogiorgi, L.C. Tzarouchi, A. Zikou, P. Kosta, M.I. Argyropoulou,

S. Konitsiotis; loannina/GR (Itzar@cc.uoi.gr)

Purpose: Excessive daytime sleepiness (EDS) is an important non-motor symptom of Parkinson's disease (PD). The underlying neuropathological mechanism accounting for EDS is not well understood. The purpose of the present study was to determine regional grey matter (GM) volume changes in PD patients with EDS. Methods and Materials: Seventeen PD patients with EDS (Epworth Sleepiness Scale ESS ${ }^{3}$ 12) (EDS-PD), 17 age and disease duration-matched PD patients (Epworth Sleepiness Scale ESS ${ }^{2}$ 6) and 17 age-matched healthy controls were enrolled in the study. The $\chi^{2}$ and Student's $t$ tests were used to test for differences in demographic and clinical characteristics between groups. Differences in GM volume between groups were evaluated by applying the voxel-based morphometry (VBM) method.

Results: Total calculated levodopa equivalent dose was higher in EDS-PD when compared to PD patients $(p<0.05)$. Comparison of EDS-PD with PD patients and controls showed increased GM volume bilaterally in the hippocampus, the parahippocampal gyrus, the fusiform gyrus and in cortical areas in the temporal, frontal and parietal lobes $(p<0.001)$.

Conclusion: EDS-PD patients present increased regional GM volume in the mesolimbic/mesocortical dopamine pathway, which is activated during sleep. Drug-induced GM volume increase through a process of neuronal plasticity may represent the underlying mechanism.

\section{B-0407 15:03}

Olfactory tract evaluation in patients with Parkinson disease (PD) and hyposmia: a diffusion tensor imaging (DTI) study

F. Pitocco, L. Di Biase, A. Giona, L. Scarciolla, C. Mallio, Y. Errante, J. Melgari, B. Beomonte Zobel, C. Quattrocchi; Rome/IT (y.errante@unicampus.it)

Purpose: Olfactory dysfunction is the second most frequent feature of $\mathrm{PD}$, occurring in $70-90 \%$ of patients. Recent studies suggest that the olfactory system is among the earliest brain regions involved in PD. Diffusion-weighted imaging (DWI) and diffusion tensor imaging (DTI) consent quantitative measures of the microstructural integrity of nuclei and fibre tracts. Our aim was to assess fractional anisotropy (FA) changes of the olfactory tract in early PD patients (Hoehn ed Yahr stage 1-2.5) and to correlate FA values with the clinical score of the olfactory deficits

Methods and Materials: A case control study was conducted on 15 PD patients responding to UK PD Society Brain Bank for probable PD and 13 healthy controls that underwent MRI (1.5T, Avanto, Siemens, Erlangen, Germany). We performed T1 weighted MPRAGE, T2 and PD weighted TSE and EPI (on 12 non-collinear directions at $b 0$ and $1000 \mathrm{~s} / \mathrm{mm} 2$ to assess DTI) sequences. FA was computed with standard formulas. All subjects underwent UPSIT (University of Pennsylvania Smell Identification Test)

Results: We found a significant difference between FA values of the gyrum rectum subcortical white matter of PD patients and healthy controls $(0.27 \pm$ vs. $0.39 \pm ; p$ $<0.05$ ). A significant difference was also found in UPSIT values between the two groups $(p<0.05)$.

Conclusion: Our results suggest that early olfactory dysfunction in patients with PD may be a primary consequence of damage to the olfactory tract. Diffusion tensor Imaging reaches the diagnostic sensitivity to assess early olfactory dysfunction in $\mathrm{PD}$ patients
B-0408 15:12

The neural correlates of anomia in the conversion from mild cognitive impairment to Alzheimer's disease

E. Pravata', J. Tavernier', R. Parker', H. Vavro ${ }^{3}$, J.E. Mintzer', M. Spampinato 1 ; ${ }^{1}$ Charleston, SC/US, ${ }^{2}$ Gainesville, FL/US, ${ }^{3}$ Zagreb/HR (emanuele.pravata@gmail.com)

Purpose: Language impairment is frequently observed in patients with Alzheimer's disease $(A D$; in this study, we investigated the extent and distribution of brain atrophy in subjects with conversion from mild cognitive impairment (MCl) to $A D$ with and without naming difficulties.

Methods and Materials: This study was approved by the Institutional Review Board and was HIPAA compliant. All subjects or their legal representatives gave informed consent for participation. Ninety-one subjects from the Alzheimer's disease neuroimaging initiative $(A D N I)$ with $(\mathrm{N}=51)$ and without $(\mathrm{N}=40)$ naming impairment as per the Boston naming test (BNT) underwent brain magnetic resonance (MR) imaging 12 months before and at AD diagnosis. Structural MR images were processed using voxel-based morphometry. Longitudinal and cross-sectional comparisons between groups were performed to assess differences in gray matter (GM) loss patterns. Results: During the 12-month prior to diagnosis, $A D$ patients with naming difficulties showed distinct areas of greater GM loss in the temporal lobes, cingulate gyri, and precunei than patients without naming difficulties. GM volume in the left fusiform gyrus (Brodmann area 20) was greater in patients without naming impairment. Conclusion: This study provided evidence of distinct patterns and dynamics of brain atrophy in $A D$ patients with naming difficulties when compared with those with intact language, as early as 12 months prior to AD diagnosis. Language deficits and their neuroanatomical correlates should be taken into account in the design of a structural imaging biomarker for the early detection of $A D$.

\section{B-0409 15:21}

Gender differences in grey matter atrophy patterns in the mild cognitive and early Alzheimer's disease

M. Spampinato ${ }^{1}$, E. Pravata'1, B. Langdon ${ }^{1}$, K. Patrick', R. Parker ${ }^{2}$;

${ }^{1}$ Charleston, SC/US, ${ }^{2}$ Gainesville, FL/US (emanuele.pravata@gmail.com)

Purpose: To investigate gender-specific differences in grey matter (GM) volume loss patterns in amnestic mild cognitive impairment $(\mathrm{aMCl})$ patients with and without progression to Alzheimer's disease (AD) using MR voxel-based morphometry (VBM). Methods and Materials: We included $186 \mathrm{aMCl}$ patients (128 males, 58 females; age $77 \pm 7$ years), with $(N=91)$ and without $(N=95)$ conversion to $A D$, from the Alzheimer's disease neuroimaging initiative (ADNI) cohort. Three-dimensional MPRAGE brain scans obtained one year before conversion from $\mathrm{aMCl}$ to $\mathrm{AD}$ and at $A D$ diagnosis were available. The general linear model was performed to evaluate for differences over time in cognitive decline from the clinical dementia rating (CDR) scale. Optimised VBM with SPM8 was used to assess GM volume differences by performing cross-sectional and longitudinal group comparisons with two-sample and paired t-tests.

Results: Both male and female AD converters showed significant cognitive decline at $C D R(p<0.001)$. Total GM volumes significantly differed between all groups of males and females $(p<0.01)$. Compared to stable aMCl, male AD converters showed atrophy progression one year prior to AD diagnosis in the limbic, temporal, parietal, frontal, insular lobes and deep gray nuclei, while female $A D$ converters showed only one area of significant GM loss in the right uncus $(p<$ 0.05 FWE-corrected).

Conclusion: The extent and distribution of regional GM volume loss in aMCl to $A D$ converters is strongly influenced by the gender. This finding has potential implications for therapeutic approaches in $A D$ and should be taken into account in clinical trials. 


\section{Cardiac}

\section{SS 603a}

\section{CT and MRI: risk stratification}

\section{Moderators:}

E.A. Mershina; Moscow/RU

M.R. Rees, Gwynedd/UK

\section{B-0410 14:00}

Updated algorithms using "in-office-evaluation" and calcium score may reduce referral of stable angina patients to computed tomography coronary angiography

A.S. Dharampal, A. Rossi, A. Dedic, S.L. Papadopoulou, A. Weustink,

K. Nieman, E. Boersma, P.J. de Feijter, G.P. Krestin; Rotterdam/NL

(anoesh_dh@hotmail.com)

Purpose: The use of CT-scans in assessment of coronary artery disease is rapidly growing. More selective referral of stable angina patients with intermediate probability of coronary artery disease (CAD) to CT coronary angiography (CTCA) may temper the health care spending on cardiac CT-scans. The value of updated algorithms using "in-office-evaluation" and calcium score (CaSc) is investigated. Methods and Materials: We evaluated retrospectively 2042 symptomatic stable angina patients who underwent "in-office-evaluation", unenhanced CT-scan for calculation of $\mathrm{CaSc}$ and CTCA. Outcome of our study was non-invasive diagnosis of obstructive CAD $(350 \%$ lumen diameter narrowing) by CTCA. Two algorithms were developed. First algorithm used "in-office-evaluation" consisting of chest pain typicality, sex, age, risk factors and ECG. The second algorithm used both "in-office-evaluation" and CaSc. Discrimination of both models was tested using the area under the ROC curves (AUC). Patients were classified to low $(<10 \%)$, intermediate (10-90\%) and high pre-test probability ( $390 \%)$ of obstructive CAD. Testing with $\mathrm{CaSc}$ or CTCA was deemed appropriate in patients intermediate probability of CAD. Net

reclassification improvement (NRI) of algorithm 2 was calculated in patients with intermediate probability of CAD according to algorithm 1 .

Results: Discrimination of CAD was significantly higher using algorithm 2 compared with algorithm 1 (AUC: 0.90 vs. 0.80 , respectively). The "in-office-evaluation" algorithm reduced both unenhanced CT-scan and CTCA by $12 \%$. An additional $32 \%$ of CTCA-scan was reduced using algorithm 2 subsequently with NRI of $66 \%$. Conclusion: Updated algorithms using both "in-office-evaluation" and CaSc may reduce referral of stable angina patients to CTCA by $44 \%$.

\section{B-0411 14:09}

\section{Dynamic CT perfusion imaging for the detection of myocardial} ischaemia: a first experience with a 3D semi-automated evaluation software

U. Ebersberger ${ }^{1}$, R.P. Marcus ${ }^{2}$, U.J. Schoepf ${ }^{1}$, P. Blanke', Y. Wang ${ }^{3}$, L. Geyer ${ }^{1}$, A.D. McQuiston ${ }^{1}$, D. Bernhardt", F. Bamberg ${ }^{2} ;{ }^{1}$ Charleston, SC/US, ${ }^{2}$ Munich/DE, ${ }^{3}$ Beijing/CN, ${ }^{4}$ Forchheim/DE (ebersberger@gmx.net)

Purpose: To evaluate the performance of a 3D semi-automated evaluation software for its accuracy and time saving potential for CT perfusion myocardial blood flow (MBF) and blood volume (MBV) assessments compared to manual evaluation as well as to SPECT.

Methods and Materials: 37 dynamic, time-resolved myocardial perfusion CT datasets acquired using a dual-source CT system (Somatom Definition Flash, Siemens Healthcare, Forchheim, Germany) were assessed both manually and by use of the semi-automated prototype for MBF and MBV, based on the AHA 16-segment model. Time required for each assessment was recorded. Results were compared to SPECT as the standard of reference.

Results: 592 segments were evaluated. 19 (3.21\%) were excluded due to insufficient coverage. A comparison of MBF and MBV measurements using the prototype versus manual assessment showed high correlation (Spearman's rank correlation coefficient $=0.85 / 0.83, p<0.0001$ ). Mean evaluation time for MBF and MBV analysis was $49.1 \pm 11.2$ minutes with manual assessment and $16.5 \pm 3.7$ minutes with the use of the prototype $(p<0.001)$.

Conclusion: 3D semi-automated assessment of dynamic myocardial perfusion CT highly correlates with manual assessment. Significantly decreased assessment time appears promising for the integration of myocardial perfusion CT in clinical workflows.
Author Disclosures:

U.J. Schoepf: Grant Recipient; Bayer-Schering, Bracco, General Electric, Medrad, and Siemens. D. Bernhardt: Employee; Siemens.

\section{B-0412 14:18}

Quantitative evaluation of myocardial perfusion reserve at 3 and 1.5 Tesla in comparison to invasive measurement of fractional flow reserve for detection of coronary artery disease T. Walcher, K. Ikuye, W. Rottbauer, J. Woehrle, P. Bernhardt; UIm/DE (peter.bernhardt@uniklinik-ulm.de)

Purpose: Fractional flow reserve (FFR) is considered as the standard invasive diagnostic tool to determine haemodynamic significance of coronary artery disease (CAD). Adenosine-perfusion cardiac magnetic resonance imaging (CMR) at 1.5 and 3 Tesla has been proven to be capable to noninvasively determine myocardial perfusion reserve, but has not been compared against each other and validated against FFR as standard reference.

Methods and Materials: 34 patients $(62.0 \pm 10.9$ years) with suspected CAD were included into the study. All patients were scanned at both field strengths, 1.5 and 3 Tesla, including adenosine and rest perfusion imaging. Coronary $x$-ray angiography including FFR measurement in the left anterior descending (LAD), circumflex $(C X)$ and right coronary artery (RCA) was then performed in all patients. Myocardial perfusion reserve was calculated in 544 myocardial segments for each field strength and compared to the FFR measurement of the supplying coronary artery. FFR 20.8 was regarded as relevantly reduced.

Results: In 38 coronary arteries ( 19 LAD, 8 CX, 11 RCA) a FFR ${ }^{2} 0.8$ was found. Receiver operator curve analysis yielded higher area under the curve for $3 \mathrm{~T}$ in comparison to 1.5 T CMR ( 0.963 vs. $0.645, p<0.001)$ resulting in higher sensitivity (0.91 vs. 0.62 ) and specificity (1.0 vs. 0.77 ).

Conclusion: Quantitative evaluation of myocardial perfusion reserve at 3 and 1.5 Tesla is capable to detect haemodynamic significance of coronary artery stenosis. In our cohort, the diagnostic accuracy at $3 \mathrm{~T}$ seems to be superior to $1.5 \mathrm{~T}$.

\section{B-0413 14:27}

Dynamic stress computed tomography perfusion imaging for the detection of functionally significant coronary lesions A. Rossi', A. Dharampal', S.E. Petersen'2, E. Klotz ${ }^{3}$, G.P. Krestin', K. Gruszczynska ${ }^{4}$, E. Capuano 2 , P.J. de Feyter ${ }^{1}$, F. Pugliese ${ }^{2} ;{ }^{1}$ Rotterdam/NL, ${ }^{2}$ London/UK, ${ }^{3}$ Forchheim/DE, ${ }^{4}$ Katowice/PL (a.rossi@erasmusmc.nl)

Purpose: To evaluate the performance of hyperaemic myocardial blood flow (MBF) derived from stress computed tomography perfusion (CTP) imaging in the detection of functionally significant coronary lesions defined by fractional flow reserve (FFR) ${ }^{2} 0.75$ in a prospective cohort of patients with stable chest pain.

Methods and Materials: Coronary computed tomography angiography (CTCA) and CTP were performed in 50 patients ( 35 males/ 15 females; mean age $58 \pm 9$ years) referred to invasive angiography. A second-generation dual-source CT scanner (Somatom Definition Flash, Siemens) with a dynamic ECG-triggered axial shuttle mode was used. This technique provides an arterial input function and myocardial time-attenuation curves fitted to a 2-compartment model to give MBF. Hyperaemia was induced by infusion of adenosine (140 $\mathrm{gg} / \mathrm{kg}$ body weight). Three to 4 minutes into the infusion, $60 \mathrm{ml}$ of contrast were injected. Gantry rotation time was 285 $\mathrm{ms}$, collimation $64 \times 0.6 \mathrm{~mm}$, tube voltage $100 \mathrm{kV}$ and the tube current-time product was $300 \mathrm{mAs} /$ rotation.

Results: FFR ${ }^{2} 0.75$ was found in 22/61 (36\%) vessels. MBF was 70 (61-75) $\mathrm{ml} / 100$ $\mathrm{ml} / \mathrm{min}$ in lesions with FFR 20.75 and $99(82-127) \mathrm{ml} / 100 \mathrm{ml} / \mathrm{min}$ in lesions with FFR $>0.75(p<0.001)$. The optimal cut-off value of MBF to discriminate functionally significant coronary lesions was 79 (61-75) $\mathrm{ml} / 100 \mathrm{ml} / \mathrm{min}$. MBF had $91 \%$ sensitivity and $80 \%$ specificity and an area under the receiver operating characteristics curve (AUC) of $0.86(95 \% \mathrm{Cl}, 0.76-0.95, \mathrm{p}<0.001)$. By comparison, visual CTCA analysis yielded $73 \%$ sensitivity, $51 \%$ specificity and an AUC of $0.62(95 \% \mathrm{Cl}$, $0.47-0.77, p<0.001$ ).

Conclusion: $\mathrm{MBF}$ performs better than visual CTCA analysis to predict functionally significant coronary lesions (FFR ${ }^{20.75)}$ ).

Author Disclosures:

E. Klotz: Employee; Siemens Healthcare. 


\section{B-0414 14:36}

In vivo quantification of total atherosclerotic burden: prognostic accuracy of whole body CTA in relation to traditional cardiovascular risk index and 5-year follow-up

F. Zaccagna, A. Napoli, G. Cartocci, V. Noce, F. Boni, C. Catalano; Rome/IT (fulvio.zaccagna@libero.it)

Purpose: To investigate if mid-term prognostic value of WB-CTA to predict cardiovascular (CV) events in asymptomatic patients with $\mathrm{CV}$ risk factors can be superior to traditional method of risk stratification.

Methods and Materials: 341 pts with CV risk factors (mean age 63.39 $\pm 10.4[34-$ 89]) underwent WB-CTA $(64 \times 0.6 \mathrm{~mm})$ with an adapted contrast injection protocol (Iomeprol-400, $400 \mathrm{mgl} / \mathrm{ml} ; 70+50 \mathrm{ml} @ 4 \mathrm{ml} / \mathrm{s}$ ). Coronary arteries were divided into 15 segments and the extra-coronary arteries into 32 segments, detected stenoses were graded using a 5-point scale. An atherosclerosis burden score (ABS) was generated for each individual and correlated to traditional CV (Framingham risk index; FRI). ABS and FRI were compared using Kaplan-Meier analysis, ROC analysis and stepwise multivariable Cox regression models.

Results: At baseline mean ABS was $19.5 \pm 20.1$ and mean FRI was $12 \pm 10.7$; $64.5 \pm 11.3 \mathrm{~m}$ after WB-CTA all patients received an interview to determine health status. According to Kaplan-Meier curves, mean event-free time was of $62.3 \pm 2.4 \mathrm{~m}$ for $A B S<10,57.6 \pm 1.9 m$ for $10<A B S 20$, with a statistically significant difference between the three groups $(p=0.012)$. Mean event-free time was $57 \pm 5.6 \mathrm{~m}$ for FRI mild-risk group and $52.5 \pm 4.2 \mathrm{~m}$ for high-risk group without significant differences between the two groups $(p=0.072)$. For the prediction of CV events, in the highrisk group ABS showed an accuracy of 0.89 , meanwhile FRI of $0.69(p=0.007)$. Multivariable analysis showed that only age $(p=0.011)$, ABS score $(p=0.000)$ and therapy $(p=0.001)$ were independent predictors of hard $C V$ events.

Conclusion: WBCTA-derived ABS reflects real atherosclerotic burden and provides superior risk stratification and event prediction with respect to FRI; hard event prediction was significantly associated with age, ABS and therapy.

\section{B-0415 14:45}

Diagnostic performance of computed tomography coronary angiography to detect or exclude angiographic 'high-risk-CAD' A.S. Dharampal, S.L. Papadopoulou, A. Rossi, W.B. Meijboom, E. Boersma, K. Nieman, P.J. de Feijter, G.P. Krestin; Rotterdam/NL (anoesh_dh@hotmail.com)

Purpose: Detection of patients with "high-risk-CAD" consisting of left main (LM) and/or three-vessel CAD (3VD) is important because optimal medical treatment combined with revascularisation has shown to improve prognosis in these patients. Diagnostic performance of CT coronary angiography (CTCA) to detect or exclude "high-risk-CAD" is assessed. The discrimination of "high-risk-CAD" by CTCA is compared with Duke risk score and calcium score.

Methods and Materials: We evaluated retrospectively 1136 symptomatic patients with stable angina without prior revascularisation ( $61 \pm 11$ years, $31 \%$ women) who underwent invasive coronary angiography (ICA) and CTCA between 2004 and 2011. Vessels were considered obstructive in the presence of $350 \%$ lumen diameter stenosis.

Results: "High-risk-CAD" determined by ICA was present in 17\% (196/1136). Sensitivity of CTCA to detect "high-risk-CAD" was $95 \%$ (95\% Cl: $91 \%-97 \%)$, specificity was $83 \%(81 \%-86 \%)$, positive predictive value was $54 \%(49 \%-59 \%)$ and negative predictive value was $99 \%$ (98\%-99\%). The "high-risk-CAD" discrimination by CTCA was significantly (AUC: 0.90 ) higher compared with Duke risk score (AUC: 0.69, $p<0.0001$ ) and calcium score (AUC: $0.73, p<0.0001$ )

Conclusion: CTCA accurately rules out "high-risk-CAD" in patients with stable angina. CTCA provides incremental value in the discrimination of "high-risk-CAD" compared with the Duke risk score and calcium score.

\section{B-0416 14:54}

Cost effectiveness and rule-out strategy of MDCT-CA in patients with undifferentiated chest pain in emergency department

R. Malago, M. Tezza, C. Barbiani, A. Pezzato, G. Sala, G. Taioli,

R. Pozzi Mucelli; Verona/IT (robertomalag@yahoo.it)

Purpose: To determine whether a negative coronary CT angiography could accurately rule out patients with undifferentiated chest pain, low TIMI risk score, for a safe release from the ED. The second endpoint is to determine the prognostic value at 2 years in case of negative MDCT-CA.

Methods and Materials: Between January 2009 and August 2012, 176 consecutive patients undergoing CA-MDCT in an Emergency Department were considered. $\mathrm{Pa}-$ tients with undifferentiated chest pain were addressed into an accelerated diagnostic protocol (ADP): clinical examination, MDCT-CA; the positive cases were recovered and underwent $C A$. If MDCT-CA was negative for significant CAD the patient was dismissed from the ED and taken under follow-up for 2 years. The costs of every protocol and the amount of costs for individual methods were calculated. Based on the results, the two diagnostic paths were compared about cost/effectiveness ratio. Results: At TIMI score only $30 \%$ of the patients resulted with high risk, whereas MDCT-CA was judged positive in $40 \%$ of the cases. MDCT-CA compared to CA revealed an accuracy of $97.8 \%$, PPV and NPV were $99 \%$ and $100 \%$. The ADP revealed lower costs than the protocol with only hospitalization (11645€ against $5322 €$ ), and showed better cost/effectiveness ratio. At 2-year follow-up $99.3 \%$ of the patients resulted well being.

Conclusion: The ADP can help better than TIMI score to rule out patients with undifferentiated chest pain. Moreover, cost analysis demonstrated a better cost effectiveness ratio in comparison to the hospitalization, also confirmed with a long-term follow-up.

\section{B-0417 15:03}

Transient ischaemic dilation of the left ventricle on SPECT: correlation with findings at coronary $\mathrm{CT}$ angiography U. Ebersberger, W.T. Halligan, P.B. Morris, B.T. Mischen, S.A. Chiaramida, U.J. Schoepf; Charleston, SC/US (ebersberger@gmx.net)

Purpose: Transient ischaemic dilation (TID) in the setting of abnormal SPECT myocardial perfusion imaging (MPI) has been linked with increased cardiovascular risk. However, the significance of TID in the setting of an otherwise normal MPI study has not been clearly established.

Methods and Materials: Coronary computed tomography angiography (cCTA) was utilised to evaluate the prevalence of atherosclerotic lesions and the severity of coronary stenosis in patients with TID with or without associated myocardial perfusion defects on SPECT-MPI. The study population consisted of 1553 patients who had undergone both CCTA and stress/rest cardiac SPECT. In patients with TID coronary CTA was used to evaluate each coronary segment for the presence and composition of atherosclerotic plaque and the degree of coronary stenosis. Findings were compared with a 2:1 matched-pair control cohort without TID.

Results: TID was identified in a total of 30 patients from a screening population of 1553 consecutively imaged patients. Findings demonstrated that compared to TID negative risk-factor matched controls $(n=60)$, TID positive patients had no significant differences in the presence and extent of atherosclerosis or the degree of coronary artery stenosis as evaluated by CCTA. Similarly, there were no significant differences in CCTA measures of coronary artery disease (CAD) in TID positive patients with normal perfusion compared to TID negative patient with a normal perfusion study $(n=48)$.

Conclusion: These results suggest that the presence of TID with an otherwise normal SPECT study does not reliably predict an increased risk for or the extent and severity of coronary artery disease.

Author Disclosures:

U.J. Schoepf: Grant Recipient; Bayer-Schering, Bracco, General Electric, Medrad, and Siemens.

\section{B-0418 15:12}

Relationship between myocardial scar and coronary artery plaque in diabetes patients: from preliminary results of assessment with cardiac computed tomography angiography and magnetic resonance imaging in patients with type 2 diabetes for detection of unrecognised myocardial scar in subclinical coronary atherosclerosis (ACCREDIT) study

J.-W. Kang ${ }^{1}$, S. Choi', S. Ko', Y. Choe', B. Choi', W. Lee', T.-H. Lim'; ${ }^{1}$ Seoul/KR, ${ }^{2}$ Seongnam/KR (jwonkang@amc.seoul.kr)

Purpose: To evaluate the correlation between occult myocardial scar (OMS) on delayed-enhancement MRI (DE-MRI) and coronary atherosclerosis on CT coronary angiography (CTCA) in asymptomatic patients with type 2 diabetes.

Methods and Materials: In this prospective, multicentre, and open-label study, 347 patients with type $2 \mathrm{DM}$ were included who had 2 or more risk factors of coronary artery disease (CAD). DE-MRI and CTCA were performed on 322 patients. The prevalence of OMS and total number of involved myocardial segments on DE-MRI were evaluated. Association of the presence of OMS with clinical parameters and coronary calcium score (CCS) and the association of OMS and the degree of stenosis and plaque characteristics of coronary artery on CTCA per patient were also evaluated.

Results: OMS was detected by CMR in $23(7.1 \%)$ of the 322 patients and the number of myocardial scar is 49 (median 2 , range $1-4$ per patient). The prevalence of CAD was $74.5 \%$, and more than $50 \%$ stenosis was found in $24.9 \%$ of the subjects. 
Using logistic regression, CCS more than 100 was the predictor of presence of OMS (odds ratio $7.484,2.459-22.776$ in $95 \%$ confidence interval). The prevalence of OMS was significantly higher in patients with more than $50 \%$ stenosis and in patients with mixed and non-calcified plaques.

Conclusion: The prevalence of OMS on DE-MRI and significant coronary disease on CTCA are not uncommon even in asymptomatic patients with type 2 diabetes. CCS more than 100 is the only predictor of OMS.

\section{Author Disclosures:}

J. Kang: Research/Grant Support; Guerbet Group, France. S. Choi: Research/ Grant Support; Guerbet Group. S. Ko: Research/Grant Support; Guerbet Group. Y. Choe: Research/Grant Support; Guerbet Group. B. Choi: Research/Grant Support; Guerbet Group. W. Lee: Research/Grant Support; Guerbet Group. T. Lim: Research/Grant Support; Guerbet Group.

\section{B-0419 15:21}

\section{Epicardial fat volume and coronary artery disease at CT: racial} differences in African American and white patients with acute chest pain P. Apfaltrer ${ }^{1}$, A. Schindler ${ }^{2}$, U.J. Schoepf ${ }^{2}$, J. Nance ${ }^{2}$, U. Ebersberger ${ }^{2}$, R. Vliegenthart ${ }^{2} ;{ }^{1}$ Mannheim/DE, ${ }^{2}$ Charleston, SC/US (paul.apfaltrer@umm.de)

Purpose: Thoracic adipose tissue likely promotes coronary artery disease (CAD). Possibly, differences in CAD risk between races are influenced by racial differences in thoracic adipose tissue. We compared CT-derived measures of thoracic fat and potential correlations with CAD between African American and white patients.

Methods and Materials: 372 age-and-gender-matched black and white patients (186 black: mean age $54 \pm 11$ yrs, $50 \%$ men; 186 white: mean age $54 \pm 11$ yrs, $50 \%$ men) who underwent thoracic dual-source CT for chest pain evaluation were retrospectively included. Epicardial adipose tissue (EAT) volume and mediastinal adipose tissue (MAT) volume were quantified using an automated analysis algorithm. Peri-coronary fat thickness (PFT) was measured on reconstructed sections orthogonal to the right coronary artery centreline. Non-calcified and calcified plaque volume was quantified by applying a threshold-based automated algorithm. CAD was defined as $50 \%$ or greater coronary artery stenosis.

Results: Mean body mass index was $31 \pm 7 \mathrm{~kg} / \mathrm{m}^{2}$ for blacks and $30 \pm 6 \mathrm{~kg} / \mathrm{m}^{2}$ for whites ( $p>0.05)$. EAT and MAT were significantly lower in blacks than in whites (59[25 th $-75^{\text {th }}$ percentile, 48-108] versus $97[67-132] \mathrm{cm}^{3}$, and $44[27-77]$ versus $87[52-157] \mathrm{cm} 3$, for both $p<0.001)$. Mean PFT in blacks and whites showed no significant difference $(p>0.05)$. Patients with CAD showed significantly higher EAT than those without CAD $\left(97.9 \pm 52.6 \mathrm{~cm}^{3}\right.$ vs. $\left.77.4 \pm 43.5 \mathrm{~cm}^{3}, p<0.05\right)$. Significant correlations were observed between EAT measurements and calcium volume $(r=0.221 ; p<0.05)$. The relationship between race and extent of adipose tissue remained significant after adjustment for cardiovascular risk factors.

Conclusion: Measures of thoracic fat were generally higher for symptomatic white than for black patients suggesting a differential relationship between thoracic adipose tissue and CAD pathophysiology by race.

Author Disclosures:

U.J. Schoepf: Research/Grant Support; Bayer-Schering, Bracco, General Electric, Medrad, Siemens.

\section{Interventional Radiology}

\section{SS 609a}

\section{Oncologic ablation and guided interventions}

\section{Moderators:}

P. Almeida; Coimbra/PT

A. Basile; Catania/IT

\section{B-0420 14:00}

Radiation exposure in CT-guided interventions

R. Kloeckner, D. Pinto dos Santos, C. Düber, M. Pitton;

Mainz/DE (roman.kloeckner@googlemail.com)

Purpose: This study aimed at investigating the radiation exposure in CT-guided interventions, to establish reference levels and discuss strategies for dose reduction. Methods and Materials: 1576 consecutive CT-guided procedures in 1284 patients performed over 4.5 years were analysed including drainage placements, biopsies of different organs, radiofrequency-/ microwave-ablations of liver, bone and lung tumours, pain blockages and vertebroplasties. Data were analysed with respect to overall radiation doses and individual doses of planning CT-series, CTintervention and control CT-series. Additionally, the impact of different fluoroscopy settings (kV/mAs) and fluoroscopy modes (single slice acquisition vs. continuous CT-fluoroscopy) on the radiation dose was investigated.

Results: $85 \%$ of the total radiation dose was applied during the pre- and postinterventional CT-series leaving only $15 \%$ to being applied by the CT-guided intervention itself. Single slice acquisition was associated with lower doses compared to continuous CT-fluoroscopy $\left(37 \mathrm{mGy}^{*} \mathrm{~cm}\right.$ vs. $\left.153 \mathrm{mGy}{ }^{*} \mathrm{~cm}, \mathrm{p}<0.001\right)$. Overall, the use of $80 \mathrm{kV}$ during the interventions led to significantly lower radiation doses compared with $120 \mathrm{kV}$ (mean: $86 \mathrm{mGy}^{*} \mathrm{~cm}$ vs. $215 \mathrm{mGy}^{*} \mathrm{~cm}, \mathrm{p}<0.001$ ). Third quartile of radiation doses varied considerably for different interventions depending on complexity.

Conclusion: To our knowledge, these data represent the largest sample of CTguided interventional procedures. Therefore, we suggest preliminary reference levels for each subgroup based on the third quartiles of our analysis. The radiation dose applied can be reduced using $80 \mathrm{kV}$ instead of $120 \mathrm{kV}$ for the intervention and by preferring single-slice acquisition over CT-fluoroscopy whenever possible.

\section{B-0421 14:09}

Percutaneous image-guided cryotherapy: a new therapeutic possibility for local recurrence of soft tissue sarcomas? N. Lippa, A. Italiano, P. Sargos, M. Kind, E. Stoeckle, B. N'Guyen Bui, F. Cornelis; Bordeaux/FR (nicolippa@gmail.com)

Purpose: To determine the outcomes after treatment for patients with local recurrence of soft tissue sarcoma (STS) of the trunk and the limbs and to identify patients who could be candidates for percutaneous image-guided cryoablation.

Methods and Materials: Between 2000 and 2010, 46 patients (57 tumours) presenting local recurrences of STS of the trunk or limbs were selected from our institutional database. Two radiologists visually assessed possibility of cryotherapy according to common inclusion criteria: maximal diameter size of the tumour ${ }^{210}$ $\mathrm{cm}$, distance to skin $>5 \mathrm{~mm}$, distance to neurovascular structures at least $3 \mathrm{~mm}$, potential access, absence of articular involvement and planned cryoablation covering the entire lesion volume. Characteristics of patients eligible for cryoablation were comparing with not selected patients.

Results: Mean tumour size was $64 \mathrm{~mm}(10-178)$. Most frequent histological subtypes were unclassified sarcomas $(n=16)$, liposarcomas $(n=15)$, or myxofibrosarcomas $(n=7)$. Surgical treatment consisted in wide excision $(n=16)$, or combined treatment $(n=24)$. After surgery, the severe complications rate was $47.5 \%$. Since relapse, mean follow-up was 42.8 months with a median overall survival of 57.6 months and median recurrence-free survival of 39.5 months. A subgroup of 13 patients was identified and comparison with other patients showed that trunk, shoulder and pelvic girdle locations $(p=0.002)$, locally aggressive $(P=0.016)$ and deep ( $\mathrm{P}=0.002)$ tumours, $25 \mathrm{~cm}(\mathrm{P}=0.044)$, and liposarcoma and myxofibrosarcoma subtypes $(P=0.007)$ were indicative of eligibility for cryoablation.

Conclusion: Eligibility criteria as defined are useful for plan cryoablation as a technically feasible alternative to surgery for treatment of local relapse of STS.

\section{B-0422 14:18}

Robot-assisted radiofrequency ablation of liver tumours: early experience B.J.J. Abdullah, C.H. Yeong, K.L. Goh, B.K. Yoong, G.F. Ho, C. Yim; Kuala Lumpur/MY (basrij@ummc.edu.my)

Purpose: We report our preliminary experience of RFA of the liver tumours using a robotic-assisted guidance system.

Methods and Materials: 6 patients with 8 lesions were treated using the Robio Ex (Perfint Healthcare, MA, USA) robotic positioning system. All the lesions were smaller than $2.5 \mathrm{~cm}$ diameter. All the procedures were performed under general anaesthesia. Following baseline CT scans, the lesions were identified. The puncture site and trajectory were then determined on Robio Ex workstation. The angulation of the needle, depth of lesion, the fluoroscopic dose as well as the accuracy of placements was determined. The performance level of procedures was documented for each biopsy on a five-point scale (5-1: Excellent-Poor).

Results: All the tumours were targeted successfully. One lesion in 2 patients required minor readjustment. 4 patients (5 lesions) had needle placement with no cranio-caudal angulation, whereas 2 patients ( 3 lesions) had cranio-caudal angulation of up to 200 . The orbital angulation ranged from 60 to 1180 . The depth of needle ranged from 12.2 to $4.7 \mathrm{~cm}$ from skin surface. Performance levels were all 5 except in one patient (4). The fluoroscopic (DLP) and total dose (CTDIvol) for the entire procedure were $316 \pm 92 \mathrm{mGy} . \mathrm{cm}$ and $260.83 \pm 70.02 \mathrm{mGy}$, respectively. Post-RFA CT scans showed successful ablation of the targeted lesions. No immediate complications were noted.

Conclusion: Robotic-assisted planning and needle placement appear to be technically easier, it requires fewer number of needle passes, fewer check scans 
and has lower dose. Study with large sample size is needed to confirm these preliminary findings.

\section{B-0423 14:27 \\ Image-guided percutaneous thermal ablation of hepatic and renal tumours using high-frequency jet ventilation to minimise respiratory motion \\ D.M.L. Tse, D.Y.F. Chung, S. Scott, P. Boardman, F.V. Gleeson, E.M. Anderson; Oxford/UK (donald.tse@gmail.com)}

Purpose: High-frequency jet ventilation minimises respiratory motion during general anaesthesia (GA-HFJV). We report our 5-year experience with GA-HFJV in therma ablation of hepatic and renal tumours, and evaluate the procedural time, rates of technical success and complications.

Methods and Materials: Between November 2007 and September 2012, 76 patients (24 females and 52 males, mean 69.6years) underwent 107 sessions of radiofrequency (RF) or microwave (MW) ablation. Tumour size, time under anaesthesia, ablation time, technical success and complications were recorded. Procedural time was calculated by subtracting ablation time from time under anaesthesia. Results: 127 hepatic tumours (24 primary, 103 metastasis), 7-80 mm in diameter (mean \pm SD, $34.2 \pm 16.0 \mathrm{~mm}$ ) were treated in 99 sessions and 8 primary renal tumours, $18-37 \mathrm{~mm}$ in diameter (mean $\pm S D, 30.4 \pm 5.7 \mathrm{~mm}$ ) were treated in 8 sessions. Mean procedural time for sessions ablating single hepatic lesions was 77 minutes (range 32-144), 110 minutes (range 48-157) for sessions ablating multiple hepatic lesions, and 94 minutes (range 57-123) for ablations of renal lesions. There is no significant difference in procedural time between CT-guided alone and combined CT and USguided ablations of single hepatic lesions $(p=0.27)$. Technical success was achieved in all sessions. No major complications were observed. Minor complications in 15 sessions (14\%) included 4 cases of immediate peri-hepatic haematoma, 3 cases of haemothorax, none requiring further treatment.

Conclusion: GA-HFJV is a safe technique which reliably minimises respiratory motion. This facilitates the procedure directly by producing ideal target immobilisation, which may reduce procedural time particularly for small or anatomically challenging lesions in the liver or kidney.

\section{B-0424 14:36}

Radiofrequency ablation of hepatocellular carcinoma associated with chronic liver disease without cirrhosis: long-term experience L. Sottocornola, A. Salmi, A. Rossini, R. Maroldi, P. Cabassa; Brescia/IT (lara.sottocornola@gmail.com)

Purpose: This study aimed at determining the efficacy of radiofrequency ablation in the treatment of patients with hepatocellular carcinoma (HCC) in patients with chronic liver disease without cirrhosis.

Methods and Materials: From the database of HCC treated by RF ablation in two referring centres $56 \mathrm{HCCs}$ were found in 49 patients (age range, 62-90 years) with chronic liver disease (mainly $\mathrm{HCV}$ ) without cirrhosis. The mean lesion diameter was $24.6 \mathrm{~mm}$ (range, $10-50 \mathrm{~mm}$ ). Patients were treated percutaneously or by laparoscopic approach. Follow-up was performed by CT at 1, 4, and 6 months and every 6 months after that. Cumulative survival and disease-free survival rates of patients were analyzed using the Kaplan-Meier method. Any complications during follow-up were reported.

Results: Cumulative survival rates were $100 \%, 82 \%$, and $59 \%$ at 1,3 , and 5 years, respectively. Median survival time was 49 months (range, 1-120 months). Maximum tumour diameter was associated with the probability of survival. During follow-up 7 (14\%) out 49 patients developed new nodules. Median disease-free survival rates were $96 \%$, at 1 year and $93 \%$ at 2 and 3 years. No major complication occurred. Conclusion: Radiofrequency ablation of HCCs in patients without cirrhosis shows long-term survival rates and a low recurrence rate.

\section{B-0425 14:45}

Safety and efficacy of sonographically guided percutaneous radiofrequency ablation of left lobe hepatocellular carcinoma M.I.M.A. Ibrahim; Sohag/EG (mandw20022002@hotmail.com)

Purpose: To assess the safety and effectiveness of percutaneous radiofrequency ablation (RFA) of left lobe hepatocellular carcinoma (HCC).

Methods and Materials: We performed a retrospective analysis of 157 patients who underwent percutaneous ultrasound-guided RFA for treatment of HCC. Forty-eight HCCs in left lobe were compared with 152 HCCs in right lobe. Patients underwent regular computed tomography imaging of the liver during follow-up and the treatment effectiveness and safety were evaluated. Incomplete ablation, recurrence, complications, and mortality were documented.
Results: The sub-capsular lesions at the time of diagnosis of HCC was significantly higher in the left lobe group (38 vs. $11, p=0.02$ ). The percentages of multiple lesions of $\mathrm{HCC}$ was significantly high in right lobe group ( 6 patients had three lesions vs. $0, p=0.03)$. The percentage of patients experiencing major complication was significantly higher in the RFA left lobe group $(p=0.01)$. There was no difference in the percentage of complete ablation rate in both groups ( $96 \%$ vs. $100 \%, p=0.11$ ). In multivariate analysis, site and number of lesions were factors associated with major complication ( $\mathrm{p}=0.04$ and 0.03 , respectively).

Conclusion: Left lobe location of HCC is an important independent risk factor of major complication after percutaneous RFA of HCC.

\section{B-0426 14:54}

Percutaneous, ultrasound-guided IRE ablations of unresectable pancreatic cancer

A. Nilsson, C. Månsson, J. Krause, R. Brahmstaedt, P. Nygren, B.-M. Karlson; Uppsala/SE (anders.nilsson@radiol.uu.se)

Purpose: To evaluate if percutaneous, ultrasound-guided irreversible electroporation (IRE) ablations can be performed safely in patients with unresectable pancreatic cancer.

Methods and Materials: Between October 2011 and June 2012, 10 patients with pancreatic cancer were treated with IRE ablation. All patients had undergone chemotherapy and radiation treatment without tumour reduction to a point where they became resectable or were not candidates for such treatment. Unresectability in all cases was due to tumour growth around the mesenteric vessels. Tumour size was $2-4.5 \mathrm{~cm}$. Ablations were performed under ultrasound guidance with the patients under general anaesthesia. 3 needles were placed around the tumour, ablations performed according to the inbuilt software in the IRE generator and the needles then replaced until the entire tumour area had been covered.

Results: Contrast-enhanced ultrasound (CEUS) the day after the ablation showed ablation zones in the tumour area, size $2.5-4 \mathrm{~cm}$ in diameter. The average postoperative stay was 10 days. 8 of the patients initially had pain at the treatment site but only post-ablation inflammation could be seen on CT. 3 patients developed a pancreatitis with elevated serum amylase. No other serious complications were noted and check-up with CT and CEUS 1 month after the treatments showed no adverse effects.

Conclusion: Percutaneous IRE ablations can be performed safely in patients with locally advanced pancreatic cancer.

\section{B-0427 15:03}

High-intensity focused ultrasound ablation of pancreatic cancer: a totally non-invasive treatment for tumour control of locally advanced lesions (stage III)

F. Zaccagna, A. Napoli, G. Cartocci, G. Brachetti, L. DI Mare, F. Boni, V. Noce, L. Bertaccini, C. Catalano; Rome/IT (napoli.alessandro@gmail.com)

Purpose: To evaluate safety and effectiveness of non-invasive high-intensity MR-guided focused ultrasound (MRgFUS) treatment for tumour control of locally advanced pancreatic cancer.

Methods and Materials: 5 patients with locally advanced pancreatic cancer underwent MRgFUS treatment using the ExAblate 2100 system (InSightec). Treatments were done in a single session, in an ambulatory setting. To evaluate tumour control, perfusion T1w-images after administration of gadobenate dimeglumine (MultiHance; Bracco, Milan, Italy) were obtained pre- and post-treatment in order to determine the non-perfused sonication-related area. Follow-up examinations were scheduled at 1, 3, 6 and 12 months after treatment. After treatment, all patients underwent $\mathrm{CHT}$ with the same chemotherapy scheme.

Results: All patients well tolerated the treatment and no heating-related adverse event was recorded during this clinical application. Immediately after treatment all patients showed coagulative necrosis with a mean non-perfused volume of $65.4 \pm 16.6$ [45 - 90]. All patients showed a significative decrease of pain due to cancer infiltration. At follow-up tumour recurrence in treatment site was MRI evident but no local progression were recorded. After MRgFUS 2 patients underwent RT meanwhile one patients required another MRgFUS treatment.

Conclusion: Our preliminary clinical experience indicates that focused ultrasound under MR guidance could be a promising non-invasive treatment modality to obtain a reduction of tumour size in patients with pancreatic adenocarcinoma not suitable for surgery. 


\section{B-0428 15:12}

Fusion imaging-guided percutaneous biopsy of focal hepatic lesions with poor conspicuity on conventional ultrasonography

H. Park, M. Lee, M. Kim, M. Lee, J. Hwang, T. Kang, S. Lim, H. Lim, H. Lim; Seoul/KR (mydr.kim@gmail.com)

Purpose: To evaluate the effectiveness of real-time US and CT/MR image fusion, for percutaneous US-guided biopsy of focal hepatic lesions with poor sonographic conspicuity.

Methods and Materials: This study was conducted as a retrospective analysis of a prospective database, and was approved by the Institutional Review Board. Patients who had target lesions with poor conspicuity on B-mode US for percutaneous biopsy were enrolled in the study. Lesion conspicuity was assessed using conventional B-mode US first, and then using fusion imaging later in the same session. We compared lesion conspicuity and detection rates between B-mode US and fusion imaging and evaluated how many cases of initially invisible lesions on B-mode US became visible after image fusion. The technical success rate was evaluated based on the final diagnoses, which were established by pathological examination of the biopsy specimen as well as through clinical and radiological follow-up examination.

Results: A total of 22 patients were enrolled in this study. After image fusion, lesion conspicuity was increased in $63.6 \%$ (14/22) of focal hepatic lesions. Moreover, $66.7 \%$ (6/9) of lesions which were invisible through B-mode US became visible after image fusion. The true positive detection rate was significantly different between B-mode US and fusion imaging (9/22 vs. 19/22, $p=0.0044)$. Percutaneous biopsy was performed for all lesions, including 3 target lesions which were invisible even after image fusion. The technical success rate was $95.5 \%$ (21/22).

Conclusion: The fusion imaging technique is effective for percutaneous biopsy of focal hepatic lesions with poor sonographic conspicuity.

\section{B-0429 15:21}

CTNAV: a prospective randomised clinical trial of a navigation system for computer assisted CT guided interventions

P. Durand, A. Moreau-Gaudry, J. Frandon, M. Medici, E. Chipon, C. Sengel,

I. Bricault; Grenoble/FR (pdurand1@chu-grenoble.fr)

Purpose: The Imactis Navigation System with electromagnetic needle holder tracking was developed to allow interactive display of the needle trajectory in a 3D volumetric CT volume, allowing the radiologist to choose optimal entry point and track to the target during CT-guided percutaneous interventions. The CTNAV trial compares the accuracy of the needle placement using standard CT procedure (CT group) and using the navigation system procedure (NAV group).

Methods and Materials: Prospective, randomised, comparative, open, controlled study conducted between June 2010 and January 2012 in Grenoble University Hospital on 120 patients who had to undergo a percutaneous $\mathrm{CT}$ procedure. $\mathrm{Pa}$ tients were randomised to have normal procedure (CT group) or Navigation System assisted procedure (NAV group). The main outcome was the distance between the planned trajectory and the effective needle trajectory after the first placement attempt. Significance was set at 0.01

Results: The comparison between the two groups shows a significant difference concerning the main outcome splitted in distance and angle: distance in $\mathrm{mm}[95 \%$ $\mathrm{CI}])$ : CT: $9.49[4.92 ; 15.18]$ NAV :3.82 [2.68;8.33], $(p<1 \mathrm{e}-05)$. Angle in degrees $[95 \% \mathrm{Cl}]): \mathrm{CT}: 8.19[6.11 ; 13.27]$ NAV: $4.13[2.18 ; 7.57](\mathrm{p}<1 \mathrm{e}-05)$. The number of control CT series was lower in the NAV group. Operator satisfaction and system acceptance were high.

Conclusion: Clinical trial analysis shows that the use of the navigation system improves the accuracy of the procedure when compared with conventional interventions.
14:00 - 15:30

Room E1

\section{Musculoskeletal}

SS 610a

\section{Musculoskeletal tumours}

Moderators:

R. Lalam; Oswestry/UK

E. Quaia; Trieste/lT

\section{B-0430 14:00}

Magnetic resonance imaging parameters predictive for malignancy: a prospective study of 100 consecutive soft-tissue musculoskeletal masses

V. Vasilevska Nikodinovska, M. Samargiski, V. Janevska, S. K.Kunovska; Skopje/MK (v_vasilevska@yahoo.com)

Purpose: To evaluate magnetic resonance imaging (MRI) parameters that contribute the most in differentiation of benign vs. malignant soft tissue musculoskeletal tumours.

Methods and Materials: Prospectively, 100 consecutive patients (53 males, 47 females, median age 51 years) with a musculoskeletal soft-tissue mass underwent magnetic resonance imaging (1.5 T,GE). Fifteen MRI parameters were evaluated for making a radiologic diagnosis. Multivariate logistic regression analysis was used for imaging parameters predictive for malignancy. Histopathology was correlated with radiologic diagnosis in all patients.

Results: There were 70 benign lesions (53 benign tumours and 17 tumour-like lesions) and 30 malignant lesions (26 malignant tumours and 4 metastases). Radiological diagnosis for primary malignant tumour was established in a total of 33 lesions, of which $24(72.7 \%)$ had histological confirmation for primary malignant tumours. Nine cases $(9 \%)$ were false-positive ( 4 benign tumours, one tumour-like lesion and 4 metastases) and two (2\%) were false-negative. Radiological diagnosis for benign and malignant tumours showed identical sensitivity of $92.5 \%$ and $92.3 \%$. High specificity of $95.7 \%$ was found in benign tumours. Global accuracy in determining the presence of benign tumour was $94 \%$ and of primary malignant tumour $89 \%$. Multivariate logistic regression analysis identified the following MRI parameters to be independent significant predictive for primary malignant tumour: extracompartmental location, presence of peritumoural oedema, inhomogeneous signal on T2 WI. After i.v. application of contrast agent, peripheral and inhomogeneous enhancement significantly more often pointed to malignant tumour.

Conclusion: A combination of individual MR imaging parameters improved radiologic imaging diagnosis in differentiation between benign and malignant soft-tissue musculoskeletal lesions.

\section{B-0431 14:09}

Evaluation of features and role of ultrasound and MR imaging in large lipomas and well-differentiated liposarcomas

H. Gupta', V. Bhat'², S. Edward', E. Hensor', P. Robinson'; ' 'Leeds/UK, ${ }^{2}$ Cardiff/UK (harun.gupta@nhs.net)

Purpose: To review surgically proven well-differentiated liposarcomas and large lipomas for significantly different imaging or clinical features.

Methods and Materials: Retrospective review of surgically treated extremity/ truncal lipomas and well-differentiated liposarcomas. 3 groups identified: lipomas imaged by ultrasound and MRI, lipomas imaged by ultrasound only (size $35 \mathrm{~cm}$ ), well-differentiated liposarcoma by ultrasound and/or MRI. Imaging was independently evaluated by 2 radiologists blinded to history and case proportion. Ultrasound scored for location, depth, size, shape, echogenicity, homogenicity, septation and Doppler flow. MRI scored for location, depth, size, margins, signal characteristics, fat suppression, septation, non-adipose areas, nodules and contrast enhancement. Fischer's exact and Mann-Whitney $U$ tests were performed.

Results: 114 lipomas ( $70^{35} \mathrm{~cm}$ US only; 44 US and MRI irrespective of size) and 27 liposarcomas were evaluated. Majority of lipomas were subcutaneous. Majority of liposarcomas were lower limb (sensitivity $88 \%$, specificity $75 \%$ ). Median lipoma volume was significantly smaller than liposarcoma ( 58.5 vs $201 \mathrm{~mm} 3$ ). No significant difference in other ultrasound criteria was found. Fat suppression was complete in $91 \%$ lipomas but only $31 \%$ liposarcomas. For liposarcomas nodular areas were moderately specific [65.9\%] and sensitive [ $80.8 \%]$ but non-adipose areas specific [93.2\%] but not sensitive [42.3\%].

Conclusion: 1. Lipomas can be large and size criteria $(>5 \mathrm{~cm})$ for specialist referral is inefficient for those with typical benign imaging appearances. A size $>7 \mathrm{~cm}$ may be more effective in such cases. 2 . Ultrasound can effectively evaluate large 
subcutaneous lesions. 3. Size, depth, heterogenicity, incomplete fat suppression and nodular areas are more sensitive for liposarcoma but there is considerable overlap with benign lipomas.

\section{B-0432 14:18}

Comparison of efficacy of surgical-, CT- and PET-CT-guided biopsy in musculoskeletal lesions

J.L.M.A. Gielen, A.M. De Schepper, S. Ceyssens, D.H. Creytens, J. Somville, P. Van Dyck, P. Pauwels, M. Peeters, P.M. Parizel; Antwerp/BE (jan.gielen@uza.be)

Purpose: To compare the efficacy of surgical-, CT- and PET-CT-guided biopsy of musculoskeletal (pseudo)tumours.

Methods and Materials: Between January 2011 and July 2012, we biopsied156 musculoskeletal lesions. A new PET-CT guidance technique, targeting the area of most intense radio-labelling (AMIR), is introduced. We included 149 lesions, 36 soft tissue lesions (STL) and 113 bone lesions (BL). All patients were randomly distributed over the surgical- or CT-guided biopsy systems: CT (n68), PET-CT (n40), surgical (n41). In each case, appropriate biopsy needles were used.

Results: Correct characterisation was obtained in $80.5 \%, 88.2 \%$ and $92.5 \%$ of surgical-, CT-, and PET-CT-guided techniques, Fisher's exact test: $P=0.2756$. Overall efficacy of characterisation was $88 \%$ in benign and $86 \%$ in malignant lesions, Chi-squared test $\mathrm{X}^{2}=0.1367, \mathrm{df}=1, \mathrm{P}=0.7116$. In $\mathrm{STL}$, correct characterisation was obtained in $80 \%$ (surgical), $77.8 \%$ (CT) and $88.2 \%$ (PET-CT). In BL, correct characterisation was obtained in $80.6 \%$ (surgical), $89.8 \%$ (CT) and $95.7 \%$ (PETCT), Fisher's exact test, $\mathrm{P}=0.7329$ and 0.2974 , respectively.

Conclusion: The diagnostic efficacy of PET-CT was better, compared to surgicaland CT-guided biopsy. The efficacy rates for malignant and benign lesions, for the three techniques, were comparable. The best results were obtained in BL with CT and PET-CT techniques. Although the differences are not statistically significant, (false results are too few to find statistically significant differences), it appears that the information provided by PET for CT-guided biopsy is useful, thereby reducing the false negative rate from $19.4 \%$ to $4.3 \%$ in $\mathrm{BL}$ and from $20 \%$ to $11.8 \%$ in STL.

\section{B-0433 14:27}

CT evaluation of thoracic bone metastasis: comparison of

3D volume-rendered images and combined axial and coronal multiplanar reconstruction images

K. Doo ${ }^{1}$, E.-Y. Kang ${ }^{1}$, H. Yong ${ }^{1}$, O. Woo ${ }^{1}$, K. Lee ${ }^{2}$, Y.-W. Oh'1; ${ }^{1}$ Seoul/KR,

${ }^{2}$ Ansan/KR (kwdoo@korea.ac.kr)

Purpose: Although a correct assessment of thoracic bone metastasis is important, it is time-consuming and insensitive by conventional axial and coronal CT images. The objective of this study was to evaluate the diagnostic benefits of 3D volumerendered (VR) images for the detection of thoracic bone metastasis on CT as compared with combined axial and coronal multiplanar reconstruction (MPR) images. Methods and Materials: Among 117 consecutive patients who underwent chest CT and PET-CT or bone scan, 21 patients who had thoracic bone metastasis were included. Two radiologists independently reviewed the probability of metastasis of ribs and vertebrae on combined axial and coronal MPR, 3D VR, and 3D VR with MPR images. Diagnosis of bone metastasis was determined by PET-CT, bone scan, or biopsy. Diagnostic performance parameters and interpretation time were evaluated. Results: For all thoracic metastasis (195/756), the mean sensitivity and accuracy of 3D VR with MPR and MPR images were similar (reader 1, 62.1\%, 88.6\% vs $52.8 \%, 91 \%$; reader $2,50.3 \%, 86 \%$ vs $48.7 \%, 85.6 \%, P>0.05)$. For rib metastasis, the mean sensitivity and accuracy of 3D VR with MPR were higher than those of MPR images (reader 1, 64.1\%, $92.7 \%$ vs $48.5 \%$, $89.5 \%$.; reader $2,52.4 \%, 88.9 \%$ vs $42.7 \%, 87.5 \%, \mathrm{P}<0.05)$. The mean interpretation time was decreased on 3D VR with MPR compared to MPR images (reader 1, 73.6 vs 122.5, reader 2, 103.4 vs 182.5 seconds, $\mathrm{P}<0.05)$.

Conclusion: The addition of 3D VR to MPR images performs significantly better feasibility than combined axial and coronal MPR images for rapid evaluation of thoracic bone metastasis.

\section{B-0434 14:36}

DCE-MRI for assessment of vessel remodelling associated with bone defect healing in experimental lytic bone metastases

D. Komljenovic, W. Semmler, T. Bäuerle;

Heidelberg/DE (d.komljenovic@dkfz-heidelberg.de)

Purpose: Integrin inhibition and antiresorptive therapy decelerate osteolysis in experimental breast cancer bone metastases. Here, using volumetric CT (VCT), dynamic contrast-enhanced MRI (DCE-MRI) and histology, we assessed the impact of this therapeutic approach on the tumour vasculature.

Methods and Materials: Rats bearing breast cancer bone metastases were imaged using VCT and DCE-MRI prior to and 25 days after the onset of therapy; untreated $(n=12)$ and treated (integrin inhibitor monotherapy; $n=12$ ) or together with antiresorptive agent (combination therapy; $n=11$ ) to assess osteolysis and functional MRI parameters, respectively. Mean vessel diameter and SMA and collagen-IV-positive vessel area fraction were assessed histologically.

Results: Compared to the control group, bone defect healing at the osteolytic site occurred in rats receiving monotherapy and even more frequent after combination therapy as shown by VCT. In addition, we observed a profound effect of mono- and combination therapy on the tumour vasculature determined by DCE-MRI parameters amplitude and exchange rate constant. Both therapy regimens induced elevated vessel permeability/perfusion in bone metastases, but opposite to monotherapy, combination of agents additionally elevated the blood volume. Immunofluorescence analysis of osteolytic lesions revealed more tumour blood vessels after combination therapy and equally immature vasculature as compared to monotherapy.

Conclusion: Upon treatment with integrin inhibitor monotherapy or in combination with an antiresorptive agent, vessel remodelling could be assessed by DCE-MRI. Interestingly, the degree of vessel remodelling could be correlated with bone defect healing, so we conclude the existence of a strong interaction between tumour angiogenesis and bone turnover in lytic osseous lesions.

\section{B-0435 14:45}

View-angle tilting (VAT) and slice-encoding metal artifact correction (SEMAC) for MR imaging of orthopaedic tumor prostheses P.M. Jungmann ${ }^{1}$, C. Ganter ${ }^{1}$, F. Pohlig ${ }^{1}$, C. Schaeffeler ${ }^{1}$, J.S. Bauer ${ }^{1}$, M. Nittka ${ }^{2}$, E.J. Rummeny ${ }^{1}$, H. Rechl' ${ }^{1}$, K. Woertler ${ }^{1} ;{ }^{1}$ Munich/DE, ${ }^{2}$ Erlangen/DE (pia.jungmann@tum.de)

Purpose: To evaluate the reduction of metallic artefacts produced by orthopaedic tumour prostheses in MR imaging by application of new WARP-sequences (VAT and SEMAC).

Methods and Materials: WARP turbo-spin-echo sequences and standard metal sequences were evaluated by scanning tumour-prostheses in an experimental model at 1.5 T MRI. Three different MR techniques were applied: (i) standard, (ii) VAT and (iii) combined VAT and SEMAC sequences (VAT\&SEMAC). For each technique, coronal short-tau-inversion-recovery (STIR), coronal T1-weighted and axial T1- and T2-weighted sequences were assessed. Diameters of artefacts were measured quantitatively at $n=8$ different standardised spots for $n=4$ different tumour prostheses and real diameters of prostheses were compared with artefacts. Wilcoxon and paired t-test were used for statistical analysis.

Results: Reduction of artefacts was achieved by application of WARP-techniques. For coronal STIR-sequences, diameters were smaller in the VAT\&SEMAC group (Mean \pm standard deviation, $70.1 \pm 92.9 \mathrm{~mm}$ ) compared with standard sequences (73.0 $\pm 92.6 \mathrm{~mm}$; Wilcoxon-test, $\mathrm{P}<0.001)$. The VAT group showed intermediate diameters $(72.0 \pm 93.1 \mathrm{~mm} ; \mathrm{P}=0.002)$. Compared with standard coronal T1-weighted sequences $(70.1 \pm 91.8 \mathrm{~mm})$, application of VAT $(68.3 \pm 91.1 \mathrm{~mm} ; \mathrm{P}=0.005)$ and VAT\&SEMAC $(68.2 \pm 91.0 \mathrm{~mm} ; \mathrm{P}=0.002)$ showed similar artefact reduction. Accordingly, axial VAT also reduced artefacts (T2-weighted, $\mathrm{P}=0.008$; $\mathrm{T} 1$-weighted, $\mathrm{P}=0.006)$. However, real diameters $(27.2 \pm 18.2 \mathrm{~mm})$ were significantly smaller than artefact diameters for all techniques (Standard STIR, 32.5 $\pm 21.4 \mathrm{~mm}$; VAT, 30.7 \pm 21.3 $\mathrm{mm}$; VAT\&SEMAC, $29.5 \pm 20.3 \mathrm{~mm}$; all $\mathrm{P}<0.05$ ).

Conclusion: In the experimental model, significant reduction of metallic artefacts was achieved by application of WARP-techniques (VAT and SEMAC) for all pulse sequences. Thus, this new technique appears to be applicable to postoperative follow-up of patients with orthopaedic tumour prostheses.

Author Disclosures:

M. Nittka: Other; Development of the WARP-Sequences, Siemens AG.

\section{B-0436 14:54}

Association of QCT bone mineral density and bone structure with vertebral fractures in patients with multiple myeloma

J. Borggrefe ${ }^{1}$, S. Giravent ${ }^{2}$, F. Thomsen ${ }^{2}$, J. Peña ${ }^{2}$, A. Wulff' ${ }^{2}$ A. Günther ${ }^{1}$,

M. Heller ${ }^{2}$, C.C. Glüer ${ }^{1} ;{ }^{1}$ Cologne/DE, ${ }^{2}$ Kiel/DE (Jan.Borggrefe@uk-koeln.de)

Purpose: Computed tomography is widely used for staging osteolyses and detecting fractures in patients with multiple myelioma (MM). We investigated the risk of vertebral fractures for MM patients in dependence of QCT trabecular bone density (VBMD trab), median trabecular separation (Tb.Sp). and cortical thickness of vertebral bodies T11 and T12.

Methods and Materials: We evaluated CT scans from the OLYMP-CT study with a cohort of $61 \mathrm{MM}$ patients that underwent CT for clinical staging. A QCT software 
(Structural Insight) was adapted in order to allow calibrated bone density and bone structure analyses of the vertebral bodies, using an underlying light weight calibration phantom with design for coronary calcium scoring (Image Analysis Phantom ${ }^{\circledR}$ ). Results: The prevalence of vertebral fractures was $48 \%$. Patients with fractures were not significantly older than patients without fractures (65 vs 62 years of average, $p>0.25)$. The trabecular vBMD in patients with fractures was significantly reduced from $200 \mathrm{mg} / \mathrm{cc}(\mathrm{SEM} \pm 9.3)$ to $166 \mathrm{mg} / \mathrm{cc}(\mathrm{SEM} \pm 9.6)(p<0.05)$. In the age-adjusted logistic regression, the OR per SD for VBMD trab. was 1.9 (1.1-3.6). The OR for Tb.Sp. was 2.3 (1.3-4.0) per SD. In a model combining both parameters, Tb.Sp. remained significant without additional predictive value. Cortical parameters did not provide significant predictive value for fracture events.

Conclusion: In MM patients, the loss of trabecular density is associated with the risk of vertebral fractures. The detection of increased trabecular separation as caused by plasmacell-infiltration appears to be of value for fracture risk assessment.

\section{B-0437 15:03}

Imaging patterns in multiple myeloma: observations at whole body MRI

N.M. Long, O.M. Drumm, P. O' Gorman, S.J. Eustace; Dublin/IE

(nevelong@gmail.com)

Purpose: To categorise imaging patterns in multiple myeloma and correlate with patient demographics and outcome.

Methods and Materials: 200 consecutive whole body MRI studies of patients with a proven diagnosis of multiple myeloma were included for review. Images were reviewed by 2 experienced radiologists with discrepancies resolved by consensus. In each case an attempt was made to characterise patterns of marrow infiltration. Correlation was subsequently performed with markers of disease activity including percentage plasma cells, LDH, immunological markers and Bence-Jones protein. Results: Four distinct patterns of marrow infiltration were identified in multiple myeloma. Pattern A: Normal marrow with superimposed plasmacytoma. Pattern B: Normal marrow with multiple superimposed plasmacytomata. Pattern C: Diffuse marrow infiltration/marrow packing. Pattern D: Diffuse marrow infiltration/marrow packing with superimposed plasmacytomata. Type A patients showed optimum outcome with improved treatment response and reduced early mortality. Marrow patterns type B, C and D correlated with progressively increasing disease severity with type $D$ patients demonstrating the poorest treatment response and outcome. Conclusion: Four distinct patterns of marrow disease can be identified in patients with multiple myeloma and these should be evaluated at diagnosis as they ultimately dictate outcome and correlate with treatment response.

\section{B-0438 15:12}

Efficacy of whole body low-dose CT (WBLDCT) in the staging of patients with early multiple myeloma: comparison with whole body magnetic resonance imaging (WBMRI)

V. Besostri, D. Ippolito, P.A. Bonaffini, A. Masetto, A. Cuccia, S. Sironi; Milan/IT (valeriabesostri@gmail.com)

Purpose: To compare WBLDCT (whole body low dose CT) study with WBMRI (whole body magnetic resonance imaging) in the identification of bone marrow involvement for the detection of bone lesions and for risk evaluation of pathological fractures.

Methods and Materials: A total of 25 patients with histologically confirmed myeloma underwent WBLDCT and WBMRI. Entire skeleton was divided into five districts (skull, spine, sternum and ribs, pelvis, upper and lower limbs) in terms of pattern, size and distribution of disease. Unenhanced WBLDCT was performed on 256-slice scanner (Philips, ICT, 256): voltage $120 \mathrm{KV}$, tube current time product $40 \mathrm{mAs}$, collimation $128 \times 0.65$. MRI protocol was performed on a $1.5 \mathrm{~T}$ (Achieva Philips) and consisted of T1 TSE and T2 STIR on coronal and sagittal plane and DWIBS on axial plane. WBLDCT findings were compared with those of WBMRI. The disease was staged according to Salmon and Durie System.

Results: On WBMRI 9 patients showed no involvement; in 16 patients, 65 regions were affected by a total of 400 focal lesions: 14 patients were stage I, 4 were stage II and 6 were stage III. On WBLDCT 8 patients showed no involvement; in 17 patients, 49 regions were affected by a total of 396 focal bone lesions: 14 patients were stage I, 2 were stage II and 8 were stage III. WBLDCT overstaged 5 patients compared with MRI. The infiltration patterns were concordant in both techniques: focal in 9 patients, diffuse in 5 patients and combined in 10 patients; 1 patient showed no disease.

Conclusion: WBLDCT is a fast and feasible technique for detecting small osteolytic lesions, but presents poor sensitivity for evaluation of abnormalities in early focal marrow infiltrations. Conversely, MRI protocol is more specific to assess low degrees of diffuse bone marrow infiltration.

\section{B-0439 15:21}

Role of apparent diffusion coefficient (ADC) maps in the evaluation of tumour response in chemotherapy in patients with multiple myeloma P.A. Bonaffini, D. Ippolito, V. Besostri, A. Casiraghi, A. Di Lelio, S. Sironi; Monza/lT (pa.bonaffini@gmail.com)

Purpose: To assess the diagnostic value of ADC maps in the evaluation of response to chemotherapy in patients with multiple myeloma (MM)

Methods and Materials: 25 patients with MM underwent a 1.5 T WB-MRI (Achieva, Philips), before and 4-6 months after chemotherapy, with the following protocol: coronal and sagittal short tau inversion recovery (STIR) T2 and T1 TSE and axial DWIBS (b values: $0,500,1000 \mathrm{~mm} 2 / \mathrm{sec}$ ). DWIBS were compared with T1 and STIR sequences in five districts (skull, spine, sternum and ribs, pelvis, upper and lower limbs) to evaluate response to treatment. On ADC maps ROI were drawn along contours of bone lesions. The absolute values and percentage variation of ADC ( $\triangle A D C$ ) before (MR1) and after (MR2) chemotherapy were calculated and compared between responders and non-responders patients.

Results: 30 lesions (12 focal, 18 diffuse) were evaluated and correctly detected on DWIBS as areas with high signal intensity as compared to normal bone marrow; response to chemotherapy was established as signal intensity decrease after treatment. The mean ADC values were: $0.63 \pm 0.24 \times 10-3 \mathrm{~mm}^{2} / \mathrm{sec}$ on MR1 and $1.04 \pm 0.46 \times 10-3 \mathrm{~mm}^{2} / \mathrm{sec}$ on MR2 for responders (18/25); $0.61 \pm 0.05 \times 10-3$ $\mathrm{mm}^{2} / \mathrm{sec}$ on MR1 and $0.69 \pm 0.09 \times 10-3 \mathrm{~mm}^{2} / \mathrm{sec}$ on MR2 for non-responders $(7 / 25)$. The mean $\triangle A D C$ in responders $(\Delta=66 \%)$ was significantly different $(\mathrm{p}<$ $0.05)$ than that in non-responders $(\Delta=15 \%)$

Conclusion: WB-MRI is a non-invasive tool able to evaluate response to treatment in patients with $\mathrm{MM}$, by determining individual changes of $A D C$ values, related to biological changes in tumour tissue.

14:00 - 15:30

Room F1

\section{Abdominal Viscera}

\section{SS 601}

\section{Liver steatosis: imaging and quantification}

Moderators:

I. Bargellini; Pisa/IT

B. Van Beers; Clichy/FR

\section{B-0440 14:00}

Presence/absence of hepatic steatosis after percutaneous intraportal pancreatic islet transplantation in 108 allo- and auto-transplanted patients: can ultrasound predict the clinical outcome?

G. Agostini, M. Venturini, G. Querques, P. Maffi, C. Gremizzi, A. Palmisano, F. De Cobelli, A. Secchi, A. Del Maschio; Milan/IT (venturini.massimo@hsr.it)

Purpose: Islet transplantation is a less invasive/safer/repeatable option than surgical pancreas transplantation for type 1 diabetic patients; it can be performed after kidney transplantation (IAK), alone (ITA) in patients without chronic renal insufficiency, or as an autotransplantation (IAT) after pancreatectomy (immunosuppression unnecessary). Steatosis is a consequence of islet engraftment: its meaning is controversial. Our longitudinal study aimed to assess steatosis incidence at ultrasound after islet auto- and allo-transplantation, and to identify any relationship with graft function.

Methods and Materials: Ultrasound was performed at baseline/6/12/24 months in 108 islet-transplanted patients (33 IAK/50 ITA/25 IAT). Steatosis first detection/ prevalence/duration/distribution were recorded. Steatosic (S) and non-steatosic patients (NS) were compared for the following parameters at baseline/6/12/24 months: insulin independence rate, B-score, C-peptide, glycated-haemoglobin, exogenous insulin requirement, fasting plasma glucose, infused islet mass.

Results: Steatosis was found in 21/108 patients, 24\% (20/83) allo-transplanted, $4 \%$ (1/25) auto-transplanted (better outcome), with first detection at 6 months, highest prevalence at 1 year (18 cases). S-patients had a significantly higher infused islet mass (IE $/ \mathrm{kg}: \mathrm{S}=10.822 ; \mathrm{NS}=6.138)$ and worse basal metabolic conditions ( $B$-score: $\mathrm{S}=1.7 \pm 1.6 ; \mathrm{NS}=2.8 \pm 2.8$ ), but better islet function at the time of steatosis first detection ( $B$-score: $\mathrm{S}=3.9 \pm 2.0 ; \mathrm{NS}=2.9 \pm 2.3$ ), after which a progressive islet exhaustion, along with steatosis disappearance, was observed. Conversely, in NS patients these parameters remained more stable in time.

Conclusion: Steatosis at ultrasound is significantly less detectable in auto-transplanted-patients with better clinical outcome. It seems to be related to stressed islets' overworking activity (higher B-score values when steatosis appears), and a higher infused islet mass. Steatosis presence precedes metabolic alterations and 
therefore could predict graft dysfunction addressing therapeutic decisions before islet exhaustion. Steatosis absence does not allow any conclusion.

\section{B-0441 14:09}

Phosphorus magnetic resonance spectroscopy (31P-MRS) in nonalcoholic fatty liver disease (NAFLD)

J.M. Abrigo, V.-S. Wong, D.-W. Yeung, G.-H. Wong, A.-h. Chan, H.-Y. Chan, W.-W. Chu; Shatin/HK (jillabrigo@cuhk.edu.hk)

Purpose: To describe hepatic biochemistry in biopsy-proven NAFLD states using ${ }^{31} \mathrm{P}-\mathrm{MRS}$ and to evaluate its diagnostic performance for distinguishing non-alcoholic steatohepatitis (NASH)

Methods and Materials: The cohort consisted of healthy controls $(n=19)$, and 132 NAFLD patients with liver biopsy, as follows: simple steatosis (SS, $n=37$ ), $\mathrm{NASH}$ with non-significant fibrosis (NASH-NSF, $\mathrm{n}=49$ ) and $\mathrm{NASH}$ with significant fibrosis (NASH-SF, $n=46$ ). ${ }^{31} \mathrm{P}-\mathrm{MRS}$ was performed on a $3 \mathrm{~T} \mathrm{MRI}$ to obtain signal intensities for phosphomonoesters (PME), phosphodiesters (PDE) and component glycerophosphocholine (GPC), total adenosine triphosphate (ATP) and component $\mathrm{a}-\mathrm{ATP}$, and inorganic phosphate $(\mathrm{Pi})$. These were expressed as percentages relative to total phosphate and compared using one-way ANOVA with post hoc Bonferroni test. Receiver operating curve (ROC) analysis was performed to determine cut-off values with highest Youden index. Statistical significance was declared at $\alpha<0.05$. Results: Compared with controls, all NAFLD groups had higher PDE $(p<0.049)$. SS and NASH-NSF had decreased PME ( $p=0.001-0.015)$, increased GPC $(p<0.001)$, normal total ATP $(p=0.067-1.000)$ and decreased $\mathrm{Pi}(p=0.018-0.021)$. NASH-SF had normal PME $(p=0.764)$ normal GPC $(p=0.727)$, decreased total ATP $(p=0.035)$ and normal $\mathrm{Pi}(\mathrm{p}=0.443)$. a-ATP was significantly decreased in both NASH groups and identified as a potential diagnostic marker. The computed area under the ROC was 0.705 , and an a-ATP threshold of $<13.13 \%$ gave a $65 \%$ sensitivity and $73 \%$ specificity for the identification of NASH.

Conclusion: ${ }^{31} \mathrm{P}-\mathrm{MRS}$ shows observable spectroscopic patterns within the NAFLD spectrum. a-ATP is a potential diagnostic marker for distinguishing NASH from SS.

\section{Author Disclosures:}

V. Wong: Research/Grant Support; Health and Health Services Research Fund sponsored by the Government of Hong Kong SAR and Research Grants Council of Hong Kong SAR. W. Chu: Research/Grant Support; Health and Health Services Research Fund sponsored by the Government of Hong Kong SAR and Research Grants Council of Hong Kong SAR.

\section{B-0442 14:18}

Clinical implications of fatty liver on hepatic diffusion-weighted imaging studied in healthy subjects

H. Dijkstra, A. Handayani, P. Kappert, M. Oudkerk, P.E. Sijens; Groningen/NL (h.dijkstra01@umcg.nl)

Purpose: To evaluate the diagnostic implications of fatty liver on DWI by assessing the hepatic fat fraction (HFF) and T2* of healthy subjects in eight liver segments in order to eliminate patient-related confounding factors such as cirrhosis and fibrosis. Methods and Materials: Eleven healthy volunteers were examined with DWI (seven b-values) and two series of in- and out-of-phase dual-echo spoiled gradient echo sequences (SPGR) to obtain the HFF (Dixon's method). A third SPGR series was acquired to calculate T2* relaxation. DWI data were analyzed using the IVIM model: molecular diffusion (Dslow), microperfusion (Dfast) and the respective fractions (fslow/fast). For each subject, four circular regions-of-interest (ø22.3 mm) were drawn in each segment according to the Couinaud-Bismuth classification. Data with low SNR $<5$ were excluded. IVIM parameters were compared between groups of low $(22.5 \%)$, intermediate $\left(2.5^{2} \mathrm{HHF}<10 \%\right)$ and high HHF $(>10 \%)$. Linear correlations between HHF, DWI parameters, and T2* were assessed.

Results: In each segment, Dslow decreased with increasing HFF. Dslow averaged over all segments differed significantly $(p<0.001)$ between low $\left(0.99 \times 10^{-3} \mathrm{~mm}^{2} / \mathrm{s}\right)$, intermediate $\left(0.74 \times 10^{-3} \mathrm{~mm}^{2} / \mathrm{s}\right)$ and high HFF $\left(0.32 \times 10^{-3} \mathrm{~mm}^{2} / \mathrm{s}\right)$. Both Dslow and $\mathrm{T}^{*}$ decreased with HFF $\left(\mathrm{R}^{2}=0.89 ; \mathrm{R}^{2}=0.72\right)$. Dfast and fslow/fast showed small intergroup differences $(p<0.04)$.

Conclusion: Increasing hepatic fat fractions caused significant reduction of the molecular diffusion and shortening of $\mathrm{T}^{*}$. This implies that hepatic DWI hindered by fat leads to apparent fluctuations of the molecular diffusion and hypointensity on DWI images; this may induce false diagnoses such as fibrosis or cirrhosis.

\section{B-0443 14:27}

Liver fat volume fraction quantification in patients with chronic liver diseases with separated estimation of fat and water relaxation times at 1.5 and $3.0 \mathrm{~T}$

B. Leporq, F. Pilleul, O. Beuf; Villeurbanne/FR

(benjamin.leporq@creatis.insa-lyon.fr)

Purpose: To validate a magnitude-based method, transposable on any imaging system and for all current clinical fields $(1.5$ and $3.0 \mathrm{~T}$ ) for liver fat volume fraction (FVF) quantification without dominant component ambiguity problems.

Methods and Materials: MR imaging was performed at 1.5 and $3.0 \mathrm{~T}$ using a multiple-angle multiple-gradient echo acquisition. A quantification algorithm correcting for relaxation time effects using a disjointed estimation of T1 and T2* of fat and water and accounting for the NMR spectrum of fat was developed. Validations were performed in a prospective comparative study with histology on 28 patients with chronic liver diseases.

Results: MR-estimated FVF and histological results were strongly correlated $(\rho=0.96)$. ROC analysis showed that FVF allowed the diagnosis of a mild (cut-off $=5.5 \%$ ) and a moderate steatosis (cut-off $=15.2 \%$ ) with a sensitivity/specificity of $100 \%$. Water relaxation times ( $\mathrm{T} 1$ and $\mathrm{T} 22^{*}$ ) were found significantly reduced in patients with iron deposition $(p<0.01)$, whereas water T1 was found significantly increased in patients with cirrhosis $(p<0.01)$.

Conclusion: This method that can be performed at 1.5 and $3.0 \mathrm{~T}$ on all imaging systems provide an accurate quantification of the FVF in patients with chronic liver disease. FVF could be a relevant biomarker for the clinical follow-up of patients with NAFLD as well as to quantify steatosis in the full spectrum of chronic liver diseases. Interestingly, thanks to the separated estimation of fat and water relaxation times, detection of cofactors such as iron deposition or cirrhosis in patients with NAFLD could be achieved.

\section{B-0444 14:36}

Combination of liver intravoxel incoherent motion imaging with MR fat quantification at 3.0 T to distinguish between pure steatosis and NASH B. Leporq ${ }^{1}$, F. Pilleul ${ }^{1}$, J. Dumortier ${ }^{2}$, O. Guillaud ${ }^{2}$, T. Lefort ${ }^{2}$, O. Beuf'; ${ }^{1}$ Villeurbanne/FR, ${ }^{2}$ Lyon/FR (benjamin.leporq@creatis.insa-lyon.fr)

Purpose: To evaluate the combination of liver intra-voxel incoherent motion imaging (IVIM) and a MRI fat quantification method at 3.0 T to make the distinction between NASH and pure steatosis.

Methods and Materials: A multi-angle multi-gradient echo acquisition combined with a dedicated algorithm was used to quantify liver fat volume fraction (FVF), corrected for water and fat relaxation times and accounting for the NMR spectrum of fat. IVIM was performed using a single-shot SE-EPI sequence, in free breathing, with 11 b-values (0-20-40-60-80-100-200-300-400-600-800s. $\left.\mathrm{mm}^{-2}\right)$ and a weighted signal averaging procedure. Three orthogonal diffusion gradients were sequentially applied. A $2000 \mathrm{~ms}$ TR, $54 \mathrm{~ms}$ minimum TE; 21 axial slices of $8 \mathrm{~mm}$ thick; $400 \times 300$ $\mathrm{mm}^{2} \mathrm{FOV} ; 128 \times 96$ matrix were used. IVIM parameters (pure molecular diffusion coefficient (DSlow), perfusion-related diffusion coefficient (DFast) and perfusion fraction ( $f)$ ) were estimated. MR imaging was performed on a healthy volunteers group ( $n=33)$, and on patients with histologically confirmed pure steatosis $(n=16)$ or NASH $(n=4)$

Results: Wilcoxon test showed that DSlow was significantly lower when fat is present $(F V F>5.5 \%)(p<0.01)$. DSlow and DFast were found significantly reduced between subjects with pure steatosis (Brunt $=0)$ and NASH (Brunt $>0)(p<0.05)$. Conclusion: Fat vesicles in pure steatosis restrict molecular diffusion without modification of micro-perfusion. In NASH, fibrosis still increase the restriction of molecular diffusion compared to patients with pure steatosis and decrease the microperfusion as illustrated by the decrease of perfusion-related diffusion coefficient. These preliminary results suggest that combining MRI fat quantification and IVIM could be a none invasive mean to distinguish between pure steatosis and NASH.

\section{B-0445 14:45}

Quantification of steatosis hepatitis and liver iron overload in a population-based study using magnetic resonance imaging J.P. Kühn¹, D. Hernando ${ }^{2}$, H. Völzke', S.B. Reeder², N. Hosten'1, B. Mensel'1, K. Hegenscheid ${ }^{1}$, M. Evert ${ }^{1}$, S. Baumeister ${ }^{1} ;{ }^{1}$ Greifswald/DE, ${ }^{2}$ Madison, WI/US (kuehn@uni-greifswald.de)

Purpose: To determine the proton-density fat fraction (PDFF), and the apparent $R 2^{*}\left(=1 / T 2^{*}\right)$ of the liver and to explore its relationship with metabolic parameters in a large-scale epidemiological study.

Methods and Materials: Between 2009 and 2012, 3,198 volunteers, 1,586 men and 1,612 women, aged 52.6 \pm 13.7 years were participated in a population-based 
study. MRI was performed in a $1.5 \mathrm{~T}$ system using a pre-clinical T2* corrected three-echo chemical shift-encoded sequence. Offline reconstruction of the PDFF (corrected for $\mathrm{T} 1, \mathrm{~T}^{*}$ relaxation, noise bias, and multi-peak spectrum of fat) and the apparent $\mathrm{R}^{*}$ (fat corrected) were performed. PDFF and $\mathrm{R} 2^{*}$ were previously calibrated by histopathologically determined fat and hepatocellular iron content, respectively. PDFF and $\mathrm{R}^{*}$ were graduated in a 4-point scale system: no-, mild-, moderate-, and high content.

Results: 2,762 MRIs were successfully performed. Median PDFF was $4.0 \%$, range $0-41.7 \%$. Liver steatosis was observed in $40.0 \%$, composed of mild: $27.6 \%$ (762 subjects), moderate: $10.7 \%$ (295 subjects), high fat content: $1.7 \%$ (48 subjects). Median apparent $\mathrm{R} 2{ }^{*}$ was $34.4 \mathrm{~s}^{-1}$, range $14.0-331.4 \mathrm{~s}^{-1}$. Subjects were grouped in no iron overload: $82.4 \%$ (2,276 subjects), and with iron overload in $17.6 \%$, composed of mild: $14.9 \%$ (412 subjects), moderate: $0.6 \%$ (19 subjects), high iron content: $1.9 \%$ (55 subjects).

Conclusion: In North-East Germany the frequency of steatosis hepatitis assessed by MRI is $40.0 \%$ (median PDFF of $4.0 \%$ ), and hepatocellular iron overload of $17.6 \%$ (apparent $\mathrm{R}^{*}$ of $34.4 \mathrm{~s}^{-1}$ ), respectively.

\section{B-0446 14:54}

Effect of Gd-EOB-DTPA on hepatic fat quantification using highspeed T2-corrected multiecho acquisition (HISTO) at 1H MR spectroscopy Y. Park ${ }^{1}$, C. Lee ${ }^{1}$, I. Kim ${ }^{1}$, B. Kiefer ${ }^{2}$, K. Kim¹ , J. Choi ${ }^{1}$, J. Lee' ${ }^{1}$, C. Park'; ${ }^{1}$ Seoul/KR, ${ }^{2}$ Erlangen/DE (pys797979@naver.com)

Purpose: To determine whether administration of Gd-EOB-DTPA affects hepatic fat quantification in a fast breath-hold high-speed T2-corrected multiecho acquisition (HISTO) MR spectroscopy.

Methods and Materials: Sixty-two patients underwent 15-second breath-hold HISTO MR spectroscopy, which enables quantification of water and fat T2, and subsequently to provide corrected measure of hepatic fat fraction (FF). All patients performed HISTO twice before and after administration of Gd-EOB-DTPA, respectively. Post-contrast HISTO was performed between 2-minute equilibrium phase and 20-minute hepatobiliary phase. We evaluated variability of FF calculation during precontrast HISTO and post-contrast HISTO and effect of contrast material on mean FF calculation, using paired t test and intraclass correlation coefficient (ICC). We also evaluated whether effect of contrast material on mean FF calculation depends on FF level ( $\left.\mathrm{FF}<5 \%, 5 \%{ }^{2} \mathrm{FF}<10 \%, 10 \%{ }^{2} \mathrm{FF}\right)$, by comparing ICC using the $\mathrm{z}$-test. Results: High reproducibility of FF calculation before and after contrast administration was observed ( $\mathrm{ICC}=0.888$ vs 0.885 , respectively). Mean FF on pre-contrast HISTO was $5.06 \% \pm 2.90$ and mean FF on post-contrast HISTO was $5.20 \% \pm 3.02$. Mean FFs were not significantly different before and after administration of contrast agent $(P=0.50)$. ICC for assessment of effect of administration of contrast agent was $0.848(P<0.001)$. ICC did not significantly change according to FF level (ICC=0.353, 0.450, and 0.730, respectively; $P=0.44$ ).

Conclusion: HISTO is known as a simple, accurate MR spectroscopic sequence of quantifying hepatic fat noninvasively. Gd-EOB-DTPA administration did not change the FF calculation of HISTO. HISTO after Gd-EOB-DTPA administration can be used for hepatic fat quantification, without spending time for additional MR sequence.

Author Disclosures:

I. Kim: Employee; Seimens. B. Kiefer: Employee; Siemens.

\section{B-0447 15:03}

Brown adipose tissue influences body fat composition and hepatic steatosis

M.K. Werner, S. Ripkens, M. Schmadl, I. Stef, A. Deckert,

C.D. Claussen, C. Würslin, N. Stefan, C. Pfannenberg;

Tübingen/DE (Matthias.Werner@med.uni-tuebingen.de)

Purpose: Brown adipose tissue (BAT) mass and activity is considered a significant factor for the development of adiposity and metabolic syndrome in humans, and represents a natural target for the modulation of energy expenditure. We evaluated the correlation of BAT activity and active volume as detectable on FDG-PET/CT scans with abdominal body fat composition and liver fat estimation.

Methods and Materials: 4852 FDG-PET/CT examinations in 3680 individual patients were screened for BAT, resulting in 601 patients and controls for subsequent analysis, matched for exam date and therefore ambient temperature, age, and gender. In addition to BAT volume and activity, an automated segmentation was used to determine visceral fat and subcutaneous fat as well as lean tissue in the abdomen. Liver fat was estimated in 430 patients with non-contrast-enhanced CT. Spearman rank correlation between parameters and a multi-variate model for liver fat excluding influencing parameters was calculated.

Results: BAT volume and activity showed a significant inverse correlation with total adipose tissue (activity $p<0.001 /$ volume $p=0.022$ ) and visceral adipose tissue $(p<0.001 / p<0.001)$, whereas there was no significant correlation between BAT volume and the amount of subcutaneous adipose tissue $(p=0.70)$. BAT activity was an independent, negatively influencing variable for liver fat content $(p=0.027)$. Conclusion: BAT significantly correlates with body fat composition, especially visceral fat mass considered relevant for the development of metabolic syndrome, and seems to be an independent regulator of hepatic steatosis.

\section{B-0448 15:12}

In-phase/opposed-phase MRI and MR spectroscopic measurements for the quantification of liver fat content in morbidly obese patients H.H. Bertram ${ }^{1}$, G. Thörmer', N. Garnov², T. Schütz², V. Peter ${ }^{2}$, T. Kahn², H. Busse ${ }^{2} ;{ }^{1}$ Munich/DE, ${ }^{2}$ Leipzig/DE (henriette.bertram@tum.de)

Purpose: Different MR techniques have been explored to quantify the liver fat content (LFC). The goal of this study is to compare the LFC values between MRS and in-phase/opposed-phase MRI in extremely obese patients.

Methods and Materials: 30 obese patients (mean BMI $48 \mathrm{~kg} / \mathrm{m} 2$ ) were imaged at 1.5 T. MR spectra were acquired in liver segments II, IV and VII. In-phase/ opposed-phase (IP/OP) MRI data were corrected for T1 and T2* effects and used to calculate LFC maps. To compare IP/OP MRI and MRS, regions of interests of the size of the MRS voxels were placed in the fat parameter map at positions that matched the location of the spectroscopic voxel. Tissue samples were taken for histopathological analysis.

Results: Mean overall intrahepatic fat content from IP/OP imaging was $14.3 \pm$ $2.0 \%$. Lipid content ranged from 0.2 to $35.7 \%$ with the IP/OP technique and from 0.0 to $71.2 \%$ with MRS. MRI and MRS values were characterised by an insignificant $(p=0.36)$ bias of $-3.6 \%$ and upper/lower limits of agreement of $3.8 /-11.0 \%$. Agreement for the three individual liver segments (II, IV and VII) had upper/lower limits of $+5.0 /-11.9 \%,+1.9 /-9.6 \%$ and $+4.8 /-12.0 \%$ and insignificant biases of $3.4 \%$ $(p=0.14),-3.9 \%(p=0.25)$ and $-3.6 \%(p=0.27)$, respectively.

Conclusion: IP/OP imaging and MRS are reliable methods for the assessment of LFC. IP/OP imaging is superior in terms of processing time and liver coverage and provides information for cases with larger regional fat differences and is helpful for lipid assessment in the left liver lobe where MRS is impractical.

\section{B-0449 15:21}

Dynamic contrast enhanced-MRI (DCE-MRI) functional assessment of vascular events occurring during the intra-hepatic engraftment of pancreatic islets may predict long-term outcome of islets transplantation A. Palmisano, A. Esposito, G. Ironi, P. Maffi, F. De Cobelli, T. Canu, A. Secchi, A. Del Maschio; Milan/IT (a.palmisano@live.it)

Purpose: Pancreatic islets transplantation (islets-tx) is a promising treatment for patients with type-1 diabetes. Neoangiogenesis of transplanted islets is a crucial phenomenon for engraftment and was investigated with DCE-MRI in mice. The aim was to use DCE-MRI in clinical islets-tx to evaluate the relationship between early vascular changes, islets loss and long-term clinical outcome.

Methods and Materials: 6 diabetic pts were enrolled. DCE-MRI was performed at baseline and 7 days after islet-tx with a 3D-T1w-TFE dynamic sequence (temporal resolution: $4 \mathrm{sec}$; 45 dynamics) acquired during gadolinium bolus injection. K-trans was assessed by DCE-MRI datasets. Three patients received Ferucarbotran labelled islets and were monitored with T2*sequences (before islets-tx, 7 days and each month after). Unlabelled islets were infused in the remaining three patients. Glycated haemoglobin, blood glucose, daily insulin request and stimulated C-peptide were monthly assessed in order to calculate beta-score (0-8) as index of graft function.

Results: Variation of liver perfusion from pre-transplantation to 7 days after islet-tx were evaluated by k-trans changes at DCE-MRI ([[Unable to Display Character: \&\#8710;]]k-trans0-7days). [[Unable to Display Character: \&\#8710;]]k-trans0-7days was strongly correlated to mean beta-score achieved during the subsequent clinical monitoring $(R=0.835 ; p<0.05)$; in particular, a [[Unable to Display Character: \&\#8710;]]k-trans0-7days decrease was observed in case of early graft impairment (Pt\#6: $\Delta$ ktrans0-7days $=-11 \%$, mean $\beta$-score:2) or complete graft failure after 1 (Pt\#2: $\Delta$ ktrans0-7days $=-32 \%$, mean $\beta$-score:1) and 6 months (Pt\#4: $\Delta$ ktrans07 days $=-28 \%$; mean $\beta$-score:1). Moreover, in pts with labelled islets, a k-trans decrease ([[Unable to Display Character: \&\#8710;]]k-trans0-7days: -28\%) was associated with a faster rate of labelled islets disappearance at $\mathrm{T}^{*}$ follow-up. Conclusion: DCE-MRI assessment of the vascular events occurring in the earliest phases of islets engraftment may predict graft outcome. 


\section{Breast}

\section{SS 602}

\section{Breast imaging after neoadjuvant therapy and surgery}

Moderators:

G. Gennaro; Padua/IT

P. Martinez-Miravete; Logroño/ES

\section{B-0450 14:00}

Early response monitoring of neoadjuvant chemotherapy in breast cancer patients using magnetic resonance imaging: a systematic review M. Lobbes ${ }^{1}$, R. Prevos ${ }^{1}$, M. Smidt'1, V. Tjan-Heijnen ${ }^{1}$, M. van Goethem ${ }^{2}$, R.G.H. Beets-Tan', J. Wildberger ${ }^{1} ;{ }^{1}$ Maastricht/NL, ${ }^{2}$ Antwerp/BE (marc.lobbes@mumc.nl)

Purpose: To assess whether MRI can identify pretreatment differences or monitor early response in breast cancer patients receiving neoadjuvant chemotherapy. Methods and Materials: Pubmed, Cochrane library, Medline, and Embase databases were searched for publications until January 1, 2012. After primary selection, studies were selected based on predefined inclusion/exclusion criteria. Two reviewers assessed study contents using an extraction form.

Results: In 15 studies, which were mainly underpowered and of heterogeneous study design, 31 different parameters were studied. Most frequently studied parameters were tumour diameter or volume, Ktrans, Kep, Ve, and apparent diffusion coefficient (ADC). Other parameters were analysed in only two or less studies. Tumour diameter, volume, and kinetic parameters did not show any pretreatment differences between responders and non-responders. In two studies, pretreatment differences in $A D C$ were observed between study groups. At early response monitoring significant and non-significant changes for all parameters were observed for most of the imaging parameters.

Conclusion: Evidence on distinguishing responders and non-responders to neoadjuvant chemotherapy using pretreatment MRI, as well as using MRI for early response monitoring, is weak and based on underpowered study results and heterogeneous study design. Thus, the value of breast MRI for response evaluation has not yet been established.

\section{B-0451 14:09}

The role of magnetic resonance imaging in assessing residual disease and pathologic complete response in breast cancer patients receiving neoadjuvant chemotherapy: a systematic review R. Prevos ${ }^{1}$, M. Smidt'1 V. Tjan-Heijnen 1 , M. van Goethem ${ }^{2}$, R.-J. Schipper ${ }^{1}$, R.G.H. Beets-Tan ${ }^{1}$, J. Wildberger ${ }^{1}$, M. Lobbes ${ }^{1} ;{ }^{1}$ Maastricht/NL, ${ }^{2}$ Antwerp/BE (marc.lobbes@mumc.nl)

Purpose: This systematic review aimed to assess the role of magnetic resonance imaging (MRI) in the evaluation of residual disease extent and the ability to detect pathologic complete response $(\mathrm{pCR})$ after neoadjuvant chemotherapy for invasive breast cancer.

Methods and Materials: Pubmed, Cochrane library, Medline, and Embase databases were searched for relevant studies published until July 1, 2012. After primary selection, two reviewers independently assessed content of each eligible study using a standardised extraction form and pre-defined inclusion and exclusion criteria. Results: 35 eligible studies were selected. Correlation coefficients of residual tumour size assessed by MRI and pathology were good, with a median value of 0.698 . Reported sensitivity, specificity, positive predictive value, and negative predictive value for predicting pCR with MRI ranged from 25 to $100 \%, 50$ to $97 \%$, 47 to $90 \%$, and 44 to $100 \%$, respectively. Both overestimation and underestimation were observed. MRI proved more accurate in determining residual disease than physical examination, mammography, and ultrasound. Recent studies suggest that diagnostic accuracy of MRI after neoadjuvant chemotherapy might be influenced by treatment regimen and breast cancer subtype.

Conclusion: Breast MRI accuracy for assessing residual disease after neoadjuvant chemotherapy is good and probably surpasses other diagnostic means. However, both overestimation and underestimation of residual disease extent can be observed.
B-0452 14:18

Radiological evaluation of complete pathological response after neoadjuvant chemotherapy treatment of breast carcinoma N.M.A. Abdel Razek, H.A. El Ghazaly, O.Z. Yousef; Cairo/EG (naglaabdelrazek@yahoo.com)

Purpose: Neoadjuvant chemotherapy followed by locoregional therapy and postoperative systemic chemotherapy is now an accepted strategy. More than $70 \%$ of patients achieve an objective response (including pathological complete remission in $10 \%-25 \%$ of cases), and many patients experience downstaging through primary chemotherapy. This study aimed to assess the complete pathological response by different imaging modalities including digital mammography, sonography, dynamic MRI, MRS and PET-CT.

Methods and Materials: 125 female patients were enrolled in the study; all were histopathologically proven breast carcinoma and underwent full-course neoadjuvant chemotherapy. 25/125 showed complete pathological response indicated by histopathological examination of the surgical specimen. The pathological complete response was retrospectively evaluated by different imaging modalities done prior surgery including digital mammography, high-resolution breast sonography, dynamic MR mammography and MR spectroscopy as well as PET CT. In all patients, metallic clips werer lodged in the tumour prior neoadjuvant treatment and preoperative wire localisation was done to all the 25 cases showing complete pathological response. Results: Of 25 of 125 patients who showed complete pathological response, 14/25 has residual asymmetric density with or without microcalcifications by mammography. 17/25 patients showed complete disappearance of the lesion by sonography. Dynamic MRM showed complete change in the kinetics of the lesions from type 3 to type 1 and type 2a curves in 24/25 cases and MRS showed complete disappearance of the choline peak in 23/25 cases. PET CT shows absence of FDGB uptake in 21/25 cases.

Conclusion: Dynamic MRM and MR proton spectroscopy offered good tools to monitor the response to chemotherapy and are the most sensitive indicators of complete pathological response even more sensitive than the PET CT.

\section{B-0453 14:27}

Breast MRI at $3 \mathrm{~T}$ : a pilot study estimating the role of unenhanced MRI (DWI combined with T2 IDEAL sequence) vs CE-MRI in the assessment of response to neo-adjuvant chemotherapy

F. Vasselli, F. Pediconi, M. Telesca, M. Luciani, V. Casali, E. Miglio, A. Castrignanò, C. Catalano; Rome/lT (federica.vasselli@gmail.com)

Purpose: To assess the role of contrast-agent-free diagnostic approach combining DWI with ueMRI and compare the results with CE-MRM in the assessment of response to NAC using a 3 T Magnet.

Methods and Materials: Consecutive patients undergoing neo-adjuvant chemotherapy for biopsy-proved breast cancer $>2 \mathrm{~cm}$ underwent MR at $3 \mathrm{~T}$ prior to chemotherapy and before surgical excision.MR protocol comprised an FSE-T2IDEAL and DWI sequences and a Vibrant-3D-T1W sequence acquired before and after contrast media. Two blinded observers rated ueMRI and then CE-MRI for evaluation of tumour response. Lesion size, ADC values and FSE-IDEAL descriptors were assessed. Tumour size measurements on UE-MR were compared with those of CE-MR with statistic T-test. Response at MRI after chemotherapy were compared to histological specimen after surgery.

Results: 31 patients were enrolled. Sensitivity of ueMRI was $92 \%$ (observer1) and $84 \%$ (observer2). Sensitivity of CE-MRI was $100 \%$ for both observers. Specificity was $82 \%$ (ueMRI) and $86 \%$ (CE-MRI) for both observers. The differences between methods and observers were not statistically significant $\left(\mathrm{P}^{3} 0.09\right)$. Lesion size measurements did not differ significantly between all sequences. Lesion conspicuity was assessed quantitatively by measuring lesion Contrast/Noise Ratio (CNR). No statistically significant difference was observed between CNR of CEMRI and DWI $(p<0.009)$ while CNR on T2 IDEAL was significantly lower respect to CE-MRI and DWI (>0.009).

Conclusion: Combination of DWI and T2 IDEAL have similar values of sensitivity and specificity of CE-MRI in the evaluation of tumour response to neo-adjuvant chemotherapy. ADC values could be useful for differentiating responders from non-responders earlier during the treatment. 


\section{B-0454 14:36}

Radiological findings in breast autologous fat injection: a retrospective review in 12-year follow-up

L. Camera, I. Baglio, G. Barbazeni, A. Bianchin, M. Valdo, S. Montemezzi; Verona/lT (camera.lfmad@gmail.com)

Purpose: To describe the radiological appearance of normal and pathological findings resulting from mammary autologous fat injection (lipofilling).

Methods and Materials: We retrospectively reviewed all the patients who undergone breast lipofilling at our Institution from January 2000 to June 2012. All patients performed mammography and ultrasound examination; $1 / 3$ of those completed the follow-up with MRI (until February 2011 with 1.5 T scanner, from March 2011 with 3.0 T scanner).

Results: 320 Patients (range 25 - 82 years) were included. 280 underwent lipofilling after mastectomy, 40 after wide local excision. 83/320 patients had MRI to complete standard senological examination in cases of prothesis, suspicious recurrence or uncertain fat necrosis; after MRI, the patients underwent US - second look. No severe complications were observed after treatment. 232/320 were negative for all the follow-up including in this group "oil cysts" like normal findings due to lipofilling. Liponecrosis (64/88 cases), the most frequently observed complication, was detected in 43/64 using US and 21/64 with MRI. In 8/88 cases mammography showed calcifications, which underwent biopsy with diagnosis of fat necrosis. 16/88 patients developed homolateral recurrence ( $7 / 16$ discovered with mammography and US, 9/16 confirmed with MRI).

Conclusion: Lipofilling is an important reconstructive surgical application in oncologic patients. This long follow-up demonstrates that this method is safe because in our experience the majority of patients remained negative after surgery. The eventual complications are easily observed with radiological examinations and do not interfere with breast cancer recurrence early diagnosis.

\section{B-0455 14:45}

Invasive ductal carcinoma with marked necrosis on neoadjuvant chemotherapy: RECIST or mRECIST in MRI assessment of tumour response?

M.M. Nadrljanski, Z.C. Milosevic; Belgrade/RS (dr.m.nadrljanski@gmail.com)

Purpose: To assess the use of RECIST, mRECIST and volumetric measurements in patients with Invasive ductal carcinoma (IDC) with initially marked necrosis on neoadjuvant chemotherapy (NACT).

Methods and Materials: In prospective analysis of 71 patients, 14 patients were selected based on marked intralesional necrosis and examined 3 times on MRI before the beginning, after the $2^{\text {nd }}$ cycle and upon completion of NACT (anthracycline/taxane-based regimen). The lesions were measured in axial plane according to RECIST and mRECIST. The volumes of the entire lesion and of the viable tissue were computed.

Results: The longest diameters measured according to RECIST and mRECIST and the appropriate volumes were significantly different initially, after the $2^{\text {nd }} \mathrm{cycle}$ and upon the completion of NACT ( $p<0.001$ for all). The size of the lesions was significantly different with/without the necrotic areas. Differences in tumour diameter and volume change (delta; \%) between RECIST and MRECIST were not significant after the $2^{\text {nd }} c y c l e$ and upon completion of NACT ( $p>0.01$ for both). According to either RECIST or mRECIST, including the volume measurements, the same number of patients were classified in the same response category, i.e., early response - PR (21.4\%); late response - PR (50.0\%).

Conclusion: To our knowledge, this is the first trial testing the use of mRECIST in extrahepatic setting. There was no significant difference in changes of diameters or the corresponding volumes according to RECIST Vs. mRECIST. Further research in larger groups needs to assess the use of the tools in the follow-up of new NACT protocols.

\section{B-0456 14:54}

\section{CAD-based assessment of therapy monitoring in neoadjuvant} chemotherapy of breast cancer

J.S. Grueneisen', S. Maderwald', T. Heusner², S. Kuemmel',

E.A. Hauth ${ }^{1}$, M. Forsting ${ }^{1}$, T.C. Lauenstein ${ }^{1}$, L. Umutlu ${ }^{1} ;{ }^{1}$ Essen/DE,

${ }^{2}$ Düsseldorf/DE (johannes.grueneisen@uk-essen.de)

Purpose: To evaluate computer-aided detection analysis in dynamic breast MRI for (a) volume determination and analysis of (b) contrast kinetic and (c) pharmacokinetic changes of malignant lesions under neoadjuvant chemotherapy.

Methods and Materials: 85 patients with histopathologically proven breast cancer were studied. Breast MRI was performed on a 1.5 T scanner (Magnetom Espree) (a) before and (b) after the third cycle of chemotherapy. For dynamic imaging gadobutrol (Gadovist $\circledast$ ) was injected intravenously at a dose of $0.1 \mathrm{mmol} / \mathrm{kg} \mathrm{BW}$ and six dynamic T1w GRE sequences (VIBE) were collected. The contrast kinetics of all malignant lesions were analysed quantitatively on a pixel by pixel basis using a computer-aided detection system (iCAD). The signal enhancement pattern was coded by three colour intensities and three colour hues. Pharmacokinetic changes were analysed based on the Tofts model including vascular permeability and extracellular volume fraction.

Results: In comparison to the baseline MRI, follow-up examinations showed a significant tumour size reduction of $57 \%$ on average. A decrease of colour hues and colour intensities demonstrated significant changes in contrast kinetics. Vascular permeability revealed a statistically significant decrease $(p<0.05)$. However, extracellular volume fraction changes revealed only a slight decrease under chemotherapy (mean decline of $16 \%$ ).

Conclusion: The CAD analysis provides a reliable evaluation of tumour size, contrast kinetics, vascular permeability and extracellular volume fraction within a reasonable time. This form of CAD analysis provides a fast and reliable assessment of early treatment response, enabling a fast modulation of therapy for non-responders.

\section{B-0457 15:03}

Breast MRI for assessment of primary systemic chemotherapy (PSC): computer assisted diagnosis (CAD) predicts degree of tissue response and residual tumour mass

P.A.T. Baltzer' ${ }^{1}$ M. Dietzel ${ }^{2}$, W.A. Kaiser ${ }^{3} ;{ }^{1}$ Vienna/AT, ${ }^{2}$ Erlangen/DE, ${ }^{3}$ Jena/DE

Purpose: Dynamic contrast-enhanced breast MRI (bMRI) is used for assessment of primary systemic chemotherapy (PSC), revealing functional information about tumour vasculature. Application of CAD systems to bMRI allows for volumetric enhancement pattern assessment. This study investigates the performance of CAD-assisted bMRI to assess the degree of tissue response to PSC and to identify residual tumour mass prior to surgery.

Methods and Materials: bMRI examinations (1.5 T, according to EUSOMA recommendations) of 67 patients with biopsy proven invasive breast cancer lesions were examined before and after PSC. Maximum diameter reduction (D), lesion volume reduction $(\mathrm{V})$ and most suspect enhancement curve $(\mathrm{C})$ were obtained using a commercial available CAD system (CADstream). Histopathologic assessment of residual tumour mass (RT) and tumour tissue vitality (TV) was performed on open surgery specimen. Prediction of RT and TV by bMRI was analysed.

Results: Before PSC, 108 malignant lesions were identified. After PSC 85 lesions remained. CAD revealed 108 lesions before and 48 lesions after PSC. Pathology showed no RT:44, reduced TV:53, TV not reduced: 11 cases. ROC-based accuracy for RT was highest for $\mathrm{C}$ and $\mathrm{D}(76.5 \%$ and $76.7 \%$, respectively) whereas $\mathrm{V}$ showed lower diagnostic performance $(70.7 \%)$. Regarding TV, accuracy was highest for $\mathrm{V}(85.7 \%)$, whereas $\mathrm{C}$ and $\mathrm{D}$ showed equal diagnostic use $(80.9 \%$ and $81.7 \%$, respectively)

Conclusion: Our investigation shows that functional information both of curve type and size of enhancement are predictors of breast cancer response to PSC. Although bMRI is specific in the described setting, it shows only moderate sensitivity.

\section{B-0458 15:12}

Evaluation of residual breast tissue post-mastectomy using breast MRI V. Tsehmaister Abitbol, A. Rundstein, A. Shalmon, E. Konen, M. Sklair-Levy; Tel Hashomer/L

Purpose: To measure the residual breast tissue in patients who underwent mastectomy with implant reconstruction using breast MRI.

Methods and Materials: Retrospective review of the breast MRI examinations performed at our institute between January and November 2011, in 45 women who had mastectomy with implant reconstruction. Residual breast thickness was evaluated at 4 points $(3,6,9,12$ o'clock). Breast MRI was performed on 1.5 Tesla with a dedicated breast coil and a standard dynamic implant bilateral breast MRI protocol. Statistics included average, standard deviation, t-test and Kendall's Tau and Spearman's rank correlation coefficient.

Results: 45 women were evaluated, the number of breasts evaluated was 88 . Mean age was 43.6 (range 26-61years). We compared residual breast tissue post-mastectomy due to carcinoma $(n=45)$ vs. preventive mastectomy $(n=43)$. The average width of residual breast tissue at $3,6,9,12$ o'clock was $8.8 \mathrm{~mm}, 13.3 \mathrm{~mm}$, $9.3 \mathrm{~mm}$, and $13.3 \mathrm{~mm}$, respectively. The mean width of residual breast tissue was smaller in the mastectomy due to carcinoma $(10.63 \mathrm{~mm})$ compared to preventive mastectomy $(11.86 \mathrm{~mm})$, the difference was not statistically significant $(\mathrm{p}=0.09)$. Conclusion: In women undergoing mastectomy either for breast carcinoma or prevention we have shown residual breast tissue measuring between 0 and 53.4 $\mathrm{mm}$, with the maximum thickness at 12 o'clock. As far as we know this is the first 
attempt to evaluate residual breast tissue in patients following mastectomy and reconstruction. This study emphasizes the need for follow-up after mastectomy due to the possible risk of recurrence in the residual tissue.

\section{B-0459 15:21}

Breast cancer recurrence in DIEP flap reconstructed breasts: a 12year retrospective study

J.A. Farras Roca, A. Luciani, R. Bosc, E. Meyblum, P. Beaussart,

F. Pigneur, Y. Belkacemi, A. Rahmouni, T.-H. Dao; Créteil/FR

(josep.farras@etu.parisdescartes.fr)

Purpose: There is no consensus on surveillance strategies of reconstructed breasts after breast cancer, and it is routinely based on clinical examination alone. This study aims to assess the incidence and radiological presentation of breast cancer recurrences after deep inferior epigastric perforator (DIEP) flap reconstruction for women with a history of breast cancer.

Methods and Materials: 265 consecutive women who underwent DIEP flap reconstruction after breast cancer from 1997 to 2009 were included in this retrospective study. All had annual screening for recurrence and we reviewed their follow-up records in our institution. The date and site of recurrences - ipsilateral or controlateral to the reconstructed breast, regional and metastatic disease - were retrieved. Clinical and radiological presentations - on mammography, ultrasound, and MRI - were further analysed.

Results: 31 of 265 patients presented 34 relapses, with a mean delay of 4.7 years $(S D=2.9)$ after DIEP reconstruction; $14(41 \%)$ were ipsilateral, while $6(18 \%)$ were controlateral and $14(41 \%)$ metastatic. Ipsilateral recurrences had a tendency to occur earlier (3.8 years, $S D=2.4, p=0.11$ ) and 12 presented at a clinically patent stage. Conclusion: Cancer recurrence rate after DIEP flap reconstruction was $12 \%$ in our cohort, $41 \%$ of them occurring in the reconstructed breast. Standardised radiological screening of DIEP flap reconstructed breasts should be encouraged.

14:00 - 15:30

Room G/H

\section{Cardiac}

\section{SS $603 b$}

\section{Cardiomyopathy and fibrosis}

Moderators:

P. Donato; Coimbra/PT

R. Maksimović; Belgrade/RS

\section{B-0460 14:00}

Potential value of fibrosis quantification in dilated cardiomyopathy

A. Jacquier, A. Kallifatidis, B. Maurel, A. Flavian, J. Gaubert, J. Bartoli,

G. Moulin; Marseille/FR (alexis.jacquier@ap-hm.fr)

Purpose: To assess the potential value of segmental quantification of myocardial fibrosis using T1 mapping in dilated cardiomyopathy (DCM) in comparison to late contrast enhancement imaging and circumferential strain.

Methods and Materials: Seventeen patients with DCM, and 11 controls subjects were prospectively included. All patients underwent cardiac MR with the following sequences (parameters set according to recommendation of the SCMR): 1) cine sequence, 2) tagging sequence CSPAMM TR/TE=37.1 ms/1.4 ms; matrix 256, FOV 340, temporal resolution $37.1 \mathrm{~ms}$, distance between tag: $6 \mathrm{~mm}$, short axis. 3) Molli sequence (TR/TE=3.0 ms $/ 1.5 \mathrm{~ms}$; matrix 144-150, thickness: $7 \mathrm{~mm}$ ) acquired before and 5, 7, $9 \mathrm{~min}$ after gadolinium injection (DOTAREM, $0.2 \mathrm{mmol} / \mathrm{kg}$ ). 4) Late gadolinium enhanced sequence $10 \mathrm{~min}$ after injection. T1 values were assessed in blood and in the 6 segments of the mid-LV slice. Statistical analysis were performed for patients and for segments with (+) and without (-) enhancement on LGE. R1, $\Delta R 1, \Delta R 1$ ratio were calculated (partition coefficient of $G d, \lambda$ ).

Results: In DCM, $\lambda$ was not significantly different between patient without $(0.50 \pm 0.05)$ and with at least one segment showing enhancement on LGE $(0.52 \pm 0.06 ; p=0.06)$. In all DCM, $\lambda$ was significantly higher compared with the value obtained in control subject $(p<0.0001)$. We found a significant correlation $\lambda$ and circumferential strain in each segment $(r=0.55 ; \mathrm{Cl}(0.39 ; 0.68) ; p<0.0001)$, and between $\lambda$ and LVEF $(r=-0.56$; $\mathrm{Cl}(-0.82 ;-0.11) ; \mathrm{p}<0.01)$.

Conclusion: In DCM, fibrosis was a diffuse process involving all the myocardium. There was a correlation between the quantity of fibrosis, circumferential strain and LVEF.
B-0461 14:09

Potential value of fibrosis quantification in hypertrophic cardiomyopathy

A. Jacquier, A. Kallifatidis, B. Maurel, A. Flavian, J. Gaubert, J. Bartoli,

G. Moulin; Marseille/FR (alexis.jacquier@ap-hm.fr)

Purpose: To assess the potential value of fibrosis quantification using T1 mapping in hypertrophic cardiomyopathy ( $\mathrm{HCM})$ in comparison to late contrast enhancement imaging, circumferential strain and LV thickness.

Methods and Materials: Seventeen patients with $\mathrm{HCM}$, and 11 controls subjects were prospectively included. All patients underwent cardiac MR with the following sequences: 1) cine sequence, 2) tagging sequence CSPAMM TR/TE=37.1 ms/1.4 ms; matrix 256, FOV 340, temporal resolution $37.1 \mathrm{~ms}$, distance between tag: $6 \mathrm{~mm}$, short axis. 3) Molli sequence (TR/TE=3.0 ms/1.5 ms; matrix 144-150, thickness:7 $\mathrm{mm}$ ) acquired before and 5, 7, 9 min after gadolinium injection (DOTAREM, 0.2 $\mathrm{mmol} / \mathrm{kg}$ ). 4) Late gadolinium enhanced sequence $10 \mathrm{~min}$ after injection. T1 values were assessed in blood and in the 6 segments of the mid-LV slice. Statistical analysis were performed for patients and for segments with (+) and without (-) enhancement on LGE. $R 1, \Delta R 1, \Delta R 1$ ratio were calculated (partition coefficient of $G d, \lambda$ ). Results: In $\mathrm{HCM}, \lambda$ was significantly different between patient with $(0.54 \pm 0.08)$ and without $(0.45 \pm 0.05)$ enhancement on LGE $(p<0.0001)$. No significant difference was measured in $\lambda$ value between control and HCM without delayed enhancement $(p=0.1)$. We did not found any correlation between lambda and circumferential strain $(r=0.11 ; \mathrm{Cl}(-0.12 ; 0.34) ; \mathrm{p}=0.3)$. On the other hand, we found a significant correlation between LV wall thickness and circumferential strain $(0.40$; Cl $(0.20 ; 0.56) ; p<0.0001)$.

Conclusion: In HCM, fibrosis is a focal process. In our study, there were no correlation between the presence of fibrosis and circumferential strain, but we found a significant correlation between LV wall thickness and circumferential strain.

\section{B-0462 14:18}

Extent of myocardial scar detected by late Gadolinium enhancementcardiac magnetic resonance (LGE-CMR) as a predictor of major adverse cardiac events (MACE) in $\mathrm{HCM}$ patients

S. Bertugno, G. Ligabue, F. Fiocchi, L. Nocetti, S. Vasaturo, V. Morini, A. Barbieri, R. Lugli; Modena/IT

Purpose: To investigate the prognostic value of LGE-CMR, throughout the association between LGE extension and the occurrence of MACE in HCM patients. Methods and Materials: 90 consecutive HCM patients ( 60 males and 30 females), aged between 14 and 79 years, underwent 1.5T CMR. Indexed end-diastolic left ventricular mass (IEDLV mass) was assessed at end-diastole. LGE-MR images were performed $15 \mathrm{~min}$ after injection of $0.2 \mathrm{mmol} / \mathrm{kg}$ of Gadolinium. A threshold of 6 SD exceeding the mean signal intensity from normal myocardium was used to define areas of fibrosis and LGE extent was expressed as percentage to the total IEDLV myocardial mass. Clinical adverse events were prospectively assessed during a follow-up period of $40.2 \pm 11.1$ months. MACE included were atrial fibrillation, non-sustained and sustained ventricular tachyarrhythmias, implantable cardioverter defibrillator implant and shock, progression of NYHA functional classes III or IV, surgery for obstructive HCM (myomectomy and mitral valve replacement), unscheduled hospitalisation, death from heart failure and sudden cardiac death. Results: The mean LGE\% was $8.11 \pm 6.63 \mathrm{~g} / \mathrm{m}^{2}$. During the follow-up one or more MACE were recorded in 35 (38.9\%) patients; 23 (25.5\%) patients experienced two or more MACE. We found a significant association between LGE\% and overall MACE (86). Patients who experienced one or more MACE had significantly higher

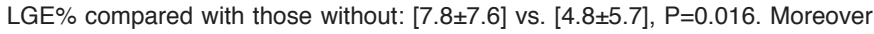
LGE\% was significantly higher in patients who presented two or more MACE than in patients with only one or without MACE: [10.1 \pm 7.6$]$ vs. [4.6 \pm 5.7$], P=0.0002$. Conclusion: Extension of LGE is a significant predictor for the occurrence of MACE in HCM patients.

\section{B-0463 14:27}

Myocardial scar extension detected by late Gadolinium enhancement-cardiovascular magnetic resonance (LGE-CMR) for arrhythmic risk stratification of $\mathrm{HCM}$ patients

S. Bertugno, G. Ligabue, F. Fiocchi, L. Nocetti, S. Vasaturo, V. Morini, A. Barbieri, R. Lugli, P. Torricelli; Modena/IT

Purpose: To investigate the role of myocardial fibrosis detected by LGE-CMR as a potential arrhythmogenic substrate in $\mathrm{HCM}$, throughout the association between LGE extension and the occurrence of ventricular tachyarrhythmias (VT) and implantable cardioverter defibrillator shock (ICDs).

Methods and Materials: LGE-CMR was performed in 90 consecutive HCM patients (mean age: $58.4 \pm 15.3 ; 66.6 \%$ males) using a 1.5T scanner. LGE-MR images were 
obtained $15 \mathrm{~min}$ after injection of $0.2 \mathrm{mmol} / \mathrm{kg}$ of Gadolinium. LGE was quantified automatically and defined as area of myocardium with signal intensity 6SD above the mean signal of remote myocardium. Indexed end-diastolic left ventricular mass (IEDLV mass) was assessed and LGE extension was defined as percentage of total IEDLV myocardium mass. Patients were followed prospectively for a mean of $40.2 \pm 11.1$ months, during which period occurrence of VT and ICDs were recorded. Results: The mean IEDLV mass was $89.5 \pm 28.4 \mathrm{~g} / \mathrm{m}^{2}$. On LGE-CMR, 70 (80\%) patients showed myocardial scar (mean LGE\%: $8.11 \pm 6.63$ of IEDLV mass). During the follow-up period 18 (20\%) NSVT and 10 (11.1\%) SVT were recorded. $9(10 \%)$ patients underwent ICD implantation and 4 of them experienced one or more appropriate ICDs. LGE\% was significantly higher in patients who exhibited NSVT and SVT than in patients who did not: $[11.6 \pm 7.5]$ vs. $[4.6 \pm 5.6], P=0.00001$ and $[13.9 \pm 7]$ vs. $[5.9 \pm 5.1], P=0.00001$. There was no significant relationship between LGE extension and ICDs: $P=0.075$.

Conclusion: Extension of fibrosis on LGE-CMR predicts the occurrence of NSVT and SVT in HCM patients.

\section{B-0464 14:36}

Prognostic value of RV remodelling in pulmonary hypertension: cardiac magnetic resonance study

N. Galea, M. Francone, I. Carbone, I. Iacucci, P. David, C. Vizza, C. Catalano; Rome/IT (nicogale2000@yahoo.it)

Purpose: In Pulmonary arterial hypertension (PH),increase of pulmonary vascular resistances results in severe right ventricular $(\mathrm{RV})$ dysfunction due to increased afterload and myocardial remodelling.

The aim of our study was to investigate how the morphological adaptive changes in myocardium (concentric vs eccentric hypertrophy,myocardial fibrosis at late enhancement) and RV ejection fraction (RVEF) impact survival in $\mathrm{PH}$.

Methods and Materials: Our study population included 83 consecutive patients with $\mathrm{PH}$ (56 idiopathic, 9 CHD-related, 16 secondary) who underwent to clinical setting, right heart catheterisation, CMR, and 6-min walk testing at the baseline. CMR protocol included cine imaging and LE acquisitions after contrast-administration (0.1 $\mathrm{mmol} / \mathrm{kg}$ Gd-BOPTA) in order to measure volumes and detect fibrosis. Population was divided in groups on the basis of median value of RV mass/diastolic volume ratio (RV M/V=Gr1<0.60,Gr2>0.60), RVEF (GrA<34\%,GrB > 34\%) and LE presence $(\mathrm{y} / \mathrm{n})$ and followed up for deaths (average:4.3 $\pm 1.6 \mathrm{yrs}$ ).

Results: Increase of RV M/V ratio ( $>0.60$ ) was related to lower RV systolic and diastolic volume (respectively $p=0.006$ and $<0.005$ ), but similar RV mass $(p=n s)$. In spite of significant differences in clinical status, effort capacity and haemodynamics between the two groups, Gr2 patients have a lower mortality according to KaplanMayer curve (mean survivor time Gr1:925dys,Gr2:1339dys, $p=0.008$ ). Baseline RVEF was predictor of mortality $(p=0.002)$. DE was demonstrated in $74 / 85$ pts and predominantly confined to RV insertion points (70/74). Multivariate analysis excluded negative impact of LE as prognostic indicators of death.

Conclusion: In $\mathrm{PH}, \mathrm{RV}$ hypertrophy and mild diastolic volume increase (high $\mathrm{M} /$ Vratio and high RVEF) is associated with low rate of deaths, suggesting a favourable prognostic role of this parameter.

\section{B-0465 14:45}

Evaluation of Brugada syndrome by cardiac magnetic resonance

L. Salvatori ${ }^{1}$, C. Tessa ${ }^{1}$, i. Del Meglio' ${ }^{1}$, S. Diciotti ${ }^{2}$, C. Vignali ${ }^{1}$, G. Casolo';

${ }^{1}$ Lido di Camaiore/IT, ${ }^{2}$ Firenze/IT (I.salvatori71@gmail.com)

Purpose: Brugada syndrome (BrS) has been originally considered to occur in structural normal hearts. However, recent pathological and imaging data suggest that structural and functional changes may be present in this syndrome. This study was designed to elucidate whether any macroscopic heart abnormality is detectable in patients with $\mathrm{BrS}$. For this purpose we used cardiac magnetic resonance (CMR). Methods and Materials: Twenty-nine patients displaying the BrS type-1 ECG pattern and 29 healthy controls underwent CMR (1.5 Tesla). Left (LV) and right ventricular $(\mathrm{RV})$ dimensions, function and regional contractility were evaluated. Late-gadolinium-enhancement (LGE) imaging was obtained in 24 patients.

Results: We found no difference between BrS patients and controls regarding LV and RV dimensions and ejection fraction. RV wall motion abnormalities (WMA) were detected in 19 patients $(65.5 \%$ ) and in 22 control subjects $(75.9 \%)$. The majority of these WMA were attributable to areas of hypokinesia and found in the RV inferior wall. None of the patients showed LGE. No differences were detected between controls and the different subgroups of BrS patients according to symptoms, family history and spontaneous type-1 ECG pattern.

Conclusion: $\mathrm{BrS}$ patients do not differ from normal subjects with regard to dimensions and global function of both LV and RV. BrS patients may show RV-WMA; however, similar changes are also present in healthy subjects and may therefore represent a physiological behaviour of RV. The lack of LGE further indicate the absence of myocardial structural damage. Our results indicate that $\mathrm{BrS}$ seems to occur in individuals with structurally and functionally normal heart.

\section{B-0466 14:54}

Sensitivity of cardiac magnetic resonance varies with clinical presentation of biopsy-proven acute myocarditis: correlation with necrosis/apoptosis patterns of viral aetiology

G. Cannavale, M. Francone, R. Rosati, P. David, A. Frustaci, C. Catalano; Rome/lT (giuseppe_cannavale@hotmail.it)

Purpose: Our aim was to evaluate CMR sensitivity in patients presenting with acute myocarditis with various clinical presentations and correlate results with histological, immunohistochemical and ultrastructural analysis.

Methods and Materials: We retrospectively evaluated CMR sensitivity in comparison with clinical presentation in 57 patients who had in the last two years an endomyocardial biopsy diagnosis of active myocarditis and clinical history 23 months. All subjects underwent at time of diagnosis a CMR and invasive cardiac examination. Immunohistochemistry and viral genomes detection by PCR were also performed.

Results: Three types of clinical patterns of myocarditis were recognised: 1 ) Infarct-like (fever, chest pain, ST segment deflection at ECG and serum Troponin I elevation). 2) Cardiomyopathic (left ventricular dysfunction in absence of ECG or systemic abnormalities). 3) Arrhythmic (sudden occurrence of arrhythmias in absence of systemic evidence of inflammation). Delayed enhancement suggesting a myocarditis was documented in $71 \%$ of patients $(n=15 / 21)$ of pattern $1,57 \%$ $(n=12 / 21)$ of pattern 2 and $47 \%(n=7 / 15)$ of pattern 3 . Tissue oedema was observed in $81 \%(n=17 / 21)$ of pattern $1,28 \%(n=6 / 21)$ in pattern 2 and $27 \%(n=4 / 15)$ in pattern 3. Early enhancement was positive in $71 \%$ (15/21) of the cases with pattern $1,67 \%$ (14/21) with pattern 2 and $40 \%$ (6/15) with pattern 3 . Myocardial damage due to different viral agents detected for each pattern was correlated with CMR findings. Conclusion: Sensitivity of CMR is high for infarct-like myocarditis, low for cardiomyopathic and very low for arrhythmic pattern. These variations are likely due to the different viral agents involved reflecting different histological patterns, causing a more extensive vascular disease in pattern 1 compared with focal myocyte and conduction tissue damage of patterns 2 and 3.

\section{B-0467 15:03}

\section{Cardiac magnetic resonance vs echocardiography for the} assessment of cardiac volumes and function in thalassemia intermedia patients

C. Tudisca1, A. Meloni' ${ }^{2}$, C. Ascioti ${ }^{3}$, S. Renne ${ }^{3}$, V. Santamaria ${ }^{4} ;{ }^{1}$ Palermo/IT, ${ }^{2}$ Pisa/IT, ${ }^{3}$ Lamezia Terme $/ I T,{ }^{4}$ Vibo Valentia/IT

Purpose: We aimed to compare the agreement of left ventricular (LV) volumes and ejection fraction (EF) by Cardiac Magnetic Resonance (CMR) and Echocardiography (US) in a moderately large cohort of Thalassaemia Intermedia (TI) patients. Methods and Materials: $74 \mathrm{TI}$ patients ( $38 \mathrm{M} ; 36.7 \pm 10.9$ years) enrolled in the MIOT (Myocardial Iron Overload in Thalassaemia) network were studied by both echo and CMR (1.5 T) within 3 months of each other. CMR cine images were acquired to assess LV parameters in a standard way. LV parameters were measured by two-dimensional echocardiography using the biplane Simpson's formula. Paired-samples t-test or Wilcoxon test, correlation coefficient, intraclass correlation (ICC) and Bland \& Altman plot were used to compare CMR and US parameters. Results: All US volumes were significantly underestimated, especially the LV enddiastolic volume index $(69.9 \pm 18.1 \mathrm{ml} / \mathrm{m} 2$ vs $96.3 \pm 17.9 \mathrm{ml} / \mathrm{m} 2, P<0.0001)$, while the US LV EF was significantly higher than the CMR EF $(63.7 \pm 6.6 \%$ vs $62.0 \pm 7.4 \%$, $P=0.051)$. The correlation between US and CMR end-dyastolic and stroke volume indexes was significant but with a very low coefficient, while the correlation for the ejection fraction was acceptable. The ICC was unsatisfactory for all volumes and good for the ejection fraction. The widest Bland Altman plot range was found for the end-diastolic volume index.

Conclusion: In conclusion, metrics of LV volume index and function showed significant systematic inter-modality differences. In particular, the US volumes were systematically underestimated. This suggests that serial measurements of volumes and function in $\mathrm{Tl}$ should be performed using the same method and, if available, the more reproducible CMR technique.

Author Disclosures:

C. Tudisca: Research/Grant Support; The MIOT project receives "no-profit support" from industrial sponsorships (Chiesi Farmaceutici S.p.A., ApoPharma Inc. and Bayer-Shering). This study was also supported by: "Ministero della Salute". . 


\section{B-0468 $\quad 15: 12$}

Right heart involvement in thalassaemia major $\left.{ }^{\mathrm{TM}}\right)$ patients: a cardiac MR (CMR) assessment

F. Pitocco, I. Di Giampietro, A. De Vivo, P. Cianciulli, C. Liguori,

B. Beomonte Zobel; Rome/IT (f.pitocco@unicampus.it)

Purpose: ${ }^{\mathrm{TM}}$ patients should develop right heart failure caused by right ventricle (RV) impairment related to chronic anaemia. It is still unknown if iron myocardial deposition and iron overload can influence the RV functional/volumetric parameters. Our aim was to define normal ranges for $\mathrm{RV}$ volumes and $\mathrm{RV}$ ejection fraction in $\mathrm{TM}_{\mathrm{M}}$ with and without myocardial iron overload.

Methods and Materials: We performed CMR examination (Scanner 1.5T, Avanto, Siemens-Germany) in a setting of 220 patients with ${ }^{\mathrm{TM}}$ under iron chelation therapy and without clinical or instrumental signs of right heart failure (mean age $37.8 \pm$ 12.8 ys; mean body surface area $\mathrm{kg} / \mathrm{m}^{2} 1.64 \pm 0.24 ; 13 \%$ ). Myocardial iron overload was considered $\mathrm{T}^{*}<20 \mathrm{~ms}$. RV-EF, volumes and mass index were evaluated.

Results: Myocardial iron overload in $13 \%$ of population was noted. A significant difference was found between mean RVEF in patients with iron overload and RVEF in patients without iron overload $(60 \pm 6.7 \%$ vs $60 \pm 11 \%, p<0.01)$, with no differences between sex. A significant difference was found in RV stroke, end-diastolic and end-systolic volume indices between the two groups $(45,30.75 \mathrm{vs} 49,31,76 \mathrm{ml} /$ $\mathrm{m} 2, \mathrm{p}<0.01)$. No significant difference was found in right ventricle mass values ( $19 \pm 5$ vs $15 \pm 13 \mathrm{~g} / \mathrm{m} 2)$.

Conclusion: Iron overload can damage right heart myocardium in the same way of left heart myocardium, showing similar functional impairment and volumetric enlargement. CMR is an useful technique for a correct evaluation of heart function and iron myocardial deposition in these kinds of patients.

\section{B-0469 15:21}

$\beta$-thalassaemia as a new potential risk factor for left ventricular noncompaction (LVNC): a cardiac MRI evaluation

C. Liguori, I. Di Giampietro, F. Pitocco, P. Cianciulli, B. Beomonte Zobel;

Rome/IT (c.liguori@unicampus.it)

Purpose: Noncompaction of the left ventricle myocardium (LVNC) is a rare cardiomyopathy characterised by multiple and excessive trabeculations, deep intertrabecular recesses and thickened ventricular myocardium. This condition is considered mostly congenital but acquired forms have also been described. Aim of the present study is to assess LVNC prevalence in $\beta$-thalassaemia patients and its potential association with cardiac siderosis

Methods and Materials: 229 transfusion-dependent patients with $\beta$-thalassaemia (160 major and 69 intermedia, mean age $37.8 \pm 12.8$ years, mean BSA $1.64 \pm$ $0.24 \mathrm{Kg} / \mathrm{m}^{2}$ ) presenting for cardiac and abdominal iron assessment by MRI (1.5T Scanner, Avanto, Siemens-Germany) were studied. Cardiac iron overload was assessed by $\mathrm{T}^{*}$ Black-Blood gradient-multiecho sequences; cardiac functional and morphological characteristics were evaluated using SSFP acquisitions. Risk factors for LVNC were noted and none of the patients had neuromuscular or congenital heart disease.

Results: 69 patients (30\%) fulfilled the preassigned strict criteria for LVNC on cardiac MRI. There were no statistically significant differences between patients with and without LVNC with respect to demographics, haemoglobin levels, splenectomy status, systemic, hepatic, and cardiac iron overload indices, iron chelation therapy. Patients with LVNC showed a significant lower cardiac functional performance (Ejection Fraction; volumes impairment).

Conclusion: Patients with $\beta$-thalassaemia have a higher prevalence of LVNC than normal individuals suggesting an underlying iron deposition mechanism to explain this condition in absence of conventional risk factors. These data seem to be crucial regarding an entity with adverse cardiac outcomes, especially in patients with $\beta$-thalassaemia where cardiac disease remains a primary cause of mortality.
14:00 - 15:30

Room L/M

\section{Head and Neck}

\section{SS 608}

Technical innovations, TMJ disease and rare entities

Moderators:

J. Frühwald-Pallamar; Vienna/AT

J. Widelec; Brussels/BE

\section{B-0470 14:00}

CT numbers of $\mathrm{CBCT}$ regarding position dependence on different size of field-of-view

M. Imura' ${ }^{1}, \mathrm{~T}$. Todoroki ${ }^{2} ;{ }^{1}$ Kyoto/JP, ${ }^{2}$ Osaka/JP (minori.imura@gmail.com)

Purpose: Quality-controlled x-ray beam CBCT (QX-CBCT) is equipped with calibration function for $\mathrm{HU}$ measurements in cone beam $\mathrm{CT}$ for the first time. In this study, we examined its characteristics of CT numbers in terms of cone angle effect represented as different field-of-view.

Methods and Materials: 1) Reproducibility and possible variation of CT numbers of QX-CBCT within exposure area on three different FOV were examined. Water phantom attached to QX-CBCT for calibration was scanned four times. Mean CT numbers of region of interest $(\mathrm{ROI})$ at the centre and twenty peripherals in each axial image were measured. 2) Varying concentrations of iodinated solution phantoms $(0-4700 \mathrm{HU})$ were scanned at $100 \mathrm{kV}$ for QX-CBCT and 80 to $140 \mathrm{kV}$ for MDCT on all FOV. ROIs were placed at the centre of each phantom on the basis of water phantom study. Correlation of CT numbers between QX-CBCT and MDCT were examined by the Pearson's correlations analysis.

Results: 1) Mean CT numbers of QX-CBCT at each independent ROI were reproducible for all FOV $(R=0.86-0.95, P<0.0001)$. CT numbers at the centre region were consistent regardless of the size of FOV, whereas the values tended to be higher and heterogeneous at the outer edge of exposure area. The consistent area was sphere like shape within exposure area. 2) CT numbers of QX-CBCT exhibited significant correlation to those of MDCT under $2500 \mathrm{HU}(\mathrm{R}=0.98, \mathrm{P}<$ 0.0001) for all FOV.

Conclusion: When handing the $\mathrm{CT}$ numbers of $\mathrm{QX}-\mathrm{CBCT}$, region-dependence within exposure area due to the effect of cone angle should carefully be considered.

\section{B-0471 14:09}

Dual-source 128-slice MDCT neck: radiation dose and image quality estimation of a clinically relevant dual-energy, high-pitch, standard single-energy protocol

J. Paul, E. Mbalisike, T.J. Vogl; Frankfurt a. Main/DE (jijopaul1980@gmail.com)

Purpose: To estimate the radiation dose and image quality of a clinically relevant single-source (SSCT), high-pitch (HPCT), and dual-energy (DECT) protocols of an innovative dual-source (DSCT) system for the examination of neck.

Methods and Materials: 180 patients were randomised to one of the three protocols: 60 patients (age: $55.4 \pm 12$ years; range: $44-84$ years) were examined with a SSCT, other $60(59.5 \pm 16.4$ years; R: $40-85)$ with HPCT, and the last 60 $(61.1 \pm 14.9$ years; $R: 47-84)$ were examined with a DECT protocol. All examinations were performed using a DSCT system. The used protocols: group-1 (SSCT: $120 \mathrm{kV}$; effective mAs: $185.4 \pm 17.7$ ), group-2 (HPCT: $120 \mathrm{kV}$; eff.mAs: $97.7 \pm 11.8$ ), and group-3 (DECT: $80 \mathrm{kV} / 140 \mathrm{kV}$ with tin-filter; eff.mAs: $248.5 \pm 25.7 ; 187 \pm 21.2$ ). A $100-\mathrm{ml}$ lomeprol non-ionic contrast material was injected in to the patients during examination.

Results: Insignificant results were yielded regarding SNR and CNR between the groups (group-1 vs. 2: 0.3125 , group-1 vs. $0.6 \mathrm{~W}: 0.6875$, group-2 vs.0.6 W: 0.3125 ), except DECT-80 (group-1 vs. 80 kV: 0.04289 , group-2 vs. 80 kV: 0.025, group-0.6 $\mathrm{W}$ vs. $80 \mathrm{kV}: 0.04567$ ) and $140 \mathrm{kV}$ data; moreover, qualitative analysis yielded the same results. Mean effective-dose was significantly lower $(p<0.05)$ in group-2 $(1.06 \pm 0.16 \mathrm{mSv})$ compared to group $-1(2.05 \pm 0.22 \mathrm{mSv})$ or group $-3(1.76 \pm 0.2 \mathrm{mSv})$. Single- and dual-energy comparison showed a significant difference (group-1 vs.3: $p=0.00001$ and group-2 vs. $3: p=0.00001$ ) for CTDIvol (percent difference: $16 \%$, $64 \%$ ) or DLP (PD: $15.5 \%, 50.5 \%$ ).

Conclusion: Quantitative and qualitative analyses showed similar results for SSCT, HPCT, and DECT-0.6 W datasets regarding quality. HPCT yielded lower dose compared to other groups; however, the DECT achieved a lower and significant dose difference from the SSCT protocol. HPCT and DECT can be used with similar image quality and lower radiation dose compared to SSCT for the scans and can be utilised various clinical advantages. 


\section{B-0472 14:18}

Performance of filtered back-projection (FBP), statistical (ASIR) and model-based (VEO) iterative reconstruction of neck CT staging examinations

F.G. Mück, L. Geyer, J. Grimm, Z. Deak, M.F. Reiser, S. Wirth; Munich/DE (fgmueck@gmail.com)

Purpose: To compare image quality of FBP, ASIR and VEO reconstructed CT images of the neck in tumour patients with a contrast-enhanced scan.

Methods and Materials: Consecutive regular contrast-enhanced tumour staging CT-scans (Discovery HD750, GE; helical, 0.8s, 120 kV, 50-400 mA mod., NI 33) of the neck were reconstructed using FBP, ASIR and VEO; $n=27$. Image quality at three defined heights (lymph node levels=LNL $2,3,7$ ) were independently rated by 2 radiologists blinded to the reconstruction algorithm. Readers rated image quality including artefacts, contrast and noise using a semi-quantitative scale of 0 to 10 (lower than 5 was defined as non-diagnostic) yielding a summary score for each height. Wilcoxon's test was used for statistical testing. ICC was calculated. Results: Patient weight was $78.2 \pm 11.3 \mathrm{~kg}$, BMI was $25.7 \pm 4.7$ and CDTI was $9.7 \pm 2.8$ mGy. Reconstruction-time was $58 \pm 12$ s for ASIR and FBP and 3780 s \pm 180 s for VEO $(p<0.01$ ). Image rating at $L N L 2$ was $7.9 \pm 0.5$ (VEO), $6.2 \pm 0.6$ (ASIR) and $5.6 \pm 0.5$ for FBP ( $p<0.01$, respectively). Rating at LNL 3 was $8.5 \pm 0.3$ (VEO), $7.4 \pm 0.4$ (ASIR) and $6.8 \pm 0.8$ for FBP $(p<0.01)$. LNL 7 score was $7.9 \pm 0.4$ (VEO), 5.5 \pm 0.4 (ASIR) and $5.1 \pm 0.4$ for FBP $(p<0.01)$. ICC was 0.81 .

Conclusion: Contrast-enhanced CT investigations of the neck VEO generally performed better then ASIR and FBP. Especially, at levels where artefacts (e.g. teeth) or high overlay of image noise (e.g. shoulder) have a strong impact on image quality, Veo clearly outperformed the other algorithms. As VEO requires 75 times higher data reconstruction-time, the process has to be sped up to allow integration into normal clinical routine work.

\section{B-0473 14:27}

Maxillary sinus pyocele: a new radiological entity

A. Lacan-Melki, S. Espinoza-Boireau, I. Khettab, P. Halimi; Paris/FR

(alacanmelki@hotmail.fr)

Purpose: Maxillary sinus pyocele is a true chronic abscess found in drugrefractory sinusitis. The purpose of this study is to define this new entity and its MRI-characteristics.

Methods and Materials: We retrospectively reviewed MRI datasets for 23 patients with "pseudotumoral sinusitis" on CT (including 2 bilateral cases). The MRI datasets were scored by two radiologists for morphological features, T1 and T2-weighted signal intensities, contrast enhancement and the degree of impact on the sinus mucosa and the retromaxillary fat. These features were compared with per-operative findings.

Results: We observed a content presenting T1 low signal (92\%), heterogeneous T2 low signal $(92 \%)$, a capsule with contrast enhancement (100\%) containing microabscesses $(52 \%)$, a mass effect on the lateral nasal wall (100\%) and retromaxillary fat stranding ( $88 \%$ ). Eighteen pyoceles were treated endoscopically, with confirmation of a maxillary sinus abscess in all cases. Furthermore, we suggest a staging system for maxillary sinus pyocele.

Conclusion: Maxillary sinus pyocele presents specific MRI characteristics. This entity must be suspected on sinus CT scan and recognised on MRI. Surgical endoscopic sinus surgery is needed. These findings therefore merit further investigation.

\section{B-0474 14:36}

Carotid blowout syndrome in patients with nasopharyngeal carcinoma treated with radiotherapy: a review of 12-year experience V.K. Fung, J.S. Shum, B.M. Lai, C. Chu, W.K. Kan; Hong Kong/HK (vincentkpfung@hotmail.com)

Purpose: To describe the clinical and angiographic features of carotid blowout syndrome (CBS) in patients with nasopharyngeal carcinoma (NPC) treated with radiotherapy (RT).

Methods and Materials: 14 patients with RT-treated NPC who underwent digital subtraction angiography (DSA) from 2000 to 2012 for management of CBS were retrospectively reviewed. The initial T and N staging of NPC, duration between diagnosis of NPC to CBS, number of radiotherapies (RT) received for recurrence were studied. Distributions and sizes of pseudoaneurysms were analysed.

Results: 9 patients (64\%) with CBS had initial T3 disease or above while 11 patients $(79 \%)$ presented with $\mathrm{N} 1$ stage or above. Time interval between diagnosis of NPC and presentation of CBS varied from 4 months to 468 months (mean = 181 months). Most patients $(n=8,57 \%)$ had recurrence and all received further RTs. Pseudoaneurysms were identified in 11 patients $(79 \%)$ with the maximum diameter ranged from $2.3 \mathrm{~mm}$ to $34.3 \mathrm{~mm}$ ( $\mathrm{mean}=11.3 \mathrm{~mm}$ ). Involvement of internal carotid artery $(n=9,64 \%)$ was more common than external carotid artery $(n=5,36 \%)$. Petrous segment of ICA was the most commonly involved segment $(n=5,36 \%)$, followed by cervical segment of ICA $(n=4,29 \%)$ and maxillary artery $(n=3,21 \%)$. Conclusion: Carotid blowout syndrome following RT were more common in our study subjects with advanced T and N stage of NPC, local recurrence and repeated RTs. Pseudoaneurysms were identified in most patients. ICA involvement was more common and petrous segment of ICA was the most commonly involved segment.

\section{B-0475 14:45}

Nasal-type NK/T-cell lymphoma of the nasal cavity and PNS: CT and MR imaging findings

K. Kang', S. Suh', K. Kim', K. Son', Y. Lee ${ }^{2}$, H. Seo ${ }^{2}$, N. Lee ${ }^{1}$, H. Seol'; ${ }^{1}$ Seoul/KR, ${ }^{2}$ Ansan/KR (slothgw@hotmail.com)

Purpose: To describe the computed tomography (CT) and magnetic resonance (MR) imaging findings of nasal-type NK/T-cell lymphoma of the nasal cavity and PNS.

Methods and Materials: During the period between July 2007 and June 2012, the CT $(n=25)$ and MR $(n=14)$ images of 26 patients with biopsy-proven nasaltype NK/T-cell lymphoma of the nasal cavity and paranasal sinus (PNS) were retrospectively reviewed.

Results: The cases of nasal-type NK/T-cell lymphoma consisted of primary tumours arising from the nasal cavity in 20 patients $(77 \%)$, PNS in 1 patient $(4 \%)$ and simultaneous involvement in the nasal cavity and PNS in 5 patients (19\%). Of the 6 patients with involvement of PNS, one presented with orbital extension. Nasopharyngeal and oropharyngeal involvement was seen in two cases. In 20 cases $(77 \%)$, the primary tumours seen as diffuse enhancing mucosal thickening. In five cases (19\%), the primary tumours seen as an enhancing mass lesion. In one case $(4 \%)$, the primary tumour was undetectable. Bone destruction was noted only in 3 cases $(12 \%)$.

Conclusion: Nasal-type NK/T-cell lymphomas of the sinonasal regions often presents with diffuse mucosal thickening rather than mass lesion. And they can mimic inflammatory disease such as sinusitis. Radiologists should be familiar with these findings indicative of the possibility of NKTL, and should perform a histopathologic confirmation to establish a diagnosis.

\section{B-0476 14:54}

Temporomandibular joint internal derangement: correlation of MRI findings with clinical symptoms

K. Šurlan Popovič, U. Lamot, M. Vrabec, R. Klavžar; Ljubljana/SI

(katarina.surlan@gmail.com)

Purpose: To determine whether the morphological manifestations detected by MRI correlate with the signs and symptoms of TMJ dysfunction according to gender and age of the patients.

Methods and Materials: 144 subjects with at least unilateral TMJ dysfunction underwent a MRI investigation of both TMJ to establish the presence of various degrees of disk displacement, osteoarthritis and effusion. Chi-square or Fisher's exact test to analyse the relationship between descriptive variables and logistic regression analysis was preformed. For the comparison of mean values between samples we used T-test. For describing the strength of association between two binary values we used odds ratios (OR).

Results: A significant relationship between symptoms and morphological manifestations was found (disk displacement $(p<0.001)$, osteoarthritis $(p<0.001)$ and effusion $(p=0.001))$. In the group without reduction there were significant increases in the risk of experiencing symptoms $(p=0.002)$ in the group with reduction the risk was marginal $(p=0.071)$. Significant correlation between age and osteoarthritis $(p=0.001)$ and age and effusion $(p=0.022)$ was found. There was no correlation between sex and morphological manifestations. Significant increases in the risk of experiencing symptoms were found in the group with morphological manifestations (disk displacement $(p=0.006)$, osteoarthritis $(p<0.001)$ and effusion $(p<0.001)$ ). Statistically marginal results were verified with multivariate analysis.

Conclusion: MRI morphological manifestations of the TMJ correlate with the presence of symptoms, therefore MRI has a crucial role in the diagnosis of treatment of TMJ dysfunction. 


\section{B-0477 15:03}

An investigation of relative signal intensity of MR images in retrodiscal tissue and lateral pterygoid muscle in patients with temporomandibular joint disorder and its relation with MRI findings Z. Ghoncheh, M. Panjnosh, H. Banihashemi; Tehran/IR

(m_ghoncheh@hotmail.com)

Purpose: The purpose of this study is evaluation of correlation between relative signal intensity of MR images in retrodiscal tissue, superior and inferior heads of pterygoid muscle in TMD patients with types of anterior disc displacement and condylar flattening.

Methods and Materials: In this cross-sectional study, 31 MR images of patients who had anterior disc displacement were evaluated. After relative signal intensity measurement for retrodiscal tissue, superior and inferior head of lateral pterygoid muscle, the correlation between relative signal intensity and types of anterior disc displacement and also condylar flattening was evaluated.

Results: The correlation between relative signal intensity of MR images and type of anterior disc displacement in retrodiscal tissue ( $p$-value $=0.10$ ), superior head of lateral pterygoid muscle ( $p$-value $=0.68$ ) and inferior head of lateral pterygoid muscle $(p$-value $=0.40$ ) was not significant. There was also no statistically significant correlation between relative signal intensity of MR images and flattening of condylar head in retrodiscal tissue $(\mathrm{p}$-value $=0.75)$, superior head of lateral pterygoid muscle $(p$-value $=0.49)$ and inferior head of lateral pterygoid muscle ( $p$-value $=0.60)$.

Conclusion: We found that although MRI is a non-invasive procedure in the diagnosis of TMD, probably relative signal intensity of MR images in retrodiscal tissue, superior and inferior head of pterygoid muscle is not a good predictor for type of anterior disc displacement and flattening of condylar head. It seems that this cannot be used as a diagnostic marker for TMD progression.

\section{B-0478 15:12}

High resolution 3D MR imaging of the temporomandibular joint:

feasibility and comparison with $2 \mathrm{D}$ sequences

U. Navarro, M. Ravanelli, D. Farina, E. Botturi, R. Maroldi; Brescia/IT

(navarrougo@gmail.com)

Purpose: To assess the feasibility of high-resolution 3D MR imaging of the temporomandibular joint (TMJ) and to compare 3D and standard $2 \mathrm{D}$ techniques. Methods and Materials: Ten volunteers underwent a TMJ MR study with surface coils including a proton density (PD) fat-saturated 3D sequence "SPACE" and 2D $P D$ fat-saturated sequences acquired on parasagittal and paracoronal planes. Twenty TMJ were imaged and analysed. The contrast between articular disk (AD) and bilaminar zone signals was measured on parasagittal images and normalised by the AD signal. Capability of $3 D$ sequences to depict eight small anatomical structures of the TMJ ( 4 on parasagittal and 4 on paracoronal plane) was assessed and compared to that of standard $2 \mathrm{D}$ sequences.

Results: Contrast between bilaminar zone and $A D$ in $3 D$ and $2 D$ sequences was 2.93 and 2.61 , respectively ( $p 0.34$ ). Fifty-eight out of 80 structures assessed in parasagittal plane were detectable on $2 \mathrm{D}$ sequences, $67 / 80$ on $3 \mathrm{D}$ sequences $(\mathrm{p}$ 0.03). Among the 80 structures assessed on paracoronal images, 40 were recognised on 2D sequences, 67 on 3D sequences ( $p$ 0.0001).

Conclusion: High resolution 3D MR imaging of TMJ is feasible with 3D PD fatsaturated SPACE sequences. The detectability of anatomical structures, potentially key for joint functionality (ligaments) is enhanced in comparison to standard 2D sequences, especially on paracoronal plane.

\section{B-0479 15:21}

Radiological anatomy of the ethmoidal arteries: a cone beam CT study L. Sottocornola, M. Dal Corso, M. Ravanelli, L. Pianta, R. Maroldi; Brescia/lT (lara.sottocornola@gmail.com)

Purpose: To assess the use of high-resolution Cone Beam computed tomography (CBCT) to evaluate the radiological anatomy of the anterior and posterior ethmoidal arteries (AEA, PEA).

Methods and Materials: Retrospective review of 53 Cone Beam CT (CBCT) of paranasal sinuses acquired from December 2011 to August 2012. Image analysis was performed on 200-m-thick multiplanar reconstructions (orthogonal planes). Results: AEA was identifiable in 106/106 on the axial plane, 106/106 on coronal and $105 / 106$ on sagittal. In $43 \%$ of patients, the AEA canal run inside the skull base (condition \#1), in $57 \%$ of cases a pneumatised cell was interposed between the canal and the skull base (condition\#2), $52 \%$ of the pneumatised cells above the AEA canal were supraorbital cells, $35 \%$ supra-bullar recesses and $13 \%$ frontal sinuses. In these cases, the average distance between the AEA canal and the skull base was $2.2 \mathrm{~mm}$ (range 1.5-2.8 mm). In condition \#2, the AEA canal was contained within a continuous or incomplete lamella ( $81 \%$ and $19 \%$, respectively). $31 \%$ of AEA canals was classified as dehiscent.

PEA was identifiable in 98/106 on the axial plane, $104 / 106$ on coronal and 101/106 on sagittal. Most of PEAs (98\%) were located within the skull base; only $2 \%$ of PEAs was separated from the skull base by a posterior ethmoid cell or sphenoid sinus. Conclusion: $\mathrm{CBCT}$ allows a precise evaluation of ethmoidal arteries anatomy. This information could be valuable for treatment planning in paranasal sinus surgery and for intraoperative 3D navigation.

14:00 - 15:30 Room N/O

\section{Musculoskeletal}

\section{SS 610b}

\section{Cartilage: advanced imaging}

Moderators:

A. Karpenko; St. Petersburg/RU

P. Omoumi; Brussels/BE

\section{B-0480 14:00}

Direct comparison of MR imaging at 1.5- and 3.0-T for evaluating the articular cartilage of the knee

P. Van Dyck', C. Kenis ${ }^{1}$, V. Lambrecht' ${ }^{2}$, K. Wouters ${ }^{1}$, F.M.H.M. Vanhoenacker ${ }^{1}$, J.L. Gielen', P.M. Parizel'; ${ }^{1}$ Antwerp/BE, ${ }^{2}$ Gent/BE (pieter.van.dyck@uza.be)

Purpose: To compare MR imaging at 1.5- and 3.0-T in the same individuals for performance in the diagnosis of cartilage lesions of the knee.

Methods and Materials: This prospective study was approved by our Institutional Review Board, and informed consent was obtained from all patients. From December 2010 to February 2012, MR examinations of the knee at both 1.5- and 3.0-T were performed in 100 symptomatic patients $(71$ men, mean age $45 y$, range 17-75y) who underwent subsequent knee arthroscopy. Three blinded independent readers graded all articular surfaces seen at MR using the Noyes classification system. With arthroscopy as the reference standard, the sensitivity and specificity of the 1.5- and 3.0-T MR protocols for detecting cartilage lesions were calculated. Differences between the 1.5- and 3.0-T exams were compared using bootstrapping statistics $(p<0.05)$.

Results: For all readers and grades of cartilage lesions combined, the respective sensitivity, specificity, and accuracy of MR for detecting cartilage lesions ( $n=144)$ were $60 \%, 96 \%$, and $87 \%$, respectively, at $1.5-\mathrm{T}$ and $69 \%, 96 \%$, and $90 \%$, respectively, at 3.0- T. For detecting and correct grading of cartilage lesions, the readers performed significantly better with 3.0-T than with 1.5-T $(p=0.001)$.

Conclusion: A comprehensive MR protocol at 3.0-T significantly improves diagnostic performance for evaluating the articular cartilage of the knee in symptomatic patients, when compared with a similar protocol performed at 1.5-T.

\section{B-0481 14:09}

Biochemical imaging of the knee's cartilage: comparison of three techniques at 3 Tesla

C. Rehnitz ${ }^{1}$, J. Kupfer ${ }^{1}$, N. Streich ${ }^{1}$, I. Burkholder ${ }^{2}$, B. Schmitt ${ }^{3}$, L. Lauer ${ }^{3}$, H.-U. Kauczor', M.-A. Weber'; ${ }^{1}$ Heidelberg/DE, ${ }^{2}$ Zweibrücken/DE, ${ }^{3}$ Erlangen/DE (Christoph.Rehnitz@med.uni-heidelberg.de)

Purpose: To prospectively compare dGEMRIC (delayed gadolinium-enhanced MRI of cartilage), T2-mapping and gagCEST (glycosoaminoglycan chemical-exchange saturation-transfer) to assess the biochemical knee's cartilage composition.

Methods and Materials: Sixty-nine subjects were prospectively included (median age, 42 years; male/female=32/37) in three cohorts: 10 healthy volunteers, 40 patients with clinically suspected cartilage lesions, and 19 patients about one year after microfracture therapy. T2-mapping, dGEMRIC, and CEST were performed at a 3 Tesla scanner with an 15-channel knee coil. Parameter maps were evaluated using region-of-interest analysis of healthy cartilage, areas of chondromalacia and repair tissue. Differentiation of damaged from healthy cartilage was assessed using receiver-operating characteristic $(\mathrm{ROC})$ analysis.

Results: Chondromalacia grade 2-3 had significantly lower dGEMRIC-values ( $p$ $<0.001)$, higher T2-values $(p<0.001)$ and higher CEST-values $(p=0.001)$ when compared to the normal appearing cartilage. dGEMRIC and T2-mapping correlated negatively (Spearman coefficient $r=-0.56, p=0.0018$ ) and T2-mapping and CEST positively ( $r=0.5, p=0.007)$, while dGEMRIC and CEST did not correlate $(r=-0.311$, $p=0.07)$. Areas of repair tissue had significantly lower T1-values $(p<0.001)$ and higher CEST-values $(p<0.001)$ with a significant negative correlation $(r=-0.589$, $p=0.01$ ), whereas T2-values were not different $(p=0.54)$. In healthy volunteers' 
cartilage, CEST and dGEMRIC correlated moderately positively $(r=0.56, p=0.09)$. ROC analysis demonstrated non-significant differences of T2-mapping vs. CEST $(p=0.14)$, CEST vs. dGEMRIC ( $p=0.89)$, and T2-mapping vs. dGEMRIC $(p=0.12)$. Conclusion: All techniques can distinguish normal from damaged cartilage with non-superiority of either technique. T2-mapping may be recommended to detect or exclude early chondromalacia, while dGEMRIC might be principally useful to assess cartilage repair tissue. CEST might have a role in both issues.

\section{Author Disclosures:}

B. Schmitt: Employee; Siemens Healthcare, Erlangen, Germany. L. Lauer:

Employee; Siemens Healthcare, Erlangen, Germany.

\section{B-0482 14:18}

High-resolution flat-panel CT arthrography for cartilage defect

detection: comparison to multi-detector $\mathrm{CT}$

R. Guggenberger ${ }^{1}$, S. Winklhofer 1 , T. Redel' ${ }^{2}$, J. Korporaal ${ }^{2}$,

A. Meier', J. von Spiczak', G. Andreisek', H. Alkadhi'; ' 'Zurich/CH,

${ }^{2}$ Forchheim/DE (romanguggenberger@yahoo.de)

Purpose: To analyse the spatial resolution of different reconstruction kernels and acquisition protocols including a prototypic high-resolution protocol in flat-panel (FP) and multi-detector (MD) CT to evaluate contrast and cartilage depiction quality of FPCT and MDCT arthrography.

Methods and Materials: A cone-beam image quality phantom was used to compare resolution and different reconstruction kernels of standard MDCT (120 and $80 \mathrm{kV})$, standard binned (2x2) and prototype high-resolution unbinned (1x1) FPCT protocols (5s and 20s runs each). With the resulting FPCT kernel best matching the standard MDCT kernel (U9Ou), joint phantoms with differently sized cartilage defects were scanned. CT numbers and noise in iodine contrast and phantom cartilage tissue were measured, and contrast-to-noise ratios (CNR) were calculated. Depiction quality of cartilage defects was qualitatively rated by two independent radiologists. Results: A sharp reconstruction kernel for all FPCT protocols best matched the resolution of the standard MDCT kernel. High-resolution 20s 1 x1 binning FPCT showed superior resolution in higher frequencies than $1.6 \mathrm{lp} / \mathrm{mm}$. The CNR were highest in 20s FPCT, followed by 5s FPCT binned and MDCT protocols. Interreader agreement for depiction quality of cartilage defects was substantial and high in joint phantoms $(0.74$ and 0.8 , respectively; $p<0.001)$. The best ratings of cartilage defect depiction quality were seen in FPCT 20s, followed by FPCT $5 \mathrm{~s}$ and MDCT acquisitions.

Conclusion: FPCT arthrography offers superior CNR and cartilage defect depiction quality to MDCT, and spatial resolution for small structures is higher when applying high-resolution acquisition protocols.

Author Disclosures:

T. Redel: Employee; Siemens. J. Korporaal: Employee; Siemens. A. Meier: Employee; Siemens.

\section{B-0483 14:27}

\section{Longitudinal evaluation of $\mathrm{T} 2$ relaxation time in relation to baseline} volume of femorotibial cartilage - data from the osteoarthritis initiative P. Beringer ${ }^{1}$, L. Nardo' ${ }^{1}$ H. Liebl'1, U.R. Heilmeier'1, A. Lai', F. Liu', G.B. Joseph'1, F. Eckstein ${ }^{2}$, T.M. Link'; ${ }^{1}$ San Francisco, CA/US, ${ }^{2}$ Salzburg/AT (paula.beringer@pmu.ac.at)

Purpose: To relate longitudinal changes in femorotibial cartilage T2 relaxation times to baseline cartilage volume in subjects with $\mathrm{OA}$, at risk for $\mathrm{OA}$ and controls from the osteoarthritis initiative (OAI).

Methods and Materials: One-hundred-thirty subjects ( 85 females, 45 males; age 45-70; BMI 19-27; KL 22) were selected from the OAI database, 45 subjects with pain and radiographic evidence of osteoarthritis (OA) (progression cohort), 45 subjects with risk factors for $\mathrm{OA}$ (incidence cohort) and 40 subjects from the control cohort. Right knee 3 T MRIs were analysed at baseline and 48 months. Coronal FLASH sequence was used to measure cartilage volume at baseline, multi-echo spinecho sequence to generate T2 maps at baseline and 48 months. Regression models were adjusted for age, gender and BMI and were applied to 4 compartments (medial femur, lateral femur, medial tibia and lateral tibia).

Results: Global T2 measurements increased in all cohorts over 48 months. Baseline T2 were significantly greater in the progression cohort compared to incidence and control cohort $(P<0.001)$, whereas 48-month follow-up data showed significantly greater measurements in the Incidence cohort $(P<0.003)$. Most pronounced T2 changes over time were observed in the incidence cohort $(P<0.023)$, changes in the progression cohort were not significant. Mean volume did not significantly differ between the cohorts, but progression cohort subjects showed the highest volumes in all compartments.
Conclusion: Progression cohort subjects showed the greatest baseline femorotibial cartilage T2, but demonstrated less increase than the other 2 cohorts over time, indicating a potential ceiling effect. High baseline cartilage volume and elevated T2 measurements were associated with the presence of cartilage degeneration.

\section{B-0484 14:36}

Longitudinal increase of $\mathrm{T} 2$ relaxation time in normal subjects indicating physiological aging of the collagen network and cartilage matrix: data from the osteoarthritis initiative (OAI)

U.R. Heilmeier, P. Beringer, L. Nardo, H. Liebl, S. Lee, G. Joseph,

C.E. McCulloch, M.C. Nevitt, T.M. Link; San Francisco, CA/US

Purpose: To analyse physiological changes of cartilage matrix in healthy subjects using T2 relaxation time measurements over a period of 48 months.

Methods and Materials: Right knee 3 Tesla MRI studies of 55 subjects (17 male, 38 female, aged $45-60$, BMI $23.9 \pm 5.7$ ) without risk factors, symptoms or radiographic signs of osteoarthritis $(\mathrm{OA})$ of the osteoarthritis initiative control cohort were studied at baseline, 2- and 4-year follow-up. Knee cartilage was segmented and T2 maps were generated in five compartments (patella, medial and lateral femur, medial and lateral tibia). Mean T2 relaxation time values and changes over time were measured for each compartment. Statistical analysis was performed using a mixed random effects model adjusted for age, sex and BMI.

Results: All subjects showed significant increases in mean T2 over time (baseline24 -48 months) in each compartment. The yearly rate of T2 change was highest in the patella $(0.78 \mathrm{~ms}, p<0.001)$ and lateral femur $(0.71 \mathrm{~ms}, p<0.001)$. It was lower in the lateral tibia $(0.45 \mathrm{~ms}, \mathrm{p}<0.001)$, medial femur $(0.36 \mathrm{~ms}, \mathrm{p}<0.001)$ and medial tibia $(0.50 \mathrm{~ms}, \mathrm{p}<0.001)$.

Conclusion: Using T2 relaxation time as a sensitive biomarker for cartilage collagen network integrity, this is the first study that radiologically demonstrates over 48 months, that healthy subjects undergo age-related changes in cartilage composition resulting in continuously increasing T2 values over time. These findings need to be considered when evaluating pathologic changes of knee cartilage such as OA.

\section{B-0485 14:45}

3D delayed gadolinium-enhanced MRI of cartilage (dGEMRIC) at 3 T used to evaluate the effect of hyaluronic acid on cartilage quality in knee osteoarthritis patients

J. van Tiel, M. Reijman, K. Bos, J. Verhaar, G.P. Krestin, S. Bierma - Zeinstra, H. Weinans, G. Kotek, E. Oei; Rotterdam/NL (j.vantiel@erasmusmc.nl)

Purpose: Intra-articular hyaluronic acid ( $\mathrm{HA})$ injections are frequently used as treatment for knee osteoarthritis $(\mathrm{OA})$. It was suggested that HA injections might improve cartilage sulphated glycosaminoglycan (sGAG) content of cartilage. The cartilage sGAG content can be determined using delayed gadolinium-enhanced MRI of cartilage (dGEMRIC). This study assessed if improvement in cartilage quality can be detected with dGEMRIC in OA knees 3 months after HA injections. Methods and Materials: In 20 patients with KL grade 1-2 knee OA, dGEMRIC was acquired at $3 \mathrm{~T}$ using a 3D-SPGR sequence before and 3 months after HA treatment. To evaluate patient symptoms, the knee injury and osteoarthritis outcome score (KOOS) questionnaire was recorded at baseline and follow-up. We analysed 8 matching anatomical ROIs in the medial and lateral knee compartment in both examinations, using the $\mathrm{T} 1$ relaxation times as a measure of cartilage quality. Outcomes of dGEMRIC and KOOS at baseline and follow-up were compared using paired testing to evaluate the symptomatic and potential structural effects of HA. Results: Outcomes of dGEMRIC at follow-up (pooled median T1 $504 \mathrm{~ms}$ ) did not improve significantly compared to baseline (pooled median T1 $490 \mathrm{~ms}$ ) in any of the analysed anatomical cartilage ROIs. Patient complaints improved significantly in KOOS subscales: pain, daily-, and sports activities.

Conclusion: Using dGEMRIC, no improvement in articular cartilage quality can be detected 3 months after HA. However, patient complaints decreased significantly after HA. Therefore, the results of this study suggest that the working mechanism of $\mathrm{HA}$ is not acting through an improvement of SGAG content in the cartilage.

Author Disclosures:

G.P. Krestin: Consultant; to GE Healthcare. Grant Recipient; from GE Healthcare, from Bayer. 


\section{B-0486 14:54}

$\mathrm{T}^{\star}$ mapping and delayed gadolinium-enhanced magnetic resonance imaging in cartilage (dGEMRIC) of glenohumeral cartilage in asymptomatic volunteers at $3 \mathbf{T}$

B. Bittersohl' ${ }^{1}$, F.R. Miese ${ }^{1}$, C. Dekkers ${ }^{1}$, H. Senyurt' ${ }^{1}$, J. Kircher ${ }^{2}$,

H.-J. Wittsack', G. Antoch'1, R. Krauspe', C. Zilkens ${ }^{1}$; $^{1}$ Düsseldorf/DE,

${ }^{2}$ Cologne/DE (bbittersohl@partners.org)

Purpose: To evaluate the feasibility of $\mathrm{T}^{*}$ and $\mathrm{T} 1 \mathrm{Gd}$ mapping to assess glenohumeral cartilage, and to establish baseline values of healthy glenohumeral cartilage at $3 \mathrm{~T}$.

Methods and Materials: Forty asymptomatic volunteers (mean age: $24.8 \pm 2.2$ years) without shoulder abnormality were included. The MRI protocol comprised a three-dimensional (3D) double-echo steady-state (DESS) sequence for morphological cartilage evaluation, a 3D gradient-echo (GRE) multiecho sequence for T2* assessment, and a dual-flip-angle 3D GRE sequence for T1Gd mapping. Statistical assessment involved descriptive statistics establishing mean $\mathrm{T}^{\star}$ and T1Gd values \pm standard deviation and the one-way analysis of variance (ANOVA) to identify any significant differences between the $\mathrm{T}^{*}$ and $\mathrm{T} 1 \mathrm{Gd}$ values of various regions of the glenohumeral joint. Intra-class correlation (ICC) analysis comparing repetitive $\mathrm{T}^{*}$ and $\mathrm{T} 1 \mathrm{Gd}$ measures of ten randomly selected individuals was used to assess intra- and inter-observer reliability.

Results: Total mean values of $\mathrm{T}^{*}=24.7 \mathrm{~ms} \pm 3.7 \mathrm{~ms}$ and $\mathrm{T} 1 \mathrm{Gd}=735.3 \pm 106.2$ $\mathrm{ms}$ were assessed. Both techniques revealed significant differences between superior and inferior glenohumeral cartilage demonstrating higher T2* $(26.2 \mathrm{~ms}$ vs. $23.2 \mathrm{~ms}$, p-value $<0.001)$ and T1Gd $(750.1 \mathrm{~ms}$ vs. $720.2 \mathrm{~ms}$, $\mathrm{p}$-value $=0.014)$ values in the superior regions. High intra- and inter-observer agreement (ICC value range: 0.895-0.983) was noted for both $\mathrm{T}^{*}$ and T1Gd mapping.

Conclusion: $\mathrm{T}^{*}$ and $\mathrm{T} 1 \mathrm{Gd}$ mapping is reliable in the assessment of glenohumeral cartilage. The values of this study can be used as reference for comparison to identify cartilage degeneration in patients suffering from shoulder joint pathology.

\section{B-0487 15:03}

Validity of gradient-echo three-dimensional delayed gadoliniumenhanced magnetic resonance imaging of hip joint cartilage: a histologically controlled study

C. Zilkens ${ }^{1}$, F.R. Miese'1, M. Herten', S. Kurzidem¹, M. Jäger'², D. König ${ }^{3}$,

G. Antoch', R. Krauspe ${ }^{1}$, B. Bittersohl'; ; Düsseldorf/DE, ${ }^{2}$ Essen/DE,

${ }^{3}$ Viersen/DE (christoph.zilkens@med.uni-duesseldorf.de)

Purpose: To validate gradient-echo 3D delayed gadolinium-enhanced magnetic resonance imaging of cartilage (dGEMRIC) by means of histological analyses in the assessment of hip joint cartilage.

Methods and Materials: Twenty-one femoral head specimens collected from 21 patients ( 7 males, 14 females, mean age: $60.9 \pm 9.6$ years; range: $37.6-77.3$ years), who underwent total hip replacement for symptomatic hip joint osteoarthritis, underwent MRI and histological assessment. A region of $2 \mathrm{~cm}^{2}$ with morphologically intact cartilage was marked with four pins to enable multi-planar MRI reformatting to be matched with histological sections. MRI was performed at $3 \mathrm{~T}$ with a $3 \mathrm{D}$ double-echo steady-state (DESS) sequence for morphological cartilage assessment and 3D volumetric interpolated breathhold examination (VIBE) for T1Gd mapping. Histological sections were evaluated according to the Mankin score system. Total Mankin score, grade of toluidine staining and a modified Mankin score classification system with four sub-groups of cartilage damage were correlated with MRI data. Results: Spearman's rho correlation analyses revealed a significant correlation between T1Gd mapping and histological analyses in all categories including total Mankin score $(r=-0.658$, $p$-value $=<0.001)$, toluidine staining $(r=-0.802$, $p$-value $<0.001)$ and modified Mankin score $(r=-0.716$, $p$-value $<0.001)$. Correlation between morphological MRI and histological cartilage assessment was statistically significant but inferior to biochemical cartilage MRI ( $r$-values ranging from -0.411 to 0.525 , p-values $<0.001$ )

Conclusion: Gradient-echo dGEMRIC is reliable while offering the unique features of high image resolution and 3D biochemically sensitive MRI for the assessment of early cartilage degeneration.

Author Disclosures:

C. Zilkens: Grant Recipient; Deutsche Arthrosehilfe. . B. Bittersohl: Grant Recipient; Deutsche Arthrosehilfe.
B-0488 15:12

In-vivo sodium magnetic resonance (MR) imaging of healthy intervertebral discs during unloading

K.M. Friedrich, J. Wimmer, V. Juras, S. Zbyn, I. Nöbauer-Huhmann, S. Trattnig; Vienna/AT

Purpose: To assess changes in sodium MR imaging values of the intervertebral discs during unloading in healthy volunteers.

Methods and Materials: This study was approved by the Institutional Review Board. All subjects provided written informed consent. Sodium MR imaging values of the intervertebral discs were measured at $7 \mathrm{~T}$ in 7 female healthy volunteers (mean age, 24.20 $\pm 1.79 y e a r s$ ) at the time point zero, and 20, 40, 60, 80, 100, 120, 160 , and 180 minutes later. $P$ values less than. 05 were considered to indicate a statistically significant difference. Paired t-tests were used for statistical analysis. Results: Mean sodium signal intensity values of the intervertebral discs were as follows (time point, mean sodium signal intensity values): $0,1983.92 ; 20,2040.14$; 40, 2178.19; 60, 2267.63; 80, 2450.02; 100, 2433.31; 120, 2473.86; 160, 2438.56; $180,2554.13$. The values increased significantly until time point 40 (time point 0 versus $20, p=0.0002$; time point 20 versus $40, p=0.0001$ ); later no significant increase was observed.

Conclusion: Sodium MR imaging provided insight into the biochemical processes taking place in the intervertebral discs during unloading of the spine. The results of this study indicate that a $40-\mathrm{min}$ rest before starting the sodium measurements might be beneficial for the planning of future studies.

\section{B-0489 15:21}

T2 mapping for assessment of lumbar disc repair after autologous disc-derived chondrocyte transplantation (ADCT)

M. Glowacki, M. Ostrowska, W. Gradkowski, J. Walecki; Warsaw/PL (mostrowska@yahoo.com)

Purpose: The goal of the study was to demonstrate the benefits of T2 mapping to the assessment of lumbar disc repair after the ADCT.

Methods and Materials: 50 patients, male and female, age range 18-40, with a single-level degenerative disc disease (DDD) were studied. All patients had a standard lumbar microdiscetomy performed and 4-6 weeks after the surgery underwent the ADCT. Prior to the surgery, one month later and 3, 6 and 12 months after the ADCT each patient had a MR examination on the $1.5 \mathrm{~T}$ MR scanner. 20 patients completed a 12-month long follow-up protocol. T2 mapping revealed the level of DDD and the adjacent levels. The evaluation of signal intensity was carried out using pixel value software.

Results: Each patient during the 12-month long follow-up reported a significant relief of pain: VAS score ranged $0-3$, ODI score $0-20 \%$. In the MR examination held after the ADCT, changes in signal intensity seen on T2-weighted MR images were reported. T2 mapping showed signs of the disc repair regardless of the lack of changes in disc dimensions. Mild increase of T2 with improved homogeneity of the structural morphology of the nucleus pulposus was observed on T2 after the ADCT. Conclusion: T2 mapping may be used for evaluating the changes of the molecular composition and structural integrity of the intervertebral disc after the ADCT and other novel restorative therapies. It has also the potential to be valuable in the detection of the early degeneration in the intervertebral disc.

14:00 - 15:30 Room P

\section{Interventional Radiology}

\section{SS 609b}

\section{Gynaecological and obstetric interventions}

\section{Moderators:}

P. Andersen; Odense/DK

R. Nijenhuis; Maastricht/NL

\section{B-0490 14:00}

Non-invasive treatment of uterine fibroids using MR-guided highintensity focused ultrasound: results on quality of life, non-perfused volume ratio and size reduction over 12 months

F. Ciolina, A. Napoli, B. Cavallo Marincola, F. Boni, F. Zaccagna, V. Noce, L. Bertaccini, C. Catalano; Rome/IT (federica.ciolina@gmail.com)

Purpose: To evaluate the efficacy of MR-guided acoustic ablation in symptoms relief and volume reduction over time. 
Methods and Materials: 76 leiomyomas in 60 women (average age 39.3 years) were treated with MRI-guided focused ultrasound (MRgFUS). The treatment is carried out using the ablative properties of the HIFU system under 3 T MRI guide. Symptoms were scored using Severity Score (SS) and quality of life was determined using the UFS-QOL score. Pre-treatment measurements of leiomyoma volume were obtained by MR images. After treatment, Non-perfused Volume (NPV) was calculated from T1-w contrast-enhanced MR sequences. The average volume of treated fibroids was $90.27 \pm 90.4 \mathrm{~mm} 3$. Follow-up images were obtained 3, 6 and 12 months after treatment and served to determine leiomyoma shrinkage. Qualitative and quantitative relations between fibroid volume, NPV ratio at treatment, and 12-month shrinkage were measured.

Results: MRgFUS treated patients demonstrated a significant change in USF-QoL score: mean SS score values were $48.6 \pm 13.4$ (pre-treatment), $25.1 \pm 8.9$ (threemonths follow-up), $22.1 \pm 7.5$ (six-months follow-up) and $19.3 \pm 6.8$ (twelve-months follow-up). Fibroids volume changed from $90.27 \pm 90.4 \mathrm{~mm} 3$ (before treatment) to $54 \pm 66.1 \mathrm{~mm} 3$ (at 12 months follow-up). We encountered a statistically significant difference between the two values $(p=0.001)$. Mean post-treatment VNP was $57.65 \pm 52.9 \mathrm{~mm} 3$, about $63 \%$ of total fibroid volume $(\mathrm{p}=0.001)$.

Conclusion: MRgFUS therapy of leyomioma results in a significant relief of symptoms and greater than $50 \%$ total fibroid ablation. The procedure is carried out in a totally non-invasive manner and features a high-safety profile.

\section{B-0491 14:09}

\section{MRI-guided focused ultrasound treatment of symptomatic uterine} fibroids: impact of technology advancement on ablation volumes in 115 patients

C.G. Trumm ${ }^{1}$, R. Stahl', D.-A. Clevert', P. Herzog ${ }^{2}$, I. Mindjuk², S. Kornprobst², R.-T. Hoffmann ${ }^{3}$, M.F. Reiser ${ }^{1}$, M. Matzko ${ }^{2} ;{ }^{1}$ Munich/DE, ${ }^{2}$ Dachau/DE,

${ }^{3}$ Dresden/DE (christoph.trumm@med.uni-muenchen.de)

Purpose: To assess the impact of the advancement of MRI-guided focused ultrasound technology on treatment outcomes in patients with symptomatic uterine fibroids, as measured by the non-perfused volume ratio.

Methods and Materials: This is a retrospective analysis of 115 women (average age, 42 years; range 27 to 54 years) with symptomatic fibroids who consecutively underwent MRI-guided focused ultrasound treatment in a single centre with the new generation ExAblate system 2100 (Insightec Ltd., Haifa, Israel) from November 2010 to June 2011. Average total volume and number of the treated fibroids (per patient) were $89 \pm 94(\mathrm{SD}) \mathrm{cm} 3$ and $2.2 \pm 1.7$, respectively. Patient baseline characteristics were analysed regarding their impact on the resulting non-perfused volume ratio. Results: MRI-guided focused ultrasound treatment was technically successful in 115 of 123 patients (93.5\%). In 8 patients treatment was not possible due to bowel loops in the beam pathway that could not be mitigated $(n=6)$, patient movement $(n=1)$ and system malfunction $(n=1)$. Average non-perfused volume ratio was $88 \pm 15 \%$ (range, 38 to 100 ). Mean applied energy level was $5400 \pm 1200 \mathrm{~J}$, and mean number of sonications was $74 \pm 27$. No major complications occurred. Two cases of first degree skin burn resolved within one week after the intervention. Of the baseline characteristics analysed, only the planned treatment volume had a statistically significant impact on the non-perfused volume ratio.

Conclusion: With technological advancement, outcome of MRI-guided focused ultrasound treatment in terms of the non-perfused volume ratio can be enhanced with a high safety profile, markedly exceeding results reported in previous clinical trials.

Author Disclosures:

D. Clevert: Speaker; Siemens Healthcare, Philips Healthcare, Bracco Imaging.

R. Hoffmann: Speaker; Sirtex Medical Limited, Boston Scientific Corporation.

M. Matzko: Speaker; GE Healthcare / Insightec Limited.

\section{B-0492 14:18}

Efficacy of MRI-guided focused ultrasound (MRgFUS) treatment of uterine fibroids: evaluation of non-perfused volume (NPV), fibroid shrinkage and clinical improvement at 6-month follow-up

I. Invernizzi' ${ }^{1}$, M. Vaiani', P. Colombo' ${ }^{1}$, F. Zucconi ${ }^{1}$, S. Sironi ${ }^{2}$, C. Ticca ${ }^{1} ;{ }^{1}$ Milan/IT, 2Monza/IT (fibromauterino.niguarda@gmail.com)

Purpose: To determine the efficacy of MRgFUS treatment of uterine fibroids evaluating fibroid shrinkage and clinical improvement after 6 months and to assess the predictive role of non-perfused volume (NPV).

Methods and Materials: A total of 74 symptomatic uterine fibroids in 60 women (range 39-51 years) underwent MRgFUS between September 2010 and July 2012 using the ExAblate 2000 system (InSightec). Before treatment T2-weighted multiplanar MRI images were obtained to measure uterine fibroids volume. Immediately after treatment $\mathrm{T} 1$-weighted contrast-enhanced fat-suppressed multiplanar MR images were used to measure NPV. Similar images obtained $6 \pm 1$ months after treatment were used to determine fibroids shrinkage. The Symptom Severity Score (SSS) and Quality of Life Score (QOLS) were examined before treatment and after 6 months. Post-treatment NPV and 6 months fibroids shrinkage were correlated and compared with SSS and QOLS results.

Results: The average NPV was $62 \% \pm 19 \%$ after treatment. Six months after treatment the treated fibroids average volume decreased from $136 \pm 142 \mathrm{~cm}^{3}$ to $98 \pm 116$ $\mathrm{cm}^{3}(n=49)(p<0.00005)$ with an average volume reduction of $36 \% \pm 22 \%$. A linear regression model showed highly significant correlation between post-treatment NPV and fibroid shrinkage at 6 months $(p=0.001)$. The mean SSS value before MRgFUS was $53 \pm 17$ and after 6 months it significantly decreased to a mean value of $22 \pm 13(p<0.001)$. Similarly the mean QOLS value before treatment was $48 \pm 22$ and it significantly increased to $76 \pm 19(p<0.001)$.

Conclusion: MRgFUS is a non-invasive, efficacy treatment for uterine fibroids. The NPV after treatment resulted predictive of uterine fibroid shrinkage after 6 months.

\section{B-0493 14:27}

Uterine adenomyosis treated by MRgFUS: technical approach and clinical results

F. Ferrari, A. Miccoli, F. Arrigoni, A.V. Giordano, E. Fascetti, G. Mascaretti, C. Masciocchi; L'Aquila/lT (fabianaferrari@hotmail.it)

Purpose: To evaluate the efficacy of the treatment of uterine adenomyosis using magnetic resonance imaging-guided focused ultrasounds (MRgFUS) with a new specific technical plan.

Methods and Materials: Twenty-four patients aged between 28 and 47 years (mean age 37.5), with symptomatic adenomyosis and uterine fibroids were treated with MRgFUS in our department, from October 2011 to July 2012. This study evaluates the findings on 6 patients affected by adenomyosis alone. Symptomatology was assessed through the symptom severity score questionnaire. One single treatment was performed with an average energy delivered to each patient equal to $2840 \mathrm{~J}$ (with a minimum value of $1300 \mathrm{~J}$ and maximum of $4800 \mathrm{~J}$ ). The longest treatment duration was 120 minutes. The plan employed was characterised by high density and reduced size of the spot length (ranging from 4 to $6 \mathrm{~mm}$ ) with the shortest cooling time between the sonifications.

Results: The evaluation of the non-perfused volume on the c.e. T1-weighed sequences performed 6 weeks after treatment, measuring each single slice, showed a maximum retraction of the necrotic area of $73 \%$ and a minimum of $52 \%$ with a mean value of $66.5 \%$. After six weeks, the symptomatic score showed a reduction of about $80 \%$ if compared to the pre-treatment one.

Conclusion: The treatment with MRgFUS allows a resolution of the symptoms and a reduction of the treated area, maintaining the integrity of the uterus, in a pathology which, up to now, has limited therapeutic perspectives.

\section{B-0494 14:36}

Is uterine artery embolisation in large sized myoma as efficient as in normal sized myoma: a retrospective comparative study in 263 patients H. Choi', G. Jeon', M. Kim², J. Lee', J. Yoon', S. Hwang ${ }^{1} ;{ }^{1}$ Seongnam/KR, ${ }^{2}$ Seoul/KR (piscean84@hanmail.net)

Purpose: The purpose of this study was to evaluate the effectiveness, safety and complications of uterine fibroid embolisation (UFE) when applied to myomas of large volume.

Methods and Materials: A total of retrospectively collected 323 patients who underwent UFE for symptomatic uterine fibroids without adenomyosis between January 2005 and February 2011 were included in this study. Patients were divided into two groups: 63 patients were selected with large fibroid burden group, defined as a dominant fibroid with a longest axis $310 \mathrm{~cm}$ or a uterine volume of $3700 \mathrm{~cm}^{3}$ and remaining 260 patients with control group. Fibroid infarction and volume reduction with MRI, complications and patients' satisfaction score were evaluated.

Results: The large fibroid group consisted of 63 (mean age $37.10 \pm 5.62$ ) women and 260 (mean age $37.33 \pm 5.62$ ) women included in control group. There were no statistical difference in average \% volume reductions of dominant fibroid as $46 \%$ in large group and $41.19 \%$ in control group, the mean satisfaction score at immediate follow-up (average 2.94 months) and mid-term follow-up (average 32.63 months) and procedure related complications or major surgery following UFE.

Conclusion: Uterine fibroid embolisation is a safe and effective treatment method which can be utilised in the treatment of myomas of large volume. The size of myoma is not a definite contraindication or poor prognosis factor for embolisation. 


\section{B-0495 14:45}

Usefulness of pelvic artery embolisation: a comparison between caesarean section and vaginal delivery in 176 patients

H. Lee, G. Jeon; Seongnam-si/KR (hyunjoo17@naver.com)

Purpose: To evaluate the efficacy and safety of transcatheter arterial embolisation (TAE) of the pelvic arteries for the treatment of postpartum haemorrhage (PPH) associated with caesarean section as compared with vaginal delivery.

Methods and Materials: A retrospective analysis of all 176 patients undergoing TAE of the pelvic arteries for PPH from January 2006 through August 2011 was conducted at two institutions. The mean patient age was 33.9 years, with a range from 24 to 46 years. Delivery details, haematology and coagulation results, embolisation details, and clinical outcomes were collected. Technical success was defined as the cessation of bleeding upon angiography and/or the angiographically successful embolisation of the bleeding artery. Clinical success was defined as the obviation of repeated embolisation or surgical intervention

Results: The technical success rate was $98.8 \%(n=174)$, and the clinical success rate was $89.7 \%(n=158)$. Among the 176 patients, 71 had caesarean sections, and 105 underwent normal vaginal deliveries. Of the 105 patients who underwent normal vaginal deliveries, $11(10.5 \%)$ required re-embolisation or surgical intervention. Of the 71 patients who had caesarean sections, seven $(9.8 \%)$ required re-embolisation or surgical intervention. The clinical success and complication rate was not related to the mode of delivery. All women resumed menses after TAE, and the majority of them $(n=125)$ described their menses as unchanged. Thirteen women had spontaneous pregnancies.

Conclusion: The caesarean mode of delivery is not a predictor of poorer outcomes of TAE; however, further study is needed to clarify this relationship.

\section{B-0496 14:54}

Transarterial embolisation in the treatment of postpartum haemorrhage

Z. Wang, X. Li, Z. Jin; Beijing/CN (zhiweiwang1981@sina.com)

Purpose: To evaluate the effectiveness and safety of transarterial embolisation in the treatment of postpartum haemorrhage (PPH).

Methods and Materials: All patients who underwent embolisation for PPH in our hospital between January 2005 and March 2012 were included. Primary PPH was defined as haemorrhage occurred within the first 24 hours after delivery, and secondary PPH was defined as haemorrhage occurring more than 24 hours after delivery. The complications, control of haemorrhage and outcome of subsequent pregnancies were assessed.

Results: A total of 143 patients (mean age, $31.2 \pm 5.9$ years) underwent embolisations for PPH. Of these patients, 109 (76\%) underwent embolisations for primary $\mathrm{PPH}$ and $34(24 \%)$ for secondary PPH. Uterine arteriovenous malformations and retaining products of conception were associated with secondary PPH $(\mathrm{P}<0.05)$. $112(78 \%)$ patients underwent bilateral uterine arteries embolisations and $31(22 \%)$ patients underwent bilateral uterine arteries and other collateral arteries (including branches of internal iliac artery, branches of external iliac artery and ovarian arteries) embolisations. Embolisation was performed using gelatin sponge. The technical success rate was $100 \%$. Bleeding was controlled in 137 patients (95.8\%) after embolisation. Six patients experienced recurrent bleeding. Two patients underwent repeated embolisations and angiography revealed uterine collaterals arising from branches of internal iliac artery. One patient with placenta percreta and three patients with persistent coagulation disorders required emergency hysterectomies. Seventeen patients became pregnant and eight uneventful intrauterine pregnancies carried to term at a median follow-up of 28 months.

Conclusion: Transarterial embolisation is a safe and effective treatment for patients with PPH allowing uterine conservation.

\section{B-0497 15:03}

Uterine artery embolisation for management of secondary postpartum haemorrhage associated with placenta accreta

Z. Wang, Z. Jin, X. Li; Beijing/CN (zhiweiwang1981@sina.com)

Purpose: To retrospectively evaluate the efficacy and safety of uterine artery embolisation (UAE) for the management of secondary postpartum haemorrhage associated with placenta accrete (PA).

Methods and Materials: 55 women with PA which was found during delivery were conservatively managed in our hospital between January 2005 and May 2012. They did not experience severe bleeding during delivery. 15 patients (mean age, $31.4 \pm 6.1$ years) developed secondary postpartum haemorrhage and they underwent UAE. The complications, control of haemorrhage and outcome of the placenta left inside the uterus were retrospectively reviewed.
Results: All patients underwent embolisation of bilateral uterine arteries. The median time between delivery and UAE was 11 days (range 3-76 days). The technical success rate of embolisation was $100 \%$. Bleeding was controlled in all patients during follow-up (11.3 \pm 6.9 months, range $3-24$ months), and no further bleeding occurred. One patient developed lower extremity deep venous thrombosis after UAE and no other major complications occurred. Placenta left inside the uterus gradually decreased in size except one during follow-up. 14 patients resumed normal menstruation. One patient subsequently became pregnant and had uneventful intrauterine pregnancies carried to term.

Conclusion: UAE is safe and effective for the management of secondary postpartum haemorrhage associated with PA.

\section{B-0498 15:12}

Placenta percreta: role of bilateral occlusion balloons insertion in to internal iliac arteries to prevent haemorrhage and hysterectomy M. Teixidor Viñas, A.-M. Belli, E. Chandraharan; London/UK (mireia.teixidor@hotmail.com)

Purpose: Placenta percreta (PP) is a recognised cause of severe postpartum haemorrhage, with high risk of blood transfusions and emergency hysterectomy with maternal morbility and mortality. Our purpose is to evaluate the role of bilateral occlusion balloon catheter insertion in to both internal iliac arteries (IIA) before elective caesarean to prevent haemorrhage and to preserve the uterus. This is one of the longest cohort of women treated with prophylactic balloon occlusion and prevents caesarean hysterectomy in abnormal placentation.

Methods and Materials: Twenty-two pregnant women who were identified as having a PP by US were treated prophylactically with bilateral occlusion balloon catheters placed in the anterior trunk of both IIA prior to elective caesarean. The balloons were inflated immediately after the baby delivery and deflated four hours later. Patients with peri-operative bleeding were treated with uterine artery embolisation (UAE), blood transfusion or hysterectomy. For each patient, estimated volume of blood lost and transfusion required was recorded.

Results: Abnormal placentation was confirmed histologically as percreta (13), acreta (7) and increta (2). Estimated median blood lost during the delivery was noted 1.8L (range 0.5-12L). Postpartum haemorrhage occurred in nine patients, six had successful embolisation and three required hysterectomy. No foetal or mother death occurred.

Conclusion: Prophylactic occlusion balloon catheter insertion in both IIA prior to elective caesarean deliveries in patients with abnormal placenta contributes to successful treatment, reduced blood lost and preserved the uterus in this high risk group of women.

Author Disclosures:

M. Teixidor Viñas: Research/Grant Support; Fundacion Alfonso Martin Escudero.

\section{B-0499 15:21}

Efficacy of bilateral uterine artery embolisation as a first-line therapeutic option for acquired uterine arteriovenous malformations J. Shin, H.-K. Yoon, G.-Y. Ko, D.-I. Gwon; Seoul/KR (jhshin@amc.seoul.kr)

Purpose: To evaluate the efficacy of bilateral uterine artery embolisation (UAE) as a first-line therapeutic option for the treatment of uterine bleeding associated with acquired uterine arteriovenous malformations (AVMs).

Methods and Materials: Ten patients were diagnosed with acquired uterine AVMs. The clinical characteristics, embolisation details, technical and clinical success, procedure-related complications, and follow-up data were assessed. Clinical success was defined as immediate resolution of vaginal bleeding without recurrence of symptoms and resolution of AVM seen on subsequent imaging studies.

Results: A total of 11 transcatheter embolisation procedures of bilateral uterine arteries with or without extra-uterine feeding arteries were performed as the firstline treatment, and with a technical success rate of $82 \%(9 / 11)$. Embolisation was incomplete in two patients who had an extra-uterine blood supply or a dominant draining vein; the former recovered from menorrhagia during our close observation, while the latter underwent a successful second embolisation procedure. Clinical success was achieved in the remaining eight patients after a single procedure and there was no recurrence of bleeding. Only one minor complication occurred: rupture of the proximal UA. Recovery to their normal menstrual cycle was seen in all eight of the clinically successful patients, one of whom subsequently had an uneventful intrauterine pregnancy carried to term.

Conclusion: Bilateral UAE is safe and effective for the treatment of acquired uterine AVMs while it preserves uterine function. However, the presence of an extra-uterine blood supply or a dominant draining vein may result in incomplete embolisation and, consequently, less successful clinical outcome. 


\section{Molecular Imaging}

\section{SS 606}

\section{MR/PET and PET/CT}

Moderators:

P.R. Ros; Cleveland, OH/US

N. Belcari; Pisa/IT

\section{B-0500 14:00}

Simultaneous ${ }^{68} \mathrm{Ga}$-DOTATOC PET/MRI for whole body staging in patients with neuroendocrine tumours: preliminary results

K.J. Beiderwellen ${ }^{1}$, T.D. Poeppel' ${ }^{1}$ V. Hartung-Knemeyer ${ }^{1}$,

C. Buchbender', H. Kuehl', A. Bockisch', T.C. Lauenstein ${ }^{1} ;{ }^{1} E s s e n / D E$,

2Düsseldorf/DE (karsten.beiderwellen@uk-essen.de)

Purpose: Comparison of diagnostic efficiency of simultaneously acquired ${ }^{68} \mathrm{Ga}-$ DOTATOC PET/MRI with a triple phase contrast-enhanced ${ }^{68} \mathrm{Ga}$-DOTATOC PET/ $\mathrm{CT}$ in patients with neuroendocrine tumours (NET).

Methods and Materials: 25 patients (12 women, 13 men; aged $53 \pm 15$ years) with histologically confirmed NET underwent a triple phase contrast-enhanced PET/CT with ${ }^{68} \mathrm{Ga}$-DOTATOC (Biograph mCT 128, Siemens, Erlangen, Germany). The patients subsequently underwent a whole body contrast-enhanced PET/MRI (Biograph mMR, Siemens, Erlangen, Germany). The resulting datasets were rated separately by two radiologists. The criteria comprised lesion count, lesion localisation and characterisation using a 4-point ordinal scale (0: not visible, I: benign, II: indeterminate, III: malignant). All available data (PET/CT, PET/MRI, history) and a clinical follow-up (mean: 95 days) served as standard of reference. Additionally, a case-based descriptive data analysis of PET/CT, PET/MRI and the submodalities was performed.

Results: In 15 of the 25 patients malignant NET lesions were present. While PET/ CT allowed for identification of 13 patients, PET/MRI offered correct identification of all 15 patients. All lesions classified as malignant in PET/CT (74 lesions) could equally be visualised in PET/MRI. With PET/MRI 12 additional liver metastases could be detected.

Conclusion: This is the first study showing the feasibility of whole body staging with ${ }^{68} \mathrm{Ga}$-DOTATOC PET/MRI in patients with NET. Our results demonstrate the superiority of PET/MRI in detecting malignant liver lesions compared to PET/CT. Whole body PET/MRI with ${ }^{68} \mathrm{Ga}$-DOTATOC bears the potential of replacing PET/ $\mathrm{CT}$ as the current gold standard in the staging of NETs.

\section{B-0501 14:09}

Diffusion-weighted imaging as part of hybrid FDG-PET/MRI protocols for whole body cancer staging: does it benefit lesion detection? T.A. Heusner ${ }^{1}$, P. Heusch'1, V. Hartung-Knemeyer ${ }^{2}$, K. Beiderwellen²,

T. Lauenstein ${ }^{2}$, M. Forsting ${ }^{2}$, G. Antoch ${ }^{1}$, C. Buchbender ${ }^{1} ;{ }^{1}$ Düsseldorf/DE, ${ }^{2}$ Essen/DE (heusner@med.uni-duesseldorf.de)

Purpose: To evaluate if diffusion-weighted imaging (DWI) results in a diagnostic benefit for lesion detection in oncologic patients if added to a whole body [18 F]FDG PET/MRI protocol.

Methods and Materials: 25 patients (mean age: $57 \pm 12$ years) with [18 F]-FDG PET positive tumours prospectively underwent whole body [18 F]-FDG-PET/MRI including DWI on a hybrid PET/MRI scanner. Two readers assessed [18 F]-FDG PET/MRI without DWI for primary tumours and metastases in consensus. In a second session, now considering DWI, readers reassessed [18 F]-FDG PET/MRI accordingly. Additionally, the lesion-to-background contrast on [18 F]-FDG PET and DWI was rated qualitatively on a 4-point scale. McNemar's test and Wilcoxon's signed-rank test were performed to test for differences in the number of detected lesions and the lesion-to-background contrast.

Results: 54 lesions were detected ( 5 primaries, 49 metastases). 51 of 54 lesions were concordantly detected by [18 F]-FDG PET/MRI alone and [18 F]-FDG PET/ MRI with DWI. [18 F]-FDG PET/MRI with DWI detected 3 additional lymph node metastases. There was no significant difference between the number of lesions detected by [18 F]-FDG PET/MRI alone and [18 F]-FDG PET/MRI with DWI ( $p=$ $0.25)$. The lesion-to-background was significantly higher on [18 F]-FDG PET compared to DWI $(p=0.001)$

Conclusion: DWI does not increase the number of detected lesions when added to a whole body [18 F]-FDG PET/MRI protocol. Compared to [18 F]-FDG PET it provides a lower lesion-to-background contrast. In order to optimise PET/MRI protocols with regard to patient comfort and scan duration, DWI seems dispensable for whole body staging, as it provides redundant information and does not aid the detection of PET positive malignancies.

\author{
B-0502 14:18 \\ Molecular imaging of the prostate using multiparametric functional \\ [11C]-acetate PET/MRI: proof of concept \\ S. Polanec, T. Helbich, K. Pinker-Domenig, S. Leitner, B. Füger, G. Karanikas, \\ M. Margreiter, S. Kommata, P. Brader; Vienna/AT
}

Purpose: The potentials of multiparametric functional PET/MRI (mfPET/MRI) are not yet explored. We hypothesise that $\mathrm{mfPET} / \mathrm{MRI}$, which assesses multiple pathophysiologic, enables an improved diagnosis prostate cancer $(\mathrm{PCa})$. Therefore, the purpose of this study was to demonstrate the feasibility of multiparametric functional MRI and [ $\left.{ }^{11} \mathrm{C}\right]$-Acetate PET/CT for molecular imaging of the prostate. Methods and Materials: 21 patients (mean age of 68.1 years range from 54 to 79 years; mean PSA level: $8.1 \mathrm{ng} / \mathrm{ml}$ range $0.9-23.4 \mathrm{ng} / \mathrm{ml}$ ) were included in this IRB-approved prospective study. $\left[{ }^{[1} \mathrm{C}\right]-A$ cetate PET/CT as well as $3 \mathrm{~T}$ MRI of the prostate using T2-weighted, dynamic contrast-enhanced and diffusion-weighted MR imaging was performed. mfPET/MRI data were coregistered, fused and colourcoded images were generated. mfPET/MRI was assessed for lesion morphology, acetate-avidity and nodal status. Lesions within the prostate gland were classified as positive when $\left[{ }^{11} \mathrm{C}\right]$-Acetate uptake was greater than blood-pool activity. The lesions were classified in the MRI with the PIRADS classification system and in 21 patients histopathologically verified.

Results: 21 lesions were detected by both MRI and mfPET/MRI. MRI classified 5 lesions as benign and 16 lesions as probably malignant. PET/MRI upgraded the 16 suspect lesions to definite malignant, which were all histopathologically proved to be malignant. Sensitivity and specificity of PET/MRI was 100 and $95 \%$, respectively. In one patient mfPET/MRI revealed a lymph node metastasis and in two patients an additional skeletal metastasis, which was missed by MRI alone. Conclusion: Molecular imaging of the prostate with mfPET/MRI is feasible and improves diagnostic accuracy. Additionally, an improved assessment of nodal status and bone metastasis is enabled.

\section{B-0503 14:27}

PET/CT guided therapy planning in prostate cancer patients eligible for radiotherapy: results from a bicentric clinical study

E. Lopci', B.A. Jereczek-Fossa², M. Rodari', G. Pepe', C. Fodor'²,

D. Zerini ${ }^{2}$, O. De Cobelli ${ }^{2}$, R. Orecchia ${ }^{2}$, A. Chiti' $;{ }^{1}$ Rozzano/IT, ${ }^{2}$ Milan/IT (egesta.lopci@gmail.com)

Purpose: To analyse the role of 11C-Choline PET/CT (Cho-PET) in the management of prostate cancer (PC) patients eligible for radiotherapy (RT).

Methods and Materials: We analysed data from 82 Cho-PET scans, out of 74 PC patients (median age 67.7years; range 50-83), referred to our Institutions for RT planning in case of biochemical relapse after previous treatment (26 surgery; 39 surgery \pm systemic), or at initial diagnosis (9 patients). All Cho-PET were reviewed and the outcome was classified as positive in prostate/prostatic bed (T), pelvic lymph nodes (N), distant metastases (M) or negative (neg). Median PSA at Cho-PET was $1.6 \mathrm{ng} / \mathrm{mL}$ (range $0.1-150 \mathrm{mg} / \mathrm{mL}$ ). In 23 cases, PET was performed during androgen deprivation therapy (ADT). Cho-PET findings were confirmed with other diagnostic techniques, clinical data and/or follow-up.

Results: Cho-PET was positive in 45 exams (55\%), of which $22 \mathrm{~T}$ ( $49 \%), 4 \mathrm{~N}(9 \%)$, and 19M in combination with $\mathrm{T}$ and/or N (42\%). The median pre-PET PSA in T-pos and N/M-pos cases were $7.4 \mathrm{ng} / \mathrm{mL}$ (range $2.7-150$ ) and $13.7 \mathrm{ng} / \mathrm{mL}$ (range 5.3-139), respectively. According to Cho-PET findings, in 28 cases patients proceeded with $\mathrm{RT} \pm$ systemic therapy, whereas in the remaining $17(48 \%)$ the therapeutic approach changed. In 6 cases patients underwent surgery followed $\pm R T$, in 4 cases patients received systemic therapy only, or other treatment in 7 cases. When considering all scans, PET impact on treatment planning reaches $21 \%(17 / 82)$.

Conclusion: Despite the suboptimal detection rate, Cho-PET comes out an important imaging modality for treatment planning in PC patients. The method results capable of influencing more than $1 / 5$ of all patients and almost half of those with a PET-pos result. 


\section{B-0504 14:36}

Detection of lung nodules in an integrated whole body MR/PET scanner: comparison to PET/CT

N.F. Schwenzer, C. Schraml, S. Gatidis, C. Brendle,

H. Schmidt, C. Pfannenberg, C.D. Claussen;

Tübingen/DE (nina.schwenzer@med.uni-tuebingen.de)

Purpose: To investigate the diagnostic performance of a whole body 3 T MR/PET in the detection of lung nodules.

Methods and Materials: Lung imaging of PET/CT and MR/PET in 20 patients with oncologic diseases was evaluated. PET/CT comprised whole body scans in expiration (CTex) and inspiration (CTin). In MR/PET, axial T1 3D-VIBE sequences of the lung were performed both in inspiration (VIBEin) and in expiration (VIBEex). Furthermore, in-/opposed-phase sequence (Dixon) used for attenuation correction in MR/PET were evaluated. In all images, the diagnostic confidence in identification of nodules was rated on a 4-point scale (1=certain; 4=uncertain). Additionally, the involvement of each lung lobe (primary/metastatic) was assessed. CT scan in inspiration served as reference. The diagnostic performance of each modality was compared in a lesion- and lobe-based analysis. The diagnostic confidence was compared using Wilcoxon test.

Results: 48 lung nodules were evaluated. In the lesion-based analysis, sensitivity (false negative rate) of CTex, VIBEin, VIBEex and Dixon was $77 \%(23 \%), 52 \%$ $(48 \%), 48 \%(52 \%)$ and $25 \%(75 \%)$. In the lobe-based analysis reflecting the clinical assessment, sensitivity (false negative rate) was $94 \%(6 \%), 89 \%(11 \%), 89 \%$ $(11 \%)$ and $53 \%(47 \%)$, respectively. In CT and MRI diagnostic confidence was rated higher in inspiration (mean score CTin/CTex 1.1/1.3; VIBEin/VIBEex 1.2/1.5) although no statistical significance was reached.

Conclusion: Results suggest that for reliable detection of lung nodules a CT scan of the lung is still recommended. Concerning clinical assessment of lung lobe involvement, VIBE sequences offered fair results especially. Lesion detection and diagnostic confidence of MRI can be improved if an inspirational examination is performed.

\section{Author Disclosures:}

N.F. Schwenzer: Other; Research contract Siemens Healthcare.

\section{B-0505 14:45}

Assessment of tumour aggressiveness with quantitative perfusion imaging and tumour volume in patients with lung cancer: correlation with metabolic parameters of 18 FDG-PET/CT

D. Fior, D. Ippolito, P.A. Bonaffini, C. Capraro, C. Messa, S. Sironi; Monza/IT (davidefior85@gmail.com)

Purpose: To evaluate the relationship between quantitative CT-perfusion parameters and tumour volume with glucose metabolism parameters, in staging of lung cancer patients with 18 FDG PET/CT whole body study.

Methods and Materials: Thirty-one patients with biopsy-proven lung cancer, referred for cePET/CT staging, were prospectively enrolled in our study. CTp was performed with following parameters: $120 \mathrm{Kv} ; 80 \mathrm{mAs}$; scan 30, during intravenous injection of $50 \mathrm{ml}$ of contrast-agent, flow rate: $5 \mathrm{ml} / \mathrm{sec}$. CTp and cePET/CT data were used to calculate a range of tumour vascularity parameters (blood flow, blood volume and mean transit time) and tumour glucose uptake (SUVmax), and finally correlated with tumour volume using a commercially available software. Four patients were excluded due to the advanced stage of the tumour. CT perfusion parameters were then statistically analysed and compared with those of FDG-PET. Results: Perfusion parameters were calculated with a dedicated software (Perfusion 3.0; Advantage; GE) and a quantitative map of arterial perfusion was generated. Patients were divided into two groups according to tumour volume, with a cut-off of $14 \mathrm{~cm} 3$. Linear regression was performed among two groups and statistically significant correlations $(P<0.05)$ were found between BF and SUVmax (negative correlation) and between MTT and SUVmax (positive correlation) for tumours with volume $>14 \mathrm{~cm} 3$. These findings may be due to the inadequate blood inflow typical of larger tumours, with a significant increase of anaerobic metabolism and glucose consumption.

Conclusion: CePET/CT perfusion is a feasible technique that offers informations related to the biological tumour behaviour that can be used for planning treatment and for an accurate evaluation of tumour response after therapy.

\section{B-0506 14:54}

FDG-PET/CT for the early prediction of histopathological complete response to neoadjuvant chemotherapy in breast cancer patients T.A. Heusner ${ }^{1}$, C. Riegger ${ }^{1}$, S. Kuemmel ${ }^{2}$, M. Forsting ${ }^{2}$, V. Hartung-Knemeyer ${ }^{2}$, G. Antoch ${ }^{1}$, C. Buchbender ${ }^{1} ;{ }^{1}$ Düsseldorf/DE, ${ }^{2}$ Essen/DE (heusner@med.uni-duesseldorf.de)

Purpose: To test if FDG-PET/CT can early differentiate between breast cancer lesions with and without pathological complete response ( $\mathrm{pCR} / \mathrm{npCR}$ ) after two cycles of neoadjuvant chemotherapy (NACT).

Methods and Materials: 26 breast cancer patients (mean age, 46.9 years \pm 9.9 years) underwent a pre-therapeutic FDG-PET/CT scan and a FDG-PET/CT after the second cycle of NACT. Histopathology served as the reference standard. SUVmax of cancer lesions before and after the second cycle of NACT were measured. Two evaluation algorithms were used: (a) pCR: Sinn Score 3 and 4, npCR: Sinn Score 0-2; (b) pCR: Sinn Score 4, npCR: Sinn Score 0-3. The absolute and relative decline of the SUVmax $(\Delta S U V \max , \triangle S U V \max (\%))$ was calculated. Differences of the SUVmax and of the SUVmax decline between pCR and npCR lesions were tested for statistical significance $(p<.05)$. To identify the optimal cut-off value of $\triangle S U V \max (\%)$ to differentiate between $\mathrm{pCR}$ lesions and $\mathrm{npCR}$ lesions a receiver operating curve $(\mathrm{ROC})$ analysis was performed.

Results: Using evaluation algorithm A the $\triangle$ SUVmax was 13.5 (pCR group) and 3.9 (npCR group) ( $P=0.006)$; the $\triangle S U V \max (\%)$ was $79 \%$ and $47 \%$, respectively $(P=0.001)$. On ROC analysis an optimal cut-off $\triangle S U V \max (\%)$ of $66 \%$ was found. Using evaluation algorithm $B$ the $\triangle$ SUVmax was 17.5 (pCR group) and 4.9 (npCR group) $(P=0.013)$; the $\triangle S U V \max (\%)$ was $89 \%$ and $51 \%$, respectively $(P=0.003)$. On ROC analysis an optimal cut-off $\triangle$ SUVmax (\%) of $88 \%$ was found.

Conclusion: FDG-PET/CT early differentiates between $\mathrm{PCR}$ and npCR after two cycles of NACT.

\section{B-0507 15:03}

Diagnostic accuracy of 11C-choline PET (CHO-PET) in patient affected by hepatocellular carcinoma (HCC): comparison with CT/MRI

E. Lopci, G. Torzilli, A. Palmisano, M. Scorsetti, A. Chiti; Rozzano/IT (egesta.lopci@gmail.com)

Purpose: To assess the diagnostic role of 11C-choline PET/CT (CHO-PET), compared to conventional imaging with CT/MRI, in patients affected by hepatocellular carcinoma (HCC).

Methods and Materials: In our study we prospectively enrolled 23 consecutive patients ( $M: F=20: 3$, mean age 70years) affected by HCC: 6 patients at presentation and 17 at restaging. In all cases we performed a whole body CHO-PET and compared findings with referring CT $(n=20)$ and/or MRI scanning $(n=11)$, for a total of 25 paired scans. The reference standard for validating imaging results was either histology (5 patients), or a multidisciplinary work-up. For every modality we determined diagnostic accuracy on a scan-based and on a site-based analysis. Results: On a scan-based analysis CHO-PET and CT/MRI demonstrated an accuracy of $86.4 \%$ and $88 \%$, respectively, whereas on a site-based analysis the two modalities demonstrated a sensitivity and accuracy, respectively, of $67.6 \%$ and $65.9 \%$ for PET, and $71.7 \%$ and $69 \%$ for CT/MRI. In both analyses, no statistically significant difference was found on T-test. In 7/23 patients, HCC showed either loco-regional involvement $(n=3)$ or distant metastases $(n=6)$. All extra-hepatic lesions were properly detected by CHO-PET (accuracy 100\%), whereas CT/MRI missed a large part of them (accuracy 58.8\%) and this most likely due to the limited field of view during acquisition. In 7 cases (30.4\%), PET information determined a change in treatment planning.

Conclusion: According to our findings, CHO-PET has a comparable accuracy with CT/MRI in HCC. The principle advantage of the modality appears its ability to detect extra-hepatic lesions, which can lead to treatment modification.

\section{B-0508 15:12}

PIB-PET as a pathological and functional marker in Alzheimer's disease V. Abreu', A. Nordberg ${ }^{2} ;{ }^{1}$ Lorenskog/NO, ${ }^{2}$ Stockholm/SE

Purpose: Amyloid imaging using positron emission tomography (PET) with the tracer PIB is widely used in Alzheimer's disease (AD) research. FDG-PET is a well-established method for measuring cerebral glucose metabolism (CMRglc), assessing functional status. We investigated the possibility to use early frames (ePIB) of PIB scans as a rough index of CBF comparing the normalised early PIB values with $\mathrm{rCMRgIc}$ in $\mathrm{AD}$ patients, mild cognitive impairment $(\mathrm{MCl})$ subjects and healthy controls.

Methods and Materials: PIB-PET and FDG-PET was performed in 37 AD patients, $21 \mathrm{MCl}$ subjects and 6 healthy controls. Patients were divided based on their PIB 
retention as PIB positive (PIB+) or PIB negative (PIB-). Data of the unidirectional influx $K_{\_} 1$ from a subset of $7 \mathrm{AD}$ patients and 3 controls were used for correlative analysis. Data were analysed using regions of interest $(\mathrm{ROI})$ analysis.

Results: We found a strong, positive correlation across brain regions between $\mathrm{K} \_1$ and ePIB $(r=0.70 ; p<0.001)$. ePIB values were significantly lower in posterior cingulate $(p<0.001)$ and parietal cortices $(p=0.002)$ in PIB+ subjects compared with PIB- although the group difference were stronger for rCMRglc in cortical areas $(p<0.001)$. Strong positive correlations between ePIB and rCMRglc were observed in all cortical regions analysed, especially posterior cingulate and parietal cortices $(p<0.001)$.

Conclusion: This study suggests that a single dynamic PIB-PET scan may provide clinical estimates of pathological (amyloidosis) and functional changes (impaired blood flow) in AD. This dual information might be valuable both in early diagnosis of $A D$ and evaluation of treatment strategies, but our results emphasise the need for further research. 


\section{Saturday, March 9}




\section{Neuro}

\section{SS 911}

\section{New insights into brain gliomas}

Moderators:

L.C. Tzarouchi; loannina/GR

P. Vilela; Almada/PT

\section{B-0509 10:30}

Data-driven grading of brain gliomas: a multiparametric MRI study V. Panara, D. Tortora, P. Mattei, S. Salice, C. Briganti, A. Cotroneo, A. Tartaro, M. Caulo; Chieti/IT (valepana@libero.it)

Purpose: To grade brain gliomas using data-driven analysis of multiparametric MRI and to compare these results with qualitative and semi-quantitative evaluations. Methods and Materials: 110 patients with histologically confirmed brain gliomas were retrospectively evaluated. Conventional and advanced MR sequences (DWI, PWI, MRS, DTI) were performed. Three evaluations were done: semiquantitative (radiological report), qualitative (conventional MRI) and quantitative. For the latter regions of interest (ROI) placed in 4 areas: with contrast enhancement (CE), highest T2 signal intensity (T2+), lowest T2 signal intensity (T2-), and most restricted diffusion (RD). rCBV, rMTT, Cho/Cr, Cho/NAA, Lip/Cr, Lac/Cr, ADC and FA were measured. Statistical analysis included t-test $(p<0.05), R O C$ analysis, discriminant analysis, and Kendall coefficient of concordance, where appropriate.

Results: Significant differences between grades were noted in age, rCBV in CE (cut off $>2.59$, sensitivity $80 \%$, specificity $91 \%, A U C=0.937, p=0.0001$ ), T2- (cut off $>2.45$, sensitivity $57 \%$, specificity $97 \%, A \cup C=0.852, p=0.0001$ ), RD (cut off $>$ 2.61 , sensitivity $54 \%$, specificity $97 \%, A U C=0.808, p=0.0001$ ), Cho/Cr in T2- (cut off $>2.07$, sensitivity $49 \%$, specificity $88 \%, A \cup C=0.685, p=0.0007$ ). Discriminant analysis including age, $\mathrm{rCBV}$ in CE, in T2- and in $\mathrm{RD}$ and $\mathrm{Cho} / \mathrm{Cr}$ in T2- correctly classified $95 \%$ of patients. Quantitative analysis showed a higher concordance with histologic examination than qualitative and semiquantitative methods $(p<0.0001)$. Conclusion: The integration of multiple biomarkers with a quantitative approach significantly increased the diagnostic accuracy in discriminate between high and low grade gliomas.

\section{B-0510 10:39}

Combining diffusion kurtosis imaging, dynamic susceptibilityweighted MR imaging and short echo time chemical shift imaging for grading gliomas

S. Van Cauter', F. De Keyzer', D. Sima', J. Veraart'², F. D'Arco'1, R. Peeters', S. Van Gool', S. Sunaert', U. Himmelreich ${ }^{1} ;{ }^{1}$ Leuven/BE, ${ }^{2}$ Antwerp/BE (sofie.vancauter@uz.kuleuven.be)

Purpose: To assess the diagnostic accuracy of diffusion kurtosis imaging (DKI), dynamic susceptibility-weighted imaging (DSC) and short echo time chemical shift imaging (CSI) for grading gliomas, as separate techniques and in a multimodal approach in order to determine the best discriminative parameter (s).

Methods and Materials: 35 patients with cerebral gliomas (12 F/23M; age range: 22-78, median age: 55) underwent DKI, DSC and CSI on a 3 T MR scanner. Diffusion parameters: mean diffusivity (MD), fractional anisotropy (FA), mean kurtosis (MK); perfusion parameters: mean relative regional cerebral blood volume (mean rrCBV), mean relative regional cerebral blood flow (mean rrCBF), mean transit time (MTT) and relative decrease ratio (rDR), and twelve CSI metabolite ratios were compared between 22 high-grade gliomas and 14 low-grade gliomas (Mann-Whitey-U, $p<0.05$ significance, Bonferroni corrected). Sensitivity, specificity, diagnostic accuracy and optimal thresholds were determined for the techniques separately and combined. Results: MK, MD, mean rrCBV, mean rrCBF, rDR, Lips/Cho, Lips/Cre, Myo/sum and Cre/sum showed statistically significant differences among tumour grades, with mean $\mathrm{rrCBF}$ as the best discriminative parameter. The combination of mean $\mathrm{rrCBF}, \mathrm{MK}$ and $\mathrm{Myo} /$ sum to grade gliomas showed diagnostic accuracy, sensitivity and specificity of $100 \%$, whereas combining mean $\mathrm{rrCBV}, \mathrm{MD}$ and Lips/Cho gave a diagnostic accuracy, sensitivity and specificity of $91 \%, 93 \%$ and $82 \%$, respectively. Conclusion: Combining the parameters mean rrCBF, MK and Myo/sum to determine the glioma grade was pathognomonic in this study. The best performing parameters, when used individually, were found to be MK and mean rrCBF.
B-0511 10:48

Dynamic contrast-enhanced (DCE) MRI of cerebral gliomas: assessment of tumoral angiogenesis with low gadolinium-based contrast agent dose

D. Gadda, L. Mazzoni, M. Moretti, S. Chiti, G. Giordano; Firenze/IT (davgadda@tin.it)

Purpose: DCE-MRI biomarkers are related to angiogenesis, a crucial process for the neoplastic growth of high-grade gliomas (HGG). A dose of $0.1-0.2 \mathrm{mmol} / \mathrm{kg}$ body weight gadolinium-based contrast agent (GBCA) is generally administered for DCE imaging. We hypothesized that DCE-MRI parameters obtained with the lower dose of $0.05 \mathrm{mmol} / \mathrm{kg}$ of a high-relaxivity GBCA (gadobenate dimeglubine) used as preload for rCBV quantification with dynamic-susceptibility contrast (DSC) MRI, may differentiate HGG from low-grade gliomas (LGG).

Methods and Materials: The GBCA low-dose DCE-MRI studies of $11 \mathrm{HGG}$ and 6 LGG were retrospectively evaluated. DCE-MRI had been followed by DSC-MRI for quantification of rCBV. Tofts model was utilised for DCE quantitative analysis. Ktrans, Kep, Ve, iAUC and rCBV estimates of HGG were compared to LGG with Student's t-test. ROC analyses were also done for each parameter.

Results: Significant differences were found for Ve (HGG 0.140 0.12 , LGG $0.010 \pm 0.009$, p0.005, AUC 0.924), iAUC (HGG 3.15 $\pm 2.01, L G G 0.43 \pm 0.4$, p 0.0012 , AUC 0.939) and rCBV (HGG 3.67 \pm 2.42 , LGG 1.27 \pm 0.33 , p0.011, AUC 0.860), whereas no significant differences were found for Kep (HGG 3.91 \pm 5.9 , LGG 9.31 \pm 6.55, p 0.1, AUC 0.803 ) and Ktrans (HGG 0.096 \pm 0.05 , LGG 0.054 \pm 0.036 , p0.11, AUC 0.742)

Conclusion: DCE-MRI with the dose of $0.05 \mathrm{mmol} / \mathrm{kg}$ of gadobenate dimeglubine can differentiate HGG, a tumour with generally high angiogenic activity, from the slow-growing LGG. The diagnostic performance of DCE parameters Ve and iAUC was higher than rCBV. The preload dose of gadolinium for DSC-MRI may be utilised for DCE-MRI, making feasible the estimation of blood volume and permeability in a single examination.

\section{B-0512 10:57}

Correction of CBV perfusion maps from T1 leakage effect allows a better correlation of perfusion DSC MR imaging with histopathologic glioma grading and an estimation of vascular permeability

F. Viry', D. Galanaud ${ }^{1}$, A. Mitulescu², Y. Chaibi' ${ }^{2}$, V. Reina' ${ }^{1}$, D. Leclercq ${ }^{1}$,

D. Dormont ${ }^{1} ;{ }^{1}$ Paris/FR, ${ }^{2}$ La Ciotat/FR (viryflore@yahoo.fr)

Purpose: Capillary proliferation is a major histopathological criterion in glioma grade determination, known to correlate with CBV. However, in case of blood brain barrier (BBB) disruption, contrast agent extravascular leakage increases the signal in voxels inducing under-estimation of CBV. Sophisticated mathematical models taking into account BBB disruption and arterial input function (AIF) can be used to correct this misestimation and enable permeability constant K2 calculation. We hypothesized that CBV corrected from vascular leakage can better differentiate glioma grade than uncorrected_CBV and that K2 increases with glioma grade. Methods and Materials: Fifty-four patients with steretotaxic biopsy-confirmed gliomas ( 20 WHO grade II, 13 grade III and 21 grade IV) explored by multimodal MR imaging including DSC gradient-echo perfusion-MR (1.5 T-3 T) were retrospectively included. Preprocessing included motion correction, automatic AIF determination and leakage correction (Perfscape/Neuroscape-Olea Medical). Perfusion maps including relative CBV (corrected/uncorrected), relative CBF and K2 were calculated. Regions-of-interest were delineated within the tumour (biopsy site), and in the symmetrical contralateral normal-appearing region.

Results: Differences in corrected_rCBV were statistically significant $(p<0.05)$ between grades IIIIV, III-IV, II-III (non-parametric Steel-Dwass test). Uncorrected_rCBV does not statistically differentiate grade II-III. K2 could only differentiate grade II-IV. Corrected_rCBV had a stronger correlation to glioma grade than uncorrected_rCBV (Spearman $r=0.73$ and $r=0.6$, respectively, $p<0.01$ ). $\mathrm{K} 2$ was weakly correlated to glioma grade $(r=0.34, p<0.01)$.

Conclusion: Taking into account BBB disruption in DSC perfusion imaging improves glioma grades differentiation with rCBV. K2 has a weak correlation with glioma grade, which could partly be explained by the heterogenous parameters of DSC sequences in this study.

Author Disclosures:

F. Viry: Equipment Support Recipient; Using Perfscape/Neuroscape software (Olea Medical). D. Galanaud: Equipment Support Recipient; Using Perfscape/ Neuroscape software (Olea Medical). A. Mitulescu: Employee; VP Clinical Affairs at Olea Medical. Y. Chaibi: Employee; Clinical \& Scientific Research Engineer at Olea Medical. V. Reina: Equipment Support Recipient; Using Perfscape/Neuroscape software (Olea Medical). D. Leclercq: Equipment Support Recipient; Using Perfscape/Neuroscape software (Olea Medical). 
D. Dormont: Equipment Support Recipient; Using Perfscape/Neuroscape software (Olea Medical).

\section{B-0513 11:06}

Paradoxical imaging features of high-grade gliomas with oligodendroglial component: quantitative analysis on diffusion and DSC perfusion MR imaging

L. Sunwoo, S. Choi, T. Kim, S.-H. Lee, C.-K. Park, J.-H. Kim, C.-H. Sohn, S.-H. Park, I. Kim; Seoul/KR (leonard.sunwoo@gmail.com)

Purpose: The aim of this study is to retrospectively investigate characteristic imaging features of high-grade gliomas with oligodendroglial component, which was compared with pure astrocytic high-grade gliomas on diffusion and DSC perfusion MR imaging using quantitative analysis.

Methods and Materials: The study was approved by the Institutional Review Board and was HIPAA compliant. Newly diagnosed high-grade glioma patients were enrolled ( $n=52 ; 21$ pure astrocytic glioblastomas (GBMs), 10 glioblastomas with oligodendroglial component (GBMOs), 9 anaplastic astrocytomas (AAs), 8 anaplastic oligodendrogliomas (AOs), and 4 anaplastic oligoastrocytomas (AOAs)). All participants underwent standard $b$-value $(b=0,1000 \mathrm{~s} / \mathrm{mm} 2)$ diffusion and DSC perfusion MR imaging within 2 weeks before surgery. All tumours were manually segmented based on T2 hyperintense portion. Apparent diffusion coefficient (ADC) and relative $\mathrm{CBV}$ ( $\mathrm{rCBV}$ ) values were analysed using histogram approach. The $5^{\text {th }}, 10^{\text {th }}$ percentile values of $A D C$ and $90^{\text {th }}, 95^{\text {th }}$ percentile values of $\mathrm{rCBV}$, as well as mean values were derived, and their mean values according to each tumour subtypes and grades were analysed.

Results: For grade III tumours, AOs showed significantly higher rCBV compared to AAs $(P<0.01)$. Derivatives of $r C B V$ values of $A O A s$ were not significantly different from those of AAs or AOs. For grade IV tumours, GBMOs showed significantly lower rCBV than GBMs $(P<0.01)$. Also, the $5^{\text {th }}$ percentile ADC values of the GBMOs were significant higher than those of GBMs $(P=0.046)$.

Conclusion: We have firstly demonstrated paradoxical lower rCBV and higher $A D C$ values in GBMOs compared to GBMs, which was a contrary finding to grade III tumours.

\section{B-0514 11:15}

Survival predictive value of perfusion-weighted MRI relative cerebral blood volume ( $\mathrm{rCBV}$ ) in glioblastoma multiforme adjusted by multiple biomarkers including MGMT promoter methylation

G. Viteri, P. Slon, J. Etxano, G. Aldave, R. García-Eulate, J.L. Zubieta, S. Tejada R. Díez-Valle, P.D. Dominguez; Pamplona/ES (pslon@unav.es)

Purpose: The survival predictive value of perfusion MRI in glioblastoma multiforme has been investigated with contradictory results. Several biomarkers have been isolated that may confound or interact with the predictive value of perfusion MRI as they imply significant therapeutical response changes. The purpose of this study is to evaluate retrospectively whether the tumoral maximum $\mathrm{rCBV}$ on pretreatment perfusion MRI has prognostic value in patients with glioblastoma multiforme, considering several biomarkers including the presence or the absence of MGMT promoter methylation.

Methods and Materials: Between December 2007 and July 2012, 42 patients (24 men, 18 women; age range, 34-78 years) with newly diagnosed supratentorial glioblastoma multiforme underwent MRI before treatment. The patient age, sex, Karnofsky Performance Scale (KPS), preoperative/postoperative tumoral volume, MGMT methylation and maximum rCBV were assessed. A multivariate Cox proportional hazards model was used to evaluate prognostic factors.

Results: Maximum rCBV $(0.984 ; 95 \% \mathrm{Cl}, 0.825-1.173)$, MGMT promoter methylation $(1.658 ; 95 \% \mathrm{Cl}, 0.627-5.529)$ and the other factors (age, KPS, preoperative volume, postoperative volume and MGMT methylation) did not significantly predict survival $(p>0.05)$. The only independent prognostic factor that was statistically significant was sex $(\mathrm{HR}=3.672 ; 95 \% \mathrm{Cl} 1.184-11.395)$.

Conclusion: In this series, the maximum rCBV at pretreatment perfusion MRI in patients with glioblastoma multiforme did not predict survival, adjusted by MGMT promoter methylation and other biomarkers.
B-0515 11:24

Additive value of arterial spin labeling in differentiating primary central nervous system lymphoma from glioblastoma multiforme: an observer performance study

K. Yamashita, T. Yoshiura, A. Hiwatashi, O. Togao, K. Kikuchi, K. Yoshimoto,

H. Honda; Fukuoka/JP (yamakou@radiol.med.kyushu-u.ac.jp)

Purpose: To determine whether ASL has an additive diagnostic value to the conventional MR images in differentiating primary central nervous system lymphomas (PCNSLs) from glioblastoma multiformes (GBMs).

Methods and Materials: MR images of 48 patients including 12 with PCNSL and 36 with GBM were retrospectively studied. Two independent neuroradiologists took part in 2 reading sessions. In the first session, the observers read only conventional MR images (T1-weighted image, T2-weighted images, post-contrast images, and DWI with apparent diffusion coefficient maps). In the second session, they read ASL cerebral blood flow (CBF) maps along with the conventional images. The confidence level for the diagnosis (PCNSL versus GBM) was graded on a 5-point scale. Interobserver agreement as well as sensitivity, specificity, accuracy, positive predictive value (PPV), and negative predictive value (NPV) were assessed for the two readers.

Results: Substantial interobserver agreement was shown for both sessions ( $\mathrm{K}=0.62$ and 0.60 , respectively). For both observers, reading with ASL was associated with higher sensitivity (92.3-100\%), specificity (100-100\%), accuracy (94.1-100\%), PPV (100-100\%), and NPV (80.0-100\%) than those without ASL (sensitivity, 92.3-100\%; specificity, 87.5-87.5\%; accuracy 91.2-97.1\%; PPV 96.0-96.3\%; NPV 77.8-100\%). Conclusion: ASL has an additive diagnostic value to the conventional MR images in differentiating PCNSLs from GBMs.

\section{B-0516 11:33}

Glioma grading using pharmacokinetic parameters in T1-weighted dynamic contrast-enhanced perfusion MR

S.C. Jung, S. Choi, S. Kim, H. Shin, J. Yeom, I. Ryoo; Seoul/KR (dynamics79@gmail.com)

Purpose: To investigate the feasibility of pharmacokinetic parameters $\left(\mathrm{K}^{\text {tras }}, \mathrm{Ve}, \mathrm{Vp}\right)$ derived from T1-weighted dynamic contrast-enhanced (DCE) perfusion magnetic resonance (MR) imaging in terms of glioma grading.

Methods and Materials: Thirty-two patients (16 males, 16 females; mean age, 48.6 years; age range, 25-72 years; 32 tumours) with histologically confirmed gliomas (WHO grade II, 6; grade III, 9; grade IV, 17) were examined with conventional MR imaging and $\mathrm{T} 1$-weighted dynamic contrast-enhanced imaging at $3 \mathrm{~T}$ before surgery or biopsy. $\mathrm{K}^{\text {tras }}$, Ve, and $\mathrm{Vp}$ were calculated and correlated with glioma grading (high grade versus low grade) on a pixel-by-pixel basis. Various percentile values of cumulative histogram were compared and assessed with receiver operating characteristic (ROC) curve according to glioma grading. We rated the percentile with highest area under the ROC curve.

Results: There were significant differences between high- and low-grade gliomas in $\mathrm{K}^{\text {tras }}$, $\mathrm{Ve}$, and $\mathrm{Vp}(\mathrm{p}<0.05)$. $\mathrm{K}^{\text {tras }}$ showed highest association according to glioma grading $(p<0.01)$. The 95 percentile in $\mathrm{K}^{\text {tras }}$ presented highest area under the ROC curve (sensitivity of $80.95 \%$, specificity of $66.67 \%$, positive predictive value of $89.49 \%$, negative predictive value of $49.97 \%$, accuracy of $77.79 \%$ ).

Conclusion: The parameters derived from DCE perfusion MR imaging were useful values for differentiating between low- and high-grade gliomas. $\mathrm{K}^{\text {tras }}$ showed best results in grading gliomas.

\section{B-0517 11:42}

Intravoxel incoherent motion MR imaging with biexponential and stretched-exponential model in grading cerebral gliomas Y. Bai ${ }^{1}$, D. Shi' ${ }^{1}$, S. Dou ${ }^{1}$, F. Sun ${ }^{2}$, M. Wang ${ }^{1} ;{ }^{1}$ Zhengzhou/CN, ${ }^{2}$ Beijing/CN (resonance2010@126.com)

Purpose: To evaluate the diagnostic accuracy of intravoxel incoherent motion (IVIM) MR imaging with biexponential and stretched-exponential models in grading cerebral gliomas.

Methods and Materials: IVIM MR imaging using biexponential and stretchedexponential models with 15 different b values range from 0 to $5000 \mathrm{sec} / \mathrm{mm}^{2}$ at 3 T MRI was performed on 17 high-grade gliomas and 14 low-grade gliomas proved by histopathology after MR examination. The derived parameters of diffusion coef[[Unable to Display Character: \&\#64257;]]cient (ADCslow), and pseudodiffusion coef[[Unable to Display Character: \&\#64257;]]cient (ADCfast) were calculated by biexponential analysis. Water diffusion heterogeneity index a and distributed diffusion coef[[Unable to Display Character: \&\#64257;]]cient (DDC) were obtained from stretched-exponential model. All the parameters were measured in 
the solid parts of gliomas and compared between high- and low-grade gliomas. All the parameters were also normalised to the corresponding values in contralateral normal appearing white matter (NAWM) and compared between high- and lowgrade gliomas. A receiver operating characteristic (ROC) analysis was performed for statistical analysis.

Results: There were significant differences in ADCfast and ADCslow values between high- and low-grade gliomas $(P<0.05)$. The a value was significantly lower in high-grade gliomas than that in low-grade gliomas $(P<0.001)$. In addition, the ADCfast, ADCslow and a values that were normalised to the values in the contralateral NAWM were all significantly different between high-grade and low-grade gliomas $(P<0.05)$

Conclusion: IVIM imaging with biexponential and stretched-exponential model is a useful tool in grading cerebral gliomas. The a value may be the best parameter for distinguishing high- from low-grade cerebral gliomas.

\section{B-0518 $11: 51$}

Apparent diffusion coefficient obtained by magnetic resonance imaging as a prognostic marker in glioblastomas: correlation with MGMT promoter methylation status

L. Calabria ${ }^{1}$, M. Rossi Espagnet ${ }^{1}$, F. Tavanti ${ }^{1}$, A. Boellis ${ }^{1}$, V. Coppola ${ }^{2}$,

M. Federici ${ }^{1}$, A. Romano 1 , L. Fantozzi ${ }^{1}$, A. Bozzao ${ }^{1} ;{ }^{1}$ Rome $/ I T,{ }^{2}$ Ronciglione/IT

Purpose: To evaluate whether apparent diffusion coefficient (ADC) values can predict the status of MGMT of glioblastoma multiforme (GBM) and correlate with overall survival (OS) and progression-free survival (PFS).

Methods and Materials: This retrospective study included 47 patients with pathologically proven glioblastoma. All of them underwent MR DWI study before surgery (mean time 1 week) and the status of methylguanine-DNA-methyltransferase (MGMT) promoter methylation was searched for. Minimum apparent diffusion coefficient (ADC) values were evaluated. OS and PSF parameters were calculated, and Student's t-test, Kaplan-Meier curves, linear and Cox regression were performed. Results: Twenty-five patients showed positive methylation of the MGMT promoter. Patients showing MGMT promoter methylation had higher minimum ADC values, and they survived longer than those without MGMT promoter methylation. The median ADCmin value of 0.80 represents the cut off value able to distinguish between methylated and un-methylated patients. Patients showing minimum ADC values higher than 0.80 survived longer than patients with minimum ADC values lower than 0.80. A linear correlation between minimum ADC values vs. the OS and PFS was observed.

Conclusion: Minimum ADC values in glioblastoma multiforme could be used as a preoperative parameter to estimate the status of MGMT promoter methylation and the survival of patients. Room D1

Chest

\section{SS 904}

\section{Image quality and dose reduction}

Moderators:

P.A. Grenier; Paris/FR

J. Vlahos; London/UK

\section{B-0519 10:30}

Clinical evaluation of an automatic tube voltage selection in chest

CT angiography

T. Niemann, S. Henry, F. Pontana, K. Yasunaga, J.-B. Faivre, J. Rémy,

M. Rémy-Jardin; Lille/FR (martine.remy@chru-lille.fr)

Purpose: To evaluate the clinical impact of automatic tube voltage selection in chest CT angiography (CTA).

Methods and Materials: 93 consecutive patients (mean BMI $25.1 \mathrm{~kg} / \mathrm{m} 2$ ), referred for a follow-up chest CTA examination, were prospectively evaluated with a scanning protocol aimed at comparing two successive CTAs acquired in similar technical conditions except for the $\mathrm{kV}$ selection: (a) the initial examination (i.e., T0 scan) was systematically obtained at $120 \mathrm{kVp}$ and 90 ref $\mathrm{mAs}$, (b) the follow-up examination (i.e., T1 scan) was obtained with an automatic selection of the kilovoltage that proposes optimised $\mathrm{kV}$ and $\mathrm{mA}$ settings according to the planned examination type (Care KV; Siemens Healthcare) which was preset for optimised CTA in this study. Results: At T1, 90 patients (97\%) were scanned with reduced tube voltage: 100 $\mathrm{kV}(\mathrm{n}=26 ; 28 \%), 80 \mathrm{kV}(\mathrm{n}=64 ; 69 \%)$ resulting in a significant DLP reduction at $\mathrm{T} 1$ (T1: 87.27; T0: 141.88 mGy.cm; $p<0.0001$ ) (mean dose reduction: $38.5 \%$ ) and a significant increase in CNR at T1 (T1: $11.5 \pm 3.5 \mathrm{HU}$; T0: $10.9 \pm 3.7 \mathrm{HU} ; \mathrm{p}=0.03)$. The increase in objective image noise at T1 $(25.4 \pm 4.7 \mathrm{HU}$ vs $17.9 \pm 3.4 \mathrm{HU})$ did not alter the diagnostic value of lung and mediastinal images with a mean score of image quality of $1.73 \pm 0.39$ in a 4 -point scale ranging from 1 (excellent) to 4 (poor). Conclusion: The automatic tube voltage selection reduced by $38.5 \%$ the radiation dose delivered during chest CT angiograms while improving the contrast-to-noise ratio of the examinations.

\section{B-0520 10:39}

Organ-based tube-current modulation: impact of breast position in clinical practice

S. Taylor ${ }^{1}$, D. Tack ${ }^{2}$, V. De Maertelaer ${ }^{3}$, P. Gevenois ${ }^{3} ;{ }^{1}$ Montigny-le-Tilleul/BE, ${ }^{2}$ Baudour/BE, ${ }^{3}$ Brussels/BE (stephengtaylor@gmail.com)

Purpose: Organ-based tube-current modulation (OBTCM) is a technique developed to lower CT radiation dose to anterior dose-sensitive organs. This IRB-approved observational study aimed to establish the location of breast tissue in a female patient in supine position with arms raised over their shoulders. A secondary purpose was to look for possible thresholds of chest diameter and woman's age below or above which the breasts lie in a central location with subsequent possible benefit from OBTCM

Methods and Materials: Informed consent was obtained in 200 consecutive women included. From standard chest CT acquisitions, one $5 / 5 \mathrm{~mm}$ image set with FOV of $500 \mathrm{~mm}$ and $\mathrm{X}$ and $\mathrm{Y}$ coordinates at 0 were obtained. From the gantry rotation centre $(x=y=0)$, the outer and inner angles limiting the breast tissue were measured and compared to the angles $\left(60^{\circ}\right)$, where OBTCM delivers lowered and increased dose to the organs.

Results: Outer angles of breast tissue were $<60^{\circ}$ for one breast in only two patients. Inner breast angles were $>60^{\circ}$ in $14 / 200$ patients for at least one breast. No threshold of chest diameter or age was found to predict a central enough location of the breast for OBTCM to be beneficial.

Conclusion: Our data show that the angular position of breast tissue is higher than the limit where reduced versus increased radiation dose is delivered in OBTCM. This system should therefore not be applied to CT chest examinations in adult women lying supine.

Author Disclosures:

D. Tack: Board Member; ESR SP Committee.

\section{B-0521 10:48}

Reduced radiation exposure of the female breast during low-dose chest CT using an organ-based tube-current modulation and bismuth shield: comparison of image quality and radiation dose Y. Kim, Y. Sung, E. Kim, H. Kim, J. Choi; Incheon/KR (skyhi95@naver.com)

Purpose: To evaluate the effect of an organ-based tube-current modulation (OBTCM) and bismuth shielding on image quality and breast radiation dose in women undergoing low-dose chest CT.

Methods and Materials: Low-dose chest CT was obtained from 80 women, who were randomly assigned to a control, OBTCM, breast shield, or breast shield plus OBTCM group. Axial slice images from aortic arch, carina, and inferior pulmonary vein were used to analyse image quality. Three radiologists scored the image quality in terms of artefact and noise, and then designated the overall image quality as optimal (does not affect the diagnostic accuracy) or suboptimal (affects the diagnostic accuracy). Noise levels were measured in the anterior and posterior lung in each image. A phantom dose study was conducted to measure the radiation dose. Results: Images with artefacts or noise were more frequently obtained in the breast shielded groups. The overall image quality was not significantly different among the four groups. Measured noise levels in the anterior lung were significantly higher in the breast shielded groups than the control group; however, no statistical significance was found among the four groups in the posterior lung. In the phantom dose study, a 16-37.5\% dose reduction in the breast was achieved using the breast shield and/or OBTCM protocol.

Conclusion: The radiation dose in the female breast may be reduced using the breast shield or OBTCM during low-dose chest CT with acceptable image quality. The OBTCM reduced the radiation dose in the breast without inducing image quality deterioration. 


\section{B-0522 10:57}

Evaluation of automated attenuation-based tube potential selection in combination with organ-specific dose reduction for clinical chest CT P. Kröpil, L. Schimmöller, S. Dietrich, P. Heusch, J. Aissa, G. Antoch, R.S. Lanzman; Düsseldorf/DE

Purpose: To evaluate the influence of attenuation-based tube potential selection (TPS) combined with organ-specific dose reduction (ODSR) on radiation dose and image quality (IQ) of contrast-enhanced chest-CT examinations in clinical routine. Methods and Materials: 68 consecutive patients (mean age $58.3 \pm 12.7$ years) undergoing clinical contrast-enhanced $(100 \mathrm{ml})$ chest CT on a 128-row scanner were included in this study. 34 chest CTs were performed using the novel dose saving algorithms TPS and ODSR (CareKV, XCare; Siemens). ODSR protects radiosensitive tissues such as breast parenchyma by lowering the tube current for anterior projections. TPS adapts semiautomatically the tube potential depending on attenuation of the subject and type of examination. Objective and subjective IQ and radiation dose (CTDIvol) were compared with 34 standard protocol chest CTs $(120 \mathrm{kVp})$. Subjective IQ (lesion detectability, diagnostic confidence) was rated on a 4-point scale (1_non diagnostic, 4_excellent). Signal-to-noise ratios (SNR) were determined from ROIs placed in the contrast-enhanced lumen of the aorta and the surrounding air outside the body.

Results: CTDIvol of chest CTs using TPS and ODSR was significantly lower than at standard protocol $(3.1 \pm 1.0 \mathrm{mGy}$ versus $6.2 \pm 2.3 \mathrm{mGy} ; \mathrm{p}<0.001)$. Although noise level was slightly elevated when using dose saving algorithms $(14.7 \pm 5.7 \mathrm{vs.} 11.4 \pm 3.8$ $\mathrm{HU} ; \mathrm{p}<0.01)$, no significant differences in SNR $(17.0 \pm 4.6$ vs. $16.5 \pm 4.5)$ or subjective IQ (mean score 3.88 versus 3.93) were observed between the two protocols. Conclusion: Attenuation-based TPS in combination with OSDR essentially lowers the dose of chest $\mathrm{CT}$ in clinical routine while maintaining high subjective and objective image quality.

\section{B-0523 11:06}

Thoracic imaging with low-dose MDCT at low tube voltages using iterative image reconstruction: where is the limit of diagnostic confidence compared to the clinical standard?

W. Stiller' ${ }^{1}$, G. Pahn' , J.L. Mayer', M. Dadrich'1, J. Hirsch², S. Böhme ${ }^{3}$, K. Markstaller ${ }^{3}$, G. Szabót, H.-U. Kauczor ${ }^{1} ;{ }^{1}$ Heidelberg/DE, ${ }^{2}$ Witten/DE, 3Vienna/AT (wolfram.stiller@med.uni-heidelberg.de)

Purpose: This study aimed to determine which combination of acquisition and iterative reconstruction parameters for low-dose MDCT at low tube voltages yields image quality and diagnostic confidence equivalent to current clinical standards in thoracic imaging

Methods and Materials: Thoracic images of eight sedated and mechanically ventilated pigs were acquired with MDCT during mid-inspiratory breath hold using standard (120 kVp, $180 \mathrm{mAs}$ ) and low-dose protocols at low tube voltages (100/80 $\mathrm{kVp}$ ) and current-time products (120/60/30 mAs). Using lung and soft-tissue kernels, images of all acquisitions were reconstructed with filtered backprojection (FBP); low-dose acquisitions were also reconstructed iteratively (levels 2 and 5, iDose, Philips Healthcare). Three independent blinded readers sorted the resulting $19 \mathrm{im}$ age data sets per animal according to diagnostic image quality (best to worst), and sorted images were statistically clustered. Additionally, CT numbers and noise have been determined in lung parenchyma, liver, muscle and aorta of all image data sets. Results: Interreader-agreement was moderate-high ( $\mathrm{k}=0.56-0.66)$. For any fixed set of acquisition parameters FBP-images were ranked lowest while those reconstructed iteratively (iDose5) were ranked highest independent of reconstruction kernel. The 19 image series could be clustered into 3 homogeneous clusters of equivalent diagnostic quality: Iteratively reconstructed images acquired at 100 $\mathrm{kVp} / 120$ mAs (iDose2/5), $100 \mathrm{kVp} / 60 \mathrm{mAs}$ (iDose5) or $80 \mathrm{kVp} / 120 \mathrm{mAs}$ (iDose5) were ranked equivalent to $\mathrm{FBP}$-images acquired using standard protocol. Iterative reconstruction did only affect CT numbers at very low dose $(80 \mathrm{kVp} / 30 \mathrm{mAs})$ while image noise could be reduced by up to $\sim 60 \%$.

Conclusion: Using low-kVp and low-mAs protocols combined with iterative image reconstruction, radiation exposure of thoracic MDCT could be reduced by $50-75 \%$.

Author Disclosures:

W. Stiller: Equipment Support Recipient; Philips Healthcare. Research/Grant Support; Philips Healthcare. H.-U. Kauczor: Advisory Board; Siemens Medical Solutions. Investigator; Boehringer Ingelheim. Speaker; Siemens Medical Solutions, Boehringer Ingelheim, Bayer, Bracco.

\section{B-0524 11:15}

Effect of acquisition parameters and reconstruction algorithm on lesion conspicuity in low-dose MDCT of the thorax

V. Yaghmai, H.G. Töre, M.P.F. Botelho, F.D. Gonzalez-Guindalini, S.K. Patel, K. Dill, C.B. Harmath; Chicago, IL/US (v-yaghmai@northwestern.edu)

Purpose: To evaluate lesion conspicuity in chest CT obtained with different tube voltage and current settings and reconstructed with iterative reconstruction (IR) and filtered back projection (FBP).

Methods and Materials: Torso phantom was scanned with 64-slice CT, 80 and $120 \mathrm{kVp}$ and a range of tube currents $(110,75,40,20$ and $10 \mathrm{mAs})$. Images were reconstructed with FBP and IR. Two blinded thoracic radiologists evaluated overall image quality: $1=$ excellent, $2=$ good, $3=$ adequate, $4=$ suboptimal, and $5=$ unacceptable. Conspicuity of ground glass opacity, ground glass nodule, soft tissue nodule, tree-in-bud nodules and emphysema was evaluated: $1=$ excellent, $2=$ good, $3=$ subtle lesion, 4=probably an artefact mimicking a lesion, $5=$ no discrimination from artefact. The lowest score was accepted as the consensus score. Image noise was measured with circular ROI. Effective dose (ED) for each acquisition was recorded. Results: Radiologists found $80 \mathrm{kVp} / 40 \mathrm{mAs}$ with IR as the lowest dose acquisition setting with adequate overall image quality (ED: $0.4 \mathrm{mSv}$ ). Objective noise was lower with IR ( $p<0.0001)$. The lowest dose acquisition settings with lesion conspicuity score of at least 4: $120 \mathrm{kVp} / 20 \mathrm{mAs}$ (ED:0.74mSv) with both FBP and IR for ground-glass opacity; $120 \mathrm{kVp} / 20 \mathrm{mAs}$ (ED: $0.74 \mathrm{mSv}$ ) with FBP vs $80 \mathrm{kVp} / 40 \mathrm{mAs}$ (ED:0.40m vv) with IR for ground-glass nodule; $80 \mathrm{kVp} / 20 \mathrm{mAs}$ (ED:0.21 mSv) with FBP vs $80 \mathrm{kVp} / 10 \mathrm{mAs}$ (ED:0.09mSv) with IR for soft tissue nodule; $80 \mathrm{kVp} / 110$ $\mathrm{mAs}$ (ED:1.13mSv) with FBP vs $120 \mathrm{kVp} / 40 \mathrm{mAs}$ (ED:1.49mSv) with IR for tree-inbud nodule; $80 \mathrm{kVp} / 110 \mathrm{mAs}$ (ED:1.13mSv) with both FBP and IR for emphysema. Conclusion: Lesion conspicuity is affected by $\mathrm{CT}$ acquisition settings. Iterative reconstruction may allow further dose reduction in implementation of low-dose CT protocols.

Author Disclosures:

H.G. Töre: Grant Recipient; Educational Grant Support by Siemens Healthcare. M.P.F. Botelho: Grant Recipient; Educational grant Support by Siemens Healthcare. F.D. Gonzalez-Guindalini: Grant Recipient; Educational Grant Support by Siemens Healthcare.

\section{B-0525 11:24}

Evaluation of an iterative reconstruction algorithm (iDose) to reduce image noise and improve image quality in submillisievert CT scans of the thorax

M. Henninger, A.A. Fingerle, C. Markus, T. Zahel, P.B. Noël, E.J. Rummeny, M. Dobritz; Munich/DE

Purpose: To evaluate objective and subjective image quality of submillisievert CT scans of the thorax reconstructed with an iterative reconstruction algorithm termed iDose.

Methods and Materials: 15 patients were prospectively included in this IRB approved study. Native CT scans were performed on a 256-MSCT (Brilliance iCT, Philips, Cleveland, $\mathrm{OH}$ ) with a tube voltage of $120 \mathrm{kV}$ and a tube current of 20 $\mathrm{mAs}$. The images were reconstructed with a sharp filter (YB, Philips) using standard filtered backprojection (FBP) and the iterative reconstruction algorithm iDose at a Level of 4 and 7 with a slice thickness of $0.6 \mathrm{~mm}$. Three thorax radiologists assessed images for overall image quality ( $1=$ excellent to $5=$ unacceptable), delineation of small anatomic details and abnormal findings ( $1=$ excellent demarcation $-6=$ not applicable), image noise (1=unacceptable image noise $-5=$ none) and artefacts (1=substantial artifacts $-4=$ none)

Results: Overall image quality was rated 4.5 for FBP, 3.3 for iDose Level 4 and 2.3 for iDose Level 7, anatomic details 4.6 for FBP, 3.3 for iDose Level 4 and 2.3 for iDose Level 7, abnormal findings 4.5 for FBP, 3.3 for iDose Level 4 and 2.2 for iDose Level 7, image noise 1.7 for FBP, 2.7 for iDose Level 4 and 3.7 for iDose Level 7 and artefacts 1.8 for FBP, 2.8 for iDose Level 4 and 3.3 for iDose Level 7 . Average radiation dose was $0.78 \pm 0.07 \mathrm{mSv}$.

Conclusion: The iDose reconstruction algorithm significantly improves image quality and reduces image noise and artefacts in submillisievert CT-scans of the thorax. In the future this may allow a further dose reduction in clinical day-to-day routine. 


\section{B-0526 11:33}

Phantom study for adaptive iterative dose reduction 3D (AIDR 3D) for low-dose chest CT examination: utility for identification of pulmonary ground glass opacity and nodule on 320-detector row CT using different scanning methods

S. Seki', H. Koyama ${ }^{1}$, Y. Ohno ${ }^{1}$, M. Nishio ${ }^{1}$, T. Yoshikawa ${ }^{1}$, S. Matsumoto', N. Sugihara' ${ }^{2}$ H. Inokawa ${ }^{2}$, K. Sugimura ${ }^{1} ;{ }^{1}$ Kobe/JP, ${ }^{2}$ Ohtawara/JP (klfsiena@yahoo.co.jp)

Purpose: To determine utility of adaptive iterative dose reduction using threedimensional processing (AIDR 3D) for pulmonary nodule identification on low-dose 320-detector-row CT using different scanning methods in phantom study.

Methods and Materials: A chest CT phantom including simulated pulmonary nodule was scanned on a 320-detector-row CT using non-helical wide volume scan (WVS) and helical scans as 64-detector-row CT (64HS) and 160-detector-row CT (160HS) at nine different tube currents. All data sets were reconstructed using AIDR 3D and filter back projection (FBP). To determine the capability of overall identification, two chest radiologists independently evaluated lesion conspicuities by five-point scoring system. At the each scanning method, gold standard was defined as the data set of standard tube current $(270 \mathrm{~mA})$ reconstructed by FBP. Interobserver agreements of detection and the capability for radiation dose reduction on each scan method were assessed by kappa statistics and ROC analyses.

Results: Interobserver agreements were almost perfect or substantial at all data sets $(K>0.60)$. When applied AIDR 3D, area under the curves of data sets equal to or higher than $30 \mathrm{~mA}$ on WVS, $40 \mathrm{~mA}$ on $64 \mathrm{HS}$, and $40 \mathrm{~mA}$ on $160 \mathrm{HS}$ had no significant difference with standard data sets $(p>0.05)$. On the other hand, when applied FBP, radiation dose reduction was limited in comparison with the data sets applied AIDR 3D at all scanning methods.

Conclusion: AIDR 3D was more useful for low-dose chest CT examination for nodule screening than FBP, and WVS can reduce more radiation dose than $64 \mathrm{HS}$ and $160 \mathrm{HS}$ on 320 -detector-row CT.

Author Disclosures:

N. Sugihara: Employee; Toshiba Medical Systems Corporations. H. Inokawa: Employee; Toshiba Medical Systems Corporations.

\section{B-0527 11:42}

The lateral scan projection radiograph (topogram) in CT pulmonary angiography: the effect on scan length and radiation dose

J.C.L. Rodrigues, I. Negus, N.E. Manghat, M.C.K. Hamilton; Bristol/UK

Purpose: To investigate the effect of a lateral topogram on excess scan length in CTPA and to quantify the impact on effective dose.

Methods and Materials: 50 CTPA studies planned with frontal topogram only (Protocol 1) were compared with 50 CTPA studies planned with frontal and lateral topograms (Protocol 2). Optimal scan length was defined from lung apex to lung base. Excess scan length beyond these landmarks was measured. Mean organ doses to thyroid, liver and stomach were estimated using standard conversion factors. Results: The mean excess scan length was significantly lower in Protocol 2 compared with Protocol $1(19.5 \pm 5.3 \mathrm{~mm}$ Vs $39.1 \pm 5.3 \mathrm{~mm}, \mathrm{P}<0.0001)$. The mean excess scan length below lung bases was significantly lower in Protocol 2 cohort compared with Protocol $1(7.5 \pm 4.2 \mathrm{~mm}$ Vs $23.0 \pm 4.2 \mathrm{~mm}, \mathrm{P}<0.0001)$, as were the mean organ doses to stomach $(4.24 \pm 0.26 \mathrm{mGy} V s 5.22 \pm 0.26 \mathrm{mGy}, \mathrm{P}<$ $0.0001)$ and liver $(5.60 \pm 0.21 \mathrm{mGy} V \mathrm{~s} 6.38 \pm 0.21 \mathrm{mGy}, \mathrm{P}<0.0001)$. A trend towards reduced over-scanning above the apices in Protocol 2 was observed compared with Protocol $1(12.0 \pm 3.2 \mathrm{~mm}$ Vs $16.2 \pm 3.2 \mathrm{~mm}, P=0.07)$, with a trend towards lower mean thyroid organ dose in ( $3.28 \pm 0.71 \mathrm{mGy} V s 4.11 \pm 0.71 \mathrm{mGy}, P=0.104)$. Conclusion: Incorporation of a lateral topogram into scan protocol in CTPA reduces excess scan length, significantly reducing dose to liver, stomach and lowers thyroid dose, without compromising image quality. This simple dose saving technique can be applied to all CT investigations of the chest on all scanners with immediate effect.

\section{B-0528 11:51}

Accuracy of lung nodule volumetry using adaptive iterative dose reduction 3D for ultra-low dose of 320-detector row CT: a phantom study K. Doo' ${ }^{1}$, E.-Y. Kang', H. Yong', O. Woo', K. Lee ${ }^{2}$, Y.-W. Oh'; ${ }^{1}{ }^{1}$ Seoul/KR, ${ }^{2}$ Ansan/KR (kwdoo@korea.ac.kr)

Purpose: Volumetric measurement of a nodule is more sensitive in the detection of tumour growth than axial diameter measurement. The purpose of this study was to assess the accuracy of lung nodule volumetry using adaptive iterative dose reduction (AIDR) 3D in comparison with filtered back-projection (FBP) in an anthropomorphic thoracic phantom on 320-detector row CT.
Methods and Materials: Ten artificial spherical nodules (diameter: 3, 5, 8, 10 and $12 \mathrm{~mm}$; CT densities: -630 and $+100 \mathrm{HU}$ ) were randomly placed inside a thoracic phantom. The phantom was scanned with low $(50 \mathrm{mAs})$ and ultra-low dose (10 mAs). They were reconstructed with FBP and AIDR 3D. Nodule volumes were estimated by a semi-automated software, which were compared with a reference standard by calculating the absolute percentage error (APE).

Results: There was no significant difference between FBP and AIDR for all nodules in low dose and for nodules of $25 \mathrm{~mm}$ in ultra-low dose. Under ultra-low dose for nodules of $38 \mathrm{~mm}$, AIDR showed significantly lower APE than FBP $(10.9 \pm 5.3 \%$ vs $16 \pm 7.8 \%, P<0.05)$ in non-solid $(-630 \mathrm{HU})$ nodules. However, in solid $(+100 \mathrm{HU})$ nodules, AIDR showed higher APE than FBP $(4.2 \pm 1.8 \%$ vs $3.3 \pm 1.5 \%, P<0.05)$. APE on ultra-low dose with AIDR was comparable with low dose with FBP $(6.4 \pm 3.1 \%)$ for non-solid nodules of ${ }^{3} 8 \mathrm{~mm}$.

Conclusion: AIDR 3D reconstruction improves the accuracy of volumetry for non-solid nodules in ultra-low dose CT, but not for solid nodules and nodules of 25 $\mathrm{mm}$. For accurate volumetry of a lung nodule, it is critical to apply reconstruction algorithm and tube current appropriate for the size and density.

$10: 30-12: 00$

Room D2

Interventional Radiology

SS 909

\section{Thoracic interventions}

Moderators:

J. Tacke; Passau/DE

K. Zelenak; Martin/SK

\section{B-0529 10:30}

CT-guided lung biopsy: core needle coaxial technique with autologous blood needle tract embolisation compared with fine needle aspiration in diagnostic yield and post-biopsy pneumothorax rate S. Lee ${ }^{1}$, A. Montaser ${ }^{2}$, L. Nardo', U.R. Heilmeier'1, B.A. Aronson'2. ${ }^{1}$ San Francisco, CA/US, ${ }^{2}$ Canton, OH/US (Sonia.Lee@ucsf.edu)

Purpose: To compare two different lung biopsy techniques in diagnostic yield and post-biopsy pneumothorax rate: CT-guided 18-gauge core needle coaxial technique (CNCT) with autologous blood needle tract embolisation (ABNTE) versus fine needle aspiration (FNA).

Method and Materials: CT-guided lung biopsies using 18-gauge CNCT with ABNTE were performed on 147 patients (79 male and 68 female, age ranging from 31 to 87 . The diagnostic yield was compared with 22-gauge fine needle aspiration lung biopsy performed on 145 patients by reviewing cytology and pathology report results. Hypocelluar specimen or non-diagnostic cell morphology was considered non-diagnostic results. Presence of pneumothorax was determined on CT or follow-up radiograph for CNCT with ABNTE group and compared with FNA pneumothorax rate statistics based on 140 cases. Pearson's chi-square test, Spearman rank correlation test (SRC) and Kendall's tau (KT) were used to compare the two biopsy techniques. Differences of pneumothorax rate were compared using oneway analysis of variance (ANOVA) and $t$ tests.

Results: Non-diagnostic result was seen in $3.9 \%$ of CNCT with ABNTE group compared with $20.7 \%$ of FNA ( $p<0.001$ ). $72.8 \%$ of CNCT and $69.3 \%$ of FNA groups were diagnosed with malignancy. No significant difference was seen in pneumothorax rate. $20.4 \%$ of the CNCT with ABNTE developed pneumothorax, $7.4 \%$ of which needed chest tube. For FNA biopsies, $18.6 \%$ had pneumothorax and $8.6 \%$ needed chest tube.

Conclusion: CT-guided lung 18-gauge CNCT with ABNTE is comparable to FNA method in post-procedure pneumothorax rate and superior in diagnostic yield.

\section{B-0530 10:39}

Computed tomography-guided lung biopsy with the patient in lateral position and the biopsy side down: effect on pneumothorax rate and clinical significance

G. Velonakis, D. Filippiadis, A. Mazioti, S.I. Argentos, K. Malagari, A. Kelekis, N. Kelekis; Athens/GR

Purpose: The purpose of the study was to assess pneumothorax rate and clinical significance in CT-guided lung biopsies with the patient in lateral position and the biopsy side down.

Methods and Materials: We retrospectively reviewed 214 percutaneous lung biopsies performed under $C T$ guidance during the past 40 months. In 173 biopsies, patients were placed in prone or supine position (Group A). In 41 biopsies, patients 
were placed in lateral position with the biopsy side down (Group B). Statistical analysis was performed comparing pneumothorax rate between the two groups. The rate of pneumothorax requiring intervention was also assessed. The effect of other variables (patients demographic characteristics, lesion characteristics, lesion distance from the skin and biopsy technique) was evaluated in both groups. Results: 27 pneumothorax occurred in Group A (15.61\%) versus 1 (2.44\%) in group $B(p=0.025)$. Pneumothorax requiring treatment was noticed in 10 cases $(5.78 \%)$ in Group A, versus $0(0 \%)$ in Group B. Emphysema and lesion size were independent risk factors for pneumothorax in Group A. Emphysema was also independent risk factor for pneumothorax necessitating a drainage catheter insertion in Group A. No independent risk factor was recognised for pneumothorax or pneumothorax requiring intervention in Group.

Conclusion: CT-guided percutaneous lung biopsies in lateral position with the biopsy side down are associated with reduced rate of pneumothorax and pneumothorax requiring treatment comparing to biopsies in other positions. Lateral position with the biopsy side down reduces also the impact of known risk factors for pneumothorax.

\section{B-0531 10:48}

C-arm cone-beam computed tomography (CBCT) needle path

overlay for percutaneous biopsy of pulmonary nodules

C. Floridi, F. Fontana, A. lerardi, G. DeMarchi, L. Nocchi-Cardim, C. Pellegrino,

G. Carrafiello, C. Fugazzola; Varese/IT (chiarafloridi@libero.it)

Purpose: The aim of this study was to evaluate the feasibility of percutaneous transthoracic needle biopsy of pulmonary nodules under XperGuide CBCT navigation guidance.

Methods and Materials: Between February 2010 to July 2011, we evaluated 70 patients with a pulmonary lesion. All patients had a diagnostic conventional chest CT and after underwent percutaneous transthoracic needle biopsies of pulmonary nodules under XperGuide CBCT navigation guidance. The mean size of the lesion was on average $5.5 \mathrm{~cm}$. Immediately after FNAB an additional CBCT was performed to evaluate any complications; moreover, a follow-up expiratory chest radiograph was obtained. Calculation of radiation dose of the procedure and statistical analysis were performed.

Results: Technical success rate was achieved in $100 \%$ of cases. Forty-six lesions were diagnosed as malignant, 20 were benign, and 4 were indeterminate. All malignancies diagnosed by FNAB were confirmed by history and imaging. The overall accuracy, sensitivity, specificity, positive predictive value and negative predictive value were $94.2 \%, 92 \%, 100 \%, 100 \%$ and $92 \%$, respectively. In the 34 patients with a lesion equal or less to $3 \mathrm{~cm}$ undergoing XperCT-guided lung biopsy the average effective dose was $11.14 \mathrm{mSV}$ (range 1.39-27.29 mSv), while in the 36 patients with lesions larger than $3 \mathrm{~cm}$ the average effective dose was $12.10 \mathrm{mSv}$ (range 2.89-49.46 mSv). The pneumothoraces rate in our study was $22.8 \%$ (16/70). Conclusion: Our study reports the diagnostic accuracy, safety of CBCT-navigation for percutaneous trans-thoracic lung biopsy which combines CT-like images for target and path location and fluoroscopy for real-time needle navigation.

\section{B-0532 10:57}

Computed tomography guided trans-thoracic fine needle aspiration biopsy of pulmonary nodule: pulmonary haemorrhage, complication or advantage?

M. De Filippo, L. Saba, G. Concari, C. Rossi; Parma/IT

Purpose: Evaluation of the incidence and the clinical significance of pulmonary haemorrhage that may occur during the computed tomography (CT)-guided transthoracic fine needle aspiration (TTFNA).

Methods and Materials: From January 2007 to September 2011 we retrospectively reviewed the CT images without contrast of 538 patients of both sexes ( $364 \mathrm{M}$ and $174 \mathrm{~F}$, average age 70 years, age range $36-90$ ) used for the guidance of CT-guided TTFNA of pulmonary nodules with suspected primitive and/or secondary neoplasm. The variables analysed were: distance between the nodule and the pleural surface, presence of emphysema, pulmonary haemorrhage (Type 1: along the needle track; Type 2: perilesional, Grade 1: $<6 \mathrm{~mm}$, Grade2: $6<x<12 \mathrm{~mm}$, Grade 3: $>12 \mathrm{~mm}$ ), presence of pneumothorax.

Results: For lesions distant more than $3 \mathrm{~cm}$ from the pleural surface, in the presence of type 1 and high Grade ( $>6 \mathrm{~mm}$ ) of pulmonary haemorrhage, pneumothorax occurred in 12 out of 62 cases (26\%); in the absence of pulmonary haemorrhage, pneumothorax occurred in 30 out of 42 cases $(71.4 \%)$. For lesions distant less than $3 \mathrm{~cm}$ from the pleural surface, in the presence of type 1 and high Grade $(>6 \mathrm{~mm}$ ) of pulmonary haemorrhage, pneumothorax has never occurred $(0 \%)$. Emphysema was present in 192 cases (35.7\%), pulmonary haemorrhage in 144 cases $(26.8 \%)$, pneumothorax in 154 cases $(28.6 \%)$.
Conclusion: Pulmonary haemorrhage during fine-needle lung biopsy is a frequent event. It protects against pneumothorax in patients with pre-existing comorbidities, especially for long needle trajectory, for bleeding greater than $6 \mathrm{~mm}$ developing along the needle track.

\section{B-0533 11:06}

Feasibility and safety of CT-guided percutaneous radiofrequency, microwave or cryoablation of the pulmonary and mediastinal unresectable tumours adjacent to the heart and large vessels C. Pusceddu, L. Melis, G. Meloni; Cagliari/IT (clapusceddu@gmail.com)

Purpose: To verify the feasibility and safety of radiofrequency ablation (RFA), microwave ablation (MWA) and cryoablation (CRA) under CT guidance in the treatment of cancer adjacent to the heart and large vessels.

Methods and Materials: 27 patients (mean age of 64 years) with unresectable primary and secondary pulmonary and mediastinal tumours (12 NSCLC, 1 invasive thymoma, 1 mesothelial sarcoma and 13 metastases) were treated by CT-guided RFA ( 14 cases), MWA (7 cases) and CRA (6 cases). All the tumours were in contact or distant from the large vessel wall or the pericardium less than $1 \mathrm{~cm}$. All ablations were performed under conscious sedation and local anaesthesia. Vital signs of the patients were non-invasively monitored continuously. The therapeutic outcomes were evaluated by contrast-enhanced CT after 1 month. In two patients, an electrode is penetrated in the pericardium without any consequences.

Results: In all cases, the procedure was technically successful. No intraprocedural arrhythmia occurred. Morbidity consisted of 7 cases of pneumothorax, 3 cases of pleural effusion and 1 case of asymptomatic thickening of the pericardium. In the CT control after 1 month, there was a complete necrosis in 22 cases and a partial (from 70 to $90 \%$ ) necrosis in the remaining 5 cases.

Conclusion: Indifferently from the energy source used to obtain the tumour necrosis, the treatment of the pulmonary and mediastinal unresectable tumours adjacent to the heart and large vessels is feasible and safe because the blood flow cause a "heat-sink" effect adjacent to such structures that prevent undesired thermal damage.

\section{B-0534 11:15}

Factors influencing local tumour control in patients with neoplastic pulmonary nodules treated with microwave ablation (MWA): a risk factor analysis

T.J. Vogl, T.S. Worst, N.N.N. Naguib, N.-E.A. Nour-Eldin;

Frankfurt a. Main/DE (t.vogl@em.uni-frankfurt.de)

Purpose: To evaluate risk factors predicting local tumour control after microwave ablation (MWA) of primary and secondary lung malignancies $23 \mathrm{~cm}$ in maximum diameter

Methods and Materials: In this retrospective study, 91 index tumours (ITs) in 57 patients were treated with single antenna MWA. Time-to-local-tumour progression was monitored using CT over a median follow-up of 10.2 months \pm 6.2 (6.0-29.2 months). An overall estimated time-to-local-tumour progression was performed via Cox regression model. Factors hypothesized to correlate with ablation response included: tumour diameter ( $<>15.5 \mathrm{~mm}$ ), tumour shape (round/oval vs. irregular), clear vs. ill-defined tumour margin, adjacency to the pleura, adjacency to bronchi, vessels of ${ }^{3} 3 \mathrm{~mm}$ in diameter located at a maximum of $5 \mathrm{~mm}$ from the IT, energy applied to IT $\left(<>26.7 \mathrm{~J} / \mathrm{mm}^{3}\right)$ and occurrence of cavernous formations after ablation. A logistic regression model was used to correlate the data.

Results: Local tumour progression occurred in 30/91 (33\%) ITs, seen in 21/57 $(36.8 \%)$ patients. Mean time-to-local tumor progression was 8.3 months $( \pm 5.5$, range 2.1-25.2) (median: 22.6 months \pm 12.4 months). Risk factors significantly correlating with local tumour progression were $>15.5 \mathrm{~mm}(\mathrm{p}<0.01)$ in diameter, irregular IT shape $(p<0.01)$, pleural contact $(p=0.02)$ and $<26.7 \mathrm{~J} / \mathrm{mm}^{3}$ applied to the IT $(p<0.001)$. IT shape $(p=0.03)$ and energy deployed per IT volume $(p=0.001)$ were found to be independent risk factors after regression analysis. Tumour margin definition $(p=0.06)$, cavernous formations $(p=0.19)$, juxtatumoral vessels $(p=0.08)$ and bronchi $(p=0.89)$ did not impact upon tumour progression post-ablation. Conclusion: Independent predicting factors for local tumour progression in primary and secondary lung neoplasms ${ }^{2} 3 \mathrm{~cm}$ in diameter were irregular IT shape and $<$ $26.7 \mathrm{~J} / \mathrm{mm}^{3}$ per IT volume. 


\section{B-0535 11:24}

Percutaneous microwave ablation of lung tumours

C. Pusceddu, L. Melis, G. Meloni; Cagliari/IT (clapusceddu@gmail.com)

Purpose: To assess the feasibility and safety of percutaneous CT-guided lung tumour microwave ablation.

Methods and Materials: 28 consecutive patients (18 men, 10 women, mean age 62 years) underwent CT-guided percutaneous microwave ablation (MWA) of unresectable lung tumours (11 primary NSCLC and 17 metastases) mean size $3.2 \mathrm{~cm}(2.5-8 \mathrm{~cm})$. The study cohort was selected according to the following criteria: 1) maximum tumour size less than $9 \mathrm{~cm}$ in diameter, 2) less than 5 metastatic tumors, 3) patients with a normal coagulation status and 4) provision of written informed consent. All procedures were performed with $\mathrm{CT}$ guidance under conscious sedation and local anesthesia. One or two straight microwave antenna (14 or 16-gauge) were placed directly into the tumour for 8-12 minutes. Follow-up included contrast-enhanced CT at 1, 3, 6 months and then at 6-month intervals; stable or reduction size and the absence of tumour enhancement CT images were considered indicative of complete tumour necrosis.

Results: In all cases, the procedural was technically successful. Morbidity consisted of 8 cases of partial pneumothorax $(28.6 \%)$ which were resolved spontaneously within 7 days. At a mean follow-up of 8 months (range 2-15), we recorded a $67.8 \%$ of complete response (tumour necrosis $=100 \%$ ) and a $32.2 \%$ of partial response (tumour necrosis range 65\%-92\%).

Conclusion: Our preliminary results show that percutaneous CT-guided microwave ablation is feasible and safe for the treatment of primary and secondary lung tumour. Further follow-up and a prospective controlled trial is necessary to validate the procedure.

\section{B-0536 11:33}

MRI chest parameters used in the assessment of tumour response post microwave ablation of pulmonary metastases

N.-E.A. Nour-Eldin, N.N.N. Naguib, T. Gruber-Rouh, T. Lehnert, M. Sadegi,

T.J. Vogl; Frankfurt a. Main/DE (nour410@hotmail.com)

Purpose: To determine the value contrast enhanced (CE-MRI) follow-up in the assessment of tumour response of microwave (MW) ablated pulmonary metastases by correlating the results with CE-CT.

Methods and Materials: This prospective study included 130 ablation sessions for pulmonary metastases in 80 patients. CE-MRI Chest scanning was performed 1 week before the ablation and at 24hours, 3, 6, 9 and 12 months post ablation. Thin section CT Volumetric measurement of the lesions was performed at the same time periods as a second parameter for comparison. The lesion MRI enhancement intensity in each study was estimated, and the ratio to the paraspinal muscle enhancement intensity at the same level was measured (Lesion Muscle Signal (LMS ratio). The correlations between post ablation follow-up CT volume of tumours and CE-MRI LMS ratio at the follow-up periods were assessed.

Results: The preablation tumour volumes ranged between 0.30 and $6.1 \mathrm{~cm}$ (mean: $\left.1.5 \mathrm{~cm}^{3}, \mathrm{SD}: 1.3\right)$. LMS ratio 1 was noted in preablation due to high contrast enhancement of the tumour and in 24-h post ablation due to the inflammatory response associated with the thermal ablation and due to tumour residue or progress. Weak correlation was detected between the LMS-ratios and CT-volumetric changes in 24-h post ablation. Strong correlation between the LMS ratios was estimated between the follow-up periods of 3 months (SpearmanR:0.62, $p=0.0021$ ), 6 months (SpearmanR:0.66, $p=0.001$ ), 9 months (SpearmanR:0.61, $p<0.001$ ) and 12 months (Spearman R:0.7, $\mathrm{p}<0.00001$ ).

Conclusion: CE-MRI follow-up of the MW ablated lung tumours can be used effectively to assess the tumour response to ablation using LMS ratio as a parameter of assessment.

\section{B-0537 11:42}

Transpulmonary chemoembolisation (TPCE) and transpulmonary chemoperfusion (TPCP) as a treatment for unresectable primary and secondary lung tumours: local tumour control and survival

T.J. Vogl, A. Müller, S. Zangos, T. Lehnert;

Frankfurt a. Main/DE (t.vogl@em.uni-frankfurt.de)

Purpose: To evaluate the effect of transpulmonary chemoembolisation (TPCE) and transpulmonary chemoperfusion (TPCP) in unresectable primary lung tumours and lung metastases on progression-free survival and overall survival depending on the indication (palliative, symptomatic, neoadjuvant).

Methods and Materials: In a retrospective study from 12/2006 to 10/2011, 119 patients (51 men (43\%), 68 women (57\%)) mean age 57 years with unresectable lung metastases (colorectal carcinoma: $n=35(29.41 \%)$, breast cancer: $n=11$
(9.24\%), other primaries: $n=48(40.34 \%))$ and primary lung tumours $(n=25(21.01 \%))$ were treated with repetitive (3-19) TPCE or TPCP. Response since initial treatment with TPCE and TPCP are evaluated according to the RECIST and WHO criteria. Results: The therapy was well tolerated without severe complications. The overall tumour response according to RECIST was partial response (PR) in $3.4 \%(n=4)$, stable disease (SD) in $54.6 \%(n=65)$ and progressive disease (PD) in $42.0 \%(n=50)$. After WHO the overall tumour response was PR in $3.36 \%(n=4)$, SD in $51.26 \%$ $(n=61)$ and $P D$ in $45.38 \%(n=54)$. Overall survival rate since initial intervention was 517 days (median), 590.4 days (mean) (range, 80-1742 days), the survival rate of the patients with palliative indication was 556 days (median), 614.3 days (mean) (range, 122-1742 days), of those with symptomatic indication 202 days (median), 206.5 days (mean) (range, 80-397 days) and of those with neoadjuvant indication 595 days (median), 717.4 days (mean) (273-1647 days).

Conclusion: TPCE and TPCP are effective therapy options for treatment of unresectable lung tumours. TPCE proved superior in lung metastases, TPCP superior in primary lung cancer.

\section{B-0538 11:51}

Endovascular treatment of haemoptysis of pulmonary artery origin: a single-centre experience

A. Khalil, S. Haddad, M. Tassart, A. Parrot, M.-F. Carette; Paris/FR

(antoine_khalil@yahoo.fr)

Purpose: This study aimed at reviewing the results and the safety of embolisation in patients affected by haemoptysis of pulmonary arterial (PA) origin.

Methods and Materials: All consecutively patients treated for haemoptysis of PA origin during 12 years (1999 to 2011) were reviewed. Age, underlying disease, and haemoptysis volume were recorded. CT-scan and pulmonary angiography of each patient were reviewed for the description of the PA lesion (aneurysm, false aneurysm, irregularity of the PA or abrupt interruption) and the material of embolisation used for the treatment.

Results: Forty-three patients (10 women, 33 men; mean age: 53 years) were treated during this period. Aetiologies were tuberculosis $(n=13)$, lung cancer $(n=8)$, necrotizing pneumonitis $(n=8)$, aspergillosis $(n=5)$, Behcet disease $(n=5)$ and others $(n=4)$. Five abnormalities of PA were found: pseudo aneurysm of the PA (PAPA) ( $n=28)$, aneurysm of the PA (APA) $(n=5)$, irregularity of PA $(n=4)$, early interruption of $P A(n=3)$, one PAVM, and no abnormalities in 2 patients. Materials used for embolisation were coils $(n=25)$, Onyx $(n=14)$ and covered stent $(n=2)$. Two early recurrences, 5 short-term recurrences, and one long term recurrence after embolisation were recorded. They were treated by surgery $(n=4)$ or by embolisation $(n=4)$. [[Unable to Display Character: \&\#8232;]]Three patients died during the hospitalisation, one of them by haemoptysis recurrence.

Conclusion: Embolisation is a safe and an effective method for the treatment of severe haemoptysis of PA origin.

\section{$10: 30-12: 00$} Room E1

\section{Musculoskeletal}

\section{SS 910}

\section{Spine: advancing the use of CT and MRI}

Moderators:

P.M. Cunningham; Navan/IE

J. Labuscagne; Bunbury, WA/AU

\section{B-0539 10:30}

Cervical nerve root infiltration under CT and MRI guidance: a cost comparison

M.H. Maurer, M. deBucourt, T. Hartwig, B. Hamm, F. Streitparth; Berlin/DE (martin.maurer@charite.de)

Purpose: To compare the costs of CT-guided and MR-guided cervical nerve root infiltration for minimally invasive treatment of cervical radicular pain.

Methods and Materials: From September 2009 until April 2012, twenty-two patients (9 men, 13 women; mean age: 48 years) underwent MR-guided single-site periradicular cervical nerve root infiltration with $40 \mathrm{mg}$ triamcinolon acetonide. A further 64 patients (34 men, 30 women; mean age: 50 years) were treated under CT-fluoroscopic guidance. Prorated costs of equipment use (purchase, depreciation, and maintenance) and expenditure for disposables were identified for MR- and CTguided procedures. For CT-guided interventions the effective dose (in millisievert, $\mathrm{mSV}$ ) was calculated based on the dose length product. 
Results: Mean intervention time was 24.9 min (range, 12-36 min) for MR-guided and $19.6 \mathrm{~min}$ (range, 5-54 $\mathrm{min}$ ) for CT-guided treatment. The average total costs per patient were EUR 197 for MR-guided and EUR 98 for CT-guided interventions. These were (MR/CT guidance) EUR 98/32 for equipment use, EUR 47/41 for staff, and EUR 52/25 for disposables. The mean effective dose for CT-guided interventions was $0.48 \pm 0.51 \mathrm{mSv}$.

Conclusion: Cervical nerve root infiltration using MRI guidance is still about twice as expensive as infiltration using CT guidance. However, not involving radiation exposure for patients and personnel, MR-guided nerve root infiltration may become a promising alternative to the $\mathrm{CT}$-guided procedure, especially as a further decrease in prices is expected for MRI devices and MR-compatible disposables.

\section{B-0540 10:39}

Fast inner volume imaging of the lumbar spine with a zoomed SPACE sequence using parallel transmit

P. Riffel' ${ }^{1}$ S. Kannengiesser ${ }^{2}$, D. Paul2 ${ }^{2}$, S. O. Schönberg ${ }^{1}$, H. Michaely'; $^{1}$ Mannheim/DE, ${ }^{2}$ Erlangen/DE (philipp.riffel@umm.de)

Purpose: Inner volume parallel transmit imaging (Zoom) allows reduction of scan time while maintaining spatial resolution in the targeted $\mathrm{ROI}$. Aliasing artefacts from body regions outside the reduced FOV can be suppressed via local excitation with the use of multidimensional RF pulses. The purpose of this study was to evaluate the feasibility and technical quality of a zoomed SPACE (Sampling Perfection with Application optimised Contrasts using different flip-angle Evolutions) sequence of the lumbar spine.

Methods and Materials: In this IRB-approved study 9 volunteers underwent an MRI exam of the lumbar spine including a conventional 3D T2w SPACE sequence with Generalized Autocalibrating Partially Parallel Acquisitions parallel imaging (GRAPPA) (acceleration factor: 2) and zoomed 3D T2w SPACE sequence with a reduced FOV (reduction factor: 2). Images were evaluated with regard to image sharpness, homogeneity and artefacts. For qualitative analysis SNR values were detected.

Results: Image sharpness of anatomical structures was significantly better with zoomed SPACE $(p<0.0001)$. Image homogeneity was significantly better with conventional SPACE $(p=0.0003)$. There was no significant difference regarding artefacts. Acquisition times were $8.19 \mathrm{~s}$ for conventional SPACE and $6.3 \mathrm{~s}$ for zoomed SPACE, respectively. In 9 of 18 cases (9 volunteers/2 readers), zoomed SPACE was selected as the preferred sequence. In 5 of 18 cases conventional SPACE was selected as the preferred sequence. SNR values showed no significant difference. Conclusion: Using parallel transmit zoomed SPACE images of the lumbar spine can be acquired about $25 \%$ faster with an increased sharpness and comparable SNR values.

\section{B-0541 10:48}

The importance of ispection of true FISP sequences during MRI of the lumbar spine

M. Papavasilopoulou, M. Raissaki, C. Voulgaris, A. Karantanas; Iraklion/GR (mariapapavasilopoulou@gmail.com)

Purpose: True FISP sequence is routinely applied as scout during lumbar spine MRI (LS MRI) in our institution. We investigated whether this sequence contributes in the diagnostic performance of LS MRI or in depicting other incidental disorders. Methods and Materials: 272 consecutive patients, $79 \%$ of whom aged between 40 and 85 years old, underwent LS MRI accordingly: true FISP fat saturation in coronal plane, T1w, T2w, STIR in sagittal plane and T2 sequences in axial plane. Two readers by consensus recorded abnormalities exclusively or better depicted at True FISP images and characterised them as potentially important, either clinically and /or radiologically.

Results: True Fisp images showed abnormalities in 240 patients (88\%) and pathology not visualised elsewhere in $98(36 \%)$. True FISP increased conspicuity of or revealed spinal, sacroiliac or paravertebral lesions including scoliosis assessment in 72 patients $(26.5 \%)$. Scoliosis was noted in 62 patients $(22.8 \%)$, was more accurately characterised and/or was depicted only on coronal views in 52 . Other abnormalities included 111 renal lesions in 110 patients (36.7\%), solid abdominal organ lesions/organomegaly in $37(13.6 \%)$, chest abnormalities in $13(4.8 \%)$ and genital in $14(5.15 \%)$. Out of the above, there were 27 lesions $(9.9 \%)$ with direct impact on patient management, 113 lesions $(41 \%)$ that could potentially affect patient health in the future if reported and 106 incidentalomas (38.9\% of True FISP), with equivocal clinical importance.

Conclusion: Careful inspection of TRUE FISP sequence during LS MRI may result in depiction of additional abnormalities that can potentially alter patient management and may increase diagnostic confidence.

\section{B-0542 10:57}

Computed tomography of the cervical spine: comparison of image quality between standard-dose filtered back-projection and low-dose iterative reconstruction protocols

F. Becce ${ }^{1}$, Y. Ben Salah², F.R. Verdun', B.C. Vande Berg' ${ }^{2}$, F.E. Lecouvet ${ }^{2}$, R. Meuli', P. Omoumi'; ; ${ }^{2}$ Lausanne/CH, ${ }^{2}$ Brussels/BE (fabio.becce@chuv.ch)

Purpose: To compare image quality between standard-dose filtered back-projection (FBP) and low-dose iterative reconstruction (IR) protocols in computed tomography (CT) of the cervical spine.

Methods and Materials: Forty patients investigated by CT of the cervical spine for chronic neck pain and/or cervical radiculopathy were prospectively randomised into two groups: standard-dose CT (120 kVp, $275 \mathrm{mAs}$ ) with FBP, or low-dose CT $(120 \mathrm{kVp}, 150 \mathrm{mAs})$ with IR. Image noise, signal-to-noise (SNR) and contrast-tonoise (CNR) ratios were measured. Two radiologists, blinded to CT parameters, independently assessed the following anatomical structures at C3-C4 and C6-C7 levels, using a four-point scale: intervertebral disc, content of neural foramina and dural sac, ligaments, soft tissues and vertebra. They subsequently rated overall image quality using a ten-point scale. Volume CT dose index (CTDIvol) and doselength product (DLP) were recorded.

Results: No significant difference in noise $(\Delta=0 \mathrm{HU})$, SNR $(\Delta=0.1)$ and CNR $(\Delta=-0.5)$ was noted between standard-dose FBP and low-dose IR protocols (all $\mathrm{p}^{3} 0.39$ ). With low-dose IR, semi-quantitative scores were significantly higher for the intervertebral discs, neural foramina and ligaments (all $\mathrm{p}^{2} 0.05$ ), comparable for the dural sac (all $p^{3} 0.24$ ), and significantly lower for the soft tissues and vertebrae (all $\mathrm{p}^{2} 0.02$ ), at both disc levels. Overall image quality scores were significantly higher with low-dose IR (all $\mathrm{p}<0.01)$. Radiation exposure $(\Delta=8.1 \mathrm{mGy}$ and $99.2 \mathrm{mGy} \cdot \mathrm{cm})$ was significantly lower with low-dose IR (all $p<0.01$ ).

Conclusion: Low-dose CT of the cervical spine with IR provides better image quality for the intervertebral discs, neural foramina and ligaments compared to standarddose CT with FBP, while reducing radiation exposure by approximately $40 \%$.

\section{B-0543 11:06}

Imaging quality of reduced radiation dose lumbar spine CT with iterative reconstruction

C.-J. Lin, S. Hung, W. Guo, T. Wu; Taipei/TW (bcjlin@gmail.com)

Purpose: The purpose of our study was to investigate the diagnostic accuracy of CT scans of the lumbar spine (CTLS) acquired with lower mAs and kilovoltage reconstructed with an IR algorithm.

Methods and Materials: We prospectively recruited 120 patients for CTLS. Patients were classified into three groups. The radiation dose reduction was set to $50 \%$ of the original estimated dose with alternative tube currents and $120 \mathrm{KV}$ in group $\mathrm{A}$, and $100 \mathrm{KV}$ in group $\mathrm{B}$. Images were reconstructed with iDOSE ${ }^{4}$ level 4 reconstruction algorithm. For group $\mathrm{C}$, the standard $\mathrm{mAs}$ and $120 \mathrm{KV}$ with filtered back projection (FBP) algorithm was employed. The SNR of the intervertebral disc (IVD), dural sac (DS) and psoas muscle (PM), and the CNR of the IVD and DS were compared across groups. Two radiologists independently rated the quality of each reduced-dose image as either equivalent (' 1 ') or inferior (' 0 ') to the rater's subjective 'standard' CTLS.

Results: There were decreased SNRs of all ROIs in group A but only DS showed statistical significance. There were significant decreased SNRs in IVD, DS, and PS in group B. There was a weak correlation between SNR and BMI in all 3 groups. The CNRs for groups A, B and C were $0.08+0.05,0.15+0.09$ and $0.12+0.06$, respectively. The mean image quality scores for groups $\mathrm{A}, \mathrm{B}$ and $\mathrm{C}$ were 0.63 , 0.83 , and 0.87 , respectively.

Conclusion: With the aid of the IR algorithm, it is feasible to provide diagnostic CTSL imaging with a $50 \%$ radiation reduction using lower $\mathrm{mAs}$.

\section{B-0544 11:15}

Multidetector computed tomography of the cervical spine: optimisation of iterative reconstruction strength levels P. Omoumi', F.R. Verdun'², Y. Ben Salah', B.C. Vande Berg', F.E. Lecouvet ${ }^{1}$, R. Meuli ${ }^{2}$, F. Becce ${ }^{2} ;{ }^{1}$ Brussels/BE, ${ }^{2}$ Lausanne/CH (patrick.omoumi@uclouvain.be)

Purpose: To determine the optimal strength level of iterative reconstructions (IRs) in multidetector computed tomography (MDCT) of the cervical spine.

Methods and Materials: Twenty patients ( 14 women, mean age 54.5 years) investigated by cervical spine MDCT for refractory cervical pain and/or cervicobrachial neuralgia were prospectively studied. MDCT raw data were reconstructed using both filtered back-projection and IR (sinogram-affirmed iterative reconstruction; strength levels 1 to 5) techniques. The image noise, signal-to-noise (SNR) and 
contrast-to-noise (CNR) ratios were measured. Two radiologists independently and blindly evaluated the following anatomical structures at both $\mathrm{C} 3-\mathrm{C} 4$ and $\mathrm{C} 6-\mathrm{C} 7$ levels, using a four-point scale: intervertebral discs, content of neural foramina and dural sac, ligaments, soft tissues and vertebrae. They ultimately rated the overall diagnostic image quality using a ten-point scale.

Results: As the strength level of IRs increased, the image noise decreased linearly, while the SNR and CNR both increased linearly (all $p^{2} 0.001$ ). For the intervertebral discs, the content of neural foramina and dural sac, and for the ligaments, the subjective image quality scores increased linearly with increasing IR strength levels (all $\mathrm{p}^{2}$ 0.03). In contrast, for the soft tissues and vertebrae, the image quality scores decreased linearly with increasing strength level of IRs (all $p<0.001$ ). Finally, the overall diagnostic image quality scores increased linearly with increasing IR strength levels (both $\mathrm{p}<0.001$ ).

Conclusion: The optimal strength level of IRs in cervical spine MDCT depends on the anatomical structure to be analysed. For the intervertebral discs and the content of neural foramina, high IR strength levels are recommended.

\section{B-0545 11:24}

Relationship among facet joint fluid (FJF) at MRI, age, and disk degeneration in patients affected with low back pain

C. Ottonello ${ }^{1}$, A. Giardino' ${ }^{2}$, G.D.E. Papini³, A. Sharipova ${ }^{3}$, G. Di Leo ${ }^{3}$,

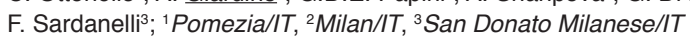

Purpose: To estimate the relationship among FJF at MRI, age, and disk degeneration in patients affected with low back pain.

Methods and Materials: Ninety-six consecutive patients (M/F 48/48, aged 49 \pm 15 years) affected with low back pain underwent MRI of lumbar spine using sagittal T1- and T2-weighted fast spin-echo and axial T2*-weighted gradient-echo sequences. For each level from L3 to S1, disk degeneration was graded using the Thompson scale (1-5), while the FJF was graded as 0 (absent), 1 (monolateral), or 2 (bilateral): per-patient disk degeneration and FJF were calculated summing the scores of the 3 spine levels. Spearman correlation coefficient, multivariate regression analysis, Friedman McNemar tests were used. Data were reported as median and interquartile interval (II).

Results: The degeneration of L3/L4 (median 3; II 2-3) was lower than that of both L4/L5 (median 3; II 2-3; $p=0.017$ ) and L5/S1 (median 3; II 3-4; $p=0.002$ ). The FJF of L5/S1 (median 0; II 0-1) was lower than that of both L3/L4 (median 1; II 0-2; $\mathrm{p}=0.002$ ) and $\mathrm{L} 4 / \mathrm{L} 5$ (median $1 ; \| \mathrm{O}-2 ; \mathrm{p}=0.019$ ). At bivariate per patient analysis, age was correlated negatively with $F J F(r=-0.199 ; p=0.052)$ and positively with disk degeneration $(r=0.582 ; p 0.142)$.

Conclusion: This preliminary study suggests that, differently from what expected, young patients undergoing MRI for low back pain show higher FJF than older patients, regardless of the disk degeneration. Further prospective studies should investigate the role of FJF on the low back pain.

\section{B-0546 11:33}

Typification of posterior instability in patients with spondylolysis or isthmic spondylolisthesis

P. Niggemann ${ }^{1}$, J. Kuchta ${ }^{2}$ H. Urbach ${ }^{2}$, H.H. Schild2; ${ }^{1}$ Mannheim/DE,

Bonn/DE (pniggemann@hotmail.com)

Purpose: To characterise different pathologic movements (posterior instability) encountered in the spondylolytic cleft in patients with spondylolysis using positional MRI. The findings are discussed with regard to possible mechanisms leading to the development of instability.

Methods and Materials: 178 segments of the lumbar spine with spondylolysis and isthmic spondylolisthesis were examined using positional MRI. Scans were obtained in neutral sitting, flexion and extension position. No visible movement in the cleft was defined as type A, fluid displaced into the cleft as type $\mathrm{Bl}$, displacement of the flava ligaments at the level of the cleft as type BII, and intraspinal cysts arising from the spondylolytic cleft as type BIII. The movements were characterised by a radiologist and a neurosurgeon experienced in positional MRI.

Results: A high agreement was found between the two observers. 133 segments were characterised as type $\mathrm{A}$, six as type $\mathrm{BI}$, twenty-four as type BII and ten as type BIII. In five segments, the type differed between the right and the left side. In two patients a mixed type BI/II, in another two patients a mixed type BII/III and in one patient a mixed type BI/III were found.

Conclusion: Imaging findings in the spondylolytic cleft are similar to findings in facet joint degeneration suggesting a similar pathomechanism. Fluid displaced in the spondylolytic cleft may lead to bulging of the flava ligament or the development of intraspinal cysts, thus explaining nerve root compression. The reported imaging findings might help understanding the connection of posterior instability and unstable slips.
B-0547 11:42

Differentiation of benign and malignant vertebral body fractures with dynamic contrast-enhanced MRI

T. Geith' ${ }^{1}$, A. Biffar', G. Schmidt', S. Sourbron², H.R. Duerr', M.F. Reiser',

A. Baur-Melnyk'; ${ }^{1}$ Munich/DE, ${ }^{2}$ Leeds/UK (tobias.geith@med.uni-muenchen.de)

Purpose: Evaluating the ability of dynamic contrast-enhanced MRI (DCE-MRI) in differentiating between acute benign and malignant vertebral body fractures. Methods and Materials: 46 patients with acute vertebral body fractures (20 malignant, 26 osteoporotic) were included. STIR, T1w, T2w and DCE-MRI (SRTurbo-Flash 2D) were acquired at 1.5 T. Parameter maps for mean-transit-time (MTT) and plasma flow (PF) were created. 2-compartment tracer kinetic modelling was applied to provide quantitative perfusion data in selected regions of interest. Results: 44 fractures were evaluated. Spots of increased PF showed significant differences ( $p=0.0003$ ) between osteoporotic (mean 28.62) and malignant (mean 11.73 ) fractures (sensitivity $63.2 \%$, specificity $96.0 \%$ ), extracellular volume (ECV) was significantly different $(\mathrm{p}=0.002$, osteoporotic mean 52.68 , malignant mean 36.71 , sensitivity $63.2 \%$, specificity $92.0 \%$ ), extraction flow (EF) was significantly different ( $p=0.03$, osteoporotic mean 15.19 , malignant mean 23.67 , sensitivity $57.9 \%$, specificity $92.0 \%$ ). K-trans, $\mathrm{PF}$ and plasma volume (PV) in spots of increased $\mathrm{PF}$, and all quantitative perfusion parameters in regions of increased MTT did not show any significant differences between benign and malignant fractures. Conclusion: Measurement of IV, ECV and EF in spots of increased PF with DCEMRI can help to differentiate between benign and malignant vertebral body fractures.

Author Disclosures:

T. Geith: Research/Grant Support; Deutsche Forschungsgemeinschaft (DFG), grant no. DI 1413/1-1. A. Biffar: Research/Grant Support; Deutsche Forschungsgemeinschaft (DFG), grant no. DI 1413/1-1. G. Schmidt: Research/ Grant Support; Deutsche Forschungsgemeinschaft (DFG), grant no. DI 1413/11.M.F. Reiser: Research/Grant Support; Deutsche Forschungsgemeinschaft (DFG), grant no. DI 1413/1-1. A. Baur-Melnyk: Research/Grant Support; Deutsche Forschungsgemeinschaft (DFG), grant no. DI 1413/1-1.

\section{B-0548 11:51}

Trabecular bone structure analysis of the spine using clinical MDCT: can it predict vertebral bone strength?

T. Baum 1 , M. Gräbeldinger ${ }^{1}$, E. Grande Garcia', R. Burgkart ${ }^{1}$, J. Patsch², E.J. Rummeny ${ }^{1}$, T.M. Link ${ }^{3}$, J.S. Bauer ${ }^{1} ;{ }^{1}$ Munich/DE, ${ }^{2}$ Vienna/AT, ${ }^{3}$ San Francisco, CA/US (thbaum@gmx.de)

Purpose: It was reported previously that trabecular bone structure parameters could add significant information for predicting bone strength beyond bone mineral density. In the past, trabecular bone structure analysis was limited by the spatial resolution of clinically available MDCT scanners. Recent technical improvements have made it possible to determine trabecular bone structure parameters of the spine using clinical MDCT. Therefore, the purpose of this study was to assess trabecular bone structure parameters obtained from clinical MDCT as well as Xtreme-CT (hr-pQCT) as standard of reference and to investigate whether clinical MDCT can predict vertebral bone strength.

Methods and Materials: Fourteen thoracic vertebral bodies were harvested from formalin-fixed human cadavers ( 11 women and 3 men; age: $84 \pm 10$ years). All specimens were examined using Xtreme-CT (isotropic voxel size of $41 \mu \mathrm{m}^{3}$ ) and

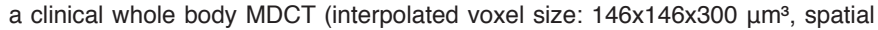
resolution: 250x250x600 $\mu^{3}$ ). Trabecular bone structure analyses (histomorphometric and texture measures) were performed in the Xtreme-CT as well as MDCT images. Vertebral failure load was determined in an uniaxial biomechanical test. Results: Xtreme-CT and MDCT-derived trabecular bone structure parameters showed correlations ranging from $r=0.60$ to $r=0.90(p<0.001)$. Correlations between trabecular bone structure parameters and failure load amounted up to $r=0.86(p<$ $0.001)$ using the Xtreme-CT images, and up to $r=0.79(p<0.001)$ using the MDCT images. Correlation coefficients obtained with Xtreme-CT and MDCT were not significantly different $(p>0.05)$.

Conclusion: The spatial resolution of clinically available whole body MDCT scanners is suitable for trabecular bone structure analysis of the spine and to predict vertebral bone strength. 


\section{Oncologic Imaging}

\section{SS 916}

\section{Whole-body imaging: how to do it}

\section{Moderators:}

T.F. Hany; Zurich/CH

D.M.J. Lambregts; Maastricht/NL

\section{B-0549 10:30}

Cross over PET/MRI vs PET/CT study in oncological patients: does it change management?

J. Al-Kalbani, A. Afaq, K. Alnabhani, R. Syed, C. O'Meara, A. Barnes,

J. Boumanji; London/UK (jalkalbani@yahoo.com)

Purpose: To evaluate the feasibility of using whole body 18 F-FDG PET/MRI in assessing patients with a proven primary tumour.

Methods and Materials: 42 consecutive patients with different tumour types underwent PET/CT (GE) with low-dose CT for attenuation correction followed by PET/MRI (Siemens Biograph mMR) using 2-point Dixon technique for attenuation correction. Three radiologists reviewed all studies and recorded the number of primary and non-primary lesions on PET CT and PET MRI. The location of each lesion was then using recorded using a confidence on a scale of 1 to 3 ( $1=$ uncertain, $2=$ probable, $3=$ definite). The degree of confidence for anatomical localisation of the lesions and degree of inter-observer agreement were measured.

Results: A total of 18218 F-FDG avid lesions were identified on PET/MRI and PET/ CT. MRI helped in accurate identification of FDG activity in 11 lesions that were missed on CT component of PET/CT (2 rectal, 1 bladder, 2 prostate, 2 vagina, 2 cervix, 1 liver, 1 nerve). $22 \%$ of the patients had a change in management following PET/MRI. The overall inter-observer agreement for both the modalities was $98 \%$ for primary and $95 \%$ for non-primary lesions. There was overall improvement in the confidence in anatomical localisation on PET/MRI (6\% primary lesions and $12 \%$ non-primary lesions).

Conclusion: Whole body PET/MRI improves the confidence in anatomical localisation of sites of disease and also provides additional value to patient management compared with PET/CT.

\section{B-0550 10:39}

Whole body magnetic resonance imaging, including diffusionweighted imaging, for staging malignant lymphoma: comparison to computed tomography in 135 patients

T.C. Kwee ${ }^{1}$, M.A. Vermoolen', E.A. Akkerman ${ }^{2}$, M. Kersten², R. Fijnheer ${ }^{3}$, F.J. Beek ${ }^{1}$, M.S. van Leeuwen ${ }^{1}$, M.B. Bierings ${ }^{1}$, R.A.J. Nievelstein ${ }^{1} ;{ }^{1}$ Utrecht/NL, ${ }^{2}$ Amsterdam/NL, ${ }^{3}$ Amersfoort/NL (thomaskwee@gmail.com)

Purpose: To compare whole body magnetic resonance imaging (MRI), including diffusion-weighted imaging (DWI), to computed tomography (CT) for staging newly diagnosed lymphoma.

Methods and Materials: One hundred and thirty-five consecutive patients with newly diagnosed lymphoma prospectively underwent whole body MRI (T1-weighted and T2-weighted short inversion time inversion recovery [ $n=135]$, and DWI $[n=130]$ ) and CT. Ann Arbor stages were assigned according to whole body MRI and CT findings. Disagreements in staging between whole body MRI (without and with $\mathrm{DWI}$ ) and CT were resolved using bone marrow biopsy, $18 \mathrm{~F}$-fluoro-2-deoxyglucose positron emission tomography (FDG-PET), and follow-up FDG-PET, CT, and whole body MRI studies as reference standard.

Results: Staging results of whole body MRI without DWI were equal to those of CT in $66.7 \%$ (90/135), higher in 25.2\% (34/135), and lower in $8.1 \%(11 / 135)$ of patients, with correct/incorrect/unresolved overstaging and incorrect/unresolved understaging relative to CT in 18/10/6 and 10/1 patient (s), respectively. Staging results of whole body MRI with DWI were equal to those of CT in $65.4 \%$ (85/130), higher in $29.2 \%(38 / 130)$, and lower in $6.2 \%(8 / 130)$ of patients, with correct/incorrect/unresolved overstaging and incorrect/unresolved understaging relative to $\mathrm{CT}$ in 22/10/6 and 7/1 patient (s), respectively.

Conclusion: Staging of newly diagnosed lymphoma using whole body MRI (without and with $\mathrm{DWI}$ ) equals staging using $\mathrm{CT}$ in the majority of patients. Disagreements between whole body MRI and CT are mostly caused by overstaging of the former relative to the latter, with the number of correctly and incorrectly overstaged cases being approximately equal. The potential advantage of DWI is still unproven.
B-0551 10:48

Combination of continuously moving table MRI and PET including image fusion: improved lesion detection in local and distant recurrence of rectal cancer

T. Baumann, A.-O. Schäfer, M. Langer;

Freiburg/DE (tobias.baumann@uniklinik-freiburg.de)

Purpose: Comprehensive and accurate staging of recurrent rectal cancer is a prerequisite to correctly assign patients to potentially curative treatment options. Therefore, it was the aim of this study to investigate the incremental utility of highresolution pelvic MRI with continuously moving table MRI (CMT-MRI) of the chest and abdomen and 18 F-FDG-PET using image fusion.

Methods and Materials: Thirty-one patients with suspected recurrence of rectal cancer received $\mathrm{MRI}$ and PET within a maximum interval of 30 days. MRI alone, PET alone and MRI and PET combined including fusion images were separately analysed by two observers. All detectable lesions were noted and the likelihood of malignancy was rated on a 5 -point scale ( $1=$ definitely benign to $5=$ definitely malignant). The standard of reference consisted of all available follow-up imaging and histopathology. Confidence ratings were subjected to JAFROC analysis and sensitivities were computed with ratings of 4 and 5 being considered as positive test results.

Results: The reference standard comprised 150 malignant lesions with 48 being considered local and 102 considered distant (35 lung, 17 liver, 40 lymph nodes, 10 other). JAFROC analysis revealed overall figures-of-merit of 0.73 for MRI, 0.63 for PET, and 0.83 for the combined approach (all differences significant). The sensitivities of MRI, PET and the combined approach were $85.4,52.1$, and $95.8 \%$ for local recurrence and $61.8,47.1$, and $81.4 \%$ for distant recurrence, respectively. Conclusion: The combination of local high-resolution MRI, thoracoabdominal CMT-MRI and FDG-PET by means of image fusion improves lesion detection in recurrent rectal cancer.

\section{B-0552 10:57}

Does positron emission tomography data acquisition impact simultaneous diffusion-weighted imaging in a whole body PET/MRI system?

R.S. Lanzman ${ }^{1}$, C. Buchbender ${ }^{1}$, P. Heusch ${ }^{1}$, V. Hartung-Knemeyer ${ }^{2}$, T. Lauenstein ${ }^{2}$, M. Forsting ${ }^{2}$, G. Antoch 1 , T. Heusner ${ }^{1} ;{ }^{1}$ Düsseldorf/DE, ${ }^{2}$ Essen/DE (Rotem.Lanzman@med.uni-duesseldorf.de)

Purpose: To investigate whether the acquisition of PET has an impact on simultaneous diffusion-weighted imaging (DWI) on an integrated PET/MRI system.

Methods and Materials: Fourteen oncological patients ( 9 men, 5 women; age $54 \pm 13$ years) underwent DWI (EPI; $b=0-500-1000 \mathrm{~s} / \mathrm{mm}^{2}$ ) on a whole body $3 \mathrm{~T}$ PET/MRI system twice in one session; first with simultaneous PET acquisition and second with the PET component switched off. The apparent diffusion coefficient (ADC) and the SNR at $b=1000 \mathrm{~s} / \mathrm{mm}^{2}$ of the myocardium, paraspinal muscle, liver, spleen, renal cortex and tumour tissue (if present) were measured. Student's t-test was performed to test for differences between the two DWI acquisitions.

Results: ADC did not differ significantly between DWI acquisitions with and without simultaneous PET for the myocardium $\left(2572 \pm 44110^{-6}{ }^{*} \mathrm{~mm}^{2} / \mathrm{s}\right.$ and $2586 \pm 376$ $10^{-6 *} \mathrm{~mm}^{2} / \mathrm{s}$, respectively) $(\mathrm{P}=0.817)$, liver $\left(1245 \pm 15810^{-6 *} \mathrm{~mm}^{2} / \mathrm{s}\right.$ vs. $1254 \pm$ $\left.17110^{-6 *} \mathrm{~mm}^{2} / \mathrm{s}\right)(\mathrm{P}=0.848)$, spleen $\left(980 \pm 12210^{-6} * \mathrm{~mm}^{2} / \mathrm{s}\right.$ vs. $1000 \pm 18710^{-6}$ $\left.{ }^{*} \mathrm{~mm}^{2} / \mathrm{s}\right)(\mathrm{P}=0.676)$, renal cortex $\left(1951 \pm 22610^{-6 *} \mathrm{~mm}^{2} / \mathrm{s}\right.$ vs. $1930 \pm 27310^{-6}$ * $\left.\mathrm{mm}^{2} / \mathrm{s}\right)(\mathrm{P}=0.730)$ and lymph node metastases $(\mathrm{n}=6)\left(853 \pm 17410^{-6 *} \mathrm{~mm}^{2} / \mathrm{s}\right.$ vs. $\left.865 \pm 17010^{-6 *} \mathrm{~mm}^{2} / \mathrm{s}\right)(\mathrm{P}=0.675)$. There was no significant difference regarding the SNR between the two DWI acquisitions.

Conclusion: Our results indicate that simultaneous acquisition of DWI and PET on an integrated PET/MRI system does not impact ADC quantification of normal and tumour tissue.

\section{B-0553 11:06}

Standardised uptake values for [18 F]-FDG in normal organ tissues: comparison of whole body PET/CT and PET/MRI

C. Buchbender ${ }^{1}$, P. Heusch ${ }^{1}$, V. Hartung-Knemeyer ${ }^{2}$, H. Kühl²,

T. Lauenstein ${ }^{2}$, M. Forsting ${ }^{2}$, G. Antoch 1 , T.A. Heusner ${ }^{1} ;{ }^{1}$ Düsseldorf/DE, ${ }^{2}$ Essen/DE (philipp.heusch@med.uni-duesseldorf.de)

Purpose: To compare standardised uptake values (SUVmax/mean) of normal organ tissues derived from PET/MRI using MR attenuation correction (MRAC) (DIXON-based 4-segment $\mu$-map) with PET/CT using CT-based attenuation correction (CTAC).

Methods and Materials: In 25 oncologic patients (15 men, 10 women; age 57 \pm 13 years) after PET/CT (60 minutes after injection of $292 \pm 40 \mathrm{MBq}[18 \mathrm{~F}]-\mathrm{FDG}$ ), 
whole body PET/MRI was additionally performed (Magnetom Biograph $\mathrm{mMR}^{\mathrm{TM}}$, Siemens Healthcare, Erlangen, Germany). $3 \mathrm{~cm} 3$ regions of interest were drawn in seven organ sites in MRAC-PET and the corresponding CTAC-PET images. Pearson correlation coefficients were calculated to compare MRAC- and CTACbased SUV values; Student's T-test for paired samples was performed to test for potential differences.

Results: The mean delay between PET/CT and PET/MRI was $152 \pm 42$ minutes. Near-perfect correlations of SUV values were found for the myocardium (SUVmax/ mean: $R=0.98 / 0.98)$; good correlations were found for the liver $(R=0.6 / 0.7)$, subcutaneous fat $(R=0.6 / 0.8)$, bone marrow $(R=0.6 / 0.5)$, psoas muscle $(R=$ $0.6 / 0.5)$ and the SUVmax of the blood-pool $(R=0.6)$. Correlations between MRAC and CTAC were non-significant for SUVmax and SUVmean of the lung and the SUVmean of the blood-pool. The mean SUVmax and SUVmean in MRAC-PET compared to the respective CTAC values was significantly lower in all organs $(p<$ 0.05 ) but in the myocardium (SUVmax) and the psoas muscle (SUVmax/mean). Conclusion: Although SUVmax and SUVmean values of normal organ tissues in PET/MRI and PET/CT are generally well correlated, the absolute values differ significantly and, for a quantitative comparison, have to be adjusted.

\section{B-0554 11:15}

Impact of FDG PET-CT on patient management and radiotherapy

treatment planning in locally advanced cervical carcinoma

S. Fleming, R. Cooper, S. Swift, F. Chowdhury, A. Scarsbrook, C. Patel; Leeds/UK

Purpose: Evaluation of the clinical impact of FDG PET-CT on management of patients with locally advanced cervical carcinoma initially staged with pelvic MRI. Methods and Materials: Retrospective analysis of consecutive patients undergoing FDG PET-CT for cervical carcinoma, between April 2008 and August 2011 in a single tertiary referral centre. Comparison was made between findings on PET-CT and pelvic MRI. Clinical impact of PET-CT was divided into major (detection of occult disease or characterisation of indeterminate lesions on MRI, as benign or malignant), minor (confirmation of suspicious MRI findings or additional findings not altering management), or no impact (concordance between PET-CT and MRI with no additional findings).

Results: Sixty-three patients were referred for a pre-treatment FDG PET-CT; age range was $21-78$ years (mean age 47 years). PET-CT had a major impact on patient management in $32 \%$, a minor impact in $8 \%$ and no impact in $60 \%$. In patients with a major impact; 12 had unsuspected nodal involvement, 5 had occult metastases, 2 had synchronous tumours and 1 had negative nodes on PET-CT. These findings either altered radiotherapy treatment planning or treatment intent. During the follow-up period until August 2012, 25\% developed disease recurrence following completion of therapy ( $11 \%$ isolated local relapse and $14 \%$ distant ( \pm local) disease). Conclusion: FDG PET-CT can have a major impact on patient management in locally advanced cervical carcinoma, by detecting occult disease or characterising indeterminate findings on MRI, resulting in an alteration in radiotherapy treatment planning and/or treatment intent in up to one-third of patients.

\section{B-0555 11:24}

Is there a role for standard uptake value in the prognostic evaluation of lung nodules?

A. Bazzocchi, S. Brocchi, G. Facchini, V. Ambrosini, S. Ascanio, G. Battista,

S. Fanti; Bologna/IT (giancarlo.facchini@gmail.com)

Purpose: Criticisms and limits about the measurement and use of standard uptake value (SUV) in PET imaging are well known. Our aim was to investigate the aid of SUV in the detection of malignancy of solitary lung nodules and to study its prognostic value.

Methods and Materials: We retrospectively analysed the clinical history of 136 patients ( 81 males, 55 females, $70.3 \pm 11.2$ year old) undergoing PET/CT for lung nodules, in a single-centre experience. The analysis included images evaluation and physicians' reports of PET/CT exams, anamnesis of patients and subsequent diagnostic examinations in their follow-up (imaging, biopsy specimen and/ or pathological analysis after surgical resection). Patient's follow-up ended with survival control in September 2012. Receiver-operating-characteristic curves and Kaplan-Meier method were used for statistical analysis.

Results: Mean follow-up was $26.3 \pm 18.0$ months. In 58/136 (42.6\%) subjects, a non-small cell lung cancer was diagnosed (35/58-adenoCa, 23/58-squamousCa); $30 / 136$ (22.1\%) patients underwent surgery. In all other patients (78/136-57.4\%), lesions were classified as non-oncologic diseases or benign. The best combination to properly characterise the neoplastic nature of nodules found a SUVmax cut-off value of 2.5 , resulting in $93.1 \%$ and $71.8 \%$, sensitivity and specificity, respectively. Nodules with higher SUVmax value $(>2.5)$ were associated with lower survival rate $(p=0.0001)$, independently from surgical/non-surgical approach. For SUV $>$ 2.5 and $<2.5$, the survival rates 1 year after PET imaging were $72.5 \%$ and $94.0 \%$, while survival expectancies after 2 years were $56.5 \%$ and $86.3 \%$, respectively. Conclusion: In conclusion, prognostic value of SUVmax was proved, independently from nature of lesions; a cut-off value of 2.5 seems to offer good reliability and accuracy.

\section{B-0556 11:33}

Detection of underlying malignancy in patients with paraneoplastic neurological syndromes: comparison of 18 F-FDG-PET/CT and contrast-enhanced $\mathrm{CT}$

N. Schramm, A. Rominger, C. Schmidt, C. Schmid-Tannwald, F.G. Meinel, M.F. Reiser, C. Rist; Munich/DE (nicolai.schramm@med.uni-muenchen.de)

Purpose: To determine the value of 18 F-FDG-PET/CT with contrast-enhanced CT (CECT) in detecting malignancies in patients with paraneoplastic neurological syndromes (PNS) in comparison with CECT alone.

Methods and Materials: PET/CTs of 66 patients with PNS were retrospectively evaluated. Two blinded readers initially reviewed the CECT. In a second session, they analysed the combined PET/CT examinations. Findings were assessed using a 4 -point-scale $(1=$ normal; $2=$ inconclusive; $3=$ malignant; $4=$ inflammatory $)$. Sensitivity and specificity for malignant findings were calculated. Data from clinical follow-up (including histopathology, follow-up imaging; mean follow-up: 25.6 months) served as standard of truth.

Results: Both readers classified 12 findings in 10 patients (15\%) as malignant on PET/CT (two patients with two primary tumours). One imaging finding was false positive. The most common tumours were bronchial carcinoma $(n=3)$, lymphnode metastases of gynaecological tumours $(n=3)$ and tonsillar carcinoma $(n=2)$. $3 / 12$ findings were not detected by CECT alone (cervical and tonsillar carcinoma, lymph-node metastasis). Sensitivity and specificity for malignant findings were 100 and $90 \%$ (PET/CT) and 78 and $88 \%$ (CECT). In $24 \%$ of the patients inconclusive $\mathrm{PET} / \mathrm{CT}$ findings were identified. $57 \%$ of these findings were only diagnosed with PET (e.g. focal uptake of the GI tract). On follow-up none of these findings corresponded to malignancy.

Conclusion: In this cohort of patients with PNS, PET/CT exhibited improved detection of underlying malignancy versus CECT alone. While hybrid imaging produces a greater number of inconclusive findings, sensitivity is increased for the detection of head and neck and gynaecological malignancies as well as metastatic lymph-node involvement.

\section{B-0557 11:42}

FDG-avid duodenal lesions: a retrospective analysis of their significance in oncology practice

S. Puri, L. Jones, J. Strang; Rochester, NY/US

(savita_puri@urmc.rochester.edu)

Purpose: To determine the significance of focal or segmental 18 F-FDG uptake within the duodenum on PET/CT.

Methods and Materials: Computerized text search for the word duodenum appearing anywhere within the PET/CT report was conducted on all patients undergoing F-18 fluorodeoxyglucose (FDG) PET/CT at the University of Rochester Medical Center from January 1, 2009 to July 30, 2012. Three expert radiologists reviewed those cases and classified duodenal uptake as focal, multifocal, segmental or diffuse. Results were correlated with histopathology, other imaging studies and clinical follow-up. Exclusion criteria included use of the word duodenum unassociated with FDG uptake, extra-duodenal tumors invading the duodenum, duplicated follow-up studies, lack of histopathology or adequate clinical follow-up.

Results: Of 8725 PET/CT reports, 95 contained the word duodenum on 83 patients. Twenty-seven of 83 patients (33\%) had duodenal FDG uptake (19 focal, 1 multifocal, 6 segmental and 1 diffuse). Eighteen of 20 with focal or multifocal pattern were malignant (90\%): 11 known biopsy-proven primary or metastatic malignancy and 7 newly identified by PET and subsequently proven malignant either by biopsy or by response to targeted chemotherapy. Of the 9 non-malignant lesions, 2 had focal uptake ( 1 biopsy-proven duodenitis, $2^{\text {nd }}$ nonspecific), 6 had segmental pattern, 1 of which was biopsy-proven Crohn's disease, the remaining 5 segmental and 1 diffuse uptake were presumed physiologic.

Conclusion: Focal duodenal FDG uptake is highly predictive of an underlying malignancy with a positive predictive value of $90 \%$ (P-value $<0.01$ ) 


\section{B-0558 11:51}

Feasibility and first clinical experiences with simultaneous PET/MR imaging of cervical cancer: comparison of image quality with MRI and PET/CT

P. Brandmaier, S. Purz, M. Reinhardt, M. Gawlitza, H. Barthel, O. Sabri, T. Kahn,

R. Kluge, P. Stumpp; Leipzig/DE (philipp.brandmaier@medizin.uni-leipzig.de)

Purpose: We report about our approach in imaging patients with cervical cancer using an integrated simultaneous PET/MRI device. Goals were the introduction of a stable and effective protocol for whole body and dedicated pelvic examination and rating of image quality.

Methods and Materials: Ten patients with primary $(n=4)$ cervical carcinoma or tumour recurrence $(n=6)$ were included. Patients underwent a simultaneous PET/ MR, PET/CT and conventional MR examination. MR and PET image quality were rated on a Likert score system (0-4) by three radiologists and a nuclear physician. PET data were simultaneously visually compared by two nuclear physicians and a radiologist.

Results: Conventionally acquired images showed adequate to good image quality for T2w-TSE and T1w-TSE (mean values $2.5 \pm 0.58$ and 2.7 \pm 0.49 ). PET/MR images of the pelvis were rated slightly better $(p>0.05)$ with a mean value of $2.8 \pm 0.71$ (T2w-TSE) and 2.8 $\pm 0.73($ T1w-TSE). Superior results $(p<0.05)$ were seen in fused T2w-PET images (mean 3.4 \pm 0.62 ). For whole body PET/MR imaging quality was good to excellent in head, pelvis and thigh. DWI showed the highest variations of values ranging from $3.32 \pm 0.67$ to $0.43 \pm 0.88$. Visual comparison of the PET component revealed adequate image quality with a tendency towards improved delineation of small foci in PET/MRI.

Conclusion: This work shows the feasibility of a combined protocol with a whole body simultaneous PET/MR examination plus a dedicated simultaneous PET/MR of the pelvis. This examination can be done within approximately one hour and provides good image quality for both, the PET and the MRI.

\section{$10: 30-12: 00$}

Room F2

\section{Breast}

\section{SS 902}

\section{Increased risk of breast cancer}

Moderators:

L.A. Carbonaro; San Donato Milanese/IT

L. Ollivier; Paris/FR

\section{B-0559 10:30}

Individual radiosensitivity: a key-issue to reconsider high breast cancer risk patient screening modalities

C. $\underline{\text { Colin }}^{1}$, N. Foray ${ }^{2}$, F. De Vathaire ${ }^{3}$, M. Bourguignon ${ }^{4}$,

P.-J. Valette ${ }^{1} ;{ }^{1}$ Pierre-Bénite/FR, ${ }^{2}$ Lyon/FR, ${ }^{3}$ Villejuif/FR, ${ }^{4}$ Paris/FR (catherine.colin01@chu-lyon.fr)

Purpose: BRCA1 and BRA2 mutation carriers may be more susceptible to radiationinduced cancer because of their role of tumour suppressor genes in radio-induced DNA damage repair and signalling. Heterozygous mutations of $\mathrm{A}^{\mathrm{TM}}$, a major kinase initiating the response to radio-induced DNA double-strand breaks, is likely to be involved in lymphoma as well as in breast cancer in high-risk patient (HRP) treated for a Hodgkin disease. Thus the standard two-view mammographic screening annually recommended with MRI in HRP must be adapted.

Methods and Materials: Recent literature data were analysed. Individual radiosensitivity was assessed by radiobiological effects and epidemiological studies. Radiophysical and radiological mammographic data about calcifications detection and reduction of exposures were also taken into account.

Results: Radio-induced DNA damage in breast epithelial cells were demonstrated ex vivo with a Low and Repeated Dose effect reflecting an increase of unrepaired DSB after 2-views mammography, exacerbated in high family risk women. Recent epidemiological data highlighted mammographic radio-induced breast cancer in BRCA1-BRCA2 mutation carriers. Compared with other technologies, Full-Field Digital Mammography is the most accurate technology to detect calcifications and/ or ductal carcinoma in situ, while delivering the lowest doses.

Conclusion: Mammographic modalities in HRP must be up-dated since pathogenic alleles in DNA repair and signalling genes carriers have an increased risk of radio-induced breast cancer. A drastic limitation of the number of systematic mammographic views to one single view (oblique) in addition to MRI may be a good compromise to detect both infiltrative and carcinoma in situ, using Full-Field Digital Mammography.
B-0560 10:39

Which screenings program should be offered to women with BRCA1 or BRCA2 mutations? A simulation of comparative cost-effectiveness G.H. De Bock', K.M. Vermeulen', L. Jansen', J.C. Oosterwijk', S. Siesling ${ }^{1}$, T. Feenstra', N. Houssami ${ }^{2}$, M.J.W. Greuter ${ }^{1} ;{ }^{1}$ Groningen/NL, ${ }^{2}$ Syndey/AU (g.h.de.bock@umcg.nl)

Purpose: There is no consensus on the most effective strategy for screening women with BRCA1 or BRCA2 mutations and women with a strong family history of breast cancer but unknown mutation status (BRCAu). The Dutch strategy uses annual MRI for age 25-30 years, annual mammography and MRI for age 30-60, and biennial mammography-only for age 60-75. The UK strategy uses annual mammography and MRI from age 30 , and mammography-only from age 50 every three years. The US strategy includes annual mammography and MRI from age 25. The effectiveness and cost-effectiveness of these screening strategies were modelled in high-risk women.

Methods and Materials: To compare various screening strategies, we integrated outcomes and cost parameters, from published data and cancer registry data into a validated simulation screening model. Model main outcomes were life-years gained and incremental cost-effectiveness rate (ICER). Analyses were performed according to the US and European guidelines with a discount-rate of $3 \%$.

Results: For BRCA1 as well for BRCA2 women, the Dutch strategy was preferred as this strategy was equally effective and cheaper than the US strategy. The three screening strategies were relatively less effective for the BRCAu group: the lifeyears gained were approximately twice in BRCA mutation carriers. For women in the BRCAu group, UK strategy was cheapest, but about $6 \%$ less effective.

Conclusion: From a cost-effectiveness perspective, the Dutch strategy for screening is preferred in women with BRCA1 or BRCA2 mutations.

\section{B-0561 10:48}

MRI of hereditary breast cancer: can hyper T2 signal intensity be considered as a new semeiotic parameter of higher aggressiveness? G. Trecate, R. Agresti, S. Viganò, L. Suman, D. Vergnaghi,

C. Ferranti, M. Marchesini, G. Scaperotta, P. Panizza; Milan/IT (giovanna.trecate@istitutotumori.mi.it)

Purpose: To compare MRI of 43 hereditary and 40 sporadic breast cancers, observe the differences and evaluate the pathological reports to investigate the differences. Methods and Materials: We positioned a ROI on the hyperT2STIR carcinomas to quantify the optical impression of signal intensity, evaluated the presence of necrosis, grading and triple negative status. Cox model was applied for statistical evaluation.

Results: The surprising main difference was the high signal intensity on T2 weighted-images (T2SI) that we registered for hereditary cancer. We found $79 \%(34 / 43)$ high T2SI in hereditary cancers and only $8 \%(3 / 40)$ hyperT2 lesions in sporadic cancers $(p<0.01)$. Over the highT2 hereditary neoplasm $53 \%$ were BRCA1 and $85 \%$ were Invasive carcinoma. The triple negative status was confirmed in $34 \%$, while not calculated for 5 . Interestingly, none of the hiperT2 lesions was G1, while $58.8 \%$ were G3 and $29 \%$ G2. Necrosis was registered in $30 \%$ and not calculated in 6. Medullary aspect were present in 3. All hyperT2 sporadic lesions were G3, while necrosis and triple-negative were found in $2 / 3$ with no mucinous variants. Conclusion: Several authors investigated the reason of hyperT2SI in breast cancers. Some described the presence of necrosis also as index of rapid growth rate and unfavourable prognosis. Some evaluated the combination with triplenegative status while some others discovered a relationship between high-grade and BRCA1 germline. Nowadays only necrosis can reliably explain the raise of T2SI, but if further investigations should confirm that triple-negative status and high-grade are mostly combined with hyperT2SI, then we can point-at hyperT2SI as an MR marker of higher aggressiveness.

\section{B-0562 10:57}

Fully automated MRI breast density (BD) measurement: comparison to standard mammographic density estimation

G.J. Wengert, W.-D. Vogl, T.H. Helbich, H. Bickel, W. Bogner,

K. Pinker-Domenig; Vienna/AT (georg.wengert@meduniwien.ac.at)

Purpose: To develop a fully automated quantitative BD measurement system with MRI based on the Dixon sequence and to compare it with qualitative and quantitative mammographic BD estimation.

Methods and Materials: 35 healthy women were included in this prospective IRB-approved study. Breast density was measured qualitatively using the ACR BIRADS categories, quantitatively from digitized mammograms using Cumulus, a user-assisted interactive thresholding technique and with radiation- and 
compression-free MRI using the Dixon sequence at $3 \mathrm{~T}$ Tesla, which acquired two datasets one representing fatty and the other fibroglandular tissue. The fully automated MRI breast density measurement system calculated the percentage of fatty and fibroglandular tissue (\%) and the total volume (cm3) of the breast. Mean values were compared using student's T-test, and descriptive statistics using the $25-75 \%$ percentile were used to define the typical range of MRI density readings corresponding to the 4-BIRADS categories.

Results: Percentage MRI breast density correlated with percentage mammographic breast density $(r=0.83 ; P<0.0001)$. MRI measurements ranged from $1.70 \%$ to $61.9 \%(29.05 \%)$ and mammographic measurements from $5.42 \%$ to $74.33 \%(40.23)$. Compared with the BIRADS categories, $25-75 \%$ percentile assessment demonstrated an interquartile range of $2.4-9.4 \%$ for BIRADS-1, 7.27-26.88\% for BIRADS-2, 26.1-48.43\% for BIRADS-3 and 39.7-53.9\% for BIRADS-4. Quantitative MRI breast density readings were consistently lower than qualitative BIRADS assessment. Conclusion: Fully automated quantitative MRI BD measurement correlates well with mammography-based breast density readings; it is fast, user-independent and radiation- and compression-free and a reliable way of breast density estimation.

\section{B-0563 11:06}

Fully automated quantitative MRI breast density (BD) measurement confirms higher breast density in breast cancer patients K.J. Pinker-Domenig, G.J. Wengert, H. Bickel, H.F. Magomatschnigg, M. Curda, T.H. Helbich; Vienna/AT (katja.pinker-domenig@meduniwien.ac.at)

Purpose: To measure and compare BD in breast cancer patients and age-matched healthy controls using a fully automated MRI BD measurement system.

Methods and Materials: 19 breast cancer cases and 19 age-matched healthy controls (age range of 34 to 68; mean 50 years) were included in this IRB-approved prospective study. BD was measured separately in all participants. The fully automated MRI BD measurement system calculated the total volume of the breast (cm3), the percentage of fatty tissue (\%) and the percentage of fibroglandular tissue (\%). In each group normal distribution (Kolmogorov-Smirnov test; $\mathrm{P}=0.12$ ) and variance homogeneity (Levene's test, $\mathrm{P}=0.69$ ) was determined to accomplish independent t-test $(P=0.316)$.

Results: Fully automated quantitative MRI BD measurements were successfully performed in both groups. Breast density ranged from $1.7 \%$ to $58.4 \%$, mean density for all women was $21.24 \%$. Mean percentage density was higher in breast cancer patients as compared with healthy controls with an average of $24 \%$ (SD $16.9 \%$ ) vs. $18.4 \%$ (16.6). Breast density decreased gradually with age in both cancer patients ( $r 2=0.237)$ and healthy controls $(r 2=0.259)$.

Conclusion: Fully automated quantitative MRI BD measurements confirm higher breast density in cancer patients as compared to healthy controls. Although not statistically significant due to the small sample size, these results are entirely consistent with the fact that breast density is a strong risk factor for breast cancer.

\section{B-0564 11:15}

To excise or not to excise? Management of high-risk breast lesions in a symptomatic population

J.D. Cunningham, A. Corr, E. Kelliher, A. O'Shea, J. Kerr, D. Duke, N. Hambly; Dublin/IE (janecunningham0708@gmail.com)

Purpose: Management of high-risk breast lesions detected by image-guided biopsy is controversial. We aim to determine the upgrade rate of high-risk lesions in a symptomatic population where surgical excision is currently the standard practice in all cases.

Methods and Materials: All ultrasound, stereotactic and MR-guided biopsies performed in our symptomatic breast unit between 01/01/2009 and 30/06/2012 were reviewed. High-risk lesions were identified from our pathology database. Currently, standard practice is surgical excision of all high-risk lesions following multidisciplinary discussion. For all lesions, post-operative histopathology was determined and malignancy upgrade rates were calculated. Correlation was made with lesion morphology, R imaging category and biopsy modality.

Results: 3157 image-guided biopsies were performed. Of these, 103 (3.3\%) were high-risk lesions, excluding fibroepithelial lesions. The distribution was as follows: 47 $(45.6 \%)$ papillary lesions, $22(21.4 \%)$ atypical ductal hyperplasia, $17(16.5 \%)$ radial scars, $14(13.6 \%)$ lobular neoplasia, $3(2.9 \%)$ flat epithelial lesions. The upgrade rate at surgical excision was $11 \%$ for papillary lesions, $18 \%$ for radial scars, $50 \%$ for atypical ductal hyperplasia, 36\% for lobular neoplasia and 33\% for flat epithelial neoplasia. The overall upgrade rate was $23 \%$.

Conclusion: The distribution of high-risk lesions in a symptomatic breast unit is different to a screening population. Diagnosis of a high-risk breast lesion at imageguided core biopsy in a symptomatic woman warrants surgical excision given the upgrade rate of $23 \%$.

\section{B-0565 11:24}

The diagnostic value of 3 Tesla breast MRI in the diagnosis of in situ and invasive breast cancer

L.G. Merckel ${ }^{1}$, H.M. Verkooijen ${ }^{1}$, N.H.G.M. Peters' ${ }^{1}$, R.M. Mann², W.B. Veldhuis ${ }^{1}$, T. van Dalen ${ }^{1}$, W.P.T.M. Mali ${ }^{1}$, P.H.M. Peeters ${ }^{1}$, M.A.A.J. van den Bosch'; ${ }^{1}$ Utrecht/NL, ${ }^{2}$ Nijmegen/NL (L.G.Merckel-2@umcutrecht.nl)

Purpose: To investigate the added diagnostic value of $3 \mathrm{~T}$ breast MRI over conventional breast imaging in the diagnosis of in situ and invasive breast cancer and to explore the role of routine versus expert reading.

Methods and Materials: Between January 2006 and May 2009, 207 patients with nonpalpable BI-RADS 3-5 lesions underwent contrast-enhanced 3 T breast MRI in a multicentre randomised trial (MONET trial). Initially, MRI scans were read by radiologists in a routine clinical setting. All histologically confirmed index lesions were re-evaluated by an expert breast radiologist according to the BI-RADS ${ }^{\circledR}$ lexicon. Added diagnostic value of breast MRI was assessed using ROC analysis. Results: Overall 78/207 (38\%) patients had malignant lesions, $31(15 \%)$ patients had pure DCIS and $47(23 \%)$ had mixed or pure invasive breast cancer. After routine MRI reading, $48 \%$ of BI-RADS $3,66 \%$ of BI-RADS 4 , and $95 \%$ of BI-RADS 5 lesions were proven malignant. After expert reading, positive predictive values were $35 \%, 63 \%$, and $100 \%$ for BI-RADS 3, 4 , and 5 lesions, respectively. In the routine setting, MRI was of added diagnostic value over baseline characteristics and conventional imaging, as the AUC increased from $0.78(95 \% \mathrm{Cl} 0.71-0.86)$ to 0.81 (95\% $\mathrm{Cl}$ 0.74-0.89). Expert MRI reading led to a strong improvement in AUC from $0.78(95 \% \mathrm{Cl} 0.71-0.86)$ to 0.89 (95\% Cl 0.83-0.95).

Conclusion: $3 \mathrm{~T}$ breast MRI of nonpalpable breast lesions is of added diagnostic value in the diagnosis of in situ and invasive breast cancer, in particular when performed by expert radiologists.

\section{B-0566 11:33}

Positive enhancement integral values in diagnostic magnetic resonance imaging of breast carcinoma: ductal carcinoma in situ (DCIS) vs invasive ductal carcinoma (IDC)

M.M. Nadrlianski, O.S. Radulovic, Z.C. Milosevic; Belgrade/RS (dr.m.nadrljanski@gmail.com)

Purpose: Positive enhancement integral (PEI) of the area under the curve after the contrast injection on breast MRI, presented as the time-intensity graph and maps, provides insight into tumour perfusion. The purpose of this paper was to contribute to the standardisation of the PEI values in IDC, DCIS and breast parenchyma and to compare the PEI values: IDC Vs. parenchyma, DCIS Vs. parenchyma and IDC Vs. DCIS.

Methods and Materials: In the prospective analysis of the 60 consecutive patients (performed between March 2010 and September 2012), with histologically confirmed DCIS $(n=30)$ or IDC $(n=30)$ (DCE-MRI, DWI, 1.5 T, gadoxetic acid), we analysed the intralesional and the parenchymal PEI values and calculated the tumour-to-parenchyma ratio. Range values, graphs and maps are provided by the software-package for diagnostic use.

Results: In our series, the PEI values in the IDC group $(748.7 \pm 32.2)$ were significantly different than those in the DCIS group (428.0 \pm 25.0$),(p<0.0001)$. The differences were also significant between the IDC and the parenchyma $(748.7 \pm 32.2$ Vs. $74.6 \pm 17 ; p<0.0001)$ and between the DCIS and the parenchyma $(428.0 \pm 25.0$ Vs. $66.0 \pm 10.6 ; p<0.0001$ ). Average breast parenchyma PEI value in both groups $(n=60)$, was 70.3+-/15.2. The tumour-to-parenchyma ratio was also significantly different between the groups IDC Vs. DCIS: $10.1 \pm 2.4$ Vs. $6.6 \pm 1.4(p<0.0001)$. Conclusion: As the quantitative parameter, $\mathrm{PEI}$ and tumour-to-parenchyma ratio may be helpful in differential diagnosis of DCIS Vs. IDC, with the standard DCEMRI exam and other parameters of fMRI. To our knowledge, this is the first paper testing the PEI values in the differential diagnosis: DCIS Vs. IDC.

\section{B-0567 11:42}

DCIS at image-guided needle biopsy: can breast MRI pre-operatory predict invasivity?

M. Marcon, V. Bertani, P. Clauser, E. Di Gaetano, V. Londero, A. Linda, R. Girometti, C. Zuiani, M. Bazzocchi; Udine/IT (magda.marcon@gmail.com)

Purpose: To assess MRI features predictive of IDC in patients with a diagnosis of DCIS at percutaneous biopsy.

Methods and Materials: We enrolled 72 patients (72 lesions) with biopsy-proven DCIS who underwent dynamic-MRI and subsequent surgical excision between 2009 and 2012. Two experienced readers in consensus retrospectively evaluated $\mathrm{MRI}$ images considering lesion morphology (mass-like vs non mass-like), size (210 $\mathrm{mm}$ vs $>10 \mathrm{~mm})$, enhancement-rate $(2100 \%$ vs $>100 \%)$ and dynamic curves (I, 
II and III). We compared the association of the above features in two groups of patients: those with pure DCIS and those with DCIS and an invasive component (DCIS+IDC) at surgical specimens. Analysis was performed using Chi-square test. Results: Patients underwent MRI after and before percutaneous biopsy in 51 $(70.8 \%)$ and $21(29.2 \%)$, respectively. Final diagnoses were pure DCIS in 47 patients $(65.0 \%)$ and DCIS+IDC in 25 patients $(35.0 \%)$. The presence of IDC was significantly $(p<0.05)$ associated with a contrast-enhancement rate $>100 \%$ $(65.2 \%$ in IDC vs $27.0 \%$ in DCIS). Invasion was higher in mass-like $(52.0 \%$ in IDC vs $29.7 \%$ in DCIS) than in non-mass-like lesions (48.0\% in IDC versus $70.3 \%$ in DCIS), although the difference was not significant ( $p>0.05)$. No further significant differences were found between the two groups ( $p>0.05$ ). Of note, $12 / 72$ lesions (8.0\% of DCIS+IDC and $21.3 \%$ of pure DCIS) were excluded from the analysis because of the absence of contrast-enhancement.

Conclusion: IDC in patients with a bioptic diagnosis of DCIS is associated with an enhancement rate $>100 \%$.

\section{B-0568 11:51}

Breast dynamic MRI in patient with DCIS percutaneous biopsy diagnosis: are different grades associated with specific features? V. Bertani, M. Marcon, P. Clauser, A. Linda, V. Londero, R. Girometti, C. Zuiani, M. Bazzocchi; Udine/IT (valeriabert@hotmail.it)

Purpose: To assess MRI features predictive of low and intermediate grade versus high grade and microinvasive DCIS.

Methods and Materials: We enrolled 47 patients (47 lesions) with biopsy-proven DCIS who underwent dynamic-MRI and subsequent surgical excision between 2009 and 2012. Two experienced readers in consensus retrospectively evaluated MRI images for the presence/absence of contrast-enhancement, lesion morphology (mass-like vs non mass-like), size $(210 \mathrm{~mm}$ vs $>10 \mathrm{~mm})$, enhancement rate $\left({ }^{2} 100 \%\right.$ vs $>100 \%$ ) and dynamic curves (I, II and III). We compared the association of the above features in two groups of patients: those with low and intermediate grade DCIS (set-1) and those with high grade and microinvasive DCIS (set-2) at surgical specimens. Analysis was performed using Chi-square test.

Results: Final diagnoses were 3 low grade (6.4\%), 17 intermediate grade (36.2\%), 18 high grade $(38.3 \%)$ and 9 microinvasive (19.1\%) DCIS. Although no significant differences in the above features were found in the two groups, a tendency to an enhancement rate $>100 \%$ and lesions size $>10 \mathrm{~mm}$ was observed in set- $2(31.8 \%$ in set- 2 vs $20.0 \%$ in set- 1 for enhancement rate; $86.4 \%$ in set- 2 vs $73.3 \%$ in set- 1 for size lesions). Of note, $10 / 47$ lesions $(25.0 \%$ in set- 1 and $18.5 \%$ in set-2) were excluded from the analysis because of the absence of contrast enhancement. Conclusion: We observed no MRI features predictive of low and intermediate grade vs high and microinvasive DCIS.

\section{B-0569 12:00}

Triple negative breast cancers (TNBCs) versus non-triple negative breast cancers (non-TNBCs) diagnosed during a high-risk screening program: sensitivity of imaging modalities and patient outcome R.M. Trimboli ${ }^{1}$, F. Podo ${ }^{2}$, F. Santoro ${ }^{2}$, F. Sardanelli3; ${ }^{1}$ San Donato Milanese/IT, ${ }^{2}$ Rome/IT, ${ }^{3}$ Milan/IT (trimboli.rm@gmail.com)

Purpose: To compare TNBCs with non-TNBCs diagnosed during a high-risk screening program

Methods and Materials: Forty-four patients were diagnosed with invasive cancers, $14(32 \%)$ TNBCs and $30(68 \%)$ non-TNBCs. Sensitivities of modalities, age at diagnosis, grade, size, nodal status, loco-regional relapse, disease-free survival (DFS), and breast cancer related death (BCRD) were evaluated. Statistics: MannWhitney, Fisher exact, $\mathrm{X}^{2}$.

Results: While of 14 TNBCs, 13 (93\%) were invasive ductal carcinomas (IDCs), of 30 non-TNBCs, $15(50 \%)$ included lobular subtype and/or DCIS component $(p=0.005)$. All 3 interval cancers were TNBCs. Sensitivity for mammography was $43 \%$ for TNBCs and $59 \%$ for non-TNBCs ( $p=n . s$ )., for ultrasound $57 \%$ and $62 \%$ ( $p=n . s)$., for $M R I 75 \%$ and $96 \%(p=0.043)$. MRI was always more sensitive than mammography or ultrasound. Of 14 TNBCs, $10(71 \%)$ were observed in BRCA1 $1^{+}$, of 30 non-TNBCs, only $9(30 \%)(p=0.028)$. Median age at diagnosis was 49 and 53 years, mean tumor size was 1.6 and $1.2 \mathrm{~cm}$, respectively. Eleven TNBCs (79\%) and 8 non-TNBCs $(27 \%)$ were grade $3(p=0.002)$. Nodal status was negative for $12 / 14$ TNBCs $(86 \%)$ and $16 / 30$ non-TNBCs $(53 \%)(p=0.038)$. Loco-regional relapses were observed for $1 / 13$ TNBCs (8\%) and $5 / 27$ non-TNBCs (19\%), 7 years DFS in $8 / 13(62 \%)$ and $17 / 27(63 \%)$, BCRD for $2 / 13(15 \%)$ and $3 / 27(11 \%)$, respectively. Distant recurrence was reported for 2 non-TNBCs only.

Conclusion: MRI outperformed mammography/ultrasound in both TNBCs and non-TNBCs. TNBCs were associated with BRCA $1^{+}$and grade 3 . Loco-regional relapse, DFS and BCRD were similar. These data support entering women at high risk of TNBC $\left(B R C A 1^{+}\right)$in screening programs including MRI.
10:30 - 12:00

Room I/K

Abdominal Viscera

SS 901

Cross-sectional pancreatic imaging and biomarkers

Moderators:

B. Marincek; Cleveland, OH/US

G.A. Zamboni; Verona/lT

\section{B-0570 10:30}

Dynamic contrast MR imaging of the pancreas under free breathing in combination with navigator technique

T. Masui', M. Katayama ${ }^{1}$, K. Sato ${ }^{1}$, K. Terauchi', K. Tsukamoto', K. Mizuki', Y. Iwadate ${ }^{2}$, H. Sakahara 1 , H. Kabasawa $2{ }^{2}{ }^{1}$ Hamamatsu/JP, ${ }^{2}$ Hino/JP (masui@sis.seirei.or.jp)

Purpose: In aged patients who cannot hold breaths, image quality of dynamic contrast MR might not be acceptable. The purpose was to evaluate feasibility of free breathing dynamic contrast MR imaging using navigator technique for pancreas. Methods and Materials: Twenty-six patients (age 73 years, pancreatic cystic lesions: 19 cases 40 lesions, solid 2 cases 2 lesions, none 5) underwent Gdcontrast-enhanced MR imaging for pancreas at 3 T (Discovery750, GEHC). Pre and 5 phases of postcontrast images (Gd-chelate, $0.1 \mathrm{mmol} / \mathrm{kg}$, injection $3 \mathrm{ml} / \mathrm{sec}$ ) were obtained using 3D fatsat FSPGR (LAVA) under free breathing with navigator technique. Image quality, blurring, recognition of each organ, and lesions were evaluated using five-point scale (5 good-1 poor). Imaging time for each phase, signal intensities (SIs) of aorta, portal vein, pancreas, and spleen, and sizes of lesions were measured. Contrast ratios were defined as SI on postcontrast/precontrast image. Existence of septa and nodules in lesions was evaluated.

Results: All images with navigator were diagnostic (image quality 4.4-4.7, blurring 4.3-4.7, lesion recognition 4.5-4.6). Imaging time was 38 to $46 \mathrm{sec}$. The enhancement was observed initially in aorta followed by spleen, pancreas. All cystic (19 cases, 40 lesions), and solid lesions (2 cases, 2 lesions) were identified (diameter $13.7 \mathrm{~mm}, 1 \mathrm{~mm}$ to $52 \mathrm{~mm}$ ). Septa in cysts were recognised in 10 of 19 cases. There was no nodule in cystic lesions.

Conclusion: With navigator technique, dynamic contrast MR imaging of pancreas can be successfully obtained with acceptable image quality and lesion recognitions under free breathing. Shorter acquisition times may be required.

Author Disclosures:

Y. Iwadate: Employee; GE Healthcare. . H. Kabasawa: Employee; GE Healthcare.

\section{B-0571 10:39}

Diffusion-weighted magnetic resonance imaging of healthy pancreas: a study to investigate the effect of age on apparent diffusion coefficient values

C. Ma, C. Pan, H. Zhang, H. Wang, J. Wang, S. Chen, J. Lu; Shanghai/CN (mengqihi@gmail.com)

Purpose: To investigate and characterise the effect of age on apparent diffusion coefficient (ADC) values in healthy pancreas.

Methods and Materials: A total of 559 patients with healthy pancreas, ranging from 20 to 81 years of age, were included in this study. Breath-hold single-shot echo-planar diffusion weighted imaging (DWl; $b$-value $=0,500 \mathrm{~s} / \mathrm{mm} 2$ ) was employed to determine the ADCs across all patients. Dependency of ADCs on age was characterised using a Spearman rank-order correlation test.

Results: Across the age spectrum, it is shown that there is no significant correlation between the mean $A D C s$ with age (correlation values $=0.03706, P=0.3818$ $>0.05$ ) or with age groups (correlation values $=0.03048, P=0.4721>0.05$ ) Conclusion: Our findings suggest that the effect of age can be excluded from the clinical diagnosis of pancreatic diseases, and that changes in ADCs are likely indicative of pancreatic pathology. 


\section{B-0572 10:48}

Pancreatic cystic lesion fluid content virtual analysis by means of acoustic radiation force impulse (ARFI) ultrasound imaging: fact or fiction?

S. Canestrini, M. D'Onofrio, R. De Robertis, S. Crosara, E. Demozzi, G. Puntel, R. Pozzi Mucelli; Verona/IT (mirko.donofrio@univr.it)

Purpose: To prospectively evaluate the application of ARFI implemented with virtual touch tissue quantification in the study of pancreatic cystic lesions, using different analysis methods, compared with the final diagnosis (pathological or by MRI/EUS findings).

Methods and Materials: 38 patients with pancreatic cystic focal lesion (diameter $>3 \mathrm{~cm}$ and located at a depth $<5.5 \mathrm{~cm}$ ) were included in the study and underwent conventional US. For every patient 5 measurements with virtual touch quantification $\mathrm{ROI}$ were performed. In order to distinguish mucinous (potentially malignant) from serous cystic lesions (mainly benign) the result "XXXX/0" was considered meaning simple liquids (reportable to water), and the accuracy of virtual touch tissue quantification in differentiating cystic-pancreatic lesions was calculated. To consider the lesion as containing complex fluids (potentially mucinous), two different reading methods have been applied: at least two numerical values when performing five measurements; prevalence of numerical values irrespective of the number of measurements. Sensitivity, specificity, positive and negative predictive values and accuracy were calculated for the differential diagnosis between mucinous versus non-mucinous cystic lesions.

Results: Sensitivity, specificity, PPV, NPV and accuracy in the group of cystic lesions using the first reading method were, respectively, $68.8 \%, 77.3 \%, 68.8 \%$, $77.3 \%, 73.7 \%$. Sensitivity, specificity, PPV, NPV and accuracy in the group of cystic lesions using the second reading method were, respectively, $37.5 \%, 100 \%$, $100 \%, 68.8 \%, 73.3 \%$.

Conclusion: ARFI with virtual tissue touch quantification can have a role in noninvasive characterisation of pancreatic cystic lesion during the conventional US examination.

\section{B-0573 10:57}

Role of perfusion $\mathrm{CT}$ in the evaluation of pancreatic masses

R. Vasudevan, M. Kang, R. Gupta, D. Bhasin, S. Rana, N. Khandelwal; Chandigarh/IN (ravisankarv85@gmail.com)

Purpose: To evaluate the efficacy of CT perfusion for detection of malignancy in pancreatic masses.

Methods and Materials: 27 patients (pts) who presented with a mass in the pancreas were enrolled in this Institute Review Board-approved prospective study after informed consent and underwent biphasic CECT with perfusion CT of the pancreas. Pts were followed up and results of cytopathological diagnosis obtained. Final diagnosis consisted of malignancy (adenocarcinoma) in 11, various benign masses in 13 and neuroendocrine tumour in 3 pts. Control group consisted of 15 pts who were referred for $\mathrm{CT}$ for an indication other than pancreatic pathology. 11 perfusion parameters were calculated using software MIStar 3.2 (Apollo imaging software). CT perfusion parameters were compared between the control and study groups as well as results of cytopathological diagnosis using ANOVA and Dunnett 2-sided t-test for statistical analysis.

Results: All perfusion parameters except extraction (E) were significantly different between adenocarcinoma and benign groups. All perfusion parameters except permeability (PS) were also statistically significantly different between adenocarcinoma group and controls. Blood flow (BF), blood volume (BV), extraction fraction product (EFP), PS and peak enhancement intensity (PEI) showed decreasing trend in values, whereas rest of the parameters showed an increasing trend from normal to benign to adenocarcinoma. Neuroendocrine tumours had different perfusion values with significantly elevated BF, BV, PEI and earlier time to peak (TTP). One patient with pancreatic tuberculosis had perfusion values paralleling adenocarcinoma but with relatively earlier TTP.

Conclusion: Perfusion CT seems to be a useful tool for differentiating between malignant and benign pancreatic masses.

\section{B-0574 11:06}

Comparison between CT and CEUS in the diagnosis of pancreatic adenocarcinoma

S. Crosara, M. D'Onofrio, R. De Robertis, S. Canestrini, R. Pozzi Mucelli; Verona/lT (mirko.donofrio@univr.it)

Purpose: To compare CEUS and CT features of pancreatic ductal adenocarcinoma in relation to tumour size.
Methods and Materials: 133 patients with pathological diagnosis of pancreatic adenocarcinoma, studied with CEUS and MDCT, have been enrolled. Two radiologists evaluated tumour size, site and imaging appearance. Sensitivity of the two methods was compared using McNemar test.

Results: In 9/133 patients neither MDCT nor US/CEUS could identify the lesion; in 9/133 patients only MDCT and in 13/133 only US/CEUS could identify the lesion. In the remaining 102 patients, both MDCT and US/CEUS were positive. US/CEUS sensitivity was $86.47 \%$, while MDCT was $83.58 \%$, with no statistically significant difference ( $p=0.523$ ). For lesions smaller than $2 \mathrm{~cm}$ US/CEUS sensitivity was $100 \%$, while MDCT sensitivity was $73.33 \%$ with no significant difference $(p=0.125)$. For lesions between 2.1 and $3 \mathrm{~cm}$ US/CEUS had a sensitivity of $95.35 \%$, while MDCT had a sensitivity of $83.72 \%$ with no significant difference, $p=0.180$ ). For lesions between 3.1 and $4 \mathrm{~cm}$, US/CEUS had a sensitivity of $87.88 \%$, while MDCT had a sensitivity of $93.94 \%$ with no significant difference $(p=0.688)$. For lesions larger than $4 \mathrm{~cm}$, US/CEUS had a sensitivity of $90.91 \%$, while MDCT had a sensitivity of $100 \%$ with no statistically significant difference $(p=0.250)$.

Conclusion: US/CEUS sensitivity in diagnosing pancreatic adenocarcinoma does not statistically differ from that of MDCT. US/CEUS sensitivity seems to be higher for small lesions, while MDCT sensitivity is higher for large lesions. By combining both the imaging methods a higher accuracy in diagnosing pancreatic carcinoma can be expected.

\section{B-0575 11:15}

High-resolution diffusion-weighted imaging of pancreatic ductal adenocarcinoma using 2D reduced field of view single-shot echo planar imaging at 3.0 Tesla

C. Ma, Y. Li, H. Wang, S. Chen, J. Lu; Shanghai/CN (mengqihi@gmail.com)

Purpose: To demonstrate the feasibility of high-resolution DWI of pancreas using $2 \mathrm{D}$ reduced field of view (RFOV) single-shot echo-planar sequence in the evaluation of pancreatic ductal adenocarcinoma.

Methods and Materials: 50 normal volunteers and 50 patients with histopathologically proven pancreatic ductal adenocarcinoma by surgery were included in the study. High-resolution DWI of pancreas was obtained using a multi-section 2D RFOV single-shot echo-planar sequence on the axial plane (FOV $16 \times 8 \mathrm{~cm}^{2}$, matrix 128×64). ADC of the normal pancreas in control group and pancreatic ductal adenocarcinoma group were measured. Mann-Whitney U-test has been used to compare ADC values between tumoral tissues and normal pancreatic tissues of the volunteers.

Results: On the high-resolution DW images, all pancreatic ductal adenocarcinoma demonstrated high signal intensity relative to the surrounding pancreatic parenchyma. The mean and standard deviations of the ADC values $\left(\times 10^{-3} \mathrm{~mm} 2 / \mathrm{s}\right)$ were as follows: pancreatic ductal adenocarcinoma $(n=50), 1.53 \pm 0.26(1.02-2.23)$, and normal pancreas in normal volunteers $(n=50), 1.98 \pm 0.25(1.60-2.73)$. ADC of the pancreatic ductal adenocarcinoma were significantly higher compared with those of normal pancreas $(U=230.000, P=0.000)$.

Conclusion: RFOV DWI shows advantages in higher resolution $\left(1.25 \times 1.25 \mathrm{~mm}^{2}\right)$ and blurring reduction compared to full FOV DWI of the pancreas. It makes a statistically significant difference on the ADC measurements of the pancreatic ductal adenocarcinoma when comparing to the control group. This approach is promising to benefit the early diagnosis of pancreatic cancers.

\section{B-0576 11:24}

Imaging features of acinar cell cystadenoma: can we differentiate them from branch duct IPMNs?

C. Delavaud ${ }^{1}$, G. D'Assignies ${ }^{1}$, J. Cros ${ }^{1}$, P. Ruszniewski', P. Hammel', A. Couvelard ${ }^{2}$, V. Vilgrain 1 , M.-P. Vullierme ${ }^{1 ;}{ }^{1}$ Clichy sur Seine/FR, ${ }^{2}$ Paris/FR (christophe.delavaud@hotmail.fr)

Purpose: Acinar cystic cystadenoma (ACC) of the pancreas is a rare benign entity first described in 2002, defined by histological criteria. Radiographic appearance had almost not been described so far. Most of the patients underwent surgical resection under the preoperative diagnosis of intraductal papillary mucinous neoplasms. The aims of this study are to define imaging diagnostic criteria of ACC based on radiopathological confrontation and to compare clinical, biological and imaging data between patients with ACC and with branch ducts IPMN.

Methods and Materials: All patients with ACC who underwent pancreatic surgery for suspicion of IPMN and the 20 last patients with histologically proven branch ducts IPMN were retrospectively included. Clinical and biological information were collected from the medical reports. Radiological and histological documents were reviewed in order to define imaging diagnostic criteria of ACC. Data were compared using the Chi-square test or Fisher's exact test. 
Results: ACC was symptomatic in all but one patient. There were no statistical difference between ACC and IPMN group with regard to clinical and biological data. Combination of four radiological criteria allowed differentiating ACC from IPMN: cyst calcification, presence of more than 5 cysts, clustered peripheral small cyst, and absence of communication with main pancreatic duct. Sensibility and specificity were, respectively, 75 and $100 \%$ with combination of at least three of these criteria. Conclusion: ACC is a rare benign pancreatic tumour with specific imaging features despite some similarities with IPMN. Recognition of this entity may help us to propose the diagnosis and prevent extensive surgery.

\section{B-0577 11:33}

Diffusion-weighted 3 T MR imaging with b-multiple SE-EPI in the differential diagnosis of cystic pancreatic lesions

P. Boraschi, F. Donati, R. Gigoni, G. Gherarducci, U. Boggi, F. Falaschi,

C. Bartolozzi; Pisa/IT (p.boraschi@do.med.unipi.it)

Purpose: To evaluate the usefulness of diffusion-weighted 3 T MR imaging with b-multiple SE-EPI in providing an objective value for the differentiation of cystic pancreatic lesions.

Methods and Materials: A series of eighty-six patients with cystic pancreatic lesions of at least $1 \mathrm{~cm}$ in size identified at US and/or CT and twenty normal subjects underwent MR imaging at 3 T device (GE DISCOVERY MR750; GE Healthcare). After the acquisition of axial T1w and T2w sequences and coronal MRCP, diffusionweighted MR imaging was performed using an axial respiratory-triggered spin-echo echo-planar sequence with multiple b values $\left(150,500,1000,1500 \mathrm{sec} / \mathrm{mm}^{2}\right)$ in all diffusion directions. ADC value was calculated by using a dedicated software fitting the curve obtained from the corresponding $A D C$ for each $b$ value. Fitted $A D C$ values were calculated by two observers in conference for each cystic pancreatic lesion and for normal pancreatic parenchyma. Imaging results were correlated with surgery, ERCP, and/or imaging follow-up.

Results: Final diagnoses included intraductal papillary mucinous tumour (IPMT) $(n=58)$, serous cystoadenoma $(n=18)$, and mucinous cystoadenoma $(n=10)$. Fitted $A D C$ value was $1.33 \times 10-3 \mathrm{~mm} 2 / \mathrm{sec}$ for normal pancreatic parenchyma, $3.10 \times 10-3$ $\mathrm{mm} 2 / \mathrm{sec}$ for IPMT, $2.57 \times 10-3 \mathrm{~mm} 2 / \mathrm{sec}$ for serous cystoadenoma, $2.93 \times 10-3 \mathrm{~mm} 2 /$ $\mathrm{sec}$ for mucinous cystoadenoma. Fitted ADC values were significantly higher in mucinous neoplasms than in serous cystoadenomas $(p<0.05)$.

Conclusion: Our results suggest that diffusion-weighted $3 \mathrm{~T}$ MR imaging with b-multiple SE-EPI may be helpful to differentiate mucinous from serous cystic pancreatic lesions. Further investigations in larger series of patients are necessary to confirm our data.

\section{B-0578 $11: 42$}

Cystic fibrosis in juveniles and adults: association of fatty involution of the pancreas at MDCT with CFTR genetic mutation and insulindependent diabetes mellitus

U.G. Mueller-Lisse, N. Ashoori, H.A. Strube, M. Schmitz, R. Fischer, R.M. Huber, M.F. Reiser, E.M. Coppenrath; Munich/DE

Purpose: Fatty involution of the pancreas (FIP) and insulin-dependent diabetes mellitus (IDDM) are characteristic features of cystic fibrosis (CF). We hypothesised that there was no association of FIP or IDDM with particular mutations at the CFTR gene locus.

Methods and Materials: MDCTs of the chest (4-64 rows, $120 \mathrm{KVp}, 10-150 \mathrm{mAs} /$ slice, CTDIw appr. 1.0-10.0 mGy, effective dose appr. 0.5-5.0 mSv) of 111 consecutive juvenile and adult CF-patients (52 females, 59 males, age, 14-51 years, mean \pm sd, $33 \pm 8$ years) were retrospectively reviewed for complete FIP versus remnant (RP) or normal pancreas (NP). Association with different CFTR-genelocus-mutations, IDDM state and patient age was statistically evaluated with Fisher's-exact-test, chi-square-test, McNemar's-chi-square-test, or Student's-t-test (two-tailed, $p<0.05$ )

Results: FIP was found in 88 CF-patients, RP in 10 , and NP in 13 , respectively. FIP was significantly associated with homozygous $(61 / 66)$ rather than heterozygous (17/34) dF508-CFTR-mutation (chi-square, 21.1287, p < 0.001). RP and NP were similarly frequent in homozygous dF508-CFTR-mutation and other CFTR-gene constellations ( 3 and 2 versus 10 and 8 , respectively, $p=0.3825$ ). RP and NP were significantly associated with heterozygous R347P-CFTR-mutation (4/4) versus homozygous dF508-CFTR-mutation $(5 / 66, p=0.00014)$. FIP and IDDM were not independent of each other (McNemar's-chi-square, 0.0167, p>0.5). FIP was not age-dependent $(\mathrm{p}=0.1239)$.

Conclusion: In juvenile and adult CF-patients, FIP is frequently found, particularly along with homozygous dF508-CFTR-mutation. Heterozygous R347P-CFTR-mutation may be pancreas-protective. FIP and IDDM are statistically not independent of each other. Pancreatic imaging may help to detect early signs of FIP in children with CF, although it is unclear if this would help to prevent IDDM.

\section{B-0579 11:51}

Pancreatic atrophy in $\beta$-thalassaemia population: morphological MR evaluation and correlation with pancreatic functional parameters C. Liguori, F. Pitocco, I. Di Giampietro, P. Cianciulli, B. Beomonte Zobel; Rome/IT (c.liguori@unicampus.it)

Purpose: To assess correlation between MRI iron deposition of the pancreas with those of the heart, liver and adrenals in patients with $\beta$-thalassaemia. To assess pancreas parenchyma atrophy prevalence coupling results with pancreatic endocrine and exocrine functional levels and ferritin levels.

Methods and Materials: We retrospectively evaluated $113 \beta$-thalassaemia patients ( 83 major and 30 intermedia, mean age $37.8 \pm 12.8$ years, mean BSA $1.64 \pm 0.24$ $\left.\mathrm{Kg} / \mathrm{m}^{2}\right)$ using $\mathrm{T} 2^{*} \mathrm{MRI}$ to assess iron content in the heart $\left(\mathrm{T}^{*}<20 \mathrm{~ms}\right)$, liver $(<$ $6.3 \mathrm{~ms})$, pancreas and adrenals ( $<26 \mathrm{~ms})$. MRI results were compared with one another and with endocrine and exocrine pancreatic serum enzyme levels and with Ferritin levels. Moreover pancreatic atrophy (TrueFISP sequences; qualitative grading) was evaluated.

Results: $\mathrm{T}^{*}$ measurements were possible in all patients regarding heart, liver and adrenals; pancreas $\mathrm{T}^{*}$ evaluation was impossible in case of moderate-severe atrophy. Cardiac and pancreatic values were very poorly correlated $(r=0.3, p<$ $0.001)$. Stronger correlation between pancreas and liver $(r=0.6, p<0.001)$ and adrenals $(r=0.6, p<0.001)$ was found. High prevalence of pancreas atrophy was noted $(29$ pts, $25 \%)$ with a majority of severe pancreatic atrophy $(70 \%)$ in young subjects. Pancreatic T2* was weakly correlated to ferritin levels. Good correlation between pancreatic $\mathrm{T}^{*}$ and pancreatic enzyme levels was noted. All patients with pancreatic atrophy showed an impaired pancreatic function.

Conclusion: Pancreatic iron overload do not correlate significantly with cardiac siderosis whether shows a better correlation with abdominal organs iron deposition. $\beta$-thalassaemia patients show higher prevalence of pancreatic atrophy with consequential pancreatic functional impairment.

10:30 - 12:00 Room N/O

\section{Cardiac}

\section{SS 903}

\section{Myocardial perfusion and coronary artery disease}

Moderators:

F. Cademartiri; Monastier di Treviso/IT

G.I. Kirova-Nedialkova; Sofia/BG

\section{B-0580 10:30}

Dual bolus dynamic contrast-enhanced cardiac perfusion imaging: comparison of eight reported analysis methods

B.M. Müller-Bierl, K. Tanaka, N. Buls, Y. Fierens, T. van Cauteren, I. Willekens, R. Luypaert, J. De Mey; Brussels/BE (bernd.mueller-bierl@uzbrussel.be)

Purpose: To compare eight reported methods for the analysis of cardiac perfusion flow data from 3 Tesla MRI on a porcine model.

Methods and Materials: An anaesthetized healthy minipig was repeatedly scanned (x 5) with a 14-day interval with Turbo FLASH. Voxel size was $2.00 \times 2.47 \times 8 \mathrm{~mm}^{3}$ and prepulse delay time was $\mathrm{Tl}=110 \mathrm{~ms}$. Total scan time for 120 scans was 2:00 min. Prebolus and bolus CA were $1.2 \mathrm{ml}$ and $6 \mathrm{ml} 0.5 \mathrm{mmol} / \mathrm{ml}$ GdDTPA (Magnevist) with an injection speed of $4 \mathrm{ml} / \mathrm{sec}$. Perfusion was assessed in 6 segments of the mid-ventricle short axis view from the heart with eight reported methods: (1) fermi, (2) model-free deconvolution, (3) modified tofts, (4) exchange, (5) uptake, (6) tofts, (7) patlak, and (8) upslope. We assessed the concordance of the methods by regression analysis and by comparing medians and distribution of the data. Results: The highest correlation $\left(R^{2}>0.9\right)$ can be observed for methods 1-3, 5, 6 . Those were considered as a reference. Method 7 underestimates the flow (slope 0.34 ). The median values of the methods $1-3,5$ match within an interval of \pm 2 SD the median of the reference $(56.0 \mathrm{ml} / 100 \mathrm{ml} / \mathrm{min})$. The $S D(3.05 \mathrm{ml} / 100 \mathrm{ml} / \mathrm{min})$ was computed from the 5 methods. Perfusion flow assessed by the methods 1-3, 8 was observed to be not significantly different from the reference $(p<0.01$, related samples Wilcoxon rank test).

Conclusion: Perfusion analysis methods can yield different results. Our data suggest that the methods $1-3$ are most concordant. 


\section{B-0581 10:39}

Image quality and radiation exposure of low-dose dual-source dynamic CT stress myocardial perfusion Imaging: a randomised

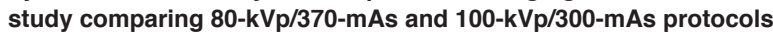
M. Fujita, K. Kitagawa, T. Ito, Y. Shiraishi, Y. Kurobe, M. Nagata, M. Ishida, H. Sakuma, K. Dohi; Tsu/JP (fujitam@clin.medic.mie-u.ac.jp)

Purpose: We determined the effect of reduced $80 \mathrm{kVp}$ tube voltage with increased $370 \mathrm{mAs}$ tube current on the radiation dose, image quality and estimated myocardial blood flow (MBF) of dynamic CT stress myocardial perfusion imaging (CTP) in patients with a normal body mass index (BMI).

Methods and Materials: Thirty consecutive patients with a normal BMI $(<25 \mathrm{~kg} /$ m2) with known or suspected coronary artery disease underwent adenosine-stress dynamic CTP using a dual-source CT scanner with alternating table positions (shuttle mode). Patients were randomised to $100-\mathrm{kVp} / 300-\mathrm{mAs}$ imaging $(\mathrm{n}=15)$ or $80-\mathrm{kVp} / 370-\mathrm{mAs}$ imaging $(\mathrm{n}=15)$. CT-estimated MBF map was generated using a model-based parametric deconvolution method. Two blinded readers graded image quality of the MBF map using a 4-point scale. Peak signal and noise of the left ventricular (LV) cavity and MBF of non-ischaemic myocardium were also compared. Results: Imaging with 80-kVp/370-mAs instead of $100-\mathrm{kVp} / 300-\mathrm{mAs}$ was associated with $40 \%$ lower radiation dose (mean dose length product, $359 \pm 66$ [range, 287-482] vs 628 \pm 112 [493-830] mGy[[Unable to Display Character: \&\#8901;]]cm; p $<0.001)$ with no significant difference in image quality of the MBF map $(3.3 \pm 0.6 \mathrm{vs}$ $3.4 \pm 0.9, p=0.627)$ or MBF of non-ischaemic myocardium $(0.93 \pm 0.27$ vs $1.01 \pm 0.32$ $\mathrm{ml} / \mathrm{min} / \mathrm{g} ; \mathrm{p}=0.543)$. Studies obtained using $80-\mathrm{kVp} / 370-\mathrm{mAs}$ were associated with $33 \%$ higher peak LV enhancement ( $803 \pm 203$ vs $603 \pm 104 \mathrm{HU} ; \mathrm{p}=0.002$ ), and $23 \%$ greater noise $(22.3 \pm 3.5$ vs $18.1 \pm 3.6 ; p=0.001)$.

Conclusion: Dynamic CTP using 80-kVp/370-mA instead of $100-\mathrm{kVp} / 300-\mathrm{mAs}$ allowed $40 \%$ reduction in radiation dose with no significant difference in image quality or MBF. The use of $80 \mathrm{kVp}$ tube voltage should be considered in dose-reduction strategies for dynamic CTP of individuals with a normal BMI.

Author Disclosures:

H. Sakuma: Research/Grant Support; Siemens Japan.

\section{B-0582 10:48}

Feasibility of CT-based dynamic stress perfusion imaging to differentiate ischaemic and infarcted myocardium as compared with magnetic resonance imaging

F. Bamberg, R. Marcus, K. Hildebrandt, F. Schwarz,

D. Theisen, K. Bauner, A. Becker, T. Johnson, K. Nikolaou;

Munich/DE (fabian.bamberg@med.uni-muenchen.de)

Purpose: To determine the feasibility of CT-based myocardial perfusion imaging to differentiate ischaemic and infarcted myocardium as compared to magnetic resonance imaging (MRI).

Methods and Materials: We prospectively enrolled subjects with suspected coronary artery disease, who underwent adenosine-mediated stress myocardial perfusion imaging by dynamic dual-source CT ( $100 \mathrm{kV}, 320 \mathrm{mAs} / \mathrm{rot})$ and 3 T-MRI. Myocardial blood flow and volume (MBF and MBV, respectively) were derived from CT images, using a model-based parametric deconvolution method for 16 myocardial segments. These were related to perfusion defects visually assessed during rest/stress and delayed MRI acquisitions. Conventional measures of diagnostic accuracy and differences in MBF/MBV were determined in a blinded fashion. Results: Among 41 enrolled subjects, 31 (mean age $70 \pm 9$ years, $74 \%$ men) completed the protocol. The prevalence of ischaemic and infarcted segments detected by MRI was moderately high ( $11.6 \%$ and $12.4 \%$, respectively). Applying a MBF cut-point $(88 \mathrm{ml} / 100 \mathrm{ml} / \mathrm{min})$, the diagnostic accuracy of CT-MPI in detecting segmental ischaemic perfusion defects and infarcted segments, as defined by MRI, was good (sensitivity: 88.89\% [0.76-0.96], NPV: $80.77 \%$ [0.6-0.93] and sensitivity: 85.25\% [0.74-0.93], NPV: $97.05 \%$ [0.94-0.99], respectively), both showing moderate PPV ( $42 \%$ and $29 \%$, respectively). While in segment with excellent image quality there was no difference in MBF between ischaemic and infarcted myocardium $(p=0.8635), M B V$ was significantly lower in infarcted than in ischaemic segments $(p$ $<0.0001$ ). Adding MBV as a co-indicator to pathological MBV (cut-point $16 \mathrm{ml} / 100$ $\mathrm{ml}$ ) detected 23/31 transmural infarcted segments (sensitivity: 74.19\% [0.55-0.88]). Conclusion: MBV derived from dynamic CT perfusion imaging may help to differentiate infarcted from ischaemic myocardium.

\section{Author Disclosures:}

F. Bamberg: Research/Grant Support; Siemens Healthcare, Bayer Healthcare. Speaker; Siemens Healthcare, Bayer Healthcare. . K. Nikolaou: Research/ Grant Support; Siemens Healthcare, Bayer Healthcare. Speaker; Siemens Healthcare, Bayer Healthcare.

\section{B-0583 10:57}

Adenosine perfusion and late gadolinium enhancement at 3 Tesla in comparison to 1.5 Tesla for the detection of coronary artery stenosis T. Walcher, K. Ikuye, W. Rottbauer, J. Woehrle, P. Bernhardt; UIm/DE (peter.bernhardt@uniklinik-ulm.de)

Purpose: Cardiac magnetic resonance imaging (CMR) using a comprehensive approach consisting of adenosine perfusion and late gadolinium enhancement (LGE) at 1.5 Tesla has established for detection of relevant coronary artery disease (CAD). Little is known about the potential advantages of 3 Tesla CMR to detect relevant CAD. Aim of our prospective study was to compare a clinical routine CMR protocol performed at both 1.5 and 3 Tesla in patients with suspected CAD undergoing coronary $\mathrm{x}$-ray angiography.

Methods and Materials: 52 patients $(62.3 \pm 10.2$ years) with suspected CAD were included into the study. All patients were scanned at both 1.5 and 3 Tesla including functional imaging, adenosine stress and rest perfusion, and LGE. CMR images were analysed by two blinded readers in consensus. X-ray angiography served as the reference method.

Results: Diagnostic accuracy of the combined analysis of perfusion and LGE imaging yielded better values at 1.5 and 3 Tesla than the analysis of perfusion images alone. Sensitivity and specificity at 3 Tesla were superior to 1.5 Tesla in detection of coronary stenoses $350 \%$ ( 0.90 vs. 0.75 and 0.84 vs. 0.75 ) and in detection of coronary stenoses $370 \%$ ( 0.88 vs. 0.80 and 0.96 vs. 0.89 ).

Conclusion: 3 Tesla CMR seems to be superior to 1.5 Tesla in detection of significant CAD, as shown in our routine clinical setting in a prospective study. Hence, 3 Tesla might become the preferred CMR field strength for evaluation of CAD in clinical practice.

\section{B-0584 11:06}

Myocardial perfusion defects and late gadolinium enhancement in asymptomatic subjects with subclinical atherosclerosis and mild coronary calcifications - results of the Heinz Nixdorf recall MRI substudy T. Schlosser, K. Nassenstein, P. Hunold, K.-H. Joeckel, J. Barkhausen, R. Erbel, B. Sievers; Essen/DE (thomas.schlosser@uni-due.de)

Purpose: The purpose of this study was to evaluate the prevalence of pathological cardiac MRI findings in subjects with mild coronary calcifications.

Methods and Materials: 101 asymptomatic volunteers were selected from a large population-based screening study in accordance with their Agatston calcium score (group 1: Agatston score: $0, n=51$, age $60.10+-6.41$; group 2: Agatston score: $1-100, n=50$, age $63.9+-6.33$ ). All subjects underwent cardiac MRI at $1.5 \mathrm{~T}$. The MRI protocol included a) functional assessment of the left ventricle, b) assessment of adenosine stress-/rest perfusion and c) quantification of late gadolinium enhancement (LGE).

Results: A stress-induced perfusion deficit was present in 1/101 subjects (1.0\%), whereas LGE was seen in 17/101 subjects (16.8\%). Group 1 (Agatston score: 0 ): In this group none of the subjects showed a perfusion deficit, whereas in seven subjects LGE was observed (13.7\%). Two of the seven subjects showed an ischaemic-type pattern of LGE (3.9\%) indicating chronic infarction. Group 2: median Agatston score of group 2 was 19 (Q1, 11; Q3, 43). In this group a perfusion deficit could be observed in one subject $(2.0 \%)$, whereas LGE was observed in ten subjects (20.0\%). However, all of these LGE patterns were assessed as non-ischaemic. Conclusion: The prevalence of stress-induced myocardial ischaemia in subjects with mild coronary calcifications score is low, whereas the frequency of non-ischaemic LGE is relatively high. Long-term follow-up will clarify whether these cardiac MRI findings contribute to cardiovascular risk stratification in asymptomatic subjects.

\section{B-0585 11:15}

Late enhancement multidetector computed tomography (LE-MDCT) assessment of scar substrate in recurrent ventricular tachycardia (VT) C. Colantoni, A. Esposito, A. Palmisano, F. De Cobelli, F. Baratto, G. Maccabelli, P. Della Bella, A. Del Maschio; Milan/IT (colantoni.caterina@hsr.it)

Purpose: Radiofrequency ablation (RFa) guided by electroanatomic-mapping (EAM) is a useful therapy in patients with recurrent-VT. Pre-treatment scar substrate assessment with cardiac-MR is important for EAM/RFa planning (subendocardial/ subepicardial), but most of these patients have ICD, contraindicating MR imaging. This study aimed to evaluate the effectiveness of LE-MDCT in the assessment of scar substrate in patients with recurrent-VT, comparing imaging findings with EAM. Methods and Materials: 26 patients with recurrent-VT underwent 64-slice-MDCT before EAM/RFa, including angiographic-scan and low-energy ( $80 \mathrm{kV})$ delayedscan (10 minutes after high-concentration iodine). MDCT scans were merged with EAMs and scar distribution at imaging was compared with scars at EAM. EAM/RFa 
approach (endocardial/epicardial) was chosen based on the prevalent distribution of scars at LE-MDCT.

Results: MDCT scans were prospectively ECG-triggered in 18 patients and retrospectively gated in 8.10 patients showed subepicardial LE suggestive for post-myocarditis scars; 14 patients had LE suggestive of post-ischaemic scars with subendocardial (4 patients) or transmural (10 patients) involvement; 1 patient had a large transmural area of LE in myocardial dystrophy; 1 did not show any scar at imaging. EAM (voltage $<0.5 \mathrm{mV}$ ) confirmed scars identified at LE- MDCT in $22 / 26$ pts (85\%): in 10 cases (45\%) there was perfect match between LE areas and low-voltage areas in EAM, in $12(55 \%)$ there was partial match and in $4(15 \%)$ there was no match. In all 22 patients with scars at imaging, RFa was always performed within LE areas.

Conclusion: LE-MDCT may represent an effective alternative for scar substrate assessment in patients with recurrent-TV, especially when ICD was previously implanted to prevent sudden death

\section{B-0586 11:24}

Dynamic CT perfusion imaging of the myocardium using a wide detector CT scanner: stress/rest or stress only

A.M. Huber ${ }^{1}$, V. Leber ${ }^{1}$, B. Gramer ${ }^{1}$, M. Vembar ${ }^{2}$, A. Leber ${ }^{1}$, E. Hoffmann ${ }^{1}$

E.J. Rummeny ${ }^{1} ;{ }^{1}$ Munich/DE, ${ }^{2}$ Cleveland, OH/US (armin.huber@tum.de)

Purpose: To determine the diagnostic accuracy of dynamic CT perfusion imaging of the myocardium in comparison to coronary angiography $(\mathrm{CA})$ and fractional flow reserve (FFR).

Methods and Materials: 32 consecutive patients were examined by dynamic CT perfusion imaging (14 consecutive data sets) using a 256-slice scanner with 8-cm detector without table movement under adenosine stress $(80 \mathrm{kV}, 250 \mathrm{mAs}$ per data set). The semiquantitative parameters time-to-peak (TTP), area-under-the-curve (AUC), upslope and peak-enhancement (PE) were determined after calculation of time-density curves. In addition, myocardial blood flow (MBF) was quantitatively determined. Results were compared with CA and FFR.

Results: The area under the ROC curve, sensitivity and specificity were 0.73 $41.7 \%$ and $90.0 \%$ for TTP, $0.67,58.3 \%$ and $83.3 \%$ for AUC, $0.90,83.3 \%$ and $93.1 \%$ for US, $0.85,83.3 \%$ and $93.3 \%$ for PE and $0.88,75.0 \%$ and $100 \%$ for MBF, respectively. The thresholds, determined by Youden's index, were $148.5 \mathrm{HU}^{*} \mathrm{~s}, 12$ $\mathrm{s}, 2.5 \mathrm{HU} / \mathrm{s}, 34 \mathrm{HU}$ and $1.64 \mathrm{ml} / \mathrm{g} / \mathrm{min}$, respectively.

Conclusion: The semiquantitative parameters PE, US and the quantitative parameter MBF showed a similar, high diagnostic accuracy. Therefore, currently it is unclear, if a dynamic examination can be considered as superior compared to a single shot examination at a potentially lower radiation dose.

\section{B-0587 11:33}

Advanced age and apnoea-hypopnoea index predict subclinical atherosclerosis in patients with obstructive sleep apnoea syndrome B. Arık ${ }^{1}$, C. Gümüş ${ }^{1}$, M. İnci ${ }^{2}$, M. Ege ${ }^{3}$, A. Zorlu ${ }^{4}$, K. Varol ${ }^{1}$, Ö. Doğan ${ }^{1} ;{ }^{1}$ Sivas/TR, ${ }^{2}$ Kahramanmaraş/TR, ${ }^{3}$ Ankara/TR, ${ }^{4}$ Malatya/TR (drfatihinci@gmail.com)

Purpose: Both obstructive sleep apnea syndrome (OSAS) and coronary artery calcification (CAC) are considered to be related with the presence of coronary artery disease. In this present study, we evaluate the association between OSAS and the presence of subclinical atherosclerosis assessed by tomographic coronary calcium score in patients who had OSAS but no history of known coronary artery disease. Methods and Materials: Seventy-three patients who were asymptomatic for coronary artery disease and had suspected OSAS were referred to overnight attended polysomnography. Patients were classified into 4 groups according to the Apnoea-Hypopnoea Index $(\mathrm{AHI})$. All patients underwent computed tomographic examination for tomographic coronary calcification scoring. Physical examination, sleep study recordings, complete blood count and serum biochemistry were obtained from all patients.

Results: There were mild to moderate, moderate to severe and severe correlation between the coronary calcium score and $\mathrm{AHI}$, basal oxygen saturation and oxygen desaturation index respectively. In univariate analysis, age, $\mathrm{AHI}$, basal oxygen saturation, oxygen desaturation index and diabetes mellitus were found to be univariate predictors of coronary artery calcification in patients with OSAS. In multivariate logistic regression model, age $(p=0.007, O R=1.082)$ and $A H I(p=0.013$, $\mathrm{OR}=1.034$ ) were only independent predictors of coronary artery calcification in patients with OSAS with a sensitivity of $88.9 \%$ and $77.8 \%$ and a specificity of $54.3 \%$ and $56.5 \%$, respectively.

Conclusion: Our findings suggest that in patients with moderate or severe OSAS and advanced age, physicians should be alert for the presence of subclinical atherosclerosis.

\section{B-0588 $11: 42$}

Prevalence of severe subclinical coronary artery disease on cardiac CT and MRI in patients with extra-cardiac arterial disease M.A.M. den Dekker', J.J.A.M. van den Dungen', I.F.J. Tielliu', R.A. Tio', R.M.J.J. Jaspers ${ }^{2}$, M. Oudkerk ${ }^{1}$, R. Vliegenthart ${ }^{1} ;{ }^{1}$ Groningen/NL, ${ }^{2}$ Deventer/NL (m.a.m.Dekker@rad.umcg.nl)

Purpose: Patients with extra-cardiac arterial disease (ECAD) are at high risk of coronary heart disease. We examined the prevalence of silent, significant coronary artery disease in patients with stenotic or aneurysmatic ECAD.

Methods and Materials: Cardiac asymptomatic ECAD patients without contraindications for computed tomography (CT) and magnetic resonance imaging (MRI) underwent coronary CT angiography (CCTA) and adenosine perfusion MRI (APMR). Calcium scoring was performed based on non-contrast CT. CCTA was positive when $350 \%$ lumen diameter stenosis in a coronary artery was found. Patients were referred to a cardiologist when CCTA showed a stenosis in the left main coronary artery (or equivalent), or when APMR showed inducible myocardial ischaemia.

Results: In total, 115 patients (mean age $65 \mathrm{yrs} ; 76 \%$ male) were included. Patients were divided into 2 categories: stenotic $(N=87)$ and aneurysmatic ECAD $(N=28)$. CCTA was positive in $38 \%$ in stenotic and $29 \%$ in aneurysmatic ECAD. No cCTA was performed in $20 \%$ due to excessive calcium. Two patients, 1 stenotic and 1 aneurysmatic ECAD had left main stenosis. APMR was positive in $12 \%$ in stenotic and $11 \%$ in aneurysmatic ECAD. Eighteen percent of stenotic and $21 \%$ in aneurysmatic ECAD have been referred to the cardiologist. A cardiac intervention was performed in $43 \%$ of stenotic and $33 \%$ in aneurysmatic ECAD. Significant stenosis or high calcium score was found in $58 \%$ in stenotic, compared to $50 \%$ in aneurysmatic ECAD. None of these outcomes were statistically significant. Conclusion: Silent, significant coronary artery disease is highly prevalent in patients with ECAD, either stenotic or aneurysmatic.

\section{B-0589 11:51}

Dual-energy CT with non-linear image blending improves visualisation of delayed myocardial contrast enhancement in acute myocardial infarction

A.H. Mahnken ${ }^{1}$, P. Bruners ${ }^{2}$, K. Kartje ${ }^{2}$, B. Schmidt ${ }^{3} ;{ }^{1}$ Marburg/DE, ${ }^{2}$ Aachen/DE, ${ }^{3}$ Forchheim/DE (mahnken@med.uni-marburg.de)

Purpose: To evaluate non-linear image blending in comparison with linear image blending in late phase dual-energy computed tomography (DECT) for the visualisation of delayed myocardial contrast enhancement in acute myocardial infarction (MI) and to identify optimal parameters for non-linear image blending.

Methods and Materials: Acute reperfused MI was induced in seven pigs by temporary occlusion of the left anterior descending or left circumflex artery. Two hours after reperfusion contrast-enhanced late phase DECT $(80 \mathrm{kV} / 140 \mathrm{kV})$ was performed. DECT data were post-processed with linear and non-linear image blending techniques. Contrast and percentage signal difference between healthy and infarcted myocardium as well as the blood pool of the left ventricle were computed for the linear and non-linear techniques including the low and high $\mathrm{kV}$ images. Data were compared using repeated measures ANOVA and post hoc t-tests.

Results: Non-linear blending showed the highest signal differences for all contrasts and analyses. Repeated measure ANOVAs confirmed these differences to be statistically significant for the different post-processing techniques ( $p$-value ranging from $<0.001$ to 0.003 ). Paired-samples post hoc t-tests proved the significance of these results ( $p$-value ranging from $<0.001$ to 0.037 ). The ideal settings for nonlinear image blending can be deduced from the CT values of the regions of interest in the linearly blended images with the weighting factor 0.3 .

Conclusion: Non-linear image blending improves the visualisation of acute $\mathrm{MI}$ in DECT. Non-linear image blending in late phase DECT of acute MI is superior to linearly blended images as well as source images obtained at $80 \mathrm{kV}$ or $140 \mathrm{kV}$.

Author Disclosures:

A.H. Mahnken: Research/Grant Support; Siemens Healthcare. Speaker; Bayer Pharma.B. Schmidt: Employee; Siemens Healthcare. 


\section{Paediatric}

\section{SS 912}

\section{Paediatric body and bones}

Moderators:

K.J. Johnson; Birmingham/UK

L.-S. Ording-Müller; Tromsø/NO

\section{B-0590 10:30}

Accuracy of ultrasound using a computerised technique to evaluate nonalcoholic fatty liver disease in obese and eutrophic adolescents as compared with MRI

J.H.R.N. Nascimento, R.B.S. Soder, M.E. Epifanio, M.B. Baldisserotto; Porto Alegre/BR (josehermesnascimento@gmail.com)

Purpose: To compare the accuracy of ultrasound (US) with computerised analysis of the hepatorenal gradient (HRG) to MRI for the evaluation of NAFLD in adolescents. Methods and Materials: This prospective transversal study evaluated 50 adolescents (aged 11-17 years), including 24 obese and 26 eutrophic individuals. All adolescents underwent computer-based US, MRI, laboratory exams, and anthropometric evaluation. Posterior generation of the receiver operating characteristic (ROC) curve was performed, and the area under the ROC curve was calculated to determine the cutoff point for the HRG and to predict levels of steatosis. The sensitivity, specificity, positive and negative predictive values and accuracy of US with computerised analysis were compared with those of NMRI as the gold standard. Results: The obese group Included $29.2 \%$ girls and $70.8 \%$ boys, and the eutrophic group included $69.2 \%$ girls and $30.8 \%$ boys. The prevalence of NAFLD was $19.2 \%$ for the eutrophic group and $83 \%$ for the obese group. The ROC generated for the HRG, with a cutoff point of 13 , had $100 \%$ sensitivity and $100 \%$ specificity. Use of this same cutoff point for the eutrophic group led to a false-positive rate of $9.5 \%$ ( $90.5 \%$ specificity) and false-negative rate of $0 \%$ (100\% sensitivity).

Conclusion: Ultrasound with computerised analysis of the HRG is a simple and noninvasive technique for the quantitative evaluation of hepatic ecogenicity that could be used to help identify obese and eutrophic adolescents with NAFLD. This method could be used in population tracking for NAFLD and for by clinical issues.

\section{B-0591 10:39}

Can ultrasonography reliably differentiate between ileo-colic and small bowel intussusception?

N. Simanovsky ${ }^{1}$, N. Lioubashevsky ${ }^{2}$, K. Rozovsky ${ }^{2}$, N. Hiller ${ }^{2} ;{ }^{1}$ Beth Horon/lL,

2Jerusalem/IL (natalias@hadassah.org.il)

Purpose: Sonographic findings for ileo-colic intussusception (ICI) and small bowel intussusception (SBI) are similar. We sought clinical and sonographic criteria that can be used to confidently differentiate these lesions.

Methods and Materials: We retrospectively evaluated ultrasound and clinical data for children diagnosed with intussusception in 2007-2011. Diameter of the intussusception, fat core diameter, outer bowel wall thickness, and the presence/ absence of lymph nodes inside the intussusception and mesentery were noted. The ratio of inner fat core (IFC) diameter to outer wall thickness (core-to-wall index [CWI]) was calculated. Characteristics for ICI and SBI were compared using the T-test, and Mann-Whitney, Levene, Chi-square, and Fisher's exact tests.

Results: There were 200 cases of intussusception in 174 consecutive patients (126 males/48 females; mean age 17.2 months; 57 SBI [28.5\%] $143 \mathrm{ICI}[71.5 \%]$ ). Mean ICl lesion diameter was $2.63 \mathrm{~cm}(1.4-4)$ vs $1.42 \mathrm{~cm}(0.8-3)$ for SBI. Mean ICI fat core diameter measured $1.32 \mathrm{~cm}(0.6-2.2)$ vs $0.1 \mathrm{~cm}(0-0.75)$ for SBI. The CWI was ${ }^{31}$ in all ICl, and [[Unable to Display Character: \&\#706;]]1 in all SBI (p[[Unable to Display Character: \&\#706;]]0.0001). Lymph nodes inside the lesion were seen in $90.7 \%$ of ICl vs $14.4 \%$ of $\mathrm{SBI}$ (p[[Unable to Display Character: \& $\# 706 ;]] 0.0001)$. Children with $\mathrm{ICI}$ had a more severe clinical picture with more vomiting $(p=0.003)$, leucocytosis $(p=0.003)$, and blood in the stool $(p=0.0005)$.

Conclusion: The presence of a central fatty core in the intussusception, its diameter and wall thickness, the ratio of fatty core thickness to outer wall thickness, and the presence of lymph nodes may reliably differentiate between ileo-colic and small bowel intussusceptions.

\section{B-0593 10:48}

Early-stage disease activity of the wrist in juvenile idiopathic arthritis: assessment with MRI in a pilot study

C.M. Nusman, R. Hemke, D. Schonenberg, J.M. van den Berg, K.M. Dolman, T.W. Kuijpers, M.A.J. van Rossum, M. Maas; Amsterdam/NL (c.m.nusman@amc.uva.nl)

Purpose: To assess feasibility and reliability of the Juvenile arthritis MRI scoring system (JAMRIS) for the wrist to evaluate early-stage disease activity, and to compare reliability of JAMRIS for the wrist for early-stage disease with the validated paediatric-targeted MRI scoring system (PTMRIS) for children with more progressive stage of disease.

Methods and Materials: MRI datasets from 20 juvenile idiopathic arthritis (JIA) patients with wrist involvement were evaluated independently by three readers using JAMRIS and PTMRIS. Assessment of early-stage disease activity in JAMRIS was achieved through extending soft tissue scoring items (synovial hypertrophy, tenosynovitis) and specifying the grading of bone scoring items affecting $<25 \%$ of the bone (bone marrow changes, bone erosions). Intraclass correlation coefficient (ICC) or Cohen's kappa was used to assess inter- and intrareader reliability. ICC of each JAMRIS scoring item was compared with the ICC of its equivalent in PTMRIS. Results: Three JAMRIS scoring items (synovial hypertrophy, bone marrow changes and bone erosions) showed moderate to good reliability: ICC varied from 0.62 to 0.89 for interreader reliability and 0.28-0.95 (reader 1 ) and 0.88-0.97 (reader 2) for intrareader reliability. Reliability of tenosynovitis was moderate to poor with Cohen's kappa varying from 0.24 to 0.48 (interreader) and 0.41 to 0.64 (intrareader). JAMRIS and PTMRIS showed similar results of interreader reliability.

Conclusion: Early JIA disease activity in the wrist can be reliably evaluated with three-out-of-four features of the simple and accurate JAMRIS scoring system. Correlating clinical disease assessment and responsiveness to change will be needed to validate JAMRIS for the wrist as outcome measure and disease monitoring tool.

\section{B-0594 10:57}

Clavicle radiographs in children: a waste of time and radiation?

M.R. Jackson, M.-P. Lirette, P. Leonard; Edinburgh/UK

(m.jackson@doctors.org.uk)

Purpose: Fractured clavicles are a common childhood injury but, in our experience, rarely require operative intervention. The purpose of this study was to evaluate if clavicle radiographs make any tangible difference to clinical management in the context of possible traumatic injury.

Methods and Materials: Retrospective analysis of all clavicle radiographs performed in our institution over the 4.5-year period since the introduction of PACS was undertaken. Radiographs performed for traumatic injury were reviewed to ascertain if a fracture was demonstrated, if follow-up radiographs were performed and if orthopaedic fixation was performed. Prospective subgroup analysis to correlate clinician confidence of the likelihood of fracture is ongoing at the time of submission. Results: Between Feb 2008 and August 2012, 995 clavicle radiographs were performed, of which 934 were taken in the context of possible acute traumatic injury. 625 demonstrated an identifiable clavicle fracture (67\%), with 22 radiographs showing equivocal findings or a bone injury outwith the clavicle. In those found to have clavicle fractures, 32 follow-up radiographs were performed in 26 individuals (4\%). Orthopaedic fixation was not performed in any of the identified cases. Conclusion: Despite a high pick-up rate of fractures, clavicle radiographs do not appear to substantially alter clinical management. In our centre, immobilization is the usual management for suspected traumatic clavicle injury, regardless of radiographic appearance. We suggest that, in the context of an experienced paediatric Emergency Department team, radiographs are reserved for patients causing concern at follow-up and are not required routinely at presentation.

\section{B-0595 11:06}

A follow-up study of a delayed approach to managing developmental hip dysplasia (DDH)

L. Woods, M. Khalil, A. Witwit, S. Manickam, M. Churchill, V. Cook; London/UK (liamwoods@doctors.org.uk)

Purpose: To assess the results of a delayed approach to managing DDH, a local system of 23 years duration.

Methods and Materials: 262 patients over 4 years with at least 3 ultrasound examinations of the hips were included, to identify follow-up patients. DDH type was recorded and treatment determined by patient records. The treatment group underwent follow-up radiography for up to 5 years.

Results: There were 12,840 live births, of which between 28 and $32 \%$ each year underwent ultrasonography of the hips based on inclusion criteria. Less than $0.06 \%$ 
( $n=221)$ had DDH necessitating 3 or more ultrasound examinations, of which $23 \%$ $(n=50)$ required treatment. There was one case of missed hip dislocation, representing $0.0001 \%$ of unexamined patients. No patients with IIC hips at presentation with conservative management represented with a dislocated hip. Radiographs in the treated group demonstrated a satisfactory outcome in $94 \%$. One patient represented with a dislocated hip following erroneous cessation of harness treatment and went on to develop early avascular necrosis. Two patients were lost to follow-up.

Conclusion: A delayed approach to managing DDH in infancy allows those patients with ultrasonographically immature hips the opportunity to develop normally without invasive treatment and identifies patients which would most benefit from treatment. This reduces the demand on resources that a DDH screening programme which treated patients more promptly would incur. Within the same financial constraints, this allows more patients to be selected for screening. A delayed approach results in a satisfactory outcome with no subsequent late dislocation in those treated appropriately.

\section{B-0596 11:15}

Value of lower-limb MRI in the staging and re-staging of post-treatment osteonecrosis in paediatric patients with lymphoproliferative disease

A. Masetto, D. Ippolito, P.A. Bonaffini, L. Longoni, A. Sala, A. Rovelli, S. Sironi; Monza/IT (dr.masetto@gmail.com)

Purpose: To assess the diagnostic value of $\mathrm{MRI}$ in detecting and monitoring post-treatment osteonecrotic lesions in paediatric patients with lymphoproliferative disease.

Methods and Materials: 109 patients (60 males, mean age 12 years) with lymphoproliferative disease treated with chemotherapy (71) or bone marrow transplant (38), and with clinical suspicion of lower limbs osteonecrosis, underwent a lowerlimb MRI examination between November 2006 and August 2012. The study was performed on a $1.5 \mathrm{~T}$ scanner (Philips, Achieva), acquiring short tau inversion recovery (STIR) long TE and T1-weighted sequences in the coronal plane from the hips to the ankles, with an average acquisition time of 18 minutes. Additional sequences were performed in selected cases (e.g. restaging known osteonecrosis of the hip with articular deformity).

Results: On the baseline or follow-up MRI, 72/109 patients showed osteonecrosis, with a total of 424 lesions (5 hips, 195 femurs, 19 patellae, 159 tibiae, 7 fibulae, 39 ankle and foot bones). 151/424 involved articular surface and 33 lesions had articular deformity. 58/109 patients had osteonecrosis on the baseline study: 35/58 underwent follow-up and 7 of them developed articular deformity. 51/109 patients did not show osteonecrosis at baseline examination and 15 of them developed at follow-up examination osteonecrotic lesions (4 with articular deformity). Moreover, 8 patients showed concomitant neoplastic lesions.

Conclusion: Our MRI protocol proved to be quick and feasible in detecting and monitoring osteonecrosis in haematologic paediatric patients, impacting both their prognosis and their management in terms of treatment and follow-up.

\section{B-0597 11:24}

Painful paediatric hip: frog-leg lateral view only

J. Bomer, F. Klerx, H.C. Holscher; Den Haag/NL (j.bomer@hagaziekenhuis.nl)

Purpose: In cases of hip complaints in the paediatric population it is common practice to acquire both $\mathrm{AP}$ and frog-leg lateral (FL) radiographs. The combination of these views has a diagnostic yield, but also doubles the radiation exposure. In our observation, the FL view alone is often diagnostic. We therefore investigated the diagnostic accuracy of obtaining a solitary FL view as compared to a combination of the AP and FL views.

Methods and Materials: Children aged 2-15 years who were sent in for hip radiographs between September 2007 and January 2011 were included in the study. Cases of related trauma or a history of hip pathology were excluded. Radiographs were retrospectively assessed by two independent radiologists. Radiologists were blinded for the AP radiograph while assessing the FL radiograph and vice versa and radiographs were presented in a random order. We used the Kappa test to calculate interobserver agreement and agreement between the assessment of both views and the solitary FL view.

Results: 524 children (mean age $8.1 \mathrm{yrs}$ ) met the study criteria and were included for analysis. 477 children had a normal exam and 47 were considered abnormal. Calculated interobserver agreement using the Kappa test was 0.96. Agreement between the solitary FL view and the combination of the AP/FL view was 0.989 . Conclusion: The diagnostic accuracy of the FL radiograph in case of hip complaints in children is as high as the current standard of both AP and FL views.

\section{B-0598 11:33}

DXA as a key instrument for the evaluation and the follow-up of adolescents affected by anorexia nervosa

F. Ciccarese, G. Facchini, E. Di Pietro, F. Moscano, L. Iero, E. Franzoni, G. Battista, A. Bazzocchi; Bologna/IT

Purpose: Anorexia nervosa (AN) is an eating disorder characterised by alteration of bone metabolism (osteopenia) and of body composition (reduction and abnormal distribution of fat mass-FM and lean mass-LM), due to oestrogen deficiency and self starvation. The aim of our study was to address bone metabolism and body changes in a cohort of patients with AN by using Dual-energy x-ray-Absorptiometry (DXA). Methods and Materials: Prospective study on 75 patients with AN, submitted to DXA at baseline-T0 and after 12months-T12. We evaluated total and regional FM and LM percentages, as well as lumbar bone mineral density-BMD and Z-score, linking them to clinical variables: menarche/amenorrhea/hormonal therapy and physical activity.

Results: Among the 46/79-58.2\% patients that completed the study the T0 clinical condition was as follows: $\mathrm{BMI}=16.4 \pm 1.4 \mathrm{~kg} / \mathrm{m} 2$, low levels of $\mathrm{FM}$ percentage $(21.7 \pm 5.7 \%)$, osteopenia (Z-score:-1.21 $\pm 1.27 \mathrm{SD}$, with higher values related to physical activity- $p=0.001$ ). At T12: significant increase in BMI- $p=0.001$, with LM reduction and FM increase (more evident in the trunk- $p<0.001$ ); regarding bone, no significant changes were observed, though a tendency in terms of improvement associated with resumption of menses was manifested.

Conclusion: After one year, significant conclusions on bone metabolic condition could not be reached. By contrast, weight recovery was associated with the increase of FM and a distortion of its distribution, more evident in trunk region (potential and adjunctive risk factor for the relapse of the psychiatric condition). The complexity of these clinical findings suggested that DXA, a low-dose and low-cost technique, could have a key role in long-term monitoring of AN patients.

\section{B-0599 11:42}

Replacing conventional spine radiographs with dual-energy x-ray absorptiometry (DXA) in children with suspected reduction in bone density E. Adiotomre, L. Summers, P. Broadley, I. Lang, G. Morrison, A. Offiah; Sheffield/UK (eseadio@googlemail.com)

Purpose: Children have greater lifetime risks of radiation-induced complications compared to adults. In children with osteogenesis imperfecta conventional radiographs are obtained to assess spine morphometry, while DXA assesses bone density. In adults DXA is used for both. We aim to establish whether iDXA can replace spine radiographs in assessment of paediatric vertebral morphometry. Methods and Materials: An 18-month prospective study of 200 consented children (5 to 15 years old) with and 50 without suspected reduction in bone density, who had lateral spine radiographs and lateral DXA scans on the same day, was conducted. Three observers will independently assess all images (blinded to corresponding results of radiographs and DXA) using a modified $A B Q$ technique. 100 random images will be interpreted twice. Diagnostic accuracy and inter- and intraobserver reliability of DXA will be compared to the gold standard of radiography. Patient/carer experience, DXA radiation dose compared to radiographs and health economics will be assessed.

Results: Interim analysis of the first 50 recruited patients showed 26 had 1 or more vertebral fractures. The fracture detection sensitivity for DXA was $62.7 \%$ and specificity $87.8 \%$. The overall accuracy for vertebral fracture detection with DXA was $83.5 \%$. The overall agreement between radiographs and DXA was $68.7 \%$. Conclusion: The modified $A B Q$ technique has limitations in children and a more reliable scoring system is required. Compared to radiography DXA does not appear to be worse for morphometry and given the reduced dose and comparable image quality, DXA should be recommended for morphometry in children pending more robust analyses. 


\section{Radiographers}

\section{SS 914}

\section{Dose optimisation as daily challenge}

Moderators:

P. Blackburn Andersen; Kolding/DK

A. Petakovic; Novo Mesto/SI

\section{B-0600 10:30}

Estimating organs cancer incidence related to patient radiation exposure following $\mathrm{PCl}$ for acute and chronic coronary total occlusion

D. Maccagni; Milan/IT (maccagni.davide@gmail.com)

Purpose: To estimate the lifetime attributable risk (LAR) of cancer incidence for individual organs following radiation exposure during $\mathrm{PCl}$ in the context of two opposite sides of angiographic spectrum of coronary occlusive disease: ST-elevation myocardial infarction (STEMI) and chronic coronary total occlusion (CTO).

Methods and Materials: We identified all consecutive patients treated with $\mathrm{PCl}$ for STEMI $(n=555)$ and for CTO $(n=543)$ in a tertiary care centre in 6 years. The LARs of cancer incidence for six organs (colon, liver, lung, red bone marrow, stomach and thyroid) were estimated using the Biological Effects of lonising Radiation (BEIR) VII model.

Results: The estimated LARs of cancer incidence for individual organs was found to markedly increase as the age of the patient decreases and was significantly higher for the lung (additional risk up to 18/100.000 person's lifetime exposure in CTO and 9/100.000 in STEMI patients, respectively, $p<0.0001)$ and for the red bone marrow (up to $3.5 / 100.000$ and $1.5 / 100.000$, respectively, $p<0.0001$ ). In both the groups, the estimated LAR of cancer incidence for stomach, colon, liver and thyroid was similar and very low.

Conclusion: In $\mathrm{PCl}$ procedures, the lung was the organ with the highest radiation absorbed. According to BEIR-VII model, the number of additional cancer cases for individual organs was on average two times higher in patients treated with $\mathrm{PCl}$ for CTO and the highest LARs were for lung and red bone marrow cancers.

\section{B-0601 10:39}

Project retake: quality assurance of radiation hygiene by maintaining image quality

\section{S.M. Khan; OsIo/NO (SAHIRA.M.KHAN@HOTMAIL.COM)}

Purpose: To reduce the extent of retake while maintaining image quality. Methods and Materials: The research was conducted in two digital $x$-ray labs, data for six months were analysed by quantitative method. Patient data: id, information of retakes was stored at modality, post-examination. 750 random patients, undergoing standard $\mathrm{x}$-ray examinations, were selected each month. A standard examination contains two or three exposures, front oblique and/or lateral. Radiographers were encouraged to choose a cause for retake. Analysis contained: type of examination, number of projections, number of exposures and retake reason per patient. The causes are systemised in the modality, ready to select if there is a retake. Reject options were radiographer, patient or student failure.

Results: There was an average of 234 retaken images out of 750 examinations. That is to say, $30 \%$ margin of error. $28 \%$ of the standard examinations were retakes; the reject option was radiographer failure. Rest $2 \%$ was patient or student failure. As a result of this a PGMI test is established for the digital labs to test quality assurance. Results after this quality assurance give a reduction of 110 retakes per month. A reduction of $50 \%$ retakes due to radiographer failure.

Conclusion: Environment, method, monitoring and feedback, influence radiographer result and radiation dose to patients. Radiographers using digital $\mathrm{x}$-ray need quality assurance as in mammography. PGMI usage will give feedback to maintain image quality and reduce radiation dose.

\section{B-0602 10:48}

Implementation of a quality control program in a radiology department M. Leão, L.P.V. Ribeiro, A.F.C.L. Abrantes, J. Pinheiro, R.P.P. Almeida, K.B. Azevedo, S.I. Rodrigues; Faro/PT (rui_ppa@hotmail.com)

Purpose: To evaluate the importance that radiographers give to quality control programs, radiological protection and the verification of radiological protection criteria. Methods and Materials: A 30-item quality control assessment and radiological protection questionnaire was applied to radiographers working on public hospitals and healthcare centres. Statistical analysis was made using descriptive statistics, Mann-Whitney and Kruskal-Wallis tests in order to verify correlation between items. Results: Radiographers consider that it is essential the implementation of a quality control program in the radiology department, but there is lack of information in this field, and $53.2 \%$ do not know what a quality control program is. $96 \%$ of radiographers claim their institutions do not have quality control programmes or accreditation. However, $25.5 \%$ had received education on quality and $85 \%$ make regular personnel radiation measures. Also, despite there is no lack of radiological protection equipment in most departments, $77.4 \%$ claim they are in poor condition. Conclusion: Quality control programs are essential to the radiology department and radiation safety guidelines must be implemented. Despite that some radiology departments have radiological protection equipment often applied by radiographers, their effectiveness and condition must be checked regularly.

\section{B-0603 10:57}

Assessment of radiological imaging conformities based in quality control charts

S. Cândido, A.M. Ribeiro, L.P.V. Ribeiro, A.F.C.L. Abrantes, J. Pinheiro, K.B. Azevedo, R.P.P. Almeida; Faro/PT (susanacandido@msn.com)

Purpose: Applying control charts to demonstrate the importance of implementing a radiological imaging quality control system.

Methods and Materials: Research conducted in a public hospital comprising a total of 1110 randomly selected exams (640 chest studies, 340 abdominal studies and 130 foot studies) grouped in 37 samples, each one with 30 exams. The conformities and non-conformities found were used to establish three types of quality control charts based on the evidence collected, namely the proportion of conformities and non-conformities $(p)$, the total number of non-conformity exams $(\mathrm{np})$ and the total number of non-conformities in each sample (c) in order to suggest corrective actions for improvement.

Results: Considering all exams, $57 \%$ are non-conformities and $43 \%$ are within conformities. Of 640 chest studies, $40 \%$ were within conformities and $60 \%$ were non-conformities; of 340 abdominal studies, $46 \%$ were within conformities and $54 \%$ were non-conformities. Foot studies had an almost even distribution of conformities and non-conformities with 51 and $49 \%$, respectively.

Conclusion: This research allowed the identification of different types of nonconformities found in radiological images which have impact on imaging quality. Therefore, the existence of adequate quality control of radiographic imaging is essential.

\section{B-0604 11:06}

A review of diagnostic imaging frequency to aid research exploring the issue of consent for higher dose paediatric examinations J.L. Portelli', J. McNulty ${ }^{2}$, S. Mohan ${ }^{2}$, P. Bezzina', L. Rainford ${ }^{2} ;^{1}$ Msida/MT, ${ }^{2}$ Dublin/IE (jonathan.portelli@um.edu.mt)

Purpose: Concerns have grown in recent years in relation to potential radiationrelated risks associated with diagnostic imaging examinations performed on paediatrics ( $<18$ years). This retrospective cohort study aims to provide an insight into the use of potentially higher dose computed tomography (CT) and fluoroscopyguided examinations in paediatrics, in two national clinical centres of comparable catchment areas in Malta and Ireland.

Methods and Materials: Retrospective data, of higher dose diagnostic imaging examinations performed in paediatrics in 2011, was collected from departmental logbooks and radiology information system (RIS) databases of the participating centres. This data included details pertaining to patient's age, type of examination and relevant examination $\mathrm{kV}, \mathrm{mAs}$, DAP, fluoroscopy time, CTDI and DLP (if available). Data were categorised according to age and examination type, and effective dose estimates for CT were calculated.

Results: In 2011 alone, at least 2,093 CT examinations, 1,532 fluoroscopy-guided procedures and 438 cardiac catheterisation interventions were performed on paediatrics across both centres. Particular complex fluoroscopy-guided interventions had an associated DAP exceeding 10,000cGy.cm2, while effective dose estimates for CT examinations indicated that some paediatric patients may receive a moderate $(>3-20 \mathrm{mSv})$ or high radiation dose (>20-50mSv).

Conclusion: Paediatric patients may undergo examinations that potentially involve a high dose of ionising radiation. Further research to explore the debated issue of benefit-risk communication and consent in paediatric imaging is therefore justified and encouraged, particularly as the study's findings also indicate that repeat or multiple imaging examinations of different anatomical areas are commonly performed in CT. 


\section{B-0605 $11: 15$}

An approach for Portuguese diagnostic reference levels for bedside chest radiography

A. Sousa, S. Serém, J. Santos, G. Paulo; Coimbra/PT

(sarajoanacs@hotmail.com)

Purpose: Bedside chest radiography represents the most commonly performed examination. Taking into account the different protocols and equipments it is important to develop diagnostic reference levels, in order to promote a patient safety culture and consequently health care best practice.

Methods and Materials: This research was carried out in two imaging department centres from the same geographic region (Hospital A and Hospital B). In phase one, the exposure parameters and entrance skin dose (ESD) value of 40 bedside standard patients' chest radiography were collected. To optimise the obtained dose values in Hospital $B$ more 15 bedside standard patients' chest radiography were collected on phase two, using parameters of Hospital A, that had already went through an optimisation process.

Results: In phase one Hospital A ESD value was statistically lower $(16.45 \mu \mathrm{Sv})$ in comparison to Hospital B $(21.94 \mu \mathrm{Sv})$. In phase two Hospital B decreased significantly the ESD value $(17 \mu \mathrm{Sv})$ in comparison with the phase one, but with no significant differences with Hospital A.

Conclusion: Patients in Hospital B at phase one were $25 \%$ more exposed to ionising radiation than patients from Hospital A for the same procedure. The implementation of optimised protocols at phase two reduced the dose levels in $29.1 \%$ in the Hospital B. The regional DRL for bedside chest radiography was set at $17 \mu \mathrm{Sv}$.

\section{B-0606 11:24}

An investigation into the relationship between the exposure index value and image quality

M.-L. Butler, L. Rainford; Dublin/IE (marielouise.butler@ucd.ie)

Purpose: 'Exposure creep' is a documented phenomenon in computed radiography (CR) where radiation dose is given unnecessarily to patients due to CR's processing capabilities. Manufacturers have responded by developing an exposure index (EI), which is a measurement of detector dose and hence relates to noise in the image. This study aimed to identify how the El value relates to image quality in clinical practice.

Methods and Materials: Three imaging suites and five radiographic examinations were included: ankle and wrist series (antero-posterior (AP) and lateral); AP and lateral lumbar spine; AP pelvis and postero-anterior (PA) chest projections. In total, 306 examinations were documented with a minimum of 10 patients for each projection. Twelve patient images representative of each projection were selected according to their Agfa lgM value; 3 low, median and high lgM values and images recording $3 \mathrm{lgM}$ values closest to the manufacturers' guidelines. Three observers, experienced radiographers (minimum of 6 years working clinically), reviewed the images for noise and overall exposure of the image. In addition the observers indicated what El value they judged appropriate to image presentation.

Results: No significant difference in image quality was noted across the categories of $\operatorname{lgM}$ when considering noise (Chi-Square $(12)=19.265, p=0.082$ ) or exposure (Chi-Square (12) $=18.012, \mathrm{p}=0.115)$. IgM values judged by observers as representative of image quality were lower than those recorded clinically.

Conclusion: No correlation with IgM and clinical image quality was identified in this study. Further research is recommended to include additional manufacturers and radiographic examinations.

\section{B-0607 11:33}

Attenuation of anode heel effect with an aluminum filter and their influence on patient dose in lumbar spine radiography

J. Soares, R. Dores, P. Sousa, S.I. Rodrigues, L.P.V. Ribeiro, A.F.C.L. Abrantes, R.P.P. Almeida; Faro/PT (joana.soares.8@hotmail.com)

Purpose: Study the attenuation of anode heel effect with an aluminum filter, thereby optimising the image quality, without increasing the patient dose exposure. Methods and Materials: This study was performed in a radiology department with a conventional radiology equipment. The filter construction was based on the radiographic study of the lumbar spine in a lateral position, and used the following technical parameters: $80 \mathrm{kV}, 10 \mathrm{~mA}, 100 \mathrm{~ms}$ and $90.2 \mathrm{~cm}$ of SID. Experiments were performed to study the behaviour of anode effect along the longitudinal anodecathode axis. Measurements of dose rate using the Detetor Unfors Xi RF was carried out. This detector also allowed performing the equipment quality control. In order to standardise the variation in beam intensity of $x$-ray filters were used with different thicknesses of aluminum to reduce the value of the dose rate and to determine the thickness of the filter construction. After construction of the filter, viability was tested by performing two tests on the lateral position of the lumbar spine, with a phantom.

Results: The results obtained using the aluminum filter resulted in a reduced variation in relative dose rate $(9 \%)$ compared to not using the filter as well as a $32 \%$ reduction in dose to the entrance surface of the skin.

Conclusion: Regarding the image quality, it has been found that the use of filter presents significant reasons the optimisation of this parameter. These results indicate that the use of the filter should be considered as a good practice in the lumbar spine radiography to achieve dose reductions.

\section{B-0608 11:42}

Antero-posterior (AP) pelvic radiography: collimator errors and their effects on radiation dose

H. Brookfield 1 , A.S. Manning-Stanley ${ }^{2}$, A. England $1{ }^{1} ;{ }^{1}$ Liverpool/UK, ${ }^{2}$ Salford/UK (aengland@liverpool.ac.uk)

Purpose: To investigate the range of collimator errors in $x$-ray rooms and to calculate their possible effects on the radiation dose for AP pelvis examinations. Methods and Materials: A collimator test tool was suspended at three heights (14, 21 and $28 \mathrm{~cm}$ ) above the table bucky in nine $x$-ray rooms. Heights corresponded to the patient thickness (mean, $\pm 2 S D$ ) in 100 patients. The $x$-ray beam was visually collimated to the inner boundary of the test tool and exposed to radiation. Differences between the visualised field size and the resultant $x$-ray field size (corrected for magnification) would indicate any collimator errors. On the pelvic phantom minimum textbook collimation was set and then changed in order to simulate a range of possible collimator errors. Phantom examinations used a standard technique with exposure termination using outer AEC chambers. Dose-area-product (DAP) was recorded.

Results: Out of nine $x$-ray rooms all but one produced a smaller irradiated area than was visually set. Errors ranged from a $16 \%$ reduction in irradiated field size to a slight over irradiation by $0.4 \%$. With the possibility that these errors could be larger in other institutions a range of $(-27 \%$ to $+18 \%)$ errors were simulated. Increasing the field size by $1 \mathrm{~cm}$ (superior/inferiorly) increased the DAP by $5 \%$. Laterally, a 1 $\mathrm{cm}$ increase caused a $4 \%$ rise. Increases of $1 \mathrm{~cm}$ in both planes raised DAP by $4 \%$. Conclusion: In this study collimator errors were minimal and favoured under irradiation. Small errors can affect DAP and are more dose significant in the superior/inferior plane.

\section{B-0609 11:51}

Breast shielding significantly reduces breast dose during lumbar spine radiography

N. Mekiš, D. Žontar, D. Škrk; Ljubljana/SI (nejc.mekis@zf.uni-lj.si)

Purpose: As use of protective shielding for the patient in conventional radiography varies widely in practice, the study aimed at providing objective answer about the effect of lead rubber sheet shielding for a specific case of breast dose during the lumbar spine radiography. Influence of the body mass index on the breast dose was also investigated.

Methods and Materials: The study was conducted both on an anthropomorphic phantom and on a cohort of 100 female patients, randomly divided into two equal groups. In both cases lumbar spine imaging was conducted in AP and lateral projection with and without the lead shield (in case of the patient study the shielding was used in one group only). The dose was measured by thermoluminescent dosimeters (TLD) placed at the centre of the breast.

Results: Using the lead shielding dose to the right breast was reduced from $0.45 \pm 0.25 \mathrm{mGy}$ to $0.09 \pm 0.07 \mathrm{mGy}(p<0.001)$ and the dose to the left breast from $0.26 \pm 0.14$ mGy to $0.06 \pm 0.04$ mGy $(p<0.001)$, compared to the unshielded breast. On average, the breast dose was reduced by approximately $80 \%$. No correlation between the body mass index (BMI) and the breast dose was found.

Conclusion: Based on the results, we can conclude that the use of breast shielding is recommended in lumbar spine radiography. Despite the low-dose exposure even without shielding, the dose can be further reduced. Moreover, the quality of the x-ray image is not affected by the use of the lead shield. 


\section{Computer Applications}

\section{SS 905}

\section{Computer-aided diagnosis}

Moderators:

T. Mang; Vienna/AT

D. Regge; Turin/IT

\section{B-0610 10:30}

Automated characterisation of pulmonary nodules in thoracic CT images using a segmentation-based classification system

C. Jacobs ${ }^{1}$, E.M. van Rikxoort ${ }^{2}$, J.-M. Kuhnigk' ${ }^{1}$ E.T. Scholten ${ }^{3}$, P.A. de Jong ${ }^{4}$, C. Schaefer-Prokop ${ }^{5}$, M. Prokop ${ }^{2}$, B. van Ginneken ${ }^{2} ;{ }^{1}$ Bremen/NL, ${ }^{2}$ Nijmegen/NL, ${ }^{3}$ Haarlem/NL, ${ }^{4}$ Utrecht/NL, ${ }^{5}$ Amersfoort/NL (colin.jacobs@mevis.fraunhofer.de)

Purpose: Clinical guidelines for follow-up of pulmonary nodules depend on nodule type and therefore accurate characterisation of nodules is important. A novel computer-aided diagnosis (CAD) system to distinguish solid, part-solid and nonsolid nodules is presented and evaluated on a large data set from a lung cancer screening trial.

Methods and Materials: The automated characterisation system is based on a previously published nodule segmentation algorithm. Four different parameter settings were used to extract the solid part, non-solid part and solid core of the lesion. For each segmentation, volume, mass, average density, $5^{\text {th }}$ percentile and $95^{\text {th }}$ percentile of densities inside the segmentation were used as features. A k-nearestneighbour classifier was used to classify nodules. The accuracy of the system to differentiate between solid and subsolid nodules, between solid, part-solid and non-solid nodules and between part-solid and non-solid nodules was evaluated. A data set consisting of 137 low-dose chest CT scans (16x0.75 mm, 120-140 kVp, $30 \mathrm{mAs}$ ) with 52 solid, 50 part-solid and 50 non-solid nodules was collected from a screening trial. The nodule type recorded in the screening database was used as the reference standard. Experiments were performed in leave-one-nodule-out cross-validation.

Results: The accuracy of CAD to differentiate between solid and subsolid nodules was 0.88 . Differentiation into solid, part-solid and non-solid nodules gave an accuracy of 0.72 . CAD had an accuracy of 0.71 in differentiating part-solid from non-solid nodules.

Conclusion: Automated characterisation of pulmonary nodules shows good performance. This can aid radiologists to decide on appropriate workup in clinical practice.

\section{Author Disclosures:}

C. Jacobs: Research/Grant Support; MeVis Medical Solutions AG.

\section{B-0611 10:39}

Semiautomatic estimation of right ventricular parameters from MR

images: a new stereological approach

M. Mazonakis, K. Pagonidis, C. Varveris, J. Damilakis; Iraklion/GR

(mazonak@med.uoc.gr)

Purpose: Right ventricular (RV) volume and ejection fraction (EF) assessment is of clinical value. This procedure, based on manual delineation of the endocardial contours on MR images, is time-consuming due to ventricle's complex shape. We introduce a stereological method for estimating RV parameters from MRI data without the need for image segmentation.

Methods and Materials: Twenty-two consecutive patients with coronary artery disease underwent cardiac MRI. Stereological estimations involved the automatic placement of a systematic grid of test points with random orientation over shortaxis images. All points hitting the RV were counted using the semiautomatic point selection process. The optimum point spacing of the grid was defined. The software automatically provided the RV volumes and EF. Two independent observers evaluated the measurement reproducibility. Stereological estimations were compared with those determined by manually tracing RV contours.

Results: Acceptable estimations of RV parameters with a mean coefficient of error of $6.9 \pm 1.3 \%$ were obtained by counting an average of only 12 points per image. The mean time of stereological analysis was $3.7 \pm 0.4 \mathrm{~min}$, whereas the intraobserver and interobserver variability was $3.2-5.2 \%$ and $4.6-7.8 \%$, respectively. The mean differences between stereology and manual tracing for estimating the end-diastolic volume, end-systolic volume and EF were small and equal to $4.1 \pm 8.0 \mathrm{ml}, 2.1 \pm 4.6$ $\mathrm{ml}$ and $-0.4 \pm 3.5 \%$, respectively. The parameters obtained by the two methods were not significantly different ( $p>0.05)$, and they were strongly correlated $(r>0.88)$.
Conclusion: The stereological approach enables the quick estimation of RV parameters with high accuracy and reproducibility from MR images.

\section{B-0612 10:48 \\ Automated computerized software for diameter and volume measurements of pulmonary metastatic disease: preliminary evaluation} E. Lotan, D. Aharoni, S. Raskin, B. Boursi, R. Berger, E. Konen; Ramamt Gan/IL

Purpose: Serial CT scans of metastatic tumours are vital in assessing the efficacy of cancer treatment. We evaluated the performance of a novel automated computerized software system that integrates registration, segmentation and tumour measurement into a single process to expedite RECIST measurement with the addition of volume measurement.

Methods and Materials: Measurements of long and short-axis of 50 lung metastatic lesions in 18 sequential patients with metastatic renal cell carcinoma were manually obtained twice by 3 radiologists. Those measurements were compared with automated software measurements (Carestream Health, Rochester, NY). In addition, the software automatically identified the lesions' contours and volumes; the readers could accept the automated measurement or correct it manually. Intraobserver correlation was assessed by intraclass correlation coefficient (ICC), and interobserver reliability by the concordance correlation coefficient (CCC) and Bland-Altman limits of agreement.

Results: The intraobserver agreements (ICC) for long/short axis and volume were in range of 0.878-0.943/0.905-0.948 and 0.984-0.995, respectively. The interobserver agreement (CCC) between radiologists' and software measurements for the long axis ranged from 0.836 to 0.947 vs 0.820 to 0.989 between different radiologists, for the short axis $0.923-0.974$ vs 0.8390 .955 , and for volume $0.911-0.941$ vs 0.968-0.991. The mean relative difference of software vs radiologists' measurements corresponds to $2.5 \%$ overestimated volume ( $95 \% \mathrm{Cl}=98.4 \%, 106.8 \%)$ of the software compared to radiologists' evaluation.

Conclusion: Automated computerized software might have an important and reliable role in current RECIST evaluations, expanding its availability and efficiency, as well as providing potential additional benefit of automated volume determination for other future response criteria.

\section{B-0613 10:57}

Computerised morphological estimation of sex and age - from subjective investigation to computer-assisted analysis

A. Vlcek, F. Kanz, W. Weninger, J. Streicher, D. Risser, F. Kainberger; Vienna/AT (alexander.vlcek@meduniwien.ac.at)

Purpose: The aim of this feasibility-study was to develop a computerised method for sex estimation with its age-associated changes to the skeleton on macerated skulls for comparison with subjective assessments applying the osteoscopic Knußmann scheme.

Methods and Materials: 24 skulls of Europeans, 12 males (aged 23 to 77 years, mean 45.58 years) and 12 females (aged 22 to 75 years, mean 46.17 years) from local historical collections, were scanned with a 64-row multidetector CT (pitch=0.64) and modelled with AMIRA 5.4. to calculate the percentual deviation of the local concavity or convexity of 5 sex specific traits (frontal bone zygomatic bone, mastoid process, planum nuchale and mentum) as measured with the shape index. Based on that different surface shapes and two indices were defined and sex differentiating formulas were developed with the regression analysis. This computer-assisted technique was compared with the Knußmann scheme and the documented sex with the two-sided Fisher's exact test.

Results: Performing the regression analysis an accuracy of $100 \%$ was reached at the frontal bone, the mentum, the planum nuchale and the right zygomatic bone, but it ranged between 75 and $100 \%$ at the left zygomatic bone and the mastoid process bilateral. The application of the Knußmann scheme ranged between 4.2 and $100 \%$. Besides that changes to the surface could be demonstrated partly as a feature during adultery.

Conclusion: Results show, that the computerised technique may be superior to subjective definitions. The lower accuracies may be reasoned by the variability of existing sutures and age.

\section{B-0614 11:06}

Semiautomatic evaluation of quantitative pharmacokinetic parameters - systematic evaluation of a new CAD-tool and evaluation of clinical performance at high field $3 \mathrm{~T}$ MRI M. Dietzel, A. Dörfler; Erlangen/DE

Purpose: Widespread use of quantitative perfusion-MRI is still hampered by sophisticated post-processing and lack of commercially available semi-automatic software 
solutions. Accordingly, dedicated software was developed. We evaluated its clinical performance in a series of brain tumours imaged with state-of-the-art 3 T-MRI.

Methods and Materials: Seventeen enhancing brain tumours were investigated (Magnetom Trio/3 T; dynamic T1-weighted-sequence/TWIST: temporal-resolution: 1.77s, in-plane-resolution: 1.2*1.1*1.1 mm³ $10 \mathrm{ml}$ Dotarem@2 ml/s; anatomicsequence/MPRAGE: in-plane-resolution: $1.0 \mathrm{~mm}^{3}$ ). Post-processing was performed to test a dedicated commercially available CAD-tool. Amongst other pre-processing features it implements [1] Elastic 3D-Motion-correction (dynamic-contrastenhanced scans), [2] co-registration of DCE- and anatomic-scans (3D and 4D), [3] identification of regions-of-interest (3D-ROI), [4] identification of appropriate arterial-input-function (AIF). Output data include quantitative pharmacokineticparameters (two-compartment Tofts-model: $k^{\text {trans }}, k^{\mathrm{ep}}$, Ve, etc). and fitted/unfitted enhancement-curves ([mmolGd/l]). All 4 pre-preprocessing features and the quality of fitted curve-data were evaluated by an experienced neuroradiologist and scored on confidence-scales ( $0=$ not appropriate to $10=$ excellent). Pharmacokineticparameters were correlated with final diagnosis (benign/malignant: ROC-analysis). Results: Motion-correction was technically successful in all cases. Co-registration in $3 \mathrm{D}$ (mean-score[ms]:9.4) and 4D (ms=9.3) as well as identification of the AIF (ms: 9.6) reached excellent ratings. Placement of $3 D$-ROIs was difficult in one lesion $(s c o r e=6)$. Yet, in total it was performed appropriately $(m s=9.0)$. Overall quality of fitted curve-data reached good scores $(m s=9)$, resulting into a high diagnosticaccuracy for the differentiation of benign vs. malignant tumors (AUC=89\% $\left[k^{e p}\right]$ ). Conclusion: The software showed an excellent clinical performance. This should help to further promote quantitative pharmacokinetic-analyses and might broaden its application in clinical practice in the future.

\section{B-0615 11:15}

Rapid semi-automated volumetry of pleural effusion in MDCT

M.P.F. Botelho, F.D. Gonzalez-Guindalini, H. Chalian, V. Yaghmai;

Chicago,IL/US (v-yaghmai@northwestern.edu)

Purpose: To evaluate precision and reproducibility of volumetric semi-automated quantification of pleural effusions in MDCT.

Methods and Materials: This study was IRB approved. We evaluated 29 consecutive pleural effusions in 20 adult patients undergoing contrast-enhanced MDCT of the chest. Two independent blinded readers used a semi-automated segmentation software for estimation of volume and recorded quantification time. Manual volumetry was obtained as the reference. We evaluated the volumetry for precision, time for assessment and inter-rater agreement, as well as percentage of over- and under-segmentation. Statistical analysis encompassed paired t-test, Bland-Altman plot and Lin's concordance correlation coefficient.

Results: The mean semi-automated and manual volumes were $1040.3 \mathrm{~mL}$ (SD $964.6 \mathrm{~mL}$ ) and $1033.1 \mathrm{~mL}(\mathrm{SD} 979.0 \mathrm{~mL})$, respectively $(P=0.379)$. Semi-automated volumetry showed significant inter-rater agreement, with Bland-Altman precision of $8.2 \%$ (upper and lower limits of $18.5 \%$ and $-13.7 \%$, respectively). The mean semi-automated volumetry was accurate when compared to manual volumetry, with Bland-Altman precision of $5.0 \%$ (upper and lower limits of $12.1 \%$ and $-7.7 \%$, respectively), and Lin's concordance correlation coefficient $(\rho c)=0.998$. There was a mean of $2.3 \%$ over-segmentation using the semi-automated software. The average user time for semi-automated volumetry was $105.3 \mathrm{sec} / \mathrm{case}$, whereas those for manual volumetry were $336 \mathrm{sec} /$ case $(P<0.001)$

Conclusion: Rapid and accurate semi-automated volumetry of pleural effusions in MDCT is feasible and reproducible.

Author Disclosures:

M.P.F. Botelho: Grant Recipient; Educational Grant Support by Siemens Healthcare. F.D. Gonzalez-Guindalini: Grant Recipient; Educational Grant Support by Siemens Healthcare. H. Chalian: Grant Recipient; Educational Grant Support by Siemens Healthcare.

\section{B-0616 11:24}

Software-supported evaluation of small-bowel motility using freebreathing cine MRI

S. Bickelhaupt ${ }^{1}$, R. Cattin ${ }^{2}$, S. Raible ${ }^{2}$, J.M. Froehlich ${ }^{1}$, H. Bouquet ${ }^{3}$, U. Bill ${ }^{3}$, M.A. Patak ${ }^{1} ;{ }^{1}$ Zurich $/ \mathrm{CH},{ }^{2} \mathrm{Biel} / \mathrm{CH},{ }^{3} \mathrm{Berne} / \mathrm{CH}$

Purpose: MRI acquisitions for analyses of small bowel motility are commonly performed in breath-hold resulting in relatively short cine sequences that limit reliable quantitative analyses. A new dedicated motility software (Motasso) permits automatic measurement of small bowel motility in free-breathing MRI allowing acquisition of long motility sequences. The aim was to validate the software prototype by comparing it to manual measurements.

Methods and Materials: 25 patients (15m/10f; mean 39 years) were included in this IRB-approved, retrospective study. MRI (1.5-T, Siemens-Sonata) was performed after standardised preparation. 2D-motility-acquisitions (dynamic-2DT2-FIESTA; TR 247.07/TE 1.23/FOV500/15 mm) covering the entire small bowel were performed in free breathing over $75 \mathrm{sec}$. Image analysis for the assessment of small bowel motility was performed both manually and with the software. The time consumption and reproducibility were compared using coefficient of variance and paired Student's t-test.

Results: 25 single regions of interest were analysed two times by hand and two times using Motasso with a dedicated displacement provider. Mean small bowel contraction frequency was 5.25 contractions per minute (SD+-2.74) for manual and 4.86 (SD+-2.48) for Motasso analyses; the mean luminal diameter was $17.88 \mathrm{~mm}$ (manual, SD+-9.31) and $18.00 \mathrm{~mm}$ (Motasso, SD+-9.13), respectively. The variability of repetitive measurements (coefficient of variation) was $9.77 \%$ (SD+-11.08) for manual and significantly lower $(p<0.01)$ for Motasso-assisted measurements (3.90\%, SD+-5.69). The mean duration needed for a single small-bowel motility assessment was $17.48 \mathrm{~min}$ for manual (SD+-1.75 min, range 14-22 min) and high significant lower $(p<0.001)$ for Motasso-assisted measurements $(4.52 \mathrm{~min}$, SD+-1.58, range $2-8 \mathrm{~min})$.

Conclusion: The use of Motasso proves highly reliable and fast measurements of small bowel motility in free breathing MRI. Reproducibility was significantly higher for Motasso-assisted than for manual measurements, while the time needed for the single measurement could be tremendously reduced using Motasso.

Author Disclosures:

H. Bouquet: Employee; Former employee of SOHARD AG. U. Bill: Employee; Fromer Employee of SOHARD AG.

\section{B-0617 11:33}

Automated system for CT carotid plaque characterisation into symptomatic and asymptomatic classes

L. Saba' ${ }^{1}$, U. Acharia ${ }^{2}$, M. Anzidei ${ }^{3}$, M. Piga ${ }^{1}$, J.S. Suri ${ }^{4} ;{ }^{1}$ Cagliari/IT,

${ }^{2}$ Singapore/SG, ${ }^{3}$ Rome/IT, ${ }^{4}$ Pocatello, ID/US (lucasaba@tiscali.it)

Purpose: Plaque treatments carry considerable risk, and hence, adjunct techniques are necessary to help doctors in making a confident decision about the necessity of such procedures for a patient. Mostly, symptomatic patients face a greater risk of cardiovascular diseases, and hence, classification of the plaque into symptomatic and asymptomatic is necessary. Therefore, we have developed a non-invasive computer-aided diagnostic technique for the same.

Methods and Materials: Carotid artery images were acquired using multi-detector row CT angiography (MDCTA). The region of interest (ROI) was segmented manually from the images, and the ROIs were used to extract texture-based and discrete wavelet transform-based features. Significant features were used to train and test support vector machine (SVM) classifiers of various kernel configurations. Results: The SVM classifier with a polynomial kernel of order 3 presented the highest accuracy of $90 \%$, sensitivity of $95.6 \%$, and specificity of $84.4 \%$. We formulated a RiskScore, which is a combination of all the features, in order to monitor the variations in features, and also to more objectively classify the two classes. Conclusion: A technique that uses the CT images to identify symptomatic and asymptomatic plaques would add more value to this modality in the area of atherosclerosis management. We have proposed and demonstrated the efficiency of one such technique in this work. The classification accuracy is high, and the technique is non-invasive, fast, highly objective, cost-effective and user-friendly. Therefore, it can serve as a valuable adjunct tool that can be used by clinicians in deciding treatment options for patients.

\section{B-0618 11:42}

Automated labelling framework applied on full and partial spine CT scans D. Major, J. Hladuvka, F. Schulze, K. Bühler; Vienna/AT (david.major@vrvis.at)

Purpose: The spine is often a frame of reference for diagnosis. For this purpose its parts have to be labelled in upper body CT scans which is a time-consuming task. An automated framework is introduced for detecting intervertebral disk and vertebra centre points and its labels in CT data containing the spine fully or partly considering clinical resource constraints.

Methods and Materials: The framework uses a machine learning-based classification approach for detecting spinal canal centre point candidates as a first step. Intervertebral disk-, rib- and sacrum centre point candidates are extracted next to the spinal canal by the same classification approach. Intevertebral disk centre point labelling is started relative to either ribs or sacrum depending on detection certainty. The labelling run is done iteratively along the spine by obtaining best matches of trained local appearance models to detected three intervertebral disk and spinal canal centre point candidate clusters. Vertebra centres/labels are derived from the intervertebral disk ones. The framework has been trained on 16 and tested on 36 
CT scans from different vendors constructed from 102 up to 5966 axial 512x512 slices containing spine-related diseases (scoliosis, fractures and osteoporosis).

Results: A correct labelling was achieved within 2 minutes on average in 35 out of 36 test cases with $99 \%$ precision and $95.5 \%$ recall of intervertebral disk centre point placements.

Conclusion: A high-throughput, memory-efficient and robust framework for automated spine labelling was developed which copes with both full and partial spine CT scans in a clinically reasonable time.

Author Disclosures:

D. Major: Research/Grant Support; This work has been supported by the Austrian Funding Agency (FFG) and AGFA Healthcare within the scope of the COMET K1 program. J. Hladuvka: Research/Grant Support; This work has been supported by the Austrian Funding Agency (FFG) and AGFA Healthcare within the scope of the COMET K1 program. F. Schulze: Research/Grant Support; This work has been supported by the Austrian Funding Agency (FFG) and AGFA Healthcare within the scope of the COMET K1 program. K. Bühler: Research/Grant Support; This work has been supported by the Austrian Funding Agency (FFG) and AGFA Healthcare within the scope of the COMET K1 program.

\section{B-0619 11:51}

Segmentation-free scheme for computer-assisted image interpretation: application to CT colonography

F. Chandelier ${ }^{1}$, T. Cabrera ${ }^{2}$, P. Kocsis ${ }^{1}$, L. Stein ${ }^{2}$, V. Demers ${ }^{2} ;{ }^{1}$ Granby, QC/CA,

${ }^{2}$ Montreal, QC/CA (virginie.demers@mail.mcgill.ca)

Purpose: To present a paradigm shift in computer-assisted image interpretation and to assess its application in CT colonography. A novel segmentation-free scheme has been developed with the hypothesis that accurate colon segmentation is neither necessary nor an end clinical objective for delivering efficient support such as colon centreline, electronic colon cleansing (ECC) and computer-aided detection of lesions (CAD).

Methods and Materials: Thick regions are expanded until they encompass statistically significant representations of the colon wall and its surroundings (Air, Tag, Soft/Hard tissue). From these regions, a coarse colon centreline is inferred through topological analysis guided by anatomic constraints; ECC is obtained by determining a transposing function depicting the distribution of voxels in a given Air-region, and applying said function to Tag-regions depicting statistically similar colon wall surroundings; unlike surface shape analysis, a CAD identifies local hyper-density concentrations from implicit surfaces flux, at a given depth related to the targeted lesion size. The complete schemes were retrospectively tested on 111 and 135 patients datasets for \{centreline; electronic cleansing and $C A D$, respectively.

Results: Colon centreline extraction was accurate in $85 \%$ of combined cathartic and tagged datasets. CAD performance was similar to that in the literature, specifically a sensitivity of $80 \%$ [70-85] for lesions at least $7 \mathrm{~mm}$ featuring a false-positive rate less than 5 per-patient.

Conclusion: The presented novel paradigm in computer-assisted image interpretation prevents traditional limitations arising from "accurately segmenting" a specific organ for further processing. To our best knowledge, this is the first attempt to design and validate such a paradigm shift in CT colonography.

Author Disclosures:

F. Chandelier: Employee; Cadens Imaging. Patent Holder; Canada Patent

2727736. P. Kocsis: Employee; Cadens Imaging. 


\section{Sunday, March 10}




\section{Oncologic Imaging}

\section{SS 1316}

\section{Rectal cancer imaging: the next step}

Moderators:

L. Curvo-Semedo; Coimbra/PT

C. Hoeffel; Reims/FR

\section{B-0620 10:30}

Predictive value of combined quantitative parameters obtained with ADC map and 18-FDG/PET study in assessment of treatment response of patients with advanced rectal cancer: histopathologic correlation

D. Ippolito, A. Cadonici, C. Trattenero, L. Monguzzi, C. Capraro, S. Sironi; Monza/IT (davide.atena@tiscalinet.it)

Purpose: To investigate the combined role of quantitative parameters of functional imaging by analysing ADC and SUVmax values before and after chemo-radiation therapy (CRT) in prediction of tumour response of patients with LARC, correlating with histologic examination (tumour regression grade/TRG).

Methods and Materials: 40 patients, with biopsy-proven rectal carcinoma, underwent a whole body 18 FDG PET/CT scan and pelvic MR examination including DWI for staging (PET 1,RM1) and after CRT (PET 2,RM 2). Subsequently, all patients underwent total mesorectal excision and the histological results were compared with imaging findings. MR scanning, performed on 1.5 T magnet (Philips, Achieva), comprised T2-weighted multiplanar imaging and DW images with b-value 0 and $1000 \mathrm{~mm}^{2} / \mathrm{sec}$. On PET/CT, the SUVmax of rectal lesion was calculated on PET1 and PET2. Percentage decrease of SUVmax ( $\triangle S U V)$ and ADC ( $\triangle A D C)$ values from baseline to pre-surgical scan were assessed and correlated with pathologic response (Mandard's criteria:TRG $1=$ complete regression, TRG5=no regression). Results: 27 tumours $(68 \%)$ showed complete (TRG1) or subtotal histological regression (TRG2) and were classified as responders; 13 tumours (32\%) were classified as non-responders (TRG3-5). Considering all patients mean values of SUVmax on PET 1 was higher $(p<0.001)$ than those on PET 2, whereas mean ADC values was lower $(p<0.001)$ on RM 1 than RM 2, with a $\triangle S U V$ and $\triangle A D C$ of $67 \%$ and $69 \%$, respectively. The most sensitive and specific predictors for TRG response were SUV2 (threshold 4.4) and ADC2 (1.29×10-3 mm2/s). Combining in a single analysis both the obtained values, positive predictive value (PPV) in different group category response related to TRG system, presented an AUC of $97 \%$. Conclusion: Combination of DWI and PET/CT may be the most feasible method to evaluate LARC patients, with accuracy values higher than those reported for other conventional imaging techniques.

\section{B-0621 10:39}

\section{Intrinsic gradient of the vascular function in rectal tumours}

M.H. Martens, E. Kluza, S. Subhani, M. Maas, C. Jeukens, G.L. Beets,

R.G.H. Beets-Tan; Maastricht/NL (milou.martens@mumc.nl)

Purpose: Spatial vascular heterogeneity decreases from periphery towards tumour core in solid tumours and is related to cancer viability. This pattern is not present in rectal tumours. The aim of this study was to determine whether rectal tumour spread into layers of rectum, affects tumour vascular function compared to that of normal rectal wall.

Methods and Materials: Seven patients with cT3- tumours received DCE-MRI. Tumours were segmented into luminal, mural and extramural regions. Normal tissue counterparts were defined (mucosa, muscularis propria and mesorectal fat). Initial slope (slopei) and area under the first 120s of enhancement curve (AUC120) were determined from relative signal enhancement-time curves. Pixel parameter values and mean parameter values were compared per patient and for entire patient group. The correlation between kinetic parameters of tumour segments and of corresponding normal tissues was determined.

Results: For each patient, both the slopei and AUC120 were significantly higher for luminal tumour pixels compared to mural and extramural tumour pixels $(p<$ 0.05). Mural pixel parameter values were significantly higher than the extramural pixels $(p<0.05)$. For the patient group the mean AUC120 for the luminal, mural and extramural tumour region was $240 \pm 46,178 \pm 20$ and $129 \pm 18$, respectively. There was a strong and significant correlation between the parameters of tumour segments and those of corresponding normal tissues (Pearson's correlation coefficient $=0.84, p=0.0001$ )
Conclusion: Using DCE-MRI-based kinetic parameters, we showed a decreasing tumour vascular function from the luminal towards extramural tumour invasion. This spatial vascular heterogeneity brings new insights into rectal cancer pathophysiology and may have important implications for treatment-strategies.

\section{B-0622 10:48}

Enhanced vascular structure and function in the tumoursurrounding mesorectum: potential risk factors for patients with rectal cancer

M.H. Martens, E. Kluza, J.-P.J.E. Kleijnen, M. Maas, C.R.L.P. Jeukens, W.H. Backes, G.L. Beets, R.G.H. Beets-Tan; Maastricht/NL (milou.martens@mumc.nl)

Purpose: The role of mesorectal vasculature in rectal cancer is poorly understood. Aim of our study was to determine impact of tumour growth as well as neoadjuvant chemoradiotherapy on mesorectal vasculature.

Methods and Materials: Ten patients with locally advanced rectal cancer underwent (re)staging MRI. Dynamic contrast-enhanced MRI with blood pool contrast agent (gadofosveset) was performed. Maximal intensity projections (MIPs) were used to assess macrovascular structure. Number, diameter and length of vascular branches were determined in mesorectum surrounding tumour and normal rectal wall. Same parameters were used to compare pre- and post-chemoradiotherapy MIPs. The mesorectal microvascular function was studied using relative enhancement-time curves, and expressed as area under the initial 2 minutes of the enhancement curve (AUC).

Results: A significantly higher number of vessel branches was found in tumourneighbouring mesorectum compared to mesorectum around normal rectal wall $(p=0.001)$. Furthermore, vessel diameter in tumour surroundings was $0.2 \mathrm{~mm}$ larger $(p=0.025)$. The average AUC in tumour-neighbouring mesorectum was also higher (6.35 and 0.58 , respectively $(p=0.01))$. Comparison of pre- and post-CRT MIPS revealed $40 \%$ lower vessel branching after CRT $(p=0.002)$. Moreover, the vascular diameter of $1.7 \pm 0.2 \mathrm{~mm}$ at the primary staging was significantly decreased to 1.3 $\pm 0.2 \mathrm{~mm}$ after CRT $(\mathrm{p}=0.007)$. In contrast, CRT resulted in significantly increased AUC values.

Conclusion: There is a significantly enhanced vascular structure and function in the tumour-surrounding mesorectum, which may have an important prognostic value for rectal cancer patients. Furthermore, chemoradiotherapy induces remodelling of the mesorectal vasculature.

\section{B-0623 10:57}

Value of 3D FSE Cube sequence at $3 \mathrm{~T}$ MRI in preoperative local staging of rectal cancer

R. Scandiffio, P. Vagli, P. Bemi, A. Mantarro, L. Faggioni, R. Balestri, P. Buccianti, E. Neri, C. Bartolozzi; Pisa/IT (rossellascandiffio@libero.it)

Purpose: To compare a standard T2w imaging, based on 2D acquisitions, with 3D FSE Cube sequence in the local staging of rectal cancer at 3 T MRI.

Methods and Materials: 32 patients with rectal cancer were prospectively evaluated at 3 T MRI before and after neoadjuvant chemotherapy. Sagittal, coronal, axial and oblique 2D T2w FSE images and an additional 3D T2 FSE Cube sequence were obtained. Each 3D dataset was processed with multiplanar and curved planar reconstructions along the rectal centreline. Two experienced radiologists, using, respectively, $2 \mathrm{D}$ or $3 \mathrm{D}$ sequences evaluated $\mathrm{T}$ and $\mathrm{N}$ parameters, with specific attention to measurement of the distance lesion-puborectal muscle and mesorectal fascia, and lesion extension. All T and N parameters were evaluated independently and grouped for statistical analysis. Linear Cohen $\mathrm{k}$ values were calculated to quantify agreement between MRI and histopathological data. Image quality was evaluated using a linear qualitative scale.

Results: Global sensitivities for T parameters were $67 \%$ and $62 \%$ for $2 \mathrm{D}$ and 3D images, while $\mathrm{N}$ category lesion accuracy values were $70 \%$ and $68 \%$, respectively. Accuracy in measuring the distance from pubo-rectal muscle to lesion border was $60 \%$ and $98 \%$ for $2 \mathrm{D}$ and $3 \mathrm{D}$, respectively. Image quality was considered very good on 2D images, and good on 3D images.

Conclusion: No significant differences between 2D and 3D images were observed for $\mathrm{T}$ and $\mathrm{N}$ staging. T2w FSE Cube demonstrated higher accuracy than $2 \mathrm{D}$ in determining the distance between lesion and pubo-rectal muscle, which represents a key factor in planning surgical resection. 


\section{B-0624 11:06}

Prediction of subsequent metastatic disease by dynamic contrast MRI in locally advanced rectal cancer

E.M. Anderson, J.M. Franklin, L. Tanner, M. Brady, F.V. Gleeson; Oxford/UK (ewan.anderson@ouh.nhs.uk)

Purpose: To predict development of metastatic disease in patients with rectal cancer by dynamic contrast-enhanced (DCE) MRI.

Methods and Materials: Twenty-three patients with locally advanced rectal cancer were treated with long course chemotherapy on an IRB-approved clinical trial. For 20/23 patients, analysable DCE MRI was acquired on a $1.5 \mathrm{~T}$ magnet. Images included repeated T1-weighted, gradient-echo, fat suppressed sequences (LAVA, TR $4.5 \mathrm{~ms}$, TE $2.2 \mathrm{~ms}, 4.0 /-2.0 \mathrm{~mm}$ thickness/gap, matrix, mm FoV) repeated every 12-15 seconds for six minutes. After 4 initial acquisitions, gadobenate dimeglumine was injected, at a dose of $0.1 \mathrm{mg} / \mathrm{kg}$ at $3 \mathrm{ml} / \mathrm{sec}$, followed by a $10-\mathrm{ml}$ normal-saline chaser. Ktrans, Kep, Ve were calculated from Toft's two-compartment kinetics model. Patients were followed for three years, or death, and the presence of metastases was assessed with contrast-enhanced CT. ROC curve analysis assessed the performance of DCE-MRI to predict development of metastases. For categorical variable comparison, the Kruskal-Wallis test was used.

Results: 17 of 23 patients had adequate follow-up, one died during chemoradiotherapy, one died post-operatively and one patient was diagnosed as metastatic during neo-adjuvant treatment. All patients were treated with adjuvant chemotherapy post-surgery. Six patients developed metastases. The median Ktrans and median Ve for all patients that developed metastases were greater than 0.6 and 0.5 , respectively. Area under ROC curve was $0.848(p=0.021)$ for Ktrans and $0.864(\mathrm{p}=0.016)$ for $\mathrm{Ve}$.

Conclusion: DCE MRI (Ktrans and Ve) values can categorise patients as likely or unlikely to develop subsequent metastatic disease, independent of the disease at presentation and the use of adjuvant therapy.

\section{B-0625 11:15}

Features on MRI after transanal endoscopic microsurgery in patients with rectal cancer

L.A. Heijnen ${ }^{1}$, M. Maas', M.H. Martens', D.M.J. Lambregts', J.W.A. Leijtens ${ }^{2}$, W. Deserno ${ }^{2}$, G.L. Beets', R.G.H. Beets-Tan ${ }^{1} ;{ }^{1}$ Maastricht/NL, ${ }^{2}$ Roermond/NL (luc.heijnen@mumc.nl)

Purpose: Transanal endoscopic microsurgery (TEM) is a minimal invasive technique for local resection of T1 and selected T2 tumours and is also an emerging option for good-responders after chemoradiation. In most centres follow-up includes regular MRI. This study aimed to describe the MR morphology of the rectal wall during follow-up in patients that received TEM.

Methods and Materials: Forty-nine patients underwent a post-TEM MRI in our centre. For 21 patients only one post-operative MRI was available. For 28 patients $>1$ MRIs were available. The MR morphology of the TEM-site was studied on the consecutive MR examinations. 32 patients were primary treated with TEM, 17 patients underwent chemoradiation followed by TEM.

Results: We identified three morphological patterns: (1) rectal wall thickening with or without fibrosis, (2) a notch at the TEM-location, and (3) irregular delineation of the rectal wall. Multiple patterns could occur within one patient. 32 patients $(65 \%)$ had rectal wall thickening, 17 patients $(35 \%)$ a notch, and 28 patients $(57 \%)$ irregular delineation of the rectal wall. In addition to these patterns, oedema (due to chemoradiation) persisted in post-chemoradiation TEM-patients. Ten patients had dehiscence after TEM post-chemoradiation $(n=58 \%)$. Six luminal recurrences occurred; 3 had rectal wall thickening, 3 a notch, and 5 an irregular rectal wall. Conclusion: Three patterns were identified on MRI after TEM. This enables radiologists to monitor this group of patients more accurately. Since minimal invasive techniques are gaining, it is very important for radiologists and surgeons to have knowledge about the normal follow-up findings after TEM.

\section{B-0626 11:24}

Role of perfusional MRI and DWI to predict pathological complete response to neoadjuvant chemoradiotherapy in rectal cancer

C.N. De Cecco, M. Ciolina, M.M. Maceroni, G. Muscogiuri, F. lafrate, A. Laghi; Rome/IT (mciolina@yahoo.com)

Purpose: To determine the ability of perfusional MRI (pMRI) and diffusion-weighted imaging (DWI) to predict pathological complete response ( $\mathrm{pCR}$ ) in patient treated with chemoradiotherapy.

Methods and Materials: Eleven consecutive patients underwent pre-, during and post-treatment dynamic contrast-enhanced MRI performed at $3 \mathrm{~T}$. Gadolinium 0.1 $\mathrm{mmol} / \mathrm{kg}$ was injected at a rate of $3 \mathrm{~mL} / \mathrm{s}$. Treatment protocol consisted of neoad- juvant chemoradiotherapy with oxaliplatin and 5-fluorouracile. Ktrans, Kep, Ve and IAUGC90 were calculated. Tumoral dimension and apparent diffusion coefficient (ADC) were analysed. Surgical specimens were the gold standard. For variable comparison, Spearman's rank correlation and Kruskal-Wallis test were used. $\mathrm{P}<$ 0.05 was considered significant.

Results: Six patients showed cPR. Baseline Ktrans, IAUGC90 and Ve were significantly higher in non-responder (NR) patients $(1.22 \pm 1.03 \mathrm{~min}-1,1.36 \pm 1.07 \mathrm{mM} / \mathrm{s}$, $0.7 \pm 0.4)$ in comparison to cPR $(0.92 \pm 0.91 \mathrm{~min}-1,0.69 \pm 0.6 \mathrm{mM} / \mathrm{s}, 0.37 \pm 0.15)$; Kep was significantly lower in NR (1.6 $\pm 0.65 \mathrm{~min}-1$ vs $2.93 \pm 2.71 \mathrm{~min}-1)$. A significant reduction in Ktrans, IAUGC90 and Ve was observed in NR during $(0.68 \pm 0.58$, $0.62 \pm 0.33,0.52 \pm 0.1)$ and after treatment $(0.047 \pm 0.016,0.052 \pm 0.025,0.19 \pm 0.28)$; instead in CPR patient a significant increase in perfusional values was reported $(1.24 \pm 1.15,1.21 \pm 0.96,0.63 \pm 0.26$ and $1.13 \pm 1.36,1.24 \pm 1.34,0.59 \pm 0.34)$. No difference in baseline ADC value was observed between cPR $(0.88 \times 10-3 \mathrm{~mm} 2 / \mathrm{sec} \pm 0.22)$ and NR $(0.85 \times 10-3 \mathrm{~mm} 2 / \mathrm{sec} \pm 0.09)$. In CPR mean tumour ADC significantly increase during treatment comparing to NR $(1.22 \times 10-3 \mathrm{~mm} 2 / \mathrm{sec} \pm 0.6$ vs $0.9810-3 \mathrm{~mm} 2 /$ $\mathrm{sec} \pm 0.12)$. No correlation was found between ADC values and pMRI parameters. Conclusion: $\mathrm{pMRI}$ and $\mathrm{ADC}$ values are two independent parameters to predict rectal tumour response to chemoradiotherapy.

\section{B-0627 11:33}

Additional value of diffusion-weighted (DWI) MRI for predicting complete tumour response (TONO) in rectal cancer treated with neoadjuvant chemoradiation therapy (CRT)

S. Sassen ${ }^{1}$, M. de Booij', M.N. Sosef', G. Lammering ${ }^{2}$, C.M.M. Bakker', R. Clarijs', R.C.M. Berendsen', J. Wals', R.F.A. Vliegen ${ }^{1} ;{ }^{1}{ }^{1}$ Heerlen/NL, ${ }^{2}$ Maastricht/NL (sandersassen@yahoo.com)

Purpose: Patients with complete response (CR) after CRT might be considered for less aggressive treatment like a wait-and-see strategy. Few studies investigated the value of DWI-MRI for predicting CR after CRT, but none included lymph nodes in the analysis (ypTONO). The aim of the present study was to retrospectively determine the additional value of DWI-MRI to conventional (T2-weighted) MRI for predicting CR after CRT.

Methods and Materials: Eighty locally advanced rectal cancer patients underwent CRT followed by restaging MRI and operation. MRI consisted of conventional sequences and DWI. Two readers with different levels of experience independently scored conventional images for $\mathrm{CR}$ and, in a second reading, combined conventional and DWI-MRI images. A 5-point confidence level score was used to generate ROC curves. Differences in performance were calculated by comparing areas under the ROC curves (AUC). Interobserver agreement, sensitivity, specificity and positive predictive values (PPV) were calculated. Histology served as reference standard. Results: Ten of 80 patients (13\%) had a pathologic complete response (yPTONO). Comparison of the ROC curves showed significant improvement of the AUC only for the experienced reader 1 from 0.77 to $0.88(p=0.009)$. Sensitivity improved from $20-30 \%$ to $40-70 \%$. Specificity and PPV improved only for reader 1 from 87 to $93 \%$, resp., 25 to $58 \%$. Interobserver agreement improved from 0.14 to 0.27 . Conclusion: Adding DWI to conventional MRI improves diagnostic performance of experienced readers and increases interobserver agreement for identification of CR. Sensitivity and PPV remain low, with a considerable risk of over- and undertreatment.

\section{B-0628 $11: 42$}

Advanced characterisation of rectal cancer prior surgery:

preliminary results on the evaluation of multimodality functional MRI techniques in correlation to histopathology

D. Hausmann, D. Dinter, P. Kienle, F. Doyan, S.O. Schönberg, U.I. Attenberger; Mannheim/DE

Purpose: High-resolution MRI has evolved to the leading imaging method for locoregional staging in rectal cancer prior treatment. Besides, functional parameters such as perfusion and diffusion-weighted imaging (DWI) could be valuable parameters for tumour characterisation. The aim of this preliminary data analysis was to compare reader variability of quantitative parameters for different stages of primary rectal cancer.

Methods and Materials: 54 patients (mean age $63 \mathrm{yrs}$ ) with biopsy proven rectal cancer (RC) underwent a multi-parametric examination protocol on a 3 T MR-system prior treatment, consisting of high-resolution T2 sequences as well as functional imaging techniques including DWI with calculation of $A D C$ (b-Werte angeben) and perfusion (turboFLASH). Perfusion values (plasma flow, PF; mean transit time, MTT) in the tumour were calculated.

Results: $\mathrm{N}=45 \mathrm{RC}$ s with complete data sets were included in this preliminary analysis. Distribution of the histopathologically proven tumour stages were as follows: 
stage I $n=8$, stage II $n=16$, stage III $n=18$. Depending upon tumour stage, ADCmean (10-3 mm2/s) ranged from 0.86-0.9 (I), 0.9-0.99 (II) and 0.84-0.88 (III). PFmean $(\mathrm{ml} / 100 \mathrm{ml} / \mathrm{min}$ ) ranged from 257-295 (I), 222-454 (II) to 80-195 (III). MTTmean (s) ranged from 56-57 (I), 55-57 (II) to 45-49 (III). No significant difference was found for the functional parameters between both readers $(p>0.11)$. Interreader correlation was good $(\mathrm{rADC}=0.8, \mathrm{rPF}=0.9, \mathrm{rMTT}=0.8 ; \mathrm{p}<0.0001)$.

Conclusion: Functional tumour characterisation is feasible with a good interobserver correlation, allowing for an objective tumour evaluation prior to treatment. These parameters can be used for functional response evaluation in neoadjuvant radiochemotherapy

\section{B-0629 $11: 51$}

ColoCare: compartmental quantification of obesity on $\mathrm{CT}$ as risk factor for colorectal cancer

J. Welzel, H. Hogenauer, J. Staffa, D. Scherer, L. Grenacher, C. Ulrich,

H.-U. Kauczor; Heidelberg/DE (johannawelzel@gmx.de)

Purpose: Obesity is associated with an increased incidence and mortality in colorectal cancer (CRC). The ColoCare study has been set up in order to determine prognostic factors following CRC diagnosis. Within this study we analysed the impact of total adipose tissue (TAT), visceral adipose tissue (VAT) and subcutaneous adipose tissue (SAT) on mortality as alternative measures to BMI.

Methods and Materials: In 77 patients with CRC (21 females, 45 males; stage 1-4; grade 1-4; mean BMI male 26.6 and female $25.8 \mathrm{~kg} / \mathrm{m}^{2}$; mean age male $59 \mathrm{y}$ and female 61y), who underwent multi-detector-CT, quantification of TAT, VAT, SAT and ratio VAT/SAT was performed on the levels of $L 3 / 4$ and $L 4 / 5$ using a compartimental densitometric technique (Syngo Volume, Siemens). Reproducibility was tested by interreadership analysis. Correlation with gender, age, tumour stage and grade, localisation, CRP and fat distribution was performed.

Results: Interreadership analysis showed high correlation of 0.95 to 1.0. VAT and VAT/SAT were significantly higher in men than in women and increased with age. BMI had significance predictive value for TAT and SAT, but not for VAT. No meaningful correlation with tumour stage and grade, localisation and CRP was found. Conclusion: Regional densitometric quantification of fat compartments on CT is highly reproducible and indispensable to obtain accurate data on visceral fat not provided by BMI. Men with $\mathrm{CRC}$ have a larger volume of visceral than women.

\section{Chest}

\section{SS 1304}

\section{CTPA techniques in lung perfusion and pulmonary hypertension}

\section{Moderators:}

I. Hartmann; Rotterdam/NL

E.J. Stern; Seattle, WA/US

\section{B-0630 10:30}

Impact of iterative reconstructions on the diagnosis of acute pulmonary embolism (PE) on low-dose CT angiograms: clinical experience in $\mathbf{5 3}$ patients

F. Pontana, S. Henry, J.-B. Faivre, N. Tacelli, S. Bendaoud, M. Rémy-Jardin,

J. Rémy; Lille/FR (martine.remy@chru-lille.fr)

Purpose: To evaluate the impact of iterative reconstructions on the diagnosis of acute PE on low-dose CT angiograms.

Methods and Materials: 53 patients were prospectively enrolled in a study designed to compare low-dose and full-dose images simultaneously available from the same dataset. The examinations were acquired with (a) both tubes set at similar energy $(120 \mathrm{kVp})$ and $(\mathrm{b})$ the total reference mAs (i.e., $110 \mathrm{mAs}$ ) split up in a way that $40 \%$ was applied to tube A while $60 \%$ was applied to tube B. Three series of images were generated: (a) full-dose images (generated from both tubes), reconstructed with FBP (group 1), used as the standard of reference, (b) low-dose images (generated from tube A; $60 \%$ dose reduction) reconstructed with FBP (group 2 ), then with an iterative reconstruction algorithm (SAFIRE) (group 3).

Results: In group 1: (a) acute PE was diagnosed in 8 patients (15\%) with 82 clots identified within central $(n=5)$, segmental $(n=39)$ and subsegmental $(n=38)$ arteries, (b) the mean level of objective noise was $41.41 \mathrm{HU}$. In group 2, the significant increase in objective noise compared to group 1 (48.37 HU; $p<0.0001$ ): (a) hampered detection of acute PE in one patient and (b) altered detection of peripheral clots (12 false-negative and 2 false-positive results). Comparing group 3 to group
1, there was: (a) no significant difference in objective noise $(p=0.9131)$ and $(b)$ similar depiction of acute PE $(p=1)$.

Conclusion: Reconstruction of low-dose images with SAFIRE provided similar diagnostic information compared to full-dose FBP images.

\section{B-0631 10:39}

Comparison of image quality and radiation dose between 64-MDCT and high pitch 128-DSCT protocols in triple rule-out studies A. Fernandez del Valle, C. Delgado, R. Oca, N. Silva, G. Tardáguila; Vigo/ES (anafdelvalle84@hotmail.com)

Purpose: To compare the image quality and radiation dose between 64-MDCT retrospective-ECG and 128-DSCT high pitch acquisition in triple rule-out protocol in acute chest pain

Methods and Materials: An homogeneous group of 60 patients with triple rule-out protocol was retrospectively revised. 30 underwent retrospective acquisition with $64-M D C T$ (120kv, pitch 0.2) and 30 high pitch acquisition in a 128-DSCT (2x120kv, pitch 3.4). All patients had heart rates below 65 bpm during acquisition. A triple contrast injection protocol was used in all patients. Vessel enhancement and image noise were quantified; signal to-noise ratio (SNR) and contrast-to-noise ratio (CNR) were calculated. Artefacts in superior cava vein, aortic root and coronary arteries were assessed by two radiologists in consensus. Effective dose was calculated on the basis of dose length product and volume CT dose index. Results were compared using the Chi-quadrate and Student's t tests.

Results: High pitch protocol had significantly lower image noise $(11.8 \pm 2.2$ vs $16.5 \pm 3.6 \mathrm{HU})$, SNR $(19.5 \pm 4.9$ vs $26.2 \pm 8.6)$ and CNR $(16.2 \pm 4.8$ vs $21.7 \pm 7.6) ; p<$ 0.01 . Vessel attenuation was similar in both protocols $(296.3 \pm 63.1$ vs $308.9 \pm 58.1$ $\mathrm{UH} ; \mathrm{p}=0.42$ ). No significant differences were found in artefacts measurements. In high pitch protocol only 3 coronary artery segments were non-diagnostic versus 34 in 64-MDCT protocol. Mean effective dose for high pitch protocol was significantly lower than $64-\mathrm{MDCT}$ protocol $(2.77 \pm 0.6$ vs $13.77 \pm 4 \mathrm{mSv} ; \mathrm{p}<0.001)$.

Conclusion: 128-DSCT high pitch acquisition compared to 64-MDCT reduces radiation dose drastically and also improves image quality in triple rule-out studies.

\section{B-0632 10:48}

Sinogram-affirmed iterative reconstructed simulated ECG-gated ultra high pitch CT pulmonary angiography in the acute setting: effect on dose and image quality

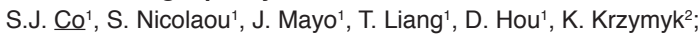

${ }^{1}$ Vancouver, BC/CA, ${ }^{2}$ Topanga, CA/US (co.steve@gmail.com)

Purpose: To evaluate the effect of a simulated ECG-gated computed tomographic pulmonary angiography (CTPA) at $100 \mathrm{kV}$ on image quality and radiation dose. Methods and Materials: Retrospective study includes 100 patients referred for CTPA with pitch 3.2. 50 patients were scanned with a standard $100 \mathrm{kV}$ protocol and 50 patients were scanned with a simulated ECG-gated $100 \mathrm{kV}$ protocol that introduces a specialized cardiac bowtie filter. All other scanning parameters were kept constant. Images were reconstructed with filtered back projection and iterative reconstruction (SAFIRE, Siemens). Central pulmonary vessel attenuation and background noise were quantitatively measured and signal-to-noise (SNR) and contrast-to-noise (CNR) were calculated. Two radiologists performed qualitative assessment grading visualisation of the pulmonary vasculature and noise level (Likert scale). Volume CT dose index and dose length product were recorded and effective dose was calculated.

Results: CTDIvol, DLP and effective dose were significantly $(p<0.0001)$ lower in the simulated ECG-gated protocol $(2.3 \pm 0.5 \mathrm{mGy}, 78.4 \pm 16.5 \mathrm{mGycm}, 1.4 \pm 0.3 \mathrm{mSy}$, respectively) compared to standard protocol $(4.3 \pm 0.5 \mathrm{mGy}, 152.0 \pm 19.6 \mathrm{mGycm}$, $2.7 \pm 0.3 \mathrm{mSy}$, respectively). Simulated ECG-gated protocol had significantly ( $p<$ $0.0001)$ higher noise than the standard protocol $(23.8 \pm 6.9 \mathrm{HU}$ vs $36.8 \pm 7.3 \mathrm{HU})$ and resulted in lower SNR $(11.8 \pm 3.7 \mathrm{HU}$ vs $19.2 \pm 8.1 \mathrm{HU})$ and CNR $(10.3 \pm 3.7 \mathrm{HU}$ vs $24.9 \pm 13.4 \mathrm{HU}$ ) but there was no significant difference in the subjective diagnostic accuracy. Post-processing with iterative reconstruction significantly $(p<0.0001)$ improved noise $(29.4 \pm 5.5 \mathrm{HU}$ from $36.8 \pm 7.3 \mathrm{HU})$.

Conclusion: The simulated ECG-gated CTPA resulted in $48 \%$ dose reduction without significantly affecting diagnostic image quality. Furthermore, the use of iterative reconstruction improves image quality by reducing noise permitting the possibility for further dose reduction strategies.

Author Disclosures:

K. Krzymyk: Employee; Siemens. 


\section{B-0633 10:57}

$70 \mathrm{kV}$ CT pulmonary angiography in clinical routine: initial experience, radiation dose and image quality

R.W. Bauer, F. Al-Butmeh, C. Frellesen, J.A. Wichmann, T. Lehnert, T.J. Vogl, M. Kerl; Frankfurt a. Main/DE (ralfwbauer@aol.com)

Purpose: To investigate the general applicability of a $70 \mathrm{kV}$ protocol for routine CT pulmonary angiography (CTPA) in unselected patients.

Methods and Materials: Thirteen patients suspected of having pulmonary embolism underwent CTPA with a novel $70 \mathrm{kV}$ protocol (208 mAs, pitch 1.2) on a 64-slice CT device. Ten patients who were investigated with the standard protocol (100 $\mathrm{kV}, 120 \mathrm{mAs}$, pitch 1.2) served as the control group. Images were reconstructed with sinogram affirmed iterative reconstruction with medium regulation strength in both groups. $75 \mathrm{ml}$ of contrast material $(350 \mathrm{mgl} / \mathrm{ml})$ was injected at $4 \mathrm{ml} / \mathrm{s}$. CTDIvol, DLP, signal intensity in the pulmonary trunk and segmental arteries and corresponding SNR values were compared. Images were assessed for diagnostic quality and artefacts.

Results: Chest diameter was not significantly ( $p>0.05$ ) different between the groups. CTDlvol (2.3 vs. $6.6 \mathrm{mGy})$ and DLP ( 71 vs. $193 \mathrm{mGycm})$ were by $65 \%$ and $63 \%$ lower at $70 \mathrm{kV}(\mathrm{p}<0.05)$; iodine attenuation was significantly higher (e.g. segmental arteries 554 vs. $313 \mathrm{HU}$ ), but so was image noise, resulting in no significant differences in SNR. Photon starvation artefacts from dense contras material in the venous system and high image noise in bigger patients were the main drawbacks at $70 \mathrm{kV}$.

Conclusion: $70 \mathrm{kV}$ CTPA seems feasible in clinical routine and results in a dose reduction of more than $60 \%$ compared to a $100 \mathrm{kV}$ protocol at equal SNR; however, patient selection is advisable. Artefacts from inflowing contrast material will require dedicated injection protocols or the use of low-iodine contrast material.

\section{B-0634 11:06}

Automated quantification of pulmonary perfused blood volume in acute pulmonary embolism using dual-energy CTPA

F.G. Meinel, A. Graef, F. Bamberg, S.F. Thieme, C. Neurohr, C. Kupatt, M.F. Reiser, T.R.C. Johnson; Munich/DE (felix.meinel@med.uni-muenchen.de)

Purpose: To determine whether automated quantification of pulmonary perfused blood volume (PBV) in dual-energy computed tomography pulmonary angiography (DE-CTPA) is of diagnostic value in assessing the severity of acute pulmonary embolism (PE).

Methods and Materials: Ethical approval and informed consent were waived by the responsible Institutional Review Board for this retrospective study. Automated quantification of PBV was performed in 71 consecutive patients (mean age $62 \pm 16$ years) with DE-CTPA positive for acute PE and no cardiopulmonary co-morbidities. PBV values adjusted for age and gender were correlated with the Qanadli obstruction score, morphological CT signs of right heart dysfunction, serum levels of troponin as well as the necessity for ICU admission.

Results: DE-CTPA-derived PBV values inversely correlated with Qanadli score $(r=-0.46, p<0.001)$, RV/LV ratio $(r=-0.52, p<0.001)$ and troponin I $(r=-0.45$, $p=0.001$ ). Patients with global PBV values $<60 \%$ were significantly more likely to require admission to an ICU than patients with global pulmonary $\mathrm{PBV}^{3} 60 \%$ (47\% vs. $11 \%, p=0.003$, PPV $47 \%$, NPV $89 \%$ ). The area under the receiver operating characteristic curve (AUC) for the prediction of ICU admission was 0.75 for pulmonary PBV, 0.83 for the Qanadli obstruction score, 0.68 for CT signs of right heart dysfunction (interventricular septal bowing and/or contrast reflux) and 0.76 for the RV/LV diameter ratio.

Conclusion: DE-CTPA can be used for an immediate, reader-independent estimation of global pulmonary PBV in acute PE, which inversely correlates with thrombus load and laboratory parameters of PE severity and can be used to predict the necessity for ICU admission.

Author Disclosures:

T.R.C. Johnson: Equipment Support Recipient; Siemens Healthcare. Research/ Grant Support; Bayer Healthcare.

\section{B-0635 11:15}

Dual-source CT perfusion maps: do they increase the diagnostic confidence in the diagnosis of pulmonary embolism by CT?

E. Stiefsohn, S. Scharer, H. Prosch, C. Neumann, K. Osinger, M. Weber, G. Mostbeck; Vienna/AT (elisabeth.stiefsohn@wienkav.at)

Purpose: The aim of this study was to assess the image quality and the diagnostic value of dual-source CT perfusion maps (DS-CT-P) compared to pulmonary dualsource CT pulmonary angiography (CT-PA) with regard to the confidence of the diagnosis of acute pulmonary embolism (PE).
Methods and Materials: This retrospective study included 100 consecutive patients (63 females, 28 to 97 years) who underwent CT-PA and DS-CT-P of the lung (Somatom Definition, Siemens, Forchheim, DE, $140 \mathrm{kV}$ and $80 \mathrm{kV}$, collimation $64 \times 1.2 \mathrm{~mm} ; 100 \mathrm{ml}$ Jopamiro 370 , Bracco, IT, flow rate $5 \mathrm{ml} / \mathrm{s}, 50 \mathrm{ml} \mathrm{NaCl}$ ) for the diagnosis of suspected PE. Two radiologists (A, B) blinded to patient information (except suspicious PE) independently analysed the CT-PA-data in random order twice, with and without DS-CT-P images. Both radiologists assessed CT-PA and DS-CT-P for image quality, presence of pulmonary emboli at main, lobar, segmental and sub-segmental arterial levels and perfusion defects, respectively. Confidence in PE diagnosis was scored on a 10-point scale.

Results: $P E$ was diagnosed in 21 and 22 patients by readers $A$ and $B$, respectively, in concordance in 20 patients. There was no significant change in diagnostic confidence with additional DS-CT-P data analysis (A: 9.4 to 9.4 ; B: 9.0 to 9.6 ), B scored one study neg. for PE on CT-PE + DS-CT-P, which was pos. by CT-PA alone. Interobserver variability with kappa $=0.882($ CT-PA) and $0.910($ CT-PA+DS-CT-P) for diagnosis of $\mathrm{PE}$ was excellent.

Conclusion: DS-CT-P data do not improve diagnostic confidence in CT-PA diagnosis of acute PE, because low image quality in CT-PA parallels low image quality in DS-CT-P.

\section{B-0636 11:24}

CT-pulmonary angiography follow-up in acute pulmonary embolism: resolution of emboli and change of CT signs of right heart dilatation and pulmonary hypertension

C. Neumann, H. Prosch, K. Osinger, D. Vedrilla, M. Weber, G. Mostbeck; Vienna/AT (christian.neumann.nch@wienkav.at)

Purpose: To determine the resolution of pulmonary emboli (PE) by CT-pulmonary angiography (CT-PA) in correlation with CT parameters of right heart load and pulmonary hypertension.

Methods and Materials: Two independent radiologists analysed initial and follow-up (F/U) CT-PA data (time interval 1-6 weeks, clinical indication for F/U) from 81 consecutive patients $(37 \mathrm{~m} / 44 \mathrm{f}, 54 \pm 36$ years) with $\mathrm{PE}$ in a retrospective study between 12/2008 and 4/2012. Clot load was assessed by Qanadli-score (QS), right (RV) and left ventricular (LV) short axis and diameters of pulmonary trunk and ascending aorta were measured to calculate the RV/LV-ratio (RLR) and vessel diameter ratio (AOPA).

Results: All 81 patients showed significant decrease of QS (mean 17.4 to 9.8, $p$ $<.001$ ), RLR and AOPA between initial and F/U CT-PA. In 10/81 patients complete $\mathrm{PE}$ lysis was found. There was good correlation between reduction of $\mathrm{QS}$ and regression of $R L R(r=-.506 ; p<.001)$ and AOPA $(r=-.458 ; p<.001)$, respectively. $R L R$ and AOPA changes $(r=.330 ; p=.003)$ correlated poorly. Patients with initial QS > 20 showed higher reduction of QS at F/U, but no significant difference was found concerning the reduction of RLR between patients with initial QS $>20$ and $220(p=.921)$. The time interval between initial and F/U CT-PA differed between patients with and without complete lysis ( 3.5 vs. 2.14 weeks, $p=.016$ ).

Conclusion: Clot resolution is associated with improvements of RLR and AOPA, but the time to complete resolution is variable and not predictable. RLV and AOPA are independent markers of right heart burden in patients with PE, but they correlate poorly with each other.

\section{B-0637 11:33}

Clinical significance of high density thrombi on non-enhanced CT scan in patients with pulmonary thromboembolism

K.S. Beck, B. Lee, H. Kim, D. Han; Seoul/KR (sallahbar@gmail.com)

Purpose: To evaluate the incidence and significance of occasionally detected high density thrombi of pulmonary embolism (PE) on non-enhanced chest CT scans. Methods and Materials: We retrospectively evaluated the non-enhanced and enhanced CT findings of 76 patients with pulmonary embolism, and the incidence of high density thrombi was calculated. Then in both iso-density thrombi (group 1) and high-density thrombi (group 2) groups, embolic burden score, ventricular septal bowing, and diameter of right ventricle (RV) and left ventricle (LV) were evaluated and compared using independent sample $\mathrm{T}$ tests.

Results: 19 patients $(25 \%)$ showed high density thrombi in pulmonary arteries on MDCT. Embolic burden score was significantly different between group 1 and 2 $(7.3 \pm 5.4 \& 12.3 \pm 6.6)(p=0.007)$. Ventricular septal bowing was also significantly more frequent in group 2. Diameter of RV $(44.0 \pm 9.6 \& 45.8 \pm 8.8)(p=0.433)$ or diameter of $L V(41.3 \pm 6.9 \& 40.9 \pm 5.8)(p=0.821)$ did not show significant difference between group 1 and 2 .

Conclusion: High density thrombi detected on non-enhanced scan in patients with PE are relatively frequent and suggest more dangerous condition. Radiologists should be aware of the possibility of thrombi on non-enhanced CT scans. 


\section{B-0638 $11: 42$}

Automated quantification of pulmonary perfused blood volume by dual-energy CTPA in chronic thromboembolic pulmonary hypertension F.G. Meinel, A. Graef, M. Armbruster, C. Neurohr, M.F. Reiser, T.R.C. Johnson; Munich/DE (felix.meinel@med.uni-muenchen.de)

Purpose: To determine whether automated quantification of pulmonary perfused blood volume (PBV) in dual-energy computed tomography pulmonary angiography (DE-CTPA) is of diagnostic value in assessing the severity of chronic thromboembolic pulmonary hypertension (CTEPH).

Methods and Materials: Ethical approval and informed consent were waived by the responsible Institutional Review Board for this retrospective study. Automated quantification of $\mathrm{PBV}$ was performed in 25 consecutive $C T E P H$ patients undergoing DE-CTPA. PBV values were correlated with cardiac index and pulmonary vascular resistance quantified by right heart catheterization and walking distance in the 6-minute walk test using Pearson's correlation coefficient and multivariate linear regression analysis to control for age and gender.

Results: DE-CTPA-derived PBV values inversely correlated with systolic $(r=-0.64$, $p=0.001)$ and mean $(r=-0.57, p=0.004)$ pulmonary arterial pressure. There was a trend for PBV values to inversely correlate with pulmonary vascular resistance $(r=-0.20, p=0.35)$. No significant correlation was found between PBV values and cardiac index or 6-minute walking distance. These correlations were confirmed to be independent of age and gender on multivariate linear regression analysis. Conclusion: DE-CTPA can be used for an immediate, reader-independent estimation of global pulmonary PBV in chronic thromboembolic pulmonary hypertension, which inversely correlates with systolic and mean pulmonary arterial pressure.

Author Disclosures:

T.R.C. Johnson: Equipment Support Recipient; Siemens Healthcare. Research/ Grant Support; Bayer Healthcare.

\section{B-0639 11:51}

Non-invasive diagnosis of pulmonary hypertension with dynamic contrast-enhanced computed tomography

M. Pienn' ${ }^{1}$, G. Kovacs ${ }^{1}$, M. Tscherner ${ }^{1}$, T.R.C. Johnson², P. Kullnig',

R. Stollberger ${ }^{1}$, A. Olschewski ${ }^{1}$, H. Olschewski', Z. Bálint ${ }^{1} ;{ }^{1}$ Graz/AT,

${ }^{2}$ Munich/DE (michael.pienn@lvr.lbg.ac.at)

Purpose: Pulmonary hypertension $(\mathrm{PH})$ is a disease characterised by an elevated pulmonary arterial pressure (PAP) and is diagnosed invasively via right heart catheterization $(\mathrm{RHC})$. We explored whether timing measures from dynamic contrast-enhanced computed tomography (CT) can be used as a non-invasive method to determine $\mathrm{PH}$.

Methods and Materials: 20 patients ( 15 with and 5 without $\mathrm{PH}$ ) were examined with a novel dynamic contrast-enhanced CT sequence following their diagnostic or follow-up RHC. X-ray attenuation over time curves were recorded for several regions of interest (ROIs) in the main pulmonary artery and in the ascending aorta and subsequently fitted with a spline fit. Time differences between the contrastmaterial bolus peaks were measured. Distances between the ROls were measured in a simultaneously acquired thorax CT to calculate the bolus propagation speeds. We compared our results to haemodynamic parameters acquired during RHC.

Results: Time differences between peaks showed good correlation (Spearman correlation coefficients ${ }^{3} 0.65$ ) with mean PAP, pulmonary vascular resistance and gas exchange parameters. Discrimination between patients with and without $\mathrm{PH}$ was achieved by evaluating time differences (sensitivity/specificity $100 \% / 80 \%$, 95\% confidence intervals: $72-100 \%$ and $28-99 \%$, respectively) and bolus speeds (sensitivity/specificity 100\%, 95\% confidence intervals: $72-100 \%$ and $48-100 \%$, respectively).

Conclusion: Time differences between bolus peaks and bolus speeds in the pulmonary artery can be utilised to identify $\mathrm{PH}$ patients with only little additional dose, contrast material and post-processing. This method can reduce the number of invasive right heart catheterizations.
10:30 - 12:00

Room D2

Interventional Radiology

SS 1309

\section{Skeletal and endocrinologic interventions}

Moderators:

L. Crocetti; Pisa/IT

D.K. Tsetis; Iraklion/GR

\section{B-0640 10:30}

Selective arterial embolisation for bone tumours

G. Rossi', E. Rimondi', A.F. Mavrogenis ${ }^{2}$, A. Andreone ${ }^{1}$, P. Spinnato ${ }^{1}$,

G. Garzillo', P. Ruggieri', D. Vanel', A. Bazzocchi'; ${ }^{1}$ Bologna/lT, ${ }^{2}$ Athens/GR

(andreanselmo99@yahoo.it)

Purpose: To present the experience of a single institution on selective arterial embolisation for primary and metastatic bone tumours.

Methods and Materials: 365 patients were treated by 454 embolisations as primary treatment for benign tumors (81 tumors), adjuvant treatment to surgery for bening and malignant bone tumours (41 tumors), and palliative treatment for bone sarcomas and metastases (243 metastatic carcinomas). The indications for repeat embolisation included pain or imaging evidence of progressive disease; 105 patients had repeat embolisation at the same location, at an interval of 1-3 months; 260 patients had one embolisation, 78 patients had two embolisations, and 29 patients had three or more embolisations.

Results: 419 of the 454 embolisations (93\%) were technically successful. In 35 cases, embolisation was not feasible because of poor vascularisation of the lesions, origin of Adamkiewicz artery in the embolisation field, atheromatosis-arteriosclerosis, and anatomical and technical problems such as small caliber vessels, many brenches, and acute angles of the vessels. A clinical response was achieved in 406 of the 419 procedures $(97 \%)$. Complications included post-embolisation syndrome in 81 patients (22\%), transient paresthesias in 41 patients (11\%), sink breakdown and subcutaneous necrosis in five patients (1.4\%), and paresis of the sciatic nerve in one patient $(0.3 \%)$.

Conclusion: Embolisation can be recommended as primary or palliative treatment, or adjunct to surgery of bone tumours. Arteries feeding the tumor and collaterals must be evaluated carefully and catheterised superselectively to protect the normal tissues.

\section{B-0641 10:39}

Radiofrequency ablation of osteoid osteoma: technique with special tricks for difficult anatomical locations and atypical nidus

A.K. Janu, B. Jankharia, K. Pilania, P. Thakrar; Mumbai/IN

(amiteasy10@gmail.com)

Purpose: 1. To identify osteoid osteoma, on the basis of clinical features and radiological findings. 2 . To describe the technique for performing radiofrequency ablation (RFA), with special considerations in difficult anatomical locations and atypical nidus. 3. To evaluate the efficacy and complications of ablation.

Methods and Materials: In this prospective study, RF ablation of 50 osteiod osteoma was carried out under CT guidance using a RITA uni-ablate electrosurgical device. Special approach planning, to minimize to complications and morbidity, was required for large lesions and lesions in difficult locations like in spine, superficial lesions and lesions adjacent to joint and growth plates (15 patients).

Results: Intranidal localisation of the needle and complete ablation was achieved in every patient accounting for a technical success rate of $100 \%$. Most of the patients were pain free within the first week and almost all had complete pain relief within a month after RFA, accounting for a primary clinical success rate of $95 \%$ and secondary clinical success rate of $100 \%$. Recurrent lesions were treated successfully with a second ablation. Mild to moderate pain, within first 48 hours, was controlled by oral analgesics. Complications seen were mild local swelling (1), transient weakness of leg (1), chip fracture (1), superficial skin infection (2) and osteomyelitis (1). Conclusion: Percutaneous CT-guided radiofrequency ablation is a safe, quick and minimally invasive method for treatment of osteoid osteoma with a high technical and clinical success rate, and minimal complications and morbidity, even in patient with atypical nidus and difficult locations. 


\section{B-0642 10:48}

Osteoid osteomas of the elbow: a challenging location

A. Bazzocchi, G. Facchini, G. Bettelli, E. Castiello, M. Cavaciocchi, R. Rotini, U. Albisinni; Bologna/IT (giancarlo.facchini@gmail.com)

Purpose: The elbow joint is a site deserving special consideration in the radiofrequency thermal ablation (RFA) of osteoid osteomas (OO). OO affecting the intracapsular space often involve articular ends; moreover, the proximity to the skin as well as the presence of vascular and nervous structures call for technical skills and caution. The purpose of this study was to report our experience in the treatment of $\mathrm{OO}$ of the elbow (e-OO).

Methods and Materials: Between 2002 and 2010, 27 patients with e-OO were candidate to RFA as first choice treatment at our Institution. Extra-articular and juxta-articular OOs were not included. We retrospectively analysed outcomes of the procedure in terms of ablation efficacy, incidence of complications and functiona results measured by Mayo-Elbow-Performance-Score (MEPS), with a minimum follow-up of 24 months.

Results: Twenty males and 7 females with age ranging from 13 to 45 years (average 26.4) showed the onset of symptoms 5 months to 6 years (average 28.0 months) before diagnosis. All patients complained about pain, while joint function was impaired in $24 / 27-88.9 \%$ cases. The average improvement of MEPS after RFA was $39.8( \pm 15.8)$ points, with $25 / 27-92.6 \%$ patients scoring 90 to $100(/ 100)$ points at the end of follow-up. No complications were recorded. One (the first treated) patient presented with recurrence of the disease 9 months after the procedure, and this was successfully healed by a second RFA. All patients were free from disease at an average follow-up of 49 months (range 2-8 years).

Conclusion: RFA of e-OO is a safe and an effective procedure, allowing excellent functional recovery.

\section{B-0643 10:57}

MR-guided high-intensity focused ultrasound for non-invasive treatment of osteoid osteoma

M. Anzidei, A. Napoli, G. Brachetti, B. Cavallo Marincola, G. Cartocci, F. Boni,

V. Noce, L. Bertaccini, C. Catalano; Rome/IT (michele.anzidei@gmail.com)

Purpose: To determine the feasibility and initial clinical efficacy of MR-guided Focused Ultrasound (MRgFUS) for treatment of patients with osteoid osteoma. Methods and Materials: This prospective, IRB approved study involved 7 consecutive patients $(6 \mathrm{~m} ; 1 \mathrm{f}$; mean age, 21$)$ with clinical and imaging diagnosis of Osteoid Osteoma; all patients underwent MRgFUS ablation (ExAblate, InSightec). Lesions located in the vertebral body were excluded, while lesions in proximity to joints or neurovascular bundles were included. Treatment success was determined at clinical and imaging follow-up at 1, 3, and 6 months post-treatment. A visual Analog Pain Score (VAS) was used to assess changes in symptoms.

Results: Treatment was carried out using a variable number of sonications (mean $4 \pm 1.8$ ) with a mean energy deposition of $866 \pm 211 \mathrm{~J}$. There were no treatment- or anaesthesia-related complications. A statistically significant $(p=0.001)$ difference was noted between the overall pre- and post-treatment mean VAS scores $(8.3 \pm 1.6$ and $0.6 \pm 1.5$, respectively). Six of the 7 treatments were conducted on patients with typical osteoid osteoma and all were completely clinically successful. The patient with giant osteoma had pain recurrence after 2 weeks, requiring surgery. At imaging, oedema and hyperemia associated with typical osteoid osteoma gradually disappeared in all lesions. No apparent relationship between nidus vascular extinction and successful outcome was found.

Conclusion: MRgFUS ablation represents an effective and totally non-invasive therapeutic option for osteoid osteoma management, without treatment-related adverse events.

\section{B-0644 11:06}

MR-guided focused ultrasound ablation on bone metastases: role of dynamic contrast-enhanced MRI in the evaluation of treatment response A. Napoli, V. Noce, M. Anzidei, F. Boni, G. Brachetti, G. Cartocci, L. Bertaccini, C. Catalano, F. Ciolina; Rome/IT (alessandro.napoli@uniroma1.it)

Purpose: To quantitatively evaluate perfusional MRI findings in skeletal metastases treated with MR-guided Focused Ultrasound (MRgFUS) ablation and compare imaging parameters with clinical pain assessment by visual analogue scale (VAS). Methods and Materials: Eleven patients treated with MRgFUS ablation of bone metastases were examined with a Dynamic Contrast-Enhanced $3 \mathrm{~T}$ MR-scan (DCE-MRI; Discovery 750, GE; gd-BOPTA, Bracco) before and 3 months after the sonication procedure. Quantitative perfusional parameters were obtained by means of DCE-MR transfer rate constant (Ktrans) and extravascular volume frac- tion (ve) with a dedicated analysis software. Every subject was monitored over the following three months assessing clinical outcome by means of pain relief with VAS. Results: Eight of eleven subjects clinically demonstrated a complete treatment response $(C R)$, with a VAS score mean reduction of $4.3(47.8 \% . p<0.001)$, whereas three patients showed a partial clinical response $(\mathrm{PR})$ with fractional relief according to VAS scale. Compared perfusional analysis of metastatic lesions in CR patients showed a significant reduction of gadolinium extraction with a 2.14/min Ktrans mean reduction ( $-\Delta \mathrm{Kt}=52.65 \%$. p0.01), while $\mathrm{PR}$ patients showed no substantial modification in Ktrans value $(\Delta \mathrm{Kt}=+0.042 / \mathrm{min},+11.39 \%$. $\mathrm{p}<0.01)$ or increase in extravascular volume. Spearman test revealed a significant relationship between Ktrans quantitative parameters and clinical evaluation by VAS scale $(p<0.001)$ in both $\mathrm{CR}$ and PR patients.

Conclusion: Perfusional quantitative MRI is a sensitive predictor of MRgFUS bone metastases treatment response if compared with clinical outcome datas. Ktrans negative modifications $(-\Delta \mathrm{Kt})$ may reflect effectiveness of ultrasound ablation procedure, as direct expression of decreased tumoural cell metabolism, and positively correlate to clinical response.

\section{B-0645 11:15}

Palliative treatment of bone metastases: analysis of biological effects of MR-guided focused ultrasound (MRgFUS) versus external beam radiation therapy (EBRT). A randomised comparative trial using functional diffusion maps as molecular activity indicator A. Napoli, G. Brachetti, D.S. De Oliveira, G. Cartocci, F. Boni, V. Noce, C. Marsecano, V. Tombolini, C. Catalano; Rome/IT (alessandro.napoli@uniroma1.it)

Purpose: This study aimed to investigate and compare the response to MRgFUS and EBRT treatments of painful bone metastases by using diffusion-weighted (DW) magnetic resonance (MR) imaging with apparent diffusion coefficient (ADC).

Methods and Materials: This prospective, double arm, randomised study with EBRT serving as control arm, received IRB approval. 36 consecutive patients (female:15 male:21 mean age:60.3) with painful bone metastases were enrolled. 18 patients underwent MRgFUS treatment, using ExAblate 2100 system (InSightec), and 18 patients underwent EBRT. Pain palliation was evaluated by visual analog scale (VAS), pain questionnaires and changes in the patients' medication. All patients underwent 3 T ce-MRI (Discovery 750, GE; gd-BOPTA, Bracco) before treatment and at 1,2 , and 3 months afterwards. MRI protocol included DWI sequences with five $b$ factors $(0-800 \mathrm{sec} / \mathrm{mm} 2)$ and ADC maps were obtained. The average $A D C$ values for each lesion were analyzed in comparison between pre- and post-treatment.

Results: No treatment-related adverse events were recorded for both arms. Statistically significant difference between baseline and follow-up VAS values and medication intake for both MRgFUS and EBRT patients ( $\mathrm{p} 0.5$ ) was observed. Progressive decrease in VAS values positively correlated to an increase in mean ADC values $(p>0.01)$ for both treatment modalities.

Conclusion: MRgFUS is a promising non-invasive treatment modality for successful palliation of bone metastasis and has potential for tumour control as compared with ERBT.

\section{B-0646 11:24}

Percutaneous laser ablation of metastatic lymph nodes in the neck from papillary thyroid carcinoma: 1-year results

G. Mauri ${ }^{1}$, T. Tondolo ${ }^{1}$, L. Cova ${ }^{1}$, T. Ierace ${ }^{1}$, N.S. Goldberg ${ }^{2}$, L. Solbiati ${ }^{1}$;

Busto Arsizio/IT, 2Jerusalem/IL (vanni.mauri@gmail.com)

Purpose: To assess the feasibility, safety and effectiveness of PLA in the treatment of metachronous lymph node metastases from papillary thyroid carcinoma in the neck.

Methods and Materials: 24 lymph nodes (mean size $1.2 \mathrm{~cm}$, range $0.6-2.6 \mathrm{~cm}$ ) in 15 patients were treated. A 1,064 nm Nd:YAG laser was used. All cases were negative at 131 l whole body scan, had positive 18 F-FDG PET and CEUS and elevated serum levels of thyroglobulin $(\mathrm{Tg})$ or anti-thyroglobulin antibodies ( $\mathrm{TgAb})$. Nodes were treated with $3 \mathrm{~W}$ power for $400-600$ sec (total energy 1,200-1,800 joules). Follow-up was performed at 6 and 12 months with CEUS, 18 FDG-PET/ CT and assessment of serum levels of $\mathrm{Tg}$ and of $\mathrm{TgAb}$.

Results: Laser ablation was technically feasible and well tolerated in all patients, with no complications. At 6-month follow-up complete ablation was demonstrated in $19 / 24(79.1 \%)$ lymph nodes in $10 / 15(66.6 \%)$ patients. $6 / 15(40.0 \%)$ patients also had negative $\mathrm{Tg}$ and $\mathrm{TgAb}$ serum levels. One patient with four treated lymph nodes developed distant metastases and did not undergo the 12-month 18 FDG-PET. At 12 months complete ablation was demonstrated in 16/20 (80\%) lymph nodes 
in $10 / 14(71.4 \%)$ patients. $4 / 15(26.6 \%)$ patients also had negative $\mathrm{Tg}$ and $\mathrm{TgAb}$ serum levels. $3 / 15$ (20\%) developed distant metastases.

Conclusion: Percutaneous US-guided interstitial laser ablation is potentially safe and effective in the treatment of metachronous nodal metastases from papillary thyroid carcinoma in the neck. With further study this may help reducing or delaying a large number of highly invasive radical neck dissection.

\section{B-0648 11:33}

Arterial spin labelling (ASL) MRI for monitoring kidney perfusion

before and after renal denervation: initial results

C. Schleich, D. Blondin, P. Kröpil, O. Vonend,

P. Heusch, H.-J. Wittsack, G. Antoch, R.S. Lanzman;

Düsseldorf/DE (Rotem.Lanzman@med.uni-duesseldorf.de)

Purpose: The purpose of our study was to investigate changes in kidney perfusion following renal denervation using arterial spin labelling (ASL) MRI at 1.5 T.

Methods and Materials: 7 patients (mean age $58 \pm 13$ years) undergoing interventional renal denervation were included in this study. In each patient, between 6 and 8 ablations were performed per renal artery. ASL MRI was performed at $1.5 \mathrm{~T}$ using a single-slice FAIR TrueFisp sequence in the coronal plane (30 averages of label and control pairs, inversion time $1200 \mathrm{~ms}$, slice thickness $8 \mathrm{~mm}$, matrix 128x128) prior to and within 24 hours following renal denervation. Patients refrained from fluid intake for at least 2 hours prior to the examination. Following post-processing, ROIs were drawn by a single reviewer on ASL parameter maps for quantification of cortical perfusion. Both kidneys of each patient were used for statistical analysis. Results: Image acquisition was completed successfully in all patients and perfusion was determined for 14 kidneys in 7 patients. In 6 patients, ASL perfusion increased by at least $10 \%$ following denervation, while in one patient ASL perfusion decreased following intervention. Mean ASL perfusion following renal denervation (245.8 \pm $43.3 \mathrm{ml} / 100 \mathrm{~g} / \mathrm{min})$ was significantly higher than prior to denervation $(273.4 \pm 56.4$ $\mathrm{ml} / 100 \mathrm{~g} / \mathrm{min})(\mathrm{p}<0.01)$.

Conclusion: Renal denervation leads to a significant increase in kidney perfusion as assessed with ASL MRI. Further studies are required to determine the predictive value of perfusion changes in patients undergoing renal denervation.

\section{B-0649 11:42}

Reliable and reproducible sampling of adrenal veins in the management of hyperaldosteronism, a technically demanding yet feasible procedure: an institutional experience

C.H. Lam, H.L. Chow, S.H. Chiu, H.Y. Cho, S. Lau; Hong Kong/HK (sunnylamsjc@yahoo.com.hk)

Purpose: To analyse the feasibility and accuracy of adrenal venous sampling (AVS) in predicting and lateralizing a surgically remedial adrenal adenoma, and to compare its findings with CT.

Methods and Materials: We compared results of AVS and computed tomography (CT) from January 2009 to May 2012. Biochemical evidence of adrenal vein cannulization was analysed with adrenal-peripheral cortisol ratios. Pathology was then reviewed in patients undergoing adrenalectomy.

Results: A total of 37 patients underwent 43 AVS during the period. The success rate was 100\% (37 out of 37 ) and $91.9 \%$ (34 out of 37 ) for left and right-sided samples, respectively. A second AVS was performed on three patients who failed the first attempt (8.1\%). There were $23(62.2 \%)$ unilateral APA and $14(37.8 \%)$ bilateral hyperplasia. All patients had a prior CT scan: 25 patients had unilateral nodules, 8 had bilateral disease, and 4 were normal scan. Concordance between CT and AVS was observed only in $21(56.8 \%)$ of the 37 patients. In the 26 patients with APA (AVS proven), 17 (65.4\%) had concordant CT findings, 7 had discordant findings, and 2 had normal scan. In the 14 patients with BAH (AVS proven), 4 (28.6\%) had concordant CT findings, 8 had discordant findings, and 2 were normal scan. The 23 patients with unilateral disease underwent adrenalectomy. Pathology all confirmed cortical adenoma.

Conclusion: We conclude that reliable and reproducible AVS result is feasible. AVS is also superior to CT in differentiation of primary aldosteronism and in lateralization of APA.
$10: 30-12: 00$

Room E1

\section{Musculoskeletal}

SS 1310

\section{Arthritis and metabolic bone disease}

Moderators:

A.J. Grainger; Leeds/UK

A. Plagou; Athens/GR

\section{B-0650 10:30}

Radiological peripheral involvement in a cohort of polyarticular juvenile idiopathic arthritis at adulthood

A. Feydy, R. Bazeli, V. Freire, M. Elhai, J. Wipff, C. Job-Deslandre, A. Kahan,

J.-L. Drapé; Paris/FR (antoine.feydy@cch.aphp.fr)

Purpose: Radiographic damage was recently identified as a feature of poor prognosis in polyarticular juvenile idiopathic arthritis (pJIA). However, most radiographic studies did not differentiate pJIA from other subtypes of JIA and little is known in PJIA persisting into adulthood. We set out, therefore, to describe radiological peripheral involvement in young adults with pJIA as compared to rheumatoid arthritis (RA) patients.

Methods and Materials: All consecutive pJIA patients followed in a transition program were included. Age, sex, disease duration, and treatment information were collected. Laboratory tests and standard radiographs of the hands and wrists, feet and hips were then analysed by two independent radiologists blinded to the diagnosis. A RA control group (<55years), matched for sex and disease duration, was recruited.

Results: Fifty-eight pJIA and 59 RA patients were included. Radiographs showed hand lesions in $78 \%$ of pJIA and $86 \%$ of RA patients, feet lesions in $67 \%$ of pJIA and $79 \%$ of RA patients and coxitis in $30 \%$ of pJIA and $17 \%$ of RA patients ( $p=N S$ ). Specificities to juvenile forms were a lower frequency of proximal interphalangeal joints involvement and a higher risk of bilateral coxitis $(81 \%$ vs. $25 \%$ ( $p=0.007$, $\mathrm{OR}=13$ [1.701-99.375]) than adult RA. Peripheral damage correlated with a more severe disease.

Conclusion: Structural peripheral damage is as common and as severe in young adults with pJIA as in adult RA. The main specific feature of pJIA seems to be a high risk of bilateral coxitis. This requires a particular monitoring of pJIA patients with unilateral hip involvement to prevent bilateralization.

\section{B-0651 10:39}

Monitoring of initial methotrexate therapy effect on cartilage composition in early rheumatoid arthritis with biochemical MRI of finger cartilage

C. Schleich, B. Ostendorf, C. Buchbender, C. Nowak, P. Sewerin, M. Schneider, G. Antoch, A. Scherer, F. Miese; Düsseldorf/DE (schleich2003@gmx.de)

Purpose: To test for improvement in cartilage glycosaminoglycan content of metacarpophalangeal joints (MCP) in patients with early rheumatoid arthritis (eRA) undergoing initial methotrexate therapy using delayed Gd (DTPA) 2-enhanced MRI of the cartilage (dGEMRIC).

Methods and Materials: MCP II/III in 19 patients with eRA and 13 healthy volunteers were examined (eRA patients: 13 females, six males, mean age 51 years; eRA six-month follow-up patients: 7 females, one male, mean age 48 years; healthy volunteers: ten females, three males, mean age 51 years). dGEMRIC was measured with manually drawn region-of-interest evaluation. Cartilage thickness was determined as a conventional measure of cartilage integrity. CRP was recorded, remission was assessed according to EULAR 2011 criteria. Statistical analysis used non-parametric Mann-Whitney-U-test to test for significant differences between the groups.

Results: dGEMRIC was significantly decreased in eRA patients compared to healthy subjects (healthy volunteers: MCP || $488 \mathrm{~ms} \pm 90 \mathrm{~ms}$, MCP III $523 \mathrm{~ms} \pm$ 100 ms; eRA patients: MCP || $414 \mathrm{~ms} \pm 119 \mathrm{~ms}$ ( $\mathrm{p}<0.05)$ MCP III $415 \mathrm{~ms} \pm 132$ $m s(p<0.05))$. There was no significant difference in cartilage thickness. Following six-month of methotrexate therapy, remission was achieved in six out of eight patients. Mean improvement of dGEMRIC index was $12 \mathrm{~ms} \pm 180 \mathrm{~ms}$ (n.s). There was no significant association of dGEMRIC with remission or change in CRP. Conclusion: Decreased glycosaminoglycan content of normal appearing finger cartilage in eRA did not significantly improve with methotrexate therapy despite clinical remission. dGEMRIC may be a possible tool for studies on cartilage protection in RA therapy. 
Author Disclosures:

G. Antoch: Speaker; Siemens AG, Bayer AG, MDS Nordion. F. Miese: Speaker; Servier.

\section{B-0652 10:48}

Quantification of synovitis in the cranio-cervical region in early rheumatoid arthritis using dynamic contrast-enhanced (DCEI) and diffusion-weighted (DWI) magnetic resonance imaging (MRI) M. Jeromel' ${ }^{1}$, V. Jevtič', M. Tomšič ${ }^{1}$, I. Pisanec ${ }^{2}$, J. Markota ${ }^{1}$, I. Serša, ${ }^{1}$, A. Ambrožič'; ; ${ }^{1}$ Ljubljana/SI, ${ }^{2} \mathrm{Celje/SI} \mathrm{(miran.jeromel@gmail.com)}$

Purpose: Early diagnosis of synovitis in the cranio-cervical region (CCR) of patients with rheumatoid arthritis (RA) is of utmost clinical importance. Quantitative evaluation of early synovitis using DCEI and DWI is introduced.

Methods and Materials: 27 patients with early RA ([ [Unable to Display Character: \&\#706;]] 24 months) and neck pain were studied at baseline and after 6 months. Standard qualitative MRI evaluation of atlantoaxial joints was coupled with two quantitative MRI methods (DCEI and DWI). Rate of early enhancement (REE), enhancement gradient (Genh) and apparent diffusion coefficient (ADC) were extracted. Results: Qualitative MRI proved synovitis around the odontoid process in 16 (59\%) patients at baseline and 14 (54\%) patients at follow-up. Quantitative DCEI and DWI confirmed active synovitis in $25(93 \%)$ patients at baseline and $24(92 \%)$ patients at follow-up. Average \pm standard error REE value was $0.72 \pm 0.05 \% / \mathrm{s}$ at the beginning and $0.66 \pm 0.06 \% / \mathrm{s}$ at the follow-up, mean Genh value was $1.42 \% / \mathrm{s}$ $(95 \% \mathrm{Cl} 1.06-1.88 \% / \mathrm{s})$ and $1.41 \% / \mathrm{s}(95 \% \mathrm{Cl} 1.04-1.90 \% / \mathrm{s})$, respectively. Average \pm standard error ADC value at the beginning was $1.79 \pm 0.07 \times 10-3 \mathrm{~mm} 2 / \mathrm{s}$ and $1.74 \pm 0.06 \times 10-3 \mathrm{~mm} 2 / \mathrm{s}$ at the follow-up.

Conclusion: DCEI and DWI enable quantification of synovitis in the CCR of patients with early RA. Qualitative and quantitative MRI demonstrated very high incidence of synovitis and confirmed active inflammatory disease in the CCR following therapy although all clinical criteria showed signs of improvement of the peripheral disease.

\section{B-0653 10:57}

Slow radiographic progression of bone destruction in the hands in early psoriatic arthritis without correlation to clinical disease activity M. Geijer ${ }^{1}$, U. Lindqvist ${ }^{2}$, T. Husmark 3 , G.-M. Alenius ${ }^{4}$, P.T. Larsson 5 , A. Teleman 6 , E. Theander ${ }^{7} ;{ }^{1}$ Lund/SE, ${ }^{2}$ Uppsala/SE, ${ }^{3} \mathrm{Falun} / \mathrm{SE},{ }^{4}$ Umeå/SE, ${ }^{5}$ Stockholm/SE, ${ }^{6}$ Oskarström/SE, ${ }^{7}$ Malmö/SE (ecr2012@geijer.info)

Purpose: To describe early $\mathrm{x}$-ray findings in the hands and evaluate progression of destruction after five years correlated to clinical parameters in early psoriatic arthritis (PsA).

Methods and Materials: In 31 men and 46 women with PsA fulfilling the CASPAR criteria hand $x$-rays were available from the 5 -year follow-up and either inclusion or the 2-year visit. X-rays were scored by the Wassenberg scoring system.

Results: Median symptom duration at inclusion was 12 months. $58 \%$ had polyarticular and 38\% mono/oligoarticular PsA. At the first evaluation $60 \%$ had Wassenberg score zero. At the 5 -year visit median score was 2 . In 34 patients (44\%) the score was still 0 , in 28 between 1 and 5 , in 5 between 6 and 10, in 6 between 11 and 15 , and 1 patient each had a score of 19,22, 39, and 42. At baseline women had significantly higher disease activity than men, but similar $x$-ray score. At 5 years men had improved considerably in function and disease activity score, but had greater $x$-ray progression ( $58 \%$ vs $28 \%, p=0.009)$. Male gender was a significant predictor of $\mathrm{x}$-ray progression. Longer symptom duration at entry predicted $\mathrm{x}$-ray progression $(p=0.038)$ and prevented minimal disease activity $(p<0.001)$.

Conclusion: After 5 years of PsA, most patients still have no or very little hand joint destruction. Symptom duration at inclusion and male gender were the main predictors of $\mathrm{x}$-ray progression,

preserved function at baseline was protective. Despite worse clinical outcome with higher disease activity scores, women had less $\mathrm{x}$-ray progression than men.

\section{B-0654 11:06}

Role of dynamic MRI in predicting outcome of patients affected with early stage arthritis

C.A. Mattiuz¹, L.M. Sconfienza1, M. Cimmino², G. Garlaschi², G. Di Leo',

F. Sardanelli'; ${ }^{1}$ Milan/IT, ${ }^{2}$ Genoa/IT (chiara.mattiuz@gmail.com)

Purpose: To estimate the association between curve parameters of dynamic MRI of the wrist and outcome of patients affected with arthritis.

Methods and Materials: Thirty-nine patients ( $M / F$ 10/29; age range 25-79 years) affected with early stage arthritis ( $<9$ months) in at least one wrist were prospectively enrolled. Each patient underwent MRI using a dedicated unit (Artoscan 0.2 T). Imaging protocol included spin-echo sequences before/after injection of $0.2 \mathrm{ml} / \mathrm{kg}$ of Gd-DTPA (Omniscan, Schering, Berlin). Starting from injection, 20 acquisitions were acquired of 3 slabs of the wrist, the first of which positioned tangentially to the radio. A ROI was placed on the most enhancing region of the synovial membrane. The dynamic curve was quantified in terms of rate of early enhancement (REE) and relative enhancement (RE). Patients were classified as remitting or not-remitting. Mann-Whitney test was used for comparisons.

Results: The mean duration of follow-up was 38 months (range 4-84 months). The final diagnosis was: 12 rheumatoid arthritis, 3 psoriatic arthritis, 3 connectivitis, and 12 other pathologies (2 carcinomas, 1 palindromic rheumatism, 2 fibromyalgia, 3 seronegative arthritis, 3 oligoarthritis, 1 reactive arthritis). Nine patients were completely remitting. Mean REE in remitting patients $(0.85 \pm 0.62)$ was not significantly different $(p=0.300)$ from that of non-remitting patients $(1.12 \pm 0.80)$; instead, a significant difference was observed in the RE $(53 \pm 37 \%$ versus $92 \pm 53 \% ; p=0.020)$. REE was negatively correlated to the follow-up duration ( $p=0.026$ ).

Conclusion: Dynamic MRI is highly predictive in outcomes of early arthritis involving the wrist.

\section{B-0655 11:15}

Association of trochlear dysplasia with degenerative abnormalities in the knee: data from the osteoarthritis initiative

P.M. Jungmann ${ }^{1}$, S.-C. Tham², H. Liebl' ${ }^{3}$, M. Nevitt ${ }^{3}$, C.E. McCulloch ${ }^{3}$, J. Lynch ${ }^{3}$, T.M. Link ${ }^{3} ;{ }^{1}$ Munich/DE, ${ }^{2}$ Singapore/SG, ${ }^{3}$ San Francisco, CA/US (pia.jungmann@tum.de)

Purpose: To evaluate trochlear morphology as a potential risk factor for patellofemoral osteoarthritis, determined by morphological and quantitative measurements of cartilage degeneration using $3 \mathrm{~T}$ magnetic resonance imaging (MRI) of the knee. Methods and Materials: MR images of right knees of 304 randomly selected subjects, aged $45-60$ years, from the osteoarthritis initiative (OAl) progression cohort were screened for trochlear dysplasia, defined by a pathological trochlear depth. Out of 304 subjects, $n=85$ demonstrated a shallow trochlea (depth $23 \mathrm{~mm} ; 28 \%$ ). In these, and also in a random sample of controls with normal trochlear depth $(n=50)$, we calculated the facetal ratio and assessed knee structural abnormalities using a modified Whole-Organ-MR-Imaging Score (WORMS). Cartilage segmentation was also performed and T2 relaxation times and patellar cartilage volume were determined. ANOVA and multivariate regression models were used for statistical analysis of the association of MRI structural measures and trochlear morphology. Results: Knees with a shallow trochlea showed higher patellofemoral degeneration (WORMS mean \pm standard deviation, $11.2 \pm 4$.6 versus $5.7 \pm 4.2$; multivariate regression, $\mathrm{P}<0.001)$ and lower patellar cartilage volume than controls $(900 \pm 664$ $\mathrm{mm} 3$ versus $1671 \pm 671 \mathrm{~mm} 3, \mathrm{P}<0.001)$. Knees with a pathological facetal ratio showed increased patellofemoral WORMS scores $(8.3 \pm 4.6$ versus $2.3 \pm 6.3, \mathrm{P}<$ 0.001 ). T2 values at the patella were significantly lower in the dysplasia group. However, significance was lost after adjustment for cartilage volume (multivariate regression, $\mathrm{P}=0.673$ )

Conclusion: Trochlear dysplasia, defined by a shallow trochlea, is associated with higher WORMS scores and lower cartilage volume, indicating more advanced osteoarthritis at the patellofemoral joint.

\section{B-0656 11:24}

Reliability of tomosynthesis for semiquantitative assessment of knee osteoarthritis features by radiologists with different levels of expertise

D. Hayashi', L. Xu', J. Gusenburg', F.W. Roemer', D.J. Hunter', L. Li', A. Guermazi'; ' ${ }^{1}$ Boston, MA/US, ${ }^{2}$ Sydney/AU (guermazi@bu.edu)

Purpose: To assess intra-reader reliability for semiquantitative (SQ) scoring of osteophytes (OPs) and subchondral cysts (SCs) using tomosynthesis among radiologists with various levels of expertise.

Methods and Materials: 40 subjects (aged 40 or above) had both knees imaged by tomsynthesis. Three readers individually assessed these 80 knees: A, expert musculoskeletal radiologist with 13 years of experience in radiographic $S Q$ scoring of osteoarthritis features; B, board-certified musculoskeletal radiologist with 5 years of clinical experience and 1 year of research experience involving SQ scoring of

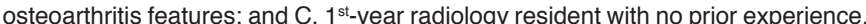
OPs were scored based on the OARSI atlas from 0 to 3 , and presence of SCs in medial/lateral femur and tibia was noted. 20 knees were randomly chosen and re-read 1 month later. Intra-reader reliability was assessed across the knee by calculating the overall exact \% agreement and the weighted $\mathrm{k}$.

Results: Readers A, B and C detected 178, 167 and 127 OPs, and 31, 28, and 37 SCs, respectively. Reader A had substantial to perfect intra-reader agreement for OPs $(100 \%$ agreement, $\mathrm{K}=1.00[95 \% \mathrm{Cl} 1.00-1.00])$ and SCs $(90 \%, \mathrm{~K}=0.86[0.65-$ $1.00])$. Reader $B$ also showed substantial intra-reader agreement for OPs $(90 \%$, 
$\mathrm{K}=0.93[0.83-1.00])$ and SCs $(95 \%, \mathrm{~K}=0.83$ [0.50-1.00]). Reader $\mathrm{C}$ showed perfect agreement for SCs $(100 \%, \mathrm{k}=1.00$ [1.00-1.00]) but agreement for OPs was notably lower than two experienced readers $(75 \%, \mathrm{~K}=0.79[0.61-0.96])$.

Conclusion: TS seems to offer excellent intra-reader reliability regardless of the reader experience for SCs. However, reader experience seems important for reliable SQ assessment of OPs using tomosynthesis.

\section{Author Disclosures:}

F.W. Roemer: Consultant; Merck Serono, NIH. Shareholder; Boston Imaging Core Lab, LLC. D.J. Hunter: Grant Recipient; ARC Future Fellowship; DonJoy; $\mathrm{NIH}$; NHMRC. A. Guermazi: Consultant; Merck Serono, Novartis, Stryker, Genzyme; AstraZeneca. Shareholder; Boston Imaging Core Lab, LLC.

\section{B-0657 11:33}

Scoring hip abnormalities using MR images (SHAMRI): a novel hip whole joint osteoarthritis evaluation system

S. Lee ${ }^{1}$, L. Nardo', A. Lai', D. Kumar', C.R. Wyatt' ${ }^{1}$, R.B. Souza', N.E. Lane ${ }^{2}$, S. Majumdar', T.M. Link'; ' San Francisco, CA/US, ${ }^{2}$ Sacramento, CA/US (Sonia.Lee@ucsf.edu)

Purpose: To develop a semi-quantitative osteoarthritis evaluation scoring system for degenerative hip abnormalities using MR images and to test its reproducibility and validity.

Methods and Materials: Radiographs and non-contrast 3 T MR images were acquired from hips of forty patients ( 20 males and 20 females; aged 25 to 61 years). Intermediate weighted fast spin echo images were acquired in 3 planes. Radiographs were classified using the Kellgren-Lawrence (KL) grading and MR studies were analysed using a new whole-joint MRI scoring system evaluating cartilage, labrum, bone, synovial structures and tendons of the hip. Reproducibility was determined using weighted-kappa statistics. Cochran-Armitage test was used to test associations between new MRI classification scores and radiographic $\mathrm{KL}$ scores. Results: Radiographic $K L$ grading distribution was: $K L 0=10, K L 1=10, K L 2=10$, $\mathrm{KL} 3=10$. Intra- and inter-observer reproducibilities per single feature had $\mathrm{K}$ values between 0.68 to 0.82 and 0.60 to 0.95 , respectively. Kappa values were lower for subregional cartilage evaluation (between 0.60 and 0.70 ), and higher for labral, bone marrow, synovial abnormalities and greater trochanter insertion tendinopathy (greater than 0.80 ). The overall percent agreement was above 0.80 for both intra- and interobserver reproducibilities. Amongst the abnormalities, full thickness cartilage defects had the highest association with KL scores $(p<0.001)$.

Conclusion: The novel MRI evaluation system of hip osteoarthritis evaluated a wide range of pathology and showed high intra- and inter-observer reproducibilities.

\section{B-0658 11:42}

Cortical thickness mapping reveals effects of age, weight and osteophytes in the proximal femur

T.D. Turmezei, G.M. Treece, A.H. Gee, C.J. Tonkin, K.E.S. Poole; Cambridge/UK (tom@diagnosticradiology.eu)

Purpose: Cortical thickness mapping is a technique that uses clinical computed tomography (CT) imaging to plot the distribution of cortical bone thickness in 3D. Given our understanding of their relationships with bone mineral density, we performed a study to investigate the effects of age, weight, and osteophytes on cortical bone distribution in the proximal femur.

Methods and Materials: We analysed CT imaging data from a cohort of 230 women aged $66 \pm 17$ years, selecting one hip from each randomly according to side. A DICOM analysis tool (Stradwin) was used to contour each hip semi-automatically and create a 3D surface mapped with individual cortical thickness values. Statistical parametric mapping was then performed to investigate model effects of age, weight and osteophytes on cortical thickness.

Results: Cortical thickness was generally thinner with advancing age, but was preserved in characteristic load-bearing regions at the medial femoral neck and lateral subtrochanteric cortex. Greater weight was also associated with significantly thicker cortex per kilogram at these same sites. Including osteophytes in the model showed that increasing severity was detected as increasingly thick cortical bone around the articular margin at the femoral head-neck junction.

Conclusion: These results demonstrate, for the first time, the effects of age, weight and osteophytes on cortical bone thickness in the proximal femur. It will be essential for future analysis of bone in this region to take these effects into account.

\section{Author Disclosures:}

G.M. Treece: Patent Holder; Co-inventor on a related GB patent application "A method of determining the cortical thickness of a patients bone".K.E.S. Poole: Patent Holder; Co-inventor on a related GB patent application "A method of determining the cortical thickness of a patients bone".

\section{B-0659 11:51}

Multifocal endosteal thickening of the femur in patients on long-term bisphosphonate therapy presenting with atypical femoral fractures M.A. Png, P.C. Mohan, J.S.B. Koh, T.S. Howe; Singapore/SG (gdrpma@gmail.com)

Purpose: To describe the incidence and radiological characteristics of multifocal endosteal thickening in patients on bisphosphonate therapy.

Methods and Materials: A retrospective study of 68 patients with atypical femoral fractures (AFF) whilst on bisphosphonate therapy was performed. Available femoral radiographs were assessed for: focal endosteal thickening (at least $1 \mathrm{~mm}$ in length and width), number of lesions, lesion location, femoral bowing, periosteal beak and black line. Multifocal endosteal thickening was defined as multiple foci of discrete endosteal thickening. Medical records were reviewed to obtain relevant clinical data. Results: Forty-eight lesions with multifocal endosteal thickening were detected in seven patients ( 2 unilateral, 5 bilateral), affecting $11.8 \%$ of femora. Location was mainly diaphyseal (95.8\%), upper (10.4\%), middle (58.3\%) and lower femur (31.3\%), involving the lateral $(85.4 \%)$, anterior $(6.3 \%)$, anterolateral $(2.1 \%)$ and posterior cortices (6.3\%). Femoral bowing was present in $85.7 \%$. Associated findings of a periosteal beak and/or a black line, seen in $14.6 \%$, were associated with increased fracture risk ( $100.0 \%$ sensitivity, $93.2 \%$ specificity).

Conclusion: Multifocal endosteal thickening is a new finding seen in patients with low bone mineral density on bisphosphonate therapy. In our cohort of patients, who presented with AFF, it was present in $11.8 \%$ of femora. It was frequently bilateral, predominantly diaphyseal in location involving the lateral cortex and often associated with bowing. Caution is advised when seen in association with periosteal beak and/or black line because of a high rate of progression to complete fracture.

\begin{tabular}{ll}
\hline $10: 30-12: 00$ & Room E2
\end{tabular}

\section{Cardiac}

\section{SS 1303}

\section{Towards improved image quality and detection}

Moderators:

G. Feuchtner; Innsbruck/AT

C. Herzog; Munich/DE

\section{B-0660 10:30}

Stenosis quantification in coronary CT angiography: impact of an integrated circuit detector with iterative reconstruction

F. Morsbach', L. Desbiolles ${ }^{2}$, A. Plass ${ }^{1}$, S. Leschka ${ }^{2}$, B. Schmidt ${ }^{3}$, V. Falk ${ }^{1}$ H. Alkadhi', P. Stolzmann'; ' 'Zurich/CH, ${ }^{2}$ St. Gallen/CH, ${ }^{3}$ Forchheim/DE

Purpose: To assess an integrated circuit (IC) detector, improving spatial resolution by means of reduced cross-talk between detector channels, in coronary CT angiography regarding image quality and stenosis quantification.

Methods and Materials: In the ex vivo part of the study, a coronary phantom including 63 defined stenoses were scanned with 128-section dual-source CT equipped with IC and with conventional detector technology. Data were reconstructed with filtered-back projection (FBP) and with iterative reconstruction (IR) at a slice-thickness of $0.6 \mathrm{~mm}$ and for data acquired with the IC detector with a slice-thickness of $0.5 \mathrm{~mm}$. Phantom observations were validated in 30 consecutive patients (11female;mean age 62) undergoing coronary CT angiography with IC. The patient images were reconstructed with FBP (slice-thickness:0.6 mm) and with IR (slice-thickness:0.5 mm). All images were assessed for image quality and degree of stenosis.

Results: Image quality was significantly $(P<0.001)$ higher with the IC detector as compared with the conventional detector. The IC detector yielded significantly more accurate results regarding stenosis grading: FBP: $12.1 \pm 7.6 \%$ vs. $13.7 \pm 7.6 \%$ $(P=0.043)$ and IR:10.5 $\pm 6.6 v s .13 .0 \pm 6.9 \%(P<0.001)$. Images with a slice thickness of $0.5 \mathrm{~mm}$ reconstructed with IR $(8.8 \pm 5.9 \%)$ obtained by the IC detector significantly $(P<0.001)$ improved measurement accuracy in the phantom as compared with FBP with a slice thickness of $0.6 \mathrm{~mm}(12.1 \pm 7.6 \%)$. We found a significantly $(\mathrm{P}<$ 0.001 ) higher image quality in patients and stenoses were quantified significantly $(\mathrm{P}=0.009)$, more severe with FBP $(47.6 \%)$ as compared with IR $(42.1 \%)$.

Conclusion: Our study indicates more accurate stenosis quantification in coronary $\mathrm{CT}$ angiography when acquiring data using an IC detector and combining IR with high-resolution images as compared with conventional detector technology and FBP reconstructions.

Author Disclosures:

B. Schmidt: Employee; Siemens Healthcare, Germany. 


\section{B-0661 10:39}

CT coronary angiography: effect of iodine CONcentration on vascular attenuation. The $\mathrm{CT}$-CON multicentric study preliminary results M. Rengo' ${ }^{1}$ A.S. Dharampal', D. Caruso', K. Nieman², A. Laghi', G.P. Krestin²; ${ }^{1}$ Latina/IT, ${ }^{2}$ Rotterdam/NL (marco.rengo@gmail.com)

Purpose: To explore the relative impacts of iodine concentration versus iodine delivery rate on intra-coronary attenuation. To describe the effect of iodine concentration on contrast bolus characteristics.

Methods and Materials: Two hundred fifty patients were prospectively randomised in 4 groups and all underwent CT Coronary Angiography (CTCA). Four CM with different iodine concentrations $(300,350,370,400 \mathrm{mgl} / \mathrm{ml})$ were delivered at a fix iodine delivery rate $(2.0 \mathrm{mgl} / \mathrm{s})$. Intracoronary attenuation values were measured and grouped on a per-vessel and per-segment bases. Time-to-peak, and pressure curves during $\mathrm{CM}$ injection were evaluated and compared.

Results: Injection fluxes were $6.7 \mathrm{ml} / \mathrm{sec}, 5.7 \mathrm{ml} / \mathrm{sec}, 5.4 \mathrm{ml} / \mathrm{sec}$ and $5 \mathrm{ml} / \mathrm{sec}$ for group A, B, C and D, respectively. Group A [300] and C [370] provided significantly higher intravascular attenuation values compared with group $B$ [350] and $D[400](P$ $<0.05$ ) in both per segment and per vessel analysis. Time-to-peak was significantly earlier in group $\mathrm{A}(15.3 \mathrm{sec})$ than in the other three groups. The injection peak pressure was significantly lower in group $A(185.16 \mathrm{psi})$ and $\mathrm{C}(189.05 \mathrm{psi})$ than in group B (215.89 psi) and D (243.33 psi). No extravasations were noted in all groups. Conclusion: Despite that the four CM were injected at the same IDR, intravascular attenuation values were not equal. The influence of chemico-physical characteristics influenced also the other injection parameters. In particular a non correspondence between the injection flux and the injection pressure was observed among groups.

\section{B-0662 10:48}

MDCT coronary angiography evolution of phasic critical stenosis in myocardial bridges

R. Malago, M. Tezza, G. Sala, A. Pezzato, C. Barbiani, R. Pozzi Mucelli; Verona/IT (robertomalago@gmail.com)

Purpose: MDCT-CA can detect the presence of myocardial bridges, through qualitative evaluation of coronary diameter, measured from the end-diastolic to the end-systolic phase; an objective evaluation can also be performed through quantitative coronary CT (QCTA). The aim is to evaluate the significance of stenosis in case of myocardial bridge.

Methods and Materials: Sixty-nine cases (mean age $62.3 \mathrm{YO}, 48 \mathrm{M}$ ) with MB identified at MDCT-CA in the period from 04/2008 to 02/2012 were retrospectively evaluated. All patients underwent CA that was performed to treat critical coronary artery stenosis. Bridges were evaluated and divided into 3 categories according to Mohlenkamp classification. Through curved MPR and axial reconstruction of the bridge the diameter of the vessel was measured before the intramyocardial stretch (a), at the middle portion (b) and at the end (c) of the bridge, using the data from the $10 \%$ of the RR interval in all datasets. Measurements were performed manually and using QCTA and compared with QCA at end-systolic and end-diastolic phase. Correlation between the results obtained by manual measurements, QCTA and QCA were calculated according to Spearman test.

Results: Type I was retrieved in 34/69 patients and type 3 in 2/69 patients. Minimum diameter in the middle of the bridge recorded was $2 \mathrm{~mm}$. Significant stenosis was recorded in 8/69 patients and confirmed with QCA in 6/69 of them. Correlation between manual score and QCA resulted $r=0.84, p>0.001$.

Conclusion: Both manual and QCTA measurements can evaluate the presence and the type of bridges and discriminate the phasic stenosis.

\section{B-0663 10:57}

Quantitative CT coronary angiography: does it predict functionally significant coronary stenoses?

A. Rossi ${ }^{1}$, S. Papadopoulou' ${ }^{1}$, F. Pugliese ${ }^{2}$, B. Russo' ${ }^{1}$, A. Dharampal ${ }^{1}$,

P.H. Kitslaar ${ }^{3}$, S.E. Petersen ${ }^{2}$, P. de Feyter ${ }^{1}$, G.P. Krestin ${ }^{1} ;{ }^{1}$ Rotterdam/NL,

${ }^{2}$ London/UK, ${ }^{3}$ Leiden/NL (a.rossi@erasmusmc.nl)

Purpose: CT coronary angiography (CTCA) interpreted visually is often inaccurate in identifying functionally significant coronary lesions in patients with stable angina. Several quantitative cross-sectional parameters can be derived from CTCA. The aim of this study was to compare the diagnostic performance of quantitative CTCA with visual CTCA in the detection of functionally significant coronary lesions using fractional flow reserve (FFR) as the reference standard.

Methods and Materials: CTCA and FFR measurements were obtained in 99 symptomatic patients. In total, 144 coronary lesions detected on CTCA were visually graded for stenosis severity. Quantitative CTCA cross-sectional parameters included minimal area diameter (MLA), percentage area stenosis (\%AS) and plaque burden [(vessel area - lumen area)/vessel area*100]. Optimal cut-off values of
CTCA-derived parameters were determined and their diagnostic performance for the detection of flow-limiting coronary lesions (FFR $\left.{ }^{2} 0.80\right)$ was compared to visual CTCA. Results: Optimal cut-off values to predict flow-limiting coronary lesion were 1.8 mm2 for MLA, $71 \%$ for $\%$ AS and $76 \%$ for plaque burden. Significantly higher specificity for MLA (68\%; 95\%Cl: 58-78; $p=0.001)$, \%AS $(68 \%, 95 \% \mathrm{Cl}: 58-77$; $\mathrm{p}<$ $0.001)$ and plaque burden $(63 \% ; 95 \% \mathrm{Cl}: 53-73 ; p=0.004)$ were found compared to visual CTCA (42\%; $95 \%$ Cl: 32 to 52$)$. No significant difference in sensitivity was found between visual CTCA and quantitative CTCA cross-sectional parameters. Conclusion: Quantitative CTCA cross-sectional parameters improve the prediction of functionally significant coronary lesions compared to visual CTCA assessment but remain insufficient. Functional assessment is still needed in lesions of moderate severity to guide patient's management.

\section{Author Disclosures:}

P.H. Kitslaar: Employee; Medis medical imaging systems B.V.

\section{B-0664 11:06}

Impact of iterative CT image reconstruction on calcium score measurements

J.A.C. van Osch ${ }^{1}$, M. Mouden ${ }^{1}$, J.A. van Dalen ${ }^{1}$, J.R. Timmer ${ }^{1}$, S. Reiffers ${ }^{1}$, M.J.W. Greuter ${ }^{2}$, J.P. Ottervanger ${ }^{1}$, S. Knollema ${ }^{1}$, P.L. Jager ${ }^{1} ;{ }^{1}$ Zwolle/NL, $^{2}$ ${ }^{2}$ Groningen/NL (j.a.c.van.osch@isala.nl)

Purpose: To investigate the effect of iterative reconstruction (IR) on the coronary calcium score in cardiac computed tomography (CT).

Methods and Materials: In 111 patients with suspected coronary artery disease and a low to intermediate pre-test likelihood the total Agatston calcium scores (TAS) were assessed using a 64-slice CT (Lightspeed VCT XT, GE). The IR algorithm used was adaptive statistical iterative reconstruction (ASIR). Comparisons were performed between scores, obtained with full-dose scans, using traditional filtered back projection (FBP) and use of $100 \%$ IR. Also, the resulting shift of patients into different risk categories was evaluated.

Results: Comparison of the FBP and IR images showed a significant reduction of the TAS $(411 \pm 39$ versus $327 \pm 31, P<0.001)$. For all patients, the TAS calculated on the IR images was consistently lower than the TAS calculated on the FBP images (reduction ranging from $3 \%$ to $100 \%$ ). As a result, almost $30 \%$ of patients classified in a lower risk category when using IR, compared to FBP. Significantly more patients had a TAS of zero when using $100 \%$ IR ( 26 vs. $11, \mathrm{P}<0.01)$.

Conclusion: The TAS decreased significantly using IR compared to FBP. This underestimation, compared to FBP, is present over the entire range of the calcium score, but most pronounced in the lower range of calcium scores. Subsequently, more patients were classified in the zero calcium score category. This effect may have a significant impact on clinical decision making and should be taken into account when using IR.

\section{B-0665 11:15}

Accuracy of prospectively ECG-triggered ultra low-dose coronary dual-source $\mathrm{CT}$ angiography using iterative reconstruction M. Lell, C. Layritz, T. Pflederer, W. Wuest, S. Achenbach, M. Uder; Erlangen/DE (michael.lell@uk-erlangen.de)

Purpose: To evaluate the image quality and diagnostic accuracy of very-low-dose CT angiography (CTA) for evaluation of coronary artery stenosis.

Methods and Materials: 50 symptomatic patients with an intermediate likelihood for coronary artery disease underwent coronary CTA prior to invasive coronary angiography. CTA images were reconstructed both using standard filtered back projection (FBP) and a raw-data based IR algorithm. Subjective image quality, image noise, signal-to-noise ratio, contrast-to-noise ratio as well as the presence of coronary stenosis $>50 \%$ were independently determined by two observers. Results: Mean estimated effective dose was $0.66 \mathrm{mSv}$. IR led to significantly improved objective image quality compared to FBP (image noise: $41 \pm 12$ vs. $49 \pm 11$ HU, CNR: $16 \pm 8$ vs. $12 \pm 4$, SNR: $13 \pm 7$ vs. $10 \pm 3$; all $p<0.001)$. Four coronary segments were not evaluable on FBP data, where as all segments showed diagnostic image quality with IR. To detect significant coronary stenosis, sensitivity, specificity, PPV and NPV were $69 \%, 97 \%, 69 \%, 97 \%$ per vessel with FBP, the corresponding values with IR were $81 \%, 97 \%, 68 \%, 98 \%(p=0.617)$.

Conclusion: Raw-data-based iterative reconstruction significantly improved image quality in very-low-dose prospectively ECG-triggered coronary DSCT angiography when compared to standard reconstruction using FBP. Along with substantial reduction of radiation exposure, iterative reconstruction also shows a trend towards higher accuracy in coronary stenosis detection compared to FBP.

Author Disclosures:

M. Lell: Grant Recipient; Bayer, Siemens. Speaker; Bayer, Siemens.

S. Achenbach: Grant Recipient; Bayer, Siemens. Speaker; Siemens. M. Uder: Grant Recipient; Siemens. 


\section{B-0666 11:24}

Influence of iterative reconstruction on coronary calcium score in cardiac computed tomography

K. Jaspers ${ }^{1}$, J.A.C. Van Osch' ${ }^{2}$, J.M. Groen ${ }^{1}$, M.J.W. Greuter ${ }^{1} ;{ }^{1}$ Groningen/NL, ${ }^{2} Z$ wolle/NL (m.j.w.greuter@umcg.nl)

Purpose: To investigate the relation between the percentage of iterative reconstruction (IR) and coronary calcium score in cardiac computed tomography (CT). Methods and Materials: A phantom containing 100 cylindrical calcifications with varying size and density was inserted into a thorax phantom (QRM Thorax, QRM, Germany) with artificial lungs and spine. The phantom was scanned with a clinical acquisition protocol on a 64-slice CT (Lightspeed VCT XT, GE). Each scan was repeated five times. The images were reconstructed with filtered backprojection (FBP) and increasing percentages of IR of $20-100 \%$. The amount of calcium was determined as an Agatston score. The influence of percentage IR on both total Agatston score (TAS) of the phantom as well as the individual calcium score (IAS) for each calcification was investigated.

Results: The average TAS decreased with increasing percentage of $\operatorname{IR}(p<0.001)$. TAS was $124 \pm 22$ at $0 \%$ IR and $76 \pm 16$ at $100 \%$ IR. IAS were underestimated when IR was used compared to pure FBP. The underestimation was relatively larger for calcifications with a low calcium score and increased for increasing percentage IR. Increasing numbers of calcifications were missed at increasing percentages of IR. At $20 \%$ IR, $4 \%$ of the calcifications were missed, whereas at $100 \%$ IR, $26 \%$ of the calcifications could not be detected.

Conclusion: Iterative reconstruction in CT results in an underestimation of coronary calcium scores compared to pure filtered backprojection. With increasing iterative reconstruction more calcifications yield a zero calcium score.

\section{B-0667 11:33}

Magnetic resonance velocity mapping-based evaluation of elevated mean pulmonary arterial pressure: the impact of visualisation techniques U. Reiter ${ }^{1}$, G. Reiter ${ }^{1}$, G. Kovacs ${ }^{1}$, A. Stalder ${ }^{2}$, M.A. Gulsun ${ }^{3}$, A. Greiser ${ }^{2}$, H. Olschewski', M.H. Fuchsjäger'; ${ }^{1}$ Graz/AT, ${ }^{2}$ Erlangen/DE, ${ }^{3}$ Princeton, NJ/US (ursula.reiter@klinikum-graz.at)

Purpose: Elevated mean pulmonary arterial pressure (mPAP) correlates linearly with the relative period of existence of vortical blood flow in the main pulmonary artery (PA), which can be investigated by time-resolved 3-dimensional (3D) magnetic resonance phase-contrast imaging (MRPCI). Ability of different visualisation tools to estimate elevated mPAP from complex flow patterns in the PA was analysed. Methods and Materials: 28 patients with elevated mPAP (11 male, mean age $60 \pm 14$ years) underwent right heart catheterization and time-resolved 3D-MRPCI of the PA. 4D-Flow software (Siemens) was used to visualise 3D velocity vector fields on 2-dimensional anatomical images (3D-vectors), vector fields projected on 2-dimensional cut-planes by multiplanar reconstruction (MPR) and 3D streamline representation. Relative period of existence of vortical blood flow within the cardiac cycle (tvortex) was evaluated with all visualisation techniques and compared with Bland-Altman and linear regression analysis.

Results: tvortex derived from different visualisations strongly correlated $(r=0.97$, 0.96 and 0.94 for 3D-vectors versus MPR, 3D-vectors versus streamlines and MPR versus streamlines, respectively). No bias was observed for tvortex determined from different techniques, 95\%-limits-of-agreement were \pm 0.11 between tvortex determined from 3D-vectors versus MPR, and \pm 0.12 in both other comparisons. In all techniques tvortex showed strong correlation with MPAP with small standard errors from regression lines $(r=0.97, \mathrm{SE}=3.2 \mathrm{mmHg}$ for $3 \mathrm{D}$-vectors, $r=0.96, \mathrm{SE}=3.6$ $\mathrm{mmHg}$ for streamlines, and $\mathrm{r}=0.92, \mathrm{SE}=4.8 \mathrm{mmHg}$ for MPR).

Conclusion: Relative periods of existence of vortical blood flow in the main pulmonary artery can be determined from vector field and streamline visualisations. 3D-vectors, however, yield best estimates for elevated mPAP.

\section{Author Disclosures:}

G. Reiter: Employee; Siemens. A. Stalder: Employee; Siemens. M.A. Gulsun: Employee; Siemens. A. Greiser: Employee; Siemens.

\section{B-0668 11:42}

Undersampled real time cine SSFP with through-time radial GRAPPA: evaluation of RV function in breath-hold and free breathing

B.J. Wintersperger ${ }^{1}$, A. Pellow', G. Bastarrika', M.A. Griswold ${ }^{2}$,

N. Seiberlich ${ }^{2}$, M. Sussman ${ }^{1} ;{ }^{1}$ Toronto, ON/CA, ${ }^{2}$ Cleveland, OH/US

(Bernd.Wintersperger@uhn.ca)

Purpose: To evaluate real-time imaging using the Through-time Radial GRAPPA reconstruction in free-breathing and breath-hold evaluation of right ventricular (RV) function.
Methods and Materials: 12 patients underwent evaluation of the RV function on $1.5 \mathrm{~T}$ employing a 32-element coil. Acquisition was performed using a real-time radial SSFP sequence (16 projections) in a multislice breath-hold and free-breathing setting with a spatial resolution of $2 \times 2 \mathrm{~mm}^{2}$ (matrix 128; slice $6 \mathrm{~mm}$ ) and a temporal resolution of $46 \mathrm{~ms}$ (TR $2.9 \mathrm{~ms}$ ). Through-time radial GRAPPA reconstruction was performed off-line (MATLAB) incorporating fully sampled (128 projections) calibration data acquired prior to the undersampled datasets. Segmented Cartesian cine SSFP was acquired at identical slice positions in multiple consecutive breath-holds with a spatial resolution of 1.3-1.5x1.3-1.5 mm, $6 \mathrm{~mm}$ slices and $35 \mathrm{~ms}$ temporal resolution (TR $3 \mathrm{~ms}$ ). Free-breathing and breath-hold real-time datasets were evaluated for RV size and function and compared with segmented cine SSFP.

Results: Breath-hold real-time imaging with through-time radial GRAPPA did not show significant difference in EDV ( $285.3 \pm 102.2 \mathrm{ml}$ vs. $292.8 \pm 107.2 \mathrm{ml} ; \mathrm{P} 0.12)$, ESV $(161.3 \pm 85.0 \mathrm{ml}$ vs. $163.2 \pm 78.7 \mathrm{ml} ; \mathrm{P}=0.6)$ and $\mathrm{EF}(45.2 \pm 9.6 \%$ vs. $45.5 \pm 6.6 \% ; \mathrm{P}=0.73)$ with low systematic and random errors as compared with respective segmented cine SSFP data. Free-breathing real-time imaging demonstrates a significant offset for ESV $(177.6 \pm 89.2 \mathrm{ml}$ vs. $163.2 \pm 78.7 \mathrm{ml} ; P=0.02)$ resulting in lower $E F$ results $(42.3 \pm 9.6 \%$ vs. $45.5 \pm 6.6 \% ; \mathrm{P}=0.05)$ and higher scatter for random error.

Conclusion: Real-time imaging using through-time radial GRAPPA allows for adequate temporal and spatial resolution to ensure adequate volumetric analysis of the RV. Although it enables artefact-free free-breathing cine data acquisition, inconsistency of slice location in transverse orientation may affect accuracy of volumetric results.

\section{Author Disclosures:}

B.J. Wintersperger: Research/Grant Support; Siemens Healthcare. Speaker; Bayer Pharma, Siemens Healthcare.M.A. Griswold: Equipment Support Recipient; Siemens Healthcare. Patent Holder; ThroughTime GRAPPA. Research/Grant Support; Siemens Healthcare. N. Seiberlich: Patent Holder; ThroughTime GRAPPA.

\section{B-0669 11:51}

Diagnostic accuracy of delayed phase dual-energy CT for the detection of chronic myocardial infarction compared to 3-T MRI late gadolinium enhancement

J.L. Wichmann, J.M. Kerl, T. Lehnert, M. Doss, T.J. Vogl, R.W. Bauer; Frankfurt a.Main/DE (docwichmann@gmail.com)

Purpose: To compare the performance of delayed phase cardiac dual-energy CT (DECT) with different grayscale image contrasts and selective myocardial iodine mapping for the detection of chronic myocardial infarction compared to 3 T MRI. Methods and Materials: This HIPAA-compliant study was approved by the Institutional Review Board and patients gave informed consent. 20 patients underwent delayed phase cardiac CT on a second-generation DECT scanner. Late iodine enhancement data were compared with late gadolinium enhancement images from 3 T cardiac MRI. DECT greyscale images were reconstructed as $100 \mathrm{kV}, 140 \mathrm{kV}$ and weighted-average (WA; linear blending) images from low and high $\mathrm{kV}$ data incorporating $80 \%, 60 \%$ or $30 \%$ of $100 \mathrm{kV}$ data. In addition, a colour-coded map of myocardial iodine distribution was calculated from DECT data. Two independent blinded radiologists reviewed all images for late enhancement areas in standard cardiac views and rated subjective image quality.

Results: DECT data from $100 \mathrm{kV}, \mathrm{WA}-80 \%$ and WA- $60 \%$ showed identical results for the correct identification of myocardial scarring ( $89 \%$ sensitivity, $98 \%$ specificity and $96 \%$ accuracy). However, WA- $60 \%$ received the best subjective image quality rating and average measured infarct size correlated best with MRI. Colour-coded iodine distribution maps were prone to artefacts ( $52 \%$ sensitivity, $88 \%$ specificity, $81 \%$ accuracy), overestimating quantity of scars while underestimating lesion size by $55 \%$ compared to MRI.

Conclusion: Linear blending of delayed phase cardiac DECT data improves image quality for the detection of myocardial scar tissue compared to MRI. Colour-coded myocardial iodine distribution maps were prone to artefacts and showed inferior diagnostic performance.

Author Disclosures:

J.M. Kerl: Consultant; Research Consultant, Siemens AG Speakers Bureau, Siemens AG. R.W. Bauer: Consultant; Research Consultant, Siemens AG Speakers Bureau, Siemens AG. 


\section{Neuro}

\section{SS 1311}

\section{Brain tumours: imaging and therapy}

\section{Moderators:}

X. Golay; London/UK

A. Zimny; Wroclaw/PL

\section{B-0670 10:30}

Intra-arterial ophthalmic artery chemotherapy for group D

retinoblastoma in children: 4-years Lausanne experience

S. Binaghi, J.-B. Zerlauth, M. Beck-Popovic, F. Munier, R. Meuli; Lausanne/CH (stefano.binaghi@chuv.ch)

Purpose: To evaluate prospectively the efficacy of supra-selective ophthalmic artery chemotherapy with Melphalan as a tumoricidal agent in children with advanced retinoblastoma to avoid surgical enucleation and/or secondary radiotherapy. Methods and Materials: From November 2008 to August 2012, 27 children (mean age 25 months) affected by group $D$ retinoblastoma underwent intraarterial superselective chemotherapy using Melphalan $(0.35 \mathrm{mg} / \mathrm{kg})$ into the ophthalmic artery. This procedure was performed under general anesthesia and repeated for a maximum of 3 sessions separated by an interval of 3-4 weeks in between sessions. A total of 72 procedures were performed. Every endovascular treatment was coupled with local intravitreal Melphalan injection, thermotherapy and/or cryotherapy.

Results: Technical success was achieved in all but two patients without major systemic or thromboembolic complications. 25 patients showed dramatic regression of tumor volume, vitreous and subretinal seeds. In the other 2 cases, severe vasospasm of the femoral artery in one case and of the ophthalmic artery in the other case prevented the successful technical realization of the procedure. Enucleation and external beam radiotherapy could be avoided in all but two treated patients, with a mean follow-up of one year. Local complications consisted of retinal detachment (2), transient ptosis (18), conjunctival and lid edema (6), local skin pigmentation (1), transient carotid artery vasospasm (1) and sectoral choroidal occlusive vasculopathy (2).

Conclusion: Transarterial ophthalmic artery chemotherapy using Melphalan has an important tumoricidal action in advanced retinoblastoma, and can avoid secondary enucleation and/or external beam radiotherapy.

\section{B-0671 10:39}

Neurocutaneous melanosis in children with giant congenital melanocytic nevi

O. Szczygielski, M. Boczar, E. Sawicka, M. Bekiesinska-Figatowska, J. Madzik, A. Romaniuk-Doroszewska, M. Uliasz, T. Klepacka, E. Michalak; Warsaw/PL (m.figatowska@mp.pl)

Purpose: To assess the incidence of neurocutaneous melanosis (NCM) in children with giant congenital melanocytic nevi.

Methods and Materials: Material consists of 21 children with giant melanocytic nevi. Brain MRI was performed at a $1.5 \mathrm{~T}$ scanner with 16 -channel head coil. All the children were operated on, the nevi were excised and histopathologically assessed. Results: Melanin deposits in the brain were found in 7 children (33.3\%). Six patients were asymptomatic and one suffered from epileptic seizures. The lesions were located in the temporal and frontal lobes, thalamus, cerebellum and pons. Two patients showed leptomeningeal spread of the disease. In one patient multilevel nerve roots neurofibromas were found on MRI and neurofibromatosis type 1 was diagnosed. No statistically significant difference of the localisation of skin nevi was found between children with and without NCM. In all the patients with NCM the excised nevi showed the picture of compound nevus with neurofibromatic component. Additionally in one patient superficial spreading melanoma was diagnosed. In the remaining 14 children various histological types of nevi were found with predominance of compound nevus with neurofibromatic component ( $8 / 14$ cases $=57.1 \%)$. Conclusion: In every case of giant melanocytic nevus brain MRI should be performed to confirm or rule out the co-existence of CNS lesions typical of NCM. Every child with NCM requires neurological and ophthalmological follow-up for monitoring the lesions. NCM seems to be correlated with histopathological type of compound nevus with neurofibromatic component. Localisation of skin nevi is not correlated with presence of NCM.

\section{B-0672 10:48}

Surveillance neuroimaging in patients with medulloblastoma should include diffusion-weighted imaging

L. van den Hauwe, C. Gidding, E. van Lindert, P. Wesseling, B. Goraj; Nijmegen/NL (lucvdhauwe@mac.com)

Purpose: To demonstrate that diffusion-weighted imaging (DWI) is able to pick up intracranial recurrences of medulloblastoma (MB) with higher sensitivity than contrast-enhanced (CE) series.

Methods and Materials: All MRI examinations of 22 children with histologically proven MB were retrospectively evaluated. Routine follow-up examinations were performed initially 3 months after operation and thereafter, every 6 months for the next 2 years. Then, controls were done yearly. Concomitant cranial and spinal MRI included T1, T2, and FLAIR in the axial plane, followed by T1 after gadolinium (Gd) in 3 orthogonal planes for the brain. Additional DWI was performed. Subsequent spinal imaging included sagittal T1- en T2-weighted images.

Results: Eleven recurrences were observed in 10 patients. In 5 patients recurrent disease was observed at the resection site in 2 of which subarachnoid spread was also observed, with recurrence in loco only in 3 patients. Five patients had recurrent disease away from the resection cavity, 2 of them in the frontal areas and 4 intraventricular recurrences at the supratentorial level. In 2 patients CE-series scored better than DWI; in 3 patients results on DWI and CE-series were equivocal. In 5 patients, recurrences were better demonstrated on DWI; some of them were only observed in retrospect.

Conclusion: The results of our retrospective study demonstrate that DWI is a powerful technique allowing for early diagnosis of recurrent disease in MB patients. DWI especially demonstrates non-enhancing metastatic lesions earlier than CElesions. DWI should therefore be included in surveillance imaging protocols of $\mathrm{MB}$ patients, especially in the group of high-risk patients.

\section{B-0673 10:57}

Preoperative evaluation of consistency of meningiomas: diagnostic efficacy of MRI

A. Hiwatashi, T. Yoshiura, O. Togao, K. Yamashita, K. Kikuchi, K. Yoshimoto, M. Mizoguchi, S.O. Suzuki, H. Honda; Fukuoka/JP

Purpose: To evaluate the value of conventional magnetic resonance imaging (MRI), diffusion-weighted imaging (DWI) and perfusion imaging (PI) in predicting the consistency of intracranial meningiomas.

Methods and Materials: This study included 44 patients ( 30 females and 14 males, age range; $20-83$ years, median 60 ) with pathologically proven meningiomas. All patients underwent preoperative T2-weighted imaging (T2WI), DWI, PI including arterial spin labelling (ASL), and dynamic contrast enhancement imaging and postcontrast 3D T1-weighted imaging on $3 \mathrm{~T}$-MRI. The ratio of signal intensity of T2WI (T2R) to adjacent normal cortex that of minimum ADC (ADCR) to normal appearing white matter, tumour blood flow (TBF), $\mathrm{K}$ trans , and tumour volume were calculated. These were compared with the consistency of meningiomas reported by neurosurgeons. Statistical analysis was performed with Mann-Whitney U test and logistic regression analysis.

Results: Twenty-five meningiomas were soft and the other 19 were hard (including mixed consistency). Compared to hard meningiomas, the soft meningiomas had higher T2R $(1.39 \pm 0.24$ vs. $1.15 \pm 0.33, P<0.05)$ and higher ADCR $(1.05 \pm 0.18$ vs. $0.97 \pm 0.19, P<0.05)$. There were no statistically significant differences in $T B F, K$ trans or tumour volume between soft and hard meningiomas $(P>0.05)$. Logistic regression analysis resulted in T2R as a causative factor to predict consistency $(P<0.01)$. Conclusion: Among various imaging techniques, T2WI is the most feasible in predicting the consistency of meningiomas.

\section{B-0674 11:06}

Radiation-induced telangiectasia in the long-term survivors of intracranial germ cell tumours: whole-ventricle vs whole-brain radiation L. Li, S. Mugikura, T. Murata, T. Kumabe, K. Jingu, T. Fujii, E. Mori, S. Takahashi; Sendai/JP (liliyanzhen05@gmail.com)

Purpose: Telangiectasia on MR images were reported to be seen in patients who received intracranial radiation. To determine the relationship between the radiation field/dose and the prevalence of telangiectasia on MR images, we compared whole-ventricle alone (WV) and whole-ventricle plus whole-brain (WB) radiation groups in the long term survivors (more than 10 years) of intracranial germ cell tumours (GCT).

Methods and Materials: We compared the number of telangiectasia on $\mathrm{T}^{*}$ weighted MR images $\left(T 2^{*} \mathrm{WI}\right)$ between WV $(n=12)$ and WB $(n=11)$ groups. Total dose of radiation between two groups was not significantly different (54.1 Gy in WV 
and 51.3 Gy in WB groups). In the WB group, axial sections of the ventricular level were considered to have received significantly higher dose of radiation (51.3 Gy, high-dose field) than the other upper and lower levels (24.0 Gy, low-dose fields). In the WB group, we compared the number of telangiectasia on $\mathrm{T}^{*} \mathrm{WI}$ between ventricular level and the other upper and lower levels.

Results: Telangiectasias were observed in 22 (96\%) patients. The number of telangiectasia in the WB group was significantly higher than the WV group (average 8.5 in WB and 3.6 in WV, $P=0.019$ ). In WB group, the number of telangiectasia was significantly higher in the ventricular level than the other upper and lower levels $(P<0.01)$

Conclusion: Radiation-induced telangiectasia appears to occur in at least $96 \%$ of patients who undergo cranial irradiation. Larger radiation exposure and higher radiation dose were associated with higher number of telangiectasia lesions.

\section{B-0675 11:15}

Dynamic sequences in Intraoperative MRI during transsphenoidal resection of pituitary adenomas: quantitative analysis

M. Rossi Espagnet ${ }^{1}$, A. Boellis ${ }^{1}$, V. Coppola ${ }^{2}$, A. Romano ${ }^{1}$, S. Pugliese ${ }^{1}$,

F. Tavanti ${ }^{1}$, L. Calabria ${ }^{1}$, L. Fantozzi ${ }^{1}$, A. Bozzao ${ }^{1} ;{ }^{1}$ Rome $/ I T,{ }^{2}$ Ronciglione/IT

Purpose: During transsphenoidal pituitary removal of macroadenomas, Intraoperative Magnetic Resonance Imaging (iMRI) can assess the presence of residual tumour. The purpose of this prospective study was to demonstrate the efficacy of intraoperative dynamic contrast-enhanced (DCE) sequences compared with conventional $\mathrm{CE}$ in evaluating tumour removal.

Methods and Materials: Inclusion criteria were complete tumour removal at iMR as subsequently demonstrated by normal 12-month MRI follow-up or-demonstration of residual tumour at $\mathrm{MRI}$ as assessed by DCE and CE images subsequently confirmed by immediate second look surgery. 19 patients were thus selected. They all underwent iMRI (1.5T) acquisitions during transsphenoidal resection of macroadenomas. For each patient, DCE and CE images were acquired directly in the operating room when neurosurgeon indicated complete tumour removal. These sequences were evaluated by measuring the volume of the surgical cavity. The mean values of these volumes were statistically compared through Student $t$-test analysis ( $p$ value was set $<0.05$ )

Results: No patient showed recurrence at follow-up indicating complete removal of the tumour, 2 showed tumour residual. Intraoperative analysis demonstrated that the mean value of the surgical cavities was significantly bigger in DCE than in CE images (2955 mm3 vs 1963 mm3, respectively, $p=0.022$ ).

Conclusion: This study demonstrated underestimation of surgical cavity by conventional $\mathrm{iMRI}$, simulating possible residual tumour and potentially leading to unnecessary surgical revision. This may be related to post-operative changes with subsequent delayed enhancement. Because of this, DCE sequences are mandatory in the intraoperative evaluation of surgical cavity during transsphenoidal pituitary resection.

\section{B-0676 11:24}

Preoperative classification of cerebral tumours by applying whole brain VPCT: which parameter to use in order to achieve the highest prognostic value?

A. Xyda ${ }^{1}$, E. Klotz ${ }^{2}$, U. Haberland ${ }^{2}$, R. Schramm ${ }^{3}$, M. Knauth ${ }^{3}$, P. Schramm ${ }^{3}$. ${ }^{1}$ Iraklion/GR, ${ }^{2}$ Forchheim/DE, ${ }^{3}$ Göttingen/DE (iroxida@yahoo.com)

Purpose: To evaluate the preoperative diagnostic power and classification accuracy of perfusion parameters derived from whole brain volume perfusion CT (VPCT) in patients with cerebral tumours.

Methods and Materials: Sixty-three patients (31 males, 32 females; mean age $55.6 \pm 13.9$ years), with MRI findings suggestive of cerebral lesions, underwent VPCT. Two readers independently evaluated VPCT data. Volumes of interest (VOIs) were delineated around the tumour according to maximum intensity projection volumes, and then mapped automatically onto the cerebral blood volume (CBV), flow (CBF) and permeability (Ktrans) perfusion datasets. A second VOI, cortically pronounced, was placed in the contra lateral hemisphere, as control. Correlations among perfusion values, tumour grade, cerebral hemisphere and VOIs were evaluated. Moreover, the diagnostic power of VPCT parameters, by means of positive and negative predictive value, was analysed.

Results: Our cohort included 32 high-grade gliomas WHO III/IV, 18 low-grade I/ II, 6 primary cerebral lymphomas, 4 metastases and 3 tumour-like lesions. Ktrans demonstrated the highest sensitivity, specificity and positive predictive value, with a cut-off point of $2.21 \mathrm{~mL} / 100 \mathrm{~mL} / \mathrm{min}$, for both the comparisons between high-grade versus low-grade and low-grade versus primary cerebral lymphomas. However, for the differentiation between high-grade and primary cerebral lymphomas, CBF and CBV proved to have $100 \%$ specificity and $100 \%$ positive predictive value, identifying preoperatively all the histopathologically proven high-grade gliomas. Conclusion: All the three perfusion parameters play an important role in the preoperative classification of cerebral tumour entities with high rate of histopathologic correlation.

Author Disclosures:

E. Klotz: Investigator; Principal Scientist of the CT Division in Siemens Healthcare Sector. U. Haberland: Employee; CT division, Siemens Healthcare Sector.

\section{B-0677 11:33}

Apparent diffusion coefficient in determination of histological subtype of meningioma

D.A. Stojanov; Nis/RS (drstojanov@gmail.com)

Purpose: The aim of our study was to verify the reliability of apparent diffusion coefficient $(A D C)$ measurements to differentiate benign from atypical/malignant meningiomas and among different sub-types.

Methods and Materials: Thirty patients (20 women and 10 men, average age $53 \pm 15$ years) with meningiomas were prospectively studied using routine MR imaging and diffusion-weighted imaging. All MR examinations were performed on 1.5 T MR (Avanto, Siemens, Erlangen, Germany). ADC values of the neoplastic tissue (avoiding calcifications and cystic or necrotic areas) were obtained as the mean of measurements from three regions of interests within the mass. MRI findings were compared with the histopathologic findings after resection using World Health Organization criteria

Results: Meningiomas were histologically graded as benign and malignant. The meningothelial subtype was found in $15(50 \%)$ patients, fibroblastic in $10(33.33 \%)$ patients and cystic $5(16.67 \%)$ patients. There was no statistical difference between meningothelial, fibroblastic and cystic meningiomas when considering mean ADC values $(0.000411 \pm 0.000066 \mathrm{~mm} 2 / \mathrm{s}$ vs. $0.000750 \pm 0.001045 \mathrm{~mm} 2 / \mathrm{s}$ vs. 0.000688 $\pm 0.000063 \mathrm{~mm} 2 / \mathrm{s})$ (p[[Unable to Display Character: \&\#707;]]0.05).

Conclusion: ADC measurement does not seem reliable in grading meningiomas or identifying histological sub-types.

\section{B-0678 11:42}

Evaluation of diffusivity in pituitary adenoma in the Sella Turcica with 3D turbo field echo with diffusion-sensitized driven-equilibrium preparation: initial experience

A. Hiwatashi ${ }^{1}$, T. Yoshiura' ${ }^{1}$, O. Togao ${ }^{1}$, K. Yamashita ${ }^{1}$, K. Kikuchi ${ }^{1}$, H. Honda' M. Obara ${ }^{2} ;{ }^{1}$ Fukuoka/JP, ${ }^{2}$ Tokyo/JP

Purpose: To evaluate the feasibility of 3D turbo field echo (TFE) with diffusionsensitized driven-equilibrium (DSDE) preparation, which is a novel non-EPI technique for diffusion-weighted imaging, for pituitary adenoma in sella turcica. Methods and Materials: This prospective study included thirteen patients ( 9 females and 4 males, age 19-82 years) with pituitary adenomas were imaged with 3D TFE with DSDE preparation. Among them four were prolactin-producing (PRL), three were growth hormone-producing $(\mathrm{GH})$ and six were non-functioning tumours (NON). Motion probing gradients were conducted at one direction (A-P) with $b$ values of 0 and $500 \mathrm{~s} / \mathrm{mm}^{2}$. The imaging voxel size was $1.5 \times 1.5 \times 1.5 \mathrm{~mm}^{3}$. The apparent diffusion coefficients (ADCs) were measured in the pituitary adenoma and the normal pituitary gland.

Results: In each patient, a pituitary adenoma was clearly visualised on 3D TFE with DSDE preparation and ADC maps without obvious geometrical distortion. ADC of the pituitary adenoma ranged from 0.57 to $2.90 \times 10^{-3} \mathrm{~mm}^{2} / \mathrm{s}$ (mean \pm standard deviation, $1.37 \pm 0.72 \times 10^{-3} \mathrm{~mm}^{2} / \mathrm{s}$ ), and was higher than that of the norma pituitary gland $\left(1.34 \pm 0.23 \times 10^{-3} \mathrm{~mm}^{2} / \mathrm{s}\right)$ without statistically significant difference $(\mathrm{P}>0.05)$. ADC in $\mathrm{PRL}\left(2.07 \pm 0.92 \times 10^{-3} \mathrm{~mm}^{2} / \mathrm{s}\right)$ was significantly higher than that in GH $\left(0.86 \pm 0.31 \times 10^{-3} \mathrm{~mm}^{2} / \mathrm{s}\right)$ and in NON $\left(1.17 \pm 0.32 \times 10^{-3} \mathrm{~mm}^{2} / \mathrm{s}\right)(P<0.05)$. Conclusion: With its insensitivity to field inhomogeneity and high spatial resolution, 3D TFE with DSDE preparation was feasible to evaluate the diffusivity in the pituitary gland. ADC measurement using this new technique may help characterise hormone activity of pituitary adenomas.

Author Disclosures:

M. Obara: Employee; Makoto Obara. 


\section{Breast}

\section{SS 1302}

\section{How to get more from breast imaging modalities}

\section{Moderators:}

N. Houssami; Sydney/AU

M. Nadrljanski; Belgrade/RS

\section{B-0679 10:30}

Magnetic resonance imaging of fibroadenomas in the female breast: new insights on dynamic and morphologic profiles and differential diagnosis from malignant breast lesions

M. Dietzel ${ }^{1}$, P.A.T. Baltzer ${ }^{2}$, W.A. Kaiser ${ }^{3} ;{ }^{1}$ Erlangen, Jena/DE,

${ }^{2} J e n a / D E$, Vienna/AT, ${ }^{3} J e n a / D E$ (dietzelmatthias2@hotmail.com)

Purpose: Fibroadenomas are amongst the most common benign breast tumours. Surprisingly, there is still little systematic data on their typical MRI characteristics. Accordingly, this breast MRI study investigates morphologic and dynamic profiles of fibroadenomas in detail and aims to identify overall accuracy regarding the differentiation from breast cancer.

Methods and Materials: Consecutive patients receiving breast MRI (standardised protocols: dynamic-T1w-GRE before/after Gd-DTPA [0.1 mmol/kgBW]; T2w-TSE) with subsequent surgico-pathological verification were enrolled. For the detailed assessment of morphologic and dynamic profiles two experienced radiologists (> 500 MRM examinations; blinded to surgico-pathological verification) performed prospective evaluation of breast MRI in consensus applying 17 predefined breast MRI descriptors.

Out of this database all patients showing fibroadenomas $(n=103)$ or breast cancer $(n=647)$ were further evaluated statistically: univariate (association of singledescriptors with fibroadenomas/breast cancer: contingency table statistics) as well as multivariate analyses were performed to identify accurate descriptor combinations and overall accuracy of breast MRI for differential diagnosis of fibroadenomas vs. breast-cancer (Chi-squared automatic interaction detection [CHAID], 10-fold cross-validation, receiver operating characteristics $[\mathrm{ROC}]$, area under the ROC curve [AUC], Bonferroni correction).

Results: Typical features of fibroadenomas were "lobulated shape" (OR=8.6), "sharp margin" $(\mathrm{OR}=14.0)$, "internal septations" $(\mathrm{OR}=17.1)$, "hyperintense signal in T2w" (OR=17.6) and "persistent curve-type" (OR=16.2; all: $\mathrm{P}<0.001)$. Diagnostic accuracy increased significantly $(P<0.001)$, if features were assessed in combination. Highest values were then observed, if the following feature combinations were present: "sharp margin and persistent curve type" (OR=140.3), "plateau and internal septations" $(O R=18.1)$. Overall accuracy of breast MRI for the differential diagnosis of fibroadenomas vs. breast cancers revealed excellent AUC (0.95, cross-validated sample).

Conclusion: In breast MRI fibroadenomas show typical morphologic and dynamic characteristics. Particularly, if multiple breast MRI characteristics are assessed in combination, differential diagnosis vs. breast cancer is possible with excellent accuracy.

\section{B-0680 10:39}

Texture analysis of malignant breast tumours: is a differentiation of ductal carcinoma in situ, invasive ductal and invasive lobular breast cancer possible?

T. Knogler' ${ }^{1}$ K. Pinker-Domenig', N. Perry², S. Milner ${ }^{2}$,

K. Mokbel ${ }^{2}$, M.E. Mayerhoefer ${ }^{1} ;{ }^{1}$ Vienna/AT, ${ }^{2}$ London/UK

(thomas.knogler@meduniwien.ac.at)

Purpose: To evaluate the ability of texture features (TF), to differentiate between ductal carcinoma in situ (DCIS), invasive ductal carcinoma (IDC) and invasive lobular carcinoma (ILC) of the breast on full-field digital mammograms (FFDM).

Methods and Materials: 110 screen detected and histopathologically verified breast cancers (27 DCIS, 73 IDC, 10 ILC) imaged with FFDM in standard views were included in this study. For each lesion, a region of interest (ROI) was manually defined, which covered the lesion as well as a rim ( $1 \mathrm{~cm}$ width) of normal-appearing breast tissue around the lesion in the view, where the lesion was depicted in largest diameter. TF derived from the grey-level histogram, co-occurrence matrix (COC), run-length matrix (RLM), absolute gradient (AG), autoregressive model (ARM) and wavelet transform were calculated for the ROIs. Fisher coefficients were calculated to determine which TF were best-suited for distinguishing between DCIS, IDC and ILC. Lesion classification was performed using linear discriminant analysis in conjunction with a k-nearest neighbour classifier, based on the combination of the 10 TF with the highest Fisher coefficients. Classification accuracy was used as the primary outcome measure.

Results: The accuracy of texture-based lesion classification was $84.33 \%$ (70 of 83 lesions) for IDC vs. ILC, $81.1 \%$ (30 of 37 lesions) for ILC vs. DCIS, but only of $70 \%$ (70 of 100 lesions) for IDC vs. DCIS.

Conclusion: TF derived from FFDM may be of value for differentiating between ILC and IDC, and ILC and DCIS, but of limited value for differentiating between IDC and DCIS.

\section{B-0681 10:48}

Variation of apparent diffusion coefficients (ADC) measured in the normal breast using diffusion-weighted MR mammography: effects of menopausal status, anthropometric measures and hormone intake K. Hegenscheid, R. Seipel, R. Laqua, C.O. Schmidt, H. Völzke, N. Hosten, B. Mensel; Greifswald/DE (katrin.hegenscheid@uni-greifswald.de)

Purpose: To assess variations of ADC values of normal breast parenchyma and effects of menopausal status, menstrual cycle phase, age, body mass index (BMI), oral contraceptives (OC), and postmenopausal hormone therapy (HT).

Methods an Materials: A total of 705 women from a population-based study $(49.2 \pm 13.0$ years) underwent diffusion-weighted imaging (DWI) of the breast at $1.5-T$ using a four-channel breast coil with five $b$ values $[50,200,500,800,1000$ $\mathrm{mm} 2 / \mathrm{s}$ ( $7900 \mathrm{~ms} / 91 \mathrm{~ms}$ (repetition time/echo time); $340 \mathrm{~mm}$ field of view; $1.8^{*} 1.8^{*} 4$ $\mathrm{mm}$ voxels)]. Quantitative analysis was performed in normal fibroglandular tissue by manually tracing regions of interest and calculating ADC. The effects of menopausal status, $\mathrm{BMI}, \mathrm{OC}$, and $\mathrm{HT}$ were studied using univariate analysis. Difference in mean $A D C$ values between the proliferative (day 1-14) and secretory menstrual cycle phase (day 15-31) was evaluated by unpaired T-test.

Results: Mean ADC values were significantly higher in the secretory $(1.56 \pm 0.29 * 10$ $3 \mathrm{~mm} 2 / \mathrm{s})$ than in the proliferative menstrual cycle phase $\left(1.42 \pm 0.28^{*} 10-3 \mathrm{~mm} 2 / \mathrm{s}\right)$ $(P=0.012)$. No significant difference was seen in $A D C$ values in relation to menopausal status $(P=0.744)$. Age significantly affected mean $A D C$ values only in postmenopausal women $(P=0.013)$, while $B M I$ had no effects $(P=0.400)$. Mean $A D C$ values were significantly higher in OC users $\left(1.57 \pm 0.29^{*} 10-3 \mathrm{~mm} 2 / \mathrm{s}\right)$ than in non-OC users $\left(1.47 \pm 0.28^{*} 10-3 \mathrm{~mm} 2 / \mathrm{s}\right)$ regardless of menstrual cycle phase $(P=0.044)$ but were similar between HT users and non-HT users $(P=0.814)$. Conclusion: $\mathrm{ADC}$ measurements for breast lesion differentiation and evaluation of treatment response should be interpreted with consideration of normal variability, menstrual cycle phase and hormone intake.

\section{B-0682 10:57}

Second-look US with MR volume navigation (V Nav) of breast lesions: a way to make it objective

A. Fausto, P. Mercuri, L. Mantovani, L. Volterrani; Siena/IT (alfofa@yahoo.it)

Purpose: To investigate the clinical impact of second-looK US with and without MR volume navigation ( $\mathrm{V}$ Nav) as reference.

Methods and Materials: Forty-one patients (59 \pm 22 years, range 27-91) with additional lesions at conventional MR underwent bilateral contrast-enhanced (0.1 mmol/Kgbw Gd-BOPTA, Bracco Diagnostics) breast MR in supine position (1.5 T, Achieva, Philips Healthcare and 1.5 T Signa HDxt, GE Healthcare) using T1-weighted high-resolution isotropic examination volume and spectral fat suppression with body coil. Three fiducial pills of vitamin $\mathrm{E}$ on the corresponding skin markers were used. US and MR co-registration was obtained by means of a magnetic transmitter positioned close to the patient and two small magnetic receivers positioned on a linear probe's bracket (LOGIQ E9, GE Healthcare). The same radiologist performed second look US without and thereafter with VNav. Images were reviewed by consensus by two radiologists. Pathologic examination was the standard of reference. McNemar test was used.

Results: Forty-seven additional lesions were detected in both MR exams. Pathologic examination showed 29 malignant and 18 benign lesions. Lesions diameter was $2.2 \pm 1.3 \mathrm{~cm}$ (mean $\pm \mathrm{SD}$, range $0.6-6.2 \mathrm{~cm}$ ). Finally, $62 \%$ (18/29) of malignant and $44 \%(8 / 18)$ of benign lesions were detected only using VNav $(p=0.0015)$. MR-guided biopsy was performed in three cases. Pathology demonstrated a 9-mm DCIS, an 8-mm sclerosing adenosis and a 12-mm atypical ductal hyperplasia. Conclusion: Second-look US with VNav showed a significant higher detection rate thus suggesting the efficacy of this new technique in reviewing additional lesion detected after MR imaging. 


\section{B-0683 11:06}

Dense breasts as a new standard indication for MR mammography: prospective analysis of 1488 consecutive patients

C.G. Kaiser ${ }^{1}$, C. Reich², K. Wasser', S.O. Schönberg', W.A. Kaiser²;

${ }^{1}$ Mannheim/DE, ${ }^{2} J e n a / D E$ (clemens.kaiser@umm.de)

Purpose: To evaluate the role of MRM in patients with "extended indications", i.e. dense breasts, in which under standard circumstances either biopsy or further diagnostic efforts would have been the next step.

Methods and Materials: Between 04/2006 and 12/2011 a consecutive total of 1492 were examined resulting in a study population of 1488 patients after dropout. Results: 124 patients were diagnosed with suspicious findings by MRM, resulting in 76 true positive findings, 48 false positive cases, 971 true negative cases and 0 false negative cases. True negative cases were either confirmed by histology upon recommendation of external physicians (18 patients), follow-up by MRM or patient questionnaires over the next 5 years by mail ( 1737 cases). 393 patients were lost to follow-up. This resulted in a sensitivity of $100 \%$, specificity of $95.2 \%$, positive predictive value of $61.3 \%$, a negative predictive value of $100 \%$ and an accuracy of $95.5 \%$. For the calculation of invasive cancers only (without DCIS cases), the results were 63 true positives, 27 false positives for malignant findings, 971 true positives and 0 false positives for benign findings.

Conclusion: The results for "dense breasts" as an extension to the standard indications for MRM are encouraging, as an excellent negative predictive value along with a satisfactory positive predictive value can be achieved. However, these results are strongly dependent on reader experience and adequate technical standards as prerequisites for optimal diagnoses. In this setting, "dense breasts" are an indication for MRM in case of unclear findings.

\section{B-0684 11:15}

Is $2 \mathrm{D}$ central projection obtained with digital breast tomosynthesis qualitatively equivalent to digital mammmography?

P. Clauser'1, V. Londero', C. Molinari', R. Girometti', A. Taibi ${ }^{2}$, S. Vecchio ${ }^{3}$

C. Zuiani', M. Bazzocchi ${ }^{1} ;{ }^{1}$ Udine/IT, ${ }^{2}$ Ferrara/ITT, ${ }^{3}$ Bologna/IT

(clauser.p@hotmail.it)

Purpose: Based on a novel approach, digital breast tomosynthesis (DBT) may simultaneously acquire 2D and 3D images of the breast. Variable dose geometry is used to give a sufficient dose in the central projection for that image to be a 2D mammogram (2D-CP). We investigated whether 2D central projection (2D-CP) obtained with DBT is equivalent to digital mammography (DM) in terms of image quality. Methods and Materials: We retrospectively evaluated 45 asymptomatic patients who underwent DM and DBT with 2D-CP for screening. Two experienced radiologists in consensus reviewed the DM and 2D-CP images in separate sessions. Readers were asked to subjectively score image quality on a 1-5 scale in terms of a) artefacts ( $1=$ absent to $5=$ significant artefacts $)$, b) noise $(1=$ absent to $5=$ very high noise) and c) fibro-glandular-breast-tissue-to-fat-contrast (1=poor to $5=$ excellent). The comparison between $2 \mathrm{D}-\mathrm{CP}$ and DM scores was performed with a Wilcoxon signed-rank-test on a per-breast basis.

Results: There was no significant difference between DM and 2D-CP in terms of artefacts, with median scores of 2.0 for both techniques ( $p>0.05)$. A significant difference $(p<0.05)$ was found between DM and 2D-CP concerning noise and contrast (median were 2 vs 4 and 5 vs 4 , respectively).

Conclusion: Although DM is superior to $2 \mathrm{D}-\mathrm{CP}$ in terms of image noise and contrast, both techniques showed a reduced incidence of artefacts. Clinical studies should be performed to assess whether this qualitative difference has clinical relevance.

\section{B-0685 11:24}

The role of additional ultrasound and tomosynthesis after normal digital mammography: comparison between both techniques P. Slon, J. Etxano, I. Simon-Yarza, G. Viteri, A. Elizalde, L.J. Pina Insausti; Pamplona/ES (ljpina@unav.es)

Purpose: To assess and compare the detection rate of additional cancers by means of ultrasound (US) or tomosynthesis (TS).

Methods and Materials: From October 2011 to September 2012 we conducted a prospective study at our reference centre. During this time, 3615 consecutive women and one man (mean age 52.3, range 29-88) underwent digital mammography (Inspiration, Siemens, Erlangen, Germany). After the evaluation of the breast density by a radiologist, routine US and tomosynthesis (oblique mediolateral view) were performed in all patients with ACR density patterns 2 (901), 3 (1165) and 4 (224). Out of these 2290 patients, 76 had suspicious lesions on conventional mammography (BI-RADS categories 4 and 5) and among them 41 had breast cancer.
Finally, 2214 patients with normal or benign mammograms (BI-RADS categories 1 to 3 ) were included in this study. Statistical analysis was performed to compare the rate of additional cancers detected on US and TS, calculating a McNemar's test (SPSS 15.0).

Results: 41 breast tumours were detected on mammography (detection rate $1.79 \%$ ). US detected 15 additional cancers (additional detection rate $0.65 \%$ ) after normal digital mammograms. TS detected 12 additional cancers $(0.40 \%)$. No statistical differences were found $(p=0.51)$.

Conclusion: Both, TS and US, increased the detection rate, without significant difference between them.

\section{B-0686 11:33}

Lesion detection and characterisation of one-view digital breast tomosynthesis combined with one-view mammography compared to two-view mammography

G. Gennaro', E.R. Hendrick², A. Toledano ${ }^{3}$, R. Chersevani', C. di Maggio', M. La Grassa ${ }^{5}$, L. Pescarini', I. Polico ${ }^{1}$, A. Proietti'; ${ }^{1}$ Padua/IT, ${ }^{2}$ Denver, CO/US,

${ }^{3}$ Washington, DC/US, ${ }^{4}$ Gorizia/IT, ${ }^{5}$ Aviano/IT (gisella.gennaro@ioveneto.it)

Purpose: To compare lesion detection and characterisation by the combination of one-view breast tomosynthesis (DBT) and one-view digital mammography with two-view digital mammography (MX).

Methods and Materials: The study included 250 consenting women with breast lesions classified as suspicious at mammography and/or ultrasound. A clinical performance study comparing two-view (CC, MLO) mammography, and the combination of DBT in MLO view and MX in CC-view (DBT+MXCC) was conducted with six breast radiologists. Data were analysed per-lesion, using lesion localisation fraction - LLF (the percentage of lesions correctly localised). Lesion detection was determined by calculating the total number of lesions rated above BI-RADS 1. Lesion characterisation was evaluated by counting malignant lesions rated above BI-RADS 3 and benign lesions scored BI-RADS 2 or 3 . Lesion detection and characterisation for all lesions, and stratified for malignant and benign lesions, were compared between DBT+MXCC and MX using analysis of variance (ANOVA). $\mathrm{P}$-values $<0.05$ were considered statistically significant. The non-inferiority margin was set at $5 \%$.

Results: Overall, DBT+MXCC was superior to MX alone in terms of number of lesions correctly detected and characterised (LLF difference: $+6.85 \%,-95 \% \mathrm{Cl}$ : $+4.02 \%$; $p$-value $=0.0007$ ). DBT+MXCC superiority was confirmed for benign lesions (LLF difference: $+7.93 \%$, $-95 \% \mathrm{Cl}:+5.71 \%$; $p$-value $<0.0001$ ), while noninferiority was achieved for malignant lesions (LLF difference: $+3.03 \%,-95 \% \mathrm{Cl}$ : $-4.59 \%$; p-value $<0.369$ ).

Conclusion: The combination of $\mathrm{DBT}+\mathrm{MXCC}$ significantly improved lesion detection and characterisation, reducing the number of false positives, while keeping the number of false negatives unchanged.

Author Disclosures:

E.R. Hendrick: Consultant; GE Healthcare. A. Toledano: Consultant; GE Healthcare.

\section{B-0687 11:42}

Does the adjunct of digital breast tomosynthesis (DBT) increase inter-reader reproducibility of two-dimensional digital mammography (2D-DM)?

G. Di Leo' ${ }^{1}$, L.A. Carbonaro'1, M. Bazzocchi², V. Londero², A. Dal Col'²,

R.M. Trimboli', F. Sardanelli'; ' San Donato Milanese/IT, ${ }^{2}$ Udine/IT (gianni.dileo77@gmail.com)

Purpose: To estimate the inter-reader reproducibility of DBT added to 2D-DM in comparison to that of 2D-DM alone.

Methods and Materials: A series of 65 breasts (65 women, aged $58 \pm 9$ years) underwent DBT (Giotto, IMS, Italy) as an adjunct to 2D-DM. After an agreement on how to report 2D-DM and DBT, 3 independent readers (R1, R2, R3) with > 6 years of experience in 2D-DM and $>1$ year of experience in DBT evaluated all studies. Each reader evaluated the breast density and assigned a BI-RADS score using only 2D-DM. After 30 days, they repeated the evaluation adding DBT to $2 \mathrm{D}$-DM. The inter-reader reproducibility was estimated using the quadratically weighted Cohen's kappa.

Results: The breast density reported at $2 \mathrm{D}-\mathrm{DM}$ by the most experienced reader was: $75 \%$ in $1(1 \%)$. Considering the B1, B2, B3, B4, and B5 BI-RADS scores, the same reader assigned the followings: $25,0,10,20$, and 10 using 2D-DM; 31, 1, 7,13 , and 13 using 2D-DM plus DBT. For each pair of readers, 2D-DM plus DBT resulted in a higher inter-reader reproducibility than that of 2D-DM alone: kappa value for 2D-DM ranged from 0.418 to 0.566 while that for 2D-DM plus DBT ranged from 0.656 to 0.758 . 
Conclusion: While the inter-reader reproducibility of 2D-DM resulted only moderate, the adjunction of DBT allowed to reach a substantial reproducibility. The use of DBT added to 2D-DM can reduce inter-reader variability and allow for more reliable readings from different radiologists.

\section{Author Disclosures:}

G. Di Leo: Consultant; IMS.

\section{B-0688 11:51}

One-to-one comparison between digital mammography and digital breast tomosynthesis using a fully automated software: breast density underestimation on digital breast tomosynthesis varies in different BI-RADS classes

A. Tagliafico, S. Airaldi, F. Cavagnetto, B. Bingotti, S. Tosto, D. Astengo,

M. Calabrese; Genoa/lT (albertotagliafico@gmail.com)

Purpose: To compare breast density on digital mammography (FFDM) and tomosynthesis (DBT) according to different BI-RADS classes (four classes from 1 to 4 ) with an automated software.

Methods and Materials: IRB approval and written informed consent were obtained. Digital breast tomosynthesis and digital mammography were obtained in the same patient. A total of 160 consecutive patients (mean age years: $50 \pm 14$; mean BMI: $22 \pm$ 3) were included. One-to-one comparison between FFDM and DBT was made with a fully automated software previously validated. Statistical analysis was performed with two-tailed t-test for paired data using statistical software.

Results: In BI-RADS class 1, digital mammography overestimated breast density of a $16 \%$. In BI-RADS class 2 , digital mammography overestimated breast density of a $11.9 \%$. In BI-RADS class 3, digital mammography overestimated breast density of a $3.5 \%$. In BI-RADS class 4 , digital mammography overestimated breast density of a $18.1 \%$. The differences resulted highly statistically significant $(p<0.0001)$. There was a good correlation between BI-RADS categories and the density evaluated with digital mammography and digital breast tomosynthesis $(r=0.56, p<0.01$ and $r=0.48 p<0.01$ ).

Conclusion: Breast density values were underestimated by DBT in comparison to FFDM with a non-linear relationship in the different BI-RADS classes. This data should influence clinical and research studies dealing with breast density as a qualitative biomarker.

\begin{tabular}{ll}
\hline 10:30 - 12:00 & Room G/H \\
\hline
\end{tabular}

\section{Genitourinary}

\section{SS 1307}

\section{The bladder and below}

Moderators:

M. Baramia; Tbilisi/GE

R.H. Oyen; Leuven/BE

\section{B-0689 10:30}

The role of $\mathrm{CT}$ as a staging tool and prognostic biomarker in the pretreatment evaluation of intra-diverticular bladder tumours P.L. Di Paolo, H.A. Vargas, H. Hricak; New York, NY/US (dipaolop@mskcc.org)

Purpose: To evaluate the use of $\mathrm{CT}$ for staging intradiverticular bladder tumours, and to determine the associations between CT tumour features, pathology and overall survival.

Methods and Materials: Between 2001 and 2010, we retrospectively identified 34 patients ( $32 \mathrm{men}$; median age $=65 \mathrm{y}$ ) with intra-diverticular bladder tumours who underwent pre-treatment $\mathrm{CT}$. In addition to $\mathrm{CT}$ stage (dichotomised as ${ }^{2} \mathrm{~T} 1$ and ${ }^{3} \mathrm{~T} 2$ ), the following $\mathrm{CT}$ features were evaluated by 2 independent readers: length of contact between the tumour and diverticular wall, tumour width (measured perpendicular to the diverticular wall), tumour morphology (flat vs polypoid) and diverticular neck involvement. Pathology findings constituted the standard of reference. Spearman correlation coefficients and hazard ratios (HRs) were calculated to determine the association between $\mathrm{CT}$ features, pathology and overall survival. Inter-reader agreement was evaluated with weighted-kappa statistics.

Results: All patients had cystoscopy-guided biopsy/resection, and underwent CT before treatment. On pathology, 30/34 patients had high-grade lesions. There was a significant association between $\mathrm{CT}$ stage and pathology stage for both readers $(p<0.001)$. However, neither CT nor pathology stage was associated with overall survival $(p=0.20-0.55)$. Both the length of contact between the tumour and diverticular wall and the tumour width were significantly associated with overall survival for both readers (HRs 1.31-1.62, $p<0.001-0.04)$. No significant associations between any of the other tumour features and overall survival were identified. Inter-reader agreement for was fair to moderate $(k=0.34-0.78)$.

Conclusion: In patients with intradiverticular bladder tumours, the length of contact between the tumour and diverticular wall and the tumour width are significantly associated with overall survival.

\section{B-0690 10:39}

Usefulness of diffusion-weighted magnetic resonance imaging in patients with muscle-invasive bladder cancer selected for radical cystectomy: lymph node staging prospective study and comparison with CT scan

J. Borrego Gómez, J. Martel Villagrán, E. De la Peña, J. Culebras, C. Llorente; Madrid/ES (jesusborregoes@yahoo.es)

Purpose: To evaluate the feasibility and accuracy of preoperative DW-MRI for the detection of metastatic lymph nodes in patients with muscle-invasive bladder cancer (MIBC) who were selected for radical cystectomy with extended lymphadenectomy as well as to compare with $\mathrm{CT}$.

Methods and Materials: Prospective study opened in January 2010 including 28 patients to date with MIBC selected for radical cystectomy with extended lymphadenectomy. Before surgery, DW-MRI and CT scan were performed. Two independent radiologists with experience in urogenital radiology interpreted the DW-MRI and CT images. MR images were read at the time of patient inclusion with the DW image findings. The measurement of the ADC value was calculated for selected studies and in nodes $>5 \mathrm{~mm}$. Diagnostic accuracy of DW-MRI was compared with CT and with histopathological findings. The sensitivity, specificity, positive and negative predictive values of diagnostic tests were analysed.

Results: 26 patients were male. The median rate of age was 69.8 years. Eight of 28 patients had lymph node metastasis. The average number of nodes in the lymphadenectomy was 12.1 . The sensitivity of DW-MRI was higher than CT: $62.5 \%$ vs $25 \%$. CT alone is not considered suitable for nodal metastasis staging because of its high false negative rate. The specificity, PPV and NPV values were similar for both DW-MRI and CT.

Conclusion: The diagnostic methods for tumour staging in MBIC should be improved to select the appropriate treatment. DW-MRI can contribute effectively in the nodal staging of patients with MIBC, but it is necessary to continue evaluation.

\section{B-0691 10:48}

Use of FLAIR sequences for detection and local staging of bladder tumours with MRI

M. Di Girolamo, G. Muscogiuri, M. Cappucci, V. Catena, R. Persechino, V. David; Rome/IT (digirolamomarco@hotmail.com)

Purpose: To increase the diagnostic accuracy of MRI in the detection and local staging of bladder tumours by using Fluid-attenuated Inversion Recovery (FLAIR) sequences.

Methods and Materials: 32 patients with bladder tumours detected by US underwent MRI using 1.5 T superconductive magnet. We performed TSE T1-weighted and T2-weighted and FLAIR sequences on axial scans. The contrast to lesion ratio was always evaluated. All the patients underwent cystoscopy with transurethral biopsy and 14 had subsequent cystectomy.

Results: In comparison with other sequences, FLAIR sequence was more sensitive in the detection of bladder neoplasms. This sequence demonstrates the hyperintense signal of bladder neoplasms from the filled bladder lumen with no signal. The sensitivity in the identification of bladder neoplasms was $100 \%$ with FLAIR sequences, $89.6 \%$ with TSE T2-weighted sequences and $86.2 \%$ with TSE T1-weighted sequences. That was due to the higher signal-to-lesion ratio of FLAIR sequences in comparison with the others. In fact, on FLAIR sequences the mean value of contrast to lesion ratio of bladder neoplasm was 33.1 while on SE T1-weighted sequences and TSE T2-weighted sequences was, respectively, 15.2 and 29.2. FLAIR sequences allowed the detection of small papillomas $(<2$ $\mathrm{mm}$ ). TSE T2-weighted sequences were more sensitive than other sequences in the study of bladder wall infiltration.

Conclusion: FLAIR sequences were more sensitive in the detection of bladder neoplasms, thanks to their higher contrast-to-lesion ratio and were helpful in the visualisation of small papillomas, especially when multifocal. 


\section{B-0692 10:57}

Magnetic resonance virtual cystoscopy versus conventional cystoscopy in the detection of urinary bladder cancer

S. Elangovan; Puducherry/IN (elangovansundar@hotmail.com)

Purpose: The non-invasive investigation of urinary bladder carcinoma by magnetic resonance (MR) virtual cystoscopy was evaluated by us and compared with conventional cystoscopy.

Methods and Materials: Thirty consecutive patients, presenting with gross haematuria and trans abdominal ultrasound suggestive of mass in the bladder were taken up for study. All patients underwent conventional cystoscopy and CISS 3D MR imaging and the images reconstructed for virtual cystoscopy using volume rendering technique. Lesions were detected and the number, size, location and morphologic features of lesions were noted. Subsequently, patients underwent conventional cystoscopy, and the findings were noted. The final diagnosis of the cases was established by histopathology.

Results: Sixty-five lesions were seen in MR virtual cystoscopy and 69 in conventional cystoscopy. The smallest lesion identified on MR virtual cystoscopy was 3 $\mathrm{mm}$. Overall sensitivity and specificity of MR virtual cystoscopy for lesion detection were 94.2 and $100 \%$, respectively. For lesions greater than $5 \mathrm{~mm}$, the detection rate was $98 \%$.Pearsons correlation test showed a good between virtual and conventional cystoscopy for maximum and minimum diameters of the lesion (0.98 and 0.92 , respectively). Location of the lesions and their morphological characteristics were found to correspond by both methods.

Conclusion: MR virtual cystoscopy is an effective tool in the detection of urinary bladder tumours, especially those larger than $5 \mathrm{~mm}$. When bladder lesion is less than $5 \mathrm{~mm}$ a sessile conventional cystoscopy is better than MR virtual cystoscopy.

\section{B-0693 11:06}

Voiding MR cystourethrography: a new diagnostic imaging

technique for the evaluation of male lower urinary tract

M. Di Girolamo, C. Cantone, E.M. Pandolfi, R. Persechino, F. Caporilli Razza,

V. David; Rome/IT (digirolamomarco@hotmail.com)

Purpose: To evaluate the diagnostic accuracy of a diagnostic imaging technique called voiding MR cystourethrography.

Methods and Materials: 10 normal volunteers and 86 male patients with bladder outlet obstruction (evaluated with urine-flow velocity recording) underwent voiding MR cystourethrography. The MR examination was performed with 1.5 $T$ superconductive magnet. The filling of the urinary bladder with paramagnetic contrast agent was obtained by the i.v. administration of furosemide followed by $3 / 4$ of the normal dose of a paramagnetic contrast agent. During the micturition two consecutive T1-weighted spoiled 3D gradient-echo acquisitions on sagittal plane were performed. 3D row images were post-processed with MIP algorithm. 25 patients performed retrograde and micturating conventional cystourethrography in the month preceding MRI.

Results: Homogeneous opacification of the bladder lumen was always obtained. 10 patients were unable to perform the MR examination. In all the volunteers and in all the patients studied (76 pts), a perfect evaluation of the male urethra with MRI was obtained and its visualisation with MIP reconstructed images was considered comparable to that obtained with conventional cystourethrography. Site, length and number of urethral strictures were accurately determined. The analysis of $3 \mathrm{D}$ sagittal scans allowed a better evaluation of the morphology of the urethral strictures in comparison with conventional cystourethrography.

Conclusion: Voiding MR cystourethrography demonstrates the morphology of the bladder neck and urethra during the micturition and can substitute standard cystourethrogram, avoiding radiation exposure to the gonads and urinary catheterization.

\section{B-0694 11:15}

Micturition MRI: voiding assessment in continent ileal urinary reservoir

J. Lopater', A. Scherrer', V. Delmas², S. Gey², Y. Neuzillet', T. Lebret';

${ }^{1}$ Suresnes/FR, ${ }^{2}$ Paris/FR (a.scherrer@hopital-foch.org)

Purpose: The aim of this study was to assess micturition MRI voiding characteristics in continent ileal urinary reservoir performed after cystectomy for bladder cancer. Methods and Materials: A total of 51 patients with continent ileal urinary reservoir were included prospectively. Reference standard micturition evaluation with maximum urine flow rate (Qmax), urinated volume and ultrasound post-void-residual (PVR) measurements were performed before or after micturition MRI. Qmax results were conclusive when urinated volume was superior to $150 \mathrm{~mL}$.

Results: Reference standard evaluations voiding mean results in the full population were satisfying $(\mathrm{Qmax}=20.59 \pm 15.91 \mathrm{~mL} / \mathrm{sec}$, urinated volume $=286.98 \pm$
190.24 mL and PVR $=94.45 \pm 167.99 \mathrm{~mL}$ ). Micturition MRI mean results were not as good as reference standard with 13 patients who could not void. MRI sagittal surface-derived Qmax results were biased in the full population with MRI systematically inferior to reference standard $(-39.3 \%, 95 \%$ confidence interval was -60.2 to -18.5). Urinated volume was calibrated between two different techniques; however, both methods disagreed on measurements (mean $=142.41 \pm 146.2 \mathrm{~mL}$, intraclass correlation was $0.18(-0.05,0.41)$, PValue $=0.07)$. Qmax performance depended on the threshold value, AUC from $0.64(0.28,1.00)$ to 1.00 in conclusive results. Conclusion: Micturition MRI allowed continent ileal urinary reservoir functional assessment. Micturition MRI was biased, agreed with reference standard on Qmax and PVR measurement but disagreed on urinated volume. Functional evaluation of micturition with MRI is complete with non-invasive outlet anomaly detection and urine flow assessment.

\section{B-0695 11:24}

Clinical relevance of magnetic resonance dynamic spongiourethrotomography of male urethral stricture detection E. Banchik, V. Dombrovsky, M. Kogan, V. Mitusov; Rostov-on-Don/RU (emma.mail@mail.ru)

Purpose: To evaluate clinical relevance of urethral stricture detection and staging using magnetic resonance dynamic spongiourethrotomography (MRDSUT). Methods and Materials: The series of 65 patients, aged 19-71 years (mean, 39.6) with urethral stricture different localisations were included. Retrograde urethrography (RUG) and voiding cystourethrography (VCUG) were also done. All patients were undergone to urethroplasty. Radiological results were compared with morphological data. MRDSUT using 2D Fast Imaging Employing Steady-State Acquisition pulse sequence in sagittal plane were done. This technique is usually used for evaluation of coronary and other vessels. This method was conducted under retrograde injection of normal saline $(0.1-0.3 \mathrm{ml} / \mathrm{sec})$ into the urethra and during micturition. Penis was taped midline to the abdominal wall. Urethral caliber, urethral elasticity, the presence of stricture, its length and spongiofibrosis extent were assessed.

Results: In all cases $(n=65)$ MRDSUT provided exact delineation of the urethral stricture and its length. The sensitivity, specificity and overall accuracy were $100 \%$, $92.6 \%$ and $96.6 \%$, respectively, compared to $90 \%, 92.2 \%$ and $90.9 \%$, respectively, for RUG and VCUG. MRDSUT sensitivity, specificity and overall accuracy in the spongiofibrosis detection were $97.4 \%, 94.5 \%$ and $96.2 \%$, respectively. RUG and VCUG cannot detect the spongiofibrosis.

Conclusion: MRDSUT can define the length of urethral stricture and its localisation more accurately than RUG in combination with VCUG, but in contrast to them it allows to detect a cause of urethral stricture, spongiofibrosis. In our clinic, MRDSUT became crucial study for diagnostic of urethral stricture disease and in the preoperative determining of a surgery type.

\section{B-0696 11:33}

Urinary bladder neck dysfunction in male patients: evaluation with MRI and with voiding MR-cystourethrography

M. Di Girolamo, A. Trucchi, S. Mariani, G. Muscogiuri, I. Casazza, V. David; Rome/IT (digirolamomarco@hotmail.com)

Purpose: This study aimed to evaluate with MRI male patients with urinary bladder neck dysfunction, studying the anatomy of bladder neck and performing voiding MR-cystourethrography.

Methods and Materials: We have evaluated with MRI 21 male patients with urinary bladder neck dysfunction diagnosed with pressure-flow study. All patients had undergone US in the month preceding MRI, excluding patients with BPH. The MR examinations were performed with an $1.5 \mathrm{~T}$ magnet. The patients had urine-filled bladders and sagittal and oblique coronal TSE T2-weighted scans were performed. The oblique coronal scans were parallel to the plane of the bladder neck. $15 \mathrm{pa}-$ tients underwent also voiding MR-cystourethrography performed with T1-weighted spoiled 3D gradient-echo acquisitions on sagittal plane performed after the filling of bladder lumen with contrast-material-enhanced urine.

Results: The entire MR examination lasted no longer than 10 minutes. We detected 18patients with abnormality of smooth muscular structures of the bladder neck and 3 with bladder neck cyst. MRI allowed a perfect evaluation of the different smooth detrusor muscles of the bladder neck. In patients with urinary bladder neck dysfunction, we detected hypertrophy of posterior smooth muscular structures of bladder neck and kyphosis of prostatic urethra. 6 patients were able to perform voiding MR-cystourethrography that showed the characteristic radiological features. Conclusion: MRI with voiding MR-cystourethrography could be performed in male patients with bladder outlet obstruction in order to visualise the aspect of the 
bladder neck. These anatomical information are useful to determine the causes of voiding obstruction and diagnose urinary bladder dysfunction.

\section{B-0697 11:42}

MRI findings of radiation-induced changes in the urethra and periurethral tissues in patients with prostate cancer

C. Marigliano ${ }^{1}$, O.F. Donati ${ }^{2}$, O. Akin ${ }^{2}$, D. Goldman², J. Eastham ${ }^{2} ;{ }^{1}$ Rome/IT, ${ }^{2}$ New York, NY/US

Purpose: The aim of this study was to assess radiotherapy (RT)-induced changes in the urethra and periurethral tissues in patients with prostate cancer $(\mathrm{PCa})$. Methods and Materials: This retrospective study included 108 men (median age, 65y; range, 43-87y) who underwent external-beam radiotherapy (EBRT) and/or brachytherapy for $\mathrm{PCa}$. On endorectal-coil MRIs of the prostate obtained within 180d before RT and 2-63 months (median, 20 months) after RT, two readers independently measured the urethral length (UL) and graded the margin definition (MD) and signal intensities (SIs) of the urethral wall, the levator ani and obturator internus muscles and the muscles of the genitourinary diaphragm on 4-point scales. Results: Mean UL decreased significantly from pre- to post-RT MRI for both readers (from 15.2 to $12.6 \mathrm{~mm}$ and 14.4 to $12.9 \mathrm{~mm}$, [p < 0.0001 , both]). Inter-reader agreement for UL was excellent for pre- and post-RT MRI $(I C C=0.89$ and 0.90 , respectively). Both readers found significantly decreased MD and increased SI in the urethral wall and pelvic muscles on post-RT MRI (p?0.008). Inter-reader agreement was fair for MD (?=0.31, pre- and post-RT) and SI of the urethral wall $(?=0.33$, pre- and post-RT) and ranged from slight to moderate for SI of the pelvic muscles $(?=0.11-0.56$, pre-RT and $?=0.11-0.46$, post-RT). Sl changes in the obturator internus muscle were greater after EBRT $(p=0.0003-0.01)$, while SI changes in the levator ani muscle were greater after brachytherapy $(p=0.03)$.

Conclusion: After RT of the prostate, MRI shows urethral shortening, decreased urethral margin definition and increased SI of the urethral wall and pelvic muscles.

\section{B-0698 11:51}

MRI anatomic evaluation in patients with persistent urinary incontinence after advance male sling: a work in progress study with a 3 T system

V. Zampa, D. Pistolesi, S. Ortori, L. Faggioni, M. Marletta, C. Bartolozzi; Pisa/IT (virnazampa@hotmail.com)

Purpose: Retrourethral transobturator sling is a new functional treatment of stress urinary incontinence after prostatectomy consisting in repositioning the urethral sphincter into the pelvis. Overall success rate is $54-80 \%$; however, a failure rate of $20-45 \%$ is reported. The aim of this study was to retrospectively analyse morphologic changes visible on MRI after sling procedure in incontinent patients and compare with the continent ones, in order to detect possible factors explaining the different clinical outcome.

Methods and Materials: 16 patients treated with advance sling were enrolled: 8/16 had clinical recovery while $8 / 16$ had persistent incontinence. MR protocol performed with a 3 T system included 3D T2w sequence (cube) and a high temporal resolution Fiesta for dynamic MRI. On the T2w sequence, the length of the bulbus posterior to the sling and the distance of the sling from a line parallel to the symphysis pubis (LSP), were measured. Mann-Whitney test was used to analyse the results. Results: In the 8 incontinent patients the length of the bulbus posterior to the sling was $<8 \mathrm{~mm}$ (range $4-8 \mathrm{~mm}$ ) while in the continent ones was $>12 \mathrm{~mm}(12-23 \mathrm{~mm}$ ) $(p<0.001)$. The sling position was located posterior to the LSP in $7 / 8$ incontinent patients $(4-12 \mathrm{~mm})$ and almost coincident in 6/8 continent patients $(p=0.04)$. Conclusion: Based on our results, the length of the bulbus posterior to the sling seems to strongly correlate with continence. Although our results must be confirmed in a larger series, surgeons should consider this element in planning the proper position of the device.
$10: 30-12: 00$

Room I/K

\section{Abdominal Viscera}

SS 1301

\section{Abdominal CT: dose reduction and technical improvements}

Moderators:

D. Tack; Baudour/BE

A. Taibbi; Palermo/IT

\section{B-0699 10:30}

Hybrid iterative reconstruction for abdominal CT: can the radiation dose be decreased while preserving the low-contrast detectability? S.T. Schindera ${ }^{1}$, D. Odedra ${ }^{2}$, S. Arsalan Raza ${ }^{2}$, T.K. Kim², H.-J. Jang ${ }^{2}$ Z. Szucs-Farkas ${ }^{3}$, P. Rogalla ${ }^{2} ;{ }^{1}$ Basle/CH, ${ }^{2}$ Toronto, ON/CA, ${ }^{3} \mathrm{Biel} / \mathrm{CH}$ (sschindera@aol.com)

Purpose: To compare the low-contrast detectability and image quality of abdominal $\mathrm{CT}$ at different radiation dose levels reconstructed with iterative reconstruction (IR) and filtered back projection (FBP).

Methods and Materials: A liver phantom with 12 simulated tumours (diameters of $5,10,15$ and $20 \mathrm{~mm}$; tumour-to-liver contrast of $-10,-20$, and $-40 \mathrm{HU}$ ) was designed. The phantom was scanned with a standard abdominal CT protocol (equivalent $100 \%$ dose) and four low-dose protocols $(20 \%, 40 \%, 60 \%$, and $80 \%$ of the standard protocol dose). CT datasets were reconstructed with hybrid IR (AIDR 3D, Toshiba) and FBP. Image noise was measured, and the tumours' CNRs were calculated. Tumour detection was assessed by three blinded radiologists. A total of 840 tumours were presented to the radiologists.

Results: IR yielded image noise reduction of $44-64 \%$ and a CNR increase of $74-$ $180 \%$ compared to FBP at the same dose level $(P<0.001)$. The overall sensitivity for tumour detection was $64.7-85.3 \%$ for IR and $66.3-85.7 \%$ for FBP at the $20 \%$ to $100 \%$ doses, respectively. There was no significant difference in the sensitivity between IR and FBP at the same dose level $(P=1.0)$. The sensitivity of the protocol at the $20 \%$ dose using FBP and IR was significantly lower compared to the protocol at the $100 \%$ dose using FBP and IR $(P<0.05)$

Conclusion: Although hybrid IR at a reduced radiation dose provides the same or better quantitative image quality compared with FBP at standard protocol dose, the low-contrast detectability is decreased which presents a risk of missing subtle pathologies.

\section{B-0700 10:39}

Image quality on liver CT based on sinogram-affirmed iterative reconstruction algorithm

B. Schulz, B. Bodelle, P. Siebenhandl, M. Beeres, F. Al-Butmeh, C. Frellesen, T.J. Vogl; Frankfurt a. Main/DE (mail@borisschulz.com)

Purpose: To evaluate efficiency of sinogram-affirmed iterative reconstruction technique, regarding noise and image quality on contrast-enhanced computed tomography (CT) of the liver.

Methods and Materials: CT examinations were performed upon 32 patients (128 slice CT, $120 \mathrm{kV}, 180 \mathrm{mAs}$, activated tube current modulation, $0.6 \mathrm{~mm}$ collimation). Each examination was reconstructed at standard filtered back projection (FBP) and 5 different SAFIRE strengths in $5 \mathrm{~mm}$ images in transversal direction with soft tissue kernel. Image noise was defined as standard deviation (SD) of Hounsfield units (HU) in air, and signal-to-noise ratio (SNR) of the liver was defined as mean liver HU per liver SD. Subjective image quality was evaluated by three raters using a 5-point scale (1=non-diagnostic image quality, $5=$ excellent image quality). Results: Average image noise was 6.2 HU (FBP), vs. 5.7 HU (SAFIRE 1), vs. 5.0 (SAFIRE 2) $4.4 \mathrm{HU}$ (SAFIRE 3), 3.8 HU (SAFIRE 4), 3.1 HU (SAFIRE 5). SNR of the liver consecutively increased when using the iterative reconstruction algorithms from 8.4 (FBP) to 9.3 (SAFIRE 1) to 10.4 (SAFIRE 2) to 12.2 (SAFIRE 3) to 15.1 (SAFIRE 4) to 17.5 (SAFIRE 5). The differences in image noise and SNR of each SAFIRE-strength to FBP was statistically significant $(p<0.05)$. Subjective image quality was voted 3.9 (FBP) vs. 4.3 (SAFIRE 1) vs. 3.7 (SAFIRE 2) vs. 2.8 (SAFIRE 3) vs. 1.8 (SAFIRE 2) vs. 1.2 (SAFIRE 5)

Conclusion: Sinogram-affirmed-based iterative reconstruction technique significantly reduces image noise and increases SNR for examinations of the liver. However, subjective image quality decreases with strong iterative strengths. 


\section{B-0701 10:48}

Efficacy of a liver detection algorithm for noise reduction in abdominal CT N. Ardley ${ }^{1}$, K. Buchan ${ }^{2}$, K. Lau' ${ }^{1}{ }^{1}$ Clayton/AU, ${ }^{2}$ Melbourne/AU

(Nicholas.Ardley@southernhealth.org.au)

Purpose: The liver, an inherently low contrast structure, appears 'noisy' on routine CT of abdomen. This may impede liver lesion detection. The development of a specific CT liver detection algorithm delivers higher radiation exposure to the liver with the aim of improving quantum noise, and correspondingly less radiation to the remaining abdomen. The aim of this study was to assess the efficacy of this algorithm on reducing noise in the liver.

Methods and Materials: 80 consecutive patients for abdominal CT with same level of iterative reconstruction were included. There was no exclusion. The CT liver detection algorithm was enabled in the last 40 of these patients. Region of interest in Hounsfield units and standard deviation (SD) were measured in the left and right lobes of liver, spleen, psoas and bladder for all patients. Radiation doses were also recorded. The results of the two groups were compared.

Results: SD reflected noise. In the liver detection algorithm group, there was a SD reduction by $10.1 \%$ in the right liver, $15.61 \%$ in the left liver and $15.83 \%$ in spleen. No significant change of SD was seen in psoas and bladder. A $5.6 \%$ overall radiation dose reduction was noted in this group.

Conclusion: The application of this newly developed CT liver detection algorithm demonstrated a reduction in noise in the liver and spleen with no significant noise changes in the remaining abdomen. There was an added benefit of overall radiation dose reduction.

\section{Author Disclosures:}

K. Buchan: Employee; Philips Healthcare.

\section{B-0702 10:57}

Impact of modified abdominal CT protocols for obese patients with filtered back projection and hybrid iterative reconstruction technique on image quality, radiation dose and low-contrast detectability: a phantom study

S.T. Schindera', D. Odedra², D. Mercer², S. Thipphavong², P. Chou², Z. Szucs-Farkas ${ }^{3}$, P. Rogalla ${ }^{2} ;{ }^{1} \mathrm{Basle} / \mathrm{CH},{ }^{2}$ Toronto, $\mathrm{ON} / \mathrm{CA},{ }^{3} \mathrm{Biel} / \mathrm{CH}$ (sschindera@aol.com)

Purpose: To assess the impact of modified CT protocols for obese patients, using filtered back projection (FBP) and hybrid iterative reconstruction (IR), on image quality, radiation dose and low-contrast detectability.

Methods and Materials: A liver phantom with 12 different tumours was designed, and two fat rings were added to mimic intermediate-sized and large-sized patients. The intermediate and large phantoms were scanned with our standard abdominal CT protocol (standard deviation [SD] 15) (Aquilion ONE, Toshiba) and were reconstructed with FBP. The large phantom was also scanned with four modified protocols using different SD values $(10,12.5,17.5$ and 20$)$ and was reconstructed with FBP and IR technique. The image noise and the CNR were assessed. Tumour detection was independently performed by three radiologists.

Results: The IR algorithm in the large phantom decreased the image noise (range -14.5 to $-37.0 \%$ ) and increased the CNR (range 26.7 to $70.6 \%$ ) compared to the FBP algorithm at the same targeted SD value $(P<0.001)$. Applying a targeted $\mathrm{SD}$ of 15 , the overall sensitivity for tumour detection in the intermediate and large phantom with FBP measured $87.7 \%$ and $75.5 \%$, and the radiation dose $42.0 \mathrm{mGy}$ and $23.7 \mathrm{mGy}$, respectively. IR did not improve the sensitivity in the large phantom compared to $F B P$ at the same $S D$ value $(P>0.05)$.

Conclusion: The same quantitative image quality yields decreased low-contrast detectability in large-sized compared to intermediate-sized patients. Compared to FBP, IR significantly improves the quantitative image quality in large-sized patients, but no improvement is observed for the low-contrast detectability.

\section{B-0703 11:06}

Automated attenuation-based tube potential selection for abdominal

CT examinations: impact on image quality and dose reduction

P. Kröpil, P. Heusch, L. Schimmöller, F. Miese, S. Dietrich, G. Antoch,

R.S. Lanzman; Düsseldorf/DE

Purpose: To evaluate the impact of attenuation-based tube potential selection (TPS) on radiation dose and image quality (IQ) of contrast-enhanced abdominal CT examinations.

Methods and Materials: 80 consecutive patients undergoing clinical contrastenhanced $(120 \mathrm{ml})$ abdominal CT examinations on a 128-row scanner were included in this study. $40 \mathrm{CT}$ scans were performed using the novel attenuation-based TPS for dose saving (CARE KV, Siemens), which adapts the tube potential to attenuation of the patient and type of examination semiautomatically. In addition, 40 abdominal CT examinations were performed with a standard tube potential of $120 \mathrm{kVp}$. Radiation doses (CTDIvol) were noted for all examinations. Signal-to-noise ratio (SNR) was determined in the contrast-enhanced lumen of the abdominal aorta (A), liver (L) and spleen (S). Subjective IQ (lesion detectability, diagnostic confidence) was rated on a 4-point scale (1_non diagnostic; 4_excellent) by two readers.

Results: Whereas noise level was slightly elevated when using TPS algorithm compared to the standard protocol (10.2 vs. $8.0 \mathrm{HU}, \mathrm{p}<0.01)$, no significant differences in SNR $(15.2 \pm 5.4$ vs. $16.5 \pm 5.2$ [A], $10.5 \pm 3.3$ vs. $11.7 \pm 3.9$ [L], $10.8 \pm 3.8$ vs. $12.0 \pm 3.9[\mathrm{~S}]$ ) were observed between the two protocols. Subjective IQ (mean score of 3.9 versus 3.8 , respectively) was good to excellent in all examinations. TPS lowered the CTDIvol significantly as compared to standard abdominal CT $(6.1 \pm 1.7 \mathrm{mGy}$ vs. $11.0 \pm 3.9 \mathrm{mGy}, \mathrm{p}<0.01)$.

Conclusion: Attenuation-based TPS lowers the dose of abdominal CT in clinical routine by up to $45 \%$ while maintaining high subjective and objective image quality.

\section{B-0704 11:15}

Second generation dual-energy $\$ \mathrm{~T}$ of the abdomen: radiation dose comparison with 64- and 128-row single energy acquisition

C.N. De Cecco, A. Darnell, N. Macias, G. Muscogiuri, C. Ayuso, A. Laghi; Rome/IT (g.muscogiuri@gmail.com)

Purpose: This study was designed to compare the radiation dose in abdominal dual-energy (DE) and single energy (SE) acquisitions obtained with a dual-energy CT (DECT) and a 64-row CT (SECT).

Methods and Materials: A total of 130 patients divided into two groups underwent precontrast and portal abdominal 128-row CT examination. In group A, DE portal acquisition was performed; group B underwent SE portal acquisition. Group C was compound of 32 subjects from group A studied with 64-row SECT. In each group, the portal phase dose length product (DLP) and radiation dose (mSv) were calculated. Results: A significant radiation dose increment $(p<0.05)$ was observed in group A $10.2 \pm 2.3 \mathrm{mSv}$ in comparison to group B $8.9 \pm 2.4$ and group C $8.8 \pm 1.9 \mathrm{mSv}$. No significant difference ( $p>0.05$ ) was reported between SE 64- and 128-row acquisitions. A significant positive correlation between radiation dose and $\mathrm{BMI}$ was observed in each group (group A, r2 $=0.59, p<0.0001$; group B, r2 $=0.35 p<$ 0.0001 ; group $C, r 2=0.20, p=0.0098$ ).

Conclusion: In clinical practice, abdominal DE modality delivers a significant higher radiation dose to the patient compared to 64- and 128-row SE acquisition. The radiation increment can be justified if a real advantage in patient diagnosis is obtained using DE information.

\section{B-0705 11:24}

Can iterative reconstructions improve the detection of small hypervascular liver nodules with dual-energy CT?

L. Facchetti' ', L. Berta'2, L. Mascaro', F. Pittiani', L. Romanini', R. Maroldi'; 'Brescia/IT, ${ }^{2}$ Milan/IT (facchettil@gmail.com)

Purpose: To optimise Dual-Energy CT (DECT) protocols with Sinogram Iterative REconstruction (SAFIRE) algorithms for improving small hepatocellular carcinoma (HCC) detection.

Methods and Materials: A DECT arterial acquisition of a cirrhotic patient was reconstructed using the standard filtered back projection (FBP-B2Of) and 3 iterative reconstructions both for the whole dose protocol (DE composition 0.5) and for 80$\mathrm{kV}$ acquisitions. Each one of the iterative medium-smooth filter (I26-I30-I31) was reconstructed at 3 different strengths (S3-S4-S5) for a total of 20 image series. For each series, 8 virtual spherical lesions were simulated in random positions on the liver. A lesion-to-liver contrast (LLC) of $+20 \mathrm{HU}$ was selected for DE-composition 0.5 and $+30 \mathrm{HU}$ for $80 \mathrm{kV}$, according to a preliminary study on $20 \mathrm{HCCs}$ acquired with DECT. Four expert radiologists performed a detectability test and rated the overall quality, noise and sharpness (5-point scale) in all images.

Results: 2/32 lesions were detected with FBP-B20f. In all the iterative reconstructions, the strength- 5 kernels led to detect $23 \pm 1.2 / 32$ lesions. The highest image quality ratings were $3.3 \pm 1.3$ (B20f), $3.5 \pm 0.7$ (I30-S3 DE-composition 0.5) and $3.1 \pm 1.2$ for 130 -S5 DE-composition 0.5, without a statistical difference (ANOVA test). All subjective ratings in the $80 \mathrm{kV}$ series were defined as suboptimal $(2.3 \pm 0.3)$. Conclusion: Iterative reconstructions increase the sensitivity of the detection of sub-centimetric low-contrast lesions, even in the $80-\mathrm{kV}$ series delivering half dose. The I30-S5 reconstruction was considered the best for the routine protocol. 


\section{B-0706 11:33}

Low-voltage CT of the abdomen: correlation between patient diameters and subjective image quality, toward identifying cutoff patient diameters for patient selection.

G.A. Zamboni, M. Ambrosetti, R. Pozzi Mucelli; Verona/lT (gzamboni@hotmail.com)

Purpose: To correlate patient diameters and subjective image quality in low-voltage and standard-voltage CT of the upper abdomen in the same patient population, with the goal of identifying cutoff patient diameters for selecting patients for lowvoltage scans.

Methods and Materials: 32 patients underwent MDCT of the abdomen with arterial phase at $80 \mathrm{kV}$ with angular dose modulation on 64-row MDCT (test group). This was compared to a previous $120-\mathrm{kV}$ scan on the same scanner. Mean interval between scans was 139 days. Patient transverse and sagittal diameters were measured at celiac axis level, and the mean was calculated. Two radiologists by consensus graded image quality on a 5 -point scale ( $5=$ =xcellent; $4=$ good; $3=$ moderate; $2=$ poor; $1=$ non-diagnostic; 3 was the chosen cutoff quality). Image quality was correlated to the transverse, sagittal and mean diameter by means of an ANOVA test.

Results: Patient diameters were unchanged across exams (all $p=n s$ ). In 80-kV scans, image quality was significantly correlated to sagittal $(p=0.034)$ and mean diameters $(p=0.025)$, while a trend to significance was observed for transverse diameter $(p=0.053)$. In $120-k V$ scans, image quality was not significantly correlated to patient diameters (all $\mathrm{p}=\mathrm{ns}$ ), and all patients received grade 4 or 5 . In $80-\mathrm{kV}$ scans, a subjective grade 3 corresponded to a transverse diameter of $329 \mathrm{~mm}$ and a sagittal diameter of $267 \mathrm{~mm}$.

Conclusion: Subjective image quality in low-voltage scans appears to be more influenced by patient size than at standard voltage scans. For our protocol, cutoff diameters for adequate image quality are transverse $329 \mathrm{~mm}$ and sagittal $267 \mathrm{~mm}$.

Author Disclosures:

G.A. Zamboni: Speaker; Guerbet.

\section{B-0707 11:42}

Preliminary application of spectral CT imaging in the differentiation of hepatic tumours

W. Chen, X. Ye, C. Zhou; Beijing/CN (wenchen0629@yahoo.com.cn)

Purpose: To investigate the value of spectral CT imaging in differential diagnosis of hepatic tumours.

Methods and Materials: This prospective study was Institutional Review Board approved with patient consent. Thirty-eight patients with hepatic tumours (22 men, 16 women, 63 lesions), including 11 patients with hepatocellular carcinoma (HCC), 17 with hemangioma $(\mathrm{HH})$ and 10 with metastatic tumour $(\mathrm{MT})$, underwent plain scan of spectral CT imaging and conventional contrast CT scan. Contrast-to-noise ratio (CNR) of different energy levels and homologous keV of optimal CNR were calculated by the spectral analysis software. The authors also chose three regions $(60-80 \mathrm{keV}, 50-90 \mathrm{keV}$ and $40-100 \mathrm{keV})$ and calculated the slope rates of spectral curves. One-way ANOVA followed by LSD post hoc tests were performed to compare parameters among $\mathrm{HCC}, \mathrm{HH}$ and MT groups.

Results: The homologous keV of optimal CNR of HCC, HH and MT groups were $68.71 \pm 1.72,67.23 \pm 1.93$ and $67.16 \pm 2.79$, respectively. The slope rate of $60-80$ $\mathrm{keV}$ region in $\mathrm{HCC}$ group $(0.446 \pm 0.201)$ was significantly higher than that in $\mathrm{HH}$ group $(0.205 \pm 0.209)$. There were significant differences between $\mathrm{HCC}, \mathrm{HH}$ and MT groups in the slope rates of $50-90-\mathrm{keV}$ and $40-100-\mathrm{keV}$ regions $(p<0.05)$. The slope rates were $0.481 \pm 0.199,0.202 \pm 0.213$ and $0.344 \pm 0.229$, respectively, in $50-90-\mathrm{keV}$ region and $0.565 \pm 0.231,0.222 \pm 0.289$ and $0.396 \pm 0.260$, respectively, in $40-100-k e V$ region.

Conclusion: The $65-70-\mathrm{keV}$ monochromatic imaging of spectral CT imaging is the best for detection of hepatic tumours and slope rates of spectral curves may be useful in differentiation of hepatic tumours.

\section{B-0708 11:51}

Ultra-high-field imaging of the biliary tract at 7 Tesla: initial results of Gd-EOB-DTPA-enhanced magnetic resonance cholangiography

A. Fischer, O. Kraff, S. Orzada, F. Nensa, L.C. Schäfer, M.E. Ladd, L. Umutlu,

T.C. Lauenstein; Essen/DE (anja.fischer@uk-essen.de)

Purpose: To assess the feasibility of magnetic resonance cholangiography (MRC) using biliary secreted gadoxetic acid at 7 Tesla (T) and to compare it to T2-weighted (w) MRC at $3 \mathrm{~T}$.

Methods and Materials: 10 healthy volunteers were examined on a 7T MR-system. T2w TSE, T1w VIBE and FLASH with inversion recovery (IR) were acquired in coronal orientation. For dynamic imaging gadoxetic acid was administrated and data were collected in a time period of 5-40 minutes for the T1w sequences. Volunteers underwent subsequent T2w respiratory-gated MRC on a 3 T MRI. For qualitative analysis a 5-point scale was used. Contrast ratios were calculated for quantitative assessment.

Results: Contrast-enhanced T1w MRC at 7T showed a homogeneous depiction of the intra- and extrahepatic biliary tract with a maximum enhancement 20 minutes post-contrast. VIBE and FLASH IR provided a good image quality for the intra- (VIBE 3.60, FLASH IR 3.67) and extrahepatic bile ducts (VIBE 3.50, FLASH IR 3.72). Quantitative analysis revealed high CR values for FLASH IR (CR 0.41 intrahepatic and 0.45 extrahepatic) due to a strong signal decrease of hepatic tissue and vessels. T2W TSE at 7T revealed a poor image quality without diagnostic potential (2.22 intrahepatic, 1.93 extrahepatic). $7 \mathrm{~T} \mathrm{VIBE}$ and FLASH provided superiority in the depiction of the intrahepatic bile ducts, while 3 T MRC was superior in the delineation of the extrahepatic biliary tract.

Conclusion: Our results demonstrate the feasibility of contrast-enhanced imaging of the biliary ducts at 7T, particularly T1w FLASH with inversion recovery enables high-quality assessment of the biliary tract.

$10: 30-12: 00$

Room L/M

\section{Head and Neck}

\section{SS 1308}

\section{Advanced imaging of the ear, orbit and glands}

Moderators:

R. Elias; Rotterdam/NL

B.F. Schuknecht; Zurich/CH

\section{B-0709 10:30}

Volume-rendering 3D-MRI for detection of auditory pathways in

children under evaluation for cochlear implantation

I. Burck, N.N. Nagy, M. Harth, T. Stöver, T.J. Vogl, S. Strieth;

Frankfurt a. Main/DE (iris.burck@gmx.de)

Purpose: Volume rendering 3D-magnetic resonance imaging (MRI) is able to show auditory pathways between the cochlea and the cerebellopontine angle in children with negative auditory brainstem responses and cochlear nerve structures undetectable by conventional high-resolution (HR) MRI.

Methods and Materials: Twelve children (mean age $\pm 43.8 \mathrm{mo}$ ) with confirmed severe hearing loss by ABR (auditory evoked brainstem responses) underwent HR$\mathrm{CT}$ and HR-MRI of the petrous bone. In addition, three-dimensional T2-weighted turbo spin-echo axial slices were obtained at $1.5 \mathrm{~T}$ and maximum intensity projections and $3 \mathrm{D}$ volume reconstructions were performed for visualisation of the cochlear anatomy.

Results: Abnormal cochleovestibular anatomic findings such as incomplete partition type I $(n=1)$, incomplete partition type II $(n=1)$, narrow internal auditory canal $(n=3)$, splitting of the internal auditory canal and concomitant anomalies of the posterior labyrinth $(n=2)$ were detected by HR-CT and HR-MRI. Interestingly, volume-rendered $3 \mathrm{D}$ inner ear reconstructions detected varying but communicating structures of the cochlea and the brainstem in all 12 cases. After cochlear implantation all twelve children showed obvious hearing reactions.

Conclusion: In contrast to conventional HR-MRI, 3D-MRI volume-rendering reconstructions are useful to detect aberrant and even tiny auditory path structures derived from the cochlea. Children with severe hearing loss and HR-MRI-based findings of missing cochlear nerve structures should be further screened with volume rendering 3D-MRI during cochlear implantation evaluation.

\section{B-0710 10:39}

Congenital sensorineural hearing loss and $A B I$ surgery: preoperative radiological protocol and considerations after 12 years of experience

M. Barillari, R. Cerini, F. Spagnolli, N. Cardobi, M. Carner, L. Colletti, V. Colletti, R. Pozzi Mucelli; Verona/IT (marco-barillari@hotmail.it)

Purpose: Cochlear nerves aplasia represents a rare but possible congenital cause of Sensori Neural Hearing Loss (SNHL) in children. Early identification and accurate diagnosis are mandatory to correctly select auditory brainstem implantation $(\mathrm{ABI})$ candidates. The purpose of the study was to evaluate the contribution of a complete pre-operative radiological assessment based on CT and MRI to prevent misdiagnosis and erroneous treatments.

Methods and Materials: Since 2000, 38 SNHL patients have been treated with an ABI in our ENT Department. Six patients had already been operated elsewhere 
with cochlear implantation without benefits. All the children performed pre-operative functional tests, temporal bone CT and brain/cerebellum-pontine angle (CPA) MRI. Results: MRI showed in all cases the absence of the cochlear branch of the eighth nerve bilaterally (38/38). Unilateral facial nerve aplasia was present in one patient. Associated inner ear malformations were present in 21/38 patients, in particular common cavity in $8 / 21$, incomplete partition type 1 in $6 / 21$ and semicircular canals malformations in $3 / 21$. No brain anomalies were showed. CT scans better depicted the labyrinthine malformations, adding information about facial nerve canal and internal auditory canal (IAC) size. 2/38 CT showed normal inner ear structures and IAC size.

Conclusion: Providing accurate information about cochlear nerve and brain anomalies is crucial in the pre-operative assessment of SNHL patients. MRI is the only radiological technique that is able to directly demonstrate the presence or the absence of cochlear nerves. CT is not fundamental and provides complementary information; a negative $\mathrm{CT}$ pattern cannot exclude a cochlear nerve malformation.

\section{B-0711 10:48}

Three dimensional and multiplanar reconstruction of the internal auditory canal using high-resolution MR imaging before cochlear implantation: does the size of the cochlear nerve affect the results of speech recognition outcome after implantation?

N.N.N. Naguib, N.-E.A. Nour-Eldin, T. Lehnert, T. Lehnert, M. Harth, T.J. Vogl, C. Hey; Frankfurt a. Main/DE (nagy3n@yahoo.com)

Purpose: To test whether the size of the cochlear nerve measured in the internal auditory canal using three dimensional (3D) and multiplanar reconstruction (MPR) high-resolution MRI affects the speech recognition outcome following cochlear implantation.

Methods and Materials: The study was retrospectively performed on 51 patients with longstanding hearing loss (mean age of 54.5 years). MRI was performed using a T2-weighted SPACE sequence. MPR and 3D reconstructions were performed using $3 \mathrm{D}$ and InSpace Applications, respectively. The preoperative cross-sectional surface area of the cochlear nerve was measured by drawing a region-of-interest around the circumference of the nerve. Speech recognition (SR) was tested using the Freiburger-Monosyllabic-Words (MSWT) and Numbers-tests (NT) preoperatively, postoperatively, at 3-, 6- and 12-month follow-up. SR and size assessment were performed in a double-blinded fashion. The correlation between the SR and nerve size were tested using Spearman's rank correlation test.

Results: The mean cross-sectional surface area of cochlear nerve in MPR and 3D was $1.27 \mathrm{~mm} 2$ (SD: 0.41, range: 0.38-2.1) and $0.6 \mathrm{~mm} 2$ (SD: 0.197, range: 0.25-1.3). The mean percentages of the SR-MSWT were 5.6, 27.16, 41.25, 51.84 and 57.1, while the mean percentages of SR-NT were 37.35, 65.88, 81.4, 85.1 and 88.47 in the preoperative, postoperative, at 3-, 6- and 12-month follow-up, respectively. There was no statistically significant correlation $(p>0.05)$ between the preoperative size of the cochlear nerve (both at MPR and $3 \mathrm{D}$ ) and the results of the SR assessment at any time point of the study.

Conclusion: The preoperative size of the cochlear nerve does not correlate significantly with the outcome of the speech recognition tests following cochlear implantation.

\section{B-0712 10:57}

Local tumour control in uveal melanoma (UM) after gamma knife radiosurgery (GKR): quantitative assessment of tumour response with contrast-enhanced ultrasound (CEUS)

C. Colantoni, M. Venturini, G. Modorati, A. Colucci, M. Di Nicola, G. Agostini, F. De Cobelli, F. Bandello, A. Del Maschio; Milan/IT (colantoni.caterina@hsr.it)

Purpose: GKR efficacy in the treatment of UM, a hypervascular tumour, is conventionally assessed by clinical and ultrasound findings. Previous reports showed that, in case of hypervascular lesions (HCC, melanoma metastasis, etc). changes in vascularization precede diameters reduction after treatment. Hence, our aim was to evaluate CEUS in the quantitative assessment of tumour response to GKR. Methods and Materials: Ten patients, who underwent GKR, were enrolled. CEUS was performed (SonoVue Bracco) with iU-22 ATL (Philips) and 5-9 MHz linear probe at baseline, at 3 and 6 months after GKR (bGKR, 3-GKR, 6-GKR). Two main tumour diameters and different quantitative parameters related to blood volume (area under the curve in the wash-in phase; wash-in perfusion index - WiPI; peak enhancement - PE) and blood flow (mean transit time; wash-in rate - WiR; rise time - RT; time to peak) were evaluated using a dedicated software (SonoTumor Bracco). Results: Mean diameters were: $b G K R=10 \times 8 \mathrm{~mm}$, 3-GKR=9×9 mm (Wilcoxon bGKR/3-GKR p > 0.05), 6-GKR=7×6 mm (Wilcoxon b-GKR/6-GKR=p < 0.05). PE values were: $b-G K R=2^{*} 10^{7}$ a.u. $3-G K R=5^{*} 10^{7}$ a.u. $(p>0.05), 6-G K R=2^{*} 10^{6} a . u$ $(p<0.05)$; WiR values were: $b-G K R=5^{*} 10^{6}$ a.u., $3-G K R=9^{*} 10^{6}$ a.u. $(p>0.05)$,
6 -GKR $=2^{*} 10^{5}$ a.u. $(p<0.05)$; WiPI values were: $b-G K R=8^{*} 10^{7} \mathrm{~cm}^{3} / \mathrm{sec}$, $3-G K R=2^{*} 10^{8} \mathrm{~cm}^{3} / \mathrm{sec}(p>0.05), 6-G K R=6^{*} 10^{6} \mathrm{~cm}^{3} / \mathrm{sec}(p<0.05)$. 6-GKR mean diameters decreased in 8/10 patients, 6-GKR CEUS parameters (PE, WiR, WiPI) decreased in all patients.

Conclusion: A reduction in PE, WiR and WiPI at 6 months after GKR suggested a depletion in tumour vascularization due to radiotherapy-induced necrosis. Our preliminary results showed the feasibility of CEUS perfusion analysis for UM, which could provide reproducible, reliable and earlier parameters for the assessment of tumour response to GKR

\section{B-0713 11:06}

Evaluation of lacrimal drainage system obstruction using combined multidetector $\mathrm{CT}$ and instillation dacryocystography M. Shweel, A. EIShafaey, M. Nasar, R. MohyEIDien; EIMinia/EG (mohshweel@yahoo.com)

Purpose: To assess the use of combined multidetector computed tomography and instillation dacryocystography (CTDCG) in detection of the level of lacrimal drainage system (LDS) obstruction.

Methods and Materials: Twenty-one patients with one-sided epiphora were our candidates for CTDCG. Axial source and post-processing images were assessed for clear viewing and the ability to detect the level of LDS obstruction. To our knowledge, no previous studies used this combination in the assessment of the LDS obstruction.

Results: All patients tolerated the examination well. Various levels of LDS obstruction were detected, common canaliculus in $4(19 \%)$ patients, lacrimal sac in 3 (14.2\%), junction between lacrimal sac and NLD in $9(42.8 \%)$ and NLD obstruction in $5(23.8 \%)$ patients. The most common CTDCG findings were dilated opacified lacrimal sac with no opacification of the nasolacrimal duct (NLD) in 9/21 (42.8\%) patients. Curved planner reformation (CPR) was excellent to detect the nasolacrimal duct (NLD) obstruction.

Conclusion: CTDCG is a non-invasive patient friendly procedure that adds benefit in documentation and preoperative planning.

\section{B-0714 11:15}

Parotid gland tumours shear wave elastography: a preliminary study

S. Espinoza-Boireau, I. Khettab, A. Lacan Melki, P. Halimi; Paris/FR (sophie.espinoza@gmail.com)

Purpose: To assess the feasibility of parotid gland tumours shear wave elastography. To establish normal parotid gland elasticity. To evaluate elasticity for the most common benign parotidian tumours (pleomorphic adenoma, Warthin's tumour) and malignant tumours.

Methods and Materials: Prospective study including 30 consecutive patients with parotid gland tumour. They all underwent share wave elastography to measure tumour elasticity and contralateral normal parotid gland elasticity. The results were confronted with histological analysis or fine-needle aspiration cytology.

Results: Elasticity measure was feasible in all cases. Mean normal parotid gland elasticity was $7 \mathrm{kPa}(n=25 ; 3-15)$. Benign tumours elasticity was $17.8 \pm 8 \mathrm{kPa}(n=18)$. Malignant tumours elasticity was $37 \pm 18 \mathrm{kPa}(\mathrm{n}=3)$. Pleomorphic adenomas elasticity was $15.3 \pm 8 \mathrm{kPa}(n=12)$. Warthin's tumours elasticity was $21.8 \pm 7.4 \mathrm{kPa}(n=6)$. Conclusion: Benign and malignant tumours seem to behave differently under shear wave elastography. Complementary studies are required to establish relevant threshold values allowing proper elastography lesions discrimination.

\section{B-0715 11:24}

Does heterogeneous echogenicity of the thyroid parenchyma influence the detection of multifocality and bilaterality for papillary thyroid carcinoma on preoperative ultrasound staging?

S. Herh, E.-K. Kim, H. Moon, J. Kwak; Seoul/KR (jinnyhs@hanmail.net)

Purpose: This study was to evaluate whether heterogeneous echogenicity of the thyroid parenchyma can influence the diagnostic performances of ultrasonography (US) in detection of multifocality and bilaterality of papillary thyroid carcinoma (PTC). Methods and Materials: Between December 2010 and January 2011, 240 patients had preoperative staging US for PTC, and underwent total or near-total thyroidectomy. Seven experienced radiologists performed preoperative US for T and $\mathrm{N}$ staging. Underlying parenchymal echogenicity of the thyroid gland, multifocality, and bilaterality of the thyroid nodules were also evaluated. Patients were divided into two groups according to the underlying echogenicity of thyroid parenchyma on US. To evaluate the diagnostic accuracy of preoperative staging US according to the underlying thyroid echogenicity, diagnostic performances including sensitivity, 
specificity, positive predictive value (PPV), negative predictive value (NPV), and accuracy were calculated and compared among the two groups.

Results: Of the 240 patients, underlying echogenicity of thyroid was heterogeneous in 55 patients (22.9\%), and homogenous in 185 patients $(77.1 \%)$. Forty-four (18.3\%) of the 240 patients had multifocal lesions in one lobe, and $77(32.1 \%)$ had bilateral lesions on pathologic examination. Diagnostic performances of preoperative staging US did not show significant differences in detecting multifocality and bilaterality between patients with homogenous and heterogeneous parenchymal echogenicity. Conclusion: Heterogeneous echogenicity of underlying thyroid parenchyma does not significantly influence the detection of multifocality and bilaterality for PTC on preoperative US staging

\section{B-0716 11:33}

Role of elastography in the characterisation of thyroid nodules F. Sogaro, P. Tessitore, A. Scrimieri, F. Pittiani, A. Borghesi, R. Maroldi; Brescia/IT (fsogaro@alice.it)

Purpose: This study aimed at evaluating the reliability of elastography in predicting malignancy of thyroid nodules.

Methods and Materials: 59 patients with thyroid nodules, who underwent to grey-scale ultrasonography (US), elastography and FNC (Fine-Needle Cytology), were prospectively enrolled from February to August 2012. For each nodule, grey-scale US parameters (hypoechogenicity, absence of halo sign, absence of colloid) were examined. Findings at elastography were classified according to Rago criteria (2010). Cytological diagnosis were classified according to the SIAPEC-IAP criteria in Tir1, Tir2, Tir3, Tir4 and Tir5. The diagnostic performance of grey-scale US and elastography were compared with cytological findings. Tir1 and Tir3 with indeterminate cytology were excluded from the study.

Results: 48 nodules were included: 41 Tir2, 3 Tir4 and 4 Tir5. According to Rago criteria 32 nodules were score 1, 2 nodules score 2 and 14 nodules score 3 . The sensitivity, specificity, positive predictive value (PPV), negative predictive value (NPV) of elastography were $71 \%, 78 \%, 35 \%, 94 \%$, respectively. The combination of grey-scale US parameters (hypoechogenicity with absence of halo sign and colloid) showed $57 \%$ sensitivity, $76 \%$ specificity, $28 \%$ PPV and $91 \%$ NPV.

Conclusion: Elastography is not useful in the differentiation of malignant and benign thyroid nodules. However, elastography had a high probability in predicting cytological benign diagnosis (high VPN). Moreover, elastography demonstrated a superior performance than the combination of grey-scale US parameters.

\section{B-0717 $11 \cdot 42$}

The new approach to thyroid elastosonography - time-strain curves may aid the differentiation of nodules

R.Z. Slapa, B. Migda, W.S. Jakubowski, J. Bierca, J. Slowinska-Srzednicka; Warsaw/PL (rz.slapa@gmail.com)

Purpose: To evaluate a new linear/non-linear approach to strain elastosonography of thyroid nodules, based on the analysis of time-strain curves and to compare it with classical elasticity score and thyroid strain ratio methods.

Methods and Materials: During 2009-2011, 67 patients scheduled for thyroidectomy (62 with multinodular goitre and 5 with single thyroid nodule) were evaluated with B-mode and power Doppler ultrasound of the whole thyroid. During ultrasound examination, 96 dominant nodules were examined with strain elastosonography with Aplio XG with linear 5-17 MHz transducer. The stiffness of each thyroid nodule was evaluated with classical features of strain elastosonography qualitatively (with elasticity scores) and semi-quantitatively with thyroid tissue strain/nodule strain ratios with application of elasto $Q$. Moreover, a novel, original approach to elasticity data based on evaluation of time-strain curves was applied.

Results: 7 papillary carcinomas, 89 benign nodules. Classical elastosonographic analysis with elasticity score and elasticity ratio on statistical analysis did not show significant difference between cancer and benign nodules ( $p$-value, respectively, 0.431 and 0.156 ). On linear/non-linear analysis of time-strain curves excellent differentiation $\left(p=5.6 \times 10^{-9}\right)$ was possible with new parameter: the Relative Length of Non-Linear Relaxation. With threshold 0.5 : sensitivity $100 \%$, specificity $85.4 \%$, area under $\mathrm{ROC}=0.975$.

Conclusion: The analysis of linear and non-linear elastosonography data may greatly improve differential diagnosis of thyroid nodules. Further large-scale studies evaluating the usefulness of linear/non-linear elastosonography phenomena (involving evaluation of vioscoelasticity, e.g. shear wave spectroscopy) in differential diagnostics of thyroid cancer are warranted.

Author Disclosures:

R.Z. Slapa: Research/Grant Support; Grant Nr N N402 476437 of Ministry of Science Poland. W.S. Jakubowski: Research/Grant Support; Grant Nr N N402
476437 of Ministry of Science Poland. J. Bierca: Research/Grant Support; Grant Nr N N402 476437 of Ministry of Science Poland.

\section{B-0718 11:51}

Semiquantitative strain elastography for the evaluation of chronic thyroiditis

F. Ozkan, M. Sahin, K. Gul, M.F. Inci, M. Yuksel; Kahramanmaras/TR (drfozkan@yahoo.com)

Purpose: To determine the value of semiquantitative strain elastography for the evaluation of chronic thyroiditis.

Methods and Materials: The study was conducted on 48 consecutive patients with chronic autoimmune thyroiditis and 53 healthy controls. Semiquantitative strain elastography was performed to calculate the strain index value (strain ratio of the strap muscles to the thyroid parenchyma) to assess thyroid stiffness. For each lobe, measurements were obtained from upper, middle and lower part of the thyroid parenchyma. Mean strain ratio values of patients and healthy controls were compared by the Mann-Whitney U-test. Validity was analysed by receiver operating characteristic (ROC) curves.

Results: The median strain ratio value of patients with chronic autoimmune thyroiditis (2.22) was significantly higher than that of healthy controls $(1.22 ; p<0.001)$. The optimal cut-off value for the prediction of diffuse thyroid pathology was 1.41 . For this cut-off value, thyroid stiffness had $90 \%$ sensitivity, $71.7 \%$ specificity, $75 \%$ positive predictive value (PPV) and $88.4 \%$ negative predictive value (NPV) for the presence of diffuse thyroid pathology. This cut-off point was exceeded by $89.6 \%$ of the patients with and $28.3 \%$ of the subjects in control group. SI values showed mild positive correlation with the TSH levels (Spearman $r$ coefficient $=0.265$ ). However, this difference did not reach statistical significance $(p=0.069)$.

Conclusion: Semiquantitative Strain Elastography seems to be a useful method for the assessment of Chronic Thyroiditis. Further studies are warranted in larger patient groups to determine the reliability of sonoelastography in patients with chronic thyroiditis.

$10: 30-12: 00$

Room N/O

\section{Emergency Radiology}

\section{SS 1317}

\section{Technical issues and clinical results}

Moderators:

M. Brink; Nijmegen/NL

G. Schueller; Bülach/CH

\section{B-0719 10:30}

Advanced visualisation of intracranial hematomas of cranial computed tomography

H. Ringl, F. Stiassny, W. Schima, M. Toepker, C. Czerny,

G. Schueller, U. Asenbaum, J. Furtner, C.J. Herold; Vienna/AT

(helmut.ringl@meduniwien.ac.at)

Purpose: To retrospectively assess the detection rate of intracranial hematomas achieved with the use of curved MIPs of the meningeal spaces, compared to reading transverse CT sections only.

Methods and Materials: This retrospective study was approved by the Institutiona Review Board of our institution, which waived informed consent. 314 consecutive patients who underwent CT imaging for cranial trauma $(n=314 ; 155$ men and 159 women; mean age, $58 \pm 24$ y; range, 2 - 98 y) were included. Four curved MIPs of the meningeal spaces with different thicknesses were rendered for each patient. Four radiologists independently evaluated all cases. Hematomas of less than 3 $\mathrm{mm}$ thickness were considered 'thin' throughout this study. The radiologists were blinded to patient names, and patient and group order was randomised. The results were compared to a standard of reference built by two experts. Logistic regression with repeated measurements was used for statistical analysis.

Results: 121 intracranial hematomas were confirmed in 39 patients. For all readers, the reading time for hematoma detection was significantly shorter ( 3 - 5 times, $P$ $<.001)$ ) for curved MIPs. The mean lesion-based detection rate of all readers was $80 \%(193 / 242)$ for transverse sections and $83 \%$ (200/242) for curved MIPs. For thin hematomas, the mean detection rate rose from $20 \%(8 / 40)$ with transverse sections to $83 \%(33 / 40)$ with curved MIPs. 
Conclusion: Curved MIPs of the meningeal spaces have the potential to shorten the detection time for epi- and subdural hematomas, increase sensitivity, especially for thin hematomas, and reduce the required operator experience for detection.

Author Disclosures:

H. Ringl: Patent Holder.

\section{B-0720 10:39}

Simple and easy way using time-intensity curve of perfusionweighted images to find penumbra in stroke patients within 4.5 hours of onset due to the carotid artery occlusion

T. Mori, T. Iwata, Y. Miyazaki, M. Nakazaki, Y. Takahashi; Kamakura/JP (morit-koc@umin.net)

Purpose: The aim of our study was to investigate whether or not time-intensity curve (TIC) of PWI can find penumbra in stroke patients with acute carotid artery occlusion. Methods and Materials: Included were patients 1) who were admitted within 4.5 hours of onset between January 2006 and January 2011, and 2) in whom emergency MRA suggested the affected carotid artery occlusion. We assessed, NIHSS on admission (NIH adm), DWI-ASPECT score, TIC types, successful recanalization (SR), NIHSS on the $7^{\text {th }}$ day $\left(\mathrm{NIH} 7^{\text {th }}\right)$, and in-hospital death. Early neurological improvement $(\mathrm{ENI})$ was defined as $\mathrm{NIH}$ adm-NIH $7^{\text {th }}>0$. TICs were generated on region of interests set at symmetrical positions of the bilateral MCA territories. According to the time to peak (TP) and the peak signal (PS) comparing the affected side (a) with the contralateral side (c), we classified TIC into four types and defined type 1 as TPa $>$ TPc and PSa $<$ PSc/2, type 2 as TPa $>$ TPc and PSc/2<PSa $<P S c$, type 3 as TPa $>$ TPc and PSa>PSc, and type 4 as TPa=TPc. Relationship between TIC types, in-hospital death and ENI were assessed.

Results: Eighty-seven patients were analysed. There were 36, 39, 12 and 0 patients in TIC type 1, 2, 3 and 4, 39 patients underwent reperfusion therapy (RT) and SR was achieved in 18 patients, and 29 patients died. TIC type 1 was the only determinant of in-hospital death $(p<0.01)$ and SR coupled with TIC type 2 was the determinant of ENI $(p<0.01)$.

Conclusion: TIC type 2 means penumbra and type 1 predetermines poor clinical outcome.

Author Disclosures:

T. Mori: Advisory Board; Kaneka medixs. Board Member; ASNR.

\section{B-0721 10:48}

CT imaging in an emergency setting is not substantially delayed by iterative reconstruction

M.J. Willemink, A.M.R. Schilham, T. Leiner, W.P.T.M. Mali, P.A. de Jong, R.P.J. Budde; Utrecht/NL (m.willemink@umcutrecht.nl)

Purpose: Iterative reconstruction (IR) is a promising noise reducing technique with the potential to reduce radiation-dose with preserved study interpretability or improve image-quality at similar radiation-dose. One of the major drawbacks of IR is a longer reconstruction time which may be problematic in the emergency setting. The purpose of the current study was to compare reconstruction time and speed of IR and filtered back-projection (FBP) in two commonly encountered emergency imaging scan-protocols: total body trauma CT and pulmonary CTA.

Methods and Materials: Fifteen patients underwent a total body CT after a traumatic event and twenty-five adults underwent a CTA for evaluation of pulmonary embolisms on a 256-slice CT-scanner. All data were reconstructed using FBP and two IR-levels (iDose ${ }^{4}$, Philips Healthcare). Quantification of reconstruction time and speed was done with a self-written plug-in for ImageJ (US National Institutes of Health).

Results: The mean delay in reconstruction time on total body trauma CTs was $44.4 \pm 8.1$ and $44.9 \pm 7.0$ seconds for iDose ${ }^{4}$-levels 1 and 6 , respectively, and on pulmonary CTAs $10.1 \pm 9.6$ and $12.0 \pm 11.8$ seconds for iDose ${ }^{4}$-levels 2 and 4 , respectively. The mean reconstruction time and speed for total body trauma CTs were $87.3 \pm 14.6,131.7 \pm 16.7$ and $132.2 \pm 17.9$ seconds, and $20.1 \pm 1.6,13.2 \pm 0.8$ and $13.2 \pm 0.6$ slices/s for FBP, iDose ${ }^{4}$-levels 1 and 6 , respectively, and for pulmonary CTAs $25.5 \pm 7.0,35.6 \pm 9.0$ and $37.6 \pm 12.0$ seconds, and $26.7 \pm 5.6,18.7 \pm 2.3$ and $18.0 \pm 2.8$ slices/s for FBP, iDose 4 -levels 2 and 4, respectively.

Conclusion: CT image reconstruction in an emergency setting is not delayed substantially by IR. Furthermore, reconstruction time and speed did not differ substantially between different IR-levels.

\section{B-0722 10:57}

Patient-tailored automatic $\mathrm{kVp}$ selection with sinogram-affirmed iterative reconstruction (SAFIRE) to reduce dose in patients undergoing emergent CT imaging of abdomen and pelvis: an intrapatient comparison

F.D. Gonzalez-Guindalini, M.P.F. Botelho, H. Chalian, V. Yaghmai; Chicago, IL/US (v-yaghmai@northwestern.edu)

Purpose: To assess the combined effect of automated kVp selection and Sinogram Affirmed Iterative Reconstruction (SAFIRE) on patient radiation dose and image quality in abdomen and pelvis CT.

Methods and Materials: This HIPAA compliant retrospective study was IRB approved. We included 52 patients who had undergone emergent CT scans of the abdomen and pelvis for abdominal pain with tube current modulation, automated $\mathrm{kVp}$ selection and SAFIRE (Scan A) and compared their abdomen and pelvis CT scans with tube current modulation, $120 \mathrm{kVp}$, filtered back projection reconstruction algorithm (Scan B). CTDIvol, DLP, effective dose (DLP x k) and image noise values were compared between two protocols. Paired samples Wilcoxon test was used for analysis and $\mathrm{P}<0.05$ was considered significant.

Results: The median CTDIvol were 12.73 mGy and 18.26 mGy for Scans A and $B$, respectively $(P=0.0002)$; the median DLPs for Scans $A$ and $B$ were 583.00 mGycm and 888.50 mGycm $(P<0.0001)$, respectively. The median effective dose was $34.38 \%$ lower with Scan A compared with Scan B (8.74 mSv vs. $13.32 \mathrm{mSv}$, $P<0.0001)$. The median image noise was significantly lower with Scan $A$ using SAFIRE (11.05 vs. $14.15, \mathrm{P}<0.0001)$.

Conclusion: Automated $\mathrm{kVp}$ selection with SAFIRE results in significantly lower dose and image noise in patients undergoing emergent $\mathrm{CT}$ examinations of the abdomen and pelvis.

Author Disclosures:

F.D. Gonzalez-Guindalini: Research/Grant Support; Educational Grant Support by Siemens Healthcare. M.P.F. Botelho: Research/Grant Support; Educational Grant Support by Siemens Healthcare. H. Chalian: Research/Grant Support; Educational Grant Support by Siemens Healthcare.

\section{B-0723 11:06}

Automated selection of tube potential in thoraco-abdominal trauma CT results in significant dose savings

C. Frellesen, J. Kerl, T. Lehnert, M. Harth, B. Schulz, B. Bodelle, M. Beeres,

T.J. Vogl, R.W. Bauer; Frankfurt/DE (c.frellesen@gmail.com)

Purpose: To investigate the impact of automated attenuation-based tube potential selection on image quality and radiation dose in polytrauma patients undergoing thoraco-abdominal trauma CT.

Methods and Materials: 2x100 age- and sex-matched polytrauma patients underwent whole body CT. The first group was examined on a 16-slice device at fixed 120 $\mathrm{kV}$ with 190 ref.mAs and automated exposure control (AEC) modulating only $\mathrm{mA}$ output. The second group underwent 128-slice CT with AEC and topogram-based automated tube potential selection (autokV) by the scanner software with either $100 \mathrm{kV} / 248$ ref.mAs, $120 \mathrm{kV} / 180$ ref.mAs or $140 \mathrm{kV} / 145$ ref.mAs. CTDIvol, DLP, body diameters, organ enhancement and image noise were compared between the groups and sub-groups.

Results: $100 \mathrm{kV}$ were automatically selected in 82 patients, $120 \mathrm{kV}$ in 12 patients and $140 \mathrm{kV}$ in 6 patients of the autokV group. Patient diameters increased with higher kV settings. The average CTDIvol (9.4 vs. $12.9 \mathrm{mGy}$ ) and DLP (669 vs. $925 \mathrm{mGycm} ; \mathrm{p}<0.05$ ) in the entire autokV group were $27 \% / 28 \%$ lower than in the group with fixed $120 \mathrm{kV}$. This effect was even more pronounced in the patients in whom $100 \mathrm{kV}$ were selected (CTDIvol $8.1 \mathrm{mGy},-37 \%$; DLP $571 \mathrm{mGycm},-38 \%$ ). Enhancement of parenchymal organs gradually increased with lower $\mathrm{kV}$, while image noise was at a stable level.

Conclusion: Software-based automated selection of the tube potential allows for significant dose savings in thoraco-abdominal trauma CT while image quality is maintained or even improved. Especially younger patients involved in accidents may benefit the most from this development.

\section{B-0724 11:15}

Usefulness of low-dose CT with or without adaptive statistical iterative reconstruction (ASIR) in the diagnosis of acute appendicitis

J.J. Fondevila, M.S. Carreras, I. Arrieta, J. Mesa, A.R. Gil,

T. Marquina, A. Castrillo, I. Aguirregoicoa, L. Martinez; Barakaldo/ES (jonfonmon@hotmail.com)

Purpose: To determine the usefulness of low-dose CT in the diagnosis and exclusion of acute appendicitis and to compare with low-dose CT taken with ASIR. 
Methods and Materials: This retrospective study includes 138 patients under 65 years-of-age with clinically suspected acute appendicitis, who underwent unenhanced low-dose CT on a 64 MDCT. 84 patients (group A) underwent a low-dose CT 34 patients (group B) underwent a low-dose CT with ASIR.

Results: Normal appendix was identified in $52(61.9 . \%)$ in A versus $26(76.5 \%)$ in B. In A $22(26.2 \%)$ versus $6(17.6 \%)$ in B were diagnosed with appendicitis. In A $10(11.9 \%)$ studies versus $2(5.9 \%)$ studies in B the appendix was not identified. In those where the appendix was not identified a conventional CT was performed. No one had appendicitis during one month follow-up for negative studies. The sensitivity and specificity of CT low-dose were $88.1 \%$ and $100 \%$, respectively, and for low-dose CT with ASIR $94.6 \%$ and $100 \%$, respectively. The sensitivity increased to $100 \%$ for the total protocol for both techniques. The effective dose mean was $2.4 \mathrm{mSv} \pm 0.8$ instead of $2.62 \mathrm{mSv} \pm 0.9$.

Conclusion: The low-dose CT is a suitable technique for the diagnosis or exclusion of acute appendicitis in young or middle-aged people, making possible inclusion as a radiological imaging test in the diagnostic algorithm of acute appendicitis. Introduction of the ASIR improves the low-dose CT technique with a higher sensitivity for appendix evaluation which increased the diagnostic confidence, slightly lowering the dose.

\section{B-0725 11:24}

The diagnostic yield of MSCT in the detection of obscure infection focuses

S. Schleder, L. Luerken, L.-M. Dendl, P. Wiggermann, C. Stroszczynski,

A. Schreyer; Regensburg/DE (Stephan.Schleder@klinik.uni-regensburg.de)

Purpose: To evaluate the diagnostic yield of multislice computed tomography (MSCT) in the detection of obscure infection focuses.

Methods and Materials: Retrospectively, 525 consecutive patients (326 male, mean age 59 years) over a period of 24 months were included in this study. All patients underwent a MSCT of the thorax $(\mathrm{N}=96)$, of the abdomen $(\mathrm{N}=101)$ or of thorax and abdomen $(\mathrm{N}=328)$ because of an clinical uncertain infection without obvious focus.

Results: In 121 patients (23\%) the clinical suspected diagnosis was approved, in 170 patients $(32.4 \%)$ a diagnosis which had not been suspected was revealed and in 234 patients $(44.6 \%)$ no infection focus could be located. Furthermore, in 169 cases $(32.2 \%)$ the therapy was changed due to MSCT findings (surgery, percutaneous abscess drainage, change of antiobiosis), while in 356 cases $(67.8 \%)$ therapy was held on.

Conclusion: The findings of MSCT are of great relevance in the detection of obscure infection focuses and can constitute an important contribution in optimising the therapy.

\section{B-0726 11:33}

Impact and appropriateness of emergency department ultrasonography requests: a prospective study

E.S. Ventura, Y. Costa, F.V. Gomes; Faro/PT (fvgomes@gmail.com)

Purpose: The aim of this study was to assess the appropriateness and clinical impact of Emergency Department ultrasonography requests in Hospital Central de Faro, Portugal.

Methods and Materials: In this prospective study, ultrasonography examinations requested by the Pediatric and General Emergency Departments of Hospital Central de Faro, between $19^{\text {th }}$ and $26^{\text {th }}$ of March 2012 were evaluated. A description of the patient's demographic parameters and medical histories was undertaken with questionnaires and the consultation of the hospital database. Examinations were classified according to clinical impact and, based on the American College of Radiology Criteria and the Portuguese Direcção Geral de Saúde Guidelines, to appropriateness. Clinical impact of the examinations was correlated with emergency department's provisional diagnosis and appropriateness according to the guidelines. The associations were statistically analysed with the Chi-square independence test and Fisher exact test.

Results: The vast majority of the ultrasonography examinations performed did not agree with the provisional diagnosis $(73.6 \%)$ and a considerable number did not have any clinical impact (37.9\%). According to the American College of Radiology and Direcção Geral de Saúde, $57.5 \%$ and $10.3 \%$ of the scans were inappropriate, respectively. Clinical impact of the ultrasound scans had a statistically significant $(P$ $<0.05$ ) association both with the appropriateness of the requests and with having a well-defined provisional diagnosis.

Conclusion: The clinical impact of ultrasound examinations in the emergency department increases both by following the American College of Radiology and Direcção Geral de Saúde guidelines and also when a well-defined provisional diagnosis is suggested by the prescribing doctors.
B-0727 11:42

Post mortem computed tomography (PMCT) quality management in critical care?

S. Kirchhoff, M.F. Reiser, O. Peschel, M. Graw, K.-G. Kanz;

Munich/DE (sonja.kirchhoff@med.uni-muenchen.de)

Purpose: In the past years the number of clinical-pathological autopsies decreased significantly due to various reasons. Forensic autopsies are disposed not only with regional differences but, however, also with on an average decreasing frequency. Especially in patients who died during trauma room management or emergency operation during critical care, no imaging could possibly be performed. Therefore, after termination of all clinical procedures a critical analysis of death and evaluation of the standards of medical care is often not possible, although especially regarding medical quality management it is highly desirable. At least to partly close this information gap we performed a post mortem Computed tomography (pmCT)study. Methods and Materials: In this prospective study whole body pmCT was performed in patients who died during trauma room management or emergency surgery leaving all installed foreign material, e.g. tube, drainage in place. The pmCT findings were compared with clinical findings and if available with autopsy reports as well. Results: Between 1999 and 200846 patients were enrolled, 18 of whom underwent additional autopsy. The major clinical diagnosis which had finally led to the termination of resuscitation procedures was confirmed by the $\mathrm{PmCT}$ findings. The autopsy report showed a high correlation in the corresponding cases to the $\mathrm{PmCT}$-findings. Conclusion: PmCT offers the possibility to improve quality management during trauma room management regarding the decision for terminating resuscitation efforts by verifying the important clinical findings. In addition, pmCT findings support specifically the autoptic reporting.

\section{B-0728 11:51}

Portrayal of radiology in major medical television series: does it influence the perception of radiology in the general population?

T. Heye $^{1}$, E.M. Merkle' ${ }^{2}$, J.R. Leyendecker ${ }^{3}$, D.T. Boll', R.T. Gupta';

1Durham, NC/US, ${ }^{2}$ Basle/CH, ${ }^{3}$ Winston-Salem, NC/US (tobias.heye@web.de)

Purpose: To assess how the portrayal of radiology in medical TV shows is perceived by the general population.

Methods and Materials: A survey was conducted among adult patients scheduled for a radiological examination, technologists and radiologists. The survey gathered information regarding TV watching habits and interest in medical TV shows. Questions addressing the accuracy in portraying radiology in comparison to reality (1-10 scale), appearance of radiological examinations and radiological staff were asked in regard to House M.D., ER, and Grey's Anatomy. The online survey conducted among radiologists and technologists from two different academic institutions served as reference standard.

Results: A total of 126 patients and 240 professionals (133 technologists, 107 radiologists) participated. A moderate and significant correlation was found between the interest in medical TV shows and the perception that clinical reality was accurately portrayed in the group of patients $(r=0.49, P=0.001)$ and technologist $(r=0.38, P=0.001)$ but no correlation was found for radiologist $(r=0.01)$. While $>60 \%$ in all survey groups noted the appearance of radiological examinations regularly to $>1 /$ show, the appearance of radiological staff was perceived by $45.2-54.0 \%$ of patients, $31.6-47.4 \%$ of technologists and $15.9-23.4 \%$ of radiologists. ER was ranked more accurate than Grey's Anatomy or House M.D.

Conclusion: The more interest in medical TV shows prevails in patients and technologists, the more are modern medical TV shows perceived as accurate in the portrayal of radiology. Radiological examinations are frequently utilised, but imaging is less often performed by radiological staff. 
10:30 - 12:00

Room P

\section{Paediatric}

\section{SS 1312}

\section{Chest, heart and radiation awareness}

\section{Moderators:}

W. Hirsch; Leipzig/DE

P.D. Humphries; London/UK

\section{B-0729 10:30}

Radiation dose levels in paediatric chest CT: experience in 506 children evaluated with dual-source $\mathrm{CT}$

T. Santangelo, L. Colas, T. Niemann, A. Simeone, M. Rémy-Jardin, J. Rémy; Lille/FR (martine.remy@chru-lille.fr)

Purpose: To assess the radiation dose delivered during dual-source chest CT examinations.

Methods and Materials: We prospectively recorded the dose-length-product (DLP) of 506 consecutive children, evaluated in 4 age groups: group $1(<1 \mathrm{yr} ; n=109$; 21\%) (mean weight: $5.33 \pm 1.99 \mathrm{~kg}$ ), group 2 (1-5 yr: $\mathrm{n}=169 ; 33 \%)$ (mean weight: $13.35 \pm 3.92 \mathrm{~kg}$ ), group $3(5-10 \mathrm{yr}: \mathrm{n}=136 ; 27 \%$ ) (mean weight: $23.77 \pm 6.36 \mathrm{~kg}$ ) and group 4 (10-15yr; $\mathrm{n}=92 ; 18 \%$ ) (mean weight: $36.83 \pm 7.78 \mathrm{~kg}$ ). All CT examinations were performed with a dual-source CT system using a dual-source, single-energy scanning protocol obtained with thin collimation, high pitch and high temporal resolution, a weight-adapted selection of the kilovoltage and milliamperage, and systematic reconstruction of images using filtered-back projection.

Results: Patients were scanned with a pitch of $2.0(n=165 ; 33 \%)$ or $3.0(n=341$; $67 \%)$, at $80 \mathrm{kV}(\mathrm{n}=499 ; 99 \%)$ or $100 \mathrm{kV}(\mathrm{n}=7 ; 1 \%)$ with a milliamperage ranging between 40 and $90 \mathrm{mAs}$. The mean duration of data acquisition was $525.8 \pm 192.8$ ms. CT examinations consisted of contrast-enhanced $(n=190)$ and noncontrast $(n=316)$ studies, obtained in the context of acquired $(n=280 ; 55 \%)$ or congenital $(n=46 ; 9 \%)$ bronchopulmonary disease, cystic fibrosis $(n=60 ; 12 \%)$, cardiovascular disease $(n=91 ; 18 \%)$, oesophageal atresia $(n=8 ; 1 \%)$ and miscellaneous causes $(n=21 ; 4 \%)$. All examinations were of diagnostic image quality. The mean DLP value in each age category was $14.29 \pm 3.38$ mGy.cm (group 1), 17.64 \pm 4.44 mGy. $\mathrm{cm}$ (group 2), $23.87 \pm 5.80 \mathrm{mGy} . \mathrm{cm}$ (group 3) and $34.61 \pm 10.80 \mathrm{mGy} . \mathrm{cm}$ (group 4). Conclusion: The doses delivered in the above-mentioned conditions are lower than those of published recommendations.

\section{B-0730 10:39}

Multi-detector CT studies in children: a comparison of radiation

doses between paediatric and non-paediatric radiology units.

Results from the first 2011 Italian SIRM survey

C. Granata ${ }^{1}$, F. Palorini ${ }^{2}$, D. Origgi ${ }^{2}$, D. Matranga ${ }^{3}$, S. Salerno ${ }^{3} ;{ }^{1}$ Genoa/lT,

${ }^{2}$ Milan/IT, ${ }^{3}$ Palermo/IT (cgranata@sirm.org)

Purpose: To provide a comparison of exposure levels in children from multi-detector computed tomography (MDCT) studies (> 16 slices) carried out in paediatric and non-paediatric Italian radiology units.

Methods and Materials: All Italian radiology departments executing MDCT studies in children were asked to collect data from typical scanning investigations (trauma, infection, staging) in three anatomical regions (head, thorax and abdomen). For each examination, the participating centres recorded the delivered dose in term of CTDIvol and DLP, previously verified with standard quality assurance tests. The study was endorsed by SIRM (Società Italiana di Radiologia Medica).

Results: We show the results from 908 examinations performed between January and June 2011 in 25 radiology units. $513(56.5 \%)$ of all exams were executed in paediatric centres. The $75^{\text {th }}$ percentiles of CTDIvol (mGy) and DLP (mGy ${ }^{*} \mathrm{~cm}$ ) distributions by three age groups (1-5, 6-10 and 11-15 years) in paediatric centres are, respectively, $28,32,34$ and $460,640,580$ for the "head" protocol; 1.6, 2.9, 3.8 and 40, 70, 130 for "thorax"; 5.5, 5.3, 9.2 and 190, 350, 490 for "abdomen". While, in non-paediatric centres we obtain: $35,58,60$ and $710,1080,1110$ for "head"; $4.7,5.0,8.4$ and $110,130,330$ for "thorax"; 8.3, 10.5, 15.4 and $330,560,860$ for "abdomen". CTDIvol and DLP distributions between paediatric and non-paediatric centres were significantly different for each age group and protocol $(p<0.001$; non-parametric Wilcoxon rank sum test).

Conclusion: These results suggest that a nation-wide program for optimisation of MDCT imaging protocols in children should be implemented.

Author Disclosures:

C. Granata: Research/Grant Support; Grant support by Società Italiana di
Radiologia Medica (SIRM). S. Salerno: Research/Grant Support; Grant support by Società Italiana di Radiologia Medica (SIRM).

\section{B-0731 10:48}

Is paediatric chest CT achievable at $70 \mathrm{kV}$ ? A feasibility study in 130 children

T. Niemann, S. Henry, L. Colas, J.-B. Faivre, T. Santangelo, J. Rémy,

M. Rémy-Jardin; Lille/FR (martine.remy@chru-lille.fr)

Purpose: To evaluate image quality of paediatric chest CT acquired at $70 \mathrm{kV}$. Methods and Materials: 130 consecutive children undergoing chest CT studies on a dual-source CT system were prospectively evaluated with a $70-\mathrm{kV}$ scanning protocol. All scanning parameters were kept similar to those usually selected for standard $80-\mathrm{kV}$ protocols, except the milliamperage. Always selected according to the patient's weight, ref mAs were increased by a factor of 1.6 to maintain comparable levels of radiation dose between newly introduced $70-\mathrm{kV}$ and routine $80-\mathrm{kV}$ protocols. Image quality at $70 \mathrm{kV}$ (group 1) was compared to that of a paired paediatric population (group 2) based on the age ( $\pm 1 \mathrm{yr})$, weight $( \pm 1 \mathrm{~kg})$ and administration of contrast material, previously scanned at $80 \mathrm{kV}$ on the same CT unit and prospectively stored in our database. Group 1 and group 2 images were reconstructed with filtered-back projection.

Results: Objective noise in group 1 was significantly lower than that measured in group $2(9.9 \pm 6.3 \mathrm{HU}$ vs $11.3 \pm 6.6 \mathrm{HU} ; \mathrm{p}=0.03)$. Subjective image quality of lung and mediastinal images in group 1 did not significantly differ from that of group $2(p=0.18)$. In group 1 , the mean dose-length-product was $15.4 \pm 2.3$ mGy.cm $(<$ 1yr; mean weight: $5.3 \mathrm{~kg})(\mathrm{n}=35) ; 18.3 \pm 2.1$ mGy.cm (1-5yr; mean weight: $13.4 \mathrm{~kg})$ $(n=44), 24.4 \pm 3.9$ mGy.cm (5-10yr; mean weight: $24.7 \mathrm{~kg})(n=33)$ and $31.7 \pm 4.7$ mGy.cm (10-15yr; mean weight: $33.1 \mathrm{~kg})(\mathrm{n}=18)$.

Conclusion: Paediatric chest CT at $70 \mathrm{kV}$ provides similar objective and subjective image quality to that achievable at $80 \mathrm{kV}$.

\section{B-0732 10:57}

Anthropometry of children and mathematical phantoms applied to dose reconstruction in paediatric radiology

M.C. Seidenbusch, K. Schneider;

Munich/DE (michael.seidenbusch@med.uni-muenchen.de)

Purpose: Reconstruction of organ doses achieved during paediatric x-ray examinations is usually done by performing Monte Carlo simulations in mathematical phantoms. For reconstruction of valid organ doses, a close-to-reality anatomical construction of the mathematical phantoms is essential.

Methods and Materials: At Dr. von Hauner's Children's Hospital, University of Munich, since 1976 all conventional $x$-ray examinations and since 2003 all computed tomographic (CT) examinations have been stored in a database. The database encompasses now clinical and dosimetric data of more than 310000 conventional and more than $3000 \mathrm{CT}$ examinations of children of all age groups.

Results: From the records in the database, various anthropometric data were extracted, e.g. body mass and body length of more than 33000 patients, thoracic diameters and the volumes, densities and masses of lungs and mammalian glands of more than 2000 patients of all age groups. These data were compared to the anthropometric data of the mathematical MIRD phantoms provided by the Finnish centre for radiation safety STUK. It can be shown that in mathematical MIRD phantoms of all age groups, body mass, body length and thoracic diameters are overestimated, whereas lung volumes and lung masses are underestimated.

Conclusion: A revision of the anthropometry of the mathematical phantoms currently in use for dose reconstruction in paediatric radiology seems necessary. [Research project of the Federal Republic of Germany, Bundesministerium für Bildung und Forschung, Förderkennzeichen 02NUK016 A)]

\section{B-0733 11:06}

Radiation exposure during scanograms (scoutviews) in paediatric computed tomography (CT)

K. Schneider, V. Teusch, M.C. Seidenbusch;

Munich/DE (michael.seidenbusch@med.uni-muenchen.de)

Purpose: Radiation exposure of patients during computed tomographic (CT) examinations is determined not only by the scan protocol but also by the scanogram (scoutview) parameters. Especially scanograms that exceed the body region of interest cause an unnecessary radiation exposure.

Methods and Materials: Since 2003, exposure and image data of all CT examinations performed at the Dr. von Hauner's Children's Hospital, University of Munich, have been stored in a database. For 3.300 thoracic and abdominal CT examinations of children of all age groups anatomical localisation and length of CT scanograms 
were analysed and compared with the corresponding real scan length. For selected CT scanners, radiation doses achieved during the scanograms were reconstructed using mathematical MIRD phantoms.

Results: Depending on the patients' age, many scanograms considerably exceed the real scan region, thus unjustifiably raising the radiation exposure of the patient. The radiation doses due to $\mathrm{CT}$ scanograms are not neglectible as, e.g. the whole body dose achieved during a thorax scanogram can exceed the whole body dose caused by a conventional chest x-ray examination by a factor of 10 .

Conclusion: An optimisation of scanogram lengths by defining specific anatomical landmarks for standard CT protocols, the optimisation of scanogram exposure parameters in paediatric CT examinations and their implementation into national and international guidelines seems to be necessary to fulfil the ALARA concept in paediatric CT. [Research project of the Federal Republic of Germany, Bundesministerium für Bildung und Forschung, Förderkennzeichen 02NUK016 A]

\section{B-0734 11:15}

Reference values for cardiac valve diameters in extreme preterm infants A. Adams, N. Wesseling, J.L.M. Strengers, W.B. de Vries, M.C. Molenschot; Utrecht/NL (a.adams@umcutrecht.nl)

Purpose: To establish reference values for heart valve diameters, assessed by routine echocardiography, in a cohort of extreme preterm infants.

Methods and Materials: Neonates under 32 weeks' gestation underwent routine echocardiography 24-72 hours after birth. Patients with evident congenital cardiac abnormalities were excluded. Heart valve diameters were retrospectively assessed in static images by measuring the maximal distance between hinge points at the level of the annulus. Measurements were performed by two investigators and repeated on another day. Diameters were then related to length, weight, body surface area (BSA), gestational age, and head circumference by linear regression. Results: A total of 198 infants (103 males) were included, with a median gestational age of 29.7 weeks (range 24.3-31.9), mean birth weight of $1.29 \pm 0.40 \mathrm{~kg}$ (range $0.54-2.75$ ), head circumference $27 \pm 2.5 \mathrm{~cm}$ (range 21-39), and BSA $0.11 \pm 0.02 \mathrm{~m}^{2}$ (range 0.06-0.18). Average valve diameters were $5.2 \pm 0.6 \mathrm{~mm}$ for aortic valves $(\mathrm{AV}), 8.3 \pm 1.1 \mathrm{~mm}$ for mitral valves $(\mathrm{MV}), 6.1 \pm 0.8 \mathrm{~mm}$ for pulmonary valves (PV), and $7.9 \pm 1.4 \mathrm{~mm}$ for tricuspid valves (TV), respectively. BSA best correlated to heart valve diameters (Pearsons-R 0.75 (AV), 0.61 (MV), $0.66(\mathrm{PV}), 0.59(\mathrm{TV})$ ). Prediction formulas for individual predictions with $95 \%$ confidence intervals of valve diameters (in $\mathrm{mm}$ ): $\mathrm{AV}=2.8(2.0-3.6)+21.6^{\star} \mathrm{BSA}, \mathrm{MV}=5.0(3.2-6.7)+30.4^{*} \mathrm{BSA}$ $\mathrm{TV}=4.2(2.0-6.3)+34.7^{\star} \mathrm{BSA}$ and $\mathrm{PV}=3.6(2.4-4.7)+22.7^{\star} \mathrm{BSA}$. Reproducibility was excellent, with intra- and inter-observer intraclass correlation coefficients of 0.97 and 0.93-0.96, respectively.

Conclusion: We established reference values for cardiac valve diameters in extreme preterm infants, based on BSA. Biological variation, inaccuracy in assessment of patient characteristics, inter-operator variability, and limited spatial resolution may account for substantial variability in heart valve diameters found in this cohort.

\section{B-0735 11:24}

Asymmetric lung perfusion in congenital heart disease: impact of differential pulmonary arterial anatomy and pulmonary vascular resistance O. Kondrachuk, T. Yalynska, R. Tammo, I. Iershova, N. Rokytska; Kiew/UA

Purpose: Asymmetry of lung perfusion is relatively common in patients with congenital heart disease. The aim of the study was to evaluate the influence of differential pulmonary arterial anatomy and pulmonary vascular resistance on blood flow distribution in patients with asymmetric lung perfusion.

Methods and Materials: We retrospectively identified 17 consecutive patients with congenital heart disease who had asymmetry of lung perfusion on phase-contrast MRI. The MRI examinations were performed on 1.5 T scanner.

Results: Fourteen patients had left lung hypoperfusion (blood flow to the left lung < $35 \%$ ) and three patients right lung hypoperfusion (blood flow to the right lung $<40 \%$ ). Branch pulmonary artery (BPA) regurgitant fraction (RF) was significantly greater in lung with decreased perfusion $(16.2 \pm 19.0 \%$ vs. $1.8 \pm 2.8 \%, P<0.001)$. There was moderate negative correlation between BPA RF and BPA differential pulmonary blood flow $(R=-0.517, P=0.002)$. Moderate positive linear relationship between BPA size and BPA differential pulmonary blood flow was also found $(R=0.669$, $P=0.003$ ). There was no significant correlation between BPA RF and BPA size. Conclusion: Both BPA anatomy and BPA RF are the significant factors for determining right and left lung flow split. According to prior reports, BPA RF reflects differential pulmonary vascular resistance.

\section{B-0736 $11: 33$}

Whole heart 4D haemodynamics in patients with transposition of the great arteries after switch procedure

J. Geiger ${ }^{1}$, M. Markl ${ }^{2}$, D. Hirtler ${ }^{1}$, B. Jung ${ }^{1}$, B. Stiller ${ }^{1}$, M. Langer ${ }^{1} ;{ }^{1}$ Freiburg/DE,

${ }^{2}$ Chicago, IL/US (julia.geiger@uniklinik-freiburg.de)

Purpose: To characterise pulmonary and aortic blood flow alterations and their correlation to postsurgical anatomy in patients with transposition of the great arteries (D-TGA) after arterial switch by 4D MRI.

Methods and Materials: Flow-sensitive 4D MRI (venc $=200 \mathrm{~cm} / \mathrm{s}$, spatial/temporal resolution $\sim 2.5 \mathrm{~mm}^{3} / 38 \mathrm{~ms}$ ) was performed in $17 \mathrm{D}$-TGA patients and 12 healthy controls (age $=12 \pm 5.4$ vs. $23 \pm 1.6$ years). Patients were divided into two groups: anterior $(n=10)$ and right anterior $(n=7)$ position of the pulmonary trunk (TP) in relation to the ascending aorta (AAo). Blood flow was visualised by time-resolved 3D particle traces. Visual grading (ranking 0-2) of flow disturbances (vortices, helices) was performed. The flow ratio right and left pulmonary artery (RPA:LPA) and flow velocity were quantified at the valves and in the RPA and LPA.

Results: Patients with anterior TP had increased TP vortices $(6 / 10$, mean grade 1.6) compared with right anterior TP position (1/7). Increased helices were seen in the LPA of both patient groups (9/17, grade 1.3), less frequent in the RPA (4) and TP (3). Systolic peak velocity in TP was significantly higher in patients $(1.74 \mathrm{~m} /$ $\mathrm{s} \pm 0.63 \mathrm{~m} / \mathrm{s}$ vs. $0.95 \mathrm{~m} / \mathrm{s} \pm 0.12 \mathrm{~m} / \mathrm{s}$ in volunteers), in the RPA $2.04 \mathrm{~m} / \mathrm{s} \pm 0.43 \mathrm{~m} / \mathrm{s}$ vs. $0.98 \mathrm{~m} / \mathrm{s} \pm 0.14 \mathrm{~m} / \mathrm{s}$, in the LPA $1.78 \mathrm{~m} / \mathrm{s} \pm 0.54 \mathrm{~m} / \mathrm{s}$ vs. $0.89 \mathrm{~m} / \mathrm{s} \pm 0.13 \mathrm{~m} / \mathrm{s}$. The flow ratio RPA:LPA was more heterogenous in patients (RPA:LPA $=1.56 \pm 0.78$ vs. $1.09 \pm 0.15$ in volunteers). 11 patients had increased helices in the AAo (grade 1.6).

Conclusion: 4D flow evaluation of postsurgical blood flow in TGA patients revealed flow disturbances with frequent helices in the LPA and AAo and enhanced vortices for the haemodynamically unfavourable anterior TP position.

\section{B-0737 11:42}

Chest-CT protocol standardisation for multicentre trial in cystic fibrosis (CF) infants

P. Ciet $^{1}$, K. Gonzalez Graniel ${ }^{1}$, S. Stick ${ }^{2}$, M. de Bruijne ${ }^{1}$, H.A.W.M. Tiddens ${ }^{1}$,

M. Van straten ${ }^{1} ;{ }^{1}$ Rotterdam/NL, ${ }^{2}$ Perth/AU (pierluigi.ciet@libero.it)

Purpose: To standardise the scan protocol for CT scanners of participating centres in a multi-centre study (clinicaltrials.gov NCT01270074) for the prevention of radiologically defined bronchiectasis in $\mathrm{CF}$ infants by ensuring the maximum image quality at the minimum radiation dose.

Methods and Materials: Three different sized phantoms (QRM, Germany) were used to assess scanners' performance of automatic exposure control (AEC). CTDI and DLP were recorded. The phantoms contained various inserts to assess slice-sensitivity-profile, in-plane spatial resolution, noise and the Hounsfield Unit (HU) scale. Scans were made for several dose levels and reconstruction kernels. Images were analysed with custom-made software (MatLab, USA) to obtain the standard deviation of the noise, point-spread-function (PSF) and slice thickness. Results: Eight different scanners with 64 slices or more from 4 manufacturers (GE, Philips, Siemens and Toshiba) were assessed. Despite differences in AEC's performance, we obtained approximately the same dose level at each center by recommending site and age-specific AEC reference levels. A constant image quality was only possible by matching the different reconstruction kernels measured PSFs at full-width-at-half-maximum. In fact, large part of the differences between scanners was related to reconstruction kernels. The relatively high noise images corresponded with reconstructions using a kernel with edge enhancement such as the Siemens B70 kernel or the GE lung kernel that are routinely used in chest imaging. Conclusion: Objective measurements on CT images allowed for matching of scan protocols among CT scanners of different manufacturers. Use of routine protocols might introduce a bias in the (automated) image analysis

\section{B-0738 $11: 51$}

Cystic fibrosis lung disease in children: correlation between MRI and HRCT scores

C. Sileo, H. Corvol, P.-Y. Boelle, E. Blondiaux, A. Clement, H. Ducou Le Pointe; Paris/FR (chiara.sileo@trs.aphp.fr)

Purpose: High-resolution computed tomography (HRCT) is known to be a sensitive means of detecting lung disease in cystic fibrosis (CF), with a significant radiation exposure. Magnetic resonance imaging (MRI) becomes increasingly important in the assessment of CF lung disease. The aim of this study was to establish the agreement between MRI and HRCT scores in a paediatric population.

Methods and Materials: HRCT and T2 and post-contrast T1 MRI images were acquired on the same day from $17 \mathrm{CF}$ patients (median age 12.7 years; range 9.1-20.2 years). Two radiologists with either $>20$ (HDLP) or 3 years (CS) of ex- 
perience in paediatric pulmonary disease scored independently HRCT, using the scoring system developed by Helbich et al, and MRI using two scoring systems developed by Helbich et al and Eichinger et al. Readers were blinded to clinical and functional data and to the other reader's findings. The agreements were assessed using the intraclass correlation coefficient (ICC) and the Bland-Altman plot for the two readers and the two methods.

Results: The inter-reader agreement was excellent for the HRCT scores (ICC=96\%), good for MRI with the Eichinger score (ICC=84\%), but less for MRI with the Helbich score $(\mathrm{ICC}=68 \%)$. There was a very good correlation between HRCT and MRI-Eichinger scores (ICC=91\%), but less between HRCT and MRIHelbich scores (ICC=78\%)

Conclusion: The good inter-reader agreement and the high correlation between HRCT and MRI-Eichinger scores support the use of lung MRI for the follow-up of lung disease in paediatric CF patients.

\begin{tabular}{ll}
\hline $10: 30-12: 00$ & Room Z
\end{tabular}

\section{Contrast Media}

\section{SS 1306}

\section{New agents and protocols}

Moderators:

L. Grazioli; Brescia/IT

T.C. Lauenstein; Essen/DE

\section{B-0739 10:30}

Magnetic resonance imaging diagnosis of metastatic lymph nodes: efficacy of the novel ultrasmall superparamagnetic iron oxide agent with monodisperse iron oxide core and multiple-interaction ligands R.-E. Yoo ${ }^{1}$, S. Choi ${ }^{1}$, H. Cho' ${ }^{1}$, E.-g. Kim² ${ }^{2}$ J. Park², W.-J. Myeong ${ }^{2} ;{ }^{1}$ Seoul/KR, ${ }^{2}$ Daejeon/KR (ry_jjang@hotmail.com)

Purpose: To evaluate feasibility of detecting lymph node metastasis using the novel USPIO in MR imaging of a rabbit model.

Methods and Materials: A novel USPIO with monodisperse iron oxide core size of $14 \pm 1 \mathrm{~nm}$ and multiple-interaction ligands inspired from mussel adhesive protein was prepared and its core size, hydrodynamic diameter, and T2 relaxivity were measured. To make metastatic lymph nodes, VX2 carcinomas were implanted into thighs of 24 rabbits 3 or 4 weeks before MR imaging. In each rabbit, MR imaging was performed before and 24 hours after the injection of the USPIO $(5.2 \mathrm{mg} \mathrm{Fe} / \mathrm{kg}$ [N=6], $7.8 \mathrm{mg} \mathrm{Fe} / \mathrm{kg}[\mathrm{N}=6]$, and $10.4 \mathrm{mg} \mathrm{Fe} / \mathrm{kg}[\mathrm{N}=12])$. MR images were correlated with pathologic results and sensitivity, specificity, and accuracy were calculated. Results: The novel USPIO demonstrated high stability without aggregation in the saline. The USPIO had a hydrodynamic diameter of $25 \sim 30 \mathrm{~nm}$ and T2 relaxivity of $281 \mathrm{mM}^{-1} \mathrm{~s}^{-1}$ at $3 \mathrm{~T}$. All USPIO dosages provided good contrast between metastatic lymph nodes without signal drop and normal lymph nodes with signal drop attributable to USPIO uptake. At $10.4 \mathrm{mg} \mathrm{Fe} / \mathrm{kg}$, the MR imaging showed $100 \%$ accuracy with $100 \%$ sensitivity (29/29) and 100\% specificity (12/12). At $5.2 \mathrm{mg} \mathrm{Fe} /$ $\mathrm{kg}$, sensitivity, specificity, and accuracy were 100\% (10/10), 55\% (6/11), and 76\% (15/21), respectively. At $7.8 \mathrm{mg} \mathrm{Fe} / \mathrm{kg}$, sensitivity, specificity, and accuracy were $100 \%(4 / 4), 83 \%(5 / 6)$, and $90 \%(9 / 10)$, respectively.

Conclusion: The novel USPIO MR imaging agent is expected to be a promising agent in the oncologic field, by enabling clear demonstration of the lymph node metastasis with high sensitivity and specificity.

Author Disclosures:

S. Choi: Grant Recipient; Hanwha Chemical Corp. E. Kim: Employee; Hanwha Chemical Corp. Research/Grant Support; Hanwha Chemical Corp. J. Park: Employee; Hanwha Chemical Corp. Research/Grant Support; Hanwha Chemical Corp. W. Myeong: Employee; Hanwha Chemical Corp. Research/Grant Support; Hanwha Chemical Corp.

\section{B-0740 10:39}

Gold as a potential contrast agent for dual-energy $\mathrm{CT}$

R. Krissak, M. Elgert, B. Kusch, R. Hünerbein;

Bad Langensalza/DE (r.krissak@hufeland.de)

Purpose: The K-edge of gold $(81 \mathrm{keV})$ is located within the energy range of diagnostic CT. This might be advantageous for dual-energy CT (DECT). The aim of this in vitro study was to compare the differentiation between iodine or gold and body tissues using DECT at different kV spectra.

Methods and Materials: A phantom containing specimens with iodine (iopamidol), gold (sodium aurothiomalate), compact bone and porcine muscle was scanned consecutively at $80 \mathrm{kVp}, 100 \mathrm{kVp}, 120 \mathrm{kVp}$ and $140 \mathrm{kVp}$, respectively, with constant $\mathrm{mAs}$ settings using a dual-source CT. Differences in calculated dual-energy ratios (DEratio) between the tissues and iodine or gold were determined for different DE spectra.

Results: The attenuation of gold has increased at higher kVp-settings compared to $80 \mathrm{kVp}$, while the attenuation of all other specimens decreased. The calculated $\mathrm{DE}$ ratios at $80 / 100 \mathrm{kVp}, 80 / 120 \mathrm{kVp}$ and $80 / 140 \mathrm{kVp}$, respectively, were $1.31,1.63$ and 1.91 for iodine, $0.90,0.88$ and 0.92 for gold, $1.20,1.30$ and 1.45 for compact bone, $1.01,1.03$ and 1.08 for muscle. The difference between the DE ratios 80/100 $\mathrm{kVp}, 80 / 120 \mathrm{kVp}$ and $80 / 140 \mathrm{kVp}$, respectively, were $0.11,0.23$ and 0.46 for iodine and bone, $0.31,0.51$ and 0.53 for gold and bone, $0.29,0.59$ and 0.83 for iodine and muscle, $0.12,0.15$ and 0.16 for gold and muscle.

Conclusion: DE ratio of gold remains relatively stable along the energy spectrum of diagnostic CT and allows a reliable material differentiation between gold and bone at contiguous low tube voltage settings ( $80 \mathrm{kV}$ and $100 \mathrm{kV})$. Thus, gold might have potential as a contrast agent for DECT.

\section{B-0741 10:48}

Potential use of intra-articular diluted high-relaxivity gadoliniumbased contrast agent for magnetic resonance arthrography (MRA): an in-vitro study

L.M. Sconfienza, A. Aliprandi, P. Cannaò, S. Sdao, M. Bandirali, F. Sardanelli; Milan/IT (io@lucasconfienza.it)

Purpose: Gadobenate dimeglumine (Gd-BOPTA) is a high-relaxivity contrast agent that tightly binds to plasmatic proteins, currently used in several clinical applications. Our aim was to test in-vitro different concentrations of Gd-BOPTA to be potentially used to perform MRA.

Methods and Materials: Gd-BOPTA (Multihance, Bracco) was diluted in saline $(\mathrm{NaCl} 0.9 \%$ ) to achieve different concentrations $(4 \mathrm{mmol} / \mathrm{l}, 2 \mathrm{mmol} / \mathrm{l}, 1 \mathrm{mmol} / \mathrm{l}$, $0.67 \mathrm{mmol} / \mathrm{l}, 0.5 \mathrm{mmol} / \mathrm{l})$. Five $\mathrm{mL}$ of these solutions was injected in sterile pipes and was added with $0.5 \mathrm{~mL}$ of fresh synovial fluid withdrawn from a 71-year-old male knee. In a separate pipe (reference), $0.5 \mathrm{~mL}$ of synovial fluid and $5 \mathrm{~mL}$ of gadopentetate dimeglumine $2 \mathrm{mmol} / \mathrm{l}$ (Gd-DTPA; Magnevist) were injected. Pipes were imaged using a T1-weighted sequence (TE $=9.8 \mathrm{msec}, T R=678 \mathrm{msec}$, slice thickness $3.5 \mathrm{~mm}$, FOV $320 \times 320 \mathrm{~mm}$ ) at 1.5 T. For each pipe on each slice, signal intensity (SI) was calculated.

Results: Mean Gd-DTPA SI was $1290 \pm 48$. Mean Gd-BOPTA SI was $1406 \pm 36$ $(+9 \%$ to reference) at $4 \mathrm{mmol} / \mathrm{l} ; 1532 \pm 36(+15 \%)$ at $2 \mathrm{mmol} / \mathrm{l} ; 1583 \pm 36(+19 \%)$ at $1 \mathrm{mmol} / \mathrm{l} ; 1494 \pm 44(+14 \%)$ at $0.67 \mathrm{mmol} / \mathrm{l} ; 1235 \pm 13(-4 \%)$ at $0.5 \mathrm{mmol} / \mathrm{l}(\mathrm{P}<.001)$. At post hoc analysis, all one-to-one comparisons were significantly different with exception of 2 and $4 \mathrm{mmol} / \mathrm{l}(\mathrm{P}=.105)$.

Conclusion: Versus Gd-DTPA, a $+19 \%$ peak SI was achieved with half Gd-BOPTA concentration. A similar SI between the two agents was achieved at $0.5 \mathrm{mmol} / \mathrm{l}$. Gd-BOPTA high relaxivity and its potential binding with synovial fluid glycoproteins could explain these results. This may improve visibility of intra-articular tears when evaluated using Gd-BOPTA MRA

Author Disclosures:

L.M. Sconfienza: Author; Springer. F. Sardanelli: Consultant; Bracco Group. Research/Grant Support; Bracco Group, Bayer AG, IMS International Medical Scientific.

\section{B-0742 10:57}

Feasibility of contrast agent volume reduction on 640 -slice CT coronary angiography in patients with low heart rate

Z.-X. Ding, Z. Wang; Hangzhou/CN (wang12345zhen@sina.com)

Purpose: To optimise the contrast injection protocol for CT coronary angiography (CTCA) to achieve more efficient utilisation of contrast agent by investigating the impact of reducing the volume of contrast agent on the attenuation of coronary arteries and sinus.

Methods and Materials: A total of 105 subjects with low heart rate ( ${ }^{2} 65$ beats $\left./ \mathrm{min}\right)$ and suspected coronary artery disease were randomly divided into three groups with 35 subjects in each group. The patients in Groups A, B and C were injected with a volume of $0.8,0.7$, and $0.6 \mathrm{ml} / \mathrm{kg}$ of contrast agent, respectively. In all cases, injection rate was $5.0 \mathrm{ml} / \mathrm{s}$, followed by $30 \mathrm{ml}$ saline flush at the same rate. CT values within the ascending and descending aorta, left main and right coronary artery orifices and coronary sinus were measured and the differences of contrast enhancement among the three groups were evaluated.

Results: The mean injection volume of contrast agent in Groups A, B and C were $54.1,47.9$ and $41.1 \mathrm{ml}$, respectively $(P<0.001)$, and the scan delay time were 23.7, 23.4 and $22.2 \mathrm{~s}$, respectively $(P<0.05)$. The $C T$ values within the coronary sinus were 112.7, 93.7 and 81.2 HU for Groups A, B and C, respectively $(P<0.001)$, 
and CT values within the ascending and descending aorta, and the coronary artery were higher than 300 Hounsfield units in all three groups.

Conclusion: Application of the $0.6 \mathrm{ml} / \mathrm{kg}$ protocol in CTCA, a steady contrast enhancement in coronary artery, can be achieved using 640-slice volume CT.

\section{B-0743 11:06}

The influence of the contrast media protocol and the scan mode on arterial enhancement in cardiac CT

E. Talakic, D. Stocker, P. Täubl, R. Maderthaner, F. Quehenberger,

H. Schoellnast, M. Tillich; Graz/AT (emina.talakic@medunigraz.at)

Purpose: To assess the influence of contrast media protocol and scan mode on arterial enhancement in cardiac CT.

Methods and Materials: 175 patients (mean age, 62; range, 18-86 years) who underwent cardiac CT were retrospectively divided into 4 groups related to scan mode (64-slice helical CT or 320-slice volume CT) and contrast media concentration (320, 350 or $400 \mathrm{mgl} / \mathrm{mL})$ - Group 1:, helical, $320(75 \mathrm{~mL})$; group 2: helical, 350 (90 mL); group 3: helical, $400(70 \mathrm{~mL})$, and group 4: volume, 400 (70 mL). Injection rate was $5 \mathrm{~mL} / \mathrm{sec}$ in all patients. ROI measurements were performed in the aorta (coronary arteries level) and cardiac chambers to assess enhancement, and in the superior vena cava (SVC) to measure the standard deviation of the attenuation values for assessment of inflow artefacts. Spearman Correlation and Wilcoxon test were used for comparison.

Results: Patients in group 3 showed significantly higher mean aortal enhancement than patients in group 1 or 2 (334 HU versus $286 \mathrm{HU}$ and $306 \mathrm{HU})$. Group 4 showed significantly higher aortal enhancement than group 3 (497 HU). Group 4 showed significantly lower enhancement in the right atrium and ventricle than group 3. No significant difference in the standard deviation of attenuation was seen within the SVC.

Conclusion: In cardiac CT, the volume mode led to significantly higher aorta enhancement and lower contrast within right atrium and ventricle compared with helical mode. Scan mode, iodine dose and flow rate had no influence on heterogeneity of contrast within the SVC.

\section{B-0744 11:15}

Multislice CT angiography with direct intra-arterial ultra-low-dosecontrast injection for the evaluation of renal graft failure: initial study M. Guzinski, J. Kurcz, J. Garcarek, M. Sasiadek; Wrocław/PL (guziol@wp.pl)

Purpose: To present initial experience with direct intraarterial ultra-low-dosecontrast CT angiography (IA-CTA) and its application in assessing renal graft artery in patients with renal graft failure.

Methods and Materials: IA-CTA of renal graft was performed in 18 patients with renal graft failure (GFR2m/s. Pig-tail catheter $4 \mathrm{~F}$ was placed in ipsilateral common iliac artery via common femoral artery. Subsequently, spiral CTA of pelvis was performed simultaneously to intraarterial administration at $4 \mathrm{ml} / \mathrm{s}$ of $6-8 \mathrm{ml}$ of contrast diluted with saline to $18-24 \mathrm{ml}$.

Results: Excellent contrast enhancement of renal graft arterial tree was observed in all patients. No complications associated with arterial access occurred. Mean creatinine/GRR levels prior to the IA-CTA were $3.30 \mathrm{mg} / \mathrm{dl}$ and $27.4 \mathrm{ml} / \mathrm{min}$, respectively. On the $5^{\text {th }}$ day following the study, mean creatinine/GFR levels amounted to $3.36 \mathrm{mg} / \mathrm{dl}$ and $27.8 \mathrm{ml} / \mathrm{min}$, respectively. Renal function deterioration was demonstrated on the $3^{\text {rd }}$ day after the procedure (creatinine $3.54 \mathrm{ml} / \mathrm{dl}$, GFR 26.8 $\mathrm{ml} / \mathrm{min})$; nevertheless, it was not statistically relevant $(p<0.71)$. Contrast-induced nephropathy occurred in only one patient $(5.6 \%)$ with transient creatinine elevation by $92 \%$. After detailed vessel analysis graft arterial stenting was performed in 10 patients, which improved renal function. The remaining 8 patients were treated conservatively.

Conclusion: Trans-catheter ultra-low-dose-contrast IA-CTA for imaging of renal graft arteries can be an effective method with low-risk of nephrotoxicity, which allows detailed graft arterial tree imaging, especially prior to interventional treatment of arterial-related renal graft insufficiency.

\section{B-0745 11:24}

Feasibility in low kV CT angiography of the abdominal aorta: assessment of image quality, radiation exposure and contrast material volume

C.R.G.L. Talei Franzesi, D. Ippolito, P.A. Bonaffini, V. Bartolo, C. Trattenero, S. Sironi; Monza/IT (ctfdoc@hotmail.com)

Purpose: To assess the image quality, dose reduction and amount of iodinate contrast injected using low-kV CT angiography protocol for the study of abdominal aorta disease.
Methods and Materials: A total of 74 consecutive patients, with clinical vascular aortic disease, were evaluated on MDCT16 slices scan (Philips-Brilliance16P, $\mathrm{NL})$ using low-kV protocol (90 kV), high pitch (1.063) and automated tube current modulation. Low contrast medium volume $(50 \mathrm{~mL})$ was administered at $4 \mathrm{ml} / \mathrm{s}$. Density measurements were performed at abdominal aorta, renal arteries and common iliac arteries. We also calculated the radiation dose exposure (dose length product, DLP). Our results were compared with 40 patients investigated with standard contrast medium volume $(100 \mathrm{~mL})$ and standard CT-angiography protocol (120 kV; $350 \mathrm{mAs}$ ). The noise level (signal-to-noise ratio, SNR), the radiation dose (expressed as DLP) and amount of iodinate contrast were analysed and statistically compared between two different groups.

Results: In all of the patients we obtained an adequate vessel opacification of aorta and renal arteries. Mean attenuation value in aorta was $305 \mathrm{HU}$, in iliac arteries was $288 \mathrm{HU}$ and in renal arteries was $276 \mathrm{HU}$, without significant attenuation difference with the control group (aorta $339 \mathrm{HU}$; iliac arteries $358 \mathrm{HU}$; renal arteries $303 \mathrm{HU}$ ). The radiation dose exposure (meanDLP: 509) was significantly lower (reduction of the $48 \%$ ) in the low-kV protocol than standard examinations at $120 \mathrm{kV}$ (meanDLP: 952). The noise level calculated in low-kV protocol (meanSNR 20) was not significantly higher than the value obtained in standard protocol (meanSNR 28). Conclusion: Low-dose CT-angiography protocol represents a feasible technique that allows a significant reduction of contrast material and radiation dose exposure without losing diagnostic accuracy being useful also in these patients with renal failure.

\section{B-0746 11:33}

CT coronary angiography with low concentration (240 mg lodine/ml) contrast material

C. Mihl ${ }^{1}$, J.F. Kalafut ${ }^{2}$, M. Yanniello ${ }^{2}$, G. Muehlenbruch ${ }^{3}$, F.F. Behrendt ${ }^{3}$, J.E. Wildberger ${ }^{1}$, M. Das ${ }^{1} ;{ }^{1}$ Maastricht/NL, ${ }^{2}$ Pittsburgh, PA/US, ${ }^{3}$ Aachen/DE (caspermihl@gmail.com)

Purpose: The use of low concentrated contrast material might be advantageous in terms of viscosity. Therefore, the purpose of this study was to evaluate the use of low concentrated contrast material $(240 \mathrm{mg}$ lodine $/ \mathrm{ml}$ ) for coronary CT angiography in routine patients.

Methods and Materials: 29 consecutive patients who were referred for routine coronary CTA were scanned on a $2^{\text {nd }}$ generation DSCT scanner (Definition Flash, Siemens). Patients received a standard contrast injection protocol as follows: lopromide $240 \mathrm{mg} / \mathrm{ml}$ (Ultravist, Bayer) with an injection rate of $9 \mathrm{ml} / \mathrm{s}$ (iodine delivery rate $2.16 \mathrm{~g} \mathrm{l} / \mathrm{s}$ ) followed by a saline chaser with the same flow rate. Contrast enhancement in the coronaries was measured proximal and distal in all coronaries. Results were compared to our standard protocol with lopromide $300 \mathrm{mg} / \mathrm{ml}$ and flow rate of $7.2 \mathrm{ml} / \mathrm{s}$ (iodine delivery rate $2.16 \mathrm{~g} \mathrm{l} / \mathrm{s}$ ). Start delay was evaluated for both groups using the test bolus method. Comparison between both groups was performed using sample t-test.

Results: Coronary enhancement reached diagnostical attenuation in both protocols. Overall comparison between enhancement with $240 \mathrm{mg} / \mathrm{ml}$ (mean $445 \mathrm{HU} \pm 114$ $\mathrm{HU}$ ) revealed no statistical significant differences with $300 \mathrm{mg} / \mathrm{ml}$ (mean $412 \mathrm{HU}$ $\pm 92 \mathrm{HU})(p=0.101)$. Attenuation levels in LAD dist and $\mathrm{Cx}$ dist were significantly higher in the low concentrated contrast material group (LAD dist 387 vs $292 \mathrm{HU}$, $\mathrm{P}<0.0001$ and $\mathrm{Cx}$ dist 392 vs $329 \mathrm{HU}, \mathrm{P}=0.003$, respectively).

Conclusion: The use of low concentrated contrast material is feasible in coronary CTA. Higher flow rates are necessary, but lower viscosity might improve attenuation in the distal segments.

\section{B-0747 11:42}

The impact of MR contrast agent concentration on bolus geometry G. Jost, H. Pietsch; Berlin/DE (gregor.jost@bayer.com)

Purpose: In contrast-enhanced MR-angiography, the luminogram is obtained during the first passage of gadolinium-based contrast agents (GBCA). The bolus concentration in conjunction with the $\mathrm{r} 1$-relaxivity determines the vascular signal enhancement. The aim was to investigate the impact of a 1 molar GBCA concentration on bolus geometry compared to a 0.5 molar agent, systematically.

Methods and Materials: 6 Göttingen minipigs were injected intravenously with gadobutrol and Gd-DTPA in a crossover setting $(0.1 \mathrm{mmol} / \mathrm{kg}, 1 \mathrm{ml} / \mathrm{s})$. Dynamic CT measurements ( $80 \mathrm{kV}, 485 \mathrm{mAseff}, \Delta \mathrm{t}=0.3 \mathrm{~s}$ ) at the thoracal region were performed without table feed. Time-density curves were determined and fitted by a gamma-variate function in the vena cava, the pulmonary artery and the ascending and descending aorta. CT-signals were transformed to Gd-concentrations on the basis of phantom measurements

Results: The administration of 1 molar gadobutrol led to a narrower (significantly lower full width half maximum) and steeper (significantly higher peak values) bolus 
shape compared to 0.5 molar Gd-DTPA in all investigated vessels. The respective $\mathrm{Gd}$-concentrations ( $\mathrm{mgGd} / \mathrm{mL}$ ) for gadobutrol vs. Gd-DTPA were $9.5 \pm 1.9$ vs. $6.8 \pm 1.2$ (vena cava), $3.4 \pm 0.4$ vs. $2.4 \pm 0.3$ (pulmonary artery) and $2.2 \pm 0.2$ vs. $1.7 \pm 0.2$ (ascending and descending aorta). The area under the bolus curve analysis yielded no significant differences between the GBCAs.

Conclusion: A 1 molar GBCA concentration leads to a more compact bolus shape with significant higher peak concentrations in the major thoracal vessels compared to a 0.5 molar agent. This might be especially relevant for the acquisition of $4 \mathrm{D}$ angiograms and for quantification of tissue perfusion.

Author Disclosures:

G. Jost: Employee; Bayer Pharma AG. H. Pietsch: Employee; Bayer Pharma AG.

\section{B-0748 11:51}

Transarterial chemoembolisation (TACE) in malignant liver metastases: evaluation of a fast C-arm CT acquisition protocol with a robotic multi-axis $\mathbf{C}$-arm system

T.J. Vogl ${ }^{1}$, M. von Roden ${ }^{2}$, E. Mbalisike ${ }^{1}$, S. Zangos ${ }^{1} ;{ }^{1}$ Frankfurt a. Main/DE,

${ }^{2}$ Forchheim/DE (t.vogl@em.uni-frankfurt.de)

Purpose: To evaluate potential contrast savings and reduction of motion artefacts in C-arm CT acquisitions with a higher rotational speed during interventional procedures.

Methods and Materials: 24 patients (mean 56.8 yrs, range 33-83) were treated with transarterial chemoembolisation (TACE). Prior to the injection of the embolisation material, a C-arm CT with a new, fast acquisition protocol was performed. A robotic multi-axis $\mathrm{C}$-arm angiographic system (Artis zeego, Siemens-Healthcare, Forchheim/Germany) was used with a rotational speed of up to $100 \%$ s. In this configuration, a C-arm-CT with a rotational trajectory of $200^{\circ}$ was acquired in $3 \mathrm{~s}$ versus $5 \mathrm{~s}$ in a commercially available $\mathrm{C}$-arm system. Two different injection protocols were applied and evaluated regarding sufficient tumour visualisation and spatial resolution of the vessel tree in the resulting 3D-dataset.

Results: Injection protocol $1(6 \mathrm{ml}$ contrast, $12 \mathrm{ml} \mathrm{NaCl}$, flow rate $3 \mathrm{ml} / \mathrm{s}$, x-ray delay $3 \mathrm{~s}$, injection site: common hepatic artery) resulted in $3 \mathrm{D}$ images with a very good spatial resolution of the vessel trees but poor visualisation of tumours/metastases. Injection protocol 2 ( $7 \mathrm{ml}$ contrast, $20 \mathrm{ml} \mathrm{NAaCl}$, x-ray delay $6 \mathrm{~s}$ ) resulted in 3D images with excellent spatial resolution and good visualisation of tumours/ metastases. From prior experience with a standard $5 \mathrm{~s}$ protocol the corresponding C-arm CT acquisition would have required a contrast load of $8 \mathrm{ml}$ and $8.5 \mathrm{ml}$, respectively. Thus, the contrast saving of the fast acquisition protocol was $25 \%$ and $18 \%$, respectively.

Conclusion: The fast $\mathrm{C}$-arm CT acquisition protocol 2 provides an interesting possibility to save contrast media during TACE with excellent spatial resolution of the vessel tree and good visualisation of hypovascular tumours.

Author Disclosures:

M. von Roden: Employee; Employee at Siemens/Forchheim. 
Monday, March 11 


\section{Neuro}

\section{SS 1711}

\section{Brain ischaemia: perfusion and diffusion}

\section{Moderators:}

L. Oleaga Zufiría; Bilbao/ES

M. Vernooij; Rotterdam/NL

\section{B-0749 10:30}

Prognostic impact of perfusion CT data in the early period after subarachnoid haemorrhage

C. Mathys, D. Martens, D.C. Reichelt, J. Aissa, K. Rybacki, K.-G. Klein,

G. Antoch, B. Turowski; Düsseldorf/DE (mathys@uni-duesseldorf.de)

Purpose: To assess the prognostic relevance of perfusion CT (PCT) examinations in the early phase after subarachnoid haemorrhage (SAH).

Methods and Materials: 312 patients were retrospectively interrogated with a questionnaire one year after spontaneous subarachnoid haemorrhage. The modified Rankin Scale (mRS) was determined, respectively. Perfusion data from the early period of the disease were available for all patients.

Results: MTT (mean transit time) values in the early period were significantly correlated $\left(p^{2} 0.001, r=0.422\right)$ with the clinical long-term outcome (mRS). Corresponding to our linear regression analysis, MTT is the second most important regressor (behind WFNS grade, which is a measure for the clinical severity of the haemorrhage) for the prediction of the long-term mRS. An MTT value of $4.11 \mathrm{~s}$ could be identified as a threshold predicting an unfavourable long-term outcome (mRS ${ }^{3} 2$ ). An MTT value ${ }^{3} 4.39$ s indicated a poor long-term outcome (mRS 4 -6). Intraarterial spasmolysis with nimodipine (70 endovascular procedures in 51 of the included patients) could reduce the average MTT value by $0.89 \mathrm{~s}$.

Conclusion: The presented data corroborate the relevance of PCT data for clinical long-term results of SAH patients and may assist risk-benefit assessment in the planning of invasive procedures (e.g. intraarterial spasmolysis).

\section{B-0750 10:39}

Advanced MRI sequences in the acute evaluation of transient ischaemic attack (TIA). A comparison between DTI, DWI and ASL including 8 weeks follow-up scans: preliminary results from the Copenhagen TIA-MRI-study

A. Christensen, C. Ovesen, J. Damm, J. Nielsen, H. Christensen;

Copenhagen/DK (anderschristensenemail@hotmail.com)

Purpose: Sensitive documentation of ischaemia is needed in clinical TIA. We aimed to compare the sensitivity of DTI, DWI and ASL in the detection of acute ischaemia and further to investigate if chronic ischaemic changes after 8 weeks in the form of gliosis always follow.

Methods and Materials: The first 40 consecutive patients included during first 3 months of an MRI-protocol (DWI, DTI and ASL within 72 hours of symptoms) and 8 weeks follow-up protocol (T2 and DWI). Patients were included by stroke consultants. Lesion volumes on DWI, DTI and follow-up T2 were compared. Hyper- or hypoperfusion on ASL was recorded. One senior neuroradiologist assessed the sequences in random order to prevent bias.

Results: Fourteen patients had acute ischaemic lesions: 2 in the posterior fossa and 12 in the cerebral hemispheres corresponding to $35 \%$ of referred patients. Thirteen of these developed gliosis in the affected area and of these 1 was noted only on DTI and 1 only as post-ischaemic hyper-perfusion on ASL. Mean lesion volume was $1.2 \mathrm{~mL}$. Mean difference between volume on DWI and gliose volume was $0.42 \mathrm{~mL}=71 \%$ and between DTI and gliose volume $0.29 \mathrm{~mL}=40 \%$ and DTI volume were significantly closer $(\mathrm{p}=0.042)$.

Conclusion: These preliminary results indicate that TIA causes a gliosis lesion consistently, and that the initial lesion as well as the extent of the permanent changes is better described using DTI. ASL seems to be able to contribute to the detection of TIA as this may be sole MRI-sign in some patients increasing detection rates with $5 \%$.

\section{B-0751 10:48}

Normal laterality index of cerebrovascular reserve of the middle cerebral artery: a 100 volunteers' BOLD fMRI study

A. Krainik, A. Attye, I. Tropres, L. Lamalle, J.-F. Le Bas; Grenoble/FR (akrainik@chu-grenoble.fr)

Purpose: Functional MRI of cerebrovascular reserve (CVR fMRI) using vasoreactivity to hypercapnia may identify patients at risk of haemodynamical stroke among those with severe stenosis of the middle cerebral artery (MCA). However, quantification remains challenging because of numerous methodological settings and their limitations. We aimed to provide a laterality index (LIMCA) to better identify abnormal CVR in clinical practice.

Methods and Materials: 100 volunteers (40 females; $49.5 \pm 21.4$ years) without cervico-encephalic arterial stenosis had a CVR fMRI using BOLD contrast with a block-design hypercapnic challenge (CO2 7\% mixed with: O2 93\% ( $n=83)$ or air $(n=17))$, using nasal canula $(n=57)$ or mask $(n=43), 1.5 T(n=46)$ or $3 T(n=54)$. Averaged end-tidal $\mathrm{CO} 2$ pressure (EtCO2) was used as a physiological regressor for statistical analyses with a general linear model (SPM8). We conducted regions of interest (ROI) measures of \%BOLD signal change $/ \mathrm{mmHg} \mathrm{EtCO} 2$ on segmented grey matter of the MCA territories. We calculated a laterality index with LIMCA=(Left_CVRMCA-Right_CVRMCA)/(Left_CVRMCA +Right_CVRMCA). $99 \%$ confidence intervals $(99 \% \mathrm{Cl})$ were calculated.

Results: No adverse reaction to hypercapnia, including panic, anxiety, headache, or fatigue, was detected during and after CVR fMRI. CVR values ranged from 0.14 to 0.48 with $\mathrm{m} \pm \mathrm{sd}=0.26 \pm 0.07 \% \mathrm{BOLD} / \mathrm{mmHg}$ EtCO2. The mean laterality index was 0.01 with an interval of fluctuation that covered 0.00 . The standard deviation was 0.026 . Thus, $99 \% \mathrm{Cl}$ was \pm 0.07 for MCA. LI values were independent of age, gas mixture, sex, inhalation modality, and MR strength.

Conclusion: Subjects without cervico-encephalic arterial stenosis have an absolute value of LIMCA below 0.08 with a $99 \%$ probability.

\section{B-0752 10:57}

In-vivo validation of velocity measurements by quantitative phase contrast MR angiography of the brain supplying arteries: a comparison with Doppler sonography

K.W. Hsieh, F. Kellner-Weldon, C. Zubler, R. Verma, M.-L. Mono, M. Reinert, J. Gralla, G. Schroth, M. El-Koussy; Berne/CH (kety.hsieh@insel.ch)

Purpose: Non-invasive quantitative phase contrast MR angiography (qMRA) has been utilised for several clinical indications, e.g. intracranial stenosis and aneurysms. The aim of this study is to validate the flow measurements of qMRA (NOVA, VasSol Inc., Chicago) by comparing the obtained velocities with qMRA and Doppler ultrasonography (DUS) in-vivo. To our knowledge, no previous reports have addressed this issue.

Methods and Materials: We retrospectively examined consecutive 24 patients ( 9 women, 15 men; mean age 63 years, range 24-80 years) with stenosis of arteries supplying the brain using qMRA and DUS. Velocities were measured in a total of 209 arterial segments (110 extra- and 99 intracranial).

Results: Overall there was a good correlation between velocity measurements with both techniques being statistically significant in all six extracranial and five out of seven intracranial segments. Velocities were $56 \%$ higher in extracranial and $45 \%$ higher in intracranial segments obtained with DUS comparing to those with qMRA. The reversal of flow direction in the ACA was detected consistently with both methods.

Conclusion: This preliminary study shows a rather good correlation between the velocities employed with GMRA and DUS. QMRA represents a robust MRI technique for flow measurements in a reasonable time, which can be integrated in the cerebrovascular MRI work-up, especially when intracranial DUS is technically not feasible. However, further in-vivo and in-vitro studies are needed to assess the need for technical optimisation of this technique.

\section{Author Disclosures:}

F. Kellner-Weldon: Grant Recipient; SPUM (Swiss National Science Foundation). M. El-Koussy: Grant Recipient; SPUM (Swiss National Science Foundation). 


\section{B-0754 11:06}

Eligibility of 3-dimensional CT perfusion blood flow-volume mismatch to predict time from symptom onset in acute ischemic stroke K.M. Thierfelder, L. von Baumgarten, F.G. Meinel,

A.B. Baumann, K. Nikolaou, M.F. Reiser, W.H. Sommer;

Munich/DE (kolja.thierfelder@med.uni-muenchen.de)

Purpose: Our aim was to evaluate if the extent of volumetrically determined blood flow-volume mismatch in recently introduced whole brain CT perfusion (WB-CTP) can predict if a stroke is younger than 4.5 hours (time window for thrombolysis). Methods and Materials: We retrospectively analysed cerebral blood flow (CBF) and volume (CBV) maps and clinical data of 103 acute stroke patients. CT perfusion images were acquired with extended brain coverage of $10 \mathrm{~cm}$ in the z-axis. Mismatch extent was defined as the fraction of $\mathrm{CBF}$ perfusion deficit without corresponding CBV abnormality and was determined volumetrically using Osirix V.4.0 imaging software. A logistic regression analysis was performed to predict time from symptom onset of $<4.5$ hours. Four mismatch extent categories, CBF perfusion deficit and CBV infarction volume were entered as predictors into the model.

Results: Mean time from symptom onset was $187 \mathrm{~min} \pm 101 \mathrm{~min}$. Mean mismatch extent was $59.5 \% \pm 31.1 \%$. After correcting for age and gender, a mismatch extent of $390 \%$ was highly predictive for a symptom onset of $<4.5 \mathrm{hrs}(\mathrm{n}=18$, OR: 8.70 , $\mathrm{p}=0.03$ ). Other categories of mismatch extend ( ${ }^{3} 80 \%$ (OR: $\left.6.67, n=31, p=0.10\right)$, $<$ $10 \%(n=13$, OR: $0.90, p=0.89),<20 \%(n=19$, OR: $1.36, p=0.66)$ as well as CBF perfusion deficit volume $(p=0.86)$ and CBV infarction volume $(p=0.77)$ failed to predict time from symptom onset.

Conclusion: A large (390\%) volumetrically determined mismatch in WB-CTP is a predictor for the time from symptom onset in the critical time window. WB-CTP mismatch might, in these cases, help to identify patients eligible for thrombolysis when symptom onset is unknown (e.g. wake up stroke).

\section{B-0755 11:15}

Whole brain CT perfusion: volumetric assessment of perfusion

deficits in patients with acute ischemic stroke

K.M. Thierfelder ${ }^{1}$, L. von Baumgarten', E. Klotz ${ }^{2}$, A.B. Baumann ${ }^{1}$

K. Nikolaou', M.F. Reiser', W.H. Sommer'; ${ }^{1}$ Munich/DE,

²Erlangen/DE (kolja.thierfelder@med.uni-muenchen.de)

Purpose: Whole brain CT perfusion (WB-CTP) eliminates the major drawback of restricted brain coverage in standard 2-slice CTP. Our aim was to assess reliability and reproducibility of a newly introduced volumetric assessment method of perfusion deficits in WB-CTP in patients with acute ischemic stroke.

Methods and Materials: 24 consecutive patients underwent 128-row WB-CTP with extended scan coverage of $10.0 \mathrm{~cm}$ in the z-axis using adaptive spiral scanning technique. Volumetric analysis of cerebral blood volume (CBV), cerebral blood flow (CBF), mean transit time (MTT), time to peak (TTP), and time to drain (TTD) was performed twice by two experienced readers using dedicated imaging software with at least 2-week intervals between readings. Inter- and intrareader agreement for individual perfusion parameter maps was assessed by intraclass correlation coefficients (ICC) and Bland-Altman analysis.

Results: Interreader agreement was highest for TTD (ICC: 0.985), followed by CBF (0.969), MTT (0.949), CBV (0.935), and TTP (0.851). Intrareader agreement was also highest for TTD (ICC: 0.995), followed by MTT (0.979), CBF $(0.977)$, CBV (9.942), and TTP (0.944). The perfusion deficits showed the highest absolute mean volume in the time-related parametric maps and did not differ significantly within this group, while mean CBF perfusion deficit volume was significantly smaller (each with $\mathrm{p}<0.01$ ).

Conclusion: Volumetric assessment in WB-CTP in acute stroke patients is reliable and reproducible. It therefore might serve for a more accurate assessment of prognosis of stroke outcome and definition of flow-volume mismatch. TTD had the highest agreement and therefore might be a suitable parameter to define tissue at risk.

\section{B-0756 11:24}

Time-to-peak (TTP) maps using whole brain CT perfusion in minor stroke: a diagnostic tool beyond penumbra measurement

S. Chakraborty, M.E. Ahmad, J.K. Wasserman, K. Keyhanian, D. Dowlatshahi, G. Stotts; Ottawa, ON/CA (santanoo@gmail.com)

Purpose: The use of thrombolysis in minor stroke or fluctuating symptoms is controversial. Treatment decisions may be influenced by identifying patients with large areas of tissue at risk and distinguishing stroke mimic. Whole brain CT perfusion in Toshiba 320 Aquilon ${ }^{\circledR}$ CT scanner allows possible assessment of these parameters and is not limited to selective brain coverage as in previous generations of CT scanners. Time-to-peak (TTP) maps have been identified as a sensitive parameter for assessment of ischaemia. We analysed the ability of TTP maps to predict eventual infarcts in patients with mild stroke presentations.

Methods and Materials: We retrospectively reviewed 164 consecutive patients presenting with acute stroke in less than 6 hours from onset. Patients presenting with a NIH Stroke Scale Score $<5$ were subjected to subgroup analysis. TTP perfusion deficit with delays of more than 3 seconds was considered significant for underlying ischaemia. The perfusion parameters were compared to follow-up imaging at 24 to 48 hours.

Results: 41 patients met inclusion criteria. 7 patients had identifiable thrombus. The TTP map had the highest sensitivity in detecting the small infarcts (92\%), followed by CBV and CBF. Importantly, TTP analysis provided very high negative predictive value (93\%). However, there was a relatively lower specificity (64\%) and a tendency to overestimate the infarct size.

Conclusion: TTP perfusion is highly sensitive in predicting small regions of ischaemia. High negative predictive value is helpful in differentiating stroke from mimics.

Author Disclosures:

S. Chakraborty: Speaker; GE, Novartis.

\section{B-0757 11:33}

Reliability of flow-volume mismatch assessment in whole brain coverage $C T$ perfusion in acute stroke patients

K.M. Thierfelder, L. von Baumgarten, F.G. Meinel,

K. Nikolaou, F.F. Strobl, M.F. Reiser, W.H. Sommer;

Munich/DE (kolja.thierfelder@med.uni-muenchen.de)

Purpose: While flow-volume mismatch is being increasingly used in the selection of stroke patients for thrombolytic therapy, there is no consensus on the method of mismatch estimation. Our aim was to determine reliability and reproducibility of different ways of mismatch assessment in patients who underwent recently introduced whole brain CT perfusion.

Methods and Materials: We included 27 consecutive stroke patients who underwent 128-row CTP with extended coverage of $10.0 \mathrm{~cm}$ in the z-axis. Two experienced readers assessed the extent of cerebral flow-volume mismatch (MM) twice and in three different ways: (1) visually estimated in steps of 10\% (Est-MM), (2) performing a volumetric mismatch analysis using Osirix software (Vol-MM), and (3) using the 10-point Alberta Stroke Programme Early CT Score (ASPECTS) scale for CTP (ASP-MM). Inter- and intrareader variabilities were determined by intraclass correlation coefficients (ICCs) and Kappa values.

Results: Est-MM showed poor interreader and moderate intrareader agreement (ICCs: 0.311/0.726). Vol-MM agreement was excellent in large (350 ml) perfusion deficits (ICCs: 0.962/0.986), while it was poor to moderate in small $(<50 \mathrm{ml})$ perfusion deficits (ICCs: 0.568/0.732). ASP-MM agreement was poor (Kappa: $0.343 / 0.540$ ), but there was no case where ASP-MM points difference between readings was ${ }^{3}$. Using the information of Vol-MM would have substantially changed the mismatch estimation and lead to a reclassification in 4/27 cases in Est-MM and in 10/27 cases in ASP-MM.

Conclusion: Volumetric mismatch assessment can improve the evaluation of whole brain CT perfusion maps, especially in patients with large perfusion deficits. It might serve for a better selection of stroke patients who may profit by thrombolytic therapy.

\section{B-0758 11:42}

Influence of temporal sampling rate of CTP acquisitions on cerebral perfusion maps using a digital phantom

M. Oei', R. van den Boom ${ }^{1}$, L.J. Oostveen', H.O.A. Laue², B. van Ginneken', R. Manniesing ${ }^{1}$, M. Prokop'; ${ }^{1}$ Nijmegen/NL, ${ }^{2}$ Bremen/DE (m.oei@rad.umcn.nl)

Purpose: To study the influence of the temporal sampling rate of CTP acquisitions on cerebral perfusion maps with noise-free synthetic data sets created with a digital phantom.

Methods and Materials: Noise-free synthetic data sets were created with a digital phantom which consists of a skull derived from a human skull phantom combined with arterial input and venous output functions, white matter (WM) and grey matter (GM) time-attenuation curves (TAC) obtained from 5 patients. By manipulating the time tags CTP protocols with a total scan duration of 60 s and a temporal sampling rate of 1-, 2-, 3- and $4 \mathrm{~s}$ were simulated. The 1-s protocol was used as reference standard. The 2-, 3- and 4-s protocols were shifted with 1s to measure the influence of missing peak points of the curve, resulting in 1, 2, 3 extra data sets, respectively. CBF, CBV and MTT maps were calculated using PMA (ASIST-Japan). Mean values of GM and WM of the protocols were compared with the reference protocol. Results: By increasing temporal resolution, CBF values decreased, MTT increased and CBV stayed relatively the same for WM and GM: CBF values were within 63 , 75 and $85 \%$ of the reference standard for $2 \mathrm{~s}, 3 \mathrm{~s}$ and $4 \mathrm{~s}$ protocols, respectively. MTT values were within 69,156 and $238 \%$ of the reference standard for 2 s, 3 s 
and 4 s protocols, respectively. CBV values stayed within $50 \%$ of the reference standard for all protocols.

Conclusion: Cerebral perfusion values are strongly influenced by temporal sampling rate.

Author Disclosures:

R. van den Boom: Research/Grant Support; Toshiba Medical Systems Corporation. R. Manniesing: Research/Grant Support; Toshiba Medical Systems Corporation. M. Prokop: Research/Grant Support; Toshiba Medical Systems Corporation.

10:30 - 12:00

Room D1

Chest

SS 1704

Technological refinements: from x-rays to microimaging

Moderators:

A.P. Parkar; Bergen/NO

C.M. Schaefer-Prokop; Amersfoort/NL

\section{B-0759 10:30}

Evaluation of pulmonary nodules by serial radiographs obtained by a flat-panel detector

Y. Nakano, K. Maeda, S. Kitahara, T. Toyama, M. Kuwabara; Kusatsu/JP (ynakano@aurora.dti.ne.jp)

Purpose: The aim of this study was to compare the detectability of pulmonary nodules by serial radiographs obtained by a flat-panel detector (FPD-SR) and chest computed radiography (CR) images.

Methods and Materials: An FPD-SR was assembled from a cassette-type detector and a portable $\mathrm{x}$-ray system. The portable system could generate pulse $\mathrm{x}$-rays of up to 5 pulses/sec; the pulse width was approximately 70 msec. Twenty FPD-SR images from full inspiration to full expiration were obtained in $4 \mathrm{sec}$. The total dose administered for FPD-SR was twice that administered for CR. Twenty-seven patients with 33 CT-confirmed lung nodules were included in this study. Receiver operating characteristic $(\mathrm{ROC})$ analysis using a five-point ordinal categorical scale by five radiologists was used to evaluate the lung nodule detectability by both modalities. Results: Analysis of variance for ROC analysis showed that FPD-SR was significantly better than $C R$ in detecting nodules $(P=0.025)$. The estimated mean $A z$ values were $0.7143 \pm 0.076$ with FPD-SR and $0.5586 \pm 0.048$ with $\mathrm{CR}$. The mean diameter of the 33 nodules was $13.1 \mathrm{~mm}$ (range, 5-31 mm). FPD-SR showed better detectability of 2 mixed ground-glass opacities and 28 solid opacities. Furthermore, two nodules detected by FPD-SR were stable in cine-mode display. Severe pleural adhesions were confirmed intraoperatively.

Conclusion: FPD-SR is superior to CR in detecting pulmonary nodules and is recommended during follow-up evaluation. FPD-SR provides a noninvasive mean values for evaluating pleural adhesions before endoscopic surgery.

Author Disclosures:

Y. Nakano: Grant Recipient; Canon Inc.

\section{B-0760 10:39}

Function ventilation analysis in dynamic chest $\mathrm{x}$-ray examination: in comparison with $133 \mathrm{Xe}$ lung ventilation scintigraphy

T. Abe, T. Izuka, N. Motohashi, Y. Shiraishi, N. Koyanagi, A. Kurosaki, M. Ito, H. Ogata, S. Kudou; Kiyose, Tokyo/JP (takehikoabe@hotmail.com)

Purpose: This study aimed to evaluate the regional ventilation functional volume using dynamic chest $\mathrm{x}$-ray examination in comparison with $133 \mathrm{Xe}$ scintigraphy. Methods and Materials: Dynamic chest $\mathrm{x}$-ray and the 133Xe scintigraphy were performed in 41 patients. The institutional review board approval and informed consent were obtained in all persons. The dynamic x-ray was obtained in the upright position at 7.5 frames per second, synchronised with the pulsed $\mathrm{x}$-ray. The each extracted and divided small area's inspiratory/expiratory lung functional signal extracted by use of a low-pass filter was compared with 133 Xe lung scintigraphy. Results: The functional volumetric signals of the dynamic chest $x$-ray in each upper, middle, lower and subphrenic fields were $12.6 \pm 2.4 \%, 19.1 \pm 3.4 \%, 20.7 \pm 5.2 \%$ $2.8 \pm 3.0 \%$ in the right, and $11.8 \pm 4.2 \%, 16.2 \pm 3.6 \%, 13.5 \pm 3.2 \%, 2.9 \pm 2.9 \%$ in the left in inspiratory phase, $11.8 \pm 3.0 \%, 18.7 \pm 3.7 \%, 20.9 \pm 5.7 \%, 3.3 \pm 3.2 \%$ of right, and $11.3 \pm 4.5 \%, 16.0 \pm 4.2 \%, 14.3 \pm 4.9 \%, 3.4 \pm 3.2 \%$ of left in expiratory phase. The functional differential volumetric signals in each fields were $12.4 \pm 1.5 \%, 19.7 \pm 2.6 \%$,
$20.9 \pm 3.7 \% 2.1 \pm 2.7 \%$ in the right, and $12.1 \pm 3.0 \%, 17.1 \pm 2.6 \%, 13.1 \pm 2.9 \%, 2.4 \pm 2.5 \%$ in the left in inspiratory phase, $12.1 \pm 1.7 \%, 19.3 \pm 2.5 \%, 21.7 \pm 4.1 \%, 2.6 \pm 2.8 \%$ of right, and $11.7 \pm 2.8 \%, 16.5 \pm 2.5 \%, 13.2 \pm 2.9 \%, 2.8 \pm 3.0 \%$ of left in expiratory phase 133 Xe lung sinchigraphy signal rate was $10.6 \pm 2.6 \%, 19.2 \pm 4.1 \%, 16.8 \pm 4.2 \%$ and $5.6 \pm 1.6 \%$ in the right, and $11.0 \pm 3.3 \%, 18.8 \pm 4.7 \%, 12.9 \pm 3.3 \%, 5.2 \pm 1.5 \%$ in the left. The each function in tidal respiration dynamic chest $x$-ray was correlated with 133Xe lung ventilation scintigraphy $(R=0.81,0.82,0.85$ and 0.82$)$.

Conclusion: Dynamic chest $\mathrm{x}$-ray examination for regional ventilation function must be equivalent to lung scintigraphy.

\section{B-0761 10:48}

Performance evaluation of automatic chest radiograph reading for detection of tuberculosis (TB): a comparative study with clinical officers and certified readers on TB suspects in sub-Saharan Africa P. Maduskar ${ }^{1}$, L. Hogeweg ${ }^{1}$, B. van Ginneken ${ }^{1}$, H. Ayles ${ }^{2} ;{ }^{1}$ Nijmegen/NL, ${ }^{2}$ London/UK (p.maduskar@rad.umcn.nl)

Purpose: Digital chest radiography (CXR) is used in high burden countries for suspect screening, active case finding and in prevalence surveys for TB diagnosis. An observer study was conducted to compare performance of automatic software with that of clinical officers and certified expert readers.

Methods and Materials: A dataset of 166 digital CXRs $(2048 \times 2048,0.25 \mathrm{~mm}$, Delft Imaging Systems, The Netherlands) was collected at Kanyama Clinic, Lusaka, Zambia. Sputum culture was used as reference. An observer study was conducted with four clinical officers who read x-rays in Kanyama Clinic, and with two readers certified to read CXRs according to CRRS standard (University of Cape Town, South Africa). A software system for detection of TB (CAD4TB-1.08, Diagnostic Image Analysis Group, The Netherlands) analysed all the cases. Human readers and software scored all the images between 0 and 100 . We report area under the receiver operating characteristics curve $(\mathrm{Az})$ with 95\% confidence intervals and pairwise comparisons from bootstrap estimates. $p<0.05$ was considered significant. Results: The dataset contained 99 positive and 67 negative cases. Az for the software was $0.72(0.63-0.80)$. Az values of CRRS certified readers were 0.71 (0.63-0.79) and 0.72 (0.64-0.78). Mean Az for clinical officers was 0.70 (0.65-0.76). There was no significant difference between any reader and the software, except for one clinical officer who performed significantly worse than automatic reading. Conclusion: Automatic computer reading has similar performance as clinical officers and certified readers. The software has potential of being used as a point-of-care decision tool, to diagnose TB or select subjects that should undergo further tests.

\section{B-0762 10:57}

Diagnostic imaging costs before and after digital tomosynthesis implementation in patient management after suspected thoracic lesions on chest radiography

E. Quaia, E. Baratella, G. Grisi, R. Cuttin, G. Poillucci, S. Kus, M. Cova; Trieste/IT (quaia@units.it)

Purpose: To evaluate diagnostic imaging costs before and after digital tomosynthesis (DTS) implementation in patients with suspected thoracic lesions on chest radiography (CXR).

Methods and Materials: Over a period of four years four-hundred-and-sixty-five patients ( 263 males, 202 females; age, $72.47 \pm 11.33$ years) with suspected thoracic lesion (s) after CXR underwent DTS. Two readers prospectively analysed in consensus DTS images. Each patient underwent CT when a pulmonary non-calcified lesion was identified by DTS, while CT was not performed when a benign pulmonary or extrapulmonary lesion or pseudolesion was identified. Diagnostic imaging costs (including material, staff, and imaging system depreciation) were evaluated during the12-month preceding DTS implementation and during the12-month after DTS implementation. The average imaging cost per patient was calculated by normalising the costs before and after implementation of DTS by the number of indeterminate CXR examinations in each of these periods.

Results: In 229/465 patients who underwent DTS after suspicious CXR, DTS showed 193 thoracic lesions and 36 pleural lesions, while in the remaining 236/465 patients lesions were ruled-out as pseudolesions of CXR. In the 12-month preceding DTS implementation, the proportion of patients who presented suspected thoracic lesions and underwent CT was 271/811; after DTS implementation, this proportion became 39/800. The average differential per-patient costs of CXR, DTS and CT examinations were $15.15,41.55$, and 113.66 euros, respectively. The perpatient change in diagnostic imaging costs before vs after DTS implementation was -24.79 euros.

Conclusion: Per-patient diagnostic imaging costs decreased after DTS implementation in patients with suspected thoracic lesions.

Author Disclosures:

E. Quaia: Consultant; GE Healthcare. 


\section{B-0763 11:06}

Lung ultrasound in the elderly population: investigating the limit between physiological and pathological patterns

A. Chiesa, F. Ciccarese, G. Gardelli, F. Feletti, U. Regina, M. Zompatori; Bologna/IT (ciccarese.f@gmail.com)

Purpose: The senile lung undergoes paraphysiological changes which have been described in several radiological and CT studies, but never investigated through ultrasound. The aim of the present study was to evaluate a group of elderly-nonsmoker-healthy patients in comparison with young subjects, by using ultrasound examination.

Methods and Materials: 100 elderly subjects ( $>70$ years-mean age: $79.4 \pm 6.9$ ) and 50 young subjects (<55years-mean age: $32.5 \pm 12.1)$ were submitted to lung ultrasound examination. We analysed the anterior, lateral and posterior surface of each lung to evaluate the presence/absence of A-lines and B-lines. Fisher test was chosen to compare data obtained in the two groups.

Results: Absence of A-lines was found in $94 / 100-94.0 \%$ of elderly versus $2 / 50-4.0 \%$ of young subjects $(p<0.0001)$. Presence of $B$-lines was detected in $37 / 100-37.0 \%$ of elderly, with a higher prevalence in posterior lung fields; the number of B-lines was ${ }^{2} 3$ per field (pf) in $27 / 37-73.0 \%$, > 3pf in 2/37-5.4\%; both 23 and > 3pf-depending on the evaluated field in $8 / 37-21.6 \%$. By contrast, $5 / 50-10.0 \%$ young subjects showed B-lines (23pf in all of cases)- $p=0.0004$.

Conclusion: To avoid misdiagnosis, we should consider that physiological ultrasound lung findings could be different among old and young people. The majority of old individuals did not present A-lines; moreover in a high percentage, B-lines were observed (23pf in most of them). These results could be explained by a reduction of impedance between lung parenchyma and soft tissue (absence of A-lines) and by an increased thickness of interlobular septa (presence of B-lines). Further studies should be proposed to define a cut-off between normal and pathological pattern.

\section{B-0764 11:15}

Digital $x$-ray optical densitometry in assessment of respiratory

function in patients with COPD exacerbation

N. Gorbunov, V. Laptev; Novosibirsk/RU (n_gorbunov@hotmail.com)

Purpose: To evaluate respiratory function in patients with exacerbation of chronic obstructive pulmonary disease (COPD) before and after treatment by means of a digital $x$-ray optical densitometry.

Methods and Materials: The study group comprised 563 patients (140 females, 423 males; mean age $58 \pm 1.3$ years, range $36-81$ years). All of them were admitted to the clinic with an exacerbation of COPD with different (1-3) stage. High-resolution digital radiographic device with a line $x$-ray sensitive silicate receiver was used. Lowdose digital $\mathrm{x}$-ray examination of the chest was performed both in inspiration and in expiration phases before and after treatment. Regional and common pulmonary optical density was evaluated with further quantitative analysis of respiratory function. For these purposes the optical density gradient (ODG) was used. All results were verified by high-resolution computed tomography (HRCT).

Results: The optical density gradient before and after treatment has been changed. There was a 1.5-1.6 times increasing value of optical density gradient due to treatment of COPD exacerbation. For patients with the first stage of COPD the $64.7 \%$ ODG increasing was estimated. For the second stage of COPD ODG increasing was $63.1 \%$ and for the third stage of COPD it was $52.1 \%$. There was a significant correlation between pulmonary optical density gradient and HRCT density gradient before and after treatment $(r>0.3 ; p<0.05)$.

Conclusion: A digital x-ray optical densitometry by means of functional highresolution digital radiography allows optimising an evaluation of therapy response in patients with COPD exacerbation combined with radiation exposure reduction.

\section{B-0765 11:24}

Diffusion-weighted echo-planar MRI with multiple b-values

in primary lung cancer: a new approach to the non-invasive differentiation of histological subtypes?

M. Regier, D. Schwarz, A. Laqmani, F.O.G. Henes, G. Adam; Hamburg/DE (mregier@uke.de)

Purpose: To investigate the feasibility of diffusion-weighted echo-planar imaging (DW-EPI) with multiple b-values and assessment of the apparent diffusion coefficient (ADC) for the differentiation of histological subtypes in primary lung cancer. Methods and Materials: Using a DW-EPI sequence at 1.5 T (TR/TE 2000/66 ms; slice thickness, $5 \mathrm{~mm}$; b-factors, 0, 50, 100, 250 and $600 \mathrm{~s} / \mathrm{mm}^{2}$ ) the lungs of 38 consecutive patients suffering from bronchial carcinoma (adenocarcinoma, $n=19$; squamous cell carcinoma, $n=14$; small cell carcinoma, $n=5$ ) were examined. By placing a Region of Interest (ROI) encovering the entire tumour manifestation within each cross-sectional image the mean (ADCmean), minimum (ADCmin) and maximum ADC (ADCmax) was determined by two independent radiologists. An additional ROI was placed into the cerebrospinal liquid and set as the reference tissue. Statistical analysis included the Wilcoxon test and $p$-values $<0.05$ indicated statistical significance.

Results: The highest ADC values were determined for the group of adenocarcinomas (ADCmean, 1.98x10-3 mm2/s; ADCmax, 2.44x10-3 mm2/s). Significantly lower mean ADCs were found for the squamous cell carcinomas (ADCmean, 1.18x10-3 $\mathrm{mm} 2 / \mathrm{s} ; \mathrm{p}<0.0001$ ) and small cell lung cancers (ADCmean, 1.29 $\times 10-3 \mathrm{~mm} 2 / \mathrm{s}$; $p=0.01$ ). Determination of the $A D C$ values did not reveal any difference between squamous cell and small cell carcinomas ( $p>0.05$ ).

Conclusion: Diffusion-weighted MRI with ADC measurement at $1.5 \mathrm{~T}$ reliably enables the differentiation of adenocarcinoma from other histological subtypes in primary lung cancer and might therefore be of distinct value in the preoperative management of patients suffering from primary lung cancer.

\section{B-0766 11:33}

Non-contrast-enhanced perfusion MRI for preoperative assessment of lung function in patients with non-small-cell lung cancer (NSCLC) G. Sommer, G. Bauman, C. Draenkow, C.-P. Heussel,

H.-U. Kauczor, H.-P. Schlemmer, M. Puderbach; Heidelberg/DE (g.sommer@dkfz-heidelberg.de)

Purpose: Knowledge about lung function defects is important for predicting functional outcome and optimising surgery in NSCLC. Fourier decomposition MRI (FDMRI) has been introduced as a method to assess regional lung perfusion without contrast agents. This study evaluates FD-MRI for preoperative assessment of lung perfusion in NSCLC patients with dynamic contrast-enhanced MRI (DCE-MRI) as standard of reference.

Methods and Materials: 15 patients with NSCLC were examined at $1.5 \mathrm{~T}$. Timeresolved images of the lungs were acquired in coronal and sagittal plane using a 2D-bSSFP sequence. Fourier decomposition was used to detect and separate periodic changes of lung proton density caused by respiratory and cardiac cycles. Perfusion-weighted images were calculated by pixel-wise integration of the cardiac spectral line. FD and DCE data were analysed visually for perfusion defects. Perfusion proportions of pulmonary lobes were calculated quantitatively by regions-of-interest analyses.

Results: FD-MRI provided diagnostic quality in 14 cases, but failed in one patient. Sensitivity, specificity and accuracy of FD-MRI for visual detection of perfusion defects were $78 \%, 92 \%$, and $90 \%$. Quantitative evaluation of perfusion proportions provided good linear correlation between FD-MRI and DCE-MRI for both upper lobes and both entire lungs $(r=0.70-0.85)$. Accuracy of FD-MRI in the lower lobes $(r=0.02-0.03)$ and the middle lobe $(r=0.32)$ was compromised by artefacts from cardiac pulsation.

Conclusion: FD-MRI is useful to detect regional lung perfusion defects in NSCLC patients before surgery. For perfusion quantification, FD-MRI is sufficiently accurate in both upper lobes and for bilateral comparison, but, in its present form, inaccurate in the middle lobe and both lower lobes.

Author Disclosures:

C. Heussel: Author; Editor of "Medinische Klinik, Intensivmedizin und Notfallmedizin", at Dr. Dietrich Steinkopff (Springer) publishing. Board Member; Chief executive chest working group of the German Roentgen society; Consultant of the European Conference on Infections in Leukemia (ECIL-3); Faculty member European Society of Thoracic Radiology. Consultant; ScheringPlough, Pfizer, Basilea, Boehringer Ingelheim, Novartis, Roche, Astellas, Gilead, MSD, Lilly. Employee; Head of Diagnostic and Interventional Radiology / Nuclear Medicine of Thoraxklinik Heidelberg; Member of the German Center for Lung Research. Founder; Founder member of the working team in infections in immunocompromized hosts of the German society of Hematology/Oncology. Patent Holder; Method and Device For Representing the Microstructure of the Lungs. IPC8 Class: AA61B5055 FI, PAN:20080208038, Inventors: W Schreiber, U Wolf, AW Scholz, CP Heussel. Research/Grant Support; Siemens 2012, Novartis 2012, Pfizer 2012. Shareholder; Stada, GSK. Speaker; Gilead, Essex, Schering-Plough, AstraZeneca, Lilly, Roche, MSD, Pfizer, Bracco, MEDA Pharma, Intermune, Chiesi, Siemens, Covidien, Pierre Fabre, Boehringer Ingelheim, Grifols. 


\section{B-0767 11:42}

Comparison of assessment of preoperative pulmonary vasculature in non-small cell lung cancer patients by non-contrast-enhanced and $4 \mathrm{D}$ contrast-enhanced MR angiography at $3 \mathrm{~T}$ and by contrastenhanced thin-section MDCT using a 64-detector row system

Y. Ohno ${ }^{1}$, S. Seki', M. Nishio', H. Koyama ${ }^{1}$, T. Yoshikawa ${ }^{1}$, S. Matsumoto', S. Satou ${ }^{2}$, K. Sugimura ${ }^{1} ;{ }^{1}$ Kobe/JP, ${ }^{2}$ Ohtawara/JP (yosirad@kobe-u.ac.jp)

Purpose: To prospectively and directly compare pulmonary vasculature assessment before surgical treatment of non-small cell lung cancer (NSCLC) patients by non-contrast-enhanced (non-CE) MR angiography, 4D CE-MR angiography and thin-section CE-CT.

Methods and Materials: A total of 77 consecutive pathologically proven and clinically assessed stage I NSCLC patients ( 41 males, 36 females; mean age: 71 years) underwent thin-section CE-CT, non-CE-MR angiography and CE-MR angiography, and surgical treatment. The capability for anomaly assessment of the three methods was independently evaluated by two reviewers using a 5-point visual scoring systems, and final assessment for each patient was made by consensus of the two readers. Interobserver agreements for pulmonary arterial and venous assessment were evaluated with the kappa statistic. Assessment of variations in overall pulmonary vasculature by the three methods was compared by means of receiver operating characteristic analyses on a per-patient basis. Finally, sensitivity, specificity and accuracy for detection of anomalies were directly compared among the three methods by means of McNemar's test.

Results: Interobserver agreements for pulmonary artery and vein assessment were substantial or almost perfect $\left(0.72^{2} \mathrm{~K}^{2} 0.86\right)$. For pulmonary arterial and venous variation assessment, non-CE-MR angiography, 4D CE-MR angiography and thinsection CE-CT showed no significant differences $(p>0.05)$ in terms of area under the curve, sensitivity, specificity and accuracy.

Conclusion: Pulmonary vascular assessment of NSCLC patients before surgical resection by non-CE-MR angiography can be considered equal to that by $4 \mathrm{D} C E-$ MR angiography and thin-section CE-CT.

\section{Author Disclosures:}

Y. Ohno: Research/Grant Support; Toshiba Medical Systems. M. Nishio: Research/Grant Support; Toshiba Medical Systems. T. Yoshikawa: Research/ Grant Support; Toshiba Medical Systems. S. Matsumoto: Research/Grant Support; Toshiba Medical Systems. S. Satou: Employee; Toshiba Medical Systems.

\section{B-0768 11:51}

In vivo micro-CT and ${ }^{18}$ F-FDG micro-PET imaging of SPC-raf and $\mathrm{SPC}$-myc transgenic mouse models of lung adenocarcinoma

T. Rodt, C. von Falck, M. Luepke, K. Hueper, R. Halter, S. Dettmer, C. Boehm,

J. Borlak, F. Wacker; Hannover/DE (rodt.thomas@mh-hannover.de)

Purpose: To report on micro-CT and micro-PET imaging of spontaneously developing lung tumour in SPC-raf and SPC-myc transgenic mice. Technical considerations regarding periprocedural management and imaging technique are addressed; tumour quantification techniques are presented. Dosage measurements are reported and potential adverse effects discussed.

Methods and Materials: 4 wild-type control, 25 SPC-raf and 7 SPC-myc transgenic mice were examined in vivo using micro-CT $(n=180)$ and ${ }^{18}$ F-FDG micro-PET $(n=14)$. Specific periprocedural management was established. Different respiratory gated and ungated micro-CT protocols were compared. A quantification routine for tumour load and growth was validated and examined in a follow-up study. ${ }^{18} \mathrm{~F}$-FDG micro-PET of orthotopic lung tumour was obtained; quantification was performed on lesion and lung region basis. Findings were correlated to histology. Thermoluminescence dosemeter measurements and dosage calculations for micro-CT and micro-PET were performed.

Results: No procedure-related deaths occurred for up to 547 days of follow-up. Respiratory gated micro-CT protocols yielded better image quality compared with ungated protocols. Intra- and inter-observer variability of the quantification routine were 5.1 and $6.5 \%$, respectively. Tumour quantification showed significant difference $(\mathrm{p}=0.043$ ) between SPC-raf transgenic and control animals in follow-up. Longitudinal tumour development correlated to cross-sectional histological data from literature. Micro-PET yielded a tumour-to-non- tumour ratio of 2.47 in SPC-myc transgenic animals. Micro-CT dosage ranged from 174 to 277 mGy. The estimated dosage calculated for micro-PET was $140 \mathrm{mGy}$.

Conclusion: Micro-CT and ${ }^{18} \mathrm{~F}-\mathrm{FDG}$ micro-PET provide quantitative data on morphology and metabolism in orthotopic lung tumour. The techniques can safely be applied in animal model phenotyping and therapeutic studies.
$10: 30-12: 00$

Room D2

Interventional Radiology

SS 1709

Abdominal interventions: from TIPS to bile ducts

Moderators:

R.F. Dondelinger; Liège/BE

B. Sekovsky; Split/HR

\section{B-0769 10:30}

Clinical relevance and interventional-radiological management of early complications after pancreatic surgery: a 12-year single-centre experience on 1285 patients

G. Agostini, M. Venturini, F. De Cobelli, S. Cappio, M. Salvioni, E. Di Rosa,

G. Balzano, P. Maffi, A. Del Maschio; Milan/IT (venturini.massimo@hsr.it)

Purpose: Despite the improvement in technique/expertise, pancreatic surgery remains burdened with a high complication rate. Our aim was to report our 12year single-centre experience about the clinical relevance and the interventionalradiological management of the early postoperative complications (treatment/ prevention) on 1285 patients submitted to pancreatic surgery.

Methods and Materials: In 2000-2012, 1285 patients were submitted to pancreatic surgery (duodenum-cephalopancreatectomy, total-pancreatectomy, distal-pancreatectomy). Patients were classified on the basis of the complication severity into 5 classes (Clavien-Dindo-classification): class- $0=$ none, class$1 / 2=$ conservative treatment, class-3a=endoscopic/interventional-radiological, class-3b=surgery, class-4=intensive care, class- $5=$ death. Interventional-radiological management was as follows: PTC/biliary-drainage in case of biliary-fistulas (bile in surgical drainage, normal bilirubin levels, undilated biliary ducts at US) under US/fluoroscopic-guidance (right approach, puncturing along the course of the sixth-segment portal branch with 21G Chiba-needle, or left approach if aerobilia/ adequate volume of left hepatic lobe); embolisation (microcoils/PVA-particles) or covered-stenting (Viabahn-Gore) in case of bleedings of gastroduodenal/splenic arteries; percutaneous drainage (US/CT-guidance) of liquid/infected collections. Pancreatic-islet-intraportal-autotransplantation was performed in case of totalpancreatectomy to prevent diabetes.

Results: Patients were classified as follows: 524/1285 (40\%) class-0, 210/1285 (16\%) class-1, 361/1285 (28\%) class-2, 72/1285 (6\%) class-3a, 55/1285 (4\%) class-3b, 24/1285 (2\%) class-4, 39/1285 (3\%) class-5. 72/1285 (class-3a) and were successfully treated as follows: $30 / 72$ PTC/biliary-drainages, $27 / 72$ liquid/infected collection percutaneous-drainages, $9 / 72$ bleedings ( 7 embolisations, 2 coveredstenting), 6/72 endoscopic-procedures. 25/1285 underwent pancreatic-isletintraportal-autotransplantation. One bleeding/ 1 biliary-fistula needed 2 treatments. Conclusion: In experienced centres of pancreatic surgery, complication rate can be reduced. Complications can be, however, successfully diagnosed, managed and prevented by interventional-radiological procedures, limiting their clinical relevance and avoiding a high-risk surgical re-treatment.

\section{B-0770 10:39}

Management of post-surgical biliary leakage with retrievable covered stent: preliminary results

A. Gioppo, A. Campari, J. Parraga, C. Michelozzi, L. Rahali, A. Sacrini,

F. Melchiorre, G. Cornalba; Milan/IT (andrea.gioppo@unimi.it)

Purpose: To evaluate technical feasibility and clinical efficacy of retrievable covered stents in treating post-surgical biliary leakage.

Methods and Materials: From April 2008 to date, 16 patients ( 9 males, 7 females; mean age 52 years, range 18-83 years) were retrospectively studied. Patients underwent placement of retrievable covered stents for treat post-surgical bile leaks. In 12 cases the procedure was performed by interventional radiologist using percutaneous trans hepatic access and in 4 cases by means of hybrid procedure (PTBD + ERCP) with both interventional radiologist and endoscopist. The leaks involved cystic duct stump $(n=6)$, common bile duct anastomosis $(n=6)$, left extra hepatic duct $(n=1)$, right extra hepatic duct $(n=2)$ and biliary confluence $(n=1)$. Results: Technical success with correct stent releasing was achieved in 15 cases; 1 case required post-dilatation ballooning. Immediate clinical and lab test improvements were observed in all patients. Post-operative imaging proved leak resolution in all cases. No early complications were observed. In 1 case late stent migration was reported 21 days after the procedure. Twelve stents were removed without complication after a mean time of 59 days, with complete leak resolution. One stent 
was removed 4 days after the procedure, during an open re-do for intra-abdominal bleeding. Three stents are still in place.

Conclusion: Placement and removal of retrievable covered stents appear to be technically feasible and could represent an excellent option in treating biliary disorders.

\section{B-0771 10:48}

Bioabsorbable biliary stent in the percutaneous treatment of benign biliary stricture: preliminary experience

G. Mauri, C. Michelozzi, V. Pedicini, D. Poretti, M. Tramarin, F. Melchiorre,

G. Cornalba, G. Brambilla; Milan/IT (vanni.mauri@gmail.com)

Purpose: To report the outcome of patients treated with a novel bioabsorbable biliary stent for benign biliary strictures.

Methods and Materials: Ten bioabsorbable polydioxanone biliary stents (Ella-DV biliary stent, ELLA-CS, Czech Republic) were deployed in 9 patients with increased level of bilirubin due to postsurgical benign biliary stricture. This stent is made of polydioxanone, a material that allows to obtain a high radial force, but that is reabsorbed by the body within 6 months. All patients had stricture recurrence after multiple standard bilioplasty ( 2 to 5 ). All patients were followed with clinical visit, ultrasound and serum bilirubin level.

Results: The procedure was successfully performed in all patients and immediate restoration of the normal caliber of the biliary duct was obtained. No immediate or late complications occurred and no patients had recurrence of the initial stricture (median follow-up 13 months, range 5-18 months).

Conclusion: Percutaneous bioabsorbable biliary stent placement is feasible and effective in the treatment of benign biliary strictures and in which standard bilioplasty has failed. In the future, this novel treatment may replace percutaneous bilioplasty a retrievable biliary stent placement as soon as long-term follow-up data will be available.

\section{B-0772 10:57}

Patient doses in liver-transplanted children with biliary strictures treated with interventional radiology procedures: a single centre survey L. Maruzzelli, G. Mamone, R. Miraglia, L. Indovina, A. Luca; Palermo/IT (gmamone@ismett.edu)

Purpose: To report the radiation doses in liver-transplanted children with biliary strictures treated with interventional radiology procedures in a single centre. Methods and Materials: 29 liver transplant recipients with biliary strictures underwent 125 procedures, the dose-area product (DAP) and fluoroscopy time (FT) were measured. Mean age 71 months (4 - 192), mean weight $17 \mathrm{~kg} \mathrm{(4-41).} \mathrm{Procedures}$ were classified into percutaneous trans-hepatic cholangiogram and biliary catheter placement (PTBD), cholangiogram and balloon dilatation (CBD), cholangiogram and biliary catheter change or removal (BCC). 92 procedures performed in an angiographic suite with image intensifier digital system, 33 procedures performed using a flat-panel detector angiographic suite.

Results: With the image intensifier digital system PTBD $(n=22), \operatorname{CBD}(n=32)$, BCC $(\mathrm{n}=38)$, mean DAP was: PTBD $3089 \pm 2196 \mathrm{cGy} \times \mathrm{cm}^{2}, \mathrm{CBD} 1406 \pm 1340 \mathrm{cGy} \times$ $\mathrm{cm}^{2}$ and BCC $1038 \pm 1420 \mathrm{cGy} \times \mathrm{cm}^{2}$; mean FT was: PTBD $807 \pm 436 \mathrm{sec}, \mathrm{CBD}$ $470 \pm 317 \mathrm{sec}, B C C 344 \pm 401 \mathrm{sec}$. With the flat panel system PTBD $(n=9), C B D$ $(n=9)$, BCC $(n=15)$, mean DAP was: PTBD $1941 \pm 2999 \mathrm{cGy} \times \mathrm{cm}^{2}$, CBD $652 \pm$ $453 \mathrm{cGy} \times \mathrm{cm}^{2}$ and BCC $111 \pm 183 \mathrm{cGy} \times \mathrm{cm}^{2}$; mean FT was PTBD $1043 \pm 760 \mathrm{sec}$, CBD $466 \pm 134 \mathrm{sec}, \mathrm{BCC} 126 \pm 85 \mathrm{sec}$. Mean DAP in the 3 categories was major in the image intensifier digital system group; however, statistical significance, likely due to the low number of procedures, was reached only for the group of patients who underwent $B C C(P=0.01)$.

Conclusion: The use of flat panel angiography equipment decreases the radiation dose.

\section{B-0773 11:06}

Endoluminal RFA with subsequent ductoplasty for biliary and pancreatic duct malignant block recanalisation

M. Mizandari' ${ }^{1}$ N. Habib 2 ; ${ }^{1}$ Tbilisi/GE, ${ }^{2}$ London/UK (mgmizandari@gmail.com)

Purpose: This study aimed to demonstrate the efficacy of percutaneous intraductal RFA in treating patients with malignant biliary and Wirsung duct obstruction. Methods and Materials: 35 patients with biliary (14 pancreatic cancer, 11 cholangiocarcinoma, 2 metastatic invasion, 5 gallbladder cancer, 2 hepatocellular carcinoma and 1 -papilla of Vater tumour) unresectable malignant block received percutaneous endoluminal RFA. All biliary patients finally underwent the stent placement. 5 of them (3- cholangiocarcinoma and 2- pancreatic tumour) were after preliminary balloon ductoplasty. Wirsung duct recanalisation was performed in 2 patients (both pancreatic cancer). 10 Watts for 2 minutes was applied using bipolar endoluminal RF device (Habib ${ }^{T M}$ EndoHPB, EMcision Ltd., London, UK), placed in a block using guidewire technique. RFA is followed by self-expanding or balloon-mounted metal-stent (MS) placement. Drainage catheter was repositioned for follow-up. Results: Duct patency restorated in $34(97.1 \%)$ cases; in $1(2.9 \%)$ case procedure filed because of guidewire conduction failure. All cases of balloonoplasty required the repeated procedure followed by stenting. 34 biliary patients maintained stent patency as long as they are alive. The mean stent patency was $115.9 \pm 64.8$ (50 - 240) days. There was no 30-day mortality, haemorrhage or pancreatitis following RFA. One complication (bile duct perforation) was observed in a post-balloonoplasty patient on repeated (RFA\&Stenting) procedure; this case was managed percutaneally. Conclusion: Percutaneous RFA treatment appears to be safe and effective in the management of inoperable biliary and Wirsung duct block. Randomised studies with prolonged follow-up are warranted.

Author Disclosures:

N. Habib: Shareholder; EMcision Itd.

\section{B-0774 11:15}

Effectiveness of therapeutic lymphography on lymphatic leakage

T. Gruber-Rouh, N.N.N. Naguib, T. Lehnert, A. Thalhammer, S. Zangos, B. Schulz, V. Jacobi, T.J. Vogl; Frankfurt/DE

Purpose: To evaluate the effectiveness of lymphography as minimally invasive treatment option for lymphatic leakage in terms of local control and to investigate the volume of lymphatic drainage per day which influences the success rate.

Methods and Materials: The study protocol was approved by the ethical committee, and informed consent was obtained from all patients prior to treatment. Between June 1988 and January 2012, 66 patients (25 women, 41 men, mean age, 52.4 years; age range 42-75 years) with lymphatic leakage underwent lymphography. Sixty-one patients $(92.4 \%)$ had successful lymphangiography while lymphangiography failed in the other 5 cases. The lymphatic leakage was in form of lymphatic fistulas $(n=31)$, lymphocele $(n=7)$, chylothorax $(n=13)$ and chylous ascites $(n=10)$. Therapeutic success was evaluated and correlated to the volume of lymphatic leakage.

Results: Signs of leakage or contrast extravasation were directly detected in 61 $(100 \%)$ patients. Based on the lymphangiography findings, 19 patients (19/61; $31.2 \%$ ) underwent surgical intervention with a complete occlusion of the lymphatic leakage. Of 61 patients, 42 patients $(42 / 61 ; 68.8 \%)$ were cured after lymphography. The lymphatic leakage could be completely occluded in $96.8 \%(30 / 31)$ of patients when the lymphatic drainage volume was less than $200 \mathrm{~mL} /$ day $(n=31)$. Even when lymphatic drainage was higher than $200 \mathrm{~mL} /$ day $(\mathrm{n}=30)$, therapeutic lymphography was still successful in $56.7 \%(17 / 30)$ of the patients.

Conclusion: Lymphography is an effective, minimally invasive method in the detection and treatment of lymphatic leakage. The volume of lymphatic drainage per day is a significant predictor of the therapeutical success rate.

\section{B-0775 11:24}

Tips for TIPS: 12-year experience

C. Zini, F. Fanelli, M. Bezzi, E. Boatta, M. Corona, P. Lucatelli, A. Cannavale,

C. Cirelli, F.M. Salvatori; Rome/IT

Purpose: Transjugular portosystemic shunt (TIPS) materials and techniques are dramatically changed during the past decade to become more effective and reliable. We report 12-year experience with e-PTFE covered stent-graft evaluating technical aspects, results, and complication rate.

Methods and Materials: From January 2000 to January 2012, 379 consecutive patients had de novo TIPS with VIATORR (W.L. Gore and Associates-FlagstaffAZ-USA). 348 patients (226 men; mean age 53 years) with a minimum follow-up of 12-months were included in this study. Patients were Child-Pugh class A (18.4\%), B $(62.6 \%)$, C (19\%) with acute/recurrent variceal bleeding (54.6\%), refractory ascites (37.9\%), hepatic hydrothorax (1.2\%) and Budd-Chiari Syndrome (6.3\%).

Results: Technical success was achieved in all cases with haemodynamic success (PSG $<12 \mathrm{mmHg}$ ) in $90.1 \%$ patients. Porto-systemic gradient mean value dropped from $21.4 \pm 5.4 \mathrm{mmHg}$ to $7.5 \pm 2.9 \mathrm{mmHg}(\mathrm{p}=0.0001)$. Reduction of varices was observed at one-month follow-up (F2: 104 vs. 29 - F3: 142 vs. 6) $(p=0.001)$. 52 re-interventions were necessary in 42 patients; primary patency rate was 87.93 $\%$ and secondary patency rate was $97.41 \%$. Mortality rate was $9.2 \%$ (early) and $35.4 \%$ (late). TIPS was a "bridge to transplant" in 66 patients. Hepatic encephalopathy occurred in $49.4 \%$ of patients; 23 cases refractory to conventional medical therapy were managed with shunt-reduction.

Conclusion: VIATORR plays an important role in TIPS scenario but its outcomes are strictly related to technical aspects such as intrahepatic tract completely covered, no concern about liver transplant, device never advanced in the portal vein for more than $3 \mathrm{~cm}, 10-\mathrm{mm}$ VIATORR has to be considered the best compromise. 


\section{B-0776 11:33}

A new trial with a newly designed Y-shaped covered stent for the palliative treatment of hilar malignant obstruction of liver: mid-term result B. Kang', G. Jung ${ }^{2} ;{ }^{1}$ Seoul/KR, ${ }^{2}$ Busan/KR (kangbc@ewha.ac.kr)

Purpose: To investigate the efficacy of a newly designed Y-shaped covered stent for the hilar malignant biliary obstruction.

Methods and Materials: From June 2011 to June 2012, we inserted the Y-shaped covered stents to treat palliatively in the patients with the hilar malignant biliary obstruction. To have a Y-configuration for the biliary drainage of both lobes of liver, we used two pieces of covered stent system: one covered main piece stent and one contra-lateral covered stent. Before and 3 days after $Y$-covered stent insertion, cholangiograms were obtained through the IHD for the evaluation of biliary drainage of both lobes of liver. We checked the blood bilirubin levels before, 1 week after and every 1 month after the stenting.

Results: They were 16 patients including 12 women and 4 men. They had inoperable intrahepatic cholangiocarcinomas in 15 and two metastatic biliary obstructions due to breast and stomach cancers. Obstructive types were 2 in I, 7 in II, 5 in IIla and 2 in IV. The placement of the $Y$-shaped covered stent was successful in all cases. The mean follow-up period was about 6 months. All cases had showed adequate biliary drainages in the follow-up tubograms. The bilirubin levels had been normalised 2 weeks after being the Y-covered stent inserted except one patient. There were no clinical infectious signs during the follow-up period. Mean patency rate was about 6.2 months ( 185 days)

Conclusion: A newly designed Y-shaped covered stent would be clinically effective for the palliative treatment of hilar obstructive malignant carcinoma of liver.

\section{B-0777 11:42}

The comparison of balloon-occluded retrograde transvenous obliteration for gastric varices using liquid and foam sclerosants

J. Koizumi' ${ }^{1}$, K. Myojin', C. Itou', N. Mori', T. Sekiguchi', T. Hara',

T. Ichikawa', Y. Imai', B. Janne d'Othée ${ }^{2} ;{ }^{1}$ Isehara/JP, ${ }^{2}$ Baltimore, MD/US

(jkoizumi@is.icc.u-tokai.ac.jp)

Purpose: Liquid ethanolamine oleate which has been used traditionally for balloonoccluded retrograde transvenous obliteration (BRTO) of the gastric varices (GV) may cause severe complications including haemolysis, allergy, etc. if overdosed. Thus, we introduced foam sclerotherapy to reduce the dose and compared the safety and efficacy of BRTO using liquid and foam sclerosants.

Methods and Materials: Forty-three patients with gastric varices were performed BRTO since October '01. Of these, three patients were excluded because simultaneous TACE or PSE was performed. Twenty patients using liquid ethanolamine oleate with iodine contrast (EOI) before March '05 and twenty patients using polidocanol foam (POF) after May '05 were included in this study. The success rates, side effects and complication rates were compared among the two groups. Results: Complete stasis of the gastric varices was obtained in all patients of both groups. Abdominal symptoms during BRTO were significantly $(p<0.05)$ higher in EOI (55\%) than POF (15\%). Postprocedure fever $(>38.0)$ was also significantly $(p<$ 0.01 ) higher in $\mathrm{EOI}(55 \%)$ than in POF $(0 \%)$. In both groups total bilirubin increased and platelet counts decreased significantly $(p<0.01)$. However, the decrease ratio of platelet counts was significantly $(p<0.05)$ higher in EOI $(-21.1 \pm 15.8 \%)$ than POF $(-9.4 \pm 13.1 \%)$. Although lactate dehydrogenase increased significantly $(p<0.01$ ) from $366.1 \pm 168.2$ to $969.4 \pm 420.7$ in EOI group, it was stable (from $229.7 \pm 36.5$ to $244.6 \pm 86.5$ ) in POF group. One patient died of ARDS one week after BRTO using EOI.

Conclusion: Foam polidocanol provided less invasive BRTO than liquid ethanolamine oleate with comparative clinical success.

\section{B-0778 11:51}

Comparison of outcomes of endovascular treatment with stent placement in patients with early and late hepatic venous outflow obstruction (HVOO)

G. Viteri, J. Etxano, P. Slon, F. Rotellar, J.I. Herrero, A. Benito, J.I. Bilbao; Pamplona/ES (gviteri@unav.es)

Purpose: To retrospectively evaluate the long term results of stent placement in patients with early and late $\mathrm{HVOO}$ after orthotopic liver transplantation.

Methods and Materials: 8 patients with early HVOO (A) and 8 patients with late HVOO (B) were recruited from 1996 to 2011. All patients were treated with self expandable stents and pre/post procedural pressure gradients were measured. Follow-up included venographic studies, cross-sectional imaging and laboratory tests. Pearson's chi-squared test was used to compare patient and graft survival, technical and clinical success, recurrence and complication rates. Log rank test was used to compare primary patency rates. (SPSS 15.0)

Results: Non significant differences were found related to technical success (A 87.5\%; B 100\% P: 1), clinical success (A 87.5\%; B 75\% P: 1), major complications (A 25\%; B 25\% P: 1), recurrence rate, (A 0\%; B 25\% P: 0.467), patient survival (A 87.5\%; B 87.5\% P: 1) and graft survival (A 100\%; B $87.5 \%$ P: 1). Regarding to the primary patency rates $(3 / 6 / 12 / 60$ months), they were slightly higher in group A (A 1.00.1.00.1.00, 1.00.; B 0.93, 0.93, 0.93, 0.70), and showed trend to signification (P: 0.097)

Conclusion: 1. Endovascular treatment with stent placement in patients with HVOO shows excellent outcomes. 2. There are no significant differences of long-term outcomes in patients with early and late HVOO were found. 3 . There might be a trend to a slightly higher primary patency rate in the group of patients with early HVOO.

10:30 - 12:00

Room E1

Musculoskeletal

\section{SS 1710}

\section{Hip: CT and MRI applications}

Moderators:

M.H. Maurer; Berlin/DE

A. Vieira; Porto/PT

\section{B-0779 10:30}

Retrospective analysis of $\mathrm{CT}$ as secondary imaging in the diagnosis of occult femoral neck fracture

K.J. Partington, P. Robinson, H. Gupta; Leeds/UK (kjpartington@doctors.org.uk)

Purpose: Improvements in CT technology, reconstruction algorithms and availability may allow modern CT to have a similar accuracy to MRI in the diagnosis of occult femoral neck fracture. We analysed the accuracy of $\mathrm{CT}$ as second line imaging in the diagnosis of occult hip fracture.

Methods and Materials: Retrospective review of $96 \mathrm{CT}$ examinations with standard multi-planar reconstructions was performed in two centres over 2 years for occult hip fracture on 64 (90 studies) and 8 ( 6 studies) slice MDCT scanners. Diagnosis, third-line and follow-up imaging was documented. Surgery or healing on follow-up imaging was considered positive for fracture. Negative radiological or clinical follow-up was considered negative for fracture.

Results: Fifteen patients (16\%) had a femoral neck fracture with 14 confirmed at surgery; and 1 managed conservatively (subsequent healing on radiographs). 1 patient $(1 \%)$ had an equivocal CT (8 detector scanner with MRI performed due to continuing clinical concern showing an undisplaced subcapital fracture. The 64-slice MDCT demonstrated 100\% sensitivity and specificity for femoral neck fracture. Mean follow-up for those with a negative CT of 223 days was achieved with 3 patients undergoing further imaging, all of which were negative. 45 patients (47\%) had other abnormalities demonstrated including pubic rami, acetabular or greater trochanter fractures.

Conclusion: CT is an accurate technique for ruling out an occult neck of femur fracture. Given its availability, rapid acquisition, familiarity to clinicians and lower costs it should be used first in the investigation of occult femoral neck fracture, with MRI reserved for cases of ongoing uncertainty.

\section{B-0780 10:39}

MRI or CT for occult hip fracture: Do either miss clinically relevant fractures?

K. Sneddon, H. Rehman, Z. Davis, F. Perks, T.O. White, R.G.E. Clement; Edinburgh/UK (crabbykerry@hotmail.com)

Purpose: There are 75,000 patients that sustain a fractured neck of femur (\#NOF) in the UK, annually, with up to $10 \%$ missed on initial plain radiographs. Additional imaging is recommended where there remains high index of suspicion. In some circumstances, MRI may be unavailable or contraindicated, thus CT is used. It is suggested (not prove) MRI is superior. We aim to review the use of CT and MRI scans for suspected \#NOF.

Methods and Materials: Retrospective review of all patients presenting to The Royal Infirmary of Edinburgh with a suspected occult hip fracture, negative initial plain radiographs, undergoing CT or MRI, over a 5-year period.

Results: A total 201 patients were included in this study. 118 underwent MRI and 83 had CT. The average time to further imaging was 8.31 and 6.94 days for MRI and CT, respectively. Occult \#NOF was identified in $37 / 118$ (31.4\%) of MRI scans and $38 / 83(45.8 \%)$ on CT. $78.4 \%$ of patients with positive MRI and $73.7 \%$ with 
positive CT underwent surgery. Occult acetabular fractures were detected in $15.9 \%$ of patients with negative scans for \#NOF, and pubic rami fractures in $17.5 \%$. No patient with a negative scan was readmitted for surgery within 12 months.

Conclusion: CT and MRI can both be equally effective tools in assessing occult \#NOF. Clinically significant occult fractures are not missed by either modality. In order to achieve targets for surgery within 48 hours of admission, the imaging modality that can be completed within the shortest time-frame should be selected.

\section{B-0781 10:48}

Digital tomosynthesis in diagnosis of occult hip fractures

M. Geijer ${ }^{1}$, D. Collin² , J.H. Göthlin²; ${ }^{1}$ Lund/SE, ${ }^{2}$ Mölndal/SE (ecr2013@geijer.info)

Purpose: Digital linear tomosynthesis has recently been introduced not only for mammography but also for chest and musculoskeletal indications. The clinical utility in musculoskeletal applications has been validated in only a few reports. The purpose of the current study was to evaluate the clinical utility of tomosynthesis in suspect occult hip fracture.

Methods and Materials: In a retrospective review 70 tomosynthesis hip examinations on 49 women and 21 men performed for further evaluation of suspect hip fracture were retrieved from the radiology archive. Three observers scored the tomosynthesis scans separately from the radiography examinations, into the categories no proximal femur fracture, suspect proximal femur fracture (femoral neck, trochanteric, or greater trochanteric avulsion) or definite proximal femur fracture. $\mathrm{CT}, \mathrm{MRI}$ or clinical outcome was used as reference.

Results: Observer variation was moderate to substantial for three observer pairs regarding radiography $(\mathrm{k}=0.56-0.8)$ and improved to substantial to almost perfect for tomosynthesis $(k=0.76-0.93)$. Eleven patients were operated on for femoral neck fracture. Tomosynthesis detected ten of these, and the eleventh patient was operated on without diagnostic confirmation from any imaging modality. One patient had been operated with dynamic hip screw for intertrochanteric fracture. The remaining trochanteric fractures were incomplete and were treated conservatively. There were no false-positive tomosynthesis diagnoses reported. There were no significant falsenegative tomosynthesis diagnoses. The distinction between incomplete/complete trochanteric fractures and greater trochanteric avulsions is sometimes difficult. Conclusion: Tomosynthesis has the possibility to aid in diagnosis and strengthen the diagnosis in suspect occult hip fracture.

\section{B-0782 10:57}

Advanced core decompression for patients with avascular necrosis of the hip: therapeutic success monitored by MRI

A. Lazik, O. Kraff, T. Claßen, S. Landgraeber, F. Grabellus, T.C. Lauenstein, J.M. Theysohn; Essen/DE (andrea.lazik@uk-essen.de)

Purpose: Advanced core decompression (ACD) is in clinical evaluation for patients with avascular necrosis (AVN) of the hip. In opposition to standard core decompression, ACD uses an expandable reamer allowing optimal debridement of necrosis after drilling a core to the femoral head. The bone defect is filled with a bone graft substitute. We aimed to constitute the therapeutic success of ACD based on MRI. Methods and Materials: Twenty patients (17 males, 3 females, mean age 49.9 years) underwent $3 \mathrm{~T} \mathrm{MRI}$ of the hip prior to, 30 days and $0.5-2$ years after ACD. The protocol included TIRM, PD/T2w TSE, high-resolution T1w, DESS and T1w VIBE sequences. Sequences were evaluated regarding delineation of necrosis, bone, graft and transformation zone in between. The volume of necrosis was measured before and after ACD.

Results: AVN prior to ACD as well as the defect filled with the graft after ACD and the residual necrosis could be imaged with reproducible high quality at $3 \mathrm{~T}$. $\mathrm{T} 1 \mathrm{w}$ and PDw sequences provided best contrast to measure the volume of necrosis. Each patient showed a reduction of necrosis after ACD (17.7 \% to $90.8 \%$, mean 43.0 , SD 24.2). The granulation zone around the graft could especially be visualised by T2w TIRM and PDw sequences. The arrangement of different layers correlated with histological findings.

Conclusion: Structural changes and reduction of necrosis after ACD can be monitored by MRI. ACD does significantly reduce the volume of necrosis in AVN of the hip.

\section{B-0783 11:06}

Lower-limb MRI examination as a predictive factor of articular collapse in haematological young patients with femoral head osteonecrosis: lesion surface versus lesion volume A. Masetto, D. Ippolito, P.A. Bonaffini, D. Fior, A.S. Casiraghi, S. Sironi; Monza/lT (dr.masetto@gmail.com)

Purpose: To compare the reliability of the femoral head volume (FHV) versus femoral head surface (FHS) to predict articular collapse in paediatric patients with osteonecrosis and lymphoproliferative disease.

Methods and Materials: 109 patients with lymphoproliferative disease and clinical suspicion of femoral head osteonecrosis following therapy underwent a lower-limb MRI examination between November 2006 and August 2012. The study was performed on a 1.5 T scanner (Philips, Achieva) with step-table technique, acquiring short tau inversion recovery (STIR) and T1-weighted sequences in the coronal plane, from the hips to the ankles. 13 patients ( 7 males, mean age $15.2 \pm 4$. 8 years) developed femoral head osteonecrosis, without articular deformity (23 heads affected). During the follow-up (9.2 \pm 2 months), 13 femoral heads developed articular deformity. On baseline MRI two radiologists measured FHV, FHS, the osteonecrosis volume (ONV) and the articular surface involved by osteonecrosis (ONS). The relative involvement of the femoral heads in terms of volume (RV: ONV/FHV) and articular surface (RS: ONS/FHS) was then calculated.

Results: Using ROC curve analysis, and considering a threshold of $24 \%$ of volume involvement, RV predicted articular deformity in 13/13 femoral heads, with sensitivity $=100 \%$, specificity $=90 \%$, accuracy $=95 \%$, PPV $=93 \%$ and NPV $=100 \%$. Considering a threshold of $50 \%$ of articular involvement, RS predicted articular deformity in 10/13 femoral heads, with sensitivity sensitivity $=77 \%$, specificity $=100 \%$, accuracy $=87 \%, \mathrm{PPV}=100 \%$ and NPV $=77 \%$.

Conclusion: RV proved to be a more reliable parameter than RS, with a high prognostic value, in predicting femoral head deformity, and can be considered a valid complementary---diagnostic tool in the follow-up of haematologic paediatric patients with osteonecrosis.

\section{B-0784 11:15}

Alpha angles and herniation pits: revisited on MRI in $\mathbf{8 0}$ asymptomatic hip joints

E. Lee, J.-A. Choi, G. Lee, H. Kang; Seongnam-si/KR

(silvercherry20@gmail.com)

Purpose: To evaluate alpha angles and herniation pits on MRI in asymptomatic hip joints and analyse their associations with demographic variables and with each other.

Methods and Materials: 80 hip joints of 44 adults who underwent hip MRI due to trauma or unilateral pelvic symptoms from September 2011 through June 2012 were retrospectively studied, among the initial 114 hips of 57 patients.

Results: The range of alpha angles was 27.73-65.03 degrees with average 41.97 degrees. 11 out of $80(13.75 \%)$ hip joints showed alpha angle of greater than 50 degrees. There was no significant correlation between alpha angle and age, gender, and laterality of hip joint. There was no significant difference of prevalence of alpha angle greater than $50^{\circ}$ in joints with herniation pit and joints without herniation pit. The intraobserver agreement of alpha angle measurements was excellent $(0.808$ in right, 0.823 in left). Of 80 hip joints, 19 (23.75\%) joints had herniation pits, with no difference according to age, gender, or laterality of hip joint.

Conclusion: The prevalence of herniation pit in asymptomatic patients was $23.75 \%$, similar to those reported in FAI. Alpha angle may be reliably evaluated on oblique axial MR images and shows a wide range in asymptomatic patients. Therefore, the presence of herniation pit and increased alpha angle may not be necessarily associated with FAI.

\section{B-0785 11:24}

Gluteus tendinopathy in femoroacetabular impingement (FAI): findings with MR-artrography of the hip

E. Lanza ${ }^{1}$, G. Pozzi ${ }^{1}$, C. Garcia Parra ${ }^{2}$, R. Bisogno ${ }^{1}$, A. Zerbi ${ }^{1} ;{ }^{1}$ Milan/IT, ${ }^{2}$ Bergamo/IT

Purpose: Femoroacetabular impingement (FAI) is suspected in patients with groin, hip or gluteal pain. Magnetic Resonance Arthrography (MRA) of the hip is used for diagnosis of FAl and study of extra-articular tissues. We incidentally observed a relevant number of gluteus medius and minimus tendinopathy (GT). Our purpose was to study associations between FAI and GT.

Methods and Materials: We retrospectively reviewed 59 symptomatic patients (26 women,41\%; 36 men, 59\%; mean age $42 \pm 11.6$ ) who underwent MRA of the 
hip for clinical suspect of FAI in a seven-month period. Intra-articular gadolinium injection (10 cc) was obtained under fluoroscopic guidance.

Results: MRA confirmed FAl in 45 patients (76\%): 7 (15.6\%) showed a PINCER impingement, $26(57.8 \%)$ patients had a CAM type and $12(26.7 \%)$ showed a mixed type. No impingement was found in $14(23.8 \%)$ patients. In 30 patients $(51 \%)$ we found degenerative alterations of medius and minimus gluteus tendons; $22(37.3 \%)$ had a concurrent diagnosis of FAl; $8(13.6 \%)$ had no alterations. Overall, 22 patients $(37.3 \%)$ showed both FAI and GT; 23 patients (39\%) had FAI alone; 8 patients $(13.6 \%)$ had isolated GT; 6 patients $(10 \%)$ had a negative examination. GT showed to be more common in a $40-60$ age range $(p<0.0001)$ and in females $(53.3 \%)(p$ $<0.044$ ). Younger patients (<40years) did not show alterations in $82.8 \%$ of cases. Conclusion: Gluteus medius and minimum tendinopathy occurs among patients with clinically suspected FAI and may contribute to pain. In some patients it may be the only responsible for symptoms. Careful study of the great trochanteric entheses should not be undervalued when looking for FAl.

\section{B-0786 11:33}

Evaluation of a new method for the assessment of anterior acetabular coverage and hip joint space narrowing

R. Ferré, E. Gibon, A. Feydy, H. Guerini, R. Campana, N. Zee, C. Bourdet,

M. Hammadouche, J.-I. Drapé; Paris/FR (kn638@yahoo.fr)

Purpose: The Lequesne's false profile (LFP) view is commonly used to evaluate hip joint space narrowing (JSN) and anterior acetabular coverage using the verticalcenter-anterior margin (VCA) angle. A novel low-dose biplanar slot scanner (SS) allows simultaneous acquisitions of weight-bearing oblique views of both hip joints. The aim was to compare LFP views versus biplanar oblique views obtained by SS. Methods and Materials: LFP views were obtained on 56 hips on a computed radiography system. On the same hips, simultaneous oblique views of both hips were acquired in the SS, with the patient's pelvis positioned at $45^{\circ}$ from each acquisition plane. Two independent observers measured VCA angle and JSN on each acquisition. JSN was evaluated through the joint space/femoral head diameter ratio. Measurements from both techniques were compared using the student t-test and the Pearson's correlation coefficient. Interobserver agreement of VCA angle and JSN assessments were calculated with the intraclass correlation coefficient (ICC). Results: VCA angle was $33.5^{\circ}\left(\mathrm{SD}=6.2^{\circ}\right)$ with SS and $35^{\circ}\left(5.6^{\circ}\right)$ with LFP views $(p<0.05)$. Pearson correlation coefficient between two techniques was $0.78(p<$ $0.01)$. JSN was $0.114\left(S D=0.03^{\circ}\right)$ with $S S$ and $0.108\left(S D=0.03^{\circ}\right)$ with $L F P$ views $(p<0.05)$. Pearson correlation coefficient was $0.85(p<0.01)$. ICCs for VCA angle were 0.91 with SS and 0.69 with LFP views. ICCs for JSN evaluation were 0.76 for both SS and LFP views.

Conclusion: SS is a reliable, easy, and low-dose evaluation of JSN and VCA angle despite a slight angular positioning compared with LFP.

\section{B-0787 11:42}

Diagnostic value of MR enterography (MRE), after oral administration

of glucose-polyethylene, in the determination of the activity of

disease in patients with known Crohn's disease: a prospective single centre study

V. Bartolo, D. Ippolito, C. Trattenero, P.A. Bonaffini, V. Besostri, S. Sironi; Monza/lT (bartolo.valentina@yahoo.it)

Purpose: To evaluate the role of magnetic resonance enterography (MRE) after oral administration of isotonic glucose-polyethylene glycol (PEG) in monitoring disease activity in Crohn's disease (CD).

Methods and Materials: Forty-eight patients ( $M 32$, mean age 43) with histological diagnosis of $C D$ and suspicion of recurrence based on clinical data and $C D$ activity index (CDAI) underwent an MRE study 45 minutes after the oral administration of 1.5-2 litres of PEG, on 1.5 T magnet and using a surface phased-array body coil. The study protocol included axial and coronal T1-weighted, sSShT2 and SBTFE sequences and dynamic contrast-enhanced THRIVE. The intestinal and extraintestinal manifestations of disease were evaluated by two radiologists blinded to clinical and pathological findings, analysing: the degree of bowel distention, wall thickening and enhancement, lymph-node enlargement, stranding of fat tissue, presence of fistulas or abscesses.

Results: Oral administration of PEG solution was well tolerated by patients, without major complications (moderate diarrhoea in 4 patients). A good relaxation of the small intestine was obtained in 44 patients $(91 \%)$. The MRE study showed recurrence in 37 patients $(77 \%$ ) as mural enhancement in 36 cases $(74 \%)$, layered enhancement in $24(50 \%)$, comb sign in $14(29 \%)$, stranding of fat tissue in 10 $(20 \%)$, mesenteric node involvement with intense enhancement in $18(37 \%)$ and extra-intestinal manifestations (fistulas and sclerosing cholangitis) in $14(29 \%)$.
Conclusion: The MRE study after oral administration of PEG solution provides an accurate assessment of small-bowel involvement, allowing an evaluation of disease activity in patients with known $\mathrm{CD}$, reducing the radiation exposure in young patients.

\section{B-0788 11:51}

Metal artefact reduction in hip prosthesis with iterative reconstructions

F. Morsbach ${ }^{1}$, S. Bickelhaupt ${ }^{1}$, G. Wanner' ${ }^{1}$ A. Krauss ${ }^{2}$; B. Schmidt², H. Alkadhi', ${ }^{1}$ Zurich $/ \mathrm{CH},{ }^{2}$ Forchheim/DE

Purpose: To evaluate the effect of a novel iterative frequency split-normalised (IFS) metal artefact reduction (MAR) on image quality of computed tomography (CT) in hip-prosthesis.

Methods and Materials: In the first study-part, an artificial hip-phantom with steel and titan-based shafts, using three inlays of water, fat and contrast-media placed in the pelvis, was used to test and optimise the IFS algorithm. Image quality and accuracy of CT number measurements of inlays were determined. In the second study-part, 41 consecutive patients with unilateral $(n=32)$ or bilateral $(n=9)$ hipprosthesis were included. Datasets using filtered back projection (FBP), IFS and a previously published MAR algorithm using linear-interpolation were reconstructed. Two readers evaluated axial, coronal and sagittal $\mathrm{CT}$ image reformations for overall image quality and evaluated image quality of pelvic organ depiction; CT numbers in the urinary bladder, gluteal muscle and subcutaneous fat were measured.

Results: Ex-vivo experiments revealed the best image quality for IFS. Measurements of CT numbers of the inlays were more accurate for IFS. In patients, we found a significant improvement in overall image quality for all reformations $(P<$ $0.001)$ and a significantly increased image quality of pelvic organs $(P<0.001)$ for the IFS algorithm. CT numbers of the bladder $(P<0.001)$ and muscle $(P=0.043)$ were significantly less variable for IFS as compared with images reconstructed with FBP and the linear interpolation MAR algorithm, whereas we found no significant difference for fat $(P=0.159)$.

Conclusion: The IFS algorithm significantly reduces metal artefacts from hipprostheses, increases image quality and improves measurement accuracy of CT numbers as compared with FBP and linear interpolation MAR algorithms.

Author Disclosures:

B. Schmidt: Employee; Siemens Healthcare, Germany.

10:30 - 12:00

Room E2

GI Tract

\section{SS 1701a}

CT/MR colonography: technical issues and clinical

\section{studies}

Moderators:

D. Cano; Pamplona/ES

D.Z. Saranovic; Belgrade/RS

\section{B-0789 10:30}

CT colonography: accurate registration of prone and supine endoluminal surfaces of the colon

T.E. Hampshire, H.R. Roth, E. Helbren, A. Plumb, D. Boone, G. Slabaugh,

S. Halligan, D.J. Hawkes; London/UK (thomas.hampshire.09@ucl.ac.uk)

Purpose: Computed tomographic (CT) colonography is a technique for detecting bowel cancer or potentially precancerous polyps. Because retained fluid and stool can mimic pathology, CT data are acquired with the patient in both prone and supine positions. Radiologists then match endoluminal locations between the two acquisitions to determine whether pathology is real. This process is hindered by the fact that the colon can undergo large deformations that often occur during repositioning of the patient. Automated registration between datasets could potentially improve efficiency and diagnostic accuracy.

Methods and Materials: We have developed software to establish correspondence between prone and supine endoluminal surfaces. An initialisation step generates image patches at the positions of haustral folds using depth map renderings and is optimised by virtual camera registration. Additional neighbourhood information is then included in a Markov Random Field model to establish landmark-based correspondences. Subsequently, the complexity of the registration task is reduced by mapping both prone and supine surfaces onto a cylindrical domain in which correspondence is established using non-rigid image registration.

Results: The registration was applied to 17 CTC cases including cases exhibiting luminal collapse, achieving fold matching accuracy of $96 \%$. Providing an accurate 
initialisation, the method significantly improved the cylindrical registration $(p<$ 0.001 ), achieving a mean error of $6.0 \mathrm{~mm}$ measured at 1743 reference points.

Conclusion: The proposed method can successfully establish correspondence between prone-supine locations on the endoluminal surface derived from CT colonography. The ability to rapidly and automatically match polyps between acquisitions will facilitate CT colonography interpretation.

\section{Author Disclosures:}

T.E. Hampshire: Research/Grant Support; MedicSight sponsered early work of CMIC project. G. Slabaugh: Employee; Ex-employee of MedicSight. S. Halligan: Grant Recipient; Holds NIHR Programme Grant in clinical radiology (CTC).

\section{B-0790 10:39}

\section{A multi-centre randomised double-blind placebo-controlled trial} to evaluate the value of a single bolus intravenous alfentanil in CT colonography

T.N. Boellaard, M.P. van der Paardt, M.W. Hollmann, S. Eberl, J. Peringa, G. Kavaliauskiene, J.H. Runge, J.A.W. Tielbeek, J. Stoker; Amsterdam/NL (t.n.boellaard@amc.uva.nl)

Purpose: To evaluate whether a single intravenous alfentanil bolus has a clinically relevant analgesic effect compared with placebo in patients undergoing elective CT colonography.

Methods and Materials: A prospective multi-centre randomised double-blind placebo-controlled trial was performed in 90 consenting symptomatic or surveillance patients scheduled for elective CT colonography. Patients were randomised to receive either a bolus of $7.5 \mu \mathrm{g} / \mathrm{kg}$ alfentanil $(n=45)$ or placebo $(n=45)$. The primary outcome was the difference in maximum pain during colonic insufflation on an 11-point numeric rating scale (one-sided t test). We defined a clinically relevant effect as a maximum pain reduction of at least 1.3 points. Secondary outcomes included total pain and burden of CT colonography (5-point scale), most burdensome aspect, side effects and recovery time measured by the Aldrete score.

Results: Maximum pain scores during insufflation were lower with alfentanil as compared with placebo, 5.3 versus $3.0(p<0.001)$. Total CT colonography pain and burden were also significantly lower with alfentanil (2.0 vs. 1.6 and 2.1 vs. 1.7 , respectively). With alfentanil fewer patients rated the insufflation as most burdensome aspect $(56.1 \%$ vs. $18.6 \%$; $p=0.001)$. No clinically relevant desaturations occurred. Episodes with desaturations $<90 \% \mathrm{SpO} 2$ and dizziness were more common with alfentanil ( $4.4 \%$ vs. $37.8 \%$; $<<0.001$ and $8.1 \%$ vs. $44.4 \% ; p<$ $0.001)$. Aldrete scores in the alfentanil group were significantly lower immediately after the procedure, but similar at 30 and 60 minutes.

Conclusion: A low-dose intravenous alfentanil bolus provides a clinically relevant reduction of maximum pain during $\mathrm{CT}$ colonography. Alfentanil may improve the acceptance of CT colonography.

\section{B-0791 10:48}

The possible role of contrast material-enhanced computed tomographic colonography (CTC) in the surveillance after surgery for colorectal cancer

M. Morone, G. Mazza, T. Natilla, G. Baiocchi, M. Ghedi, E. Dettori, B. Frittoli, L. Grazioli; Brescia/IT (mariomorone@libero.it)

Purpose: To evaluate the accuracy of contrast material-enhanced computed tomographic colonography (CTC) for follow-up after curative surgery for colorectal cancer without evidence of disease recurrence.

Methods and Materials: 86 patients underwent CTC and colonoscopy (within 4 months) after colorectal cancer surgery. Patients with the suspicion of disease recurrence underwent pathologic confirmation. Patients performed bowel cleansing and faecal tagging; bowel distention was achieved using $\mathrm{CO} 2$. Both prone and supine scans, one after intravenous contrast material administration during the portal phase, were obtained using a 64-detector CT ("Brilliance" Philips with iDose). Images were reconstructed with a section thickness of $0.75 \mathrm{~mm}$, transferred to a workstation and interpreted by one radiologist with experience reading several hundred CTC cases. Sensitivity, specificity, positive predictive value (PPV), and negative predictive value (NPV) of CTC for anastomotic and non-anastomotic colonic lesions (metachronous or flat lesions and polips) were determined and compared to endoscopy.

Results: CTC detected all 2 anastomotic recurrences, all 3 metachronous lesions (sensitivity and specificity $=100 \%$ ) and a perianastomotic recurrence. In 13 patients CTC depicted $16 / 17$ polips $>5 \mathrm{~mm}$ (sensitivity $=94.1 \%$, specificity $=100 \%$, $\mathrm{VPP}=100 \%, \mathrm{VPN}=98.6 \%$, accuracy $=98.8 \%$ ) and in 1 patient detected 1 of 3 flat lesions (sensitivity $=33.3 \%$, specificity $=100 \%$ ). CTC enabled detection of extracolonic recurrences in 3 patients ( 2 hepatic and 1 lung metastases) but missed a $7 \mathrm{~mm}$ sessile polyp and 2 flat lesions. CTC quality was inadequate in the $11 \%$ of patients. Conclusion: CTC can be effective and represents a valid alternative to colonoscopy for surveillance for colorectal cancer recurrence after surgery.

\section{B-0792 10:57}

CT colonography: comparative study of experienced vs nonexperienced radiologists using 3D flythrough approach with and without CAD software

F. Vecchietti, M. Rengo, D. Caruso, C.N. De Cecco, D. Bellini, A. Laghi; Latina/IT (fabrizio.vecchietti@gmail.com)

Purpose: To compare performances of different experienced readers using a primary 3D flythrough approach with and without the use of a CAD analysis. Methods and Materials: Three readers evaluated 50 patients with 100 endoscopically proved polyps (ranging from $3 \mathrm{~mm}$ to $40 \mathrm{~mm}$ ) and different colonic preparations (18 fluid tagging, 32 full cathartic preparation). Dataset analyses were performed on a GE ADW 4.6 workstation equipped with VCAR colon software. Per-polyp sensitivity, inter-reader agreement, mean reporting time and false positive were evaluated for each approach.

Results: Less experienced readers increased per-polyp sensitivity, respectively, from $75 \%$ and $64 \%$ for $3 D$ analysis to $86 \%$ and $74 \%$ for primary $3 D+C A D$ analysis with a significant difference $(p=0.001$ and $p=0.04)$ while no significant differences were found for the expert reader $(p=0.06)$. Less experienced readers were faster when assisted by CAD but no significant differences were found on mean reading time for all readers $(p=0.5 / 0.07 / 0.1)$. Mean false-positive findings for CAD standalone analysis were $12(+-13)$. All readers decrease false positive when assisted by CAD especially less experienced ones. Inter-reader agreement was higher among all readers when assisted by $\operatorname{CAD}(0.33$ to $0.63 / 0.39$ to $0.62 / 0.58$ to 0.65 ) Conclusion: Our study demonstrated that if less experienced readers were assisted by CAD they increase their sensitivity significantly. They have other non-significant advantages like the reduction of false positive rate and mean reporting time.

\section{B-0793 11:06}

CT colonography bowel preparation: influence of a laxative agent on a fluid tagging preparation

M. Rengo, D. Bellini, M.M. Maceroni, F. Vecchietti, D. Caruso, P. Lucchesi, A. Laghi; Latina/IT (marco.rengo@gmail.com)

Purpose: To compare two different bowel preparation in terms of patient acceptance and image quality.

Methods and Materials: Seventy-five consecutive patients were prospectively randomised into two groups. Both groups followed a low residue diet for two days before the examination. Both preparations were based on fluid tagging, using a iodinate contrast medium (gastromiro). Group 1 received $180 \mathrm{ml}$ of fluid tagging agent the day before the study. Group 2 received $60 \mathrm{ml}$ of fluid tagging agent the day of the exam and $500 \mathrm{ml}$ of a laxative agent (Lovol-esse) the day before the study. Patient acceptance was evaluated using a visual assessment scale. Quality of bowel preparation was evaluated using quantitative (tagged fluid density and number of untagged residue per segment) and qualitative (homogeneity per segment on a 4 point scale) scores.

Results: Bowel preparation was complete in all patients. No statistical significant differences for tagged fluid density, number of residue and tagging homogeneity between the two groups were founded. A significant higher patient acceptance was founded in group $2(\mathrm{P}=0.02)$.

Conclusion: The preparation based on the combination of a laxative and a fluid tagging agents was better accepted. Adding a laxative agent to a fluid tagging preparation did not improved image quality but was associated with an higher patient acceptance. Moreover, the if a laxative agent is added to a fluid tagging bowel preparation the amount of iodine can be reduced.

\section{B-0794 11:15}

Flat lesions: CTC with and without CAD

G. Mazza, T. Natilla, M. Morone, B. Frittoli, G. Baiocchi, L. Grazioli; Brescia/IT (dottormazza@gmail.com)

Purpose: To retrospectively evaluate the diagnostic accuracy of computed tomography colonography (CTC) to identify the flat lesions (reference standard: optical colonscopy OC) by an expert reader and a novice reader. To evaluate the contribution provided by a CAD system as a second reader.

Methods and Materials: From 2010 to 2011, 90 patients underwent over a period not exceeding four months both examinations of CTC and OC. Considering the latter as a reference method, CTC examinations were retrospectively and blindly 
reviewed by two readers separately respectively having a degree of initial and advanced experience (for both primary 3D view). Calculated values were sensitivity, specificity, NVP, PVP for patient-to-segment and both without and with the use of the CAD system (im3D-Turin) as the second reader.

Results: The OC showed 54 flat lesions in 23 patients. The values [[Unable to Display Character: \&\#8203;]][Unable to Display Character: \&\#8203;]]of sensitivity, specificity, NVP, PVP reported by the novice radiologist were respectively: $55 \%$, $76 \%, 55 \%$ and $80 \%$, while the values obtained by the radiologist with advanced experience were equal to $67 \%, 89 \%, 75 \%, 91 \%$. With the use of CAD as a second reader the novice player has achieved an improvement of the sensitivity to $62 \%$, while the skilled reader did not obtain a significant increase of' diagnostic accuracy. Conclusion: The sensitivity and NPV of CTC for flat lesions depend on the experience of the radiologist reader. CAD seems to be an effective aid for the novice radiologist only.

\section{B-0795 11:24}

MR colonography to screen for colorectal neoplasia in asymptomatic adults

A. Graser, A. Melzer, E. Lindner, P. Stieber, M.F. Reiser, B. Göke, F.T. Kolligs; Munich/DE (anno.graser@med.uni-muenchen.de)

Purpose: To test whether high field MR colonography could serve as a screening test for adenoma ${ }^{3} 6 \mathrm{~mm}$ and advanced adenoma in asymptomatic adults.

Methods and Materials: 286 asymptomatic adults aged $40-82$ years underwent FOBT testing, same-day 3 Tesla MR colonography and colonoscopy. Segmental unblinding of the colonoscopist to the results of MR colonography. MR was performed after injection of Gadolinium contrast agent using high resolution 3D VIBE sequences. All detected lesions were removed or biopsied during colonoscopy. For each of six colonic segments, presence or absence of polyps was assessed. Per-polyp sensitivities and per-patient sensitivities, specificities, positive and negative predictive values including $95 \%$ confidence intervals $(\mathrm{Cl})$ were determined for adenoma of various size categories and advanced adenoma.

Results: 133 adenomas and 2 cancers were diagnosed in 86 patients. 37 adenomas were ${ }^{3} 6 \mathrm{~mm}$ and 20 adenomas were advanced. Sensitivities of MR colonography and colonoscopy for adenoma ${ }^{3} 6 \mathrm{~mm}$ were $78.4 \%(95 \% \mathrm{Cl}, 61.8-90.2)$ and $97.3 \%$ (85.8-99.9), and $75 \%$ (50.9-91.3) and 100\% (83.2-100.0) for advanced adenoma, respectively. MR colonography, colonoscopy, and FOBT identified $86.7 \%$ (96.396.2), $96.7 \%(82.8-99.9)$, and $6.9 \%(0.8-22.8)$ of individuals with adenoma ${ }^{3} 6 \mathrm{~mm}$, and $82.4 \%$ (56.6-96.2), $100 \%$ (80.5-100.0), and 12.5 (1.6-38.3) of individuals with advanced adenoma, respectively. Specificity of MR colonography, colonoscopy, and FOBT for individuals with advanced adenoma was $81.0 \%$ (75.8-85.5), $48.5 \%$ (42.4-54.7), and $92.2 \%(88.2-95.2)$

Conclusion: 3 Tesla MR colonography has high sensitivity and specificity for adenoma ${ }^{3} 6 \mathrm{~mm}$ and advanced adenoma. Its sensitivity and strongly outperforms FOBT. MR colonography is a potent radiation-free screening tool for colorectal neoplasia.

\section{Author Disclosures:}

A. Graser: Advisory Board; Siemens AG Healthcare. Consultant; Siemens AG Healthcare, Bayer AG. Grant Recipient; Bayer Healthcare. Investigator; Pfizer Pharma. Speaker; Siemens AG Healthcare, Bayer AG, Bracco, Pfizer Pharma.

\section{B-0796 11:33}

Retrospective evaluation of diverticular disease in a large cohort of 1091 patients who underwent CT colonography

M. Ciolina' ${ }^{1}$, C. De Cecco2, P. Baldassari', M. Iannitti', A. Pichi', F. lafrate', A. Laghi'; ${ }^{1}$ Rome/IT, ${ }^{2}$ Latina/lT (mariaciolina@gmail.com)

Purpose: To evaluate the prevalence of diverticular disease according to age, gender, colonic segments, disease severity and symptoms in a group of patients who underwent CT colonography (CTC).

Methods and Materials: Population included 1091 consecutive Caucasian patients who underwent CTC examination. Patients with diverticular disease were retrospectively stratified into 7 groups according to age (25-85 yrs). Each group was further stratified according to sex, symptoms and colonic segment involvement. Severity of diverticular disease was evaluated using a 3-point scale: grade 1 (rare< 6 diverticula), grade 2 (multiple 6-20) and grade 3 (several> 20).

Results: Diverticular disease was observed in 561 patients (321 women and 240 men, mean age $68 \pm 12 \mathrm{yrs}$ ). Symptomatic disease was present in $47.4 \%$ cases. In $60 \%$ of patients ${ }^{2} 45$ years at least one diverticulum in the sigmoid colon was observed. Disease severity increased over time in all colonic segments, especially in sigmoid colon where grade 3 ranged from $2.7 \%$ at $25 \mathrm{yrs}$ to $24.4 \%$ at $85 \mathrm{yrs}$. Also in advanced age, diverticula prevalence remains significant higher in sigma than right colon (cecum $7.8 \%$, ascending 6.7\%).No significant difference in diverticula prevalence and distribution was related to patient gender ( $p>0.05$ ). In sigma, advanced disease (grade 2/3) shows a significant positive correlation with reduced colonic distension $(p<0.05)$. No correlation was found between severity of sigmoid diverticula and symptoms onset.

Conclusion: Prevalence and severity of diverticular disease increased over time in all colonic segments. An elevated number of asymptomatic diverticula is present in the right colon. An high percentage of patients with severe sigmoid diverticular disease remain asymptomatic.

\section{B-0797 11:42}

Accuracy of water enema-MDCT in colon cancer staging: a prospective study

C. Ridereau-Zins ${ }^{1}$, E. Sibileau' ${ }^{1}$, A. Pavageau', D. Vanel${ }^{2}$,

E. Lermite' ${ }^{1}$, E. Metivier-Cesbron', C. Aube $1 ;{ }^{1}$ Angers/FR, ${ }^{2}$ Bologna/IT

(Caridereauzins@chu-angers.fr)

Purpose: To assess the accuracy of water-enema multi-detector computed tomography (WE-MDCT) in extra-rectal colon cancer staging.

Methods and Materials: Fifty-three patients (mean age 70 years) with extra-rectal colon cancer proven by colonoscopy and biopsy were prospectively evaluated by preoperative WE-MDCT. CT scans were both intraluminally (water enema or WE) and intravenous (iodinated) contrast enhanced (CE). All patients underwent surgery. Tumours were classified with the TNM staging system. Noted CT features were: tumour size and location; tumour form and edges; spread to the pericolic fat or neighbouring organs; thickening of retroperitoneal fascia; number, size and enhancement of the peritumoral lymph nodes. Tumours were classified on CT into $3 \mathrm{~T}$-stage groups: T1/T2, T3 and T4. Lymph nodes were classified by their density after injection (positive over 100 Hounsfield units (HU)).

Results: Tumour localisation to the specific colon segment was correct in all the cases. The agreement between WE-MDCT staging and histopathology staging was good $(\mathrm{k}=0.64)$. A stiff aspect of the external edges of tumours provided excellent sensitivity for T3/T4 inclusion (Se 97.7\%, NPV 85.7\%). Thickening of a fascia or the abdominal wall provided good specificity for T4 stage (Sp 88.1\%, NPV 94.9\%). Enhancement over $100 \mathrm{HU}$ of at least one peritumoral lymph node was the best criterion of $\mathrm{N}+$ staging (Sp 67.7\%, NPV 87.5\%).

Conclusion: WE-MDCT permits good staging of colon cancer based on objective features.

\section{B-0798 11:51}

Feasibility study of low-dose CT colonography using model-based iterative reconstruction: preliminary findings in 20 patients

V. Vardhanabhuti, C. Roobottom; Plymouth/UK (vvar@me.com)

Purpose: To compare image quality on computed tomographic (CT) images acquired at standard dose (SD) and low dose (LD) using adaptive statistical iterative reconstruction (ASIR) and model-based iterative reconstruction (MBIR) techniques. Methods and Materials: 20 patients were prospectively recruited for the study with informed consent. They underwent standard dose (SD) and low-dose (LD) CT colonography. Low-dose parameters were based on our phantom study with using vendor-specific automatic tube current modulation known as noise index. Both sets of scans were reconstructed with ASIR and MBIR. Objective and subjective image qualities were assessed as well as diagnostic accuracies for significant lesions (e.g. polyps, cancer, etc). Effective doses for each scans were recorded. Results: Objective image analysis supports significant noise reduction and superior contrast-to-noise ratio with low-dose scans using MBIR technique $(p<0.05)$ despite being acquired at lower doses. Subjective image parameters were equivalent for LD MBIR and SD ASIR for both colonic and extra-colonic findings. Diagnostic accuracies for polyp detection and other significant lesions were comparable. Dose recorded were substantially lower for MBIR (range $45-75 \%$ reduction compared with ASIR) with mean average being $4.2 \mathrm{mSv}$ in our population group.

Conclusion: MBIR shows superior reduction in noise whilst maintaining image quality and most importantly substantial dose reduction can be achieved. More patients are being recruited to substantiate diagnostic accuracies data with full results to be presented at the ECR 2013. 


\section{Oncologic Imaging}

\section{SS 1716}

\section{Response evaluation in oncology: beyond RECIST}

\section{Moderators:}

T. Denecke; Berlin/DE

M.I. Furmanek; Warsaw/PL

\section{B-0799 10:30}

Calibration of computed tomography (CT) volumetric measurements for assessing tumour response to drug therapy in a randomised multicentre oncology study

R. Avila' ${ }^{1}$, D. Yankelevitz ${ }^{2}$, V. Archer ${ }^{3}$, G. Zahlmann ${ }^{1}$ Clifton Park, NY/US, ${ }^{2}$ New York, NY/US, ${ }^{3}$ Welwyn Garden City/UK, ${ }^{4}$ Basle/CH (rick.avila@kitware.com)

Purpose: Quantification of tumour diameter and volumetric measurement variability, due to CT acquisition properties, for patients participating in a clinical NSCLC trial. Methods and Materials: A validated 'pocket phantom' was scanned with patients; image acquisition was standardised. Volumetric and diameter measurement performance in $\mathrm{X}, \mathrm{Y}$, and $\mathrm{Z}$ axes was evaluated. Fully automated software detected and analysed phantoms. A precision-manufactured $15.88 \mathrm{~mm}$ diameter teflon sphere within each phantom was segmented, using constant thresholding, and measured. Results: Data were acquired from 77 patients at various time points during the trial using 13 different scanner models across three manufacturers. The mean, standard deviation (SD) and coefficient of variation (CV) were calculated in the $X$, $\mathrm{Y}$ and $\mathrm{Z}$ axes of the scanner. Mean, SD and $\mathrm{CV}$ for diameter measurements were $15.7 \mathrm{~mm}, 0.13 \mathrm{~mm}$ and $0.8 \%$, respectively (X axis); $15.6 \mathrm{~mm}, 0.15 \mathrm{~mm}$ and $1.0 \%$ ( $\mathrm{Y}$ axis); $15.8 \mathrm{~mm}, 0.71 \mathrm{~mm}$ and $4.5 \%$ ( $\mathrm{Z}$ axis). Mean, $\mathrm{SD}$ and $\mathrm{CV}$ of volumetric measurements were $1,989 \mathrm{~mm}^{3}, 99 \mathrm{~mm}^{3}$ and $5.0 \%$, respectively. Variability of $Z$-axis diameter measurements was $>4$ times that of $X$ and $Y$ axes. For 45 patients with $>1 \mathrm{scan}$, volumetric measurement $\mathrm{CV}$ was $5.3 \%$. Maximum volumetric change for consecutive CT scans was $44 \%$. Exclusion of six patients with maximum variation reduced $\mathrm{CV}$ to $2.2 \%$. All six of these patients' images were acquired using 16 - and 8-row scanners from a single manufacturer.

Conclusion: Axial diameter measurements had consistently low variability across all scanners and acquisition parameters. Large variation occurred for $Z$ diameter measurements and volumetric measurements.

\section{Author Disclosures:}

R. Avila: Employee; Kitware Inc. Shareholder; Kitware Inc. Other; Consultant to Roche. D. Yankelevitz: Research/Grant Support; F. Hoffmann-La Roche sponsored this research. David Yankelevitz had no personal gain from the sponsorship. Other; David Yankelevitz is a named sponsor on related patents and patent applications. V. Archer: Employee; Venice Archer is an employee of F. Hoffmann-La Roche. G. Zahlmann: Employee; Gudrun Zahlmann is an employee of F. Hoffmann-La Roche.

\section{B-0800 10:39}

Evaluating the agreement between tumour volumetry and the estimated volumes of tumour lesions using an algorithm M. D'Anastasi, R.P. Laubender, V. Heinemann, M.F. Reiser, M. Schlichting, A. Graser; Munich/DE (melvin.danastasi@med.uni-muenchen.de)

Purpose: To evaluate the agreement between true tumour volume and tumour volume derived from (i) a new formula based on longest lesion (RECIST) diameter, (ii) a new formula based on longest diameter and longest orthogonal (WHO) diameter. Methods and Materials: 89 baseline and follow-up CTs were available in 20 patients with metastastic colorectal cancer from the randomised phase II multicenter $\mathrm{ClOX}$ trial. Target lesions were defined at baseline and followed over time. Lesions were evaluated by (i) semi-automated volumetry using Siemens Syngo.via and (ii) volumetric assement using a newly developed formula based on manual measurement of the longest diameter and the longest orthogonal diameter. True, WHO- and RECIST-based volumes were calculated. We compared the agreement of the true volume to the WHO- and RECIST-based volume. We also compared the agreement between 'true' and WHO-based volume relative changes by means of the intraclass correlation.

Results: A total of 151 lesions were evaluated. Using a variance components model it was shown that the difference between true and RECIST-based volume is statistically significant $(p<0.001)$ indicating a substantial constant bias. The same model showed a difference between true and WHO-based volume, which was not statistically significant $(p=0.50)$, indicating no substantial constant bias. Scatter-plots show that the RECIST-based volume overestimates lesion volume. The intraclass correlation between 'true' and WHO-based volume relative changes was 0.95 , showing nearly perfect agreement between methods.

Conclusion: Our proposed formula, if based on WHO-measurements, allows for a very good estimate of relative volume changes (RECIST-based formula overestimates the true volume).

\section{Author Disclosures:}

M. D’Anastasi: Research/Grant Support; Merck KGaA. R.P. Laubender: Research/Grant Support; Merck KGaA. V. Heinemann: Research/Grant Support; Merck KGaA. M. Schlichting: Employee; Merck KGaA. A. Graser: Speaker; Siemens.

\section{B-0801 10:48}

Chemotherapy response evaluation with magnetic resonance (MR) and FDG-PET/CT in gastroesophageal tumours (GT): apparent diffusion coefficient (ADC) and standardised uptake volume (SUV) changes compared with tumour regression grade (TRG) at histology F. Giganti, F. De Cobelli, C. Canevari, E. Orsenigo, L. Albarello, E. Mazza, C. Staudacher, L. Gianolli, A. Del Maschio; Milan/IT

Purpose: To compare ADC and SUV modifications during neoadjuvant chemotherapy (NC) with TRG (5 grade-histological indicator of treatment response) to evaluate if their modifications may help to differentiate between Responders (R) and Non Responders (NR) to NC

Methods and Materials: 17 patients with GT ( 3 oesophageal, 3 GEJ and 11 gastric cancer) were evaluated on a 1.5-T MR system. MR protocol included DiffusionWeighted Imaging (DWI) with b value $0-600 \mathrm{~s} / \mathrm{mm}^{2}$, before and 3 weeks after the end of NC and ADC was calculated. Patients underwent FDG-PET/CT scans before and after NC, obtaining SUV each time. All patients were then submitted to radical surgery and the results were compared with TRG. TRG 1, 2 or 3 patients were classified as R; TRG 4 or 5 patients as NR.

Results: 8 patients were considered $R$ and 9 as NR. $8 / 17$ patients showed an increase, 5/17 a reduction of ADC while no significant changes for 4/17. ADC significantly increased in $R$ group compared with NR $(p<0.01)$. ADC showed significant inverse correlation with TRG $(R=-0.79 ; p<0.01)$. Concerning PET findings, $3 / 17$ patients showed an increment and 10/17 a reduction of SUV while no significant changes for $4 / 17$; SUV and TRG were not correlated $(R=0.21$; $p>$ $0.05)$. No correlation was found between SUV and ADC $(R=-0.18 ; p>0.05)$. Conclusion: In evaluating tumour response, DWI-MR seems to be more accurate than FDG-PET and SUV. ADC modifications represent a reproducible tool to assess tumour response to NC.

\section{B-0802 10:57}

Treatment response assessment in Hodgkin lymphoma: in search for morphological correlates of metabolic activity

T. Knogler, G. Karanikas, M. Weber, K. El-Rabadi, M.E. Mayerhoefer; Vienna/AT (thomas.knogler@meduniwien.ac.at)

Purpose: To predict early treatment response with three-dimensional texture features (TF) in patients with Hodgkin lymphoma $(\mathrm{HL})$ after radio-chemotherapy extracted from contrast-enhanced CT.

Methods and Materials: 21 patients with histologically proven $\mathrm{HL}$ were included in this study. Contrast-enhanced (18)F-FDG PET/CT was obtained on a dedicated $\mathrm{PET} / \mathrm{CT}$ scanner. Volumes-of-interest and long- and short-axis diameter were manually defined on $48 \mathrm{HL}$ manifestations prior and post-radio-chemotherapy on the CT image stack. Three-dimensional texture features derived from the grey-level histogram, co-occurrence matrix, run-length matrix and absolute gradient were calculated for the VOls. A stepwise logistic regression with forward selection was performed to find classic radiologic features (i.e. lesion diameter, lesion volume) and TF, which correctly classify treatment response (i.e. full response and partial response). Classification in $\mathrm{PET/CT}$ was used as reference.

Results: Difference in short axis diameter best fit as classic feature with a sensitivity of $100 \%$, a specificity of $54.5 \%$ and an accuracy of $89.6 \%$. Combination of "S_0_0_1_Entrp" and difference of "vertl_fraction" best fit as TF with a sensitivity of $97.3 \%$, a specificity of $72.7 \%$ and an accuracy of $91.7 \%$.

Conclusion: Texture features extracted from contrast-enhanced CT in patients with $\mathrm{HL}$ are superior in differentiation between responders and non-responders without the need for PET examinations, compared to classical radiological features. However, PET/CT as state-of-the-art imaging technique has a sensitivity and specificity $>90 \%$, so that further research with larger patient number is needed to investigate this new method. 


\section{B-0803 11:06}

Predictive values of MRI parameters for the progression-free survival after radioembolisation in patients with metastases of neuroendocrine tumours

W.H. Sommer, F. Ceelen, P. Paprottka, M.F. Reiser, D. Theisen;

Munich/DE (wieland.sommer@med.uni-muenchen.de)

Purpose: The aim of this study was to define the role of MRI in the pretherapeutic prediction of treatment response in patients with liver metastases of neuroendocrine tumours (NET) undergoing radioembolisation.

Methods and Materials: In 45 patients with proven hepatic metastases NET, MRI was performed at baseline (3 T MRI scanner, Gd-EOB-DTPA). Furthermore, PET-CT examinations with somatostatin-specific tracer ${ }^{68} \mathrm{Ga}$-DOTATATE were performed at baseline. The following imaging predictors were defined: patient age/gender, proliferation marker Ki-67, tumourload (\%), vascularisation of metastases, tumour necrosis and haemorrhage. The status of somatostatin receptor was defined by analysing mean and maximum SUV in PET-CT. As primary end point we defined the progression-free survival (PFS) using RECIST criteria in MRI follow-up examinations (every 3 months). The effects of the predictors on the progression-free survival were analysed using Kaplan-Meier statistics.

Results: The mean follow-up time was $445 \pm 411$ days. The mean PFS was 699 days (95\% Cl 326-964). Hypovascular metastases showed significant earlier progress ( 255 vs. 727 days; $p<0.01$ ). A proliferation marker $<2 \%$ was significantly associated with a longer PFS than a proliferation marker between $2-20 \%$ and $>20 \%$ ( $p<$ 0.001 ). Patient age, gender, tumour load in the liver, tumour necrosis, haemorrhage as well as radioreceptor status pre-SIRT did not show any impact on PFS ( $p>0.05)$. Conclusion: Radioembolisation in neuroendocrine liver metastases is most efficient in hypervascular metastases with low proliferation index. Tumour-load as well as radioreceptor status do not have influence on the progression-free survival in contrast to other therapeutic options in NETs.

\section{Author Disclosures:}

W.H. Sommer: Consultant; Bayer.

\section{B-0804 11:15}

One-month apparent diffusion coefficient-determined response at diffusion-weighted MRI is an independent predictor of response to transarterial chemoembolisation for hepatocellular carcinoma

V. Vandecaveye, K. Michielsen, F. De Keyzer, W. Laleman, M. Komuta,

F. Nevens, T. Roskams, C. Verslype, G. Maleux; Leuven/BE

(vincent.vandecaveye@uzleuven.be)

Purpose: To evaluate 1 month diffusion-weighted (DWI) magnetic resonance imaging (MRI) for predicting response to transarterial chemo-embolisation (TACE) of unresectable hepatocellular carcinoma (HCC).

Methods and Materials: Thirty-eight HCC patients underwent 1.5 Tesla MRI, including DWI and dynamic contrast-enhanced sequences, before and 1 month after the first TACE. One month response was assessed using RECIST 1.1, EASL, mRECIST and apparent diffusion coefficient changes (ADCratio). Eight patients were transplanted 2 months post-TACE which additionally allowed correlation of 1-month imaging with histopathological necrotic ratio using a Mann-Whitney $U$ test. In the remaining 30 patients, the univariate effect on progression-free survival (PFS) and overall survival (OS) of RECIST, EASL, mRECIST, ADCratio and pretreatment variables was examined with the Kaplan-Meier and log-rank tests finalized by multivariate analysis. Comparative accuracy of 1-month response assessment was determined by receiver operator characteristic curves.

Results: In the 8 transplanted patients, correlation was only significant between the ADCratio and the pathologically determined percentage tumoral necrotic ratio $(p=0.03)$. In the 30 followed patients, median PFS and OS were, respectively, 5 and 48 months. Univariate analysis showed significant correlation of EASL and ADCratio with PFS $(p<0.05)$ but multivariate analysis showed significant correlation between ADCratio and PFS $(p<0.001)$. ADCratio correlated substantially with OS ( $p=0.07$ ). For predicting 5-month PFS, sensitivity was $91 \%$ and specificity $79 \%$ for the ADCratio, $46 \%$ and $74 \%$ for mRECIST and EASL and $82 \%$ and $32 \%$ for RECIST 1.1

Conclusion: One month ADCratio post-TACE is an independent predictor of PFS. The ADCratio shows near-significant correlation with OS.

\section{B-0805 11:24}

Real-time imaging of the therapeutic response of tumours to antivascular treatment in mice by gadofosveset-enhanced MRI A.J. Höink ${ }^{1}$, T. Persigehl' ${ }^{2}$, J. Ring ${ }^{1}$, R.M. Mesters ${ }^{1}$, W.E. Berdel ${ }^{1}$, W.L. Heindel ${ }^{1}$, C. Bremer ${ }^{1}$, C. Schwöppe ${ }^{1} ;{ }^{1}$ Münster/DE, ${ }^{2}$ Cologne/DE (anna.knauer@ukmuenster.de)

Purpose: Tumour treatment by retargeted truncated protein derivatives of the human tissue factor (tTF) results in tumour vessel infarction, necrosis and subsequent tumour growth retardation and regression. A novel method of gadofosvesetenhanced MRI, that enables the visualisation of the response to this treatment in real-time, is introduced.

Methods and Materials: Dynamic gadofosveset-enhanced MRI $(3 \mathrm{~T})$ was performed in eight tumour-bearing (U87) nude mice. In the course of an ultrafast T1-weighted gradient echo sequence, a gadolinium-based blood pool contrast agent (gadofosveset trisodium) was injected intravenously via a tail vein catheter. After the maximum contrast intensity inside the tumour was obtained, $150 \mu \mathrm{l}$ of tTF-NGR (a retargeted tTF derivative which binds to CD13-overexpressing tumour endothelial cells) were injected intravenously via a second tail vein catheter ( $\mathrm{n}=$ 4; saline solution as control: $n=4$ ), and the contrast behaviour of the tumour was monitored by region of interest (ROI) analysis.

Results: Following the injection of tTF-NGR (approx. $500 \mathrm{sec}$. after the beginning of the sequence), the contrast intensity inside the tumours of the treated mice decreased more instantly and more strongly than in the tumours of the control mice (AUC $1121.3 \pm 0.65$ vs. $1164.4 \pm 0.52 \mathrm{AU}$ ).

Conclusion: Gadofosveset-enhanced MRI allows for the visualisation of the therapeutic response of tumours to anti-vascular treatment in real-time. Considering the intended clinical application of tTF-NGR, this method might be a simple and quick surrogate parameter for the monitoring of the therapeutic response to vascular disrupting agents in humans.

\section{B-0806 11:33}

Reproducibility of therapy response evaluation between experienced and less experienced readers of pleural mesothelioma by MRECIST, RECIST 1.0, RECIST 1.1, and WHO

L. Stahlhut ${ }^{1}$, A. Bunck ${ }^{1}$, Y. Tan², M. Steen ${ }^{1}$, X. Guo ${ }^{2}$, D. Maintz', L. Schwartz ${ }^{2}$, B. Zhao ${ }^{2}$, T. Persigehl ${ }^{1} ;{ }^{1}$ Cologne/DE, ${ }^{2}$ New York, NY/US (leandra.stahlhut@uk-koeln.de)

Purpose: The specific growth pattern of pleural mesothelioma along the pleural surface makes therapy monitoring using common measurement criteria, such as RECIST 1.0, difficult. Therefore, Byrne et al. developed modified RECIST criteria for pleural mesothelioma. In this study we evaluated the accordance between experienced and less experienced readers for RECIST 1.0, RECIST 1.1, mRECIST, and WHO.

Methods and Materials: A total of 43 baseline and follow-up-CT scans from 15 patients from a clinical multi-centre pleural mesothelioma trial were retrospectively evaluated by different experienced radiologist (about 1 year and more than 10 years), who selected target lesions independently according to mRECIST, RECIST 1.0, RECIST 1.1 and WHO criteria. CT scans were then re-measured after an interval of greater than one month. The inter- and intra-reader variability using the different response criteria was analysed.

Results: Both, the intra-reader- and inter-reader variability were best when using mRECIST (95\% limits of agreement of $-28.4 / 12.9$ and of $-32.1 / 20.7$ ) compared 'with RECIST 1.0, RECIST 1.1 and WHO. Further, regarding the objective response $(\mathrm{PR}=$ partial response, $\mathrm{SD}=$ stable disease and $\mathrm{PD}=$ progressive disease $), \mathrm{mRE}$ CIST showed the most reliable intra-observer outcome with only one differently classified examination and the most reliable inter-observer outcome with four differently classified examinations.

Conclusion: Our results suggest that mRECIST is most reliable for treatment response evaluation of pleural mesothelioma between experienced and less experienced readers compared with RECIST 1.0, RECIST 1.1 and WHO. To confirm these findings, the inclusion of further readers and more patient data is warranted.

\section{B-0807 11:42}

Use of multimedia structured reporting for tumour response assessment

D.J. Vining ${ }^{1}$, A. Pitici ${ }^{2}$, I. Aghenitei², C. Popovici², M. Jurca²,

R. Rosu ${ }^{2}$, A. Tsimberidou $1 ;{ }^{1}$ Houston, TX/US, ${ }^{2}$ Chapel Hill, NC/US

(dvining@mdanderson.org)

Purpose: Response Evaluation Criteria in Solid Tumours (RECIST) is used to assess patient response to anticancer therapy. However, radiologists often fail to report target lesions, perform tumour measurements and accurately compare serial 
exams. We present a structured reporting system, called ViSion, which captures a radiologist's measurements and key images in an efficient manner to generate disease timelines and automate the calculation of RECIST.

Methods and Materials: We analysed serial radiology reports from ten patients with cancer to determine if radiologists reported tumour measurements, saved measurements in PACS, and whether there was correlation of tumour metrics between exams. In addition, we created a structured reporting solution that uploads screen captures and audio descriptions of image findings to a computer server where each finding is labelled with anatomy and pathology terms, presented in a multimedia structured report, and can be used to calculate RECIST.

Results: The analysis of baseline and subsequent radiology reports revealed that radiologists reported measurements in $90 \%$ of cases, saved measurements in $20 \%$ of baseline and $50 \%$ of follow-up studies, and correlated measurements between exams in only $40 \%$. The application of ViSion enabled a means to record tumour measurements in $100 \%$ of cases by providing a visual record of where target lesions were previously measured, facilitating the linking of image findings between examinations and displaying the findings in disease timelines.

Conclusion: The ViSion structured reporting system provides a means for more reproducible tumour response assessment.

Author Disclosures:

D.J. Vining: Founder; VisionSR, Inc. A. Pitici: Employee; Eloquentix, Inc. I. Aghenitei: Employee; Eloquentix, Inc. I. Aghenitei: Employee; Eloquentix, Inc. C. Popovici: Employee; Eloquentix, Inc. M. Jurca: Employee; Eloquentix, Inc. R. Rosu: CEO; Eloquentix, Inc.

\section{B-0808 $11: 51$}

Comparison of breast-MRI vs. standard prognostic factors for the prediction of local recurrence after treatment of primary breast cancer

P.A.T. Baltzer ${ }^{1}$, M. Dietzel ${ }^{2}$, H. Habrecht ${ }^{3}$, R. Zoubi ${ }^{4}$, C. Jerowski ${ }^{3}$,

I.B. Runnebaum ${ }^{3}$, W.A. Kaiser ${ }^{3} ;{ }^{1}$ Jena/DE, Vienna/AT, ${ }^{2}$ Erlangen/DE, Jena/DE ${ }^{3}$ Jena/DE, ${ }^{4}$ Bielefeld/DE, Jena/DE

Purpose: To identify the potential of breast MRI vs. standard prognostic factors to predict tumour recurrence after successful treatment of primary breast cancer. Methods and Materials: Consecutive patients receiving staging MRI and subsequent guideline-approved therapy of primary breast cancer at our academic breast centre were investigated. Standard prognostic factors including TNM staging, tumour typing, tumour grading, progesterone/estrogen receptors, HER2NEU score and associated DCIS component were assessed. Follow-up was performed (endpoint: "tumour-recurrence" after complete remission). For analysis of breastMRI a dedicated CAD software was used (computer-aided analysis). It enables semi-automatic analysis of enhancement characteristics of the whole tumour (3D analysis of early vs. delayed phase) and the "hot-spot" (i.e. voxel with highest washin/wash-out ratio). Potential of breast MRI characteristics vs. standard prognostic factors to stratify the endpoint separately and in combination was investigated using logistic regression. To identify significant and independent predictors, backward feature selection was applied ( $p^{\text {entry }} /$ removal: $<0.05 />0.01$ )

Results: 223 patients were included (endpoint: $n=13 / 5.8 \%$, loss-to follow-up: $\mathrm{n}=7 / 3.1 \%$ ). If tested separately, logistic regression identified moderate potential for breast MRI (AUCMRI=0.79, $\mathrm{p}=0.001$ ) and standard prognostic factors (AUCstandard $=0.71 ; p=0.01$ ) to stratify the endpoint. If breast $\mathrm{MRI}$ characteristics and standard prognostic factors were combined, predictive performance could be increased considerably (AUCcombined $=0.90, p<0.001$, sensitivity $=84.6 \%$, specificity=87.3\%). Feature selection identified one standard prognostic factor (M-Stage) and four breast MRI characteristics (hot-spot: Wash-in, washout-ratio 3D-analysis: tumour voxels showing wash-out plus weak wash-in, tumour voxels showing plateau and weak wash-in) as the most significant and independent predictors of tumour recurrence.

Conclusion: As standalone methods both breast MRI characteristics and standard prognostic factors have limited potential to predict local tumour recurrence. Yet, they have additive prognostic effect on each other: If used in combination, they showed promising potential to predict this important endpoint ( $\mathrm{AUC}=0.90)$
$10: 30-12: 00$

Room F2

\section{Breast}

\section{SS 1702}

\section{Tomosynthesis: a role in clinical practice?}

Moderators:

S. Allen; Sutton/UK

E.M. Jung; Regensburg/DE

\section{B-0809 10:30}

The role of additional tomosynthesis combined with digital mammography P. Martínez ${ }^{1}$, J. Echano², M. Sainz', I. Simon², G. Viteri²,

A. Garcia Lallana', C. Minguillon', L. Pina'; ' Zaragoza/ES, ${ }^{2}$ Pamplona/ES

(paulamartinezmiravete@yahoo.es)

Purpose: To evaluate the detection rate of breast cancer using digital tomosynthesis as an additional tool combined with digital mammography in ACR density patterns 2, 3 and 4 in a clinical setting.

Methods and Materials: From December 2010 to September 2012, 9301 combo retrospective studies (2D-digital mammography plus tomosynthesis) were performed in ACR density patterns 2, 3 and 4 to evaluate the role of the tomosynthesis in the detection of additional cancers. Statistical analysis was performed calculating the Pearson Chi-square test and using the SPSS software.

Results: 118 breast tumours were detected on conventional digital mammography (detection rate 1.26\%). Seventy-one additional cancers were detected on tomosynthesis and not seen on standard mammograms: 14 cases on ACR 2 (the detection rate was increased by $0.61 \%), 27$ cases in ACR $3(0.74 \%)$ and 30 cases in ACR $4(0.87 \%)$. No statistical differences were found in the detection rate among the three patterns $(p=0.78)$. Tomosynthesis increased the detection rate by $0.76 \%$, so the total detection rate using the combo studies was $2.03 \%$.

Conclusion: The addition of digital tomosynthesis increases the detection rate from $1.26 \%$ to $2.03 \%$. There are no significant differences among the three ACR density patterns, so tomosynthesis can be useful not only in dense patterns but also in pattern 2 .

\section{B-0810 10:39}

Does breast tomosynthesis combined with 2D digital mammography increase the detection rate of breast cancer?

R. Saiz-Mendiguren ${ }^{1}$, C. Marginet-Mangas ${ }^{1}$, A. Garcia-Lallana ${ }^{2}$,

E. Martinez ${ }^{1}$, C. Ibarburen ${ }^{1}$; L. Pina Insausti ${ }^{3} ;{ }^{1}$ Palma de Mallorca/ES,

${ }^{2}$ Santurce/ES, ${ }^{3}$ Pamplona/ES (saiz.ramon@gmail.com)

Purpose: To evaluate the incidence of breast cancer screening with digital mammography combined with breast tomosynthesis in the ACR density patterns 1 to 4 . Methods and Materials: Retrospective study: from September 2011 to August 2012, 2778 consecutive women were examined retrospectively with a Selenia ${ }^{\circledR}$ Dimensions System 3D (Hologic $\left.{ }^{\circledR}\right)$. The distribution among the ACR density patters was studied. All women underwent a "combo" study, which consisted of a 2D-DM ( 2 projections) and 3D-DBT (2 projections). The increase in the detection of additional breast cancers due to the 3D-tomosynthesis studies has been evaluated. Chi-square test with SPSS for windows (15.0 Chicago, IL) has been used to evaluate if there were any statistically significant differences $(p<0.05$ was considered statistically significant). The clinical, radiologic and pathologic findings are described. Results: Among all ACR density pattern, 15 breast cancers were diagnosed with the 2D-DM studies (detection rate $0.54 \%$ ). 7 additional cancers were diagnosed due to the 3D-DBT imaging findings (four in ACR density patter 2, one in ACR density pattern 3 and two in ACR density pattern 4). The global detection rate increased to $0.79 \%$. No statistical differences were found regarding the detection rate among all density patterns.

Conclusion: In our group of patients the use of 3D-DBT combined with conventional 2D-DM increased the rate of breast cancer diagnosis from 0.54 to $0.79 \%$. No statistical significant differences were found among all ACR density patterns. 3D-DBT may be useful not only in dense breasts (ACR 3 and 4 ) but also in medium density breasts (ACR 2). 


\section{B-0811 10:48}

Detection rate for suspicious lesions of digital breast tomosynthesis in combination with digital mammography or $2 \mathrm{D}$ central projection imaging

C. Zuiani ${ }^{1}$, P. Clauser ${ }^{1}$, V. Londero', C. Molinari', R. Girometti ${ }^{1}$,

A. Taibi ${ }^{2}$, S. Vecchio ${ }^{3}$, M. Bazzocchi'; ${ }^{1}$ Udine $/ I T,{ }^{2}$ Ferrara/lT, ${ }^{3}$ Bologna/lT (clauser.p@hotmail.it)

Purpose: Based on a novel approach, Digital Breast Tomosynthesis (DBT) may simultaneously acquire 2D and 3D images of the breast. Variable dose geometry is used to give a sufficient dose in the central projection (2D-CP) for that image to be a $2 \mathrm{D}$ mammogram. We aimed to assess the detection rate for suspicious lesions (ACR-BIRADS 3-5) of DBT associated with Digital Mammography (DM) or 2D-CP. Methods and Materials: 45 asymptomatic women who performed DM and DBT with 2D-CP as screening examinations were included. Two experienced radiologists, blinded to final diagnosis, evaluated images in separate sessions according to two different strategies of reading: DM+DBT (set-A) and 2D-CP+DBT (set-B). They assessed breast lesions as benign or suspicious in case of ACR-BI-RADS scoring of 1-2 and 3-5, respectively. Discordant cases were reviewed in consensus. Agreement was assessed with weighted-kappa statistic.

Results: Both modalities detected 31 lesions that appeared as 22 masses, 7 microcalcifications and 2 masses with microcalcifications. Lesions assessed as suspicious were $83.8 \%$ (Cl95\%=67.4-92.9\%) for set-A and $74.2 \%$ (CI95\%=56.7$86.3 \%)$ for set-B, respectively, corresponding to an excellent agreement $(k=0.86)$. Set-A detected as suspicious three small masses that were classified as benign with set-B.

Conclusion: An all-in-one, dose-sparing examination using DBT with 2D-CP provides a good agreement in detecting suspicious lesions compared to combined $\mathrm{DM}+\mathrm{DBT}$. Of note, a difference in the classification of small masses was identified.

\section{B-0812 10:57}

Digital mammography in comparison with digital breast tomosynthesis in detection of multicentric breast cancer N. Prvulovic, D. Djilas, M. Prvulovic, D. Bogdanovic, K. Koprivsek; Novi Sad/RS (prvulovicn@gmail.com)

Purpose: The aim of this study was to demonstrate the diagnostic significance of digital mammography with tomosynthesis (3D) versus digital mammography (2D) in the group of histologically proven multicentric breast cancer.

Methods and Materials: In the group of 110 patients examined in this study, 2-D mammography showed suspicious breast lesions. All patients were sent to the Oncology Institute of Vojvodina for further examination and biopsy. At our institution after initially clinical breast exam, 2D and 3D mammography were performed in the same act. All imaging procedures were performed on Hologic Selenia Dimensions equipment. Two radiologists independently read mammograms and core needle biopsy were performed in all BIRADS 4 and 5 groups. All patients had fibroglandular or heterogeneously dense breasts (ACR2 and ACR3).

Results: In the group of 110 patients, pathologic findings revealed 80 unifocal and 30 multicentric cancers for a total of 152 malignant foci. All unifocal cancers were detected by both methods. With 2D mammography, additionally 15 suspicious lesions were found and using 3D mammography 31 suspicious mass were found. Both 2D and 3D mammography showed the same 15 lesions but 3D mammography showed 16 "new" lesions which were obscured on 2D mammography. Overall sensitivity was 82\% (125/152) for 2D mammography and 94\% (141/152) for 3D mammography $(p<0.05)$. Results showed that in $10 \%$ of multicentric foci were in situ carcinomas (DCIS).

Conclusion: 3D mammography was more sensitive than 2D mammography for the detection of multicentric foci in fibroglandular or heterogeneously dense breasts.

\section{B-0813 11:06}

The role of tomosynthesis after normal mammography according to ACR density patterns

J. Etxano, P. Slon, I. Simon-Yarza, R. Zalazar, A. Elizalde, L. Pina; Pamplona/ES (jetxano@unav.es)

Purpose: To assess the detection rate of breast cancer using tomosynthesis (TS) as an adjunct tool after normal mammography regarding the ACR density patterns. Methods and Materials: This prospective study was performed from October 2011 to September 2012. During this time, 3615 consecutive women (mean age 52.3, range 29-88) underwent digital mammography (Inspiration, Siemens, Erlangen, Germany) in a reference centre. After the evaluation of the breast density by a radiologist, tomosynthesis (TS) was performed using the oblique mediolateral view in all patients with ACR density patterns 2 (901), 3 (1165) and 4 (224). Out of these 2290 patients, 76 were found to have suspicious lesions on conventional mammography (BI-RADS categories 4\&5), among them 41 had breast cancer. Thus, 2214 patients with normal or benign mammograms (BI-RADS categories 1 to 3) were included in this study. Statistical analysis was performed to compare the rate of additional cancers detected on TS with regards to the ACR density patterns, calculating a Pearson Chi-square test (SPSS 15.0).

Results: Conventional digital mammography detected 41 cancers (detection rate $1.79 \%$ ). TS after normal or benign digital mammography detected 13 additional malignant cases (additional detection rate 12/2290: 0.40\%): 5 cases in ACR 2 $(0.17 \%) ; 7$ cases in dense patterns ACR $3 \& 4(0.23 \%)$. No statistical differences were found between the ACR density patterns $(p=0.35)$.

Conclusion: In our experience, additional TS in oblique mediolateral view after normal mammograms increases the detection rate from $1.37 \%$ to $1.77 \%$. Moreover, it can be useful not only in dense patterns (3\&4) but also in scattered fibroglandular densities (pattern 2).

\section{B-0814 11:15}

Influence of breast density on diagnostic performance of digital

breast tomosynthesis (DBT) after digital mammography (DM) focusing on recall rate reduction

L.A. Carbonaro ${ }^{1}$, R.M. Trimboli', N. Verardi', M. Fedeli², G. Di Leo ${ }^{1}$,

F. Sardanelli'; ' San Donato Milanese/IT, ${ }^{2}$ Milan/IT (luca.carbonaro@gmail.com)

Purpose: To estimate the influence of breast density on diagnostic performance of DBT added to DM (DM+DBT), focusing on recall rate reduction.

Methods and Materials: Seventy-five women were recalled for 77 findings on DM screening examination and further investigated according to usual protocols (additional DM views, ultrasonography, and/or core needle biopsy). The final conventional assessment was: 7 cancers ( 4 invasive ductal carcinoma, 2 invasive lobular carcinomas) and 68 negative/benign cases. Before this conventional work-up, 1- or 2-view DBT was obtained (Giotto, IMS, Italy) for an off-line further evaluation. Two independent readers, blinded to final conventional assessment, retrospectively assigned a BI-RADS score to each lesion on both DM only and DM+DBT. Breast density was scored according to ACR categories from 1 to $4 . X^{2}$ test was used. Results: None of the 7 cancers was missed on DM+DBT by each of the two readers; of 14 readings, $7(50 \%)$ had an increased BI-RADS score. Of 136 readings of 68 benign/negative cases, density was ACR1 in $24(18 \%)$, ACR2 in $60(44 \%)$, ACR3 in $42(31 \%)$ and ACR-4 in $10(7 \%)$; the BI-RADS score was increased in $3(2 \%)$, unchanged in $61(45 \%)$ and decreased in $72(53 \%)$. BI-RADS was decreased for 11/24 (46\%) ACR1, 34/60 (53\%) ACR2, 25/42 (59\%) and 4/10 (40\%) ACR4 $(p=0.595)$.

Conclusion: Breast density does not influence the capability of DBT, as added to $\mathrm{DM}$, to decrease the BI-RADS score of negative/benign cases recalled for further assessment at screening DM.

\section{B-0815 11:24}

Recall rate reduction by adding double reading digital breast tomosynthesis (DBT) to digital mammography (DM)

L.A. Carbonaro ${ }^{1}$, R.M. Trimboli', N. Verardi', K. Khouri Chalouhi' ${ }^{2}$, G. Di Leo ${ }^{1}$,

F. Sardanelli'; ' ${ }^{1}$ San Donato Milanese/IT, ${ }^{2}$ Milan/IT (Iuca.carbonaro@gmail.com)

Purpose: To estimate the reduction in recall rate induced by adding double reading DBT to DM.

Methods and Materials: During 21 weeks of screening-activity, women with at least one mammographic suspicious finding were further investigated according to usual protocols (additional views, ultrasonography, and core needle biopsy). Before this standard exams, 1- or 2-view DBT was acquired (Giotto, IMS, Italy). Of a total of $1,286 \mathrm{DM}$ screening-exams, 74 were initially recalled (recall rate of $5.8 \%$ ) for 77 suspicious findings. Two independent readers (who did not perform initial standard reading and blinded to final diagnosis) retrospectively evaluated DM and DBT. Each lesion was scored according to BI-RADS classification, considering $3,4 a-b-c$, and 5 as recalls.

Results: Two DBTs (two findings) were excluded for technical problems. At combined DM+DBT evaluation each of the two readers recalled $37 / 72$ (51\%) women; considering each case judged as BI-RADS $3-5$ by at least one of the two readers as recalled (double reading), a total of 49 cases were recalled with a reduction in recall rate of $23 / 72(32 \%)$. Of 14 readings of 7 cancers (5 IDCs, 2 ILCs), we had BI-RADS upgrade for $7(50 \%)$, confirmation for $6(43 \%)$, and downgrade for $1(7 \%)$. Of 136 readings of 68 negative cases, we had a BI-RADS upgrade in 3 $(2 \%)$, confirmation in $61(45 \%)$, and downgrade in $72(53 \%)$.

Conclusion: No cancers were missed. The use of double reading DBT as an adjunct to DM could allow for a reduction of recall rate by $32 \%$, from $5.8 \%$ to $3.9 \%$. 


\section{B-0816 11:33}

Breast tomosynthesis versus digital mammography: evaluation of diagnostic potential in women with abnormal screening mammograms M.A. Shaaban; Cairo/EG (marwaadelmm@hotmail.com)

Purpose: To compare the diagnostic potential of digital mammography with tomosynthesis.

Methods and Materials: 100 women with abnormal screening mammograms, 187 findings, and definite imaging or histopathological diagnosis were included. The screening mammography interpretation consisted of direct comparison of the tomosynthesis (DBT) examination with full field digital mammography (FFDM) images. The study radiologists subjectively rated the equivalence of the image quality of both the DBT and the FFDM examinations with no use of additional mammography, magnification views or comparison mammography. The next step was categorising the findings in both FFDM and DBT separately according to BIRADS classification and the statistical analysis evaluated the $p$ values of DBT and FFDM as well as their sensitivities.

Results: All findings categorised as BIRADS 3 according to mammography showed reduction in number,particularly for masses, when examined with tomosynthesis. The image quality of tomosynthesis was subjectively rated as equivalent $(53.5 \%)$ or superior $(43.3 \%)$ to digital mammography in $96.8 \%$ of the total of findings; the test was highly significant $(p<0.001)$. For masses, tomosynthesis image quality was rated as equivalent in $40.5 \%$ or superior in $58.1 \%$ of findings. Masses were $53 \%$ of findings in which tomosynthesis had superior image quality. The FFDM Sensitivity was of $91.9 \%$ compared with $95.2 \%$ for DBT.

Conclusion: The diagnostic potential of digital breast tomosynthesis is superior to that of full-field digital mammography by evaluating their image quality and their sensitivity. Based on a subjective analysis, DBT showed a significantly higher image quality compared with digital mammography in all finding types, especially in the characterisation of masses.

\section{B-0817 11:42}

Analysis of discordant screening-detected cancers at FFDM (2D) versus $2 \mathrm{D}$ plus tomosynthesis (combo mode) in a population-based screening program: results from the Oslo tomosynthesis screening trial P. Skaane, R. Gullien, M. Krager, U. Haakenaasen, M. Izadi, I.N. Jebsen, U. Ekseth, E.B. Eben; Oslo/NO (perska@ous-hf.no)

Purpose: To compare discordant screen-detected cancers in FFDM ("2D-mode") versus $2 \mathrm{D}$ plus tomosynthesis (2D+3D or "combo-mode") in a population-based screening program.

Methods and Materials: Oslo Tomosynthesis Screening Trial (OTST), a large prospective study comparing FFDM with 2D plus tomosynthesis in breast cancer screening, is part of the Norwegian Screening Program. Women 50-69 are invited biannually to mammography using independent double reading. The two main arms of OTST have independent double reading. One 2D arm is modified with CAD and one combo-arm with synthetic $2 \mathrm{D}$. The four arms are read by independent radiologists. From 22.11.2010 to 31.12.2011, 121 screen-detected cancers were found. Results: Cancers detected: Arm A (conventional FFDM) 77, arm B (2D+CAD) 77, arm C (2D+tomo) 101, and arm D (synthetic 2D+tomo) 94. Merging arm A and B ("2D-mode") and C+D ("combo-mode"), 90 cancers were detected by "2D-mode" and 119 by "combo-mode" (32.2\% relative increase). Discordant rate (screendetected cancer with TP score by only one reader) was $28.9 \%$ (26/90) for "2D-mode" and $36.1 \%(43 / 119)$ for "combo-mode". For cancers manifesting as spiculated mass or distortion ( $n=63$ ) the discordant rate was 31.0\% (13/42) for "2D-mode" and 30.6\% $(19 / 62)$ for "combo-mode". For cancers with microcalcifications the values were $25.7 \%(9 / 35)$ and $39.5 \%(17 / 43)$, respectively.

Conclusion: Combined FFDM and tomosynthesis showed a $32 \%$ relative increase in cancer detection as compared with FFDM. Discordant cancer rate was approximately the same or higher for "combo-mode" as compared with "2D-mode". Independent double reading should be the standard of care when tomosynthesis is implemented into mammography screening.

\section{Author Disclosures:}

P. Skaane: Equipment Support Recipient; Equipment support by Hologic. Research/Grant Support; Hologic supported with equipment and financial support for overtime reading. M. Krager: Research/Grant Support; Financial support from Hologic for overtime reading. U. Haakenaasen: Research/ Grant Support; Financial support from Hologic for overtime reading. M. Izadi: Research/Grant Support; Financial support from Hologic for overtime reading I.N. Jebsen: Research/Grant Support; Financial support from Hologic for overtime reading. U. Ekseth: Research/Grant Support; Financial support from Hologic for overtime reading. E.B. Eben: Research/Grant Support; Financial support from Hologic for overtime reading.

\section{B-0818 11:51}

The STORM trial of screening mammography: screening with tomosynthesis or standard mammography

S. Ciatto (posthumous) ${ }^{1}$, N. Houssami ${ }^{2}$, D. Bernardi', F. Caumo ${ }^{3}$, P. Macaskill²;

${ }^{1}$ Trento/IT, ${ }^{2}$ Sydney/AU, ${ }^{3}$ Verona/IT (nehmath@med.usyd.edu.au)

Purpose: To examine the incremental effect of adding 3D-mammography with tomosynthesis to standard 2D digital mammography in population breast screening. Methods and Materials: STORM is a prospective comparative study of integrated $2 \mathrm{D} / 3 \mathrm{D}$ and $2 \mathrm{D}$-only mammography that investigated screening in two phases [2D relative to 2D/3D obtained with Combo ${ }^{\circledast}$, yielding paired data for each screening examination. Women aged 349 years who attended population-based screening services in Trento and Verona (Italy), from September 2011 to March 2012, were included. Screens were interpreted sequentially using standard double-reading, with recall of a positive screen at either reading phases. Paired proportions were compared using McNemar's Chi-square test.

Results: Based on 5000 screens, 33 breast cancers were detected: 23 cancers were detected at both $2 \mathrm{D}$ and $2 \mathrm{D} / 3 \mathrm{D}$ reading, 10 cancers were detected only with integrated $2 \mathrm{D} / 3 \mathrm{D}$ screening, and no cancers were detected only at $2 \mathrm{D}$ reading $(\mathrm{p}=0.002)$; incremental detection attributable to $2 \mathrm{D} / 3 \mathrm{D}$ screening was $2.0 / 1000$ screens (95\% Cl 0.8-3.2/1000) or an incremental 30.3\% (95\% Cl 15.6-48.7\%) of cancers. There were 299 false positive (FP) recalls: 141 were FP at both reading phases, 106 were FP at $2 \mathrm{D}$-only, and 52 were FP at 2D/3D reading $(p<0.001)$. Had recall been conditional to 2D/3D screen-positivity, FP recalls would have been reduced by $18.1 \%$ (95\% Cl 13.9-22.9\%) without missing any of the breast cancers identified to date in the study population.

Conclusion: Integrated 2D/3D mammography has the potential to enhance breast screening outcomes by improving cancer detection as well as by reducing FP recalls. Randomised trials of 2D/3D screening versus standard 2D screening are needed.

Author Disclosures:

N. Houssami: Grant Recipient; Receives research support via National Health and Medical Research Council program grant 633003 to the Screening \& Test Evaluation Program. Other; Travel support was provided by Hologic (USA/ltaly) for attendance at collaborators/research meetings. D. Bernardi: Equipment Support Recipient; Technologic support for trial from Hologic USA/ttaly. Other; Travel support from Hologic to attend collaborators/research meetings. F. Caumo: Equipment Support Recipient; Technologic support for the trial received through Hologic. Other; Travel support to collaborators meetings was received through Hologic. P. Macaskill: Grant Recipient; Receives research support through National Health and Medical Research Council program grant 633003 to STEP.

10:30 - 12:00

Room G/H

Genitourinary

\section{SS 1707}

\section{Hints on haematuria and adrenals for all}

Moderators:

O. Nikolic; Novi Sad/RS

H. Sugimura; Miyazaki/JP

\section{B-0819 10:30}

Impact of patient mobilisation on interpretation of upper urinary tract filling defects seen on MDCT urography

J.M. Froehlich, A. Joeres, P.J. Probst, F. Nussberger, H.C. Thoeny; Berne/CH (froehlich@klus-apotheke.ch)

Purpose: To evaluate whether patient mobilisation before the excretory phase has an impact on the misinterpretation rate of upper urinary tract filling defects seen on MDCT urography.

Methods and Materials: 641 consecutive patients undergoing contrast-enhanced CT urography (Philips ASH,120 kVp,250 mA,rotation time $0.75 \mathrm{sec}$ ) examinations for various reasons between Oct 2009 and Dec 2011 were reviewed for upper urinary tract filling defects and lesion location by two readers. After August 2010 all patients ( $n=246$ ) were systematically mobilised after accomplishing the early nephrographic scan. After a standardised preparation with $1 \mathrm{~L}$ of oral water over $60 \mathrm{~min}$, the patient is asked to empty the bladder. Initially the patient receives intravenously (iv) $20 \mathrm{mg}$ of furosemide. Contrast administration consists of a fixed iv volume of $110-120 \mathrm{ml}$ lobitridol $300 \mathrm{mgl} / \mathrm{ml}$ followed by $20 \mathrm{ml}$ saline solution injected at a rate of $2-3 \mathrm{ml} / \mathrm{s}$. After $10 \mathrm{~min}$ and subsequently every $2 \mathrm{~min}$ first low-radiation scouts are acquired 
in order to optimise the depiction of the excretory phase. Standard of reference for comparison was histology or follow-up of at least 6 months.

Results: 63 endoluminal filling defects were detected: 26 of these occured in the group with movement, while 37 were detected in the group without mobilisation. 29 / 63 of the lesions were subsequently confirmed to be tumours, while 27 endoluminal filling defects were contrast media attributed leading to misinterpretation; 23 of these occured in the group without mobilisation, while only 4 were encountered after mobilisation $(p<0.0001)$.

Conclusion: Patient mobilisation is a prerequisite to avoid misinterpretation of filling defects during MDCT.

\section{Author Disclosures:}

J.M. Froehlich: Consultant; Guerbet.

\section{B-0820 10:39}

Evaluation of diagnostic strategies for hematuria using risk stratification N.C. Cowan; Birmingham/UK (nccowan.uro@gmail.com)

Purpose: To evaluate diagnostic imaging strategies for hematuria using a system of risk stratification to determine the choice of imaging technique.

Methods and Materials: The clinical cohort consisted of 840 consecutive patients presenting with nonvisible $(\mathrm{NVH})$ and visible $(\mathrm{VH})$ hematuria. The low-risk cohort consisted of Groups 1\&2: < 40-years with NVH and VH and Group 3: > 40-years with NVH. Group 4 (high-risk) was $>40$-years with VH. Low-risk cohort underwent unenhanced CTKUB and/or ultrasonography; high-risk cohort underwent CT urography. The prevalence of disease according to cohort and diagnostic accuracy of each imaging technique for causative disease were calculated. The reference standard was cystoscopy, histopathology, unenhanced CT, histopathology and imaging follow-up (1-year).

Results: Disease prevalence for Groups 1-4, respectively, was stone disease; 11/83 (13\%), 8/49 (16\%), $61 / 419(15 \%)$ and 56/289 (19\%), renal cell cancer (RCC): $0 \%$ (0/83), 2/49 (4.1\%), 2/419 (0.5\%) and 4/289 (1.4\%). Upper urinary tract urothelial cancer (UTUC) and bladder cancer (BCa) were present only in groups 3 and 4 . The prevalence of UTUC for groups 3 and 4 was $2 / 419(0.5 \%)$ and $3 / 289(1.0 \%)$ and for $\mathrm{BCa}, 4 / 419$ (1.0\%) and 49/289 (17.0\%). For high-risk cohort, CT urography was positive in $46 \%(118 / 256)$ overall and for tumours in $70 / 256(27 \%)$. Sensitivity of CT urography for RCC was $100 \%$, (4/4) and UTUC, $100 \%(3 / 3)$, and specificity was $99 \%(248 / 251)$ and $98 \%(247 / 253)$, respectively.

Conclusion: Risk stratification of clinical cohorts for urothelial cancer reduces redundant diagnostic testing, maintains diagnostic accuracy and reduces costs. CT urography for investigating hematuria should be reserved for populations at high-risk for urothelial cancer.

\section{B-0821 10:48}

\section{Use of novel iterative reconstruction in CT KUB: approach on} improving image quality

V. Vardhanabhuti, C. Roobottom, S. Ilyas; Plymouth/UK (sumaira.ilyas@nhs.net)

Purpose: To compare image quality on computed tomographic (CT) images acquired with filtered backprojection (FBP), adaptive statistical iterative reconstruction (ASIR) and model-based iterative reconstruction (MBIR) techniques in CT KUB examination.

Methods and Materials: 20 patients were scanned with standard protocol CT KUB at our institution. Same raw data were reconstructed using FBP, ASIR and MBIR. Objective and subjective image qualities were assessed. Quantitative data such as objective image noise and mean attenuation were analysed by comparing standard deviations, 95\% confidence interval and calculating percentage difference. Mean image noise values and attenuation values were compared between different reconstruction algorithms using ANOVA. The interobserver variation and percentage agreement between the two radiologists for each of the assessed subjective image quality and lesion assessment parameters were estimated by using weighted k-statistics. Kruskal-Wallis rank sum test was used to test for equality of median scores among all subjective parameters.

Results: Objective image analysis supports significant noise reduction and superior contrast-to-noise ratio with new MBIR technique $(p<0.05)$. Subjective image parameters were maximally rated for MBIR followed by ASIR then FBP and show statistical significance differences $(p<0.05)$.

Conclusion: MBIR shows superior reduction in noise and improved image quality (both objective and subjective analysis) compared with ASIR and FBP in the context of CT KUB scanning which is inherently a noisy study. At same dose, we have shown that image quality can be improved by applying MBIR in clinical practice, and if available should be utilised.

\section{B-0822 10:57}

Renal cell carcinoma subtype differentiation on single-phase contrast-enhanced CT

F. Veloso Gomes' ${ }^{1}$, J. Palas², A. Matos², V. Mascarenhas ${ }^{3}$, V. Herédia ${ }^{4}$, S. Duarte ${ }^{3}$, M. Ramalho ${ }^{2} ;{ }^{1}$ Faro/PT, ${ }^{2}$ Almada/PT, ${ }^{3}$ Lisbon/PT, ${ }^{4}$ Évora/PT (fvgomes@gmail.com)

Purpose: To decrease the radiation dose, many abdominal computed tomography (CT) protocols exclude the unenhanced phase. Our purpose was to compare the ability to differentiate renal cell carcinoma (RCC) subtype between multiphasic and arterial single-phase post-contrast acquisitions, using previously described and novel indices using the psoas muscle as a surrogate.

Methods and Materials: A multicentric, retrospective study was performed. At surgery, 80 renal tumours were identified (53 clear-cell, 13 papillary and 14 chromophobe). Region-of-interest measurements within tumour, uninvolved renal cortex and the ipsilateral psoas muscle, on unenhanced and corticomedullary phases were used to calculate tumour-percentage-enhancement (TE) and tumourto-cortex-enhancement (TCl) indices. One-way ANOVA for independent samples was used to test for mean differences between the three tumour subtypes. Receiver operating characteristic $(\mathrm{ROC})$ curve analysis was performed for the comparison of clear cell and papillary RCCs.

Results: Multi-phase contrast-enhanced-CT revealed significantly lower mean TE in papillary tumours in comparison with clear-cell $(p<0.01)$. The TCl was also lower for papillary tumours $(p<0.01)$. Papillary tumours can be distinguished from clear-cell tumours with $\mathrm{TE}<53 \%$ and $\mathrm{TCK}<0.22$ (area under ROC curve of 0.997 and 0.995, respectively). Single-phase contrast-enhanced-CT revealed a lower mean TE for papillary tumours when compared with both clear-cell and cromophobe $(p<0.01)$. Papillary tumours can be distinguished from clear-cell with a $T E<23 \%$ (AUC under ROC curve 0.954), but not with TCl.

Conclusion: Subtype RCC differentiation can be assessed on a single-phase contrast-enhanced-CT study when unenhanced $\mathrm{CT}$ is not available. Papillary tumours can be distinguished from clear-cell tumours with high sensitivity and specificity.

\section{B-0823 11:06}

To assess parameters within $\mathrm{CT}$ findings to aid distinction of histological subtype of renal cell carcinoma within our institute M.J. Conneely, D. Ferguson, C. Donagh, M. Quinlan, T. McHale, P. McCarthy; Galway/IE (m.conneely11@nuigalway.ie)

Purpose: Early determination of histological subtype of renal cell carcinoma optimises patient management. Using CT, we aim to assess parameters to aid diagnosis of histological subtype within our institute.

Methods and Materials: A retrospective chart review was performed to identify patients who underwent nephrectomy, between 2009 and 2011, for renal cell carcinoma. Review of associated imaging was performed, by a consultant uroradiologist. Note was made of the presence of necrosis, calcification and degree of arterial and delayed enhancement present on CT studies performed preoperatively. Comparison with histological findings was undertaken.

Results: 115 patients were identified of which $84 \%, 10.4 \%$ and $5.6 \%$ of patients were diagnosed with clear cell, papillary and chromophobe subtype, respectively. Necrosis was present in $80 \%$ of tumours with necrosis most prevalent in clear cell subtype. Calcification was more prevalent in papillary subtype $(25 \%)$ when compared with clear cell $(14.4 \%)$. Using combined values of increased arterial phase enhancement of $<25 \mathrm{HU}$ and decreased delayed phase attenuation of $<$ $10 \mathrm{HU}$, the sensitivity and specificity for differentiating papillary cell subtype were $80 \%$ and $100 \%$, respectively. Using the converse attenuation values (i.e. $>25 \mathrm{HU}$ and $>10 \mathrm{HU}$ ), the sensitivity and specificity for differentiating clear cell carcinoma within our study were $79 \%$ and $67 \%$. Chromophobe tumours demonstrated a more uniform enhancement pattern within the tumour, but with varying values of enhancement/washout.

Conclusion: Using attenuation values stated, CT can aid the determination of papillary renal cell carcinoma histological subtype, within our institute, given their reduced levels of enhancement/washout. 


\section{B-0824 11:15}

MDCT in differentiating PT1-2 from $\mathrm{pT} 3$ tumours in clear cell RCC

O. Le, C. Wood, P. Tamboli, R. Vikram; Houston, TX/US

(ott.le@mdanderson.org)

Purpose: This study aimed at determining the accuracy of multi-detector computed tomography (MDCT) in distinguishing pT1-2 from pT3 clear cell renal cell cancer (cRCC).

Methods and Materials: 66 consecutive patients who had radical nephrectomy for CRCC with preoperative staging CT using dual injection technique were included. The study included non-contrast, corticomedullary and combined nephrographic/ excretory phases (from two separate contrast injections; 0.625-1.25 mm). Sagittal and coronal reconstructions were performed. Involvement of calyces, renal sinus fat, perinephric fat \& renal vein were assessed by two blinded readers and correlated with final pathology results. Distortion or clear invasion and not mere abutment were considered to indicate invasion of calyces or renal sinus fat. Presence of a tumour thrombus or encasement by more than 180 degrees of the cross-sectional circumference of renal vein or its branches was considered a sign of renal vein invasion. Results: The sensitivities and specificities on CT were renal sinus fat $97 \%$ and $24 \%$; calyces $100 \%$ and $15 \%$; perinephric fat $46 \%$ and $72 \%$; renal vein $90 \%$ and $59 \%$. Conclusion: MDCT has high sensitivity but poor specificity in predicting renal calyceal, renal sinus fat and renal hilar vein involvement. CT is poor in predicting perinephric fat involvement.

\section{B-0825 11:24}

Reader variability in urinary stone size estimation: training does not help M. Lidén, T. Andersson, H. Geijer; Örebro/SE (matsliden@yahoo.com)

Purpose: Within narrow limits, the CT estimated size influences the prognosis for spontaneous passage of a ureteral calculus. In the present experiment, we tried to reduce the inter-reader variability in urinary calculus size estimation by measuring the variability before, immediately after and late after a training session.

Methods and Materials: A retrospectively generated anonymous urinary calculi CT image data bank consisting of 50 unenhanced CT-stacks demonstrating ureteral or kidney calculi was used. Eleven readers, radiologists and residents (mean experience 13 years) independently estimated the largest axial diameter of the calculi in three sessions: baseline, post-training and follow-up. Immediately before the post-training session each reader received training on eight calculi aimed at reducing inter-reader variability without affecting the mean reader size estimate. Follow-up session was performed four to eight weeks later without further training. Results: Per-calculus standard deviation between readers was $0.44,0.42$ and 0.51 $\mathrm{mm}$ and inter-reader limits of agreement were $\pm 1.3, \pm 1.2$ and $\pm 1.5 \mathrm{~mm}$ for baseline, post-training and follow-up session, respectively. Friedman test showed significant difference for per-calculus standard deviation $(p<0.001)$. Pairwise Wilcoxon signedranks test showed increased variability in follow-up session compared to baseline $(p<0.001)$ and post-training session $(p<0.001)$. The small reduction in variability in post-training session compared to baseline was not statistically significant.

Conclusion: The small statistically non-significant reduction of inter-reader variability from the tested training protocol had no long-term effect, where an increase in variability was seen. Other approaches such as reader-independent urinary calculus size estimation seem more promising for reduction of inter-reader variability.

\section{B-0826 11:33}

Contrast-enhanced CT and chemical shift MRI: accuracy for lipidpoor adenoma characterisation

Y.A. Choi, B.K. Park, J.M. Seo, S.Y. Park, C.K. Kim; Seoul/KR

(ya84.choi@samsung.com)

Purpose: To compare the accuracy of contrast-enhanced CT and chemical shift MRI for diagnosing lipid-poor adenoma.

Methods and Materials: Fifty-six hyperattenuating adrenal masses $(>10 \mathrm{HU})$ on unenhanced CT images were found in 56 patients ( 41 men and 15 women; mean age, 54.6 years) by reviewing radiologic reports. These lesions were retrospectively analysed with contrast-enhanced CT and chemical shift MR. The diagnostic accuracy of CT using absolute percentage loss of enhancement (PLE) and relative PLE and of chemical shift MRI using adrenal-to-spleen ratio (ASR) or signal intensity index (SII) were obtained to determine which modality was more accurate for lipid-poor adenoma. For CT, an adenoma was diagnosed if a mass had an absolute PLE $>60 \%$ or a relative PLE $>40 \%$. For MR, an adenoma was diagnosed if a mass had an ASR $<0.71$ or an SII $>16.5 \%$. Either follow-up imaging or pathologic review was considered standard reference. McNemar test was used to compare $\mathrm{CT}$ and $\mathrm{MR}$ regarding accuracy for adenoma.
Results: Hyperattenuating adrenal masses included 40 adenomas and 16 nonadenomas. The sensitivity, specificity, and accuracy for adenoma at CT were 100\% (40/40), $75 \%$ (12/16), and $93 \%$ (52 of 56), respectively, and at MRI were $75 \%$ (30/40), 56\% (9/16), and 70\% (39/56), respectively. CT helped identify 10 more adenomas and three more non-adenomas than did MR. There was significant difference between CT and MRI regarding accuracy for adenoma $(p=0.0044)$. Conclusion: Contrast-enhanced CT is better than chemical shift MRI for characterising lipid-poor adenoma.

\section{B-0827 11:42}

Functional MRI with diffusion-weighted imaging and ADC map in characterisation of undetermined adrenal lesions: comparison with chemical shift imaging

C.R.G.L. Talei Franzesi, D. Ippolito, P.A. Bonaffini, C. Capraro, V. Besostri, S. Sironi; Monza/lT (ctfdoc@hotmail.com)

Purpose: To evaluate the diagnostic performance of diffusion-weighted imaging and apparent diffusion coefficient value, in the characterisation of indeterminate adrenal lesions.

Methods and Materials: A prospective study was conducted in 83 patients with different adrenal lesions (adenomas, myelolipomas, adrenal metastasis and primitive carcinomas). All patients underwent MRI scan (1.5 T, Achieva, Philips) with a phased array multi-coil using a conventional protocol that included also diffusion-weighted imaging (DWI) with b-value of $0,300,500$ and $1000 \mathrm{~mm} 2 / \mathrm{sec}$. DWI images were compared with T1 FFE in- and out of phase. The mean ADC value of adrenal lesions were calculated and correlated with MRI findings. ROI were manually drawn along the contours of adrenal lesion on ADC maps. The obtained data were compared and correlated with chemical shift images and then statistical analysis was performed.

Results: The diagnosis of the adrenal lesions was proved by follow-up examination (size increase of over a period of time) or through biopsy (oncologic patients). All adrenal lesions showed restriction of diffusion and motion of water protons on DWI images. There was no significant difference in obtained ADC values between adenomas (mean value: $1150 \pm 95.4 \mathrm{~mm} 2 / \mathrm{sec}$ ), myelolipomas (mean value: $1263 \pm 210.8$ $\mathrm{mm} 2 / \mathrm{sec}$ ) and primitive tumour (mean value: $1124 \pm 78.5 \mathrm{~mm} 2 / \mathrm{sec}$ ). The adrenal metastases (mean value: $1050 \pm 87.5 \mathrm{~mm} 2 / \mathrm{sec}$ ) presented lower ADC value in comparison to the other adrenal lesions but not statistical significant $(p>0.05)$. Conclusion: The diffusion-weighted images and ADC map were not useful to differentiate between benignant and malignant adrenal lesions. The chemical shift imaging technique is still the most helpful sequences in typing of indeterminate adrenal lesions.

\section{B-0828 11:51}

Non-contrast-enhanced MR adrenal venography for adrenal venous sampling

H. Ota, K. Takase, N. Satani, T. Kimura, K. Seiji, Y. Ono, R. Morimoto, F. Sato,

S. Takahashi; Sendai/JP (h-ota@rad.med.tohoku.ac.jp)

Purpose: In patients with primary aldosteronism, adrenal venous sampling (AVS) is essential to distinguish unilateral aldosterone producing adenoma from bilateral idiopathic adrenal hyperplasia. Identification of bilateral adrenal veins, especially the right adrenal vein that has anatomical variations and is smaller than the left one, is important for successful AVS. This study aims to identify the right adrenal vein using non-contrast-enhanced MR imaging at $3 \mathrm{~T}$.

Methods and Materials: Consecutive 28 patients scheduled for AVS underwent MR scans at $3 \mathrm{~T}$. A three-dimensional respiratory-triggered true fast imaging with steady-state precession sequence with transverse acquisition was used for MR adrenal venography. The image quality of the right adrenal vein was evaluated on a five-point scale (1=invisible, $5=$ excellent). The level of orifice was documented as upper, middle or lower third of a vertebra. Presence of a common trunk of the right adrenal vein with an accessory hepatic vein was documented. MR findings were compared with catheter venography at AVS.

Results: MR venography demonstrated right adrenal veins in all the patients $(100 \%)$. AVS was successful in all the patients $(100 \%)$. Mean image quality was 3.8 (range 2-5). The right adrenal vein formed a common trunk with the accessory hepatic vein in two cases $(7 \%)$, which were confirmed by catheter venography. The orifices on MR venography were located between T11 and Th12 vertebrae. Differences of the orifice levels between MR and catheter venography ranged within two thirds of vertebrae, which were explained by respiratory motion.

Conclusion: Non-invasive MR adrenal venography is useful for planning of AVS. 
10:30 - 12:00

Room I/K

\section{Abdominal Viscera}

\section{SS 1701b}

\section{DWI in pancreatic and hepatobiliary diseases}

Moderators:

G.J. Munneke; London/UK

D. Weishaupt; Zurich/CH

\section{B-0829 10:30}

Feasibility of abdominal diffusion Kurtosis imaging compared to standard diffusion weighted imaging at 1.5 and 3 Tesla

J. Hansmann, A. Lemke, J. Wambsganss, S.O. Schönberg, U. Attenberger;

Mannheim/DE (jan.hansmann@medma.uni-heidelberg.de)

Purpose: To demonstrate the feasibility of diffusion kurtosis imaging (DKI) for the depiction and differentiation of liver and kidney abnormalities in comparison to standard diffusion weighted imaging (DWI) at 1.5 and $3 \mathrm{~T}$.

Methods and Materials: 109 consecutive patients underwent a routine abdominal MR protocol including standard DWI and DKI. 1.5 and $3 \mathrm{~T}$ b-values for DKI were identical: $b=0-100-500-1000-1500-2000 \mathrm{~s} / \mathrm{mm}^{2} .55$ liver and 36 kidney lesions were evaluated at $1.5 \mathrm{~T}$, and 16 liver and 11 kidney lesions, respectively, at $3 \mathrm{~T}$. DKI was assessed by an in-house built software. Kurtosis values were quantified by region-of-interest analysis and compared between lesions and normal parenchyma by a Wilcoxon rank sum test.

Results: Mean kidney kurtosis values were identical at 1.5 and $3 \mathrm{~T}$ in normal parenchyma $(K=0.5)$ and cysts $(K=0.4)$. Differences dependent on field strength were only found in malignant tumours ( 0.8 vs 0.5$)$. At 1.5 as well as $3 \mathrm{~T}$, cysts, tumours and normal kidney parenchyma could be differentiated with significance $(p<0.0413)$ using the kurtosis values. At both field strengths, cysts, benign and malignant liver tumours could be differentiated with statistical significance from normal parenchyma $(p<0.001, p<0.0071, p<0.001)$. However, malignant and benign liver tumours were significantly different at $1.5 \mathrm{~T}(\mathrm{p}=0.0382)$. Abnormalities and normal parenchyma could be visually differentiated on both, DWI and DKI. Conclusion: DKI of kidney and liver abnormalities is feasible at both, 1.5 and $3 \mathrm{~T}$ and allows for a quantified differentiation between normal parenchyma and pathologic lesions.

\section{Author Disclosures:}

U. Attenberger: Consultant; Bayer Healthcare.

\section{B-0830 10:39}

Differential diagnosis of benign from malignant distal biliary strictures: value of adding diffusion-weighted imaging to conventional magnetic resonance cholangiopancreatography R.-E. Yoo, J. Lee, J. Yoon, J. Kim, J. Han, B. Choi; Seoul/KR (ry_jjang@hotmail.com)

Purpose: To determine the value of adding diffusion-weighted imaging (DWI) to magnetic resonance cholangiopancreatography (MRCP) in differentiating benign from malignant distal biliary strictures.

Methods and Materials: Two independent readers reviewed three image sets (1: MRCP alone, 2: MRCP and DWI combined, and 3: MRCP, DWI, and contrastenhanced T1-weighted imaging (T1WI) combined) of sixty patients with suspected distal biliary strictures at two-week interval and rated the probability of malignancy. Diagnostic performance (Az value) and accuracy were compared using the ROC curves and McNemar test. Additionally, accuracy was also analysed for two subgroups (group 1: sphincter of Oddi stricture and group 2: distal bile duct stricture). $\mathrm{K}$ coefficients were calculated to assess the interobserver agreement.

Results: Az value and accuracy improved significantly after additional review of DWI for both readers: $A z=0.780$ vs. $0.916(P=.003)$ for reader 1 and 0.784 vs. $0.853(P=.037)$ for reader 2 ; accuracy $=69 \%$ vs. $93 \%$ for reader $1(P<.001)$ and $57 \%$ vs. $85 \%$ for reader $2(P<001)$. For both readers, no significant difference in the Az value and accuracy was found between MRCP and DWI combined and MRCP, DWI, and contrast-enhanced T1WI combined (P>.200). In subgroups, additional review of DWI improved the accuracy of both readers with or without statistical significance. There was substantial interobserver agreement in all three image sets ( $\mathrm{k}=0.695-0.732$ ).

Conclusion: Addition of DWI to MRCP significantly improved diagnostic accuracy in the characterisation of distal biliary strictures. The diagnostic performance of $\mathrm{MRCP}$ and DWI combined was comparable with that of MRCP, DWI, and contrastenhanced MRI combined.

\section{B-0831 10:48}

Correlation of measurements of diffusion-weighted MR imaging and Gd-EOB-DTPA-enhanced MR imaging in hepatic malignant tumours: $A D C$ versus signal intensity on hepatobiliary phase S. Choi, Y. Park, C. Lee, K. Kim, J. Choi, J. Lee, C. Park; Seoul/KR (monetoile84@hanmail.net)

Purpose: To evaluate correlation of apparent diffusion coefficient (ADC) on diffusion-weighted imaging (DWI) and signal intensity (SI) on hepatobiliary phase with Gd-EOB-DTPA-enhanced MRI in malignant hepatic tumours.

Methods and Materials: A total 63 patients with malignant hepatic tumours (30 hepatocellular carcinoma ( $\mathrm{HCC}$ ), 9 cholangiocarcinoma (CCC), 41 colorectal cancer metastasis (CM), 9 breast cancer metastasis (BM)) underwent both DWI and GdEOB-DTPA-enhanced MRI. Mean ADC of tumours was measured by averaging three $\mathrm{ROI}$ measurements. Lesion-to-liver SI ratio of tumour on hepatobiliary phase was evaluated with ROI measurement. EOB-cloud of tumour was considered when lesion-to-liver SI ratio on hepatobiliary phase was $>0.6$. We evaluated incidence of EOB-cloud of malignant hepatic tumour. Mean ADCs between hepatic tumours with EOB-cloud and without EOB-cloud were evaluated using t-test. We also evaluated difference of mean ADC between malignant hepatic tumours with EOB-cloud using Kruskal-Wallis test.

Results: EOB-cloud was noted in 34 cases $(34 / 89,38.2 \%)$, which included 16 $\mathrm{HCC}(16 / 30), 8 \mathrm{CCC}(8 / 9), 7 \mathrm{CM}(7 / 41)$, and $3 \mathrm{BM}(3 / 9)$. Mean ADC was $1.22 \pm 0.29$ $\left(\times 10^{-3} \mathrm{~mm}^{2} / \mathrm{sec}\right)$ in 34 tumours with EOB-cloud and $1.25 \pm 0.46$ in 55 tumours without EOB-cloud. Mean ADC was not significantly different between these two groups $(P=0.07)$. Among malignant hepatic tumours with EOB-cloud, mean ADC was $1.12 \pm 0.23$ in $\mathrm{HCC}, 1.32 \pm 0.33$ in CCC, $1.18 \pm 0.24$ in $\mathrm{CM}$, and $1.56 \pm 0.42$ in BM. There was no significant difference between these tumours $(P=0.22)$.

Conclusion: EOB-cloud is not infrequently observed in malignant hepatic tumours. Mean ADC values were not significantly different between tumours with EOB-cloud and without EOB-cloud. Therefore, SI on hepatobiliary phase might not reflect ADC related to perfusion and diffusion factors.

\section{B-0832 10:57}

Non-invasive assessment of solid benign and malignant focal liver lesions by quantitative analysis of functional MRI with diffusionweighted imaging

M. Colombo, D. Ippolito, P.A. Bonaffini, C. Trattenero, V. Bartolo, S. Sironi; Monza/IT (maddalenacolombo1982@gmail.com)

Purpose: To assess the added value of functional sequences, using diffusionweighted imaging and apparent diffusion coefficient value, to conventional MRI imaging examination in the differential diagnosis from benign and malignant focal liver lesions.

Methods and Materials: A total of 53 patients with known focal liver lesions including 36 benign ( 25 angiomas, $8 \mathrm{FNH}, 2$ abscesses) and 18 malignant (9 metastases, 7 HCCs, 2 cholangiocarcinomas) underwent 1.5-T MRI examination (Achieva, Philips). The study was performed with a phased array multi-coil using a conventional protocol that included also diffusion-weighted imaging (DWI) with different $b$-values. The ROls were manually drawn on focal liver lesions and on surrounding normal hepatic parenchyma in ADC maps, and statistical analysis of $A D C$ mean values was performed.

Results: Lesions were proved either by follow-up (benign lesions) or biopsy (malignant lesions). Following mean ADC values were obtained for different types of benign lesions: angiomas (1794.27 $\pm 125,2 \times 10-3 \mathrm{~mm} 2 / \mathrm{sec}), \mathrm{FNH}(1486.74 \pm 156$, $8 \times 10-3 \mathrm{~mm} 2 / \mathrm{sec})$, abscesses $(1133 \pm 145,5 \times 10-3 \mathrm{~mm} 2 / \mathrm{sec})$ while in the neoplastic lesions were: metastases $(1025,6 \pm 93 \times 10-3 \mathrm{~mm} 2 / \mathrm{sec}), \mathrm{HCCs}(887,6 \pm 71,10 \times 10-3$ $\mathrm{mm} 2 / \mathrm{sec}$ ) and cholangiocarcinomas (1285 $\pm 25 \times 10-3 \mathrm{~mm} 2 / \mathrm{sec})$. In normal hepatic parenchyma, mean perfusion values were $(1158,4 \pm 158,7 \times 10-3 \mathrm{~mm} 2 / \mathrm{sec})$. A statistical higher values $(p<0.001)$ were obtained in diffusion parameters among benign than those of malignant lesions (1563 \pm 142 , 4 vs $1066 \pm 138$, 3x10-3 mm2/sec). Conclusion: Diffusion-weighted images offer useful quantitative information (by evaluation of $A D C$ maps) that differs significantly in solid and malignant liver lesions, due to tumour different vascularity and cellularity. 


\section{B-0833 11:06}

Quantitative data analysis of the diffusion-weighted MR-images in the differential diagnosis of metastatic liver disease of colorectal and pancreatic aetiology

Y. Savchenkov, S. Bagnenko, G. Trufanov, V. Fokin; St. Petersburg/RU (yura_savchenkov@mail.ru)

Purpose: To develop differential diagnosis metastatic liver disease on the basis of quantitative data analysis of DWI.

Methods and Materials: 57 metastatic liver lesions colorectal (37 lesions) and pancreatic (20 lesions) aetiology were analysed in 20 patients. We used DWI with the factors b-50,300,800,1200,1500s $/ \mathrm{mm} 2$. All measurements were carried out within a single axial slice (for each pulse sequence separately). We used the coefficients, calculated on the basis of the ratio measured values in several organs, in different parts of the liver or in one and the same region of the liver.

Results: The most informative in the native study is the ratio of $\mathrm{SI}_{\text {esionb300 }} / \mathrm{SI}_{\text {aorta b300, }}$, $\mathrm{SI}_{\text {lesionb50 }} / \mathrm{SI}_{\text {aortab50 }}$ и SI $\mathrm{I}_{\text {eesion50 }} / \mathrm{SI}_{\text {liverb50 }}(\mathrm{p}<0.001)$. Somewhat less informative demonstrated is the ratio of $\mathrm{SI}_{\text {lesionb300 }} / \mathrm{SI}_{\text {liverb300 }}$ n $\mathrm{ADC}_{\text {lesion50-300-800 }}(\mathrm{p}<0.001$ and $\mathrm{p}=0.003)$. Evaluation of the mean values for group informative indicator ratios on the maps of the ADC (b-50,3008,800s/mm2) revealed the distinctive features. The metastatic lesions of pancreatic aetiology characterised by higher value $A D C$ in comparison with colorectal metastases are (115.75 \pm 10.9$) \times 10-5 \mathrm{~mm} 2 / \mathrm{s}$ and $(77.46 \pm 6.81) \times 105$ $\mathrm{mm} 2 / \mathrm{s}$ accordingly. Similar changes were observed in the following relations: $\mathrm{SI}_{\text {lesionb50 }} / \mathrm{SI}_{\text {aortab5050 }}, \mathrm{SI}_{\text {lesionb300 }} / \mathrm{Sl}_{\text {aortab30 } 0}, \mathrm{SI}_{\text {lesionb50 }} / \mathrm{SI}_{\text {liverb50 }}, \mathrm{SI}_{\text {lesionb300 }} / \mathrm{SI}_{\text {liver b300 }}$ (pancreatic aetiology $-5.39 \pm 0.72,4.12 \pm 0.52,3.76 \pm 0.55,2.78 \pm 0.35$, accordingly; colorectal aetiology $-2.71 \pm 0.19,2.17 \pm 0.13,1.88 \pm 0.14,1.7 \pm 0.1$.

Conclusion: DWI is a promising additional method allowing to make progress in the issue of the differential diagnosis of metastatic liver lesion of colorectal and pancreatic aetiology.

\section{B-0834 11:15}

Does the ADC-map eradicate the T2-shine-through effect affecting DW images of focal liver lesions? A study with exponential ADC-maps

R. Girometti, M. Maieron, S. Pullini, E. Minighin, L. Cereser, G. Como,

M. Bazzocchi, C. Zuiani; Udine/IT (rgirometti@sirm.org)

Purpose: In clinical practice, the Apparent Diffusion Coefficient (ADC)-map is supposed to eradicate the T2-shine-through effect (T2-STE) potentially affecting the Diffusion-weighted (DW) signal of focal liver lesions (FLLs) at higher b-value. We investigated whether this assumption is valid.

Methods and Materials: Included were forty-five patients with 73 focal liver lesions (54 malignant and 19 benign) detected on a $1.5 \mathrm{~T}$ system (cysts and haemangiomas were excluded from analysis). DW examination was performed using a single-shot Echoplanar sequence with b values of 50,500 and $800 \mathrm{sec} / \mathrm{mm}^{2}$, leading to the generation of (a) the ADC-map, using a 3-point regression analysis and (b) the exponential ADC-map (E-ADC-map), using the b800/b50 images ratio, which provides a diffusion-weighted image with complete eradication of T2-relaxation time effects. By performing an analysis of differential signal patterns, two experienced radiologists in consensus estimated the prevalence of the T2-STE when combining DW images with the ADC-map or E-ADC-map.

Results: The number of FLLs affected by the T2-STE was significantly larger $(p<$ 0.05 ;McNemar test) as assessed by the E-ADC-map (41/73; $56.7 \%$; $95 \%$ C.I. 44.0 $67.7)$ than the ADC-map (35/73; $47.9 \%$, 95\%C.I. 36.1-59.9). Additional T2-STE was found by E-ADC-map in the case of 1 benign ( 1 focal nodular hyperplasia) and 5 malignant ( 3 hepatocarcinomas and 2 metastases) FLLs, respectively.

Conclusion: The ADC-map is unsuited to correct for all cases of T2-STE affecting DW images. E-ADC-map should be alternatively used in the visual analysis of FLLs.

\section{B-0835 11:24}

Prevalence of the T2-shine-through effect on DW images of focal

liver lesions: a study with exponential ADC-maps at $1.5 \mathrm{~T}$

R. Girometti, M. Maieron, E. Minighin, S. Pullini, L. Cereser, G. Como,

M. Bazzocchi, C. Zuiani; Udine/IT (rgirometti@sirm.org)

Purpose: The aim of this study was to assess the prevalence of the T2-shinethrough effect (T2-STE) when imaging focal liver lesions (FLLs) with Diffusion Weighted (DW) Magnetic Resonance Imaging at 1.5 T.

Methods and Materials: We retrospectively enrolled forty-five patients with 73 focal liver lesions (54 malignant and 19 benign) detected on a highly performing $1.5 \mathrm{~T}$ system (cysts and haemangiomas were excluded from analysis). DW examination was performed using a single-shot Echoplanar sequence with $b$ values of 50 , 500 and $800 \mathrm{sec} / \mathrm{mm}^{2}$. Exponential ADC-map (E-ADC-map) was then generated using the b800/b50 DW images ratio, which provides a diffusion-weighted image with complete eradication of T2-relaxation time effects. T2-STE was assessed as present when lesion hyperintensity on b800 DW image corresponded to iso- to hypo-intensity on the E-ADC-map. Analysis of prevalence was stratified for benign and malignant FLLs.

Results: The prevalence of the T2-STE was high, involving 41 of 73 FLLs $(56.7 \%$; $95 \%$ C.I. 44.0-67.7). No significant difference ( $p>0.05$; Fisher exact test) was found between the prevalence of T2-STE in benign (10/19; $52.6 \%$, 95\%C.I. 28.8-75.5) vs. malignant $(31 / 54 ; 57.4 \%, 95 \%$ C.I. 43.2-70.7) FLLs. Among malignant FLLs, the T2-STE occurred with a comparable frequency both in hepatocarcinoma $(n=17)$ and metastases $(n=14)$.

Conclusion: The T2-STE occurred in more than one-half of lesions in our series, suggesting that contamination of DW signal from the T2 relaxation time still occurs at higher b-values currently used at $1.5 \mathrm{~T}$.

\section{B-0836 11:33}

DWI magnetic resonance imaging in the evaluation of $\mathrm{HCC}$ recurrence after trans-arterial chemoembolisation (TACE): comparison with contrast-enhanced MR imaging

M. Di Martino, R. Di Miscio, M. Del Monte, C. Lombardo, D. Geiger, C. Catalano; Rome/IT (dimase81_6@hotmail.com)

Purpose: To qualitatively compare contrast-enhanced MR Imaging and DWI MR Imaging in the evaluation of HCC recurrence in patients treated with TACE in patients in list for liver transplantation.

Methods and Materials: Thirty cirrhotic patients with $80 \mathrm{HCCs}$ underwent MR Imaging at 1.5 T. MR acquisitions comprised unenhanced breath-hold T2W images and volumetric 3D Gd-BOPTA-enhanced ( $0.1 \mathrm{mmol} / \mathrm{kg}$; MultiHance ${ }^{\circledR}$, Bracco) T1W GRE images acquired at $25 \mathrm{~s}, 60 \mathrm{~s}, 180 \mathrm{~s}$ (dynamic phase). DWI was performed by a breath-hold SSEPI sequence (TR $=2028, T E=76$, matrix $=192 \times 125$, slice thickness $5.00 \mathrm{~mm}$, GRAPPA, b-values $0,50,500,800 \mathrm{sec} / \mathrm{mm} 2)$. Three datasets of images were independently read by two readers: 1) Precontrast sequences plus DWI, 2) precontrast and post-contrast sequences, 3) precontrast plus post-contrast and DWI sequences. All patients underwent liver transplantation. Sensitivity, specificity and diagnostic were calculated for statistical analysis and compared with each other. Results: Good interobserver agreement was found. The third dataset of images reported a trend to a better mean sensitivity ( $8 \%$ vs $85 \%$ ) specificity ( $92 \%$ vs $89 \%$ ) and diagnostic accuracy (.89 vs.87) compared to the second datasets. The first dataset was significantly inferior to second and third one.

Conclusion: The combined interpretation of post-contrast and DWI images helps to a better diagnostic accuracy in the evaluation of tumour recurrence after TACE.

\section{B-0837 11:42}

MRI of pancreatic adenocarcinoma including diffusion-weighted imaging: assessment of tumour conspicuity and pathologic correlation L. Legrand, V. Duchatelle, V. Molinié, I. Boulay-Coletta, E. Sibileau, M. Zins; Paris/FR (legrand.lau@gmail.com)

Purpose: To identify the best MR sequences for pancreatic adenocarcinoma conspicuity and determine whether MR signal and apparent diffusion coefficient (ADC) values correlate with pathological findings.

Methods and Materials: 25 consecutive patients with pathologically proven pancreatic adenocarcinoma who underwent MRI (1.5 or $3 \mathrm{~T}$ ) before surgical resection were included. Fat-suppressed (FS) T1-weighted gradient-echo (GRE), FS T2-weighted fast-recovery fast spin echo, 3D FS dynamic T1-weighted gadolinium-enhanced GRE during arterial, portal and delayed phases and diffusion-weighted imaging (DWI) with b values of 0 and 600 or $800 \mathrm{sec} / \mathrm{mm} 2$ were obtained. Two observers reviewed MR sequences for size, signal characteristics and lesion conspicuity using a four-point rating scale. ADC of tumours were calculated. Histological characteristics (differentiation, fibrosis, necrosis) were correlated with MRI features. Results: $95 \%$ of pancreatic adenocarcinomas were hypointense on 3D FS T1 GRE arterial phase, which was the best sequence for tumour conspicuity, followed by portal phase. DWI was not useful for delineating $26 \%$ of tumours. Maximum diameter at pathological examination ranged from 18 to $60 \mathrm{~mm}$ (mean $33 \mathrm{~mm}$ ). It was best correlated with MR tumour size on DWI. No correlation was found between ADC (mean value : $1.76 \times 10-3 \mathrm{~mm} 2 / \mathrm{sec}$ ) and differentiation, fibrosis or necrosis. All homogeneous hyperintense lesions on delayed phase ( $23 \%$ of tumours) were predominantly fibrous tumours.

Conclusion: 3D FS T1 GRE arterial phase sequence is superior to DWI for pancreatic adenocarcinoma conspicuity but it underestimates the size of the tumour. DWI is the best sequence for size evaluation when the tumour is correctly delineated. 


\section{B-0838 11:51}

Evaluation of diffusion-weighted MR imaging for detection of infection in acute pancreatic collections and/or necrosis: preliminary results

F. Islim, A.E. Salik, S. Bayramoglu, K. Guven, H. Alis, A.N. Turhan; Istanbul/TR (fislim@yahoo.com)

Purpose: To evaluate the contribution of diffusion-weighted magnetic resonance imaging (DW MRI) for detection of infection that has a critical role in the prognosis of acute pancreatitis.

Methods and Materials: 21 DW MRI at b values of 0,500 and 1000 were prospectively performed on 20 patients with acute pancreatitis. The collections and necrosis with peripheral bright signal on DW MR Images were accepted as positive. Apparent diffusion coefficient (ADC) values of peripheral (if DW MRI was positive) and central parts of the collections and/or necrosis were measured by three radiologists. Intra and inter-observer reliabilities were analyzed. Student's t-test was used to compare the ADC values between infected and sterile groups. Results: Except 1 false positive result DWI could successfully estimate the presence of infection. The mean ADC value of peripheral diffusion restricted areas was found to be $1.16 \pm 0.14 \times 10^{-3} \mathrm{~mm}^{2} / \mathrm{s}$. The mean ADC values measured from central parts of the collections and/or necrosis in infected and sterile groups were significantly different $(p<0.05)\left(2.58 \pm 0.36 \times 10^{-3} \mathrm{~mm}^{2} / \mathrm{s}\right.$ and $2.86 \pm 0.29 \times 10^{-3}$ $\mathrm{mm}^{2} / \mathrm{s}$ respectively).

Conclusion: Diffusion weighted MRI may be helpful for detection of infection in acute pancreatic collections and/or necrosis. Room L/M

\section{Physics in Radiology}

\section{SS 1713}

\section{Breast imaging and novel CT techniques}

\section{Moderators:}

N. Karssemeijer; Nijmegen/NL

M. Koutalonis; Colchester/UK

\section{B-0839 10:30}

Comparative detector evaluation of full field digital mammography (FFDM) systems using the Fourier definition of the Hotellingobserver signal-to-noise ratio (SNR)

R. Wise, I. Delakis, E. Kulama, D. McRobbie; London/UK

Purpose: Evaluation and optimisation of image quality plays an important role in enhancing the diagnostic value of mammography studies. Mammographic image quality is typically assessed on the basis of analysing images of the CDMAM contrast-detail phantom for mammography. However, CDMAM analysis can suffer from intra-observer variations and limited statistics. The purpose of this work was to apply Hotelling-observer SNR analysis in a comparative evaluation of FFDM detectors.

Methods and Materials: The generalised normalised noise power spectrum (GNNPS) was measured by collecting flat field images of $4 \mathrm{~cm}$ PMMA phantom. The generalised modulation transfer function (GMTF) was then measured by placing a $0.02-\mathrm{mm}$ Tungsten edge in the middle of the $4 \mathrm{~cm}$ PMMA phantom. This setup allowed for scatter and focal-spot unsharpness to be incorporated in the measurements. The Hotelling-observer SNR was calculated for input signal originating from gold discs of varying thicknesses and diameters, to replicate CDMAM analysis. Results: For each FFDM detector, Hotelling-observer SNR increased with the diameter and thickness of the gold disc. The Hotelling-observer SNR results showed variations in the performance of FFDM detectors evaluated, demonstrating the use of this metric as a differentiator. In addition, Hotelling-observer SNR values were higher for systems displaying better CDMAM analysis results, demonstrating the relation between the two metrics.

Conclusion: We used Hotelling observer-SNR to evaluate the performance of FFDM detectors, on the basis of a methodology accounting for focal-spot unsharpness and scatter. The calculated task SNR values varied across the detectors evaluated, in a manner commensurate with CDMAM analysis results.
B-0840 10:39

Spectral reconstruction algorithm for digital breast tomosynthesis I. Sechopoulos, V. Mejia-Bustamante, S.S.J. Feng, J. Nagy; Atlanta, GA/US (isechop@emory.edu)

Purpose: To develop a novel spectral reconstruction algorithm for digital breast tomosynthesis (DBT) that results in reduced artifacts and improved image quality. Methods and Materials: Current reconstruction algorithms assume that the $\mathrm{x}$ ray source emits a mono-energetic $\mathrm{x}$-ray beam, resulting in artifacts and possibly sub-optimal image quality. To avoid this, a mathematical model of DBT acquisition that includes the spectral nature of the $x$-ray source was developed, resulting in a large scale non-linear inversion problem which we solve using an iterative, gradient descent method. The resulting algorithm was tested using breast phantoms with lesions resembling masses and calcifications. The breast phantoms were imaged with a clinical DBT system using the automatic exposure control system. The resulting spectral reconstructions were compared to the system's filtered backprojection (FBP) reconstruction, which assumes a mono-energetic $x$-ray source.

Results: The signal difference-to-noise ratios of the masses in the spectral reconstruction were all superior to those of FBP by a mean factor of 2.6. In addition, the masses exhibited better homogeneity and no artifacts in the direction of $\mathrm{x}$-ray tube travel, typically seen in DBT images. The microcalcifications also resulted in improved signal difference and without artifacts seen in the FBP reconstructions. Subjective comparison of images of heterogeneous breast phantoms showed an overall improvement in image quality, substantial reduction of artifacts, and lower noise.

Conclusion: Spectral reconstruction can improve image quality and reduce artifacts in DBT images. This new algorithm also allows for investigation of novel acquisition techniques that could result in further improvements in DBT imaging.

Author Disclosures:

I. Sechopoulos: Research/Grant Support; Hologic, Inc., Fuji.

\section{B-0841 10:48}

Contrast-detail visibility analysis for digital breast tomosynthesis versus digital mammography

S. Elie, W.J.H. Veldkamp, R.E. van Engen, M.J.M. Broeders; Nijmegen/NL (s.elie@Ircb.nl)

Purpose: Digital breast tomosynthesis (DBT) is expected to improve visibility of lesions by reducing overprojection of tissue. This phantom study investigates the image quality of DBT images by comparing in-plane contrast and detail performance to digital mammography (DM) images in simulated mammographic background conditions.

Methods and Materials: Contrast-detail visibility was compared by imaging a 0.1 $\mathrm{mm}$ aluminium contrast-detail phantom (CDmam 3.4) that was positioned between two layers containing a breast-simulating material with comparable scatter and primary transmission properties. To obtain increased phantom thickness PMMA was added. The related simulated breast thicknesses were 32,60 and $90 \mathrm{~mm}$. Images were acquired with the following systems: Sectra Microdose L30 (DM, Sectra prototype DBT) and Hologic Selenia Dimensions (both DM and DBT mode). For each imaging mode and thickness eight images were acquired. X-ray spectra corresponded to clinical practice, where the mean glandular dose (MGD) of the DM mode was matched to the MGD of the DBT imaging mode. The CDmam images were scored by human observers and contrast and detail performance was evaluated. Results: Results showed that the contrast resolution (object diameters $0.4-2.0 \mathrm{~mm}$ and object thicknesses 0.03-0.16 $\mu \mathrm{m}$ ) improves in DBT. However, DBT showed a loss in spatial resolution (object diameters $0.06-0.31 \mathrm{~mm}$ and object thicknesses 0.2-2.0 $\mu \mathrm{m}$ ) compared to DM.

Conclusion: The in-plane DBT images are less affected by overprojecting structures resulting in better contrast resolution. However, smaller objects are better visualised with DM, due to superior spatial resolution in DM images compared to in-plane DBT images.

\section{B-0842 10:57}

Can a colour representation of grey-scale images improve object detectability in mammography?

E. Salvagnini', L. Cockmartin ${ }^{1}$, K. Michielsen ${ }^{1}$, J. Jacobs ${ }^{2}$, N.W. Marshall', L. Struelens ${ }^{3}$, H. Bosmans ${ }^{1} ;{ }^{1}$ Leuven/BE, ${ }^{2}$ Heverlee/BE, ${ }^{3} \mathrm{Mol} / \mathrm{BE}$ (hilde.bosmans@uzleuven.be)

Purpose: To examine the influence of display colour scales on human detection thresholds using a contrast-detail (c-d) phantom imaged with structured background. Methods and Materials: This study used a CDMAM phantom imaged in combination with PMMA and the CIRS BR3D phantom to add structured background to the 
images. Four thicknesses of the combined phantoms were imaged with a digital mammography unit. Of each thickness, four images were acquired under automatic exposure control. Images were displayed under standard clinical conditions: first on two 5 megapixel medical grade monitors calibrated to the DICOM GSDF standard, then on a 6 megapixel colour monitor, displaying the images in green-scale. Greenscale was chosen as this wavelength matches a peak in human visual response. Reading was performed with the Sara ${ }^{2}$ software which displays each CDMAM cell under random rotation, reducing reader memorization. Readers performed a 4-alternative forced choice task; five readers scored all images.

Results: Threshold gold thickness (TGT) increased as beam quality and thickness increased, indicating a reduction in object detectability with increasing object thickness. Results obtained for the grey-scale display had a higher TGT compared to green-scale results; TGT values obtained with green-scale display were between $7 \%$ and $31 \%$ lower than grey-scale display TGT. P values for the different diameters obtained from Wilcoxon matched pairs tests ranged from $<0.01$ to 0.68 for $2 \mathrm{~cm}$ and $<0.01$ to 0.44 for $7 \mathrm{~cm}$

Conclusion: Observer performance measured by c-d curves was equal or better for images displayed in green-scale rather than grey-scale, indicating potential benefit for using green-scale displays in medical applications.

\section{B-0843 11:06}

Volumetric breast-density measurement using spectral mammography

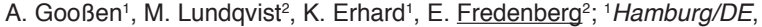

${ }^{2}$ Solna/SE (erik.fredenberg@philips.com)

Purpose: Volumetric breast density (VBD) quantification is useful for assessing diagnostic accuracy and the risk of developing breast cancer. The purpose of this study is to evaluate spectral imaging as a method to improve the accuracy of VBD measurements compared to conventional non-spectral methods.

Methods and Materials: The VBD is the volumetric fraction of fibro-glandula tissue in the breast. To measure VBD in a conventional non-energy resolved mammogram, assumptions have to be made on the breast thickness. Spectral imaging, on the other hand, enables simultaneous measurement of breast thickness and glandularity by employing the spectral difference in x-ray attenuation between adipose and fibro-glandular tissue. Spectral mammograms were acquired in a single exposure using a photon-counting mammography system. The VBD was measured by material decomposition based on calibration to tissue-equivalent material. For reference, VBD was measured with a conventional non-spectral method in a similar group of patients.

Results: Spectral mammograms of 75 patients, aged 50-69 years, were examined. The correlation in VBD between contralateral breasts was $r=0.97 .246$ patients were examined with conventional methods, which returned a correlation between contralateral breasts of $r=0.92$. The VBD distribution was similar in the two groups as measured with the two different methods.

Conclusion: Spectral imaging provides an efficient measure of VBD. The VBD distributions over the group of patients were comparable in the spectral and nonspectral measurements, but the correlation between contralateral breasts was tighter using spectral imaging, which indicates higher consistency.

Author Disclosures:

A. Gooßen: Employee; Philips. M. Lundqvist: Employee; Philips. K. Erhard: Employee; Philips. E. Fredenberg: Employee; Philips.

\section{B-0844 11:15}

Monochromatic dual-energy $\mathrm{x}$-rays for precise measurement of bone mineral density: preliminary results

C.-H. Lee, K.-H. Yoon, Y.-H. Lee, E. Kang, D. Kang; Iksan/KR

(99 Ich99@hanmail.net)

Purpose: The feasibility of monochromatic dual-energy x-ray absorptiometry (DEXA) using multilayer reflector for precise measurement of bone mineral density (BMD) was examined.

Methods and Materials: A multilayer mirror was fabricated to select an x-ray peak with an energy of $40 \mathrm{keV}$ and $59.3 \mathrm{keV}$ generated from an x-ray tube with a tungsten target. Experimental set-up of monochromatic DEXA was installed by dual-energy of monochromatic $\mathrm{x}$-rays source and CZT detector. The performance of the system was evaluated using a DEXA phantom, spectrometer and radiation dosimeter. We compared the image quality and the attenuation reduction characteristics of the phantom to determine precision of BMD on conventional polychromatic and monochromatic DEXA.

Results: The monochromatic beam from the multilayer reflector had a full-widthat-half-maximum of $2.69 \mathrm{keV}$ at $40 \mathrm{keV}$ and $5.8 \mathrm{keV}$ at $59.3 \mathrm{keV}$, the reflectivity was 0.60 and 0.58 , respectively. Conventional polychromatic and monochromatic DEXA showed radiation doses of $0.32 \mathrm{mGy}$ and $0.05 \mathrm{mGy}$, respectively. The image contrast of the monochromatic X-rays was better than that of the polychromatic $\mathrm{x}$-rays. Mean values of standard deviation of linear attenuation reduction characteristics of the phantom were $2.78 \%$ and $0.87 \%$ on conventional polychromatic and monochromatic DEXA, respectively.

Conclusion: Monochromatic DEXA using a multilayer mirror would be provided a precise measurement of bone mineral density by achieving the better performance with lower radiation dose than that of the conventional polychromatic DEXA

\section{B-0845 11:24}

Phase contrast imaging using grating interferometer: preliminary results

D. Kang, K.-H. Yoon, Y.-H. Lee, E. Kang, C.-H. Lee; Iksan/KR

(sharp8588@naver.com)

Purpose: Phase-contrast radiography is an emerging technique for producing medical imaging with more soft tissue contrast than traditional absorption radiography. The goal of this study is to evaluate the feasibility of phase contrast imaging using grating interferometer for clinical use.

Methods and Materials: We developed a prototype of phase contrast x-ray imaging system using micro-focus $\mathrm{x}$-ray source, grating interferometer and CMOS detector. The source contains a tungsten anode $\mathrm{x}$-ray tube that can operate from 38 $\mathrm{kV}$ at $0.35 \mathrm{~mA}$ and focal spot size of $0.3 \mathrm{~mm}$. A specification of phase grating was 4.714um period, Ni 6.08 um height and $30 \times 70 \mathrm{~mm}^{2}$ area; and analyser grating was 2.4um period, Au $20.3 \mathrm{um}$ height and $20 \times 60 \mathrm{~mm}^{2}$ area. With application of phase retrieval algorithm, phantoms such as acryl ball, honey bee and goldfish images were obtained. We compared with images of conventional absorption radiography and evaluated the phase intensity by phase stepping method.

Results: Phase contrast image shows a detailed view of the edge of the acryl ball. On the phase contrast image there are dark and light lines running along the hole. These lines represent the phase-contrast edge enhancement effect. A similar effect is evident at the honeybee and goldfish images. The phase contrast edge enhancement effect is visible in the phase contrast radiography, but not in either of the absorption contrast image.

Conclusion: The phase contrast imaging using grating interferometer will ultimately provide a novel $x$-ray imaging system that enables with high contrast, which can be valuable in medical imaging such as breast, cartilage and vessels.

\section{B-0846 11:33}

First in-vitro results of microbubbles as a scattering contrast agent for x-ray dark-field imaging measured at a first pre-clinical x-ray phase-contrast and dark-field CT scanner

A. Velroyen ${ }^{1}$, M. Bech ${ }^{1}$, A. Tapfer ${ }^{1}$, M. Ingrisch², C.C. Cyran²,

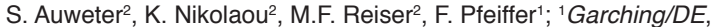

${ }^{2}$ Munich/DE (astrid.velroyen@ph.tum.de)

Purpose: Dark-field and phase-contrast imaging, which generate contrast from ultra-small angle scattering and refraction of $x$-rays in matter, have been shown to increase soft-tissue contrast and provide complementary information to conventional absorption-based imaging. To further improve image quality and explore possible alternatives to commonly applied iodine-based contrast agents, the use of microbubble-based ultrasound contrast agents as an x-ray scattering dark-field contrast medium was investigated.

Methods and Materials: Using the first pre-clinical phase-contrast and dark-field CT scanner with polychromatic $x$-ray source and grating interferometer installed on a rotating gantry, absorption, phase-contrast and dark-field images of microbubblebased contrast-agent samples were acquired in-vitro. For quantitative comparison, the contrast-to-noise ratio relative to a reference was calculated for varying detector resolutions (29-928 micrometer) in absorption and dark-field images.

Results: It was possible to generate an improved contrast-to-noise ratio in the darkfield image compared to the absorption-based image (6.88 versus 1.41 , pixel size 29 micrometer) by exploiting the $x$-ray scattering through microbubbles. With larger pixel sizes (928 micrometer), the relative contrast gain increased to 78.82 for dark field, whereas, with 1.06, it remained in the same order of magnitude for absorption. Conclusion: The proved feasibility of microbubble-based dark-field contrastenhancement with clinically compatible pixel sizes, together with the large potential of the introduction of grating interferometry into a clinical environment as shown by the successful implementation of the technology into a rotating gantry, strongly promote the use of microbubbles as a dedicated dark-field $\mathrm{x}$-ray contrast agent and warrant further investigation in an experimental in-vivo setting. 


\section{B-0847 11:42}

In vivo urinary stones chemical compound identification using dualenergy CT

T. Kraśnicki, M. Guziński, P. Podgórski, J. Bladowska, A. Czarnecka,

K. Tupikowski, M. Sąsiadek; Wrocław/PL (tomasz.krasnicki@gmail.com)

Purpose: The aim of the study was to identify in vivo urinary stones composition by analysing $Z$ effective (Zeff -weighted mean atomic number) values and to compare obtained results to the chemical analysis of the stones.

Methods and Materials: Twenty-eight patients with urolithiasis symptoms were examined using dual-energy CT (pitch 0.5, tube voltage with fast kVp switching $80 / 140 \mathrm{kVp}$, DFOV $5 \times 5 \mathrm{~cm}$ ). Data obtained during the examination were analysed on a clinical workstation with dedicated software (GSI Viewer). Urinary stones were evaluated separately by measuring Zeff value using small ROI method. The Zeff values were assigned to the corresponding composition and were compared to the results of chemical analysis after the removal of stones from the urinary tract. Results: The Zeff analyses revealed 3 types of calculi: homogenous stones (22 cases) with the high Zeff value: $13.52( \pm 0.6)$; homogenous stones ( 3 cases) with the low Zeff value (from 6.9 to 7.1, mean 6.98); heterogeneous stones ( 3 cases) with Zeff values 11.23 to 11.9 (mean 11.45) and 14.50 to 14.90 (mean 14.74). The cases of first group correspond to calcium oxalate stones. The second group Zeff values correspond to uric acid stones, while the third group was compatible the stones containing mixed struvite and apatite crystals. Urinary stones chemical analysis conducted after urinary tract removal confirmed the Zeff measurements results. Conclusion: Dual-energy CT is able to identify in vivo chemical composition of urinary stones and it may be useful in planning the optimal patient management.

\section{B-0848 11:51}

Value of monoenergetic low-keV dual-energy CT datasets for improved image quality of abdominal CT angiography

S. Sudarski, D. Schneider, P. Apfaltrer, S.O. Schönberg, T. Henzler;

Mannheim/DE (sonja_sudarski@gmx.de)

Purpose: High vessel attenuation and a high contrast-to-noise (CNR) ratio are prerequisites for highly diagnostic abdominal CT angiography (CTA) examinations. We compared image-quality of standard $120 \mathrm{keV}$ polyenergetic images (PEIs) with calculated low-keV monoenergetic images (MEls) in dual-energy CTA studies of the abdomen.

Methods and Materials: Datasets of fifty patients (42 men, $71 \pm 10$ years) who underwent abdominal dual-energy CTA were retrospectively evaluated. The 80 - and $140-\mathrm{keV}$ dual-energy image data was used to calculate MEls in 10-keV intervals from $120 \mathrm{keV}$ to $40 \mathrm{keV}$. Vessel attenuation and image noise were measured in three regions-of-interest (infrarenal aorta, external iliac artery and superior mesenteric artery) and the signal-to-noise ratio (SNR) and CNR were subsequently calculated. Differences between measurements in MEls and PEls were evaluated using the Student's t-test.

Results: SNR and CNR of 70-keV MEls were highest compared to MEls at other low-keV levels. When comparing MEls at $70 \mathrm{keV}$ to PEls, vessel attenuation and image noise were significantly higher in MEls in all three regions-of-interest $(+14 \%$, $+13 \%,+14 \%$; $p<0.05$ and $+12 \%,+6 \%,+9 \%$; all $p<0.05$ ). SNR was slightly increased in $70-k e V$ MEls $(+4 \%,+7 \%,+6 \% ; p<0.05)$. CNR was also significantly higher in MEls when compared to PEIs $(+18 \%,+16 \%$ and $+18 \%$; $p<0.05)$. Conclusion: Calculated 70-keV MEls show improved vessel attenuation, SNR and CNR in abdominal dual-energy CTA studies when compared to the standard 120-keV PEls. Thus, lowering the total amount of iodinated contrast material might become feasible with monoenergetic low-keV imaging.
$10: 30-12: 00$

Room N/O

\section{Cardiac}

\section{SS 1703}

Biomarkers, tissue characterisation and remodelling

Moderators:

M. Das; Maastricht/NL

K. Gruszczynska; Katowice/PL

\section{B-0849 10:30}

Biomarkers of atherosclerotic and myocardial remodelling: correlation with plaque volume in cardiac $\mathrm{CT}$

L.L. Geyer', A. Krazinski', J.R. Silverman'1, F. Bamberg'2,

U. Ebersberger', P. Costello', U.J. Schoepf'; ${ }^{1}$ Charleston, SC/US,

2Munich/DE (Lucas.Geyer@med.Imu.de)

Purpose: We aimed at correlating the plasma levels of circulating biomarkers in the context of atherosclerotic disease activity and myocardial remodelling with coronary dual-source CT angiography (CCTA) findings.

Methods and Materials: 75 patients with suspected coronary artery disease (CAD) underwent cCTA. Patients were evaluated for coronary plaque type and the presence and the severity of CAD. A semi-automated software was used for measuring non-calcified and mixed plaque volumes; lipid-rich and fibrous contents were discriminated. Cardiac function parameters were obtained. Plasma samples were collected and a cytokine and protease profiling panel was performed by multiplex analysis. The plasma concentrations of seven biomarkers were measured: TNFa, IL-6, IL-8, matrix metalloproteinase (MMP)-2, MMP-3, MMP-7, MMP-8. Data were analysed using Spearman's rank correlation and Mann-Whitney-U-test.

Results: Data of 75 patients ( $81 \%$ male, $59 \pm 10$ years) were evaluated. $60 / 75$ patients showed atherosclerotic changes in at least one vessel based on CCTA. 46 non-calcified, 129 calcified, and 86 mixed plaques were identified. We found a statistically significant $(p<0.05)$ positive correlation between the plasma concentrations of MMP-8 and the lipid-rich, fibrous, and total plaque volumes per patient. Moreover, IL-8 and TNFa showed a significant $(p<0.05)$ positive correlation with myocardial mass. Patients with diabetes mellitus showed significantly reduced MMP-7 (2.9 $\pm 1.6 \mathrm{ng}, 4.9 \pm 3.0 \mathrm{ng}, \mathrm{p}<0.05)$. MMP-2, MMP-3, and MMP-9 did not show any significant correlations.

Conclusion: Our study suggests that elevated levels of MMP-8 are associated with evidence of greater plaque volume in CCTA. IL- 8 and TNFa may also indicate more active myocardial remodelling with higher myocardial mass in CT.

Author Disclosures:

L.L. Geyer: Speaker; GE Healthcare, Germany.F. Bamberg: Research/ Grant Support; Bayer Healthcare (Germany). Speaker; Siemens Healthcare (Forchheim, Germany).U.J. Schoepf: Consultant; Bayer-Schering, Bracco, General Electric, Medrad, Siemens, TeraRecon. Research/Grant Support; Bayer-Schering, Bracco, General Electric, Medrad, Siemens.

\section{B-0850 10:39}

\section{Morpho-functional and metabolic assessment of the heart in} adult-onset growth hormone (GH) deficiency patients with cardiac magnetic resonance (CMR) and 31P MR spectroscopy (31P-MRS) M. Cava, F. De Cobelli, A. Esposito, G. Perseghin, A. Rossini, R. Lanzi, M. Losa, T. Canu, A. Del Maschio; Milan/IT

Purpose: Growth Hormone seems to play an important role in cardiovascular system function: structural and morpho-functional alterations could be related to its deficiency, with an increased mortality risk. In recent years some studies reported echocardiographic evaluations in patients with $\mathrm{GH}$-deficiency, but no significant data concerning CMR and 31P-MRS are available now.

Methods and Materials: CMR study (before and after Gadolinium injection) and 31P-MRS were performed on a $1.5 \mathrm{~T}$ magnet (Philips Intera Master) in 16 adult patients with adult onset GH-deficiency (GHD:10 males and 6 females, 49.3 \pm 13.7 years). Left and right ventricle morpho-functional evaluation, structural (with lateenhancement assessment) and metabolic feature estimation (as PCr/ATP ratio) were performed. 16 healthy individuals were the matched control group (Co). Results: GHD showed normal biventricular function. Median left ventricular mass (Mass/BSA GHD $49.7 \pm 18.3$ vs CO $80 \pm 20 p<0.001$ ) and mass/volume ratio were reduced $(0.68 \pm 0.15$ vs $1.09 \pm 0.13 p<0.001)$ with normal volumes. DT (trans-mitral deceleration time) was shortened ( $148.5 \pm 38.4$ vs $192.5 \pm 35.8 p=0.003)$. GHD had slightly lower right ventricle median volume $(p=0.03)$. GHD pts showed lower $\mathrm{PCr} /$ 
ATP ratio $(1.9 \pm 0.28$ vs $2.15 \pm 0.3 p=0.03)$. In late enhancement sequences there were no structural abnormalities.

Conclusion: Our data suggest that CMR in association with 31P-MRS could to identify early myocardial alterations in morphology, function, structure and metabolism in patients with adult onset GH-deficiency. Overall myocardial mass reduction could be related to a reduced energy load, maybe due to an anabolic impairment: even if at preliminary stages ventricular function could be normal, this process may anticipate subsequent functional loss

\section{B-0851 10:48}

Right ventricular remodelling after ST-elevation myocardial infarction

M. Tille, M. Gutberlet, H. Thiele, M. Grothoff;

Leipzig/DE (mandy.tille@herzzentrum-leipzig.de)

Purpose: Right ventricular (RV) injury is common after ST-elevation myocardial infarction (STEMI) and can be accurately detected by cardiac magnetic resonance (CMR). In this longitudinal study we sought to analyse RV myocardial remodelling after STEMI.

Methods and Materials: We studied 41 patients with RVI 1-4 days after primary angioplasty in STEMI. T2-weighted and late-gadolinium-enhancement (LGE) CMR was used for visualising oedema and necrosis. Cine-imaging was performed to access volumes and wall motion abnormalities (WMA), which, in combination with oedema were considered diagnostic for RVI. A second CMR examination was performed with a mean follow-up period of 5.2 months.

Results: The initial examination showed $17 / 41$ patients with LGE. The remaining 24 patients demonstrated oedema and WMA only. There was no difference in RV ejection fraction (RV-EF) between these two groups $(43.9 \pm 7.4 \%$ vs. $49.5 \pm 4.6 \%$, $\mathrm{p}<0.001$ ); however, RV-EF was decreased in both. In follow-up, no oedema was detectable and mean RV-EF was within a normal range again in both, patients with LGE $(49.5 \pm 5.6 \%)$ and patients without LGE $(48.8 \pm 5.6 \%, p=0.69)$. LGE was significantly smaller in follow-up compared to the first examination $(p=0.023)$. In multivariate regression analysis, RV oedema was the only parameter that was associated with a significant improvement of RV-EF.

Conclusion: The significant decrease of LGE in the follow-up examination implies that the area of hyperenhancement might represent necrosis. We furthermore conclude that the extend of RV scar is too small to permanently impair RV-EF function in the majority of patients in follow-up.

\section{B-0852 10:57}

Influence of periprocedural myonecrosis assessed by magnetic resonance delayed enhancement in patients with bifurcation lesions angioplasty on prognosis at one-year follow-up

R. Gil, J. Walecki, D. Vassilev, M. Furmanek, A. Michałek, M. Odyniec-Nowacka, A.J. Piliszek; Warsaw/PL (agnieszka.piliszek@gmail.com)

Purpose: The factors governing periprocedural myocardial injury (PMI) and its influence on the prognosis at follow-up are not completely clear. We used delayed enhancement magnetic resonance imaging (LGE MRI) to assess myonecrosis after PCl.

Methods and Materials: Forty-three patients with stable coronary artery disease were studied with $1.5 \mathrm{~T}$ MRI before and after (within 72h) stenting of bifurcation lesions. The left ventricular function and LGE were assessed. We performed angiographic measurements of the vessel diameters, and bifurcation angles pre- and post-intervention.

Results: After interventional procedure $24(56 \%)$ patients had troponin I elevation and $14(32.6 \%)$ patients had evidence of new myocardial enhancement $(4.5 \pm 2.9$ grams, $4.4 \% \pm 4.5 \%$ of left ventricle mass). Troponin I cut-off value for new LGE was $0.7 \mathrm{ng} / \mathrm{ml}(\mathrm{p}=0.018)$. Ten (23.3\%) patients had new LGE at the area supplied by SB, eight (18.6\%) patients at the area of distal embolisation and four $(9.3 \%)$ patients in both localisations. There was clear correlation between LGE localisation and geometry of the bifurcation (smaller modified distal bifurcation angle in SB area LGE group $22 \pm 9^{\circ}$ vs $\left.41 \pm 13^{\circ}, p=0.001\right)$. At 13 moths' follow-up the MACE rate was $14 \%$. There was no correlation between periprocedural myonecrosis expressed as a new area of LGE and MACE.

Conclusion: Occurrence of $\mathrm{PMl}$ after coronary bifurcation stenting is high; it is possible to predict the PMI localisation based on the bifurcation geometry; but PMI mass is small, usually $<5 \mathrm{~g}$ and has no influence on serious cardiac events at follow-up.

\section{B-0853 11.06}

Exposure to a high fat diet during early development increases the susceptibility to cardiac lipid accumulation

P.A. van Ewijk, S. Paglialunga, J. Slenter, J.E. Wildberger, J. Glatz,

M.K.C. Hesselink, P. Schrauwen, V.B. Schrauwen-Hinderling, M.E. Kooi;

Maastricht/NL (eline.kooi@mumc.nl)

Purpose: It was shown in mice the exposure to a high fat (HF) diet during early development increased the susceptibility to high-fat diet-induced hepatic steatosis and decreased markers of hepatic mitochondrial function. We aim to investigate whether early exposure to a HF diet also increases the susceptibility to cardiac fat storage and diminishes cardiac mitochondrial function and ejection fraction. Methods and Materials: Male and female C57BL6 mice were fed a HF diet (45\% kcal fat) or standard chow diet ( $9 \% \mathrm{kcal}$ fat (LF)), starting at least 6 weeks before conception and continuing during gestation and lactation. From weaning onwards, all offspring were fed the HF diet, generating two groups: HF/HF and LF/ HF. Cardiac function and myocardial fat content were measured in male offspring at 15 weeks of age $(n=26)$ using magnetic resonance imaging and spectroscopy on a 7T MR System (Bruker Biospin $\mathrm{GmbH}$ ). Cardiac mitochondrial respiration was determined in parallel groups $(n=11)$, using high resolution respirometry (OROBOROS Instruments)

Results: At 15 weeks of age, the HF/HF group showed significantly higher cardiac lipid content $(p=0.02)$ compared to the LF/HF group. No significant differences in cardiac mitochondrial function and ejection fraction were detected.

Conclusion: Mice exposed to HF feeding during early development were more susceptible for high-fat diet-induced cardiac lipid accumulation. This was not (yet) accompanied by decreased mitochondrial function and/or decreased ejection fraction.

\section{B-0854 11:15}

Cardiac and hepatic iron and heart function by MR in thalassaemia major patients treated with combined deferiprone and desferrioxamine regimen versus monotherapies: a multi-centre, observational and prospective study

C. Tudisca' ${ }^{1}$ P. Ricchi ${ }^{2}$, A. Meloni ${ }^{3}$, E. Grassedonio ${ }^{1}$, M. Santodirocco ${ }^{4}$; ${ }^{1}$ Palermo/IT, ${ }^{2}$ Naples/IT, ${ }^{3}$ Pisa/IT, ${ }^{4}$ San Giovanni Rotondo/IT

Purpose: Due to the limited data available in literature, the aim of this multicentre study was to prospectively assess in thalassaemia major ( ${ }^{\mathrm{TM}}$ ) the efficacy of combined deferiprone (DFP) and desferrioxamine (DFO) regimen versus DFP and DFO in monotherapy by quantitative magnetic resonance imaging (MRI) over a follow-up of 18 months.

Methods and Materials: Among the first $1135^{\mathrm{TM}}$ patients in the MIOT (Myocardial Iron Overload in Thalassaemia) network, we evaluated those who had been received combined regimen $(\mathrm{N}=51)$ or $\mathrm{DFP}(\mathrm{N}=39)$ and $\mathrm{DFO}(\mathrm{N}=74)$ monotherapies between the two MRI scans. Iron overload was measured by $\mathrm{T}^{*}$ multiecho technique. Biventricular function parameters were quantitatively evaluated by cine images.

Results: The percentage of patients that maintained a normal global heart T2 ${ }^{*}$ value was comparable between DFP+DFO versus both groups. Among the patients with myocardial iron overload at baseline, the changes in the global heart $\mathrm{T2}^{\star}$ and in biventricular function were not significantly different in DFP+DFO versus the DFP group. The changes in the global heart $\mathrm{T}^{*}$ were significantly higher in the DFP+DFO versus DFO group, without a difference in biventricular function. Among the patients with hepatic iron at baseline the increase in liver $\mathrm{T}^{*}$ values was significantly higher in combination versus both groups.

Conclusion: In TM patients at the dosages used in the real world, combined DFP+DFO regimen was more effective in removing cardiac iron load only versus the DFO group and in clearing hepatic iron versus both groups. Combined therapy did not show an additional effect on heart function.

\section{Author Disclosures:}

C. Tudisca: Author; Dr. Pepe Alessia received speaker's honoraria from Chiesi, ApoPharma Inc. and Novartis. Research/Grant Support; The MIOT project receives "no-profit support" from industrial sponsorships (Chiesi Farmaceutici S.p.A., ApoPharma Inc. and Bayer-Shering). This study was also supported by: "Ministero della Salute". 


\section{B-0855 11:24}

Are the preferential patterns of myocardial iron overload preserved at the CMR follow-up?

C. Tudisca ${ }^{1}$, A. Meloni ${ }^{2}$, G. Giuffrida ${ }^{3}$, E. Grassedonio', E. Chiodi ${ }^{4}$, A. Pietrapertosa ${ }^{5}$, M. Lombardi ${ }^{2}$, M. Midiri', A. Pepe ${ }^{2} ;{ }^{1}$ Palermo/IT, ${ }^{2}$ Pisa/IT, ${ }^{3}$ Catania/IT, ${ }^{4}$ Ferrara/IT, ${ }^{5}$ Bari/lT

Purpose: We aimed to determine if a preferential pattern of myocardial iron overload (MIO) was preserved between two Cardiac Magnetic Resonance (CMR) scans in thalassaemia major ${ }^{\mathrm{TM} M}$ patients.

Methods and Materials: Three short-axis views of the left ventricle were acquired and analysed using a 16-segment standardised model. The T2* value on each segment and the global value were calculated. Four main circumferential regions (anterior, septal, inferior and lateral) were defined. 259 TM patients with a CMR follow-up (FU) study at $18 \pm 3$ months and with significant MIO at baseline (global $\left.\mathrm{T}^{*}<26 \mathrm{~ms}\right)$ were selected and divided into two groups: severe $\left(\mathrm{N}=80\right.$, global $\mathrm{T}^{*}<$ $10 \mathrm{~ms})$ and mild-moderate MIO ( $\mathrm{N}=179$, global T2* $10-26 \mathrm{~ms})$.

Results: For each group there was a significant improvement in the global as well as in regional $\mathrm{T}^{*}$ values. For the whole patient population as well as for both groups, at basal the mean $\mathrm{T}^{*}$ value over the anterior region was significantly lower than the mean $\mathrm{T}^{*}$ values over the other regions, and the mean $\mathrm{T}^{*}$ over the inferior region was significantly lower than the $\mathrm{T}^{*}$ values over septal and lateral regions. The same pattern was present at the FU, with a little difference for patients with mild-moderate MIO.

Conclusion: In conclusion, a preferential pattern of iron store in anterior and inferior regions was present at both basal and FU CMRs, with an increment of T2* values at FU due to a basal CMR-guided chelation therapy. The anterior region seems to be the region in which the iron accumulates first and is removed later.

\section{B-0856 11:33}

The effect of partial volume averaging on peak velocity measurements in phase contrast magnetic resonance angiography (PCMRA)

J.C.L. Rodrigues ${ }^{1}$, K. Minhas ${ }^{1}$, G. Pieles ${ }^{1}$, C. Bucciarelli-Ducci', R. Tulloh ${ }^{1}$, N.E. Manghat ${ }^{1}$, C.J. Occleshaw ${ }^{2}$, M.C.K. Hamilton ${ }^{1} ;{ }^{1}$ Bristol/UK, ${ }^{2}$ Auckland/NZ

Purpose: ECG-gated PCMRA is an established non-invasive in vivo method to measure blood flow. By default, the Siemens Argus PCMRA flow analysis software calculates a voxel averaged peak velocity. The voxel with the highest velocity in the data set is first determined. This value is then averaged with this the velocities of its neighbouring 4 voxels to generate the voxel averaged peak velocity. This may underestimate peak velocity. The aim of this study was to determine if voxel averaging leads to a lower calculated peak velocity.

Methods and Materials: 145 peak velocity measurements in 14 different anatomical locations in 37 subjects (healthy volunteers, congenital and acquired heart disease patients) were analysed by default voxel averaging method and single voxel technique and compared. The effects of flow velocity, scan protocol (breath hold versus free breathing) and scanner type (Siemens Symphony versus Siemens Avanto) were also assessed. Statistical significance was defined as $\mathrm{P}<0.05$.

Results: There was a highly significant mean percentage increase in peak velocity of $7.1 \%$ when peak velocity was calculated by single voxel compared with voxel averaging technique $(P<0.0001)$. Significant increases in peak flow were observed by single voxel compared with voxel averaging regardless of patient type, anatomical location, CMR scanner or scan technique in terms of breathing command $(p<0.05)$. Disabling the voxel averaging technique had no effect on the volume of flow recorded.

Conclusion: The use of voxel averaging produces a consistent underestimation of peak velocity. At peak velocities $>300 \mathrm{~m} \cdot \mathrm{s}^{-1}$, the error may be clinically significant, e.g. misclassifying the severity of aortic stenosis. For this reason, it is recommended that for the assessment of peak velocity by PCMRA voxel averaging should not be used.

\section{B-0857 11:42}

Different patterns of delayed cardiac enhancement in symptomatic patients with post-total correction of tetralogy of fallot assessed by cardiac MRI and its correlation with right ventricular function: a report from Iran

F. Akhavan, M. Motevalli, N. Abolfat-h-Zadeh Ghale Joughi; Tehran/IR (akhavan79@yahoo.com)

Purpose: The underlying mechanisms that contribute to global right ventricular (RV) dysfunction in patients with repaired TOF are incompletely understood. We sought to quantify delayed enhancement (DE) as a possible factor in development of RV failure after repairing TOF to deliberate correlations between DE and RV function when evaluating by cardiac magnetic resonance (CMR).

Methods and Materials: In a retrospective cohort study, the clinical and CMR data of 110 Iranian symptomatic patients with repaired TOF during 35 years ago were collected. Cardiac function indexes were compared in two groups of patients with and without DE.

Results: Patient's mean age was $21.9 \pm 6.9$ years. $65(59.1 \%)$ patients were male and $45(40.9 \%)$ were female. DE was positive in $103(93.6 \%)$ and negative in 7 (6.4\%) patients. The position of DE was "right ventricular outflow tract (RVOT) without other sites" in 81 (78.6\%) and "RVOT with other sites" in 22 (21.4\%) patients. Mean right ventricle end diastolic volume (RVEDV) in DE positive and DE negative was $258.2 \mathrm{~mL} / \mathrm{m} 2 \pm 91.8$ and $185.5 \mathrm{~mL} / \mathrm{m} 2 \pm 38.2$, respectively $(P=0.047)$. Mean of right ventricle end systolic volume (RVESV) in DE positive and DE negative was $162.8 \mathrm{~mL} / \mathrm{m} 2 \pm 71.1$ and $103.3 \mathrm{~mL} / \mathrm{m} 2 \pm 35.9$, respectively $(P=0.045)$ and mean of right ventricle ejection fraction (RVEF) in DE positive and DE negative was $38.2 \% \pm 7.7$ and $44.8 \% \pm 9.1$, respectively $(P=0.010)$.

Conclusion: DE is a common finding in symptomatic patients with repaired TOF. Using CMR modality, we found DE as an indicative of ventricular scarring or fibrosis in these patients showing a direct correlation with RV dysfunction.

\section{B-0858 11:51}

Diastolic versus systolic acquisition of T1 maps of normal human myocardium at $1.5 \mathrm{~T}$ magnetic resonance imaging

U. Reiter ${ }^{1}$, G. Reiter ${ }^{1}$, K. Dorr' 1 A. Greiser ${ }^{2}$, R. Maderthaner ${ }^{1}$, M.H. Fuchsjäger'; ${ }^{1}$ Graz/AT, ${ }^{2}$ Erlangen/DE (ursula.reiter@klinikum-graz.at)

Purpose: Variation of myocardial blood volume during the cardiac cycle suggests dependence of longitudinal myocardial T1 relaxation time with cardiac phase. Differences and relations between diastolic and systolic T1 times of normal myocardium were investigated.

Methods and Materials: 40 healthy subjects ( 20 females and 20 males, age $23 \pm 3$ years, age range 20-35 years) underwent $1.5 \mathrm{~T}$ magnetic resonance $\mathrm{T} 1$-mapping with motion-corrected MOLLI (Modified Look Locker Inversion Recovery) acquired in three short axes (basal, mid-ventricular and apical) in systole and end-diastole. Regional T1 values were determined in all 16 AHA-segments; their mean was interpreted as myocardial T1 time. Resulting T1 values were analyzed employing t-test, analysis of variance and correlation analysis.

Results: Mean myocardial T1 times $(984 \pm 28 \mathrm{~ms}$ in diastole and $959 \pm 21 \mathrm{~ms}$ in systole) as well as all segmental mean T1 values in diastole and systole differed significantly ( $p<0.0001$ for all comparisons). Correlations between diastolic and systolic $\mathrm{T} 1$ times were, however, strong $(r=0.98$ for myocardial and $r=0.81$ for segmental T1 times). Mean diastolic versus systolic T1 values in different AHAsegments differed significantly $(p<0.0001$ in both phases), but there was a strong correlation between segmental mean T1 values in diastole and systole $(r=0.83)$. Systolic-to-diastolic increase of T1 was above average in lateral $(p<0.0001)$ and apical $(p<0.0001)$ segments.

Conclusion: T1 relaxation times of normal myocardium differ significantly between diastole and systole at $1.5 \mathrm{~T}$. Their strong correlation facilitates translation of diastolic and systolic T1 normal values. Regional deviations from the mean systolic-to-diastolic increase of $\mathrm{T} 1$ might be associated with regional differences in myocardial perfusion.

Author Disclosures:

G. Reiter: Employee; Siemens. A. Greiser: Employee; Siemens. 
10:30 - 12:00

Room P

\section{Paediatric}

\section{SS 1712}

\section{Foetal and neonatal imaging}

\section{Moderators:}

N. De Graaf; Rotterdam/NL

H. Ringertz; Linköping/SE

\section{B-0859 10:30}

Antenatal blood flow quantification by phase-contrast MRI using a new triggering method: comparison with Doppler ultrasound in a sheep model

B.P. Schönnagel, C. Much, J. Yamamura, G. Adam, F. Ueberle,

M. Tavares de Sousa, H. Kooijman, U. Wedegärtner;

Hamburg/DE (b.schoennagel@uke.de)

Purpose: To demonstrate feasibility of antenatal flow velocimetry using phasecontrast MRI in the foetal aorta for the first time using a newly developed Doppler ultrasound device for triggering the foetal heart beat and to compare MR measurements with standard Doppler ultrasound in a foetal sheep model.

Methods and Materials: Four pregnant sheep carrying singleton foetuses (gestational age 123 days) were anaesthetized to undergo foetal MR examination on a $1.5 \mathrm{~T}$ imager. A newly developed MR-compatible Doppler ultrasound sensor was placed on the abdomen of the ewe, above the foetal heart. The recorded signal of the foetal heart was transferred to the ECG trigger unit of the MR scanner and used for foetal cardiac triggering. Blood flow velocity measurements of the foetal descending aorta were assessed and compared with Doppler ultrasound measurements performed following MR examination. Time-volume curves were assessed and mean peak velocities were calculated.

Results: Triggering of the foetal heart rate was possible in all MR examinations allowing high quality flow measurements of the foetal aorta. Comparison of both methods revealed concordant results, both revealing characteristic arterial flow waveforms and average peak flow velocities of $60 \mathrm{~cm} / \mathrm{s}( \pm 3.4)$ and $62 \mathrm{~cm} / \mathrm{s}( \pm 9.2)$, respectively.

Conclusion: Phase-contrast MRI flow velocimetry of the foetal aorta was successfully performed using the newly developed MR-compatible Doppler-ultrasound device for foetal cardiac triggering in a foetal sheep model. Comparison with foetal Doppler ultrasound revealed concordant results.

\section{Author Disclosures:}

H. Kooijman: Employee; Philips Healthcare.

\section{B-0860 10:39}

Relationship between body growth and regional brain volume changes in extremely premature small for gestational age babies L.C. Tzarouchi, A. Drougia, A. Zikou, A. Charisiadi, P. Kosta, L. Astrakas, S. Andronikou, M.I. Argyropoulou; Ioannina/GR (Itzar@cc.uoi.gr)

Purpose: Extremely low birth weight and/or low gestational age has been associated with alterations in brain development. The purpose of the study was to assess whether early body growth catch-up reflects regional brain volume (rBV) changes by evaluating small for gestational age (SGA) extremely premature babies (gestational age $<32$ weeks) at term equivalent age.

Methods and Materials: Twenty-five extremely premature infants (GA: 29.8 \pm 2.1 weeks) with normal brain US studies and normal MRI at term equivalent age $(41.5+1.6$ weeks) were enrolled. Thirteen were appropriate for gestational age at birth and at term (AGA), 5 SGA at birth and AGA at term (SGA+) and 7 SGA at birth and term (SGA-). T1-weighted high-resolution 3D images were segmented using the SPM 5.0 and volumes of 90 individual GM areas were calculated using the IBASPM toolbox. Two-tailed Student's t-test was performed to test for differences in rBV between groups.

Results: There was no difference in rBV between AGA and SGA+ (body growth catch-up at term). In SGA- (persistent body growth restriction at term), areas of late maturation (frontal lobes, caudate nucleus) presented lower rGMv. There was no difference in rBV between AGA and SGA- in brain areas of early maturation (occipital cortex, thalami) $(p<0.05)$.

Conclusion: In extremely premature babies, body growth catch-up is associated with rBV catch-up at term equivalent age. RBV changes start from areas with great potential for early maturation.
B-0861 10:48

Do vascular events impact brain maturation of twin pregnancies complicated with twin-to-twin transfusion syndrome?

S. Bargy; Paris/FR (sophiebargy@gmail.com)

Purpose: To evaluate foetal brain maturation during twin pregnancies complicated with twin-to-twin transfusion syndrome (TTTS).

Methods and Materials: Over a period of 4 years, we retrospectively evaluated $44 \mathrm{MRIs}$ of women with monochorionic twin pregnancies complicated with TTTS. MRI scans were realized at an average of 31 weeks of gestation. All pregnancies complicated with TTTS and successfully treated with fetoscopic laser were included. Eighty-eight foetal brains were analysed and compared with an MRI brain atlas and with the co-twin. Biparietal brain diameter, cerebellar transverse diameter, sulcation, operculation, and myelination were analysed.

Results: Among the 88 foetuses, $4(4.2 \%)$ developed severe brain damage (unilateral brain atrophy, clastic lesion of corpus callosum with cyst) and showed unusual delay of brain maturation. In others cases of successfully treated TTTS, we found no difference in brain

maturation between twins.

Conclusion: Brain maturation during twin pregnancies with treated TTTS is well preserved except in cases with a severe vascular complication.

\section{B-0862 10:57}

Microstructural changes of white matter tracts in small for gestational age very preterm infants evaluated at term equivalent age L.C. Tzarouchi, A. Drougia, A. Zikou, P. Kosta, L. Astrakas, S. Andronikou, M.I. Argyropoulou; loannina/GR (Itzar@cc.uoi.gr)

Purpose: Very preterm infants suffer a wide range of neurodevelopmental disabilities. The purpose of the study was to assess the relationship between body growth and white matter (WM) microstructure maturation, in very low-birth weight, extremely preterm infants.

Methods and Materials: Twenty-seven extremely premature infants (gestational age: $29.11 \pm 1.9$ weeks) with normal brain US studies and normal MRI at term equivalent age ( $41.1 \pm 1.03$ weeks) were enrolled in the study. Using a diffusion-weighted sequence, fractional anisotropy (FA) was measured bilaterally in 16 fibre tracts with the region-of-interest method. The effect of sex and the relationship between somatometric measurements (birth and body weight, crown to heel length and head circumference) and FA was evaluated using a general linear model. Two-tailed Student's t-test was used to assess differences between small for gestational age (SGA) and appropriate for gestational age (AGA) infants.

Results: A positive correlation was found between body weight and FA in the anterior corona radiata, the posterior limb of internal capsule, the external capsule, the inferior fronto-occipital fasciculus, the superior longitudinal fasciculus, the middle cerebellar peduncles and the fornix. Decreased FA was observed in the superior longitudinal fasciculus and the fornix in SGA infants $(p<0.05)$.

Conclusion: In extremely premature infants body growth correlates with changes in WM microstructure. Compromised growth at term equivalent age is associated with microstructural abnormalities in areas related to attention, language, memory and executive functioning.

\section{B-0863 11:06}

The "tail sign" in the differential diagnosis of vermian pathologies in foetuses under 24 weeks: role of foetal MRI

P. Sollazzo, M. Sergi, V. Vinci, S. Bernardo, M. Saldari, L. Manganaro; Rome/IT (paolosollazzo@hotmail.it)

Purpose: To define by foetal MRI the "tail sign" and it's meaning in the differential diagnosis of vermian pathologies.

Methods and Materials: We performed 357 foetal MRI and including 41 cases under 24 weeks of gestation with the US suspect of posterior cranial fossa pathology. Foetal MRI was performed with a 1.5-T Magnet. We evaluated the biometrical parameters of the cerebellum, vermis morphology and biometry, IV ventricle, cerebellopontine angle, cisterna magna and tentorium insertion. We highlighted the presence of a linear hypointensity on T2 images in correspondence of the inferior part of the vermis, the "tail sign"; this feature corresponds histologically to a thickness of the $4^{\text {th }}$ ventricle roof that appears raised and dysplastic.

Results: Foetal MRI detected alterations of the posterior cranial fossa in $38 / 41$ cases and excluded the US suspicion of pathologies in the remaining 3 cases. MRI results were compared with post-mortem results in 20/38 cases or after birth follow-up in the remaining. Results were confirmed in $36 / 38$ cases and disconfirmed in 2 cases. We identified the "tail sign" in the 100\% of Dandy-Walker malformation 
and in 6/10 cases of partial vermis agenesis. We had no evidence of "tail sign" in all cases of vermis hypoplasia, vermis malrotation and rhombencephalosynapsis. Conclusion: Foetal MRI represents a useful imaging technique for the diagnosis of posterior cranial fossa pathologies to confirm and characterise different cerebellar malformations.

\section{B-0864 11:15}

Real time cine-MRI in children and foetuses with head and neck pathology T. Feygin, A. Pollock, J. Stein, T. Victoria; Philadelphia, PA/US

(Joel.Stein@uphs.upenn.edu)

Purpose: To determine the feasibility and applications of real-time cine-MRI in functional evaluation of selected pathologic entities of head and neck in children and foetuses.

Methods and Materials: For the foetal assessment, cine-MRI evaluations were performed in 370 patients in addition to routine foetal MR evaluation of different organ systems. Different patterns of foetal swallowing were identified in foetuses with head and neck pathologies. Real time MR techniques were applied in 12 volunteers and 5 patients with $22 \mathrm{q}$ deletion for swallowing and phonation evaluation. Preliminary comparison was done for compatibility of value of MR cine technique and barium swallowing with video recording.

Results: Real-time cine MRI was technically feasible in evaluation of various prenatal and postnatal head and neck pathologies. Foetal swallowing in patients with head and neck masses, cleft lip and palate, micrognathia differs from control group of foetuses with variety of other entities, not involving head and neck. The various phase of swallowing were identifiable, providing information that could be used in planning of foetal delivery. The MR patterns of swallowing and phonation in healthy volunteers and children with $22 \mathrm{q}$ deletion were identifiable and the obtained information was seemingly compatible with fluoroscopy data.

Conclusion: Cine-MRI methods are technically feasible and valuable tools in both prenatal and postnatal assessment, with increasing applications in the functional evaluation of head and neck pathology.

\section{B-0865 11:24}

Magnetic-resonance-imaging-based ratio of fetal lung volume to foetal body volume as a new prognostic marker in congenital diaphragmatic hernia

M. Weidner, C. Hagelstein, A. Debus, A. Walleyo, S.O. Schönberg, T. Schaible, K.A. Büsing, S. Kehl, K.W. Neff; Mannheim/DE (Meike.Weidner@gmx.de)

Purpose: To evaluate prognostic accuracy of the magnetic resonance imaging (MRI)-based ratio of fetal lung volume (FLV) to fetal body volume (FBV) concerning survival and need for extracorporeal membrane oxygenation (ECMO) therapy in congenital diaphragmatic hernia $(\mathrm{CDH})$

Methods and Materials: The local research ethics committee approved this study and written informed consent was obtained. MRI was performed in 96 fetuses with $\mathrm{CDH}$ and in 44 fetuses without any affection of lung and body volume. FLV and FBV were measured and used to calculate the ratio of FLV/FBV. Additionally, subgroup analysis of all fetuses whose growth development was less than $80 \%$ of the expected value was performed. Logistic regression analysis was used to model the dependence of survival and ECMO requirement on the ratio. Prognostic accuracy was evaluated by applying the area under the curve (AUC).

Results: 78 (81\%) fetuses survived and 35 (36\%) neonates needed ECMO therapy. The ratio of FLV/FBV was significantly higher in surviving fetuses $(0.013 \pm 0.006$ vs. $0.007 \pm 0.004 ; p<0.0001 ; A \cup C=0.829)$. Neonates receiving ECMO therapy showed prenatally a significant lower ratio $(0.009 \pm 0.006$ vs. $0.014 \pm 0.005 ; p=0.0001$ $A \cup C=0.811)$. According to subgroup analysis for fetuses with growth restriction $(n=13), A U C$ increased to $A \cup C=0.875$ for prediction of survival.

Conclusion: The ratio FLV/FBV is able to predict neonatal survival and ECMO requirement in children with $\mathrm{CDH}$. Unlike other prognostic parameters (i.e. observed/ expected MR-FLV, ultrasound observed/expected lung-to-head ratio) it is independent of reference to a control group and may enhance prognostic accuracy especially in patients whose growth development differs from expectation.

\section{B-0866 11:33}

Repetitive MR measurements of lung volume in foetuses with congenital diaphragmatic hernia to investigate the individual development of pulmonary hypoplasia during pregnancy

C. Hagelstein, M. Weidner, A. Debus, A. Walleyo, S.O. Schönberg, T. Schaible, S. Kehl, K.A. Büsing, K.W. Neff; Mannheim/DE (Claudia.Hagelstein@umm.de)

Purpose: Little is known about the individual development of lung hypoplasia in foetuses with congenital diaphragmatic hernia $(\mathrm{CDH})$ during pregnancy. The purpose of this study was to investigate changes in foetal lung volume (FLV) in $\mathrm{CDH}$ patients and to calculate weekly growth rates of the FLV using serial MRI.

Methods and Materials: MR-FLV was measured in 89 foetuses with $\mathrm{CDH}$ between 19 and 39 weeks gestation. All foetuses received two MR examinations with at least four weeks in between. In addition to the absolute MR-FLV (aMR-FLV) and the observed to expected MR-FLV (o/eMR-FLV) a weekly growth rate of the aMRFLV was determined for each foetus.

Results: In all but two foetuses the aMR-FLV increased between the two examinations (mean $11.1 \mathrm{ml}$, range 1.5-37.7 ml). Mean o/eMR-FLV at the first MRI (mean $26.0 \pm 3.1$ weeks gestation) was $33.3 \pm 12.2 \%$ (range $10.5-79.1 \%$ ) and $29.5 \pm 10.9 \%$ (range 10.8-63.7\%) at the second MRI (mean 33.9 \pm 2.4 weeks gestation). 54/89 foetuses $(60.7 \%)$ showed a decrease of the o/eMR-FLV during pregnancy with a mean reduction of $9.4 \%$ (range $2.2-31.7 \%$ ), 23 foetuses $(25.8 \%$ ) increasing o/eMR-FLVs with a mean increase of $7.7 \%$ (range $2.2-26.2 \%$ ) and 12 foetuses $(13.5 \%)$ revealed stable values. CDH patients had lower weekly growth rates with an average growth rate of only $26 \%$ compared to healthy foetuses.

Conclusion: The individual development of FLV in $\mathrm{CDH}$ patients during pregnancy is variable with decreasing, stable and increasing o/eMR-FLVs. In individual cases, the o/eMR-FLV relatively changed by more than $80 \%$ during pregnancy. Follow-up examinations are advisable prior to decision-making about pre- and postnatal therapeutic options.

\section{B-0867 11:42}

Periventricular nodular heterotopia: prenatal ultrasound findings

E. Blondiaux, C. Sileo, C. Nahama-Allouche, M.-L. Moutard,

A. Gelot, J.-M. Jouannic, H. Ducou le Pointe, C. Garel; Paris/FR

(eleonore.blondiaux@trs.aphp.fr)

Purpose: To describe the prenatal sonographic findings suggestive of periventricular nodular heterotopia (PVNH).

Methods and Materials: This retrospective study included foetuses referred for magnetic resonance imaging (MRI) and diagnosed with $\mathrm{PVNH}$, which was confirmed by post-natal MRI or autopsy. The type of $\mathrm{PVNH}$, associated ventriculomegaly and associated malformations were reported.

Results: 11 foetuses were included (9 females, 2 males) with a mean gestational age at diagnosis of 31 weeks' gestation (23-34). PVNH were small and diffuse $(n=7)$, large and multiple $(n=1)$ or single $(n=3)$. A targeted ultrasonographic (US) scan performed before foetal MRI missed the diagnosis in 4 cases [diffuse $(n=1)$ and single $(n=3)]$. Another US scan performed after MRI diagnosed PVNH in 2 of these 4 cases. Ventriculomegaly was present in 6 cases [uni- $(n=4)$ or bilateral $(n=2)]$. All PVNH appeared as nodules of intermediate echogenicity protruding into the ventricular lumen. In all cases of diffuse PVNH, the frontal horns and bodies of the lateral ventricles displayed a square shape with irregular borders. Associated cerebral malformations were observed in 7 cases and included corpus callosum agenesis ( $n=4$ with additional malformations in 2 cases) and retrocerebellar cyst $(n=3)$. Extracerebral malformations were also present $(n=2)$. Maternal MRI was performed in 5/6 cases of isolated small and diffuse PVNH in female foetuses and demonstrated PVNH in 2 cases.

Conclusion: PVNH are underdiagnosed at prenatal US even on targeted scans. Indented ventricular borders on axial views and irregular square shaped lateral ventricles on coronal views are suggestive of PVNH at prenatal US.

\section{B-0868 $11: 51$}

Role of foetal MR in the evaluation of gastrointestinal tract anomalies S. Bernardo, V. Vinci, P. Sollazzo, M. Sergi, M. Saldari, L. Manganaro; Rome/IT (silviabernardo@live.it)

Purpose: To determine if foetal MRI may confirm, complete or change the diagnosis in cases of sonographically detected GI tract abnormalities.

Methods and Materials: This is a prospective study (December 2010-June 2012) of 27 foetal abdominal MRI scans. The exam was performed between 24 and 38 weeks of gestation on a 1.5-T magnet. MRI exam was performed within two weeks from the US examination. US diagnoses included: omphalocele $(n=3)$; bowel dilatation $(n=7)$, associated with ascites in 4 cases, foetal abdominal cyst $(n=5)$, oesophageal atresia $(n=2)$, hyperechoic bowel $(n=3)$, associated with ascites in 1 case; meconium peritonitis $(n=3)$, only ascites $(n=2)$. MRI protocol included T1 $\mathrm{W}$ fast gradient-echo (Flash $2 \mathrm{D}$ ) and T2 W half-Fourier acquired single-shot turbo spin-echo (HASTE) sequences in axial, coronal and sagittal planes.

Results: Foetal MRI examination made correct diagnoses in 25 patients, it changed US diagnoses in 5 cases of foetal abdominal cysts and in one case of hyperechoic bowel it excluded any intestinal abnormalities. It provided more informations than US in the cases of intestinal dilatation, where foetal MRI was able to identify the level of obstruction and in 2 patients where it revealed the presence of a microcolon. 
Finally, MRI made correct diagnosis in 4 of 6 foetuses with expansive formations, whereas in one of these cases we suspected a choledochal cyst and it was a gastric duplication and in another patient we diagnosed an ascites and it was an intrabdominal cystic lymphangioma.

Conclusion: Our preliminary results suggest that MRI has a supplemental value to sonography in anatomic evaluation and characterisation of GI abnormalities. Ultrafast imaging sequence MRI is helpful to confirm, refine or exclude US diagnosis. It also provides useful informations in prenatal counselling and perinatal management.

\section{$10: 30-12: 00$}

Room Q

\section{Radiographers}

\section{SS 1714}

\section{Mammography}

Moderators:

S.J. Foley; Dublin/IE

P. Vahtramae; Pärnu/EE

\section{B-0869 10:30}

Online portfolio for radiographers attending the breast cancer screening mammography certificate in Switzerland

N. Richli Meystre, L. Flaction, R. Le Coultre; Lausanne/CH

(nicole.richli@hesav.ch)

Purpose: In order to achieve high image quality with breast cancer screening mammography, the European guideline recommends training programs dedicated to radiographers. Those training programs are very helpful for practitioners but back at their workplace it might be difficult for them to apply the achieved knowledge in their daily practice. To support radiographers to transfer good mammography practice in their daily screening activity, an online portfolio is realized by each radiographer during the following 6 months after the training session.

Methods and Materials: Every month, the trainee radiographer posts about 4 mammography examinations including the quality self-assessment on a dedicated electronic platform. Experienced radiographers from the screening program of western Switzerland comment the picture quality as well as the accuracy of the self assessment. Tips and tricks, to improve quality, are also given.

Results: Actually 26 portfolios have been completed. The majority of the radiographers appreciated the personalized comments given by the experts. They found the tips very helpful and they say the portfolio helped them to continuously improve the quality of the performed mammograms. Nevertheless, some radiographers were upset because of the high time investment necessary to the realization of this kind of portfolio.

Conclusion: In western Switzerland, where there are many decentralized breast cancer screening units, the online portfolio is an interesting tool to support radiographers with the development and the transfer of competences in their daily practice.

\section{B-0870 10:39}

Inter-observer agreement among PGMI radiographer in Cambridge/

$\mathrm{UK}$ and Oslo/NO in assessing each other's and their own

mammography screening exams

R. Gullien ${ }^{1}$, E. Rostad', A.E. Haakull', R.L. Hammond'1, M. Hauge ${ }^{1}$, K. Taylor ${ }^{2}$, J.G. Andersen ${ }^{1} ;{ }^{1}$ Oslo/NO, ${ }^{2}$ Cambridge/UK (uxroeb@ous-hf.no)

Purpose: To evaluate the inter-observer agreement between PGMI radiographers in UK/Cambridge and Norway/Oslo in the PGMI classification of mammograms using the recommendations stated in their own national quality assurance manual. Methods and Materials: Four experienced PGMI radiographers from both locations (UK/Cambridge and Norway/Oslo) independently assessed and classified a selection of 112 exams (CC and MLO of each breast) from each centre, by giving each image a separate PGMI score and an overall score for the exam: $P=$ perfect=3, $\mathrm{G}=$ good $=2, \mathrm{M}=$ moderately good $=1$, and $\mathrm{I}=$ inadequate $=0$. The selected exams contained all four categories of PGMI. We assessed the overall score for the exam and additionally chose to assess the MLO score. Inter-observer agreement was assessed for both groups (Kappa analysis).

Results: Kappa analyses of overall score gave poor agreement (Mean Kappa < 0.2 between the PGMI radiographers in Cambridge and Oslo when both Cambridge and Oslo exams were assessed. Analyses of the score for MLO images gave fair agreement (Kappa 0.2-0.29) between the two centres assessing the Cambridge images. Analyses of the Oslo MLO images gave a poor Kappa agreement. Individual Kappa agreement of the MLO varied from moderate (Kappa 0.48 ) to poor agreement $(<0.2)$ between the radiographers at both centres.
Conclusion: It was moderate to poor agreement between the two centres, for the overall score and the MLO score. A possible reason for the poor agreement might be because of the differences in the national quality assurance manual. It is important to further analyse and investigate the reasons for the low accordance.

\section{B-0871 10:48}

Radiation dose levels assessment in mammography

C. Lourenço, P. Sousa, S.I. Rodrigues, A.F.C.L. Abrantes, L.P.V. Ribeiro,

K.B. Azevedo, R.P.P. Almeida; Faro/PT (a35589@ualg.pt)

Purpose: To measure radiation dose levels in mammography of two different institutions ( $A$ and $B$ ) and to compare them with international guidelines.

Methods and Materials: A survey of exposure parameters ( $\mathrm{kV}$ and $\mathrm{mAs}$ ) and sociodemographic and anthropometric characteristics (age, gender, weight and height) of patients was used to perform the calculation of entrance skin dose (ESD) medium glandular dose (MGD) of the 548 examinations observed. For viability measurements, the dose rate and quality control using the RF detector Unfors Xi were carried out. Through mathematical formulas, the values of ESD (Gy) and MGD (mGy) were calculated.

Results: The values [[Unable to Display Character: \&\#8203;]][Unable to Display Character: \&\#8203;]]of the ESD for a full examination were $6.59 \pm 3.61 \mathrm{mGy}$ to institution $A$ and $6.27 \pm 3.15 \mathrm{mGy}$ to $B$ institution. Also, the values [[Unable to Display Character: \&\#8203;]][[Unable to Display Character: \&\#8203;]] of MGD obtained for a full examination were $1.40 \pm 0.43$ mGy for institution $A$ and $1.39 \pm 0.57$ mGy for institution $B$. About ESD, only the value of institution $B$ was slightly higher than the same in France and was lower than all other international guidelines. The MGD was lower than the international guidelines in both institutions.

Conclusion: The ESD values were generally lower than international guidelines, with only one exception. This exception must be analysed to achieve the lowest possible dose. MGD values [[Unable to Display Character: \&\#8203;]][Unable to Display Character: \&\#8203;]]calculated were found below the diagnostic reference levels, but it is important to keep reconsidering the protocols used, to optimise them.

\section{B-0872 10:57}

Quality control in digital mammography: radiographers practice at 5 hospitals of Lisbon

C. Tomás, C. Gonçalves, C.I.S. Reis; Lisbon/PT (claudiatomas90@gmail.com)

Purpose: To investigate quality control/(QC) guidelines in use for digital mammography/(DM) in 5 hospitals, to identify radiographer's practice in QC concerning DM and to identify the causes of exam rejection/repetition.

Methods and Materials: Questionnaires were developed and applied to 30 radiographers working in DM (computed-radiography or direct-digital-mammography/ (DDM)) in 5 hospitals to characterise the QC practices (guidelines in use, performed tests, tests frequency). Rejected/repeated analyses was performed in one hospital with DDM equipment using the checklist proposed by the International Atomic Energy Agency/(IAEA).

Results: Guidelines for QC are followed by $70 \%$ (20) of the radiographers: $55 \%$ follow American-guidelines, $20 \%$ the Portuguese, $15 \%$ the European/EUREF and $10 \%$ other guidelines. There is more than one guideline followed by radiographers in three institutions. The $\mathrm{QC}$ tests performed by radiographers in the same institution (proposed by IAEA to test image acquisition and display systems) were not the same and in the right frequency. The main causes for mammography image rejection/repetition were improper positioning in $85 \%$ of the analysed images due to non-inclusion of pectoral muscle in mediolateral oblique projection and inner and outer quadrants asymmetry for craniocaudal projection.

Conclusion: In this group of hospitals with DM, there is no uniformity of QC practices. This can have impacts in mammography costs. This may be justified by the absence of certification programs concerning mammography departments. Continuous education and training programs frequency to work with DM are not mandatory and also QC is not required by law in Portugal.

\section{B-0873 11:06}

An investigation into the psychological anxiety of Maltese women before and after a mammogram

C. Falzon; Sta Lucija/MT (valeview@maltanet.net)

Purpose: To investigate whether there is a difference in anxiety levels between Maltese women with and without a family history of breast cancer, before and after a mammogram.

Methods and Materials: The study involved the participation of 150 Maltese women aged 35 years and over. The women were divided into two groups; group 1 without and group 2 with family history of breast cancer. Anxiety levels pre- and 
post-mammogram were measured using the well-established State and Trait Inventory for Adults (STAI) developed by C.D. Spielberger.

Results: There was a significant difference (p0.05). A negative correlation was obtained when the mean S-anxiety scores (pre: group1, $r=-0.123$ and group 2, $r=-0.245$ and post: group1, $r=-0.046$ and group $2, r=-0.78$ ) of both groups were compared with age.

Conclusion: The factor causing anxiety in women was mainly related to the procedure because their anxiety diminished significantly after the mammogram. The correlation showed that those who scored high pre-mammogram anxiety also scored high post-mammogram anxiety. No significant difference in the anxiety levels between the two groups existed before and after the mammogram. A negative correlation existed between age and S-anxiety of the women, resulting in younger women being more anxious than older women.

\section{B-0874 11:15}

Ergonomic and environmental assessment in digital mammography room: impact on radiographers' activity

S. Costa, E. Oliveira, F. Serranheira, S. Viegas, C.I.S. Reis; Lisbon/PT (stefaniebcosta@gmail.com)

Purpose: To investigate ergonomic-characteristics of the equipment, environmental conditions and its impact on radiographers' activity and to identify opportunities to optimise the practice and reduce the probability of WRMSDs occurrence.

Methods and Materials: Radiographer's mammography procedures were observed and organized in tasks time-scale-graphic. Interviews were applied to 7 radiographers working in digital-mammography to characterise the practice (work patterns, mammographic equipment, exam room, environmental conditions) and to identify the presence of WRMSDs symptoms. Postural analyses were evaluated through video and pictures acquired during mammography positioning. Measurements of angles were done with Meazure2.0.158 software and classified according to the European Standard/EN100-4:2005+A1:2008.

Results: The task-time scale showed that the average duration of each exam is 5 minutes and also that the task which took more time was the indications given to the patient. $45.5 \%$ of the radiographers referred that the most exhaustive procedure is the positioning of MLO-view and short-stature patients (22.7\%). In this specific case, the anatomical areas that can suffer more WRMSDs are neck, arms and back presenting an angle of $87.2^{\circ}, 118.6^{\circ}$ and $63.6^{\circ}$, respectively. The visual contact with the patient was compromised only during the right-MLO-view acquisition. Considering the environmental conditions the illuminance values are not in accordance with the guidelines.

Conclusion: The ergonomic characteristics of the equipment do not provide comfort to the radiographers. In order to reduce the probability of work-related musculoskeletal disorders in mammography positioning and patient safety, new equipment features should be developed. Environmental conditions are adequate concerning the international references for temperature and humidity but not for illuminance.

\section{B-0875 11:24}

Accuracy of students, and radiographers, with and without, mammography post-graduation in interpretation of mammography images R.B.J. $\underline{\text { Cópio }}^{1}$, K.B. Azevedo', A.F.C.L. Abrantes ${ }^{1}$, L.P.V. Ribeiro ${ }^{1}$, R.P.P. Almeida1 , C.A. Silva ${ }^{2} ;{ }^{1}$ Faro/PT, ${ }^{2}$ Evora/PT (rita.copio.rc@gmail.com)

Purpose: To evaluate the sensitivity, specificity and diagnostic accuracy of radiographers with and without mammography post-graduation and last year students of the Radiography Course in the interpretation of mammography images.

Methods and Materials: A grid of socio-demographic characteristics and ViewDEX software (Viewer for Digital Evaluation of $x$-ray Images) for the interpretation of mammography images by participants were used. Each of the 16 cases was built with the four basic images. After viewing images, the 22 participants showed their level of confidence about the presence of abnormal radiological findings in relation to the case.

Results: The results obtained showed values of sensitivity of $81 \%, 89 \%$ and $80 \%$ for the last year students, radiographers without post-graduation and post-graduate radiographers, respectively. For specificity, the values were $63 \%, 75 \%$ and $50 \%$ for the same groups, respectively. With regard to diagnostic accuracy, the values obtained were $80 \%$ for the group of students in the last year, $88 \%$ for the group of radiographers without post-graduation and $78 \%$ for post-graduate radiographers. The mean response time was 46.9 seconds to the last year students, 59.8 seconds for radiographers without post-graduation and 64.6 seconds to post-graduate radiographers.

Conclusion: The sensitivity, specificity and diagnostic accuracy varies between each group. However, there are not significant differences between them. Also, participants with a higher formation have higher average response time.
B-0876 11:33

Patient satisfaction assessment in mammography exams

L. Borralho, L.P.V. Ribeiro, C.A. Silva, A.F.C.L. Abrantes, J. Pinheiro,

R.P.P. Almeida; Faro/PT (leilapatricia90@hotmail.com)

Purpose: This study aimed at measuring patient satisfaction in mammography exams and satisfaction-related factors.

Methods and Materials: Research was conducted in several private practice clinics with a total of 183 subjects using the Myasthenia Gravis questionnaire for assessing patient satisfaction concerning four dimensions: structure (physical environment), process (performing the exam), discomfort (physical and psychological) and general satisfaction (present and future).

Results: Overall patient satisfaction was high $(90 \%)$, with technical performance (process) and psychological discomfort having the major influence on patient satisfaction. Exam costs and age had very low, or no impact, on satisfaction. Also, the greater patient's education levels, the greater were their demands for process requirements, unlike patients with lower education levels which presented higher satisfaction levels.

Conclusion: The great amount of patients of high age groups and respective pathologies may have influenced results when related to satisfaction and education level. The numbers of mammography exams have increased over the years and therefore it is essential to assess patient satisfaction so that improvements can be made.

\section{B-0877 11:42}

Impacts of digital mammography in radiographers practice

L. Painho, T. Fernandes, C.I.S. Reis; Lisbon/PT (tiagojpfernandes@gmail.com)

Purpose: To characterise the techniques in use by radiographers for digital mammography/(DM) utilisation and compare analogue with digital technologies.

Methods and Materials: A total of 1492 images obtained with DM equipment, considering patient's characteristics, exposure parameters, compression-force, were analysed. The image quality/(IQ) was evaluated according to PGMI-scale. Results: The thickness increases on mediolateral-oblique view/(MLO)/( $r=0.818)$. The compression-force increased when compared to the craniocaudal/(CC) to MLO view/( $r=0.36)$. The mean energies were 29.4 and $29.6 \mathrm{kVp}$ for CC and MLO, respectively, while mean intensities were 79.5 for $\mathrm{CC}$ and $83.4 \mathrm{mAs}$ for MLO. The mean-glandular-dose/(MGD) was variable (0.40-3 mGy). The anode-filter combination always used was W/Rh. Global IQ was $P$ (perfect) for CC, while in MLO the mean was $G$ (Good). The radiographers experience varied between 2 and 28 years. Conclusion: The compression force depends on breast compressed thickness. It can vary between 6 and $10 \mathrm{~N}$ for breast thickness range of 45 to $76 \mathrm{~mm}$. The technique suffered changes when the analogue was compared with digital technologies: the energy is higher, the intensity is lower and the anode-filter has changed from $\mathrm{Mo} / \mathrm{Mo}$ to $\mathrm{Rh} / \mathrm{Rh}$ and W/Rh. Breast symmetry absence on CC projection, inframammary folds absence and skin folds presence were the criteria not achieved more frequently in this study, according to European Guidelines on Quality Criteria for Diagnostic Radiographer Images. IQ of all exams was classified as appropriated (perfect and good (362 out 373)). Nevertheless, it is noticeable that some variation exists concerning the radiographer practice when comparing breasts with the same features. The radiographers experience and training can also affect the results.

\section{B-0878 11:51}

\section{Imaging ethics}

B. Hofmann; Oslo/NO (b.m.hofmann@medisin.uio.no)

Purpose: The benefits of modern medical imaging technology are obvious. However, the ethical challenges are less acknowledged. The purpose of the study is to highlight and to address core ethical challenges with modern medical imaging. Methods and Materials: A review of literature in radiology reveals a series of ethical challenges in modern medical imaging that are analysed with standard methods in modern bioethics.

Results: The ethical challenges are divided into four categories, i.e. challenges with 1) existing, and 2) new imaging techniques, 3) altered use of existing imaging techniques, and 4) challenges with basic concepts and end points in diagnostic imaging. A series of ethical challenges are identified within these categories, such as (radiation) safety, rationing, and justice (1), implementation without evidence-based outcome (2), reduced diagnostic accuracy, patient autonomy (3), and challenges with underuse, overuse, and futile imaging (4).

Conclusion: Behind great benefits of modern imaging, a series of ethical challenges can be identified. While praising the benefits, the challenges need to be appraised to maintain confidence and trust in the profession, as well as a sound and fruitful development in the field. 


\section{Molecular Imaging}

\section{SS 1706}

\section{Molecular imaging in cancer and degenerative diseases}

\section{Moderators:}

J. Hakumäki; Kuopio/FI

E. Lopci; Rozzano/IT

\section{B-0879 10:30}

Improving current preclinical therapy monitoring methods by multiparametric MR using a hNIS-expressing tumour xenograft mouse model

E.J. Koziolek ${ }^{1}$, I. Apostolova ${ }^{2}$, A. Tarkowska ${ }^{1}$, U. Schumacher ${ }^{1}$, W. Brenner ${ }^{2}$, G. Adam ${ }^{1}$, M.G. Kaul ${ }^{1} ;{ }^{1}$ Hamburg/DE, ${ }^{2}$ Berlin/DE (ekoziole@uke.de)

Purpose: Preclinical methods for validation of tumour therapy response are generally limited to manual tumour size estimation using a calliper. The purpose of our study was to establish tumour xenograft mouse models allowing non-invasive, multimodal tumour imaging and monitoring. To further characterise tumour tissue for early therapy response, multiparametric MR was established.

Methods and Materials: Tumour cells of human origin (colon, mamma) were stably transfected with the human sodium iodine symporter (hNIS) gene. Mouse tumour xenograft models were established. Imaging was performed on 7T MR (ClinScan, Bruker) and SPECT/CT (NanoSPECT, Mediso). To monitor tumour growth by MRI, size was measured using a T2-weighted sequence. Additionally, diffusion-weighted imaging (DWI) and dynamic contrast-enhanced (DCE) MRI (0.15 $\mathrm{mmol} / \mathrm{kg}$ Gd-DTPA) were performed. To analyse tracer accumulation and retention time, SPECT was performed using Tc99m (100 MBq).

Results: hNIS expression in both tumour entities was visualised by a strong Tc99m-uptake via SPECT-imaging when compared to hNIS-negative tumour xenografts. MR imaging allowed a precise delineation of tumour mass and volume. Signal enhancement measured by DCE was predominantly found in the tumour periphery, whilst homogenous tumour tissue showed a more uniform contrast agent distribution. Increased apparent diffusion coefficient (ADC) in the tumour centre may indicate necrotic tissue.

Conclusion: The hNIS-transfected tumour xenograft model is a promising tool for tumour imaging and monitoring. Tumour tissue characterisation can be analysed by MR parameters such as diffusion, perfusion and permeability in order to predict therapy response. A further characterisation is possible by pharmacokinetic modelling of tumour-specific tracer uptake.

\section{B-0880 10:39}

\section{In vivo assessment of the anti-apoptotic effect of c-kit+ pluripotent} bone marrow cells on ischaemic myocardium in mice with hybrid fluorescence molecular tomography - x-ray computed tomography M. Wildgruber, A. Ale, K. Kosanke, R. Braren, R. Meier, E.J. Rummeny, V. Ntziachristos; Munich/DE

Purpose: To investigate the anti-apoptotic effect of pluripotent c-kit+ bone marrow cells after murine myocardial infarction by hybrid fluorescence molecular tomography - x-ray computed tomography targeting Annexin V (FMT-XCT).

Methods and Materials: Myocardial ischemia (MI) was induced in kit+/+ mice, c-kit-deficient KitW/W-v mice and bone marrow reconstituted KitW/W-v mice by transient ligation of the left anterior descending artery. Mice were injected with an Annexin-V-targeted molecular imaging probe 4 hours prior to imaging. Hybrid FMTXCT was performed 6 hours, 24 hours and 7 days after induction of ischemic injury. Molecular imaging signal for Annexin-Vivo750 was validated by ex-vivo cryoslicing, immunohistochemistry and flow cytometry.

Results: Successful image acquisition of FMT and CT was achieved in all mice. In control kit+/+ mice, the FMT signal for Annexin V peaked at 6 hours after myocardial infarction with subsequent decrease at 24 hours and 7 days after onse of ischemic injury. KitW/W-v mice revealed an increased and prolonged in-vivo apoptosis signal, which was associated with progressive decline in heart function. Bone marrow reconstitution was able to reduce cardiomyocyte apoptosis and rescue KitW/W-v mice from progressive heart failure. In-vivo apoptosis signal was verified after killed by apoptosis staining using immunohistochemistry (TUNEL) and flow cytometry (Caspase-3). Hybrid acquisition of $x$-ray computed tomography allowed exact localisation of the FMT signal to the left-ventricle and facilitated organ segmentation and attenuation correction.
Conclusion: Hybrid FMT-XCT targeting Annexin $\mathrm{V}$ enables monitoring of the beneficial effects of $c$-kit cell treatment after myocardial ischemia in a murine ischemia-reperfusion model.

\section{B-0881 10:48}

Magnetic resonance imaging of murine myocardial infarction with an elastin-binding contrast agent

M. Wildgruber ${ }^{1}$, M. Settles ${ }^{1}$, I. Bielicki ${ }^{1}$, K. Kosanke ${ }^{1}$, A.M. Huber ${ }^{1}$

E.J. Rummeny ${ }^{1}$, R.M. Botnar ${ }^{2} ;{ }^{1}$ Munich/DE, ${ }^{2}$ London/DE

Purpose: To study elastin-targeted magnetic resonance imaging (MRI) of murine myocardial infarction.

Methods and Materials: Myocardial infarction (MI) was induced in C57BL/6J mice by permanent ligation of the left anterior descending artery (LAD). Elastin-target ed MRI was performed on day 7 following myocardial infarction using CP-1052 in comparison to conventional Gd-DTPA-enhanced cardiac MRI. Competition experiments were carried out with La-1052, in which the paramagnetic gadolinium core is replaced by lanthanum. Further, delayed enhancement studies were compared to ex-vivo 2.3,5-triphenyltetrazolium chloride (TTC) staining.

Results: CNR after Gd-DPTA injection peaked at 10 minutes and returned to preinjection values at 60 minutes. After injection of CP-1052, the CNR peaked at 45 minutes and was significantly higher than Gd-DTPA at 30 minutes $(p=0.04), 45$ minutes $(p=0.02)$ and 60 minutes $(p=0.02)$. Competition experiments with La-1052 revealed a significantly reduced CNR indicating specific binding. Ex-vivo TTC staining of explanted hearts revealed good correlation for infarct size determination with elastin-targeted MRI.

Conclusion: Elastin-targeted cardiac MRI enables visualisation of injured myocardium in an experimental mouse model of myocardial infarction with a higher CNR and enhanced binding characteristics. Improved dynamic MRI with CP-1052 is attributed to its higher relaxivity and its affinity for elastin.

\section{B-0882 10:57}

Comparison of nanobodies and conventional monoclonal antibodies for in vivo fluorescence imaging of lymphomas

P. Bannas, A. Lenz, V. Kunick, W. Danquah, F. Haag, M. Trepel, G. Adam, H. Ittrich, F. Koch-Nolte; Hamburg/DE (p.bannas@uke.de)

Purpose: To compare different formats of llama-derived nanobodies as alternatives to monoclonal antibodies for specific in vivo near-infrared fluorescence (NIRF) imaging of lymphomas in a mouse model.

Methods and Materials: We used ecto-enzyme ART2 on lymphoma cells as model target and compared three different formats of ART2-specific nanobodies with a conventional antibody (150kD): a monovalent llama nanobody (15kD), a bivalent diabody $(30 \mathrm{kD})$, and a bivalent $\mathrm{Fc}$-fusion protein $(80 \mathrm{kD})$. All constructs were labeled with AlexaFluor680 and injected in nude mice, bearing ART2-positive and ART2negative tumors. Circulating and excreted conjugates were monitored in plasma and urine and in vivo NIRF-imaging was performed over $24 \mathrm{~h}$. Tumor tissue penetration and target binding were analysed by FACS and fluorescence microscopy. Results: In vivo NIRF-imaging revealed specific labeling of ART2-positive tumors but not of ART2-negative tumors with all AF680-conjugates. The smaller nanobody and diabody revealed rapid renal elimination with highest signal-to-background ratios of ART2-positive tumors. The larger Fc-fusion protein and conventional antibody revealed long circulation times in plasma, resulting in unspecific background signals. FACS and Immunohistochemistry of tumors revealed deeper penetration and more homogenous labeling of tumor tissue with the nanobody and diabody than with the Fc-fusion protein and conventional antibody.

Conclusion: Renally excreted small nanobody-formats $(<60 \mathrm{kD})$ can be used for specific in vivo imaging of lymphomas with a significantly higher signal-tobackground ratio than larger antibody-constructs $(>60 \mathrm{kD})$ that are retained in circulation. Therefore, single domain and bivalent nanobodies seem particularly suited for short-term diagnostic imaging, whereas reformatted nanobody-Fc fusion proteins appear suited for long-term applications.

\section{B-0883 11:06}

The design of a dual-functional imaging contrast agent for targeting to tumour endothelial marker (TEM8)

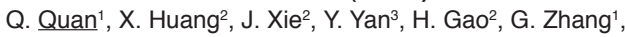

X. Chen'; ${ }^{1}$ Shanghai/CN, ${ }^{2}$ Bethesda, MD/US, ${ }^{3}$ Madison, WI/US (quanquan527@gmail.com)

Purpose: We have previously designed QQM peptide that binds specifically to the extracellular domain of TEM8. In this study, we would like to develop a QQM-based nanoplatform for dual-modality-targeted imaging of TEM8 expression. 
Methods and Materials: Near-infrared dye ZW-800 (Z) and QQM peptide (Q) were conjugated onto the surface of iron oxide nanoparticles (IO). The Z-IO-Q particles were characterised by TEM, DLS, fluorescence intensity. MR and optical imaging were carried out on $4 \mathrm{~T} 1$ cells in vitro and tumour xenografts. The receptor specificity of the dual-modality imaging probe was also confirmed by a blocking experiment in the presence of excess amount of non-labelled QQM peptide.

Results: The Z-IO-Q conjugate had an overall hydrodynamic particle size of $47.8 \mathrm{~nm}$. Incubation of $4 \mathrm{~T} 1$ cells leads to more significant T2* signal drop (82.18 $\pm 2.84 \%)$ than $Z-I O(17.48 \pm 0.50 \%)(P<0.01)$. In vivo MR imaging showed significantly higher accumulation of Z-IO-Q than Z-IO in $4 \mathrm{~T} 1$ tumour xenograft, with T2* signal drop of $47.51 \pm 10.26 \%$ versus $15.02 \pm 5.13 \%$ at $2 \mathrm{~h}$ and $41.80 \pm$ $6.94 \%$ versus $2.93 \pm 9.0 \%$ at $24 \mathrm{~h}$ post-injection for $Z-I O-Q$ and $Z-I O$, respectively. The higher fluorescence intensity in the optical imaging of $Z-I O-Q$ group was also observed. Both MR enhancement and optical imaging contrast were effectively blocked in the presence of QQM peptide.

Conclusion: We have successfully developed QQM peptide as a tumour TEM8 targeting ligand that can promote both MR and optical imaging contrast enhancement in TEM8 positive tumours.

\section{B-0884 11:15}

Folic acid-modified dendrimer-entrapped gold nanoparticles as nanoprobes for targeted computed tomography imaging of human lung adenocarcinoma

L.-F. Zheng, H. Wang, C. Peng, M.-W. Shen, X.-Y. Shi, G.-X. Zhang;

Shanghai/CN (zhenglinfeng04@yahoo.com.cn)

Purpose: To study the new usage of folic acid-modified dendrimer-entrapped gold nanoparticles (Au DENPs-FA) as nanoprobes for in vitro and in vivo targeted computed tomography (CT) imaging of human lung adenocarcinoma

Methods and Materials: Au DENPs prepared using amine-terminated generation 5 poly (amidoamine) dendrimers as templates were covalently linked with $\mathrm{FA}$, followed by an acetylation reaction to neutralise the remaining dendrimer surface amines. The formed Au DENPs-FA was used for both in vitro and in vivo targeted CT imaging of human lung adenocarcinoma cells (SPC-A1 cells) and the xenograft tumour model, which express folic acid receptors (FAR) verified by immunohistochemical staining.

Results: Micro-CT images show that SPC-A1 cells can be detected under $\mathrm{x}$-ray after incubation with the Au DENPs-FA in vitro and the xenograft tumour model can be imaged after intravenous, intratumoral, and intraperitoneal administration of the particles. Transmission electron microscopy data confirm that the $\mathrm{Au}$ DENPs-FA is able to be uptaken dominantly in the lysosomes of the cells. Combined morphological observation of cells after hematoxylin and eosin staining, MTT (3-(4.5-dimethylthiazol-2-yl)-2.5-diphenyltetrazolium bromide) assay of cell viability, and flow cytometric analysis of cell cycle and apoptosis shows that the Au DENPs-FA does not affect cell morphology, viability, and cell cycle and apoptosis, indicating their good biocompatibility at the given concentration range.

Conclusion: These findings suggest that the developed Au DENPs-FA have a great potential to be used as imaging probes for targeted $\mathrm{CT}$ imaging of human lung adenocarcinoma.

Author Disclosures:

L. Zheng: Research/Grant Support; the Shanghai Jiao Tong University medical engineering crossover Fund Project (No. YG2011MS47) and Natural Science Foundation of Shanghai Science and Technology Commission (No. 12ZR1424900). H. Wang: Research/Grant Support; the National Natural Science Foundation of China (30901730). G. Zhang: Research/Grant Support; the Key Program of Science and Technology Commission of Shanghai Municipality (No.11JC1410500).

\section{B-0885 11:24}

Imaging of the therapeutic efficiency of photodynamic therapy with a new designed fluorescence optical annexin probe

K. Haedicke, S. Gräfe, F. Lehmann, W.A. Kaiser, I. Hilger;

Jena/DE (Katja.Haedicke@med.uni-jena.de)

Purpose: Photodynamic therapy (PDT) destroys tumour tissue via generating reactive oxygen species after administration of a photosensitizer and illuminating the tumour with light. Next to many other molecular processes, apoptosis occurs in the tumour after this therapy. We designed a new fluorescence optical probe based on annexin $V$ to detect this therapeutic effect after PDT in vivo via non-invasive near-infrared fluorescence optical imaging.

Methods and Materials: Annexin V was labelled with the near-infrared dye DY734-NHS and characterised spectroscopically. Binding affinity of the probe to apoptotic tongue-squamous epithelium carcinoma (CAL-27) cells was analysed by flow cytometry and determining the dissociation constant. For detecting apoptosis in vivo, CAL-27 tumour-bearing mice were treated with PDT and injected with the annexin $\mathrm{V}$ probe two days and two weeks after therapy. The fluorescence intensity of the tumours was evaluated semiquantitatively over time.

Results: The annexin $V$ probe showed nearly the same absorption $(715 \mathrm{~nm})$ and emission $(750 \mathrm{~nm})$ spectrum like the free dye and possessed a high binding affinity to apoptotic cells (KD-value $20 \mathrm{nM}$ ). Therapeutic efficiency of PDT could be detected in vivo in the tumours via apoptotic cells shortly after therapy. Our probe accumulated significantly stronger in treated tumours at two days in contrast to two weeks after therapy.

Conclusion: In summary, we could successfully image the therapeutic efficiency of PDT with the new designed fluorescence optical annexin $V$ probe. Apoptotic cells could be detected in the tumour short time periods ( 2 days) but not longer time (2 weeks) after PDT.

\section{B-0886 11:33}

Bimodal NIRF nanoparticles as an optical and MRI contrast agent for imaging cells of the mononuclear phagocyte system

J. Domey ${ }^{1}$, C. Bergemann ${ }^{2}$, I. Krumbein ${ }^{1}$, J.R. Reichenbach ${ }^{1}$, S. Bremer-Streck', W.A. Kaiser', I. Hilger'; ${ }^{1}$ Jena/DE, ${ }^{2}$ Berlin/DE (Jenny.Domey@med.uni-jena.de)

Purpose: Even though approaches for multimodal imaging of targeted structures like in tumours have been suggested, fewer investigations have been made on bimodal optical and MR-imaging of inflammatory diseases via passive targeting of cells of the mononuclear phagocytic system (MPS). However, non-targeted nanoparticles (NP) could represent a simple and effective labelling tool being rapidly phagocytized from the blood stream by mononuclear cells which are then migrating to the site of inflammation.

Methods and Materials: For the production of bimodal NP iron oxide cores were enclosed by a near infrared (NIR)-fluorochrome (IR780) and surrounded by a starchmatrix. The spectroscopic and MR-imaging properties of the bimodal particles were determined in agarose phantoms and in vivo using a near infrared fluorescence (NIRF) small animal imaging system and 1.5 and $3 \mathrm{~T}-\mathrm{MRI}$, respectively. In vivo optical and MR-imaging was performed on mice with hind leg oedema.

Results: A specific NIRF- (average fluorescence signal 0.004 scaled counts/s for $25 \mu \mathrm{g} / \mathrm{ml} \mathrm{NP}$ ) and MR-signal (1.5 T: r1=6 mM-1 * sec-1, r2=583 mM-1 * sec-1, $\mathrm{r}^{*}=613 \mathrm{mM}-1$ * sec-1) was detected for the bimodal NP in vitro. In vivo-MR and NIRF-imaging studies confirmed specific signalling of the particles within oedema. Conclusion: Our bimodal non-targeted fluorescent iron oxide NP successfully labelled cells of the MPS in vitro and in vivo without specific target recognition by antibodies. Additionally, the ability of specific NIR-signalling and MR-tracking of mononuclear cells makes them suitable for application as a simple and potent NIRF-optical and MRI contrast agent for visualisation of inflammatory diseases.

\section{B-0887 11:42}

Assessment of in vivo cell viability of Gd or SPIO cells using MRI and BLI J. Guenoun, A. Ruggiero, G. Doeswijk, R. Janssens, G.A. Koning, G. Kotek, G.P. Krestin, M.R. Bernsen; Rotterdam/NL (j.guenoun@erasmusmc.nl)

Purpose: To assess whether MRI can distinguish viable from non-viable transplanted cells in vivo, using either Gd-liposomes or SPIO as cell labelling agent. Methods and Materials: Gd-DTPA was incorporated in cationic liposomes. Rat mesenchymal stem cells (rMSCs) were transduced to constitutively express firefly luciferase and labelled with either SPIO $(215 \mu \mathrm{g} \mathrm{Fe} / \mathrm{ml})$ for $24 \mathrm{~h}$ or $125 \mu \mathrm{M}$ Gd-liposomes ( $4 \mathrm{~h}$ ). A cell concentration of $5 \times 105$ viable or non-viable rMSCs were injected in rat musculoskeletal tissue. MRI and BLI were performed on days 2, 5, 10 and 15 after injection. MRI T1 mapping (TR variation) and T2 and T2* mapping (TE variation) were performed on a $3 \mathrm{~T}$ clinical scanner using customised coils. Bioluminescence imaging was performed after intraperitoneal injection of $D$-Luciferin $(100 \mathrm{mg} / \mathrm{kg}$ ). At end-point (day 15) animals were sacrificed for immunofluorescent staining on iron and macrophages.

Results: While MRI signal from non-viable Gd-MSCs disappeared $<1$ day postinjection, signal void from non-viable SPIO-MSCs remained visible up to at least 2 weeks. Histology showed the iron to remain in situ. Viable Gd-MSCs could be distinguished from non-viable Gd-MSCs, based on both visible contrast changes and $\mathrm{r} 1$ and $\mathrm{r} 2$ relaxivity measurements. Differences in viability of SPIO-MSCs, however, could not be detected visually or by $\mathrm{r} 2$ measurements.

Conclusion: Gd-liposomes can be used as a cell labelling tool to distinguish between viable and dead transplanted MSCs in vivo. For this purpose Gd-liposomes are favoured over SPIO nanoparticles, due to the low specificity of the latter. 


\section{B-0888 11:51}

Interaction of magnetically labelled multipotent mesenchymal stromal cells and E-/P-selectins monitored by magnetic resonance imaging in mice

J. Salamon, K. Peldschus, D. Wicklein, C. Lange, H. Ittrich, U. Schumacher, G. Adam; Hamburg/DE (j.salamon@uke.de)

Purpose: This study's purpose was to analyse the influence of E- and P-selectins on the migratory pattern of magnetically labelled multipotent mesenchymal stromal cells (MSC) in E-/P-selectin deficient (KO) and wildtype (WT) mice using MRI and fluorescence microscopy.

Methods and Materials: Murine MSC were labelled with fluorescent iron-oxide micro-particles and carboxyfluorescein succinidylester. The ability to bind selectins and the expression of typical MSC markers were assessed by flow cytometry. Labelled MSC were injected into KO- and WT-mice applying doses of $5 \times 104$ cells intracardially, $1 \times 106$ cells intravenously and $5 \times 106$ cells intraperitoneally. Mice underwent sequential MRI at 3.0 T using high-resolution $\mathrm{T} 2^{*} 3 \mathrm{D}$ and $2 \mathrm{D}$ coherent gradient-echo sequences and histological evaluation after 7 days.

Results: Expression of typical MSC markers and binding abilities to E-/P-selectins were found similar after labelling. After intravenous cell injection in KO-mice significant SNR decrease was assessed solely in the liver from day 0 to 1 ; WT-mice revealed an increasing SNR decrease of liver, spleen and bone marrow within 7 days. After intraperitoneal injection no significant SNR changes were observed in KO-mice; WT-mice showed an increasing SNR decrease of liver, spleen and bone marrow within 7 days. After intracardiac injection multiple susceptibility artefacts could be detected no longer then day 4 in $\mathrm{KO}$ mice but persisted up to day 7 in WT-mice. Fluorescence microscopy confirmed MRI findings.

Conclusion: This study demonstrates that MSC retain their binding ability to $\mathrm{E}$ /P-selectins after magnetic labelling. E-/P-selectin deficiency in mice significantly alters the distribution of magnetically labelled MSC.

Room B

\section{Neuro}

\section{SS 1811a}

\section{Latest developments in neuroimaging}

Moderators:

S. Haller; Geneva/CH

E. Papadaki; Iraklion/GR

\section{B-0889 14:00}

Improved image quality for higher diagnostic accuracy of cranial computed tomography using iterative image reconstruction H. Haubenreisser ${ }^{1}$, C. Fink ${ }^{2}$, P. Apfaltrer ${ }^{1}$, B. Schmidt ${ }^{3}$, M. Sedlmair ${ }^{3}$, S.O. Schönberg' 1 , T. Henzler'; ${ }^{1}$ Mannheim/DE, ${ }^{2}$ Celle/DE,

${ }^{3}$ Forchheim/DE (holger.haubenreisser@medma.uni-heidelberg.de)

Purpose: To prospectively compare the image quality of cranial computed tomography (cCT) with thin slice widths using traditional filtered back projection (FBP) and sinogram-affirmed iterative image reconstruction (SAFIRE).

Methods and Materials: 40 consecutive studies (19 men; $71.6 \pm 16.6$ years) referred for $\mathrm{CCT}$ were prospectively included. Each cranial $\mathrm{CT}$ raw data set was reconstructed with FBP and SAFIRE with decreasing slice widths $(5 \mathrm{~mm}-1 \mathrm{~mm})$. Objective image quality was assessed by measuring image noise in three predefined regions of the brain (white matter, thalamus, cerebellum) using identical regions of interest (ROIs). Subjective image quality was assessed by 2 experienced radiologists by ranking the reconstructed data sets with respect to overall image quality. The Mann-Whitney U-test and Cohen's Kappa were used for statistical analysis. Results: Image noise was statistically significantly reduced in all SAFIRE images at identical slice widths when compared to the images reconstructed with FBP $(4.26 \pm 0.43 \mathrm{HU}$ vs. $7.67 \pm 1.19 \mathrm{HU}$ at $1 \mathrm{~mm}$ slice width) $(p<0.001)$. Mean signal attenuation for each region and slice width remained constant between the two reconstruction methods ( $p>0.5$ ). SNR was comparable between $1 \mathrm{~mm}$ SAFIRE images and $5 \mathrm{~mm}$ FBP images. Subjective image quality of SAFIRE images was rated consistently higher than that of the FBP images $(p<0.001)$. Interobserver agreement was excellent between both radiologists (Cohen's $K=0.79-0.86$ ). Conclusion: Iterative image reconstruction significantly reduces image noise, while increasing image quality. In CCT this may be used to decrease slice width and thus reduce partial volume effects, which may lead to increased diagnostic accuracy.

\section{Author Disclosures:}

B. Schmidt: Employee; Siemens Healthcare. M. Sedlmair: Employee; Siemens Healthcare.
B-0890 14:09

Synchrotron radiation micro-CT imaging of the mouse brain

A. Chabrol' ${ }^{1}$ H. Rositi', M. Marinescu', M. Langer ${ }^{2}$, C. Olivier ${ }^{2}$, F. Peyrin ${ }^{2}$,

C. Frindel ${ }^{1}$, M. Wiart ${ }^{1}$, Y. Berthezène ${ }^{1} ;{ }^{1}$ Lyon/FR, ${ }^{2}$ Grenoble/FR

(aurelie_chabrol@yahoo.fr)

Purpose: MRI coupled with the injection of ultrasmall particles of iron oxide (USPIO) has been successfully applied for pre-clinical and clinical studies of cerebral inflammation following stroke. Current limitations of this approach are represented by the difficulty of interpreting MR signal changes in terms of exact USPIO Iocalisation. To overcome this limitation, we introduce Synchrotron Radiation phase micro-computerized tomography (SR-PCT) as a new method to visualise USPIO distribution into the whole brains of mice with cerebral ischaemia.

Methods and Materials: The sensitivity of the technique was assessed by performing SR-PCT and MRI back-to-back in 10 mice stereotaxically injected with a range of USPIO concentrations. Eight mice with cerebral ischaemia were then intravenously injected with USPIOs and imaged back-to-back with MRI and SR-PCT. Results: SR-PCT showed hyperintense areas in the regions of MR signal loss. In stereotaxically injected animals, SR-PCT proved sensitive enough to detect iron in nanomolar quantities. In ischaemic animals, some of these hyperintense areas consisted of bright spots about 40- $\mu \mathrm{m}$ in diameter, compatible with USPIO-labelled macrophages. Immunohistochemistry demonstrated the presence of iron-labelled cells, most probably macrophages, at the same location. The SR-PCT pipeline, moreover, identified brain anatomy as clearly as histology, without the need for sectioning or staining, with an examination time of 44 minutes per brain at an isotropic spatial resolution of $8 \mu \mathrm{m}$.

Conclusion: Phase-contrast $x$-ray tomography may become an invaluable complement to USPIO-enhanced MRI to study post-ischaemic inflammation in intact brain, with unequaled neuroanatomic analysis.

\section{B-0891 14:18}

Effect of dose reduction on cerebral CT perfusion maps: results

from a hybrid digital perfusion phantom

R. van den Boom ${ }^{1}$, M. Oei', L.J. Oostveen ${ }^{1}$, H. Laue ${ }^{2}$,

B. van Ginneken', R. Manniesing 1 , M. Prokop ${ }^{1}{ }^{1}$ Nijmegen/NL,

2Bremen/DE (R.vandenBoom@rad.umcn.nl)

Purpose: To quantitatively evaluate the effect of dose reduction of a standard head CTP protocol on the perfusion maps.

Methods and Materials: A hybrid digital perfusion phantom was used to simulate dose reduction in a CT perfusion acquisition. Noise patterns were obtained by scanning an epoxy filled human skull phantom on multiple mAs levels on a 320row scanner and combined with tissue attenuation curves obtained from 5 patient data for grey matter (GM), white matter (WM), artery and vein. We simulated a real clinical CTP head protocol available on the Toshiba CT console (1 scan at $200 \mathrm{mAs}, 13$ scans at $100 \mathrm{mAs}, 5$ scans at $75 \mathrm{mAs}$ and all at $80 \mathrm{kV}$ ) with a total duration of $55 \mathrm{~s}$ ( $5 \mathrm{~mm}$ slab thickness) and artificially reduced the dose from $100 \%$ to $10 \%$ in steps of $10 \%$. CBF, CBV and MTT maps were calculated using PMA (ASIST-JAPAN). Mean values of GM and WM were evaluated.

Results: Absolute CBF and CBV values stay within $20 \%$ of the values at original dose for CTP acquisitions at more than $50 \%$ of the original dose for both GM and WM. At $10 \%$ of the original dose, WM CBV values were overestimated with 24$107 \%$, GM CBV values with $4-32 \%$, and WM CBF values with $42-106 \%$. GM CBF values were within $20 \%$ of the values at $100 \%$ of original dose. MTT values were less affected by dose reduction.

Conclusion: Absolute CBF and CBV values require sufficient dose to be correct: values at low dose are overestimated.

Author Disclosures:

R. van den Boom: Research/Grant Support; Toshiba Medical Systems Corporation. R. Manniesing: Research/Grant Support; Toshiba Medical Systems Corporation. M. Prokop: Research/Grant Support; Toshiba Medical Systems Corporation.

\section{B-0892 14:27}

iDose hybrid iterative reconstruction algorithm improves image quality in brain $\mathrm{CT}$ and partly compensates for radiation dose reduction A. Löve, R. Siemund, P. Höglund, D. van Westen, L. Stenberg, C. Petersen, I.M. Björkman-Burtscher; Lund/SE (askell.love@skane.se)

Purpose: To evaluate the potential of the reconstruction algorithm iDose to preserve image quality in brain-CT acquired with $30 \%$ reduced radiation dose and to evaluate image quality assessment methods. 
Methods and Materials: 40 patients (mean age 77 years, range 65-93) gave informed consent for this prospective research ethic committee-approved study and underwent two consecutive brain CT-scans, with normal radiation dose (ND) (CTDlvol $57 \mathrm{mGy}, 2.6 \mathrm{mSv}$ ) and $30 \%$ reduced dose (RD). ND scans were reconstructed using filtered backprojection (FBP), while RD scans were reconstructed using FBP and two iDose levels, ID2 and ID4. Image quality was assessed with grading and ranking by six neuroradiologists. Mean Hounsfield units $(\mathrm{HU})$ and noise were measured. Data were analysed using linear mixed models, and the Tukey method for multiple comparisons.

Results: For all reconstructions image quality was graded adequate for clinical use. Image quality was graded good/excellent in $93 \%$ of cases for ND, $83 \%$ of cases for ID4, 79\% for ID2, and $67 \%$ for RD. For all quality parameters ID4 and ID2 were graded better than RD $(p<0.0055$ and $p<0.035)$ but worse than ND ( $p$ $<<0.001)$. Pooled ranking results were equivalent despite considerable variation in individual assessments. HU were identical between reconstructions. Image noise was similar in ND, ID2 and ID4 but higher in RD.

Conclusion: The iterative reconstruction algorithm improves image quality of reduced-dose images and consistently delivers sufficient clinical image quality, although it does not fully compensate for $30 \%$ dose reduction. Pitfalls related to subjective assessment can be addressed with careful study design.

\section{B-0893 14:36}

MRI susceptibility-weighted imaging (SWI): role in the differentiation of inflammatory pathologies from high-grade glioma

S. Chu' ${ }^{1}$, Y. Wang 1 , Z. Yang 1 , F. Xv' ${ }^{1}$, O. Boyko ${ }^{2} ;{ }^{1}$ Shanghai/CN,

2Los Angeles, CA/US (chushu1018@hotmail.com)

Purpose: To determine whether MRI susceptibility-weighted imaging can assist in discriminating between inflammatory pathologies and high-grade glioma.

Methods and Materials: Susceptibility-weighted imaging (SWI) was performed at 3.0 T (Siemens Medical Systems) and images were reviewed retrospectively in 17 patients with cerebral inflammatory pathologies and 25 patients with histologically proven high-grade gliomas. Only gliomas without MR findings of haemorrhage on T1WI were included in the study. The morphological pattern of the susceptibility signal and the anatomic positional relationship between the contrast enhancement areas and susceptibility signals were retrospectively reviewed by consensus of 2 reviewers. The SWI morphological pattern was scored as: patchy, nodular, dot-like or linear and designated as inside or outside the zone of contrast enhancement. Results: In total, 17 clinically proven inflammatory diseases ( 7 parasitic granulomas, 3 inflammation-demyelinating and 7 neurovasculitis) and 23 lesions were reviewed. Patchy, nodular, dot-like susceptibility were demonstrated in both inflammatory and neoplastic lesions, whereas linear susceptibility signals were only identified in the inflammatory diseases. Nine of $23(39 \%)$ susceptibility signals were situated outside the contrast enhancement areas in inflammatory pathologies with no susceptibility signals found outside the border of the contrast enhancement areas among all the high-grade gliomas.

Conclusion: On SWI, linear susceptibility signal and/or susceptibility signal located outside the area of contrast enhancement was more frequently presented in inflammatory processes and not found with the high-grade gliomas. This finding may reflect a different pathophysiologic mechanism and distinction of bleeding/ microbleeding occurrence and presentation in inflammatory pathologies compared to high-grade gliomas.

\section{B-0894 14:45}

Inter-session reproducibility of cerebral blood flow (CBF) in the primary motor cortex as assessed in the VESPA (vendor-specific features of ASL-MRI) study

R.M.E. Steketee ${ }^{1}$, H.-J.M.M. Mutsaerts², G.C. Houston ${ }^{3}$, C.B.L.M. Majoie' ${ }^{2}$, A.J. Nederveen ${ }^{2}$, M. Smits $1 ;{ }^{1}$ Rotterdam/NL, ${ }^{2}$ Amsterdam/NL, ${ }^{3}$ Zeist/NL (r.steketee@erasmusmc.nl)

Purpose: Arterial spin labelling (ASL) is a non-invasive magnetic resonance imaging (MRI) technique to quantify cerebral blood flow (CBF). To reliably use quantitative $\mathrm{CBF}$ in clinical practice, inter-session variability needs to be established. The current study assesses reproducibility of resting and delta $\mathrm{CBF}$ in the primary motor cortex measured with pseudocontinuous-ASL ( $p-C A S L)$ during rest $(R)$ and fingertapping $(\mathrm{FT})$ in healthy volunteers.

Methods and Materials: Ten participants (mean age 22y, 5 male) underwent 3D p-CASL and T1w scanning at $3 \mathrm{~T}$ (GE Healthcare, US) twice. Sessions were separated by $1-4$ weeks. During p-CASL participants performed 2 min of $1-\mathrm{Hz}$ auditory paced bimanual FT alternated with 2 min of $R, 4$ times per session. Data were realigned, coregistered and normalised using SPM8 (London, UK). CBF values from gray matter only were extracted from the bilateral primary motor cortex using MarsBaR (Marseilles, FR). CBF values were compared between conditions (FT, R), and resting and delta CBF (CBF[FT]-CBF[R]) values between sessions using paired t-tests.

Results: $\mathrm{CBF}$ values were significantly higher during $\mathrm{FT}$ than $\mathrm{R}$ in both sessions ( 52.30 versus $47.70 \mathrm{ml} / 100 \mathrm{cc} \mathrm{GM} / \mathrm{min}, \mathrm{p}<0.01 ; 52.32$ versus $48.45 \mathrm{ml} / 100 \mathrm{cc}$ $\mathrm{GM} / \mathrm{min} \mathrm{p}<0.01)$. There was no significant difference in resting $(47.70$ versus $48.45 \mathrm{ml} / 100 \mathrm{cc}$ GM/min, $p>0.05)$ or delta CBF between sessions $(4.60 \mathrm{ml} / 100$ cc $\mathrm{GM} / \mathrm{min}$ versus $3.87 \mathrm{ml} / 100 \mathrm{cc} \mathrm{GM} / \mathrm{min}, \mathrm{p}>0.05$ ).

Conclusion: Mean resting and delta CBF as measured with p-CASL were consistent over sessions, while a significant increase of CBF during motor activity could be quantified. Current efforts are directed towards assessing inter-vendor reproducibility in a larger population: the VESPA study.

\section{B-0895 14:54}

Are quantitative pharmacokinetic data of dynamic contrastenhanced MRI influenced by observer-related bias? New data with special focus on reproducibility, precision, accuracy, reliability, repeatability and systematic bias

M. Dietzel' ${ }^{1}$, M. Saake ${ }^{1}$, P.A.T. Baltzer ${ }^{2}$, S. Lang ${ }^{1}$, B. Volbers ${ }^{1}$, T. Struffert' ${ }^{1}$, T. Engelhorn ${ }^{1}$, A. Dörfler ${ }^{1} ;{ }^{1}$ Erlangen/DE, ${ }^{2}$ Vienna/AT (dietzelmatthias2@hotmail.com)

Purpose: Quantitative T1-weighted perfusion imaging is promising. However, current software solutions are still semi-automatic and results are therefore potentially prone to observer-related bias. Surprisingly, this aspect of DCE-MRI is still poorly investigated. We aimed to systematically address this matter using a dedicated imaging protocol and semi-automatic workflow for quantitative pharmacokinetic analysis.

Methods and Materials: An ultra-fast high-spatial and high-temporal resolution protocol was used for T1-weighted perfusion imaging (Magnetom Aera @1.5 T; dynamic view-sharing 3D gradient-echo-sequence/TWIST: temporal-resolution: 1.81s, in-plane resolution: $1.1 \mathrm{~mm}^{3}, 6 \mathrm{ml}$ Gadovist @2 ml/s, acquisition time: $62 \mathrm{~s}$ ). For the assessment of pharmacokinetic parameters ( $\mathrm{k}^{\text {trans }}$, two-compartment Tofts-model, fast Arterial-Input-Function) a dedicated semi-automatic software solution was used. Enhancing intracranial tumours were independently evaluated two times by one experienced neuroradiologist ( $>4000 \mathrm{MRI}, 8$-year experience in DCE-MRI). Results of pharmacokinetic parameters for each reading were documented in a database. Variability of $k^{\text {trans }}$ measurements was assessed by means of reproducibility (concordance correlation coefficient), precision (Pearson $\rho$ ), accuracy (Bias correction factor/Cb), reliability (intraclass correlation coefficient), repeatability (coefficient of repeatability/Cr) and systematic bias (Bland-Altmann plot: regression coefficient/Rc).

Results: 15 malignant and 8 benign intracranial tumours were included. Appropriate values for median $\left(1^{\text {st }} / 2^{\text {nd }}\right.$ measurement: $\left.0.113 / 0.104\right)$, range $\left(1^{\text {st }} / 2^{\text {nd }}\right.$ measurement: $0.518-0.054 / 0.520-0.061)$ and standard deviation $\left(1^{\mathrm{st}} / 2^{\text {nd }}\right.$ measurement: $0.099 / 0.100)$ of $k^{\text {trans }}$ were identified within both measurements. Reproducibility (concordance correlation coefficient $=0.993$ ), precision (Pearson $\rho=0.993$ ), accuracy (Bias correction factor $/ \mathrm{Cb}=0.999$ ), reliability (intraclass correlation coefficient $=0.996$ ), repeatability (coefficient of repeatability/CR $=0.045$ ) reached excellent values. With a regression coefficient of -0.020 there was no substantial evidence of systematic bias.

Conclusion: T1-weighted perfusion imaging with subsequent pharmacokinetic analysis showed excellent reproducibility, precision, accuracy, reliability and repeatability. According to our results it is a robust quantitative MRI method and not influenced by observer-related or systematic bias.

\section{B-0896 15:03}

MR elastography (MRE) for non-invasive differentiation of intracranial tumours: results of a histology correlated study M. Simon'1, J. Guo², M. Bonsanto1, D. Petersen 1 , I. Sack², J. Würfell;

${ }^{1}$ Lübeck/DE, ${ }^{2}$ Berlin/DE, ${ }^{3}$ Göttingen/DE (martin.simon@radiologische-allianz.de)

Purpose: We investigated the potential of MR elastography (MRE) to non-invasively differentiate intracranial tumours by quantifying the tumour tissue viscoelasticity or "firmness" in a preoperative setting and subsequently correlated MRE parameters to histology.

Methods and Materials: Twelve patients with suspected intracranial malignoma and planned surgery were selected from routine. Tumours showing signs of bleeding, aberrant vessels or aneurysms were excluded. MRE was performed on an 3 Tesla MRI scanner, applying motion encoding EPI sequences in three directions and monofrequent shear wave generation by a head cradle as previously described. Viscoelasticity was measured for tumour tissue as well as for healthy white matter as reference. 
Results: The study comprised of $n=12$ intracranial tumours, among these meningeomas $(n=2)$, solitary metastasis $(n=3)$, as well as WHO grade II - IV tumours $(n=7)$. Compared to reference tissue, meningeomas were significantly stiffer. Metastases were either slightly firmer or softer than reference tissue. WHO grade II and III tumours $(n=4)$ were softer than white matter in all cases. The highest loss of tissue stiffness was represented by WHO grade IV tumours. Viscoelasticity parameters were supplemented by a histopathology work-up. In summary, high WHO grade tumours were significantly softer than tumours of lower staging, the range of metastatic viscoelasticity was slightly higher than primary brain tumours. MRE was well tolerated by all patients.

Conclusion: MR elastography of cerebral tumours at 3 Tesla reliably quantifies tissue firmness for preoperative planning. According to our histology correlated preliminary results, tumour viscoelasticity may be an additional diagnostic parameter for non-invasive tumour differentiation.

\section{B-0897 15:12}

Intra- and inter-scanner test-retest reliability of whole-brain arterial spin labeling perfusion MRI

B. Wu, X. Wu, X. Lou, M. Ge, L. Ma; Beijing/CN (646173826@qq.com)

Purpose: Perfusion MRI can be used to identify and monitor patients with acute ischaemic stroke. The objective of this study was to investigate whether it is possible to pool together whole brain arterial spin labelling (ASL) perfusion imaging data at different times, or from different scanners, located at two different sites.

Methods and Materials: Eight healthy subjects were scanned on two different 3.0 T scanners with 3D pseudo-continuous labelling technique. Scans were repeated intra-session and inter-session after 7-30 days. The ASL data with two postlabelling delay time (PLD) of 1.5 and 2.5 seconds were acquired. High-spatial-resolution whole brain $\mathrm{T} 1$-weighted images were also acquired for image registration and normalisation into a standardised space within the SPM8. The cerebral blood flow (CBF) values of grey matter, white matter, frontal lobe, parietal lobe, occipital lobe, temporal lobe, putamen, posterior cingulate, and thalamus were extracted for comparison, using WFU Pickatlas tool. The intra- and inter-scanner reliability was evaluated with the intraclass correlation coefficient (ICC). For this study, two-way model, single-measures ICC were calculated using SPSS.

Results: The CBF data demonstrated high measurement reliability (total ICC: 0.910; intra-scanner ICC: 0.923; inter-scanner ICC: 0.862) for 1.5s PLD, compared with 2.5s PLD (total ICC: 0.928; intra-scanner ICC: 0.944; inter-scanner ICC: 0.884). Conclusion: Although inter-scanner reliability is lower than intra-scanner, there is a very high similarity of the outcomes at different times and from two different scanners. Our findings support the feasibility of cross-site pooling of whole-brain ASL data, and CBF values with longer PLD time could be more reliable.

\section{B-0898 15:21}

Software for the radiological view: fully automated CT-based quantification of microangiopathic density reduction in white matter in comparison to gold standard MRI

U. Hanning ${ }^{1}$, I. Noelte' ${ }^{2}$, G. Homann', T. Niederstadt'1 ${ }^{1}$, V. Hesselmann',

W. Heindel', A. Kemmling ${ }^{3} ;{ }^{1}$ Münster/DE, ${ }^{2}$ Mannheim/DE,

${ }^{3}$ Hamburg/DE (uhanning@uni-muenster.de)

Purpose: Assessment of white matter lesions is primarily a domain of magnetic resonance imaging (MRI); however, computed tomography (CT) is the more frequently used diagnostic procedure. The reduction of density of white matter in CT images can be used as a surrogate for the severity of microangiopathic WM changes. We present a new CT-based fully automated rater-independent method for quantification of microangiopathic white matter changes.

Methods and Materials: Tissue segmentation was performed in 600 normal brains MRI (3.0 Tesla, T1-3D-Turbo-Field-Echo) of two large population studies (BiDirect and SEARCH-Health Study) to obtain a probabilistic WM-tissue-map in standard $\mathrm{MNI}-152$ space (published previously).The tissue-specific density (Hounsfield Unit, $\mathrm{HU}$ ) within WM-space was determined by the mean of all voxel densities weighted by WM content: $\Sigma$ (HUxyz $\times$ Pxyz (WM))/ $\Sigma$ (Pxyz (WM); (HUxyz = density of voxelxyz; Pxyz = partial WM content at voxelxyz). The reduction of $\mathrm{HU}$ over WM-space in CT images was correlated with gold standard MR-based WM lesion volume measurements. Results were compared with rater-based Fazekas scores for severity of WM disease

Results: The process of CT-based tissue-specific segmentation was reliable in 103 cases with varying occurrence of microangiopathy. In comparison with MRI FLAIR-based WM-lesion volume, the CT- based HU-weighted reduction of the white matter showed a significant correlation coefficient $(0.87)$.
Conclusion: The presented method allows fully automated observer-independent quantification of microangiopathic $\mathrm{HU}$-induced reduction of the white matter in CT with high correlation to gold standard MRI.

14:00 - 15:30 Room C

Neuro

SS 1811b

Spinal imaging

Moderators:

M. Buruian; Targu-Mures/RO

A. Cianfoni; Lugano/CH

\section{B-0899 14:00}

Diagnosis of lumbosacral transitional vertebrae on lumbar MRI: role of spinal and paraspinal anatomic markers and value of additional whole-spine localiser

N. Tokgoz, M. Ucar, B. Erdogan Sendur, K. Kılıc, C. Ozcan; Ankara/TR (niltokgoz@yahoo.com)

Purpose: Diagnosis of lumbosacral transitional vertebrae (LSTVs) is very important to avoid wrong level surgery. The aim of this study with a large patient group was to evaluate the role of spinal and paraspinal anatomic markers in identification of LSTVs on lumbar MRI and to assess the need for whole-spine localiser (WSL). Methods and Materials: Lumbar MRI of 1049 adult patients was studied. The morphology of S1-2 disc, L5 and S1 body and lumbar spinous processes were evaluated. The positions of aortic bifurcation (AB), right renal artery (RRA), superior mesenteric artery (SMA) and conus medullaris (CM) were described. Comparing with WSL, the diagnostic error in numbering vertebral segments on lumbar MRI was evaluated.

Results: In lumbarisation, all patients revealed a well-formed S1-2 disc with squared $\mathrm{S} 1$ body. A rhombus-shaped L5 body in sacralisation and a rectangular-shaped $\mathrm{S} 1$ body in lumbarisation were found. L3 had the longest spinous process. The most common sites of spinal and paraspinal structures were $A B$ at $L 4$ body $(71.1 \%), R R A$ at L1 body (53.6\%) and L1-2 disc (34.1\%), SMA at L1 body (55.1\%) and T12-L1 disc $(31.6 \%)$, and CM at L1 body ( $44.8 \%)$. They were located higher in sacralisation and lower in lumbarisation. On lumbar MRI, the diagnostic error in evaluation of vertebral segmentation was $14.1 \%$.

Conclusion: The morphologic features and locations of the spinal and paraspinal structures on lumbar MRI are not completely reliable in diagnosis of LSTVs and identification of the vertebral levels. On lumbar MRI, accurate localisation of the vertebral segments should be done with the help of additional WSL.

\section{B-0900 14:09}

Intravertebral gas in the cervical spine

P. Slon, J. Etxano, G. Viteri, I. Simón-Yarza, M. Páramo, P. García, J.C. Pueyo, J. Larrache; Pamplona/ES (pslon@unav.es)

Purpose: The presence of intravertebral gas in the lumbar and thoracic spine, usually secondary to trauma, is a well-recognised radiological phenomenon (Kümmel's Disease). However, intravertebral gas within a cervical vertebral body is a common but unknown process. We present a series of 27 patients with gas in the cervical vertebral bodies, not associated with traumatism. The aim of this study is to describe this radiological finding and its possible aetiology.

Methods and Materials: Our study group included 27 patients who underwent a routine neck $C T$ examination for reasons not related with the cervical spine. All the patients presented air within a cervical vertebral body. We analysed several factors such as age, sex, location of the gas, history of radiotherapy, chemotherapy, the presence of degenerative disease, previous infections or corticosteroid therapy. Results: The mean age was 67 years (age range 29-82), 21 men and 6 women. The main affected body was C6 (48\%), followed by C5 (33\%). Most of the lesions were adjacent to the inferior endplate of the vertebral body. Most subjects showed degenerative changes (96\%), although none had gas within intervertebral discs. $34 \%$ of the patients received radiotherapy. None of the patients had a history of trauma, infection or treatment with corticosteroids.

Conclusion: The presence of gas within a cervical vertebral body seems to be a common but unknown process. This phenomenon could be related with cervical degenerative disease. Further studies should be conducted to verify this hypothesis and to elucidate the nature of this finding. 


\section{B-0901 14:18}

The importance of craniovertebral and cervicomedullary angle on cervicogenic headache

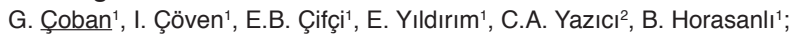
${ }^{1}$ Konya/TR, ${ }^{2}$ Ankara/TR (drgokcencoban@gmail.com)

Purpose: Many studies indicated that cervicogenic headache $(\mathrm{CH})$ may originate from cervical structures innervated by the upper cervical spinal nerves and the trigeminal nerve branches. Studies to date have not investigated whether the narrowing of the craniovertebral angle (CVA) and cervicomedullary angle (CMA) affects the nerve branches and causes pain. The aim of this study was to investigate the effect of the narrowing of the CVA and CMA values on the occurrence $\mathrm{CH}$. Methods and Materials: Between January 2011 and May 2012, 205 patients with the diagnosis of $\mathrm{CH}$ were included in the study. The pain scores in patients were carried out using a visual analogue scale from 1 to 4 (pain groups). Non-headache control group consisted of 40 volunteers. Brain MRI was performed in all. CVA and CMA were measured on the sagittal T2-weighted MRI. Each CVA and CMA values were measured on two occasions by two radiologists in four-week intervals. Results: Ages of the pain groups and control group were homogeneous $(p=0.538)$. Intraclass correlation coefficients were found to be over $98 \%$ of all measurements. Pain scores showed an increase with decreasing CVA and CMA values and it was statistically significant $(p<0.001)$. For multiple comparisons, Bonferroni's test was applied. The relationship between the CMA and CVA values [[Unable to Display Character: \&\#8203;]][Unable to Display Character: \&\#8203;]]and pain scores were statistically significant for each group $(p<0.05)$.

Conclusion: Our study showed that the narrowing of CVA and CMA values affects the occurrence $\mathrm{CH}$. Also there is an inverse relationship between the angle values and pain scores.

\section{B-0902 14:27}

Acute low back/leg pain due to lumbar disc herniation: intradiscal and intraforaminal injections of oxygen-ozone vs steroid and anaesthetics A. Alexandre', G. Izzo', G.G. Giardina', L. Coro'2, A. Alexandre'2; ${ }^{1}$ Rome/IT, ${ }^{2}$ Treviso/IT

Purpose: Prospective comparison of the clinical effectiveness of intraforaminal and intradiscal injections of a mixture of steroid and a local anaesthetic, with respect to intraforaminal and intradiscal oxygen-ozone (O2-O3) administration, in the management of radicular pain related to acute lumbar disk herniation.

Methods and Materials: Medical Ethical Committee approval and informed consent were obtained. Two hundred fifty-nine patients (186 men, 73 women; age range, 18-66 years) were included and were randomly assigned to two groups. 127 patients (group A) underwent intradiscal and intraforaminal injections of a steroid and an anaesthetic, and 132 patients (group B) underwent intraforaminal and intradiscal treatment with $\mathrm{O} 2-\mathrm{O} 3$ mixture. Procedures were performed under fluoroscopy. Roland Morris Questionnaire and VAS pain evaluation were administered before treatment and at intervals, the last at 12-month follow-up. Patients and clinicians were blinded as to which treatment was performed. Results were compared with the $\mathrm{X}^{2}$ test.

Results: After 12 months, treatment was successful in $47 \%$ patients of group A and in $74 \%$ patients in group $B$. The difference was significant $(P<0.01)$.

Conclusion: Intraforaminal and intradiscal injections of $\mathrm{O} 2-\mathrm{O} 3$ are more effective at 12 months than injections of only a steroid and an anaesthetic in the same sites.

\section{B-0903 14:36}

Optimising a BLADE sequence for T2-weighted MRI of the cervical spine in transverse orientation: is there a chance for artefact reduction? C. Fellner ${ }^{1}$, C.M. Wendl', C. Stroszczynski', T. Finkenzeller ${ }^{2} ;{ }^{1}$ Regensburg/DE, ²Nürnberg/DE (claudia.fellner@ukr.de)

Purpose: Due to their intrinsic in-plane motion correction BLADE (synonym: PROPELLER) sequences are advantageous for sagittal T2-weighted MRI of the cervical spine by reducing motion and flow artefacts. For transverse images, however, severe CSF flow artefacts have been described. The aim of this study was to evaluate if BLADE with an optimised parameter setting might overcome those limitations.

Methods and Materials: 15 volunteers (age: $26-50$ years) were examined on a 1.5 T scanner (Magnetom Avanto, Siemens Healthcare). Our routine turbo spin echo sequence with conventional k-space filling (TSE) was compared with TSE with rotating and partially overlapping filling (BLADE). 4 BLADE sequences with different parameter settings were applied in all subjects: with additional flow compensation in slice selection direction, with an increased coverage of "blades" (186\%), and with only 1 concatenation (instead of 2 of the basic version). Delineation of spinal cord and CSF flow artefacts were graded by consensus of 2 experienced readers in comparison to the TSE sequence.

Results: The basic BLADE version with 1 or 2 concatenations was significantly inferior to TSE (2-sided Wilcoxon test, $\mathrm{P}<0.05)$. Using additional flow compensation or an increased $k$-space coverage resulted in no significant differences of BLADE compared with TSE - with a minor advantage in favour of the BLADE technique with increased k-space coverage.

Conclusion: The value of the BLADE technique for artefact reduction in transverse imaging of the cervical spine is strongly dependent on dedicated parameter setting. We present a setting which might be promising for application in a patient study.

\section{B-0904 14:45}

Role of 3D MRI with ProSet technique in the evaluation of lumbar radiculopathy

D. Grasso, C. Borreggine, C. Bristogiannis, L.P. Stoppino, D. Melchionda,

L. Macarini; Foggia/IT (daniela.grasso@hotmail.it)

Purpose: The aim of our study is to demonstrate the effectiveness of 3D MRI in the diagnosis of lumbar radiculopathy in comparison with clinical and neurophysiological data.

Methods and Materials: 32 patients with L5 or $\mathrm{S} 1$ monoradiculopathy caused by a posterolateral or intraforaminal disk herniation underwent MRI examination after preliminary clinical assessment and electromyography (EMG). We performed conventional spin-echo and 3D coronal FFE sequences with selective water excitation (ProSet imaging). Using 3D coronal FFE sequences indentation, swelling and tilting angle of the nerve root were evaluated. The tilting angle was compared with the asymptomatic contralateral side of the nerve root. All the data were processed using Epi Info 3.3 software (CDC, Atlanta, GA, USA) and were compared by means of Fisher exact test.

Results: ProSet sequence revealed abnormal tilting angle in 18 patients $(57.2$ $\% ; p<0.05)$, monolateral swelling or indentation of roots and spinal nerves in 28 patients $(85.7 \%$; $p$ 0.001). In 10 patients EMG demonstrated an involvement of more than one nerve roots, whereas the ProSet revealed only one involved root. In 2 patients the nerve alterations were revealed only by EMG.

Conclusion: EMG has an improved ability to highlight the early changes of the roots caused by mechanical and biochemical alterations due to compression. ProSet Imaging demonstrates high sensitivity in identifying the exact level of the involved root and can provide a useful tool to plan surgical treatments.

\section{B-0905 14:54}

Assessing severity of peripheral neuropathy with DTI

C. Mathys, J. Aissa, G. Meyer zu Hörste, D.C. Reichelt, G. Antoch,

B. Turowski, H.-P. Hartung, K.A. Sheikh, H.C. Lehmann;

Düsseldorf/DE (mathys@uni-duesseldorf.de)

Purpose: To investigate the utility of DTI in detecting nerve injury or neuropathic changes in proximal nerve segments in patients with peripheral neuropathy. Methods and Materials: Twenty-four age-matched individuals with $(n=14)$ and without $(n=10)$ peripheral neuropathy underwent DTI of a defined sciatic nerve segment. Patients and controls were evaluated by clinical examination and nerve conduction studies at baseline and six months after the initial DTI scan. Four patients were scheduled for a second scan six months after the initial MRI.

Results: The mean FA value was significantly lower in sciatic nerves from patients with peripheral neuropathy as compared to controls. Sciatic nerve FA values positively correlated with clinical disability scores and electrophysiological parameters of axonal damage, i.e. the compound muscle action potential amplitudes of the peroneal and tibial nerve at baseline and six months after MRI scan. No correlation was found with axial and radial diffusivities.

Conclusion: DTI-derived FA values are a sensitive measure to discriminate healthy from functionally impaired human sciatic nerve segments. This technique might be potentially useful in estimating the proximal axonal degeneration burden in patients with peripheral neuropathies.

\section{B-0906 15:03}

Leukodystrophy with brainstem and spinal cord involvement and lactate elevation (LBSL): assessment of the involved white matter tracts by MRI

H.M.H. Kassem ${ }^{1}$, S. Radwan', M.H.I. Elmahdy ${ }^{1}$, A.A.S.W. Wafae ${ }^{2}$, H.R.S. Farghaly'; ${ }^{1}$ Riyadh/SA, ${ }^{2}$ Cairo/EG (kassem_hassan60@yahoo.com)

Purpose: The purpose is to describe the imaging features of the involved brainstem and spinal cord tracts in cases of leukodystrophy with brainstem and spinal cord involvement and brain lactate elevation (LBSL). 
Methods and Materials: We retrospectively reviewed the imaging features and enhancement pattern of the selectively involved white matter tracts in sixteen (16) genetically proven cases of leukodystrophy with brainstem and spinal cord involvement and elevated brain lactate (LBSL). All patients were presented with slow progressive cerebellar sensory ataxia with spasticity and dorsal column dysfunction. MRI of the brain and spine and MRS of the brain were done to all patients. Results: In all cases, MRI showed signal abnormalities in the sensory and pyramidal white matter tracts in addition to inhomogeneous signal abnormalities in the periventricular and deep white matter, brain stem, cerebellar connections and dorsal columns of the spinal cord. The subcortical $U$ fibres were spared in all cases. Proton MRS showed consistent elevation of the lactate within the abnormal white matter. In eleven cases, diffusion restriction was found in most of the length of the corticospinal and sensory tracts in addition to involvement of the superior and inferior cerebellar peduncles and the mesencephalic trigeminal tracts. These parts of the white matter tracts also displayed heterogeneous enhancement after gadolinium administration.

Conclusion: Distinct diffusion restriction and characteristic enhancement of the selectively involved white matter tracts should lead to the diagnosis LBSL with the help of the other MRI and MRS diagnostic criteria.

\section{B-0907 15:12}

Diffusion tensor imaging of the spondylotic cervical spinal cord: a new biomarker in cervical spinal cord myelopathy? A preliminary study

F.W. Cartes-Zumelzu, S. Ingorokva, H. Kostron, G.M. Feuchtner,

C. Kremser, R. Granata, G. Broessner, C. Thomé; Innsbruck/AT

(fabiola.cartes-zumelzu@i-med.ac.at)

Purpose: The purpose of this study was to evaluate the role of DTI in assessing cervical spondylotic myelopathy (CSM).

Methods and Materials: We prospectively evaluated 65 Patients with symptomatic CSM. The JOA (Japanese Orthopedic Association) myelopathy score was used to assess the clinical situation, dividing patients into groups with minimum myelopathy (MIM), moderate myelopathy (MOM) and severe myelopathy (SEM). All patients underwent examination on a 3.0 T MRI scanner (Verio, Siemens). DTI of the spinal cord was performed in 30 directions (b-value of 0 and 900). Fractional anisotropy (FA), apparent diffusion coefficients (ADC), Eigenvalues (E1, E2.3) were measured. Results: Mean patient age was 58. 8 y/- 12, 2 SD. According to the JOA Classification, 33 patients were classified into the MIM Group and 29 into the MOM and 3 into the SEM Group. Significant differences were seen in ADC $(0.9 \pm 0.2$ vs $1.1 \pm 0.2)$ values and $F A(0.7 \pm 0.1$ vs $0.5 \pm 0.1)$ when comparing normal with pathological levels as well as in E1 (1.9 \pm 0.4 vs $2.3 \pm 0.4)$ and E2.3 $(0.37 \pm 0.24$ vs $0.5 \pm$ 0.29), respectively. Significant elevation of ADC and reduction of FA was seen in absolute vertebrostenotic segments. Significant Elevation of E1 and E2.3 were seen in vertebrostenotic segments compared with normal.

Conclusion: Our results suggest a high sensitivity of DTI metrics in the assessment of CSM. Significant increases in longitudinal and transverse diffusion were seen in our results which might represent a novel biomarker of CSM.

\section{B-0908 15:21}

Diffusion tensor imaging and magnetisation transfer imaging of spinal cord in MS patients

M. Gencturk, Y. Anik, H. Efendi, A. Demirci; Kocaeli/TR

(drm.gencturk@hotmail.com)

Purpose: The aim of this study was to evaluate the spinal cord involvement in multiple sclerosis (MS) patients with diffusion tensor imaging (DTI) and magnetisation transfer imaging (MTI) and to compare FA, ADC and MTR values among MS patients with and without spinal plaque with that of the control group.

Methods and Materials: Sixteen of thirty-two MS patients that had plaque in the spinal cord and 16 that did not have plaque in the spinal cord were included. As a control group 16 healthy volunteered patients were integrated. All patients were examined via 3 T MR. DTI, dynamic MTI and conventional were obtained. ROI localisations were set as plaque, periplaque, normal-appearing spinal cord, from central/posterior and both lateral sides.

Results: In MS patients with spinal plaque FA and MTR values were significantly decreased compared with periplaque $(p=0.041, p=0.012)$, and normal-appearing cord $(p=0.010, p<0.0001)$. Comparisons of normal-appearing spinal cord among MS patients with/without spinal plaque and control group revealed a significant decrease of FA and MTR in MS patients with plaque $(p<0.01$, and at significance limit in MS patients without plaque $(p=0.051, p=0.050)$ compared with that of the control group.

Conclusion: FA and MTR values alter in plaque and periplaque regions and even normal-appearing spinal cord which can be attributed to disease burden. DTI FA and MTR values seem to be a promising in assessment of spinal involvement in MS patients.
$14: 00-15: 30$

Room D1

\section{Cardiac}

\section{SS 1803}

\section{Cardiac imaging: into the future}

Moderators:

R.W. Bauer; Frankfurt a. Main/DE

E. Canet Soulas; Bron/FR

\section{B-0909 14:00}

Ablation of atrial flutter guided by magnetic resonance imaging

M. Grothoff, P. Sommer, C. Piorkowski, J. Hoffmann, G. Hindricks, M. Gutberlet; Leipzig/DE (grothoff@gmx.de)

Purpose: Fluoroscopy-guided radiofrequency (RF) catheter ablation is the standard therapy in atrial flutter and is associated with substantial radiation exposure. We introduce the method of RF ablation using real-time magnetic resonance (MR) guidance.

Methods and Materials: RF ablation was performed in 10 patients with typical atrial flutter in a standard 1.5 Tesla MRI. Fully MR compatible $8.5 \mathrm{~F}$ ablation catheters and an MR compatible electrophysiology (EP) recording system (Imricor Medical Systems, Burnsville, Minnesota) were used to map/ablate the cavotricuspid isthmus. MR-guidance was performed using a standard cine steady-state-free-precession real-time sequence with a frame-rate of $8 / \mathrm{s}$ for passive tracking in two orthogonal planes, comparable to right anterior oblique and left anterior oblique projections. Ablation was performed with RF pulses of $40 \mathrm{~W}$ and 60 seconds burning time. Postablation imaging was performed using T2-weighted and late gadolinium enhancement imaging for visualisation of oedema and necrosis.

Results: Time in the MRI scanner was $72 \pm 12$ minutes. A total isthmus block could be achieved in one patient and was confirmed in the conventional EP environment. In the remaining 9 patients a partial isthmus block was verified. No adverse events occurred during the procedures or within the first 30 days to follow-up. Postablation imaging clearly showed oedema and necrosis of the cavotricuspid isthmus. Conclusion: We demonstrated the feasibility of MRI-guided cavotricuspid isthmus ablation using a standard 1.5 Tesla MRI and a standard real-time SSFP sequence for passive catheter tracking. Further development will concentrate on materials to optimise the steerability of the catheter

\section{B-0910 14:09}

Quantitative BOLD response of the left ventricular myocardium to hyperoxic respiratory challenge at $1.5 \mathrm{~T}$ and $3.0 \mathrm{~T}$ cardiac magnetic resonance imaging

S. Winklhofer, S. Pazahr, R. Manka, H. Alkadhi, A. Boss, P. Stolzmann; Zurich/CH (sebastian.winklhofer@usz.ch)

Purpose: To quantify the changes of myocardial transverse relaxation times $\left(\Delta \mathrm{T} 2^{*}\right)$ induced by hyperoxic respiratory challenge at different field strengths in an intraindividual comparison of healthy volunteers.

Methods and Materials: Blood oxygen level-dependent (BOLD) cardiovascular magnetic resonance (CMR) data were acquired in ten healthy volunteers ( 5 women; 5 men; median age 29 years, range 22-35 years) at $1.5 \mathrm{~T}$ and $3.0 \mathrm{~T}$. Medical air $(21 \% \mathrm{O} 2)$, pure oxygen and carbogen $(95 \% \mathrm{O} 2,5 \% \mathrm{CO} 2)$ were alternatively administered in a block-design temporal pattern to induce normoxia, hyperoxia and hyperoxic hypercapnia, respectively. Parametrical T2* relaxation time maps were computed, average $\mathrm{T}^{*}$ values were derived from Region-of-Interest analysis by two independent and blinded readers in 16 standard myocardial segments on 3 short axis slices per patient.

Results: Inter- and intra-reader correlations of $\mathrm{T}^{*}$ measurements were good (ICC $=0.75$ and $I C C=0.79$, both $P<0.001$ ). Under normoxia, the mean $\mathrm{T}^{*}$ values were $30.6+7.0 \mathrm{~ms}$ at $1.5 \mathrm{~T}$ and $27.6+7.6 \mathrm{~ms}$ at $3.0 \mathrm{~T}$. Both hyperoxic gases induced significant $(\mathrm{P}<0.001)$ relaxation time increases $\Delta \mathrm{T} 2^{*}$ (oxygen: $1.5 \mathrm{~T}, 3.5+5.9 \mathrm{~ms}$; 3.0 T, 2.1+6.3 ms; carbogen: $1.5 \mathrm{~T}, 2.9+7.1 \mathrm{~ms} ; 3.0 \mathrm{~T}, 4.3+7.6 \mathrm{sm})$. As opposed to $1.5 \mathrm{~T}$, the magnitude of $\Delta \mathrm{T}^{*}$ response was higher at $3.0 \mathrm{~T}$ and under carbogen as compared with oxygen breathing $(p<0.01)$.

Conclusion: The myocardial $\triangle T 2^{*}$ response to hyperoxic respiratory challenge can reliably be quantified with BOLD CMR. The magnitude T2* increases significantly with a higher field strength as well as with carbogen as compared to oxygen breathing. Future studies are warranted to investigate the potential diagnostic role of BOLD response in assessing patients with coronary cardiomyopathy. 


\section{B-0911 14:18}

High-resolution susceptibility-weighted magnetic resonance imaging of rat heart microvasculature

E. Blondiaux, L. Pidial, G. Autret, D. Balvay, C.-A. Cuenod, H. Ducou le Pointe, O. Clément; Paris/FR (eleonore.blondiaux@trs.aphp.fr)

Purpose: To demonstrate that myocardial microvessels detection is feasible at high-resolution susceptibility-weighted MR imaging (SWI)

Methods and Materials: Fourteen Wistar rats underwent ex vivo cardiac MR imaging on a 4.7 Tesla scanner using a cryogenic device. Thin, tubular hypointensities within the myocardium of normal rat hearts at high-resolution susceptibility-weighted MR imaging (group 1, $\mathrm{n}=6$ ) were presumed to be intramyocardial microvessels. To prove that these "strand-like" structures were microvessels, a reference map of the myocardial macrovessels (group 2, n=4) after direct intracoronary injection of barium sulphate, a viscous contrast media was defined. Then, a superparamagnetic contrast agent (ferumoxsil, Lumirem ${ }^{\circledR}$ ), an intravascular contrast agent, was injected to stack up the distribution of intramyocardial microvessels (group $3, n=4$ ). For groups 2 and 3, 3D fast imaging with steady state in precession (3D FISP) sequences was performed. The results of images in group 1 (spatial resolution: 39 $\mu \mathrm{m}$ ) and group 3 (spatial resolution: $39 \times 39 \times 39 \mu \mathrm{m}^{3}$ ) were qualitatively compared. Results: After intracoronary artery injection of barium sulphate, the main coronary arteries were visible up to the third-order branches (group 2). Beyond this level, the coronary microvasculature was opacified with ferumoxsil (group 3) as demonstrated by the presence of ferumoxsil inside the microvessels on MR-matched histological sections. The regional distribution of microvessels was similar between groups 1 and 3 .

Conclusion: Myocardial microvessels appear as "strand-like" structures on highresolution MRI without injection of contrast media. This provides new methods for evaluation of neovascularization in preclinical models of myocardial infarction.

\section{B-0912 14:27}

T2-weighted dark blood cardiac imaging using a dual-source parallel radiofrequency transmission with $\mathrm{RF}$ shimming at $3 \mathrm{~T}$ compared with standard MR imaging at $3 \mathrm{~T}$

M. Rasper, M. Settles, B. Gramer, M. Souvatzoglou, E.J. Rummeny, A. Huber; Munich/DE (armin.huber@tum.de)

Purpose: To prospectively compare the image quality and homogeneity of cardiac T2-weighted dark blood images using a dual-source parallel radiofrequency (RF) transmission MR system with RF shimming at 3.0 T and conventional MR imaging at 3.0 T.

Methods and Materials: Dual-source parallel RF transmission 3.0 T MR system with local RF shimming was used to obtain T2-weighted dark blood images in 31 patients and compared with standard MRI at 3.0 T. SNR was determined in the interventricular septum (IS), the lateral wall (LW), the right ventricular cavity (RV) and left ventricular cavity (LV) in the anterior wall (AW), posterior wall (PW) and in the left ventricular cavity (LV) in two-chamber orientation.

Results: Mean SNR values were 15.2 (IS), 8.4 (LW), 16.9 (AW), 22.5 (PW), 3.2 (RV) and 6.6 (LV) using dual-source RF transmission with RF shimming. Mean SNR values were 10.7 (IS), 7.5 (LW), 21.2 (AW), 12.7 (PW), 2.2 (RV) and 6.5 (LV) with conventional shimming. Mean CNR values were 4.8 (IS-RV) and 3.5 (PW-LV) using dual-source parallel radiofrequency transmission with RF shimming. Mean CNR values were 3.3 (IS-RV) and 3.3 (PW-LV) for conventional RF shimming. Mean subjective image quality was $3.8 / 3.7$ (reader $1 /$ reader 2) for dual-source parallel RF transmission with RF shimming and $2.9 / 2.7$ for conventional MRI $(p<0.05)$. Conclusion: Dual-source parallel RF transmission with RF shimming at $3.0 \mathrm{~T}$ improves homogeneity of cardiac T2-weighted dark blood images based on SNR and CNR measurements and improves subjective image quality.

\section{B-0913 14:36}

Myocardial T1-mapping and myocardial extracellular volume (ECV) fraction: intraindividual comparison of gadobutrol and gadoterate meglumine in an animal model

K.U. Bauner ${ }^{1}$, T. Sander', D. Theisen ${ }^{1}$, H. Pietsch², G. Jost², M.F. Reiser ${ }^{1}$, K. Nikolaou', B.J. Wintersperger ${ }^{3} ;{ }^{1}$ Munich/DE, ${ }^{2}$ Berlin/DE, ${ }^{3 \top}$ oronto, ON/CA (kerstin.bauner@med.uni-muenchen.de)

Purpose: To assess dynamic T1 shortening effects of equimolar doses of gadobutrol and gadoterate meglumine in myocardium and the effect on extracellular volume fractions (ECV) in an animal model.

Methods and Materials: 10 rabbits underwent cardiac MRI under general anaesthesia on a 1.5 Tesla scanner using an 8-element knee coil. Exams included SSFP cine imaging as well as pre- and post-contrast myocardial T1-mapping using a modified look locker inversion recovery (MOLLI) technique. Each animal was scanned twice with a 24-h interval after receiving either $0.1 \mathrm{mmol} / \mathrm{kg}$ gadobutrol
(Bayer Pharma) or gadoterate meglumine (Guerbet Group) in a random order. MOLLI was performed at a mid-ventricular slice location pre- and 4-20 min (2 min intervals) post-contrast administration. T1 values of the blood pool and the myocardium were measured for each time point and ECV assessment was based on myocardial $\Delta R 1$ assessment (pre and post) in relation to $\Delta R 1$ blood pool and incorporation of the haematocrit.

Results: While pre-contrast $T 1$ values were similar $(p=0.3)$, post-contrast $T 1$ values were significantly shorter at any measured time point post-injection after gadobutrol application $(p<0.047)$. Myocardial ECV data though calculated for both agents did not show significant differences ( $p>0.21$ ). In addition, ECV data related to both agents did not show any significant differences over the assessed time course of 4-20 min p.i. ( $p>0.14$, gadobutrol; $p>0.09$, gadoterate meglumine).

Conclusion: Gadobutrol leads to a significantly higher shortening of myocardial $T 1$ values as compared to gadoterate meglumine. The ECV assessment which is independent of the CA properties can be adequately evaluated as early as 4 min p.i. in normal myocardium.

Author Disclosures:

K.U. Bauner: Equipment Support Recipient; Bayer Pharmaceuticals. T. Sander: Equipment Support Recipient; Bayer Pharmaceuticals. H. Pietsch: Employee; Bayer HealthCare Pharmaceuticals. G. Jost: Employee; Bayer HealthCare Pharmaceuticals.B.J. Wintersperger: Equipment Support Recipient; Bayer HealthCare Pharmaceuticals

\section{B-0914 14:45}

Noninvasive diagnosis of pulmonary hypertension via magnetic resonance vortex flow detection: definition of cut-off criteria

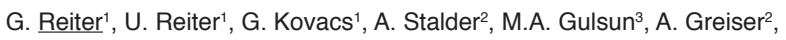
H. Olschewski', M.H. Fuchsjäger'; ${ }^{1} G r a z / A T,{ }^{2}$ Erlangen/DE, ${ }^{3}$ Princeton, NJ/US (gert.reiter@siemens.com)

Purpose: Existence of vortical blood flow in the main pulmonary artery as diagnostic criterion for diagnosis of pulmonary hypertension was analysed in 3-dimensional (3D) vector field and streamline representations.

Methods and Materials: 50 subjects with known or suspected pulmonary hypertension ( 23 patients with and 27 without pulmonary hypertension, age $58 \pm 13$ years) underwent right heart catheterisation and time-resolved, 3D phase contrast imaging of the main pulmonary artery. Blood flow patterns were visualised as 3D velocity vector fields projected on 2-dimensional anatomical images (3D-vectors) and as 3D streamlines using 4D-Flow software (Siemens). Relative period of existence of vortical blood flow within the cardiac cycle (tvortex) was evaluated. Comparison of methods for using tvortex as diagnostic criterion for pulmonary hypertension was performed by receiver operating characteristic analysis.

Results: Areas under the curve for diagnosis of pulmonary hypertension did not differ significantly $(\mathrm{p}=0.30)$ and were 0.998 (95\%-confidence interval 0.983 to 1.000$)$ in case 3D-vector vortex detection and 0.991 (95\%-confidence interval 0.948 to 0.999 ) in case of streamline vortex detection. The optimal tvortex cut-off value maximising sum of sensitivity and specificity was tvortex $=0.15$ for both visualisations. Resulting $95 \%$-confidence intervals for sensitivity and specificity were 0.86 to 1.00 and 0.82 to 0.99 in case 3D-vector vortex detection and 0.79 to 0.99 and 0.82 to 0.99 in case $3 \mathrm{D}$ streamline vortex detection.

Conclusion: Main pulmonary artery blood flow patterns provide an accurate tool for the diagnosis of pulmonary hypertension with the optimal tvortex cut-off value 0.15 .

Author Disclosures:

G. Reiter: Employee; Siemens.A. Stalder: Employee; Siemens. M.A. Gulsun: Employee; Siemens. A. Greiser: Employee; Siemens.

\section{B-0915 14:54}

Feasibility study for an image-based enhancement of low-dose intraprocedural 4D C-arm cardiac flat-detector CT (4D-FD-CT)

Y. Kyriakou' ${ }^{1}$, C. Koehler ${ }^{1}$, S. de Buck'2, D. Nuyens ${ }^{2}$, H. Heidbuchel'; ${ }^{1}$ Forchheim/DE, ${ }^{2}$ Leuven/BE (yiannis.kyriakou@siemens.com)

Purpose: To assess the image quality after image-based processing of low-dose single-rotation intra-procedural 4D-FD-CT (SR 4D-FD-CT).

Methods and Materials: Multiple rotation contrast-enhanced 4D-FD-CT is associated with many C-arm projections ( $\sim 1000$ projections) and a high amount of contrast agent (CA). In this feasibility study, we used a single-rotation of the $\mathrm{C}$-arm (Artis zee biplane, Siemens AG, Healthcare Sector, Germany) accompanied by cardiac pacing acquiring a reduced number of low-dose projections ( 100-400). However, fewer projections per single cardiac phase may cause sampling and streak artefacts. An image-based temporal gradient is calculated to generate a respective motion map which after appropriate frequency-space analysis separates cardiac motion from other temporal changes, e.g. sampling artefacts. Then a motion-map-dependent merging of different cardiac phases is performed aiming 
for better trade-off of high temporal resolution, less noise and streak artefacts. This is combined with an automatic segmentation and suppression of catheter-induced streaks. This preliminary study included 5 in vivo porcine models. Images were evaluated by experienced cardiologists with respect to noise, temporal resolution and artefact susceptibility.

Results: An enhancement of temporal resolution was shown by a decreased fullwidth at half maximum of catheter cross sections using SR 4D-FD-CT as compared to static reconstructions. Motion-map-processing of individual phases increased the contrast-to-noise ratio for each heart phase, e.g. in the left ventricle by an average of $30 \%$. Visual impression confirmed a significant streak reduction.

Conclusion: First results show the potential of SR 4D-FD-CT for pragmatic 4Dinterventional imaging. SR 4D-FD-CT allows for dynamic segmentation of cardiac chambers and valves.

Author Disclosures:

Y. Kyriakou: Employee; Siemens AG. C. Koehler: Employee; Siemens AG.

S. de Buck: Research/Grant Support; Siemens AG. D. Nuyens: Consultant;

Siemens AG. H. Heidbuchel: Consultant; Siemens AG.

\section{B-0916 15:03}

Assessment of HU-value stability in dynamic CT-scans for quantitative estimation of myocardial perfusion

M. Handler ${ }^{1}$, T. Rienmüller ${ }^{1}$, P. Ourednicek², V. Makarenko ${ }^{3}$, L. Bockeria ${ }^{3}$,

S. Zhorzholiani ${ }^{3}$, I. Krestinich ${ }^{3}$, R. Rienmüller ${ }^{4}$, C. Baumgartner ${ }^{1} ;{ }^{1}$ Hall in Tirol/AT,

${ }^{2}$ Prague 5/CZ, ${ }^{3}$ Moscow/RU, ${ }^{4}$ Graz/AT (michael.handler@umit.at)

Purpose: For quantitative measurement of left ventricular myocardial perfusion, stability of HU-values in Dynamic CT-scans is essential. A phantom study is used to evaluate this stability over slices and time using the Philips iCT256-slice scanner. Methods and Materials: Seven time series of CT-images with 11 slices $(5 \mathrm{~mm})$ over 30 seconds of a CATPHAN phantom were acquired using the iCT256 at 100 $\mathrm{kVp} / 100 \mathrm{mAs}$. Six different regions of interest (ROI) were placed into the water regions of the phantom for all slices and time steps. Five ROls were arranged in a star-like pattern in order to enable evaluation of intra slice variability. One ROI covered the entire water-region. Stability over time and slices as well as within each slice was statistically examined.

Results: The absolute difference in the mean values of the entire water $\mathrm{ROI}$ in all seven phantom studies was maximum 5.4 HU. The examination of the inter-slice variability revealed a drift of maximal $0.057 \mathrm{HU} /$ slice. Over time, a maximal drift of $0.043 \mathrm{HU} / \mathrm{s}$ could be observed. The maximum intra-slice difference of the mean values was at $7 \mathrm{HU}$. This slightly higher value is due to beam hardening artefacts in the ROls close to the high-density /bone simulating/ structure in the phantom. Conclusion: The absolute difference of the mean values in all ROls over time/ slices remained below 5.4 HU. Hence, the HU variation of the Philips iCT256slice scanner lies within an acceptable range for the quantitative estimation of left ventricular myocardial perfusion.

\section{B-0917 15:12}

Impact of iterative image reconstruction algorithms on the feasibility of automated plaque assessment in coronary computed tomographic angiography

S. Puchner, M. Ferencik, M. Károlyi, S. Do, P. Maurovich-Horvat, U. Hoffmann, C. Schlett; Boston, MA/US (stefan.puchner@meduniwien.ac.at)

Purpose: To evaluate the impact of iterative image reconstruction algorithms including adaptive statistical (ASIR) and model based (MBIR) on the feasibility of automated plaque assessment in coronary computed tomography angiography (CCTA) as compared to filtered-back-projection (FBP) reconstructed algorithm. Methods and Materials: Three ex-vivo human hearts were imaged by CCTA and reconstructed with FBP, ASIR and MBIR. An automated plaque quantification software (Vitrea Cardiac Solutions, MN) was applied for each of the algorithms to fit the outer and inner vessel-wall boundaries. Each coronary cross section, in which the automated software detected wrongly the boundaries, was corrected in a random and blinded fashion. The percentages of corrections were compared between the reconstruction algorithms using Chi-square-test.

Results: A total of 2295 CCTA cross sections with $0.5 \mathrm{~mm}$ increments were assessed which equals to 765 co-registered triplets (FBP/ASIR/MBIR). Any boundary corrections were performed in a total of 712 cross sections $(31.0 \%)$, including outer $(\mathrm{N}=400)$ and inner $(\mathrm{N}=381)$ vessel-wall. The percentage of corrected cross sections was lower for MBIR (24.1\%) as compared to ASIR $(32.4 \%, p=0.0003)$ and FBP $(36.6 \%, p<0.0001)$, and marginal between ASIR and FBP $(p=0.09)$. Regional agreement of performed corrections existed between reconstruction algorithms (kappa=0.53). The benefit of MBIR above FBP was associated to the presence of moderate and severe calcification (OR: 2.9 and 5.7, $p<0.0001$, respectively).
Conclusion: Using MBIR significantly reduced the need for vessel-wall boundary corrections compared to other reconstruction algorithms, particularly at the side of calcifications. Thus, MBIR improves the feasibility of automated plaque assessment in CCTA and potentially its clinical applicability.

\section{B-0918 15:21}

Calcium score of small coronary calcifications on multi detector computed tomography: a phantom study

J.M. Groen ${ }^{1}$, K.F. Kofoed ${ }^{2}$, M. Zacho ${ }^{2}$, R. Vliegenthart ${ }^{1}$, T.P. Willems ${ }^{1}$, M.J.W. Greuter ${ }^{1} ;{ }^{1}$ Groningen/NL, ${ }^{2}$ Copenhagen/DK (m.j.w.greuter@umcg.nl)

Purpose: To assess the feasibility of a new phantom which enables establishment of a calcium scoring protocol for multidetector computed tomography (MDCT) that yields calcium scores comparable to electron beam tomography (EBT) values and to physical mass.

Methods and Materials: A phantom containing 100 small calcifications ranging 0.5-2.0 mm was scanned on EBT using a standard coronary calcium protocol, and on a 320-row MDCT scanner using different scanning, reconstruction and scoring parameters (tube voltage $80-135 \mathrm{kV}$, slice thickness $0.5-3.0 \mathrm{~mm}$, reconstruction kernel FC11-FC15 and scoring threshold 110-150 HU). The Agatston and mass score were compared and the influence of the parameters was assessed.

Results: On EBT, the Agatston and mass scores were 0-20, and 0-3 mg, respectively. On MDCT, the Agatston and mass scores were 0-20, and 0-4 mg, respectively. The Agatston score on MDCT differed $52 \%$ between 80 and $135 \mathrm{kV}, 65 \%$ between 0.5 and $3.0 \mathrm{~mm}$ and $48 \%$ between FC11 and FC15. More calcifications were detected with a lower tube voltage, a smaller slice thickness, a sharper kernel and a lower threshold. An acquisition protocol with $100 \mathrm{kV}$ and two reconstructions protocols could be defined. This protocol yielded Agatston scores as close to EBT as possible, as well as mass scores as close to physical mass as possible. Conclusion: With the new phantom a calcium scoring protocol can be found which yields Agatston scores comparable to EBT values and to physical mass.

\begin{tabular}{ll}
\hline $14: 00-15: 30$ & Room D2
\end{tabular}

\section{Interventional Radiology}

\section{SS 1809}

\section{New approaches to aortic and peripheral interventions}

Moderators:

S. Kudrnova; Budapest/HU

R. Morgan; London/UK

\section{B-0919 14:00}

Paclitaxel-coated balloons for the failing dialysis access: a prospective randomised controlled trial

P.M. Kitrou, K. Katsanos, S. Spiliopoulos, N. Cristeas, M. Kuriakopoulou,

D. Karnabatidis, D. Siablis; Patras/GR (panoskitrou@gmail.com)

Purpose: To report 1-year results of a prospective randomised controlled trial investigating angioplasty with paclitaxel-coated balloons (PCB) versus plain balloon angioplasty for the treatment of failing arteriovenous dialysis access either a fistula (AVF) or a graft (AVG).

Methods and Materials: This was a non-inferiority hypothesis trial registered in clinicaltrials.gov (NCT01174472). Enrollment criteria included an angiographic and clinical diagnosis of dysfunctional dialysis access due to at least one stenotic lesion in patients with AVF or AVG circuits. Forty patients were randomised to undergo either PCB dilatation $(n=20)$ or standard PTA $(n=20)$ of a venous outflow stenotic lesion. Regular angiographic follow-up was scheduled every 2 months. Study primary endpoints included technical success (defined as residual stenosis of the treated lesion $>30 \%$ without any significant dissection) and 1-year primary patency of the treated site (defined as angiographic visualisation of a patent lesion with $<50 \%$ restenosis and no need for any additional repeat procedure within the previously treated lesion due to failing access).

Results: Baseline variables were comparably distributed among the two groups. Technical success was $100 \%$ for both groups. Additional post-dilation with a highpressure balloon was necessary in 11/20 (55\%) of the PCB-treated cases. At 1 year, cumulative target lesion primary patency was significantly higher after $\mathrm{PCB}$ application (35\% in PCB group versus 5\% in PBA group, $p<0.001, \mathrm{HR}(95 \% \mathrm{Cl})$ $=0.27(0.13-0.58)$ ).

Conclusion: PCB angioplasty might have a place in our quiver for the treatment of stenotic venous outflow lesions of failing dialysis access. 


\section{B-0920 14:09}

Lower limb multilevel treatment with drug eluting balloon: 12-month results from the "DEBELLUM" trial

F. Fanelli, A. Cannavale, P. Lucatelli, A. Wlderk, C. Cirelli, F.M. Salvatori; Rome/IT (fabrizio.fanelli@uniroma1.it)

Purpose: To report 12-month results of the "DEBELLUM"-drug-eluting balloon evaluation for lower limb multilevel treatment-trial performed to evaluate the efficacy of drug-eluting balloon-DEB versus conventional balloon catheter-NCB in the treatment of peripheral arterial disease.

Methods and Materials: Fifty consecutive patients (mean age $67 \pm 21$ years) with symptomatic peripheral arterial disease were randomised between DEB ( 25 patients-57 lesions) (Inpact ${ }^{\circledR}$-Medtronic) and NCB (25 patients- 65 lesions). Inclusion criteria were native single or multiple stenosis or occlusion (mean lesion length 7.5 $\pm 3.5 \mathrm{~cm}$ ) in the femoro-popliteal or below the knee (BTK) regions. Dilatation was performed in each group either for native lesions (26 DEB; $30 \mathrm{NCB}$ ) or post-stent implantation (31 DEB; $35 \mathrm{NCB}$ ). Primary end points were late lumen loss (LLL) at 6 and 12 months. Secondary end-points were target lesion revascularisation (TLR), amputation and thrombosis rate.

Results: One-hundred-twenty-two lesions were treated: $75.4 \%$ in the femoropopliteal area; $24.6 \%$ BTK; $21.3 \%$ total occlusions. LLL was $0.5 \pm 1.4 \mathrm{~mm}$ (DEB) vs. $1.6 \pm 0.7 \mathrm{~mm}(\mathrm{NCB})(p<0.01)$ at 6 months and $0.64 \pm 0.9 \mathrm{~mm}$ (DEB) vs. $1.81 \pm 1.1$ $\mathrm{mm}$ (NCB) $\mathrm{P}=0.001$ at 12 months. One-year results showed a reduced TLR 4/25 (16\%) vs. $10 / 25(40 \%) p<0.01$. Thrombosis was $4 \%$ (DEB) vs. $5.2 \%$ (NCB) $p=0.6$. Amputation rate $4 \%$ (DEB) vs. $7.9 \% p=0.46$. Sub-group analysis showed for native stenosis a LLL of $0.63 \pm 0.9 \mathrm{~mm}$ (DEB) vs. $1.70 \pm 0.6 \mathrm{~mm}$ (NCB) $p<0.01$. After stent implantation $0.65 \pm 0.7 \mathrm{~mm}$ (DEB) vs. $1.91 \pm 0.3 \mathrm{~mm}(\mathrm{NCB}) \mathrm{p}<0.01$. Ankle-brachialindex improved more using DEB, baseline: $0.55 \pm 0.06$; post-procedure: $0.81 \pm 0.3$ NCB, baseline: $0.57 \pm 0.05$; post-procedure: $0.68 \pm 0.13(p<0.05)$.

Conclusion: Results confirmed DEB as effective in treatment of peripheral arterial disease.

\section{B-0921 14:18}

Directional atherectomy of chronic total femoropopliteal occlusions: middle-term outcomes

M. Rossi, G.M. Varano, F. Laurino, G. Orgera, A. Zolovkins, N. Maltzeff, M. Citone, V. David; Rome/IT (gianluca.varano@gmail.com)

Purpose: This study aimed at assessing retrospectively the middle-term clinical outcome of the femoropopliteal chronic total occlusion (CTOs) treatment by means of directional atherectomy (DA).

Methods and Materials: Between January 2008 and March 2012, 47 patients (51 limbs, patient age $70 \pm 10$ years) were included in the study with mean follow-up of 20 months (6-51). 51 femoro-popliteal CTO in 47 Patients with severe intermittent claudication $(\mathrm{N}=15 ; 49.4 \%)$ or critical limb ischaemia (CLI) $(\mathrm{N}=16 ; 51.6 \%)$ were treated with SilverHawk or TurboHawk atherectomy followed by angioplasty in 10 (32.2\%) cases. Clinical and USCD follow-up was set at 3, 6 and 12 months and yearly thereafter. Immediate technical success, disease-free patient survival and target lesion revascularisation (TLR) rates were assessed with Kaplan-Meier analysis. Results: Technical success rate was $98 \%$ with a lesion length of $80 \pm 56 \mathrm{~mm}$. Limbsalvage and survival rates were 87.5 and $90.3 \%$ at 2 years, respectively. At 6, 12 and 24 months disease-free patient survival was $88.7,79.9$ and $64.6 \%$. Whereas adjunctive PTA was performed in $32 \%$ cases to improve the angiographic result, $12 \%$ required an associated tibial vessel treatment. No perioperative mortality was observed. One complication (2\%) occurred.

Conclusion: Percutaneous directional atherectomy is a safe and effective technique in lower limb revascularisation. Preliminary results supported by short-mid term data encourage use of DA with SilverHawk and TurboHawk catheter in the femoro-popliteal obstructive lesions also in traditionally "forbidden districts". Longterm data are missing.

\section{B-0922 14:27}

\section{Predictive value of angiographic scores for the integrated} management of the ischaemic diabetic foot

F.M. Turini, I. Bargellini, A. Piaggesi, A. Cicorelli, R. Cervelli, E. lacopi, R. Cioni, C. Bartolozzi; Pisa/lT (francesca_turini@libero.it)

Purpose: To retrospectively evaluate the agreement between angiographic scores and clinical outcome after endoluminal revascularization in diabetic patients with critical limb ischaemia (CLI).

Methods and Materials: Data were retrospectively collected of consecutive diabetic patients with stage IV CLI who underwent percutaneous lower limb revascularization from January 2009 to June 2011. Pre- and post-procedural angiographic images were reviewed to classify arterial involvement according to: 1) TASC II, 2) Graziani's morphological classification, 3) Joint Vascular Society Council calf and foot scores. Clinical results (healing, non-healing, major amputation) were compared to baseline clinical data and angiographic results.

Results: 202 percutaneous procedures were performed; the immediate technical success rate was $93.6 \%$. Preprocedurally, the mean \pm SD calf and foot scores were $7.8 \pm 1.6$ and $7.3 \pm 2.3$, respectively; 132 patients $(65.3 \%)$ were in Graziani's morphological classes 4 to 7 ; in $112(55.4 \%)$ cases TASC II was inapplicable, for the absence of femoro-popliteal lesions. Post-procedurally, mean calf and foot scores were $4.8 \pm 2.3$ and $5.9 \pm 2.6$, respectively, and $86.6 \%$ of cases were into the Graziani's classes 1 and 2 , whereas TASC II was inapplicable in all cases. Healing and major amputation rates were $66.8 \%$ and $3.9 \%$, respectively; only pre- and post-procedural foot scores were significantly associated with the clinical outcome $(P<.05)$. Conclusion: Endoluminal revascularization represents a valuable treatment option in diabetic patients with CLI. In this population, TASC II is inadequate to describe peripheral arterial involvement. Pre- and post-procedural foot scores represent the most significant angiographic parameter to evaluate treatment success, thus aggressive treatment of foot arteries should be attempted whenever possible.

\section{B-0923 14:36}

12 months results of a randomised trial comparing mono- or dual-antiplatelet therapy in interventionally treated patients with peripheral arterial disease

F.F. Strobl' ${ }^{1}$, J. Schmehl'2, K. Brechtel'2, T. Zeller ${ }^{3}$, C.D. Claussen², G. Tepe ${ }^{4} ;{ }^{1}$ Munich/DE, ${ }^{2}$ Tübingen/DE, ${ }^{3}$ Bad Krozingen/DE,

${ }^{4}$ Rosenheim/DE (frederik.strobl@med.Imu.de)

Purpose: In this trial, peri- and postinterventional dual antiplatelet therapy significantly reduced rate of target lesion revascularisation after 6 months. It is not known whether this effect persists after stopping clopidogrel. Thus, we conducted an additional follow-up after 12 months.

Methods and Materials: In this double-blinded, randomised trial, we enrolled 80 patients who were treated with PTA or stenting in the upper leg. 40 patients received pre- and postinterventional dual therapy with aspirin and clopidogrel. The other 40 patients received same doses of aspirin and placebo instead of clopidogrel. Clopidogrel/placebo was stopped after 6 months and patients remained on aspirin only. TLR and mortality rate were reevaluated 12 months after the intervention. Results: We initially enrolled 80 patients (42 male, $70 \pm 10 y$ ), 40 in each group in the study. 12 months after the intervention, 36 clopidogrel and 37 placebo patients could be reevaluated. At 6 months, clopidogrel patients had significantly lower rates of TLR compared to placebo patients: $2(5 \%)$ vs. $8(20 \%), p=0.04$. After stopping clopidogrel/placebo, there was no more significant difference in TLR 12 months after study inclusion with $9(25 \%)$ clopidogrel vs. $12(32.4 \%)$ placebo patients $(p=0.35)$. Mortality was 0 vs. 1 at 6 months $(p=0.32)$ and 0 vs. 3 at 12 months $(p=0.08)$. Conclusion: In contrast to the first follow-up 6 months after the intervention according reduction of TLR, this advantage of dual antiplatelet therapy does not persist after stopping clopidogrel. Prolonged dual therapy (>6 months) should be evaluated and considered in patients with high risk for restenosis.

\section{B-0924 14:45}

Comparing 2D angiography, 3D rotational angiography, and preprocedural $C T$ image fusion with $2 \mathrm{D}$ fluoroscopy for endovascular repair of thoraco-abdominal aortic aneurysm V. Tacher ${ }^{1}$, M. Lin ${ }^{2}$, P. Desgranges ${ }^{1}$, T. Grünhagen ${ }^{3}$, A. Luciani', J.-P. Becquemin ${ }^{1}$, J.-F. Deux ${ }^{1}$, A. Rahmouni ${ }^{1 ;}{ }^{1}$ Creteil/FR ${ }^{2}$ Briarcliff Manor, NY/US, ${ }^{3}$ Best/NL

Purpose: To evaluate the feasibility of pre-procedural arterial phase computed tomography image fusion with intra-procedural fluoroscopy (or Image fusion: IF) road-mapping in endovascular repair of thoraco-abdominal aortic aneurysm (EVRTAAA) compared to current road-mapping methods (2D and 3D angiographies) in terms of $x$-ray exposure, injected contrast volume, and procedure time.

Methods and Materials: Single-institution prospective study (Internal institute approved), on 37 patients, informed written consent provided, and treated for EVRTAAA. All interventions were performed on the same angiographic system. Patients were consecutively placed in three groups in a seven-month period: " $2 \mathrm{D}$ angiography" (2DA) (9 patients), "3D rotational angiography" (3DA) (14 patients) and "image fusion" (IF) (14 patients). Injected contrast volume, x-ray exposure (dose-area-product: DAP) and procedure time were recorded. To compare the groups in terms of the above measures and general patient characteristics, statistical tests using Fisher's Exact, Kruskal-Wallis and Mann-Whitney were performed. Results: Patient characteristics and stenting types were similar between all three groups with no statistically significant differences ( $p$-value> 0.05). The IF 
group showed significant reduction ( $p$-value $<0.0001$ ) in injected contrast volume compared to the other groups (2DA: $235 \pm 145 \mathrm{ml}$; 3DA: $225 \pm 119 \mathrm{ml}$; IF: $65 \pm 28$ $\mathrm{ml}$ ). Although not statistically significant ( $p$-value $=0.18$ ), DAP showed a trend in reduction for the IF group (2DA: $1188 \pm 1067 \mathrm{~Gy} \times \mathrm{cm}^{2}$; 3DA: $984 \pm 581 \mathrm{~Gy} \times \mathrm{cm}^{2}$; IF: $\left.655 \pm 457 \mathrm{~Gy} \times \mathrm{cm}^{2}\right)$. There was no statistical difference $(\mathrm{p}$-value $=0.59)$ in procedure time between groups (2DA: $233 \pm 123$ minutes; 3DA: $181 \pm 53$ minutes; IF: $189 \pm 60$ minutes).

Conclusion: Image fusion road-mapping for EVRTAAA is feasible and is associated to a statistically significant reduction in contrast volume and a trend towards DAP reduction while maintaining similar procedure time.

\section{B-0925 14:54}

\section{Endoleak complications of thoracic endovascular aortic repair} (TEVAR): long-term outcomes and survival

A. Rampoldi, P. Fratto, M. Solcia, R. Vercelli, C. Migliorisi, F. Barbosa, D. Foresti, M. Nichelatti, G. Cornalba; Milan/IT

Purpose: To assess the endoleak complications and survival after TEVAR. Methods and Materials: Between July 2005 and July 2011, 77 patients (64 male and 13 female) were underwent TEVAR. 24 were in emergency and 17 of procedures were hybrid. The medium term follow-up was 24 months. Cross-tabulations were checked with chi-squared test, or by Fisher's exact test. Survivorships were analysed by Kaplan-Meyer product limit method and by Cox semiparametric regression

Results: Technical success was achieved in 98.7\%. 14/77 presented endoleaks during the follow-up (type $1=7$, type $2=6$ and type $3=1$ ). During the follow-up there were 7 mortalities, mean time 19.82 months (range 3-48). In survival rates there was no difference between hybrid procedures group $(p=0.3387)$ and elective or emergency group $(p=0.3608)$. There was a statistical difference on survival between the group that had endoleaks during the follow-up $(p=0.0093)$ and who had had necessity of a second endovascular interventional $(p=0.0184)$. Statistical difference on survival was also seen between patients who developed type 1 and 2 endoleaks $(p=0.0336)$.

Conclusion: TEVAR is a safe and effective treatment. The presence of endoleak, special type 1 and the necessity of a second endovascular intervention reduces significantly the survival.

\section{B-0926 15:03}

Selective coverage of the left subclavian artery without recanalization in patients with patent vertebro-vertebral arterial communications during TEVAR: a single centre study M. Lee; Seoul/KR (eterna0@yuhs.ac)

Purpose: To evaluate the safety and effectiveness of selective coverage of the left subclavian artery (LSCA) without recanalization during TEVAR in patients with patent vertebro-vertebral arterial communications and to assess morphologic change of the vertebral artery (VA) after the procedure.

Methods and Materials: Among 126 patients who underwent TEVAR, 29 patients requiring LSCA coverage without pre-emptive recanalization were retrospectively analysed. The patients' ages ranged from 45 to 84 years. Vertebro-vertebral arterial communications in all patients were evaluated by contrast-enhanced computed tomography (CECT), time-of flight magnetic resonance angiography, or conventional angiography. Neurologic complications such as spinal cord ischaemia (SCI) or cerebrovascular accidents (CVA) were analysed. Pre- and post-procedural changes in VAs were evaluated on follow-Up CECT.

Results: The overall 30-day mortality was $6.9 \%$ (2/29). None of the patients had an $\mathrm{SCl}$ or stroke of posterior circulation alone. CVA from embolic infarctions occurred in two patients $(7.4 \%)$. Transient left arm ischaemic symptoms were present in 5 patients $(18.5 \%)$, but none required secondary interventions. Delayed development of type I endoleak occurred due to stent deformity in one patient, who underwent surgery. One patient required re-intervention after a pseudoaneurysm developed at the distal margin of the previously placed stent-graft at ten-month follow-up CECT. Hypertrophy of the right VA after TEVAR was seen in 7 of 27 patients (25.9\%); two patients showed bilateral hypertrophy of VAs.

Conclusion: LSCA coverage without recanalization can be safely performed during TEVAR in patients with patent vertebro-vertebral communications. Hypertrophy of the right VA was noted in $25.9 \%$ of patients after LSCA coverage.
B-0927 15:12

Completely CT-guided treatment of lumbar artery fed type 2 endoleaks after stent graft repair of abdominal aortic aneurysms with direct sac puncture and embolisation with the liquid embolic agent ethylene vinyl alcohol copolymer (Onyx)

M. Treitt' 1 M. Sadeghi' ${ }^{2}$ K. Eberhardt', D. Maxien', M.F. Reiser'; ${ }^{1}$ Munich/DE, 2Erding/DE (mtreitl@med.uni-muenchen.de)

Purpose: Treatment of lumbar artery fed type 2 endoleaks (lafT2E) after abdominal endovascular aneurysm repair (aEVAR) exhibits high failure and recurrency rates. We report our preliminary results of CT-guided direct sac puncture, followed by embolisation with the liquid embolic agent ethylene vinyl alcohol copolymer (Onyx®). Methods and Materials: 17 male patients (mean age $76 \pm 6$ yrs) with lafT2E were included after failure of conservative treatment. All received CT guided direct sac puncture under local anaesthesia, followed by $\mathrm{CT}$ fluoroscopic embolisation with Onyx $34{ }^{\circledR}$ or $20 \AA$. Follow-up examination included contrast-enhanced ultrasound (CEUS) and dual-phase CT angiography every 6 months for at least 2 years (ongoing).

Results: Technical success was achieved in all cases, requiring dual puncture of the aneurysm sac in 8 cases $(47.1 \%)$. Average duration of the procedure was 60 $\pm 12 \mathrm{~min}$. In mean $7.5 \mathrm{ml}$ of Onyx 34® were used per procedure. In 6 cases Onyx $20 \circledR$ was additionally used for complete filling of the endoleak. Repeat intervention was necessary in 3 cases (17.3\%) due to new endoleak development in the follow-up period of $12 \pm 8$ months. The procedure failed permanently in 1 case. Complications such as major bleeding, spinal ischaemia or non-target embolisations were not observed.

Conclusion: Completely CT-guided treatment of lafT2E is a feasible and safe treatment alternative. Difficulties lay in the determination of the necessary amount of Onyx and the end point of the intervention. Several punctures as well as repeat intervention might be necessary to achieve permanent endoleak occlusion.

Author Disclosures:

M. Treitl: Advisory Board; Covidien. Consultant; Covidien, Biotronik.

\section{B-0928 15:21}

Endovascular treatment of type 1 endoleaks after stent graft repair of aortic aneurysms: effectiveness and safety of the ethylene vinyl alcohol copolymer Onyx as liquid embolic agent

M. Treitt' ${ }^{1}$ M. Sadeghi ${ }^{2}$, K. Eberhardt' 1 , D. Maxien ${ }^{1}$, M.F. Reiser ${ }^{1} ;{ }^{1}$ Munich/DE, ${ }^{2} E r d i n g / D E$ (mtreitl@med.uni-muenchen.de)

Purpose: Treatment of type 1 endoleaks after endovascular aneurysm repair (EVAR) can be challenging. We describe a technique for direct embolisation with the liquid embolic agent ethylene vinyl alcohol copolymer Onyx by direct catheter access to the endoleak, and present first results.

Methods and Materials: 18 patients (16 male, 2 female, mean age $72+-7 \mathrm{yrs}$ ) with a type $1 a(n=13)$ or a type $1 b(n=5)$ endoleak were treated. In 13 cases, it was applied as stand-alone procedure, and in 5 cases in combination with stent graft extension. Access was trans-brachial for type 1a endoleaks, and trans-inguinal for type $1 \mathrm{~b}$ endoleaks. A multi-purpose catheter was placed between the aortic wall and the stent graft to access the endoleak, followed by coaxial introduction of a dimethyl sulfoxide (DMSO) compatible micro-catheter into the endoleak, and consecutive Onyx embolisation.

Results: Technical success was achieved in all cases. A mean volume of $11 \mathrm{ml}$ +- $5 \mathrm{ml}$ of Onyx 34 was used. In 5 cases, additional coils were used as an anchor for Onyx. 1 patient developed reperfusion of the endoleak during follow-up of 12 +- 8 months, which was successfully treated with the same technique. Non-target embolisation, catheter encasement, or anaphylactic reactions to DMSO were not observed.

Conclusion: The described technique is feasible, safe, and effective for treatment of type $1 \mathrm{a}$ and $1 \mathrm{~b}$ endoleaks with excellent technical success rates, and low relapse rates. It can be used as stand-alone procedure or easily be combined with stent graft extension.

Author Disclosures:

M. Treitl: Advisory Board; Covidien. Consultant; Covidien, Biotronik. 
14:00 - 15:30

Room E1

\section{Musculoskeletal}

\section{SS 1810}

\section{Knee: new horizons}

Moderators:

M. Aparisi Gomez; Valencia/ES

A. Cotten; Lille/FR

\section{B-0929 14:00}

PCL index as an objective MRI tool to evaluate anatomic ACL

reconstruction during functional activities

A. Ntoulia', F. Zampeli', E. Pappas ${ }^{2}$, S. Ristanis ${ }^{1}$, A. Georgoulis'; ${ }^{1}$ /oannina/GR,

${ }^{2}$ Brooklyn, NYIUS

Purpose: To test if the posterior cruciate ligament $(\mathrm{PCL})$ index is predictive of rotational knee kinematics during pivoting activities and anterior tibial translation (ATT) in patients after anatomic single-bundle (SB) anterior cruciate ligament reconstruction (ACLR).

Methods and Materials: Sixteen patients with SB-ACLR were prospectively evaluated with 3D-motion analysis during (1) descending and pivoting, and (2) landing and pivoting. The side-to-side difference of tibial rotation range of motion (SSDTR) between the ACLR and the contralateral intact knee was calculated. ATT SSD was measured with a KT-1000 arthrometer. MRI was used to measure the PCL index for the study group and a control group. Linear regression models were used with PCL index as predictor of SSDTR for each task and side to side anterior translation differences. Unpaired sample t-test was used to compare PCL index group means between the study and control group. The level of significance was set at $\mathrm{a}=0.05$. Results: PCL index of the ACLR knee was significantly lower compared to the healthy control knee $(p<0.001)$. The PCL index was predictive of low SSDTR during pivoting after descending and landing tasks $(R 2=0.472, p=0.003$ and $R 2=0.477$, $p=0.003$ respectively). $P C L$ index was not predictive of ATT with $134 \mathrm{~N}$ or maximum manual force $(R 2=0.13, p=0.17$ and $R 2=0.009, p=0.726$ respectively).

Conclusion: The PCL index after anatomic SB ACLR is predictive of rotational kinematics during dynamic pivoting activities. These results suggest that after anatomical ACLR the PCL index can serve as an indicator of tibial-femoral position and may predict dynamic tibial rotation.

\section{B-0930 14:09}

Microstructural evaluation of the cruciate ligaments with MR diffusion tensor imaging (DTI): correlations with knee stability L. Di Clemente', D. Tortora', V. Panara'1, M. Savastano', V. Calvisi ${ }^{2}$, P. Palumbo ${ }^{2}$, A. Tartaro', A.R. Cotroneo ${ }^{1}$, M. Caulo': ${ }^{1}$ Chieti/lT, ${ }^{2}{ }^{\prime}$ 'Aquila/lT (lorisdiclemente@gmail.com)

Purpose: The evaluation of knee stability is currently based on tibio-femoral sagittal plane motion measurement as evaluated using the KT-1000 arthrometer. Conventional MRI cannot evaluate knee stability. The aim of the study was to assess the use of MR-Diffusion Tensor Imaging (DTI) as a tool for a microstructural examination of the anterior and posterior cruciate ligaments and to correlate DTI finding with clinically evaluated stability.

Methods and Materials: 11 subjects ( 6 females) without previous history of knee injuries underwent MRI using conventional and DTI sequences. Fractional anisotropy (FA) values were calculated in each anterior and posterior cruciate ligament within 3 different ROIs placed in the lower, middle and upper portion of the ligaments. Knee stability was assessed using KT-1000 arthrometer in 4 progressive active displacements: $15 \mathrm{lbs}, 20 \mathrm{lbs}$, manual maximum displacement and under quadricipital muscle contraction. Statistical analysis was performed using Independent t-Test and Kendall tau rank correlation test, where appropriate $(p<0.05)$. Results: FAs of the ACL (mean: $0.573 \pm 0.047$ ) were significantly lower compared with the PCL (mean: $0.676 \pm 0.046)(p<0.001)$, whereas no side differences were noted. A significant negative correlation between FA and KT-1000 arthrometer values at $15 \mathrm{lbs}$ was present in the PCL $(r=-0.341 ; p=0.029)$. No correlations were observed between the FA and KT-1000 values in the ACL.

Conclusion: Functional assessment of the microstructural organisation of the cruciate ligaments with DTI expands MRI applications beyond simple morphological studies and could help bridge the gap between imaging and clinical evaluation of knee stability.

\section{B-0931 14:18}

Dynamic contrast-enhanced imaging for detection of complications after double-bundle reconstruction of the anterior cruciate ligament Y.-C. Lin', Y.-H. Juan', Y.-C. Cheung', W.-L. Yeh', C.-H. Chiu', C.-F. Tan', C.-M. Kuo'; ; ${ }^{1}$ Taoyuan/TW, ${ }^{2}$ llan/TW (yuching1221@gmail.com)

Purpose: Double-bundle reconstruction with stump preservation has revolutionised the surgical treatment for anterior cruciate ligament $(A C L)$ injury. However, poor revascularization at the osteoligamentous interface $(\mathrm{OI})$ of the tibia tunnel remains a major cause of graft complications. In this study, we applied dynamic contrastenhanced (CE) magnetic resonance imaging (MRI) to quantify the Ol enhancement values of the tibia tunnels and ACL stump. We aimed to determine the relationship between graft complications and $\mathrm{OI}$ and stump enhancements.

Methods and Materials: From October 2011 to April 2012, 34 patients were enrolled in our study (mean postoperative duration, 7.3 months). All patients underwent one 1.5-T MRI study with the imaging pulse-sequence protocol of proton density-weighted imaging (WI), T2WI, pre-enhanced and post-enhanced T1WI, and dynamic CE MRI. Graft complications, including cystic degeneration and tear, were evaluated using pre-enhanced MRI, and peak enhancement (ePeak) values were acquired from a dynamic CE study. The receiver operating characteristic $(\mathrm{ROC})$ analysis was used to obtain optimal cut-off values for complicated grafts. Results: Our study included 28 patients (mean age, 25.5 years). Nine patients $(32.1 \%)$ had cystic degeneration and $1(3.6 \%)$ had complete posterolateral $(\mathrm{PL})$ bundle tear. Mean ePeak percentages for graft with or without complications were $84.19 \% / 127.69 \%$ for anterior--medial (AM) bundle, $107.54 \% / 128.21 \%$ for PL bundle, and $171 \% / 151.06 \%$ for stump, respectively. ROC analysis yielded the optimal ePeak cut-off values of $126 \%, 104 \%$, and $35 \%$ for AM bundle, PL bundle, and stump, respectively.

Conclusion: Graft complications were directly associated with higher tibial OI values but inversely associated with higher stump values.

\section{B-0932 14:27}

Susceptibility artifacts on knee MRI: their frequency, change over time, and their relation with radiography-detected calcifications and joint space narrowing, and MR-detected meniscal tears

M. Jarraya ${ }^{1}$, D. Hayashi', A. Guermazi', C.K. Kwoh², M.J. Hannon², C.E. Moore', J.M. Jakicic ${ }^{2}$, S.M. Green ${ }^{2}$, F.W. Roemer ${ }^{4} ;{ }^{1}$ Boston, MA/US, ${ }^{2}$ Pittsburgh, PA/US, ${ }^{3}$ Houston, TX/US, ${ }^{4}$ Augsburg/DE (mohamedjarraya@gmail.com)

Purpose: We assessed the frequency of susceptibility artefacts (SA) on DESS and IW-fs, and their associations with calcifications and joint space narrowing (JSN) on $x$-ray (XR), and meniscal tears in the medial TFJ.

Methods and Materials: 346 knees of 177 subjects aged 35-65 with pain were included. 3 T MRI performed at baseline and 6-month follow-up (f/u). AP knee XR taken at baseline and calcifications within medial TFJ, and medial JSN were recorded. SA in TFJ space assessed on coronal DESS and IW fs. XR and MR images, each read on separate reading sessions. Kappa statistics applied to define agreement between findings on the baseline DESS/IW fs and XR.

Results: Mean age was $52 \pm 6$ years, $54 \%$ men, mean BMI was $29 \pm 4$. Medial meniscal tears and medial JSN were seen in 126 and 44 knees, respectively. At baseline, 13 knees showed SA on DESS, of which 6 were seen at f/u and 7 disappeared. Knees with $S A$ were more likely to have medial meniscal tears (9/13 vs. $117 / 333, p=0.017)$ and medial JSN (5/13 vs. 39/333, $p=0.016)$. On IW-fs, 4 knees showed SA at baseline. Of 7 knees with calcifications on XR, 2 demonstrated SA on DESS. Agreement between DESS and IW-fs was $\mathrm{k}=0.46(95 \% \mathrm{Cl} 0.17-0.75)$, and between SA on DESS and calcification on XR was $\mathrm{k}=0.18(95 \% \mathrm{Cl}-0.06-0.42)$. Conclusion: SA on DESS in the TFJ were seen in $<5 \%$ of knees. It rarely corresponded to calcifications on XR and commonly showed longitudinal changes, supporting the hypothesis that it represents vacuum phenomenon.

Author Disclosures:

A. Guermazi: Consultant; Genzyme, Novartis, Merck Serono, Stryker. Founder; Boston Imaging Core Lab (BICL). LLC. Shareholder; Boston Imaging Core Lab (BICL). LLC.F.W. Roemer: Consultant; National Institute of Health, Merck Serono. Shareholder; Boston Imaging Core Lab (BICL). LLC. 


\section{B-0933 14:36}

Meniscal pathology demonstraded by MRI and low level laser

therapy: a double-blinded placebo controlled trial

O. Kiritsi' ${ }^{1}$, N. Malliaropoulos ${ }^{2}$, K. Tsitas ${ }^{2}$, K. Omar';

${ }^{1}$ Gorleston on sea, Great Yarmouth/UK, ${ }^{2}$ Thessaloniki/GR (kirolga@hotmail.com)

Purpose: We performed a randomised, double blinded, placebo controlled study to assess the effectiveness of LLLT in patients with knee pain related to meniscal pathology.

Methods and Materials: The study trial number is ISRCTN24203769. We only included symptomatic patients with tiny focus of Grade 3 attenuation (seen only on 0.7 thickness sequences) or intrasubstance tears with spot of Grade 3 signal intensity approaching the articular surface. None of the patients in the study group underwent arthroscopy or new MRI investigation. A paired samples t-test was used to detect significant changes in subjective knee pain over the experimental period within groups, and ANOVA was used to detect any significant differences between the two groups.

Results: Pain was significantly improved for the LLLT group than for the placebo group $(F=154, p<0.0001)$. Pain scores were significantly better after LLLT. Four (12.5\%) patients did not respond to LLLT. At baseline, the average Lysholm score was $77 \pm 4.6$ for the LLLT group, and $77.2 \pm 2.6$ for the placebo group [ $p>0.05]$. Four weeks after LLLT or placebo therapy, the laser group reported an average Lysholm score of $82.5 \pm 4.6$, the placebo group scored $79.0 \pm 1.9$. At 6 months, the laser group had an average Lysholm score of $82.2 \pm 5.7$, an after one year they scored $81.6 \pm 6.6(\mathrm{~F}=14.82923, \mathrm{p}=0.002)$. Treatment with LLLT was associated with a significant decrease of symptoms compared to placebo.

Conclusion: In patients with small meniscal tears who do not wish to undergo surgery, LLLT should be considered as a non-invasive alternative.

\section{B-0934 14:45}

Meniscal tears are associated with adjacent tibiofemoral cartilage degeneration assessed using 3 T MRI T2 relaxation time H. Alizai, W. Lin, H. Liebl, L. Nardo, C. McCulloch, J. Lynch, M. Nevitt; San Francisco, CA/US

Purpose: The causal relationship between meniscal tears and Osteoarthritis (OA) is not clear, i.e. whether meniscal injury leads to cartilage degeneration or vice versa. Therefore, we aimed to evaluate the impact of meniscal tears on adjacent, morphologically intact knee cartilage using T2 relaxation time, a sensitive biomarker for early cartilage degeneration, and compare with healthy controls.

Methods and Materials: Sixty-nine subjects without evidence of $O A$ were recruited from the Osteoarthritis Initiative. Thirty-five Subjects (26-women) with a normal medial meniscus were used as controls (age $61 \pm 8, \mathrm{BMI} 27 \pm 5$ ). Thirty-four subjects (20-women) had either a simple (non-displaced tear; $\mathrm{n}=19$, age $61 \pm 9$, BMI $28 \pm 5$ ) or complex tear (displaced tear; $n=15$, age $60 \pm 9$, BMI $29 \pm 5$ ) of the posterior horn of the medial meniscus. None of the tear-subjects had morphological lesions of the medial tibio-femoral cartilage. T2-measurements were performed in the whole medial tibia (MT) and the medial femur compartments (MF), and in tibio-femoral subregions adjacent to the torn meniscus (SMT \& SMF). Multiple linear-regression models were used to compare mean T2-values of individuals with meniscal tears with normal controls.

Results: The sMT had significantly higher T2-values in the tear group (35 ms) compared with controls ( $32 \mathrm{~ms}, \mathrm{p}=0.01$ ). When comparing individuals with simple versus complex tears, the complex tear group had significantly higher T2-values in the MT $(p=0.04), M F(p=0.02), s M T(p=0.03)$ and sMF $(p=0.002)$. T2-values in the cartilage sMT in the tear group were significantly higher than MT T2-values $(\mathrm{p}<0.0001)$.

Conclusion: Presence and severity of meniscal injuries are associated with increased cartilage degeneration as measured with $\mathrm{T} 2$ relaxation time, most pronounced in cartilage adjacent to the tears.

\section{B-0935 14:54}

Articular depression assessed by MDCT as a predictor of soft-tissue injuries in tibial plateau fractures: intraindividual correlation to findings at magnetic resonance imaging

M. Regier, A. Spiro, F.O.G. Henes, G. Adam; Hamburg/DE (mregier@uke.de)

Purpose: The aim of the present study was to determine whether the amount of articular depression or fracture type assessed at MDCT may predict the incidence and number of associated soft-tissue injuries in tibial plateau fractures compared with Magnetic Resonance Imaging.

Methods and Materials: The medical reports of 54 patients with acute tibial plateau fractures who were admitted to the emergency department of a university medical centre were reviewed. MDCT and MRI were performed in every patient.
The Schatzker classification for tibial plateau fractures and the amount of articular depression were assessed using MDCT. Magnetic resonance images were evaluated for crucial and collateral ligament injury, meniscal tears and patellar retinaculum lesions. Statistics included logistic regression and analysis of covariance.

Results: Associated soft-tissue injuries were identified in $82 \%$ of the patients. Logistic regressions revealed a significant impact of increasing amounts of tibial plateau fracture depression on the incidence of meniscus lateralis tears $(P=0.025)$ and lesions of the anterior cruciate ligament $(P=0.018)$. Analysis of covariance demonstrated a significant correlation between the amount of articular depression and the absolute number of soft-tissue injuries $(P=0.001)$.

Conclusion: Articular depression is a potential predictor of specific meniscal and ligamentous injuries in acute tibial plateau fracture. Due to the high incidence of associated soft-tissue lesions, magnetic resonance imaging is generally recommended, especially in cases with distinct tibial plateau fracture depression at MDCT imaging.

\section{B-0936 15:03}

Anatomical risk factors in patients after patellar dislocation: a casecontrol study using MRI

T. Köhlitz, S. Scheffler, T. Jung, E. Wiener, B. Vollnberg, G. Diederichs; Berlin/DE (bernd.vollnberg@charite.de)

Purpose: To assess anatomical risk factors in patients after lateral patellar dislocation (LPD) and controls using MRI.

Methods and Materials: MR images of 186 knees after LPD and of 186 age- and gender-matched controls were analysed. The presence of trochlear dysplasia was assessed by evaluation of trochlear inclination, facet asymmetry, and trochlear depth; patella alta was evaluated by Insall-Salvati-index and Caton-Deschampsindex; and lateralized force vector was measured by the tibial tuberosity-trochlear groove (TT-TG) distance.

Results: Compared with controls, dislocators had significantly lower values for all three parameters of trochlear dysplasia $(-32 \%,-32 \%,-44 \%)$ and significantly higher values for patella alta $(+14 \%,+13 \%)$ and TT-TG $(+49 \%)$ (all $P<0.001)$. Trochlear dysplasia was observed in 112 dislocators (66\%), of whom $61(36 \%)$ additionally had patella alta and $15(9 \%)$ an abnormal TT-TG. As isolated risk factors, patella alta (15\%) and abnormal TT-TG (1\%) were rare. Only 25 dislocators (15\%) had no anatomical risk factor. Trochlear dysplasia in conjunction with abnormal TT-TG or patella alta is associated with a 37 -fold and 41 -fold higher risk.

Conclusion: Most dislocators have anatomical risk factors, varying in severity and constellation. Trochlear dysplasia is the main risk factor for LPD, while patellar alta and abnormal tibial tuberosity-trochlear groove distance may be additional factors. Patient-specific evaluation of risk factors following LPD may help future management.

\section{B-0937 15:12}

Early changes of trabecular bone structure in asymptomatic subjects with knee malalignment

T. Baum, J. Penzel, M. Sauerschnig, E.J. Rummeny, K. Wörtler, J.S. Bauer; Munich/DE (thbaum@gmx.de)

Purpose: Knee malalignment is a risk factor for knee osteoarthritis (OA). The purpose of this study was to investigate whether alterations of trabecular bone structure can already be found in young, asymptomatic subjects with knee malalignment. Methods and Materials: Forty-eight asymptomatic subjects ( 25 females, 23 males: age: $26 \pm 2$ years) without history of knee injury or surgery were included in this study. Based on MR measurements, knee joint alignment of both lower extremities was assessed and subjects were divided into four groups (each including 12 subjects, i.e. 24 knees): neutral (up to $1^{\circ}$ varus/valgus), mild varus $\left(2^{\circ}-4^{\circ}\right)$, severe varus (greater $\left.4^{\circ}\right)$, and valgus $\left(2^{\circ}-4^{\circ}\right)$. A T1-weighted 3D-FLASH sequence (spatial resolution: $0.25 \times 0.25 \times 1.2 \mathrm{~mm}^{3}$ ) was obtained of each knee at $1.5 \mathrm{~T}$ to determine histomorphometric and texture parameters of the trabecular bone in the medial/ lateral femur/tibia (MF,LF,MT,LT). The calculated parameters were compared between the four groups in each compartment using ANOVA including Bonferroni correction for multiple comparisons.

Results: Apparent bone volume fraction was significantly different between the severe varus and valgus group in the MT compartment $(0.35 \pm 0.02$ vs. $0.32 \pm 0.02$; $\mathrm{p}=0.022)$. Furthermore, apparent trabecular thickness in the MT compartment was lower in the valgus group $(0.36 \pm 0.03 \mathrm{~mm})$ compared to the mild varus $(0.40 \pm 0.08$ $\mathrm{mm} ; \mathrm{p}=0.025)$ and severe varus group $(0.40 \pm 0.04 ; \mathrm{p}=0.020)$. In the MF compartment, fractal dimension was significantly greater in the severe varus than in the valgus group ( $1.68 \pm 0.01$ vs. $1.66 \pm 0.02 ; p=0.022)$.

Conclusion: Early trabecular bone changes can be found in asymptomatic subjects with knee malalignment. This finding may have important implications for the prevention of knee OA. 


\section{B-0938 15:21}

Total knee arthroplasty MR imaging with the WARP-technique, featuring slice-encoding metal artefact correction (SEMAC) and view-angle tilting (VAT): reduction of metal artefacts for STIR and intermediate-weighted sequences

R. Sutter, R. Hodek, S.F. Fucentese, C.W.A. Pfirmann; Zurich/CH (reto.sutter@balgrist.ch)

Purpose: To compare image quality and detection of clinical findings of the new WARP sequence versus conventional optimised MR sequences in patients with total knee arthroplasty (TKA).

Methods and Materials: Forty-two patients with TKA underwent 1.5 T MRI in this prospective study. Slice-encoding metal artefact correction (SEMAC), view-angle tilting (VAT), and increased bandwidth were combined by the "WARP"-turbo spin echo sequence. Twenty-four patients underwent CT as a reference standard. Coronal STIR-WARP and sagittal intermediate-weighted (PD) WARP-sequences were compared to standard sequences optimised with high readout bandwidth (STIR-hiBW/PD-hiBW). Signal void was quantified. Qualitative criteria (depiction of anatomy, distortion, blurring, noise) were assessed on a five-point scale (1, no artefacts; 5 , severe artefacts) by two readers. Clinical findings (e.g. periprosthetic osteolysis) were noted. At-test (quantitative data) and a Wilcoxon signed rank test (qualitative data) served for statistics.

Results: Signal void areas were smaller for STIR-WARP than STIR-hiBW (mean; $\left.20.1 \mathrm{~cm}^{2} / 27.0 \mathrm{~cm}^{2}\right)$, and for PD-WARP than PD-hiBW $\left(14.2 \mathrm{~cm}^{2} / 16.5 \mathrm{~cm}^{2} ; \mathrm{p}<.001\right.$ for all comparisons). Depiction of anatomic structures was better on STIR-WARP versus STIR-hiBW (2.9-3.7 vs. 4.2-4.9; $p<.001)$, and on PD-WARP versus PDhiBW (2.5-3.5 vs. 3.1-3.8; $p<.007)$. Distortion and image noise were lower for WARP than for the standard sequences $(p<.002)$, while blurring was similar for both techniques. There was a statistically significant advantage for detection of clinical findings for STIR-WARP versus STIR-hiBW (74 and 37 findings; $p<.001$ ) and for PD-WARP versus PD-hiBW (63 and 34 findings; $p<.001$ ).

Conclusion: The WARP sequences showed a statistically significant improvement for most image criteria. Detection of clinically relevant findings was markedly increased. Room E2

\section{GI Tract}

\section{SS 1801a}

\section{Colorectal cancer: diagnostic biomarkers and response assessment}

Moderators:

A. Filippone; Chieti/lT

J. Husty; Brno/CZ

\section{B-0939 14:00}

MR imaging evaluation of rectal cancer before and after chemoradiation therapy

R. Cazzato, C.L. Piccolo, R. Del Vescovo, F. Giurazza, R.F. Grasso,

B. Beomonte Zobel; Rome/IT (C.Piccolo@unicampus.it)

Purpose: To determine the diagnostic accuracy of MR in patients with rectal carcinoma by comparing post-chemoradiation MR imaging with pathological specimens. Methods and Materials: We enrolled 39 patients with a locally advanced rectal cancer. All patients received chemoradiation therapy before surgery. Those patients underwent a neoadjuvant chemoradiation therapy followed by MR scans. The MR images were analysed by a team of two expert radiologists to whom clinical and histo-pathological findings were unknown.

Results: Following neoadjuvant chemoradiation therapy, we observed 23 (59\%) patients with a rectum disease staged ${ }^{2} \mathrm{~T} 2$ and $16(41 \%)$ patients with a disease staged > T2, after MR images analysis. Post-treatment histological staging (TNM) analysis revealed 13 patients with a disease $>\mathrm{T} 2$ and 26 patients with a disease 2 T2. By arranging the Kappa Cohen test to find the agreement value between post-chemoradiation MR staging and histological response, we found that with a disease confined to the sierosa ( ${ }^{2} \mathrm{~T} 3$ ) the agreement was $83.6 \%$. We found an agreement of $97.22 \%$ between MR and histology for a disease ${ }^{2} \mathrm{~N} 1$ and an accord of $33.33 \%$ for a disease more advanced than $\mathrm{N} 1$ (>N1).

Conclusion: MR is critical in discovering a T3 disease; moreover, with morphologic MR Imaging we do not have always the opportunity to discern a few residual cancer cells hidden in the fibrotic tissue that could cause a CRM involvement on histology.
B-0940 14:09

MRI-based selection of clinical complete and good responders after chemoradiation for rectal cancer allows for successful minimal invasive treatment

L. Heijnen'1, M. Maas', M.H. Martens', D.M.J. Lambregts' ${ }^{1}$, J.W.A. Leijtens², R.G.H. Beets-Tan', G.L. Beets ${ }^{1} ;{ }^{1}$ Maastricht/NL, ${ }^{2}$ Roermond/NL (luc.heijnen@mumc.nl)

Purpose: Patients with good or complete response after neoadjuvant chemoradiation have excellent long-term outcome. Minimal invasive treatment (i.e. transanal endoscopic microsurgery (TEM) and wait-and-see policy) are increasingly considered as an alternative to major surgery. With this prospective cohort study we aimed to evaluate long-term outcome of strictly MR-based selected patients who have been treated with minimal invasive treatment.

Methods and Materials: Eight weeks after chemoradiation, endoscopy and restaging MRI were performed (including diffusion-weighted MRI for yT-staging and gadofosveset-enhanced MRI for $\mathrm{yN}$-staging). Complete responders were selected for wait-and-see policy and good responders with small tumour remnant for TEM. Both treatment groups underwent intensive 3-to-6 monthly follow-up, using MR imaging (DWI+gadofosveset), CEA, CT of thorax and abdomen and endoscopy was performed. Long-term outcome was estimated with Kaplan-Meier curves. Results: Forty-one patients were included, thirty-three in the wait-and-see group and eight in the TEM-group. Mean follow-up was 26 months (range 6-91). For the TEM-group, 4 patients had ypT0 and 4 had ypT2. Two patients, both in the wait-and-see group, developed a local recurrence within two years and underwent surgery, leading to a 2-year local recurrence rate of $9 \%$. Both recurrences were detected on (DWI-)MRI in an early stage. The cumulative probabilities of 2-year disease-free survival and overall survival were $93 \%$ and $100 \%$, respectively. No recurrences occurred in the TEM-group.

Conclusion: Both selection and follow-up of good and complete responders after chemoradiation for rectal cancer with MRI is feasible. Long-term outcome so far is excellent. (DWI-)MRI seems to be a reliable tool for early recurrence detection.

\section{B-0941 14:18}

Diffusion-weighted-based volumetry in the assessment of response in patients with rectal cancer treated with neo-adjuvant therapy: feasibility study

S.F. Carbone, M. Palumbo, V. Ricci, L. Pelliccia, E. Cacchiarelli, L. Volterrani; Siena/lT (fracarb@gmail.com)

Purpose: To establish the reproducibility of volumetric magnetic resonance diffusion (VDWI) and compare it with the conventional volume (VC) in the assessment of response in patients with rectal cancer treated with chemoradiotherapy (CRT). Methods and Materials: We retrospectively examined 21 patients (mean age 68.9 years) with rectal cancer who have performed MRI before and after $45 \pm 15$ days of the CRT after undergoing anterior resection of the rectum. After surgery, ypTN and TRG (tumour regression grading) according to Mandard classification were obtained. We considered patients with TRG 1-2 responder and non-responder patients with TRG 3-5. Two radiologists, blinded, have extrapolated the volume VC and VDWI lesions pre-and post-CRT. The inter-observer agreement and the agreement between Vc and VDWI pre- and post-CRT were calculated by intraclasscorrelation coefficient (ICC).

Results: The inter-observer agreement for VC and VDWI was 0.948 and 0.916 respectively, while the correlation between VC and VDWI investigating pre-CRT was 0.828 and in the post-CRT in 0.151. After CRT, VC shows a correlation with the TRG of rho $=0.154(p<0542)$, while a significant linear relationship was evident between VDWI and TRG with rho $=0556(p<0.05)$. Post-CRT the VDWI shows a significant difference between responders and nonresponders $(p=0.018)$.

Conclusion: The VDWI is a reproducible quantitative technique in assessing the response to CRT in patients with rectal cancer and shows a better correlation with the residue of the disease compared with the VC.

\section{B-0942 14:27}

Therapy response assessment in locally advanced rectal cancer: comparison between functional dynamic and diffusion parameters in MRI R. Fusco, V. Granata, M. Petrillo, M. Sansone, A. Petrillo; Naples/IT (roberta.fusco@unina.it)

Purpose: We investigated the diagnostic performance of DCE-MRI and DW-MRI in evaluating neoadjuvant chemo-radiotherapy (CRT) response in LARC. We compared a semiquantitative dynamic feature, the standardised index of shape (SIS) and the diffusion parameters obtained using intravoxel incoherent method. 
Methods and Materials: 14 consecutive patients with LARC were enrolled. Patients underwent DCE-MRI and DW-MRI (1.5 T, Magnetom Simphony TIM, Siemens, Erlangen). An expert radiologist performed a manual segmentation of the whole lesion on a derived series obtained subtracting the basal and the $5^{\text {th }}$ post-contrastographic series and on DWI with $b$ value $=800$. SIS and pure diffusion coefficient, pseudo-diffusion coefficient and perfusion fraction were calculated pixel by pixel. After surgery, tumour regression grade (TRG) was obtained. Patients with TRG 1-2 were considered responders and patients with TRG 3-4-5 were considered non-responders. The value changes of each parameters from baseline to presurgical scan were assessed and correlated with the TRG. Sensitivity, specificity, ROC analysis were applied. Youden Index was used to obtain the optimal cut-off value. Mc-Nemar test was performed to underline statistical difference.

Results: Seven patients were classified as responders and 7 as non-responders at histology. The diffusion parameter (fraction perfusion) showed a sensitivity of $100 \%$, specificity of $25 \%$ and area under ROC 0.4750 (optimal cut-off value $86.3 \%$ ); SIS showed a sensitivity of $90 \%$, a specificity of $100 \%$ and area under ROC 0.7265 (optimal cut-off value $36.0 \%$ ). A p value of $<0.05$ was obtained comparing SIS vs diffusion parameter.

Conclusion: SIS can achieve the best results in discriminating patients responders from non-responders.

\section{B-0943 14:36}

Reproducibility of MRI texture analysis in primary rectal cancer

S. Gourtsoyianni', G. Ljungqvist' ${ }^{1}$, A. Khan², R. Glynne- Jones²,

B. Ganeshan ${ }^{3}$, K. Miles ${ }^{3}$, V. Goh $;{ }^{1}$ London/UK, ${ }^{2}$ Northwood/UK, ${ }^{3}$ Falmer/UK (sgty76@gmail.com)

Purpose: This study aimed at assessing the feasibility and reproducibility of MRI texture analysis in rectal cancer.

Methods and Materials: Following IRB approval, 14 patients (11 male, mean $68.3 y e a r s)$ with primary rectal cancer underwent two baseline MRIs. T2-W axial sequences were exported for texture analysis (TexRAD, University of Sussex). A Laplacian of Gaussian band-pass filter was applied to highlight different spatial scales (fine (1.0), medium (1.5-2.0) and coarse (2.5) texture). Texture was quantified as entropy $(E)$, uniformity $(U)$, kurtosis $(K)$, skewness $(S)$ and standard deviation of the histogram (SDH) and recorded as for absolute scale values for each tumour. Greater heterogeneity is represented by higher E, greater K, S and SDH and lower uniformity. Overall group median values were recorded and reproducibility (testretest agreement) was assessed using Bland-Altman statistics.

Results: All tumours were greater than Stage II; mean length $5.5 \mathrm{~cm}$. Good reproducibility was achieved across all filters $(1.0-2.5)$ for $\mathrm{E}, \mathrm{U}$ and $\mathrm{SDH}$ with mean differences ranging from -0.007 to +0.002 for $\mathrm{E} ;-0.11 \mathrm{X} 10^{-3}$ to $+0.001 \times 10^{-3}$ for $\mathrm{U}$; and +1.60 to +4.97 for $\mathrm{SDH}$; and within subject coefficients of variation from $2.09-2.55 \%$ for $\mathrm{E}, 10.3-12.7 \%$ for $\mathrm{U}$, and $14.3-16.1 \%$ for SDH. There was greater variability for $\mathrm{K}$ and $\mathrm{S}$ : mean differences ranged from +0.168 to +0.334 for $\mathrm{K}$; and +0.089 to +0.272 for $S$; within subject coefficients of variation from $31.3-93.7 \%$ for $\mathrm{K}$, and $21.5-49.6 \%$ for $\mathrm{S}$.

Conclusion: MRI texture analysis is feasible in rectal cancer and a reproducible technique in the trial setting.

\section{B-0944 14:45}

Predictors of mesorectal fascia infiltration on spectral pre-saturation inversion-recovery (SPIR) MR imaging sequence after gadolinium injection in patients with rectal carcinoma after neoadjuvant chemoand radiation therapy

E. Quaia, L. De Paoli, A. Gennari, B. Cabibbo, M. Cova; Trieste/IT (quaia@units.it)

Purpose: To retrospectively assess the value of spectral pre-saturation inversionrecovery (SPIR) MR imaging sequence after gadolinium injection to identify predictors of mesorectal fat and mesorectal fascia infiltration in patients with locally advanced rectal carcinoma after neoadjuvant chemo- and radiation therapy (CRT). Methods and Materials: Sixty-five consecutive patients (mean age: 68.93 years, range: $48-83$ years, $M: F 39: 26$ ) with locally advanced rectal carcinoma underwent CRT followed by surgery. MR imaging was performed before and after completion of CRT using T2-weighted fast spin-echo and T1-weighted SPIR sequences before and after gadolinium injection. MR images were assessed by two radiologists in consensus. The evidence of reticular (interwoven strands within the mesorectal fat creating a meshwork) or linear-shaped enhancing strands (strands travelling separately through the mesorectal fat) on MR images were retrospectively correlated to the histopathological findings.

Results: After CRT the disease was either limited to the rectal wall ( $n=31$ patients) or presented mesorectal fat infiltration $(n=34)$. In 5 patients mesorectal fascia infiltration was present on the surgical specimen. In 33 patients reticular $(n=18)$ or linear-shaped $(n=15)$ mesorectal enhancing strands were observed. Reticular-shaped mesorectal enhancing strands were predictors of mesorectal fat infiltration (odds ratio, 7.36; 95\% confidence intervals, 1.87-28.98; $P<0.05$ ) but not of mesorectal fascia infiltration $(0.86 ; 0.08-8.87 ; \mathrm{P}=0.9)$. The enhancing strands reaching the mesorectal fascia represent a predictors of mesorectal fascia infiltration $(3.61,0.99-13.15 ; P<0.05)$

Conclusion: The evidence of enhancing strands reaching the mesorectal fascia represents a predictor of mesorectal fascia infiltration and should suggest to intensify the CRT.

Author Disclosures:

E. Quaia: Consultant; Bracco.

\section{B-0945 14:54}

Neoadjuvant therapy in locally advanced colon cancer: the role of MDCT I. Gonzalez Crespo, D. Cano, J. Etxano, P. Slon, J. Arredondo; Pamplona/ES (dcanor@unav.es)

Purpose: To show the utility of MDCT assessing the tumour response in locally advanced colon cancer treated with neoadjuvant chemotherapy.

Methods and Materials: 22 consecutive patients who underwent this therapy during a 15-month period were included. All tumours were staged before treatment using MDCT 64 or PET-CT scan, and after neoadjuvant treatment other scan was performed by calculating the differences in tumour volumes making use of a dedicated software for semiautomatic volume segmentation, to assess tumour response before surgery. Maximum standard uptake value (SUV max) by PET-CT between the time of initial diagnosis and after neoadjuvant chemotherapy was also measured. Surgical-related complications and oncological outcomes were obtained. Results: Tolerance to chemotherapy was excellent in $86.4 \%$ of patients and $95.5 \%$ completed the entire chemotherapy initially planned. After neoadjuvant treatment, $69.5 \%$ reduction of tumour volume was observed by CT-scan and up to $59.9 \%$ decrease of SUVmax (Standard Uptake Value) was achieved by PET/ MDCT. None of the patients showed tumour progression during the preoperative treatment, and all of them underwent the previously planned surgical procedure. Four patients developed postoperative complications. Median time between the end of chemotherapy and surgery was 22 days.

Conclusion: Preoperative chemotherapy induces a tumour response that can be measured by imaging methods (MDCT).

\section{B-0946 15:03}

Assessment of the variation in the contrast-to-noise ratio across a range of $\mathrm{CT}$ scanners in a multicentre perfusion $\mathrm{CT}$ study of colorectal cancer (PROSpeCT): a phantom study

M. Lewis, V.J. Goh; London/UK

Purpose: To determine the effect of CT scanner models, iterative reconstruction (IR) and phantom size on CNR of perfusion CT scans.

Methods and Materials: A 22-cm diameter water phantom containing different iodine contrast inserts from $2.5 \mathrm{mg} / \mathrm{ml}$ to $25 \mathrm{mg} / \mathrm{ml}$ was scanned on seven CT scanners from four manufacturers using the Perfusion $\mathrm{CT}$ acquisition protocols of a multi-centre clinical trial in colorectal cancer (PROSPeCT). All acquisition protocols employed $100 \mathrm{kV}$ and $\sim 5 \mathrm{~mm}$ reconstructed slice. Images were reconstructed with filtered back projection and iterative reconstruction, where available. CT numbers and background noise levels were measured and displayed CTDlvol values noted. A sub-set of measurements was obtained on a $30-\mathrm{cm}$ diameter phantom. The relationship between CT number and iodine density was determined for all datasets and CNR values calculated.

Results: A linear relationship between CT number and iodine density was observed, with small variations between CT scanners. No change in linearity was observed with IR of different 'strengths'. CTDIvolvalues varied by a factor $>2$. For the lowest density insert, CNR varied from 2.9 to 4.4. With IR, CNR increased by a factor of 1.15 to 2.3 depending on IR'strength'. CNR decreased by $\sim 50 \%$ in the $30 \mathrm{~cm}$ phantom. Conclusion: The CNR varied by a factor of 1.5 across the range of scanners utilised in this mutlicentre study. The variation was largely due to image noise differences. IR does not affect contrast enhancement and can be used to achieve a given CNR at a reduced dose.

Author Disclosures:

V.J. Goh: Equipment Support Recipient; Siemens Healthcare, GE Healthcare, TexRAD. Research/Grant Support; NIHR HTA grant. 
B-0947 15:12

Diffusion-weighted-based MRI: volumetry and apparent diffusion coefficient

S.F. Carbone, M. Palumbo, T. Carfagno, V. Ricci, L. Pirtoli, L. Volterrani; Siena/IT (fracarb@gmail.com)

Purpose: To assess the diagnostic accuracy of volumetric magnetic resonance diffusion (VDWI) and Apparent Diffusion Coefficient (ADC) in the assessment of response in patients with rectal cancer treated with chemoradiotherapy (CRT). Methods and Materials: We retrospectively examined 30 patients (mean age 68.1 years) with rectal cancer, who have performed MRI before and after $45 \pm 15$ days of the CRT after undergoing to anterior resection of the rectum. After surgery, ypTN and TRG (tumour regression grading) according to Mandard classification were obtained. We considered responders patients with TRG 1-2 or with a negative follow-up recurrence in the next 12 months (only three cases). Two radiologists, in consensus and using commercial software, have extrapolated pre-and post-CRT VDWI of the lesions and calculated the ADC.

Results: The ADC did not show significant differences between responders and not-responders ( $p=0.08$ pre-CRT, $p=0.1$ post-CRT); the VDWI of responders was significantly lower in both pre-CRT $(p=0.0065)$ and in post-CRT $(p=0.006)$ compared with not-responders VDWI. The accuracy in the evaluation of response was of 70 and $91 \%$, respectively, for the ADC and VDWI.

Conclusion: The VDWI is more reliable than ADC to assess the response to CRT in patients with rectal cancer.

\section{B-0948 15:21}

Computed tomography of the bowel: a prospective comparison study between four techniques

M. Revelli, F. Paparo, L. Bacigalupo, A. Garlaschi, L. Cevasco, E. Biscaldi, G. Rollandi; Genoa/IT

Purpose: Our purposes were to compare the grade of bowel distension obtained with four different $\mathrm{CT}$ techniques dedicated for examination of small intestine (CT-enteroclysis and CT-enterography), colon (CT with water enema), or both (CT-enterography with water enema) and to assess patient tolerance towards each protocol.

Methods and Materials: We recruited four groups of 30 patients. Each group corresponded to a specific CT technique, for a total of 120 consecutive patients ( 65 male, 55 female; mean age $51.09 \pm 13.36$ years). CT studies were evaluated in consensus by two gastrointestinal-dedicated radiologists who performed quantitative and qualitative analysis of bowel distension. Presence and type of adverse effects were recorded.

Results: CT-enteroclysis provided the best distension of jejunal loops (median diameter $27 \mathrm{~mm}$; range 17-32 mm) compared with all other techniques $(p<0.0001)$. Frequency of patients with an adequate distension of the terminal ileum was not significantly different among the four groups $(p=0.0608)$. At both quantitative and qualitative analysis CT with water enema and CT-enterography with water enema determined a greater and more consistent luminal filling of the large intestine compared with the one provided by both CT-enteroclysis and CT-enterography ( $p$ $<0.0001$ for all colonic segments). Adverse effects were more frequent in patients from the CT-enteroclysis group $(p<0.0028)$.

Conclusion: CT-enteroclysis allows an optimal distension of jejunal loops, but it is the most uncomfortable CT protocol. When performing CT with water enema, an adequate retrograde distension of the terminal ileum was provided in a high percentage of patients. CT-enterography with water enema provides a simultaneous optimal distension of both small and large bowel.
14:00 - 15:30

Room F1

Breast

SS 1802a

Newer techniques in breast imaging and therapy

Moderators:

F. Chamming's; Paris/FR

M. Sklair-Levy; Tel Aviv/IL

\section{B-0949 14:00}

The impact of contrast-enhanced digital mammography on the characterisation of breast lesions

M.H. Helal, R.M.K. Fouad, M.A. Aly, R. Wessam, I. Gouda, N. AbdeIShafi,

O.M. Nada, S. Mansour, M. Elbadawy; Cairo/EG (maha_helal@yahoo.com)

Purpose: To evaluate the impact of dual-energy contrast-enhanced digital mammography (CEDM) on the characterisation of breast lesions.

Methods and Materials: In this prospective study, we performed standard mammograms and dual-energy CEDM to assess 210 breast lesions using Senobright GE Digital System. Selection of cases was based on the identification of indeterminate mammography lesions. Two different radiologists analysed the lesions' morphology and pattern of contrast uptake.

Results: The study included $132(62.8 \%)$ malignant and $78(37.2 \%)$ benign breast lesions. The calculated sensitivity, specificity, positive and negative predictive values of CEDM were $93.9 \%, 89.6 \%, 93.9 \%$ and $89.6 \%$, respectively, as compared to $85.6 \%, 55.1 \%, 76.3 \%$, and $69.3 \%$ for FFDM. False negative results were encountered in 8 cases $(3.8 \%$ ) which included 3 invasive carcinomas, 2 mucinous carcinomas and 3 duct carcinomas in situ. False positive results were encountered in 8 cases (3.8\%) including 5 abscess cavities and 1 case of operative bed fat necrosis both showing a ring pattern of contrast uptake and 2 atypical fibroadenomas. Enhancing malignant lesions included 124 cases showing different patterns of contrast uptake. A ring pattern was seen in 20 cases (16.1\%), ductal branching segmental pattern in 5 cases $(4.1 \%)$, intense homogeneous pattern in 20 cases $(16.1 \%)$ and intense heterogeneouspattern in 79 cases $(63.7 \%)$.

Conclusion: CEDM has a potential impact on the characterisation of breast lesions. Intensely enhancing lesions are strongly indicative of a malignant nature, yet, overlap of enhancement patterns still exists and therefore further studies with a larger number of studied population are recommended.

\section{B-0950 14:09}

Clinical feasibility of contrast-enhanced dual-energy mammography (CEDEM) with a tungsten (W)/titanium (Ti) anode/filter combination: a prototype report

T. Knogler ${ }^{1}$, R. Leithner'1, M. Hörnig'², F. Semturs ${ }^{1}$, M. Waitzbauer'1,

G. Langs ${ }^{1}$, P. Homolka ${ }^{1}$, K. Pinker-Domenig ${ }^{1}$, T.H. Helbich ${ }^{1}{ }^{1}$ Vienna/AT,

${ }^{2}$ Erlangen/DE (thomas.knogler@meduniwien.ac.at)

Purpose: To test the feasibility of CEDEM with a W/Ti anode/filter combination for high energy images in a clinical setting.

Methods and Materials: Fifteen female patients with 15 breast lesions were included in this study. CEDEM was performed with a Mammomat Inspiration prototype (Siemens, Germany) before and after i.v. administration of 2-ml lomeron ${ }^{\circledR}$ 400 (Bracco, Italy) per kg b.w. at a rate of $3.5 \mathrm{ml} / \mathrm{sec}$. Dual-energy images were acquired with 28-32 kVp and a W/Rhodium (Rh) anode/filter combination for lowenergy and $49 \mathrm{kVp}$ and a $\mathrm{W} / \mathrm{Ti}$ anode filter combination for high energy. Weighted subtraction images were computed for diagnostic work-up. Images were assessed by two readers with respect to lesion-enhancement and image quality. A histological work-up was performed in all lesions.

Results: Histopathology revealed eight malignant lesions and seven benign lesions (size range from 8 to $38 \mathrm{~mm}$ ). All malignant lesions enhanced and were seen from both readers on weighted subtraction images. Benign lesions did not enhance thus they were not visualised on weighted subtraction images. Image quality was rated excellent from both readers. Based on the visibility of the lesion CEDEM allowed an accurate differentiation of benign and malignant breast lesions.

Conclusion: CEDEM with a W/Ti anode/filter combination is suitable and feasible. Lesion visibility and image quality were excellent. Further research is needed to determine the value of CEDEM in a clinical setting. 


\section{B-0951 14:18}

Comparison of contrast-enhanced dual-energy mammography (CEDEM) with contrast-enhanced magnetic resonance imaging (CEMRI) in breast lesions: a prototype report

T. Knogler', R. Leithner', M. Hörnig ${ }^{2}$, M. Waitzbauer ${ }^{1}$, F. Semturs ${ }^{1}$,

G. Langs ${ }^{1}$, P. Homolka ${ }^{1}$, K. Pinker-Domenig ${ }^{1}$, T.H. Helbich ${ }^{1} ;{ }^{1}$ Vienna/AT,

${ }^{2}$ Erlangen/DE (thomas.knogler@meduniwien.ac.at)

Purpose: To test the feasibility of CEDEM, by comparing its results with CE-MRI in breast lesions.

Methods and Materials: Fifteen female patients with 15 breast lesions were included in this study. CEDEM was performed with a Mammomat Inspiration prototype (Siemens, Germany) before and after i.v. administration of $2 \mathrm{ml}$ lomeron ${ }^{\circledR} 400$ (Bracco, Italy) per kg b.w. at a rate of $3.5 \mathrm{ml} / \mathrm{sec}$. The same lesions were assessed with CE-MRI (Tim Trio, Siemens, Erlangen, Germany) using a standard protocol and the administration of $0.2 \mathrm{mmol}$ Dotarem ${ }^{\circledR}$ (Guerbet, France) per kg b.w. Images of both modalities were assessed by two readers with respect to morphology and enhancement characteristics. A histological work-up was performed in all lesions. Results: Histopathology revealed eight malignant and seven benign lesions (size range from 8 to $38 \mathrm{~mm}$ ). Morphology and enhancement characteristics were similar and comparable with both techniques. All malignant lesions showed strong contrast uptake and suspicious morphologic signs, whereas benign lesions tend to enhance less strong on both techniques. Based on the morphologic and enhancement characteristics CEDEM and CE-MRI allowed accurate differentiation of benign and malignant breast lesions.

Conclusion: CEDEM allows adequate differentiation of benign and malignant breast lesions using morphologic and enhancement characteristics. CEDEM imaging features are comparable to those of CE-MRI. CEDEM offers a feasible and low cost alternative to CE-MRI in breast lesions.

Author Disclosures:

M. Hörnig: Employee; Siemens AG, Healthcare, Germany.

\section{B-0952 14:27}

Metabolic imaging of breast tumours with dedicated breast 18 F-FDG PET-CT: comparison with contrast-enhanced MRI at 3 T (3 T CE-MRI) H.F. Magometschnigg, T.H. Helbich, H. Bickel, G. Wengert, B. Brück, K. Pinker-Domenig; Vienna/AT (h.magometschnigg@gmail.com)

Purpose: To assess metabolic imaging of breast tumours with breast 18 F-FDG PET-CT and compare it with 3 T CE-MRI.

Methods and Materials: 159 suspicious breast lesions were included in this IRB-approved prospective study. All patients were examined with 18 F-FDG PET-CT and 3 T CE-MRI of the breast. The MRI protocol consisted of a T2-W and a contrast-enhanced high-resolution 3D-T1-w sequence with a single dose of contrast agent (Dotarem ${ }^{\circledR}$, Guerbet). All patients were subjected to prone 18 F-FDG PET-CT scanning. CT data were used for attenuation correction. Breast PET-CT was assessed for 18 F-FDG-avidity of lesions. Tumours were classified as positive when $18 \mathrm{~F}$-FDG-uptake was greater than blood-pool activity. Lesions within tissues demonstrating moderate/high physiologic background activity were considered positive if the activity was greater than the adjacent physiologic activity. 3 T CE-MRI was assessed for lesion morphology and enhancement kinetics and classified according to BI-RADS ${ }^{\circledR}$. All lesions were histopathologically verified. Results: PET-CT had a sensitivity of $96 \%$, a specificity of $76 \%$ and diagnostic accuracy of $90 \%$. CE-MRI had a sensitivity of $100 \%$, a specificity of $80 \%$ and a diagnostic accuracy of $94 \%$. There were 46 benign and 113 malignant lesions (mean lesion size $26.03 \mathrm{~mm}$; range $5-90 \mathrm{~mm}$ ). The 5 false-negative lesions in PET-CT were small lesions (mean $11.2 \mathrm{~mm}$ ), had a very low SUV (mean SUVmax 1.59 ) or were adjacent to tissue with high physiologic activity or low grade IDCs. Conclusion: 3 T CE-MRI is superior to PET-CT for assessment of breast lesions, but dedicated breast PET-CT is a valid alternative in patients, who are unsuitable for MRI.

\section{B-0953 14:36}

High spatial and temporal resolution breast imaging at 7 Tesla goes clinical

K. Pinker-Domenig, P.A.T. Baltzer, T. Helbich, S. Gruber, W. Bogner,

O. Zaric, L. Minarikova, H. Moosbauer, S. Trattnig; Vienna/AT

(katja.pinker@meduniwien.ac.at)

Purpose: To validate a contrast-enhanced (CE) combined high spatial and temporal resolution imaging protocol for the assessment of breast tumours at 7 Tesla in clinical practice.
Methods and Materials: Twenty-five breast lesions classified suspicious in mammography or ultrasound (BIRADS 4/5) were included in this prospective IRB-approved study. All patients underwent 7T MRI (Siemens Magnetom, Erlangen, Germany) using a dedicated 4-channel breast coil and a high temporal and spatial resolution 3D T1-weighted sequence (TWIST, fat-sat, $0.7 \mathrm{~mm}$ isotropic, temporal resolution $14 \mathrm{sec}$, examination time $9 \mathrm{~min}$ ) with a single dose of contrast agent (Dotarem, Guerbet). One patient had to be excluded due to insufficient data quality. Two readers trained in different institutions independently assessed lesion morphology and enhancement kinetics and classified them according to BI-RADS. Lesion size, localisation and image quality were assessed. Sensitivity, specificity, diagnostic accuracy and interrater agreement (kappa statistics) were calculated for both observers. The histopathological diagnoses were used as the standard of reference.

Results: There were three benign and 21 malignant lesions. Mean histopathological size was $25 \mathrm{~mm}$ (range 8-51 mm). On the basis of lesion morphology and enhancement patterns, all lesions were correctly classified as benign $(n=3)$ and malignant $(n=21)$ by 7 T CE-MRI. Sensitivity, specificity and diagnostic accuracy were $100 \%$. Interrater agreement for BI-RADS ratings was excellent (0.928). Kappa agreement for single BI-RADS descriptors ranged between 0.709 and 1 .

Conclusion: High spatial and temporal resolution imaging in clinical practice is feasible using $7 \mathrm{~T}$ MRI, revealing excellent diagnostic accuracy and interrater agreement.

\section{B-0954 14:45}

Evaluation of $\mathrm{T} 1 / \mathrm{T} 2$ ratios in a pilot study as a potential biomarker of biopsy: proven benign and malignant breast lesions in correlation with histopathological disease stage

M.A. Malikova, J.N. Tkacz, P.J. Slanetz, A. Aakil, C.-Y. Guo, H. Jara; Boston, MA/US (mmalikov@bu.edu)

Purpose: To study a potential role of quantitative MRI (qMRI) using T1/T2 ratios to differentiate benign from malignant breast lesions, assess contralateral breast involvement and monitor response to treatment.

Methods and Materials: A cross sectional study of 41 women with 46 breast lesions were scanned with the mixed-TSE pulse sequence, which is multispectral in $\mathrm{T} 1$ and T2, therefore, affords maps of T1/T2. Patients were grouped according to histopathological stage of disease: untreated malignant tumor, treated malignancy and benign disease. qMRI assessment of the same quadrant of contralateral breast that did not have cancerous lesion was performed and compared to the breast that was subjected to chemotherapy and/or radiation therapy in order to monitor response to treatment.

Results: Elevated T1/T2 means of $6.44 \pm 1.05(\mathrm{~N}=7)$ were observed for biopsy proven malignant lesions and $5.98 \pm 0.63(\mathrm{~N}=21)$ for malignant lesions that were treated prior to $\mathrm{GMRI}$ with chemotherapy and/or radiation as compared to $3.88 \pm$ $0.43(\mathrm{~N}=18)$ for benign lesions. The higher stage of cancer determined by histopathology analysis was also strongly associated with higher $\mathrm{T} 1 / \mathrm{T} 2$ ratio $(\mathrm{p}=0.0093)$. Estrogen and progesterone receptors negative status was strongly correlated with higher $\mathrm{T} 1 / \mathrm{T} 2$ ratio $\mathrm{p}=0.060$ and $\mathrm{p}=0.0427$, respectively. Her2/neu receptor status showed no significant correlation with $\mathrm{T} 1 / \mathrm{T} 2$ ratio $(\mathrm{p}=0.1506)$.

Conclusion: The T1/T2 ratios provide measures that strongly correlate with histopathological findings. This quantitative information of tissue properties can provide basis for improving the specificity of diagnostic breast imaging and serve as a tool to assess response to treatment and contralateral breast involvement.

\section{B-0955 14:54}

Improved differentiation of breast tumours using novel imaging system based on co-registered opto-acoustic tomography and ultrasound P. . Otto ${ }^{1}$, K. Kist ${ }^{1}$, N.C. Dornbluth ${ }^{1}$, T. Stavros ${ }^{1}$, D. Herzog ${ }^{1}$, B. Clingman',

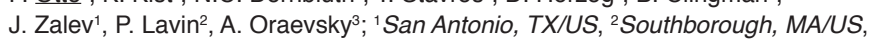
${ }^{3}$ Houston, TX/US (ottop@uthscsa.edu)

Purpose: A novel system called Imagio combines ultrasound and opto-acoustics $(\mathrm{OA})$ to more accurately distinguish malignant from benign tumours. We analysed readers' ability to assess probability of malignancy (POM) using OA versus conventional diagnostic ultrasound (DUS) alone.

Methods and Materials: 73 patients with breast masses were assessed with $\mathrm{OA}$ and DUS. All the masses were biopsied. Histology was the gold standard. OA employs near-infrared laser pulses at two different wavelengths (to provide contrast between oxygenated haemoglobin in benign lesions and de-oxygenated haemoglobin in malignant lesions) to illuminate tissues through a fibreoptic bundle incorporated into a prototype duplex hand-held ultrasound probe. It detects the laser-generated ultrasonic pressure waves that are then used for reconstruction of two-dimensional OA images. OA maps of total haemoglobin and blood oxygen 
provide functional information that is co-registered with the morphological information from B-mode grey scale ultrasound images.

Results: Five blinded readers independently assessed POM for OA and DUS. OA provided $>40 \%$ higher mean POM for all malignant lesions; a $>30 \%$ higher mean POM for malignant BIRADS 4B lesions; and $>10 \%$ higher mean POM for malignant BIRADS 5 lesions than did DUS. OA also could spare $23.7 \%$ more negative biopsies than DUS at the critical 2\% POM level.

Conclusion: The fused functional OA and grey scale anatomic information significantly improved distinction of benign from malignant breast masses compared with DUS alone, especially within the BIRADS $4 \mathrm{~b}$ category, where OA better distinguishes benign from malignant lesions and thereby prevents unnecessary biopsies.

Author Disclosures:

P. Otto: Consultant; Seno Medical Instruments.T. Stavros: Employee; Seno

Medical Instruments. D. Herzog: Employee; Seno Medical Instruments.

B. Clingman: Employee; Seno Medical Instruments. J. Zalev: Employee;

Seno Medical Instruments. P. Lavin: Consultant; Seno Medical Instruments.

A. Oraevsky: Board Member; Seno Medical Instruments. Consultant; Seno

Medical Instruments.

\section{B-0956 15:03}

Breast lesion excision system (BLES) - innovative breast biopsy technology: experience under sterotactic guidance in small microcalcification clusters

S. Vigano', G.P. Scaperrotta, C. Ferranti, L. Suman, M. Marchesini, E. Capalbo, P. Panizza; Milan/IT (sar.vigano@gmail.com)

Purpose: Stereotactic vacuum-assisted biopsy (VABB) is the first choice technique to characterise suspicious microcalcification clusters; however, considerable proportion of understaged invasive cancer (IDC) at subsequent surgical histology has been reported. BLES, thanks to a radiofrequency excisional system, remove a larger not fragmented histological sample (25×20 mm, weight $3 \mathrm{~g})$. Our aim was to prospectively assess BLES diagnostic and eventually therapeutic efficiency in a selected cohort of patients with suspicious microcalcification cluster.

Methods and Materials: From September 2010, patients with small microcalcification cluster, $<1 \mathrm{~cm}$, staged $4 \mathrm{a}-4 \mathrm{~b}$ according to BI-RADS classification system, were proposed for excisional biopsy with BLES. Written informed consent was obtained. Results: Up to now, 80 patients underwent BLES at our centre; 32 of them $(40 \%)$ had malignant lesion, 7 were IDC and 25 (78\%) in-situ (DCIS) carcinoma. Rationale of the study was to completely remove microcalcification clusters, obtaining enough surrounding tissue for histological margins' evaluation. Complete histological concordance was obtained in $100 \%$ of atypical hyperplasia. All patients with DCIS underwent surgical excision: upgrading to IDC resulted in only $3 / 25(12 \%$ underestimation proportion); among DCIS cases, surgical excision demonstrated complete removal of the lesion by BLES in $18 / 25$ patients (72\%).

Conclusion: In our experience, BLES is a valid, simple and safe alternative to VABB, providing better diagnostic performance evaluated as histologic accuracy with lower underestimation rate compared to previously reported for VABB. In selected cases, particularly in small microcalcification clusters, BLES may represent an interesting new therapeutic strategy considering the high rate of complete lesion removal.

\section{B-0957 15:12}

Percutaneous breast lesion excision system (BLES): a new tool for complete closed excision of high risk lesions

N.M. Abdel Razek; Cairo/EG (naglaabdelrazek@yahoo.com)

Purpose: The objective of the study is to assess the use of "Intact" Breast lesion excision system as the primary method for histopathology diagnosis in suspicious small and borderline lesions as well as the unclassified microcalcifications.

Methods and Materials: 58 cases were included (Feb-August 2012). Inclusion criteria: small lesion less than $20 \mathrm{~mm}$, unclassified microcalcifications, asymmetric densities, focal distortion.

Results: 52 cases were successfully done, $88.5 \%$ (46/52) cases were done under ultrasound guidance and $11.5 \%(6 / 52)$ cases were done under stereotactic guidance. $38.5 \%$ (20/52) lesions were removed using the $15 \mathrm{~mm}$ probe and $61.5 \%$ (32/52) lesions were removed using the $20 \mathrm{~mm}$ probe size. The main indication was complete removal of a small mass (20/52) followed by biopsy of unclassified microcalcifications, either without mass $(8 / 52)$ or associated with a mass $(8 / 52)$. Asymmetry and distortion were the indications in (6/52) and finally clustered micro cysts (8/52). As regards the histopathology after total excision; $90.4 \%(47 / 52)$ of lesions were benign and only $9.6 \%$ (5/52) of lesions were malignant. Fibroadenoma was the commonly encountered benign lesion in the study group, it was encountered in $38.5 \%$ (20/52), followed by papilloma found in $19.3 \%$ (10/52), followed by sclerosing adenosis in $13.5 \%$ (7/52), followed by fibrocystic changes in $11.5 \%$ (6/52) and ADH in 7.6\% (4/52). DCIS was encountered in 5.8\% (3/52) and IDC occurred in only $3.8 \%$ (2/52). Re-surgery for more margin was done in 5 cases in spite of $2 \mathrm{~mm}$ margin free after BLES.

Conclusion: BLES is not for cancer therapy but it can be an adequate method for diagnosis of problematic cases especially the unclassified microcalcifications. BLESS is an available option for the management of small NPM. BLES offers complete lesion removal thus much indicated in borderline lesions.

\section{B-0958 15:21}

Cryoablation as local therapy for patients with breast cancer bone metastases (BCBM)

C. Pusceddu' ${ }^{1}$, S. Pilleri², G. Amucano' ${ }^{1}$, L. Melis ${ }^{1}$, G. Meloni2 ${ }^{2}{ }^{1}$ Cagliari/lT,

2Sassari/lT (clapusceddu@gmail.com)

Purpose: The aim was to evaluate the safety and efficacy of CRA as local therapy for breast carcinomas with bone metastases.

Methods and Materials: Sixteen CRA breast lesions, mean size 2.4 (range 0.8$6.7 \mathrm{~cm}$ ) in thirteen consecutive patients, mean age 52 (36-81) with core-needle biopsy-proven breast carcinoma and bone metastases were enrolled in this study. 10 patients had one lesion and three patients had 2 lesions. Under local anaesthesia and mild conscious sedation, the tumour and surrounding breast tissue were ablated with percutaneous CT-guided CRA. Cryoablation consisted of 2 cycles each of 10 minutes of freezing followed by a 4-min active thawing phase and a 4-min passive thawing phase for each one. Ten patients underwent one CRA session and two patients 2 CRA sessions. One patient is treated with two sessions for the same lesion.

Results: All CRA sessions were successfully completed and all breast tumours were ablated. Morbidity consists in transient and mild ecchymotic changes and post-procedural oedema seen in two cases and alteration in skin pigmentation seen in the point of insertion of the cryoprobes in other two cases. The therapeutic outcomes were evaluated by contrast-enhanced TC or MRI at 1-, 3- and 6-month interval. The absence of tumour enhancement TC or MR image was considered to indicate complete tumour necrosis.

Conclusion: During the mean follow-up of 11 months (3-24 months) none of the patients had shown local relapses. A patient died for liver tumour progression after 16 months.

$14: 00-15: 30$ Room F2

Breast

\section{SS $1802 b$}

\section{Maximising cancer detection in breast screening}

Moderators:

M. Álvarez-Benito; Córdoba/ES

E. Szabó; Szeged/HU

\section{B-0959 14:00}

Breast cancer prediction modelling based on common mammographic findings in screening

J. Timmers ${ }^{1}$, A.L.M. Verbeek' ${ }^{1}$, R.M. Pijnappel ${ }^{2}$, J. in 't Hout ${ }^{1}$, M.J.M. Broeders ${ }^{1}$,

G.J. den Heeten ${ }^{3} ;{ }^{1}$ Nijmegen/NL, ${ }^{2}$ Utrecht/NL, ${ }^{3}$ Amsterdam/NL

(j.timmers@Ircb.nl)

Purpose: To develop a prediction model for breast cancer (nomogram) based on common mammographic findings on screening mammograms. The model is designed to reduce interobserver variation in assigning BI-RADS in the Dutch breast cancer screening programme.

Methods and Materials: We retrospectively reviewed 352 positive (digital) screening mammograms of women participating in the Nijmegen region of the Dutch screening programme (December 2006-November 2008). The following mammographic findings were assessed by consensus reading of 3 expert radiologists: masses and features of masses, calcifications, parenchymal deformity, asymmetric density and mammographic density and BI-RADS. Data on age, diagnostic workup, final diagnosis and surgical procedures were collected from patient records. Multivariable logistic regression analyses were used to build our breast cancer prediction model, presented as a nomogram.

Results: Breast cancer was diagnosed in 108 cases (31\%). The highest positive predictive value (PPV) was reported for spiculated masses $(96 \%)$ and the lowest for well-defined masses $(9 \%)$. Characteristics included in the nomogram based 
on statistical significance and clinical relevance are: age, mass, calcifications, parenchymal deformity and asymmetric density.

Conclusion: With our nomogram we developed a tool to assist screening radiologists in determining the chance of malignancy based on mammographic findings. We propose cut-off values for assigning BI-RADS categories in the Dutch screening setting based on our nomogram which will need to be validated in future research. These values can easily be adapted for use in other screening programmes.

\section{B-0960 14:09}

\section{Non-blinded versus blinded double reading of screening}

mammograms in the Netherlands: a population-based study

L.E.M. Duijm ${ }^{1}$, G.J. den Heeten², J. Nederend', M.J.M. Broeders²;

${ }^{1}$ Eindhoven/NL, ${ }^{2}$ Nijmegen/NL (LEMDuijm@hotmail.com)

Purpose: To determine screening mammography outcomes at non-blinded versus blinded double reading

Methods and Materials: We included a consecutive series of 87,487 digita screening mammograms, obtained at 3 screening units in the Netherlands between July 1, 2009 and July 1, 2011. A total of 12 certified screening radiologists participated. Every other month, screening mammograms were double read, either in a non-blinded fashion $\left(2^{\text {nd }}\right.$ reader was informed about the $1^{\text {st }}$ reader's decision) or in a blinded fashion ( $2^{\text {nd }}$ reader was not informed about the $1^{\text {st }}$ reader's decision). Women with discrepant readings were always referred for further evaluation. During 1-year follow-up, we collected the radiology reports, surgical correspondence and pathology reports of all referred women.

Results: 42,996 and 44,491 screens had been read either in a non-blinded or blinded fashion, respectively. Referral rate was significantly higher at blinded double reading $(3.3 \%$ versus $2.8 \%, p<0.001)$, whereas the cancer detection rate $(7.2$ versus $6.5, p=0.24)$ and positive predictive value (PPV) of referral $(21.5 \%$ versus $23.0 \%, p=0.36$ ) were comparable. The 2 reading strategies showed no differences in the proportion of ductal cancers in situ among screen detected cancers $(23.1 \%$ versus $25.6 \%, p=0.46)$, proportion of smaller tumours ( $(1 \mathrm{a}-\mathrm{c})$ among invasive cancers $(81.1 \%$ versus $80.0 \%, p=0.76)$ or axillary lymph node metastasis in invasive cancers $(17.6 \%$ versus $22.0 \%, p=0.45)$.

Conclusion: Cancer detection rates were similar for both reading strategies, whereas blinded double reading resulted in a significantly higher referral rate. PPV of referral and tumour stage of screen detected cancers were comparable for both reading strategies.

\section{B-0961 14:18}

\section{Discrepant screening mammography assessments at double} reading: impact of arbitration by a third reader on screening outcome

L.E.M. Duijm ${ }^{1}$, G.J. den Heeten², J. Nederend', M.J.M. Broeders²;

'Eindhoven/NL, ${ }^{2}$ Nijmegen/NL (LEMDuijm@hotmail.com)

Purpose: To determine the value of adding a third reader for arbitration of discrepant screening mammography assessments at double reading.

Methods and Materials: We included 84,927 consecutive digital screening mammograms obtained at a Dutch screening mammography programme. The mammograms were double read in a non-blinded $(41,397$ screens) or blinded $(43,530$ screens) fashion by a group of 12 screening radiologists and discrepant readings were always referred for further evaluation. Each discrepant reading was then randomly assigned to a third screening radiologist, who retrospectively decided whether he would have referred the woman. This third reader was blinded to screening outcome. During 1 year follow-up, radiology reports, surgical correspondence and pathology reports of each referred woman were collected.

Results: Discrepant readings comprised $28.9 \%(340 / 1,175)$ and $56.9 \%(834 / 1,465)$ of referrals at non-blinded and blinded double reading, respectively $(p<0.001)$. At non-blinded double reading, arbitration would have decreased the referral rate (from $2.8 \%$ to $2.3 \%, p<0.001$ ) and cancer detection rate (CDR, from 6.6 to 6.3 per 1,000 screens, $\mathrm{p}=0.9$ ) and increased the positive predictive value (PPV) of referral (from $23.1 \%$ to $27.5 \%, p=0.03$ ). At blinded double reading, arbitration would also have resulted in a decreased referral rate (from $3.4 \%$ to $2.2 \%, p<0.001$ ) and CDR (from 7.3 to $6.7, p=0.5$ ), and an increased PPV (from $21.7 \%$ to $30.7 \%, p<0.001$ ). Conclusion: Both at non-blinded and blinded double reading, arbitration would have resulted in a significantly lower referral rate and significantly higher positive predictive value, at the expense of a non-significantly reduced CDR.

\section{B-0962 14:27}

First experiences with a self-test for Dutch breast screening radiologists as a quality assurance tool

J. Timmers ${ }^{1}$, A. Verbeek ${ }^{1}$, R. Pijnappel ${ }^{2}$, M. Broeders ${ }^{1}$, G. den Heeten ${ }^{3}$;

${ }^{1}$ Nijmegen/NL, ${ }^{2}$ Utrecht/NL, ${ }^{3}$ Amsterdam/NL (j.timmers@lrcb.nl)

Purpose: To evaluate the use of a self-test as a quality assurance tool for screening radiologists in the Dutch breast cancer screening programme.

Methods and Materials: 144 screening radiologists were invited to voluntarily complete a test set of 60 screening mammograms. The following grading criteria were assigned regarding the most suspicious lesion: location, level of suspicion, BI-RADS, laterality, type (well defined mass, ill defined mass, spiculated mass, microcalcification clusters, architectural distortion and asymmetric density) and mammographic density are assigned. Also, several reader characteristics, such as years of experience and number of cases read per year, were to be completed. Case and lesion sensitivity and specificity were determined for all readers. The spearman correlation coefficient was used to determine correlation between reader characteristics and performance measured by the area under the receiver operator characteristics (ROC) curve (AUC).

Results: 112 radiologists completed the test set $(78 \%)$. The mean age was 49 (range 33-68) and on radiologists read on average 10,000 (range 700-60,000) screening mammograms per year. The median AUC value was 0.91 , case sensitivity $91 \%$, lesion sensitivity was $91 \%$ and specificity $94 \%$. The AUC was not correlated to reader characteristics. The test-set revealed interobserver variation in assigning lesion types.

Conclusion: Overall, a good performance was seen among all screening radiologists. Readers are able to determine their educational needs and compare it with peers during training or audits. It is therefore a useful quality assurance tool. Medical education should be dedicated to reducing interobserver variation.

\section{B-0963 14:36}

Transition from analogue to digital screening mammography significantly increases the proportion of women referred twice for the same lesion

L.E.M. Duijm, H. Wiersma; Eindhoven/NL (LEMDuijm@hotmail.com)

Purpose: To determine the effect of transition from analogue to digital screening on the proportion of women referred twice for the same lesion and to assess screening outcome.

Methods and Materials: We included 302,912 consecutive analogue screens (obtained between January 2000 and April 2010) and 90,288 consecutive digital screens (obtained between July 2009 and July 2011). Review of the screening mammograms of women who had been referred twice was performed to determine whether their initial and second referral comprised the same lesion. During 1-year follow-up, radiology reports, surgical correspondence and pathology reports of each referred woman were collected.

Results: The overall positive predictive value (PPV) of referral was 37.3\% at analogue screening and $22.0 \%$ at digital screening $(P<0.001)$. Among women who had only been assessed by analogue screens, 37 ( $0.9 \%$ of referrals) had been referred twice for the same lesion. These referrals included 13 malignancies (PPV 32.4\%). Among the 90,288 women who received their first digital screen following one or several previous analogue screens, $52(1.9 \%$ of digital referrals, $p<0.001)$ were referred again for a lesion for which she had been referred previously at analogue screening. These second referrals included 8 malignancies (PPV 15.4\%, $p=0.03$ ). Conclusion: Implementation of digital screening significantly increased the proportion of women referred twice for the same lesion. Their PPV was significantly lower than the one found in women who had been referred repeatedly for the same lesion during analogue screening.

\section{B-0964 14:45}

Audits as part of quality assurance in the Dutch breast cancer screening programme

T.D. Geertse, R. Holland, C.G.C.M. van Landsveld-Verhoeven, J.M.H. Timmers, K.H. Schuur, R. Pijnappel, F. Jansen, M.J.M. Broeders, G.J. den Heeten; Nijmegen/NL (t.geertse@Ircb.nl)

Purpose: To determine the impact of audits as a quality assurance tool to improve breast cancer screening performance.

Methods and Materials: The Dutch centrally organised breast cancer screening programme offers biennial mammography to women aged 50-75 years. A team of the National Expert and Training Centre for Breast Cancer Screening with peer radiologists conducts audits of all 16 reading units every 3 years as part of the quality assurance programme. Data on screening outcomes are assessed and a 
radiological review is performed (40 interval cancers, 40 screen detected stage-II cancers and 40 consecutive recalled cases). The audit is completed with a report, summarising the results and giving recommendations. This study compares four audit series (1996-2000/2001-2005/2003-2007/2010-2011).

Results: Recall rates (subsequent screens) increased from 6, 10, 12 to 14 per 1000 , respectively, in the four series. Detection rates also increased from 3.3, 4.3, 4.7 to 5.7 per 1000 . Distribution of tumour size and lymph-node status of invasive tumours remained stable $(p=0.4)$. The percentage interval and screen-detected stage II cancers classified as "missed" during the review did not change $(22 \%$ to $25 \%, p=0.1$ ). Review of consecutive recalled cases showed an increasing number of cases the audit team would not have recalled with a higher recall rate of the screening radiologists.

Conclusion: We found audits are helpful in controlling the balance between (false positive) recalls and detected breast cancers. It also serves as a learning and feedback tool as it triggers discussion between screening radiologists. Overall, it can be seen as an important quality assurance tool.

\section{B-0965 14:54}

Avoidable surgical consultations in women with a positive screening mammogram: experience from a southern region of the Dutch breast screening programme

I.L. Schreutelkamp, R.M. Kwee, M. de Booij, M.E.A.P. Adriaensen van Roij; Heerlen/NL (miraude@gmail.com)

Purpose: According to current Dutch guidelines, all women following a positive screening mammogram are referred for a full hospital assessment including surgical outpatient clinic and radiology department. Till 2007, all women with a positive screening mammogram in our screening region were only referred for further assessment to our radiology department. Purpose of this study was to determine how often surgical consultation in women with a positive screening mammogram could be avoided by a radiological pre-assessment.

Methods and Materials: All women with a positive screening mammogram, $\mathrm{n}=1014$, referred to our radiology department from 2002 to 2007 were included. Data were prospectively collected by a senior breast radiologist. In-hospital follow-up data were available till September 2012. Descriptive statistics were used. Percentage of patients only assessed by a radiologist was determined. Negative predictive value for malignancy was calculated from the in-hospital follow-up.

Results: 427 of 1014 women (42\%) were only assessed at the radiology department without further surgical consultation. During follow-up, 8 of these 427 women ( $2 \%$ ) developed a malignancy in the same breast. At least 6 of these malignancies were located at a different location than the original screening findings which led to the initial referral. The estimated negative predictive value for malignancy was $99.5 \%$. Conclusion: By referring women with a positive screening mammogram to the radiology department for a pre-assessment, a surgical consultation was avoided in $42 \%$, with an estimated negative predictive value for malignancy of $99.5 \%$.

\section{B-0966 15:03}

BI-RADS 3 category, a pain in the neck for the radiologist: which technique detects more cases?

J. Etxano, I. Simon-Yarza, G. Viteri, M. Paramo, A. Elizalde, L.J. Pina Insausti; Pamplona/ES (ljpina@unav.es)

Purpose: This study aimed at evaluating the rate of BI-RADS 3 lesions detected by digital mammography, ultrasonography and tomosynthesis.

Methods and Materials: From October 2011 to August 2012, 2256 patients underwent digital mamography, US and tomosynthesis and all of them showing ACR density patterns $2,3 \& 4$. For each imaging modality, all the patients were classified according to the BI-RADS categories 0-6. We compared the rate of BI-RADS 3 lesions detected on each technique using a Pearson Chi-square test (SPSS 15.0) Results: Mammography detected 227 BI-RADS 3 lesions (10.06\%), US detected 471 BI-RADS 3 lesions (20.88\%) and tomosynthesis detected 270 BI-RADS lesions $(11.97 \%)$. There were statistically significant differences between mamography and US ( $p<0.001)$, US vs tomosynthesis $(p<0.001)$ and mammography vs tomosynthesis $(p=0.04)$. Four cancers were diagnosed: one of them was a BI-RADS 3 lesion in all imaging modalities and the 3 remaining cases were BI-RADS 3 on mammography but BI-RADS 4 or 5 on US or tomosynthesis.

Conclusion: Our results indicate that both US and tomosynthesis detect more additional BI-RADS 3 lesions than mammography alone. US detects twice as many BI-RADS 3 as mammography. Tomosynthesis detects more BI-RADS 3 lesions than mammography; however, the ratio is smaller ( $11.97 \%$ vs $10.06 \% ; p=0.04)$.

\section{B-0967 15:12}

Adding 3D automated breast ultrasound (ABUS) to service screening mammography in dense breasts

B. Wilczek, K. Hågemo, B. Adalsteisson, M. Janicijevic, K. Thorneman,

C. Hinzer, K. Leifland; Stockholm/SE (brigitte.wilczek@unilabs.com)

Purpose: Describe the impact on recall rates by adding ABUS service. Methods and Materials: From November 2010 to February 2012, 1676 asymptomatic women with more than $50 \%$ density at visual mammographic assessment were enrolled. Bilateral ABUS (somo.vuß U-Systems, Inc. Sunnyvale, CA, USA) acquisitions were obtained and reviewed in combination with double-read twoview full-field digital mammography (FFDM). The bilateral ABUS examinations were performed by a radiographer. The image assessment was performed by a radiologist who was the first FFDM screening reader. All ABUS examinations were double read by the second reader if the FFDM findings from either reader, or ABUS findings from the first reader, led to discussion among the two readers. Results: $10 \%$ led to discussion among the two readers. In 8 cases of discussion caused by FFDM images, ABUS let to avoid recall (0.5\%). $2.3 \%$ who underwent FFDM and ABUS, were recalled compared with $3.0 \%$ of women in the year 2010 who had only FFDM in the service screening program. $0.9 \%$ were recalled because of FFDM findings, where ABUS was either normal or abnormal. $1.4 \%$ were recalled for ABUS findings with normal FFDM. Breast -cancer detection was $0.7 \%$. Conclusion: Adding ABUS to service screening mammography with FFDM did not negatively affect the recall rate in asymptomatic women with dense breast tissue and even improved the cancer detection.

\section{Author Disclosures:}

B. Wilczek: Equipment Support Recipient; Unrestricted use of the equipment from U-systems. K. Hågemo: Equipment Support Recipient; Unrestricted use of the equipment from U-systems. B. Adalsteisson: Equipment Support Recipient; Unrestricted use of the equipment from U-systems. M. Janicijevic: Equipment Support Recipient; Unrestricted use of the equipment from

U-systems. K. Thorneman: Equipment Support Recipient; Unrestricted use of the equipment from U-systems. C. Hinzer: Equipment Support Recipient; Unrestricted use of the equipment from U-systems. K. Leifland: Equipment Support Recipient; Unrestricted use of the equipment from U-systems.

\section{B-0968 15:21}

Feasibility of automated 3D breast ultrasound scanning in screening of women with high risk

J.C.M. van Zelst, R.D.M. Mus, T. Tan, N. Karssemeijer, R.M. Mann; Nijmegen/NL (janvanzelst@me.com)

Purpose: Automated 3D-breast ultrasound (ABVS) is investigated as a six-monthly addition to annual breast cancer screening with MRI+mammography (MM) in highrisk women ( $L T R>50 \%$ ). ABVS, an inexpensive radiation-free technique, allows more frequent screening and temporal comparison. This study assesses effects of additional ABVS examinations at baseline.

Methods and Materials: The study population consists of 234 women in whom ABVS and MM were performed on the same day. All ABVS and MM examinations were read by one of 4 breast radiologist. The recall rate (RR), biopsy rate (BR), cancer detection rate (CDR), sensitivity and specificity of ABVS and MM screening were analysed.

Results: Based upon MM, 28 patients were recalled for further examination (RR=12\%). With ABVS 12 of these patients were also recalled, as well as 17 other women. Consequently, the RR increased to 45/234. Biopsies were deemed necessary in 21 patients after MM and increased to 26 with ABVS added, an increase from $9 \%-11 \%$. 17 additional ABVS findings were resolved with targeted ultrasound. In total 4 cancers were found by MM (CDR $1.7 \%$, sensitivity $100 \%$, specificity $89 \%$ ). Two of these cancers were also detected by ABVS (CDR $0.9 \%$, sensitivity $50 \%$ specificity $88 \%$ ). The two missed cancers were retrospectively visible, but misinterpreted due to post-operative scarring.

Conclusion: Adding ABVS to high-risk MM screening increased RR and BR at baseline. Whether these negative effects are reduced when radiologists gain more experience and whether they are balanced by earlier detection of breast cancer due to the six-month interval of ABVS remain to be determined. 
14:00 - 15:30

Room G/H

\section{Genitourinary}

\section{SS 1807}

\section{Mixed modalities in GU imaging}

Moderators:

A.J. Beer; Munich/DE

I. Vivas; Pamplona/ES

\section{B-0969 14:00}

Simultaneous [18 F] choline PET/MRI of the prostate: initial results

A. Wetter, C. Lipponer, F. Nensa, T. Olbricht, H. Rübben, A. Bockisch,

T. Lauenstein; Essen/DE (Christine.Lipponer@uk-essen.de)

Purpose: To to present first results of simultaneous [18 F] choline PET/MR imaging of the prostate.

Methods and Materials: 13 consecutive patients were examined with simultaneous [18 F] choline PET/MRI. At the time of analysis, eight patients had histologically proven prostate cancer, 3 patients had negative prostate biopsies and two patients had suspected prostate cancer in the presence of an elevated or rising PSA level but did not have a prostate biopsy so far. Sequence protocol consisted of T2-weighted high-resolution images and diffusion-weighted images of the prostate in addition to PET imaging. Image quality was assessed by two radiologists and PET images were evaluated qualitatively and quantitatively.

Results: Simultaneous [18 F] PET/MRI of the prostate proved to be robust without technical failure in all patients. Image quality was diagnostic in all examinations. High-resolution T2-weighted images allowed exact correlation of elevated focal or diffuse choline uptake of the prostate. Whereas all prostate carcinomas were PET-positive, diffusion-weighted imaging was inconspicuous in three out of eight prostate cancers.

Conclusion: Simultaneous [18 F] PET/MRI of the prostate combines high-resolution prostate images with metabolic/molecular imaging and is feasible for routine use. The PET component is superior over DWI in identifying and localising tumor in the prostate.

\section{B-0970 14:09}

Advantage of hybrid whole body 3 T PET-MRI scanner for prostate cancer imaging

T. de Perrot, O. Rager, M. Pusztaszeri, L. Koehl, A. Figueiral, M.-F. Pelte,

C. Iselin, O. Ratib, J.-P. Vallee; Geneva/CH

Purpose: To define the benefit of 18 F-fluorocholine PET-MRI combined in a single session with pelvic MRI on a 3 Tesla PET-MRI scanner in prostate cancer patients before radical prostatectomy.

Methods and Materials: Twenty-six patients scheduled for a prostate PET-MRI before radical prostatectomy were retrospectively analysed. The examinations were performed on a Philips 3 Tesla PET-MRI with prostate MR protocol included $\mathrm{T} 2$, diffusion $(A D C)$ and perfusion imaging followed by a PET acquisition. Using histology as reference, logistic regression was performed to compute ROC curves with and without PET.

Results: A complete protocol was obtained in 23 patients, three exclusions due to prosthesis artefacts or no endorectal coil. Prostate cancer was found in $53 \mathrm{sec}-$ tors over 184 . With only 12 positive sectors in the transitional zone, analysis was focused on the peripheral zone. Following the registration of PET and T2, SUV was increased in the peripheral sectors with prostate cancer by comparison with normal sectors $(4.26 \pm 2.03$ versus $2.15 \pm 0.66 ; p<0.001)$. After stepwise parameters selection in logistic regression, best MR parameter to combine with the PET was ADC. An improved area under the curve was observed by the PET adjunction (AUCpetmr $=0.893$ versus $A U C m r=0.834 ; p=0.027$ ). From the $R O C$ analysis, the best cutoff was for the ADC $1.190^{*} 10^{-3} \mathrm{~mm}^{2} / \mathrm{sec}$ and for the SUVmax 2.882 .

Conclusion: $18 \mathrm{~F}$-fluorocholine hybrid PET-MRI of the prostate was feasible in a single session with state-of-the-art MR and PET examinations. PET registration with T2-weighted MRI allowed confident SUVmax measurements. Combined with the $A D C$ images, the PET acquisition led to a better diagnostic accuracy than MRI alone.
B-0971 14:18

Renal cell carcinoma subtype differentiation: CT vs MRI

F. Veloso Gomes ${ }^{1}$, A. Matos ${ }^{2}$, J. Palas ${ }^{2}$, V. Herédia ${ }^{3}$, V. Mascarenhas ${ }^{4}$,

M. Ramalho ${ }^{2} ;{ }^{1}$ Faro/PT, ${ }^{2}$ Almada/PT, ${ }^{3}$ Évora/PT, ${ }^{4}$ Lisbon/PT

(fvgomes@gmail.com)

Purpose: To compare the diagnostic accuracy of both multiphasic contrastenhanced computed tomography (CT) and magnetic resonance imaging (MRI) in differentiating the three most common renal cell cancer (RCC) subtypes.

Methods and Materials: A multicentric, retrospective study of all surgically removed RCCs was performed. 114 renal tumours were identified ( 53 clear cell, 13 papillary and 14 chromophobe at CT and 25 clear cell, 16 papillary and 5 chromophobe at MRI). Quantitative image analysis was performed by placing region-of-interest over the tumours and uninvolved renal cortex on unenhanced and arterial phases. Tumour percentage enhancement (TE) and tumour/cortical index (TCI) were calculated. One-way ANOVA for independent samples was used to test for mean differences between the three RCC subtypes. Receiving operating characteristic (ROC) curve analysis was performed and independent ROC curves were compared. Results: Papillary tumours showed significantly lower mean TE than clear cell tumours $(p<0.01)$ at both, CT and MRI. Similarly, the TCI was significantly lower for papillary tumours $(p<0.01)$ whilst no difference was found between cromophobe and either clear cell or papillary types at multiphase contrast-enhanced-MRI. Papillary tumours can be diagnosed with thresholds of $\mathrm{TE}<53 \%$ and $\mathrm{TCK}<0.22$ (area-under-curve of 0.997 and 0.954 ) for $\mathrm{CT}$ and thresholds of $\mathrm{TE}<55.5 \%$ and $\mathrm{TCK}<0.33$ (AUC/ROC curves of 0.885 and 0.917 ).

ROC curves comparison revealed no significant differences.

Conclusion: Similar contrast-enhancing behavior was observed in CT and MRI between RCC subtypes. CT showed higher sensitivity and specificity to differentiate papillary from clear cell tumours for both indices, but ROC curve comparison failed to demonstrate significant differences.

\section{B-0972 14:27}

Characterisation of complex cystic renal masses according to the Bosniak criteria: results after a 5-year follow-up with CEUS M. Bertolotto, V. Savoca, W. Toscano, S. Kus, C. Cercato, M.A. Cova; Trieste/IT (bertolot@units.it)

Purpose: To investigate the effectiveness of CEUS in the follow-up of complex cystic renal lesions. Category IIF cysts are presumably benign, but require follow-up as long as 5 years to prove benignity. If performed with $\mathrm{CT}$, it requires significant radiation. Although they are considered surgical lesions, also category III cysts may be followed-up in poor surgical candidates. CEUS proved effective in characterisation of complex renal cysts. No studies, however, reported data after a 5-year follow-up. Methods and Materials: Images and clips of complex cystic renal masses investigated with CEUS before August 2007 and followed-up with the same technique were retrospectively evaluated. There were 41 patients with category IIF cysts and 12 patients with category III cysts who were not operated because of patient refusal $(n=2)$ or severe comorbidities. Fourteen patients (12 category IIF, 2 category III) were ruled-out because of follow-up $<5$ years. Five category III and 2 category IIF lesions were operated after 2-3 years of follow-up. Eventually, 32 complex renal cysts (27 category IIF, 5 category III) had 5-year follow-up.

Results: 10/27 category IIF cysts increased in size, but not in complexity during the follow-up. Reduction in size was observed in $3 / 27$ category IIF cysts, while the other remained stable. Four/5 category III cysts increased in complexity (category IV). One of these patients developed metastases. One category III cyst reduced in complexity within 3 months (IIF) and then remained stable.

Conclusion: Complex cystic renal massed can be effectively followed-up with CEUS without radiation.

\section{B-0973 14:36}

Differentiation between benign Leydig cell and malignant germ cell testicular tumours with qualitative and quantitative contrastenhanced ultrasound assessments

D.Y. Huang, R.J. Eckersly, M.E. Sellars, P.S. Sidhu; London/UK (dean.huang@nhs.net)

Purpose: To evaluate the feasibility of applying qualitative and quantitative assessments with contrast-enhanced ultrasound (CEUS) in differentiating benign Leydig cell and malignant germ cell testicular tumours.

Methods and Materials: CEUS examination of 22 patients (median age 40 years, range, 21-63 years) with 22 testicular lesions (11 Leydig cell tumours: median size $5 \mathrm{~mm}$, range $3-16 \mathrm{~mm}$; 11 germ cell tumours: median size $15 \mathrm{~mm}$; range $3-38 \mathrm{~mm}$ ) was evaluated. Histopathologic confirmation was obtained following orchidectomy 
or excision biopsy. Qualitative analysis of cine loops was assessed for each CEUS examination by two observers and consensus reached on the pattern and timing of tumour enhancement. Perfusion quantification of the lesions was performed where possible, with time-intensity curves were analysed.

Results: Distinct differentiation on enhancement patterns was observed between Leydig cell and germ cell tumours. All 11 Leydig cell tumours displayed intense early enhancement which persisted for longer than the normal testicular parenchymal enhancement. The germ cell tumours showed loss of the normal linear vascular pattern, but with a more rapid washout of contrast. Quantitative analysis confirms the observation, with longer full width at maximum (FWHM) and slower contrast outflow on the time-intensity curves observed in Leydig cell tumours comparing to germ cell tumours. With improved diagnostic confidence, testicular-sparing excisions were performed for 5 lesions following pre-operative CEUS analysis and unnecessary orchidectomies avoided.

Conclusion: We describe, to date the first, consistent differentiating characteristic CEUS enhancement pattern in rare benign Leydig cell tumours. This observation increases diagnostic confidence, thus allows for testicular-preserving options to be considered.

\section{B-0974 14:45}

US and colour-Doppler findings in testicular lymphoma and their mimics

M. Bertolotto ${ }^{1}$, L.E. Derchi ${ }^{2}$, M. Secil ${ }^{3}$, M. Valentino ${ }^{4}$, P. Pavlica ${ }^{5}$, M.A. Cova';

${ }^{1}$ Trieste/IT, ${ }^{2}$ Genoa/IT, ${ }^{3}$ Izmir/TR, ${ }^{4}$ Tolmezzo/IT, ${ }^{5}$ Bologna/IT (bertolot@units.it)

Purpose: To describe US and colour-Doppler findings in testicular lymphoma and their mimics.

Methods and Materials: We reviewed the US and colour-Doppler findings in 10 pts with pathology-proven lymphoma of the testis and compared them with those in 7 patients in whom lymphoproliferative disease was suspected on clinical and US grounds and pathology showed only inflammatory changes.

Results: Lymphoma patients' age range was 34-76 years. Six had testicular involvement in systemic disease or recurrence; four had primary disease. One had bilateral involvement. Seven testes in 6 patients were diffusely involved (4 homogeneously; 3 heterogeneously); four had nodular hypoechoic lesions which, in 1 testis, were multiple. All lesions, either focal or diffuse, were hypervascular, with vessels of normal rectilinear shape. In patients with nodular lesions, there was no distorsion of vascular course at the point where vessels entered the mass. Findings suggested an infiltrative process, and were confirmed at pathology. The remaining seven patients had history and US features suggesting testicular lymphoproliferative disease. All had hypoechoic focal hypervascular lesions containing vessels with rectilinear course that were eventually proved inflammatory changes ( 2 chronic focal orchitis, 2 idiopathic granulomatous orchitis, 2 brucellosis, 1 tuberculosis). Conclusion: In patients over 60 and/or with known lymphoproliferative disease, the presence of hypoechoic, hypervascular testicular lesion with absence of vascular distorsion must suggest lymphoma. Care must be taken since, albeit rarely, inflammatory lesions may present the same imaging pattern in the absence of clinical signs and symptoms of inflammation.

\section{B-0975 14:54}

Perfusion and permeability DCE-MRI measurements in renal cell carcinoma and metastases: effect of ROI size and positioning on inter- and intraobserver variability

M. Braunagel, E. Radler, M. Ingrisch, M. Staehler, A. Crispin, K. Nikolaou, M.F. Reiser, M. Notohamiprodjo; Munich/DE (margarita.braunagel@med.Imu.de)

Purpose: To assess the influence of region of interest (ROI) size and positioning on parameters of perfusion and permeability and inter- and intraobserver variability of DCE-MRI of renal cell carcinoma (RCC) and metastases.

Methods and Materials: 33 patients with RCC and 17 patients with metastases were examined with dynamic contrast-enhanced (DCE) MRI at $1.5 \mathrm{~T}$ with a half body weight-adapted dose of gadobutrol. Analysis with a two-compartment exchange model provided four parameters: plasma flow (Fp), plasma volume (Vp), permeability-surface-product (Ps) and interstitial volume (VEE). Arterial input function and retrospective respiratory triggering were applied. ROls of the whole tumour, circular edge and an arbitrary vital region were defined on morphological sequences and on parametric plasma flow maps.

Results: Paired t-tests showed no significant differences between the results of both readers. Intraclass correlation coefficient (ICC) for interobserver correlation for whole tumour ROI were excellent for Fp (morphology: 0.97/parametric maps: $0.95), \mathrm{Vp}(0.95 / 0.89)$ but moderate for VEE $(0.6 / 0.6)$ and PS $(0.79 / 0.5)$. Correlation of circular ROIs was also excellent for Fp (0.92/0.94) and Vp (0.9/0.8), but worse for VEE and PS. Intraobserver correlation for the parameters Fp, Vp and PS were excellent (0.9/0.9), but worse for VEE (0.7/0.6). Correlations of small arbitrary ROIs were lower for all parameters.

Conclusion: Perfusion and permeability parameters of DCE-MRI of RCC are influenced by ROI-size and positioning. The best inter- and intraobserver correlation showed definition of whole tumour ROI with morphological sequences or plasma flow maps. Evaluating tumours or monitoring antiangiogenic therapy, perfusion parameters are more reliable, while permeability parameters are more susceptible to interobserver variability.

\section{B-0976 15:03}

Can a contrast-enhanced ultrasound nephrostogram be used instead of a fluoroscopic nephrostogram: preliminary findings

M. Daneshi, K. Patel, D. Huang, M. Sellars, P. Sidhu; London/UK

Purpose: The use of contrast-enhanced ultrasound (CEUS) has extended beyond traditional uses, and the possibility to delineate percutaneous tubes and drains is achievable. We have compared the traditional fluoroscopic nephrostogram using iodinated contrast agents with CEUS nephrostogram to ascertain the accuracy, utility and convenience of the CEUS nephrostogram.

Methods and Materials: The standard conventional nephrostogram was performed immediately prior to the CEUS nephrostogram. The CEUS nephrostogram technique involved diluting $0.2 \mathrm{ml}$ of SonoVue with $40 \mathrm{ml}$ of normal saline and introduced into the renal collecting system via the nephrostomy tube. Digital cineclips and still images were recorded to allow accurate retrospective comparison by two independent reviewers to the reference standard.

Results: Twelve nephrostomies in 10 patients (median age 64 yrs, range 29-91 yrs, 6 females and 4 males) were performed and reviewed. The renal pelvicalyceal system was visualised in both CEUS and fluoroscopic nephrostograms in 11/12 $(92 \%)$ with one nephrostomy tube identified as being misplaced. The entire ureter was visualised in 6/12 (50\%) with a CEUS nephrostogram compared with 8/12 (75\%) using traditional nephrostogram. Fluoroscopic nephrostogram showed drainage of contrast into the bladder in 10/12 (83\%) cases compared with 9/12 (75\%) using CEUS.

Conclusion: Preliminary results suggest that CEUS nephrostogram is a feasible method to confirm the correct positioning of the nephrostomy tube, image the ureters and determine if there is satisfactory drainage into the bladder. CEUS nephrostogram is a suitable alternative for the traditional nephrostogram in patients with contraindications to iodinated contrast agents or if the procedure needs to be performed at the bedside.

\section{B-0977 15:12}

Vena cava anomalies associated with horseshoe kidney on MDCT

T. Ichikawa, J. Koizumi, S. Kawada, Y. Imai; Isehara/JP

(tamaki-i@mars.sannet.ne.jp)

Purpose: The incidence of vena cava (VC) anomalies in horseshoe kidney (HSK) patients is relatively high because of embryogenesis. We evaluated prevalence and variation of anomalous inferior vena cava (IVC) and superior vena cava (SVC) in HSK patients detected using CT.

Methods and Materials: Seventy-one patients with HSK and 2292 patients with normal kidney (NK) who underwent chest CT were evaluated of prevalence and variation of anomalous SVC and 205 patients with HSK and 1990 patients with NK who underwent abdominal CT were evaluated of prevalence and variation of anomalous IVC. We reviewed axial CT images with 5- $\mathrm{mm}$ reconstruction interval and compared prevalence of anomalous VC between HSK and NK patients using Chi-square test.

Results: Anomalous SVC was identified in 3 patients (4.2\%) in HSK patients: 2 double SVCs and 1 persistent left SVC without right SVC and 5 patients $(0.22 \%)$ in NK patients: 3 double SVCs and 2 persistent left SVC without right SVC. Anomalous IVC was identified in 8 patients (3.9\%) in HSK patients: 1 preisthmic IVC with retrocaval ureter, 4 double IVCs, 2 left IVC, and 1 IVC with azygos continuation and 12 patients $(0.6 \%)$ in NK patients: 8 double IVCs, 3 left IVC, and one IVC with azygos continuation. There was a significantly higher prevalence of anomalous VC in patients with HSK than in those NK on MDCT $(P<0.001)$.

Conclusion: HSK patients were frequently found anomalous VC and detection of those anomalies in HSK patients is important during central venous catheter procedures and operation. 


\section{B-0978 15:21}

Comparison of dynamic transperineal ultrasound with evacuation proctography for the evaluation of patients with posterior compartment pelvic disorders

M.J. Webb, Y. Ron, S. Zelber-Sagi, E. Tiomni, Z. Halpern, E. Santo; Tel Aviv/IL (murielw1999@yahoo.fr)

Purpose: Evacuation proctography (EP) is still considered the gold standard imaging technique for patients suffering from defecation disorders. Dynamic transperineal ultrasound (DTPU) is a non-invasive imaging modality to explore such patients. To assess the value of DTPU compared with EP in patients with defecation disorders. Methods and Materials: Between January 2008 and November 2010, 102 consecutive patients that underwent both DTPU and EP for obstructed defecation were reviewed. The tests were performed by two different operators, each one unaware of the results of the other. Comparison was made between the two modalities.

Results: EP revealed a rectocele in $49 \%$, enterocele in 17\%, intussusception in $28 \%$, and anismus in $20 \%$ patients. DTPU revealed a rectocele in $53 \%$, enterocele in $15 \%$, intussusception in $37 \%$ and anismus in $27 \%$ patients. The degree of agreement of the two techniques calculated by kappa measure was for rectocele 0.69 , for enterocele 0.7 , for intussusception 0.4 and for anismus 0.53 . The accuracy of the DTPU as compared with EP was $84 \%$ for rectocele (sensitivity $88.0 \%$, specificity $81.0 \%$ ), $92 \%$ for enterocele (sensitivity $70.6 \%$, specificity $96.5 \%$ ) $74 \%$ for intussusception (sensitivity $69.0 \%$, specificity $75.3 \%$ ) and $83 \%$ for anismus (sensitivity $75 \%$, specificity $85 \%$ ).

Conclusion: The degree of concordance between the two modalities is good in the diagnosis of rectocele and enterocele, but only fair for intussusception and anismus. With regard to intussusceptions and anismus, correlation with other modalities may be warranted. These preliminary data indicates that DTPU can be an alternative to $\mathrm{EP}$ in assessing patients with defecation disorders. Room I/K

\section{Abdominal Viscera}

\section{SS $1801 b$}

\section{Liver volume, function and focal lesions}

Moderators:

B. Choi; Seoul/KR

C.M. Nyhsen; Sunderland/UK

\section{B-0979 14:00}

ROC curve analysis of diagnostic confidence of CT and MR in focal liver pathology

L. Saba', M. Di Martino ${ }^{2}$, C. Catalano ${ }^{2}$, M. Piga ${ }^{1} ;{ }^{1}$ Cagliari/lT, ${ }^{2}$ Rome/lT (lucasaba@tiscali.it)

Purpose: To evaluate and compare ROC curve of diagnostic confidence of multi-phase CT and MR after administration of Gd-EOB-DTPA in the diagnosis of malignantbenignant neoplasm of the liver.

Methods and Materials: Forty patients underwent multi-phase CT and MR after administration of Gd-EOB-DTPA. After CT and MR all patients underwent biopsy. All exams were evaluated by 2 experienced examiners independently that expressed their diagnostic confidence in a five-degree scale: 1 (certainly benign), 2 (probably benign), 3 (uncertain), 4 (probably malignant), 5 (certainly malignant). ROC curve analysis and area under the curve (AUC) were calculated for each observer and their difference was tested according to the different methodology used.

Results: In our population there were 15 malignant neoplasms and 25 benignant neoplasms. For the CT analysis AUC was 0.792 (SE $=0.088,95 \% \mathrm{Cl}$ from 0.634 to 0.903 ) and 0.701 ( $\mathrm{SE}=0.099,95 \% \mathrm{Cl}$ from 0.535 to 0.835 ) for observers 1 and 2, respectively, whereas for the MR analysis AUC was 0.923 (SE $=0.058,95 \% \mathrm{Cl}$ form 0.793 to 0.983 ) and 0.934 ( $\mathrm{SE}=0.054,95 \% \mathrm{Cl}$ from 0.808 to 0.987 ). For both observers a statistically significant difference in diagnostic confidence between the AUC of CT and MR ( $p$ value $<0.05$ ) was found.

Conclusion: Our results indicate that for both readers the diagnostic confidence using MR with Gd-EOB-DTPA is statistically significantly superior to CT with multiphase technique.
B-0980 14:09

Diagnostic accuracy of dynamic gadoxetic-acid-enhanced MRI and PET-CT in patients with liver metastases from neuroendocrine neoplasms W.H. Sommer ${ }^{1}$, C. Zech ${ }^{1}$, S. Sourbron ${ }^{2}$, M. Armbruster ${ }^{1}$, M.F. Reiser'; ${ }^{1}$ Munich/DE, ${ }^{2}$ Leeds/UK (wieland.sommer@med.uni-muenchen.de)

Purpose: To evaluate the diagnostic accuracy of dynamic gadoxetic-acid-enhanced MRI parameters in comparison to standardised uptake values (SUV) from both ${ }^{18} \mathrm{~F}$-FDG- and ${ }^{68} \mathrm{Ga}$-DOTATATE PET-CT in patients with proven liver metastases of neuroendocrine neoplasms (NEN).

Methods and Materials: Data were acquired in 47 patients with hepatic metastases from NEN. DCE-MRI was performed at $3 \mathrm{~T}$ with Gd-EOB-DTPA and a 3D sequence acquiring 48 slices of data every 2.2 seconds for 5 minutes. Up to three regions of interest (ROIs) were defined in metastases $(>2 \mathrm{~cm}$ ) and liver tissue in morphological images. Corresponding ROIs were defined in the DCE-MRI dataset and in the PET-CT dataset. Diagnostic accuracy for all perfusion parameters were evaluated for the differentiation between metastases and liver tissue: arterial and venous plasma flow (APF and VPF), extracellular mean transit time (exMTT), extracellular volume (exVol), intracellular uptake rate (UR) of Gd-EOB-DTPA and arterial flow fraction (AFF) as well as SUVs derived either from ${ }^{18} \mathrm{~F}-\mathrm{FDG}$ or from ${ }^{68} \mathrm{Ga}$-DOTATATE-PET-CT.

Results: Area under the curve for different DCE-MRI parameters was between AUC $=0.559$ (extracellular mean transit time) and 0.901 (arterial plasma flow - sensitivity: $91.9 \%$, specificity: $77.3 \%$, cut-off value: $26.0 \mathrm{ml} / \mathrm{min} / 100 \mathrm{ml}$ ). Sensitivity and specificity of SUVmean derived from ${ }^{68} \mathrm{Ga}$-DOTATATE were $92.6 \%$ and $93.8 \%$, respectively (AUC $=0.966$ ). SUVmean derived from ${ }^{18} \mathrm{~F}$-FDG-PET-CT showed a sensitivity and specificity of $95.0 \%$ and $91.3 \%$, respectively (AUC $=0.989$ ).

Conclusion: Both PET-CT parameters and DCE-MRI-perfusion parameters - especially flow and uptake-related DCE-MRI parameters show the ability to distinguish between liver metastases and normal liver tissue and may provide additional information in unclear cases.

Author Disclosures:

C. Zech: Consultant; Bayer. Speaker; Bayer. S. Sourbron: Consultant; Bayer.

\section{B-0981 14:18}

The LiMAx test as adjunct to $C T$ for assessing liver function after portal vein embolisation

A.H. Mahnken' ${ }^{1}$, A. Roeth², P.H. Alizai', C. Kuhl', R.M. van Dam²,

U. Neumann ${ }^{2}$, M. Schmeding ${ }^{2} ;{ }^{1}$ Marburg/DE, ${ }^{2}$ Aachen/DE, ${ }^{3}$ Maastricht/NL

(mahnken@med.uni-marburg.de)

Purpose: To compare CT volumetry and the LiMAx-test, a metabolic 13C-Methacetin respiratory test was performed, for examination of the effects of portal vein embolisation (PVE) on liver function over time.

Methods and Materials: 27 patients (16 males; 11 females $63 \pm 9.5$ ys) with PVE of the right portal vein prior to major liver resection for colorectal metastases $(n=11)$, Klatskin tumours $(n=8), \operatorname{HCC}(n=2), \operatorname{CCC}(n=3)$ and liver metastases of other cancers $(n=3)$ were included in this study. Liver function was investigated with the LiMAx test prior to PVE, 24 hours after embolisation and immediately prior to resection. CT volumetry of the right and left liver was performed prior to PVE and prior to resection.

Results: The average time between PVE and resection was $32 \pm 11$ days. The average LiMAx values before and after PVE were $374 \mu \mathrm{g} / \mathrm{h} / \mathrm{kg}$ and $312 \mu \mathrm{g} / \mathrm{h} / \mathrm{kg}$, respectively. Preoperatively, the LiMAx value increased to $380 \mu \mathrm{g} / \mathrm{h} / \mathrm{kg}(\mathrm{p}<0.05)$. In parallel, the average volume of the future liver remnant significantly increased from $576 \mathrm{ml}$ to $706 \mathrm{ml}(\mathrm{p}=0.006)$, whereas the mean right liver volume decreased from $1288 \mathrm{ml}$ to $1121 \mathrm{ml}(\mathrm{p}=0.001)$.

Conclusion: The postinterventional decrease in LiMAx values corresponds to the loss in function with subsequent shrinkage of the embolised right liver over time. The normalisation of the LiMAx value after regeneration proofs the functional relevance of the hypertrophy of the remnant left liver. When compared to volumetry alone, the combination of imaging and LiMAx test permits monitoring the functional relevance of liver hypertrophy. This may help identify the optimal point for resection after PVE.

Author Disclosures:

A.H. Mahnken: Research/Grant Support; Siemens. Speaker; Bayer.C. Kuhl: Speaker; Bayer. 


\section{B-0982 14:27}

Future remnant liver function after portal vein occlusion measured with 99mTc-mebrofenin SPECT/CT

I. Dudás, T. Györke, C. Korom, P. Pajor, A. Zsirka-Klein, E. Horvath, A. Bozó,

P. Kupcsulik, O. Hahn; Budapest/HU (oszkarhahn176@gmail.com)

Purpose: To compare the results of ${ }^{99 \mathrm{~m} T \mathrm{Tc}-m e b r o f e n i n ~ S P E C T / C T}$ volumetry and $\mathrm{CT}$ volumetry after portal vein occlusion techniques, i.e. portal vein embolisation (PVE), portal vein ligation (PVL) prior to extended liver resections.

Methods and Materials: Between March 2011 and September 2012 forty patients presenting with primary or secondary liver tumours were included. The estimated future liver remnant volume (FLRV) of these patients after the planned extended hepatectomy measured with CT-volumetry was less than 30 (normal liver), or $40 \%$ (cirrhosis). To increase the FLRV, portal vein occlusion techniques (32 PVE, $8 \mathrm{PVL}$ ) were performed. Eight weeks after portal occlusion CT-volumetry and to measure functional future liver remnant volume (FLRVf)-SPECT/CT volumetry with ${ }^{99 m} \mathrm{Tc}$-mebrofenin were performed. Postoperative complication rates (using the Clavien criteria) were also measured. Patients with "borderline" FLRV (25-32\%) were evaluated with special interest.

Results: Eight weeks after portal vein occlusion, FLRVf was significantly higher than FLRV (mean FLRVf: $51.2 \%$ vs. mean FLRV: $32.9 \%$; $p<0.05$ ). $32 / 40$ patients became resectable. Postoperative complication rates were lower if FLRVf was significantly higher than FLRV. In patients with "borderline" FLRV $(n=17)$ these results were even more convincing (mean FLRVf: $46.5 \%$ vs. mean FLRV: $27.8 \% ; p<0.05$ ). Conclusion: ${ }^{99 \mathrm{~m} T C} \mathrm{C}$ Mebrofenin SPECT/CT can be useful in the measurement of future liver remnant functional volume after portal vein occlusions. This can make surgeons decision easier than CT volumetry in planning extended hepatectomies.

\section{B-0983 14:36}

Comparison of percutaneous portal vein embolisation, portal vein ligation and portal vein occlusion combined with ipsilateral hepatic artery cannula implantation prior to major liver resection

P. Pajor, I. Dudás, A. Zsirka Klein, P. Kupcsulik, J. Tamás, O. Hahn; Budapest/HU (oszkarhahn176@gmail.com)

Purpose: To evaluate the results of percutaneous portal vein embolisation (PVE), portal vein ligation (PVL) and portal vein ligation combined with hepatic artery cannula implantation (PVL+can) prior to extended hepatectomy.

Methods and Materials: Between 2004 and 2012 hundred and thirty-eight patients presenting with multiple or large liver metastases or large hepatocellular carcinoma were included. The estimated residual liver volume (FLR) of these patients after the planned extended hepatectomy was less than $30 \%$ (normal liver), or $40 \%$ (cirrhotic liver). To increase the FLR portal vein occlusion techniques (50 PVE, $88 \mathrm{PVL}$ alone, or PVL combined with hepatic artery cannula implantation into the ipsilateral hepatic artery - PVL+can) were performed. They were evaluated with MDCT including volume assessment, before and 8 weeks after these procedures. Results: $110 / 138$ patients became resectable (PVE, PVL, PVL+can; $74.3 \%, 80 \%$, $88.5 \%$ ). FLR\% increase 8 weeks after PVE, PVL or PVL+can was $17.6 \%, 16.5 \%$, $19 \%$, the complication rate of the various portal occlusion techniques were $4.5 \%$, $3.6 \%, 11.5 \%$, respectively. At least 4 segments were resected during hepatectomy. Overall postoperative morbidity and mortality rates were $13 \%$ and $2.5 \%$, respectively. Complication and mortality rates did not differ significantly in the 3 groups. Conclusion: Patients with previously unresectable liver tumours can benefit from resection after all kinds of portal occlusion techniques. Although complication rate of portal occlusion combined with hepatic artery canule implantation is higher, more patients become resectable due to higher increase rate of FLR in this group.

\section{B-0984 14:45}

Hepatic parenchymal and vascular contrast improvement in superdelayed phase images of Gd-EOB-DTPA-enhanced MRI

S. Kobayashi, O. Matsui, T. Gabata, W. Koda, T. Minami, K. Kozaka, A. Kitao; Kanazawa/JP (satoshik@staff.kanazawa-u.ac.jp)

Purpose: To elucidate the parenchymal and vascular contrast improvement effect of super-delayed phase (SDP) images of Gd-EOB-DTPA (EOB)-enhanced MRI in poor hepatobiliary phase (HBP) image cases special focus on Child-Pugh (CP) classification.

Methods and Materials: 76 cases, who have examined EOB-enhanced MRI for closer examination of hepatic lesions, and taken SDP images approximately 90 minutes after iv administration of $\mathrm{EOB}$ because of poor HBP image are subjected to this study. 20 hepatobiliary disease cases who had also taken SDP images which show normal HBP images were used as control. Hepatic vascular/parenchymal enhancement ratios (ER) were defined as signal intensity (SI) of intrahepatic vessel
/ SI of liver. ER of HBP and SDP were calculated and compared between each CP class liver damage groups. Chi square test was used for statistics and $p<0.05$ was considered statistical significant.

Results: In poor HBP cases $(n=76)$, ER of HBP and SDP were $0.88 \pm 0.16$ and $0.64 \pm 0.16$. In control cases $(n=20)$, ER of HBP and SDP were $0.54 \pm 0.08$ and $0.39 \pm 0.06$. ER of HBP and SDP in CP-A poor HBP $(n=27), C P-B$ poor HBP $(n=47)$, CP-C poor HBP $(n=2)$ were $0.83 \pm 0.14$ and $0.60 \pm 0.13,0.90 \pm 0.16$ and $0.65 \pm 0.16$, $1.03 \pm 0.16$ and $0.99 \pm 0.19$, respectively (all combinations except CP-C showed significance difference).

Conclusion: In most of the poor HBP image cases, SDP image improve parenchymal and vascular contrast except CP-C liver damage cases.

\section{B-0985 14:54}

Estimation of liver function using T1 mapping on Gd-EOB-DTPAenhanced MR imaging

M. Haimerl, N. Verloh, C. Fellner, C. Nießen, C. Stroszczynski, P. Wiggermann; Regensburg/DE (michael.haimerl@ukr.de)

Purpose: To determine the ability of T1 mapping of liver on Gd-EOB-DTPAenhanced MR imaging (MRI) for estimating liver function and to compare the estimated liver function to the model for end-stage liver disease (MELD) score. Methods and Materials: 24 patients underwent Gd-EOB-DTPA-enhanced MRI on a 3 T system. Patients were classified into two groups: MELD score $<9(n=15)$ and MELD score ${ }^{39}(n=9)$. Two TurboFLASH sequences $(T I=400 \mathrm{~ms}, 1000 \mathrm{~ms})$ were acquired before and 20 minutes after Gd-EOB-DTPA administration to obtain T1 maps. T1 relaxation times were determined indicating Gd-EOB-DTPA liver-uptake and correlated to the MELD score.

Results: Significant changes between T1 relaxation times of non-enhanced MRI (794 ms $\pm 136 \mathrm{~ms}$ ) and Gd-EOB-DTPA-enhanced MRI (351 ms $\pm 141 \mathrm{~ms}$ ) were observed $(p<0.001)$. T1 relaxation time for non-enhanced MRI showed no significant differences $(p=0.387)$ between the group with MELD score $<9$ (776 ms \pm $157 \mathrm{~ms})$ and the group with MELD score ${ }^{3} 9(824 \mathrm{~ms} \pm 90 \mathrm{~ms})$. After administration of Gd-EOB-DTPA T1 relaxation time of patients with MELD score $<9(284 \mathrm{~ms} \pm$ $104 \mathrm{~ms}$ ) and patients with MELD score ${ }^{3} 9$ ( $465 \mathrm{~ms} \pm 123 \mathrm{~ms}$ ) shows a significant difference $(p=0.002)$. The shortening of T1 relaxation time was significantly lower for patients with a higher MELD score $(\mathrm{p}=0.002)$.

Conclusion: Patients with advanced liver disease showed significantly lower changes in T1 mapping. Therefore, evaluation of changes in T1 mapping of the liver parenchyma may serve as a useful method to determine whole liver function, to improve the estimation of segmental liver function and finally to define the grade of liver disease.

\section{B-0986 15:03}

Liver dysfunction induced by amiodarone therapy: evaluation by sequential CT studies

Y. Sonoyama, T. Tajima, T. Shiga, N. Hagiwara, S. Sakai; Tokyo/JP (y-sonoyama@rad.twmu.ac.jp)

Purpose: To evaluate the relationship between the sequential changes of CT attenuation of the liver (CTL) and liver damage after administration of amiodarone (AMD) for treatment of refractory ventricular arrhythmias.

Methods and Materials: This was a retrospective study of 45 patients (26 men, 19 women: age range 19-89, mean 59) with AMD administration. Serum level of drugs (AMD and desethylamiodarone (d-AMD), a metabolic product of AMD), the percentage of rises in serum alanine transaminase (ALT) level (ALT\%) and total administered dose of AMD at evaluation CT were evaluated and compared with the $C T L$. As regards to 23 patients who underwent CT before the initiation of AMD administration, the medication periods and total administered doses of AMD were compared with changes in CTL.

Results: There was a mild correlation between CTL and mean medication period $(p=0.0238, r=0.337)$. There was a strong correlation between CTL and serum level of AMD $(p<0.0001, r=0.699)$, whereas there was a moderate correlation between CTL and serum level of drugs (AMD) $(p<0.0001, r=0.574)$. There were significant correlations between total doses of administered drug and the CTL $(p=0.0008$, $r=0.480)$ and between ALT\% and the CTL $(p=0.0078, r=0.420)$.

Conclusion: CTL was demonstrated to correlate with total administered doses of AMD, ALT\%, medication periods and serum level of drugs, especially of d-AMD. Serial changes in CTL can be a sensitive less-invasive biomarker in patients treated with AMD. 


\section{B-0987 15:12}

Feasibility of a volumetric measurement of the left liver lobe with 3D ultrasound compared with MRI

E. Danse, O. Lebecque, N. Michoux, R. Reding; Brussels/BE

(etienne.danse@uclouvain.be)

Purpose: To assess the feasibility of the volumetric estimation of the left liver lobe, with 3D ultrasound, with MRI comparison and surgical specimen.

Methods and Materials: The data of 19 consecutive patients referred for giving their left liver lobe to a child were reviewed (living donor transplantation program). The measurements of the 3D volumes of the segments II and III were performed retrospectively with comparison of the same evaluation performed with MRI (the reference and routine technique for the preoperative assessment of the liver size). All the examinations were done twice by 2 experts radiologists and radiologists in training. The results were statistically analysed (variance test, Gage $R \& R$ and Bland-Altman). 3D and MRI measurements were compared and related to surgically proved volume quantification.

Results: Intra- and inter-observators reproducibility was poorly conclusive (for US and MRI): gage R\&R analysis is related to a PRR of $57 \%$ for US and $54 \%$ for MRI. Bland-Altman analysis was giving an underestimation of volume measurements with US (bias from $-11 \mathrm{ml}$ to $-44 \mathrm{ml}$ ). Agreement limits (-1.96SD to $+1.96 \mathrm{SD})$ for the 4 observators were included between $-174.3 \mathrm{ml}$ and $+103 \mathrm{ml}$ (from $-71.6 \%$ to $+43.5 \%$ ).

Conclusion: Correct estimation of the left liver lobe volume performed with 3D or MRI is related to a low repeatability and a low reproducibility for both techniques. Bland-Altman analysis is also related to significative differences of volume measurement in both techniques.

\section{B-0988 15:21}

An accurate formula for a quick estimate of liver volume in polycystic liver disease

M. Chrispijn, T.J.G. Gevers, J.P.H. Drenth; Nijmegen/NL

(melissachrispijn@gmail.com)

Purpose: Polycystic liver disease (PLD) patients have severe hepatomegaly. Primary aim of treatment is total liver volume (TLV) reduction. The gold standard to assess TLV is CT volumetry which involves manual delineating of the liver outline. This is time consuming and requires expertise. There is a clear need for an easy and fast approach to estimate TLV in routine practice.

Methods and Materials: All PLD patients with ${ }^{31}$ CT scan were considered for inclusion. We measured the liver in transverse $(\mathrm{T})$, anterioposterior (AP) and craniocaudal (CC) directions and performed CT liver volumetry. We had access to an inception and a replication cohort. We performed multiple linear regression to predict TLV using the three dimensions in the inception cohort. Liver volumes were transformed logarithmically. The goodness of fit of the prediction model was assessed and stated as $\mathrm{R}^{2}$. Finally, we validated the prediction model in our replication cohort.

Results: The inception cohort included 54 PLD patients (median TLV $3890 \mathrm{~mL}$ (range 1084-13611 mL)). All three diameters were significantly correlated with TLV $(p<0.001)$. The linear regression model resulted in the following formula: predicted $T L V=4,179+\left(0.009^{*} \mathrm{~T}\right)+\left(0.007^{\star} \mathrm{AP}\right)+\left(0.001^{*} \mathrm{CC}\right)$. Our model predicted TLV accurately in the inception cohort $\left(\mathrm{R}^{2}=0.907\right)$. We validated our prediction model in a replication cohort of 22 PLD patients (median TLV $3603 \mathrm{~mL}$, range 1261-16746 mL). TLVs predicted by our model were highly correlated with TLVs measured by CT volumetry $\left(R^{2}=0.95\right)$, indicating that this model can be reproduced in other PLD patients.

Conclusion: TLV can easily, quickly and accurately be estimated based on three liver dimensions.
14:00 - 15:30

Room L/M

Physics in Radiology

SS 1813

Patient dose management and dose surveys

Moderators:

H. Bosmans; Leuven/BE

W. Stiller; Heidelberg/DE

\section{B-0989 14:00}

European population dose and differences in radiological procedures between European countries

R. Bly ${ }^{1}$, A. Jahnen ${ }^{2}$, H. Olerud ${ }^{3}$, J. Vassileva ${ }^{4}$, H. Järvinen ${ }^{1}$, S. Vogiatzi ${ }^{5}$; ${ }^{1}$ Helsinki/FI, ${ }^{2}$ Luxembourg/LU, ${ }^{3}$ Osterås/NO, ${ }^{4}$ Sofia/BG, ${ }^{5}$ Athens/GR (ritva.bly@stuk.fi)

Purpose: Population doses from radiodiagnostic (x-ray and nuclear medicine) procedures in Europe were estimated for the first time in the recent DOSE DATAMED 2 (DDM2) project (www.ddmed.eu) launched by the European Commission. Methods and Materials: Data of 34 countries were collected to an established database.

Results: The estimated population dose per caput is $1 \mathrm{mSv}$, which is lower than corresponding dose in USA ( $3 \mathrm{mSv}$ ) or in Australia (1.7 mSv). The highest average contribution is from CT, $56 \%$. From plain radiography, fluoroscopy and interventional radiology the proportional fractions are $19 \%, 17 \%$ and $7.5 \%$ respectively. From diagnostic nuclear medicine procedures, the estimated mean effective dose per caput is $0.036 \mathrm{mSv}$. Heart and bone examinations contribute $40 \%$ and $36 \%$, respectively. Conclusion: There were high variations between countries in frequencies (for example, in thorax radiography about ten-fold between Spain or Portugal and Germany) and typical effective doses (for example, in CT head four-fold between Denmark and Luxembourg). This presentation will review the available data in Europe using graphs in which countries can be identified. Furthermore, the procedures contributing most to the population dose are highlighted.

\section{B-0990 14:09}

Radiation dose from multidetector CT examinations in adults in Italy: result of the first Italian survey

S. Salerno ${ }^{1}$, C. Granata ${ }^{2}$, D. Matranga ${ }^{1}$, F. Palorini ${ }^{3}$, D. Origgi ${ }^{3} ;{ }^{1}$ Palermo/IT, ${ }^{2} \mathrm{Genoa} / \mathrm{IT}$, ${ }^{3} \mathrm{Milan} / \mathrm{IT}$ (sergio.salerno@unipa.it)

Purpose: The first large-scale Italian survey on radiation dose delivered to adult patients in multidetector CT (MDCT) examinations was performed.

Methods and Materials: All the Italian radiology departments and the radiologists' members of the Italian society of radiology (SIRM) were invited to take part in the survey. The minimum criteria for participation were: MDCT apparatus from 16 to 64 rows, a referring radiologist and medical physicist for the survey, at least 10000 examinations per year performed. 82 radiology units were considered eligible. The data for three common CT examinations (head, chest and abdomen) were retrospectively collected during 2011 for common clinical indications: head CT for stroke, haemorrhage trauma; chest for trauma, infection, cancer diagnosis and follow-up; abdomen for trauma infection, cancer diagnosis and follow-up. Patient data and technical parameters ( $k V p, m A s$, tube rotation speed, thickness, collimation, scan length, pitch, filtration, etc). of the examinations, including CTDIvol and DLP, were collected in a database.

Results: Data of 6845 different patients were collected. 65/82 centres completed the survey. The $75^{\text {th }}$ percentiles of CTDIvol (mGy) and DLP (mGy $\left.{ }^{*} \mathrm{~cm}\right)$ distributions, respectively, are: 69 and 1382 for head CT; 16 and 754 for chest CT; 19 and 2157 for CT of the abdomen. The $31 \%$ of chest studies and the $71 \%$ of abdominal studies present more than one sequence.

Conclusion: The analysis performed constitutes the first Italian database on radiation dose in MDCT study and can aid to define new European diagnostic reference levels (DRLs) including the MDCT practice.

Author Disclosures:

S. Salerno: Research/Grant Support; Italian Society of Radiology SIRM.

C. Granata: Research/Grant Support; Italian Society of Radiology SIRM. 


\section{B-0991 14:18}

Patient-specific whole body voxel model for accurate Monte Carlo CT dose estimation

N. Saltybaeva, Y. Smal, D. Kolditz, W. Kalender;

Erlangen/DE (natalia.saltybaeva@imp.uni-erlangen.de)

Purpose: The accuracy of CT dose estimates based on Monte Carlo (MC) techniques is limited as data is only available for the scanned volume. The purpose of this study was to generate and to validate whole body voxel models using the patient CT images extended by established voxel phantoms.

Methods and Materials: Measurements were performed on a SOMATOM Definition Flash scanner (Siemens, Forchheim, Germany) using the anthropomorphic Rando-Alderson phantom. The absorbed dose was measured using a set of 90 calibrated thermoluminescent dosimeter (TLD) chips for thorax protocols with tube voltage of 80,100 and $120 \mathrm{kV}$. Whole body voxel models were built as a combination of the patient CT images and the ICRP Adult Male Reference voxel phantom. $\mathrm{MC}$ simulations were performed using the ImpactMC software (CT Imaging $\mathrm{GmbH}$, Erlangen, Germany) for only the scanned volume and for the combined whole body model. Measured and simulated dose values were compared for each TLD chip. Results: At $120 \mathrm{kV}$, the dose values obtained by MC simulations based on only CT images differed from TLD measurements by $19 \%$ on average with a maximum difference of $37 \%$ for the border slices, while the whole body model allowed for a mean difference of $6 \%$. The difference between measured and calculated results was reduced by $53 \%, 60 \%$ and $68 \%$ for 80,100 and $120 \mathrm{kV}$, respectively, when using the whole body voxel model instead of the CT-scanned volume only. Conclusion: The proposed whole body voxel model for Monte Carlo simulations significantly improves the accuracy of patient-specific CT dose estimates.

Author Disclosures:

W. Kalender: Consultant; Siemens Healthcare.

\section{B-0992 14:27}

A simple algorithm for dose estimation in $\mathrm{CT}$ imaging considering patient size

Y. Smal, N. Saltybaeva, W. Kalender;

Erlangen/DE (yulia.smal@imp.uni-erlangen.de)

Purpose: Accurate patient dose estimation using CT imaging is important particularly as the modality is being increasingly used. Body habitus varies and the use of Monte Carlo simulations for "standard man" phantoms does not provide accurate dose estimation for non-standard patient sizes. The study proposes an algorithm for dose estimation for an individual patient from simulated data from mathematical phantoms.

Methods and Materials: The algorithm interpolates doses simulated for ORNL mathematical phantoms accounting for differences on patient and phantom sizes. Four patient models were produced using 2 anthropomorphic phantoms (representing 1- and 5-year-old children) with and without an additional fat-equivalent layer. All phantoms were scanned at 80 and $120 \mathrm{kV}$ (trunk protocol). Doses were measured with 60 TLD dosimeters. Additionally, DICOM images were segmented for organs and data were used as input for dose simulation. All simulations were performed with validated tool (ImpactMC). Doses for phantoms and patients were estimated from the MC simulations using the proposed algorithm. Calculated and measured doses were compared.

Results: The algorithm improved accuracy in dose estimation by $49,35,30$, and $21 \%$ for small thin, small thick, large thin and large thick phantom sizes, respectively, compared with estimates based on ORNL phantoms only. For patient data the accuracy in lung dose estimates improved from 15 to $4 \%$, and from 108 to $15 \%$, for 2- and 6-year-old patients, respectively.

Conclusion: The proposed algorithm improves dose estimation accuracy for individual paediatric patients. It is simple and suitable for implementation in software tool for dose calculation.

Author Disclosures:

W. Kalender: Consultant; Siemens Healthcare.

\section{B-0993 14:36}

Monte Carlo tool for 3D-dose simulation in computed tomography taking into account tube current modulation

N. Saltybaeva ${ }^{1}$, D. Kolditz ${ }^{1}$, B. Schmidt ${ }^{2}$, W. Kalender ${ }^{1} ;{ }^{1}$ Erlangen/DE,

${ }^{2}$ Forchheim/DE (natalia.saltybaeva@imp.uni-erlangen.de)

Purpose: Monte Carlo (MC) methods are widely used in v-ray computed tomography (CT) for accurate 3D dose calculation; in most cases, however, they do not consider tube current modulation (TCM). The aim of this study was to include TCM effects in MC dose simulations and to validate this approach.

Methods and Materials: All measurements were performed on a SOMATOM Definition Flash scanner (Siemens Healthcare, Forchheim, Germany) using three anthropomorphic phantoms: adult Alderson-Rando, 5-year-old child and 1-yearold child. The phantoms were scanned with a trunk protocol using an online TCM system (CareDose4, Siemens Healthcare, Forchheim, Germany). Organ dose values were measured using 94 calibrated thermoluminescent dosimeters (TLD) for each phantom. MC simulations were performed using ImpactMC software (CT Imaging $\mathrm{GmbH}$, Erlangen, Germany) based on the 3D voxelised data derived from acquired CT images. The tool was modified to take TCM curves into account. The current values for different tube positions were extracted from the raw data using manufacturer software. Simulated dose values were compared with TLD measurements on a chip-by-chip basis.

Results: For MC calculations without taking TCM data into account the mean differences between measured and simulated dose values amounted to $92 \%, 83 \%$, $71 \%$ for adult, 5 -year-old child and 1 -year-old phantoms, while modelling with TCM reduced the mean differences to $5.3 \%, 7.5 \%$ and $6.4 \%$, respectively.

Conclusion: MC dose estimates including TCM data were in good agreement with measurements. This technique can significantly improve the accuracy of 3D-dose assessment.

Author Disclosures:

B. Schmidt: Employee; Siemens Healthcare. W. Kalender: Consultant; Siemens Healthcare.

\section{B-0994 14:45}

Evaluation of patient dose reduction after introduction of iterative reconstruction in routine MDCT

D. Defez ${ }^{1}$, P. Coulon', P.C. Douek ${ }^{3}$, L. Boussel ${ }^{4} ;{ }^{1}$ Pierre Benite/FR,

${ }^{2}$ Suresnes/FR, ${ }^{3}$ Bron/FR, ${ }^{4}$ Lyon/FR (didier.defez@chu-lyon.fr)

Purpose: To evaluate patient dose reduction with iterative reconstruction (IR) technique, on routinely 64-detector computed tomography (CT).

Methods and Materials: All consecutive patients addressed for a CT scanner to our University Hospital (Lyon, France) between March 2011 and March 2012 were included in this study. Dose length product (DLP) was prospectively recorded before (2438 scans) and after (2823 scans) the installation of iDose4 IR on a Brilliance 64 CT scanner (Philips HealthCare), with a software tool created by our radio physics department. Dose were compared for routinely performed brain, full dose chest, low dose chest follow-up, chest-abdomen-pelvis, inferior limbs and ECG-gated cardiac examination.

Results: The global average dose reduction over all kind of examinations was $31.9 \%$ with some variations depending mainly on the initial dose level and the clinical application. The average dose reduction measured on DLP was $19.3 \%$ for brain with $840.3 \pm 79.3$ vs $678.3 \pm 91.7$ mGy.cm $(p<0.0001)$ before and after upgrade, $33.7 \%$ for standard chest ( $357.7 \pm 184.1$ vs $237.2 \pm 118.2$ mGy.cm, $p<0.0001), 24.5 \%$ for low dose chest follow-up ( $126.7 \pm 72.2$ vs $95.7 \pm 33.3$ mGy.cm, $p=0.004), 36.3 \%$ for chest-abdomen-pelvis (683.2 \pm 290.5 vs $435.2 \pm 254.2$ mGy.cm, $p<0.0001), 36.3 \%$ for inferior limbs $(1075.0 \pm 514.5$ vs $684.3 \pm 231.8$ mGy.cm, $p=0.001), 42.7 \%$ for cardiac CTA (842.9 206.9 vs $484.7 \pm 213.3$ mGy.cm, $p<0.0001)$.

Conclusion: On Brilliance 64 CT scanner, introduction of IR reduced patient radiation dose between 19 and $43 \%$ in routine practice, depending on the clinical application.

Author Disclosures:

P. Coulon: Employee; PHILIPS.

\section{B-0995 14:54}

Adjusting and measuring CT dose in severely obese patients R. Marcus, F. Bamberg, K. Neumaier, M.F. Reiser, T.R.C. Johnson; Munich/DE (roy.marcus@med.uni-muenchen.de)

Purpose: $\mathrm{CT}$ in morbidly obese patients remains a challenge with respect to dose and image quality. Automatic adaptation of parameters results in high dose length products which do not reflect the actual dose due to the shielding by the fat. Aim of this study was to determine the actual dose and image quality.

Methods and Materials: An Alderson phantom was equipped with 116 thermoluminiscent detectors. Scans were performed using three set-ups: (I) 'Obese' phantom in $30-\mathrm{cm}$ circumferential fat (simulating $175 \mathrm{~cm} / 300 \mathrm{~kg}$ patient) with a dual--source protocol at $2 \times 140 \mathrm{kVp}$ with current modulation; (II) Obese phantom with automatic potential and current modulation; (III) Slim phantom with automatic potential and current modulation; dose was calculated according to IRCP-103 and based on DLP. Image quality was assessed subjectively and by image noise. 
Results: In set-up III, measured and calculated dose agreed well ( 3.05 vs. $3.0 \mathrm{mSv}$ ). Set-ups I and II showed a discrepancy of $72 \%$ between measured and calculated dose (9.52 vs. $34.20 \mathrm{mSv}$ and 10.69 vs. $37.43 \mathrm{mSv}$ ). Image quality in set-ups I and II was rated sufficient, compared to the excellent image quality in set-up III. Set-up.i. showed a lower image noise (9 vs. $33 \mathrm{HU}$ ) and lower dose (12\%) than set-up II. Conclusion: Morbidly obese patients receive higher dose in CT examinations, which is required to achieve diagnostic image quality. Calculations based on DLP and standard conversion factors overestimate the dose by approx. $70 \%$ in 300 $\mathrm{kg}$ patients, while the actual equivalent dose remains within the reference limits.

\section{B-0996 15:03}

The effects of head size/shape, head positioning, and bow-tie filter selection on peak tissue doses from brain perfusion 256-slice CT K. Perisinakis ${ }^{1}$, I. Seimenis ${ }^{2}$, A. Tzedakis ${ }^{1}$, A.E. Papadakis ${ }^{1}$, J. Damilakis ${ }^{1}$; ${ }^{1}$ Iraklion/GR, ${ }^{2}$ Alexandroupolis/GR (Kostas.Perisinakis@med.uoc.gr)

Purpose: To determine peak doses to skin, eye lens, brain parenchyma and cranial red bone marrow (RBM) of adult individuals subjected to low-dose brain perfusion 256-slice CT studies, and investigate the effects of patient head size/shape, head position and bow-tie filter selection.

Methods and Materials: Peak doses to radiosensitive tissues were measured in 106 individual-specific head phantoms subjected to the standard low dose brain perfusion CT on a 256-slice CT scanner using a novel Monte Carlo simulation software. The effects of head size/shape, head position during acquisition and bow-tie filter on resulting peak doses were investigated. The effects of head mis-centering and use of narrow bow-tie filter on image quality were assessed.

Results: The mean peak doses to eye lens, skin, brain and RBM were found to be 124, 120, 95 and 163 mGy, respectively. Patient head size/shape was found to have minimal effect on peak doses, since maximum differences were less than $7 \%$. Bow-tie filter selection and head mis-centering were found to have a considerable effect on peak tissue doses with minimal image quality deterioration. The use of the narrower bow-tie filter available resulted in $35 \%$ reduction of peak tissue doses. Conclusion: Typical peak doses to skin, eye lens, brain and RBM from the standard low dose brain perfusion 256-slice CT exposure are well below the corresponding thresholds for induction of deterministic effects. The use of narrow bow-tie filters may considerably reduce peak absorbed dose to all above radiosensitive tissues with minimal degradation of image quality.

\section{B-0997 15:12}

Detector dose vs image quality in radiography with digital detectors: a visual grading analysis

R. Decoster, H. Mol, D. Smits; Brussels/BE (robin.decoster@hubrussel.be)

Purpose: The introduction of digital detectors in the radiology predicted a dose reduction. Due to the dynamic range, radiographs of sufficient quality can be produced with a lower detector air kerma (DAK). However, this reduction was not observed. Some authors indicate a creep toward higher DAK, explained by better appreciation of the radiographs due to a higher contrast-to-noise ratio.

Methods and Materials: To investigate the relation between the DAK and the appreciation of image quality by radiologists, 172 anterior-posterior (AP) radiographs of the knee and 152 radiographs of the pelvis where collected randomly in 19 radiologic centres. A visual grading analysis (VGA) with a five-point scale was used to judge the image quality of seven anatomic structures. The mid-point of the scale was equalised to diagnostic image quality. Six radiologists scored both datasets, in a controlled environment, with ViewDex®. Every observer received an instruction and a training dataset. To determine intra-observer variability twenty radiographs were repeated.

Results: The intra-observer variability was not significant $(p>0.05)$ in both datasets. The knee AP obtained a VGA score of 3.92, the pelvis obtained VGA score of 3.71. In both cases, the inter-observer correlations are high and significant. The correlation between the VGAS and the DAK (CR $1.54 \mu$ Gy-5.48 $\mu$ Gy; DR $0.38 \mu$ Gy$6 \mu$ Gy) was not significant in either dataset. Neither were other analyses based on technical parameters.

Conclusion: The VGA revealed a mean image quality higher than diagnostic necessary. Based on the DAK, an overexposure is suspected. The relation between the DAK range and the appreciation of the radiograph needs further investigation.

\section{B-0998 15:21}

Active personal dosimeters to support optimisation of radiation protection in interventional radiology

R. Padovani, F. Bonutti, A. Trianni, G. Bernardi; Udine/IT

(padovani.renato@aoud.sanita.fvg.it)

Purpose: The combined availability of DICOM radiation dose structured report and active personal dosimeters in interventional radiology (IR) can support the optimisation of radiation protection, both of patient and staff.

Methods and Materials: For nine months, interventionalists performing interventional cardiology procedures have used a personal active dosimeter (DoseAware, Philips) worn over the apron. Information is displayed in real-time and transmitted to a database. The patient dosimetry report (Allura FD10, Philips) has been also automatically collected for all procedures. A Matlab routine integrates both information on procedure data and patient and staff exposures, providing a tool aiming to identify high and/or non-optimised exposures.

Results: On a sample of 45 coronary angiography and percutaneous angioplasty procedures the derived mean KAP (SD, maximum) per procedure was 69.0 (53.1, 236.2) $\mathrm{Gycm}^{2}$. The first operator mean (range) personal equivalent dose was 19 $(0.65 \div 27.3) \mu \mathrm{Sv} /$ procedure. The mean (SD) scatter equivalent dose measured on the C-arm at the machine isocentre level per unit of KAP was $2.32(0.50) \mu \mathrm{Sv} / \mathrm{Gycm}^{2}$. Conclusion: The availability of the DICOM structured patient dosimetry report and the technology advancement of personal dosimetry can conveniently support the optimisation radiation protection process in IR. The described experience supports the initiative of ISEMIR project (IAEA, Austria) that recommends the development of active dosimetry technology for a better monitoring of operators in IR and the development of a DICOM operator structured dosimetry report as prerequisite to define and develop software platforms aiming to combine patient and staff exposure information.

$\begin{array}{ll}14: 00-15: 30 & \text { Room N/O }\end{array}$

\section{Vascular}

\section{SS 1815}

\section{Vascular research}

Moderators:

S. Kuribayashi; Tokyo/JP

A. Pellegrin; Trieste/lT

\section{B-0999 14:00}

Hybrid-MRA steady state VIBE imaging - a delayed closer look at the vessels: evaluation of the additional diagnostic benefit in patients who undergo a peripheral magnetic resonance angiographic protocol M. Ong, K. Hausotter, S.O. Schönberg, H.J. Michaely;

Mannheim/DE (melissa.ong@umm.de)

Purpose: The purpose of this study was to evaluate the additional diagnostic benefit of high-resolution steady state VIBE imaging in peripheral MRA.

Methods and Materials: In this retrospective IRB-approved study 224 patients (72 women, 152 men, age $67.3 \pm 13.9$ ) were included who had undergone a peripheral MRA exam after injection of $0.1 \mathrm{mmol} / \mathrm{kg}$ gadobutrol including a large field of view MRA, time-resolved MRA of the calf station and steady state 3D VIBE sequence (3 T Siemens TimTrio, $1.3 \mathrm{~mm}$ isotropic spatial resolution, PAT 3, 1:26 min Tacq per station) prior to the time-resolved MRA. One board-certified radiologist rated image quality of the VIBE sequences on an ordinal three-point scale and analysed the images for additional diagnostic findings. Descriptive statistics and demographic patient data were used for further evaluation.

Results: The image quality of the VIBE sequences of the pelvis, upper and lower leg was excellent in up to $88 \%, 84 \%$ and $47 \%$, respectively. Poor image quality was only detected in the upper (2\%) and lower leg (6\%). The VIBE sequence yielded an additional diagnostic benefit in $44 \%$ of the patients overall. There was no significant difference in terms of additional findings between men and women ( $46 \%$ and $40 \%$, $p=0.4601$ ). The patient sample revealed a distribution of $59 \%$ inpatients and $41 \%$ outpatients with additional pathology found in approximately $44 \%$ for each group. Conclusion: Steady-state 3D VIBE sequences in peripheral MRA yield additional relevant diagnostic findings in $44 \%$ and above in patients older than 60 years irrespective of gender and patient status.

Author Disclosures:

H.J. Michaely: Consultant; Bayer Healthcare. 


\section{B-1000 14:09}

Multimodal imaging of atherosclerotic plaques using SPIO and quantum dot labelled high density lipoproteins in ApoE-/- mice C.S.L. Jung, H. Ittrich, M.G. Kaul, T. Ducic, O. Bruns, B. Freund, J. Hereren, G. Adam; Hamburg/DE

Purpose: To estimate superparamagnetic iron oxide (SPIO) and quantum dot (QD) labelled high density lipoproteins (HDL) for imaging of atherosclerotic plaques with MR and several ex vivo imaging techniques in ApoE-/- mice.

Methods and Materials: HDL nanosomes (HDL-NS) were synthesised by incorporation of QD and SPIO into the lipid core HDL-NS were characterised by dynamic light scattering (DLS), size exclusion chromatography (FPLC) and by cholesterol and fluorescent measurements. For evaluation of biodistribution and blood clearance Fe59 radioactive-labelled HDL-NS were applied intravenously (iv) and intraperitoneally (ip) into ApoE-/- mice. Radioactivity measurements of different organs were performed using a whole body y-counter (HAMCO). In vivo MRI using a T2* ${ }^{*}$-gradient echo sequence at $7 \mathrm{~T}$ (Clinscan, Bruker) was matched with ex-vivo MRI, ex vivo confocal fluorescence microscopy (CFM), $x$-ray fluorescence microscopy ( $x$-ray FM) and histological analysis (Prussian-Blue (PB) stains) to visualise the uptake of HDL-NS in atherosclerotic plaques.

Results: In vitro analyses confirmed equal size of HDL-NS compared to endogenous HDL. Radioactivity measurements at HAMCO showed a significant higher uptake of HDL-NS into atherosclerotic plaques after ip injection $(p<0.006)$ in addition to a predominant accumulation in liver and spleen. Blood concentration time curves of iv and ip application showed a biphasic progress. Ex-vivo MRI, $x$-ray FM, CFM and PB stains of aortic specimens showed an uptake of HDL-NS into atherosclerotic lesions of ApoE-/- mice.

Conclusion: Synthesis of HDL-NS with equal properties compared to native HDL was established. HDL-NS accumulation in atherosclerotic plaques can be visualised non-invasively by MRI proven by ex-vivo imaging techniques.

\section{B-1001 14:18}

Skin autofluorescence, a non-invasive marker for AGE accumulation, is elevated in subjects with evidence for subclinical atherosclerosis M.A.M. den Dekker, M. Zwiers, E.R. van den Heuvel, L.C. de Vos, A.J. Smit, M. Oudkerk, R. Vliegenthart, J.D. Lefrandt, D.J. Mulder; Groningen/NL (m.a.m.Dekker@rad.umcg.nl)

Purpose: Skin autofluorescence (AF) is a non-invasive marker for advanced glycation endproducts (AGEs) and predicts cardiovascular disease (mortality) in diabetes and renal disease. We examined whether skin AF is increased in subclinical atherosclerosis and whether it is associated with the degree of atherosclerosis independent of diabetes and renal function.

Methods and Materials: Cross-sectional study of 223 patients referred for primary $(\mathrm{PP} ; \mathrm{N}=163)$ or secondary $(\mathrm{SP} ; \mathrm{N}=60)$ prevention. Skin $\mathrm{AF}$ was measured using the AGE-reader and the degree of atherosclerosis was assessed based on ultrasonographically detected plaques in carotid and femoral arteries (in PP only), and computed tomography-derived coronary artery calcium score (CACS). PP group was divided into subjects with subclinical atherosclerosis defined as $>1$ plaque or CACS $>100$ ( $N=67$; age 53 years [interquartile range 48-56];49\% male) and those without (controls; $N=96 ; 43$ years [38-51];55\%) SP group consisted of patients with symptomatic peripheral arterial disease (PAD;64 years [58-70];73\%).

Results: Skin AF was higher in subjects with subclinical and clinical atherosclerosis compared with controls (skin AF 2.11 [1.83-2.46] and 2.71 [2.15-3.27] vs. 1.87 [1.68-2.12], respectively; $P=0.005$ and $<0.001$ ). In controls and PAD, skin AF was higher in those with CRP above median compared with below. In a multiple regression analysis, the association of skin AF with the 3 atherosclerosis categories was independent of age, gender, diabetes, Framingham Risk score, and kidney function. Conclusion: Skin AF is increased in subjects with subclinical and clinical atherosclerosis, independently of known confounders. These data suggest that AGEs are associated with the burden of atherosclerosis.

Author Disclosures:

A.J. Smit: Founder; DiagnOptics.
B-1002 14:27

Vascular enhancement and image quality of lower-extremity CT venography using $100 \mathrm{kVp}$ and ultrahigh pitch: comparison of conventional lower-extremity CT venography using $120 \mathrm{kVp}$ and standard pitch

K. Choo, J. Park, J. Kim, J. Roh, C. Park; Busan/KR (dorae007@hotmail.com)

Purpose: The objective of our study was to compare lower-extremity CT Venography (CTV) using $100 \mathrm{kVp}$ and ultra-high pitch with conventional lower-extremity CTV using $120 \mathrm{kVp}$ and standard pitch in the vascular enhancement and image quality. Methods and Materials: The 100 patients with suspected deep vein thrombosis and varicose vein were divided into two groups; one group (group A, 50 patients) was $100 \mathrm{kVp}$ and ultra-high pitch (3) and the other group (group B, 50 patients) 120 $\mathrm{kVp}$ and standard pitch (0.6). Vessel enhancement and image noise were assessed in the inferior vena cava (IVC), femoral vein, and popliteal vein. Two radiologists who were blinded to the protocol assessed image quality score by consensus using a 5-point visual scale. Effective dose was estimated using the dose-length product (DLP). The Wilcoxon rank test was used to evaluate differences between the two groups using statistics software (SPSS, version 13.0).

Results: Mean vascular enhancement of IVC, femoral vein and popliteal vein was significantly higher in the group $A$ than in the group $B$ and the images of the group A had significantly higher image noise. However, there were no significant differences in image quality score of the IVC, femoral vein and popliteal vein. The mean DLP in the group $A(402.10 \pm 94.29 \mathrm{mGy} \cdot \mathrm{cm})$ was significantly lower than that in the group $B(973.36 \pm 63.20 \mathrm{mGy} \cdot \mathrm{cm})(\mathrm{p}<0.001)$.

Conclusion: Although lower-extremity CTV using $100 \mathrm{kVp}$ and ultra-high pitch increased venous enhancement, it provided sufficient image quality and lower radiation dose.

\section{B-1003 14:36}

CT textural analysis of culprit lesions in transient ischaemic attack and/or stroke: correlation with immunohistochemistry E. Barden', L. Menezes'1, B. Ganeshan'1, M. Rodriguez-Justo', R. Endozo', C.W. Kotze' ${ }^{2}$, S.W. Yusuf ${ }^{2}$, A.M. Groves'; ${ }^{1}$ London/UK, ${ }^{2}$ Brighton/UK (edward.barden@doctors.net.uk)

Purpose: To investigate if texture analysis of computed tomography (CT) carotid angiograms can identify culprit lesions in Transient Ischaemic Attack (TIA) and/ or stroke, and correlate $\mathrm{CT}$ texture with immunohistochemistry of endarterectomy specimens, identifying neo-angiogenesis (VEGF) and macrophage density (CD68). Methods and Materials: Following institutional review board approval, 14 consecutive patients with symptomatic carotid stenosis awaiting endarterectomy were enrolled: 12 males, 2 females, mean age $( \pm S D) 67.6 \pm 6.7$ years. CT carotid angiography was performed. Axial CT images of the carotid bifurcation on each side were evaluated using $\operatorname{Tex} \mathrm{RAD}{ }^{\circledR}$, a commercially available texture analysis software algorithm. This comprised extracting texture features at fine, medium and coarse scales followed by quantification of the distribution of greyness, coarseness and heterogeneity within each lesion using mean grey-level intensity (MGI, average brightness) and proportion of positive pixels (PPP). The data were compared between symptomatic and asymptomatic sides (using Wilcoxon paired test), and correlated with quantitative immunohistochemical staining for macrophage density (CD68) and vascular endothelial growth factor (VEGF) of the endarterectomy specimens (using non-parametric Spearman's rank correlation).

Results: Medium textures differentiated between symptomatic and asymptomatic sides ( $\mathrm{MGl}$ and PPP, $\mathrm{p}=0.041$ ). Medium textures also correlated significantly (positively) with immunohistochemical markers (MGI vs CD68, $r s=0.680, p=$ 0.007 and PPP vs VEGF, $r s=0.640, p=0.014$ ).

Conclusion: CT textural analysis can identify culprit lesions in TIA and/or stroke and possibly distinguish between the pathophysiological basis (neo-angiogenesis versus macrophage infiltration) of vulnerable plaque.

\section{B-1004 14:45}

Non-contrast-enhanced MR angiography combined with peripheral cardiac gating at $3 \mathrm{~T}$ : comparison of respiratory triggered and breath held technique

T. Masui', M. Katayama ${ }^{1}$, K. Sato ${ }^{1}$, K. Terauchi', N. Takei ${ }^{2}$, K. Tsukamoto ${ }^{1}$, K. Mizuki', M. Miyoshi' ${ }^{2}$, H. Sakahara ${ }^{1} ;{ }^{1}$ Hamamatsu/JP, ${ }^{2}$ Hino/JP (masui@sis.seirei.or.jp)

Purpose: In non-contrast MRA (NCMRA) using FIESTA (GEHC) at 3 T, we found signal inhomogeneity due to systolic flow effects in aorta. The purpose was to evaluate feasibility of NC MRA at $3 \mathrm{~T}$ with peripheral pulse gating $(P G)$ for evaluation 
of renal artery using respiratory triggered $(\mathrm{RT})$ or breath held $(\mathrm{BH})$ in reference to dynamic contrast MRA (CMRA).

Methods and Materials: 33 patients were involved (median age 61 years). RT and BH NC MRAs using PG were obtained at $3 \mathrm{~T}$ (Discovery MR750, GEHC) using Inflow inversion recovery (IFIR) 3D FIESTA with fan beam k-space view ordering in a coronal plane. CMRA with 3D EFGRE was obtained as reference [0.1 mmol/kg Gd-chelate, injection $3 \mathrm{ml} / \mathrm{sec}]$. Evaluation, image quality, artefacts (1 undiagnostic- 5 excellent) and visualisation of aorta and renal arteries were ranked with 5-point scale. Contrast was calculated (SI artery/surrounding tissue). Wilcoxon signed rank test and Student's t test with Bonferroni correction was used for statistical evaluation.

Results: ALL NCMRAs were diagnostic (3.7-4.7). Scores for image quality and artefacts of RT NCMRA were better than those of BH NCMRA (NS) although visualisation of aorta was slightly better with BH than RT NCMRA. RT NCMRA provided better visualisation of distal renal arteries and better contrast than those with CMRA $(p<0.05)$. Contrasts of aorta and proximal renal arteries were slightly better in CMRA than those in NCMRAs (NS).

Conclusion: With PG, homogenous SI in aorta was obtained in NCMRA. When image quality of RT NCMRA is not enough to evaluate arteries, BH NCMRA can be used as substitute.

Author Disclosures:

N. Takei: Employee; GE Healthcare.M. Miyoshi: Employee; GE Healthcare.

\section{B-1005 14:54}

Low $\mathrm{kV}$ settings $\mathrm{CT}$-angiography with ultra low contrast medium volume for the assessment of thoracic and abdominal aorta disease: a feasibility study

C.R.G.L. Talei Franzesi, D. Ippolito, P.A. Bonaffini, D. Fior, O. Minutolo, S. Sironi; Monza/IT (ctfdoc@hotmail.com)

Purpose: To evaluate the quality, the diagnostic performance and the radiation exposure of low-kV CT angiography protocol (100 kV) with ultra low-contrast medium volume $(40 \mathrm{~mL})$ in the assessment of the aorta disease.

Methods and Materials: Seventy patients with thoracic or abdominal aortic disease were prospectively examined with 256MDCT scan (Brilliance iCT, Philips) using ultra low-dose radiation protocol (100 kV; automated tube current modulation) and ultra low-contrast volume ( $40 \mathrm{~mL} ; 4 \mathrm{~mL} / \mathrm{s} ; 350 \mathrm{mgl} / \mathrm{mL})$. In the thoracic aorta assessment we performed ECG-gated retrospective protocol, necessary for the evaluation of ascending aorta. Density measurements were performed on ascending, arch, descending, abdominal aorta, renal arteries and common iliac arteries. A control groups of 50 patients who underwent standard CT-angiography protocol (120 kV; $350 \mathrm{mAs}$ ) and standard contrast volume $(120 \mathrm{~mL})$ were also evaluated. The obtained data in terms of radiation dose exposure (dose-length product, DLP), administered contrast and intravessels density were compared and statistically analysed.

Results: In every CT-exam we could correctly visualise and evaluate main branch of thoracic and abdominal aorta. No significant difference of density measurements was achieved between the low- $\mathrm{kV}$ protocol: mean attenuation value of thoracic aorta $304 \mathrm{HU}$, abdominal aorta $343 \mathrm{HU}$ and renal arteries $331 \mathrm{HU}$; and control group: mean value of thoracic aorta $320 \mathrm{HU}$, abdominal aorta $339 \mathrm{HU}$ and renal arteries $303 \mathrm{HU}$. The radiation dose exposure in low-kV protocol was significantly reduced $(p<0.05)$ in comparison with control group, obtaining the following value for thoracic scan: 490DLP and abdominal scan: 335DLP, while in control group we obtained for the thoracic scan: DLP625 and for the abdominal scan: DLP952, respectively. Conclusion: Low-kV protocol provides a comparable diagnostic performance with standard protocol, decreasing significantly the radiation dose exposure (over $40 \%$ ) and allowing also a significant reduction of contrast material volume $(40 \mathrm{~mL})$, preserving renal function.

\section{B-1006 15:03}

$\mathrm{X}$-ray phase-contrast imaging of arterial vessel wall: translation from synchrotron radiation to a conventional lab-based $x$-ray source

H. Hetterich'1, M. Willner' ${ }^{2}$, S. Fill', F. Bamberg', J. Herzen'2,

M. Stockmar ${ }^{2}$, F. Pfeiffer ${ }^{2}$, M.F. Reiser ${ }^{1}$, T. Saam ${ }^{1} ;{ }^{1}$ Munich/DE,

${ }^{2}$ Garching/DE (holger.hetterich@med.uni-muenchen.de)

Purpose: Phase-contrast imaging $(\mathrm{PCl})$ is a novel $\mathrm{x}$-ray-based technique that provides excellent soft tissue contrast but so far was depending on synchrotron radiation. The aim of this study was to evaluate the feasibility of visualising human carotid arteries by grating-based $\mathrm{PCl}(\mathrm{gb}-\mathrm{PCl})$ at two different experimental setups: (1) applying synchrotron radiation and (2) using a conventional $x$-ray tube. Methods and Materials: Five ex-vivo carotid artery specimens were examined with $\mathrm{gb}-\mathrm{PCl}$ either at a synchrotron facility using a monochromatic $\mathrm{x}$-ray beam $(\mathrm{N}=2$;
$23 \mathrm{keV}$; pixel size $5.4 \mu \mathrm{m}$ ), or at a laboratory set-up with a conventional x-ray tube $(\mathrm{n}=3,35 \mathrm{kVp}, 70 \mathrm{~mA}$, pixel size $100 \mu \mathrm{m})$. Tomographic images were reconstructed and compared to histopathology. Independent readers determined vessel dimensions and signal-to-noise ratios (SNR) between gb-PCl and absorption images. Results: In total, 51 sections were included in the analysis. Images from both setups provided sufficient contrast to differentiate vessel layers. There was a strong positive correlation between $\mathrm{PCl}$ and histology with respect for lumen, intima and vessel wall area for both synchrotron and laboratory-based measurements (Pearson's $R>0.94$ and $p<0.01$ for all). Synchrotron-based images were characterised by significantly higher SNRs than laboratory-based images $(p<0.001)$. Both gb-PCl set-ups had superior SNRs compared to corresponding absorption-based images $(p<0.001)$. Inter-reader reproducibility was excellent, with ICC $>0.98$ for synchrotron and $>0.84$ for laboratory-based measurements.

Conclusion: Our results demonstrate the feasibility of gb-PCl for carotid atherosclerotic plaque imaging using both synchrotron and laboratory-based experimental set-ups. The technique holds promise for accurate vessel wall characterisation.

\section{B-1007 15:12}

Single contrast medium dose peripheral MR angiography is feasible

without subtraction using two-point Dixon fat saturation

T. Leiner ${ }^{1}$, J. Habets ${ }^{1}$, B. Versluis ${ }^{2}$, L. Geerts ${ }^{3}$, J. Hendrikse ${ }^{1}$,

E.-J. Vonken', H. Eggers ${ }^{4} ;{ }^{1}$ Utrecht/NL, ${ }^{2}$ Maastricht/NL, ${ }^{3}$ Best/NL,

4Hamburg/DE (t.leiner@umcutrecht.nl)

Purpose: To investigate the feasibility and image quality of subtractionless firstpass single contrast medium dose $(0.1 \mathrm{mmol} / \mathrm{kg})$ peripheral contrast-enhanced magnetic resonance angiography (MRA) using two-point Dixon fat-saturation (2DixFS) compared with conventional subtraction-based MRA with regard to vesselto-background contrast, signal-to-noise ratio (SNR) and subjective image quality. Methods and Materials: 28 patients (13M, $15 \mathrm{~F}$; mean age \pm SD, $66 \pm 16$ ) with known or suspected peripheral arterial disease underwent single contrast medium dose (0.1 mmol/kg body weight) $1.5-$ T MRA using 2DixFS during first arterial passage of contrast material. Results were compared with data obtained using a conventional, subtraction-based approach. A phantom study was performed to assess signal-to-noise ratio (SNR) of both MRA techniques. Vessel-to-background (VTB) contrast and SNR were measured and compared with the paired samples t-test. Two experienced observers scored subjective image quality. Fisher's exact test was used to compare subjective image quality. Agreement regarding subjective image quality was expressed in quadratic weighted $\mathrm{k}$ values.

Results: Patient data showed improved VTB contrast in all anatomical locations with 2DixFS versus the conventional, subtraction-based MRA method (all $P<0.001$ ). Subjective image quality was uniformly higher with 2DixFS when compared with subtracted images. In all cases this was significant $(P<0.03)$, except the aortoiliac arteries for observer $1(\mathrm{P}=0.052)$. Phantom studies indicated a $15 \%$ higher SNR with the Dixon technique (31.9 vs 27.6$)$.

Conclusion: This study demonstrates the feasibility of single contrast medium dose subtractionless lower extremity MRA using 2DixFS method. Both objective and subjective image quality are better compared with subtraction based MRA of the peripheral vascular tree.

Author Disclosures:

T. Leiner: Advisory Board; Bayer Healthcare, Philips Healthcare.L. Geerts: Employee; Philips Healthcare.H. Eggers: Employee; Philips Healthcare.

\section{B-1008 15:21}

Correlation of cardiovascular risk factors and occult atherosclerotic findings using whole body magnetic resonance imaging of the vascular system in an asymptomatic patient collective: initial results S. Mangold, E. Randrianarisoa, P. Krumm, C. Bretschneider,

A. Seeger, K. Rittig, B. Balletshofer, C.D. Claussen, U. Kramer;

Tübingen/DE (Stefanie.Mangold@med.uni-tuebingen.de)

Purpose: To evaluate the prevalence of atherosclerotic disease, myocardial infarction and cerebrovascular disease in asymptomatic patients with cardiovascular risk profile including whole body magnetic resonance imaging (WB-MRI) of the cardiovascular system into the framework of a comprehensive prevention concept. Methods and Materials: Prospectively, 52 patients without any known cardiovascular disease (34 men, 18 woman, mean age 50.1 7.4 years) but suffering from cardiovascular risk factors such as arterial hypertension [AHT], impaired glucose tolerance, hyperlipidaemia and obesity, were clinically assessed including family history, blood collection, blood pressure assessment and oral glucose tolerance test. Furthermore, the carotid intima-media thickness [CIMT], potentially indicative for atherosclerotic disease was assessed by ultrasound and a WB-MR angiography (1.5 T, Avanto, Siemens Medical Solutions) containing an examination of the 
brain and the heart was performed. A vessel score, determined from the sum of arteriosclerotic changes of all evaluated vessel divided by the number of vessels with a range from 1, normal to 6 , stenosis $>99 \%$, was introduced and score's association with risk factors was assessed.

Results: The mean MRI score was $1.51 \pm 0.18$. The WB-MR angiography-based score was significantly associated with the body mass index [BMI] $(p=0.024)$ and AHT in combination with an elevated BMI $(p=0.042)$. No significant associations were found for age $(p=0.706)$, sex $(p=0.502)$, impaired fasting glucose $(p=0.277)$, hyperlipidaemia $(p=0.218)$ and CIMT $(p=0.183)$. No patients with myocardial infarction and 2 patients with cerebrovascular disease were found.

Conclusion: WB-MR angiography allows the detection of occult atherosclerotic disease in asymptomatic patients with cardiovascular risk profile especially in cases of AHT and/or obesity.

\section{4:00 - 15:30}

Room P

\section{Paediatric}

\section{SS 1812}

\section{Paediatric brain}

Moderators:

M.A. Lucic; Sremska Kamenica/RS

M. Stenzel; Jena/DE

\section{B-1010 14:00 \\ Ultra-low-dose CT of the inner ear with adaptive statistical image reconstruction in infants candidate to cochlear prosthesis implantation: preliminary experience \\ L. Faggioni, E. Neri, L. Bruschini, V. Seccia, S. Sellari Franceschini, \\ C. Bartolozzi; Pisa/IT (Ifaggioni@sirm.org)}

Purpose: To evaluate image quality and radiation dose of ultra-low-dose CT examinations of the inner ear performed using adaptive statistical image reconstruction (ASiR) in infants candidate to cochlear prosthesis implantation.

Methods and Materials: We evaluated 24 infants (14 males, 10 females, age 4-29 months, mean 17 months) with sensorineural deafness who underwent CT of the inner ear before cochlear prosthesis implantation. Out of them, 13 were imaged on a 128-row CT scanner (Discovery CT750 HD, General Electric, Milwaukee, WI) using an ultra-low dose protocol $(80 \mathrm{kV}, 10 \mathrm{~mA}, 0.5 \mathrm{~s}$ gantry rotation time) and a model-based iterative reconstruction algorithm ( $\left.A S R^{\mathrm{TM}}\right)$, while in the remaining 11 CT had been performed on a 64-row scanner before the introduction of the 128-row CT equipment at our Institution, using a regular paediatric protocol (100 kV, $30 \mathrm{~mA}$, 0.5 s gantry rotation time) with conventional filtered backprojection. Dose-length product (DLP) values were obtained from dose reports generated from the scanner at the end of each examination. Image quality was rated in blind by two radiologists using a Likert scale ( $1=$ non-diagnostic through $5=$ excellent).

Results: None of the examinations were rated as non-diagnostic. Image quality was comparable between the 128 - and the 64-row CT group $(4.45 \pm 0.63$ vs $4.51 \pm 0.56$, respectively; $p=0.31)$. DLP was significantly lower in the 128-row CT group ( $5.46 \pm 0.35$ vs $25.69 \pm 0.83 \mathrm{mGy}^{*} \mathrm{~cm}$, respectively; $\left.p<0.001\right)$.

Conclusion: Compared with a regular paediatric protocol, ultra-low-dose CT of the inner ear with ASiR allows for markedly reduced radiation dose with preserved image quality.

\section{B-1011 14:09}

Traumatic subdural hygromas in children between 0 and 2 years: a retrospective $C T$ and $M R I$ study

M.L. Hahnemann ${ }^{1}$, A. Schmeling ${ }^{2}$, M. Schlamann ${ }^{1}$, M. Forsting ${ }^{1}$, H. Pfeiffer ${ }^{2}$, D. Wittschieber ${ }^{2} ;{ }^{1}$ Essen/DE, ${ }^{2}$ Münster/DE

Purpose: Non-accidental head injuries (NAHI) represent a leading cause of death in infants. In this context, the role of traumatic subdural hygromas (SDHy) as possible acute or chronic finding in child abuse, especially in shaken-baby-syndrome, is not well understood and still a matter of debate. By modern imaging techniques, the present study aimed to investigate the characteristics of $\mathrm{SDHy}$.

Methods and Materials: From the years 1999-2012, we retrospectively analysed the images of all children between 0 and 2 years who were suspected to have a craniocerebral injury and got an initial cranial computer tomography at the University Hospital Essen. In cases where additional MR examinations existed these MR images were also analysed. All skeletal and cerebral pathologies, demographic data, and, if existing, related clinical anamneses and findings were recorded. All $\mathrm{SDHy}$ cases were classified according to both a classification system originally developed for adult trauma patients in 2008 and a novel approach based on recent literature and our additional observations.

Results: Of the 14 years considered, 136 cases met the inclusion criteria. In 33 cases a NAHI was assumed. 17 cases showed SDHy. Only one SDHy case was assumed to be related to an accident. The new classification system for traumatic SDHy in children will be introduced and discussed.

Conclusion: As demonstrated for different SDHy appearances, neuroradiologists should be aware of the possible presentations of NAHI. In addition, investigation of SDHy's may allow for new insights into the pathogenesis of phenomena such as the shaken baby syndrome.

\section{B-1012 14:18}

Apparent kurtosis coefficient (AKC) in brain: a feasibility study in paediatric populations

I. D’Errico, A. Ciccarone, M. Esposito, M. Mortilla, C. Fonda; Florence/IT (igni77@hotmail.it)

Purpose: Diffusion-weighted imaging (DWI) depends on the b-values employed in acquisition. At low b values (lower than $500 \mathrm{~s} / \mathrm{mm} 2$ ) the signal attenuation is bi-exponential and is influenced both by diffusion and perfusion. At high $b$ values (higher than $1000 \mathrm{~s} / \mathrm{mm} 2$ ) the signal attenuation is influenced by restricted water diffusion and hence follows a non-Gaussian distribution. Diffusion Kurtosis Imaging (DKI) provides quantifiable information about the deviation from Gaussian distribution in water diffusion process. Our purpose was to apply DKI method to paediatric patients with different pathologies (tumours, ischaemia) and to establish its feasibility in detecting brain diseases.

Methods and Materials: We examined 20 patients from newborn to 16-year-old. Diffusion weighted imaging was performed with 5 b values from 500 to 2500 with step $500 \mathrm{~s} / \mathrm{mm} 2$. Fitting all b values we were able to discriminate Diffusion and Kurtosis parameters. A home-made software performed all fitting and DWI, ADC, AKC maps.

Results: AKC maps revealed additional information for tissue characterisation. In ischaemia, AKC demonstrated more details of pathologic tissue changes and provided information about prognosis. In tumours, AKC maps were used for discriminating low-grade from high-grade lesions revealing a better accuracy than conventional diffusion parameters.

Conclusion: AKC maps are non-invasive methods that are well tolerated by paediatric patients and that provide a more detailed characterisation of neural tissue in the clinical context. Their application in pathological conditions such as ischaemia and tumours provides additional information about microstructural tissue changes, differential diagnosis and prognosis.

\section{B-1013 14:27}

Which T1 pulse should be used to study the preterm brain with a 3 Tesla scanner?

D. Tortora, V. Panara, P.A. Mattei, S. Salice, M. Tagliamonte, C. Briganti, A.R. Cotroneo, A. Tartaro, M. Caulo; Chieti/IT (domenicotortora@hotmail.it)

Purpose: The loss of contrast on MR T1-weighted images obtained at 3 Tesla (3 T) may negatively influence the detection of punctate hyperintense lesions, which are indicative of periventricular leukomalacia (PVL) in preterm neonates. In this 3 T MR study we compared the sensitivity of different T1-weighted sequences in identifying $P V L$

Methods and Materials: The presence of non cystic-PVL was retrospectively evaluated in 3 Tesla (3 T) MR brain studies of 200 preterm neonates acquired at term-corrected age. In 30 neonates with PVL, T1 hyperintense punctate lesions were counted by two neuroradiologists in consensus on 4 different axial T1-weighted sequences: $3 \mathrm{~mm}$ Inversion Recovery (IR), Spin Echo (SE), reformatted 3D-Fast Field Echo (FFE) and $1 \mathrm{~mm}$ reformatted 3D-FFE. Sequences were presented randomly. Statistical differences of the number of hyperintense lesions detected by the different sequences were evaluated using the Student's paired t test $(p<0.05)$ Results: The greater number of T1 hyperintense punctate lesion was identified using the $1 \mathrm{~mm}$ axial reformatted 3D-FFE sequence (381 lesions), which resulted to be the most sensitive sequence in identifying PVL lesions $(p<0.0001)$. Axial 3 mm FFE, IR and SE sequences identified 213, 146 and 94 hyperintense punctate lesions, respectively. No statistical differences were found between $3 \mathrm{~mm}-\mathrm{T} 1$ sequences ( $p>0.05)$.

Conclusion: When using a 3 Tesla MR scanner $1 \mathrm{~mm}$ axial reformatted T1-3D-FFE is the most sensitive T1-weighted sequence for detecting punctate hyperintense lesions which may affect the brain of preterm neonates. 


\section{B-1014 14:36}

Paediatric brain tumour classification by quantitative methods of magnetic resonance imaging: combination of DTI, PWI and MRS

S. Chełstowska, E. Jurkiewicz, I. Pakuła-Kościesza, K. Nowak, K. Malczyk, S. Rączkowska, M. Armata, J. Walecki; Warsaw/PL (sylwia.chelstowska@gmail.com)

Purpose: Preoperative classification of brain tumours have limitations. Advanced imaging techniques, including diffusion tensor imaging (DTI), perfusion-weighted imaging (PWI), and magnetic resonance spectroscopy (MRS) can provide more information than conventional magnetic resonance imaging.

Methods and Materials: We retrospectively evaluated 45 patients (27 girls and 18 boys, aged 2-19 years old, mean age 13) with histologically proven 29 low- and 16 high-grade gliomas. Standard MR oncological protocol includes MRS, DTI and PWI. 2D CSI sequence was used, with short ( $30 \mathrm{~ms})$ and long ( $135 \mathrm{~ms}$ ) TE and TR $=1500 \mathrm{~ms}$. The U Mann-Whitney and Wilcoxon tests were employed to compare apparent diffusion coefficient (ADC) and fractional anisotropy (FA) generated from DTI, cerebral blood volume ( $\mathrm{rCBV}$ ) generated from PWI and MRS data, keeping the division for low and high grade tumours. $p$ value of up to 0.05 was considered statistically significant.

Results: It was observed statistically significant difference for rCBV maps calculated as a ratio of tumour and normal tissue. Moreover NAA/Cr and $\mathrm{Cho} / \mathrm{Cr}$ ratios were also statistically significant for low- and high-grade tumours. ADC maps, FA data were correlated with rCBV maps. Analysis of the metabolites ratios showed strong correlation between increased ratios of Cho/NAA and Cho/Cr and malignancy of the tumour. The presence of lactate and lipid was predominantly detected in high-grade gliomas.

Conclusion: Combination of PWI, DTI and MRS with conventional MR imaging increases the accuracy of estimation of tumour malignancy. The best statistically suitable parameter was found to be the perfusion rCBV maps.

\section{B-1015 14:45}

Perfusion parameters of pilocytic astrocytomas: additional oddities of these tumours

M. Martucci, S. Gaudino, E. Gangemi, R. Colantonio, G. Di Lella, C. Colosimo; Rome/IT (matia.martucci@gmail.com)

Purpose: To present perfusion data of 8 paediatric pilocytic astrocytomas (PA). To compare these data with those of low-grade gliomas (LGG), high-grade gliomas (HGG) and metastases (Met). To discuss their clinical relevance.

Methods and Materials: We reviewed conventional, diffusion and perfusion (DSC-GRE EPI) MRI of 8 paediatric PA. Maximum rCBV within enhancing and non-enhancing tumours, minimum and mean percentage of signal recovery (PSR) were obtained. PSR-Tmax was calculated as the percentage of signal intensity recovered at the end of acquisition (time maximum). The increasing of the curve was defined as the difference between PSRTmax and PSR. Results were compared with those from $8 \mathrm{HGG}, 8 \mathrm{LGG}$ and 8 Mets. Data were presented as mean value $\pm S \mathrm{SD}$. Statistical comparisons were performed with Student's t and Mann-Whitney tests ( $p<0.05$ significant).

Results: rCBV of PA $(1.44 \pm 0.27)$ did not show significant differences between enhancing and non-enhancing regions and resulted slightly higher than LGG $(1.2 \pm 0.35$,$) . PSR was higher than 80 \%$ in PA and LGG; Mets showed lowest PSR (PSRmean 61.07 \pm 11.17 ). At Tmax PA showed highest PSR (>90\%), and in 5 cases the curve overshot the baseline. PA demonstrated increasing of the curve closer to HGG than LGG.

Conclusion: Perfusion parameters should be considered additional oddities of PA: some perfusion behaviour resulted peculiar (curve crossing the baseline), other dissimilar to LGG (higher rCBV) and other similar to HGG (curve increasing in time). These characteristics may be explained by the peculiar vascular wall and the size of interstitial space.

Author Disclosures:

C. Colosimo: Consultant; Research Consultant Bracco Group, Research Consultant Bayer AG.
B-1016 14:54

Evaluation of metabolic changes within the normal appearing grey and white matters in children with growth hormone deficiency: magnetic resonance spectroscopy and hormonal correlation J. Bladowska, A. Zimny, A. Zacharzewska, T.M. Gondek, A. Banaszek, T. Żak, A. Noczyńska, M. Sąsiadek; Wroclaw/PL (asia.bladowska@gmail.com)

Purpose: The pathogenesis of idiopathic growth hormone deficiency (GHD) in children, including possible cerebral metabolic alterations, remains unclear. The aim of the study was to evaluate the metabolic changes within the normal appearing brain in children with GHD using MR spectroscopy (MRS) and to correlate MRS measurements with hormonal concentrations.

Methods and Materials: Seventy-one children with GHD (mean age $6.9 \mathrm{yrs}$ ) and 11 healthy controls (mean age $8.4 \mathrm{yrs}$ ) were enrolled in the study. The MRS examinations were performed on $1.5 \mathrm{~T}$ scanner. Voxels were located in the posterior cingulate gyrus (PCG) and the left parietal white matter (PWM). The NAA/Cr, Cho/Cr and $\mathrm{ml} / \mathrm{Cr}$ ratios were analysed in both groups. There were also evaluated correlations between the metabolite ratios and hormonal concentrations: growth hormone $(\mathrm{GH})$ in two stimulation tests and GH during the night, as well as IGF-1 (insulinlike growth factor) and IGFBP3 (insulin-like growth factor-binding protein) levels. Results: There was statistically significant $(p<0.05)$ decrease of the NAA/Cr ratios in PCG and PWM in children with GHD compared to the normal subjects. Other metabolite ratios showed no significant differences. We found also statistically significant positive correlations between NAA/Cr ratio in PWM and IGFBP3 level, as well as $\mathrm{GH}$ concentration in stimulation test with glucagon.

Conclusion: The reduction of NAA/Cr ratios may suggest loss of neuronal activity within normal appearing grey and white matters in children with GHD, thus MRS could be sensitive marker of cerebral metabolic disturbances associated with GHD and additional indicator for therapy with recombinant $\mathrm{GH}$.

\section{B-1017 15:03}

Functional correlation of the spastic hemiplegic patients with MR findings K. Hayakawa, S. Yoshida, H. Tatekawa, Y. Yamori, T. Kanda, N. Yoshida,

H. Hirota, M. Iwami, K. Nakamura; Kyoto/JP (hayakawakatsumi@hotmail.com)

Purpose: The objective of this study is to correlate MR findings with their clinical function of children with congenital spastic hemiplegia.

Methods and Materials: The subjects consisted of 20 male and 16 female patients diagnosed as congenital spastic hemiplegia. Their ages at MR exam ranged from 9 months to 27 years (mean age $=5.5$ years). Their MR lesion categories were classified into the three types comprising periventricular white matter lesion (PV) $(\mathrm{N}=17)$, cerebral cortical and subcortical lesion (CSC) $(\mathrm{N}=11)$, malformation (Mal) $(\mathrm{N}=8)$. We evaluated the correlation between these three lesion categories and MR findings and their clinical functions.

Results: The incidences of mental retardation were correlated with lesion category ( $25 \%$ in PV, $18 \%$ in CSC and $100 \%$ in Mal, $p=0.0004$ ). Those of epilepsy were correlated with lesion category (24\% in PV, $64 \%$ in CSC and $88 \%$ in Mal, $p=0.007)$, cerebral hemiatrophy $(p<0.001)$, brainstem hemiatrophy $(p=0.004)$ and ventriculomegaly $(p=0.04)$. Those of walking age by the 18 months were correlated with lesion category ( $82 \%$ in PV, $70 \%$ in CSC and $13 \%$ in Mal, $p=0.002$ ). Those of school performances (the percentage of handicapped class) were correlated with lesion category (14\% in PV, $11 \%$ in CSC and $100 \%$ in Mal, $p=0.001$ ). Those of the ipsilateral hand functions were not correlated with any parameter. The favourable clinical outcomes were correlated with the disease category of PV. The worse functional outcomes were correlated with the disease category of Mal.

Conclusion: The functional prognosis of spastic hemiplegia is significantly correlated with three types of brain lesion on MRI.

\section{B-1018 15:12}

Diffusion tensor tractography and fractional anisotropy in paediatric cortical abnormalities evaluation with TBSS analysis

A. Ciccarone, I. D'Errico, M. Esposito, M. Mortilla, C. Fonda; Florence/IT (a.ciccarone@meyer.it)

Purpose: The microstructure of tissues and white matter tracts in vivo may be studied with DTI. The alterations involving the cerebral cortex associated in various degrees with subcortical white matter abnormalities often affect the projection white matter tracts. The white matter in cortical brain abnormalities is evaluated with diffusion tensor imaging (DTI) and Tract-Based Spatial Statistics (TBSS).

Methods and Materials: 65 patients (1year-14year range) 39 males and 26 females) with different pattern of cortical alterations were studied. The DTI data was processed in FSL. For each DTI exam, brain extraction only of images with $b$ value equal to zero was performed so that a mask of entire brain was created. 
Templates were built taking into account the age of patients. Artefact movements and eddy current distortions were removed. Mean diffusivity (MD) and fractional anisotropy (FA) were quantified together to the three principal directions of diffusion (eigenvectors) with their own eigenvalues (tensor diagonalization). So analysis of single fibre orientation for each voxel with a bayesian algorithm was executed. Voxelwise statistical analysis of the FA, MD and axial diffusivity data was carried out using TBSS between focal cortical abnormalities sites and normal ones.

Results: Different pattern of cortical organization and white matter projections are evidenced depending of lesional load and extension, site and age. In focal cortical abnormalities the TBSS analysis revealed significant differences $(p<0.05)$ between affected cortical sites and normal ones.

Conclusion: Different FA data were collected. The reductions of FA, the associated elevation of diffusivity in altered contiguous or distal area are evidenced.

\section{4:00 - 15:30}

Room Q

\section{Radiographers}

\section{SS 1814}

\section{Challenges of different imaging techniques}

\section{Moderators:}

H.H. Hjemly; Olso/NO

J. McNulty; Dublin/IE

\section{B-1019 14:00}

Occupational exposition to electromagnetic fields in MR:

understanding the discussion around the directive 2004/40/EC

C.S.L. Santos, D.P. Rocha, M.M.C.P. Ribeiro; Lisbon/PT

(catiasantos.rm@gmail.com)

Purpose: The magnetic resonance imaging (MRI) uses the magnetic field effects to its functioning. This study aimed to clarify the EMF Directive 2004/40/EC discussion which imposes limits to electromagnetic fields (EMF) regarding the occupational exposure.

Methods and Materials: It was explored policy documents, regulations, guidelines, laws and other official documents on this subject. To understand the reason of this controversy, the evidence of consequences from the EMF exposure over professionals was analysed. From the information obtained an online survey was developed, with national and international coverage, towards 11 professional groups considered involved in the controversy. The opinions were analysed by the force field analysis diagram, through the Policymaker K4-health ${ }^{\mathrm{TM}}$ application to speculate the influences, forces, power and strategies developed in this process. Results and Conclusion: From 50 respondents $62 \%$ do not know the ESR's position, the Directive and its contents. $60 \%$ know the safety measures to apply in the MR room and report adverse events for lack of compliance with safety standards, being the levels of SAR, the most topic referred. They do not know the limits imposed and have no opinion about the Directive's controversy $(40 \%)$. The political impact is considered neutral (49\%) against the negative clinical and economic impacts $(54 \%)$ that falls over the equipment manufacturers $(62 \%)$. The professional societies have most decision, followed by physicists, doctors, manufacturers, government decision-makers and health-managers. The manufacturers, radiographers and supervisors have median influence, and the patients have weak influence over this process. On this matter influences and pressure strategies over the European Commission and Council were recognised.

\section{B-1020 14:09}

Radiographers perceptions of magnetic resonance imaging: a study of the causes that lead to the repetition of exams

T.R. Filipe ${ }^{1}$, L.P.V. Ribeiro', R.P.P. Almeida ${ }^{1}$, S.I. Rodrigues ${ }^{1}$, K.B. Azevedo', C.A. Silva ${ }^{2}$, A.F.L. Abrantes ${ }^{1} ;{ }^{1}$ Faro/PT, ${ }^{2}$ Évora/PT (tiagofilipe_@ @hotmail.com)

Purpose: To know how often repetitions of MRI exams and sequences occur in radiology departments.

Methods and Materials: A self-applied questionnaire was used as instrument and assigned to 57 radiographers who performed MRI exams to determine which were the causes that lead to the repetition. The questionnaires were interpreted and statistically analysed through descriptive statistics and Spearman's rho correlation. Results: At a 95\% confidence interval, the major results suggest that the patient's movement during the MRI exams is the main cause to repeat this exams (mean of 3.88 on a 5-point Likert scale). However, there are causes related to the radiographer's and the results showed that the introduction of wrong imaging parameters is a major cause too $(\mathrm{N}=26)$. Spearman rho correlations between radiographer's time of experience and frequency of MRI exams repetitions were poor and not significant $(r=0.141 ; p=0.297)$. The correlations between radiographer's tiredness and frequency of MRI exams repetitions were negative, weak and not significant $(r=-0.151 ; p=0.263)$.

Conclusion: The patients' movement may disrupt the examination or degrade the images with artefacts. The level of experience does not influence the repetitions of MRI exams, it seems that senior radiographers do not have improvements in performance as it should be expected. It is recommendable to do training courses regularly to improve the performance and systematically evaluate. Several features will need to be identified which would decrease the MRI exams repetitions.

\section{B-1021 14:18}

Functional mapping of the visual word form area with frequent words of the Portuguese lexicon: an fMRI study

C. Ferreira ${ }^{1}$, P. Martins ${ }^{2}$, G. Cunha ${ }^{1}$, N. Canário ${ }^{1}$, C. Nunes ${ }^{1}$, A.C. Miranda ${ }^{2}$,

J. Ribeiro ${ }^{2}$, S. Afonso ${ }^{2}$, M. Castelo-Branco ${ }^{1} ;{ }^{1}$ Coimbra/PT, ${ }^{2}$ Aveiro/PT

(c_dferreira@yahoo.com)

Purpose: The visual word form area (VWFA), part of the fusiform gyrus in the temporal lobe underlies word form encoding. Impairment of this region causes reading deficits, including alexia. The aim of this study is to functionally map VWFA at the individual subject level using frequent words of the Portuguese lexicon, to develop a paradigm potentially useful for clinical patients.

Methods and Materials: Functional magnetic resonance images (fMRI) were acquired in from nine subjects. The visual paradigm used consisted of 13 blocks (30 seconds block duration): 7 baseline (checkerboard stimuli), 3 blocks of frequent words and 3 of pseudo-words ( 40 words/pseudowords per block). General linear model analysis was performed using BrainVoyager $Q x$ to identify brain regions encoding word form.

Results: An area corresponding to VWFA could be mapped in both hemispheres in all subjects at a statistical threshold of 0.001 , corrected for multiple comparisons. Seven of the cases had predominant activation in the left hemisphere, one has equivalent activations bilaterally and one subject has predominant activation in the right hemisphere. The number of activated clusters was significantly larger in the left hemisphere at the group level ( $p=0.015$, Wilcoxon signed rank test). Conclusion: Bilateral activation was replicated in VWFA in all subjects, with a clear left hemispheric dominance demonstrating the robustness of the paradigm, making it potentially useful in a clinical setting, e.g. pre-surgical mapping.

\section{B-1022 14:27}

A survey of the various methods and techniques employed in myocardial stress testing

K. Borg Grima ${ }^{1}$, L. Rainford ${ }^{2}$, P. Bezzina ${ }^{1}$, D. O'Leary ${ }^{2} ;{ }^{1} M$ sida/MT, ${ }^{2}$ Dublin/IE (karen.borg-grima@um.edu.mt)

Purpose: Literature suggests a range of pharmacological stress agents and radioactive tracers, together with a variety of defined protocols that may be used in conducting myocardial stress testing prior to cardiac scintigraphy imaging. An online survey was performed to evaluate current practice.

Methods and Materials: A survey was developed comprising of twelve questions, by using 'Survey Monkey', which aimed to identify the variances present in conducting myocardial stress testing and in clinical protocol details if available. Professional groups working in Nuclear Medicine, such as the medical-physicsengineering community and virtual radiopharmacy, were targeted. Access to the survey remained open for eight months. Forty-three members, including both radiographers and medical physicists responded from across Europe and Australasia. SPSS was used to evaluate the results obtained, based on Chi-square tests and comparisons between multiple responses.

Results: The majority (72\%) of the participants were from United Kingdom, 18\% from other European countries and 9\% from Australasia. In 39 centres pharmacological stress testing was performed either alone or in conjunction with exercise stress testing. The results indicated that $60 \%$ of the participants were aware of local stress test protocols; however, guidelines indicating which patients were not suitable for pharmacological stress tests may require clarification.

Conclusion: Results suggest that for pharmacological stress testing there is a need for increased awareness of protocols, and across all responders the need for improved knowledge and understanding of local protocols was identified. This initial survey justifies further research towards increase protocol compliance and standardisation of practice during myocardial stress testing. 


\section{B-1023 14:36}

Importance of scout imaging in neuroradiologic studies

D.C. Martin, S.H. Erbay, M. Hakky, K.D. Erbay, N. Erbay, D. Marrero;

Burlington, MA/US (dann.martin@tufts.edu)

Purpose: Scout images are essential in neuroradiology. Though these images may have limited diagnostic quality, they visualise larger parts of the body. Therefore, certain radiologic findings may be visualised only or largely in the scout images and truly constitute unexpected radiologic findings (UF). Impact related to spinal location and imaging modality on to the incidence and distribution of UF remains unknown. Methods and Materials: 500 patients undergoing $\mathrm{CT}$ and 500 patients undergoing MRI of spinal column between 11/01/2009 and 1/31/2010 are included. UF reported in the original dictations are separated into 3 groups: scout only (I), scout+diagnostic images (II) and diagnostic images (III) only. In addition to type of imaging and spinal location, locations and organ system of these findings are recorded.

Results: There were 81 UF in CT and 113 in MRI group. In both groups, patients with UF were significantly older than the ones without UF, with a slight female preponderance. Majority of UF in CT were seen in diagnostic images. UF seen only in scout images of CT were exception although these were significant findings. UF distribution in MRI was more even. Equal numbers of UF were seen in groups II and III. Approximately $17 \%$ of UF seen in MRI were present in group I. Conclusion: Scout images of neuroradiologic studies may harbour significant number UF. This is especially true in MRI where $17 \%$ of UF seen only in scout images. Although UF seen solely in scout images of $\mathrm{CT}$ is rare, these are predominantly significant findings.

\section{B-1024 14:45}

Comparison of a 12-channel- with a 32-channel head coil using SNR measurements

C. Vandulek, D. Kaczur, E. Vinczen, I. Repa; Kaposvár/HU

(cvandulek@gmail.com)

Purpose: One of the key attributes characterising the quality of an MRI head coil is the signal-to-noise ratio (SNR). The purpose of this study was to compare a 12-channel- with 32-channel head coil using standardised SNR measurements.

Methods and Materials: The measurements were performed on a $1.5 \mathrm{~T}$ MRI scanner. The SNR measurements were performed on the coils' phantoms and on volunteers. The SNR protocol consisted of axial T1 FSE and T2 FSE measurements. Analysis of the SNR calculations was performed using ROI's positioned in the phantoms and the brain tissue of the volunteers.

Results: The results of the SNR measurements of the phantoms show that the SNR of the 32-channel coil is double that of the 12-channel head coil. However, the SNR of the volunteers showed only a $65 \%$ increase of SNR with the T1 FSE measurement, and a $30 \%$ increase with the T2 FSE on the 32-channel coil. There were no artefacts observed on either measurements of the phantoms and human volunteers.

Conclusion: This study confirmed a difference of the SNR between the 12- and 32-channel head coil. While the phantom measurements showed a twofold increase of SNR between the two coils, this rate was not confirmed on the human volunteers. The results of this study amplify the advantage of using the 32-channel coil for neuroradiological examinations (e.g. fMRI, MRS), whereas the 12-channel coil is sufficient for routine brain examinations.

\section{B-1025 14:54}

Psychological effect of chronic exposure to high magnetic field on MRI technologists

N.M. Mishah, W. Hamed; Jeddah/SA (nabeel747@hotmail.com)

Purpose: The purpose of this study is to measure the incident of depression among MRI technologists since they are chronically exposed to high magnetic fields that may carry occupational hazard on central nervous system and on their brain chemicals. This may cause a long-term psychological side effects such as depression. Methods and Materials: The data and the statistical information of this project were based on BECK Depression Inventory BDI, 1996 version which is used by healthcare professionals as depression screening tool. The questionnaire of depression screening tool was delivered to all MRI technologists working in major hospitals within large cities in Makkah region. The total completed questionnaire received was 45 and only 40 was accepted with a rejection rate of $11.11 \%$. The received data were analysed using a computer-aided statistical tool then the percentage of depression found among MRI technologists included in our study will be compared to previous study conducted on dentists in USA and among community population in Saudi Arabia.
Results: The percentage of depression among MRI technologists included in our sample was found to be $25 \%$ which is considered to be much more than the percentage (10\%) found among the dentists in USA $(6.5 \%)$ and among community population in Saudi Arabia.

Conclusion: It was found that there is a strong relationship between the incident of depression among MRI technologists and the incident is higher among technologists working for more than 5 years.

\section{B-1026 15:03}

Development and implementation of a synthetic data evaluation scenario for image fusion algorithms based on discrete wavelet transform and principal component analysis

V. Weiss; Wiener Neustadt/AT (volker.weiss@hotmail.com)

Purpose: Successful image fusion reduces the amount of data without significantly reducing the amount of relevant information. This study aims to answer the question whether the image fusion results were calculated successfully and what successful image fusion actually means.

Methods and Materials: The study presents two new approaches for the quantitative evaluation of image fusion schemes. It provides the development and implementation of a synthetic data evaluation scenario for image fusion algorithms based on discrete wavelet transform (DWT) and principal component analysis (PCA). Furthermore, it presents a medical application visualising and comparing fused axial CT slices, reconstructed from a CT volume data set with identical position but calculated with different convolution kernels. The study provides a ground truth data-based performance evaluation, as well as a comparison of the DWT and PCA to each other by computing and visualising significant differences in their performance.

Results: In the present study, the DWT, compared to the PCA, turns out to be the more powerful and therefore more suitable image fusion method, both qualitatively and quantitatively.

Conclusion: According to estimates of certain surveyed medical specialists, to whom the results have been shown, a serious diagnosis solely on the basis of fused images is conceivable. The fused images would also be conceivable for documentation purposes and printouts for the referring physicians. Finally, storing fused image series instead of individual series would reduce the amount of memory required in long-term archives by half.

\section{B-1027 15:12}

Image registration and fusion of CT and micro-CT of a sheep's cochlea S. Leitner; Berndorf/AT

Purpose: The sheep's cochlea seems to be most similar to human ones. Hence, it is of great interest in the fields of developing cochlea implants and improving operation techniques of electrode implantation in ENT- (ear-nose-throat) surgery. Methods and Materials: For investigating the sheep as a suitable large animal model, data sets of high resolution micro-CT and CT of its cochlea were acquired. The research on inner-ear diseases and ENT-surgery methods require image processing methods, such as image registration and image fusion. In this thesis, the micro-CT data set was registered with the CT data set in a common coordinate system using two different software packages.

Results: The registration was successful with both types of software. In order to evaluate the results visually, the fused multimodal data were represented by means of colour overlay as well as 3D rendering techniques.

Conclusion: The basis of image-registration of these two modalities was laid and serves as comparison between the image information of the high resolution micro$\mathrm{CT}$ and the conventional CT in order to overcome an interpretation gap between these two modalities. The appropriate $3 \mathrm{D}$ visualisation of the vestibule-cochlea system by means of the highest resolution image devices and medical image processing methods support fundamental research on macro-and micro-mechanical processes of hearing and basic understanding for developing therapeutic and preventive methods for ear diseases. 


\section{B-1028 15:21}

Evaluation of image quality optimisation using VGC and ordinal regression analysis

F. Zarb 1 , M.F. McEntee ${ }^{2}$, S. Foley ${ }^{3}$, L. Rainford ${ }^{3} ;{ }^{1} M s i d a / M T,{ }^{2} S y d n e y / A U$,

${ }^{3}$ Dublin/IE (francis.zarb@um.edu.mt)

Purpose: To evaluate observer performance involving absolute visual grading analysis of image criteria using visual grading characteristic curves (VGC) and ordinal regression analysis during CT examination optimisation.

Methods and Materials: Images obtained from current and optimised head CT protocols $(n=120)$ from 4 suites were presented using ViewDex on Advantage Workstations for evaluation by radiologists $(n=6)$, grading the visibility of anatomical structures sourced from the European guidelines on quality criteria for CT.

Results: VGC analysis in terms of the area under the curve showed no significant differences ( $p>0.05$ ) from the 0.5 threshold value, indicating similar image quality between data sets. Ordinal regression analysis indicated no significant $(p>0.05)$ change in image quality in suites 1 and 3 but discriminated between the data sets for suite 2 and $4(p<0.05)$. Analysis on individual criteria indicated a difference $(p<0.05)$ in image quality between the protocols for all criteria in suite 2 but no difference ( $p>0.05)$ in suite 4. Discussion: VGC curves demonstrated general trends between protocols. Findings of ordinal regression analysis not only indicate significant differences in image quality but also identify specific anatomical criteria contributing to those differences. The importance of selecting the most appropriate method of analysis in optimisation techniques is highlighted as findings influence protocol implementation based on clinical requirements.

Conclusion: An overall 15\% radiation dose saving was achieved as a result of optimisation of the head CT protocols. Optimised protocols were implemented for initial diagnosis for three suites and only for follow-up cases excluding initial diagnosis for the remaining suite.

\section{4:00 - 15:30} Room Z

\section{Contrast Media}

\section{SS 1806}

\section{Functional studies and safety}

Moderators:

T. Gleeson; Wexford/UK

E.M. Merkle; Basle/CH

\section{B-1029 14:00}

Measuring hepatic functional reserve using low temporal resolution Gd-EOB-DTPA dynamic contrast-enhanced MRI: a comparison study with galactosyl-human serum albumin scintigraphy and indocyanine green retention

K. Saito', J.R. Ledsam², S.P. Sourbron'2, T. Hashimoto' ${ }^{1}$, Y. Araki', S. Akata ${ }^{1}$ K. Tokuuye'; ${ }^{1}$ Tokyo/JP, ${ }^{2}$ Leeds/UK (um07jrl@leeds.ac.uk)

Purpose: To investigate if tracer kinetic modelling of low temporal resolution dynamic contrast-enhanced (DCE) MRI with gadoxetic acid (Gd-EOB-DTPA) has the potential to replace technetium $99 \mathrm{~m}$ galactosyl human serum albumin (GSA) scintigraphy and indocyanine green (ICG) retention for the measurement of liver functional reserve.

Methods and Methods: 28 patients (18 males, 10 females; mean age 67y) awaiting liver resection for various cancers underwent DCEMRI, GSA-scintigraphy and ICG as part of routine pre-operative evaluation. Examinations were conducted within a single month. Underlying liver disease was present in 4 patients. The Gd-EOBDTPA MRI sequence acquired 5 images: pre-contrast, double arterial phase, portal phase, and 4 minutes after injection. GSA-scintigraphy images were acquired every 10 s following contrast injection. Contrast uptake rate (UR) and extracellular volume (Ve) were calculated from DCE-MRI, and the ratio of GSA radioactivity of liver to heart-plus-liver and percent of contrast uptake at 15 minutes (LHL15 and LU15, respectively) from GSA-scintigraphy. ICG retention at 15 minutes, Child-Pugh cirrhosis score (CPS) and post-operative Inuyama fibrosis biopsy criteria were also recorded. Statistical analysis was with SPSS17.

Results: Significant correlations were obtained for: UR and LHL15, LU15 and ICG15 (all 0.4-0.6, p < 0.05); UR and CPS $(-0.642, \mathrm{p}<0.001)$; Ve and Inuyama (0.440, $p=0.041$ ); ICG15 and UR, LHL15, LU15, CPS and Inuyama (all 0.4-0.6, $p$ $<0.05)$; LU15/LHL15 with Inuyama $(-0.65, p=0.001)$ and CPS $(-0.492$ and -0.618 , respectively, $\mathrm{p}<0.01$ ).

Conclusion: Measures of liver function obtained by routine Gd-EOB-DTPA DCEMRI with tracer kinetic modelling may provide a suitable alternative for evaluation of liver functional reserve.
B-1030 14:09

Dynamic enhancement pattern of Gd-EOB-DTPA compared to gadobutrol in patients with $\mathrm{HCC}$

S. Kinner, C. Kloeters, L. Umutlu, N. Sippel, T.C. Lauenstein; Essen/DE (Sonja.Kinner@uni-due.de)

Purpose: Gd-EOB-DTPA as liver-specific contrast agents comprises high r1 and r2 values and seems to be well suited for dynamic MR imaging in addition to liverspecific characteristics. Aim of this study was to compare Gd-EOB-DTPA to the extracellular contrast compound gadobutrol at dynamic abdominal MRI.

Methods and Materials: Dynamic contrast-enhanced liver imaging was performed in 42 patients with $\mathrm{HCC}$ with a T1-weighed volume interpolated breath-hold examination. Gd-EOB-DTPA $(0.25 \mathrm{mmol} / \mathrm{ml})$ and gadobutrol $(1 \mathrm{mmol} / \mathrm{ml})$ were injected body weight adapted using care bolus technique and a power injector $(2 \mathrm{ml} / \mathrm{s})$ on two consecutive days. SNR values of abdominal vessels and CNR values of HCC lesions during arterial phase were evaluated. For statistical evaluation a Wilcoxonsigned-rank-test was used.

Results: All MR images were of diagnostic image quality. SNR of aorta in gadobutrol-enhanced images amounted to $106 \pm 15$, with Gd-EOB-DTPA to $77 \pm 14(p=$ 0.12 ). The portal vein (pv) and vena cava (vc) exhibited similar SNR values in the portalvenous/venous contrast phase (pv: SNR gadobutrol $=68 \pm 6$, SNR Gd-EOBDTPA $=63 \pm 3 ; p=0.6 ; v c:$ SNR gadobutrol $=59 \pm 4$, SNR Gd-EOB-DTPA $=52 \pm 3 ; p=$ 0.64). CNR values of $\mathrm{HCC}$ lesions amounted to $27 \pm 4$ for Gd-EOB-DTPA, whereas with gadobutrol $C N R=41 \pm 6(p=0.14)$.

Conclusion: Despite the higher $r 1$ value of Gd-EOB-DTPA, SNR and CNR values for gadobutrol were higher. This might be due to the higher gadolinium concentration. To achieve best image quality, the agent used in liver MR should be adapted to the clinical question: Gadobutrol should be used for vascular analysis, whereas Gd-EOB-DTPA should be used for lesion characterisation.

\section{B-1031 14:18}

Gadoxetic acid-enhanced hepatobiliary phase MRI and high b-value diffusion-weighted imaging (DWI) in the differential diagnosis between benign to malignant liver lesions

P. Arcuri, G. Fodero, S. Roccia, S. Molica, V. Arcuri; Catanzaro/IT (arppaolo@alice.it)

Purpose: The aim of our experience was to evaluate the value of gadoxetic acidenhanced hepatobiliary phase imaging and high b-value DWI in the differential diagnosis between benign and malignant liver lesions.

Methods and Materials: Twenty-four malignant liver lesions (fourteen small HCC and ten metastatic lesions) and twelve benign lesions (five $\mathrm{FNH}$, four adenomas and three regenerative nodules in cirrhotic liver) were studied. A retrospective evaluation was made. The qualitative analysis was obtained by the evaluation of two radiologists of the lesions's signal intensity compared with that of the surrounding liver parenchyma on hepatobiliary phase images and DWI (b-value=600s $/ \mathrm{mm} 2$ ). The contrast-to-noise ratio (CNR) and relative contrast enhancement of lesions on hepatobiliary phase images and the apparent diffusion coefficient (ADC) values were assessed (by ROIs) for quantitative analysis. Statistical analysis was performed by the Mann-Whitney $U$ test. $P$ value less than 0.05 was considered statistically significant.

Results: In the qualitative analysis, nineteen malignant lesions (79\%) and three benign lesions $(25 \%)$ were hypointense on hepatobiliary phase images; five malignant lesions $(21 \%)$ and nine benign lesions $(75 \%)$ were iso- or hyperintense in the same phase. Twenty malignant lesions ( $83 \%$ ) and four benign lesions (33\%) were hyperintense in DWl; four malignant lesions (17\%) and nine benign lesions $(75 \%)$ were iso-hypointense in DWI. In the quantitative analysis the mean relative contrast enhancement ratio of the malignant lesions was higher than that of benign lesions. Conclusion: Hypointensity on gadoxetic acid-enhanced hepatobiliary phase images and hyperintensity on DWI ( $b$-factor=600 s/mm2) suggest malignant lesions rather than benign hepatocellular nodules.

\section{B-1032 14:27}

Gadoxetic acid-enhanced MRI of the liver: correlation between Gadoxetic acid uptake and serum hepatic enzymes levels E. Talakic, H. Schoellnast, T. Wimmer, J. Steiner, F. Quehenberger, U. Reiter; Graz/AT

Purpose: To assess the correlation between enhancement of the liver during the dynamic phases and the hepatobiliary phase and serum hepatic enzyme levels in Gadoxetic acid-enhanced MRI of the liver.

Methods and Materials: 75 patients who underwent gadoxetic acid-enhanced MRI of the liver were enrolled. Images were obtained before contrast injection, during the 
arterial phase, portal venous phase, hepatovenous phase, equilibrium phase, and hepatobiliary phase which was 20 minutes after Gadoxetic administration. A test bolus was used to optimise the contrast injection protocol. Signal intensity of the liver parenchyma in all phases was defined using region-of-interest $(\mathrm{ROI})$ measurements for enhancement calculation. Serum hepatic enzyme levels (bilirubin; cholinesterase, $\mathrm{CHE}$; aspartat-aminotransferase, AST; alanine-aminotransferase, ALT; gamma-glutamyl-transpeptidase, GGT; alkaline phosphatase, AP) were available in all patients. Spearman correlation test was used to test the correlation between liver enhancement during the different phases and serum hepatic enzyme levels. Results: At late time points after contrast injection all serum hepatic enzymes were correlated with liver enhancement. Bilirubin and $\mathrm{CHE}$ showed stronger correlations than the other serum hepatic enzymes and they became correlated at earlier time points. The strongest correlation was observed between bilirubin and enhancement during the hepatobiliary phase and between $\mathrm{CHE}$ and enhancement during the hepatobiliary phase. Bilirubin showed negative and $\mathrm{CHE}$ showed positive correlation. The Spearman correlation coefficient was -0.64 for bilirubin and 0.61 for $\mathrm{CHE}$. Conclusion: In gadoxetic acid-enhanced MRI of the liver gadoxetic uptake during the hepatobiliary phase is strongest correlated with bilirubin and $\mathrm{CHE}$ serum levels, whereas the other serum hepatic enzymes show weak or moderate correlations.

\section{B-1033 14:36}

MRCP using hepatospecific contrast media (GD-EOB-DTPA) in biliary leaks

R. Faletti, M. Fronda, F. Barisone, C. Bogetti, A. Dominguez, A. Rapellino,

L. Marchisio, P. Fonio, G. Gandini; Turin/IT

Purpose: Aim of the study is to evaluate the use of MRCP using hepatospecific contrast media (GD-EOB-DTBA) to confirm biliary leak suspect and treatment planning. Methods and Materials: 13 consecutive patients ( 7 males, 6 females - average age 64 years), during 13 months (April 2011 to May 2012) underwent MRCP study using GD-EOB-DTBA due to clinical suspect of biliary leak. Using an 1.5 T MRI scanner, T1-weighted 3D GRE pulse sequences before and in hepatospecific phase, after GD-EOB-DTBA injection, were performed. All patients with biliary leak underwent endoscopic, interventional radiology (IR) or surgical approach for treatment.

Results: Biliary leak was clinically suspected in 13 patients and underwent abdominal surgery (7 laparoscopic cholecystectomy, 5 various hepatic resections, 1 resection of a pancreatic-duodenal mass) after 33 days ( $\mathrm{AV}$; range 4 to 107). In 11/13 biliary leak was found at 44 minutes (AV; range 20 to 76 minutes) from GD-EOB-DTBA injection, from intrahepatic ducts (6/11) site, cystic duct stump $(2 / 11)$, both intrahepatic and principal biliary duct $(2 / 11)$ and from a biliodigestive anastomosis (1/11). Biliary leak was confirmed and successfully treated by percutaneous transhepatic cholangiography (8/11), endoscopic approach (1/11), IR and endoscopic "rendez-vouz" technique (1/11) and by surgical laparotomy (1/11). In 2/13 patients, no biliary leak was found and only clinical monitoring was performed, with good outcome. Any adverse reaction or technical problem occurred during the MRCP examination.

Conclusion: MRCP with GD-EOB-DTBA is an high sensitivity, safe and fast examination to confirm the suspect and to locate precisely a biliary leak, concurring best planning for treatment.

\section{B-1034 14:45}

$\mathrm{N}$-acetylcysteine for the prevention of contrast-induced nephropathy in rats


${ }^{2}$ Sivas/TR, ${ }^{3}$ Kayseri/TR (drfatihinci@gmail.com)

Purpose: To date, there is no effective treatment of contrast-induced nephropathy (CIN). N-acetylcystein (NAC) has yielded some promising results recently in the prevention of CIN. In this study, the structural effects of NAC on CIN were analysed. Methods and Materials: Forty adult Wistar albino male rats were randomly allocated to four groups. The first group was the control group $(n=9)$ which received only distilled water; second group was the contrast group $(n=10)$ which received $\mathrm{CM}$; the third group was the contrast plus NAC group $(n=8)$ which received CM and was treated with NAC; and the last group was NAC group $(n=10)$ which received only NAC. At the end of the $3^{\text {rd }}$ day, the right and left kidneys were removed and reserved for histopathological examination. All tissue sections were examined with light microscope looking for histopathological changes by the same experienced pathologist, without knowledge of the prior treatment. Histopathological examination was conducted in a blinded fashion, and glomerular injury scores, arteriolar injury scores and tubulointerstitial injury scores were calculated.

Results: There was a significant difference among the scores of glomerular injury, arteriolar injury and tubulointerstitial injury in all groups $(p<0.05)$. The scores of glomerular, arteriolar and tubulointerstitial injury of the group-1 and group- 4 were not significantly different from each other $(p<0.05)$. Renal injury scores in group-3 group were higher than in group-1 and-4, but significantly lower than the scores of the group-2 $(p<0.05)$.

Conclusion: NAC could be useful to prevent the renal tissue from CIN, especially in high-risk patients.

\section{B-1035 14:54}

Evaluation of enhancement properties of gadolinium-labelled nanoparticles for contrast-enhanced MRI in rats with experimental liver tumours at $9.4 \mathrm{~T}$

P. Fries ${ }^{1}$, D. Morr ${ }^{1}$, A. Müller ${ }^{1}$, F. Lux², O. Tillement ${ }^{2}$, J. Palm¹, G. Schneider ${ }^{1}$,

R. Seidel ${ }^{1}$, A. Buecker ${ }^{1} ;{ }^{1}$ Homburg/DE, ${ }^{2}$ Lyon/FR (drpeterfries@googlemail.com)

Purpose: To compare a Gd-labelled nanoparticle (AGuIX) with a standard extracellular Gd-based contrast agent (Gd-DOTA) for dynamic contrast-enhanced MRI at 9.4T in rats with hepatic colorectal cancer metastases.

Methods and Materials: 10 rats with hepatic colorectal cancer metastases were subjected to MRI (9.4T, Bruker, Germany) acquiring T1w retrospectively self-gated FLASH sequences $\left(\mathrm{TR} / \mathrm{TE}=45 / 2.5 \mathrm{~ms}, \mathrm{FA}=45^{\circ}, \mathrm{TA}=1: 23 \mathrm{~min}, \mathrm{FOV}=5.12 \times 5.12\right.$ $\mathrm{cm} 2$, matrix $=256 \times 256$ ) before and at 10 consecutive time points after contrast injection. First, each animal received $0.1 \mathrm{mmol} / \mathrm{kg}$ BW Gd-DOTA intravenously. Two days later, AGulX was applied at $0.01 \mathrm{mmol} / \mathrm{kg} \mathrm{BW}$ (representing equal Gd-doses). SNR of normal liver tissue (SNRliver) and tumour (SNRtumour), CNR and lesion enhancement (LE) were calculated based on ROI measurements. Statistical analyses included paired t-tests and Wilcoxon matched-pairs signed-rank tests $(p<0.05)$. Histopathologic evaluation confirmed the presence of metastases in all animals. Results: Mean SNRliver ( \pm SD): Gd-DOTA $14.5( \pm 0.7) / A G u l X 23.3( \pm 1.7)$, SNRtumour: Gd-DOTA $18.0( \pm 1.1) / A G u l X 30.60( \pm 2.9)$, CNR: Gd-DOTA $3.5( \pm 0.5) /$ AGulX $6.7( \pm 1.2)$ and LE: Gd-DOTA $3.0( \pm 0.6) /$ AGulX $15.1( \pm 2.9)$ were each significantly higher for AGulX for all time points and groups $(p=0.001-0.04)$. Both compounds demonstrated comparable kinetics with an early peak followed by a washout without plateau.

Conclusion: The Gd-labelled nanoparticle provides higher SNR, CNR and LE at 9.4T as compared to the standard extracellular compound for equal doses of applied Gd. This is most likely based on the complex structure of AGulX and the subsequent increased interaction with protons leading to a higher relaxivity. Despite the increased molecular size, AGulX shows perfusion-kinetics comparable to the extracellular agent during the first 20 min after application.

Author Disclosures:

A. Buecker: Research/Grant Support; German Ministry of Education and Research (Grant Number: 0314101).

\section{B-1036 15:03}

Regorafenib monotherapy effects on experimental human colon carcinoma xenografts monitored by DCE-CT with immunohistochemical validation

P.M. Kazmierczak, H. Hirner, L. Havla, M. Schneider, O. Dietrich, C. Bruns, M.F. Reiser, K. Nikolaou, C.C. Cyran; Munich/DE (Philipp.Kazmierczak@med.uni-muenchen.de)

Purpose: To investigate dynamic contrast-enhanced computed tomography (DCECT) for monitoring the effects of regorafenib on experimental colon carcinomas in rats by quantitative assessments of tumour microcirculation parameters with immunohistochemical validation.

Methods and Materials: Colon carcinoma xenografts (HT-29) implanted subcutaneously in female athymic rats $(n=25)$ were imaged at baseline and after a one-week treatment with regorafenib by DCE-CT (128 slice dual source CT). The therapy group $(n=13)$ received regorafenib daily $(10 \mathrm{mg} / \mathrm{kg}$ bodyweight) via gavage. Quantitative parameters of tumour microcirculation (plasma flow, $\mathrm{mL} / 100 \mathrm{~mL} / \mathrm{min}$ ), endothelial permeability (PS, $\mathrm{mL} / 100 \mathrm{~mL} / \mathrm{min}$ ), and tumour vascularity (plasma volume, \%) were calculated using a 2-compartment uptake model. DCE-CT parameters were validated with immunohistochemical assessments of tumour microvascular density (CD-31), tumour cell apoptosis (TUNEL), and proliferation (Ki-67).

Results: Regorafenib significantly $(\mathrm{p}<0.05)$ suppressed tumour perfusion $(12.8 \pm 2.3$ to $8.8 \pm 2.9 \mathrm{ml} / 100 \mathrm{ml} / \mathrm{min})$ and tumour vascularity $(15.7 \pm 5.3$ to $5.5 \pm 3.5$ $\mathrm{ml} / 100 \mathrm{ml} / \mathrm{min}$ ). Significantly lower microvascular density was observed in the therapy group (CD-31; $48 \pm 10$ vs. $113 \pm 25, p<0.05)$. In regorafenib-treated tumors, significantly more apoptotic cells (TUNEL; $11,844 \pm 2,927$ vs. $5,097 \pm 3,463, p<0.05$ ) and significantly less proliferating cells (Ki-67; 4,277 \pm 1017 vs. $4,841 \pm 1,593$, $p<$ 0.05 ) were observed. DCE-CT tumour perfusion and tumour vascularity correlated significantly $(p<0.05)$ with microvascular density $(C D-31 ; r=0.84$ and 0.66$)$ and inversely with apoptosis (TUNEL; $r=-0.66$ and -0.71 ). 
Conclusion: Regorafenib significantly suppressed tumour perfusion and vascularity quantified by DCE-CT in experimental colon carcinomas in rats with good to moderate correlations to an immunohistochemical gold standard. Tumour response biomarkers assessed by DCE-CT might be a promising future approach to a more personalised and targeted cancer therapy.

Author Disclosures:

K. Nikolaou: Research/Grant Support; Bayer Healthcare, Germany.

C.C. Cyran: Research/Grant Support; Bayer Healthcare, Germany.

B-1037 15:12

Contrast-induced nephropathy in patients undergoing intravenous contrast-enhanced computed tomography and the relationship with risk factors: a meta-analysis

S.I. Moos, J. Stoker, S. Bipat; Amsterdam/NL (s.i.moos@amc.uva.nl)

Purpose: To summarise the incidence of contrast-induced nephropathy (CIN) and to study associations between $\mathrm{CIN}$ and risk factors in patients undergoing intravenous contrast-enhanced computed tomography (CECT).

Methods and Materials: We searched the MEDLINE, EMBASE and Cochrane databases from 2002 till July 2012. Two reviewers checked inclusion criteria and extracted data. Mean CIN incidence and associations between risk factors and CIN were pooled by random-effect approach.

Results: Twenty-three articles with 20,000 patients (mean age 64.5 years, mean eGFR at baseline $55.17 \mathrm{~mL} / \mathrm{min}$ ) were included. CIN was defined as absolute or relative $(44.2 \mu \mathrm{mol} / \mathrm{L} />25 \%)$ serum creatinine increase, mostly within 72 hours. All studies included a high proportion of patients with risk factors; chronic kidney disease (CKD) in $52 \%$ of all patients, diabetes mellitus (DM) in $14 \%$, hypertension (HT) in $54 \%$ and congestive heart failure (CHF) in $21 \%$. The mean incidence of CIN was $3.7 \%(95 \% \mathrm{Cl}: 2.5 \%-5.6 \%)$. Data analysis showed an increased risk for $\mathrm{CIN}$ in the presence of DM ((odds ratio $1.73(95 \% \mathrm{Cl}: 1.07-2.82), \mathrm{p}=0.03)$ ) and CKD (2.52 (95\% Cl: 1.56-4.08), $\mathrm{p}=0.0002)$. HT and CHF were not associated with an increased risk $(p=0.07, p=0.19)$.

Conclusion: The mean incidence of CIN after CECT was $3.7 \%$ and is associated with DM and CKD. The incidence of CIN is mostly reported in studies with a high proportion of patients with risk factors. Therefore, the reported CIN incidence and associated risk factors for CECT might be overestimated compared to the CIN incidence in a general population undergoing CECT.

\section{B-1038 15:21}

Renal MR angiography at 7 Tesla: how much gadolinium do we need?

L. Umutlu, A. Fischer, M. Forsting, M.E. Ladd, S. Maderwald, O. Kraff,

T.C. Lauenstein; Essen/DE (Lale.Umutlu@uk-essen.de)

Purpose: To determine whether contrast agent (CA) dose reduction to one-half and one-quarter of the standardised dosage allows for preserved image quality of renal MR angiography at $7 \mathrm{~T}$.

Methods and Materials: MR examinations were performed in 6 healthy subjects on a 7T MR system (Magnetom 7T), utilising a custom-built 8-channel RF body coil. Dynamic 3D FLASH data sets were obtained pre contrast and 20sec after the application of contrast agent. Examinations were performed at three different time points for injection of three dosages of CA (Gadobutrol, Bayer Healthcare): (1) $0.1 \mathrm{mmol} / \mathrm{kg}$ body weight (BW), (2) $0.05 \mathrm{mmol} / \mathrm{kg} \mathrm{BW}$ and (3) $0.025 \mathrm{mmol} / \mathrm{kg} \mathrm{BW}$. Contrast ratios (CR) were measured pre and post contrast in the aorta and both renal arteries in correlation to adjacent psoas major muscle. Qualitative analysis with regard to delineation of the pre-contrast and post-contrast renal arterial vasculature was performed by two radiologists using a five-point-scale ( $5=$ =xcellent to $1=$ non diagnostic).

Results: Non-enhanced T1w MRI provided an inherently high signal intensity of vasculature, yielding a good overall pre-contrast arterial delineation (mean 3.65). The application of contrast agent showed improved vessel delineation in qualitative and quantitative analysis for all three dosages, yielding comparable results in subjective ratings of qualitative analysis (mean0.025Gd 4.55; mean0.05Gd 4.50; mean0.1Gd mean 4.60). Quantitative analysis of contrast ratios showed only minor increase of mean values with increasing Gadolinium dosage (mean0.025Gd 0.08; mean0.05Gd 0.15; mean0.1Gd mean 0.18).

Conclusion: 7T CE-MRA allows for a significant dose reduction to one-quarter while maintaining high image quality. 